2. To: (Receiving organization) Central Files

5. Proj./Prog./Dept./Div.:

SNF Project $\backslash W-442$

8. Originator Remarks:

The attached document is submitted for release.

Pasen 1.276 NCNP $4 \mathrm{~A}$

Pases 277- 372 ANSYS 5.0A, END LHG 7/2/97

11. Receiver Remarks: 11A. Design Baseline Document? [] Yes [X] No
3. From: (Originating organization) SNF Storage Projects

6. Design Authority/ Design Agent/Cog. Engr.:

L. H. Goldmann

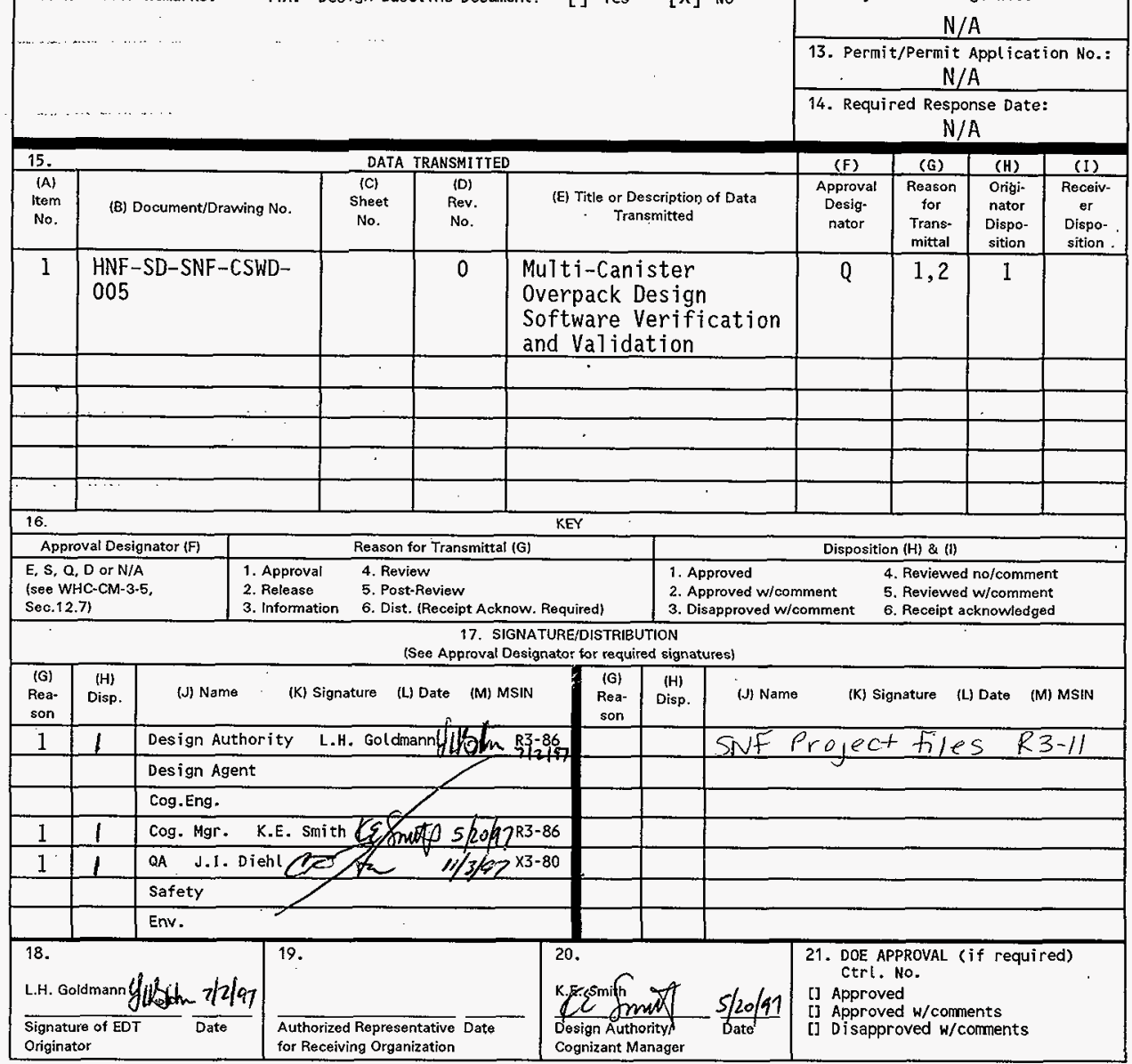




\title{
MULTI-CANISTER OVERPACK PROJECT - VERIFICATION AND VALIDATION, MCNP 4A
}

\author{
L. H. Goldmann
}

DE\&S HANFORD, INC., RichT and, WA 99352

U.S. Department of Energy Contract DE-AC06-96RL13200

EDT/ECN: 620109

Org Code: 2T340

UC: 600

B\&R Code: EW7040000

Charge Code: LE004

Tota1 Pages: 374

Key Words: Multi-Canister Overpack, Software, Validation, Spent Fuel, Ansys, MCNP

Abstract: This supporting document contains the software verification and validation (V\&V) package used for Phase 2 design of the Spent Nuclear Fuel MuIti-Canister Overpack. V\&V packages for both ANSYS and MCNP are included.

TRADEMARK DISCLAIMER. Reference herein to any specific comercial product, process, or service by trade name, trademark, manufacturer, or otherwise, does not necessarily constitute or imply its endorsement, recommendation, or favoring by the United States Government or any agency thereof or

its contractors or subcontractors.

Printed in the United States of America. To obtain copies of this document, contact: Document Control Services, P.O. Box 950, Mailstop H6-08, Richland WA 99352, Phone (509) 372-2420;

Fax (509) 376-4989.
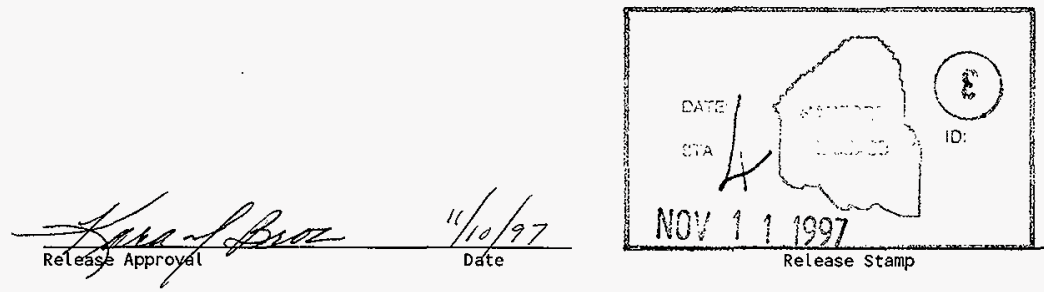
HNF-SD-SNF-CSWD-005, Rev. 0

\section{MULTI-CANISTER OVERPACK PROJECT}

\section{VERIFICATION AND VALIDATION \\ MCNP 4A}




\section{Parsons Engineering Science \\ AVLIS Program, Lawrence Livermore National Laboratory \\ Safety Engineering and Analysis Support Services, Group B Task \\ USEC Contract No. USEC-96-C-0012}

Form $1 \mathrm{~A}$

Computer Software Verification Record

Verification \# PD-A-VV-003

Software Title: MCNP 4A : A Monte Carlo N Particle Transport Code

Release Version: 4A Release Date: November 1993

Software Developed By: Los Alamos National Lab

Computer System ID (PC CPU Serial Number): 501981-801 056AVO000189 (DOE 6246264)

Additional Computer ID : 501981-801 056AVO000238 (DOE 6246271)

Additional Computer ID : 501981-801 056AVO000041 (DOE 6239600)

Additional Computer ID : $501981-801$ 056AVO000088 (DOE 6246288)

Computer System

Processor: 586 (Pentium)

Clock Speed: 166

(MHZ)

Memory: $32 \quad \mathrm{MB} \quad$ Hard Drive Capacity: 1,600

Operating System: Windows 95

Language/compiler: Lahey Fortran 77

Computer System ID (PC CPU Serial Number): 0012358

Computer System Processor: 586(Pentium) $\quad$ Clock Speed: 100

Memory: 48 MB $\quad$ Hard Drive Capacity: 1,080

Operating System: Windows 95

Language/compiler: Lahey Fortran 77

Description of Verification Run(s): This software requires that it be compiled specifically for the machine it is to be used on. Iterefore to facilitate ease in the verification process the software automatically runs 25 sample problems to ensure proper installation and compilation. Once the runs are completed the software checks for verification by performing a file comparison on the new output file and the old output file. Any differences between any of the files will cause a verification error. Due to the manner in which the verification is completed (by file comparison) a verification error does not necessarily indicate a problem. this indicates that a closer look at the output files is needed to determine the cause of the error(s).

Input File Name: See MCNP-4A documentation

Output File Name: See MCNP-4A documentation

Disk Name and Path: C:IMCNP 4A or C:IMCNP (this is computer specific)

Results of Verification Run: The verification runs produced a verification error. This occurred on all 5 systems that MCNP was installed. Upon further investigation of all 5 systems, 4 of the 25 sample problems had output files that differed from those supplied. However, these problems do not invalidate the verification muns. Using the file comparison method for verification is a good beginning, but will result in errors if any additional output is requested, thereby moving the positions of the rows to be compared causing errors even when the final results are exactly the same. This was the case with the runs that were found to have problems. It appears that additional output was requested by the code developers after the sample problems were already created. In order to confirm this the final results of these four cases were compared and no difference was found. Therefore MCNP-4A is verified and may be used as needed. Attachment $A$ has the file comparison results. Attachment $B$ has the full output of those files that showed any discrepancy

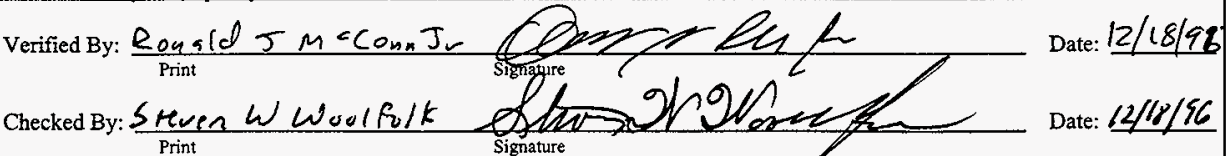

(Page 1 of 423) 


\section{Parsons Engineering Science \\ AVLIS Program, Lawrence Livermore National Laboratory \\ USEC Contract No. USEC-96-C-0012 \\ Form 1B (Continued) \\ Computer Software Validation Record}

Safety Engineering and Analysis Support Services, Group B Task

Software Model Validation (cont.):

RSIC Newsletter of October 22, 1993 (see Section 3 of the MCNP manual [CCC-200]). "The last version of MCNP teleased to RSIC. MCNP 4.2. Was so robust that in over two years of use by over a thousand users at hundreds of institutions around the world no important errors were found. MCNP 4A raises the standard of quality even higher. The code can be made to track MCNP 4.2 identically (except for MCNP 4.2 bugs). indicating that no new bugs have been added (new DBCN(20) option). The test set has been upgraded and the installation procedure has been tightened further, and include END/B-IV and ENDL data. These has been more testing and benchmarking of MCNP 4A than any prerelease MCNP version in history. The code has undergone eight months of testing with preliminary versions at Los Alamos. Many significant changes from MCNP 4.2 have been documented and can be traced. Most Software Quality Assurance guidelines of IEEE and European ISO-9000 have been applied, including for the first time multiple reviews of all coding.

The MCNP Team continues to pay cash to anyone who can find the slightest flaw in the code. There were 59 such cash awards made for MCNP 4.2."

The neutron transport portions of this software has been validated by comparison with benchmark problems as described in "MCNP: Neutron Benchmark Problems" (LA-72212) which is also included in the RSIC MCNP 4A manual (CCC-200). enchmark problem comparison for neutrons can also be found in "MCNP Neutron Benchmarks," by J.S. Hendricks, D. J. thalen, D. A. Cardon, and J. L. Uhle (see Trans. Am. Nucl. Soc. 63 426 [June 1991]) and "Benchmarking Study of the MCNP Code Against Cold Critical Experiments," by S. Sitaraman (see Trans. Am. Nucl. Soc. 63 426 [June 1991]) both of which are attached to this record.

The photon transport portion of this software has been validated by comparison with benchmark problems as described in:

- J.S. Hendricks and J.F. Briesmeister, "Recent MCNP Developments", IEEE Transactions on Nuclear Science, Vol. 39 No. 4.pg. 1035-1040, 1992.

R. Guaraldi, F. Padoani. K. W. Burn, and G. Gualdrini. "Monte Carlo Applications to Photon Radiation Protection and Dosimetry At ENEA." Progress In Nuclear Energu. Vol 24, pg. 365-376, 1990.

D. J. Whalen, D. E. Hollowell, and J. S. Hendricks, "Monte Carlo Photon Benchmark Problems," Proceedings of the International Topical Meeting on Advances in Mathmematics. Computations, and Reactor Physics, 5, 301. 5-1 (April 1991)

- S. Sitaraman, "Benchmarking Study of the MCNP Code Against Cold Critical Experiments". Transactions of the American Nuclear Society, Vol. 63, pg. 426-429, 1991.

- J.S. Hendircks, D.J. Whalen, D.A. Cardon, J.L. Uhle "MCNP Neutron Benchmarks". Transactions of the American Nuclear Society. Vol.65,pg. 262-263, 1992.

- D. J. Whalen, D. E. Hollowell, and J.S. Hendricks, "MCNP. Photon Benchmark Problems," LA-12196 (September 1991).

Copies of these papers are attached.

The validation of the beta (i.e. electron) transport portion of this software is discussed in "Monte Carlo Applications To Photon Radiation Protection and Dosimetry At ENEA" as discussed above. 
HNF-SD-SNF-CSWD-005, Rev. 0

Attachment A

File Comparison Results 
Atrached are the actual file comparison results from the five computers listed above. These are broken down by system with the files have discrepancies being listed in fully in Attachment B.

\section{Computer System ID : 501981-801 056AVO000189 (DOE 6246264)}

Difm01

Comparing files INP01M and met101

FC: no differences encountered

Difm02

Comparing files INP02M and mct102

FC: no differences encountered

Difm03

Comparing files INP03M and mct103

FC: no differences encountered

\section{Difm04}

Comparing files INP04M and mct104

FC: no differences encountered

\section{Difm05}

Comparing files INP05M and mct105

FC: no differences encountered

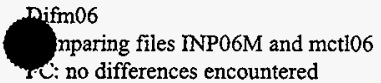

Difm07

Comparing files INP07M and mct107

FC: no differences encountered

Difm08

Comparing files INP08M and mct108

FC: no differences encountered

Difm09

Comparing files INP09M and metl09

FC: no differences encountered

Difm10

Comparing files INP10M and mctl10

FC: no differences encountered

\section{Difm11}

Comparing files INP11M and mctll1

FC: no differences encountered

Difml2

Comparing files INP12M and mot112 


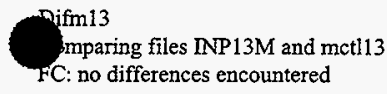

Difm14

Comparing files INP14M and mctl14

FC: no differences encountered

\section{Difm15}

Comparing files INP15M and motlis

FC: no differences encountered

\section{Difm16}

Comparing files INP16M and motl16

FC: no differences encountered

Difm17

Comparing files INP17M and mctl17

FC: no differences encountered

Difm18

Comparing files INP $18 \mathrm{M}$ and metl18

FC: no differences encountered

\section{Difm19}

Comparing files INP19M and met119

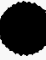

Difm20

File(s) not found : inp $20 \mathrm{~m}$

Difm21

Comparing files INP21M and mct121

FC: no differences encountered

Difm22

Comparing files INP22M and mctl22

FC: no differences encountered

\section{Difm23}

Comparing files INP23M and mctl23 FC: no differences encountered

\section{Difm24}

Comparing files INP24M and mctl24 FC: no differences encountered

\section{Difm25}

Comparing files INP25M and mctl25 FC: no differences encountered

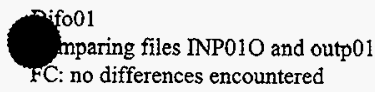


pparing files $\mathrm{INP} 02 \mathrm{O}$ and outp02

c: no differences encountered

Difo03

Comparing files INP03O and outp 03

FC: no differences encountered

Difo04

Comparing files INP040 and outp04

FC: no differences encountered

Difo05

Comparing files INP05O and outp05

FC: no differences encountered

\section{Difo06}

Comparing files INP060 and outp06

FC: no differences encountered

\section{Difo07}

Comparing files INP07O and outp07

FC: no differences encountered

\section{Difo08}

Comparing files INP080 and outp08

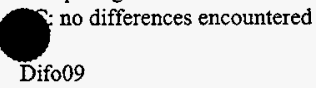

Comparing files INP090 and outp09

FC: no differences encountered

\section{Difo10}

Comparing files INP10O and outp 10 FC: no differences encountered

\section{Difol 1}

Comparing files INP11O and outp11

FC: no differences encountered

\section{Difo12}

Comparing files INP12O and outp12 FC: no differences encountered

\section{Difo13}

Comparing files NNP130 and outp 13

FC: no differences encountered

\section{Difol4}

Comparing files INP14O and outp14 FC: no differences encountered 
Tifol 6

Comparing files INP160 and outp16

FC: no differences encountered

Difol7

Comparing files INP170 and outp17

FC: no differences encountered

Difol8

Comparing files INP18O and outp18

****** INP180

cycle $5 \mathrm{k}$ (collision) 1.135994 removal lifetime(abs) $1.1136 \mathrm{E}+01$ source points generated 222

cycle $6 \mathrm{k}$ (collision) 1.077014 removal lifetime(abs) $1.2222 \mathrm{E}+01$ source points generated 204

****** outp 18

cycle $5 \mathrm{k}$ (collision) 1.135994 removal lifetime(abs) $1.1136 \mathrm{E}+01$ source points generated 222 source distribution written to file inp $18 \mathrm{~s} \quad$ cycle $=5$

cycle $6 \mathrm{k}$ (collision) 1.077014 removal lifetime(abs) $1.2222 \mathrm{E}+01$ source points generated 204

Difo19

Comparing files INP190 and outp19

FC: no differences encountered

\section{Difo20 \\ mparing files INP200 and outp20 \\ **** INP200 \\ ****** outp 20}

$\begin{array}{llll}8 & 0.000 E+00 & 0.000 E+00 & 0.000 E+00\end{array}$

$90.000 \mathrm{E}+00 \quad 0.000 \mathrm{E}+00 \quad 0.000 \mathrm{E}+00$

$10 \quad 0.000 \mathrm{E} \div 00 \quad 0.000 \mathrm{E}+00 \quad 0.000 \mathrm{E}+00$

$110.000 \mathrm{E}+00 \quad 0.000 \mathrm{E}+00 \quad 0.000 \mathrm{E}+00$

$12 \quad 0.000 \mathrm{E}+00 \quad 0.000 \mathrm{E}+00 \quad 0.000 \mathrm{E}+00$

$13 \quad 0.000 \mathrm{E}+00 \quad 0.000 \mathrm{E}+00 \quad 0.000 \mathrm{E}+00$

$14 \quad 0.000 \mathrm{E}+00 \quad 0.000 \mathrm{E}+00 \quad 0.000 \mathrm{E}+00$

$15 \quad 0.000 \mathrm{E}+00 \quad 0.000 \mathrm{E}+00 \quad 0.000 \mathrm{E}+00$

$16 \quad 0.000 \mathrm{E}+00 \quad 0.000 \mathrm{E}+00 \quad 0.000 \mathrm{E}+00$

$17 \quad 0.000 \mathrm{E}+00 \quad 0.000 \mathrm{E}+00 \quad 0.000 \mathrm{E}+00$

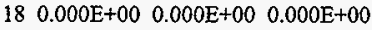

$19 \quad 0.000 \mathrm{E}+00 \quad 0.000 \mathrm{E}+00 \quad 0.000 \mathrm{E}+00$

$20 \quad 0.000 \mathrm{E}+00 \quad 0.000 \mathrm{E}+00 \quad 0.000 \mathrm{E}+00$

$21 \quad 0.000 \mathrm{E}+00 \quad 0.000 \mathrm{E}+00 \quad 0.000 \mathrm{E}+00$

$22 \quad 0.000 \mathrm{E}+00 \quad 0.000 \mathrm{E}+00 \quad 0.000 \mathrm{E}+00$

$\begin{array}{llll}23 & 0.000 \mathrm{E}+00 & 0.000 \mathrm{E}+00 & 0.000 \mathrm{E}+00\end{array}$

$\begin{array}{lllll}24 & 0.000 \mathrm{E}+00 & 0.000 \mathrm{E}+00 & 0.000 \mathrm{E}+00\end{array}$

$\begin{array}{llll}25 & 0.000 \mathrm{E}+00 & 0.000 \mathrm{E}+00 & 0.000 \mathrm{E}+00\end{array}$

$26 \quad 0.000 \mathrm{E}+00 \quad 0.000 \mathrm{E}+00 \quad 0.000 \mathrm{E}+00$

$\begin{array}{llll}27 & 0.000 \mathrm{E}+00 & 0.000 \mathrm{E}+00 & 0.000 \mathrm{E}+00\end{array}$

$\begin{array}{lllll}28 & 0.000 \mathrm{E}+00 & 0.000 \mathrm{E}+00 & 0.000 \mathrm{E}+00\end{array}$

$29 \quad 0.000 \mathrm{E}+00 \quad 0.000 \mathrm{E}+00 \quad 0.000 \mathrm{E}+00$

$30 \quad 0.000 \mathrm{E}+00 \quad 0.000 \mathrm{E}+00 \quad 0.000 \mathrm{E}+00$

$\begin{array}{llll}31 & 0.000 \mathrm{E}+00 & 0.000 \mathrm{E}+00 & 0.000 \mathrm{E}+00\end{array}$

$\begin{array}{lll}32 & 0.000 \mathrm{E}+00 \quad 0.000 \mathrm{E}+00 \quad 0.000 \mathrm{E}+00\end{array}$
$1 \quad 1-3.604 \mathrm{E}-01 \quad 4.799 \mathrm{E}-01 \quad 7.999 \mathrm{E}-01 \quad 1.000 \mathrm{E}+00 \quad 1.000 \mathrm{E}+00 \quad 0.000 \mathrm{E}+00$

$1 \quad 1-2.241 \mathrm{E}-01 \quad 3.960 \mathrm{E}-01 \quad 8.905 \mathrm{E}-01 \quad 1.000 \mathrm{E}+00 \quad 1.000 \mathrm{E}+00 \quad 0.000 \mathrm{E}+00$

I $1-2.928 \mathrm{E}-01-7.352 \mathrm{E}-01 \quad 6.114 \mathrm{E}-01 \quad 1.000 \mathrm{E}+00 \quad 1.000 \mathrm{E}+00 \quad 0.000 \mathrm{E}+00$

$1 \quad 18.260 \mathrm{E}-01 \quad 1.184 \mathrm{E}-01 \quad 5.510 \mathrm{E}-01 \quad 1.000 \mathrm{E}+00 \quad 1.000 \mathrm{E}+00 \quad 0.000 \mathrm{E}+00$

$1 \quad 17.540 \mathrm{E}-01-1.976 \mathrm{E}-01 \quad 6.264 \mathrm{E}-01 \quad 1.000 \mathrm{E}+00 \quad 1.000 \mathrm{E}+00 \quad 0.000 \mathrm{E}+00$

$1 \quad 1 \quad 8.140 \mathrm{E}-01-3.427 \mathrm{E}-01 \quad 4.690 \mathrm{E}-01 \quad 1.000 \mathrm{E}+00 \quad 1.000 \mathrm{E}+00 \quad 0.000 \mathrm{E}+00$

$1 \quad 1-1.257 \mathrm{E}-01 \quad 8.038 \mathrm{E}-01 \quad 5.814 \mathrm{E}-01 \quad 1.000 \mathrm{E} \div 00 \quad 1.000 \mathrm{E}+00 \quad 0.000 \mathrm{E}+00$

$1 \quad 1-1.464 \mathrm{E}-01-1.070 \mathrm{E}-01 \quad 9.834 \mathrm{E}-01 \quad 1.000 \mathrm{E}+00 \quad 1.000 \mathrm{E}+00 \quad 0.000 \mathrm{E}+00$

$1 \quad 1 \quad 1.310 \mathrm{E}-02-1.617 \mathrm{E}-02 \quad 9.998 \mathrm{E}-01 \quad 1.000 \mathrm{E}+00 \quad 1.000 \mathrm{E}+00 \quad 0.000 \mathrm{E}+00$

$1 \quad 1-8.843 \mathrm{E}-01-4.126 \mathrm{E}-01 \quad 2.187 \mathrm{E}-01 \quad 1.000 \mathrm{E}+00 \quad 1.000 \mathrm{E}+00 \quad 0.000 \mathrm{E}+00$

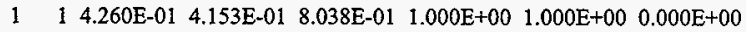

$1 \quad 1-7.311 \mathrm{E}-01-1.136 \mathrm{E}-02 \quad 6.822 \mathrm{E}-01 \quad 1.000 \mathrm{E}+00 \quad 1.000 \mathrm{E}+00 \quad 0.000 \mathrm{E}+00$

I $1-2.370 \mathrm{E}-01 \quad 3.207 \mathrm{E}-01 \quad 9.171 \mathrm{E}-01 \quad 1.000 \mathrm{E}+00 \quad 1.000 \mathrm{E}+00 \quad 0.000 \mathrm{E}+00$

$1 \quad 1 \quad 4.276 \mathrm{E}-01-2.485 \mathrm{E}-02 \quad 9.036 \mathrm{E}-01 \quad 1.000 \mathrm{E}+00 \quad 1.000 \mathrm{E}+00 \quad 0.000 \mathrm{E}+00$

$1 \quad 7.662 \mathrm{E}-01 \quad 7.908 \mathrm{E}-02 \quad 6.378 \mathrm{E}-01 \quad 1.000 \mathrm{E}+00 \quad 1.000 \mathrm{E}+00 \quad 0.000 \mathrm{E}+00$

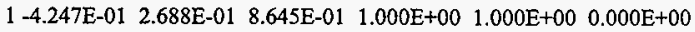

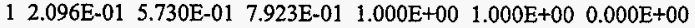

$\begin{array}{lllllll}1 & -2.665 \mathrm{E}-03 & -5.270 \mathrm{E}-01 & 8.499 \mathrm{E}-01 & 1.000 \mathrm{E}+00 & 1.000 \mathrm{E}+00 & 0.000 \mathrm{E}+00\end{array}$

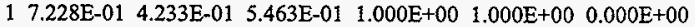

$13.484 \mathrm{E}-01-3.564 \mathrm{E}-01 \quad 8.669 \mathrm{E}-01 \quad 1.000 \mathrm{E}+00 \quad 1.000 \mathrm{E}+00 \quad 0.000 \mathrm{E}+00$

$18.706 \mathrm{E}-01-3.198 \mathrm{E}-01 \quad 3.738 \mathrm{E}-01 \quad 1.000 \mathrm{E}+00 \quad 1.000 \mathrm{E}+00 \quad 0.000 \mathrm{E}+00$

$\begin{array}{llllll}1-8.188 \mathrm{E}-01 & 2.059 \mathrm{E}-01 & 5.359 \mathrm{E}-01 & 1.000 \mathrm{E}+00 & 1.000 \mathrm{E}+00 & 0.000 \mathrm{E}+00\end{array}$

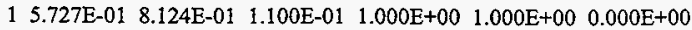

$15.390 \mathrm{E}-02 \quad 5.914 \mathrm{E}-01 \quad 8.046 \mathrm{E}-01 \quad 1.000 \mathrm{E}+00 \quad 1.000 \mathrm{E}+00 \quad 0.000 \mathrm{E}+00$

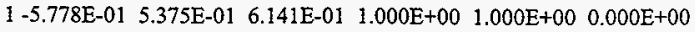


$33 \quad 0.000 \mathrm{E}+00 \quad 0.000 \mathrm{E}+00 \quad 0.000 \mathrm{E}+00$ $34 \quad 0.000 \mathrm{E}+00 \quad 0.000 \mathrm{E}+00 \quad 0.000 \mathrm{E}+00$ $35 \quad 0.000 \mathrm{E}+00 \quad 0.000 \mathrm{E}+00 \quad 0.000 \mathrm{E}+00$ $\begin{array}{lll}36 & 0.000 \mathrm{E}+00 \quad 0.000 \mathrm{E}+00 \quad 0.000 \mathrm{E}+00\end{array}$ $\begin{array}{llll}37 & 0.000 \mathrm{E}+00 & 0.000 \mathrm{E}+00 \quad 0.000 \mathrm{E}+00\end{array}$ $\begin{array}{llll}38 & 0.000 \mathrm{E}+00 & 0.000 \mathrm{E}+00 \quad 0.000 \mathrm{E}+00\end{array}$ $390.000 \mathrm{E}+00 \quad 0.000 \mathrm{E}+00 \quad 0.000 \mathrm{E}+00$ $40 \quad 0.000 \mathrm{E}+00 \quad 0.000 \mathrm{E}+00 \quad 0.000 \mathrm{E}+00$ $\begin{array}{lllll}41 & 0.000 \mathrm{E}+00 & 0.000 \mathrm{E}+00 & 0.000 \mathrm{E}+00\end{array}$ $42 \quad 0.000 \mathrm{E}+00 \quad 0.000 \mathrm{E}+00 \quad 0.000 \mathrm{E}+00$ $\begin{array}{llllll}43 & 0.000 \mathrm{E}+00 & 0.000 \mathrm{E}+00 & 0.000 \mathrm{E}+00\end{array}$ $\begin{array}{lllllll}44 & 0.000 \mathrm{E}+00 & 0.000 \mathrm{E}+00 & 0.000 \mathrm{E}+00\end{array}$ $45 \quad 0.000 \mathrm{E}+00 \quad 0.000 \mathrm{E}+00 \quad 0.000 \mathrm{E}+00$ $\begin{array}{llll}46 & 0.000 \mathrm{E}+00 & 0.000 \mathrm{E}+00 & 0.000 \mathrm{E}+00\end{array}$ $\begin{array}{lll}47 & 0.000 \mathrm{E}+00 & 0.000 \mathrm{E}+00 \quad 0.000 \mathrm{E}+00\end{array}$ $\begin{array}{llllllll}48 & 0.000 \mathrm{E}+00 & 0.000 \mathrm{E}+00 & 0.000 \mathrm{E}+00\end{array}$ $\begin{array}{llll}49 & 0.000 \mathrm{E}+00 & 0.000 \mathrm{E}+00 & 0.000 \mathrm{E}+00\end{array}$ $\begin{array}{lll}50 & 0.000 \mathrm{E}+00 \quad 0.000 \mathrm{E}+00 \quad 0.000 \mathrm{E}+00\end{array}$ problem summary

0

run terminated when 10000 particle histories were done.

photon creation tracks weight energy photon loss tracks weight energy (per source particle)

source $0 \quad 0 . \quad 0$.

$0 . \quad$ escape (per source particle)

$\begin{array}{lllllll}2.210 \mathrm{E}-01 & 2.482 \mathrm{E}-01 & 9.432 \mathrm{E}-01 & 1.000 \mathrm{E}+00 & 1.000 \mathrm{E}+00 & 0.000 \mathrm{E}+00\end{array}$

$14.339 \mathrm{E}-01 \quad 7.515 \mathrm{E}-02 \quad 8.978 \mathrm{E}-01 \quad 1.000 \mathrm{E}+00 \quad 1.000 \mathrm{E}+00 \quad 0.000 \mathrm{E}+00$

$\begin{array}{llllll}1-4.263 \mathrm{E}-01 & 8.490 \mathrm{E}-01 & 3.123 \mathrm{E}-01 & 1.000 \mathrm{E}+00 & 1.000 \mathrm{E}+00 & 0.000 \mathrm{E}+00\end{array}$

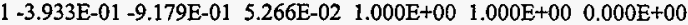

$\begin{array}{llllll}1-4.846 \mathrm{E}-01 & 4.378 \mathrm{E}-01 & 7.573 \mathrm{E}-01 & 1.000 \mathrm{E}+00 & 1.000 \mathrm{E}+00 & 0.000 \mathrm{E}+00\end{array}$

l -5.950E-01 $-5.730 \mathrm{E}-01 \quad 5.636 \mathrm{E}-01 \quad 1.000 \mathrm{E}+00 \quad 1.000 \mathrm{E}+00 \quad 0.000 \mathrm{E}+00$

$1-7.252 \mathrm{E}-01-5.143 \mathrm{E}-01 \quad 4.578 \mathrm{E}-01 \quad 1.000 \mathrm{E}+00 \quad 1.000 \mathrm{E}+00 \quad 0.000 \mathrm{E}+00$

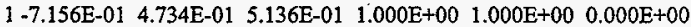

$\begin{array}{llllll}1-1.771 \mathrm{E}-01 & 9.229 \mathrm{E}-01 & 3.417 \mathrm{E}-01 & 1.000 \mathrm{E}+00 & 1.000 \mathrm{E}+00 & 0.000 \mathrm{E}+00\end{array}$

$1-1.149 \mathrm{E}-01-4.206 \mathrm{E}-01 \quad 8.999 \mathrm{E}-01 \quad 1.000 \mathrm{E}+00 \quad 1.000 \mathrm{E}+00 \quad 0.000 \mathrm{E}+00$

$1 \quad 4.509 \mathrm{E}-01 \quad 8.630 \mathrm{E}-02 \quad 8.884 \mathrm{E}-01 \quad 1.000 \mathrm{E}+00 \quad 1.000 \mathrm{E}+00 \quad 0.000 \mathrm{E}+00$

$\begin{array}{llllll}1-7.358 \mathrm{E}-01 & 2.500 \mathrm{E}-01 & 6.294 \mathrm{E}-01 & 1.000 \mathrm{E}+00 & 1.000 \mathrm{E}+00 & 0.000 \mathrm{E}+00\end{array}$

$1-8.448 \mathrm{E}-01 \quad 3.645 \mathrm{E}-01 \quad 3.917 \mathrm{E}-01 \quad 1.000 \mathrm{E}+00 \quad 1.000 \mathrm{E}+00 \quad 0.000 \mathrm{E}+00$

$1 \quad 1.958 \mathrm{E}-01-2.769 \mathrm{E}-01 \quad 9.407 \mathrm{E}-01 \quad 1.000 \mathrm{E}+00 \quad 1.000 \mathrm{E}+00 \quad 0.000 \mathrm{E}+00$

$12.788 \mathrm{E}-01-7.325 \mathrm{E}-03 \quad 9.603 \mathrm{E}-01 \quad 1.000 \mathrm{E}+00 \quad 1.000 \mathrm{E}+00 \quad 0.000 \mathrm{E}+00$

$\begin{array}{llllll}1-3.201 \mathrm{E}-01 & 4.174 \mathrm{E}-01 & 8.505 \mathrm{E}-01 & 1.000 \mathrm{E}+00 & 1.000 \mathrm{E}+00 & 0.000 \mathrm{E}+00\end{array}$

$18.960 \mathrm{E}-01-1.641 \mathrm{E}-01 \quad 4.126 \mathrm{E}-01 \quad 1.000 \mathrm{E}+00 \quad 1.000 \mathrm{E}+00 \quad 0.000 \mathrm{E}+00$

energy cutoff
time cutoff

ight window

$0 \quad 0$.

0. time cutoff

$575 \quad 5.7500 \mathrm{E}-02 \quad 1.5476 \mathrm{E}-02$

limportance

$\begin{array}{lll}0 & 0\end{array}$

0 .

weight window

$\begin{array}{lll}0 & 0 . & 0 .\end{array}$

weight cutoff

$\begin{array}{ll}0 & 0 .\end{array}$

0 .

energy importance 00 .

dxtran

00.

0 .

forced collisions

exp. transform

00.

0 .

0 .

00.

0.

$\begin{array}{lll}0 & 0 . & 0 .\end{array}$

from neutrons

$694 \quad 6.9400 \mathrm{E}-02$

p-annibilation

0 o. 0 .

$\begin{array}{ll}0 & 0 .\end{array}$

0 .

electron $x$-rays

$0 \quad 0$.

0 .

2nd fluorescence

00. cell importance

00.

0.

weight cutoff $\quad 00$.

energy importance 00.

(

dxtran $\quad 0 \quad 0.00$.

forced collisions $\quad \begin{array}{llll}0 & 0 . & 0 .\end{array}$

exp. transform $\quad \begin{array}{llll}0 & 0 . & 0 .\end{array}$

compton scatter $\quad 0 \quad 0 . \quad 6.6993 \mathrm{E}-05$

number of photons banked

$1.7315 \mathrm{E}-02$

694

total

$694 \quad 6.9400 \mathrm{E}-02 \quad 1.7315 \mathrm{E}-02$

photon tracks per source particle

$6.9400 \mathrm{E}-02$

average lifetime, shakes cutoffs

photon collisions per source particle $1.3000 \mathrm{E}-02$ capture $8.3325 \mathrm{E}-05$ eco $1.0000 \mathrm{E}-01$

total photon collisions

130 capture or escape 7.0450E-05 wc1 $0.0000 \mathrm{E}+00$

0 any termination $7.0450 \mathrm{E}-05 \quad$ wc2 $0.0000 \mathrm{E}+00$

electron creation tracks weight energy electron loss tracks weight energy

(per source particle)

source

$10000 \quad 1.0000 \mathrm{E}+00$

ight window

1 importance

weight cutoff

$\begin{array}{ccc}0 & 0 . & 0 \\ 0 & 0 . & 0 . \\ 0 & 0 . & 0 .\end{array}$

0 .

$1.0000 \mathrm{E}+00$

energy cutoff

(per source particle)

time cutoff

$\begin{array}{crr}\text { escape } & 9464 & 9.4640 \mathrm{E}-01 \\ 2046 & 2.0460 \mathrm{E}-01 & 2.0141 \mathrm{E}-02\end{array}$

$7.1762 \mathrm{E}-01$

$0 \quad 0 . \quad 0$.

weight window

00.

0. 、 cell importance 00 .

0 . weight cutoff

00 .

0 . 


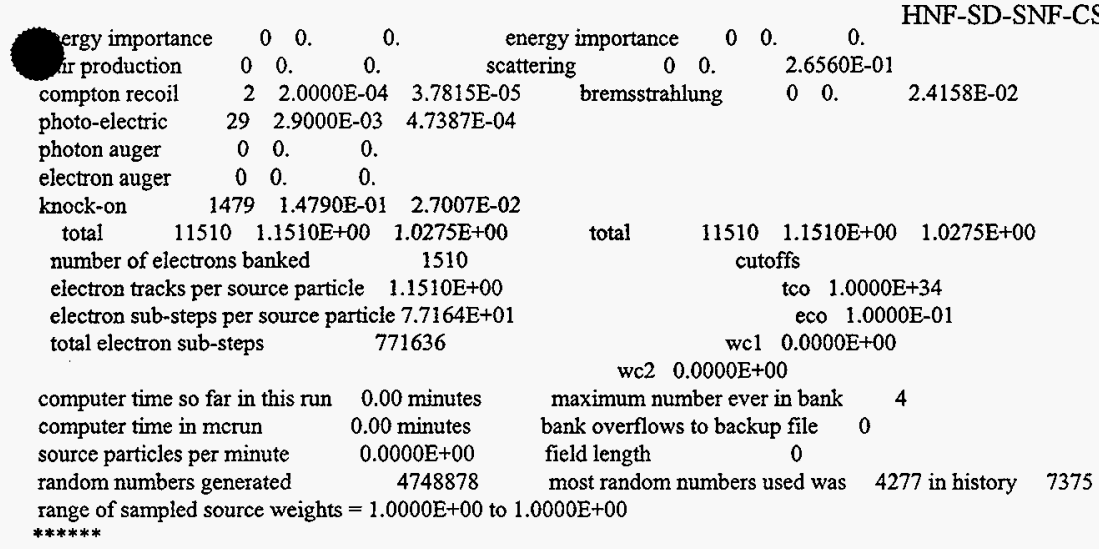

\section{Difo21}

Comparing files INP210 and outp21

FC: no differences encountered

\section{Difo22}

Comparing files INP22O and outp22

no differences encountered

\section{Difo23}

Comparing files INP23O and outp23

FC: no differences encountered

\section{Difo24}

Comparing files INP24O and outp24

\section{****** INP240}

cycle $3 \mathrm{k}$ (collision) 0.551862 removal lifetime(abs) $3.8422 \mathrm{E}+03$ source points generated 253 estimator cycle 4 ave of 2 cycles combination simple average combined average corr ******* outp 24

cycle $3 \mathrm{k}$ (collision) 0.551862 removal lifetime(abs) $3.8422 \mathrm{E}+03$ source points generated 253 source distribution written to file inp24s cycle $=3$ estimator cycle 4 ave of 2 cycles combination simple average combined average corr

\section{$* * * * * *$}

****** INP24O

tally type 6 track length estimate of heating. units mev/gram tally for neutrons

****** outp 24

tally type 6 track length estimate of heating. units mev/gram ******* tally for neutrons 


\section{Gomputer System ID : 501981-801 056AVO000238 (DOE 6246271)}

Difm01

Comparing files INP01M and met101

FC: no differences encountered

Difm02

Comparing files INP02M and met102

FC: no differences encountered

Difm03

Comparing files INP03M and meti 03

FC: no differences encountered

Difm04

Comparing files INP04M and mcti04

FC: no differences encountered

Difm05

Comparing files INP05M and met105

FC: no differences encountered

Difm06

Comparing files INP06M and mctl06

FC: no differences encountered

m07

wouparing files INP07M and met107

FC: no differences encountered

Difm08

Comparing files INP08M and met108

FC: no differences encountered

Difm09

Comparing files INP09M and meti09

FC: no differences encountered

\section{Difm10}

Comparing files INP10M and mct110

FC: no differences encountered

Difm11

Comparing files INP11M and metl11

FC: no differences encountered

\section{Difm12}

Comparing files INP12M and metl12

FC: no differences encountered

Difm13

paring files INP13M and metl13

no differences encountered 
mparing files INP14M and met114
FC: no differences encountered

Difm15

Comparing files INP15M and metl15

FC: no differences encountered

Difm16

Comparing files INP16M and met116

FC: no differences encountered

Difm17

Comparing files INP17M and metl17

FC: no differences encountered

\section{Difm18}

Comparing files INP18M and met118

FC: no differences encountered

\section{Difm19}

Comparing files INP19M and metl19

FC: no differences encountered

\section{Difm20}

File(s) not found : inp $20 \mathrm{~m}$

$\sin 21$

Comparing files NP21M and mctl21

FC: no differences encountered

\section{Difm22}

Comparing files INP22M and metl22

FC: no differences encountered

Difm23

Comparing files INP23M and mot123

FC: no differences encountered

\section{Difm24}

Comparing files NP24M and met124

FC: no differences encountered

\section{Difm25}

Comparing files INP25M and mct125

FC: no differences encountered

\section{Difo0 I}

Comparing files INP010 and outp01

FC: no differences encountered

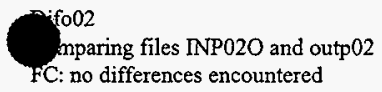


mparing files INP03O and outp03

FC: no differences encountered

Difo04

Comparing files INP04O and outp04

FC: no differences encountered

\section{Difo05}

Comparing files INP05O and outp05

FC: no differences encountered

\section{Difo06}

Comparing files INP060 and outp06

FC: no differences encountered

\section{Difo07}

Comparing files RP07O and outp 07

FC: no differences encountered

\section{Difo08}

Comparing files INP080 and outp 08

FC: no differences encountered

Difo09

Comparing files INP09O and outp09 no differences encountered

Difolo

Comparing files INP10O and outp10

FC: no differences encountered

Difoll

Comparing files INP110 and outp 11

FC: no differences encountered

Difol2

Comparing files $\mathrm{INP} 12 \mathrm{O}$ and outp 12

FC: no differences encountered

\section{Difo13}

Comparing files $\mathbb{N P} 130$ and outp 13

FC: no differences encountered

\section{Difol4}

Comparing files INP14O and outp14

FC: no differences encountered

\section{Difo15}

Comparing files INP15O and outp15

FC: no differences encountered 
Difol7

Comparing files INP17O and outp17

FC: no differences encountered

Difol 8

Comparing files INP18O and outp18

******* INP180

cycle $5 \mathrm{k}$ (collision) 1.135994 removal lifetime(abs) $1.1136 \mathrm{E}+01$ source points generated 222

cycle $6 \mathrm{k}$ (collision) 1.077014 removal lifetime(abs) $1.2222 \mathrm{E}+01$ source points generated 204

****** outp18

cycle $5 \mathrm{k}$ (collision) 1.135994 removal lifetime(abs) $1.1136 \mathrm{E}+01$ source points generated 222 source distribution written to file inp18s cycle $=5$

cycle $6 \mathrm{k}$ (collision) 1.077014 removal lifetime(abs) $1.2222 \mathrm{E}+01$ source points generated 204

Difo19

Comparing files INP19O and outp19

FC: no differences encountered

Difo20

Comparing files INP200 and outp20

******* INP200

******* outp 20

$\begin{array}{lll}8 & 0.000 \mathrm{E}+00 & 0.000 \mathrm{E}+00 \quad 0.000 \mathrm{E}+00\end{array}$

$90.000 \mathrm{E}+00 \quad 0.000 \mathrm{E}+00 \quad 0.000 \mathrm{E}+00$

$10 \quad 0.000 \mathrm{E}+00 \quad 0.000 \mathrm{E}+00 \quad 0.000 \mathrm{E}+00$

$11 \quad 0.000 \mathrm{E}+00 \quad 0.000 \mathrm{E}+00 \quad 0.000 \mathrm{E}+00$

$12 \quad 0.000 \mathrm{E}+00 \quad 0.000 \mathrm{E}+00 \quad 0.000 \mathrm{E}+00$

$13 \quad 0.000 \mathrm{E}+00 \quad 0.000 \mathrm{E}+00 \quad 0.000 \mathrm{E}+00$

$14 \quad 0.000 \mathrm{E}+00 \quad 0.000 \mathrm{E}+00 \quad 0.000 \mathrm{E}+00$

$15 \quad 0.000 \mathrm{E}+00 \quad 0.000 \mathrm{E}+00 \quad 0.000 \mathrm{E}+00$

$160.000 \mathrm{E}+00 \quad 0.000 \mathrm{E}+00 \quad 0.000 \mathrm{E}+00$

$\begin{array}{lllll}17 & 0.000 \mathrm{E}+00 & 0.000 \mathrm{E}+00 & 0.000 \mathrm{E}+00\end{array}$

$\begin{array}{lll}18 & 0.000 \mathrm{E}+00 \quad 0.000 \mathrm{E}+00 \quad 0.000 \mathrm{E}+00\end{array}$

$19 \quad 0.000 \mathrm{E}+00 \quad 0.000 \mathrm{E}+00 \quad 0.000 \mathrm{E}+00$

$20 \quad 0.000 \mathrm{E}+00 \quad 0.000 \mathrm{E}+00 \quad 0.000 \mathrm{E}+00$

$21 \quad 0.000 \mathrm{E}+00 \quad 0.000 \mathrm{E}+00 \quad 0.000 \mathrm{E}+00$

$22 \quad 0.000 \mathrm{E}+00 \quad 0.000 \mathrm{E}+00 \quad 0.000 \mathrm{E}+00$

$23 \quad 0.000 \mathrm{E}+00 \quad 0.000 \mathrm{E}+00 \quad 0.000 \mathrm{E}+00$

$24 \quad 0.000 \mathrm{E}+00 \quad 0.000 \mathrm{E}+00 \quad 0.000 \mathrm{E}+00$

$25 \quad 0.000 \mathrm{E}+00 \quad 0.000 \mathrm{E}+00 \quad 0.000 \mathrm{E}+00$

$26 \quad 0.000 \mathrm{E}+00 \quad 0.000 \mathrm{E}+00 \quad 0.000 \mathrm{E}+00$

$27 \quad 0.000 \mathrm{E}+00 \quad 0.000 \mathrm{E}+00 \quad 0.000 \mathrm{E}+00$

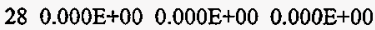

$29 \quad 0.000 \mathrm{E}+00 \quad 0.000 \mathrm{E}+00 \quad 0.000 \mathrm{E}+00$

$30 \quad 0.000 \mathrm{E}+00 \quad 0.000 \mathrm{E}+00 \quad 0.000 \mathrm{E}+00$

$310.000 \mathrm{E}+00 \quad 0.000 \mathrm{E}+00 \quad 0.000 \mathrm{E}+00$

$32 \quad 0.000 \mathrm{E}+00 \quad 0.000 \mathrm{E}+00 \quad 0.000 \mathrm{E}+00$

$33 \quad 0.000 \mathrm{E}+00 \quad 0.000 \mathrm{E}+00 \quad 0.000 \mathrm{E}+00$

$34 \quad 0.000 \mathrm{E}+00 \quad 0.000 \mathrm{E}+00 \quad 0.000 \mathrm{E}+00$

$35 \quad 0.000 \mathrm{E}+00 \quad 0.000 \mathrm{E}+00 \quad 0.000 \mathrm{E}+00$

$36 \quad 0.000 \mathrm{E}+00 \quad 0.000 \mathrm{E}+00 \quad 0.000 \mathrm{E}+00$

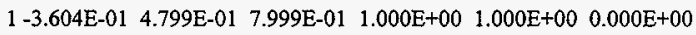
$\begin{array}{llllll}1-2.241 \mathrm{E}-01 & 3.960 \mathrm{E}-01 & 8.905 \mathrm{E}-01 & 1.000 \mathrm{E}+00 & 1.000 \mathrm{E}+00 & 0.000 \mathrm{E}+00\end{array}$

$1-2.928 \mathrm{E}-01-7.352 \mathrm{E}-01 \quad 6.114 \mathrm{E}-01 \quad 1.000 \mathrm{E}+00 \quad 1.000 \mathrm{E}+00 \quad 0.000 \mathrm{E}+00$

$18.260 \mathrm{E}-01 \quad 1.184 \mathrm{E}-01 \quad 5.510 \mathrm{E}-01 \quad 1.000 \mathrm{E}+00 \quad 1.000 \mathrm{E}+00 \quad 0.000 \mathrm{E}+00$

$17.540 \mathrm{E}-01-1.976 \mathrm{E}-01 \quad 6.264 \mathrm{E}-01 \quad 1.000 \mathrm{E}+00 \quad 1.000 \mathrm{E}+00 \quad 0.000 \mathrm{E}+00$

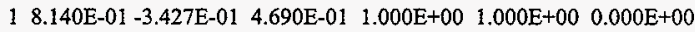

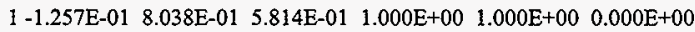

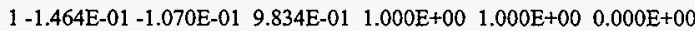

$1 \quad 1.310 \mathrm{E}-02-1.617 \mathrm{E}-02 \quad 9.998 \mathrm{E}-01 \quad 1.000 \mathrm{E}+00 \quad 1.000 \mathrm{E}+00 \quad 0.000 \mathrm{E}+00$

$1-8.843 \mathrm{E}-01-4.126 \mathrm{E}-01 \quad 2.187 \mathrm{E}-01 \quad 1.000 \mathrm{E}+00 \quad 1.000 \mathrm{E}+00 \quad 0.000 \mathrm{E}+00$

$14.260 \mathrm{E}-01 \quad 4.153 \mathrm{E}-01 \quad 8.038 \mathrm{E}-01 \quad 1.000 \mathrm{E}+00 \quad 1.000 \mathrm{E}+00 \quad 0.000 \mathrm{E}+00$

$1-7.311 \mathrm{E}-01-1.136 \mathrm{E}-02 \quad 6.822 \mathrm{E}-01 \quad 1.000 \mathrm{E}+00 \quad 1.000 \mathrm{E}+00 \quad 0.000 \mathrm{E}+00$

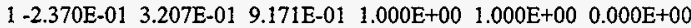

1 4.276E-01 $-2.485 \mathrm{E}-02 \quad 9.036 \mathrm{E}-01 \quad 1.000 \mathrm{E}+00 \quad 1.000 \mathrm{E}+00 \quad 0.000 \mathrm{E}+00$

$1 \quad 7.662 \mathrm{E}-01 \quad 7.908 \mathrm{E}-02 \quad 6.378 \mathrm{E}-01 \quad 1.000 \mathrm{E}+00 \quad 1.000 \mathrm{E}+00 \quad 0.000 \mathrm{E}+00$

$\begin{array}{lllllll}1-4.247 \mathrm{E}-01 & 2.688 \mathrm{E}-01 & 8.645 \mathrm{E}-01 & 1.000 \mathrm{E}+00 & 1.000 \mathrm{E}+00 & 0.000 \mathrm{E}+00\end{array}$

I $2.096 \mathrm{E}-01 \quad 5.730 \mathrm{E}-01 \quad 7.923 \mathrm{E}-01 \quad 1.000 \mathrm{E}+00 \quad 1.000 \mathrm{E}+00 \quad 0.000 \mathrm{E}+00$

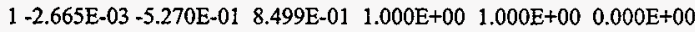

$\begin{array}{lllllllll}1 & 7.228 \mathrm{E}-01 & 4.233 \mathrm{E}-01 & 5.463 \mathrm{E}-01 & 1.000 \mathrm{E}+00 & 1.000 \mathrm{E}+00 & 0.000 \mathrm{E}+00\end{array}$

$13.484 \mathrm{E}-01-3.564 \mathrm{E}-01 \quad 8.669 \mathrm{E}-01 \quad 1.000 \mathrm{E}+00 \quad 1.000 \mathrm{E}+00 \quad 0.000 \mathrm{E}+00$

$1 \quad 8.706 \mathrm{E}-01-3.198 \mathrm{E}-01 \quad 3.738 \mathrm{E}-01 \quad 1.000 \mathrm{E}+00 \quad 1.000 \mathrm{E}+00 \quad 0.000 \mathrm{E}+00$

$\begin{array}{llllll}1-8.188 \mathrm{E}-01 & 2.059 \mathrm{E}-01 & 5.359 \mathrm{E}-01 & 1.000 \mathrm{E}+00 & 1.000 \mathrm{E}+00 & 0.000 \mathrm{E}+00\end{array}$

$15.727 \mathrm{E}-01 \quad 8.124 \mathrm{E}-01 \quad 1.100 \mathrm{E}-01 \quad 1.000 \mathrm{E}+00 \quad 1.000 \mathrm{E}+00 \quad 0.000 \mathrm{E}+00$

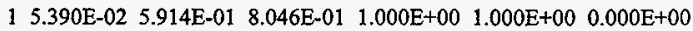

$\begin{array}{lllllll}1 & -5.778 \mathrm{E}-01 & 5.375 \mathrm{E}-01 & 6.141 \mathrm{E}-01 & 1.000 \mathrm{E}+00 & 1.000 \mathrm{E}+00 & 0.000 \mathrm{E}+00\end{array}$

$1-7.198 \mathrm{E}-01-2.890 \mathrm{E}-01 \quad 6.311 \mathrm{E}-01 \quad 1.000 \mathrm{E}+00 \quad 1.000 \mathrm{E}+00 \quad 0.000 \mathrm{E}+00$

$\begin{array}{llllll}12.210 \mathrm{E}-01 & 2.482 \mathrm{E}-01 & 9.432 \mathrm{E}-01 & 1.000 \mathrm{E}+00 & 1.000 \mathrm{E}+00 & 0.000 \mathrm{E}+00\end{array}$

$14.339 \mathrm{E}-01 \quad 7.515 \mathrm{E}-02 \quad 8.978 \mathrm{E}-01 \quad 1.000 \mathrm{E}+00 \quad 1.000 \mathrm{E}+00 \quad 0.000 \mathrm{E}+00$

$\begin{array}{llllll}1-4.263 \mathrm{E}-01 & 8.490 \mathrm{E}-01 & 3.123 \mathrm{E}-01 & 1.000 \mathrm{E}+00 & 1.000 \mathrm{E}+00 & 0.000 \mathrm{E}+00\end{array}$ 
$37 \quad 0.000 \mathrm{E}+00 \quad 0.000 \mathrm{E}+00 \quad 0.000 \mathrm{E}+00$ $\begin{array}{lllll}38 & 0.000 \mathrm{E}+00 & 0.000 \mathrm{E}+00 & 0.000 \mathrm{E}+00\end{array}$ $390.000 \mathrm{E}+00 \quad 0.000 \mathrm{E}+00 \quad 0.000 \mathrm{E}+00$ $40 \quad 0.000 \mathrm{E}+00 \quad 0.000 \mathrm{E}+00 \quad 0.000 \mathrm{E}+00$ $41 \quad 0.000 \mathrm{E}+00 \quad 0.000 \mathrm{E}+00 \quad 0.000 \mathrm{E}+00$ $42 \quad 0.000 \mathrm{E}+00 \quad 0.000 \mathrm{E}+00 \quad 0.000 \mathrm{E}+00$ $\begin{array}{llll}43 & 0.000 \mathrm{E}+00 & 0.000 \mathrm{E}+00 & 0.000 \mathrm{E}+00\end{array}$ $\begin{array}{llll}44 & 0.000 \mathrm{E}+00 & 0.000 \mathrm{E}+00 & 0.000 \mathrm{E}+00\end{array}$

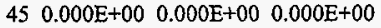
$46 \quad 0.000 \mathrm{E}+00 \quad 0.000 \mathrm{E}+00 \quad 0.000 \mathrm{E}+00$ $\begin{array}{lll}47 & 0.000 \mathrm{E}+00 \quad 0.000 \mathrm{E}+00 \quad 0.000 \mathrm{E}+00\end{array}$ $\begin{array}{llllll}48 & 0.000 \mathrm{E}+00 & 0.000 \mathrm{E}+00 & 0.000 \mathrm{E}+00\end{array}$ $\begin{array}{llll}49 & 0.000 \mathrm{E}+00 \quad 0.000 \mathrm{E}+00 \quad 0.000 \mathrm{E}+00\end{array}$

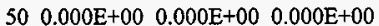

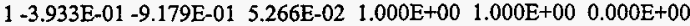

$\begin{array}{llllll}1-4.846 \mathrm{E}-01 & 4.378 \mathrm{E}-01 & 7.573 \mathrm{E}-01 & 1.000 \mathrm{E}+00 & 1.000 \mathrm{E}+00 & 0.000 \mathrm{E}+00\end{array}$

$1-5.950 \mathrm{E}-01-5.730 \mathrm{E}-01 \quad 5.636 \mathrm{E}-01 \quad 1.000 \mathrm{E}+00 \quad 1.000 \mathrm{E}+00 \quad 0.000 \mathrm{E}+00$

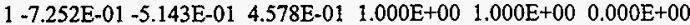

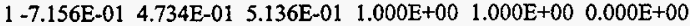

$1-1.771 \mathrm{E}-01 \quad 9.229 \mathrm{E}-01 \quad 3.417 \mathrm{E}-01 \quad 1.000 \mathrm{E}+00 \quad 1.000 \mathrm{E}+00 \quad 0.000 \mathrm{E}+00$

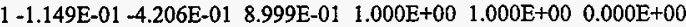

$14.509 \mathrm{E}-01 \quad 8.630 \mathrm{E}-02 \quad 8.884 \mathrm{E}-01 \quad 1.000 \mathrm{E}+00 \quad 1.000 \mathrm{E}+00 \quad 0.000 \mathrm{E}+00$

$\begin{array}{llllll}1-7.358 \mathrm{E}-01 & 2.500 \mathrm{E}-01 & 6.294 \mathrm{E}-01 & 1.000 \mathrm{E}+00 & 1.000 \mathrm{E}+00 & 0.000 \mathrm{E}+00\end{array}$

$1-8.448 \mathrm{E}-01 \quad 3.645 \mathrm{E}-01 \quad 3.917 \mathrm{E}-01 \quad 1.000 \mathrm{E}+00 \quad 1.000 \mathrm{E}+00 \quad 0.000 \mathrm{E}+00$

1 1.958E-01 -2.769E-01 9.407E-01 $1.000 \mathrm{E}+00 \quad 1.000 \mathrm{E}+00 \quad 0.000 \mathrm{E}+00$

$12.788 \mathrm{E}-01-7.325 \mathrm{E}-03 \quad 9.603 \mathrm{E}-01 \quad 1.000 \mathrm{E}+00 \quad 1.000 \mathrm{E}+00 \quad 0.000 \mathrm{E}+00$

$\begin{array}{llllll}1-3.201 \mathrm{E}-01 & 4.174 \mathrm{E}-01 & 8.505 \mathrm{E}-01 & 1.000 \mathrm{E}+00 & 1.000 \mathrm{E}+00 & 0.000 \mathrm{E}+00\end{array}$

$18.960 \mathrm{E}-01-1.641 \mathrm{E}-01 \quad 4.126 \mathrm{E}-01 \quad 1.000 \mathrm{E}+00 \quad 1.000 \mathrm{E}+00 \quad 0.000 \mathrm{E}+00$

Iproblem summary

Iun terminated when 10000 particle histories were done. 0

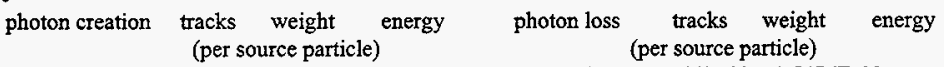

source

00.0 .

escape

$575 \quad 5.7500 \mathrm{E}-02 \quad 1.5476 \mathrm{E}-02$

energy cutoff $\quad 0 \quad 0.00$.

weight window time cutoff 00 .

0.

cell importance

$\begin{array}{lll}0 & 0 . & 0 .\end{array}$

weight window

00

0.

weight cutoff

$\begin{array}{lll}0 & 0 . & 0 .\end{array}$

00.

0 .

energy importance

00.

0 .

cell importance

00.

weight cutoff

00.

energy importance 00 .

0 .

tran $0 \quad 0$

0 .

rorced collisions

$\begin{array}{lll}0 & 0 . & 0\end{array}$

exp. transform

$\begin{array}{lll}0 & 0 . & 0 .\end{array}$

dxtran $\quad 0 \quad 0$.

forced collisions

0 .

from neutrons

bremsstrahlung

$\begin{array}{lll}0 & 0 . & 0 .\end{array}$

exp. transform

00.

0

compton 0.0.

p-annihilation

$694 \quad 6.9400 \mathrm{E}-02$

electron $\mathrm{X}$-rays

$\begin{array}{lll}0 & 0 . & 0 .\end{array}$

0.

1.7315E-02 capture

00.

pair production

00.

$119 \quad 1.1900 \mathrm{E}-02$

$\begin{array}{lll}0 & 0 . & 0 . \\ 0 & 0 . & 0 .\end{array}$

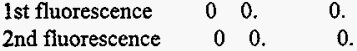

$\begin{array}{lllllllll}\text { total } & 694 & 6.9400 \mathrm{E}-02 & 1.7315 \mathrm{E}-02 & \text { total } & 694 & 6.9400 \mathrm{E}-02 & 1.7315 \mathrm{E}-02\end{array}$

number of photons banked

694 average lifetime, shakes cutoffs

photon tracks per source particle $6.9400 \mathrm{E}-02$ escape $6.7786 \mathrm{E}-05$ tco $1.0000 \mathrm{E}+34$

photon collisions per source particle 1.3000E-02 capture 8.3325E-05 eco 1.0000E-01

total photon collisions $\quad 130 \quad$ capture or escape $7.0450 \mathrm{E}-05$ wcl $0.0000 \mathrm{E}+00$

any termination $\quad 7.0450 \mathrm{E}-05 \quad$ wc2 $\quad 0.0000 \mathrm{E}+00$

0

electron creation tracks weight energy electron loss tracks weight energy

(per source particle)

source

$10000 \quad 1.0000 \mathrm{E}+00$

$1.0000 \mathrm{E}+00$ energy cutoff

time cutoff 00.0.

weight window

$0 \quad 0$.

0.

weight window

00 .

$9464 \quad 9.4640 \mathrm{E}-01 \quad 7.1762 \mathrm{E}-01$

cell importance

$\begin{array}{lll}0 & 0 . & 0 .\end{array}$

weight cutoff

energy importance

00.

0 .

$\begin{array}{lll}0 & 0 . & 0 .\end{array}$

cell importance

00.

weight cutoff

00.

energy importance

scattering 00 .

2 2.0000E-04

ir production

$3.7815 \mathrm{E}-05$

bremsstrahliung

$00 . \quad 0$

photo-electric

$29 \quad 2.9000 \mathrm{E}-03$

4.7387E-04

$2.6560 \mathrm{E}-01$

$\begin{array}{lll}0 & 0 . & 2.4158 \mathrm{E}-02\end{array}$ 


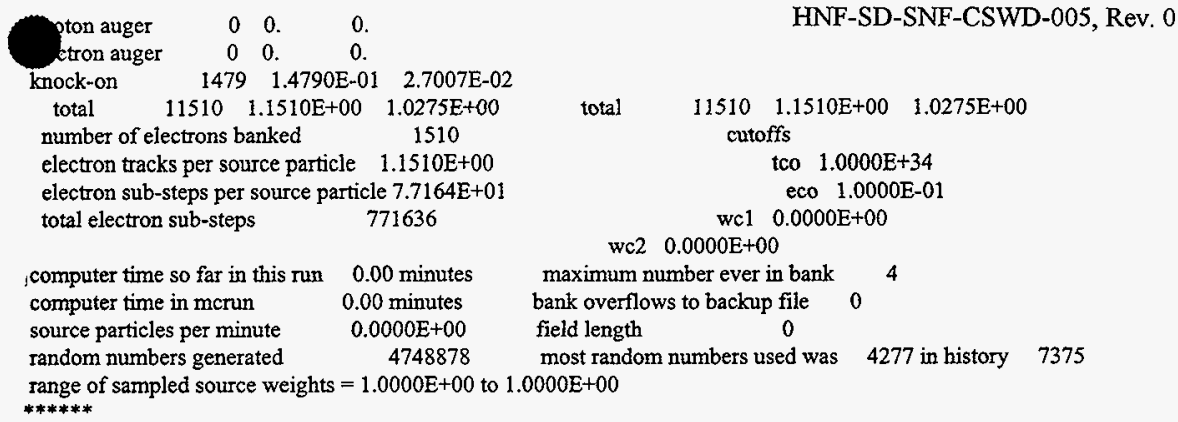

computer time so far in this run 0.00 minutes computer time in mcrun $\quad 0.00$ minutes source particles per minute $\quad 0.0000 \mathrm{E}+00$ random numbers generated $\quad 4748878$ range of sampled source weights $=1.0000 \mathrm{E}+00$ to $1.0000 \mathrm{E}+00$

$* * * * * *$

Difo21

Comparing files INP210 and outp21

FC: no differences encountered

\section{Difo22}

Comparing files INP22O and outp22

FC: no differences encountered

\section{Difo 23}

Comparing files INP23O and outp23 no differences encountered

\section{Difo24}

Comparing files INP240 and outp24

****** INP240

cycle $3 \mathrm{k}$ (collision) 0.551862 removal lifetime(abs) $3.8422 \mathrm{E}+03$ source points generated 253 estimator cycle 4 ave of 2 cycles combination simple average combined average corr ****** outp 24

cycle $3 \mathrm{k}$ (collision) 0.551862 removal lifetime(abs) $3.8422 \mathrm{E}+03$ source points generated 253 source distribution written to file inp $24 \mathrm{~s} \quad$ cycle $=3$ estimator cycle 4 ave of 2 cycles combination simple average combined average corr $* * * * * *$

\section{****** INP240}

tally type 6 track length estimate of heating. units mev/gram taily for neutrons

\section{****** outp24}

tally type 6 track length estimate of heating. units mev/gram tally for neutrons

\section{Difo25}

Comparing files INP25O and outp25

FC: no differences encountered 


\section{Computer System ID : 501981-801 056AVO000041 (DOE 6239600)}

Difm01

Comparing files INPOIM and mct101

FC: no differences encountered

\section{Difm02}

Comparing files INP02M and mctl02

FC: no differences encountered

\section{Difm03}

Comparing files INP03M and mct103 FC: no differences encountered

Difm04

Comparing files INP04M and mct104 FC: no differences encountered

\section{Difm05}

Comparing files INP05M and met105 FC: no differences encountered

Difm06

Comparing files INP06M and mct106 FC: no differences encountered n 07

.omparing files INP07M and mct107

FC: no differences encountered

\section{Difm08}

Comparing files INP08M and mct108 FC: no differences encountered

\section{Difm09}

Comparing files INP09M and mct109 FC: no differences encountered

\section{Difm10}

Comparing files INP10M and met110

FC: no differences encountered

\section{DifmI I}

Comparing files INP11M and motil1 FC: no differences encountered

\section{Difm!2}

Comparing files INP12M and mctl12 FC: no differences encountered

Difm13

\footnotetext{
paring files INP13M and met113
} no differences encountered 
$\mathrm{FC}$ : no differences encountered

\section{Difm15}

Comparing files INP15M and matl15

FC: no differences encountered

\section{Difm16}

Comparing files INP16M and met116

FC: no differences encountered

\section{Difm17}

Comparing files INP17M and mctl17

FC: no differences encountered

\section{Difm18}

Comparing files INP18M and mctl18

FC: no differences encountered

\section{Difm19}

Comparing files NP19M and metl19

FC: no differences encountered

\section{Difm20}

File(s) not found : inp20m

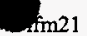

Comparing files INP21M and mct12 1

FC: no differences encountered

Difm22

Comparing files INP22M and mctl22 FC: no differences encountered

\section{Difm23}

Comparing files INP23M and inct123

FC: no differences encountered

\section{Difm24}

Comparing files INP24M and mctl24

FC: no differences encountered

\section{Difm25}

Comparing files INP25M and mct125

FC: no differences encountered

Difo01

Comparing files $\mathrm{INP} 01 \mathrm{O}$ and outp01 FC: no differences encountered

\footnotetext{
foo2

mparing files $\mathrm{INP} 02 \mathrm{O}$ and outp02

FC: no differences encountered
} 
mparing files INP03O and outp03

$F C$ : no differences encountered

\section{Difo04}

Comparing files INP04O and outp04

FC: no differences encountered

\section{Difo05}

Comparing files INP05O and outp05

FC: no differences encountered

\section{Difo06}

Comparing files INP060 and outp06

FC: no differences encountered

\section{Difo07}

Comparing files NP07O and outp07

FC: no differences encountered

\section{Difo08}

Comparing files INP08O and outp08

FC: no differences encountered

Difo09

Comparing files INP09O and outp09 no differences encountered

\section{Difo10}

Comparing files INP10O and outp 10

FC: no differences encountered

\section{Difol1}

Comparing files INP110 and outp 11 FC: no differences encountered

\section{Difo12}

Comparing files INP12O and outp12 FC: no differences encountered

\section{Difo13}

Comparing files INP13O and outp13

FC: no differences encountered

\section{Difol14}

Comparing files INP14O and outp 14 FC: no differences encountered

Difo15

Comparing files INP15O and outp15 FC: no differences encountered 
Difol7

Comparing files INP17O and outp17

FC: no differences encountered

\section{Difo18}

Comparing files INP180 and outp18

****** INP180

cycle $5 \mathrm{k}$ (collision) 1.135994 removal lifetime(abs) $1.1136 \mathrm{E}+01$ source points generated 222

cycle $6 \mathrm{k}$ (collision) 1.077014 removal lifetime(abs) $1.2222 \mathrm{E}+01$ source points generated 204

****** outp 18

cycle $5 \mathrm{k}$ (collision) 1.135994 removal lifetime(abs) $1.1136 \mathrm{E}+01$ source points generated 222 source distribution written to file inp $18 \mathrm{~s} \quad$ cycle $=5$

cycle $6 \mathrm{k}$ (collision) 1.077014 removal lifetime(abs) $1.2222 \mathrm{E}+01$ source points generated 204

*******

\section{Difo19}

Comparing files INP19O and outp19

FC: no differences encountered

\section{Difo20}

Comparing files INP20O and outp20

\section{****** INP200}

****** outp20

$\begin{array}{llll}8 & 0.000 \mathrm{E}+00 & 0.000 \mathrm{E}+00 & 0.000 \mathrm{E}+00\end{array}$

$90.000 \mathrm{E}+00 \quad 0.000 \mathrm{E}+00 \quad 0.000 \mathrm{E}+00$

$10 \quad 0.000 \mathrm{E}+00 \quad 0.000 \mathrm{E}+00 \quad 0.000 \mathrm{E}+00$

$110.000 \mathrm{E}+00 \quad 0.000 \mathrm{E}+00 \quad 0.000 \mathrm{E}+00$

$12 \quad 0.000 \mathrm{E}+00 \quad 0.000 \mathrm{E}+00 \quad 0.000 \mathrm{E}+00$

$13 \quad 0.000 \mathrm{E}+00 \quad 0.000 \mathrm{E}+00 \quad 0.000 \mathrm{E}+00$

$14 \quad 0.000 \mathrm{E}+00 \quad 0.000 \mathrm{E}+00 \quad 0.000 \mathrm{E}+00$

$15 \quad 0.000 \mathrm{E}+00 \quad 0.000 \mathrm{E}+00 \quad 0.000 \mathrm{E}+00$

$16 \quad 0.000 \mathrm{E}+00 \quad 0.000 \mathrm{E}+00 \quad 0.000 \mathrm{E}+00$

$\begin{array}{lll}17 & 0.000 \mathrm{E}+00 \quad 0.000 \mathrm{E}+00 \quad 0.000 \mathrm{E}+00\end{array}$

$\begin{array}{lll}18 & 0.000 \mathrm{E}+00 \quad 0.000 \mathrm{E}+00 \quad 0.000 \mathrm{E}+00\end{array}$

$19 \quad 0.000 \mathrm{E}+00 \quad 0.000 \mathrm{E}+00 \quad 0.000 \mathrm{E}+00$

$20 \quad 0.000 \mathrm{E}+00 \quad 0.000 \mathrm{E}+00 \quad 0.000 \mathrm{E}+00$

$21 \quad 0.000 \mathrm{E}+00 \quad 0.000 \mathrm{E}+00 \quad 0.000 \mathrm{E}+00$

$22 \quad 0.000 \mathrm{E}+00 \quad 0.000 \mathrm{E}+00 \quad 0.000 \mathrm{E}+00$

$230.000 \mathrm{E}+00 \quad 0.000 \mathrm{E}+00 \quad 0.000 \mathrm{E}+00$

$24 \quad 0.000 \mathrm{E}+00 \quad 0.000 \mathrm{E}+00 \quad 0.000 \mathrm{E}+00$

$250.000 \mathrm{E}+00 \quad 0.000 \mathrm{E}+00 \quad 0.000 \mathrm{E}+00$

$26 \quad 0.000 \mathrm{E}+00 \quad 0.000 \mathrm{E}+00 \quad 0.000 \mathrm{E}+00$

$27 \quad 0.000 \mathrm{E}+00 \quad 0.000 \mathrm{E}+00 \quad 0.000 \mathrm{E}+00$

$28 \quad 0.000 \mathrm{E}+00 \quad 0.000 \mathrm{E}+00 \quad 0.000 \mathrm{E}+00$

$29 \quad 0.000 \mathrm{E}+00 \quad 0.000 \mathrm{E}+00 \quad 0.000 \mathrm{E}+00$

$\begin{array}{llll}30 & 0.000 \mathrm{E}+00 & 0.000 \mathrm{E}+00 & 0.000 \mathrm{E}+00\end{array}$

$31 \quad 0.000 \mathrm{E}+00 \quad 0.000 \mathrm{E}+00 \quad 0.000 \mathrm{E}+00$

$32 \quad 0.000 \mathrm{E}+00 \quad 0.000 \mathrm{E}+00 \quad 0.000 \mathrm{E}+00$

$33 \quad 0.000 \mathrm{E}+00 \quad 0.000 \mathrm{E}+00 \quad 0.000 \mathrm{E}+00$

$34 \quad 0.000 \mathrm{E}+00 \quad 0.000 \mathrm{E}+00 \quad 0.000 \mathrm{E}+00$

$\begin{array}{lllll}35 & 0.000 \mathrm{E}+00 & 0.000 \mathrm{E}+00 & 0.000 \mathrm{E}+00\end{array}$

$36 \quad 0.000 \mathrm{E}+00 \quad 0.000 \mathrm{E}+00 \quad 0.000 \mathrm{E}+00$

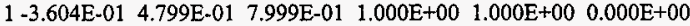

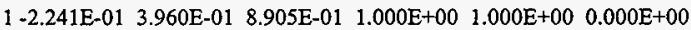

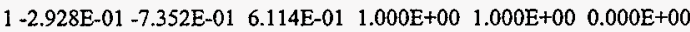
$18.260 \mathrm{E}-01 \quad 1.184 \mathrm{E}-01 \quad 5.510 \mathrm{E}-01 \quad 1.000 \mathrm{E}+00 \quad 1.000 \mathrm{E}+00 \quad 0.000 \mathrm{E}+00$

$17.540 \mathrm{E}-01-1.976 \mathrm{E}-01 \quad 6.264 \mathrm{E}-01 \quad 1.000 \mathrm{E}+00 \quad 1.000 \mathrm{E}+00 \quad 0.000 \mathrm{E}+00$

$18.140 \mathrm{E}-01-3.427 \mathrm{E}-01 \quad 4.690 \mathrm{E}-01 \quad 1.000 \mathrm{E}+00 \quad 1.000 \mathrm{E}+00 \quad 0.000 \mathrm{E}+00$

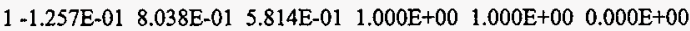
$1-1.464 \mathrm{E}-01-1.070 \mathrm{E}-01 \quad 9.834 \mathrm{E}-01 \quad 1.000 \mathrm{E}+00 \quad 1.000 \mathrm{E}+00 \quad 0.000 \mathrm{E}+00$ $1 \quad 1.310 \mathrm{E}-02-1.617 \mathrm{E}-02 \quad 9.998 \mathrm{E}-01 \quad 1.000 \mathrm{E}+00 \quad 1.000 \mathrm{E}+00 \quad 0.000 \mathrm{E}+00$

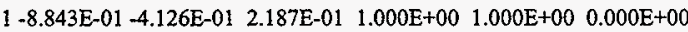

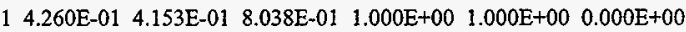
$1-7.311 \mathrm{E}-01-1.136 \mathrm{E}-02 \quad 6.822 \mathrm{E}-01 \quad 1.000 \mathrm{E}+00 \quad 1.000 \mathrm{E}+00 \quad 0.000 \mathrm{E}+00$

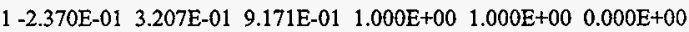
$1 \quad 4.276 \mathrm{E}-01-2.485 \mathrm{E}-02 \quad 9.036 \mathrm{E}-01 \quad 1.000 \mathrm{E}+00 \quad 1.000 \mathrm{E}+00 \quad 0.000 \mathrm{E}+00$ $17.662 \mathrm{E}-01 \quad 7.908 \mathrm{E}-02 \quad 6.378 \mathrm{E}-01 \quad 1.000 \mathrm{E}+00 \quad 1.000 \mathrm{E}+00 \quad 0.000 \mathrm{E}+00$ $\begin{array}{lllllll}1 & -4.247 \mathrm{E}-01 & 2.688 \mathrm{E}-01 & 8.645 \mathrm{E}-01 & 1.000 \mathrm{E}+00 & 1.000 \mathrm{E}+00 & 0.000 \mathrm{E}+00\end{array}$ $12.096 \mathrm{E}-01 \quad 5.730 \mathrm{E}-01 \quad 7.923 \mathrm{E}-01 \quad 1.000 \mathrm{E}+00 \quad 1.000 \mathrm{E}+00 \quad 0.000 \mathrm{E}+00$ $1-2.665 \mathrm{E}-03-5.270 \mathrm{E}-01 \quad 8.499 \mathrm{E}-01 \quad 1.000 \mathrm{E}+00 \quad 1.000 \mathrm{E}+00 \quad 0.000 \mathrm{E}+00$ $17.228 \mathrm{E}-01 \quad 4.233 \mathrm{E}-01 \quad 5.463 \mathrm{E}-01 \quad 1.000 \mathrm{E}+00 \quad 1.000 \mathrm{E}+00 \quad 0.000 \mathrm{E}+00$ $\begin{array}{llllllll}1 & 3.484 \mathrm{E}-01 & -3.564 \mathrm{E}-01 & 8.669 \mathrm{E}-01 & 1.000 \mathrm{E}+00 & 1.000 \mathrm{E}+00 & 0.000 \mathrm{E}+00\end{array}$ $18.706 \mathrm{E}-01-3.198 \mathrm{E}-01 \quad 3.738 \mathrm{E}-01 \quad 1.000 \mathrm{E}+00 \quad 1.000 \mathrm{E}+00 \quad 0.000 \mathrm{E}+00$ $\begin{array}{lllllll}1-8.188 \mathrm{E}-01 & 2.059 \mathrm{E}-01 & 5.359 \mathrm{E}-01 & 1.000 \mathrm{E}+00 & 1.000 \mathrm{E}+00 & 0.000 \mathrm{E}+00\end{array}$ $15.727 \mathrm{E}-01 \quad 8.124 \mathrm{E}-01 \quad 1.100 \mathrm{E}-01 \quad 1.000 \mathrm{E}+00 \quad 1.000 \mathrm{E}+00 \quad 0.000 \mathrm{E}+00$ $\begin{array}{llllllll}5.390 \mathrm{E}-02 & 5.914 \mathrm{E}-01 & 8.046 \mathrm{E}-01 & 1.000 \mathrm{E}+00 & 1.000 \mathrm{E}+00 & 0.000 \mathrm{E}+00\end{array}$ $\begin{array}{lllllll}1-5.778 \mathrm{E}-01 & 5.375 \mathrm{E}-01 & 6.141 \mathrm{E}-01 & 1.000 \mathrm{E}+00 & 1.000 \mathrm{E}+00 & 0.000 \mathrm{E}+00\end{array}$ $\begin{array}{llll}1-7.198 \mathrm{E}-01 & -2.890 \mathrm{E}-01 & 6.311 \mathrm{E}-01 \quad 1.000 \mathrm{E}+00 & 1.000 \mathrm{E}+00 \quad 0.000 \mathrm{E}+00\end{array}$

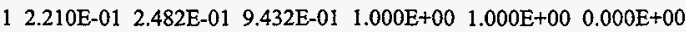
$1 \quad 4.339 \mathrm{E}-01 \quad 7.515 \mathrm{E}-02 \quad 8.978 \mathrm{E}-01 \quad 1.000 \mathrm{E}+00 \quad 1.000 \mathrm{E}+00 \quad 0.000 \mathrm{E}+00$ $\begin{array}{lllllll}1 & -4.263 \mathrm{E}-01 & 8.490 \mathrm{E}-01 & 3.123 \mathrm{E}-01 & 1.000 \mathrm{E}+00 & 1.000 \mathrm{E}+00 & 0.000 \mathrm{E}+00\end{array}$ 
HNF-SD-SNF-CSWD-005, Rev. 0

$37 \quad 0.000 \mathrm{E}+00 \quad 0.000 \mathrm{E}+00 \quad 0.000 \mathrm{E}+00$ $\begin{array}{lllll}38 & 0.000 \mathrm{E}+00 & 0.000 \mathrm{E}+00 & 0.000 \mathrm{E}+00 & 1\end{array}$

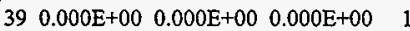
$40 \quad 0.000 \mathrm{E}+00 \quad 0.000 \mathrm{E}+00 \quad 0.000 \mathrm{E}+00 \quad 1$ $41 \quad 0.000 \mathrm{E}+00 \quad 0.000 \mathrm{E}+00 \quad 0.000 \mathrm{E}+00 \quad 1$ $42 \quad 0.000 \mathrm{E}+00 \quad 0.000 \mathrm{E}+00 \quad 0.000 \mathrm{E}+00$ $43 \quad 0.000 \mathrm{E}+00 \quad 0.000 \mathrm{E}+00 \quad 0.000 \mathrm{E}+00$ $440.000 \mathrm{E}+00 \quad 0.000 \mathrm{E}+00 \quad 0.000 \mathrm{E}+00$ $45 \quad 0.000 \mathrm{E}+00 \quad 0.000 \mathrm{E}+00 \quad 0.000 \mathrm{E}+00$ $\begin{array}{llll}46 & 0.000 \mathrm{E}+00 & 0.000 \mathrm{E}+00 & 0.000 \mathrm{E}+00\end{array}$ $47 \quad 0.000 \mathrm{E}+00 \quad 0.000 \mathrm{E}+00 \quad 0.000 \mathrm{E}+00$ $48 \quad 0.000 \mathrm{E}+00 \quad 0.000 \mathrm{E}+00 \quad 0.000 \mathrm{E}+00$ $49 \quad 0.000 \mathrm{E}+00 \quad 0.000 \mathrm{E}+00 \quad 0.000 \mathrm{E}+00 \quad 1$ $\begin{array}{lll}50 & 0.000 \mathrm{E}+00 \quad 0.000 \mathrm{E}+00 \quad 0.000 \mathrm{E}+00 \quad 1\end{array}$ Iproblem summary 0 run terminated when 10000 particle histories were done. photon creation tracks weight energy
(per source particle) source 00.

o. escape

weight window cell importance weight cutoff energy importance tran fced collisions exp. transform from neutrons bremsstrahlung p-annihilation electron $x$-rays 1 st fluorescence 2nd fluorescence

$\begin{array}{ccc}0 & 0 . & 0 . \\ 0 & 0 . & 0 . \\ 0 & 0 . & 0 . \\ 0 & 0 . & 0 .\end{array}$
time cutoff $\begin{array}{lll}0 & 0 . & 0\end{array}$

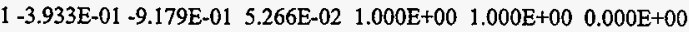
$\begin{array}{llllll}1-4.846 \mathrm{E}-01 & 4.378 \mathrm{E}-01 & 7.573 \mathrm{E}-01 & 1.000 \mathrm{E}+00 & 1.000 \mathrm{E}+00 & 0.000 \mathrm{E}+00\end{array}$ $1-5.950 \mathrm{E}-01-5.730 \mathrm{E}-01 \quad 5.636 \mathrm{E}-01 \quad 1.000 \mathrm{E}+00 \quad 1.000 \mathrm{E}+00 \quad 0.000 \mathrm{E}+00$ $1-7.252 \mathrm{E}-01-5.143 \mathrm{E}-01 \quad 4.578 \mathrm{E}-01 \quad 1.000 \mathrm{E}+00 \quad 1.000 \mathrm{E}+00 \quad 0.000 \mathrm{E}+00$

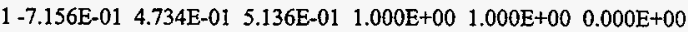

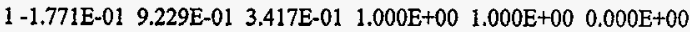

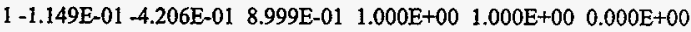

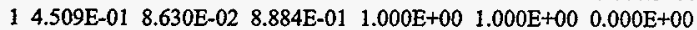
$\begin{array}{llllll}1-7.358 \mathrm{E}-01 & 2.500 \mathrm{E}-01 & 6.294 \mathrm{E}-01 & 1.000 \mathrm{E}+00 & 1.000 \mathrm{E}+00 & 0.000 \mathrm{E}+00\end{array}$ $\begin{array}{lllllll}1 & -8.448 \mathrm{E}-01 & 3.645 \mathrm{E}-01 & 3.917 \mathrm{E}-01 & 1.000 \mathrm{E}+00 & 1.000 \mathrm{E}+00 & 0.000 \mathrm{E}+00\end{array}$ $1 \quad 1.958 \mathrm{E}-01-2.769 \mathrm{E}-01 \quad 9.407 \mathrm{E}-01 \quad 1.000 \mathrm{E}+00 \quad 1.000 \mathrm{E}+00 \quad 0.000 \mathrm{E}+00$ $12.788 \mathrm{E}-01-7.325 \mathrm{E}-03 \quad 9.603 \mathrm{E}-01 \quad 1.000 \mathrm{E}+00 \quad 1.000 \mathrm{E}+00 \quad 0.000 \mathrm{E}+00$

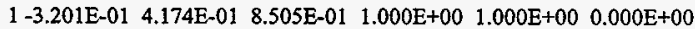
$18.960 \mathrm{E}-01-1.641 \mathrm{E}-01 \quad 4.126 \mathrm{E}-01 \quad 1.000 \mathrm{E}+00 \quad 1.000 \mathrm{E}+00 \quad 0.000 \mathrm{E}+00$ total $694 \quad 6.9400 \mathrm{E}-02$ number of photons banked $0 . \quad$ weight window 00. cell importance $\quad 0 \quad 0$. weight cutoff $\quad 0 \quad 0$. tracks weight energy photon loss tracks weight
(per source particle) $575 \quad 5.7500 \mathrm{E}-02 \quad 1.5476 \mathrm{E}-02$ photon tracks per source particle photon collisions per source particl
total photon collisions

total $694 \quad 6.9400 \mathrm{E}-02 \quad 1.7315 \mathrm{E}-02$ 694 average lifetime, shakes cutoffs

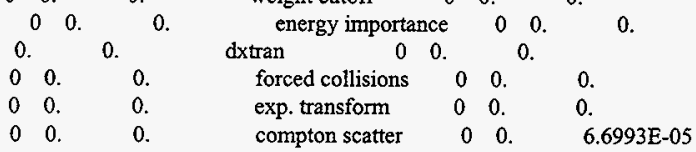
$\begin{array}{ccccccccc}694 & 6.9400 \mathrm{E}-02 & 1.7315 \mathrm{E}-02 & \text { capture } & & & 119 & 1.1900 \mathrm{E}-02 & 1.7720 \mathrm{E}-03 \\ 0 & 0 . & 0 . & \text { pair production } & 0 & 0 . & 0 . & & \end{array}$

\section{0}

electron creation tracks weight energy electron loss tracks weight energy (per source particle)

source $10000 \quad 1.0000 \mathrm{E}+00$ $6.9400 \mathrm{E}-02$ escape $6.7786 \mathrm{E}-05$ capture $8.3325 \mathrm{E}-05$ eco $1.0000 \mathrm{E}-01$ tco $1.0000 \mathrm{E}+34$ 130 capture or escape 7.0450E-05 wc1 $0.0000 \mathrm{E}+00$ any termination $7.0450 \mathrm{E}-05$ wc2 $0.0000 \mathrm{E}+00$

weight window cell importance weight cutoff energy importance

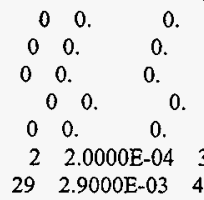

$1.0000 \mathrm{E}+00$ energy cutoff \begin{tabular}{ccc} 
& \multicolumn{2}{c}{ (per source particle) } \\
escape & 9464 & $9.4640 \mathrm{E}-01$ \\
2046 & $2.0460 \mathrm{E}-01$ & $2.0141 \mathrm{E}-02$ \\
0 & 0. & 0.
\end{tabular} time cutoff $\quad 0 \quad 0 . \quad 0$. \begin{tabular}{ccc} 
& \multicolumn{2}{c}{ (per source particle) } \\
escape & 9464 & $9.4640 \mathrm{E}-01$ \\
2046 & $2.0460 \mathrm{E}-01$ & $2.0141 \mathrm{E}-02$ \\
0 & 0. & 0.
\end{tabular} 7.1762E-01 weight window $\quad 00.0$. cell importance $\quad 0 \quad 0.0$. weight cutoff $\quad 00$. $\begin{array}{ccccc}\text { energy importance } & 0 & 0 . & 0 . \\ \text { scattering } & 0 & 0 . & & 2.6560 \mathrm{E}-01\end{array}$ inpton recoil photo-electric $29 \quad 2.9000 \mathrm{E}-03$

$3.7815 \mathrm{E}-05$ 4.7387E-04 bremsstrahlung

00. $2.4158 \mathrm{E}-02$ 


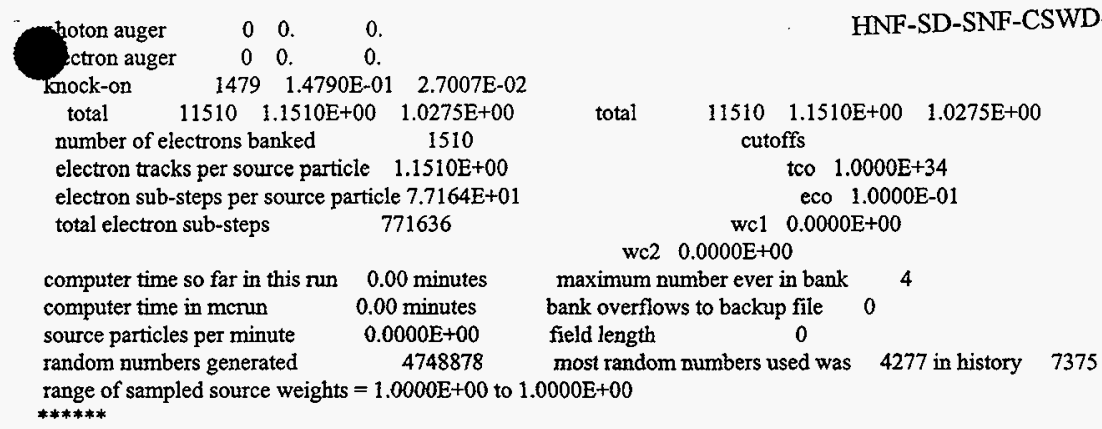

\section{Difo21}

Comparing files INP21O and outp21

FC: no differences encountered

\section{Difo22}

Comparing files INP22O and outp22

FC: no differences encountered

\section{Difo23}

Comparing files INP230 and outp23 no differences encountered

\section{Difo24}

Comparing files INP240 and outp24

****** INP240

cycle $3 \mathrm{k}$ (collision) 0.551862 removal lifetime(abs) $3.8422 \mathrm{E}+03$ source points generated 253 estimator cycle 4 ave of 2 cycles combination simple average combined average con $* * * * * *$ outp 24

cycle $3 \mathrm{k}$ (collision) 0.551862 removal lifetime(abs) $3.8422 \mathrm{E}+03$ source points generated 253 source distribution written to file inp24s cycle $=3$ estimator cycle 4 ave of 2 cycles combination ******

\section{****** INP240}

tally type 6 track length estimate of heating. units mev/gram tally for neutrons

******* outp24

tally type 6 track length estimate of heating. units mev/gram ****** tally for neutrons

\section{Difo25}

Comparing files INP25O and outp25

FC: no differences encountered 


\section{Pmputer System ID : 501981-801 056AVO000088 (DOE 6246288)}

Difm01

Comparing files INP01M and mctiol

FC: no differences encountered

Difm02

Comparing files $\mathrm{NP} 02 \mathrm{M}$ and mct102

FC: no differences encountered

Difm03

Comparing files INP03M and mct103

FC: no differences encountered

\section{Difm04}

Comparing files INP04M and mct104

FC: no differences encountered

Difm05

Comparing files INP05M and met105

FC: no differences encountered

Difm06

Comparing files INP06M and mct106

FC: no differences encountered

Comparing files INP07M and mct107

FC: no differences encountered

\section{Difm08}

Comparing files INP08M and mct108 FC: no differences encountered

Difm09

Comparing files INP09M and mot109 FC: no differences encountered

\section{Difm10}

Comparing files INP10M and mctl10

FC: no differences encountered

\section{Difm11}

Comparing files INP11M and motl11

FC: no differences encountered

\section{Difm12}

Comparing files INP12M and mctl12

FC: no differences encountered

\section{Difm13}


mparing files INP14M and met114

FC: no differences encountered

\section{Difm15}

Comparing files INP15M and mctl15

FC: no differences encountered

Difm16

Comparing files INP16M and mctl16

FC: no differences encountered

\section{Difm17}

Comparing files INP17M and mct117

FC: no differences encountered

Difm18

Comparing files INP18M and mctl18

FC: no differences encountered

Difm19

Comparing files INP19M and metl19

FC: no differences encountered

\section{Difm20}

Fijle(s) tot found : inp $20 \mathrm{~m}$

-itim21

Comparing files INP21M and mctl21

FC: no differences encountered

Difm22

Comparing files INP22M and mct 22

FC: no differences encountered

Difm23

Comparing files INP23M and mct123

FC: no differences encountered

Difm24

Comparing files INP24M and mct124

FC: no differences encountered

\section{Difm25}

Comparing files INP25M and mct125

FC: no differences encountered

\section{Difo01}

Comparing files INP01O and outp01

FC: no differences encountered

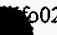

hparing files INP02O and outp02

FC: no differences encountered 
mparing files INP03O and outp03

$\overrightarrow{F C}$ : no differences encountered

Difo04

Comparing files INP04O and outp04

FC: no differences encountered

\section{Difo05}

Comparing files INP05O and outp05

FC: no differences encountered

\section{Difo06}

Comparing files INP060 and outp06

FC: no differences encountered

\section{Difo07}

Comparing files INP07O and outp07

FC: no differences encountered

\section{Difo08}

Comparing files $\mathrm{INP} 08 \mathrm{O}$ and outp 08

FC: no differences encountered

Difo09

Comparing files $\mathrm{NNP} 090$ and outp09 no differences encountered

Difol0

Comparing files INP 100 and outp 10 FC: no differences encountered

\section{Difol1}

Comparing files INP110 and outp11

FC: no differences encountered

\section{Difol2}

Comparing files INP12O and outp12

FC: no differences encountered

\section{Difol3}

Comparing files INP130 and outp13

FC: no differences encountered

\section{Difol4}

Comparing files INP140 and outp14 FC: no differences encountered

Difo15

Comparing files INP15O and outp15

FC: no differences encountered 
HNF-SD-SNF-CSWD-005, Rev. 0

no differences encountered

Difol7

Comparing files INP17O and outp17

FC: no differences encountered

Difol 8

Comparing files INP18O and outp18

****** INP18O

cycle $5 \mathrm{k}$ (collision) 1.135994 removal lifetime(abs) $1.1136 \mathrm{E}+01$ source points generated 222

cycle $6 \mathrm{k}$ (collision) 1.077014 removal lifetime(abs) $1.2222 \mathrm{E}+01$ source points generated 204

****** outp18

cycle $5 \mathrm{k}$ (collision) 1.135994 removal lifetime(abs) $1.1136 \mathrm{E}+01$ source points generated 222

source distribution written to file inp18s cycle $=5$

cycle $6 \mathrm{k}$ (collision) 1.077014 removal lifetime(abs) $1.2222 \mathrm{E}+01$ source points generated 204

*******

Difo19

Comparing files INP19O and outp19

FC; no differences encountered

Difo20

Comparing files NP200 and outp20

******* INP200

****** outp20

$8 \quad 0.000 \mathrm{E}+00 \quad 0.000 \mathrm{E}+00 \quad 0.000 \mathrm{E}+00$

$90.000 \mathrm{E}+00 \quad 0.000 \mathrm{E}+00 \quad 0.000 \mathrm{E}+00$

$10 \quad 0.000 \mathrm{E}+00 \quad 0.000 \mathrm{E}+00 \quad 0.000 \mathrm{E}+00$

$110.000 \mathrm{E}+00 \quad 0.000 \mathrm{E}+00 \quad 0.000 \mathrm{E}+00$

$12 \quad 0.000 \mathrm{E}+00 \quad 0.000 \mathrm{E}+00 \quad 0.000 \mathrm{E}+00$

$13 \quad 0.000 \mathrm{E}+00 \quad 0.000 \mathrm{E}+00 \quad 0.000 \mathrm{E}+00$

$14 \quad 0.000 \mathrm{E}+00 \quad 0.000 \mathrm{E}+00 \quad 0.000 \mathrm{E}+00$

$15 \quad 0.000 \mathrm{E}+00 \quad 0.000 \mathrm{E}+00 \quad 0.000 \mathrm{E}+00$

$16 \quad 0.000 \mathrm{E}+00 \quad 0.000 \mathrm{E}+00 \quad 0.000 \mathrm{E}+00$

$i 7 \quad 0.000 \mathrm{E}+00 \quad 0.000 \mathrm{E}+00 \quad 0.000 \mathrm{E}+00$

$18 \quad 0.000 \mathrm{E}+00 \quad 0.000 \mathrm{E}+00 \quad 0.000 \mathrm{E}+00$

$19 \quad 0.000 \mathrm{E}+00 \quad 0.000 \mathrm{E}+00 \quad 0.000 \mathrm{E}+00$

$20 \quad 0.000 \mathrm{E}+00 \quad 0.000 \mathrm{E}+00 \quad 0.000 \mathrm{E}+00$

$21 \quad 0.000 \mathrm{E}+00 \quad 0.000 \mathrm{E}+00 \quad 0.000 \mathrm{E}+00$

$22 \quad 0.000 \mathrm{E}+00 \quad 0.000 \mathrm{E}+00 \quad 0.000 \mathrm{E}+00$

$23 \quad 0.000 \mathrm{E}+00 \quad 0.000 \mathrm{E}+00 \quad 0.000 \mathrm{E}+00$

$24 \quad 0.000 \mathrm{E}+00 \quad 0.000 \mathrm{E}+00 \quad 0.000 \mathrm{E}+00$

$25 \quad 0.000 \mathrm{E}+00 \quad 0.000 \mathrm{E}+00 \quad 0.000 \mathrm{E}+00$

$26 \quad 0.000 \mathrm{E}+00 \quad 0.000 \mathrm{E}+00 \quad 0.000 \mathrm{E}+00$

$27 \quad 0.000 \mathrm{E}+00 \quad 0.000 \mathrm{E}+00 \quad 0.000 \mathrm{E}+00$

$28 \quad 0.000 \mathrm{E}+00 \quad 0.000 \mathrm{E}+00 \quad 0.000 \mathrm{E}+00$

$29 \quad 0.000 \mathrm{E}+00 \quad 0.000 \mathrm{E}+00 \quad 0.000 \mathrm{E}+00$

$30 \quad 0.000 \mathrm{E}+00 \quad 0.000 \mathrm{E}+00 \quad 0.000 \mathrm{E}+00$

$310.000 \mathrm{E}+00 \quad 0.000 \mathrm{E}+00 \quad 0.000 \mathrm{E}+00$

$32 \quad 0.000 \mathrm{E}+00 \quad 0.000 \mathrm{E}+00 \quad 0.000 \mathrm{E}+00$

$33 \quad 0.000 \mathrm{E}+00 \quad 0.000 \mathrm{E}+00 \quad 0.000 \mathrm{E}+00$

$34 \quad 0.000 \mathrm{E}+00 \quad 0.000 \mathrm{E}+00 \quad 0.000 \mathrm{E}+00$

s5 $0.000 \mathrm{E}+00 \quad 0.000 \mathrm{E}+00 \quad 0.000 \mathrm{E}+00$

$360.000 \mathrm{E}+00 \quad 0.000 \mathrm{E}+00 \quad 0.000 \mathrm{E}+00$
1 -3.604E-01 4.799E-01 7.999E-01 1.000E+00 1.000E+00 0.000E+00

$\begin{array}{lllllll}1-2.241 \mathrm{E}-01 & 3.960 \mathrm{E}-01 & 8.905 \mathrm{E}-01 & 1.000 \mathrm{E}+00 & 1.000 \mathrm{E}+00 & 0.000 \mathrm{E}+00\end{array}$

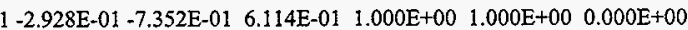

$\begin{array}{lllllll}1 & 8.260 \mathrm{E}-01 & 1.184 \mathrm{E}-01 & 5.510 \mathrm{E}-01 & 1.000 \mathrm{E}+00 & 1.000 \mathrm{E}+00 & 0.000 \mathrm{E}+00\end{array}$

$17.540 \mathrm{E}-01-1.976 \mathrm{E}-01 \quad 6.264 \mathrm{E}-01 \quad 1.000 \mathrm{E}+00 \quad 1.000 \mathrm{E}+00 \quad 0.000 \mathrm{E}+00$

$18.140 \mathrm{E}-01-3.427 \mathrm{E}-01 \quad 4.690 \mathrm{E}-01 \quad 1.000 \mathrm{E}+00 \quad 1.000 \mathrm{E}+00 \quad 0.000 \mathrm{E}+00$

$1-1.257 \mathrm{E}-01 \quad 8.038 \mathrm{E}-01 \quad 5.814 \mathrm{E}-01 \quad 1.000 \mathrm{E}+00 \quad 1.000 \mathrm{E}+00 \quad 0.000 \mathrm{E}+00$

$\begin{array}{llllll}1-1.464 \mathrm{E}-01 & -1.070 \mathrm{E}-01 & 9.834 \mathrm{E}-01 & 1.000 \mathrm{E}+00 & 1.000 \mathrm{E}+00 & 0.000 \mathrm{E}+00\end{array}$

1 1.310E-02 -1.617E-02 9.998E-01 $1.000 \mathrm{E}+00 \quad 1.000 \mathrm{E}+00 \quad 0.000 \mathrm{E}+00$

$\begin{array}{llllll}1-8.843 E-01 & -4.126 \mathrm{E}-01 & 2.187 \mathrm{E}-01 & 1.000 \mathrm{E}+00 & 1.000 \mathrm{E}+00 & 0.000 \mathrm{E}+00\end{array}$

1 4.260E-01 4.153E-01 8.038E-01 1.000E+00 1.000E+00 $0.000 \mathrm{E}+00$

$1-7.311 \mathrm{E}-01-1.136 \mathrm{E}-02 \quad 6.822 \mathrm{E}-01 \quad 1.000 \mathrm{E}+00 \quad 1.000 \mathrm{E}+00 \quad 0.000 \mathrm{E} \div 00$

$1-2.370 \mathrm{E}-01 \quad 3.207 \mathrm{E}-01 \quad 9.171 \mathrm{E}-01 \quad 1.000 \mathrm{E}+00 \quad 1.000 \mathrm{E}+00 \quad 0.000 \mathrm{E}+00$

$1 \quad 4.276 \mathrm{E}-01-2.485 \mathrm{E}-02 \quad 9.036 \mathrm{E}-01 \quad 1.000 \mathrm{E}+00 \quad 1.000 \mathrm{E}+00 \quad 0.000 \mathrm{E}+00$

$17.662 \mathrm{E}-01 \quad 7.908 \mathrm{E}-02 \quad 6.378 \mathrm{E}-01 \quad 1.000 \mathrm{E}+00 \quad 1.000 \mathrm{E}+00 \quad 0.000 \mathrm{E}+00$

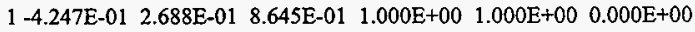

$12.096 \mathrm{E}-01 \quad 5.730 \mathrm{E}-0 \pm \quad 7.923 \mathrm{E}-01 \quad 1.000 \mathrm{E}+00 \quad 1.000 \mathrm{E}+00 \quad 0.000 \mathrm{E}+00$

$1-2.665 \mathrm{E}-03-5.270 \mathrm{E}-01 \quad 8.499 \mathrm{E}-01 \quad 1.000 \mathrm{E}+00 \quad 1.000 \mathrm{E}+00 \quad 0.000 \mathrm{E}+00$

$17.228 \mathrm{E}-01 \quad 4.233 \mathrm{E}-01 \quad 5.463 \mathrm{E}-01 \quad 1.000 \mathrm{E}+00 \quad 1.000 \mathrm{E}+00 \quad 0.000 \mathrm{E}+00$

$1 \quad 3.484 \mathrm{E}-01-3.564 \mathrm{E}-01 \quad 8.669 \mathrm{E}-01 \quad 1.000 \mathrm{E}+00 \quad 1.000 \mathrm{E}+00 \quad 0.000 \mathrm{E}+00$

1 8.706E-01 -3.198E-01 3.738E-01 1.000E +00 1.000E $+00 \quad 0.000 \mathrm{E}+00$

$\begin{array}{llllll}1-8.188 \mathrm{E}-01 & 2.059 \mathrm{E}-01 & 5.359 \mathrm{E}-01 & 1.000 \mathrm{E}+00 & 1.000 \mathrm{E}+00 & 0.000 \mathrm{E}+00\end{array}$

$\begin{array}{lllllll}1 & 5.727 \mathrm{E}-01 & 8.124 \mathrm{E}-01 & 1.100 \mathrm{E}-01 & 1.000 \mathrm{E}+00 & 1.000 \mathrm{E}+00 & 0.000 \mathrm{E}+00\end{array}$

$\begin{array}{lllllll}1 & 5.390 \mathrm{E}-02 & 5.914 \mathrm{E}-01 & 8.046 \mathrm{E}-01 & 1.000 \mathrm{E}+00 & 1.000 \mathrm{E}+00 & 0.000 \mathrm{E}+00\end{array}$

$\begin{array}{lllllll}1-5.778 \mathrm{E}-01 & 5.375 \mathrm{E}-01 & 6.141 \mathrm{E}-01 & 1.000 \mathrm{E}+00 & 1.000 \mathrm{E}+00 & 0.000 \mathrm{E}+00\end{array}$

$1-7.198 \mathrm{E}-01-2.890 \mathrm{E}-01 \quad 6.311 \mathrm{E}-01 \quad 1.000 \mathrm{E}+00 \quad 1.000 \mathrm{E}+00 \quad 0.000 \mathrm{E}+00$

$12.210 \mathrm{E}-01 \quad 2.482 \mathrm{E}-01 \quad 9.432 \mathrm{E}-01 \quad 1.000 \mathrm{E}+00 \quad 1.000 \mathrm{E}+00 \quad 0.000 \mathrm{E}+00$

$1 \quad 4.339 \mathrm{E}-01 \quad 7.515 \mathrm{E}-02 \quad 8.978 \mathrm{E}-01 \quad 1.000 \mathrm{E}+00 \quad 1.000 \mathrm{E}+00 \quad 0.000 \mathrm{E}+00$

$\begin{array}{llllll}1-4.263 \mathrm{E}-01 & 8.490 \mathrm{E}-01 & 3.123 \mathrm{E}-01 & 1.000 \mathrm{E}+00 & 1.000 \mathrm{E}+00 & 0.000 \mathrm{E}+00\end{array}$ 
$37 \quad 0.000 \mathrm{E}+00 \quad 0.000 \mathrm{E}+00 \quad 0.000 \mathrm{E}+00$

$\begin{array}{llll}38 & 0.000 \mathrm{E}+00 & 0.000 \mathrm{E}+00 & 0.000 \mathrm{E}+00\end{array}$ $39 \quad 0.000 \mathrm{E}+00 \quad 0.000 \mathrm{E}+00 \quad 0.000 \mathrm{E}+00$ $40 \quad 0.000 \mathrm{E}+00 \quad 0.000 \mathrm{E}+00 \quad 0.000 \mathrm{E}+00$ $\begin{array}{lll}41 & 0.000 \mathrm{E}+00 \quad 0.000 \mathrm{E}+00 \quad 0.000 \mathrm{E}+00\end{array}$ $42 \quad 0.000 \mathrm{E}+00 \quad 0.000 \mathrm{E}+00 \quad 0.000 \mathrm{E}+00$ $\begin{array}{lllll}43 & 0.000 \mathrm{E}+00 & 0.000 \mathrm{E}+00 & 0.000 \mathrm{E}+00\end{array}$ $44 \quad 0.000 \mathrm{E}+00 \quad 0.000 \mathrm{E}+00 \quad 0.000 \mathrm{E}+00$ $\begin{array}{llll}45 & 0.000 \mathrm{E}+00 & 0.000 \mathrm{E}+00 & 0.000 \mathrm{E}+00\end{array}$ $46 \quad 0.000 \mathrm{E}+00 \quad 0.000 \mathrm{E}+00 \quad 0.000 \mathrm{E}+00$ $\begin{array}{lll}47 & 0.000 \mathrm{E}+00 \quad 0.000 \mathrm{E}+00 \quad 0.000 \mathrm{E}+00\end{array}$ $\begin{array}{llll}48 & 0.000 \mathrm{E}+00 \quad 0.000 \mathrm{E}+00 \quad 0.000 \mathrm{E}+00\end{array}$ $\begin{array}{llll}49 & 0.000 \mathrm{E}+00 & 0.000 \mathrm{E}+00 & 0.000 \mathrm{E}+00\end{array}$ $\begin{array}{lll}50 & 0.000 \mathrm{E}+00 \quad 0.000 \mathrm{E}+00 \quad 0.000 \mathrm{E}+00\end{array}$

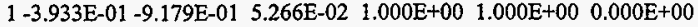

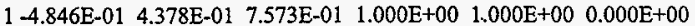

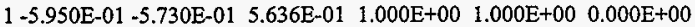

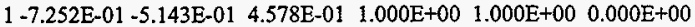
$\begin{array}{lllllll}1-7.156 \mathrm{E}-01 & 4.734 \mathrm{E}-01 & 5.136 \mathrm{E}-01 & 1.000 \mathrm{E}+00 & 1.000 \mathrm{E}+00 & 0.000 \mathrm{E}+00\end{array}$ $\begin{array}{llllll}1-1.771 \mathrm{E}-01 & 9.229 \mathrm{E}-01 & 3.417 \mathrm{E}-01 & 1.000 \mathrm{E}+00 & 1.000 \mathrm{E}+00 & 0.000 \mathrm{E}+00\end{array}$ $\begin{array}{llllll}1-1.149 \mathrm{E}-01 & -4.206 \mathrm{E}-01 & 8.999 \mathrm{E}-01 & 1.000 \mathrm{E}+00 & 1.000 \mathrm{E}+00 & 0.000 \mathrm{E}+00\end{array}$ $\begin{array}{llllllll}1 & 4.509 \mathrm{E}-01 & 8.630 \mathrm{E}-02 & 8.884 \mathrm{E}-01 & 1.000 \mathrm{E}+00 & 1.000 \mathrm{E}+00 & 0.000 \mathrm{E}+00\end{array}$ $\begin{array}{llllll}1-7.358 \mathrm{E}-01 & 2.500 \mathrm{E}-01 & 6.294 \mathrm{E}-01 & 1.000 \mathrm{E}+00 & 1.000 \mathrm{E}+00 & 0.000 \mathrm{E}+00\end{array}$ $\begin{array}{llllll}1-8.448 \mathrm{E}-01 & 3.645 \mathrm{E}-01 & 3.917 \mathrm{E}-01 & 1.000 \mathrm{E}+00 & 1.000 \mathrm{E}+00 & 0.000 \mathrm{E}+00\end{array}$ $1 \quad 1.958 \mathrm{E}-01-2.769 \mathrm{E}-01 \quad 9.407 \mathrm{E}-01 \quad 1.000 \mathrm{E}+00 \quad 1.000 \mathrm{E}+00 \quad 0.000 \mathrm{E}+00$

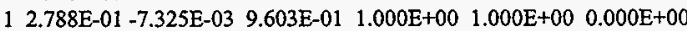

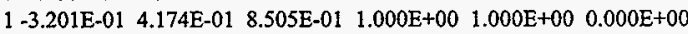
$18.960 \mathrm{E}-01-1.641 \mathrm{E}-01 \quad 4.126 \mathrm{E}-01 \quad 1.000 \mathrm{E}+00 \quad 1.000 \mathrm{E}+00 \quad 0.000 \mathrm{E}+00$

lproblem summary

run terminated when 10000 particle histories were done. 0

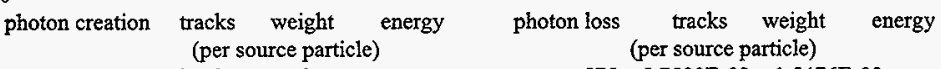

source

$00 . \quad 0$

escape
energy cutoff

$575 \quad 5.7500 \mathrm{E}-02 \quad 1.5476 \mathrm{E}-02$

time cutoff

$0 \quad 0.0$.

weight window

$0 \quad 0$.

0 . $0 \quad 0$.

0.

cell importance

00.

weight window

00.

0.

weight cutoff

00.

0 .

epergy importance

tran

00.

0.

cell importance

00.

weight cutoff

00. energy importance $0 \quad 0$.

lorced collisions

exp. transform

$\begin{array}{lll}0 & 0 . & 0 .\end{array}$

$\begin{array}{lll}0 & 0 . & 0 .\end{array}$

dxtran 00.

forced collisions $\quad 0 \quad 0 . \quad 0$.

exp. transform $\quad \begin{array}{llll}0 & 0 . & 0 .\end{array}$

from neutrons

bremsstrahlung

0.0 .0$.

$0 \quad 0 . \quad 0$.

compton scatter $\quad 0 \quad 0$.

$6.6993 \mathrm{E}-05$

p-annihilation

$6946.9400 \mathrm{E}-02$

electron $\mathrm{x}$-rays

0.

1.7315E-02 capture

$119 \quad 1.1900 \mathrm{E}-02$

pair production $\quad 0 \quad 0$.

0.

Ist fluorescence $\quad \begin{array}{lll}0 & 0 . & 0 .\end{array}$

2nd fluorescence $\quad 0 \quad 0 . \quad 0$.

$\begin{array}{llllllll}\text { total } & 694 & 6.9400 \mathrm{E}-02 & 1.7315 \mathrm{E}-02 & \text { total } & 694 & 6.9400 \mathrm{E}-02 & 1.7315 \mathrm{E}-02\end{array}$

number of photons banked 694 average lifetime, shakes cutoffs

photon tracks per source particle $6.9400 \mathrm{E}-02$ escape $6.7786 \mathrm{E}-05$ tco $1.0000 \mathrm{E}+34$

photon collisions per source particle $1.3000 \mathrm{E}-02$ capture $8.3325 \mathrm{E}-05$ eco $1.0000 \mathrm{E}-01$

$\begin{array}{llllll}\text { total photon collisions } & 130 \quad \text { capture or escape } 7.0450 \mathrm{E}-05 & \mathrm{wcl} & 0.0000 \mathrm{E}+00\end{array}$

any termination $7.0450 \mathrm{E}-05$ wc2 $0.0000 \mathrm{E}+00$

0

electron creation tracks weight energy electron loss tracks weight energy (per source particle)

source

$10000 \quad 1.0000 \mathrm{E}+00$

$1.0000 \mathrm{E}+00$

(per source particle) energy cutoff $2046 \quad 2.0460 \mathrm{E}-01 \quad 2.0141 \mathrm{E}-02$

time cutoff 00.0.

weight window

$\begin{array}{lll}0 & 0 . & 0 .\end{array}$

weight window

$\begin{array}{ll}0 & 0\end{array}$

cell importance

weight cutoff

energy importance

$\begin{array}{lll}0 & 0 . & 0\end{array}$

cell importance

00 .

weight cutoff $\quad \begin{array}{lll}0 & 0 .\end{array}$

$\begin{array}{lll}0 & 0 . & 0 .\end{array}$

energy importance

0

scattering

$0 \quad 0$.

impton recoil

$\begin{array}{ll}0 & 0 .\end{array}$

0.

bremsstrahlung

$3.7815 \mathrm{E}-05$

4.7387E-04

$29 \quad 2.9000 \mathrm{E}-03$ 


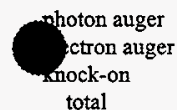

$\begin{array}{lll}0 & 0 . & 0 . \\ 0 & 0 . & 0 .\end{array}$

$1479 \quad 1.4790 \mathrm{E}-01 \quad 2.7007 \mathrm{E}-02$

$\begin{array}{lll}11510 & 1.1510 \mathrm{E}+00 \quad 1.0275 \mathrm{E}+00\end{array}$

number of electrons banked $\quad 1510$

electron tracks per source particle $1.1510 \mathrm{E}+00$

electron sub-steps per source particle 7.7164E+01

total electron sub-steps $\quad 771636$

computer time so far in this run 0.00 minutes

computer time in mcrun

0.00 minutes

source particles per minute

$0.0000 \mathrm{E}+00$

4748878 most rand
$.0000 \mathrm{E}+00$ to $1.0000 \mathrm{E}+00$

HNF-SD-SNF-CSWD-005, Rev. 0

random numbers generated

total

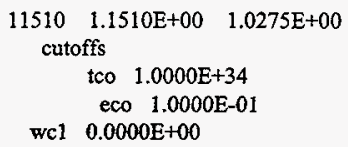

maximum number ever in bank 4

bank overflows to backup file 0

field length 0

most random numbers used was 4277 in history 7375

range of sampled source weights $=1.0000 \mathrm{E}+00$ to $1.0000 \mathrm{E}+00$

Difo21

Comparing files INP21O and outp21

FC: no differences encountered

Difo22

Comparing files INP22O and outp22

FC: no differences encountered

Difo23

Comparing files INP230 and outp23

no differences encountered

Difo24

Comparing files INP24O and outp24

****** INP24O

cycle $3 \mathrm{k}$ (collision) 0.551862 removal lifetime(abs) $3.8422 \mathrm{E}+03$ source points generated 253

estimator cycle 4 ave of 2 cycles combination simple average combined average corr

****** outp24

cycle $3 \mathrm{k}$ (collision) 0.551862 removal lifetime(abs) $3.8422 \mathrm{E}+03$ source points generated 253

source distribution written to file inp24s $\quad$ cycle $=3$

estimator cycle 4 ave of 2 cycles combination simple average combined average corr

$* * * * * *$

****** INP240

tally type 6 track length estimate of heating. units mev/gram

tally for neutrons

****** outp24

tally type 6 track length estimate of heating. units mev/gram

******* tally for neutrons

\section{Difo25}

Comparing files INP250 and outp25

FC: no differences encountered 


\section{omputer System ID : 0012358}

Difm01

Comparing files $\mathrm{INP} 01 \mathrm{M}$ and met101

FC: no differences encountered

Difm02

Comparing files INP02M and mct 102

FC: no differences encountered

\section{Difm03}

Comparing files INP03M and met103 FC: no differences encountered

\section{Difm04}

Comparing files INP04M and met104

FC: no differences encountered

Difm05

Comparing files INP05M and mcti05

FC: no differences encountered

\section{Difm06}

Comparing files INP06M and mct106 FC: no differences encountered

Comparing files INP07M and mct107

FC: no differences encountered

\section{Difm08}

Comparing files INP08M and met108 FC: no differences encountered

Difm09

Comparing files INP09M and meti09

FC: no differences encountered

Difm10

Comparing files INP10M and met110

FC: no differences encountered

Difm11

Comparing files INP11M and motl11 FC: no differences encountered

Difm12

Comparing files INP12M and mctl12 FC: no differences encountered

\section{Difm13}

mparing files INP13M and mctl13 no differences encountered 
mparing files INP $14 \mathrm{M}$ and met114
FC: no differences encountered

Difm15

Comparing files INP15M and mcti15

FC: no differences encountered

Difm16

Comparing files INP16M and motl16

FC: no differences encountered

\section{Difm17}

Comparing files INP17M and motl17

FC: no differences encountered

\section{Difm18}

Comparing files INP18M and motl18

FC: no đifferences encountered

\section{Difn19}

Comparing files INP19M and motl19

FC: no differences encountered

\section{Difm20}

File(s) not found : inp20m

$\min 21$

Comparing files INP21M and mctl21

FC: no differences encountered

\section{Difin22}

Comparing files INP22M and mct122

FC: no differences encountered

\section{Difm23}

Comparing files INP23M and met123

FC: no differences encountered

\section{Difm24}

Comparing files INP24M and mctl24

FC: no differences encountered

\section{Difm25}

Comparing files INP25M and mcti25

FC: no differences encountered

Difo01

Comparing files INP01O and outp01 FC: no differences encountered 
FC: no differences encountered

\section{Difo04}

Comparing files INP04O and outp04 FC: no differences encountered

\section{Difo05}

Comparing files INP05O and outp05 FC: no differences encountered

\section{Difo06}

Comparing files INP06O and outp06 FC: no differences encountered

\section{Difo07}

Comparing files INP07O and outp07

FC: no differences encountered

\section{Difo08}

Comparing files INP08O and outp08 FC: no differences encountered

Difo09

nomparing files INP09O and outp09 no differences encountered

Difolo

Comparing files INP10O and outp10

FC: no differences encountered

\section{Difol1}

Comparing files INP11O and outp1 1 FC: no differences encountered

\section{Difol2}

Comparing files INP12O and outp12 FC: no differences encountered

\section{Difo13}

Comparing files INP13O and outp13 FC: no differences encountered

\section{Difo14}

Comparing files INP 140 and outp14 FC: no differences encountered

\section{Difol5}

Comparing files INP15O and outp15 $F C$ : no differences encountered 
Difo17

Comparing files INP17O and outp17

FC: no differences encountered

Difo18

Comparing files NNP180 and outp18

******* INP180

cycle $5 \mathrm{k}$ (collision) 1.135994 removal lifetime(abs) $1.1136 \mathrm{E}+01$ source points generated 222

cycle $6 \mathrm{k}$ (collision) 1.077014 removal lifetime(abs) $1.2222 \mathrm{E}+01$ source points generated 204

****** outp 18

cycle $5 \mathrm{k}$ (collision) 1.135994 removal lifetime(abs) $1.1136 \mathrm{E}+01$ source points generated 222 source distribution written to file inp $18 \mathrm{~s} \quad$ cycle $=5$

cycle $6 \mathrm{k}$ (collision) 1.077014 removal lifetime(abs) $1.2222 \mathrm{E}+01$ source points generated 204

*******

Difo19

Comparing files INP19O and outp19

FC: no differences encountered

\section{Difo 20}

Comparing files INP20O and outp20

****** NNP200

******* outp 20

$\begin{array}{lll}8 & 0.000 \mathrm{E}+00 \quad 0.000 \mathrm{E}+00 \quad 0.000 \mathrm{E}+00\end{array}$

$90.000 \mathrm{E}+00 \quad 0.000 \mathrm{E}+00 \quad 0.000 \mathrm{E}+00$

$10 \quad 0.000 \mathrm{E}+00 \quad 0.000 \mathrm{E}+00 \quad 0.000 \mathrm{E}+00$

$110.000 \mathrm{E}+00 \quad 0.000 \mathrm{E}+00 \quad 0.000 \mathrm{E}+00$

$12 \quad 0.000 \mathrm{E}+00 \quad 0.000 \mathrm{E}+00 \quad 0.000 \mathrm{E}+00$

$\begin{array}{lllll}13 & 0.000 \mathrm{E}+00 & 0.000 \mathrm{E}+00 & 0.000 \mathrm{E}+00\end{array}$

$14 \quad 0.000 \mathrm{E}+00 \quad 0.000 \mathrm{E}+00 \quad 0.000 \mathrm{E}+00$

$15 \quad 0.000 \mathrm{E}+00 \quad 0.000 \mathrm{E}+00 \quad 0.000 \mathrm{E}+00$

$16 \quad 0.000 \mathrm{E}+00 \quad 0.000 \mathrm{E}+00 \quad 0.000 \mathrm{E}+00$

$17 \quad 0.000 \mathrm{E}+00 \quad 0.000 \mathrm{E}+00 \quad 0.000 \mathrm{E}+00$

$\begin{array}{lllll}18 & 0.000 \mathrm{E}+00 & 0.000 \mathrm{E}+00 & 0.000 \mathrm{E}+00\end{array}$

$19 \quad 0.000 \mathrm{E}+00 \quad 0.000 \mathrm{E}+00 \quad 0.000 \mathrm{E}+00$

$20 \quad 0.000 \mathrm{E}+00 \quad 0.000 \mathrm{E}+00 \quad 0.000 \mathrm{E}+00$

$21 \quad 0.000 \mathrm{E}+00 \quad 0.000 \mathrm{E}+00 \quad 0.000 \mathrm{E}+00$

$220.000 \mathrm{E}+00 \quad 0.000 \mathrm{E}+00 \quad 0.000 \mathrm{E}+00$

$23 \quad 0.000 \mathrm{E}+00 \quad 0.000 \mathrm{E}+00 \quad 0.000 \mathrm{E}+00$

$240.000 \mathrm{E}+00 \quad 0.000 \mathrm{E}+00 \quad 0.000 \mathrm{E}+00$

$\begin{array}{lllll}25 & 0.000 \mathrm{E}+00 & 0.000 \mathrm{E}+00 & 0.000 \mathrm{E}+00\end{array}$

$26 \quad 0.000 \mathrm{E}+00 \quad 0.000 \mathrm{E}+00 \quad 0.000 \mathrm{E}+00$

$27 \quad 0.000 \mathrm{E}+00 \quad 0.000 \mathrm{E}+00 \quad 0.000 \mathrm{E}+00$

$28 \quad 0.000 \mathrm{E}+00 \quad 0.000 \mathrm{E}+00 \quad 0.000 \mathrm{E}+00$

$\begin{array}{lll}29 & 0.000 \mathrm{E}+00 \quad 0.000 \mathrm{E}+00 \quad 0.000 \mathrm{E}+00\end{array}$

$\begin{array}{llll}30 & 0.000 \mathrm{E}+00 & 0.000 \mathrm{E}+00 & 0.000 \mathrm{E}+00\end{array}$

$310.000 \mathrm{E}+00 \quad 0.000 \mathrm{E}+00 \quad 0.000 \mathrm{E}+00$

$32 \quad 0.000 \mathrm{E}+00 \quad 0.000 \mathrm{E}+00 \quad 0.000 \mathrm{E}+00$

$33 \quad 0.000 \mathrm{E}+00 \quad 0.000 \mathrm{E}+00 \quad 0.000 \mathrm{E}+00$

$34 \quad 0.000 \mathrm{E}+00 \quad 0.000 \mathrm{E}+00 \quad 0.000 \mathrm{E}+00$

$\begin{array}{llll}35 & 0.000 \mathrm{E}+00 \quad 0.000 \mathrm{E}+00 & 0.000 \mathrm{E}+00\end{array}$

$36 \quad 0.000 \mathrm{E}+00 \quad 0.000 \mathrm{E}+00 \quad 0.000 \mathrm{E}+00$
$1 \quad 1-3.604 \mathrm{E}-01 \quad 4.799 \mathrm{E}-01 \quad 7.999 \mathrm{E}-01 \quad 1.000 \mathrm{E}+00 \quad 1.000 \mathrm{E}+00 \quad 0.000 \mathrm{E}+00$

$1 \quad 1-2.241 \mathrm{E}-01 \quad 3.960 \mathrm{E}-01 \quad 8.905 \mathrm{E}-01 \quad 1.000 \mathrm{E}+00 \quad 1.000 \mathrm{E}+00 \quad 0.000 \mathrm{E}+00$

$1 \quad 1-2.928 \mathrm{E}-01-7.352 \mathrm{E}-01 \quad 6.114 \mathrm{E}-01 \quad 1.000 \mathrm{E}+00 \quad 1.000 \mathrm{E}+00 \quad 0.000 \mathrm{E}+00$

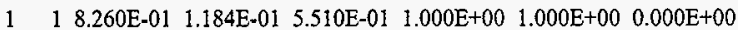

$1 \quad 1 \quad 7.540 \mathrm{E}-01-1.976 \mathrm{E}-01 \quad 6.264 \mathrm{E}-01 \quad 1.000 \mathrm{E}+00 \quad 1.000 \mathrm{E}+00 \quad 0.000 \mathrm{E}+00$

$1 \quad 1 \quad 8.140 \mathrm{E}-01-3.427 \mathrm{E}-01 \quad 4.690 \mathrm{E}-01 \quad 1.000 \mathrm{E}+00 \quad 1.000 \mathrm{E}+00 \quad 0.000 \mathrm{E}+00$

$1 \quad 1-1.257 \mathrm{E}-01 \quad 8.038 \mathrm{E}-01 \quad 5.814 \mathrm{E}-01 \quad 1.000 \mathrm{E}+00 \quad 1.000 \mathrm{E}+00 \quad 0.000 \mathrm{E}+00$

$1 \quad 1-1.464 \mathrm{E}-01-1.070 \mathrm{E}-01 \quad 9.834 \mathrm{E}-01 \quad 1.000 \mathrm{E}+00 \quad 1.000 \mathrm{E}+00 \quad 0.000 \mathrm{E}+00$

$1 \quad 1 \quad 1.310 \mathrm{E}-02-1.617 \mathrm{E}-02 \quad 9.998 \mathrm{E}-01 \quad 1.000 \mathrm{E}+00 \quad 1.000 \mathrm{E}+00 \quad 0.000 \mathrm{E}+00$

$1 \quad 1-8.843 \mathrm{E}-01-4.126 \mathrm{E}-01 \quad 2.187 \mathrm{E}-01 \quad 1.000 \mathrm{E}+00 \quad 1.000 \mathrm{E}+00 \quad 0.000 \mathrm{E}+00$

$1 \quad 14.260 \mathrm{E}-01 \quad 4.153 \mathrm{E}-01 \quad 8.038 \mathrm{E}-01 \quad 1.000 \mathrm{E}+00 \quad 1.000 \mathrm{E}+00 \quad 0.000 \mathrm{E}+00$

$1 \quad 1-7.311 \mathrm{E}-01-1.136 \mathrm{E}-02 \quad 6.822 \mathrm{E}-01 \quad 1.000 \mathrm{E}+00 \quad 1.000 \mathrm{E}+00 \quad 0.000 \mathrm{E}+00$

$1 \quad 1-2.370 \mathrm{E}-01 \quad 3.207 \mathrm{E}-01 \quad 9.171 \mathrm{E}-01 \quad 1.000 \mathrm{E}+00 \quad 1.000 \mathrm{E}+00 \quad 0.000 \mathrm{E}+00$

1 I $4.276 \mathrm{E}-01-2.485 \mathrm{E}-02 \quad 9.036 \mathrm{E}-01 \quad 1.000 \mathrm{E}+00 \quad 1.000 \mathrm{E}+00 \quad 0.000 \mathrm{E}+00$

$1 \quad 1 \quad 7.662 \mathrm{E}-01 \quad 7.908 \mathrm{E}-02 \quad 6.378 \mathrm{E}-01 \quad 1.000 \mathrm{E}+00 \quad 1.000 \mathrm{E}+00 \quad 0.000 \mathrm{E}+00$

$1 \quad 1-4.247 \mathrm{E}-01 \quad 2.688 \mathrm{E}-01 \quad 8.645 \mathrm{E}-01 \quad 1.000 \mathrm{E}+00 \quad 1.000 \mathrm{E}+00 \quad 0.000 \mathrm{E}+00$

$\begin{array}{llllllll}1 & 1 & 2.096 \mathrm{E}-01 & 5.730 \mathrm{E}-01 & 7.923 \mathrm{E}-01 & 1.000 \mathrm{E}+00 & 1.000 \mathrm{E}+00 & 0.000 \mathrm{E}+00\end{array}$

$1 \quad 1-2.665 \mathrm{E}-03-5.270 \mathrm{E}-01 \quad 8.499 \mathrm{E}-01 \quad 1.000 \mathrm{E}+00 \quad 1.000 \mathrm{E}+00 \quad 0.000 \mathrm{E}+00$

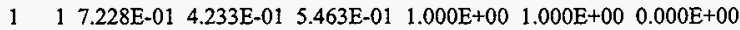

$1 \quad 1 \quad 3.484 \mathrm{E}-01-3.564 \mathrm{E}-01 \quad 8.669 \mathrm{E}-01 \quad 1.000 \mathrm{E}+00 \quad 1.000 \mathrm{E}+00 \quad 0.000 \mathrm{E}+00$

$\begin{array}{llllllll}1 & 1 & 8.706 \mathrm{E}-01 & -3.198 \mathrm{E}-01 & 3.738 \mathrm{E}-01 & 1.000 \mathrm{E}+00 & 1.000 \mathrm{E}+00 & 0.000 \mathrm{E}+00\end{array}$

$\begin{array}{llllllll}1 & 1-8.188 \mathrm{E}-01 & 2.059 \mathrm{E}-01 & 5.359 \mathrm{E}-01 & 1.000 \mathrm{E}+00 & 1.000 \mathrm{E}+00 & 0.000 \mathrm{E}+00\end{array}$

$1 \quad 1 \quad 5.727 \mathrm{E}-01 \quad 8.124 \mathrm{E}-01 \quad 1.100 \mathrm{E}-01 \quad 1.000 \mathrm{E}+00 \quad 1.000 \mathrm{E}+00 \quad 0.000 \mathrm{E}+00$

$1 \quad 1 \quad 5.390 \mathrm{E}-02 \quad 5.914 \mathrm{E}-01 \quad 8.046 \mathrm{E}-01 \quad 1.000 \mathrm{E}+00 \quad 1.000 \mathrm{E}+00 \quad 0.000 \mathrm{E}+00$

$1 \quad 1-5.778 \mathrm{E}-01 \quad 5.375 \mathrm{E}-01 \quad 6.141 \mathrm{E}-01 \quad 1.000 \mathrm{E}+00 \quad 1.000 \mathrm{E}+00 \quad 0.000 \mathrm{E}+00$

$1 \quad 1-7.198 \mathrm{E}-01-2.890 \mathrm{E}-01 \quad 6.311 \mathrm{E}-01 \quad 1.000 \mathrm{E}+00 \quad 1.000 \mathrm{E}+00 \quad 0.000 \mathrm{E}+00$

$1 \quad 1 \quad 2.210 \mathrm{E}-01 \quad 2.482 \mathrm{E}-01 \quad 9.432 \mathrm{E}-01 \quad 1.000 \mathrm{E}+00 \quad 1.000 \mathrm{E}+00 \quad 0.000 \mathrm{E}+00$

$1 \quad 1 \quad 4.339 \mathrm{E}-01 \quad 7.515 \mathrm{E}-02 \quad 8.978 \mathrm{E}-01 \quad 1.000 \mathrm{E}+00 \quad 1.000 \mathrm{E}+00 \quad 0.000 \mathrm{E}+00$

$1 \quad 1-4.263 \mathrm{E}-01 \quad 8.490 \mathrm{E}-01 \quad 3.123 \mathrm{E}-01 \quad 1.000 \mathrm{E}+00 \quad 1.000 \mathrm{E}+00 \quad 0.000 \mathrm{E}+00$ 


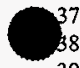
$37 \quad 0.000 \mathrm{E}+00 \quad 0.000 \mathrm{E}+00 \quad 0.000 \mathrm{E}+00$ $38 \quad 0.000 \mathrm{E}+00 \quad 0.000 \mathrm{E}+00 \quad 0.000 \mathrm{E}+00$ $39 \quad 0.000 \mathrm{E}+00 \quad 0.000 \mathrm{E}+00 \quad 0.000 \mathrm{E}+00$ $40 \quad 0.000 \mathrm{E}+00 \quad 0.000 \mathrm{E}+00 \quad 0.000 \mathrm{E}+00$ $41 \quad 0.000 \mathrm{E}+00 \quad 0.000 \mathrm{E}+00 \quad 0.000 \mathrm{E}+00$ $42 \quad 0.000 \mathrm{E}+00 \quad 0.000 \mathrm{E}+00 \quad 0.000 \mathrm{E}+00$ $\begin{array}{llll}43 & 0.000 \mathrm{E}+00 & 0.000 \mathrm{E}+00 & 0.000 \mathrm{E}+00\end{array}$ $44 \quad 0.000 \mathrm{E}+00 \quad 0.000 \mathrm{E}+00 \quad 0.000 \mathrm{E}+00$ $45 \quad 0.000 \mathrm{E}+00 \quad 0.000 \mathrm{E}+00 \quad 0.000 \mathrm{E}+00$ $46 \quad 0.000 \mathrm{E}+00 \quad 0.000 \mathrm{E}+00 \quad 0.000 \mathrm{E}+00$ $47 \quad 0.000 \mathrm{E}+00 \quad 0.000 \mathrm{E}+00 \quad 0.000 \mathrm{E}+00$ $48 \quad 0.000 \mathrm{E}+00 \quad 0.000 \mathrm{E}+00 \quad 0.000 \mathrm{E}+00$ $49 \quad 0.000 \mathrm{E}+00 \quad 0.000 \mathrm{E}+00 \quad 0.000 \mathrm{E}+00$

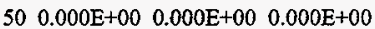
problem summary

rin terminated when 10000 particle histories were done. 0

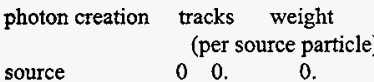

escape energy cutoff time cutoff
$1-3.933 \mathrm{E}-01-9.179 \mathrm{E}-01 \quad 5.266 \mathrm{E}-02 \quad 1.000 \mathrm{E}+00 \quad 1.000 \mathrm{E}+00 \quad 0.000 \mathrm{E}+00$

$\begin{array}{llllll}1-4.846 \mathrm{E}-01 & 4.378 \mathrm{E}-01 & 7.573 \mathrm{E}-01 & 1.000 \mathrm{E}+00 & 1.000 \mathrm{E}+00 & 0.000 \mathrm{E}+00\end{array}$

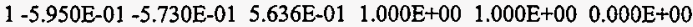

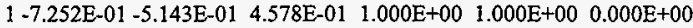

$\begin{array}{lllllll}1-7.156 \mathrm{E}-01 & 4.734 \mathrm{E}-01 & 5.136 \mathrm{E}-01 & 1.000 \mathrm{E}+00 & 1.000 \mathrm{E}+00 & 0.000 \mathrm{E}+00\end{array}$

$1-1.771 \mathrm{E}-01 \quad 9.229 \mathrm{E}-01 \quad 3.417 \mathrm{E}-01 \quad 1.000 \mathrm{E}+00 \quad 1.000 \mathrm{E}+00 \quad 0.000 \mathrm{E}+00$

$\begin{array}{llllll}1-1.149 \mathrm{E}-01 & -4.206 \mathrm{E}-01 & 8.999 \mathrm{E}-01 & 1.000 \mathrm{E}+00 & 1.000 \mathrm{E}+00 & 0.000 \mathrm{E}+00\end{array}$

$14.509 \mathrm{E}-01 \quad 8.630 \mathrm{E}-02 \quad 8.884 \mathrm{E}-01 \quad 1.000 \mathrm{E}+00 \quad 1.000 \mathrm{E}+00 \quad 0.000 \mathrm{E}+00$

$\begin{array}{llllll}1-7.358 \mathrm{E}-01 & 2.500 \mathrm{E}-01 & 6.294 \mathrm{E}-01 & 1.000 \mathrm{E}+00 & 1.000 \mathrm{E}+00 & 0.000 \mathrm{E}+00\end{array}$

$1-8.448 \mathrm{E}-01 \quad 3.645 \mathrm{E}-01 \quad 3.917 \mathrm{E}-01 \quad 1.000 \mathrm{E}+00 \quad 1.000 \mathrm{E}+00 \quad 0.000 \mathrm{E}+00$

$1 \quad 1.958 \mathrm{E}-01-2.769 \mathrm{E}-01 \quad 9.407 \mathrm{E}-01 \quad 1.000 \mathrm{E}+00 \quad 1.000 \mathrm{E}+00 \quad 0.000 \mathrm{E}+00$

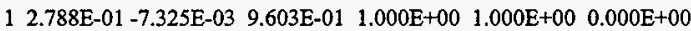

$\begin{array}{llllll}1-3.201 \mathrm{E}-01 & 4.174 \mathrm{E}-01 & 8.505 \mathrm{E}-01 & 1.000 \mathrm{E}+00 & 1.000 \mathrm{E}+00 & 0.000 \mathrm{E}+00\end{array}$

1 8.960E-01 -1.641E-01 4.126E-01 $1.000 \mathrm{E}+00 \quad 1.000 \mathrm{E}+00 \quad 0.000 \mathrm{E}+00$ weight window

cell importance

weight cutoff

energy importance ran

wrced collisions

exp. transform

from neutrons

bremsstrahlung

p-annihilation

electron $\mathrm{x}$-rays

1 st fluorescence

2nd fluorescence

$0 \quad 0$.

0 .

00.

0.

00.

0.

$\begin{array}{lll}0 & 0 . & 0 .\end{array}$

$\begin{array}{lll}0 & 0 . & 0\end{array}$

$\begin{array}{lll}0 & 0 . & 0 .\end{array}$

$694 \quad 6.9400 \mathrm{E}-02$

$\begin{array}{lll}0 & 0 . & 0 .\end{array}$

$0 \quad 0$.

0.

$\begin{array}{lll}0 & 0 . & 0 .\end{array}$

00.

$694 \quad 6.9400 \mathrm{E}-02 \quad 1.7315 \mathrm{E}-02$

694

total

$694 \quad 6.9400 \mathrm{E}-02 \quad 1.7315 \mathrm{E}-02$

number of photons banked

photon tracks per source particle

$6.9400 \mathrm{E}-02$

average lifetis

escape

capture

130

capture

total photon collisions

any termination $7.0450 \mathrm{E}-05$

cutoffs

tco $1.0000 \mathrm{E}+34$

eco $1.0000 \mathrm{E}-01$

wc1 $0.0000 \mathrm{E}+00$

wc2 $0.0000 \mathrm{E}+00$

$\begin{array}{lll}0 & 0 . & 0 .\end{array}$

0.0. tracks weight energy (per source particle)

0

electron creation tracks weight energy electron loss tracks weight energy

(per source particle)

source

$10000 \quad 1.0000 \mathrm{E}+00$

$1.0000 \mathrm{E}+00$

(per source particle)

energy cutoff $\quad 2046 \quad 2.0460 \mathrm{E}-01 \quad 2.0141 \mathrm{E}-02$

time cutoff $\quad 0 \quad 0.00$.

weight window

$0 \quad 0$.

0 .

weight window

00.

cell importance

00.

0.

cell importance

00.

0.

weight cutoff

energy importance

00.

0 .

00.

0 .

weight cutoff

00.

energy importance

0

scattering 00 .

mpton recoil

0.

2 2.0000E-04

$29 \quad 2.9000 \mathrm{E}-03$

$3.7815 \mathrm{E}-05$
$4.7387 \mathrm{E}-04$

bremsstrahlung

2.6560E-0I

photo-electric

00 .

$2.4158 \mathrm{E}-02$ 


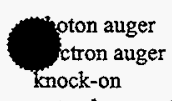

$0 \quad 0$

0.

$\begin{array}{lll}0 & 0 . & 0\end{array}$

$1479 \quad 1.4790 \mathrm{E}-01 \quad 2.7007 \mathrm{E}-02$

$\begin{array}{llll}\text { total } & 11510 & 1.1510 \mathrm{E}+00 & 1.0275 \mathrm{E}+00\end{array}$

number of electrons banked

1510

electron tracks per source particle $1.1510 \mathrm{E}+00$

electron sub-steps per source particle $7.7164 \mathrm{E}+01$

total electron sub-steps

771636

computer time so far in this sun 0.00 minutes

computer time in mcrun $\quad 0.00$ minutes

source particles per minute $\quad 0.0000 \mathrm{E}+00$

random numbers generated $\quad 4748878$

range of sampled source weights $=1.0000 \mathrm{E}+00$ to $1.0000 \mathrm{E}+00$

total

HNF-SD-SNF-CSWD-005, Rev. 0

********

\section{Difo21}

Comparing files INP210 and outp21

FC: no differences encountered

\section{Difo22}

Comparing files INP22O and outp22

FC: no differences encountered

Difo23

Comparing files INP23O and outp23

no differences encountered

\section{Difo24}

Comparing files $\mathrm{NP} 240$ and outp 24

****** INP24O

cycle $3 \mathrm{k}$ (collision) 0.551862 removal lifetime(abs) $3.8422 \mathrm{E}+03$ source points generated 253

estimator cycle 4 ave of 2 cycles combination simple average combined average corr ****** outp 24

cycle $3 \mathrm{k}$ (collision) 0.551862 removal lifetime(abs) $3.8422 \mathrm{E}+03$ source points generated 253 source distribution written to file inp $24 \mathrm{~s}$ cycle $=3$

estimator cycle 4 ave of 2 cycles combination simple average combined average corr

$* * * * * *$

****** INP240

tally type 6 track length estimate of heating. units mev/gram

tally for neutrons

****** outp24

tally type 6 track length estimate of heating. units mev/gram tally for neutrons

Difo25

Comparing files INP25O and outp25

FC: no differences encountered 
HNF-SD-SNF-CSWD-005, Rev. 0

\section{Attachment B}

\section{Full Output for In-Depth Comparison}




\section{Pmputer System ID : 501981-801 056AVO000189 (DOE 6246264)}

\section{File: Inp180}

1- prob18 - kcode in a hexagonal prism lattice.

2- c three half control rods and five whole control rods.

3- $30 \quad 0-905-19291$ fill $=1$

4- $31 \quad 0-906-19291$ fill=1 $(16.711300)$

5- $37 \quad 0-907-1929$ ) fill $=1(-16.711300)$

6. $34 \quad 0-913-1929$ fill $=1(011.91850)$

7- $\quad 32 \quad 0-914-1929$ fill $=1(10.32175 .95920)$

8- $\quad 33 \quad 0-915-1929$ fill $=1(8.3557 \quad 14.47240)$

9- $35 \quad 0.916-1929 \quad$ fill $=1(-8.355714 .47240)$

$10-\quad 36 \quad 0-917-1929$ fill=1 $(-10.32175 .95920)$

11- c universe 1: structure of control rod.

12- $38 \quad 11 \quad-2.02 \quad-880 u=1 \quad \$$ control rod core

13- $39 \quad 6 \quad-8.4 \quad 880-881 \quad u=1 \quad \$$ control rod cladding

14- $40 \quad 12-1.00 \quad 881-882 u=1 \quad \$$ control rod gap

15- $41 \quad 6 \quad-8.4 \quad 882 \quad u=1 \quad \$$ control rod sheath

16- c the space between the control tods, filled with iattice.

17- $140 \quad 0-17129-19905906907913914915916917$ fill $=2$

18. c universe 2: lattice of fuel rods with water in between.

19- $42 \quad 12-1.00 \quad-301 \quad 302-303 \quad 304-305306 \quad u=2 \quad l a t=2$ fill $=$

20- $\quad-37: 27-1: 330: 0$ \&

21- $\quad 24 r 39 r 24 r 311 r 24 r 311+24 r 39 r 2$

22- $\quad 24 r 39 \mathrm{r} 23 \mathrm{r} 312 \mathrm{r} 23 \mathrm{r} 312 \mathrm{r} 23 \mathrm{r} 39 \mathrm{r} 2 \mathrm{rr}$

23- $\quad 23 \mathrm{r} 310 \mathrm{r} 2 \mathrm{r} 313 \mathrm{r} 2 \mathrm{r} 313 \mathrm{r} 2 \mathrm{rr} 310 \mathrm{r} 2 \mathrm{tr}$

24- $23 r 357 \pi 22 \mathrm{r} \&$

25- $\quad 22 \mathrm{r} 358 \mathrm{r} 22 \mathrm{r}$

26- $\quad 22 r 316 r 22 r 317 r 22 r 316 r 23 r$

27- $\quad 22 \mathrm{r} 315 \mathrm{r} 23 \mathrm{r} 316 \mathrm{r} 23 \mathrm{rr} 15 \mathrm{r} 24 \mathrm{r}$

2 1r $315 \mathrm{r} 24 \mathrm{r} 315 \mathrm{r} 24 \mathrm{rr} 315 \mathrm{r} 24 \mathrm{r}$

$21+315 r 23 r 316 r 23 r 315 r 25 r$ $21+315 r 22 r 317 r 22 r 315 r 26 r$

$21+354 r 27+\&$

c can code remember \& thru comment?

$2355 \mathrm{r} 2 \mathrm{rr}$

$2325 \mathrm{r} 22 \mathrm{r} 325 \mathrm{r} 28 \mathrm{r}$

$2324 \mathrm{r} 23 \mathrm{r} 324 \mathrm{r} 29 \mathrm{r}$

$2323 r 24 r 323 r 210 r$

$2315 \mathrm{r} 22 \mathrm{r} 34 \mathrm{r} 23 \mathrm{r} 34 \mathrm{r} 22 \mathrm{r} 315 \mathrm{r} 211 \mathrm{r}$

$2314 r 23 r 34 r 22 r 34 r 23 r 314 r 2$ I 2 r

$2313 \mathrm{r} 24 \mathrm{r} 311 \mathrm{r} 24 \mathrm{r} 313 \mathrm{r} 213 \mathrm{r}$

2313 r $23 r 312 r 23 r 313 r 214 r$

$2313 r 22 r 313 r 22 r 313 r 215 r$

$2346 \mathrm{r} 216 \mathrm{r}$

$2345 \mathrm{r} 217 \mathrm{r}$

2344 r 2 18r

2 1 $341+220 \mathrm{r}$

$2 \operatorname{lr} 340+22$ lr

2 it $339 \mathrm{~T} 222 \mathrm{~T}$

2 2r336r 2 24r

$22 r 335 r 225 r$

$23 \mathrm{r} 332 \mathrm{r} 227 \mathrm{r}$

$24+329 \mathrm{~T} 229 \mathrm{r}$

$25 \mathrm{r} 326 \mathrm{r} 231 \mathrm{r}$

$26 r 323 r 233 r$

$28 \mathrm{r} 318 \mathrm{r} 236 \mathrm{r}$

211 r 311 r $240 \mathrm{r}$

$264 \mathrm{r}$

c universe 3: structure of fuel rod lattice elements.

$\begin{array}{lllll}154 & 2-13.75 & -58 \quad \mathrm{u}=3 & \$ & \text { fuel element }\end{array}$

$149 \quad 12 \quad-1.00 \quad 58-268 \quad u=3 \quad \$ g a p$

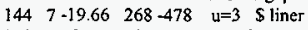

$\begin{array}{llllll}159 & 6 & -8.4 & 478 & -698 \quad u=3 & \text { \$cladding }\end{array}$

$141 \quad 12-1.00 \quad 698 \quad u=3 \quad S$ water between the fuel rods 
162 0 17:-29:19:-1 Soutside world

$* 1$ py $0 \quad \$ x-z$ plane, reflective

17 cz 29.135

19 pz $31.75 \$$ top of reactor

$29 \mathrm{pz}-31.75$ \$ bottom of reactor

$\begin{array}{lllll}58 & \mathrm{c} / 2 & 3.4414 & .8515 & .3240\end{array}$

$\begin{array}{lllll}268 & \mathrm{c} / \mathrm{z} & 3.4414 & .8515 & .3345\end{array}$

$\begin{array}{llllll}478 & \mathrm{c} / 2 & 3.4414 & .8515 & .3475\end{array}$

$\begin{array}{llllll}698 & \mathrm{c} / \mathrm{z} & 3.4414 & .8515 & .4318\end{array}$

$880 \mathrm{cz} 1.7251$

$881 \mathrm{cz} 1.8051$

$882 \mathrm{cz} 1.9051$

905 cz 2.1055

$906 \mathrm{c} / \mathrm{z} \quad 16.711302 .1055$

$907 \mathrm{c} / 2-16.711302 .1055$

$913 \mathrm{c} / \mathrm{z} \quad 011.91852 .1055$

$914 \mathrm{c} / \mathrm{z} \quad 10.32175 .95922 .1055$

$915 \mathrm{c} / \mathrm{z} \quad 8.355714 .47242 .1055$

$916 \mathrm{c} / \mathrm{z}-8.3557 \quad 14.47242 .1055$

$917 \mathrm{c} / \mathrm{z}-10.32175 .95922 .1055$

$301 \mathrm{px} 3.9330$

$302 \mathrm{px} 2.9498$

303 p 11.732050807605 .8994

304 p 11.732050807603 .9330

305 p $-11.73205080760-.9834$

306 p $-11.73205080760-2.9498$

imp:n 1 18r 0

$\begin{array}{lllllll}m 2 & 92235.40 c & -.70573 & 92238.40 c & -.23821 & 7014.40 c & -.05605\end{array}$

m6 $\quad 41093.40 c-.99000 \quad 40000.40 c-.01000$

m7 $74000.40 \mathrm{c}-.74000$

$\mathrm{mll} \quad 5010.03 \mathrm{~d}-.6870 \quad 5011.40 \mathrm{c}-.0840 \quad 6012.40 \mathrm{c}-.2290$

$\mathrm{m} 12 \quad 1001.00 \mathrm{c} 1 \quad 1002.55 \mathrm{cl} \quad 8016.40 \mathrm{cl}$

mt12 hwtr.01 lwtr.01

kcode 200156

waming. tallies are normed per fission neutron for one generation.

99. ksrc $3.2 .2 .23 .2-3.2 .2 .23 .243 .2-43.243 .1-43.2$

100 - e .01 .11 .10 .

101- fq $\mathrm{fe}$

102- fo 4 fuel rod flux in $5 y$ locations averaged over $5 x$ elements

103- f4:n $(154<(42[-10:-6-10]))$ \$ average $5 x$ elements at $j=-1$

104- $\quad(154<(42[-10:-630])) \& \$$ average $5 x$ elements at $j=3$

105- $\quad(154<(42[-10 ;-6100]))$ S average $5 x$ elements at $j=10$ \&

106- $\quad(154<(42[-10:-6210])) \quad \$$ average $5 \times$ elements at $j=21$

107- $\quad(154<(42[-10:-6290])) \$$ average $5 \times$ elements at $j=29$

$108-\quad$ sd $4 \quad 104.70890624 r \quad \$ 5$ times the volume of cell 154

109. print -98

$110-\quad$ prdmp $2 \mathrm{j}-1$

111. ptrac buffer $=20$ file $=$ asc write $=a l l$ event $=$ bnk

I initial source from ksrc card.

print table 90

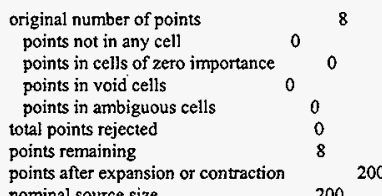

nominal source size

1.000000

initial guess for $k$ (eff.)

5

les to skip before tallying 
tally type 4 track length estimate of particle flux.

tally for neutrons

order of printing: $\mathrm{fe}$

cells $(154<(42[-10:-6-10]))(154<(42[-10:-630]))(154<(42[-10:-6100]))(154<(42[-10:-6210]))(154<(42[-10:-6$ $290]))$

\section{energy bins \\ $0.00000 \mathrm{E}+00$ to $1.00000 \mathrm{E}-02 \mathrm{mev}$ \\ $1.00000 \mathrm{E}-02$ to $1.00000 \mathrm{E}-01 \mathrm{mev}$ \\ $1.00000 \mathrm{E}-01$ to $1.00000 \mathrm{E}+00 \mathrm{mev}$ \\ $1.00000 \mathrm{E}+00$ to $1.00000 \mathrm{E}+01 \mathrm{mev}$ total bin}

Imaterial composition

the sum of the fractions of material 2 was $9.999900 \mathrm{E}-0]$

the stum of the fractions of material 7 was $7.400000 \mathrm{E}-01$

the sum of the fractions of material 12 was $3.000000 \mathrm{E}+00$

material

number component nuclide, atom fraction

$$
\begin{array}{rrr}
92235,0.37504 & 92238,0.12499 & 7014,0.49997 \\
41093,0.98982 & 40000,0.01018 & \\
74000,1.00000 & & \\
5010,0.71977 & 5011,0.08004 & 6012,0.20019 \\
1001,0.33333 & 1002,0.33333 & 8016,0.33333
\end{array}
$$

ociated thermal $s(a, b)$ data sets: hwtr.01t lwtr.01t print table 30

$5 \mathrm{x}$ elements

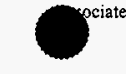

print table 40

material

number component nuclide, mass fraction

$\begin{array}{rrr}92235,0.70574 & 92238,0.23821 & 7014,0.05605 \\ 41093,0.99000 & 40000,0.01000 & \\ 74000,1.00000 & & \\ 5010,0.68700 & 5011,0.08400 & 6012,0.22900 \\ 1001,0.05300 & 1002,0.10591 & 8016,0.84109\end{array}$

waming. 3 of the materials had unnormalized fractions.

lcell volumes and masses

print table 50

cell atom gram input calculated reason volume density density volume volume mass pieces not calculated

$\begin{array}{llllllll} & 30 & 0.00000 \mathrm{E}+00 & 0.00000 \mathrm{E}+00 & 0.00000 \mathrm{E}+00 & 0.00000 \mathrm{E}+00 & 0.00000 \mathrm{E}+00\end{array}$

2 31 $0.00000 \mathrm{E}+00 \quad 0.00000 \mathrm{E}+00 \quad 0.00000 \mathrm{E}+00 \quad 0.00000 \mathrm{E}+00 \quad 0.00000 \mathrm{E}+00$

$\begin{array}{llllllll}37 & 0.00000 \mathrm{E}+00 & 0.00000 \mathrm{E}+00 & 0.00000 \mathrm{E}+00 & 0.00000 \mathrm{E}+00 & 0.00000 \mathrm{E}+00\end{array}$

$\begin{array}{llllllll}4 & 34 & 0.00000 \mathrm{E}+00 & 0.00000 \mathrm{E} \div 00 & 0.00000 \mathrm{E}+00 & 8.84370 \mathrm{E}+02 & 0.00000 \mathrm{E}+00\end{array}$

$\begin{array}{lllllll}5 & 32 & 0.00000 \mathrm{E}+00 & 0.00000 \mathrm{E}+00 & 0.00000 \mathrm{E}+00 & 8.84370 \mathrm{E}+02 & 0.00000 \mathrm{E}+00\end{array}$

$\begin{array}{llllllll}6 & 33 & 0.00000 \mathrm{E}+00 & 0.00000 \mathrm{E}+00 & 0.00000 \mathrm{E}+00 & 8.84370 \mathrm{E}+02 & 0.00000 \mathrm{E}+00\end{array}$

$\begin{array}{llllllll}7 & 35 & 0.00000 \mathrm{E}+00 & 0.00000 \mathrm{E}+00 & 0.00000 \mathrm{E}+00 & 8.84370 \mathrm{E}+02 & 0.00000 \mathrm{E}+00\end{array}$

$\begin{array}{lllllll}8 & 36 & 0.00000 \mathrm{E}+00 \quad 0.00000 \mathrm{E}+00 & 0.00000 \mathrm{E}+00 & 8.84370 \mathrm{E}+02 & 0.00000 \mathrm{E}+00\end{array}$

$\begin{array}{llllllll}9 & 38 & 1.15958 \mathrm{E}-01 & 2.02000 \mathrm{E}+00 & 0.00000 \mathrm{E}+00 & 0.00000 \mathrm{E}+00 & 0.00000 \mathrm{E}+00\end{array}$

$\begin{array}{lllllllll}10 & 39 & 5.44575 \mathrm{E}-02 & 8.40000 \mathrm{E}+00 & 0.00000 \mathrm{E}+00 & 0.00000 \mathrm{E}+00 & 0.00000 \mathrm{E}+00\end{array}$

$\begin{array}{llllllll}11 & 40 & 9.50007 \mathrm{E}-02 & 1.00000 \mathrm{E}+00 & 0.00000 \mathrm{E}+00 & 0.00000 \mathrm{E}+00 & 0.00000 \mathrm{E}+00\end{array}$

$\begin{array}{lllllll}41 & 5.44575 \mathrm{E}-02 & 8.40000 \mathrm{E}+00 & 0.00000 \mathrm{E}+00 & 0.00000 \mathrm{E}+00 & 0.00000 \mathrm{E}+00\end{array}$

$140 \quad 0.00000 \mathrm{E}+00 \quad 0.00000 \mathrm{E}+00 \quad 0.00000 \mathrm{E}+00 \quad 0.00000 \mathrm{E}+00 \quad 0.00000 \mathrm{E}+00$

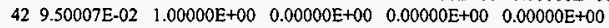

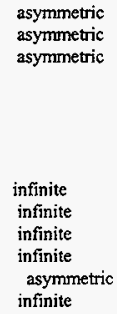




\section{HNF-SD-SNF-CSWD-005, Rev. 0}

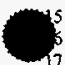

$154 \quad 6.62920 \mathrm{E}-02 \quad 1.37500 \mathrm{E}+01 \quad 0.00000 \mathrm{E}+00 \quad 0.00000 \mathrm{E}+00 \quad 0.00000 \mathrm{E}+00$ $\begin{array}{lllllll}149 & 9.50007 \mathrm{E}-02 & 1.00000 \mathrm{E}+00 & 0.00000 \mathrm{E}+00 & 0.00000 \mathrm{E}+00 & 0.00000 \mathrm{E}+00\end{array}$ $\begin{array}{llllll}144 & 6.43969 \mathrm{E}-02 & 1.96600 \mathrm{E}+01 & 0.00000 \mathrm{E}+00 & 0.00000 \mathrm{E}+00 & 0.00000 \mathrm{E}+00\end{array}$ $\begin{array}{lllllllll}18 & 159 & 5.44575 \mathrm{E}-02 & 8.40000 \mathrm{E}+00 & 0.00000 \mathrm{E}+00 & 0.00000 \mathrm{E}+00 & 0.00000 \mathrm{E}+00\end{array}$ $\begin{array}{llllllllll}19 & 141 & 9.50007 \mathrm{E}-02 & 1.00000 \mathrm{E}+00 & 0.00000 \mathrm{E}+00 & 0.00000 \mathrm{E}+00 & 0.00000 \mathrm{E}+00\end{array}$ $20 \quad 162 \quad 0.00000 \mathrm{E}+00 \quad 0.00000 \mathrm{E}+00 \quad 0.00000 \mathrm{E}+00 \quad 0.00000 \mathrm{E}+00 \quad 0.00000 \mathrm{E}+00$ isurface areas

print table 50

infinite

infinite

infinite

infinite

infinite

0 asymmetric

surface input calculated reason arez

area area not calculated

$1 \quad 10.00000 \mathrm{E}+00 \quad 0.00000 \mathrm{E}+00$ asymmetric

$2170.00000 \mathrm{E}+00 \quad 0.00000 \mathrm{E}+00$ asymmetric

$3 \quad 19 \quad 0.00000 \mathrm{E}+00 \quad 0.00000 \mathrm{E}+00$ asymmetric

$4290.00000 \mathrm{E}+00 \quad 0.00000 \mathrm{E}+00$ asymmetric

$558 \quad 0.00000 \mathrm{E}+00 \quad 0.00000 \mathrm{E}+00$ infinite

$62680.00000 \mathrm{E}+00 \quad 0.00000 \mathrm{E}+00$ infinite

$74780.00000 \mathrm{E}+00 \quad 0.00000 \mathrm{E}+00$ infinite

$86980.00000 \mathrm{E}+00 \quad 0.00000 \mathrm{E}+00$ infinite

$9880 \quad 0.00000 \mathrm{E}+00 \quad 0.00000 \mathrm{E}+00$ infinite

$108810.00000 \mathrm{E}+00 \quad 0.00000 \mathrm{E}+00$ infinite

$11882 \quad 0.00000 \mathrm{E}+00 \quad 0.00000 \mathrm{E}+00$ infinite

$129050.00000 \mathrm{E}+00 \quad 0.00000 \mathrm{E}+00$ asymmetric

$13906 \quad 0.00000 \mathrm{E}+00 \quad 0.00000 \mathrm{E}+00$ asymmetric

$14907 \quad 0.00000 E+00 \quad 0.00000 E+00$ asymmetric

$\begin{array}{lll}15 & 913 & 0.00000 \mathrm{E}+00 \quad 8.40057 \mathrm{E}+02\end{array}$

$16 \quad 914 \quad 0.00000 \mathrm{E}+00 \quad 8.40057 \mathrm{E}+02$

$17 \quad 915 \quad 0.00000 \mathrm{E}+00 \quad 8.40057 \mathrm{E}+02$

$18 \quad 916 \quad 0.00000 \mathrm{E}+00 \quad 8.40057 \mathrm{E}+02$

$19917 \quad 0.00000 \mathrm{E}+00 \quad 8.40057 \mathrm{E}+02$

$203010.00000 \mathrm{E}+00 \quad 0.00000 \mathrm{E}+00$ infinite

$213020.00000 \mathrm{E}+00 \quad 0.00000 \mathrm{E}+00$ infinite

$3030.00000 \mathrm{E}+00 \quad 0.00000 \mathrm{E}+00$ infinite

$3040.00000 E+00 \quad 0.00000 E+00$

$3050.00000 \mathrm{E}+00 \quad 0.00000 \mathrm{E}+00$

$25306 \quad 0.00000 E+00 \quad 0.00000 E+00$

1 cells

print table 60

atom
cell mat density density

l $30 \quad 0 \quad 0.00000 \mathrm{E}+000.00000 \mathrm{E}+000.00000 \mathrm{E}+000.00000 \mathrm{E}+00 \quad 0 \quad 1.0000 \mathrm{E}+00$

$23100.00000 \mathrm{E}+00 \quad 0.00000 \mathrm{E}+000.00000 \mathrm{E}+000.00000 \mathrm{E}+00 \quad 0 \quad 1.0000 \mathrm{E}+00$

$33700.00000 \mathrm{E}+000.00000 \mathrm{E}+000.00000 \mathrm{E}+000.00000 \mathrm{E}+00 \quad 0 \quad 1.0000 \mathrm{E}+00$

$43400.00000 \mathrm{E}+000.00000 \mathrm{E}+00 \quad 8.84370 \mathrm{E}+020.00000 \mathrm{E}+00 \quad 11.0000 \mathrm{E}+00$

$532 \quad 0 \quad 0.00000 \mathrm{E}+000.00000 \mathrm{E}+008.84370 \mathrm{E}+020.00000 \mathrm{E}+00$ 1 $1.0000 \mathrm{E}+00$

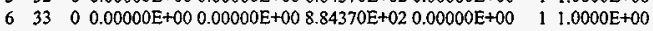

$735 \quad 0 \quad 0.00000 \mathrm{E}+000.00000 \mathrm{E}+008.84370 \mathrm{E}+020.00000 \mathrm{E}+00,1.0000 \mathrm{E}+00$

$836 \quad 0 \quad 0.00000 \mathrm{E}+00 \quad 0.00000 \mathrm{E}+00 \quad 8.84370 \mathrm{E}+020.00000 \mathrm{E}+00 \quad 11.0000 \mathrm{E}+00$

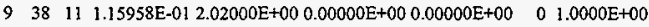

$103965.44575 \mathrm{E}-028.40000 \mathrm{E}+000.00000 \mathrm{E}+000.00000 \mathrm{E}+00 \quad 0 \quad 1.0000 \mathrm{E}+00$

$1140 \quad 12 \mathrm{~s} 9.50007 \mathrm{E}-02 \quad 1.00000 \mathrm{E}+000.00000 \mathrm{E}+000.00000 \mathrm{E}+00 \quad 0 \quad 1.0000 \mathrm{E}+00$

$1241 \quad 6 \quad 5.44575 \mathrm{E}-02 \quad 8.40000 \mathrm{E}+000.00000 \mathrm{E}+000.00000 \mathrm{E}+00 \quad 0 \quad 1.0000 \mathrm{E}+00$

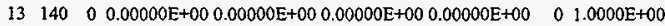

$14 \quad 4212 \mathrm{~s} 9.50007 \mathrm{E}-02 \quad 1.00000 \mathrm{E}+000.00000 \mathrm{E}+000.00000 \mathrm{E}+00 \quad 0 \quad 1.0000 \mathrm{E}+00$

$15 \quad 154 \quad 2 \quad 6.62920 \mathrm{E}-021.37500 \mathrm{E}+010.00000 \mathrm{E}+000.00000 \mathrm{E}+00 \quad 0 \quad 1.0000 \mathrm{E}+00$

$16 \quad 14912 \mathrm{~s} 9.50007 \mathrm{E}-021.00000 \mathrm{E}+00 \quad 0.00000 \mathrm{E}+000.00000 \mathrm{E}+00 \quad 0 \quad 1.0000 \mathrm{E}+00$

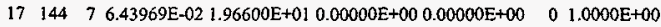

$18 \quad 15965.44575 \mathrm{E}-028.40000 \mathrm{E}+000.00000 \mathrm{E}+000.00000 \mathrm{E}+00 \quad 0 \quad 1.0000 \mathrm{E}+00$

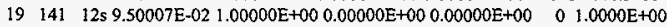

$20 \quad 162 \quad 0 \quad 0.00000 \mathrm{E}+00 \quad 0.00000 \mathrm{E}+00 \quad 0.00000 \mathrm{E}+00 \quad 0.00000 \mathrm{E}+00 \quad 0 \quad 0.0000 \mathrm{E}+00$

total

1surfaces
$4.42185 \mathrm{E}+030.00000 \mathrm{E}+00$

print table 70 
py $0.0000000 \mathrm{E}+00$

cz $2.9135000 \mathrm{E}+01$

pz 3.1750000E+01

pz $-3.1750000 \mathrm{E}+01$

$\mathrm{c} / 2 \quad 3.4414000 \mathrm{E}+00 \quad 8.5150000 \mathrm{E}-01 \quad 3.2400000 \mathrm{E}-01$

c/z $3.4414000 \mathrm{E}+00 \quad 8.5150000 \mathrm{E}-01 \quad 3.3450000 \mathrm{E}-01$

$c / 2 \quad 3.4414000 \mathrm{E}+00 \quad 8.5150000 \mathrm{E}-01 \quad 3.4750000 \mathrm{E}-01$

c/2 $3.4414000 \mathrm{E}+00 \quad 8.5150000 \mathrm{E}-01 \quad 4.3180000 \mathrm{E}-01$

cz $1.7251000 \mathrm{E}+00$

cz $1.8051000 \mathrm{E}+00$

cz $1.9051000 \mathrm{E}+00$

cz $2.1055000 \mathrm{E}+00$

$c / 2 \quad 1.6711300 \mathrm{E}+01 \quad 0.0000000 \mathrm{E}+00 \quad 2.1055000 \mathrm{E}+00$

$\mathrm{c} / 2-1.6711300 \mathrm{E}+01 \quad 0.0000000 \mathrm{E}+00 \quad 2.1055000 \mathrm{E}+00$

$\mathrm{c} / \mathrm{z} \quad 0.0000000 \mathrm{E}+00 \quad 1.1918500 \mathrm{E}+0 \mathrm{l} \quad 2.1055000 \mathrm{E}+00$

c/z $\quad 1.0321700 \mathrm{E}+01 \quad 5.9592000 \mathrm{E}+00 \quad 2.1055000 \mathrm{E}+00$

c/z $8.3557000 \mathrm{E}+00 \quad 1.4472400 \mathrm{E}+01 \quad 2.1055000 \mathrm{E}+00$

$\mathrm{c} / \mathrm{z}-8.3557000 \mathrm{E}+00 \quad 1.4472400 \mathrm{E}+01 \quad 2.1055000 \mathrm{E}+00$

$\mathrm{c} / 2-1.0321700 \mathrm{E}+01 \quad 5.9592000 \mathrm{E}+00 \quad 2.1055000 \mathrm{E}+00$

pX $3.9330000 \mathrm{E}+00$

px $2.9498000 \mathrm{E}+00$

$\begin{array}{llll}\text { p } & 1.0000000 \mathrm{E}+00 & 1.7320508 \mathrm{E}+00 & 0.0000000 \mathrm{E}+00 \quad 5.8994000 \mathrm{E}+00\end{array}$

p $\quad 1.0000000 \mathrm{E}+00 \quad 1.7320508 \mathrm{E}+00 \quad 0.0000000 \mathrm{E}+00 \quad 3.9330000 \mathrm{E}+00$

p $-1.0000000 \mathrm{E}+00 \quad 1.7320508 \mathrm{E}+00 \quad 0.0000000 \mathrm{E}+00 \quad-9.8340000 \mathrm{E}-01$

p $-1.0000000 \mathrm{E}+00 \quad 1.7320508 \mathrm{E}+00 \quad 0.0000000 \mathrm{E}+00 \quad-2.9498000 \mathrm{E}+00$

1 cell temperatures in mev for the free-gas thermal neutron treatment.

print table 72

all non-zero importance cells with materials have a temperature for thermal neutrons of $2.5300 \mathrm{E}-08$ mev.

2 warning messages so far

Icross-section tables

print table 100

HNF-SD-SNF-CSWD-005, Rev. 0

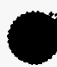

length

tables from file testlibl

I001.00c 1782 I-h-1 from endf-vi.।

$1002.55 \mathrm{c} 4102$ njoy

mat $12505 / 26 / 93$

6011.40 3229 ENDL library name: nd900719 MCNP translation: 900831 14:10:05

$7014.40 \mathrm{c} 8535$ ENDL library name: nd900719 MCNP translation: 900831 14:10:05

8016.40c 5358 ENDL libraty name: nd900719 MCNP translation: 900831 14:10:05

40000.40c 93372 ENDL library name: nd900719 MCNP translation: 900831 14:10:05

$41093.40 \mathrm{c} 42746$ ENDL library name: nd900719 MCNP translation: 900831 14:10:05

74000.40c 91244 ENDL library name: nd900719 MCNP translation: 900831 14:10:05

$92235.40 \mathrm{c} 34772$ ENDL library name: nd900719 MCNP translation: 9total nu 10:05

92238.40c 40834 ENDL library name: nd901 118 MCNP translation: 9total nu 56:49

$5010.03 d 3682$ b-10 endf $\mathrm{b}$-iv new gamma production format

hwtr.01t 10193 deuterium in heavy water at 300 degrees kelvin

oct 75

Jwtr.01t 10193 hyơrogen in light water at 300 degrees kelvin

$10010010 / 22 / 85$

900207

900207

900207

900207

900323

900208

900208

900503

901119

total 354900

waming. neutron energy cutoff is below some cross-section tables.

decimal words of dynamically allocated storage

source distribution written to file inp $18 \mathrm{~s} \quad$ cycle $=0$

3 warning messages so far.

field length $=0 \quad \mathrm{cp} 0=0.00$

print table 110

probl 18 - kcode in a hexagonal prism lattice. 
$13.000 \mathrm{E}+00 \quad 2.000 \mathrm{E}-01 \quad 2.000 \mathrm{E}-01 \quad 140$ $3.492 \mathrm{E}+00 \quad 1.051 \mathrm{E}+00 \quad 2.000 \mathrm{E}-01 \quad 42(0,-1,0)$ $3.492 \mathrm{E}+00 \quad 1.051 \mathrm{E}+00 \quad 2.000 \mathrm{E}-01 \quad 154$ $23.000 \mathrm{E}+00 \quad 2.000 \mathrm{E}-01 \quad 2.000 \mathrm{E}-01 \quad 140$ $3.492 \mathrm{E}+00 \quad 1.051 \mathrm{E}+00 \quad 2.000 \mathrm{E}-01 \quad 42(0,-1,0)$ $3.492 \mathrm{E}+00 \quad 1.051 \mathrm{E}+00 \quad 2.000 \mathrm{E}-01 \quad 154$ 3 3.000E $+00 \quad 2.000 \mathrm{E}-01 \quad 2.000 \mathrm{E}-01 \quad 140$ $3.492 \mathrm{E}+00 \quad 1.051 \mathrm{E}+00 \quad 2.000 \mathrm{E}-01 \quad 42(0,-1,0)$ $3.492 \mathrm{E}+00 \quad 1.051 \mathrm{E}+00 \quad 2.000 \mathrm{E}-01 \quad 154$

4 3.000E+00 2.000E-01 2.000E-01 140 3.492E+00 1.051E+00 2.000E-0) 42( $0,-1,0)$ $3.492 \mathrm{E}+00 \quad 1.051 \mathrm{E}+00 \quad 2.000 \mathrm{E}-01 \quad 154$ $53.000 \mathrm{E}+00 \quad 2.000 \mathrm{E}-01 \quad 2.000 \mathrm{E}-01 \quad 140$ $3.492 \mathrm{E}+00 \quad 1.051 \mathrm{E}+00 \quad 2.000 \mathrm{E}-01 \quad 42(0,-1,0)$ $3.492 \mathrm{E}+00 \quad 1.051 \mathrm{E}+00 \quad 2.000 \mathrm{E}-01 \quad 154$ $63.000 \mathrm{E}+00$ 2.000E-01 2.000E-01 140 $3.492 \mathrm{E}+00 \quad 1.05$ !E+00 2.000E-01 $42(0,-1,0)$ $3.492 \mathrm{E}+00 \quad 1.051 \mathrm{E}+00 \quad 2.000 \mathrm{E}-01 \quad 154$

$\begin{array}{ll}7.000 \mathrm{E}+00 \quad 2.000 \mathrm{E}-01 & 2.000 \mathrm{E}-01 \quad 140\end{array}$ $3.492 \mathrm{E}+00 \quad 1.051 \mathrm{E}+00 \quad 2.000 \mathrm{E}-01 \quad 42(0,-1,0)$ $3.492 \mathrm{E}+00 \quad 1.051 \mathrm{E}+00 \quad 2.000 \mathrm{E}-01 \quad 154$

8 3.000E+00 2.000E-01 2.000E-01 140 $\begin{array}{lll}3.492 \mathrm{E}+00 & 1.051 \mathrm{E}+00 \quad 2.000 \mathrm{E}-01 \quad 42(\end{array}$ $3.492 \mathrm{E}+00 \quad 1.051 \mathrm{E}+00 \quad 2.000 \mathrm{E}-01 \quad 154$ $93.000 \mathrm{E}+00 \quad 2.000 \mathrm{E}-01 \quad 2.000 \mathrm{E}-01 \quad 140$ $3.492 \mathrm{E}+00 \quad 1.053 \mathrm{E}+00 \quad 2.000 \mathrm{E}-01 \quad 42(0,-1,0)$ $3.492 \mathrm{E}+00 \quad 3.051 \mathrm{E}+00 \quad 2.000 \mathrm{E}-01154$

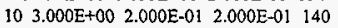
$3.492 \mathrm{E}+00 \quad 1.051 \mathrm{E}+00 \quad 2.000 \mathrm{E}-01 \quad 42(0,-1,0)$ $3.492 \mathrm{E}+00 \quad 1.051 \mathrm{E}+00 \quad 2.000 \mathrm{E}-01 \quad 154$ $3.000 \mathrm{E}+00 \quad 2.000 \mathrm{E}-01 \quad 2.000 \mathrm{E}-01 \quad 140$ $3.492 \mathrm{E}+00 \quad 1.051 \mathrm{E}+00 \quad 2.000 \mathrm{E}-01 \quad 42(0,-1,0)$ $3.492 \mathrm{E}+00 \quad 1.051 \mathrm{E}+00 \quad 2.000 \mathrm{E}-01 \quad 154$ $123.000 \mathrm{E}+00 \quad 2.000 \mathrm{E}-01 \quad 2.000 \mathrm{E}-01 \quad 140$ $3.492 \mathrm{E}+00 \quad 1.051 \mathrm{E}+00 \quad 2.000 \mathrm{E}-01 \quad 42(0,-1,0)$ $3.492 \mathrm{E}+00 \quad 1.051 \mathrm{E}+00 \quad 2.000 \mathrm{E}-01 \quad 154$ $13 \quad 3.000 \mathrm{E}+00 \quad 2.000 \mathrm{E}-01 \quad 2.000 \mathrm{E}-01 \quad 140$ $3.492 \mathrm{E}+00 \quad 3.051 \mathrm{E}+00 \quad 2.000 \mathrm{E}-01 \quad 42(0,-1,0)$ $3.492 \mathrm{E}+00 \quad 1.051 \mathrm{E}+00 \quad 2.000 \mathrm{E}-01 \quad 154$

$143.000 \mathrm{E}+00 \quad 2.000 \mathrm{E}-01 \quad 2.000 \mathrm{E}-01 \quad 140$ $3.492 \mathrm{E}+00 \quad 1.051 \mathrm{E}+00 \quad 2.000 \mathrm{E}-01 \quad 42(0,-1,0)$ $3.492 \mathrm{E}+00 \quad 1.051 \mathrm{E}+00 \quad 2.000 \mathrm{E}-0 \mathrm{I} \quad 154$ $153.000 \mathrm{E}+00 \quad 2.000 \mathrm{E}-01 \quad 2.000 \mathrm{E}-01 \quad 140$ $3.492 \mathrm{E}+00 \quad 1.051 \mathrm{E}+00 \quad 2.000 \mathrm{E}-01 \quad 42($ $3.492 \mathrm{E}+00 \quad 1.051 \mathrm{E}+00 \quad 2.000 \mathrm{E}-01 \quad 154$

$163.000 \mathrm{E}+00 \quad 2.000 \mathrm{E}-01 \quad 2.000 \mathrm{E}-01 \quad 140$ $3.492 \mathrm{E}+00 \quad 1.051 \mathrm{E}+00 \quad 2.000 \mathrm{E}-01 \quad 42(0,-1,0)$ $3.492 \mathrm{E}+00 \quad 1.051 \mathrm{E}+00 \quad 2.000 \mathrm{E}-01 \quad 154$ $173.000 \mathrm{E}+00 \quad 2.000 \mathrm{E}-01 \quad 2.000 \mathrm{E}-01 \quad 140$ $3.492 \mathrm{E}+00 \quad 1.051 \mathrm{E}+00 \quad 2.000 \mathrm{E}-01 \quad 42(0,-1,0)$ $3.492 \mathrm{E}+00 \quad 1.051 \mathrm{E}+00 \quad 2.000 \mathrm{E}-01 \quad 154$

$18 \quad 3.000 \mathrm{E}+00 \quad 2.000 \mathrm{E}-01 \quad 2.000 \mathrm{E}-01 \quad 140$ $3.492 \mathrm{E}+00 \quad 1.051 \mathrm{E}+00 \quad 2.000 \mathrm{E}-01 \quad 42($ $3.492 \mathrm{E}+00 \quad 1.051 \mathrm{E}+00 \quad 2.000 \mathrm{E}-01 \quad 154$

$193.000 \mathrm{E}+00 \quad 2.000 \mathrm{E}-01 \quad 2.000 \mathrm{E}-01 \quad 140$ $3.492 \mathrm{E}+00 \quad 1.051 \mathrm{E}+00 \quad 2.000 \mathrm{E}-01 \quad 42(0,-1,0)$ $3.492 \mathrm{E}+00 \quad 1.051 \mathrm{E}+00 \quad 2.000 \mathrm{E}-01 \quad 154$

$203.000 \mathrm{E}+00 \quad 2.000 \mathrm{E}-01 \quad 2.000 \mathrm{E}-01 \quad 140$ $3.492 \mathrm{E}+00 \quad 1.051 \mathrm{E}+00 \quad 2.000 \mathrm{E}-01 \quad 42(0,-1,0)$ $3.492 \mathrm{E}+00 \quad 1.051 \mathrm{E}+00 \quad 2.000 \mathrm{E}-01 \quad 154$

21 $3.000 \mathrm{E}+00 \quad 2.000 \mathrm{E}-01 \quad 2.000 \mathrm{E}-01 \quad 140$ $3.492 \mathrm{E}+00 \quad 1.051 \mathrm{E}+00 \quad 2.000 \mathrm{E}-01 \quad 42(0,-1,0)$ $492 \mathrm{E}+00 \quad 1.051 \mathrm{E}+00 \quad 2.000 \mathrm{E}=01 \quad 154$ $3.000 \mathrm{E}+00 \quad 2.000 \mathrm{E}-01 \quad 2.000 \mathrm{E}-01 \quad 140$ $\begin{array}{lllll}5.085 \mathrm{E}-01 & 4.733 \mathrm{E}-01 & 7.193 \mathrm{E}-01 & 2.209 \mathrm{E}+00 & 1.000 \mathrm{E}+00 \quad 0.000 \mathrm{E}+00\end{array}$ $5.085 \mathrm{E}-01 \quad 4.733 \mathrm{E}-01 \quad 7.193 \mathrm{E}-01$

0 5.085E-01 4.733E-01 7.193E-01

8.952E-01 -4.447E-01 -2.944E-02 4.904E+00 $1.000 \mathrm{E}+00 \quad 0.000 \mathrm{E}+00$ $8.952 \mathrm{E}-01-4.447 \mathrm{E}-01-2.944 \mathrm{E}-02$

0 8.952E-01 4.447E-01 -2.944E-02

$-6.184 \mathrm{E}-0 \mathrm{I}-4.495 \mathrm{E}-0 \mathrm{l} \quad 6.446 \mathrm{E}-01 \quad 3.809 \mathrm{E}-0 \mathrm{l} \quad 1.000 \mathrm{E}+00 \quad 0.000 \mathrm{E}+00$ $-6.184 \mathrm{E}-01-4.495 \mathrm{E}-01 \quad 6.446 \mathrm{E}-01$

$0-6.184 \mathrm{E}-01-4.495 \mathrm{E}-01 \quad 6.446 \mathrm{E}-01$

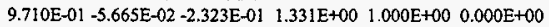
$9.710 \mathrm{E}-01+5.665 \mathrm{E}-02-2.323 \mathrm{E}-01$

0 9.710E-01 $-5.665 \mathrm{E}-02-2.323 \mathrm{E}-01$

$5.861 \mathrm{E}-01 \quad 1.496 \mathrm{E}-01-7.963 \mathrm{E}-01 \quad 1.902 \mathrm{E}+00 \quad 1.000 \mathrm{E}+00 \quad 0.000 \mathrm{E}+00$ $5.861 \mathrm{E}-01 \quad 1.496 \mathrm{E}-01-7.963 \mathrm{E}-01$

0 5.861E-01 1.496E-01 -7.963E-01

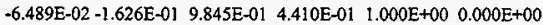
$-6.489 \mathrm{E}-02-1.626 \mathrm{E}-019.845 \mathrm{E}-01$

$0-6.489 \mathrm{E}-02-1.626 \mathrm{E}-019.845 \mathrm{E}-01$

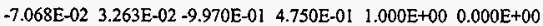
$-7.068 \mathrm{E}-02 \quad 3.263 \mathrm{E}-02-9.970 \mathrm{E}-01$

$0 \cdot 7.068 \mathrm{E}-02 \quad 3.263 \mathrm{E}-02-9.970 \mathrm{E}-01$

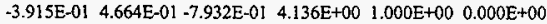
$-3.915 \mathrm{E}-01$ 4.664E-01 -7.932E-01

$0-3.915 \mathrm{E}-01 \quad 4.664 \mathrm{E}-01-7.932 \mathrm{E}-01$

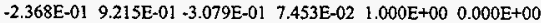
$-2.368 \mathrm{E}-01 \quad 9.215 \mathrm{E}-01-3.079 \mathrm{E}-01$

$0-2.368 \mathrm{E}-01 \quad 9.215 \mathrm{E}-01-3.079 \mathrm{E}-01$

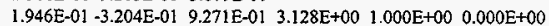
$1.946 \mathrm{E}-01-3.204 \mathrm{E}-019.271 \mathrm{E}-01$

$0 \quad 1.946 \mathrm{E}-01-3.204 \mathrm{E}-01 \quad 9.271 \mathrm{E}-01$

$-6.698 \mathrm{E}-01-7.177 \mathrm{E}-01+1.905 \mathrm{E}-01 \quad 1.014 \mathrm{E}+00 \quad 1.000 \mathrm{E}+00 \quad 0.000 \mathrm{E}+00$ $-6.698 \mathrm{E}-01-7.177 \mathrm{E}-01-1.905 \mathrm{E}-01$

$0-6.698 \mathrm{E}-01-7.177 \mathrm{E}-01-1.905 \mathrm{E}-01$

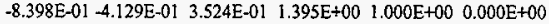
$-8.398 \mathrm{E}-01-4.129 \mathrm{E}-01 \quad 3.524 \mathrm{E}-01$

$0-8.398 \mathrm{E}-01-4.129 \mathrm{E}-01 \quad 3.524 \mathrm{E}-01$

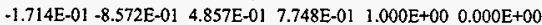
$-1.714 \mathrm{E}-01-8.572 \mathrm{E}-01 \quad 4.857 \mathrm{E}-01$

$0-1.714 \mathrm{E}-01-8.572 \mathrm{E}-01 \quad 4.857 \mathrm{E}-01$

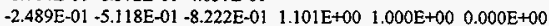
$-2.489 \mathrm{E}-01-5.118 \mathrm{E}-01-8.222 \mathrm{E}-01$

$0-2.489 \mathrm{E}-01-5.118 \mathrm{E}-01-8.222 \mathrm{E}-01$

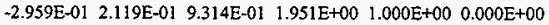
$-2.959 \mathrm{E}-01 \quad 2.119 \mathrm{E}-01 \quad 9.314 \mathrm{E}-01$

$0-2.959 \mathrm{E}-01 \quad 2.119 \mathrm{E}-01 \quad 9.314 \mathrm{E}-01$

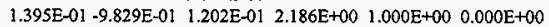
$1.395 \mathrm{E}-01-9.829 \mathrm{E}-01 \quad 1.202 \mathrm{E}-01$

$0 \quad 1.395 \mathrm{E}-01-9.829 \mathrm{E}-01 \quad 1.202 \mathrm{E}-01$ $6.909 \mathrm{E}-01-7.110 \mathrm{E}-01 \quad 1.307 \mathrm{E}-01 \quad 1.865 \mathrm{E}+00 \quad 1.000 \mathrm{E}+00 \quad 0.000 \mathrm{E}+00$ $6.909 \mathrm{E}-01-7.110 \mathrm{E}-01 \quad 1.307 \mathrm{E}-01$

$0 \quad 6.909 \mathrm{E}-01-7.110 \mathrm{E}-01 \quad 1.307 \mathrm{E}-01$

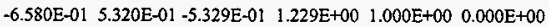
$-6.580 \mathrm{E}-01 \quad 5.320 \mathrm{E}-01-5.329 \mathrm{E}-01$

$0-6.580 \mathrm{E}-01 \quad 5.320 \mathrm{E}-01-5.329 \mathrm{E}-01$ $\begin{array}{llll}-9.903 \mathrm{E}-01 & -1.380 \mathrm{E}-01 & 1.353 \mathrm{E}-02 & 1.305 \mathrm{E}+00 \quad 1.000 \mathrm{E}+00 \quad 0.000 \mathrm{E}+00\end{array}$ $-9.903 \mathrm{E}-01-1.380 \mathrm{E}-01 \quad 1.353 \mathrm{E}-02$

$0-9.903 \mathrm{E}-01-1.380 \mathrm{E}-01 \quad 1.353 \mathrm{E}-02$

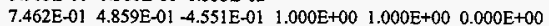
$7.462 \mathrm{E}-01 \quad 4.859 \mathrm{E}-01-4.551 \mathrm{E}-01$

$07.462 \mathrm{E}-01 \quad 4.859 \mathrm{E}-01-4.551 \mathrm{E}-01$

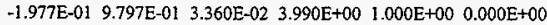
$-1.977 \mathrm{E}-01 \quad 9.797 \mathrm{E}-01 \quad 3.360 \mathrm{E}-02$

$0-1.977 \mathrm{E}-01 \quad 9.797 \mathrm{E}-01 \quad 3.360 \mathrm{E}-02$

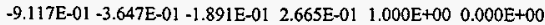


tracks population collisions collisions number flux average average cell entering - weight weighted weighted track weight track mfp (per history) energy energy (relative) (cm)

$\begin{array}{cccccccccccc}1 & 30 & 0 & 0 & 0 & 0.0000 \mathrm{E}+00 & 0.0000 \mathrm{E}+00 & 0.0000 \mathrm{E}+00 & 0.0000 \mathrm{E}+00 & 0.0000 \mathrm{E}+00 \\ 2 & 31 & 0 & 0 & 0 & 0.0000 \mathrm{E}+00 & 0.0000 \mathrm{E}+00 & 0.0000 \mathrm{E}+00 & 0.0000 \mathrm{E}+00 & 0.0000 \mathrm{E}+00 \\ 3 & 37 & 0 & 0 & 0 & 0.0000 \mathrm{E}+00 & 0.0000 \mathrm{E}+00 & 0.0000 \mathrm{E}+00 & 0.0000 \mathrm{E}+00 & 0.0000 \mathrm{E}+00 \\ 4 & 34 & 0 & 0 & 0 & 0.0000 \mathrm{E}+00 & 0.0000 \mathrm{E}+00 & 0.0000 \mathrm{E}+00 & 0.0000 \mathrm{E}+00 & 0.0000 \mathrm{E}+00 \\ 5 & 32 & 0 & 0 & 0 & 0.0000 \mathrm{E}+00 & 0.0000 \mathrm{E}+00 & 0.00000 \mathrm{E}+00 & 0.0000 \mathrm{E}+00 & 0.0000 \mathrm{E}+00 \\ 6 & 33 & 0 & 0 & 0 & 0.0000 \mathrm{E}+00 & 0.0000 \mathrm{E}+00 & 0.0000 \mathrm{E}+00 & 0.0000 \mathrm{E}+00 & 0.0000 \mathrm{E}+00 \\ 7 & 35 & 0 & 0 & 0 & 0.0000 \mathrm{E}+00 & 0.0000 \mathrm{E}+00 & 0.0000 \mathrm{E}+00 & 0.0000 \mathrm{E}+00 & 0.0000 \mathrm{E}+00 \\ 8 & 36 & 0 & 0 & 0 & 0.0000 \mathrm{E}+00 & 0.0000 \mathrm{E}+00 & 0.00000 \mathrm{E}+00 & 0.0000 \mathrm{E}+00 & 0.0000 \mathrm{E}+00 \\ 9 & 38 & 920 & 618 & 1249 & 5.9116 \mathrm{E}-01 & 3.0414 \mathrm{E}-01 & 8.9304 \mathrm{E}-01 & 6.4212 \mathrm{E}-01 & 2.6324 \mathrm{E}+00 \\ 10 & 39 & 1635 & 637 & 130 & 6.3779 \mathrm{E}-02 & 8.8236 \mathrm{E}-02 & 7.6766 \mathrm{E}-01 & 6.4931 \mathrm{E}-01 & 2.6947 \mathrm{E}+00 \\ 11 & 40 & 1728 & 663 & 184 & 8.8176 \mathrm{E}-02 & 7.6162 \mathrm{E}-02 & 7.6184 \mathrm{E}-01 & 6.4437 \mathrm{E}-01 & 2.5146 \mathrm{E}+00 \\ 12 & 41 & 1867 & 712 & 317 & 1.5811 \mathrm{E}-01 & 8.6238 \mathrm{E}-02 & 7.9306 \mathrm{E}-01 & 6.5030 \mathrm{E}-01 & 2.6940 \mathrm{E}+00 \\ 13 & 140 & 0 & 0 & 0 & 0.0000 \mathrm{E}+00 & 0.0000 \mathrm{E}+00 & 0.0000 \mathrm{E}+00 & 0.0000 \mathrm{E}+00 & 0.0000 \mathrm{E}+00 \\ 14 & 42 & 2069 & 867 & 441 & 2.1911 \mathrm{E}-01 & 8.9382 \mathrm{E}-02 & 7.8771 \mathrm{E}-01 & 6.6777 \mathrm{E}-01 & 2.5091 \mathrm{E}+00 \\ 15 & 154 & 25302 & 1253 & 8436 & 4.1390 \mathrm{E}+00 & 1.2606 \mathrm{E}-01 & 8.5005 \mathrm{E}-01 & 6.6920 \mathrm{E}-01 & 2.4712 \mathrm{E}+00 \\ 16 & 149 & 49542 & 1253 & 580 & 2.8963 \mathrm{E}-01 & 1.0795 \mathrm{E}-01 & 8.2361 \mathrm{E}-01 & 6.7129 \mathrm{E}-01 & 2.5408 \mathrm{E}+00 \\ 17 & 144 & 51224 & 1254 & 885 & 4.2652 \mathrm{E}-01 & 1.0541 \mathrm{E}-01 & 8.1599 \mathrm{E}-01 & 6.6971 \mathrm{E}-01 & 2.2391 \mathrm{E}+00 \\ 18 & 159 & 58650 & 1254 & 4019 & 2.1254 \mathrm{E}+00 & 9.8931 \mathrm{E}-02 & 8.1154 \mathrm{E}-01 & 6.6977 \mathrm{E}-01 & 2.7541 \mathrm{E}+00 \\ 19 & 141 & 73122 & 1254 & 6523 & 3.2229 \mathrm{E}+00 & 9.4499 \mathrm{E}-02 & 8.0639 \mathrm{E}-01 & 6.6810 \mathrm{E}-01 & 2.5056 \mathrm{E}+00\end{array}$

total $\quad 266059 \quad 9765 \quad 22764 \quad 1.1324 \mathrm{E}+01$

Ineutron weight balance in each cell -- external events

print table 130

cell entering source energy time exiting total cutoff cutoff

$\begin{array}{lllllll}30 & 0.0000 \mathrm{E}+00 & 0.0000 \mathrm{E}+00 & 0.0000 \mathrm{E}+00 & 0.0000 \mathrm{E}+00 & 0.0000 \mathrm{E}+00 & 0.0000 \mathrm{E}+00\end{array}$ $31 \quad 0.0000 \mathrm{E}+00 \quad 0.0000 \mathrm{E}+00 \quad 0.0000 \mathrm{E}+00 \quad 0.0000 \mathrm{E}+00 \quad 0.0000 \mathrm{E}+00 \quad 0.0000 \mathrm{E}+00$ $\begin{array}{lllllllll}37 & 0.0000 \mathrm{E}+00 & 0.0000 \mathrm{E}+00 & 0.0000 \mathrm{E}+00 & 0.0000 \mathrm{E}+00 & 0.0000 \mathrm{E}+00 & 0.0000 \mathrm{E}+00\end{array}$

$\begin{array}{lllllllll}4 & 34 & 0.0000 \mathrm{E}+00 & 0.0000 \mathrm{E}+00 & 0.0000 \mathrm{E}+00 & 0.0000 \mathrm{E}+00 & 0.0000 \mathrm{E}+00 & 0.0000 \mathrm{E}+00\end{array}$ $\begin{array}{lllllllll}5 & 32 & 0.0000 \mathrm{E}+00 & 0.0000 \mathrm{E}+00 & 0.0000 \mathrm{E}+00 & 0.0000 \mathrm{E}+00 & 0.0000 \mathrm{E}+00 & 0.0000 \mathrm{E}+00\end{array}$ $\begin{array}{lllllllll}6 & 33 & 0.0000 \mathrm{E}+00 & 0.0000 \mathrm{E}+00 & 0.0000 \mathrm{E}+00 & 0.0000 \mathrm{E}+00 & 0.0000 \mathrm{E}+00 & 0.0000 \mathrm{E}+00\end{array}$ $\begin{array}{llllllll}7 & 35 & 0.0000 \mathrm{E}+00 & 0.0000 \mathrm{E}+00 & 0.0000 \mathrm{E}+00 & 0.0000 \mathrm{E}+00 & 0.0000 \mathrm{E}+00 & 0.0000 \mathrm{E}+00\end{array}$

$\begin{array}{llllllllll}8 & 36 & 0.0000 \mathrm{E}+00 & 0.0000 \mathrm{E}+00 & 0.0000 \mathrm{E}+00 & 0.0000 \mathrm{E}+00 & 0.0000 \mathrm{E}+00 & 0.0000 \mathrm{E}+00\end{array}$

$938 \quad 4.8822 \mathrm{E}-01 \quad 0.0000 \mathrm{E}+00 \quad 0.0000 \mathrm{E}+00 \quad 0.0000 \mathrm{E}+00 \quad-3.3091 \mathrm{E}-01 \quad 1.5731 \mathrm{E}-01$

$\begin{array}{llllllll}10 & 39 & 8.4996 \mathrm{E}-01 & 0.0000 \mathrm{E}+00 & 0.0000 \mathrm{E}+00 & 0.0000 \mathrm{E}+00 & -8.4933 \mathrm{E}-01 & 6.2750 \mathrm{E}-04\end{array}$

$\begin{array}{llllllll}11 & 40 & 8.9904 \mathrm{E}-01 & 0.0000 \mathrm{E}+00 & 0.0000 \mathrm{E}+00 & 0.0000 \mathrm{E}+00 & -8.9904 \mathrm{E}-01 & 3.9460 \mathrm{E}-06\end{array}$

$\begin{array}{llllllll}12 & 41 & 9.7260 \mathrm{E}-01 & 0.0000 \mathrm{E}+00 & 0.0000 \mathrm{E}+00 & 0.0000 \mathrm{E}+00 & -9.6978 \mathrm{E}-01 \quad 2.8204 \mathrm{E}-03\end{array}$

$\begin{array}{lllllllll}13 & 140 & 0.0000 \mathrm{E}+00 & 0.0000 \mathrm{E}+00 & 0.0000 \mathrm{E}+00 & 0.0000 \mathrm{E}+00 & 0.0000 \mathrm{E}+00 & 0.0000 \mathrm{E}+00\end{array}$

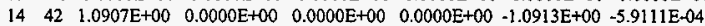

$\begin{array}{lllllllll}15 & 154 & 1.2943 \mathrm{E}+01 & 8.4067 \mathrm{E}-01 & 0.0000 \mathrm{E}+00 & 0.0000 \mathrm{E}+00 & -1.3171 \mathrm{E}+01 & 6.1278 \mathrm{E}-01\end{array}$

$\begin{array}{lllllllll}16 & 149 & 2.6508 \mathrm{E}+01 & 0.0000 \mathrm{E}+00 & 0.0000 \mathrm{E}+00 & 0.0000 \mathrm{E}+00 & -2.6508 \mathrm{E}+01 & 3.5884 \mathrm{E}-05\end{array}$

$\begin{array}{llllllllll}17 & 144 & 2.7419 \mathrm{E}+01 & 0.0000 \mathrm{E}+00 & 0.0000 \mathrm{E}+00 & 0.0000 \mathrm{E}+00 & -2.7405 \mathrm{E}+01 & 1.4572 \mathrm{E}-02\end{array}$

$\begin{array}{llllllll}18 & 159 & 3.1331 \mathrm{E}+01 & 8.0064 \mathrm{E}-02 & 0.0000 \mathrm{E}+00 & 0.0000 \mathrm{E}+00 & -3.1361 \mathrm{E}+01 & 5.0067 \mathrm{E}-02\end{array}$

$\begin{array}{llllllll}19 & 141 & 3.9111 \mathrm{E}+01 & 4.0032 \mathrm{E}-02 & 0.0000 \mathrm{E}+00 & 0.0000 \mathrm{E}+00 & -3.9150 \mathrm{E}+01 & 4.5860 \mathrm{E}-04\end{array}$

total $\quad 1.4161 \mathrm{E}+02 \quad 9.6077 \mathrm{E}-01 \quad 0.0000 \mathrm{E}+00 \quad 0.0000 \mathrm{E}+00 \quad-1.4174 \mathrm{E}+02 \quad 8.3809 \mathrm{E}-01$ 1neutron weight balance in each celt -- variance reduction events print table 130

cell weight cell weight energy dxtran forced exponential total window importance cutoff importance collision transform

l $30 \quad 0.0000 \mathrm{E}+00 \quad 0.0000 \mathrm{E}+00 \quad 0.0000 \mathrm{E}+00 \quad 0.0000 \mathrm{E}+00 \quad 0.0000 \mathrm{E}+00 \quad 0.0000 \mathrm{E}+00 \quad 0.0000 \mathrm{E}+00 \quad 0.0000 \mathrm{E}+00$

$\begin{array}{llllllllllll}2 & 31 & 0.0000 E+00 & 0.0000 E+00 & 0.0000 E+00 & 0.0000 E+00 & 0.0000 E+00 & 0.0000 E+00 & 0.0000 E+00 & 0.0000 E+00\end{array}$

$\begin{array}{llllllllll}3 & 37 & 0.0000 \mathrm{E}+00 & 0.0000 \mathrm{E}+00 & 0.0000 \mathrm{E}+00 & 0.0000 \mathrm{E}+00 & 0.0000 \mathrm{E}+00 & 0.0000 \mathrm{E}+00 & 0.0000 \mathrm{E}+00 & 0.0000 \mathrm{E}+00\end{array}$

$434 \quad 0.0000 \mathrm{E}+00 \quad 0.0000 \mathrm{E}+00 \quad 0.0000 \mathrm{E}+00 \quad 0.0000 \mathrm{E}+00 \quad 0.0000 \mathrm{E}+00 \quad 0.0000 \mathrm{E}+00 \quad 0.0000 \mathrm{E}+00 \quad 0.0000 \mathrm{E}+00$

$\begin{array}{llllllllllll}5 & 32 & 0.0000 \mathrm{E}+00 & 0.0000 \mathrm{E}+00 & 0.0000 \mathrm{E}+00 & 0.0000 \mathrm{E}+00 & 0.0000 \mathrm{E}+00 & 0.0000 \mathrm{E}+00 & 0.0000 \mathrm{E}+00 & 0.0000 \mathrm{E}+00\end{array}$ $\begin{array}{llllllllll}33 & 0.0000 E+00 & 0.0000 E+00 & 0.0000 E+00 & 0.0000 E+00 & 0.0000 E+00 & 0.0000 E+00 & 0.0000 E+00 & 0.0000 E+00\end{array}$ $\begin{array}{llllllllll}35 & 0.0000 \mathrm{E}+00 & 0.0000 \mathrm{E}+00 & 0.0000 \mathrm{E}+00 & 0.0000 \mathrm{E}+00 & 0.0000 \mathrm{E}+00 & 0.0000 \mathrm{E}+00 & 0.0000 \mathrm{E}+00 & 0.0000 \mathrm{E}+00\end{array}$ $\begin{array}{lllllllllll}36 & 0.0000 \mathrm{E}+00 & 0.0000 \mathrm{E}+00 & 0.0000 \mathrm{E}+00 & 0.0000 \mathrm{E}+00 & 0.0000 \mathrm{E}+00 & 0.0000 \mathrm{E}+00 & 0.0000 \mathrm{E}+00 & 0.0000 \mathrm{E}+00\end{array}$ 


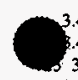
$3.492 \mathrm{E}+00 \quad 1.051 \mathrm{E}+00 \quad 2.000 \mathrm{E}-01 \quad 42(0,-1,0)$ $8.492 \mathrm{E}+00 \quad 1.051 \mathrm{E}+00 \quad 2.000 \mathrm{E}-01 \quad 154$ $3.000 \mathrm{E}+00 \quad 2.000 \mathrm{E}-01 \quad 2.000 \mathrm{E}-01140$ $3.492 \mathrm{E}+00 \quad 1.051 \mathrm{E}+00 \quad 2.000 \mathrm{E}-01 \quad 42(0,-1,0)$ $3.492 \mathrm{E}+00 \quad 1.051 \mathrm{E}+00 \quad 2.000 \mathrm{E}-01 \quad 154$ $243.000 \mathrm{E}+00 \quad 2.000 \mathrm{E}-012.000 \mathrm{E}-01 \quad 140$ $3.492 \mathrm{E}+00 \quad 1.051 \mathrm{E}+00 \quad 2.000 \mathrm{E}-01 \quad 42(0,-1,0)$ $3.492 \mathrm{E}+00 \quad 1.051 \mathrm{E}+00 \quad 2.000 \mathrm{E}-01 \quad 154$

$253.000 \mathrm{E}+00 \quad 2.000 \mathrm{E}-01 \quad 2.000 \mathrm{E}-01 \quad 140$ $3.492 \mathrm{E}+00 \quad 1.051 \mathrm{E}+002.000 \mathrm{E}-01 \quad 42(0,-1,0)$ $3.492 \mathrm{E}+00 \quad 1.051 \mathrm{E}+00 \quad 2.000 \mathrm{E}-01 \quad 154$

$262.000 \mathrm{E}-01 \quad 3.000 \mathrm{E}+00 \quad 2.000 \mathrm{E}-01 \quad 140$ $3.641 \mathrm{E}+00 \quad 4.456 \mathrm{E}-01 \quad 2.000 \mathrm{E}-01 \quad 42(-5,3,0)$ $3.641 \mathrm{E}+00$ 4.456E-01 2.000E-01 141 $27 \quad 2.000 \mathrm{E}-01 \quad 3.000 \mathrm{E}+00 \quad 2.000 \mathrm{E}-01 \quad 140$ $3.641 \mathrm{E}+00 \quad 4.456 \mathrm{E}-01 \quad 2.000 \mathrm{E}-01 \quad 42(-5,3,0)$ $3.641 \mathrm{E}+00 \quad 4.456 \mathrm{E}-01 \quad 2.000 \mathrm{E}-01 \quad 141$

$28 \quad 2.000 \mathrm{E}-01 \quad 3.000 \mathrm{E}+00 \quad 2.000 \mathrm{E}-01 \quad 140$ $3.64 \mathrm{IE}+00 \quad 4.456 \mathrm{E}-01 \quad 2.000 \mathrm{E}-01 \quad 42(-5,3,0)$ $3.641 \mathrm{E}+00 \quad 4.456 \mathrm{E}-01 \quad 2.000 \mathrm{E}-01 \quad 141$

$292.000 \mathrm{E}-01 \quad 3.000 \mathrm{E}+00 \quad 2.000 \mathrm{E}-01 \quad 140$ $3.641 \mathrm{E}+00 \quad 4.456 \mathrm{E}-01 \quad 2.000 \mathrm{E}-01 \quad 42(-5,3,0)$ $3.64 \mathrm{IE}+00 \quad 4.456 \mathrm{E}-01 \quad 2.000 \mathrm{E}-01 \quad 141$

$302.000 \mathrm{E}-013.000 \mathrm{E}+00 \quad 2.000 \mathrm{E}-01 \quad 140$ $3.641 \mathrm{E}+00 \quad 4.456 \mathrm{E}-01 \quad 2.000 \mathrm{E}-01 \quad 42(-5,3,0)$ $3.641 \mathrm{E}+00 \quad 4.456 \mathrm{E}-01 \quad 2.000 \mathrm{E}-01 \quad 141$

31 $2.000 \mathrm{E}-01 \quad 3.000 \mathrm{E}+00 \quad 2.000 \mathrm{E}-01 \quad 140$ $3.641 \mathrm{E}+00 \quad 4.456 \mathrm{E}-01 \quad 2.000 \mathrm{E}-01 \quad 42(-5,3,0)$ $3.641 \mathrm{E}+00 \quad 4.456 \mathrm{E}-01 \quad 2.000 \mathrm{E}-01 \quad 141$

32 2.000E-01 3.000E+00 2.000E-01 140 $3.641 \mathrm{E}+00 \quad 4.456 \mathrm{E}-01 \quad 2.000 \mathrm{E}-01 \quad 42(-5,3,0)$ $3.641 \mathrm{E}+00 \quad 4.456 \mathrm{E}-01 \quad 2.000 \mathrm{E}-01 \quad 141$ $2.000 \mathrm{E}-01 \quad 3.000 \mathrm{E}+00 \quad 2.000 \mathrm{E}-01 \quad 140$ $641 \mathrm{E}+00 \quad 4.456 \mathrm{E}-01 \quad 2.000 \mathrm{E}-01 \quad 42(-5,3,0)$ $3.641 \mathrm{E}+00 \quad 4.456 \mathrm{E}-01 \quad 2.000 \mathrm{E}-01 \quad 141$

$342.000 \mathrm{E}-01 \quad 3.000 \mathrm{E}+00 \quad 2.000 \mathrm{E}-01 \quad 140$ $3.641 \mathrm{E}+00 \quad 4.456 \mathrm{E}-01 \quad 2.000 \mathrm{E}-01 \quad 42(-5,3,0)$ $3.641 \mathrm{E}+00 \quad 4.456 \mathrm{E}-01 \quad 2.000 \mathrm{E}-01 \quad$ i4]

$352.000 \mathrm{E}-013.000 \mathrm{E}+00 \quad 2.000 \mathrm{E}-01 \quad 140$ $3.641 \mathrm{E}+00 \quad 4.456 \mathrm{E}-01 \quad 2.000 \mathrm{E}-0 \mathrm{I} \quad 42(-5,3,0)$ $3.641 \mathrm{E}+00 \quad 4.456 \mathrm{E}-01 \quad 2.000 \mathrm{E}-01 \quad 141$

$362.000 \mathrm{E}-01 \quad 3.000 \mathrm{E}+00 \quad 2.000 \mathrm{E}-01 \quad 140$ $3.641 \mathrm{E}+00 \quad 4.456 \mathrm{E}-01 \quad 2.000 \mathrm{E}-01 \quad 42(-5,3,0)$ $3.641 \mathrm{E}+00 \quad 4.456 \mathrm{E}-01 \quad 2.000 \mathrm{E}-01 \quad 141$

$372.000 \mathrm{E}-013.000 \mathrm{E}+00 \quad 2.000 \mathrm{E}-0 ! 140$ $3.641 \mathrm{E}+00 \quad 4.456 \mathrm{E}-01 \quad 2.000 \mathrm{E}-01 \quad 42(-5,3,0)$ $3.641 \mathrm{E}+00 \quad 4.456 \mathrm{E}-01 \quad 2.000 \mathrm{E}-01 \quad 141$

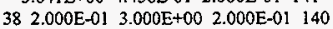
$3.641 \mathrm{E}+00 \quad 4.456 \mathrm{E}-01 \quad 2.000 \mathrm{E}-01 \quad 42(-5,3,0)$ $3.641 \mathrm{E}+00 \quad 4.456 \mathrm{E}-01 \quad 2.000 \mathrm{E}-01 \quad 141$

$392.000 \mathrm{E}-01 \quad 3.000 \mathrm{E}+00 \quad 2.000 \mathrm{E}-01 \quad 140$ $3.641 \mathrm{E}+00 \quad 4.456 \mathrm{E}-01 \quad 2.000 \mathrm{E}-01 \quad 42(-5,3,0)$ $3.641 \mathrm{E}+00 \quad 4.456 \mathrm{E}-01 \quad 2.000 \mathrm{E}-01 \quad 141$

$402.000 \mathrm{E}-013.000 \mathrm{E}+00 \quad 2.000 \mathrm{E}-01 \quad 140$ $3.641 \mathrm{E}+00 \quad 4.456 \mathrm{E}-01 \quad 2.000 \mathrm{E}-01 \quad 42(-5,3,0)$ $3.641 \mathrm{E}+00 \quad 4.456 \mathrm{E}-01 \quad 2.000 \mathrm{E}-01 \quad 141$

$41 \quad 2.000 \mathrm{E}-01 \quad 3.000 \mathrm{E}+00 \quad 2.000 \mathrm{E} * 01 \quad 140$ $3.641 \mathrm{E}+00 \quad 4.456 \mathrm{E}-01 \quad 2.000 \mathrm{E}-01 \quad 42(-5,3,0)$ $3.641 \mathrm{E}+00 \quad 4.456 \mathrm{E}-01 \quad 2.000 \mathrm{E}-01 \quad 141$

$42 \quad 2.000 \mathrm{E}-01 \quad 3.000 \mathrm{E}+00 \quad 2.000 \mathrm{E}-01 \quad 140$ $3.641 \mathrm{E}+00 \quad 4.456 \mathrm{E}-01 \quad 2.000 \mathrm{E}-01 \quad 42(-5,3,0)$ $3.641 \mathrm{E}+00 \quad 4.456 \mathrm{E}-01 \quad 2.000 \mathrm{E}-01 \quad 141$

$43 \quad 2.000 \mathrm{E}-01 \quad 3.000 \mathrm{E}+00 \quad 2.000 \mathrm{E}-01 \quad 140$ $3.641 \mathrm{E}+00 \quad 4.456 \mathrm{E}-01 \quad 2.000 \mathrm{E}-01 \quad 42(-5,3,0)$ $3.641 \mathrm{E}+00 \quad 4.456 \mathrm{E}-01 \quad 2.000 \mathrm{E}-01 \quad 141$ $2.000 \mathrm{E}-01 \quad 3.000 \mathrm{E}+00 \quad 2.000 \mathrm{E}-01 \quad 140$ $3.641 \mathrm{E}+00 \quad 4.456 \mathrm{E}-01 \quad 2.000 \mathrm{E}-01 \quad 42(-5,3,0)$
$-9.117 \mathrm{E}-01-3.647 \mathrm{E}-01-1.891 \mathrm{E}-01$

HNF-SD-SNF-CSWD-005, Rev. 0

$0-9.117 \mathrm{E}-01-3.647 \mathrm{E}-01-1.891 \mathrm{E}-01$

$-4.287 \mathrm{E}-01 \quad 8.361 \mathrm{E}-01-3.423 \mathrm{E}-01 \quad 1.156 \mathrm{E}+00 \quad 1.000 \mathrm{E}+00 \quad 0.000 \mathrm{E}+00$

$-4.287 \mathrm{E}-01 \quad 8.361 \mathrm{E}-01-3.423 \mathrm{E}-01$

$0-4.287 \mathrm{E}-01 \quad 8.361 \mathrm{E}-01-3.423 \mathrm{E}-01$

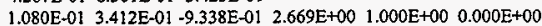

$1.080 \mathrm{E}-01 \quad 3.412 \mathrm{E}-01-9.338 \mathrm{E}-01$

$0 \quad 1.080 \mathrm{E}-01 \quad 3.412 \mathrm{E}-01-9.338 \mathrm{E}-01$

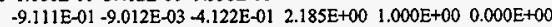

$-9.111 \mathrm{E}-01-9.012 \mathrm{E}-03-4.122 \mathrm{E}-01$

$0-9.111 \mathrm{E}-01-9.012 \mathrm{E}-03-4.122 \mathrm{E}-01$

$-2.568 \mathrm{E}-01-6.391 \mathrm{E}-01 \cdot 7.249 \mathrm{E}-01 \quad 7.280 \mathrm{E}-01 \quad 1.000 \mathrm{E}+00 \quad 0.000 \mathrm{E}+00$

$-2.568 \mathrm{E}-01-6.391 \mathrm{E}-01-7.249 \mathrm{E}-01$

$0-2.568 \mathrm{E}-01-6.391 \mathrm{E}-01-7.249 \mathrm{E}-01$

$\begin{array}{llllll}-2.912 \mathrm{E}-01 & 8.086 \mathrm{E}-01 & 5.113 \mathrm{E}-01 & 1.404 \mathrm{E}+00 & 1.000 \mathrm{E}+00 & 0.000 \mathrm{E}+00\end{array}$

$-2.912 \mathrm{E}-01 \quad 8.086 \mathrm{E}-01 \quad 5.113 \mathrm{E}-01$

$0-2.912 \mathrm{E}-01 \quad 8.086 \mathrm{E}-01 \quad 5.113 \mathrm{E}-01$

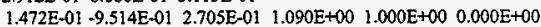

$1.472 \mathrm{E}-01-9.514 \mathrm{E}-01 \quad 2.705 \mathrm{E}-01$

$0 \quad 1.472 \mathrm{E}-01-9.514 \mathrm{E}-01 \quad 2.705 \mathrm{E}-01$

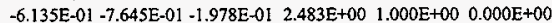

$-6.135 \mathrm{E}-01-7.645 \mathrm{E}-01-1.978 \mathrm{E}-01$

$0-6.135 \mathrm{E}-01-7.645 \mathrm{E}-01-1.978 \mathrm{E}-01$

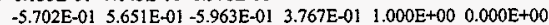

$-5.702 \mathrm{E}-015.651 \mathrm{E}-01-5.963 \mathrm{E}-01$

$0-5.702 \mathrm{E}-01 \quad 5.651 \mathrm{E}-01-5.963 \mathrm{E}-01$

$\begin{array}{lllll}-6.607 \mathrm{E}-01 & 5.373 \mathrm{E}-01 \quad-5.242 \mathrm{E}-01 & 1.019 \mathrm{E}+00 & 1.000 \mathrm{E}+00 & 0.000 \mathrm{E}+00\end{array}$

$-6.607 \mathrm{E}-01 \quad 5.373 \mathrm{E}-01-5.242 \mathrm{E}-01$

$0-6.607 \mathrm{E}-015.373 \mathrm{E}-01-5.242 \mathrm{E}-01$

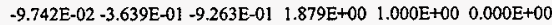

$-9.742 \mathrm{E}-02-3.639 \mathrm{E}-01-9.263 \mathrm{E}-01$

$0-9.742 \mathrm{E}-02-3.639 \mathrm{E}-01-9.263 \mathrm{E}-01$

$\begin{array}{llll}-1.965 \mathrm{E}-01+3.145 \mathrm{E}-01-9.287 \mathrm{E}-01 & 4.700 \mathrm{E}-01 & 1.000 \mathrm{E}+00 & 0.000 \mathrm{E}+00\end{array}$

$-1.965 \mathrm{E}-01-3.145 \mathrm{E}-01-9.287 \mathrm{E}-01$

$0-1.965 \mathrm{E}-01-3.145 \mathrm{E}-01-9.287 \mathrm{E}-01$

4.097E-01 8.465E-01 $-3.399 \mathrm{E}-01 \quad$ [. $530 \mathrm{E}+00 \quad 1.000 \mathrm{E}+00 \quad 0.000 \mathrm{E}+00$ 4.09\%E-01 8.465E-01 -3.399E-01

0 4.097E-01 8.465E-01 -3.399E-01

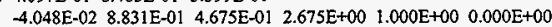
$-4.048 \mathrm{E}-02 \quad 8.831 \mathrm{E}-01 \quad 4.675 \mathrm{E}-01$

$0-4.048 \mathrm{E}-02 \quad 8.831 \mathrm{E}-01 \quad 4.675 \mathrm{E}-01$

$3.371 \mathrm{E}-01-9.269 \mathrm{E}-01-1.652 \mathrm{E}-01 \quad 9.222 \mathrm{E}-01 \quad 1.000 \mathrm{E}+00 \quad 0.000 \mathrm{E}+00$

$3.371 \mathrm{E}-01-9.269 \mathrm{E}-01-1.652 \mathrm{E}-01$

0 3.371E-01 -9.269E-01-1.652E-01

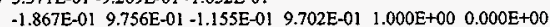

$-1.867 \mathrm{E}-01 \quad 9.756 \mathrm{E}-01-1.155 \mathrm{E}-01$

$0-1.867 \mathrm{E}-01 \quad 9.756 \mathrm{E}-01-1.155 \mathrm{E}-01$

$\begin{array}{lllll}-2.616 \mathrm{E}-01 & 2.336 \mathrm{E}-01 & -9.365 \mathrm{E}-01 & 1.230 \mathrm{E}+00 & 1.000 \mathrm{E}+00 \quad 0.000 \mathrm{E}+00\end{array}$ $-2.616 \mathrm{E}-012.336 \mathrm{E}-01+9.365 \mathrm{E}-01$

$0-2.616 \mathrm{E}-01 \quad 2.336 \mathrm{E}-01-9.365 \mathrm{E}-01$

$9.780 \mathrm{E}-01-7.641 \mathrm{E}-02-1.939 \mathrm{E}-01 \quad 1.083 \mathrm{E}+00 \quad 1.000 \mathrm{E}+00 \quad 0.000 \mathrm{E}+00$

$9.780 \mathrm{E}-01-7.641 \mathrm{E}-02-1.939 \mathrm{E}-01$

$09.780 \mathrm{E}-01-7.641 \mathrm{E}-02-1.939 \mathrm{E}-01$

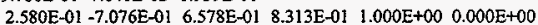

$2.580 \mathrm{E}-01-7.076 \mathrm{E}-01 \quad 6.578 \mathrm{E}-01$

$0 \quad 2.580 \mathrm{E}-01+7.076 \mathrm{E}-01 \quad 6.578 \mathrm{E}-01$

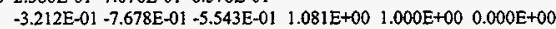
$-3.212 \mathrm{E}-01-7.678 \mathrm{E}-01-5.543 \mathrm{E}-01$

$0-3.212 \mathrm{E}-01-7.678 \mathrm{E}-01-5.543 \mathrm{E}-01$

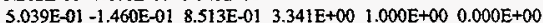
$5.039 \mathrm{E}-01+1.460 \mathrm{E}-01 \quad 8.513 \mathrm{E}-01$

0 5.039E-01 -1 . 460E-01 8.513E-01

$\begin{array}{llllll}6.080 \mathrm{E}-01 & 5.487 \mathrm{E}-01 & 5.738 \mathrm{E}-01 & 4.097 \mathrm{E}-01 & 1.000 \mathrm{E}+00 & 0.000 \mathrm{E}+00\end{array}$

$6.080 \mathrm{E}-01 \quad 5.487 \mathrm{E}-01 \quad 5.738 \mathrm{E}-01$

$0 \quad 6.080 \mathrm{E}-01 \quad 5.487 \mathrm{E}-01 \quad 5.738 \mathrm{E}-01$

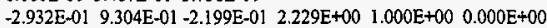
$-2.932 \mathrm{E}-01 \quad 9.304 \mathrm{E}+01-2.199 \mathrm{E}-01$ 


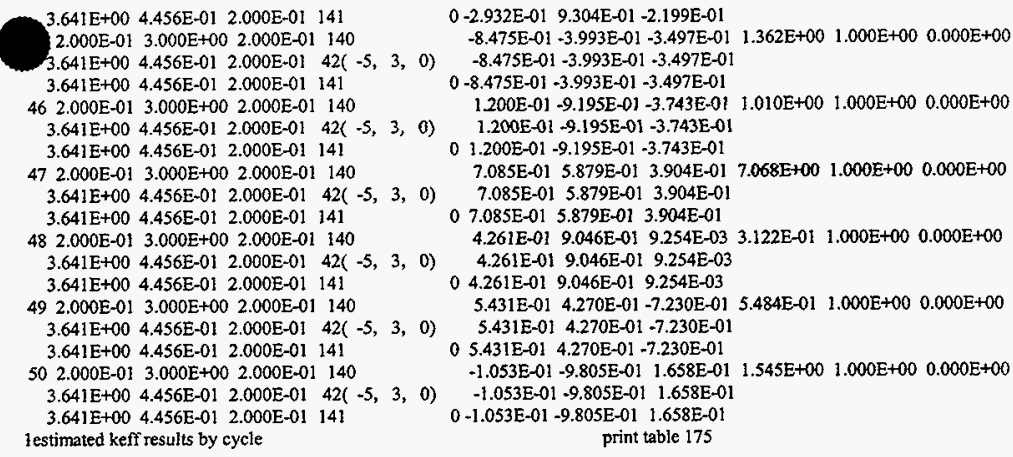

cycle $1 \mathrm{k}$ (collision) 1.253108 removal lifetime(abs) $1.7557 \mathrm{E}+01$ source points generated 257

cycle $2 \mathrm{k}$ (collision) 1.211997 removal lifetime(abs) $1.1671 \mathrm{E}+01$ source points generated 195

cycle $3 \mathrm{k}$ (collision) 1.200265 removal lifetime(abs) $9.6992 \mathrm{E}+00$ source points generated 193

cycle $4 \mathrm{k}$ (collision) 1.129530 removal lifetime(abs) $9.3720 \mathrm{E}+00$ source points generated 182

cycle $5 \mathrm{k}$ (collision) 1.135994 removal lifetime(abs) $1.1136 \mathrm{E}+01$ source points generated 222

cycle $6 \mathrm{k}$ (collision) 1.077014 removal lifetime(abs) $1.2222 \mathrm{E}+01$ source points generated 204

arce distribution written to file inp $18 \mathrm{~s} \quad$ cycle $=6$
roblem summary

0

run terminated when 6 kcode cycles were done.

neutron creation tracks weight energy neutron loss tracks weight energy (per source particle) (per source particle)

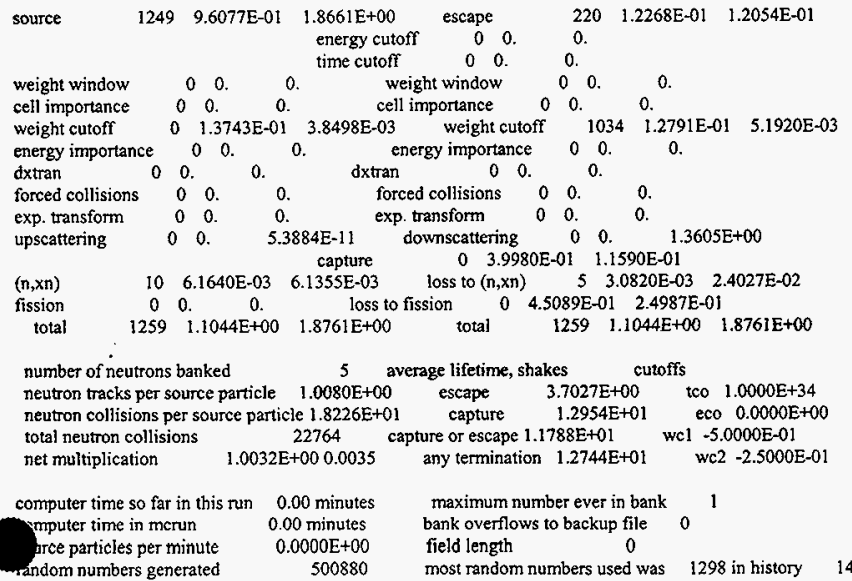


$\begin{array}{lllllllll}38 & 0.0000 \mathrm{E}+00 & 0.0000 \mathrm{E}+00 & 1.2447 \mathrm{E}-03 & 0.0000 \mathrm{E}+00 & 0.0000 \mathrm{E}+00 & 0.0000 \mathrm{E}+00 & 0.0000 \mathrm{E}+00 & 1.2447 \mathrm{E}-03\end{array}$

$\begin{array}{lllllllll}39 & 0.0000 \mathrm{E}+00 & 0.0000 \mathrm{E}+00 & 2.0097 \mathrm{E}-04 & 0.0000 \mathrm{E}+00 & 0.0000 \mathrm{E}+00 & 0.0000 \mathrm{E}+00 & 0.0000 \mathrm{E}+00 & 2.0097 \mathrm{E}-04\end{array}$

$\begin{array}{lllllllllllll}1 & 40 & 0.0000 \mathrm{E}+00 & 0.0000 \mathrm{E}+00 & 0.0000 \mathrm{E}+00 & 0.0000 \mathrm{E}+00 & 0.0000 \mathrm{E}+00 & 0.0000 \mathrm{E}+00 & 0.0000 \mathrm{E}+00 & 0.0000 \mathrm{E}+00\end{array}$

$\begin{array}{lllllllllll}12 & 4 l & 0.0000 \mathrm{E}+00 & 0.0000 \mathrm{E}+00 & 2.9100 \mathrm{E}-04 & 0.0000 \mathrm{E}+00 & 0.0000 \mathrm{E}+00 & 0.0000 \mathrm{E}+00 & 0.0000 \mathrm{E}+00 & 2.9100 \mathrm{E}-04\end{array}$

$\begin{array}{lllllllllllll}13 & 140 & 0.0000 \mathrm{E}+00 & 0.0000 \mathrm{E}+00 & 0.0000 \mathrm{E}+00 & 0.0000 \mathrm{E}+00 & 0.0000 \mathrm{E}+00 & 0.0000 \mathrm{E}+00 & 0.0000 \mathrm{E}+00 & 0.0000 \mathrm{E}+00\end{array}$

$\begin{array}{llllllllll}14 & 42 & 0.0000 \mathrm{E}+00 & 0.0000 \mathrm{E}+00 & 0.0000 \mathrm{E}+00 & 0.0000 \mathrm{E}+00 & 0.0000 \mathrm{E}+00 & 0.0000 \mathrm{E}+00 & 0.0000 \mathrm{E}+00 & 0.0000 \mathrm{E}+00\end{array}$

$\begin{array}{llllllllllll}15 & 154 & 0.0000 \mathrm{E}+00 & 0.0000 \mathrm{E}+00 & 8.0354 \mathrm{E}-03 & 0.0000 \mathrm{E}+00 & 0.0000 \mathrm{E}+00 & 0.0000 \mathrm{E}+00 & 0.0000 \mathrm{E}+00 & 8.0354 \mathrm{E}-03\end{array}$

$\begin{array}{lllllllllll}16 & 149 & 0.0000 E+00 & 0.0000 E+00 & 0.0000 E+00 & 0.0000 E+00 & 0.0000 E+00 & 0.0000 E+00 & 0.0000 E+00 & 0.0000 E+00\end{array}$

$\begin{array}{lllllllllll}17 & 144 & 0.0000 \mathrm{E}+00 & 0.0000 \mathrm{E}+0 & -3.0848 \mathrm{E}-06 & 0.0000 \mathrm{E}+00 & 0.0000 \mathrm{E}+00 & 0.0000 \mathrm{E}+00 & 0.0000 \mathrm{E}+00 & -3.0848 \mathrm{E}-06\end{array}$

$\begin{array}{llllllllll}18 & 159 & 0.0000 \mathrm{E}+00 & 0.0000 \mathrm{E}+00 & -2.5216 \mathrm{E}-04 & 0.0000 \mathrm{E}+00 & 0.0000 \mathrm{E}+00 & 0.0000 \mathrm{E}+00 & 0.0000 \mathrm{E}+00 & -2.5216 \mathrm{E}-04\end{array}$

$\begin{array}{llllllllll}19 & 141 & 0.0000 \mathrm{E}+00 & 0.0000 \mathrm{E}+00 & 0.0000 \mathrm{E}+00 & 0.0000 \mathrm{E}+00 & 0.0000 \mathrm{E}+00 & 0.0000 \mathrm{E}+00 & 0.0000 \mathrm{E}+00 & 0.0000 \mathrm{E}+00\end{array}$

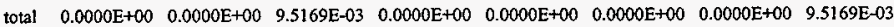
Ineutron weight balance in each cell -- physical events print table 130 cell ( $\mathrm{n}, \mathrm{xn})$ fission capture loss to loss to total

$\begin{array}{llllllll}1 & 30 & 0.0000 \mathrm{E}+00 & 0.0000 \mathrm{E}+00 & 0.0000 \mathrm{E}+00 & 0.0000 \mathrm{E}+00 & 0.0000 \mathrm{E}+00 & 0.0000 \mathrm{E}+00\end{array}$

$\begin{array}{llllllllll}2 & 31 & 0.0000 \mathrm{E}+00 & 0.0000 \mathrm{E}+00 & 0.0000 \mathrm{E}+00 & 0.0000 \mathrm{E}+00 & 0.0000 \mathrm{E}+00 & 0.0000 \mathrm{E}+00\end{array}$

$\begin{array}{lllllllllll}3 & 37 & 0.0000 \mathrm{E}+00 & 0.0000 \mathrm{E}+00 & 0.0000 \mathrm{E}+00 & 0.0000 \mathrm{E}+00 & 0.0000 \mathrm{E}+00 & 0.0000 \mathrm{E}+00\end{array}$

$\begin{array}{lllllllll}4 & 34 & 0.0000 \mathrm{E}+00 & 0.0000 \mathrm{E}+00 & 0.0000 \mathrm{E}+00 & 0.0000 \mathrm{E}+00 & 0.0000 \mathrm{E}+00 & 0.0000 \mathrm{E}+00\end{array}$

$\begin{array}{llllllllll}5 & 32 & 0.0000 \mathrm{E}+00 & 0.0000 \mathrm{E}+00 & 0.0000 \mathrm{E}+00 & 0.0000 \mathrm{E}+00 & 0.0000 \mathrm{E}+00 & 0.0000 \mathrm{E}+00\end{array}$

$\begin{array}{lllllllllll}6 & 33 & 0.0000 \mathrm{E}+00 & 0.0000 \mathrm{E}+00 & 0.0000 \mathrm{E}+00 & 0.0000 \mathrm{E}+00 & 0.0000 \mathrm{E}+00 & 0.0000 \mathrm{E}+00\end{array}$

$\begin{array}{llllllllll}7 & 35 & 0.0000 \mathrm{E}+00 & 0.0000 \mathrm{E}+00 & 0.0000 \mathrm{E}+00 & 0.0000 \mathrm{E}+00 & 0.0000 \mathrm{E}+00 & 0.0000 \mathrm{E}+00\end{array}$

$\begin{array}{llllllllll}8 & 36 & 0.0000 \mathrm{E}+00 & 0.0000 \mathrm{E}+00 & 0.0000 \mathrm{E}+00 & 0.0000 \mathrm{E}+00 & 0.0000 \mathrm{E}+00 & 0.0000 \mathrm{E}+00\end{array}$

$9 \quad 38 \quad 0.0000 \mathrm{E}+00 \quad 0.0000 \mathrm{E}+00 \quad-1.5855 \mathrm{E}-01 \quad 0.0000 \mathrm{E}+00 \quad 0.0000 \mathrm{E}+00 \quad-1.5855 \mathrm{E}-01$

$\begin{array}{lllllllllll}10 & 39 & 0.0000 \mathrm{E}+00 & 0.0000 \mathrm{E}+00 & -8.2847 \mathrm{E}-04 & 0.0000 \mathrm{E}+00 & 0.0000 \mathrm{E}+00 & -8.2847 \mathrm{E}-04\end{array}$

$\begin{array}{llllllll}11 & 40 & 0.0000 \mathrm{E}+00 & 0.0000 \mathrm{E}+00 & -3.9460 \mathrm{E}-06 & 0.0000 \mathrm{E}+00 & 0.0000 \mathrm{E}+00 & -3.9460 \mathrm{E}-06\end{array}$

$12 \quad 41 \quad 0.0000 \mathrm{E}+00 \quad 0.0000 \mathrm{E}+00 \quad-3.1114 \mathrm{E}-03 \quad 0.0000 \mathrm{E}+00 \quad 0.0000 \mathrm{E}+00 \quad-3.1114 \mathrm{E}-03$

$13 \quad 140 \quad 0.0000 \mathrm{E}+00 \quad 0.0000 \mathrm{E}+00 \quad 0.0000 \mathrm{E}+00 \quad 0.0000 \mathrm{E}+00 \quad 0.0000 \mathrm{E}+00 \quad 0.0000 \mathrm{E}+00$

$14 \quad 42 \quad 1.2461 \mathrm{E}-03 \quad 0.0000 \mathrm{E}+00 \quad-3.1955 \mathrm{E}-05 \quad-6.2306 \mathrm{E}-04 \quad 0.0000 \mathrm{E}+00 \quad 5.9111 \mathrm{E}-04$

$5 \quad 154 \quad 4.9179 \mathrm{E}-03 \quad 0.0000 \mathrm{E}+00-1.7239 \mathrm{E}-01-2.4589 \mathrm{E}-03 \quad-4.5089 \mathrm{E}-01 \quad-6.2082 \mathrm{E}-01$

$\begin{array}{llllllll}6 & 149 & 0.0000 \mathrm{E}+00 & 0.0000 \mathrm{E}+00 & -3.5884 \mathrm{E}-05 & 0.0000 \mathrm{E}+00 & 0.0000 \mathrm{E}+00 & -3.5884 \mathrm{E}-05\end{array}$

$\begin{array}{llllllll}17 & 144 & 0.0000 \mathrm{E}+00 & 0.0000 \mathrm{E}+00 & -1.4569 \mathrm{E}-02 & 0.0000 \mathrm{E}+00 & 0.0000 \mathrm{E}+00 & -1.4569 \mathrm{E}-02\end{array}$

$\begin{array}{llllllll}18 & 159 & 0.0000 \mathrm{E}+00 & 0.0000 \mathrm{E}+00 & -4.9815 \mathrm{E}-02 & 0.0000 \mathrm{E}+00 & 0.0000 \mathrm{E}+00 & -4.9815 \mathrm{E}-02\end{array}$

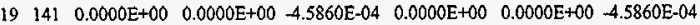

total $\quad 6.1640 \mathrm{E}-03 \quad 0.0000 \mathrm{E}+00 \quad-3.9980 \mathrm{E}-01 \quad-3.0820 \mathrm{E}-03 \quad-4.5089 \mathrm{E}-01 \quad-8.4760 \mathrm{E}-01$

Ineutron activity of each nuclide in each cell, per source particle

print table 140

cell nuclides atom total collisions weight lost weight loss weight gain fraction collisions * weight to capture to fission by $(n, x n)$

$938 \quad 5010.03 \mathrm{~d} \quad 7.1977 \mathrm{E}-01$ $5011.40 \mathrm{c}$ 8.0041E-02 $6012.40 c \quad 2.0019 E-01$

$\begin{array}{llll}10 & 39 & 41093.40 \mathrm{c} & 9.8982 \mathrm{E}-01\end{array}$ $40000.40 \mathrm{c} \quad 1.0183 \mathrm{E}-02$

$11 \quad 40 \quad 1001.00 \mathrm{c} \quad 3.3333 \mathrm{E}-01$ $1002.55 \mathrm{c} \quad 3.3333 \mathrm{E}-01$ $8016.40 \mathrm{c} \quad 3.3333 \mathrm{E}-01$

$\begin{array}{llll}12 & 41 \quad 41093.40 \mathrm{c} \quad 9.8982 \mathrm{E}-01\end{array}$ $40000.40 \mathrm{C} \quad 1.0183 \mathrm{E}-02$

$14 \quad 42 \quad 1001.00 \mathrm{c} \quad 3.3333 \mathrm{E}-01$ $1002.55 \mathrm{c} \quad 3.3333 \mathrm{E}-01$ $8016.40 \mathrm{c} \quad 3.3333 \mathrm{E}-01$

$15 \quad 154 \quad 92235,40 \mathrm{c} \quad 3.7504 \mathrm{E}-01$ $92238.40 \mathrm{c} \quad 1.2499 \mathrm{E}-01$ $7014.40 \mathrm{c} \quad 4.9997 \mathrm{E}-01$
$941 \quad 4.4212 \mathrm{E}-01 \quad 1.5853 \mathrm{E}-01 \quad 0.0000 \mathrm{E}+00 \quad 0.0000 \mathrm{E}+00$ $\begin{array}{lllll}76 & 3.6073 \mathrm{E}-02 & 1.3591 \mathrm{E}-07 & 0.0000 \mathrm{E}+00 & 0.0000 \mathrm{E}+00\end{array}$ $232 \quad 1.1297 \mathrm{E}-01 \quad 1.8446 \mathrm{E}-05 \quad 0.0000 \mathrm{E}+00 \quad 0.0000 \mathrm{E}+00$

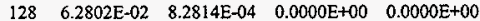
$2 \quad 9.7676 \mathrm{E}-04 \quad 3.3160 \mathrm{E}-07 \quad 0.0000 \mathrm{E}+00 \quad 0.0000 \mathrm{E}+00$

$111 \quad 5.1533 \mathrm{E}-02 \quad 3.9251 \mathrm{E}-06 \quad 0.0000 \mathrm{E}+00 \quad 0.0000 \mathrm{E}+00$ $33 \quad 1.5373 \mathrm{E}-02 \quad 2.0884 \mathrm{E}-08 \quad 0.0000 \mathrm{E}+00 \quad 0.0000 \mathrm{E}+00$ $40 \quad 2.1271 \mathrm{E}-02 \quad 0.0000 \mathrm{E}+00 \quad 0.0000 \mathrm{E}+00 \quad 0.0000 \mathrm{E}+00$

$313 \quad 1.5623 \mathrm{E}-01 \quad 3.1072 \mathrm{E}-03 \quad 0.0000 \mathrm{E}+00 \quad 0.0000 \mathrm{E}+00$ $4 \quad 1.8747 \mathrm{E}-03 \quad 4.2322 \mathrm{E}-06 \quad 0.0000 \mathrm{E}+00 \quad 0.0000 \mathrm{E}+00$

$257 \quad 1.2131 \mathrm{E}-01 \quad 4.3914 \mathrm{E}-06 \quad 0.0000 \mathrm{E}+00 \quad 0.0000 \mathrm{E}+\infty 0$ $86 \quad 4.6121 \mathrm{E}-02 \quad 6.8027 \mathrm{E}-08 \quad 0.0000 \mathrm{E}+00 \quad 6.2306 \mathrm{E}-04$

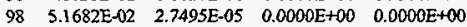

$4759 \quad 2.3227 \mathrm{E}+00 \quad 1.3244 \mathrm{E}-0 \mathrm{I} \quad 4.4247 \mathrm{E}-01 \quad 5.8410 \mathrm{E}-04$ $\begin{array}{llllll}1470 & 7.3870 \mathrm{E}-01 & 2.3272 \mathrm{E}-02 & 8.4162 \mathrm{E}-03 & 1.8748 \mathrm{E}-03\end{array}$ $\begin{array}{lllll}2207 & 1.0775 \mathrm{E}+00 & 1.6682 \mathrm{E}-02 & 0.0000 \mathrm{E}+00 & 0.0000 \mathrm{E}+00\end{array}$ 
HNF-SD-SNF-CSWD-005, Rev. 0

$\begin{array}{lllllll}1002.55 \mathrm{c} & 3.3333 \mathrm{E}-01 & 110 & 5.8433 \mathrm{E}-02 & 9.1921 \mathrm{E}-08 & 0.0000 \mathrm{E}+00 & 0.0000 \mathrm{E}+00\end{array}$

$\begin{array}{lllllll}8016.40 \mathrm{c} & 3.3333 \mathrm{E}-01 & 125 & 6.3072 \mathrm{E}-02 & 3.1305 \mathrm{E}-05 & 0.0000 \mathrm{E}+00 & 0.0000 \mathrm{E}+00\end{array}$

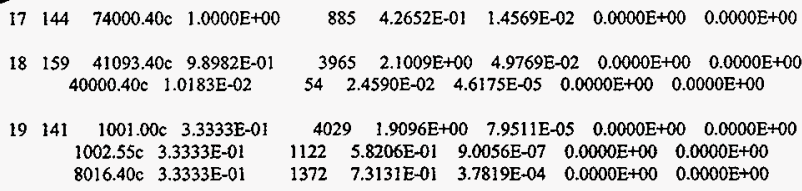

total $\quad 22764 \quad 1.1324 \mathrm{E}+01 \quad 3.9980 \mathrm{E}-01 \quad 4.5089 \mathrm{E}-01 \quad 3.0820 \mathrm{E}-03$

total over all cells for each nuclide total collisions weight lost weight loss weight gain collisions * weight to capture to fission by $(n, x n)$

$\begin{array}{lcccccc}1001.00 \mathrm{c} & 4742 & 2.2505 \mathrm{E}+00 & 9.2315 \mathrm{E}-05 & 0.0000 \mathrm{E}+00 & 0.0000 \mathrm{E}+00 \\ 1002.55 \mathrm{c} & 1351 & 7.0199 \mathrm{E}-01 & 1.0814 \mathrm{E}-06 & 0.0000 \mathrm{E}+00 & 6.2306 \mathrm{E}-04 \\ 5011.40 \mathrm{c} & 76 & 3.6073 \mathrm{E}-02 & 1.3591 \mathrm{E}-07 & 0.0000 \mathrm{E}+00 & 0.0000 \mathrm{E}+00 \\ 6012.40 \mathrm{c} & 232 & 1.1297 \mathrm{E}-01 & 1.8446 \mathrm{E}-05 & 0.0000 \mathrm{E}+00 & 0.0000 \mathrm{E}+00 \\ 7014.40 \mathrm{c} & 2207 & 1.0775 \mathrm{E}+00 & 1.6682 \mathrm{E}-02 & 0.0000 \mathrm{E}+00 & 0.0000 \mathrm{E}+00 \\ 8016.40 \mathrm{c} & 1635 & 8.6733 \mathrm{E}-01 & 4.3699 \mathrm{E}-04 & 0.0000 \mathrm{E}+00 & 0.0000 \mathrm{E}+00 \\ 40000.40 \mathrm{c} & 60 & 2.7441 \mathrm{E}-02 & 5.0739 \mathrm{E}-05 & 0.0000 \mathrm{E}+00 & 0.0000 \mathrm{E}+00 \\ 41093.40 \mathrm{c} & 4406 & 2.3199 \mathrm{E}+00 & 5.3704 \mathrm{E}-02 & 0.0000 \mathrm{E}+00 & 0.0000 \mathrm{E}+00 \\ 74000.40 \mathrm{c} & 885 & 4.2652 \mathrm{E}-01 & 1.4569 \mathrm{E}-02 & 0.0000 \mathrm{E}+00 & 0.0000 \mathrm{E}+00 \\ 92235.40 \mathrm{c} & 4759 & 2.3227 \mathrm{E}+00 & 1.3244 \mathrm{E}-01 & 4.4247 \mathrm{E}-01 & 5.8410 \mathrm{E}-04 \\ 92238.40 \mathrm{c} & 1470 & 7.3870 \mathrm{E}-01 & 2.3272 \mathrm{E}-02 & 8.4162 \mathrm{E}-03 & 1.8748 \mathrm{E}-03 \\ 5010.03 \mathrm{~d} & 941 & 4.4212 \mathrm{E}-01 & 1.5853 \mathrm{E}-01 & 0.0000 \mathrm{E}+00 & 0.0000 \mathrm{E}+00\end{array}$

the initial fission neutron source distribution used the 8 source points that were input on the ksrc card.

criticality problem was scheduled to skip 5 cycles and run a total of 6 cycles with nominally 200 neutrons per cycle. problem has run $S$ inactive cycles with 1027 neutron histories and 1 active cycles with 222 neutron histories.

this calculation has completed the requested number of keff cycles using a total of 1249 fission neutron source histories. all cells with fissionable material were sampled and had fission neutron source points.

there is no combined collision/absorption/track-length estimate for keff because only I active cycles were run. lindividual and average keff estimator results by cycle

keff neutron keff estimators by cycle average keff estimators and deviations average $k(\mathrm{c} / a / t)$ cycle histories $k$ (coll) $k$ (abs) $k$ (track) $k$ (colt) st dev $k(a b s)$ st dev $k$ (track) st dev $k$ (cia/t) st dev fom

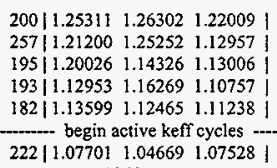

number of histories used for normalizing tallies $=200.00$

cell $a$ is $(154<42[-10-10] 42[-9-10] 42[-8-10] 42[-7-10] 42[-6-10])$

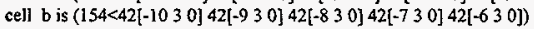

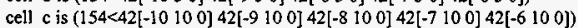

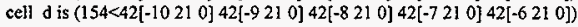

cell e is $(154<42[-10290] 42[-9290042[-8290] 42[-7290] 42[-6290])$

volumes

cell: $a$ b a $\quad d \quad$ e

$\begin{array}{llll}1.04709 \mathrm{E}+02 & 1.04709 \mathrm{E}+02 & 1.04709 \mathrm{E}+02 \quad 1.04709 \mathrm{E}+02 \quad 1.04709 \mathrm{E}+02\end{array}$ 


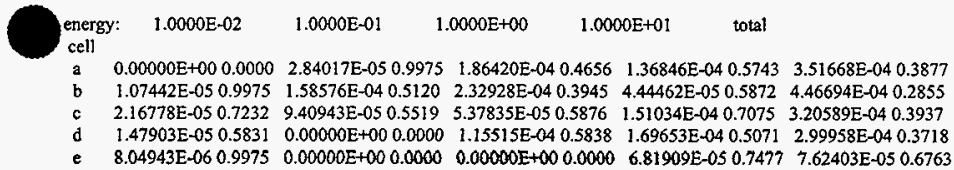

lanalysis of the results in the tally fluctuation chart bin $(\mathbb{G c})$ for tally 4 with nps $=1249$ print table 160

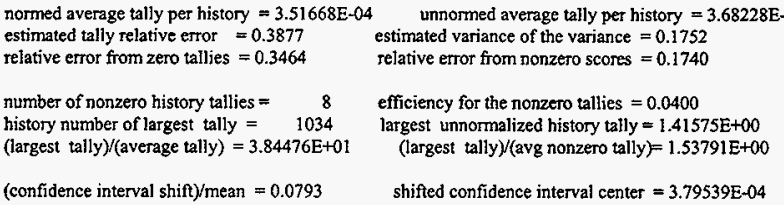

if the largest history score sampled so far were to occur on the very next history, the tfe bin quantities would change as follows: $\mathrm{nps}=222$ for this table because 5 keff cycles and 1027 histories were skipped before tally accumulation.

estimated quantities value at nps value at nps+l value(nps+1)/value(nps)-1.

$\begin{array}{lccc}\text { mean } & 3.51668 \mathrm{E}-04 & 4.10723 \mathrm{E}-04 & 0.167927 \\ \text { relative error } & 3.87653 \mathrm{E}-01 & 3.61597 \mathrm{E}-01 & -0.067213 \\ \text { variance of the variance } & 1.75161 \mathrm{E}-01 & 1.47665 \mathrm{E}-01 & -0.156976 \\ \text { shifted center } & 3.79539 \mathrm{E}-04 & 3.77169 \mathrm{E}-04 & -0.006244 \\ \text { figure of merit } & 0.00000 \mathrm{E}+00 & 0.00000 \mathrm{E}+00 & 0.000000\end{array}$

is not enough information in the largest history scores (usually less than 500 scores) for a reliable estimate of the slope. history score probability density function appears to have an unsampled region at the largest history scores: please examine.

***** the nps-dependent tfe bin check results are suspect because there are only 1 nps tally values to analyze *****

results of 10 statistical checks for the estimated answer for the tally fluctuation chart (tfc) bin of tally 4

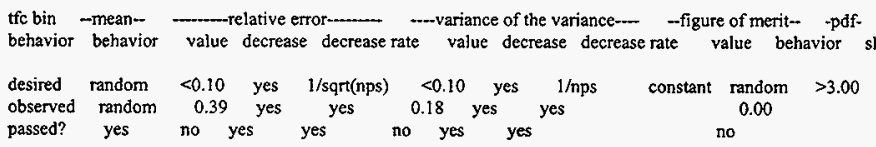

warning. the tally in the tally fluctuation chart bin did not pass 3 of the 10 statistical checks.

lunnormed tally density for tally $4 \quad$ nonzero tally mean $(m)=9.206 E-01 \quad n p s=1249$ print table 161

abscissa ordinate log plot of tally probability density function in tally fluctuation chart bin $(d=$ decade, slope $=0.0$ )

tally number num den $\log$ den:d-..

$1.00-01 \quad 12.43-01-0.614 * * * * * * * * * * * * * * * * * * * * * * * * * * * * * * * * * * * * * * * * * * * * * * * * * * * * * * * * * * * * * * * * * * * * * * * * * * * * * * * * * * * * * * * * * * * * * * * * * * * * \mid *$

$1.26-01 \quad 00.00+00 \quad 0.000$

$\begin{array}{llll}1.58-01 & 0 & 0.00+00 & 0.000\end{array}$

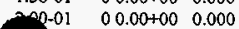

$0.00+00 \quad 0.000$

$00.00 \div 00 \quad 0.000$ 


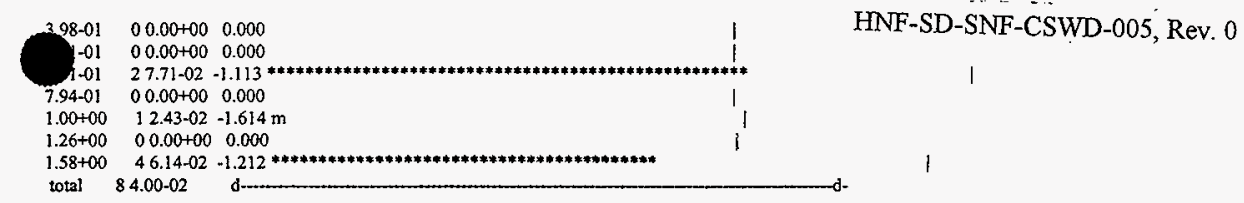

cumulative tally number for tally 4 nonzero tally mean $(m)=9.206 E-01 \quad n p s=1249$ print table 162

abscissa cum ordinate plot of the cumulative number of tallies in the tally fluctuation chart bin from 0 to 100 percent tally number cum pct:- $-10-20-30-40-50-60-100$ $1.00000 \mathrm{E}-01$ i $12.500|* * * * * * * *| * * *$

$1.25893 \mathrm{E}-0 \mathrm{I} \quad 1 \quad 12.500|* * * * * * * *| * * *$

$1.58489 \mathrm{E}-01 \quad 112.500 \mid * * * * * * * * * ; * * *$

$1.99526 \mathrm{E}-\left.01 \quad 1 \quad 12.500\right|^{* * * * * * * * *+* * *}$

$2.51189 \mathrm{E}-01 \quad 112.500]^{* * * * * * * * * \mid * * *}$

$3.16228 \mathrm{E}-01 \quad 1 \quad 12.500]^{* * * * * * * * * 1 * * *}$

$3.98108 \mathrm{E}-01$

$5.01188 \mathrm{E}-0$ !

$6.30958 \mathrm{E}-01$

$7.94329 \mathrm{E}-01$

$1.00000 \mathrm{E}+00$

$1.25893 \mathrm{E}+00$

$1.58489 \mathrm{E}+00$

total

1 1 12.500$]^{\left.* * * * * * * * * *\right|^{* * *}}$

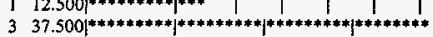

$337.500|* * * * * * * * *| * * * * * * * * *|* * * * * * * * *| * * * * * * * \mid$

101010

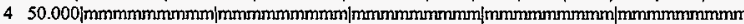

$\left.450.000\right|^{* * * * * * * * *|* * * * * * * * *| * * * * * * * * *|* * * * * * * * *| * * * * * * * * * \mid}$

$\left.8100.000|* * * * * * * * *| * * * * * * * * *\right|^{* * * * * * * * *|* * * * * * * *| * * * * * * * * *|* * * * * * * * * ; * * * * * * * *|} \mid$

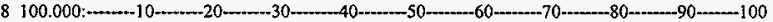

cumulative unnormed tally for tally $4 \quad$ nonzero tally mean $(m)=9.206 \mathrm{E}-01 \quad \mathrm{nps}=1249$ print table 162

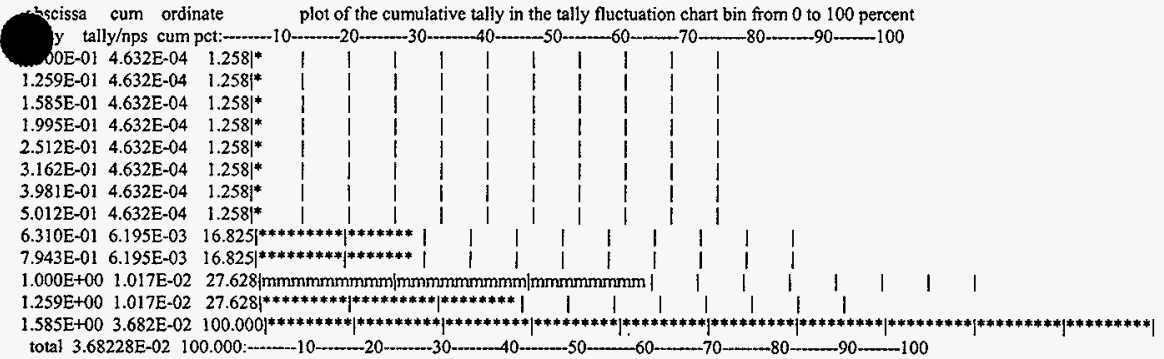

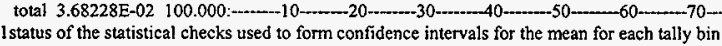

tally result of statistical checks for the tfc bin (the first check not passed is listed) and error magnitude check for all bins

4 missed 3 of 10 tfc bin checks: the relative error exceeds the recommended value of 0.1 for nonpoint detector tallies missed all bin error check: 25 tally bins had 4 bins with zeros and 21 bins with relative errors exceeding 0.10

the 10 statistical checks are only for the tally fluctuation chart bin and do not apply to other tally bins.

the tally bins with zeros may or may not be correct: compare the source, cutoffs, multipliers, et cetera with the tally bins.

warning. I of the 1 tally fluctuation chart bins did not pass all 10 statistical checks.

waming. 1 of the $\mathbf{l}$ tallies had bins with relative errors greater than recommended.

lally fluctuation charts

tally 4

mean error vov slope fom 
6 waming messages so far.

run terminated when 6 kcode cycles were done.

\section{File: Outp18}

1- prob18 - kcode in a hexagonal prism lattice.

2- c three half control rods and five whole control rods.

3- $30 \quad 0-905-1929$ ! fill $=1$

4. $\quad 31 \quad 0-906-19291$ fill $=1(16.711300)$

5- $37 \quad 0-907-1929$ ifill $=1(-16.711300)$

6. $34 \quad 0-913-1929 \quad$ fill $=1(011.91850)$

7- $\quad 32 \quad 0-914-1929$ fill $=1(10.32175 .95920)$

8- $33 \quad 0-915-1929$ fill $=1(8.3557 \quad 14.47240)$

9. $\quad 35 \quad 0-916-1929$ fill $=1(-8.3557 \quad 14.47240)$

10- $36 \quad 0-917-1929$ fill $=1 \quad(-10.32175 .95920)$

11. c universe 1: structure of control rod.

12- $\quad 38 \quad 11-2.02 \quad-880$ u=1 $\$$ control rod core

13- $39 \quad 6 \quad-8.4 \quad 880-881$ u=l \$ control tod cladding

14- $\quad 40 \quad 12-1.00 \quad 881-882 u=1 \quad S$ control rod gap

15- $\quad 41 \quad 6 \quad-8.4 \quad 882 \quad u=1 \quad \$$ control rod sheath

16- $c$ the space between the control rods, filled with lattice.

17- $\quad 140 \quad 0-17129-19905906907913914915916917$ fill $=2$

18- $\quad c$ universe 2: lattice of fuel rods with water in between.

19- $42 \quad 12-1.00 \quad-301302-303 \quad 304-305306 \quad u=2$ lat $=2$ fill= 20- $\quad-37: 27-1: 330: 0 \&$

$24 r 39 r 24 r 311 r 24 r 311 r 24 r 39 r 2$

$24 r 3$ 9r 23 r 312 Ir 2 3r 312 r 23 3r 3 9r 2 ir

2 3r 3 10r 2 2r 313 r 2 2r 313 r 2 2r 310 r 2 it

$23 r 357 r 2$ 2r \&

$22 \mathrm{r} 358 \mathrm{r} 22 \mathrm{r}$

2 2r $316 r 22 r 317 \pi 22 r 316 r 23 r$

2 r $315 \mathrm{r} 23 \mathrm{r} 316 \mathrm{r} 23 \mathrm{r} 315 \mathrm{r} 24 \mathrm{r}$

$2 \operatorname{lr} 315 \mathrm{r} 24 \mathrm{r} 31 \mathrm{sr} 24 \mathrm{r} 315 \mathrm{r} 24 \mathrm{r}$

2 1r $315 r 23 r 316 r 23 r 315 r 25 r$

$2 \operatorname{lr} 315 r 22 r 317 r 22 r 315 r 26 r$

21 r 354 r $27 \mathrm{r} \&$

c can code remember \& thru comment?

$2355 \mathrm{r} 27 \mathrm{r}$

$2325 \mathrm{r} 22 \mathrm{r} 325 \mathrm{r} 28 \mathrm{r}$

$2324 r 23 r 324 r 29 r$

$2323 \mathrm{r} 24 \mathrm{r} 323 \mathrm{r} 210 \mathrm{r}$

$2315 \mathrm{r} 22 \mathrm{r} 34 \mathrm{r} 23 \mathrm{r} 34 \mathrm{r} 22 \mathrm{rr} 315 \mathrm{rr} 211 \mathrm{r}$

$2314 \mathrm{r} 23 \mathrm{r} 34 \mathrm{r} 2 \mathrm{2r} 34 \mathrm{r} 23 \mathrm{r} 314 \mathrm{r} 212 \mathrm{r}$

$2313 r 24 r 311 r 24 r 313 r 213 r$

$2313 \mathrm{r} 23 \mathrm{r} 3 \mathrm{r}$ 12r $23 \mathrm{r} 313 \mathrm{r} 214 \mathrm{r}$

$2313 \mathrm{r} 22 \mathrm{r} 313 \mathrm{r} 22 \mathrm{r} 313 \mathrm{r} 215 \mathrm{r}$

$2346 \mathrm{r} 216 \mathrm{r}$

$2345 \mathrm{r} 217 \mathrm{r}$

$2344 \mathrm{r} 218 \mathrm{r}$

2 1 $341 \mathrm{r} 220 \mathrm{r}$

$21 \mathrm{r} 340 \mathrm{r} 221 \mathrm{r}$

$21 \mathrm{r} 339 \mathrm{r} 222 \mathrm{r}$

$22 \mathrm{r} 336 \mathrm{r} 224 \mathrm{r}$

$22 \mathrm{r} 335 \mathrm{r} 225 \mathrm{r}$

$23 \mathrm{r} 332 \mathrm{r} 227 \mathrm{r}$

$24 \mathrm{r} 329 \mathrm{r} 229 \mathrm{r}$

$25 \mathrm{r} 326 \mathrm{r} 231 \mathrm{r}$

$26.323 r 233 \mathrm{r}$

$28 \mathrm{r} 318 \mathrm{r} 236 \mathrm{r}$

$211 \mathrm{r} 311 \mathrm{r} 240 \mathrm{r}$ 
c universe 3: structure of fuel rod lattice elements

$154 \quad 2-13.75 \quad-58 \quad u=3 \quad$ S fuel element

$149 \quad 12 \quad+1.00 \quad 58-268 \quad$ u=3 $\$$ gap

$\begin{array}{lllll}144 & 7 & -19.66 \quad 268 & -478 \quad u=3 & \text { sliner }\end{array}$

$159 \quad 6 \quad-8.4 \quad 478-698 \quad u=3 \quad \$$ cladding

$141 \quad 12 \quad-1.00 \quad 698 \quad \mathrm{u}=3$ \$ water between the fuel rods

162 0 17:-29:19:-1 S outside world

*1 py 0 \$ $x-z$ plane, reflective

17 cz 29.135

19 pz 31.75 \$ top of reactor

$29 \mathrm{pz}-31.75 \$$ bottom of reactor

$\begin{array}{llllll}58 & \mathrm{c} / z & 3.4414 & .8515 & .3240\end{array}$

$\begin{array}{lllll}268 & \mathrm{c} / \mathrm{z} & 3.4414 & .8515 & .3345\end{array}$

$\begin{array}{lllll}478 & \mathrm{c} / \mathrm{z} & 3.4414 & .8515 & .3475\end{array}$

$\begin{array}{lllll}698 & \mathrm{c} / 2 & 3.4414 & .8515 & .4318\end{array}$

$880 \mathrm{cz} 1.7251$

$881 \mathrm{cz} 1.805 \mathrm{l}$

$882 \mathrm{cz} 1.9051$

$905 \mathrm{cz} 2.1055$

$906 \mathrm{c} / \mathrm{z} \quad 16.711302 .1055$

$907 \mathrm{c} / \mathrm{z}-16.711302 .1055$

$913 \mathrm{c} / \mathrm{z} \quad 011.91852 .1055$

$914 \mathrm{c} / \mathrm{z} \quad 10.32175 .95922 .1055$

$915 \mathrm{c} / \mathrm{z} \quad 8.3557 \quad 14.47242 .1055$

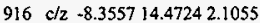

$917 \mathrm{c} / \mathrm{z}-10.32175 .95922 .1055$

301 px 3.9330

302 px 2.9498

303 p 11.732050807605 .8994

304 p 11.732050807603 .9330

$\begin{array}{llllllll}305 & -1 & 1.7320508076 & -.9834\end{array}$

306 p $-11.73205080760-2.9498$

imp:n $118 \mathrm{r} 0$

$\begin{array}{lllllll}\mathrm{m} 2 & 92235.40 c & -.70573 & 92238.40 c & -.23821 & 7014.40 c & -.05605\end{array}$

m6 $\quad 41093.40 c-.99000 \quad 40000.40 c-.01000$

$\mathrm{m} 7 \quad 74000.40 \mathrm{c}-.74000$

$\mathrm{mll} 5010.03 \mathrm{~d}-.6870 \quad 5011.40 \mathrm{c}-.0840 \quad 6012.40 \mathrm{c}-.2290$

$\mathrm{m} 12 \quad 1001.00 \mathrm{c}$ I $1002.55 \mathrm{c} 18016.40 \mathrm{c} 1$

$\mathrm{mt} 2$ hwtr.01 lwtr.01

kcode 200156

warning. tallies are normed per fission neutron for one generation.

99- ksrc $3.2 .2 .23 .2-3.2 .2 .23 .243 .2-43.243 .1-43.2$

$100-$ e .01 .11 .10 .

101- fq fe

102- fc4 fuel rod flux in 5 y locations averaged over $5 x$ elements

103- $\quad$ 4:n $(154<(42[-10:-6-10]))$ average $5 x$ elements at $j=-1$ $(154<(42[-10:-630])) \&$ \& average $5 x$ elements at $j=3$

( $154<(42[-10:-6100]))$ Saverage $5 x$ elements at $j=10$ \& $(154<(42[-10:-6210])) \$$ average $5 \times$ elements at $j=21$ (154<(42[-10:-6 290$])$ ) $\$$ average $5 x$ elements at $j=29$ sd4 $104.70890624 \mathrm{r} \quad \$ 5$ times the volume of cell 154 print -98

prdmp $2 \mathrm{j}-1$

ptrac buffer $=20$ file=asc write $=$ all event $=b$ k

111 - purac buffer-20 file-asc
initial source from ksrc card.

print table 90

original number of points

8

points not in any cell

points in cells of zero importance

points in void cells

points in ambiguous cells

1) points rejected

its remaining

points after expansion or contraction 


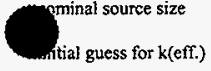

200

1.000000

cycles to skip before tallying

number of keff cycles that can be stored

total fission nubar data are being used

Itally 4

print table 30

$+$

tally type 4 track length estimate of particle flux.

fuel rod flux in 5 y locations averaged over $5 x$ elements

tally for neutrons

order of printing: $f e$

cells $(154<(42[-10:-6-10]))(154<(42[-10:-630]))(154<(42[-10:-6100]))(154<(42[-10:-6210]))(154<(42[-10:-6$ $2901)$ )

energy bins

$0.00000 \mathrm{E}+00$ to $1.00000 \mathrm{E}-02 \mathrm{mev}$

1.00000E-02 to $1.00000 \mathrm{E}-01 \mathrm{mev}$

$1.00000 \mathrm{E}-01$ to $1.00000 \mathrm{E}+00 \mathrm{mev}$

$1.00000 \mathrm{E}+00$ to $1.00000 \mathrm{E}+01 \mathrm{mev}$ total bin

Imaterial composition

print table 40

the sum of the fractions of material 2 was $9.999900 \mathrm{E}-01$

the sum of the fractions of material 7 was $7.400000 \mathrm{E}-01$

the sum of the fractions of material 12 was $3.000000 E+00$

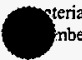

component nuclide, atom fraction

$\begin{array}{rrr}92235,0.37504 & 92238,0.12499 & 7014,0.49997 \\ 41093,0.98982 & 40000,0.01018 & \\ 74000,1.00000 & & \\ 5010,0.71977 & 5011,0.08004 & 6012,0.20019 \\ 1001,0.33333 & 1002,0.33333 & 8016,0.33333\end{array}$

associated thermal s(a,b) data sets: hwtr.01t lwtr.01t

material

number component nuclide, mass fraction

$\begin{array}{rrrr}2 & 92235,0.70574 & 92238,0.23821 & 7014,0.05605 \\ 6 & 41093,0.99000 & 40000,0.01000 & \\ 7 & 74000,1.00000 & & \\ 11 & 5010,0.68700 & 5011,0.08400 & 6012,0.22900 \\ 12 & 1001,0.05300 & 1002,0.10591 & 8016,0.84109\end{array}$

waming. 3 of the materials had unnormalized fractions.

lcell volumes and masses

print table 50

$\begin{array}{cccc}\text { cell atom } & \text { gram } & \text { input } & \text { calculated } \\ \text { density density } & \text { volume } & \text { reason volume } \\ \text { volume mass pieces not calculated }\end{array}$

l $30 \quad 0.00000 \mathrm{E}+00 \quad 0.00000 \mathrm{E}+00 \quad 0.00000 \mathrm{E}+00 \quad 0.00000 \mathrm{E}+00 \quad 0.00000 \mathrm{E}+00$

$231 \quad 0.00000 \mathrm{E}+00 \quad 0.00000 \mathrm{E}+00 \quad 0.00000 \mathrm{E}+00 \quad 0.00000 \mathrm{E}+00 \quad 0.00000 \mathrm{E}+00$

$\begin{array}{lllllll}37 & 0.00000 \mathrm{E}+00 & 0.00000 \mathrm{E}+00 & 0.00000 \mathrm{E}+00 & 0.00000 \mathrm{E}+00 & 0.00000 \mathrm{E}+00\end{array}$

$\begin{array}{lllllll}34 & 0.00000 \mathrm{E}+00 & 0.00000 \mathrm{E}+00 & 0.00000 \mathrm{E}+00 & 8.84370 \mathrm{E}+02 & 0.00000 \mathrm{E}+00\end{array}$

$\begin{array}{lllllll}32 & 0.00000 \mathrm{E}+00 & 0.00000 \mathrm{E}+00 & 0.00000 \mathrm{E}+00 & 8.84370 \mathrm{E}+02 & 0.00000 \mathrm{E}+00\end{array}$

$\begin{array}{lllllll}33 & 0.00000 E+00 & 0.00000 E+00 & 0.00000 E+00 & 8.84370 E+02 & 0.00000 E+00\end{array}$

\section{asymmetric \\ asymmetric asymmetric}


HNF-SD-SNF-CSWD-005, Rev. 0
$350.00000 \mathrm{E}+00 \quad 0.00000 \mathrm{E}+00 \quad 0.00000 \mathrm{E}+00 \quad 8.84370 \mathrm{E}+02 \quad 0.00000 \mathrm{E}+00$ $\begin{array}{llllll}36 & 0.00000 \mathrm{E}+00 & 0.00000 \mathrm{E}+00 & 0.00000 \mathrm{E}+00 & 8.84370 \mathrm{E}+02 & 0.00000 \mathrm{E}+00\end{array}$ $\begin{array}{llllll}38 & 1.15958 \mathrm{E}-01 & 2.02000 \mathrm{E}+00 & 0.00000 \mathrm{E}+00 & 0.00000 \mathrm{E}+00 & 0.00000 \mathrm{E}+00\end{array}$ $10 \quad 395.44575 \mathrm{E}-02 \quad 8.40000 \mathrm{E}+00 \quad 0.00000 \mathrm{E}+00 \quad 0.00000 \mathrm{E}+00 \quad 0.00000 \mathrm{E}+00$ $\begin{array}{lllllllll}11 & 40 & 9.50007 \mathrm{E}-02 & 1.00000 \mathrm{E}+00 & 0.00000 \mathrm{E}+00 & 0.00000 \mathrm{E}+00 & 0.00000 \mathrm{E}+00\end{array}$ $\begin{array}{lllllllll}12 & 41 & 5.44575 \mathrm{E}-02 & 8.40000 \mathrm{E}+00 & 0.00000 \mathrm{E}+00 & 0.00000 \mathrm{E}+00 & 0.00000 \mathrm{E}+\infty\end{array}$

$\begin{array}{llllllllll}13 & 140 & 0.00000 \mathrm{E}+00 & 0.00000 \mathrm{E}+00 & 0.00000 \mathrm{E}+00 & 0.00000 \mathrm{E}+00 & 0.00000 \mathrm{E}+00\end{array}$ $14 \quad 42 \quad 9.50007 \mathrm{E}-02 \quad 1.00000 \mathrm{E}+00 \quad 0.00000 \mathrm{E}+00 \quad 0.00000 \mathrm{E}+00 \quad 0.00000 \mathrm{E}+01$ $\begin{array}{llllllll}15 & 154 & 6.62920 \mathrm{E}-02 & 1.37500 \mathrm{E}+01 & 0.00000 \mathrm{E}+00 & 0.00000 \mathrm{E}+00 & 0.00000 \mathrm{E}+00\end{array}$ $\begin{array}{lllllll}16 & 149 & 9.50007 \mathrm{E}-02 & 1.00000 \mathrm{E}+00 & 0.00000 \mathrm{E}+00 & 0.00000 \mathrm{E}+00 & 0.00000 \mathrm{E}+00\end{array}$ $\begin{array}{llllllll}17 & 144 & 6.43969 \mathrm{E}-02 & 1.96600 \mathrm{E}+01 & 0.00000 \mathrm{E}+00 & 0.00000 \mathrm{E}+00 & 0.00000 \mathrm{E}+00\end{array}$ $\begin{array}{llllllll}18 & 159 & 5.44575 \mathrm{E}-02 & 8.40000 \mathrm{E}+00 & 0.00000 \mathrm{E}+00 & 0.00000 \mathrm{E}+00 & 0.00000 \mathrm{E}+00\end{array}$ $\begin{array}{llllllll}19 & 141 & 9.50007 \mathrm{E}-02 & 1.00000 \mathrm{E}+00 & 0.00000 \mathrm{E}+00 & 0.00000 \mathrm{E}+00 & 0.00000 \mathrm{E}+00\end{array}$ $\begin{array}{lllllll}20 & 162 & 0.00000 E+00 & 0.00000 E+00 & 0.00000 E+00 & 0.00000 E+00 & 0.00000 E+00\end{array}$ 1surface areas print table 50 surface input calculated reason area
area area not calculated

$1 \quad 10.00000 \mathrm{E}+00 \quad 0.00000 \mathrm{E}+00$ asymmetric

$2 \quad 170.00000 \mathrm{E}+00 \quad 0.00000 \mathrm{E}+00$ asymmetric

$3190.00000 \mathrm{E}+00 \quad 0.00000 \mathrm{E}+00$ asymmetric

$4290.00000 \mathrm{E}+00 \quad 0.00000 \mathrm{E}+00$ asymmetric

$558 \quad 0.00000 \mathrm{E}+00 \quad 0.00000 \mathrm{E}+00$ infinite

$6268 \quad 0.00000 \mathrm{E}+00 \quad 0.00000 \mathrm{E}+00$ infinite

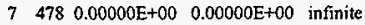

$8 \quad 698 \quad 0.00000 \mathrm{E}+00 \quad 0.00000 \mathrm{E}+00$ infinite

$9880 \quad 0.00000 \mathrm{E}+00 \quad 0.00000 \mathrm{E}+00$ infinite

$10 \quad 881 \quad 0.00000 \mathrm{E}+00 \quad 0.00000 \mathrm{E}+00$ infinite

$11882 \quad 0.00000 \mathrm{E}+00 \quad 0.00000 \mathrm{E}+00$ infinite

$12905 \quad 0.00000 \mathrm{E}+00 \quad 0.00000 \mathrm{E}+00$ asymmetric

$13906 \quad 0.00000 \mathrm{E}+00 \quad 0.00000 \mathrm{E}+00$ asymmetric

4 $9070.00000 \mathrm{E}+00 \quad 0.00000 \mathrm{E}+00$ asymmetric

$913 \quad 0.00000 \mathrm{E}+00 \quad 8.40057 \mathrm{E}+02$

$9140.00000 \mathrm{E}+00 \quad 8.40057 \mathrm{E}+02$

$\begin{array}{lll}17 & 915 & 0.00000 \mathrm{E}+00 \quad 8.40057 \mathrm{E}+02\end{array}$

$\begin{array}{lll}18 & 916 & 0.00000 \mathrm{E}+00 \quad 8.40057 \mathrm{E}+02\end{array}$

$19917 \quad 0.00000 \mathrm{E}+00 \quad 8.40057 \mathrm{E}+02$

$203010.00000 \mathrm{E}+00 \quad 0.00000 \mathrm{E}+00$ infinite

$21302 \quad 0.00000 \mathrm{E}+00 \quad 0.00000 \mathrm{E}+00$ infinite

$22 \quad 303 \quad 0.00000 \mathrm{E}+00 \quad 0.00000 \mathrm{E}+00$ infinite

$233040.00000 \mathrm{E}+00 \quad 0.00000 \mathrm{E}+00$

$24305 \quad 0.00000 \mathrm{E}+00 \quad 0.00000 \mathrm{E}+00$

$25306 \quad 0.00000 \mathrm{E}+00 \quad 0.00000 \mathrm{E}+00$

lcells

atom gram cell mat density density volume mass pieces importance
I $30 \quad 0 \quad 0.00000 \mathrm{E}+000.00000 \mathrm{E}+000.00000 \mathrm{E}+000.00000 \mathrm{E}+00$

$23100.00000 \mathrm{E}+000.00000 \mathrm{E}+000.00000 \mathrm{E}+000.00000 \mathrm{E}+00$

$337 \quad 0 \quad 0.00000 \mathrm{E}+000.00000 \mathrm{E}+00 \quad 0.00000 \mathrm{E}+000.00000 \mathrm{E}+00$

$434 \quad 0 \quad 0.00000 \mathrm{E}+000.00000 \mathrm{E}+008.84370 \mathrm{E}+020.00000 \mathrm{E}+00$

$532 \quad 0 \quad 0.00000 \mathrm{E}+000.00000 \mathrm{E}+008.84370 \mathrm{E}+020.00000 \mathrm{E}+00$

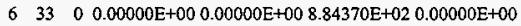

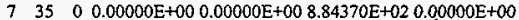

$836 \quad 0 \quad 0.00000 \mathrm{E}+00 \quad 0.00000 \mathrm{E}+00 \quad 8.84370 \mathrm{E}+02 \quad 0.00000 \mathrm{E}+00$

$938 \quad 111.15958 \mathrm{E}-012.02000 \mathrm{E}+00 \quad 0.00000 \mathrm{E}+000.00000 \mathrm{E}+\infty$

$1039 \quad 6 \quad 5.44575 \mathrm{E}-02 \quad 8.40000 \mathrm{E}+000.00000 \mathrm{E}+000.00000 \mathrm{E}+00$

I1 $40 \quad 12 \mathrm{~s} 9.50007 \mathrm{E}-021.00000 \mathrm{E}+000.00000 \mathrm{E}+000.00000 \mathrm{E}+00$

$124165.44575 \mathrm{E}-028.40000 \mathrm{E}+00 \quad 0.00000 \mathrm{E}+000.00000 \mathrm{E}+00$

$13 \quad 140 \quad 0 \quad 0.00000 \mathrm{E}+00 \quad 0.00000 \mathrm{E}+00 \quad 0.00000 \mathrm{E}+000.00000 \mathrm{E}+00$

$14 \quad 4212 \mathrm{~s} 9.50007 \mathrm{E}-021.00000 \mathrm{E}+000.00000 \mathrm{E}+000.00000 \mathrm{E}+00$

$1515426.62920 \mathrm{E}-021.37500 \mathrm{E}+010.00000 \mathrm{E}+000.00000 \mathrm{E}+00$

149 12s $9.50007 \mathrm{E}-02 \quad 1.00000 \mathrm{E}+000.00000 \mathrm{E}+000.00000 \mathrm{E}+00$

$14476.43969 \mathrm{E}-02 \quad 1.96600 \mathrm{E}+010.00000 \mathrm{E}+000.00000 \mathrm{E}+00$

$15965.44575 \mathrm{E}-02 \quad 8.40000 \mathrm{E}+000.00000 \mathrm{E}+000.00000 \mathrm{E} \div 00$
1

0 infinite

0 infinite

0 infinite

0 infinite

0

0

\section{0}

0

0

0

\section{(a) infinite}

0 asymmetric asymmetric

infinite

infinite

infinite

infinite print table 60
0 $1.0000 \mathrm{E}+00$
o $1.0000 \mathrm{E}+00$
o $1.0000 \mathrm{E}+00$
$11.0000 \mathrm{E}+00$
$1.0000 \mathrm{E}+00$
$11.0000 \mathrm{E}+00$
1 $1.0000 \mathrm{E}+00$
1 $1.0000 \mathrm{E}+00$
$01.0000 \mathrm{E}+00$
a $1.0000 E+00$
o $1.0000 \mathrm{E}+00$
$01.0000 \mathrm{E}+00$
$0 \quad 1.0000 \mathrm{E}+00$
$0 \quad 1.0000 \mathrm{E}+00$
o $1.0000 \mathrm{E}+00$
$01.0000 \mathrm{E}+00$
$01.0000 \mathrm{E}+00$
o $1.0000 \mathrm{E}+00$ 
$19 \quad[4] \quad 1259.50007 \mathrm{E}-02 \quad 1.00000 \mathrm{E}+000.00000 \mathrm{E}+000.00000 \mathrm{E}+00 \quad 0 \quad 1.0000 \mathrm{E}+00$

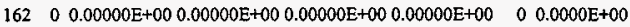

HNF-SD-SNF-CSWD-005, Rev. 0

$4.42185 \mathrm{E}+030.00000 \mathrm{E}+00$

total

1 surfaces

print table 70

surface trans type surface coefficients

$1 \quad 1$ refl. py $0.0000000 \mathrm{E}+00$

$17 \quad$ CZ $2.9135000 E+01$

319 pz $3.1750000 \mathrm{E}+01$

$29 \quad \mathrm{pz}-3.1750000 \mathrm{E}+01$

$5 \quad 58 \quad c / z \quad 3.4414000 \mathrm{E}+00 \quad 8.5150000 \mathrm{E}-01 \quad 3.2400000 \mathrm{E}-01$

$\begin{array}{lllllll}6 & 268 \quad \mathrm{c} / 2 & 3.4414000 \mathrm{E}+00 & 8.5150000 \mathrm{E}-01 & 3.3450000 \mathrm{E}-01\end{array}$

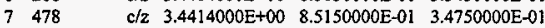

$\begin{array}{lllllll}8 & 698 & c / z & 3.4414000 \mathrm{E}+00 & 8.5150000 \mathrm{E}-01 & 4.3180000 \mathrm{E}-01\end{array}$

9880 c2 $1.7251000 \mathrm{E}+00$

$\begin{array}{llll}10 & 881 & \mathrm{CZ} & 1.8051000 \mathrm{E}+00\end{array}$

$\begin{array}{llll}11 & 882 \quad \mathrm{cz} & 1.9051000 \mathrm{E}+00\end{array}$

$\begin{array}{llll}12 & 905 \quad \mathrm{CZ} & 2.1055000 \mathrm{E}+00\end{array}$

$\begin{array}{llllll}13 & 906 \quad c / 2 & 1.6711300 \mathrm{E}+01 & 0.0000000 \mathrm{E}+00 & 2.1055000 \mathrm{E}+00\end{array}$

$14907 \quad \mathrm{c} / \mathrm{z}-1.6711300 \mathrm{E}+01 \quad 0.0000000 \mathrm{E}+00 \quad 2.1055000 \mathrm{E}+00$

I5 $913 \quad \mathrm{c} / 2 \quad 0.0000000 \mathrm{E}+00 \quad 1.1918500 \mathrm{E}+0 \mathrm{I} \quad 2.1055000 \mathrm{E}+00$

$\begin{array}{lllll}16914 \quad \mathrm{c} / 2 & 1.0321700 \mathrm{E}+01 & 5.9592000 \mathrm{E}+00 & 2.1055000 \mathrm{E}+00\end{array}$

$\begin{array}{llllll}17 & 915 & c / z & 8.3557000 E+00 & 1.4472400 \mathrm{E}+01 & 2.1055000 \mathrm{E}+00\end{array}$

$\begin{array}{llllll}18 & 916 & c / z & -8.3557000 E+00 & 1.4472400 E+01 & 2.1055000 E+00\end{array}$

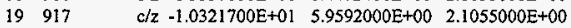

$\begin{array}{llll}20 & 301 & \mathrm{px} & 3.9330000 \mathrm{E}+00\end{array}$

$21302 \quad \mathrm{px} 2.9498000 \mathrm{E}+00$

$\begin{array}{lllllll}22 & 303 & \text { p } & 1.0000000 \mathrm{E}+00 & 1.7320508 \mathrm{E}+00 & 0.0000000 \mathrm{E}+00 & 5.8994000 \mathrm{E}+00\end{array}$

$\begin{array}{lllllll}23 & 304 & p & 1.0000000 \mathrm{E}+00 & 1.7320508 \mathrm{E}+00 & 0.0000000 \mathrm{E}+00 & 3.9330000 \mathrm{E}+00\end{array}$

$\begin{array}{lllllll}24 & 305 & p & -1.0000000 E+00 & 1.7320508 E+00 & 0.0000000 E+00 & -9.8340000 E-01\end{array}$

$\begin{array}{lllllll}25306 & p & -1.0000000 E+00 & 1.7320508 \mathrm{E}+00 & 0.0000000 \mathrm{E}+00 & -2.9498000 \mathrm{E}+00\end{array}$

11 temperatures in mev for the free-gas thermal neutron treatment. print table 72

all non-zero importance cells with materials have a temperature for thermal neutrons of $2.5300 \mathrm{E}-08$ mev.

2 warning messages so far.

lcross-section tables

print table 100

table length

tables from file testlib!

$1001.00 \mathrm{c} \quad 1782$ !-h-l from endf-vi.l

$1002.55 \mathrm{c} 4102$ njoy

mat $12505 / 26 / 93$

$5011.40 \mathrm{c} 3229$ ENDL library name: nd900719 MCNP translation: 900831 14:10:05

6012.40c 4858 ENDL library name: nd900719 MCNP translation: 900831 14:10:05

7014.40c 8535 ENDL library name: nd900719 MCNP translation: 900831 14:10:05

$8016.40 \mathrm{c} 5358$ ENDL library name: nd900719 MCNP translation: 900831 14:10:05

40000.40c 93372 ENDL library name: nd900719 MCNP translation: 900831 14:10:05

41093.40c 42746 ENDL library name: nd900719 MCNP translation: 900831 14:10:05

74000.40C 91244 ENDL library name: nd900719 MCNP translation: 900831 14:10:05

92235.40c 34772 ENDL library name: nd900719 MCNP translation: 9total nu 10:05

92238.40c 40834 ENDL library name: nd901118 MCNP translation: 9total nu 56:49

$5010.03 d \quad 3682$ b-10 endf/b-iv new gamma production format

901119 hwtr.01t 10193 deuterium in heavy water at 300 degrees kelvin

14 oct 75

Iwt.01t 10193 hydrogen in light water at 300 degrees kelvin

$\begin{array}{lll}1002 & 0 & 010 / 22 / 85\end{array}$

$10010010 / 22 / 85$

total 354900

warning. neutron energy cutoff is below some cross-section tables. 
-

3 warning messages so far.

1 starting morun. field length $=0 \quad$ cp $0=0.00$

probl 8 - kcode in a hexagonal prism lattice.

cycle $=0$
HNF-SD-SNF-CSWD-005, Rev. 0

print table 110

nps $x \quad y \quad z \quad$ cell lattice $(i, j, k)$ surface $u \quad v \quad w$ energy weight time

I 3.000E+00 2.000E-01 2.000E-0! 140 $3.492 \mathrm{E}+00 \quad 1.051 \mathrm{E}+00 \quad 2.000 \mathrm{E}-01 \quad 42($ $3.492 \mathrm{E}+00 \quad 1.051 \mathrm{E}+00 \quad 2.000 \mathrm{E}-01 \quad 154$

$23.000 \mathrm{E}+00 \quad 2.000 \mathrm{E}-01 \quad 2.000 \mathrm{E}-01140$ $3.492 \mathrm{E}+001.051 \mathrm{E}+00$ 2.000E-01 $42(0,-1,0)$ $3.492 \mathrm{E}+001.051 \mathrm{E}+00 \quad 2.000 \mathrm{E}-01154$

3 3.000E+00 2.000E-01 2.000E-01 140 $3.492 \mathrm{E}+001.051 \mathrm{E}+00 \quad 2.000 \mathrm{E}-01 \quad 42(0,-1$, $3.492 \mathrm{E}+00 \quad 1.051 \mathrm{E}+00 \quad 2.000 \mathrm{E}-01154$

$43.000 \mathrm{E}+00 \quad 2.000 \mathrm{E}-01 \quad 2.000 \mathrm{E}-01 \quad 140$ $3.492 \mathrm{E}+001.051 \mathrm{E}+002.000 \mathrm{E}-0 \mathrm{l}$ 42( $0,-1,0)$ $3.492 \mathrm{E}+00 \quad 1.051 \mathrm{E}+00 \quad 2.000 \mathrm{E}-01154$

5 3.000E+00 2.000E-01 2.000E-01 140 $3.492 \mathrm{E}+00 \quad 1.051 \mathrm{E}+00 \quad 2.000 \mathrm{E}-01 \quad 42(0,-1,0)$ $3.492 \mathrm{E}+00 \quad 1.051 \mathrm{E}+00 \quad 2.000 \mathrm{E}-01154$

$63.000 \mathrm{E}+00$ 2.000E-01 2.000E-01 140 $3.492 \mathrm{E}+00 \quad 1.051 \mathrm{E}+00 \quad 2.000 \mathrm{E}-01$ 42( $0,-1,0)$ $3.492 \mathrm{E}+00 \quad 1.051 \mathrm{E}+00 \quad 2.000 \mathrm{E}+01 \quad 154$

$73.000 \mathrm{E}+00 \quad 2.000 \mathrm{E}-012.000 \mathrm{E}-01140$ $3.492 \mathrm{E}+00$ 1.0S1E+00 2.000E-01 42( $3.492 E+001.051 E+00 \quad 2.000 E-01154$ $3.000 \mathrm{E}+00 \quad 2.000 \mathrm{E}-01 \quad 2.000 \mathrm{E}-01 \quad 140$ $3.492 \mathrm{E}+00 \quad 1.051 \mathrm{E}+002.000 \mathrm{E}-01 \quad 42(0,-1,0)$ $3.492 \mathrm{E}+00 \quad 1.051 \mathrm{E}+00 \quad 2.000 \mathrm{E}-01154$

$93.000 \mathrm{E}+00$ 2.000E-01 2.000E-01 140 $3.492 \mathrm{E}+00 \quad 1.051 \mathrm{E}+00 \quad 2.000 \mathrm{E}-01 \quad 42$ ( $3.492 \mathrm{E}+00 \quad 1.051 \mathrm{E}+00 \quad 2.000 \mathrm{E}-01154$ $10 \quad 3.000 \mathrm{E}+00 \quad 2.000 \mathrm{E}-01 \quad 2.000 \mathrm{E}-0 \mathrm{I} \quad \mathrm{I} 40$ $3.492 \mathrm{E}+00 \quad 1.051 \mathrm{E}+00 \quad 2.000 \mathrm{E}-01 \quad 42(0,-1,0)$ $3.492 \mathrm{E}+00 \quad 1.051 \mathrm{E}+00 \quad 2.000 \mathrm{E}-01 \quad 154$

11 $3.000 \mathrm{E}+00 \quad 2.000 \mathrm{E}-01 \quad 2.000 \mathrm{E}-01 \quad 140$ $3.492 \mathrm{E}+00 \quad 1.051 \mathrm{E}+00 \quad 2.000 \mathrm{E}-01 \quad 42(0,-1,0)$ $3.492 \mathrm{E}+00 \quad 1.051 \mathrm{E}+00 \quad 2.000 \mathrm{E}-01 \quad 154$

$123.000 \mathrm{E}+00 \quad 2.000 \mathrm{E}-01 \quad 2.000 \mathrm{E}-01 \quad 140$ $3.492 \mathrm{E}+00 \quad 1.051 \mathrm{E}+00 \quad 2.000 \mathrm{E}-01 \quad 42(0,-1,0)$ $3.492 \mathrm{E}+001.051 \mathrm{E}+002.000 \mathrm{E}-01154$ 13 3.000E+00 2.000E-01 2.000E-01 140 $3.492 \mathrm{E}+00 \quad 1.051 \mathrm{E}+00 \quad 2.000 \mathrm{E}-01 \quad 42(0,-1,0)$ $3.492 \mathrm{E}+00 \quad 1.051 \mathrm{E}+00 \quad 2.000 \mathrm{E}-01 \quad 154$

14 3.000E +00 2.000E-01 2.000E-01 140 $3.492 \mathrm{E}+00 \quad 1.051 \mathrm{E}+00 \quad 2.000 \mathrm{E}-0) \quad 42(0,-1,0)$ $3.492 \mathrm{E}+00 \quad 1.051 \mathrm{E}+00 \quad 2.000 \mathrm{E}-01 \quad 154$ I5 $3.000 \mathrm{E}+00 \quad 2.000 \mathrm{E}-01 \quad 2.000 \mathrm{E}-01 \quad 140$ $3.492 \mathrm{E}+00 \quad 1.051 \mathrm{E}+002.000 \mathrm{E}-01 \quad 42(0,-1,0)$ $3.492 \mathrm{E}+00 \quad 1.051 \mathrm{E}+00 \quad 2.000 \mathrm{E}-01154$

$16 \quad 3.000 \mathrm{E}+00 \quad 2.000 \mathrm{E}-01 \quad 2.000 \mathrm{E}-01 \quad 140$ $3.492 \mathrm{E}+001.051 \mathrm{E}+00 \quad 2.000 \mathrm{E}-01 \quad 42(0,-1,0)$ $3.492 \mathrm{E}+00 \quad 1.051 \mathrm{E}+00 \quad 2.000 \mathrm{E}-03 \quad 154$

$173.000 \mathrm{E}+00 \quad 2.000 \mathrm{E}-01 \quad 2.000 \mathrm{E}-01 \quad 140$ $3.492 \mathrm{E}+001.051 \mathrm{E}+00$ 2.000E-01 $42(0,-1,0)$ $3.492 \mathrm{E}+00 \quad 1.051 \mathrm{E}+00 \quad 2.000 \mathrm{E}-01 \quad 154$ $183.000 \mathrm{E}+00 \quad 2.000 \mathrm{E}-01 \quad 2.000 \mathrm{E}-01 \quad 140$ $8.492 \mathrm{E}+00 \quad 1.051 \mathrm{E}+00 \quad 2.000 \mathrm{E}-01 \quad 42(0,-1,0)$ $3.492 \mathrm{E}+00 \quad 1.051 \mathrm{E}+00 \quad 2.000 \mathrm{E}-01 \quad 154$ I9 $3.000 \mathrm{E}+00 \quad 2.000 \mathrm{E}-01 \quad 2.000 \mathrm{E}-01 \quad 140$

$0,-1,0)$

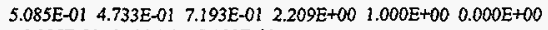
$5.085 \mathrm{E}-01 \quad 4.733 \mathrm{E}-01 \quad 7.193 \mathrm{E}-01$

0 5.085E-01 4.733E-01 7.193E-01

8.952E-01 -4.447E-01 $-2.944 \mathrm{E}-02 \quad 4.904 \mathrm{E}+00 \quad 1.000 \mathrm{E}+00 \quad 0.000 \mathrm{E}+00$ 8.952E-01-4.447E-01-2.944E-02

0 8.952E-01 -4.447E-01 -2.944E-02

$\begin{array}{llll}-6.184 \mathrm{E}-01-4.495 \mathrm{E}-01 & 6.446 \mathrm{E}-01 \quad 3.809 \mathrm{E}-01 \quad 1.000 \mathrm{E}+00 \quad 0.000 \mathrm{E}+00\end{array}$ $-6.184 \mathrm{E}-01-4.495 \mathrm{E}-01 \quad 6.446 \mathrm{E}-01$

$0-6.184 \mathrm{E}-01-4.495 \mathrm{E}-01 \quad 6.446 \mathrm{E}-0 \mathrm{l}$

9.710E-01 $-5.665 \mathrm{E}-02-2.323 \mathrm{E}-01 \quad 1.331 \mathrm{E}+00 \quad 1.000 \mathrm{E}+00 \quad 0.000 \mathrm{E}+00$ $9.710 \mathrm{E}-01-5.665 \mathrm{E}+02-2.323 \mathrm{E}-01$

0 9.710E-01 -5.665E-02 -2.323E-01

$5.861 \mathrm{E}-01 \quad 1.496 \mathrm{E}-01-7.963 \mathrm{E}-01 \quad 1.902 \mathrm{E}+00 \quad 1.000 \mathrm{E}+00 \quad 0.000 \mathrm{E}+00$ $5.861 \mathrm{E}-01 \quad 1.496 \mathrm{E}-01-7.963 \mathrm{E}-01$

0 5.861E-01 1.496E-01 -7.963E-01

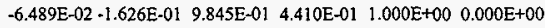
$-6.489 \mathrm{E}-02-1.626 \mathrm{E}-01 \quad 9.845 \mathrm{E}-01$

$0-6.489 \mathrm{E}-02-1.626 \mathrm{E}-01 \quad 9.845 \mathrm{E}-01$

$\begin{array}{lllll}-7.068 \mathrm{E}-02 & 3.263 \mathrm{E}-02 & -9.970 \mathrm{E}-01 & 4.750 \mathrm{E}-01 & 1.000 \mathrm{E}+00 \quad 0.000 \mathrm{E}+00\end{array}$ $-7.068 \mathrm{E}-02 \quad 3.263 \mathrm{E}-02-9.970 \mathrm{E}-01$

$0-7.068 \mathrm{E}-02 \quad 3.263 \mathrm{E}-02-9.970 \mathrm{E}-01$

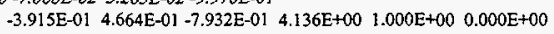

$-3.915 \mathrm{E}-01 \quad 4.664 \mathrm{E}-01-7.932 \mathrm{E}-01$

$0-3.915 \mathrm{E}-01 \quad 4.664 \mathrm{E}-01-7.932 \mathrm{E}-01$

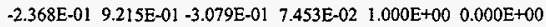
$-2.368 \mathrm{E}-019.215 \mathrm{E}-01-3.079 \mathrm{E}-01$

$0-2.368 \mathrm{E}-01 \quad 9.215 \mathrm{E}-01-3.079 \mathrm{E}-01$

$1.946 \mathrm{E}-01-3.204 \mathrm{E}-01 \quad 9.271 \mathrm{E}-01 \quad 3.128 \mathrm{E}+00 \quad 1.000 \mathrm{E}+00 \quad 0.000 \mathrm{E}+00$ $1.946 \mathrm{E}-01-3.204 \mathrm{E}-01 \quad 9.271 \mathrm{E}-01$

$0 \quad 1.946 \mathrm{E}-01-3.204 \mathrm{E}-01 \quad 9.271 \mathrm{E}-01$

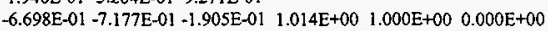
$-6.698 \mathrm{E}-01-7.177 \mathrm{E}-01-1.905 \mathrm{E}-01$

$0-6.698 \mathrm{E}-01-7.177 \mathrm{E}-01-1.905 \mathrm{E}-01$

$\begin{array}{lllll}-8.398 \mathrm{E}-01 & -4.129 \mathrm{E}-01 & 3.524 \mathrm{E}-01 & 1.395 \mathrm{E}+00 & 1.000 \mathrm{E}+00 \quad 0.000 \mathrm{E}+00\end{array}$ $-8.398 \mathrm{E}-01-4.129 \mathrm{E}-01 \quad 3.524 \mathrm{E}-01$

$0-8.398 \mathrm{E}-01-4.129 \mathrm{E}-01 \quad 3.524 \mathrm{E}-01$

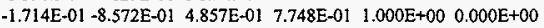
$-1.714 \mathrm{E}-01-8.572 \mathrm{E}-01$ 4.857E-01

$0+1.714 \mathrm{E}-01-8.572 \mathrm{E}-01 \quad 4.857 \mathrm{E}-01$

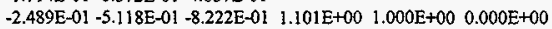
$-2.489 \mathrm{E}-01-\$ .118 \mathrm{E}-01-8.222 \mathrm{E}-01$

$0-2.489 \mathrm{E}-01-5.118 \mathrm{E}-01-8.222 \mathrm{E}-01$

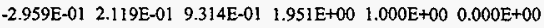
$-2.959 \mathrm{E}-01 \quad 2.119 \mathrm{E}-01 \quad 9.314 \mathrm{E}-01$

$0-2.959 \mathrm{E}-01$ 2. $119 \mathrm{E}-01 \quad 9.314 \mathrm{E}-01$

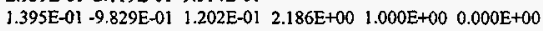
1.395E-01 -9.829E-01 1.202E-01

0 1.395E-01 $-9.829 E-01 \quad 1.202 E-01$

$6.909 \mathrm{E}-01-7.110 \mathrm{E}-01 \quad 1.307 \mathrm{E}-01 \quad 1.865 \mathrm{E}+00 \quad 1.000 \mathrm{E}+00 \quad 0.000 \mathrm{E}+00$ $6.909 \mathrm{E}-01-7.110 \mathrm{E}-01 \quad 1.307 \mathrm{E}-01$

$06.909 \mathrm{E}-01-7.110 \mathrm{E}-01 \quad 1.307 \mathrm{E}-01$

$-6.580 \mathrm{E}-01 \quad 5.320 \mathrm{E}-01-5.329 \mathrm{E}-01 \quad 1.229 \mathrm{E}+00 \quad 1.000 \mathrm{E}+00 \quad 0.000 \mathrm{E}+00$ $-6.580 \mathrm{E}-01 \quad 5.320 \mathrm{E}-01-5.329 \mathrm{E}-01$

$0-6.580 \mathrm{E}-01 \quad 5.320 \mathrm{E}-01-5.329 \mathrm{E}-01$

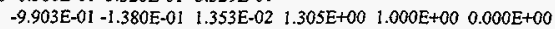



E $+002.000 \mathrm{E}-012.000 \mathrm{E}-01 \quad 140$ $3.492 \mathrm{E}+00 \quad 1.051 \mathrm{E}+00 \quad 2.000 \mathrm{E}-01 \quad 42(0,-1,0)$ $3.492 \mathrm{E}+00 \quad 1.051 \mathrm{E}+00 \quad 2.000 \mathrm{E}-01 \quad 154$

$213.000 \mathrm{E}+00 \quad 2.000 \mathrm{E}-01 \quad 2.000 \mathrm{E}-01 \quad 140$ $3.492 \mathrm{E}+00 \quad 1.051 \mathrm{E}+00 \quad 2.000 \mathrm{E}-01 \quad 42(0,-1,0)$ $3.492 \mathrm{E}+00 \quad 1.051 \mathrm{E}+00 \quad 2.000 \mathrm{E}-01 \quad 154$

$22 \quad 3.000 \mathrm{E}+00 \quad 2.000 \mathrm{E}-01 \quad 2.000 \mathrm{E}-01 \quad 140$ $3.492 \mathrm{E}+00 \quad 1.051 \mathrm{E}+00 \quad 2.000 \mathrm{E}-01 \quad 42(0,-1,0)$ $3.492 \mathrm{E}+00 \quad 1.051 \mathrm{E}+00 \quad 2.000 \mathrm{E}-01 \quad 154$

$23 \quad 3.000 \mathrm{E}+00 \quad 2.000 \mathrm{E}-01 \quad 2.000 \mathrm{E}-01 \quad 140$ $3.492 \mathrm{E}+00 \quad 1.051 \mathrm{E}+00 \quad 2.000 \mathrm{E}-01 \quad 42(0,-1,0)$ $3.492 \mathrm{E}+00 \quad 1.051 \mathrm{E}+00 \quad 2.000 \mathrm{E}-01 \quad 154$

$243.000 \mathrm{E}+00 \quad 2.000 \mathrm{E}-01 \quad 2.000 \mathrm{E}-01 \quad 140$ $3.492 \mathrm{E}+00 \quad 1.051 \mathrm{E}+00 \quad 2.000 \mathrm{E}-01 \quad 42(0,-1,0)$ $3.492 \mathrm{E}+00 \quad 1.051 \mathrm{E}+00 \quad 2.000 \mathrm{E}-01 \quad 154$

$253.000 \mathrm{E}+00 \quad 2.000 \mathrm{E}-01 \quad 2.000 \mathrm{E}-01 \quad 140$ $3.492 \mathrm{E}+00 \quad 1.051 \mathrm{E}+00 \quad 2.000 \mathrm{E}-01 \quad 42(0,-1,0)$ $3.492 \mathrm{E}+00 \quad 1.051 \mathrm{E}+00 \quad 2.000 \mathrm{E}-01154$

$262.000 \mathrm{E}-01 \quad 3.000 \mathrm{E}+00 \quad 2.000 \mathrm{E}-01 \quad 140$ $3.641 \mathrm{E}+00 \quad 4.456 \mathrm{E}-01 \quad 2.000 \mathrm{E}-01 \quad 42(-5,3,0)$ $3.641 \mathrm{E}+00 \quad 4.456 \mathrm{E}-01 \quad 2.000 \mathrm{E}-01 \quad 141$

$27 \quad 2.000 \mathrm{E}-01 \quad 3.000 \mathrm{E}+00 \quad 2.000 \mathrm{E}-01 \quad 140$ $3.64 ! \mathrm{E}+00 \quad 4.456 \mathrm{E}-01 \quad 2.000 \mathrm{E}-01 \quad 42(-5,3,0)$ $3.641 \mathrm{E}+00 \quad 4.456 \mathrm{E}-01 \quad 2.000 \mathrm{E}-01 \quad 141$

28 2.000E-01 $3.000 \mathrm{E}+00 \quad 2.000 \mathrm{E}-01140$ $3.641 \mathrm{E}+00 \quad 4.456 \mathrm{E}-01 \quad 2.000 \mathrm{E}-01 \quad 42(-5,3,0)$ $3.641 \mathrm{E}+00 \quad 4.456 \mathrm{E}-01 \quad 2.000 \mathrm{E}-01 \quad$ 14]

$292.000 \mathrm{E}-01 \quad 3.000 \mathrm{E}+00 \quad 2.000 \mathrm{E}-01 \quad 140$ $3.64 I E+00 \quad 4.456 E-01 \quad 2.000 \mathrm{E}-01 \quad 42(-5,3,0)$ $3.641 \mathrm{E}+00 \quad 4.456 \mathrm{E}-01 \quad 2.000 \mathrm{E}-01 \quad 141$ $2.000 \mathrm{E}-01 \quad 3.000 \mathrm{E}+00 \quad 2.000 \mathrm{E}-01 \quad 140$ $641 \mathrm{E}+00 \quad 4.456 \mathrm{E}-01 \quad 2.000 \mathrm{E}-01 \quad 42(-5,3,0)$ $641 \mathrm{E}+00 \quad 4.456 \mathrm{E}-01 \quad 2.000 \mathrm{E}-01 \quad 141$

$312.000 \mathrm{E}-01 \quad 3.000 \mathrm{E}+00 \quad 2.000 \mathrm{E}-01 \quad 140$ $3.641 \mathrm{E}+00 \quad 4.456 \mathrm{E}-01 \quad 2.000 \mathrm{E}-01 \quad 42(-5,3,0)$ $3.641 \mathrm{E}+00 \quad 4.456 \mathrm{E}-01 \quad 2.000 \mathrm{E}-01 \quad 141$

$32 \quad 2.000 \mathrm{E}-01 \quad 3.000 \mathrm{E}+00 \quad 2.000 \mathrm{E}-01 \quad 140$

$3.641 \mathrm{E}+00 \quad 4.456 \mathrm{E}-01 \quad 2.000 \mathrm{E}-01 \quad 42(-5,3,0)$ $3.641 \mathrm{E}+00 \quad 4.456 \mathrm{E}-01 \quad 2.000 \mathrm{E}-01 \quad 141$

33 2.000E-01 3.000E $+00 \quad 2.000 \mathrm{E}-01 \quad 140$ $3.641 \mathrm{E}+00 \quad 4.456 \mathrm{E}-01 \quad 2.000 \mathrm{E}-01 \quad 42(-5,3,0)$ $3.641 \mathrm{E}+00 \quad 4.456 \mathrm{E}-01 \quad 2.000 \mathrm{E}-01 \quad 141$

34 2.000E-01 $3.000 \mathrm{E}+00 \quad 2.000 \mathrm{E}-01 \quad 140$ $3.641 \mathrm{E}+00 \quad 4.456 \mathrm{E}-01 \quad 2.000 \mathrm{E}-01 \quad 42(-5,3,0)$ $3.641 \mathrm{E}+00 \quad 4.456 \mathrm{E}-01 \quad 2.000 \mathrm{E}-0 \mathrm{I} \quad 141$

$352.000 \mathrm{E}-0] \quad 3.000 \mathrm{E}+00 \quad 2.000 \mathrm{E}-01 \quad 140$ $3.641 \mathrm{E}+00 \quad 4.456 \mathrm{E}-01 \quad 2.000 \mathrm{E}-01 \quad 42(-5,3,0)$ $3.641 \mathrm{E}+00 \quad 4.456 \mathrm{E}-01 \quad 2.000 \mathrm{E}-01 \quad 141$

$362.000 \mathrm{E}-01 \quad 3.000 \mathrm{E}+00 \quad 2.000 \mathrm{E}-01 \quad 140$ $3.641 \mathrm{E}+00 \quad 4.456 \mathrm{E}-01 \quad 2.000 \mathrm{E}-01 \quad 42(-5,3,0)$ $3.641 \mathrm{E}+00 \quad 4.456 \mathrm{E}-01 \quad 2.000 \mathrm{E}-01 \quad 141$

$372.000 \mathrm{E}-01 \quad 3.000 \mathrm{E}+00 \quad 2.000 \mathrm{E}-01 \quad 140$ $3.641 \mathrm{E}+00 \quad 4.456 \mathrm{E}-01 \quad 2.000 \mathrm{E}-01 \quad 42(-5,3,0)$ $3.641 \mathrm{E}+00 \quad 4.456 \mathrm{E}-01 \quad 2.000 \mathrm{E}-01 \quad 141$

$38 \quad 2.000 \mathrm{E}-01 \quad 3.000 \mathrm{E}+00 \quad 2.000 \mathrm{E}-01 \quad 140$ $3.641 \mathrm{E}+00 \quad 4.456 \mathrm{E}-01 \quad 2.000 \mathrm{E}-01 \quad 42(-5,3,0)$ $3.641 \mathrm{E}+00 \quad 4.456 \mathrm{E}-0 \mathrm{I} \quad 2.000 \mathrm{E}-01 \quad 141$

$392.000 \mathrm{E}-01 \quad 3.000 \mathrm{E}+00 \quad 2.000 \mathrm{E}-01 \quad 140$ $3.641 \mathrm{E}+00 \quad 4.456 \mathrm{E}-01 \quad 2.000 \mathrm{E}-01 \quad 42(-5,3,0)$ $3.641 \mathrm{E}+00 \quad 4.456 \mathrm{E}-01 \quad 2.000 \mathrm{E}-01 \quad 141$

$40 \quad 2.000 \mathrm{E}-01 \quad 3.000 \mathrm{E}+00 \quad 2.000 \mathrm{E}-01 \quad 140$ $3.641 \mathrm{E}+00 \quad 4.456 \mathrm{E}-01 \quad 2.000 \mathrm{E}-01 \quad 42(-5,3,0)$ $641 \mathrm{E}+00 \quad 4.456 \mathrm{E}-01 \quad 2.000 \mathrm{E}-01 \quad 14 \mathrm{I}$

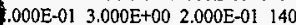
$-9.903 \mathrm{E}-01-1.380 \mathrm{E}-01 \quad 1.353 \mathrm{E}-02$ HNF-SD-SNF-CSWD-005, Rev. 0

$0-9.903 \mathrm{E}-01-1.380 \mathrm{E}-01 \quad 1.353 \mathrm{E}-02$

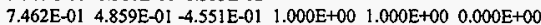

$7.462 \mathrm{E}-01 \quad 4.859 \mathrm{E}-01-4.55 \mathrm{LE}-01$

$0 \quad 7.462 \mathrm{E}-01 \quad 4.859 \mathrm{E}-01-4.55 \mathrm{IE}-01$

$-1.977 \mathrm{E}-01 \quad 9.797 \mathrm{E}-01 \quad 3.360 \mathrm{E}-02 \quad 3.990 \mathrm{E}+00 \quad 1.000 \mathrm{E}+00 \quad 0.000 \mathrm{E}+00$ $-1.977 \mathrm{E}-01$ 9.797E-01 3.360E-02

$0-1.977 \mathrm{E}-01 \quad 9.797 \mathrm{E}-01 \quad 3.360 \mathrm{E}-02$

$-9.117 \mathrm{E}-01-3.647 \mathrm{E}-01-1.891 \mathrm{E}-01 \quad 2.665 \mathrm{E}-01 \quad 1.000 \mathrm{E}+00 \quad 0.000 \mathrm{E}+00$

$-9.117 \mathrm{E}-01-3.647 \mathrm{E}-01-1.891 \mathrm{E}-01$

$0-9.117 \mathrm{E}-01-3.647 \mathrm{E}-01-1.891 \mathrm{E}-01$

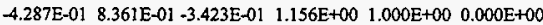
$-4.287 \mathrm{E}-01 \quad 8.361 \mathrm{E}-01-3.423 \mathrm{E}-01$

$0-4.287 \mathrm{E}-01$ 8.361E-01 -3.423E-01

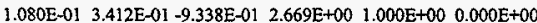
$1.080 \mathrm{E}-01 \quad 3.412 \mathrm{E}-01-9.338 \mathrm{E}-01$

$0 \quad 1.080 \mathrm{E}-01 \quad 3.412 \mathrm{E}-01-9.338 \mathrm{E}-01$

$-9.111 \mathrm{E}-01-9.012 \mathrm{E}-03-4.122 \mathrm{E}-01 \quad 2.185 \mathrm{E}+00 \quad 1.000 \mathrm{E}+00 \quad 0.000 \mathrm{E}+00$ $-9.111 \mathrm{E}-01-9.012 \mathrm{E}-03-4.122 \mathrm{E}-01$

$0-9.111 \mathrm{E}-01-9.012 \mathrm{E}-03-4.122 \mathrm{E}-01$ $-2.568 \mathrm{E}-01-6.391 \mathrm{E}-01-7.249 \mathrm{E}-01 \quad 7.280 \mathrm{E}-01 \quad 1.000 \mathrm{E}+00 \quad 0.000 \mathrm{E}+00$ $-2.568 \mathrm{E}-01-6.391 \mathrm{E}-01-7.249 \mathrm{E}-01$

$0-2.568 \mathrm{E}-01-6.391 \mathrm{E}-01-7.249 \mathrm{E}-01$

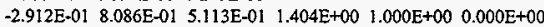
$-2.912 \mathrm{E}-01 \mathrm{t} \quad 8.086 \mathrm{E}-01$ 5.113E-01

$0-2.912 \mathrm{E}-01 \quad 8.086 \mathrm{E}-01 \quad 5.113 \mathrm{E}-01$

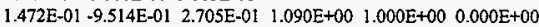
$1.472 \mathrm{E}-01-9.514 \mathrm{E}-01 \quad 2.705 \mathrm{E}-01$

$0 \quad 1.472 \mathrm{E}-01-9.514 \mathrm{E}-01 \quad 2.705 \mathrm{E}-01$ $-6.135 \mathrm{E}-01-7.645 \mathrm{E}-01-1.978 \mathrm{E}-01 \quad 2.483 \mathrm{E}+00 \quad 1.000 \mathrm{E}+00 \quad 0.000 \mathrm{E}+00$ $-6.135 \mathrm{E}-01-7.645 \mathrm{E}-01-1.978 \mathrm{E}-01$

$0-6.135 \mathrm{E}-01-7.645 \mathrm{E}-01-1.978 \mathrm{E}-01$ $\begin{array}{lllll}-5.702 \mathrm{E}-01 & 5.651 \mathrm{E}-01 & -5.963 \mathrm{E}-01 & 3.767 \mathrm{E}-01 & 1.000 \mathrm{E}+00 \quad 0.000 \mathrm{E}+00\end{array}$ $-5.702 \mathrm{E}-01$ 5.65IE-01 -5.963E-01

$0-5.702 \mathrm{E}-01 \quad 5.651 \mathrm{E}-01-5.963 \mathrm{E}-01$

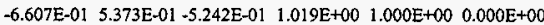
$-6.607 \mathrm{E}-01 \quad 5.373 \mathrm{E}-01-5.242 \mathrm{E}-01$

$0-6.607 \mathrm{E}-015.373 \mathrm{E}-01-5.242 \mathrm{E}-01$ $-9.742 \mathrm{E}-02-3.639 \mathrm{E}-01-9.263 \mathrm{E}-01 \quad 1.879 \mathrm{E}+00 \quad 1.000 \mathrm{E}+00 \quad 0.000 \mathrm{E}+00$ $-9.742 \mathrm{E}-02-3.639 \mathrm{E}-01-9.263 \mathrm{E}-01$

$0-9.742 \mathrm{E}-02-3.639 \mathrm{E}-01-9.263 \mathrm{E}-01$

$-1.965 \mathrm{E}-01-3.145 \mathrm{E}-01-9.287 \mathrm{E}-01 \quad 4.700 \mathrm{E}-0 \mathrm{I} \quad 1.000 \mathrm{E}+00 \quad 0.000 \mathrm{E}+00$ $-1.965 \mathrm{E}-01-3.145 \mathrm{E}-01-9.287 \mathrm{E}-01$

$0-1.965 \mathrm{E}-01-3.145 \mathrm{E}-01-9.287 \mathrm{E}-01$

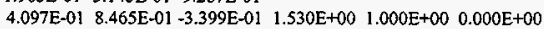
4.097E-01 8.465E-01 -3.399E-01

0 4.097E-01 8.465E-01 -3.399E-01

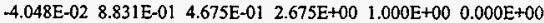
$-4.048 \mathrm{E}-02 \quad 8.831 \mathrm{E}-01 \quad 4.675 \mathrm{E}-0$

$0-4.048 \mathrm{E}-02 \quad 8.831 \mathrm{E}-01 \quad 4.675 \mathrm{E}-01$ 3.371E-01 -9.269E-01 -1.652E-01 9.222E-01 1.000E+00 $0.000 \mathrm{E}+00$ $3.371 \mathrm{E}-01-9.269 \mathrm{E}-01 \cdot 1.652 \mathrm{E}-0 \mathrm{I}$

0 3.371E-01 -9.269E-01 -1.652E-01

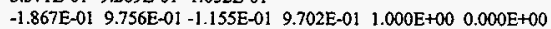
$-1.867 \mathrm{E}-019.756 \mathrm{E}-01-1.155 \mathrm{E}-01$

$0-1.867 \mathrm{E}-01 \quad 9.756 \mathrm{E}-01-1.155 \mathrm{E}-01$ $\begin{array}{lllll}-2.616 \mathrm{E}-01 & 2.336 \mathrm{E}-01-9.365 \mathrm{E}-01 & 1.230 \mathrm{E}+00 & 1.000 \mathrm{E}+00 & 0.000 \mathrm{E}+00\end{array}$ $-2.616 \mathrm{E}-01 \quad 2.336 \mathrm{E}-01-9.365 \mathrm{E}-01$

$0-2.616 \mathrm{E}-01 \quad 2.336 \mathrm{E}-01-9.365 \mathrm{E}-01$ $9.780 \mathrm{E}-01-7.641 \mathrm{E}-02-1.939 \mathrm{E}-01 \quad 1.083 \mathrm{E}+00 \quad 1.000 \mathrm{E}+00 \quad 0.000 \mathrm{E}+00$ $9.780 \mathrm{E}-01-7.641 \mathrm{E}-02-1.939 \mathrm{E}-01$

0 9.780E-01 -7.64IE-02 -1.939E-01 $2.580 \mathrm{E}-01-7.076 \mathrm{E}-01 \quad 6.578 \mathrm{E}-01 \quad 8.313 \mathrm{E}-01 \quad 1.000 \mathrm{E}+00 \quad 0.000 \mathrm{E}+00$ $2.580 \mathrm{E}-01-7.076 \mathrm{E}-01 \quad 6.578 \mathrm{E}-01$

$0 \quad 2.580 \mathrm{E}-01-7.076 \mathrm{E}-01 \quad 6.578 \mathrm{E}-01$

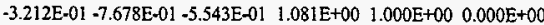
$-3.212 \mathrm{E}-01-7.678 \mathrm{E}-01-5.543 \mathrm{E}-01$ 
4.641E+00 4.456E-01 2.000E-01 141

$2.000 \mathrm{E}-0$ ? $3.000 \mathrm{E}+002.000 \mathrm{E}-01 \quad 140$

$3.641 \mathrm{E}+00 \quad 4.456 \mathrm{E}-01 \quad 2.000 \mathrm{E}-01 \quad 42(-5,3,0)$

$3.641 \mathrm{E}+00 \quad 4.456 \mathrm{E}-01 \quad 2.000 \mathrm{E}-01141$

$43 \quad 2.000 \mathrm{E}-01 \quad 3.000 \mathrm{E}+00 \quad 2.000 \mathrm{E}-01 \quad 140$

$3.641 \mathrm{E}+00 \quad 4.456 \mathrm{E}-01 \quad 2.000 \mathrm{E}-01 \quad 42(-5,3,0)$

$3.641 \mathrm{E}+00 \quad 4.456 \mathrm{E}-01 \quad 2,000 \mathrm{E}-01 \quad 141$

$442.000 \mathrm{E}-013.000 \mathrm{E}+00 \quad 2.000 \mathrm{E}-01 \quad 140$

$3.641 \mathrm{E}+00 \quad 4.456 \mathrm{E}-01 \quad 2.000 \mathrm{E}-01 \quad 42(-5,3,0)$

$3.641 \mathrm{E}+00 \quad 4.456 \mathrm{E}-01 \quad 2.000 \mathrm{E}-01 \quad 141$

45 2.000E-01 3.000E+00 2.000E-01 140

$3.641 \mathrm{E}+00 \quad 4.456 \mathrm{E}-01 \quad 2.000 \mathrm{E}-01 \quad 42(-5,3,0)$

$3.641 \mathrm{E}+00 \quad 4.456 \mathrm{E}-0 \mathrm{I} \quad 2.000 \mathrm{E}-01141$

$462.000 \mathrm{E}-01 \quad 3.000 \mathrm{E}+00 \quad 2.000 \mathrm{E}-01 \quad 140$

$3.641 \mathrm{E}+00 \quad 4.456 \mathrm{E}-01 \quad 2.000 \mathrm{E}-01 \quad 42(-5,3,0)$

$3.641 \mathrm{E}+00 \quad 4.456 \mathrm{E}-01 \quad 2.000 \mathrm{E}-01 \quad 141$

47 2.000E-01 3.000E+00 2.000E-01 140

$3.641 \mathrm{E}+00 \quad 4.456 \mathrm{E}-01 \quad 2.000 \mathrm{E}-01 \quad 42(-5,3,0)$

$3.641 \mathrm{E}+00 \quad 4.456 \mathrm{E}-01 \quad 2.000 \mathrm{E}-01 \quad 141$

$48 \quad 2.000 \mathrm{E}-01 \quad 3.000 \mathrm{E}+00 \quad 2.000 \mathrm{E}-01 \quad 140$

$3.641 \mathrm{E}+00 \quad 4.456 \mathrm{E}-01 \quad 2.000 \mathrm{E}-01 \quad 42(-5,3,0)$

$3.641 \mathrm{E}+00 \quad 4.456 \mathrm{E}-01 \quad 2.000 \mathrm{E}-01 \quad 141$

$492.000 \mathrm{E}-013.000 \mathrm{E}+00 \quad 2.000 \mathrm{E}-01140$

$3.641 \mathrm{E}+00 \quad 4.456 \mathrm{E}-01 \quad 2.000 \mathrm{E}-01 \quad 42(-5,3,0)$

$3.641 \mathrm{E}+00 \quad 4.456 \mathrm{E}-01 \quad 2.000 \mathrm{E}-01 \quad 141$

50 2.000E-01 3.000E+00 2.000E-01 140

$3.641 \mathrm{E}+00 \quad 4.456 \mathrm{E}-01 \quad 2.000 \mathrm{E}-01 \quad 42(-5,3,0)$

$3.641 \mathrm{E}+00 \quad 4.456 \mathrm{E}-01 \quad 2.000 \mathrm{E}-01 \quad 141$

lestimated keff results by cycle
$0-3.212 \mathrm{E}-01-7.678 \mathrm{E}-01-5.543 \mathrm{E}-01$ $5.039 \mathrm{E}-01-1.460 \mathrm{E}-01 \quad 8.513 \mathrm{E}-01 \quad 3.341 \mathrm{E}+00 \quad 1.000 \mathrm{E}+00 \quad 0.000 \mathrm{E}+00$ 5.039E-01 - $1.460 \mathrm{E}-01 \quad 8.513 \mathrm{E}-01$

$0 \quad 5.039 \mathrm{E}-01 * 1.460 \mathrm{E}-01 \quad 8.513 \mathrm{E}-01$

$\begin{array}{llllll}6.080 \mathrm{E}-01 & 5.487 \mathrm{E}-01 & 5.738 \mathrm{E}-01 & 4.097 \mathrm{E}-01 & 1.000 \mathrm{E}+00 & 0.000 \mathrm{E}+00\end{array}$

$6.080 \mathrm{E}-01 \quad 5.487 \mathrm{E}-01 \quad 5.738 \mathrm{E}-01$

0 6.080E-01 5.487E-01 5.738E-01

$-2.932 \mathrm{E}-01 \quad 9.304 \mathrm{E}-01-2.199 \mathrm{E}-01 \quad 2.229 \mathrm{E}+00 \quad 1.000 \mathrm{E}+00 \quad 0.000 \mathrm{E}+00$

$-2.932 \mathrm{E}-01 \quad 9.304 \mathrm{E}-01-2.199 \mathrm{E}-01$

$0-2.932 \mathrm{E}-01 \quad 9.304 \mathrm{E}-01-2.199 \mathrm{E}-01$

$-8.475 \mathrm{E}-01-3.993 \mathrm{E}-01-3.497 \mathrm{E}-01 \quad 1.362 \mathrm{E}+00 \quad 1.000 \mathrm{E}+00 \quad 0.000 \mathrm{E}+00$

$-8.475 \mathrm{E}-01-3.993 \mathrm{E}-01-3.497 \mathrm{E}-01$

$0-8.475 \mathrm{E}-01-3.993 \mathrm{E}-01-3.497 \mathrm{E}-01$

$1.200 \mathrm{E}-01-9.195 \mathrm{E}-01-3.743 \mathrm{E}-01 \quad 1.010 \mathrm{E}+00 \quad 1.000 \mathrm{E}+00 \quad 0.000 \mathrm{E}+00$

1.200E-01 -9.195E-01 -3.743E-01

$0 \quad 1.200 \mathrm{E}-01-9.195 \mathrm{E}-01-3.743 \mathrm{E}-01$

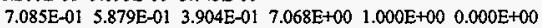
$7.085 \mathrm{E}-01 \quad 5.879 \mathrm{E}-01 \quad 3.904 \mathrm{E}-01$

$07.085 \mathrm{E}-01 \quad 5.879 \mathrm{E}-01 \quad 3.904 \mathrm{E}-01$

$\begin{array}{lllll}4.261 \mathrm{E}-01 & 9.046 \mathrm{E}-01 & 9.254 \mathrm{E}-03 & 3.122 \mathrm{E}-01 & 1.000 \mathrm{E}+00 \quad 0.000 \mathrm{E}+00\end{array}$

4.261E-01 9.046E-01 9.254E-03

$04.261 \mathrm{E}-019.046 \mathrm{E}-01 \quad 9.254 \mathrm{E}-03$ 5.431E-01 4.270E-01 -7.230E-01 5.484E-01 $1.000 \mathrm{E}+00 \quad 0.000 \mathrm{E}+00$

$5.431 \mathrm{E}-01 \quad 4.270 \mathrm{E}-01-7.230 \mathrm{E}-01$

0 5.431E-01 4.270E-01 -7.230E-01

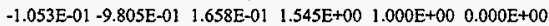

$-1.053 \mathrm{E}-01-9.805 \mathrm{E}-01 \quad 1.658 \mathrm{E}-01$

$0-1.053 \mathrm{E}-01-9.805 \mathrm{E}-01 \quad 1.658 \mathrm{E}-01$

print table 175

cycle $1 \mathrm{k}$ (collision) 1.253108 removal lifetime(abs) $1.7557 \mathrm{E}+01$ source points generated 257

$2 \mathrm{k}$ (collision) 1.211997 removal lifetime(abs) $1.1671 \mathrm{E}+01$ source points generated 195

cycle $3 \mathrm{k}$ (collision) 1.200265 removal lifetime(abs) $9.6992 \mathrm{E}+00$ source points generated 193

cycle 4 k(collision) 1.129530 removal lifetime(abs) $9.3720 \mathrm{E}+00$ source points generated 182

cycle 5 k(collision) 1.135994 removal lifetime(abs) $1.1136 \mathrm{E}+01$ source points generated 222

source distribution written to file inp $18 \mathrm{~s} \quad$ cycle $=5$

cycle $6 \mathrm{k}$ (collision) 1.077014 removal lifetime(abs) $1.2222 \mathrm{E}+01$ source points generated 204

source distribution written to file inp $18 \mathrm{~s} \quad$ cycle $=6$

Iproblem summary

0

run terminated when 6 kcode cycles were done.

neutron creation tracks weight energy neutron loss tracks weight energy (per source particle) (per source particle)

$\begin{array}{llllllll}\text { source } & 1249 & 9.6077 \mathrm{E}-01 & 1.8661 \mathrm{E}+00 & \text { escape } & 220 & 1.2268 \mathrm{E}-01 & 1.2054 \mathrm{E}-01\end{array}$

energy cutoff $\quad 0 \quad 0.00$.

time cutoff $\quad \begin{array}{llll}0 & 0 . & 0\end{array}$

weight window

$00.0 . \quad 0$ weight window

00.

0.

cell importance

$0 \quad 0 . \quad 0 . \quad$ cell importance

0.0.

weight cutoff

$\begin{array}{llll}0 & 1.3743 \mathrm{E}-01 & 3.8498 \mathrm{E}-03 & \text { weight cutoff }\end{array}$

$1034 \quad 1.2791 \mathrm{E}-01 \quad 5.1920 \mathrm{E}-03$

energy importance

dxtran

0 .

forced collisions

00

0 .

$\begin{array}{cc}\text { energy importance } \\ \text { dxtran } 0 & 0\end{array}$

00.

0.

exp. transform $\quad 0 \quad 0 . \quad 0$.

upscattering

00 .

$0 . \quad$ forced collisions 00 .

. exp. transform $\quad 0 \quad 0$.

$5.3884 \mathrm{E}-11$

$\begin{array}{lllll}\text { E-11 } & \text { downscattering } & 0 & 0 . & 1 \\ \text { capture } & 0 & 3.9980 \mathrm{E}-01 & 1.1590 \mathrm{E}-01\end{array}$

0.

$\begin{array}{lllllll}10 & 6.1640 \mathrm{E}-03 & 6.1355 \mathrm{E}-03 & \text { loss to }(\mathrm{n}, \mathrm{xn}) & 5 & 3.0820 \mathrm{E}-03 & 2.4027 \mathrm{E}-02\end{array}$

fion $\quad 0 \quad 0.00$ loss to fission $0.5089 \mathrm{E}-01 \quad 2.4987 \mathrm{E}-01$

$\begin{array}{lllllllll}\text { total } & 1259 & 1.1044 \mathrm{E}+00 & 1.8761 \mathrm{E}+00 & \text { total } & 1259 & 1.1044 \mathrm{E}+00 & 1.8761 \mathrm{E}+00\end{array}$


HNF-SD-SNF-CSWD-005, Rev. 0

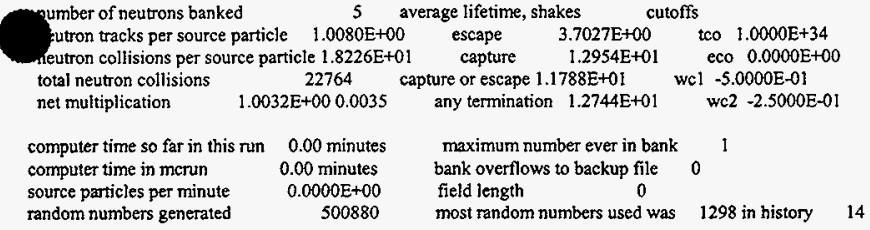

range of sampled source weights $=7.7821 \mathrm{E}-01$ to $1.0989 \mathrm{E}+00$

Ineutron activity in each cell

print table 126

tracks population coltisions collisions number flux average average

cell entering * weight weighted weighted track weight track mfp (per history) energy energy (relative) (cm)

$\begin{array}{llllllllllll}1 & 30 & 0 & 0 & 0 & 0.0000 \mathrm{E}+00 & 0.0000 \mathrm{E}+00 & 0.0000 \mathrm{E}+00 & 0.0000 \mathrm{E}+00 & 0.0000 \mathrm{E}+00\end{array}$

$\begin{array}{lllllllllll}2 & 31 & 0 & 0 & 0 & 0.0000 \mathrm{E}+00 & 0.0000 \mathrm{E}+00 & 0.0000 \mathrm{E}+00 & 0.0000 \mathrm{E}+00 & 0.0000 \mathrm{E}+00\end{array}$

$\begin{array}{lllllllllll}3 & 37 & 0 & 0 & 0 & 0.0000 \mathrm{E}+00 & 0.0000 \mathrm{E}+00 & 0.0000 \mathrm{E}+00 & 0.0000 \mathrm{E}+00 & 0.0000 \mathrm{E}+00\end{array}$

$\begin{array}{lllllllllll}434 & 0 & 0 & 0 & 0.0000 \mathrm{E}+00 & 0.0000 \mathrm{E}+00 & 0.0000 \mathrm{E}+00 & 0.0000 \mathrm{E}+00 & 0.0000 \mathrm{E}+00\end{array}$

$\begin{array}{lllllllllll}5 & 32 & 0 & 0 & 0 & 0.0000 \mathrm{E}+00 & 0.0000 \mathrm{E}+00 & 0.0000 \mathrm{E}+00 & 0.0000 \mathrm{E}+00 & 0.0000 \mathrm{E}+00\end{array}$

$\begin{array}{llllllllll}6 & 33 & 0 & 0 & 0 & 0.0000 \mathrm{E}+00 & 0.0000 \mathrm{E}+00 & 0.0000 \mathrm{E}+00 & 0.0000 \mathrm{E}+00 & 0.0000 \mathrm{E}+00\end{array}$

$\begin{array}{lllllllllll}7 & 35 & 0 & 0 & 0 & 0.0000 \mathrm{E}+00 & 0.0000 \mathrm{E}+00 & 0.0000 \mathrm{E}+00 & 0.0000 \mathrm{E}+00 & 0.0000 \mathrm{E}+00\end{array}$

$\begin{array}{lllllllllll}8 & 36 & 0 & 0 & 0 & 0.0000 \mathrm{E}+00 & 0.0000 \mathrm{E}+00 & 0.0000 \mathrm{E}+00 & 0.0000 \mathrm{E}+00 & 0.0000 \mathrm{E}+00\end{array}$

$\begin{array}{lllllllllll}9 & 38 & 920 & 618 & 1249 & 5.9116 \mathrm{E}-01 & 3.0414 \mathrm{E}-01 & 8.9304 \mathrm{E}-01 & 6.4212 \mathrm{E}-01 & 2.6324 \mathrm{E}+00\end{array}$

$\begin{array}{lllllllllll}10 & 39 & 1635 & 637 & 130 & 6.3779 \mathrm{E}-02 & 8.8236 \mathrm{E}-02 & 7.6766 \mathrm{E}-01 & 6.4931 \mathrm{E}-01 & 2.6947 \mathrm{E}+00\end{array}$

$\begin{array}{lllllllllll}11 & 40 & 1728 & 663 & 184 & 8.8176 \mathrm{E}-02 & 7.6162 \mathrm{E}-02 & 7.6184 \mathrm{E}-01 & 6.4437 \mathrm{E}-01 & 2.5146 \mathrm{E}+00\end{array}$

$\begin{array}{lllllllllll}12 & 41 & 1867 & 712 & 317 & 1.5811 \mathrm{E}-01 & 8.6238 \mathrm{E}-02 & 7.9306 \mathrm{E}-01 & 6.5030 \mathrm{E}-01 & 2.6940 \mathrm{E}+00\end{array}$

$\begin{array}{lllllllllllll}13 & 140 & 0 & 0 & 0 & 0.0000 \mathrm{E}+00 & 0.0000 \mathrm{E}+00 & 0.0000 \mathrm{E}+00 & 0.0000 \mathrm{E}+00 & 0.0000 \mathrm{E}+00\end{array}$

$\begin{array}{lllllllllll}14 & 42 & 2069 & 867 & 441 & 2.1911 \mathrm{E}-01 & 8.9382 \mathrm{E}-02 & 7.8771 \mathrm{E}-01 & 6.6777 \mathrm{E}-01 & 2.5091 \mathrm{E}+00\end{array}$

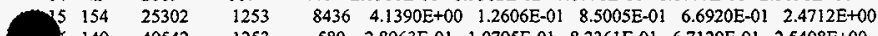

$\begin{array}{llllllllll}149 & 49542 & 1253 & 580 & 2.8963 \mathrm{E}-01 & 1.0795 \mathrm{E}-01 & 8.2361 \mathrm{E}-01 & 6.7129 \mathrm{E}-01 & 2.5408 \mathrm{E}+00\end{array}$

$\begin{array}{llllllllllll}7 & 144 & 51224 & 1254 & 885 & 4.2652 \mathrm{E}-01 & 1.0541 \mathrm{E}-01 & 8.1599 \mathrm{E}-01 & 6.6971 \mathrm{E}-01 & 2.2391 \mathrm{E}+00\end{array}$

$\begin{array}{lllllllllll}18 & 159 & 58650 & 1254 & 4019 & 2.1254 \mathrm{E}+00 & 9.8931 \mathrm{E}-02 & 8.1154 \mathrm{E}-01 & 6.6977 \mathrm{E}-01 & 2.7541 \mathrm{E}+00\end{array}$

$\begin{array}{llllllllll}19 & 141 & 73122 & 1254 & 6523 & 3.2229 \mathrm{E}+00 & 9.4499 \mathrm{E}-02 & 8.0639 \mathrm{E}-01 & 6.6810 \mathrm{E}-01 & 2.5056 \mathrm{E}+00\end{array}$

total $266059 \quad 9765 \quad 22764 \quad 1.1324 \mathrm{E}+0$ !

Ineutron weight balance in each cell - extemal events print table 130

cell entering source energy time exiting total
cutoff cutoff

$\begin{array}{llllllll}30 & 0.0000 \mathrm{E}+00 & 0.0000 \mathrm{E}+00 & 0.0000 \mathrm{E}+00 & 0.0000 \mathrm{E}+00 & 0.0000 \mathrm{E}+00 & 0.0000 \mathrm{E}+00\end{array}$

$\begin{array}{llllllllll}2 & 31 & 0.0000 \mathrm{E}+00 & 0.0000 \mathrm{E}+00 & 0.0000 \mathrm{E}+00 & 0.0000 \mathrm{E}+00 & 0.0000 \mathrm{E}+00 & 0.0000 \mathrm{E}+00\end{array}$

$\begin{array}{llllllllll}3 & 37 & 0.0000 \mathrm{E}+00 & 0.0000 \mathrm{E}+00 & 0.0000 \mathrm{E}+00 & 0.0000 \mathrm{E}+00 & 0.0000 \mathrm{E}+00 & 0.0000 \mathrm{E}+00\end{array}$

$\begin{array}{llllllll}4 & 34 & 0.0000 \mathrm{E}+00 & 0.0000 \mathrm{E}+00 & 0.0000 \mathrm{E}+00 & 0.0000 \mathrm{E}+00 & 0.0000 \mathrm{E}+00 & 0.0000 \mathrm{E}+00\end{array}$

$\begin{array}{lllllllllll}5 & 32 & 0.0000 \mathrm{E}+00 & 0.0000 \mathrm{E}+00 & 0.0000 \mathrm{E}+00 & 0.0000 \mathrm{E}+00 & 0.0000 \mathrm{E}+00 & 0.0000 \mathrm{E}+00\end{array}$

$\begin{array}{lllllllll}6 & 33 & 0.0000 \mathrm{E}+00 & 0.0000 \mathrm{E}+00 & 0.0000 \mathrm{E}+00 & 0.0000 \mathrm{E}+00 & 0.0000 \mathrm{E}+00 & 0.0000 \mathrm{E}+00\end{array}$

$\begin{array}{llllllllll}7 & 35 & 0.0000 \mathrm{E}+00 & 0.0000 \mathrm{E}+00 & 0.0000 \mathrm{E}+00 & 0.0000 \mathrm{E}+00 & 0.0000 \mathrm{E}+00 & 0.0000 \mathrm{E}+00\end{array}$

$\begin{array}{llllllllll}8 & 36 & 0.0000 \mathrm{E}+00 & 0.0000 \mathrm{E}+00 & 0.0000 \mathrm{E}+00 & 0.0000 \mathrm{E}+00 & 0.0000 \mathrm{E}+00 & 0.0000 \mathrm{E}+00\end{array}$

$\begin{array}{lllllllll}9 & 38 & 4.8822 \mathrm{E}-01 & 0.0000 \mathrm{E}+00 & 0.0000 \mathrm{E}+00 & 0.0000 \mathrm{E}+00 & -3.3091 \mathrm{E}-01 & 1.5731 \mathrm{E}-01\end{array}$

$\begin{array}{llllllll}10 & 39 & 8.4996 \mathrm{E}-01 & 0.0000 \mathrm{E}+00 & 0.0000 \mathrm{E}+00 & 0.0000 \mathrm{E}+00 & -8.4933 \mathrm{E}-01 & 6.2750 \mathrm{E}-0.4\end{array}$

$11 \quad 40 \quad 8.9904 \mathrm{E}-01 \quad 0.0000 \mathrm{E}+00 \quad 0.0000 \mathrm{E}+00 \quad 0.0000 \mathrm{E}+00 \quad-8.9904 \mathrm{E}-01 \quad 3.9460 \mathrm{E}-06$

$\begin{array}{lllllllll}12 & 41 & 9.7260 \mathrm{E}-01 & 0.0000 \mathrm{E}+00 & 0.0000 \mathrm{E}+00 & 0.0000 \mathrm{E}+00 & -9.6978 \mathrm{E}-01 & 2.8204 \mathrm{E}-03\end{array}$

$\begin{array}{lllllllll}13 & 140 & 0.0000 \mathrm{E}+00 & 0.0000 \mathrm{E}+00 & 0.0000 \mathrm{E}+00 & 0.0000 \mathrm{E}+00 & 0.0000 \mathrm{E}+00 & 0.0000 \mathrm{E}+00\end{array}$

$\begin{array}{lllllllll}14 & 42 & 1.0907 \mathrm{E}+00 & 0.0000 \mathrm{E}+00 & 0.0000 \mathrm{E}+00 & 0.0000 \mathrm{E}+00 & -1.0913 \mathrm{E}+00 & -5.9111 \mathrm{E}-04\end{array}$

$\begin{array}{llllllll}15 & 154 & 1.2943 \mathrm{E}+01 & 8.4067 \mathrm{E}-01 & 0.0000 \mathrm{E}+00 & 0.0000 \mathrm{E}+00 & -1.3171 \mathrm{E}+01 & 6.1278 \mathrm{E}-01\end{array}$

$\begin{array}{lllllllll}16 & 149 & 2.6508 \mathrm{E}+01 & 0.0000 \mathrm{E}+00 & 0.0000 \mathrm{E}+00 & 0.0000 \mathrm{E}+00 & -2.6508 \mathrm{E}+01 & 3.5884 \mathrm{E}-05\end{array}$

$17 \quad 144 \quad 2.7419 \mathrm{E}+01 \quad 0.0000 \mathrm{E}+00 \quad 0.0000 \mathrm{E}+00 \quad 0.0000 \mathrm{E}+00 \quad-2.7405 \mathrm{E}+01 \quad 1.4572 \mathrm{E}-02$

$\begin{array}{llllllll}18 & 159 & 3.1331 \mathrm{E}+01 & 8.0064 \mathrm{E}-02 & 0.0000 \mathrm{E}+00 & 0.0000 \mathrm{E}+00 & -3.1361 \mathrm{E}+01 & 5.0067 \mathrm{E}-02\end{array}$

$19 \quad 141 \quad 3.9111 \mathrm{E}+01 \quad 4.0032 \mathrm{E}-02 \quad 0.0000 \mathrm{E}+00 \quad 0.0000 \mathrm{E}+00 \quad-3.9150 \mathrm{E}+01 \quad 4.5860 \mathrm{E}-04$

ntal $\quad 1.4161 \mathrm{E}+02 \quad 9.6077 \mathrm{E}-01 \quad 0.0000 \mathrm{E}+00 \quad 0.0000 \mathrm{E}+00 \quad-1.4174 \mathrm{E}+02 \quad 8.3809 \mathrm{E}-01$ 
10 statistical checks are only for the tally fluctuation chart bin and do not apply to other tally bins.

tally bins with zeros may or may not be correct: compare the source, cutoffs, multipliers, et cetera with the tally bins.

warning. 1 of the 1 tally fluctuation chart bins did not pass all 10 statistical checks.

warning. 1 of the 1 tallies had bins with relative errors greater than recommended.

1 tally fluctuation charts

$$
\text { tally } 4
$$

nps mean error vov slope fom

$10000.0000 \mathrm{E}+000.00000 .0000 \quad 0.0$

1249 3.5167E-04 $0.38770 .1752 \quad 0.0$

tally data written to file inp $18 \mathrm{~m}$

ascii file inp $18 \mathrm{p}$ written with 5 events from 5 histories.

6 warning messages so far.

run terminated when 6 kcode cycles were done.

\section{File: Inp200}

1. prob20 - continuous energy electron version of problem prob19.

2- $\quad 1 \quad 1-19.3 \quad 1-23-45-6$

3- $20 \# 1$

5. $1 \mathrm{zO}^{2}$

6. $2 \mathrm{pz} .00635$

plane

7. $3 \mathrm{px}-10$

8. $4 \times 100$

$+$

$5 \quad y-103$

$6 \mathrm{p} 010011000101$

plane

plane

11.

12. $\mathrm{ml} \mathrm{74184.1}$

13- sdef erg=1 sur=1 vec $=001$ par $=3$

14- imp:p,e 10

15- mode $\mathrm{p} \mathrm{e}$

16- *fl:p 12

17. $\quad$ e $0.1 .191,282.373 .464 .555 .645 .736 .827 .918$ l.1

18- * $11: 012$

19- tr9 $000100010000 \quad 1$

warning. non-orthogonality of surface transformation $9>2 . \mathrm{e}-6$

20- fq ef

21- $\mathrm{f} 21: e 12$

22- *f28:p 1

23- e28 $\quad-.00101 . e-6.1 .191 .282 .373 .464 .555 .645 .736 .827 .9181 .1$

24- es8 $\quad-.00101 . e-6.1 .191 .282 .373 .464 .555 .645 .736 .827 .918 \quad 1.1$

25- f58:e 1

26- 16:p 1

27- f31:e 12

28- ft 31 elc 1

29. f4l:e 12

30- ft4l elc 2

31- f5l:e 12

32- fit 51 elc 3

33- e8 $1000 \mathrm{nt}$

34- $\quad *$ f8:p,e 1

35- nps 10000

36- ctme 30

37- prdmp 2j-1

cut:p $1 \mathrm{j} .1$

cut:e 1 j 1

print 11070 
hing. tr 9 card unused.

waming. 1 energy bins of tally 1 are below energy cutoff.

waming. tally 8 needs zero energy bin for negative $f 8$ scores. lcells

print table 60

cell mat density density volume mass pieces importance importance

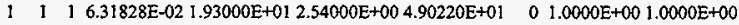

$220 \quad 0.00000 \mathrm{E}+000.00000 \mathrm{E}+000.00000 \mathrm{E}+000.00000 \mathrm{E}+00 \quad 0 \quad 0.0000 \mathrm{E}+000.0000 \mathrm{E}+00$

total

1surfaces

$2.54000 \mathrm{E}+004.90220 \mathrm{E}+0 \mathrm{I}$

print table 70

surface trans type surface coefficients

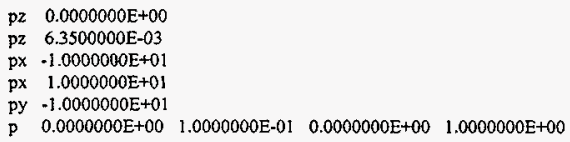

minimum source weight $=1.0000 \mathrm{E}+00 \quad$ maximum source weight $=1.0000 \mathrm{E}+00$

4 waming messages so far.

lcross-section tables

print table 100

table length

tables from file testlibl

$74000.02 \mathrm{p} \quad 755$

$01 / 15 / 93$

total 755

maximum photon energy set to 100.0 mev (maximum electron energy)

tables from file testlibl

$74000.01 \mathrm{e} \quad 478$

$11 / 16 / 88$

decimal words of dynamically allocated storage

4 warning messages so far.

I starting merun. field length $=0 \quad c p 0=0.00 \quad$ print table 110

prob20 - continuous energy electron version of problem prob19.

nps $x \quad y \quad z \quad$ cell surf $u \quad v \quad w$ energy weight time

I $0.000 \mathrm{E}+00 \quad 0.000 \mathrm{E}+00 \quad 0.000 \mathrm{E}+00 \quad 1 \quad 1 \quad 3.096 \mathrm{E}-0 \mathrm{t}-4.068 \mathrm{E}-01 \quad 8.595 \mathrm{E}-01 \quad 1.000 \mathrm{E}+00 \quad 1.000 \mathrm{E}+00 \quad 0.000 \mathrm{E}+00$

$20.000 \mathrm{E}+00 \quad 0.000 \mathrm{E}+00 \quad 0.000 \mathrm{E}+00 \quad 1 \quad 19.871 \mathrm{E}-01 \quad 1.062 \mathrm{E}-01 \quad 1.197 \mathrm{E}-01 \quad 1.000 \mathrm{E}+00 \quad 1.000 \mathrm{E}+00 \quad 0.000 \mathrm{E}+00$

$30.000 \mathrm{E}+00 \quad 0.000 \mathrm{E}+00 \quad 0.000 \mathrm{E}+00 \quad 1 \quad 1-1.347 \mathrm{E}-01 \quad 5.198 \mathrm{E}-01 \quad 8.436 \mathrm{E}-01 \quad 1.000 \mathrm{E}+00 \quad 1.000 \mathrm{E}+00 \quad 0.000 \mathrm{E}+00$

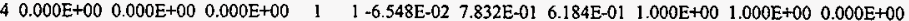

$5 \quad 0.000 \mathrm{E}+00 \quad 0.000 \mathrm{E}+00 \quad 0.000 \mathrm{E}+00 \quad 1 \quad 1 \quad 1.554 \mathrm{E}-01-9.204 \mathrm{E}-01 \quad 3.588 \mathrm{E}-01 \quad 1.000 \mathrm{E}+00 \quad 1.000 \mathrm{E}+00 \quad 0.000 \mathrm{E}+00$

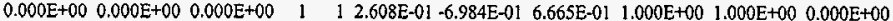

$\begin{array}{lllllllll}0.000 \mathrm{E}+00 & 0.000 \mathrm{E}+00 & 0.000 \mathrm{E}+00 \quad 1 \quad 1 & 6.931 \mathrm{E}-01 & -9.170 \mathrm{E}-02 & 7.149 \mathrm{E}-01 & 1.000 \mathrm{E}+00 & 1.000 \mathrm{E}+00 & 0.000 \mathrm{E}+00\end{array}$ 
prob20 - continuous energy electron version of problem prob 19.

2. 1 1 $1-19.31-23-45-6$

3- $20 \#$

4., 202

$+$

6- 2 pz. 00635

7- 3 px -10

8. $4 \times 100$

9. 5 y -103

10. 6 p01001100010I

plane

11.

12- $\mathrm{ml} \mathrm{74184.1}$

13. sdef erg $=1$ sur $=1$ vec $=001$ par $=3$

14- imp:p,e 10

15- mode $\mathrm{p} e$

16. *fl:p 12

$17-\quad$ e0 $\quad .1 .191 .282 .373 .464 .555$.645 $.736 .827 .918 \quad 1.1$

18- *flt: 12

19- 590001000100001

waming. non-orthogonality of surface transformation $9>2 . e-6$

20- fq ef

21- f2l:e 12

22- *f28:p 1

23- e28 $\quad .00101 . \mathrm{e}-6.1 .191 .282 .373 .464 .555 .645 .736 .827 .918 \quad 1.1$

24- es8 $\quad-.0010$ l.e-6.1 $191.282 .373 .464 .555 .645 .736 .827 .918 \quad 1 . \mathrm{I}$

25- f58:e 1

26- f6:p 1

B1:e 12

ftil elc 1

f4 $1: e^{1} 2$

30. ft4l elc 2

31. f51:e 12

32- ft51 elc 3

33- e8 $1000 \mathrm{nt}$

34- *f8:p,e 1

35. nps 10000

36- ctme 30

37- prdmp $2 \mathrm{j}-1$

38- cut:p $1 \mathrm{j} .1$

39- cut:e $1 \mathrm{j} .1$

40- print 11070

41-

warning. to 9 card unused.

warning. I energy bins of tally 1 are below energy cutoff.

waming. tally 8 needs zero energy bin for negative f8 scores. lcells

print table 60

atom gram
cell mat density density volume mass pieces importance importance

$1116.31828 \mathrm{E}-021.93000 \mathrm{E}+012.54000 \mathrm{E}+004.90220 \mathrm{E}+01 \quad 01.0000 \mathrm{E}+001.0000 \mathrm{E}+00$

$2200.00000 \mathrm{E}+00 \quad 0.00000 \mathrm{E}+000.00000 \mathrm{E}+000.00000 \mathrm{E}+00 \quad 0 \quad 0.0000 \mathrm{E}+00 \quad 0.0000 \mathrm{E}+00$

total

1surfaces

$2.54000 \mathrm{E}+004.90220 \mathrm{E}+01$

print table 70

surface trans type surface coefficients 
minimum source weight $=1.0000 \mathrm{E}+00$ maximum source weight $=1.0000 \mathrm{E}+00$

4 waming messages so far.

lcross-section tables

print table 100

table length

tables from file testlib1

$74000.02 p \quad 755$

$01 / 15 / 93$

total 755

maximum photon energy set to 100.0 mev (maximum electron energy)

tables from file testlibl

$74000.01 \mathrm{e} \quad 478$

$11 / 16 / 88$

decimal words of dynamically allocated storage

4 waming messages so far.

tarting merun. field length $=0 \quad \mathrm{cp} 0=0.00 \quad$ print table 110

prob20 - continuous energy electron version of problem probl9.

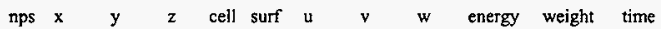

I $0.000 \mathrm{E}+00 \quad 0.000 \mathrm{E}+00 \quad 0.000 \mathrm{E}+00$

$20.000 \mathrm{E}+00 \quad 0.000 \mathrm{E}+00 \quad 0.000 \mathrm{E}+00$

$30.000 \mathrm{E}+00 \quad 0.000 \mathrm{E}+00 \quad 0.000 \mathrm{E}+00$

$40.000 \mathrm{E}+00 \quad 0.000 \mathrm{E}+00 \quad 0.000 \mathrm{E}+00$

$50.000 \mathrm{E}+00 \quad 0.000 \mathrm{E}+00 \quad 0.000 \mathrm{E}+00$

$60.000 \mathrm{E}+00 \quad 0.000 \mathrm{E}+00 \quad 0.000 \mathrm{E}+00$

$\begin{array}{lll}7 & 0.000 \mathrm{E}+00 \quad 0.000 \mathrm{E}+00 \quad 0.000 \mathrm{E}+00\end{array}$

$80.000 \mathrm{E}+00 \quad 0.000 \mathrm{E}+00 \quad 0.000 \mathrm{E}+00$

$90.000 \mathrm{E}+00 \quad 0.000 \mathrm{E}+00 \quad 0.000 \mathrm{E}+00$

$10 \quad 0.000 \mathrm{E}+00 \quad 0.000 \mathrm{E}+00 \quad 0.000 \mathrm{E}+00$

11 $0.000 \mathrm{E}+00 \quad 0.000 \mathrm{E}+00 \quad 0.000 \mathrm{E}+00$

$120.000 \mathrm{E}+00 \quad 0.000 \mathrm{E}+00 \quad 0.000 \mathrm{E}+00$

$130.000 \mathrm{E}+00 \quad 0.000 \mathrm{E}+00 \quad 0.000 \mathrm{E}+00$

$14 \quad 0.000 \mathrm{E}+00 \quad 0.000 \mathrm{E}+00 \quad 0.000 \mathrm{E}+00$

$150.000 \mathrm{E}+00 \quad 0.000 \mathrm{E}+00 \quad 0.000 \mathrm{E}+00$

$16 \quad 0.000 \mathrm{E}+00 \quad 0.000 \mathrm{E}+00 \quad 0.000 \mathrm{E}+00$

$17 \quad 0.000 \mathrm{E}+00 \quad 0.000 \mathrm{E}+00 \quad 0.000 \mathrm{E}+00$

$18 \quad 0.000 \mathrm{E}+00 \quad 0.000 \mathrm{E}+00 \quad 0.000 \mathrm{E}+00$

$190.000 \mathrm{E}+00 \quad 0.000 \mathrm{E}+00 \quad 0.000 \mathrm{E}+00$

$20 \quad 0.000 \mathrm{E}+00 \quad 0.000 \mathrm{E}+00 \quad 0.000 \mathrm{E}+00$

21 $\quad 0.000 \mathrm{E}+00 \quad 0.000 \mathrm{E}+00 \quad 0.000 \mathrm{E}+00$

$22 \quad 0.000 \mathrm{E}+00 \quad 0.000 \mathrm{E}+00 \quad 0.000 \mathrm{E}+00$

$23 \quad 0.000 \mathrm{E}+00 \quad 0.000 \mathrm{E}+00 \quad 0.000 \mathrm{E}+00$

$24 \quad 0.000 \mathrm{E}+00 \quad 0.000 \mathrm{E}+00 \quad 0.000 \mathrm{E}+00$

$25 \quad 0.000 \mathrm{E}+00 \quad 0.000 \mathrm{E}+00 \quad 0.000 \mathrm{E}+00$

$260.000 \mathrm{E}+00 \quad 0.000 \mathrm{E}+00 \quad 0.000 \mathrm{E}+00$

$270.000 \mathrm{E}+00 \quad 0.000 \mathrm{E}+00 \quad 0.000 \mathrm{E}+00$

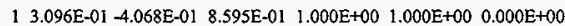
$\begin{array}{lllllll}1 & 9.871 \mathrm{E}-01 & 1.062 \mathrm{E}-01 & 1.197 \mathrm{E}-01 & 1.000 \mathrm{E}+00 & 1.000 \mathrm{E}+00 & 0.000 \mathrm{E}+00\end{array}$ $1-1.347 \mathrm{E}-01 \quad 5.198 \mathrm{E}-01 \quad 8.436 \mathrm{E}-01 \quad 1.000 \mathrm{E}+00 \quad 1.000 \mathrm{E}+00 \quad 0.000 \mathrm{E}+00$ $\begin{array}{llllll}1-6.548 \mathrm{E}-02 & 7.832 \mathrm{E}-01 & 6.184 \mathrm{E}-01 & 1.000 \mathrm{E}+00 & 1.000 \mathrm{E}+00 & 0.000 \mathrm{E}+00\end{array}$ 1 1.554E-01 -9.204E-01 3.588E-01 1.000E+00 1.000E+00 $0.000 \mathrm{E}+00$

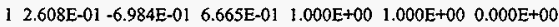
$16.931 \mathrm{E}-01-9.170 \mathrm{E}-02 \quad 7.149 \mathrm{E}-01 \quad 1.000 \mathrm{E}+00 \quad 1.000 \mathrm{E}+00 \quad 0.000 \mathrm{E}+00$

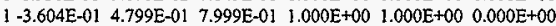

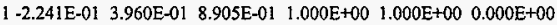
$1-2.928 \mathrm{E}-01-7.352 \mathrm{E}-01 \quad 6.114 \mathrm{E}-01 \quad 1.000 \mathrm{E}+00 \quad 1.000 \mathrm{E}+00 \quad 0.000 \mathrm{E}+00$ $1 \quad 8.260 \mathrm{E}-01 \quad 1.184 \mathrm{E}-01 \quad 5.510 \mathrm{E}-01 \quad 1.000 \mathrm{E}+00 \quad 1.000 \mathrm{E}+00 \quad 0.000 \mathrm{E}+00$ $17.540 \mathrm{E}-01-1.976 \mathrm{E}-01 \quad 6.264 \mathrm{E}-01 \quad 1.000 \mathrm{E}+00 \quad 1.000 \mathrm{E}+00 \quad 0.000 \mathrm{E}+00$ $18.140 \mathrm{E}-01-3.427 \mathrm{E}-01 \quad 4.690 \mathrm{E}-01 \quad 1.000 \mathrm{E}+00 \quad 1.000 \mathrm{E}+00 \quad 0.000 \mathrm{E}+00$ $\begin{array}{llllll}1-1.257 \mathrm{E}-01 & 8.038 \mathrm{E}-01 & 5.814 \mathrm{E}-01 & 1.000 \mathrm{E}+00 & 1.000 \mathrm{E}+00 & 0.000 \mathrm{E}+00\end{array}$ $1-1.464 \mathrm{E}-01-1.070 \mathrm{E}-01 \quad 9.834 \mathrm{E}-01 \quad 1.000 \mathrm{E}+00 \quad 1.000 \mathrm{E}+00 \quad 0.000 \mathrm{E}+00$ $1 \quad 1.310 \mathrm{E}-02-1.617 \mathrm{E}-02 \quad 9.998 \mathrm{E}-01 \quad 1.000 \mathrm{E}+00 \quad 1.000 \mathrm{E}+00 \quad 0.000 \mathrm{E}+00$ $\begin{array}{lllllll}1 & -8.843 \mathrm{E}-01 & -4.126 \mathrm{E}-01 & 2.187 \mathrm{E}-01 & 1.000 \mathrm{E}+00 & 1.000 \mathrm{E}+00 & 0.000 \mathrm{E}+00\end{array}$ $14.260 \mathrm{E}-01 \quad 4.153 \mathrm{E}-01 \quad 8.038 \mathrm{E}-01 \quad 1.000 \mathrm{E}+00 \quad 1.000 \mathrm{E}+00 \quad 0.000 \mathrm{E}+00$ $\begin{array}{lllllll}1 & -7.311 \mathrm{E}-01 & -1.136 \mathrm{E}-02 & 6.822 \mathrm{E}-01 & 1.000 \mathrm{E}+00 & 1.000 \mathrm{E}+00 & 0.000 \mathrm{E}+00\end{array}$

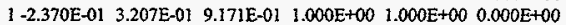
$14.276 \mathrm{E}-01-2.485 \mathrm{E}-02 \quad 9.036 \mathrm{E}-01 \quad 1.000 \mathrm{E}+00 \quad 1.000 \mathrm{E}+00 \quad 0.000 \mathrm{E}+00$ $17.662 \mathrm{E}-017.908 \mathrm{E}-02 \quad 6.378 \mathrm{E}-01 \quad 1.000 \mathrm{E}+00 \quad 1.000 \mathrm{E}+00 \quad 0.000 \mathrm{E}+00$ $1-4.247 \mathrm{E}-012.688 \mathrm{E}-01 \quad 8.645 \mathrm{E}-01 \quad 1.000 \mathrm{E}+00 \quad 1.000 \mathrm{E}+00 \quad 0.000 \mathrm{E}+00$ $12.096 \mathrm{E}-01 \quad 5.730 \mathrm{E}-01 \quad 7.923 \mathrm{E}-01 \quad 1.000 \mathrm{E}+00 \quad 1.000 \mathrm{E}+00 \quad 0.000 \mathrm{E}+00$ $1-2.665 \mathrm{E}-03-5.270 \mathrm{E}-01 \quad 8.499 \mathrm{E}-01 \quad 1.000 \mathrm{E}+00 \quad 1.000 \mathrm{E}+00 \quad 0.000 \mathrm{E}+00$ l 7.228E-01 4.233E-01 5.463E-0) $1.000 \mathrm{E}+00 \quad 1.000 \mathrm{E}+00 \quad 0.000 \mathrm{E}+00$ 1 $3.484 \mathrm{E}-01-3.564 \mathrm{E}-01 \quad 8.669 \mathrm{E}-01 \quad 1.000 \mathrm{E}+00 \quad 1.000 \mathrm{E}+00 \quad 0.000 \mathrm{E}+00$ 
$28 \quad 0.000 \mathrm{E}+00 \quad 0.000 \mathrm{E}+00 \quad 0.000 \mathrm{E}+00$ $92 \quad 0.000 \mathrm{E}+00 \quad 0.000 \mathrm{E}+00 \quad 0.000 \mathrm{E}+00$ 10 $0.000 \mathrm{E}+00 \quad 0.000 \mathrm{E}+00 \quad 0.000 \mathrm{E}+00$ 3) $0.000 \mathrm{E}+00 \quad 0.000 \mathrm{E}+00 \quad 0.000 \mathrm{E}+00$ $320.000 \mathrm{E}+00 \quad 0.000 \mathrm{E}+00 \quad 0.000 \mathrm{E}+00$ $330.000 \mathrm{E}+00 \quad 0.000 \mathrm{E}+00 \quad 0.000 \mathrm{E}+00$ $34 \quad 0.000 \mathrm{E}+00 \quad 0.000 \mathrm{E}+00 \quad 0.000 \mathrm{E}+00$ $35 \quad 0.000 \mathrm{E}+00 \quad 0.000 \mathrm{E}+00 \quad 0.000 \mathrm{E}+00$ $36 \quad 0.000 \mathrm{E}+00 \quad 0.000 \mathrm{E}+00 \quad 0.000 \mathrm{E}+00$ $370.000 \mathrm{E}+00 \quad 0.000 \mathrm{E}+00 \quad 0.000 \mathrm{E}+00$ $38 \quad 0.000 \mathrm{E}+00 \quad 0.000 \mathrm{E}+00 \quad 0.000 \mathrm{E}+00$ $390.000 \mathrm{E}+00 \quad 0.000 \mathrm{E}+00 \quad 0.000 \mathrm{E}+00$ $40 \quad 0.000 \mathrm{E}+00 \quad 0.000 \mathrm{E}+00 \quad 0.000 \mathrm{E}+00$ $410.000 \mathrm{E}+00 \quad 0.000 \mathrm{E}+00 \quad 0.000 \mathrm{E}+00$ $42 \quad 0.000 \mathrm{E}+00 \quad 0.000 \mathrm{E}+00 \quad 0.000 \mathrm{E}+00$ $430.000 \mathrm{E}+00 \quad 0.000 \mathrm{E}+00 \quad 0.000 \mathrm{E}+00$ $44 \quad 0.000 \mathrm{E}+00 \quad 0.000 \mathrm{E}+00 \quad 0.000 \mathrm{E}+00$ $45 \quad 0.000 \mathrm{E}+00 \quad 0.000 \mathrm{E}+00 \quad 0.000 \mathrm{E}+00$ $\begin{array}{llll}46 & 0.000 \mathrm{E}+00 & 0.000 \mathrm{E}+00 & 0.000 \mathrm{E}+00\end{array}$ $47 \quad 0.000 \mathrm{E}+00 \quad 0.000 \mathrm{E}+00 \quad 0.000 \mathrm{E}+00$ $\begin{array}{llll}48 & 0.000 \mathrm{E}+00 & 0.000 \mathrm{E}+00 & 0.000 \mathrm{E}+00\end{array}$ $49 \quad 0.000 \mathrm{E}+00 \quad 0.000 \mathrm{E}+00 \quad 0.000 \mathrm{E}+00$ $50 \quad 0.000 \mathrm{E}+00 \quad 0.000 \mathrm{E}+00 \quad 0.000 \mathrm{E}+00$ Iproblem summary

run terminated when 10000 particle histories were done.

photon creation tracks weight energy photon loss tracks weight energy (per source particle) (per source particle)

source

$0 \quad 0$.

0. escape
energy cutoff time cutoff $\quad 000.0$.

ght window importance

eight cutoff $\begin{array}{lll}0 & 0\end{array}$ 0. 00. energy importance dxtran $\begin{array}{lll}0 & 0\end{array}$ 00. 0 . forced collisions exp. transform from neutrons bremsstrahlung p-annihilation electron $x$-rays 1st fluorescence 2nd fluorescence total

number of photons banked photon tracks per source particle photon collisions per source particle $13000 \mathrm{E}$ total photon collisions $\begin{array}{lll}0 & 0 & 0\end{array}$ $\begin{array}{lll}0 & 0 . & 0 .\end{array}$ 0 0. 0. $694 \quad 6.9400 \mathrm{E}-02$ 0 o. 0. $\begin{array}{lll}0 & 0 . & 0\end{array}$ $0 \quad 0$. 00. $1.7315 \mathrm{E}-02$ cell importance $\quad 0 \quad 0 . \quad 0$. weight cutoff 00.0 . 0 . energy importance $\quad 0 \quad 0 . \quad 0$. forced collisions $\quad 0 \quad 0 . \quad 0$. exp. transform $\quad 0 \quad 0 . \quad 0$. compton scatter $\quad 0 \quad 0.6 .6993 \mathrm{E}-0 \mathrm{~S}$ pair production $\quad 0 \quad 0.0$.

\section{0}

electron creation tracks weight energy electron loss tracks weight energy (per source particle) (per source particle)

source

$10000 \quad 1.0000 \mathrm{E}+00$

$1.0000 \mathrm{E}+00$
cnergy cutoff
time cutoff

weight window $0 \quad 0$. 0. cell importance weight cutoff energy importance $\begin{array}{lll}0 & 0\end{array}$ 0 . 00. 0 . 00. 0. cell importance weight cutoff pair production

$0 \quad 0$. 0. scattering 2 2.0000E-04 3.7815E-05 $29 \quad 2.9000 \mathrm{E}-03 \quad 4.7387 \mathrm{E}-04$ $575 \quad 5.7500 \mathrm{E}-02 \quad 1.5476 \mathrm{E}-02$

total

$694 \quad 6.9400 \mathrm{E}-02 \quad 1.7315 \mathrm{E}-02$ $1.7720 \mathrm{E}-03$

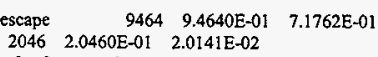

escape $\quad 9464 \quad 9.4640 \mathrm{E}-01 \quad 7.1762 \mathrm{E}-01$ $2046 \quad 2.0460 \mathrm{E}-01 \quad 2.0141 \mathrm{E}-02$ weight window 0. $0 \quad 0$. energy importance 00. 00. 00.

8.706E-01 -3.198E-01 3.738E-01 1.000E+00 $1.000 \mathrm{E}+000.000 \mathrm{E}+00$

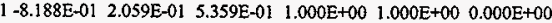
$\begin{array}{llllllll}5.727 \mathrm{E}-01 & 8.124 \mathrm{E}-01 & 1.100 \mathrm{E}-01 & 1.000 \mathrm{E}+00 & 1.000 \mathrm{E}+00 & 0.000 \mathrm{E}+00\end{array}$ $\begin{array}{llllll} & 5.914 \mathrm{E}-01 & 8.046 \mathrm{E}-01 & 1.000 \mathrm{E}+00 & 1.000 \mathrm{E}+00 & 0.000 \mathrm{E}+00\end{array}$

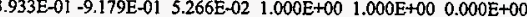
E-01 2.769E-01 9.407E-01 $1.000 \mathrm{E}+001.000 \mathrm{E}+00$ 0.000E+00 788E-01 -7.325E-03 9.603E-01 1.000E+00 1.000E+00 0.000E 00 ge lifetime, shakes cutoffs escape $6.7786 \mathrm{E}-05$ tco $1.0000 \mathrm{E}+34$ capture $8.3325 \mathrm{E}-05$ eco $1.0000 \mathrm{E}-01$ wel $0.0000 \mathrm{E}+00$ $0000 \mathrm{E}+00$

0. 2.6560E-01 $00 . \quad 2.4158 \mathrm{E}-02$ 
photon auger
tron auger
ckon total

number of electrons banked

$\begin{array}{lll}0 & 0 . & 0\end{array}$ $1479 \quad 1.4790 \mathrm{E}-01 \quad 2.7007 \mathrm{E}-02$ $11510 \quad 1.1510 \mathrm{E}+00 \quad 1.0275 \mathrm{E}+00$ electron tracks per source particle $1.1510 \mathrm{E}+00$ electron sub-steps per source particle $7.7164 \mathrm{E}+01$ total electron sub-steps $\quad 771636$

computer time so far in this run 0.00 minutes computer time in mcrun 0.00 minutes source particles per minute random numbers generated $0.0000 \mathrm{E}+00$ 4748878 total $\quad 11510 \quad 1.1510 \mathrm{E}+00 \quad 1.0275 \mathrm{E}+00$

cutoffs tco $1.0000 \mathrm{E}+34$
eco $1.0000 \mathrm{E}-01$

wcl $0.0000 \mathrm{E}+00$

wc2 $0.0000 \mathrm{E}+00$

$\begin{aligned} & \text { maximum number ever in bank } \\ & \text { bank overflows to backup file }\end{aligned}$
field length
$\begin{aligned} & 0 \\ & \text { most random numbers used was }\end{aligned}$

range of sampled source weights $=1.0000 \mathrm{E}+00$ to $1.0000 \mathrm{E}+00$

Iphoton activity in each cell

print table 126

tracks population collisions collisions number flux average average cell entering

* weight weighted weighted track weight track mfp (per history) energy energy (relative) (cm)

$\begin{array}{llllllllll}1 & 1 & 0 & 694 & 130 & 1.3000 \mathrm{E}-02 & 2.8286 \mathrm{E}-01 & 2.8286 \mathrm{E}-01 & 1.0000 \mathrm{E}+00 & 1.6113 \mathrm{E}-01\end{array}$

total $\quad 0 \quad 694 \quad 130 \quad 1.3000 \mathrm{E}-02$

lelectron activity in each cell

print table 126

tracks population substeps substeps number flux average average

cell entering

- weight weighted weighted track weight track mfp (per history) energy energy (relative) (cm)

\section{$\begin{array}{llllllllll}1 & 1 & 10000 & 11510 & 771636 & 7.7164 \mathrm{E}+01 & 7.4988 \mathrm{E}-01 & 7.6821 \mathrm{E}-01 & 1.0000 \mathrm{E}+00 & 2.5110 \mathrm{E}-04\end{array}$ \\ $\begin{array}{lllll}1 \text { tally } 1 & 10000 & 11510 & 771636 & 7.7164 E+01\end{array}$}

tally type $1^{*}$ energy crossing a surface. units mev

tally for photons

surface: 112

energy

$1.0000 \mathrm{E}-01 \quad 0.00000 \mathrm{E}+00 \quad 0.0000 \quad 0.00000 \mathrm{E}+00 \quad 0.0000$

$1.9100 \mathrm{E}-01 \quad 1.20113 \mathrm{E}-03 \quad 0.1079 \quad 2.16688 \mathrm{E}-030.0814$

$2.8200 \mathrm{E}-01 \quad 8.17217 \mathrm{E}-040.1673 \quad 2.04350 \mathrm{E}-03 \quad 0.1062$

$3.7300 \mathrm{E}-01 \quad 9.52191 \mathrm{E}-04 \quad 0.1829 \quad 1.82060 \mathrm{E}-030.1357$

4.6400E-01 $1.03815 \mathrm{E}-03 \quad 0.2001 \quad 1.00234 \mathrm{E}-03 \quad 0.2043$

5.5500E-01 5.66295E-04 0.3018 8.02842E-04 0.2503

$6.4500 \mathrm{E}-01 \quad 6.09365 \mathrm{E}-04 \quad 0.3162 \quad 6.54966 \mathrm{E}-040.3016$

$\begin{array}{llll}7.3600 \mathrm{E}-01 & 3.45345 \mathrm{E}-04 & 0.4472 & 4.20131 \mathrm{E}-040.4085\end{array}$

$8.2700 \mathrm{E}-01 \quad 7.79347 \mathrm{E}-05 \quad 0.9999 \quad 7.03770 \mathrm{E}-040.3334$

$9.1800 \mathrm{E}-01 \quad 1.70096 \mathrm{E}-04 \quad 0.7070 \quad 8.29418 \mathrm{E}-050.9999$

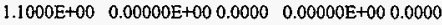

total $5.77773 \mathrm{E}-030.0811 \quad 9.69798 \mathrm{E}-030.0608$

Itally $6 \quad \mathrm{nps}=10000$

tally type 6 track length estimate of heating. units mev/gram

tally for photons

masses

$$
\text { cell: } 1
$$

$4.90220 \mathrm{E}+0$ ]

cell:

energy

$1.0000 \mathrm{E}-01 \quad 0.00000 \mathrm{E}+000.0000$

$.9100 \mathrm{E}-01 \quad 1.80395 \mathrm{E}-050.0935$

2200E-01 4.79830E-06 0.1242

$7300 \mathrm{E}-01 \quad 1.64870 \mathrm{E}-060.2061$ 
4.6400E-01 1.76412E-06 0.4116

HNF-SD-SNF-CSWD-005, Rev. 0

$.5500 \mathrm{E}-01 \quad 6.34259 \mathrm{E}-070.4736$

.4500E-01 2.47457E-07 0.3129

7.3600E-01 2.58011E-07 0.6571

$8.2700 \mathrm{E}-01 \quad 1.16245 \mathrm{E}-070.3470$

9.1800E-01 4.46610E-08 0.6403

$1.1000 \mathrm{E}+00 \quad 0.00000 \mathrm{E}+000.0000$

total 2.75512E-05 0.0722

ttally $28 \quad \mathrm{nps}=10000$

tally type 8* energy deposition units mev

tally for photons electrons

cell: $\quad 1$

energy

$-1.0000 \mathrm{E}-03-8.72550 \mathrm{E}-040.1508$

$0.0000 \mathrm{E}+00 \quad 0.00000 \mathrm{E}+000.0000$

$1.0000 \mathrm{E}-06 \quad 0.00000 \mathrm{E}+000.0000$

$1.0000 \mathrm{E}-01 \quad 1.08193 \mathrm{E}-020.0227$

$1.9100 \mathrm{E}-01 \quad 3.91016 \mathrm{E}-020.0170$

$2.8200 \mathrm{E}-01 \quad 4.36100 \mathrm{E}-020.0210$

$3.7300 \mathrm{E}-01 \quad 3.53763 \mathrm{E}-020.0286$

4.6400E-01 2.66881E-02 0.0382

$5.5500 \mathrm{E}-01 \quad 2.21833 \mathrm{E}-02 \quad 0.0468$

6.4500E-01 1.58947E-02 0.0606

$7.3600 \mathrm{E}-01 \quad 1.08330 \mathrm{E}-02 \quad 0.0790$

8.2700E-01 8.71177E-03 0.0940

$9.1800 \mathrm{E}-01 \quad 6.65495 \mathrm{E}-03 \quad 0.1136$

$1.1000 \mathrm{E}+00 \quad 4.79000 \mathrm{E}-02 \quad 0.0446$

total $2.66900 \mathrm{E}-010.0090$

1tally $11 \quad \mathrm{nps}=10000$

tally type 1* energy crossing a surface. units mev tally for electrons

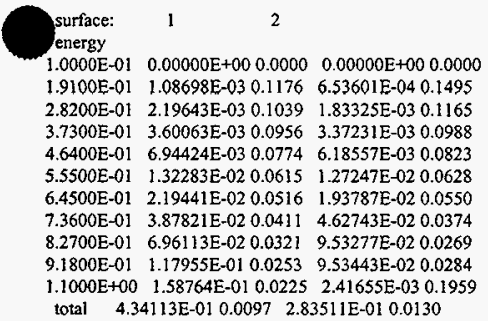

1tally 21 nps $=10000$

tally type 1 number of particles crossing a surface. tally for electrons
surface: $\quad 1 \quad 2$
energy
$1.0000 \mathrm{E}-01 \quad 0.00000 \mathrm{E}+00 \quad 0.0000 \quad 0.00000 \mathrm{E}+000.0000$
$1.9100 \mathrm{E}-01 \quad 7.70000 \mathrm{E}-03 \quad 0.1165 \quad 4.60000 \mathrm{E}-030.1471$
$2.8200 \mathrm{E}-01 \quad 9.30000 \mathrm{E}-03 \quad 0.1032 \quad 7.60000 \mathrm{E}-03 \quad 0.1158$

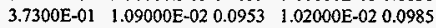
$4.6400 \mathrm{E}-01 \quad 1.65000 \mathrm{E}-02 \quad 0.0772 \quad 1.46000 \mathrm{E}-020.0822$

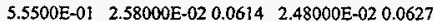

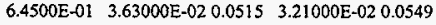

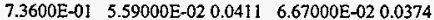

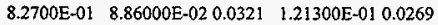
$9.1800 \mathrm{E}-0 \mathrm{I} \quad 1.34800 \mathrm{E}-01 \quad 0.0253 \quad 1.10600 \mathrm{E}-01 \quad 0.0284$
$1.1000 \mathrm{E}+00 \quad 1.65500 \mathrm{E}-010.0225 \quad 2.60000 \mathrm{E}-03 \quad 0.1959$
total $5.51300 \mathrm{E}-010.0094 \quad 3.95100 \mathrm{E}-010.0126$
ly $31 \mathrm{nps}=10000$
tally type 1 number of particles crossing a surface. 


surface: 12

energy

$1.0000 \mathrm{E}-01 \quad 0.00000 \mathrm{E}+000.0000 \quad 0.00000 \mathrm{E}+00 \quad 0.0000$

$1.9100 \mathrm{E}-01 \quad-7.70000 \mathrm{E}-03 \quad 0.1165-4.60000 \mathrm{E}-030.1471$

$2.8200 \mathrm{E}-0 \mathrm{I} \quad 9.30000 \mathrm{E}-030.1032-7.60000 \mathrm{E}-030.1158$

$3.7300 \mathrm{E}-01-1.09000 \mathrm{E}-020.0953-1.02000 \mathrm{E}-020.0985$

$4.6400 \mathrm{E}-01-1.65000 \mathrm{E}-020.0772-1.46000 \mathrm{E}-020.0822$

$5.5500 \mathrm{E}-01-2.58000 \mathrm{E}-020.0614-2.48000 \mathrm{E}-020.0627$

$6.4500 \mathrm{E}-01-3.63000 \mathrm{E}-020.0515-3.21000 \mathrm{E}-020.0549$

$7.3600 \mathrm{E}-0 \mathrm{l} \quad-5.59000 \mathrm{E}-02 \quad 0.0411-6.67000 \mathrm{E}-020.0374$

$8.2700 \mathrm{E}-01-8.86000 \mathrm{E}-020.0321-1.21300 \mathrm{E}-010.0269$

$9.1800 \mathrm{E}-01-1.34800 \mathrm{E}-010.0253-1.10600 \mathrm{E}-010.0284$

$1.1000 \mathrm{E}+00-1.65500 \mathrm{E}-010.0225-2.60000 \mathrm{E}-030.1959$

total $-5.51300 \mathrm{E}-010.0094-3.95100 \mathrm{E}-010.0126$

1 tally $41 \quad \mathrm{nps}=10000$

tally type 1 number of particles crossing a surface. tally for electrons

this tally is modified by $\mathrm{ft}$ elc

surface: 12

energy

$1.0000 \mathrm{E}-0 \mathrm{I} \quad 0.00000 \mathrm{E}+00 \quad 0.0000 \quad 0.00000 \mathrm{E}+00 \quad 0.0000$

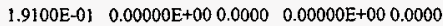

$2.8200 \mathrm{E}-0 \mathrm{I} \quad 0.00000 \mathrm{E}+000.0000 \quad 0.00000 \mathrm{E}+000.0000$

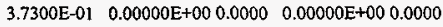

4.6400E-01 $\quad 0.00000 \mathrm{E}+00 \quad 0.0000 \quad 0.00000 \mathrm{E}+000.0000$

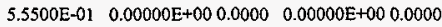

$6.4500 \mathrm{E}-01 \quad 0.00000 \mathrm{E}+000.0000 \quad 0.00000 \mathrm{E}+000.0000$

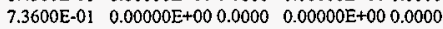

$2700 \mathrm{E}-01 \quad 0.00000 \mathrm{E}+00 \quad 0.0000 \quad 0.00000 \mathrm{E}+000.0000$

$1800 \mathrm{E}-01 \quad 0.00000 \mathrm{E}+00 \quad 0.0000 \quad 0.00000 \mathrm{E}+000.0000$

$1000 \mathrm{E}+00 \quad 0.00000 \mathrm{E}+00 \quad 0.0000 \quad 0.00000 \mathrm{E}+000.0000$ total $0.00000 \mathrm{E}+000.0000 \quad 0.00000 \mathrm{E}+00 \quad 0.0000$

surface: $\quad 1 \quad 2$

energy

1.0000E-01 $0.00000 \mathrm{E}+00 \quad 0.0000 \quad 0.00000 \mathrm{E}+00 \quad 0.0000$ $\begin{array}{llll}1.9100 \mathrm{E}+01 & 7.70000 \mathrm{E}-03 & 0.1165 & 4.60000 \mathrm{E}-030.1471\end{array}$

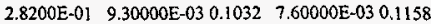

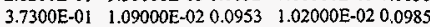

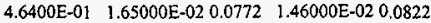

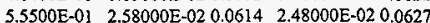

6.4500E-01 3.63000E-02 $0.0515 \quad 3.21000 \mathrm{E}-020.0549$

$\begin{array}{lllll}7.3600 E-01 & 5.59000 E-02 & 0.0411 & 6.67000 E-02 & 0.0374\end{array}$

$\begin{array}{lllll}8.2700 \mathrm{E}-01 & 8.86000 \mathrm{E}-02 & 0.0321 & 1.21300 \mathrm{E}-01 & 0.0269\end{array}$

$\begin{array}{lllll}9.1800 \mathrm{E}-01 & 1.34800 \mathrm{E}-01 & 0.0253 & 1.10600 \mathrm{E}-01 & 0.0284\end{array}$

$1.1000 \mathrm{E}+00 \quad 1.65500 \mathrm{E}-01 \quad 0.0225 \quad 2.60000 \mathrm{E}-030.1959$

total $5.51300 \mathrm{E}-010.0094 \quad 3.95100 \mathrm{E}-010.0126$

surface: 12

energy

$\begin{array}{lll}1.0000 \mathrm{E}-01 & 0.00000 \mathrm{E}+00 & 0.0000 \quad 0.00000 \mathrm{E}+000.0000\end{array}$

$1.9100 \mathrm{E}-01 \quad 7.70000 \mathrm{E}-03 \quad 0.1165 \quad 4.60000 \mathrm{E}-03 \quad 0.1471$.

$2.8200 \mathrm{E}-01 \quad 9.30000 \mathrm{E}-03 \quad 0.1032 \quad 7.60000 \mathrm{E}-03 \quad 0.1158$

$3.7300 \mathrm{E}-01 \quad 1.09000 \mathrm{E}-02 \quad 0.0953 \quad 1.02000 \mathrm{E}-02 \quad 0.0985$

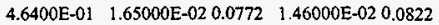

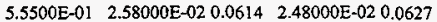

$6.4500 \mathrm{E}-01 \quad 3.63000 \mathrm{E}-02 \quad 0.0515 \quad 3.21000 \mathrm{E}-020.0549$

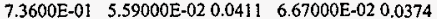

$\begin{array}{lllll}8.2700 \mathrm{E}-01 & 8.86000 \mathrm{E}-02 & 0.0321 & 1.21300 \mathrm{E}-01 & 0.0269\end{array}$

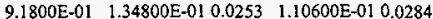

$.1000 \mathrm{E}+00 \quad 1.65500 \mathrm{E}-010.0225 \quad 2.60000 \mathrm{E}-030.1959$

total $5.51300 \mathrm{E}-01 \quad 0.0094 \quad 3.95100 \mathrm{E}-010.0126$

tly 51 $\mathrm{nps}=10000$ 


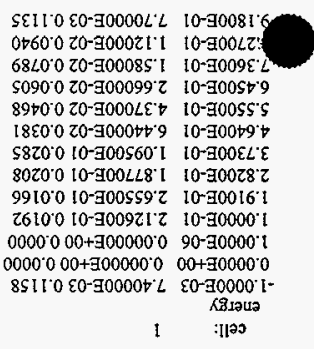

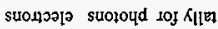
-uounqụs!̣ 14 $0000 \mathrm{~L}=\mathrm{s} d u \quad 8 \mathrm{~S}$ Kllet

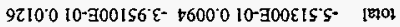

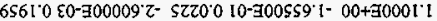

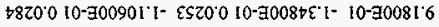

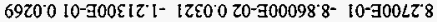

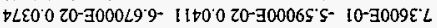

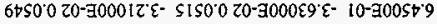

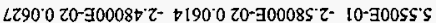

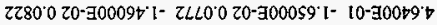

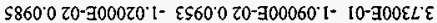

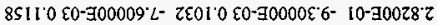

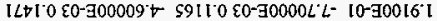
$0000^{\circ} 000+\exists 00000^{\circ} 0 \quad 0000^{\circ} 000+300000^{\prime} 0 \quad 10-30000^{\circ} \mathrm{I}$

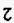
Кำนว

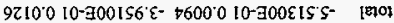

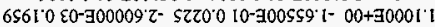

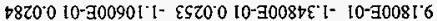

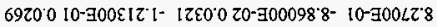

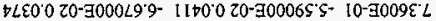

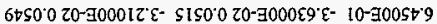

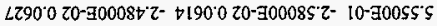

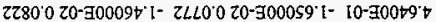

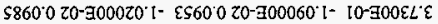

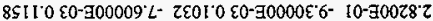

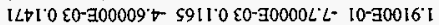
$0000^{\circ} 000+700000^{\circ} 0 \quad 0000^{\circ} 000+300000^{\circ} 0 \quad 10-90000^{\circ}$ I

$0000^{\circ} 000+300000^{\circ} 0 \quad 0000^{\circ} 000+300000^{\circ} 0$ [B] $0000000+70000000000000+300000000+3000 I^{\prime} t$ $0000^{\circ} 000+300000^{\circ} 0 \quad 0000^{\circ} 000+300000^{\circ} 0 \quad 10-30081^{\prime} 6$ $0000^{\circ} 000+900000^{\circ} 0 \quad 0000^{\circ} 000+700000^{\circ} 0 \quad 10-900<Z^{\circ} 8$ $0000^{\circ} 000+700000^{\circ} 00000000+300000^{\circ} 0 \quad 10-3009 \varepsilon^{\circ} L$ $00000^{\circ} 000+300000^{\circ} 0 \quad 0000^{\prime} 000+300000^{\circ} 0 \quad 10-300 S t^{\prime} 9$ $0000^{\circ} 000+300000^{\circ} 00000.000+300000^{\circ} 0 \quad 10-3005 \varsigma \varsigma$ $0000000+300000^{\circ} 0 \quad 0000^{\circ} 000+300000^{\circ} 0 \quad 10-30069^{\circ} t$ $0000^{\circ} 000+\$ 00000^{\circ} 0 \quad 0000^{\circ} 000+300000^{\circ} 0 \quad 10-900 \varepsilon L \varepsilon$ $0000^{\circ} 000+300000^{\circ} 0 \quad 0000^{\circ} 000+300000^{\circ} 0 \quad 10-300 z 8^{\circ} \tau$ $0000^{\circ} 000+300000^{\circ} 0 \quad 0000^{\circ} 000+300000^{\circ} 0 \quad 10-30016^{\prime} \mathrm{I}$ $0000^{\circ} 000+300000^{\circ} \quad 0000000+300000 \%$ 10-30000' I Кร1aนเa

$$
\text { l } l \text { :apejins }
$$

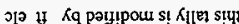

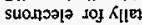

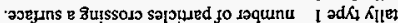


$1.1000 \mathrm{E}+00 \quad 4.79000 \mathrm{E}-020.0446$

total $1.00000 \mathrm{E}+000.0000$

y $8 \quad \mathrm{nps}=10000$

tally type $8^{*}$ energy deposition

tally for photons electrons

HNF-SD-SNF-CSWD-005, Rev. 0

cell: $\quad 1$

energy

$1.0000 \mathrm{E}+03 \quad 2.66900 \mathrm{E}-010.0090$

1status of the statistical checks used to form confidence intervals for the mean for each tally bin

tally result of statistical checks for the tfc bin (the first check not passed is listed) and error magnitude check for all bins

1 missed 1 of $10 \mathrm{tfc}$ bin checks: there is insufficient tfe bin tally information to estimate the large tally slope reliably missed all bin error check: 24 tally bins had 4 bins with zeros and 17 bins with relative errors exceeding 0.10

6 missed 1 of 10 tfc bin checks: the variance of the variance appears not to decrease as $1 / n p s$ for the last half of problem missed all bin error check: 12 tally bins had 2 bins with zeros and 8 bins with relative errors exceeding 0.10

28 passed the 10 statistical checks for the tally fluctuation chart bin result

missed all bin error check: 15 tally bins had 2 bins with zeros and 2 bins with relative errors exceeding 0.10

11 missed 1 of 10 tfe bin checks: the slope of decrease of largest tallies is less than the minimum acceptable value of 3.0 missed all bin error check: 24 tally bins had 2 bins with zeros and 5 bins with relative errors exceeding 0.10

21 missed 1 of $10 \mathrm{t} f \mathrm{c}$ bin checks: the estimated mean has a trend during the last half of the problem missed all bin error check: 24 tally bins had 2 bins with zeros and 5 bins with relative errors exceeding 0.10

31 missed 2 of $10 \mathrm{tfc}$ bin checks: the estimated mean has a trend during the last half of the problem missed all bin error check: 24 tally bins had 2 bins with zeros and 5 bins with relative errors exceeding 0.10

41 missed 1 of 10 tf $\mathrm{c}$ bin checks: the estimated mean has a trend during the last half of the problem missed all bin erTor check: 72 tally bins had 28 bins with zeros and 10 bins with telative errors exceeding 0.10

51 missed 2 of 10 tfc bin checks: the estimated mean has a trend during the last half of the problem missed all bin error check: 72 tally bins had 28 bins with zeros and 10 bins with relative errors exceeding 0.10

58 passed the 10 statistical checks for the tally fluctuation chan bin result missed all bin error check: 15 tally bins had 2 bins with zeros and 2 bins with relative enrors exceeding 0.10

8 passed the 10 statistical checks for the tally fluctuation chart bin result passed all bin erfor check: 1 tally bins all have relative enors less than 0.10 with no zero bins

the 10 statistical checks are only for the tally fluctuation chart bin and do not apply to other tally bins.

the tally bins with zeros may or may not be correct: compare the source, cutoffs, multipliers, et cetera with the tally bins.

waming. 7 of the 10 tally fluctuation chart bins dio not pass all 10 statistical checks.

warning. 9 of the 10 tallies had bins with relative errors greater than recommended.

1 tally fluctuation charts

tally 1 tally 6 tally 28

nps mean error vov slope fom mean enror vov slope fom

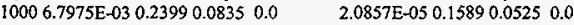

$20005.5780 \mathrm{E}-030.18840 .0641 \quad 0.0 \quad 2.4397 \mathrm{E}-050.17010 .1693 \quad 0.0$

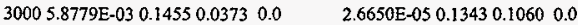

$40005.7880 \mathrm{E}-030.13170 .03110 .0 \quad 2.7894 \mathrm{E}-050.11450 .06560 .0$

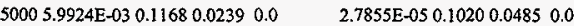

$60005.9423 \mathrm{E}-030.10550 .0195 \quad 0.0 \quad 2.7793 \mathrm{E}-050.09310 .04180 .0$

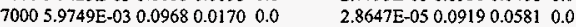

$80005.7670 \mathrm{E}-03 \quad 0.09130 .0154 \quad 0.0 \quad 2.8249 \mathrm{E}-050.08380 .052410 .0$

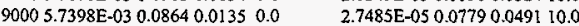

$100005.7777 \mathrm{E}-030.08110 .0120 \quad 0.0 \quad 2.7551 \mathrm{E}-050.07220 .043610 .0$ mean etror vov slope fom $2.6993 \mathrm{E}-010.02790 .004410 .0$ $2.6475 \mathrm{E}-010.01990 .002210 .0$ $2.6433 \mathrm{E}-010.01610 .001510 .0$ 2.6224E-01 0.01400 .001110 .0

$2.6175 \mathrm{E}-010.01260 .000910 .0$ $2.6496 \mathrm{E}-010.01160 .000710 .0$ $2.6691 \mathrm{E}-010.01070 .000610 .0$ 2.6739E-01 0.01000 .000510 .0

$2.6699 \mathrm{E}-010.00950 .000510 .0$

$2.6690 \mathrm{E}-010.00900 .000410 .0$ 
tally 11 nps mean error vov slope fom $10004.4504 \mathrm{E}-010.02960 .00022 .1$ $20004.4489 \mathrm{E}-010.02110 .00012 .0$ $30004.4226 \mathrm{E}-010.01730 .0001 \quad 1.8$ $40004.4011 \mathrm{E}-010.01510 .0000 \quad 1.8$ $50004.4004 \mathrm{E}-010.01350 .0000 \quad 1.9$ $60004.3549 E-010.01250 .0000 \quad 1.8$ $70004.3477 \mathrm{E}-010.01150 .00001 .9$ $80004.3339 \mathrm{E}-01 \quad 0.01080 .0000 \quad 1.8$ $90004.3428 \mathrm{E}-010.01020 .00001 .7$ $100004.3411 \mathrm{E}=010.00970 .00001 .6$

tally 41

nps mean error vov slope fom $10005.6500 \mathrm{E}-010.02860 .00044 .4$ $20005.6500 \mathrm{E}-010.02040 .0003 \quad 4.4$ $30005.6400 \mathrm{E}-010.01680 .000210 .0$ $40005.6100 \mathrm{E}-010.01470 .000210 .0$ $50005.5860 \mathrm{E}-010.01310 .000110 .0$ $60005.5433 \mathrm{E}+01 \quad 0.01210 .000110 .0$ $70005.5386 \mathrm{E}-01 \quad 0.01120 .0001 \quad 10.0$ $80005.5238 \mathrm{E}-01 \quad 0.01050 .000110 .0$ $90005.5200 \mathrm{E}-01 \quad 0.00990 .000110 .0$ $100005.5130 \mathrm{E}-010.00940 .000110 .0$ tally 21

tally 31

mean error vov slope fom 5.6500E-01 0.02860 .00044 .4 $5.6500 \mathrm{E}-010.02040 .0003 \quad 4.4$ $5.6400 \mathrm{E}-010.01680 .000210 .0$ $5.6100 \mathrm{E}-010.01470 .000210 .0$ $5.5860 \mathrm{E}-010.01310 .000110 .0$ $5.5433 \mathrm{E}-010.01210 .000110 .0$ $5.5386 \mathrm{E}-010.01120 .000110 .0$ $5.5238 \mathrm{E}-010.01050 .000110 .0$ $5.5200 \mathrm{E}-010.00990 .000110 .0$ $5.5130 \mathrm{E}-010.00940 .000110 .0$

tally 51 tally 58 mean error vov slope fom $-5.6500 \mathrm{E}-010.02860 .0004 \quad 0.0$

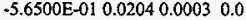

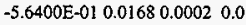
$\begin{array}{llll}-5.6100 \mathrm{E}-0 ! & 0.0147 & 0.0002 & 0.0\end{array}$ $\begin{array}{llll}-5.5860 \mathrm{E}-01 & 0.0131 & 0.0001 & 0.0\end{array}$ $\begin{array}{llllll}-5.5433 \mathrm{E}-01 & 0.0121 & 0.0001 & 0.0\end{array}$ $-5.5386 \mathrm{E}-010.01120 .00010 .0$ $\begin{array}{llll}-5.5238 \mathrm{E}-01 & 0.01050 .0001 & 0.0\end{array}$ $-5.5200 \mathrm{E}-010.00990 .00010 .0$ $\begin{array}{lll}-5.5130 \mathrm{E}-01 & 0.00940 .0001 & 0.0\end{array}$ mean error vov slope fom $-5.6500 \mathrm{E}-010.02860 .0004$
0.0 $\begin{array}{llll}-5.6500 \mathrm{E}-01 & 0.0204 & 0.0003 & 0.0\end{array}$ $\begin{array}{lll}-5.6400 \mathrm{E}-01 & 0.01680 .0002 \quad 0.0\end{array}$ $\begin{array}{llll}-5.6100 \mathrm{E}-01 & 0.01470 .0002 & 0.0\end{array}$ $\begin{array}{llll}-5.5860 \mathrm{E}-010.01310 .0001 & 0.0\end{array}$ $\begin{array}{llllll}-5.5433 \mathrm{E}-01 & 0.0121 & 0.0001 & 0.0\end{array}$ $-5.5386 \mathrm{E}-010.01120 .00010 .0$ $\begin{array}{llll}-5.5238 \mathrm{E}-01 & 0.01050 .0001 & 0.0\end{array}$ $-5.5200 \mathrm{E}-010.00990 .00010 .0$
0.5 $-5.5130 \mathrm{E}-010.00940 .00010 .0$

mean error vov slope fom $1.0000 \mathrm{E}+000.00000 .000010 .0$ $1.0000 \mathrm{E}+000.00000 .000010 .0$ $1.0000 \mathrm{E}+000.00000 .000010 .0$ $1.0000 \mathrm{E}+000.00000 .000010 .0$ $1.0000 \mathrm{E}+000.00000 .000010 .0$ $1.0000 \mathrm{E}+000.00000 .000010 .0$ $1.0000 \mathrm{E}+000.00000 .000010 .0$ $1.0000 \mathrm{E}+000.00000 .000010 .0$ $1.0000 \mathrm{E}+000.00000 .000010 .0$ $1.0000 \mathrm{E}+000.00000 .000010 .0$ tally 8

nps mean error vov slope fom $10002.6993 \mathrm{E}-010.02790 .004410 .0$ $20002.6475 \mathrm{E}-010.01990 .002210 .0$ $30002.6433 \mathrm{E}-010.01610 .001510 .0$ $40002.6224 \mathrm{E}-010.01400 .001110 .0$ $50002.6175 \mathrm{E}-010.01260 .000910 .0$ $60002.6496 \mathrm{E}-010.01160 .000710 .0$ $70002.6691 \mathrm{E}-010.01070 .000610 .0$ $80002.6739 \mathrm{E}-010.01000 .000510 .0$ $90002.6699 \mathrm{E}-010.00950 .000510 .0$ $100002.6690 \mathrm{E}-010.00900 .000410 .0$ tally data written to file inp $20 \mathrm{~m}$

6 warning messages so far.

Iun terminated when 10000 particle histories were done.

\section{File: Inp240}

1- prob 24 - reflecting lattice. $15 \times 15$ at 3.75 w/o u-235 enrichment

2- 1 I -10.182-1 u=2

3. 2 2-.001 $1-2 \mathrm{u}=2$

4- $3 \quad 3-6.552-3 u=2$

5. 4 4-1.03 $\mathrm{u}=2$

6- 5 - $4-1.0-14: 15 u=3$

7- $6 \quad 3-6.5514-15 u=3$

8- $7 \quad 4-1.0-4+5-6+7 u=1$ lat $=1$ fill $=-8: 8-8: 80: 0$

9. $\quad 117 \mathrm{r} 214 \mathrm{r} 11214 \mathrm{r} 1122322322 \mathrm{r} 322$

10- $\quad 3221126 r_{326 r 1123 r 3}$

11- $\quad 24 r 323 r 1122328 \mathrm{r} 322$

12- $\quad 11214 \mathrm{r} 1122 \mathrm{r} 322 \mathrm{r} 322 \mathrm{r}$

13- $\quad 322 \mathrm{r} 11214 \mathrm{rl} 122328 \mathrm{r} 3$

14- $\quad 221123 r 324 r 323 r 11$

15- $\quad 26 r 326 r 11223223$

$22 \mathrm{r} 32232211214 \mathrm{r} 11214 \mathrm{r} 117 \mathrm{r}$

$8 \quad 0-8 \quad-10 \quad-12 u=4$ fill $=1$

$9 \quad 5-7.98: 10 u=4$ 


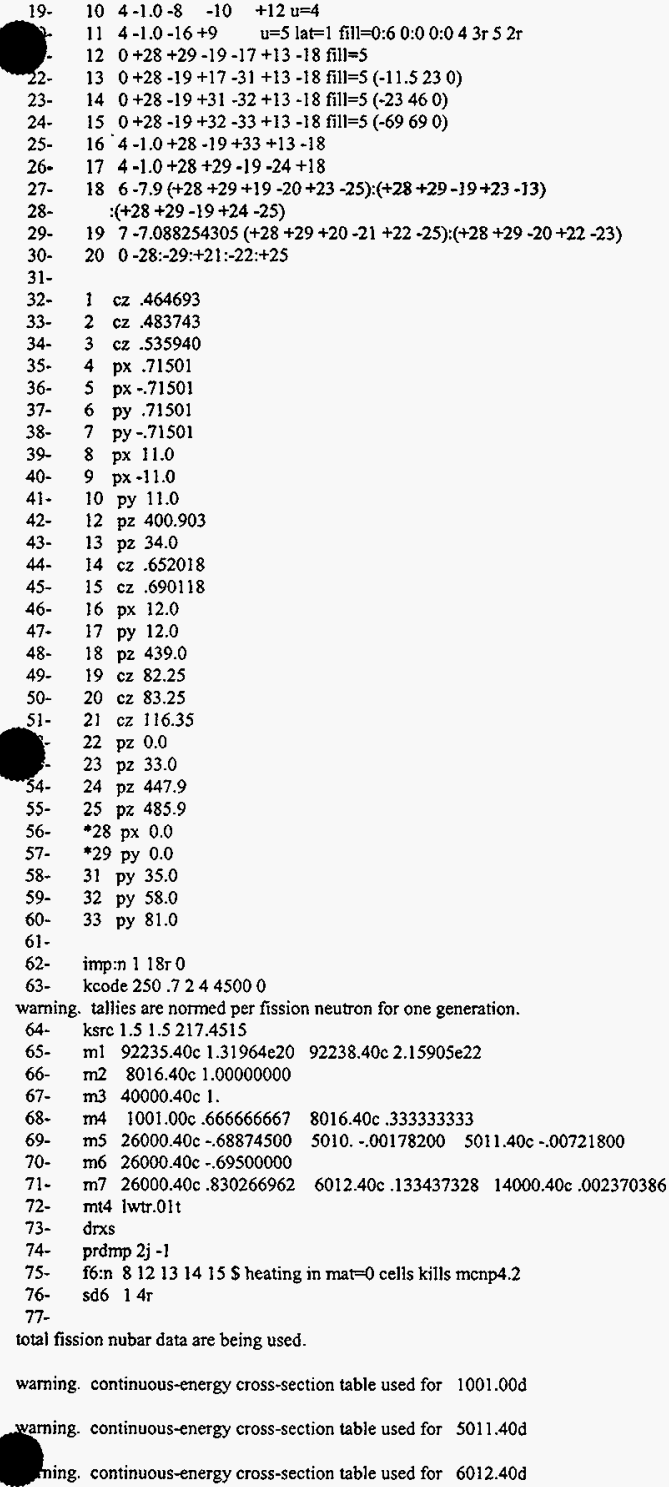




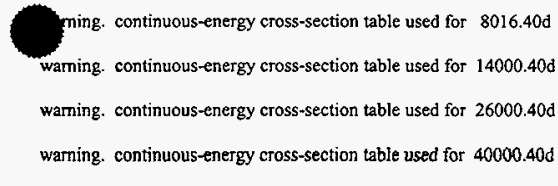

warning. continuous-energy cross-section table used for $92235.40 \mathrm{~d}$

waming. continuous-energy cross-section table used for 92238.400

warning. 4 of the materials had unnormalized fractions.

lcells

print table 60

$$
\text { cell mat density density volume mass pieces importance }
$$

\begin{tabular}{|c|c|c|}
\hline 1 & $12.57597 \mathrm{E}-021.01820 \mathrm{E}+010.00000 \mathrm{E}+000.00000 \mathrm{E}+00$ & \\
\hline 2 & $23.76497 \mathrm{E}-051.00000 \mathrm{E}-030.00000 \mathrm{E}+000.00000 \mathrm{E}+00$ & $01.0000 \mathrm{E}+00$ \\
\hline 3 & $34.32392 \mathrm{E}-026.55000 \mathrm{E}+000.00000 \mathrm{E}+000.00000 \mathrm{E}+00$ & $01.0000 \mathrm{E}+00$ \\
\hline 4 & 4s $1.00309 \mathrm{E}-011.00000 \mathrm{E}+000.00000 \mathrm{E}+000.00000 \mathrm{E}+00$ & $01.0000 \mathrm{E}+00$ \\
\hline 5 & 4s $1.00309 \mathrm{E}-011.00000 \mathrm{E}+000.00000 \mathrm{E}+000.00000 \mathrm{E}+00$ & $01.0000 \mathrm{E}+00$ \\
\hline 6 & $34.32392 \mathrm{E}-026.55000 \mathrm{E}+000.00000 \mathrm{E}+000.00000 \mathrm{E}+00$ & $01.0000 \mathrm{E}+00$ \\
\hline 7 & $4 s] .00309 \mathrm{E}+01 \quad 1.00000 \mathrm{E}+000.00000 \mathrm{E}+00 \quad 0.00000 \mathrm{E}+00$ & $0 \quad 1.0000 \mathrm{E} \div 00$ \\
\hline 8 & $0 \quad 0.00000 \mathrm{E}+00 \quad 0.00000 \mathrm{E}+00 \quad 0.00000 \mathrm{E}+00 \quad 0.00000 \mathrm{E}+00$ & $01.0000 \mathrm{E}+00$ \\
\hline 9 & $58.97738 \mathrm{E}-027.90000 \mathrm{E}+000.00000 \mathrm{E}+000.00000 \mathrm{E}+00$ & $01.0000 \mathrm{E}+00$ \\
\hline 10 & $4 \mathrm{~s} 1.00309 \mathrm{E}-011.00000 \mathrm{E}+000.00000 \mathrm{E}+000.00000 \mathrm{E}+00$ & $01.0000 \mathrm{E}+00$ \\
\hline 11 & 4s $1.00309 \mathrm{E}-01 \quad 1.00000 \mathrm{E}+000.00000 \mathrm{E}+000.00000 \mathrm{E}+00$ & $01.0000 \mathrm{E}+00$ \\
\hline 12 & $0 \quad 0.00000 \mathrm{E}+000.00000 \mathrm{E}+000.00000 \mathrm{E}+000.00000 \mathrm{E}+00$ & $01.0000 \mathrm{E}+00$ \\
\hline 13 & $00.00000 \mathrm{E}+000.00000 \mathrm{E}+000.00000 \mathrm{E}+000.00000 \mathrm{E}+00$ & $01.0000 \mathrm{E}+00$ \\
\hline 14 & $0 \quad 0.00000 \mathrm{E}+00 \quad 0.00000 \mathrm{E}+000.00000 \mathrm{E}+00 \quad 0.00000 \mathrm{E}+00$ & $01.0000 \mathrm{E}+00$ \\
\hline 15 & $0 \quad 0.00000 \mathrm{E}+000.00000 \mathrm{E}+00 \quad 0.00000 \mathrm{E}+000.00000 \mathrm{E}+00$ & $01.0000 \mathrm{E}+00$ \\
\hline 16 & 4s $1.00309 \mathrm{E}-011.00000 \mathrm{E}+000.00000 \mathrm{E}+000.00000 \mathrm{E}+00$ & $01.0000 \mathrm{E}+00$ \\
\hline 17 & is $1.00309 \mathrm{E}-011.00000 \mathrm{E}+000.00000 \mathrm{E}+000.00000 \mathrm{E}+00$ & $01.0000 \mathrm{E}+00$ \\
\hline 18 & $68.51890 \mathrm{E}=027.90000 \mathrm{E}+000.00000 \mathrm{E}+000.00000 \mathrm{E}+00$ & $01.0000 E+00$ \\
\hline 19 & $78.58502 \mathrm{E}-027.08825 \mathrm{E}+000.00000 \mathrm{E}+000.00000 \mathrm{E}+00$ & $01.0000 \mathrm{E}+00$ \\
\hline & $0 \quad 0.00000 \mathrm{E}+000.00000 \mathrm{E}+000.00000 \mathrm{E}+000.00000 \mathrm{E}+00$ & $00.0000 \mathrm{E}+00$ \\
\hline
\end{tabular}

total

$0.00000 \mathrm{E}+000.00000 \mathrm{E}+00$

11 warning messages so far.

Icross-section tables

print table 100

table length

tables from file testlibl

I001.00c 2139 1-h-1 from endf-vi.1

mat $12505 / 26 / 93$

$5010.03 \mathrm{~d} 3945$ b-10 endf/b-iv new gamma production format (1273) 14 oct 75

$5011.40 \mathrm{c} 3473$ ENDL library name: nd900719 MCNP translation: 900831 14:10:05 900207

$6012.40 \mathrm{c} 5049$ ENDL library name: nd900719 MCNP translation: 900831 14:10:05 900207

8016.40c S693 ENDL library name: nd900719 MCNP translation: 900831 14:10:05 900207

$14000.40 \mathrm{c} 8732$ ENDL library name: nd900719 MCNP translation: 900831 14:10:05 900207

26000.40 21846 ENDL library name: nd900719 MCNP translation: 900831 14:10:05 900207

40000.40c 111279 ENDL library name: nd900719 MCNP transłation: 900831 14:10:05 900323

92235.40c 40506 ENDL library name: nd900719 MCNP translation: 9total nu 10:05 900503

92238.40c 48310 ENDL library name: nd901118 MCNP translation: 9total nu 56:49 901119

$\begin{array}{lllll}\text { lwtr.01t } 10193 \text { hydrogen in light water at } 300 \text { degrees kelvin } & 1001 & 0 & 010 / 22 / 85\end{array}$

total 261165

waming. neutron energy cutoff is below some cross-section tables. 
12 warning messages so far. lestimated keff results by cycle

print table 175

cycle $1 \mathrm{k}$ (collision) 0.658210 removal lifetime(abs) $5.1517 \mathrm{E}+03$ source points generated 228

cycle $2 \mathrm{k}$ (collision) 0.549318 removal lifetime(abs) $3.7645 \mathrm{E}+03$ source points generated 209

cycle $3 \mathrm{k}$ (collision) 0.551862 removal lifetime(abs) $3.8422 \mathrm{E}+03$ source points generated 253

estimator cycle 4 ave of 2 cycles combination simple average combined average corr

$\begin{array}{llllllllll}\mathrm{k} \text { (collision) } & 0.584886 & 0.5683740 .0291 & \mathrm{k} \text { (col/abs) } & 0.000000 & 0.0000 & 0.0000000 .0000 & 0.0000\end{array}$

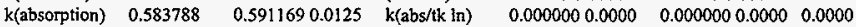

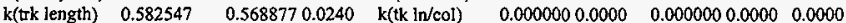

rem life(col) $3.9485 \mathrm{E}+03 \quad 3.8951 \mathrm{E}+030.0137$

rem life(abs) $4.0658 \mathrm{E}+03 \quad 3.9540 \mathrm{E}+03 \quad 0.0283 \quad \mathrm{life}(\mathrm{col} / \mathrm{abs}) \quad 0.0000 \mathrm{E}+000.0000 \quad 0.0000 \mathrm{E}+000.0000 \quad 0.0000$

source points generated 264

source distribution written to file inp24s $\quad$ cycle $=4$

Iproblem summary

run terminated when 4 kcode cycles were done.

neutron creation tracks weight energy neutron loss tracks weight energy (per source particle) (per source particle)

$\begin{array}{llllllll}\text { source } & 940 & 1.0638 \mathrm{E}+00 & 1.9444 \mathrm{E}+00 & \text { escape } & 0 & 0 . & 0 .\end{array}$

energy cutoff $\quad 00 . \quad 0$.

time cutoff 00.0.

ight window $\quad \begin{array}{lllllll}0 & 0 . & 0 . & \text { weight window } & 0 & 0 . & 0 .\end{array}$

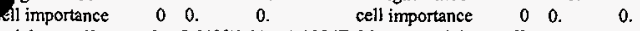

$\begin{array}{lllllllll}\text { weight cutoff } & 0 & 5.6409 \mathrm{E}-02 & 1.1284 \mathrm{E}-06 & \text { weight cutoff } & 944 & 5.2413 \mathrm{E}-02 & 7.1769 \mathrm{E}-07\end{array}$

energy importance $\begin{array}{llllllll}0 & 0 . & 0 . & \text { energy importance } & 0 & 0 . & 0 .\end{array}$

$\begin{array}{llllllllllll}\text { dxtran } & 0 & 0 . & 0 . & & \text { dxtran } & 0 & 0 . & 0 . & 0 . \\ \text { forced collisions } & 0 & 0 . & 0 . & \text { forced collisions } & 0 & 0 . & 0 .\end{array}$

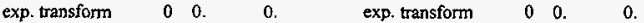

$\begin{array}{lllllllll}\text { upscattering } & 0 & 0 . & 1.4710 \mathrm{E}-07 & \text { downscattering } & 0 & 0 . & 1.8098 \mathrm{E}+00\end{array}$

$\begin{array}{rlllll} & \text { capture } & 0 & 8.1698 \mathrm{E}-01 & 1.8384 \mathrm{E}-02\end{array}$

$(n, x n) \quad \begin{array}{llllllll}8 & 6.9970 \mathrm{E}-03 & 3.6428 \mathrm{E}-03 & \text { loss to }(n, x n) & 4 & 3.4985 \mathrm{E}-03 & 2.8025 \mathrm{E}-02\end{array}$

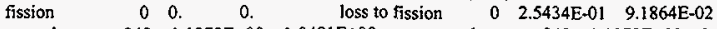

$\begin{array}{lllllllll}\text { total } & 948 & 1.1272 \mathrm{E}+00 & 1.9481 \mathrm{E}+00 \quad \text { total } & 948 & 1.1272 \mathrm{E}+00 & 1.9481 \mathrm{E}+00\end{array}$

number of neutrons banked 4 average lifetime, shakes cutoffs

neutron tracks per source particle $1.0085 \mathrm{E}+00$ escape $0.0000 \mathrm{E}+00$ tco $1.0000 \mathrm{E}+34$

neutron collisions per source particle $6.1389 \mathrm{E}+01$ capture $4.1766 \mathrm{E}+03$ eco $0.0000 \mathrm{E}+00$

total neutron collisions 57706 capture or escape $4.1766 \mathrm{E}+03$ wcl $-5.0000 \mathrm{E}-01$

net multiplication $\quad 1.0033 \mathrm{E}+000.0030$ any termination $4.4650 \mathrm{E}+03$ wc2 $-2.5000 \mathrm{E}-01$

computer time so far in this run 0.00 minutes maximum number ever in bank 1

computer time in mcrun $\quad 0.00$ minutes bank overflows to backup fije 0

source particles per minute $\quad 0.0000 \mathrm{E}+00$

random numbers generated $\quad 690539$

field length

most random numbers used was 3677 in history 21

range of sampled source weights $=9.8814 \mathrm{E}-01$ to $1.1962 \mathrm{E}+00$

Ineutron activity in each cell

print table 126

tracks population collisions collisions number flux average average

cell entering * weight weighted weighted track weight track mfp (per history) energy energy (relative) (cm) 


$\begin{array}{ccccccccccccc}4 & 4 & 44969 & 944 & 40660 & 3.0973 \mathrm{E}+01 & 3.0008 \mathrm{E}-04 & 5.9877 \mathrm{E}-01 & 8.3882 \mathrm{E}-01 & 1.4858 \mathrm{E}+00 \\ 5 & 5 & 8213 & 845 & 8775 & 6.4600 \mathrm{E}+00 & 2.0190 \mathrm{E}-04 & 5.0976 \mathrm{E}-01 & 8.1000 \mathrm{E}-01 & 1.3256 \mathrm{E}+00 \\ 5 & 6 & 4924 & 791 & 109 & 9.6195 \mathrm{E}-02 & 2.0789 \mathrm{E}-04 & 5.2658 \mathrm{E}-01 & 8.2057 \mathrm{E}-01 & 3.6398 \mathrm{E}+00 \\ 7 & 7 & 2832 & 638 & 1607 & 1.4612 \mathrm{E}+00 & 8.5166 \mathrm{E}-04 & 7.1930 \mathrm{E}-01 & 9.4163 \mathrm{E}-01 & 1.7232 \mathrm{E}+00 \\ 8 & 8 & 0 & 0 & 0 & 0.0000 \mathrm{E}+00 & 0.0000 \mathrm{E}+00 & 0.0000 \mathrm{E}+00 & 0.0000 \mathrm{E}+00 & 0.0000 \mathrm{E}+00 \\ 9 & 9 & 1333 & 607 & 1347 & 1.2836 \mathrm{E}+00 & 3.8320 \mathrm{E}-03 & 8.0017 \mathrm{E}-01 & 9.7093 \mathrm{E}-01 & 3.1777 \mathrm{E}+00 \\ 10 & 10 & 0 & 0 & 0 & 0.0000 \mathrm{E}+00 & 0.0000 \mathrm{E}+00 & 0.0000 \mathrm{E}+00 & 0.0000 \mathrm{E}+00 & 0.0000 \mathrm{E}+00 \\ 11 & 11 & 7 & 1 & 126 & 9.7846 \mathrm{E}-02 & 9.0288 \mathrm{E}-05 & 3.2866 \mathrm{E}-01 & 7.7027 \mathrm{E}-01 & 1.0087 \mathrm{E}+00 \\ 12 & 12 & 0 & 0 & 0 & 0.0000 \mathrm{E}+00 & 0.0000 \mathrm{E}+00 & 0.0000 \mathrm{E}+00 & 0.0000 \mathrm{E}+00 & 0.0000 \mathrm{E}+00 \\ 13 & 13 & 0 & 0 & 0 & 0.0000 \mathrm{E}+00 & 0.0000 \mathrm{E}+00 & 0.0000 \mathrm{E}+00 & 0.0000 \mathrm{E}+00 & 0.0000 \mathrm{E}+00 \\ 14 & 14 & 0 & 0 & 0 & 0.0000 \mathrm{E}+00 & 0.0000 \mathrm{E}+00 & 0.0000 \mathrm{E}+00 & 0.0000 \mathrm{E}+00 & 0.0000 \mathrm{E}+00 \\ 15-15 & 0 & 0 & 0 & 0.0000 \mathrm{E}+00 & 0.0000 \mathrm{E}+0 & 0.0000 \mathrm{E}+00 & 0.0000 \mathrm{E}+00 & 0.0000 \mathrm{E}+00 \\ 16 & 16 & 0 & 0 & 0 & 0.0000 \mathrm{E}+00 & 0.0000 \mathrm{E}+00 & 0.0000 \mathrm{E}+00 & 0.0000 \mathrm{E}+00 & 0.0000 \mathrm{E}+00 \\ 17 & 17 & 0 & 0 & 0 & 0.0000 \mathrm{E}+00 & 0.0000 \mathrm{E}+00 & 0.0000 \mathrm{E}+00 & 0.0000 \mathrm{E}+00 & 0.0000 \mathrm{E}+00 \\ 18 & 18 & 0 & 0 & 0 & 0.0000 \mathrm{E}+00 & 0.0000 \mathrm{E}+00 & 0.0000 \mathrm{E}+00 & 0.0000 \mathrm{E}+00 & 0.0000 \mathrm{E}+00 \\ 19 & 19 & 0 & 0 & 0 & 0.0000 \mathrm{E}+00 & 0.0000 \mathrm{E}+00 & 0.0000 \mathrm{E}+00 & 0.0000 \mathrm{E}+00 & 0.0000 \mathrm{E}+00 \\ & & & & & & & & & & & & \end{array}$

the initial fission neutron source distribution used the 1 source points that were input on the kste card.

the criticality problem was scheduled to skip 2 cycles and run a total of 4 cycles with nominally 250 neutrons per cycle.

this problem has run 2 inactive cycles with 478 neutron histories and 2 active cycles with 462 neutron histories.

this calculation has completed the requested number of keff cycles using a total of 940 fission neutron source histories. all cells with fissionable material were sampled and had fission neutron source points.

thefe is no combined collision/absorption/track-length estimate for keff because only 2 active cycles were nu.

the estimated average keffs, one standard deviations, and 68,95 , and 99 percent confidence intervals are:

\begin{tabular}{|cccccccc} 
keff estimator & keff & standard deviation & $68 \%$ confidence & $95 \%$ confidence & $99 \%$ confidence \\
collision & 0.56837 & 0.01651 & 0.53834 to 0.59841 & 0.35851 to 0.77824 & 0.00000 to 1.61951 \\
absorption & 0.59117 & 0.00738 & 0.57774 to 0.60460 & 0.49735 to 0.68498 & 0.12129 to 1.06105 \\
track length & 0.56888 & 0.01367 & 0.54401 to 0.59374 & 0.39513 to 0.74263 & 0.00000 to 1.43912
\end{tabular}

lindividual and average keff estimator results by cycle

keff neutron keff estimators by cycle average keff estimators and deviations average $k(c / a / t)$

cycle histories $k$ (coll) $k$ (abs) $k$ (track) $k$ (coll) st dev $k(a b s)$ st dev $k$ (track) st dev $k(c / a / t)$ st dev fom

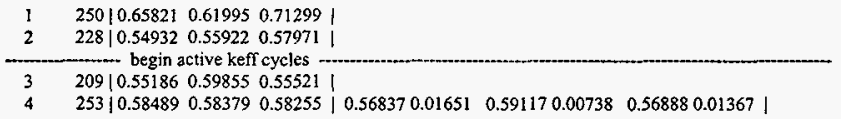

the largest active cycle keffs by estimator are: the smallest active cycle keffs by estimator are:

collision 0.58489 on cycle 4

absorption 0.59855 on cycle 3

track length 0.58255 on cycle 4

1 tally 6 nps $=940$

tally type 6 track length estimate of heating. units mev/gram

tally for neutrons

number of histories used for normalizing tallies $=\quad 500.00$

masses
ll: $\begin{array}{lllll}8 & 12 & 13 & 14 & 15\end{array}$
$1.00000 E+00 \quad 1.00000 E+00 \quad 1.00000 E+00 \quad 1.00000 E+00 \quad 1.00000 E+00$

collision 0.55186 on cycle 3

absorption 0.58379 on cycle 4

track length 0.55521 on cycle 3

corr

\section{$4.02566 E+010.0434$}


ceil 14

9.78180E- 010.4014

cell 15

1.20234E-01 0.9710

tanalysis of the results in the tally fluctuation chart bin (tfc) for tally 6 with nps $=940$ print table 160
normed average tally per history $=4.02566 \mathrm{E}+01$
estimated tally relative error $=0.0434$
relative error from zero tallies $=0.0128$
unnormed average tally per history $=4.02566 \mathrm{E}+01$
estimated variance of the variance $=0.0095$
relative error from nonzero scores $=0.0415$
number of nonzero history tallies $=\quad 462$
history number of largest tally $=\quad 641$
(largest tally)/(average tally) $=6.75049 \mathrm{E}+00$
efficiency for the nonzero tallies $=0.9240$
largest unnormalized history tally $=2.71752 \mathrm{E}+02$
(largest tally) $/$ (avg nonzero tally) $=6.23745 \mathrm{E}+00$
(confidence interval shift) $/$ mean $=0.0014$
shifted confidence interval center $=4.03113 \mathrm{E}+01$

if the largest history score sampled so far were to occur on the very next history, the tfe bin quantities would change as follows: $n p s=462$ for this table because 2 keff cycles and 478 histories were skipped before tally accumulation

estimated quantities value at nps value at nps+1 value(nps+1)/value(nps)-1

$\begin{array}{lccc}\text { mean } & 4.02566 \mathrm{E}+01 & 4.07566 \mathrm{E}+01 & 0.012420 \\ \text { relative error } & 4.34431 \mathrm{E}-02 & 4.24506 \mathrm{E}-02 & -0.022846 \\ \text { variance of the variance } & 9.52674 \mathrm{E}-03 & 1.33327 \mathrm{E}-02 & 0.399508 \\ \text { hifted center } & 4.03113 \mathrm{E}+01 & 4.03457 \mathrm{E}+01 & 0.000853 \\ \text { rgure of merit } & 0.00000 \mathrm{E}+00 & 0.00000 \mathrm{E}+00 & 0.000000\end{array}$

there is not enough information in the largest history scores (usually less than 500 scores) for a reliable estimate of the slope. the history score probability density function appears to have an unsampled region at the largest history scores: please examine.

***** the nps-dependent tfe bin check results are suspect because there are only 1 nps tally values to analyze *****

results of 10 statistical checks for the estimated answer for the tally fluctuation chart (tfc) bin of tally 6

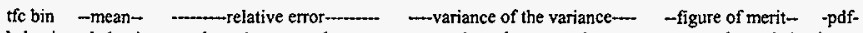

behavior behavior value decrease decrease rate value decrease decrease rate value behavior slope

$\begin{array}{lccccccccc}\text { desired random } & <0.10 & \text { yes } & \text { l/sqrt(nps) } & <0.10 \text { yes } 1 / \mathrm{nps} & \text { constant random } & >3.00 \\ \text { observed random } & 0.04 & \text { yes } & \text { yes } & 0.01 \text { yes yes } & 0.00 & \text { no }\end{array}$

observed random 0.04 yes yes 0.01 yes yes
passed? yes yes yes yes yes yes yes

waming. the tally in the tally fuctuation chan bir did not pass 1 of the 10 statistical checks.

lunnormed tally density for tally $6 \quad$ nonzero tally mean $(\mathrm{m})=4.357 \mathrm{E}+0 \mathrm{l} \mathrm{nps}=940$ print table $16 \mathrm{I}$

abscissa ordinate $\log$ plot of tally probability density function in tally fluctuation chart bin( $d=d e c a d e$, siope $=0.0$ )

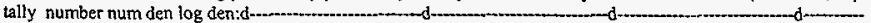

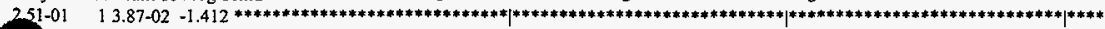

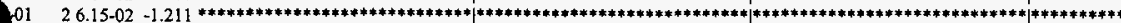

$12.44-02-1.612 * * * * * * * * * * * * * * * * * * * * * * * * * * * * *|* * * * * * * * * * * * * * * * * * * * * * * * * * * * * j ; * * * * * * * * * * * * * * * * * * * * * * * * * * *|$ 


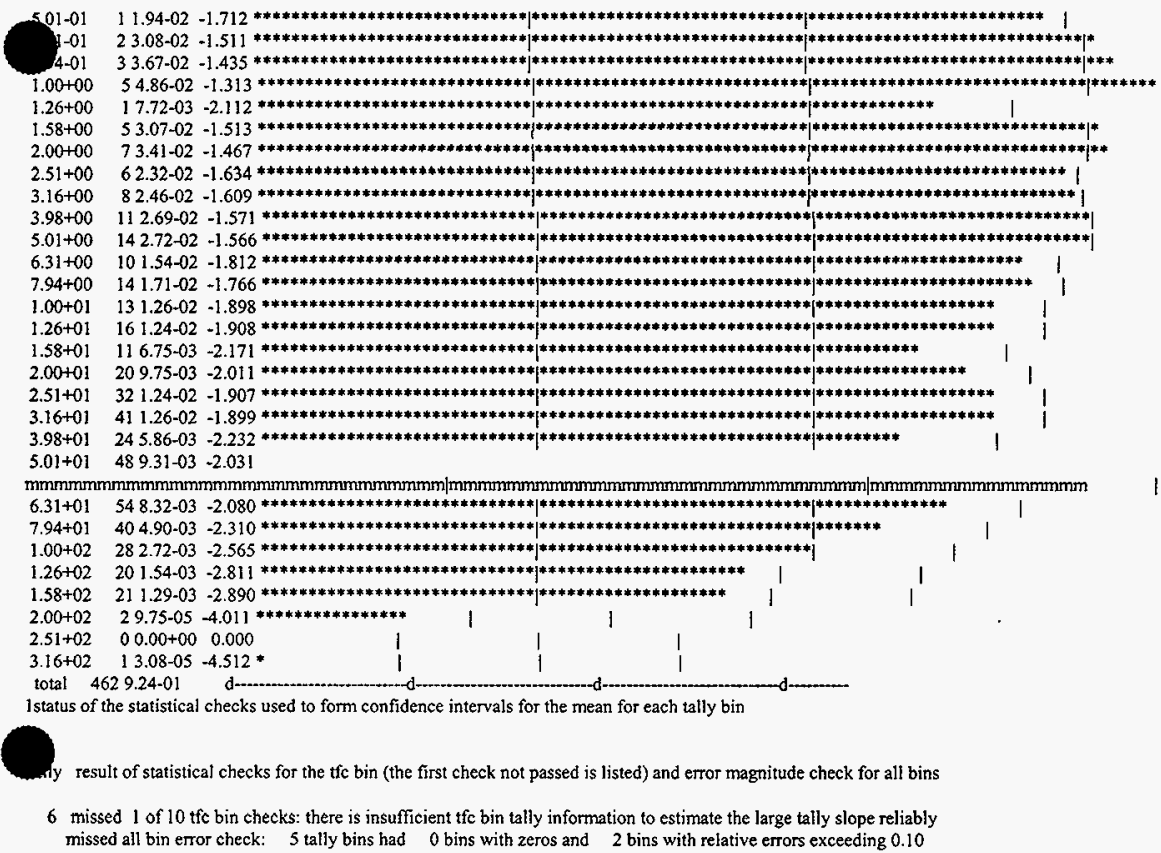

the 10 statistical checks are only for the tally fluctuation chart bin and do not apply to other tally bins.

warning. 1 of the 1 tally fluctuation chart bins did not pass all 10 statistical checks.

waming. 1 of the 1 tallies had bins with relative errors greater than recommended.

Itally fluctuation charts

tally 6

nps mean error vov slope fom

$9404.0257 \mathrm{E}+010.04340 .0095 \quad 0.0$

tally data written to file inp $24 \mathrm{~m}$

15 warning messages so far.

run terminated when 4 kcode cycles were done.

\section{File: Outp24}

1- prob 24 - reflecting lattice. $15 \times 15$ at 3.75 w/o $u-235$ enrichment.

2- $1 \quad 1-10.182-1 \mathrm{u}=2$

3- 2 2-.001 $1-2 \mathrm{u}=2$

4. $3 \quad 3-6.552-3 \quad u=2$

5- 4 4-1.03u=2

6- 5 - $4-1.0-14: 15 u=3$

$6 \quad 3-6.5514-15 u=3$

$7 \quad 4-1.0-4+5-6+7 u=1$ lat $=1$ fill $=-8: 8-8: 80: 0$

$117 \mathrm{r} 214 \mathrm{r} 11214 \mathrm{r} 1122322322 \mathrm{r} 322$ 
$3221126 r 326 r 112353$

$24 \pi 323 r 1122328 \mathrm{r} 322$

$11214 \mathrm{r} 1122 \mathrm{r} 322 \mathrm{r} 322 \mathrm{r}$

$322 \mathrm{r} 11214 \mathrm{r} 1122328 \mathrm{r} 3$

$221123 r 324 r 323 r 11$

$26 \mathrm{r} 326 \mathrm{r} 11223223$

$22 r 32232211214 \pi 11214 \pi 117 \mathrm{r}$

$\begin{array}{lllll}8 & 0 & -8 & -10 & -12 \mathrm{u}=4 \text { fill }=1\end{array}$

$9 \quad 5-7.98: 10 u=4$

19. $10 \quad 4-1.0-8 \quad-10 \quad+12 u=4$

20- $\quad 11 \quad 4-1.0-16+9 \quad u=5$ lat $=1$ fill $=0: 60: 00: 043 \mathrm{r} 52 \mathrm{r}$

21- $12 \quad 0+28+29-19-17+13-18$ fill $=5$

22- $13 \quad 0+28-19+17-31+13-18$ fill $=5(-11.5230)$

23- $140+28-19+31-32+13-18$ fill $=5(-23460)$

24- $15 \quad 0+28-19+32-33+13-18$ fill $=5(-69690)$

25. $16 \quad 4-1.0+28-19+33+13-18$

26- $17 \quad 4-1.0+28+29-19-24+18$

$27-18 \quad 6-7.9(+28+29+19-20+23-25):(+28+29-19+23-13)$ $:(+28+29-19+24-25)$

29- $197-7.088254305(+28+29+20-21+22-25):(+28+29-20+22-23)$

30. $20 \quad 0-28:-29:+21:-22:+25$

31-

32- $\quad 1$ cz .464693

33- 2 cz .483743

$34-\quad 3$ cz .535940

35- 4 px .71501

36- $5 \mathrm{px}-.71501$

37- 6 py .71501

38- 7 py -.71501

39- $8 \mathrm{px} 11.0$

40. $9 \mathrm{px}-11.0$

41. $\quad 10$ py 11.0

$12 \mathrm{pz} 400.903$

$13 \mathrm{pz} 34.0$

14 cz .652018

45- $\quad 15$ cz .690118

46- $16 \mathrm{px} 12.0$

47. $\quad 17$ py 12.0

48- $\quad 18$ pz 439.0

49. $\quad 19$ cz 82.25

50. $\quad 20 \mathrm{cz} 83.25$

51- 21 cz 116.35

52- 22 pz 0.0

53- $\quad 23$ pz 33.0

54- $\quad 24$ pz 447.9

55- $\quad 25 \mathrm{pz} 485.9$

56- $* 28 \mathrm{px} 0.0$

57. $\quad 29$ py 0.0

58- 31 py 35.0

$59-32$ py 58.0

$60-33$ py 81.0

61 -

62- imp:n $118 \mathrm{r} 0$

63- kcode 250.72445000

waming. tallies are nomed per fission neutron for one generation.

64. $\mathrm{ksrc} 1.51 .5217 .4515$

$65-\mathrm{ml} \quad 92235.40 \mathrm{c} 1.31964 \mathrm{e} 20 \quad 92238.40 \mathrm{c} 2.15905 \mathrm{e} 22$

66- $\mathrm{m} 28016.40 \mathrm{c} 1.00000000$

$67-\mathrm{m}^{3} \quad 40000.40 \mathrm{c} \mathrm{l}$.

68- $\mathrm{m} 4 \quad 1001.00 \mathrm{c} .666666667 \quad 8016.40 \mathrm{c} .3333333333$

69. m5 $26000.40 \mathrm{c}-.68874500 \quad 5010 .-.00178200 \quad 5011.40 \mathrm{c}-.00721800$

70- $m 6 \quad 26000.40 c-.69500000$

71- $\quad \begin{array}{lllll}\mathrm{m} 7 & 26000.40 \mathrm{c} .830266962 & 6012.40 \mathrm{c} .133437328 & \text { l4000.40c } .002370386\end{array}$

72- $\mathrm{mt4}$ lwtr.01t

drxs

prdmp $2 \mathrm{j}-1$

f6:n $812131415 \$$ heating in mat $=0$ cells kills menp4.2 
fission nubar data are being used.

warning. continuous-energy cross-section table used for 1001.000

waming. continuous-energy cross-section table used for $5011.40 \mathrm{~d}$

warning. continuous-energy cross-section table used for $6012.40 \mathrm{~d}$

warning. continuous-energy cross-section table used for $8016.40 \mathrm{~d}$

waming. continuous-energy cross-section table used for $14000.40 \mathrm{~d}$

warning. continuousenergy cross-section table used for $26000.40 \mathrm{~d}$

waming. continuous-energy cross-section table used for $40000.40 \mathrm{~d}$

warning. continuous-energy cross-section table used for $92235.40 \mathrm{~d}$

waming. continuous-energy cross-section table used for $92238.40 \mathrm{~d}$

waming. 4 of the materials had unnormalized fractions.

lcells

print table 60

cell mat density density volume mass pieces importance

$1 \quad 1 \quad 1 \quad 2.57597 \mathrm{E}-021.01820 \mathrm{E}+010.00000 \mathrm{E}+000.00000 \mathrm{E}+00$

$223.76497 \mathrm{E}-051.00000 \mathrm{E}-030.00000 \mathrm{E}+000.00000 \mathrm{E}+00$

$01.0000 \mathrm{E}+00$

3 4.32392E-02 $6.55000 \mathrm{E}+000.00000 \mathrm{E}+000.00000 \mathrm{E}+00$

$01.0000 \mathrm{E}+00$

44 4s $1.00309 \mathrm{E}-011.00000 \mathrm{E}+000.00000 \mathrm{E}+000.00000 \mathrm{E}+00$

$01.0000 \mathrm{E}+00$

5 4s $1.00309 \mathrm{E}-01 \quad 1.00000 \mathrm{E}+000.00000 \mathrm{E}+000.00000 \mathrm{E}+00 \quad 0 \quad 1.0000 \mathrm{E}+00$

$6334.32392 \mathrm{E}-026.55000 \mathrm{E}+000.00000 \mathrm{E}+000.00000 \mathrm{E}+00 \quad 0 \quad 1.0000 \mathrm{E}+00$

7 4s $1.00309 \mathrm{E}-01 \quad 1.00000 \mathrm{E}+00 \quad 0.00000 \mathrm{E}+000.00000 \mathrm{E}+00 \quad 0 \quad 1.0000 \mathrm{E}+00$

$8800.00000 \mathrm{E}+000.00000 \mathrm{E}+000.00000 \mathrm{E}+000.00000 \mathrm{E}+00 \quad 0 \quad 1.0000 \mathrm{E}+00$

$9958.97738 \mathrm{E}-027.90000 \mathrm{E}+000.00000 \mathrm{E}+000.00000 \mathrm{E}+00 \quad 0 \quad 1.0000 \mathrm{E}+00$

$10 \quad 10$ 4s $1.00309 \mathrm{E}-011.00000 \mathrm{E}+000.00000 \mathrm{E}+000.00000 \mathrm{E}+00 \quad 0 \quad 1.0000 \mathrm{E}+00$

11 11 4s $1.00309 \mathrm{E}-011.00000 \mathrm{E}+000.00000 \mathrm{E}+000.00000 \mathrm{E}+00 \quad 0 \quad 1.0000 \mathrm{E}+00$

$1212 \quad 0 \quad 0.00000 \mathrm{E}+00 \quad 0.00000 \mathrm{E}+000.00000 \mathrm{E}+000.00000 \mathrm{E}+00 \quad 0 \quad 1.0000 \mathrm{E}+00$

$13 \quad 13 \quad 0 \quad 0.00000 \mathrm{E}+00 \quad 0.00000 \mathrm{E}+00 \quad 0.00000 \mathrm{E}+00 \quad 0.00000 \mathrm{E}+00 \quad 0 \quad 1.0000 \mathrm{E}+00$

$14 \quad 14 \quad 0 \quad 0.00000 \mathrm{E}+000.00000 \mathrm{E}+000.00000 \mathrm{E}+000.00000 \mathrm{E}+00 \quad 0 \quad 1.0000 \mathrm{E}+00$

15 is $0 \quad 0.00000 \mathrm{E}+00 \quad 0.00000 \mathrm{E}+00 \quad 0.00000 \mathrm{E}+00 \quad 0.00000 \mathrm{E}+00 \quad 0 \quad 1.0000 \mathrm{E}+00$

$16 \quad 16 \quad 4 \mathrm{~s} 1.00309 \mathrm{E}-011.00000 \mathrm{E}+000.00000 \mathrm{E}+000.00000 \mathrm{E}+00 \quad 0 \quad 1.0000 \mathrm{E}+00$

$17 \quad 17$ 4s $1.00309 \mathrm{E}-01 \quad 1.00000 \mathrm{E}+000.00000 \mathrm{E}+000.00000 \mathrm{E}+00 \quad 0 \quad 1.0000 \mathrm{E}+00$

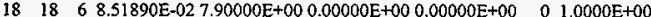

$191978.58502 \mathrm{E}-027.08825 \mathrm{E}+000.00000 \mathrm{E}+000.00000 \mathrm{E}+00 \quad 0 \quad 1.0000 \mathrm{E}+00$

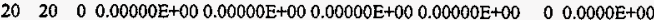

total

\section{$0.00000 \mathrm{E}+000.00000 \mathrm{E}+00$}

Il waming messages so far.

lcross-section tables

print table 100

table length

tables from file testlibl

1001.00c 2139 l-h-1 from endf-vi.

5010.03 d 3945 b-10 endf/b-iv new gamma production format

mat $12505 / 26 / 93$

(1273) $\quad 14$ oct 75

$5011.40 \mathrm{c} 3473$ ENDL library name: nd900719 MCNP translation: 900831 14:10:05

$6012.40 \mathrm{c} 5049$ ENDL library name: nd900719 MCNP translation: 900831 14:10:05

$8016.40 \mathrm{c} 5693$ ENDL library name: nd900719 MCNP translation: 900831 14:10:05

14000.40c 8732 ENDL library name: nd900719 MCNP translation: 900831 14:10:05

$6000.40 \mathrm{C} 21846$ ENDL library name: nd900719 MCNP translation: 900831 14:10:05

$900.40 \mathrm{c} 111279$ ENDL library name: nd900719 MCNP translation: 900831 14:10:05

$235.40 \mathrm{c} 40506$ ENDL library name: nd900719 MCNP translation: 9total nu 10:05

900207

900207

900207

900207

900207

900323

900503 
$92238.40 \mathrm{c} 48310$ ENDL library name: nd901118 MCNP translation: 9total nu 56:49 tr.01t 10193 hydrogen in light water at 300 degrees kelvin

total 261165

waming. neutron energy cutoff is below some cross-section tables.

decimal words of dynamically allocated storage

source distribution written to file inp24s $\quad$ cycle $=0$

12 waming messages so far.

lestimated keff results by cycle

print table 175

cycle $1 \mathrm{k}$ (collision) 0.658210 removal lifetime(abs) $5.1517 \mathrm{E}+03$ source points generated 228

cycle $2 \mathrm{k}$ (collision) 0.549318 removal lifetime(abs) $3.7645 \mathrm{E}+03$ source points generated 209

cycle $3 \mathrm{k}$ (coltision) 0.551862 removal lifetime(abs) $3.8422 \mathrm{E}+03$ source points generated 253

source distribution written to file inp24s cycle $=3$

estimator cycle 4 ave of 2 cycles combination simple average combined average corr

$\begin{array}{llllllllll}\text { k(collision) } & 0.584886 & 0.568374 & 0.0291 & \mathrm{k} \text { (col/abs) } & 0.000000 & 0.0000 & 0.0000000 .0000 & 0.0000\end{array}$

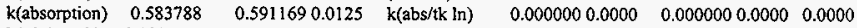

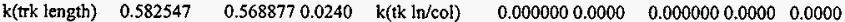

tem life(col) $3.9485 \mathrm{E}+03 \quad 3.8951 \mathrm{E}+03 \quad 0.0137$

rem life(abs) $\quad 4.0658 \mathrm{E}+03 \quad 3.9540 \mathrm{E}+03 \quad 0.0283 \quad \mathrm{life}(\mathrm{col} / \mathrm{abs}) \quad 0.0000 \mathrm{E}+000.0000 \quad 0.0000 \mathrm{E}+000.0000 \quad 0.0000$

anurce points generated 264

fce distribution written to file inp $24 \mathrm{~s} \quad$ cycle $=4$

lproblem summary

0

run terminated when 4 kcode cycles were done.

neutron creation tracks weight energy neutron loss tracks weight energy
(per source particle)
(per source particle)

$\begin{array}{llllllll}\text { source } & 940 & 1.0638 \mathrm{E}+00 & 1.9444 \mathrm{E}+00 & \text { escape } & 0 & 0 . & 0 .\end{array}$

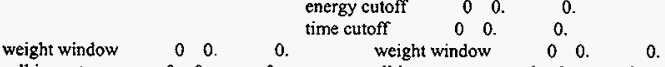

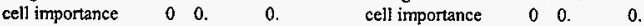

$\begin{array}{lllllllll}\text { weight cutoff } & 0 & 5.6409 \mathrm{E}-02 & 1.1284 \mathrm{E}-06 & \text { weight cutoff } & 944 & 5.2413 \mathrm{E}-02 & 7.1769 \mathrm{E}-07\end{array}$

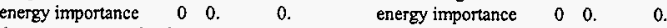

$\begin{array}{llllllllllll}\text { dxtran } & 0 & 0 . & 0 . & & \text { dxtran } & 0 & 0 . & 0 . & \\ \text { forced collisions } & 0 & 0 . & 0 . & \text { forced collisions } & 0 & 0 . & 0 .\end{array}$

$\begin{array}{llllllll}\text { exp. transform } & 0 & 0 . & 0 . & \text { exp. transform } & 0 & 0 . & 0 .\end{array}$

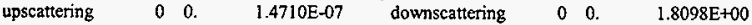

$\begin{array}{rllll} & \text { capture } & 0 & 8.1698 \mathrm{E}-01 & 1.8384 \mathrm{E}-02\end{array}$

$(n, x n) \quad \begin{array}{llllllll}8 & 6.9970 \mathrm{E}-03 & 3.6428 \mathrm{E}-03 & \text { loss to }(n, \mathrm{xn}) & 4 & 3.4985 \mathrm{E}-03 & 2.8025 \mathrm{E}-02\end{array}$

fission $\quad 0$ o. $\quad 0$. loss to fission $0 \begin{array}{ccc}0 & 2.5434 \mathrm{E}-01 & 9.1864 \mathrm{E}-02\end{array}$

$\begin{array}{lllllllll}\text { total } & 948 & 1.1272 \mathrm{E}+00 & 1.9481 \mathrm{E}+00 & \text { total } & 948 & 1.1272 \mathrm{E}+00 & 1.9481 \mathrm{E}+00\end{array}$

number of neutrons banked 4 average lifetime, shakes cutoffs

neutron tracks per source particle $1.0085 \mathrm{E}+00$ escape $0.0000 \mathrm{E}+00$ tco $1.0000 \mathrm{E}+34$

neutron collisions per source particle $6.1389 \mathrm{E}+01$ capture $4.1766 \mathrm{E}+03$ eco $0.0000 \mathrm{E}+00$

total neutron collisions 57706 capture or escape $4.1766 \mathrm{E}+03$ wcl $-5.0000 \mathrm{E}-01$

net multiplication $\quad 1.0033 \mathrm{E}+000.0030$ any termination $4.4650 \mathrm{E}+03$ wc2 $-2.5000 \mathrm{E}-01$

$\begin{array}{ll}\text { moputer time so far in this run } & 0.00 \text { minutes maximum number ever in bank } \\ \text { puter time in merun } & 0.00 \text { minutes bank overflows to backup file }\end{array}$

ce particles per minute $\quad 0.0000 \mathrm{E}+00$

field length 


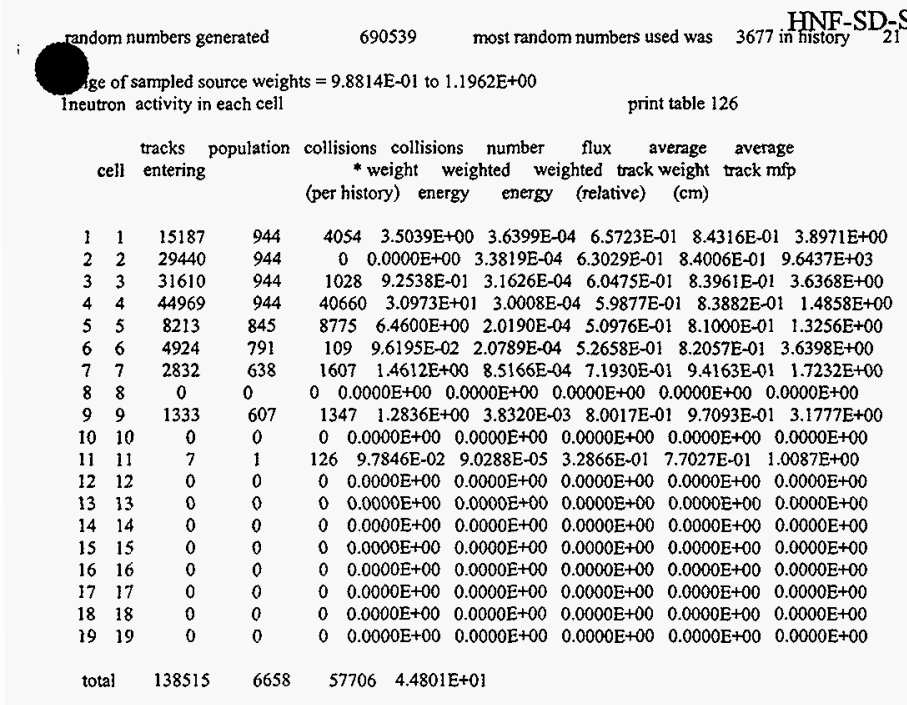

the initial fission neutron source distribution used the 1 source points that were input on the ksrc card.

he criticality problem was scheduled to skip 2 cycles and run a total of 4 cycles with nominally 250 neutrons per cycle problem has run 2 inactive cycles with 478 neutron histories and 2 active cycles with 462 neutron histories.

this calculation has completed the requested number of keff cycles using a total of 940 fission neutron source histories. all cells with fissionable material were sampled and had fission neutron source points.

there is no combined collision/absorption/track-length estimate for keff because only 2 active cycles were nun.

the estimated average keffs, one standard deviations, and 68,95 , and 99 percent confidence intervals are:

keff estimator keff standard deviation $68 \%$ confidence $95 \%$ confidence $99 \%$ confidence com

$\begin{array}{ccccccc}\text { collision } & 0.56837 & 0.01651 & 0.53834 \text { to } 0.59841 & 0.35851 \text { to } 0.77824 & 0.00000 \text { to } 1.61951 \\ \text { absorption } & 0.59117 & 0.00738 & 0.57774 \text { to } 0.60460 & 0.49735 \text { to } 0.68498 & 0.12129 \text { to } 1.06105 \\ \text { track length } & 0.56888 & 0.01367 & 0.54401 \text { to } 0.59374 & 0.39513 \text { to } 0.74263 & 0.00000 \text { to } 1.43912\end{array}$

Individual and average keff estimator results by cycle

keff neutron keff estimators by cycle average keff estimators and deviations average $k(\mathrm{c} / a / t)$ cycle histories $k$ (coll) $k(a b s) k($ track) $k$ (coll) st dev $k$ (abs) st dev $k$ (track) st dev $k(c / a / t) s t$ dev fom

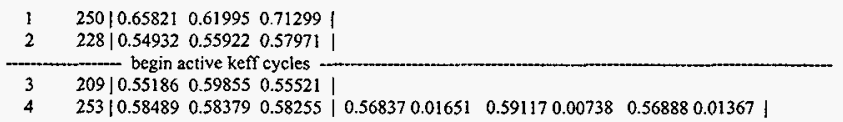

the largest active cycle keffs by estimator are:

collision 0.58489 on cycle 4

absorption 0.59855 on cycle 3

track length 0.58255 on cycle 4 the smallest active cycle keffs by estimator are:

collision 0.55186 on cycle 3

absorption 0.58379 on cycle 4

track length 0.55521 on cycle 3 
1tally 6 nps $=940$

tally type 6 track length estimate of heating. units mev/gram

tally for neutrons

HNF-SD-SNF-CSWD-005, Rev. 0

mer of histories used for normalizing tallies =

500.00

masses

cell: $\begin{array}{llllll}8 & 12 & 13 & 14 & 15\end{array}$

$1.00000 \mathrm{E}+00 \quad 1.00000 \mathrm{E}+00 \quad 1.00000 \mathrm{E}+00 \quad 1.00000 \mathrm{E}+00 \quad 1.00000 \mathrm{E}+00$

cell 8

$4.02566 \mathrm{E}+010.0434$

cell 12

$2.65224 \mathrm{E}+010.0629$

cell 13

$1.32494 \mathrm{E}+010.0904$

cell 14

$9.78180 \mathrm{E}-01 \quad 0.4014$

cell 15

$1.20234 \mathrm{E}-010.9710$

lanalysis of the results in the tally fluctuation chart bin (tfe) for tally 6 with nps $=940$ print table 160

normed average tally per history $=4.02566 \mathrm{E}+01 \quad$ unnormed average tally per history $=4.02566 \mathrm{E}+01$

estimated tally relative enror $=0.0434$

estimated variance of the variance $=0.0095$

relative eтror from zero tallies $=0.0128$

relative error from nonzero scores $=0.0415$

number of nonzero history tallies $=\quad 462$ efficiency for the nonzero tallies $=0.9240$

history number of largest tally $=\quad 641 \quad$ largest unnormalized history tally $=2.71752 \mathrm{E}+02$

(largest tally)/(average tally) $=6.75049 \mathrm{E}+00 \quad$ (largest tally) $/($ avg nonzero tally) $=6.23745 \mathrm{E}+00$

hfidence interval shift $/$ mean $=0.0014$

shifted confidence interval center $=4.03113 \mathrm{E}+01$

if the largest history score sampled so far were to occur on the very next history, the tfe bin quantities would change as follows: $\mathrm{nps}=462$ for this table because 2 keff cycles and 478 histories were skipped before tally accumulation.

estimated quantities value at nps value at nps+1 value(nps+1)/value(nps)-1.

$\begin{array}{lccc}\text { mean } & 4.02566 \mathrm{E}+0 \mathrm{~J} & 4.07566 \mathrm{E}+01 & 0.012420 \\ \text { relative enror } & 4.34431 \mathrm{E}-02 & 4.24506 \mathrm{E}-02 & -0.022846 \\ \text { variance of the variance } & 9.52674 \mathrm{E}-03 & 1.33327 \mathrm{E}-02 & 0.399508 \\ \text { shifted center } & 4.03113 \mathrm{E}+01 & 4.03457 \mathrm{E}+01 & 0.000853 \\ \text { figure of merit } & 0.00000 \mathrm{E}+00 & 0.00000 \mathrm{E}+00 & 0.000000\end{array}$

there is not enough information in the largest history scores (usually less than 500 scores) for a reliable estimate of the slope. the history score probability density function appears to have an unsampled region at the largest history scores: please examine.

***** the nps-dependent tfc bin check results are suspect because there are only 1 nps tally values to analyze *****

results of 10 statistical checks for the estimated answer for the tally fluctuation chart (tfc) bin of tally 6

tfc bin -mean-- - - - - - variance of the variance--- -figure of merit-- $\quad$ pdf-

behavior behavior value decrease decrease rate value decrease decrease rate value behavior slope

\begin{tabular}{|ccccccccc} 
diesired random & $<0.10$ & yes & $1 /$ sqrt(nps) & $<0.10$ & yes & $1 /$ nps & constant random $>3.00$ \\
fved random & 0.04 yes & yes & 0.01 yes yes & yes & 0.00 & no
\end{tabular}


warning. the tally in the tally fluctuation chart bin did not pass 1 of the 10 statistical checks.

I unnormed tally density for tally $6 \quad$ nonzero tally mean $(m)=4.357 \mathrm{E}+01 \mathrm{nps}=940$ print table 161

abscissa ordinate $\log$ plot of tally probability density function in tally fluctuation chart $b i n(d=d e c a d e$, slope $=0.0)$

tally number num den log den:d-_ —

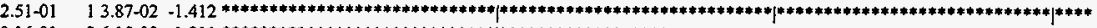

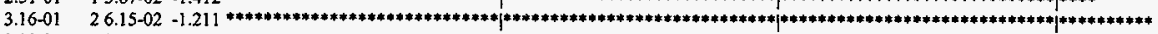

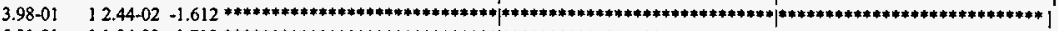

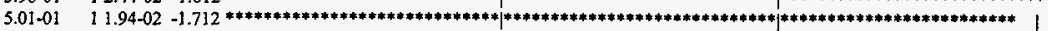

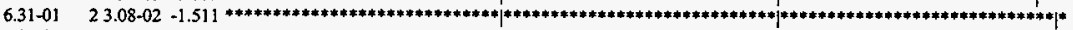

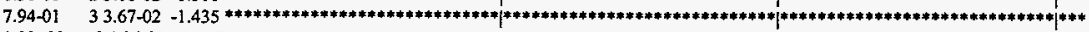

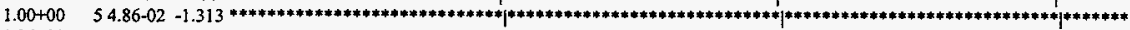

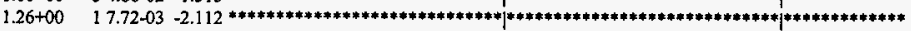

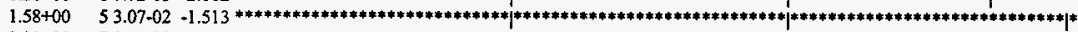

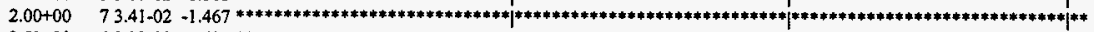

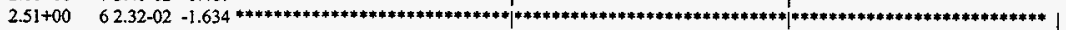

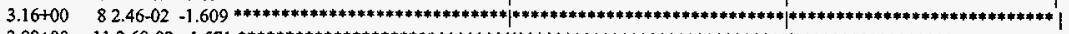

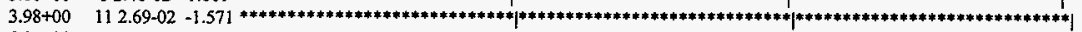

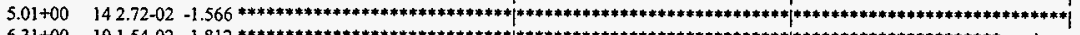

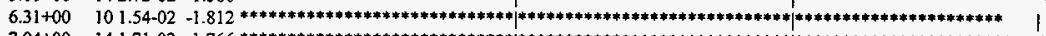

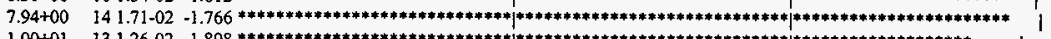

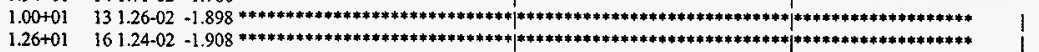

$1.58+03$

$2.00 \div 01$

$2.51+01$

$3.16+01$

$3.98+01$

$5.01+01$

$116.75-03-2.171$

$209.75-03-2.011$

$321.24-02-1.907 * * * * * * * * * * * * * * * * * * * * * * * * * * * * *|* * * * * * * * * * * * * * * * * * * * * * * * * * * * *| * * * * * * * * * * * * * * * * * * *$

$* * * * * * * * * * * * * * * * * * * * * * * * * * * * *$

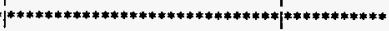

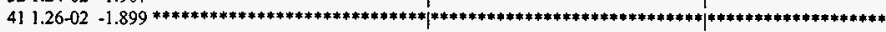

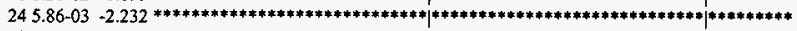

$489.31-03-2.031$

mpmmmmmmmmmmmmmmmmmmmmmmmm|

$1+01548.32-03-2.080 * * * * * * * * * * * * * * * * * * * * * * * * * * * * * * ;|* * * * * * * * * * * * * * * * * * * * * * * * * * * * * *| * * * * * * * * * * * * * * *$

S4+01 $404.90-03-2.310 * * * * * * * * * * * * * * * * * * * * * * * * * * * *|* * * * * * * * * * * * * * * * * * * * * * * * * * * * *| * * * * * * *$

$1.00+02$

$1.26+02$

$1.58+02$

$2.00+02$

$2.51+02$

$3.16+02$

$282.72-03 \quad-2.565 * 4$

$201.54-03-2.811$

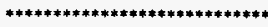

$|* * * * * * * * * * * * * * * * * * * * * * * * * * * * *|$

$211.29-03-2.890 * 4$

$29.75-05-4.011$

$00.00+00 \quad 0.000$

$13.08-05-4.512$ *

total $4629.24-0$

1status of the statistical checks used to form confidence intervals for the mean for each tally bin

tally result of statistical checks for the tfe bin (the first check not passed is listed) and error magnitude check for all bins

6 missed 1 of $10 \mathrm{tfc}$ bin checks: there is insufficient tfc bin tally information to estimate the large tally slope reliably misseô all bin error check: 5 tally bins had 0 bins with zeros and 2 bins with relative errors exceeding 0.10

the 10 statistical checks are only for the tally fluctuation chart bin and do not apply to other tally bins.

warning. 1 of the 1 tally fluctuation chart bins did not pass all 10 statistical checks.

waming. 1 of the 1 tallies had bins with relative erors greater than recommended.

I tally fluctuation charts

tally 6

nps mean etror vov slope fom

$9404.0257 \mathrm{E}+010.04340 .00950 .0$

tally data written to file inp $24 \mathrm{~m}$

15 warning messages so far.

terminated when 4 kcode cycles were done. 


\section{Computer System ID : 501981-801 056AVO000238 (DOE 6246271)}

\section{File: Inp180}

1- prob18 - kcode in a hexagonal prism lattice.

2- c three half control rods and five whole control rods.

3- $\quad 30 \quad 0-905-1929$ ] fill $=$ !

4- $31 \quad 0-906-19291$ fill $=1(16.711300)$

5. $37 \quad 0-907-1929]$ fill $=1(-16.711300)$

6- $34 \quad 0-913-1929$ fill=1 $(011.91850)$

$7-\quad 32 \quad 0-914-1929 \quad$ fill $=1(10.32175 .95920)$

8. $\quad 33 \quad 0-915-1929$ fill $=!(8.3557 \quad 14.47240)$

9. $\quad 35 \quad 0.916-1929$ fill $=1 \quad(-8.3557 \quad 14.47240)$

10. $36 \quad 0-917-1929$ fill $=1(-10.32175 .95920)$

11. c universe 1: structure of control rod.

12- $\quad 38 \quad 11 \quad-2.02 \quad-880 \quad u=1 \quad \$$ control rod core

13- $39 \quad 6-8.4 \quad 880-881 \quad \mathrm{u}=1$ \$ control rod cladding

14. $40 \quad 12-1.00 \quad 881-882 u=1 \quad \$$ control rod gap

15- $\quad 41 \quad 6 \quad-8.4 \quad 882 \quad \mathrm{u}=1 \quad \$$ control rod sheath

16- $c$ the space between the control rods, filled with lattice.

17- $\quad 140 \quad 0 \quad-17129-19905906907913914915916917$ fill $=2$

18- c universe 2: lattice of fuel rods with water in between.

19- $42 \quad 12-1.00 \quad-301 \quad 302-303 \quad 304-305306 \quad u=2$ lat $=2$ fill $=$

20- $\quad-37: 27-1: 330: 0 \&$

21- $\quad 24 r 39 r 24 r 311 r 24 r 311 r 24 r 39 r 2$

22- $\quad 24 r 39 \mathrm{r}^{2} 3 \mathrm{r} 312 \mathrm{r} 23 \mathrm{r} 312 \mathrm{r} 23 \mathrm{r} 39 \mathrm{r} 2 \mathrm{Ir}$

23. $\quad 23 r 310 r 22 r 313 r 22 r 313 r 22 r 310 r 2 \mathrm{lr}$

24- $\quad 23 r 357 r 22 r \&$

25- $\quad 22 \mathrm{r} 358 \mathrm{r} 22 \mathrm{r}$

26- $\quad 22 \mathrm{r} 316 \mathrm{r} 22 \mathrm{r} 317 \mathrm{r} 22 \mathrm{r} 316 \mathrm{r} 2 \mathrm{3r}$

$22 \mathrm{r} 315 \mathrm{r} 23 \mathrm{r} 316 \mathrm{r} 23 \mathrm{r} 315 \mathrm{r} 24 \mathrm{r}$

$2 \operatorname{lr} 315 r 24 r 315 r 24 r 315 r 24 r$

$2 \operatorname{tr} 315 r 23 r 316 r 23 r 315 r 25 r$

2 เा $315 r 2$ 2r $317 r 22 r 315 r 26 r$

2 Ir $354 r 27 r$ \&

c can code remember \& thru comment?

$2355 \mathrm{r} 27 \mathrm{r}$

$2325 r 22 r 325 r 28 r$

$2324 \mathrm{r} 23 \mathrm{r} 324 \mathrm{r} 29 \mathrm{r}$

$2323 \mathrm{r} 24 \mathrm{r} 323 \mathrm{r} 210 \mathrm{r}$

$2315 r 22 r 34 r 23 r 34 r 2$ 2r $315 r 211 \mathrm{rr}$

$2314 r_{2} 3 r_{3} 4 r_{2} 2 r_{3} 4 r_{2} 3 r_{3} 14 r 212 r$

$2313 r 24 r 311 r 24 r 313 r 213 r$

$2313 r 23 r 312 \pi 23 r 313 r 214 r$

$2313 \mathrm{r} 22 \mathrm{r} 313 \mathrm{r} 22 \mathrm{r} 313 \mathrm{r} 215 \mathrm{r}$

$2346 \mathrm{r} 216 \mathrm{r}$

$2345 \mathrm{r} 217 \mathrm{r}$

$2344 \mathrm{r} 218 \mathrm{r}$

2 rr $341 \mathrm{r} 220 \mathrm{r}$

2 ir 340 2 $21 \mathrm{r}$

$2 \operatorname{lr} 339 \mathrm{r} 222 \mathrm{r}$

$22 \mathrm{r} 336 \mathrm{r} 224 \mathrm{r}$

2 2r $335 r 225 r$

$23 r 332 r 227 r$

$24 \mathrm{r} 329 \mathrm{r} 229 \mathrm{r}$

$25 \mathrm{r} 326 \mathrm{r} 231 \mathrm{r}$

$26 \mathrm{r} 323 \mathrm{r} 233 \mathrm{r}$

$28 \mathrm{r} 318 \mathrm{r} 236 \mathrm{r}$

2 11r $311+240 \mathrm{r}$

$264 \mathrm{r}$

c universe 3: structure of fuel rod lattice elements.

$1542-13.75 \quad-58 \quad \mathrm{u}=3 \quad \$$ fuel element

$149 \quad 12 \quad-1.00 \quad 58-268 \quad$ u=3 $\$$ gap

$144 \quad 7.19 .66 \quad 268-478 \quad u=3$ s liner

$\begin{array}{llllll}159 & 6 & -8.4 & 478 & -698 \quad u=3 & \$\end{array}$ cladding

$141 \quad 12-1.00 \quad 698 \quad u=3 \quad \$$ water between the fuel rods 
162 0 17:-29:19:-1 \$ outside world

$* 1$ py $0 \quad \$ x-z$ plane, reflective

17 cz 29.135

$19 \mathrm{pz} 31.75$ \$ top of reactor

68- $\quad 29 \mathrm{pz}-31.75 \quad \$$ bottom of reactor

69- $\quad \begin{array}{llllll}58 & \mathrm{c} / z & 3.4414 & .8515 & .3240\end{array}$

$\begin{array}{llllll}70- & 268 & \mathrm{c} / \mathrm{z} & 3.4414 & .8515 & .3345\end{array}$

$\begin{array}{lllllll}71- & 478 & \mathrm{c} / \mathrm{z} & 3.4414 & .8515 & .3475\end{array}$

$\begin{array}{lllllll}72- & 698 & \mathrm{c} / 2 & 3.4414 & .8515 & .4318\end{array}$

$\begin{array}{llll}73- & 880 & \mathrm{cz} & 1.7251\end{array}$

74- $\quad 881 \quad \mathrm{cz} 1.8051$

75- $\quad 882 \mathrm{cz} \quad 1.9051$

76. 905 cz 2.1055

$77-\quad 906 \mathrm{c} / \mathrm{z} \quad 16.711302 .1055$

78- $\quad 907 \mathrm{c} / 2+16.711302 .1055$

79- $\quad 913 \quad c / z \quad 011.91852 .1055$

80- $\quad 914 \mathrm{ctz} \quad 10.32175 .95922 .1055$

$81-\quad 915 \quad c / z \quad 8.3557 \quad 14.47242 .1055$

82- $\quad 916 \quad c / z-8.3557 \quad 14.47242 .1055$

83- $\quad 917 \quad c / z-10.32175 .95922 .1055$

84- $301 \mathrm{px} 3.9330$

85- $\quad 302$ px 2.9498

86- $\quad 303$ p 11.732050807605 .8994

87- $\quad 304$ p 11.732050807603 .9330

88- $\quad 305$ p $-11.73205080760-.9834$

89- $\quad 306$ p $-11.73205080760-2.9498$

90 -

91- imp:n l $18 \mathrm{r} \quad 0$

92- $\quad \mathrm{m} 2 \quad 92235.40 \mathrm{c} \quad-.70573 \quad 92238.40 \mathrm{c}-.23821 \quad 7014.40 \mathrm{c}-.05605$

93- $\mathrm{m} 6 \quad 41093.40 \mathrm{c}-.99000 \quad 40000.40 \mathrm{c}-.01000$

94- $\mathrm{m} 7 \quad 74000.40 \mathrm{c}-.74000$

$\mathrm{mll} \quad 5010.03 \mathrm{~d}-.6870 \quad 5011.40 \mathrm{c}-.0840 \quad 6012.40 \mathrm{c}-.2290$

$\mathrm{ml2} 1001.00 \mathrm{c} 1 \quad 1002.55 \mathrm{c} 18016.40 \mathrm{cl}$

$\mathrm{mt12}$ hwt.01 lwtr.01

98- kcode 200156

waming. tallies are nomed per fission neutron for one generation.

99- ksrc $3.2 .2 .23 .2-3.2 .2 .23 .243 .2-43.243 .1-43.2$

$100-\mathrm{e} .01 .11 .10$.

101- fq $\mathrm{fe}$

102- fc 4 fuel rod flux in $5 y$ locations averaged over $5 x$ elements

103- $\quad \mathrm{f} 4$ :n $(154<(42[-10:-6-10]))$ \$average $5 \times$ elements at $j=-1$

104- $\quad(154<(42[-10:-630])) \&$ average $5 x$ elements at $j=3$

105- $\quad(154<(42[-10:-6100])) \quad S$ average $5 x$ elements at $j=10$ \&

106- $\quad(154<(42[-10:-6210]))$ \$ average $5 \times$ elements at $j=21$

107: $\quad(154<(42[-10:-6290])) \quad S$ average $5 \times$ elements at $j=29$

108- Sd4 $104.70890624 r \quad S 5$ times the volume of cell 154

109. print -98

110- prdmp $2 \mathrm{j}-1$

111- ptrac buffer $=20$ file $=$ asc write $=$ all event $=$ bnk

1 initial source from ksrc card.

print table 90

original number of points points not in any cell points in cells of zero importance points in void cells points in ambiguous cells total points rejected points remaining points after expansion or contraction nominal source size

initial guess for $k$ (eff.)

1.000000

les to skip before tallying

$$
5
$$



tally type 4 track length estimate of particle flux. tally for neutrons

order of printing: $\mathrm{fe}$

cells $(154<(42[-10:-6-10]))(154<(42[-10:-630]))(154<(42\{-10:-6100]))(154<(42[-10:-6210]))(154<(42[-10:-6$ $290 \mathrm{j})$

energy bins

$0.00000 \mathrm{E}+00$ to $1.00000 \mathrm{E}-02$ mev

$1.00000 \mathrm{E}-02$ to $1.00000 \mathrm{E}-01 \mathrm{mev}$

I. $.00000 \mathrm{E}-01$ to $1.00000 \mathrm{E}+00 \mathrm{mev}$

$1.00000 \mathrm{E}+00$ to $1.00000 \mathrm{E}+01 \mathrm{mev}$

total bin

1 material composition

print table 40

the sum of the fractions of material 2 was $9.999900 \mathrm{E}-0 \mathrm{I}$

the sum of the fractions of material 7 was $7.400000 \mathrm{E}-01$

the sum of the fractions of material 12 was $3.000000 \mathrm{E}+00$

material

number component nuclide, atom fraction

$\begin{array}{lllll}2 & 92235,0.37504 & 92238,0.12499 & 7014,0.49997\end{array}$

$641093,0.9898240000,0.01018$

$7 \quad 74000,1.00000$

$115010,0.71977 \quad 5011,0.08004 \quad 6012,0.20019$

$12 \quad 1001,0.33333 \quad 1002,0.33333 \quad 8016,0.33333$

mossociated thermal $s(a, b)$ data sets: hwtr.01t jwtr.01t

number component nuclide, mass fraction

$\begin{array}{rrrr}2 & 92235,0.70574 & 92238,0.23821 & 7014,0.05605 \\ 6 & 41093,0.99000 & 40000,0.01000 & \\ 7 & 74000,1.00000 & & \\ 11 & 5010,0.68700 & 5011,0.08400 & 6012,0.22900 \\ 12 & 1001,0.05300 & 1002,0.10591 & 8016,0.84109\end{array}$

waming. 3 of the materials had unnormalized fractions.

icell volumes and masses

print table 50

cell atom gram input calculated density density volume volume mass pieces not calculated

$\begin{array}{lllllll}30 & 0.00000 \mathrm{E}+00 & 0.00000 \mathrm{E}+00 & 0.00000 \mathrm{E}+00 & 0.00000 \mathrm{E}+00 & 0.00000 \mathrm{E}+00\end{array}$

2 31 $0.00000 \mathrm{E}+00 \quad 0.00000 \mathrm{E}+00 \quad 0.00000 \mathrm{E}+00 \quad 0.00000 \mathrm{E}+00 \quad 0.00000 \mathrm{E}+00$

$337 \quad 0.00000 \mathrm{E}+00 \quad 0.00000 \mathrm{E}+00 \quad 0.00000 \mathrm{E}+00 \quad 0.00000 \mathrm{E}+00 \quad 0.00000 \mathrm{E}+00$

$\begin{array}{llllllll}4 & 34 & 0.00000 \mathrm{E}+00 & 0.00000 \mathrm{E}+00 & 0.00000 \mathrm{E}+00 & 8.84370 \mathrm{E}+02 & 0.00000 \mathrm{E}+00\end{array}$

$\begin{array}{llllllll}5 & 32 & 0.00000 \mathrm{E}+00 & 0.00000 \mathrm{E}+00 & 0.00000 \mathrm{E}+00 & 8.84370 \mathrm{E}+02 & 0.00000 \mathrm{E}+00\end{array}$

$\begin{array}{llllllll}6 & 33 & 0.00000 \mathrm{E}+00 & 0.00000 \mathrm{E}+00 & 0.00000 \mathrm{E}+00 & 8.84370 \mathrm{E}+02 & 0.00000 \mathrm{E}+00\end{array}$

$\begin{array}{llllllll}7 & 35 & 0.00000 \mathrm{E}+00 & 0.00000 \mathrm{E}+00 & 0.00000 \mathrm{E}+00 & 8.84370 \mathrm{E}+02 & 0.00000 \mathrm{E}+00\end{array}$

$\begin{array}{lllllll}8 & 36 & 0.00000 \mathrm{E}+00 & 0.00000 \mathrm{E}+00 & 0.00000 \mathrm{E}+00 & 8.84370 \mathrm{E}+02 & 0.00000 \mathrm{E}+00\end{array}$

$\begin{array}{llllllll}9 & 38 & 1.15958 \mathrm{E}-01 & 2.02000 \mathrm{E}+00 & 0.00000 \mathrm{E}+00 & 0.00000 \mathrm{E}+00 & 0.00000 \mathrm{E}+00\end{array}$

$\begin{array}{lllllllll}10 & 39 & 5.44575 \mathrm{E}-02 & 8.40000 \mathrm{E}+00 & 0.00000 \mathrm{E}+00 & 0.00000 \mathrm{E}+00 & 0.00000 \mathrm{E}+00\end{array}$

II $40 \quad 9.50007 \mathrm{E}-02 \quad 1.00000 \mathrm{E}+00 \quad 0.00000 \mathrm{E}+00 \quad 0.00000 \mathrm{E}+00 \quad 0.00000 \mathrm{E}+00$

12 41 $5.44575 \mathrm{E}-02 \quad 8.40000 \mathrm{E}+00 \quad 0.00000 \mathrm{E}+00 \quad 0.00000 \mathrm{E}+00 \quad 0.00000 \mathrm{E}+00$

$\begin{array}{lllllll}140 & 0.00000 E+00 & 0.00000 E+00 & 0.00000 E+00 & 0.00000 E+00 & 0.00000 E & +00\end{array}$

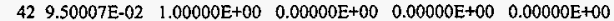

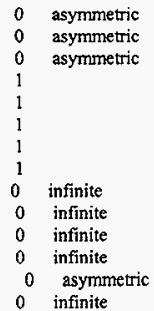


HNF-SD-SNF-CSWD-005, Rev. 0

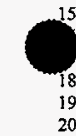

$5 \quad 154 \quad 6.62920 \mathrm{E}-02 \quad 1.37500 \mathrm{E}+01 \quad 0.00000 \mathrm{E}+00 \quad 0.00000 \mathrm{E}+00 \quad 0.00000 \mathrm{E}+00$ $\begin{array}{lllllll}149 & 9.50007 \mathrm{E}-02 & 1.00000 \mathrm{E}+00 & 0.00000 \mathrm{E}+00 & 0.00000 \mathrm{E}+00 & 0.00000 \mathrm{E}+00\end{array}$ $\begin{array}{lllllll}144 & 6.43969 \mathrm{E}-02 & 1.96600 \mathrm{E}+01 & 0.00000 \mathrm{E}+00 & 0.00000 \mathrm{E}+00 & 0.00000 \mathrm{E}+00\end{array}$ $\begin{array}{llllllll}18 & 159 & 5.44575 \mathrm{E}-02 & 8.40000 \mathrm{E}+00 & 0.00000 \mathrm{E}+00 & 0.00000 \mathrm{E}+00 & 0.00000 \mathrm{E}+00\end{array}$ $\begin{array}{llllllll}19 & 141 & 9.50007 \mathrm{E}-02 & 1.00000 \mathrm{E}+00 & 0.00000 \mathrm{E}+00 & 0.00000 \mathrm{E}+00 & 0.00000 \mathrm{E}+00\end{array}$ $\begin{array}{llllllll}20 & 162 & 0.00000 \mathrm{E}+00 & 0.00000 \mathrm{E}+00 & 0.00000 \mathrm{E}+00 & 0.00000 \mathrm{E}+00 & 0.00000 \mathrm{E}+00\end{array}$ isurface areas print table 50

infinite

infinite

infinite

infinite

infinite

0 asymmetric

surface input calculated reason area area area not calculated

$1 \quad 10.00000 \mathrm{E}+00 \quad 0.00000 \mathrm{E}+00$ asymmetric

$2 \quad 17 \quad 0.00000 \mathrm{E}+00 \quad 0.00000 \mathrm{E}+00$ asymmetric

$190.00000 \mathrm{E}+00 \quad 0.00000 \mathrm{E}+00$ asymmetric

$29 \quad 0.00000 \mathrm{E}+00 \quad 0.00000 \mathrm{E}+00$ asymmetric

$\begin{array}{lll}58 & 0.00000 \mathrm{E}+00 \quad 0.00000 \mathrm{E}+00 & \text { infinite }\end{array}$

$2680.00000 \mathrm{E}+00 \quad 0.00000 \mathrm{E}+00$ infinite

$478 \quad 0.00000 \mathrm{E}+00 \quad 0.00000 \mathrm{E}+00$ infinite

$86980.00000 \mathrm{E}+00 \quad 0.00000 \mathrm{E}+00$ infinite

$9880 \quad 0.00000 \mathrm{E}+00 \quad 0.00000 \mathrm{E}+00$ infinite

$10 \quad 881 \quad 0.00000 \mathrm{E}+00 \quad 0.00000 \mathrm{E}+00$ infinite

$11882 \quad 0.00000 \mathrm{E}+00 \quad 0.00000 \mathrm{E}+00$ infinite

$129050.00000 \mathrm{E}+00 \quad 0.00000 \mathrm{E}+00$ asymmetric

$139060.00000 \mathrm{E}+00 \quad 0.00000 \mathrm{E}+00$ asymmetric

$149070.00000 \mathrm{E}+00 \quad 0.00000 \mathrm{E} \div 00$ asymmetric

$\begin{array}{lll}15 & 913 & 0.00000 E+00 \quad 8.40057 E+02\end{array}$

$\begin{array}{lll}16 & 914 & 0.00000 \mathrm{E}+00 \quad 8.40057 \mathrm{E}+02\end{array}$

$17 \quad 915 \quad 0.00000 \mathrm{E}+00 \quad 8.40057 \mathrm{E}+02$

$18916 \quad 0.00000 \mathrm{E}+00 \quad 8.40057 \mathrm{E}+02$

$19917 \quad 0.00000 \mathrm{E}+00 \quad 8.40057 \mathrm{E}+02$

$203010.00000 \mathrm{E}+00 \quad 0.00000 \mathrm{E}+00$ infinite

$21302 \quad 0.00000 \mathrm{E}+00 \quad 0.00000 \mathrm{E}+00$ infinite

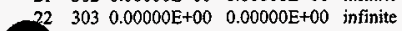

$3040.00000 \mathrm{E}+00 \quad 0.00000 \mathrm{E}+00$

$305 \quad 0.00000 \mathrm{E}+00 \quad 0.00000 \mathrm{E}+00$

$25306 \quad 0.00000 \mathrm{E}+00 \quad 0.00000 \mathrm{E}+00$

leells

print table 60

atom gram neutron

cell mat density density volume mass pieces importance

$130 \quad 0 \quad 0.00000 \mathrm{E}+00 \quad 0.00000 \mathrm{E}+00 \quad 0.00000 \mathrm{E}+00 \quad 0.00000 \mathrm{E}+00$

$231 \quad 0 \quad 0.00000 \mathrm{E}+00 \quad 0.00000 \mathrm{E}+000.00000 \mathrm{E}+000.00000 \mathrm{E}+00$

$33700.00000 \mathrm{E}+00 \quad 0.00000 \mathrm{E}+00 \quad 0.00000 \mathrm{E}+000.00000 \mathrm{E}+00$

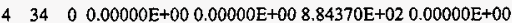

$53200.00000 \mathrm{E}+000.00000 \mathrm{E}+008.84370 \mathrm{E}+020.00000 \mathrm{E}+00$

$63300.00000 \mathrm{E}+00 \quad 0.00000 \mathrm{E}+008.84370 \mathrm{E}+02 \quad 0.00000 \mathrm{E}+00$

$735 \quad 0 \quad 0.00000 \mathrm{E}+00 \quad 0.00000 \mathrm{E}+008.84370 \mathrm{E}+02 \quad 0.00000 \mathrm{E}+00$

$836 \quad 0 \quad 0.00000 \mathrm{E}+00 \quad 0.00000 \mathrm{E}+00 \quad 8.84370 \mathrm{E}+020.00000 \mathrm{E}+00$

$938 \quad 11 \quad 1.15958 \mathrm{E}-012.02000 \mathrm{E}+000.00000 \mathrm{E}+000.00000 \mathrm{E}+00$

$10 \quad 39 \quad 6 \quad 5.44575 \mathrm{E}-02 \quad 8.40000 \mathrm{E}+000.00000 \mathrm{E}+00 \quad 0.00000 \mathrm{E}+00$

$11 \quad 40 \quad 12 s 9.50007 \mathrm{E}-02 \quad 1.00000 \mathrm{E}+000.00000 \mathrm{E}+000.00000 \mathrm{E}+00$

$124165.44575 \mathrm{E}-028.40000 \mathrm{E}+000.00000 \mathrm{E}+000.00000 \mathrm{E}+00$

$13 \quad 140 \quad 0 \quad 0.00000 \mathrm{E}+000.00000 \mathrm{E}+000.00000 \mathrm{E}+00 \quad 0.00000 \mathrm{E}+00$

$14 \quad 42 \quad 12 \mathrm{~s} 9.50007 \mathrm{E}-02 \quad 1.00000 \mathrm{E}+000.00000 \mathrm{E}+000.00000 \mathrm{E}+00$

$15 \quad 15426.62920 \mathrm{E}-021.37500 \mathrm{E}+010.00000 \mathrm{E}+000.00000 \mathrm{E}+00$

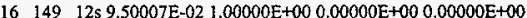

$17 \quad 14476.43969 \mathrm{E}-021.96600 \mathrm{E}+010.00000 \mathrm{E}+000.00000 \mathrm{E}+00$

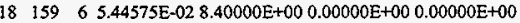

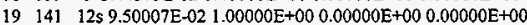

$20 \quad 162 \quad 0 \quad 0.00000 \mathrm{E}+00 \quad 0.00000 \mathrm{E}+000.00000 \mathrm{E}+00 \quad 0.00000 \mathrm{E}+00$

$01.0000 \mathrm{E}+00$

$01.0000 \mathrm{E}+00$

$0 \quad 1.0000 \mathrm{E}+00$

$11.0000 \mathrm{E}+00$

$11.0000 \mathrm{E}+00$

I $1.0000 \mathrm{E}+00$

$11.0000 \mathrm{E}+00$

$11.0000 \mathrm{E}+00$

$01.0000 \mathrm{E}+00$

$0 \quad 1.0000 \mathrm{E}+00$

$01.0000 \mathrm{E}+00$

$0 \quad 1.0000 \mathrm{E}+00$

$01.0000 \mathrm{E}+00$

$01.0000 \mathrm{E}+00$

$01.0000 \mathrm{E}+00$

$01.0000 \mathrm{E}+00$

$0 \quad 1.0000 \mathrm{E}+00$

$0 \quad 1.0000 \mathrm{E}+00$

$01.0000 \mathrm{E}+00$

$0.0000 \mathrm{E}+00$

total 4.42185E $+030.00000 E+00$

Isurfaces

print table 70

urface trans type surface coefficients 
1 cell temperatures in mev for the free-gas thermal neutron treatment.

print table 72

all non-zero importance cells with materials have a temperature for thermal neutrons of $2.5300 \mathrm{E}-08$ mev.

2 warning messages so far Icross-section tables print table 100 table length

tables from file testlib 1

$1001.00 \mathrm{c} \quad 1782 \quad 1$-h-l from endf-vi.l

$1002.55 \mathrm{c} 4102$ njoy mat $125 \quad 05 / 26 / 93$

S011.40c 3229 ENDL library name: nd900719 MCNP translation: 900831 14:10:05

$6012.40 \mathrm{c} 4858$ ENDL library name: nd900719 MCNP translation: 900831 14:10:05

$7014.40 \mathrm{c} 8535$ ENDL library name: nd900719 MCNP translation: 900831 14:10:05

8016.40c 5358 ENDL library name: nd900719 MCNP translation: 900831 14:10:05

40000.40c 93372 ENDL library name: nd900719 MCNP translation: 900831 14:10:05

41093.40c 42746 ENDL library name: nd900719 MCNP translation: 900831 14:10:05

74000.40c 91244 ENDL library name: nd900719 MCNP translation: 900831 14:10:05

92235.40c 34772 ENDL library name: nd900719 MCNP translation: 9total nu 10:05

92238.40c 40834 ENDL library name: nd901118 MCNP translation: 9total nu 56:49

$5010.03 \mathrm{~d} 3682 \mathrm{~b}-10$ endf/b-iv new gamma production format

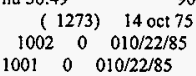

hwtr.01t 10193 deuterium in heavy water at 300 degrees kelvin

900207

900207

900207

900207

900323

900208

900208

900503

901119

total 354900

waming. neutron energy cutoff is below some cross-section tables.

decimal words of dynamically allocated storage

source distribution written to file inpl $8 \mathrm{~s} \quad$ cycle $=0$

3 waming messages so far.

1 starting morun. field length $=0 \quad 0 \quad \mathrm{cp} 0=0.00 \quad$ print table 110

robI8 - kcode in a hexagonal prism lattice. 
HNF-SD-SNF-CSWD-005, Rev. 0

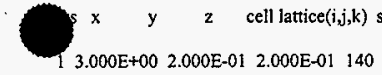
$3.492 \mathrm{E}+00 \quad 1.051 \mathrm{E}+00 \quad 2.000 \mathrm{E}-01 \quad 42(0,-1,0)$ $3.492 \mathrm{E}+00 \quad 1.051 \mathrm{E}+00 \quad 2.000 \mathrm{E}-01 \quad 154$

$23.000 \mathrm{E}+00 \quad 2.000 \mathrm{E}-01 \quad 2.000 \mathrm{E}-01 \quad 140$ $3.492 \mathrm{E}+00 \quad 1.051 \mathrm{E}+00 \quad 2.000 \mathrm{E}-01 \quad 42(0,-1,0)$ $3.492 \mathrm{E}+00 \quad 1.051 \mathrm{E}+00 \quad 2.000 \mathrm{E}-01 \quad 154$ $3 \quad 3.000 \mathrm{E}+00 \quad 2.000 \mathrm{E}-01 \quad 2.000 \mathrm{E}-01 \quad 140$ $3.492 \mathrm{E}+00 \quad 1.051 \mathrm{E}+00 \quad 2.000 \mathrm{E}-01 \quad 42($ $3.492 \mathrm{E}+00 \quad 1.051 \mathrm{E}+00 \quad 2.000 \mathrm{E}-01 \quad 154$ $43.000 \mathrm{E}+00 \quad 2.000 \mathrm{E}-01 \quad 2.000 \mathrm{E}-01140$ $3.492 \mathrm{E}+00 \quad 1.051 \mathrm{E}+00 \quad 2.000 \mathrm{E}-01 \quad 42(0,-1,0)$ $3.492 \mathrm{E}+00 \quad 1.051 \mathrm{E}+00 \quad 2.000 \mathrm{E}-01 \quad 154$

$53.000 \mathrm{E}+00 \quad 2.000 \mathrm{E}-01 \quad 2.000 \mathrm{E}-01 \quad 140$ $3.492 \mathrm{E}+00 \quad 1.051 \mathrm{E}+00 \quad 2.000 \mathrm{E}-01 \quad 42(0,-1,0)$ $3.492 \mathrm{E}+00 \quad 1.051 \mathrm{E}+00 \quad 2.000 \mathrm{E}-01 \quad 154$ $63.000 \mathrm{E}+00 \quad 2.000 \mathrm{E}-01 \quad 2.000 \mathrm{E}-01 \quad 140$ $3.492 \mathrm{E}+00 \quad 1.051 \mathrm{E}+00 \quad 2.000 \mathrm{E}-01 \quad 42(0,-1,0)$ $3.492 \mathrm{E}+00 \quad 1.051 \mathrm{E}+00 \quad 2.000 \mathrm{E}-01 \quad 154$

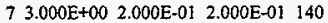
$3.492 \mathrm{E}+00 \quad 1.051 \mathrm{E}+00 \quad 2.000 \mathrm{E}-01 \quad 42(0,-1,0)$ $3.492 \mathrm{E}+00 \quad 1.051 \mathrm{E}+00 \quad 2.000 \mathrm{E}-01 \quad 154$

$83.000 \mathrm{E}+00 \quad 2.000 \mathrm{E}-01 \quad 2.000 \mathrm{E}-01 \quad 140$ $3.492 \mathrm{E}+00 \quad 1.051 \mathrm{E}+00 \quad 2.000 \mathrm{E}-01 \quad 42(0,-1,0)$ $3.492 \mathrm{E}+00 \quad 1.051 \mathrm{E}+00 \quad 2.000 \mathrm{E}-01 \quad 154$ $93.000 \mathrm{E}+00 \quad 2.000 \mathrm{E}-01 \quad 2.000 \mathrm{E}-01 \quad 140$ $3.492 \mathrm{E}+00 \quad 1.051 \mathrm{E}+00 \quad 2.000 \mathrm{E}-01 \quad 42(0,-1,0)$ $3.492 \mathrm{E}+00 \quad 1.051 \mathrm{E}+00 \quad 2.000 \mathrm{E}-01 \quad 154$ $103.000 \mathrm{E}+00 \quad 2.000 \mathrm{E}-01 \quad 2.000 \mathrm{E}-01 \quad 140$ $3.492 \mathrm{E}+00 \quad 1.051 \mathrm{E}+00 \quad 2.000 \mathrm{E}-01 \quad 42(0,-1,0)$ $3.492 \mathrm{E}+00 \quad 1.051 \mathrm{E}+00 \quad 2.000 \mathrm{E}-01 \quad 154$ $3.000 \mathrm{E}+00 \quad 2.000 \mathrm{E}-01 \quad 2.000 \mathrm{E}-01 \quad 140$ $3.492 \mathrm{E}+00 \quad 1.051 \mathrm{E}+00 \quad 2.000 \mathrm{E}-01 \quad 42(0,-1,0)$ $3.492 \mathrm{E}+00 \quad 1.051 \mathrm{E}+00 \quad 2.000 \mathrm{E}-01 \quad 154$ $123.000 \mathrm{E}+00 \quad 2.000 \mathrm{E}-01 \quad 2.000 \mathrm{E}-01 \quad 140$ $3.492 \mathrm{E}+00 \quad 1.051 \mathrm{E}+00 \quad 2.000 \mathrm{E}-01 \quad 42(0,-1,0)$ $3.492 \mathrm{E}+00 \quad 1.051 \mathrm{E}+00 \quad 2.000 \mathrm{E}-01 \quad 154$

$133.000 \mathrm{E}+00 \quad 2.000 \mathrm{E}-012.000 \mathrm{E}-0 \mathrm{I} \quad 140$ $3.492 \mathrm{E}+00 \quad 1.051 \mathrm{E}+00 \quad 2.000 \mathrm{E}-01 \quad 42(0,-1,0)$ $3.492 \mathrm{E}+00 \quad 1.051 \mathrm{E}+00 \quad 2.000 \mathrm{E}-01 \quad 154$

$143.000 \mathrm{E}+00 \quad 2.000 \mathrm{E}-01 \quad 2.000 \mathrm{E}-01 \quad 140$ $3.492 \mathrm{E}+00 \quad 1.051 \mathrm{E}+00 \quad 2.000 \mathrm{E}-01 \quad 42(0,-1,0)$ $3.492 \mathrm{E}+00 \quad 1.051 \mathrm{E}+00 \quad 2.000 \mathrm{E}-01 \quad 154$

$153.000 \mathrm{E}+00 \quad 2.000 \mathrm{E}-012.000 \mathrm{E}-01 \quad 140$ $3.492 \mathrm{E}+00 \quad 1.051 \mathrm{E}+00 \quad 2.000 \mathrm{E}-01 \quad 42(0,-1,0)$ $3.492 \mathrm{E}+00 \quad 1.051 \mathrm{E}+00 \quad 2.000 \mathrm{E}-01 \quad 154$ $16 \quad 3.000 \mathrm{E}+00 \quad 2.000 \mathrm{E}-01 \quad 2.000 \mathrm{E}-01 \quad 140$ $3.492 \mathrm{E}+00 \quad 1.051 \mathrm{E}+00 \quad 2.000 \mathrm{E}-01 \quad 42(0,-1,0)$ $3.492 \mathrm{E}+00 \quad 1.051 \mathrm{E}+00 \quad 2.000 \mathrm{E}-01 \quad 154$

$173.000 \mathrm{E}+00 \quad 2.000 \mathrm{E}-01 \quad 2.000 \mathrm{E}-01 \quad 140$ $3.492 \mathrm{E}+00 \quad 1.051 \mathrm{E}+00 \quad 2.000 \mathrm{E}-01 \quad 42(0,-1,0)$ $3.492 \mathrm{E}+00 \quad 1.051 \mathrm{E}+00 \quad 2.000 \mathrm{E}-01 \quad 154$

$18 \quad 3.000 \mathrm{E}+00 \quad 2.000 \mathrm{E}-01 \quad 2.000 \mathrm{E}-01 \quad 140$ $3.492 \mathrm{E}+00 \quad 1.051 \mathrm{E}+00 \quad 2.000 \mathrm{E}-01 \quad 42(0,-1,0)$ $3.492 \mathrm{E}+00 \quad 1.051 \mathrm{E}+00 \quad 2.000 \mathrm{E}-01 \quad 154$

$193.000 \mathrm{E}+00 \quad 2.000 \mathrm{E}-01 \quad 2.000 \mathrm{E}-01 \quad 140$ $3.492 \mathrm{E}+00 \quad 1.051 \mathrm{E}+00 \quad 2.000 \mathrm{E}-0 \mathrm{I} \quad 42(0,-1,0)$ $3.492 \mathrm{E}+00 \quad 1.051 \mathrm{E}+00 \quad 2.000 \mathrm{E}-01 \quad 154$ $20 \quad 3.000 \mathrm{E}+00 \quad 2.000 \mathrm{E}-01 \quad 2.000 \mathrm{E}-01 \quad 140$ $3.492 \mathrm{E}+00 \quad 1.051 \mathrm{E}+00 \quad 2.000 \mathrm{E}-01 \quad 42(0,-], 0)$ $3.492 \mathrm{E}+00 \quad 1.051 \mathrm{E}+00 \quad 2.000 \mathrm{E}-01 \quad 154$

21 $3.000 \mathrm{E}+00 \quad 2.000 \mathrm{E}-01 \quad 2.000 \mathrm{E}-01 \quad 140$ $\begin{array}{lllll}3.492 \mathrm{E}+00 & 1.051 \mathrm{E}+00 \quad 2.000 \mathrm{E}-01 \quad 42( & 0,-1, & 0\end{array}$ $\begin{array}{lll}3.492 \mathrm{E}+00 & 1.051 \mathrm{E}+00 \quad 2.000 \mathrm{E}-01 \quad 154\end{array}$ $3.000 \mathrm{E}+00 \quad 2.000 \mathrm{E}-01 \quad 2.000 \mathrm{E}-01 \quad 140$ $v \quad w$ energy weight time

$\begin{array}{lllll}5.085 \mathrm{E}-01 & 4.733 \mathrm{E}-01 & 7.193 \mathrm{E}-01 & 2.209 \mathrm{E}+00 \quad 1.000 \mathrm{E}+00 & 0.000 \mathrm{E}+00\end{array}$ $5.085 \mathrm{E}-01 \quad 4.733 \mathrm{E}-01 \quad 7.193 \mathrm{E}-01$

0 5.085E-01 4.733E-01 7.193E-01

$8.952 \mathrm{E}-01-4.447 \mathrm{E}-01 \quad-2.944 \mathrm{E}-02 \quad 4.904 \mathrm{E}+00 \quad 1.000 \mathrm{E}+00 \quad 0.000 \mathrm{E}+00$ $8.952 \mathrm{E}-01-4.447 \mathrm{E}-01-2.944 \mathrm{E}-02$

0 8.952E-01-4.447E-01 -2.944E-02

$-6.184 \mathrm{E}-01-4.49 \mathrm{SE}-0 \mathrm{I} \quad 6.446 \mathrm{E}-01 \quad 3.809 \mathrm{E}-01 \quad 1.000 \mathrm{E}+00 \quad 0.000 \mathrm{E}+00$

$-6.184 \mathrm{E}-01-4.495 \mathrm{E}-01 \quad 6.446 \mathrm{E}-01$

$0-6.184 \mathrm{E}-01-4.495 \mathrm{E}-01 \quad 6.446 \mathrm{E}-01$

$9.710 \mathrm{E}-01 \quad-5.665 \mathrm{E}-02-2.323 \mathrm{E}-01 \quad 1.331 \mathrm{E}+00 \quad 1.000 \mathrm{E}+00 \quad 0.000 \mathrm{E}+00$ 9.710E-01 -5.665E-02 -2.323E-01

0 9.710E-01 -5.665E-02 -2.323E-01

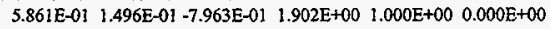
$5.861 \mathrm{E}-01 \quad 1.496 \mathrm{E}-01-7.963 \mathrm{E}-01$

$0 \quad 5.861 \mathrm{E}-01 \quad 1.496 \mathrm{E}-01-7.963 \mathrm{E}-01$

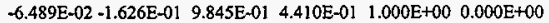
$-6.489 \mathrm{E}-02-1.626 \mathrm{E}-019.845 \mathrm{E}-01$

$0-6.489 \mathrm{E}-02-1.626 \mathrm{E}-01 \quad 9.845 \mathrm{E}-01$

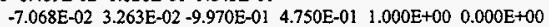

$-7.068 \mathrm{E}-02 \quad 3.263 \mathrm{E}-02-9.970 \mathrm{E}-01$

$0-7.068 \mathrm{E}-02 \quad 3.263 \mathrm{E}-02-9.970 \mathrm{E}-01$

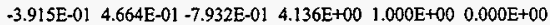

$-3.915 \mathrm{E}-01 \quad 4.664 \mathrm{E}-01-7.932 \mathrm{E}-01$

$0-3.915 \mathrm{E}-01 \quad 4.664 \mathrm{E}-01-7.932 \mathrm{E}-01$

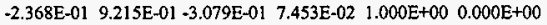

$-2.368 \mathrm{E}-01$ 9.215E-01 -3.079E-01

$0-2.368 \mathrm{E}-01 \quad 9.215 \mathrm{E}-01-3.079 \mathrm{E}-01$

$1.946 \mathrm{E}-01-3.204 \mathrm{E}-01 \quad 9.271 \mathrm{E}-01 \quad 3.128 \mathrm{E}+00 \quad 1.000 \mathrm{E}+00 \quad 0.000 \mathrm{E}+00$ $1.946 \mathrm{E}-01-3.204 \mathrm{E}-01 \quad 9.271 \mathrm{E}-01$

$0 \quad 1.946 \mathrm{E}-01-3.204 \mathrm{E}-01 \quad 9.271 \mathrm{E}-01$

$-6.698 \mathrm{E}-01+7.177 \mathrm{E}-01-1.905 \mathrm{E}-01 \quad 1.014 \mathrm{E}+00 \quad 1.000 \mathrm{E}+00 \quad 0.000 \mathrm{E}+00$

$-6.698 \mathrm{E}-01-7.177 \mathrm{E}-01-1.905 \mathrm{E}-01$

$0-6.698 \mathrm{E}-01-7.177 \mathrm{E}-01-1.905 \mathrm{E}-01$

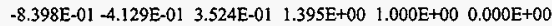
$-8.398 \mathrm{E}-01-4.129 \mathrm{E}-01 \quad 3.524 \mathrm{E}-01$

$0 \cdot 8.398 \mathrm{E}-01-4.129 \mathrm{E}-01 \quad 3.524 \mathrm{E}-01$

$\begin{array}{lllll}-1.714 \mathrm{E}-01 & -8.572 \mathrm{E}-01 & 4.857 \mathrm{E}-01 & 7.748 \mathrm{E}-01 & 1.000 \mathrm{E}+00 \quad 0.000 \mathrm{E}+00\end{array}$

$-1.714 \mathrm{E}-01-8.572 \mathrm{E}-01 \quad 4.857 \mathrm{E}-01$

$0-1.714 \mathrm{E}-01-8.572 \mathrm{E}-01 \quad 4.857 \mathrm{E}-01$

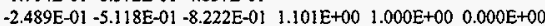

$-2.489 \mathrm{E}-01-5.118 \mathrm{E}-01-8.222 \mathrm{E}-01$

$0-2.489 \mathrm{E}-01-5.118 \mathrm{E}-01-8.222 \mathrm{E}-01$

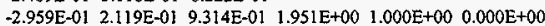
$-2.959 \mathrm{E}-012.119 \mathrm{E}-019.314 \mathrm{E}-01$

$0+2.959 \mathrm{E}-01 \quad 2.119 \mathrm{E}-01 \quad 9.314 \mathrm{E}-01$

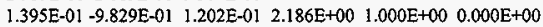
1.395E-01 $-9.829 \mathrm{E}-01 \quad 1.202 \mathrm{E}-01$

$0 \quad 1.395 \mathrm{E}-01-9.829 \mathrm{E}-01 \quad 1.202 \mathrm{E}-01$

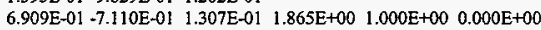
$6.909 \mathrm{E}-01-7.110 \mathrm{E}-01 \quad 1.307 \mathrm{E}-01$

$06.909 \mathrm{E}-01 \cdot 7.110 \mathrm{E}-01 \quad 1.307 \mathrm{E}-01$

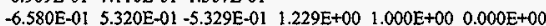
$-6.580 \mathrm{E}-01 \quad 5.320 \mathrm{E}-01-5.329 \mathrm{E}-01$

$0-6.580 \mathrm{E}-01 \quad 5.320 \mathrm{E}-01-5.329 \mathrm{E}-01$

$-9.903 \mathrm{E}-01-1.380 \mathrm{E}-01 \quad 1.353 \mathrm{E}-02 \quad 1.305 \mathrm{E}+00 \quad 1.000 \mathrm{E}+00 \quad 0.000 \mathrm{E}+00$ $-9.903 \mathrm{E}-01-1.380 \mathrm{E}-01 \quad 1.353 \mathrm{E}-02$

$0-9.903 \mathrm{E}-01-1.380 \mathrm{E}-01 \quad 1.353 \mathrm{E}-02$

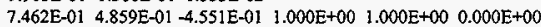
$7.462 \mathrm{E}-014.859 \mathrm{E}-01-4.551 \mathrm{E}-01$

0 7.462E-01 4.859E-01 - $4.551 \mathrm{E}-01$

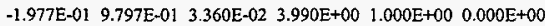
$-1.977 \mathrm{E}-01 \quad 9.797 \mathrm{E}-013.360 \mathrm{E}-02$

$0-1.977 \mathrm{E}-01 \quad 9.797 \mathrm{E}-01 \quad 3.360 \mathrm{E}-02$

$-9.117 \mathrm{E}-01-3.647 \mathrm{E}-01-1.891 \mathrm{E}-01 \quad 2.665 \mathrm{E}-01 \quad 1.000 \mathrm{E}+00 \quad 0.000 \mathrm{E}+00$ 

$3.492 \mathrm{E}+00 \quad 1.051 \mathrm{E}+00 \quad 2.000 \mathrm{E}-01154$ $3.000 E+00 \quad 2.000 E-01 \quad 2.000 E-01 \quad 140$ $3.492 \mathrm{E}+00 \quad 1.051 \mathrm{E}+00 \quad 2.000 \mathrm{E}-01 \quad 42(0,-1,0)$ $3.492 \mathrm{E}+00 \quad 1.051 \mathrm{E}+00 \quad 2.000 \mathrm{E}-01 \quad 154$

$24 \quad 3.000 \mathrm{E}+00 \quad 2.000 \mathrm{E}-01 \quad 2.000 \mathrm{E}-01 \quad 140$ $3.492 \mathrm{E}+00 \quad 1.051 \mathrm{E}+002.000 \mathrm{E}-01 \quad 42(0,-1,0)$ $3.492 \mathrm{E}+00 \quad 1.051 \mathrm{E}+00 \quad 2.000 \mathrm{E}-01154$

$253.000 \mathrm{E}+00 \quad 2.000 \mathrm{E}-01 \quad 2.000 \mathrm{E}-01 \quad 140$ $3.492 \mathrm{E}+001.051 \mathrm{E}+002.000 \mathrm{E}-01 \quad 42(0,-1,0)$ $3.492 \mathrm{E}+00 \quad 1.051 \mathrm{E}+00 \quad 2.000 \mathrm{E}-01 \quad 154$

$26 \quad 2.000 \mathrm{E}-01 \quad 3.000 \mathrm{E}+00 \quad 2.000 \mathrm{E}-01 \quad 140$ $3.641 \mathrm{E}+00$ 4.456E-01 2.000E-01 $42(-5,3,0)$ $3.641 E+00 \quad 4.456 E-01 \quad 2.000 E-01 \quad 141$

27 2.000E-01 3.000E+00 2.000E-01 140 $3.641 \mathrm{E}+00 \quad 4.456 \mathrm{E}-01 \quad 2.000 \mathrm{E}-01 \quad 42(-5,3,0)$ $3.641 \mathrm{E}+00$ 4.456E-01 2.000E-01 141

$28 \quad 2.000 \mathrm{E}-01 \quad 3.000 \mathrm{E}+00 \quad 2.000 \mathrm{E}-01 \quad 140$ $3.641 \mathrm{E}+00 \quad 4.456 \mathrm{E}-01 \quad 2.000 \mathrm{E}-01 \quad 42(-5,3,0)$ $3.641 \mathrm{E}+00 \quad 4.456 \mathrm{E}-01 \quad 2.000 \mathrm{E}-01 \quad 141$

$292.000 \mathrm{E}-01 \quad 3.000 \mathrm{E}+00 \quad 2.000 \mathrm{E}-01 \quad 140$ $3.641 \mathrm{E}+00 \quad 4.456 \mathrm{E}-012.000 \mathrm{E}-01 \quad 42(-5,3,0)$ $3.641 \mathrm{E}+00$ 4.456E-01 2.000E-01 141

$30 \quad 2.000 \mathrm{E}-01 \quad 3.000 \mathrm{E}+00 \quad 2.000 \mathrm{E}-01 \quad 140$ $-9.117 \mathrm{E}-01-3.647 \mathrm{E}-01-1.891 \mathrm{E}-01$ $0-9.117 \mathrm{E}-01-3.647 \mathrm{E}-01-1.891 \mathrm{E}-01$

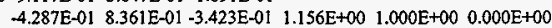
$-4.287 \mathrm{E}-01 \quad 8.361 \mathrm{E}-01-3.423 \mathrm{E}-01$

$0-4.287 E-0 ! 8.361 E-01-3.423 E-01$ $\begin{array}{llllll}1.080 \mathrm{E}-01 & 3.412 \mathrm{E}-01 & -9.338 \mathrm{E}-01 & 2.669 \mathrm{E}+00 & 1.000 \mathrm{E}+00 & 0.000 \mathrm{E}+00\end{array}$ $1.080 \mathrm{E}-01 \quad 3.412 \mathrm{E}-01-9.338 \mathrm{E}-01$

0 1.080E-01 3.412E-01 -9.338E-01 $-9.111 \mathrm{E}-01$-9.012E-03 -4.122E $-0 \mathrm{I} \quad 2.185 \mathrm{E}+00 \quad 1.000 \mathrm{E}+00 \quad 0.000 \mathrm{E}+00$ $-9.111 \mathrm{E}-01-9.012 \mathrm{E}-03-4.122 \mathrm{E}-01$

$0-9.111 \mathrm{E}-01-9.012 \mathrm{E}-03-4.122 \mathrm{E}-01$ $-2.568 \mathrm{E}-01-6.391 \mathrm{E}-01-7.249 \mathrm{E}-01 \quad 7.280 \mathrm{E}-01 \quad 1.000 \mathrm{E}+00 \quad 0.000 \mathrm{E}+00$ $-2.568 \mathrm{E}-01-6.391 \mathrm{E}-01-7.249 \mathrm{E}-01$

$0-2.568 \mathrm{E}-01-6.391 \mathrm{E}-01-7.249 \mathrm{E}-01$ $\begin{array}{llllll}-2.912 \mathrm{E}-01 & 8.086 \mathrm{E}-01 & 5.113 \mathrm{E}-01 & 1.404 \mathrm{E}+00 & 1.000 \mathrm{E}+00 \quad 0.000 \mathrm{E}+00\end{array}$ $-2.912 \mathrm{E}-01 \quad 8.086 \mathrm{E}-01 \quad 5.113 \mathrm{E}-01$

$0-2.912 \mathrm{E}-018.086 \mathrm{E}-01 \quad 5.113 \mathrm{E}-01$

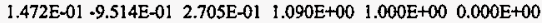
$1.472 \mathrm{E}-01-9.514 \mathrm{E}-01 \quad 2.705 \mathrm{E}-01$

$0 \quad 1.472 \mathrm{E}-01-9.514 \mathrm{E}-012.705 \mathrm{E}-01$ $-6.135 \mathrm{E}-01-7.645 \mathrm{E}-01-1.978 \mathrm{E}-01 \quad 2.483 \mathrm{E}+00 \quad 1.000 \mathrm{E}+00 \quad 0.000 \mathrm{E}+00$ $-6.135 \mathrm{E}-01-7.645 \mathrm{E}-01-1.978 \mathrm{E}-01$

$0-6.135 \mathrm{E}-01-7.645 \mathrm{E}-01-1.978 \mathrm{E}-01$

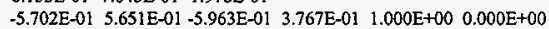

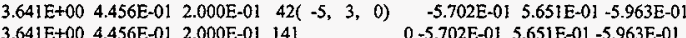

$312.000 \mathrm{E}-01 \quad 3.000 \mathrm{E}+00 \quad 2.000 \mathrm{E}-01 \quad 140$ $3.641 \mathrm{E}+00 \quad 4.456 \mathrm{E}-01$ 2.000E-01 $42(-5,3,0)$ $3.641 \mathrm{E}+00 \quad 4.456 \mathrm{E}-012.000 \mathrm{E}-01 \quad 141$

32 2.000E-01 3.000E+00 2.000E-01 140 $3.641 E+00 \quad 4.456 E-01 \quad 2.000 E-01 \quad 42(-5,3,0)$ $3.641 \mathrm{E}+00 \quad 4.456 \mathrm{E}-012.000 \mathrm{E}-01 \quad 141$ 2.000E-01 3.000E+00 2.000E-01 140 $.641 \mathrm{E}+00 \quad 4.456 \mathrm{E}-01 \quad 2.000 \mathrm{E}-01 \quad 42(-5,3,0)$ $.641 \mathrm{E}+00 \quad 4.456 \mathrm{E}-01 \quad 2.000 \mathrm{E}-01 \quad 141$

$342.000 \mathrm{E}-01 \quad 3.000 \mathrm{E}+00 \quad 2.000 \mathrm{E}-01140$ $3.641 \mathrm{E}+00$ 4.456E-01 2.000E-01 $42(-5,3,0)$ $3.641 \mathrm{E}+00 \quad 4.456 \mathrm{E}-012.000 \mathrm{E}-01 \quad 141$

$35 \quad 2.000 \mathrm{E}-01 \quad 3.000 \mathrm{E}+00 \quad 2.000 \mathrm{E}-01 \quad 140$

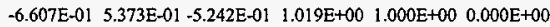
$-6.607 \mathrm{E}-01 \quad 5.373 \mathrm{E}-01-5.242 \mathrm{E}-01$

$0-6.607 \mathrm{E}-01$ 5.373E-01 - $5.242 \mathrm{E}-01$ $-9.742 \mathrm{E}-02-3.639 \mathrm{E}-01-9.263 \mathrm{E}-01 \quad 1.879 \mathrm{E}+00 \quad 1.000 \mathrm{E}+00 \quad 0.000 \mathrm{E}+00$ $-9.742 \mathrm{E}-02+3.639 \mathrm{E}-01-9.263 \mathrm{E}-01$

$0-9.742 \mathrm{E}-02-3.639 \mathrm{E}-01-9.263 \mathrm{E}-01$ $-1.965 \mathrm{E}-01-3.145 \mathrm{E}-01-9.287 \mathrm{E}-01 \quad 4.700 \mathrm{E}-01 \quad 1.000 \mathrm{E}+00 \quad 0.000 \mathrm{E}+00$ $-1.965 \mathrm{E}-01-3.145 \mathrm{E}-01-9.287 \mathrm{E}-01$

$0-1.965 \mathrm{E}-01-3.145 \mathrm{E}-01-9.287 \mathrm{E}-01$ $4.097 \mathrm{E}-01 \quad 8.465 \mathrm{E}-01 \quad-3.399 \mathrm{E}-01 \quad 1.530 \mathrm{E}+00 \quad 1.000 \mathrm{E}+00 \quad 0.000 \mathrm{E}+00$ 4.097E-01 8.465E-01 -3.399E-01

0 4.097E-01 8.465E-01 -3.399E-01

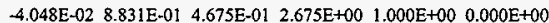
$3.641 \mathrm{E}+00 \quad 4.456 \mathrm{E}-01 \quad 2.000 \mathrm{E}-01 \quad 42(-5,3,0) \quad-4.048 \mathrm{E}-02 \quad 8.831 \mathrm{E}-01 \quad 4.675 \mathrm{E}-01$ $3.641 \mathrm{E}+00 \quad 4.456 \mathrm{E}-012.000 \mathrm{E}-01 \quad 141$ 36 2.000E-01 3.000E+00 2.000E-01 140 $3.641 \mathrm{E}+00 \quad 4.456 \mathrm{E}-01 \quad 2.000 \mathrm{E}-01 \quad 42(-5,3,0)$ $3.641 \mathrm{E}+00 \quad 4.456 \mathrm{E}-01 \quad 2,000 \mathrm{E}-01 \quad 141$

37 2.000E-01 3.000E+00 2.000E-01 140 $3.641 \mathrm{E}+00 \quad 4.456 \mathrm{E}-01 \quad 2.000 \mathrm{E}-01 \quad 42(-5,3,0)$ $3.641 \mathrm{E}+00 \quad 4.456 \mathrm{E}-01 \quad 2.000 \mathrm{E}-01 \quad 141$

38 2.000E-01 $3.000 \mathrm{E}+00 \quad 2.000 \mathrm{E}-01 \quad 140$ $3.641 \mathrm{E}+00 \quad 4.456 \mathrm{E}-01 \quad 2.000 \mathrm{E}-01 \quad 42(-5,3,0)$ $3.641 \mathrm{E}+00 \quad 4.456 \mathrm{E}-0] 2.000 \mathrm{E}-01 \quad 141$

$39 \quad 2.000 \mathrm{E}-01 \quad 3.000 \mathrm{E}+00 \quad 2.000 \mathrm{E}-01 \quad 140$ $3.641 \mathrm{E}+00 \quad 4.456 \mathrm{E}-01 \quad 2.000 \mathrm{E}-01 \quad 42(-5,3,0)$ $3.641 \mathrm{E}+00 \quad 4.456 \mathrm{E}-013.000 \mathrm{E}-01 \quad 141$

$40 \quad 2.000 \mathrm{E}-01 \quad 3.000 \mathrm{E}+00 \quad 2.000 \mathrm{E}-0 \mathrm{I} \quad 140$ 3.64]E+00 4.456E-01 2.000E-01 42( $-5,3,0)$ $3.641 \mathrm{E}+00 \quad 4.456 \mathrm{E}-01 \quad 2.000 \mathrm{E}-01 \quad 141$ $41 \quad 2.000 \mathrm{E}-01 \quad 3.000 \mathrm{E}+00 \quad 2.000 \mathrm{E}-01 \quad 140$ $3.641 \mathrm{E}+00 \quad 4.456 \mathrm{E}-01 \quad 2.000 \mathrm{E}-01 \quad 42(-5,3,0)$ $3.641 \mathrm{E}+00 \quad 4.456 \mathrm{E}-01 \quad 2.000 \mathrm{E}-01 \quad 141$ 42 2.000E-01 $3.000 \mathrm{E}+00 \quad 2.000 \mathrm{E}-01 \quad 140$ $3.641 \mathrm{E}+00 \quad 4.456 \mathrm{E}-01 \quad 2.000 \mathrm{E}-01 \quad 42(-5,3,0)$ $3.641 \mathrm{E}+00 \quad 4.456 \mathrm{E}-01$ 2.000E-01 141 43 2.000E-0! $3.000 \mathrm{E}+00 \quad 2.000 \mathrm{E}-01 \quad 140$ $3.641 \mathrm{E}+00 \quad 4.456 \mathrm{E}-01 \quad 2.000 \mathrm{E}-01 \quad 42(-5,3,0)$ $3.641 \mathrm{E}+00 \quad 4.456 \mathrm{E}-01 \quad 2.000 \mathrm{E}-01 \quad 141$ 2.000E-01 3.000E+00 2.000E-01 140 -4.048E-02 8.831E-01 4.675E-01 $3.371 \mathrm{E}-01-9.269 \mathrm{E}-01-1.652 \mathrm{E}-01 \quad 9.222 \mathrm{E}-01 \quad 1.000 \mathrm{E}+00 \quad 0.000 \mathrm{E}+00$ $3.371 \mathrm{E}-01-9.269 \mathrm{E}-01-1.652 \mathrm{E}-01$ 0 3.37! E-01 -9.269E-01 -1.652E-01 $\begin{array}{lllll}-1.867 \mathrm{E}-01 & 9.756 \mathrm{E}-01-1.155 \mathrm{E}-01 & 9.702 \mathrm{E}-01 & 1.000 \mathrm{E}+00 & 0.000 \mathrm{E}+00\end{array}$ $-1.867 \mathrm{E}-01 \quad 9.756 \mathrm{E}-01-1.155 \mathrm{E}-01$

$0-1.867 \mathrm{E}-019.756 \mathrm{E}-01-1.155 \mathrm{E}-01$ $\begin{array}{lllll}-2.616 \mathrm{E}-01 & 2.336 \mathrm{E}-01 & -9.365 \mathrm{E}-01 & 1.230 \mathrm{E}+00 \quad 1.000 \mathrm{E}+00 \quad 0.000 \mathrm{E}+00\end{array}$ $-2.616 \mathrm{E}-01 \quad 2.336 \mathrm{E}-01-9.365 \mathrm{E}-01$

$0-2.616 \mathrm{E}-01 \quad 2.336 \mathrm{E}-0 \mathrm{I}-9.365 \mathrm{E}-01$ $9.780 \mathrm{E}-01-7.641 \mathrm{E}-02-1.939 \mathrm{E}-0 ! \quad 1.083 \mathrm{E}+00 \quad 1.000 \mathrm{E}+00 \quad 0.000 \mathrm{E}+00$ $9.780 \mathrm{E}-01-7.641 \mathrm{E}-02-1.939 \mathrm{E}-01$ $0 \quad 9.780 \mathrm{E}-01-7.641 \mathrm{E}-02-1.939 \mathrm{E}-01$ $2.580 \mathrm{E}-01-7.076 \mathrm{E}-01 \quad 6.578 \mathrm{E}-01 \quad 8.313 \mathrm{E}-01 \quad 1.000 \mathrm{E}+00 \quad 0.000 \mathrm{E}+00$ 2.580E-01 -7.076E-01 6.578E-01

0 2.580E-01 -7.076E-01 6.578E-01

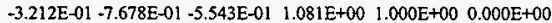
$-3.212 \mathrm{E}-01-7.678 \mathrm{E}-01-5.543 \mathrm{E}-01$

$0-3.212 \mathrm{E}-01-7.678 \mathrm{E}-01-5.543 \mathrm{E}-01$ $5.039 \mathrm{E}-01-1.460 \mathrm{E}-01 \quad 8.513 \mathrm{E}-01 \quad 3.341 \mathrm{E}+00 \quad 1.000 \mathrm{E}+00 \quad 0.000 \mathrm{E}+00$ $5.039 \mathrm{E}-01-1.460 \mathrm{E}-01 \quad 8.513 \mathrm{E}-01$

$0 \quad 5.039 \mathrm{E}-01-1.460 \mathrm{E}-01 \quad 8.513 \mathrm{E}-01$

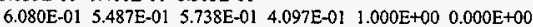
$6.080 \mathrm{E}-01 \quad 5.487 \mathrm{E}-01 \quad 5.738 \mathrm{E}-01$

$0 \quad 6.080 \mathrm{E}-01 \quad 5.487 \mathrm{E}-01 \quad 5.738 \mathrm{E}-01$ $-2.932 \mathrm{E}-01 \quad 9.304 \mathrm{E}-01-2.199 \mathrm{E}-01 \quad 2.229 \mathrm{E}+00 \quad 1.000 \mathrm{E}+00 \quad 0.000 \mathrm{E}+00$ $-2.932 \mathrm{E}-01 \quad 9.304 \mathrm{E}-01-2.199 \mathrm{E}-01$ 


\section{HNF-SD-SNF-CSWD-005, Rev. 0}

$3.641 \mathrm{E}+00 \quad 4.456 \mathrm{E}-01 \quad 2.000 \mathrm{E}-01 \quad 141$

$2.000 \mathrm{E}-01 \quad 3.000 \mathrm{E}+00 \quad 2.000 \mathrm{E}-01 \quad 140$

$6.641 \mathrm{E}+00 \quad 4.456 \mathrm{E}-01 \quad 2.000 \mathrm{E}-01 \quad 42(-5,3,0)$

$3.641 \mathrm{E}+00 \quad 4.456 \mathrm{E}-01 \quad 2.000 \mathrm{E}-01 \quad 141$

$462.000 \mathrm{E}-01 \quad 3.000 \mathrm{E}+00 \quad 2.000 \mathrm{E}-01 \quad 140$

$3.641 \mathrm{E}+00 \quad 4.456 \mathrm{E}-01 \quad 2.000 \mathrm{E}-01 \quad 42(-5,3,0)$

$3.641 \mathrm{E}+00 \quad 4.456 \mathrm{E}-01 \quad 2.000 \mathrm{E}-01141$

$47 \quad 2.000 \mathrm{E}-01 \quad 3.000 \mathrm{E}+00 \quad 2.000 \mathrm{E}+01 \quad 140$

$3.641 \mathrm{E}+00 \quad 4.456 \mathrm{E}-01 \quad 2.000 \mathrm{E}-01 \quad 42(-5,3,0)$

$3.641 \mathrm{E}+00 \quad 4.456 \mathrm{E}-01 \quad 2.000 \mathrm{E}-01 \quad 141$

$48 \quad 2.000 \mathrm{E}-01 \quad 3.000 \mathrm{E}+00 \quad 2.000 \mathrm{E}-01 \quad 140$

$3.641 \mathrm{E}+00 \quad 4.456 \mathrm{E}-01 \quad 2.000 \mathrm{E}-01 \quad 42(-5,3,0)$

$3.641 \mathrm{E}+00 \quad 4.456 \mathrm{E}-01 \quad 2.000 \mathrm{E}-01 \quad 141$

$492.000 \mathrm{E}-013.000 \mathrm{E}+00 \quad 2.000 \mathrm{E}-01 \quad 140$

$3.641 \mathrm{E}+00 \quad 4.456 \mathrm{E}-01 \quad 2.000 \mathrm{E}-01 \quad 42(-5,3,0)$

$3.641 \mathrm{E}+00 \quad 4.456 \mathrm{E}-01 \quad 2.000 \mathrm{E}-01 \quad 141$

50 2.000E-01 3.000E+00 2.000E-01 140

$3.641 \mathrm{E}+00 \quad 4.456 \mathrm{E}-01 \quad 2.000 \mathrm{E}-01 \quad 42(-5,3,0)$

$3.641 \mathrm{E}+00 \quad 4.456 \mathrm{E}-01 \quad 2.000 \mathrm{E}-01 \quad 141$

lestimated keff results by cycle
$0-2.932 \mathrm{E}-01 \quad 9.304 \mathrm{E}-01-2.199 \mathrm{E}-01$

$-8.475 \mathrm{E}-01-3.993 \mathrm{E}-01-3.497 \mathrm{E}-01 \quad 1.362 \mathrm{E}+00 \quad 1.000 \mathrm{E}+00 \quad 0.000 \mathrm{E}+00$

$-8.475 \mathrm{E}-01-3.993 \mathrm{E}-01-3.497 \mathrm{E}-01$

$0-8.475 \mathrm{E}-01-3.993 \mathrm{E}-0 \mathrm{I}-3.497 \mathrm{E}-01$

$1.200 \mathrm{E}-01-9.195 \mathrm{E}-01-3.743 \mathrm{E}-01 \quad 1.010 \mathrm{E}+00 \quad 1.000 \mathrm{E}+00 \quad 0.000 \mathrm{E}+00$

$1.200 \mathrm{E}-01-9.195 \mathrm{E}-01-3.743 \mathrm{E}-01$

0 1.200E-01 -9.195E-01 -3.743E-01

$7.085 \mathrm{E}-01 \quad 5.879 \mathrm{E}-01 \quad 3.904 \mathrm{E}-01 \quad 7.068 \mathrm{E}+00 \quad 1.000 \mathrm{E}+00 \quad 0.000 \mathrm{E}+00$

7.085E-01 5.879E-01 3.904E-01

$0 \quad 7.085 \mathrm{E}-01 \quad 5.879 \mathrm{E}-01 \quad 3.904 \mathrm{E}-01$

$\begin{array}{llllll}4.261 \mathrm{E}-01 & 9.046 \mathrm{E}-01 & 9.254 \mathrm{E}-03 & 3.122 \mathrm{E}-01 & 1.000 \mathrm{E}+00 & 0.000 \mathrm{E}+00\end{array}$

4.261E-01 9.046E-01 9.254E-03

0 4.261E-01 9.046E-01 9.254E-03

$5.431 \mathrm{E}-01 \quad 4.270 \mathrm{E}-01-7.230 \mathrm{E}-01 \quad 5.484 \mathrm{E}-01 \quad 1.000 \mathrm{E}+00 \quad 0.000 \mathrm{E}+00$

$5.431 \mathrm{E}-01 \quad 4.270 \mathrm{E}-01-7.230 \mathrm{E}-01$

0 5.431E-01 4.270E-01 -7.230E-01

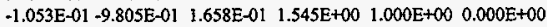

$-1.053 \mathrm{E}-01-9.805 \mathrm{E}-01$ 1.658E-01

$0-1.053 \mathrm{E}-01-9.805 \mathrm{E}-01 \quad 1.658 \mathrm{E}-01$

print table 175

cycle I k(collision) 1.253108 removal lifetime(abs) $1.7557 \mathrm{E}+0 \mathrm{l}$ source points generated 257

cycle $2 \mathrm{k}$ (collision) 1.211997 removal lifetime(abs) $1.1671 \mathrm{E}+0 \mathrm{l}$ source points generated 195

cycle $3 \mathrm{k}$ (collision) 1.200265 removal lifetime(abs) $9.6992 \mathrm{E}+00$ source points generated 193

cycle $4 \mathrm{k}$ (collision) 1.129530 removal lifetime(abs) $9.3720 \mathrm{E}+00$ source points generated 182

cycle $5 \mathrm{k}$ (collision) 1.135994 removal lifetime(abs) $1.1136 \mathrm{E}+01$ source points generated 222

cycle $6 \mathrm{k}$ (collision) 1.077014 removal lifetime(abs) 1.2222E+01 source points generated 204

rce distribution written to file inp $18 \mathrm{~s} \quad$ cycle $=6$

blem summary

0

nu terminated when 6 kcode cycles were done.

neutroncreation tracks weight energy neutron loss tracks weight energy (per source particle) (per source particle)

source

$1249 \quad 9.6077 \mathrm{E}-01 \quad 1.8661 \mathrm{E}+00$

energy cutoff $\begin{gathered}\text { escape } \\ 0\end{gathered}$

time cutoff $\quad 0 \quad 0.0$.

$\begin{array}{lllllllll}\text { weight window } & 0 & 0 . & 0 . & \text { weight window } & 0 & 0 . & 0 .\end{array}$

cell importarce $\quad 0 \quad 0 . \quad 0 . \quad$ cell importance $\quad 0.0 .0$.

$\begin{array}{lllllllll}\text { weight cutoff } & 0 & 1.3743 \mathrm{E}-01 & 3.8498 \mathrm{E}-03 & \text { weight cutoff } & 1034 & 1.2791 \mathrm{E}-01 & 5.1920 \mathrm{E}-03\end{array}$

energy importance $\quad 0 \quad 0 . \quad 0 . \quad 0 \quad$ energy importance $\begin{array}{llllll}0 & 0 . & 0 .\end{array}$

$\begin{array}{llllllllllll}\text { oxtran } & 0 & 0 . & 0 . & & \text { dxtran } & 0 & 0 . & 0 . & \\ \text { forced collisions } & 0 & 0 . & 0 . & \text { forced collisions } & 0 & 0 . & 0 .\end{array}$

exp. transform $\quad \begin{array}{lllllll}0 & 0 . & 0 . & \text { exp. transform } & 0 & 0 . & 0 .\end{array}$

$\begin{array}{llllllll}\text { upscattering } & 0 & 0 . & 5.3884 \mathrm{E}-11 & \text { downscattering } & 0 & 0 . & 1.3605 \mathrm{E}+00\end{array}$

$\begin{array}{cccc}\text { capture } & 0 & 3.9980 \mathrm{E}-01 & 1.1590 \mathrm{E}-01\end{array}$

$(n, x n) \quad \begin{array}{lllllll}10 & 6.1640 \mathrm{E}-03 & 6.1355 \mathrm{E}-03 & \text { loss to }(n, x n) & 5 & 3.0820 \mathrm{E}-03 & 2.4027 \mathrm{E}-02\end{array}$

fission $\quad 0.0 .00$ loss to fission $0 \quad 4.5089 \mathrm{E}-01 \quad 2.4987 \mathrm{E}-01$

$\begin{array}{lllllllll}\text { total } & 1259 & 1.1044 \mathrm{E}+00 & 1.8761 \mathrm{E}+00 & \text { total } & 1259 & 1.1044 \mathrm{E}+00 & 1.8761 \mathrm{E}+00\end{array}$

number of neutrons banked $\quad 5$ average lifetime, shakes cutoffs

neutron tracks per source particle $1.0080 \mathrm{E}+00$ escape $3.7027 \mathrm{E}+00$ too $1.0000 \mathrm{E}+34$

neutron collisions per source particle $1.8226 \mathrm{E}+01$ capture $1.2954 \mathrm{E}+01$ eco $0.0000 \mathrm{E}+00$

total neutron collisions 22764 capture or escape $1.1788 \mathrm{E}+01 \quad \mathrm{wcl}-5.0000 \mathrm{E}-01$

net multiplication $\quad 1.0032 \mathrm{E}+000.0035$ any termination $1.2744 \mathrm{E}+01$ wc2 $-2.5000 \mathrm{E}-01$

computer time so far in this run 0.00 minutes

$\begin{array}{lr}\text { puter time in merun } & 0.00 \text { minutes } \\ \text { ce particles per minute } & 0.0000 \mathrm{E}+00\end{array}$

maximum number ever in bank 1

500880

bank overflows to backup file 0

field length

most random numbers used was 1298 in history 
HNF-SD-SNF-CSWD-005, Rev. 0

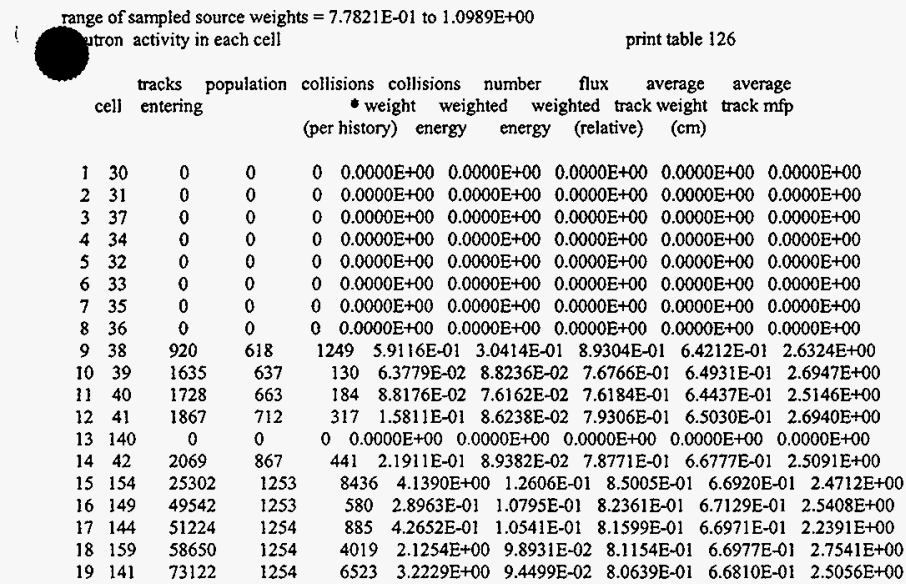

total $266059 \quad 9765 \quad 22764 \quad 1.1324 \mathrm{E}+01$

Ineutron weight balance in each cell -- external events

print table 130

cell entering source energy time exiting total

$$
\text { cutoff cutoff }
$$

$\begin{array}{lllllll}30 & 0.0000 \mathrm{E}+00 & 0.0000 \mathrm{E}+00 & 0.0000 \mathrm{E}+00 & 0.0000 \mathrm{E}+00 & 0.0000 \mathrm{E}+00 & 0.0000 \mathrm{E}+00\end{array}$

$\begin{array}{lllllllll}31 & 0.0000 E+00 & 0.0000 E+00 & 0.0000 E+00 & 0.0000 E+00 & 0.0000 E+00 & 0.0000 E+00\end{array}$

$\begin{array}{lllllll}37 & 0.0000 \mathrm{E}+00 & 0.0000 \mathrm{E}+00 & 0.0000 \mathrm{E}+00 & 0.0000 \mathrm{E}+00 & 0.0000 \mathrm{E}+00 & 0.0000 \mathrm{E}+00\end{array}$

$434 \quad 0.0000 \mathrm{E}+00 \quad 0.0000 \mathrm{E}+00 \quad 0.0000 \mathrm{E}+00 \quad 0.0000 \mathrm{E}+00 \quad 0.0000 \mathrm{E}+00 \quad 0.0000 \mathrm{E}+00$

$\begin{array}{lllllllll}5 & 32 & 0.0000 \mathrm{E}+00 & 0.0000 \mathrm{E}+00 & 0.0000 \mathrm{E}+00 & 0.0000 \mathrm{E}+00 & 0.0000 \mathrm{E}+00 & 0.0000 \mathrm{E}+00\end{array}$

$\begin{array}{llllllllll}6 & 33 & 0.0000 \mathrm{E}+00 & 0.0000 \mathrm{E}+00 & 0.0000 \mathrm{E}+00 & 0.0000 \mathrm{E}+00 & 0.0000 \mathrm{E}+00 & 0.0000 \mathrm{E}+00\end{array}$

$\begin{array}{llllllllll}7 & 35 & 0.0000 \mathrm{E}+00 & 0.0000 \mathrm{E}+00 & 0.0000 \mathrm{E}+00 & 0.0000 \mathrm{E}+00 & 0.0000 \mathrm{E}+00 & 0.0000 \mathrm{E}+00\end{array}$

$\begin{array}{llllllllll}8 & 36 & 0.0000 \mathrm{E}+00 & 0.0000 \mathrm{E}+00 & 0.0000 \mathrm{E}+00 & 0.0000 \mathrm{E}+00 & 0.0000 \mathrm{E}+00 & 0.0000 \mathrm{E}+00\end{array}$

$9 \quad 38 \quad 4.8822 \mathrm{E}-01 \quad 0.0000 \mathrm{E}+00 \quad 0.0000 \mathrm{E}+00 \quad 0.0000 \mathrm{E}+00 \quad-3.3091 \mathrm{E}-01 \quad 1.5731 \mathrm{E}-01$

$\begin{array}{lllllllll}10 & 39 & 8.4996 \mathrm{E}-01 & 0.0000 \mathrm{E}+00 & 0.0000 \mathrm{E}+00 & 0.0000 \mathrm{E}+00 & -8.4933 \mathrm{E}-01 & 6.2750 \mathrm{E}-04\end{array}$

$\begin{array}{lllllllll}11 & 40 & 8.9904 \mathrm{E}-01 & 0.0000 \mathrm{E}+00 & 0.0000 \mathrm{E}+00 & 0.0000 \mathrm{E}+00 & -8.9904 \mathrm{E}-01 & 3.9460 \mathrm{E}-06\end{array}$

$\begin{array}{llllllll}12 & 41 & 9.7260 \mathrm{E}-01 & 0.0000 \mathrm{E}+00 & 0.0000 \mathrm{E}+00 & 0.0000 \mathrm{E}+00 & -9.6978 \mathrm{E}-01 & 2.8204 \mathrm{E}-03\end{array}$

$\begin{array}{llllllllll}13 & 140 & 0.0000 \mathrm{E}+00 & 0.0000 \mathrm{E}+00 & 0.0000 \mathrm{E}+00 & 0.0000 \mathrm{E}+00 & 0.0000 \mathrm{E}+00 & 0.0000 \mathrm{E}+00\end{array}$

$\begin{array}{llllllll}14 & 42 & 1.0907 \mathrm{E}+00 & 0.0000 \mathrm{E}+00 & 0.0000 \mathrm{E}+00 & 0.0000 \mathrm{E}+00 & -1.0913 \mathrm{E}+00 & -5.9111 \mathrm{E}-04\end{array}$

$\begin{array}{lllllllll}15 & 154 & 1.2943 \mathrm{E}+01 & 8.4067 \mathrm{E}-01 & 0.0000 \mathrm{E}+00 & 0.0000 \mathrm{E}+00 & -1.3171 \mathrm{E}+01 & 6.1278 \mathrm{E}-01\end{array}$

$\begin{array}{lllllllll}16 & 149 & 2.6508 \mathrm{E}+01 & 0.0000 \mathrm{E}+00 & 0.0000 \mathrm{E}+00 & 0.0000 \mathrm{E}+00 & -2.6508 \mathrm{E}+01 & 3.5884 \mathrm{E}-05\end{array}$

$\begin{array}{lllllllll}17 & 144 & 2.7419 \mathrm{E}+01 & 0.0000 \mathrm{E}+00 & 0.0000 \mathrm{E}+00 & 0.0000 \mathrm{E}+00 & -2.7405 \mathrm{E}+01 & 1.4572 \mathrm{E}-02\end{array}$

$\begin{array}{llllllll}18 & 159 & 3.1331 \mathrm{E}+01 & 8.0064 \mathrm{E}-02 & 0.0000 \mathrm{E}+00 & 0.0000 \mathrm{E}+00 & -3.1361 \mathrm{E}+01 & 5.0067 \mathrm{E}-02\end{array}$

$19 \quad 141 \quad 3.9111 \mathrm{E}+01 \quad 4.0032 \mathrm{E}-02 \quad 0.0000 \mathrm{E}+00 \quad 0.0000 \mathrm{E}+00 \quad-3.9150 \mathrm{E}+01 \quad 4.5860 \mathrm{E}-04$

total $\quad 1.4161 \mathrm{E}+02 \quad 9.6077 \mathrm{E}+01 \quad 0.0000 \mathrm{E}+00 \quad 0.0000 \mathrm{E}+00 \quad-1.4174 \mathrm{E}+02 \quad 8.3809 \mathrm{E}-01$ Ineutron weight balance in each cell -- variance reduction events print table 130

cell weight cell weight energy dxtran forced exponential total window importance cutoff importance collision transform

$\begin{array}{llllllllll}30 & 0.0000 \mathrm{E}+00 & 0.0000 \mathrm{E}+00 & 0.0000 \mathrm{E}+00 & 0.0000 \mathrm{E}+00 & 0.0000 \mathrm{E}+00 & 0.0000 \mathrm{E}+00 & 0.0000 \mathrm{E}+00 & 0.0000 \mathrm{E}+00\end{array}$

$\begin{array}{lllllllllll}2 & 31 & 0.0000 \mathrm{E}+00 & 0.0000 \mathrm{E}+00 & 0.0000 \mathrm{E}+00 & 0.0000 \mathrm{E}+00 & 0.0000 \mathrm{E}+00 & 0.0000 \mathrm{E}+00 & 0.0000 \mathrm{E}+00 & 0.0000 \mathrm{E}+00\end{array}$

$\begin{array}{lllllllllll}3 & 37 & 0.0000 \mathrm{E}+00 & 0.0000 \mathrm{E}+00 & 0.0000 \mathrm{E}+00 & 0.0000 \mathrm{E}+00 & 0.0000 \mathrm{E}+00 & 0.0000 \mathrm{E}+00 & 0.0000 \mathrm{E}+00 & 0.0000 \mathrm{E}+00\end{array}$

$\begin{array}{llllllllll}4 & 34 & 0.0000 \mathrm{E}+00 & 0.0000 \mathrm{E}+00 & 0.0000 \mathrm{E}+00 & 0.0000 \mathrm{E}+00 & 0.0000 \mathrm{E}+00 & 0.0000 \mathrm{E}+00 & 0.0000 \mathrm{E}+00 & 0.0000 \mathrm{E}+00\end{array}$

$\begin{array}{lllllllllllllll}5 & 32 & 0.0000 \mathrm{E}+00 & 0.0000 \mathrm{E}+00 & 0.0000 \mathrm{E}+00 & 0.0000 \mathrm{E}+00 & 0.0000 \mathrm{E}+00 & 0.0000 \mathrm{E}+00 & 0.0000 \mathrm{E}+00 & 0.0000 \mathrm{E}+00\end{array}$

$\begin{array}{llllllllll}33 & 0.0000 \mathrm{E}+00 & 0.0000 \mathrm{E}+00 & 0.0000 \mathrm{E}+00 & 0.0000 \mathrm{E}+0.0 & 0.0000 \mathrm{E}+00 & 0.0000 \mathrm{E}+00 & 0.0000 \mathrm{E}+00 & 0.0000 \mathrm{E}+00\end{array}$

$\begin{array}{llllllllll}35 & 0.0000 \mathrm{E}+00 & 0.0000 \mathrm{E}+00 & 0.0000 \mathrm{E}+00 & 0.0000 \mathrm{E}+00 & 0.0000 \mathrm{E}+00 & 0.0000 \mathrm{E}+00 & 0.0000 \mathrm{E}+00 & 0.0000 \mathrm{E}+00\end{array}$

$\begin{array}{lllllllllll}36 & 0.0000 \mathrm{E}+00 & 0.0000 \mathrm{E}+00 & 0.0000 \mathrm{E}+00 & 0.0000 \mathrm{E}+00 & 0.0000 \mathrm{E}+00 & 0.0000 \mathrm{E}+00 & 0.0000 \mathrm{E}+00 & 0.0000 \mathrm{E}+00\end{array}$ 


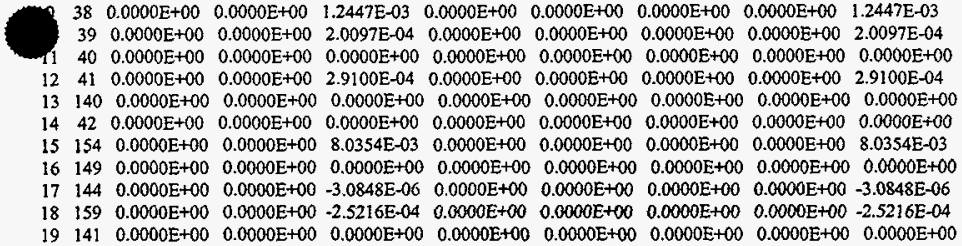

total $\quad 0.0000 \mathrm{E}+00 \quad 0.0000 \mathrm{E}+00 \quad 9.5169 \mathrm{E}-03 \quad 0.0000 \mathrm{E}+00 \quad 0.0000 \mathrm{E}+00 \quad 0.0000 \mathrm{E}+00 \quad 0.0000 \mathrm{E}+00 \quad 9.5169 \mathrm{E}-03$ ineutron weight balance in each cell - physical events print table 130

$$
\text { cell }(n, x n) \text { fission capture loss to loss to total }
$$

I $30 \quad 0.0000 \mathrm{E}+00 \quad 0.0000 \mathrm{E}+00 \quad 0.0000 \mathrm{E}+00 \quad 0.0000 \mathrm{E}+00 \quad 0.0000 \mathrm{E}+00 \quad 0.0000 \mathrm{E}+00$

$\begin{array}{lllllllll}2 & 31 & 0.0000 \mathrm{E}+00 & 0.0000 \mathrm{E}+00 & 0.0000 \mathrm{E}+00 & 0.0000 \mathrm{E}+00 & 0.0000 \mathrm{E}+00 & 0.0000 \mathrm{E}+00\end{array}$

$\begin{array}{llllllllll}3 & 37 & 0.0000 \mathrm{E}+00 & 0.0000 \mathrm{E}+00 & 0.0000 \mathrm{E}+00 & 0.0000 \mathrm{E}+00 & 0.0000 \mathrm{E}+00 & 0.0000 \mathrm{E}+00\end{array}$

$\begin{array}{lllllllll}4 & 34 & 0.0000 \mathrm{E}+00 & 0.0000 \mathrm{E}+00 & 0.0000 \mathrm{E}+00 & 0.0000 \mathrm{E}+00 & 0.0000 \mathrm{E}+00 & 0.0000 \mathrm{E}+00\end{array}$

$\begin{array}{llllllll}5 & 32 & 0.0000 \mathrm{E}+00 & 0.0000 \mathrm{E}+00 & 0.0000 \mathrm{E}+00 & 0.0000 \mathrm{E}+00 & 0.0000 \mathrm{E}+00 & 0.0000 \mathrm{E}+00\end{array}$

$\begin{array}{lllllllll}6 & 33 & 0.0000 \mathrm{E}+00 & 0.0000 \mathrm{E}+00 & 0.0000 \mathrm{E}+00 & 0.0000 \mathrm{E}+00 & 0.0000 \mathrm{E}+00 & 0.0000 \mathrm{E}+00\end{array}$

$\begin{array}{lllllllll}7 & 35 & 0.0000 \mathrm{E}+00 & 0.0000 \mathrm{E}+00 & 0.0000 \mathrm{E}+00 & 0.0000 \mathrm{E}+00 & 0.0000 \mathrm{E}+00 & 0.0000 \mathrm{E}+00\end{array}$

$\begin{array}{llllllllllll}8 & 36 & 0.0000 \mathrm{E}+00 & 0.0000 \mathrm{E}+00 & 0.0000 \mathrm{E}+00 & 0.0000 \mathrm{E}+00 & 0.0000 \mathrm{E}+00 & 0.0000 \mathrm{E}+00\end{array}$

$938 \quad 0.0000 \mathrm{E}+00 \quad 0.0000 \mathrm{E}+00+[.5855 \mathrm{E}-0] \quad 0.0000 \mathrm{E}+00 \quad 0.0000 \mathrm{E}+00+1.5855 \mathrm{E}-01$

$\begin{array}{lllllllll}10 & 39 & 0.0000 \mathrm{E}+00 & 0.0000 \mathrm{E}+00 & -8.2847 \mathrm{E}-04 & 0.0000 \mathrm{E}+00 & 0.0000 \mathrm{E}+00 & -8.2847 \mathrm{E}-04\end{array}$

$11 \quad 40 \quad 0.0000 \mathrm{E}+00 \quad 0.0000 \mathrm{E}+00 \quad-3.9460 \mathrm{E}-06 \quad 0.0000 \mathrm{E}+00 \quad 0.0000 \mathrm{E}+00 \quad-3.9460 \mathrm{E}-06$

12 4i $0.0000 \mathrm{E}+00 \quad 0.0000 \mathrm{E}+00 \quad-3.1114 \mathrm{E}-03 \quad 0.0000 \mathrm{E}+00 \quad 0.0000 \mathrm{E}+00 \quad-3.1114 \mathrm{E}-03$

$\begin{array}{llllllllll}13 & 140 & 0.0000 \mathrm{E}+00 & 0.0000 \mathrm{E}+00 & 0.0000 \mathrm{E}+00 & 0.0000 \mathrm{E}+00 & 0.0000 \mathrm{E}+00 & 0.0000 \mathrm{E}+00\end{array}$

$14 \quad 42 \quad 1.2461 \mathrm{E}-03 \quad 0.0000 \mathrm{E}+00 \quad-3.1955 \mathrm{E}-05 \quad+6.2306 \mathrm{E}-04 \quad 0.0000 \mathrm{E}+00 \quad 5.911] \mathrm{E}-04$

$5 \quad 154 \quad 4.9179 \mathrm{E}-03 \quad 0.0000 \mathrm{E}+00 \quad-1.7239 \mathrm{E}-01 \quad-2.4589 \mathrm{E}-03-4.5089 \mathrm{E}-01 \quad-6.2082 \mathrm{E}-01$

$149 \quad 0.0000 \mathrm{E}+00 \quad 0.0000 \mathrm{E}+00 \quad-3.5884 \mathrm{E}-05 \quad 0.0000 \mathrm{E}+00 \quad 0.0000 \mathrm{E}+00 \quad-3.5884 \mathrm{E}-05$

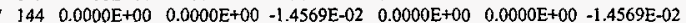

$\begin{array}{llllllll}18 & 159 & 0.0000 \mathrm{E}+00 & 0.0000 \mathrm{E}+00 & -4.9815 \mathrm{E}-02 & 0.0000 \mathrm{E}+00 & 0.0000 \mathrm{E}+00 & -4.9815 \mathrm{E}-02\end{array}$

$\begin{array}{llllllll}19 & 141 & 0.0000 \mathrm{E}+00 & 0.0000 \mathrm{E}+00 & -4.5860 \mathrm{E}-04 & 0.0000 \mathrm{E}+00 & 0.0000 \mathrm{E}+00 & -4.5860 \mathrm{E}-04\end{array}$

total 6.1640E-03 $0.0000 \mathrm{E}+00-3.9980 \mathrm{E}-01 \quad-3.0820 \mathrm{E}-03-4.5089 \mathrm{E}-01 \quad-8.4760 \mathrm{E}-01$

1neutron activity of each nuclide in each cell, per source particle print table 140

cell nuclides atom total collisions weight lost weight loss weight gain fraction collisions weight to capture to fission by $(n, \times n)$

$9385010.03 \mathrm{~d} \quad 7.1977 \mathrm{E}-01$ $5011.40 \mathrm{c} \quad 8.0041 \mathrm{E}-02$ $6012.40 c \quad 2.0019 \mathrm{E}-01$

$10 \quad 39 \quad 41093.40 \mathrm{c} \quad 9.8982 \mathrm{E}-0 \mathrm{t}$ $40000.40 \mathrm{C} \quad 1.0183 \mathrm{E}-02$

$11 \quad 40 \quad 1001.00 \mathrm{c} \quad 3.3333 \mathrm{E}-01$ $1002.55 \mathrm{c} \quad 3.3333 \mathrm{E}-0 \mathrm{I}$ $8016.40 \mathrm{c} \quad 3.3333 \mathrm{E}-01$

$12 \quad 41 \quad 41093.40 \mathrm{c} \quad 9.8982 \mathrm{E}-01$ $40000.40 \mathrm{c} \quad 1.0183 \mathrm{E}-02$

$14 \quad 42 \quad 1001.00 \mathrm{c} \quad 3.3333 \mathrm{E}-01$ $1002.55 c \quad 3.3333 \mathrm{E}-01$ $8016.40 \mathrm{c} \quad 3.3333 \mathrm{E}-01$

$\begin{array}{lll}15 & 154 & 92235.40 \mathrm{c} \quad 3.7504 \mathrm{E}-01\end{array}$ $92238.40 \mathrm{c} \quad 1.2499 \mathrm{E}-01$ $7014.40 c \quad 4.9997 E-01$
$941 \quad 4.4212 \mathrm{E}-01 \quad 1.5853 \mathrm{E}-01 \quad 0.0000 \mathrm{E}+00 \quad 0.0000 \mathrm{E}+00$

$\begin{array}{lllll}76 & 3.6073 \mathrm{E}-02 & 1.3591 \mathrm{E}-07 & 0.0000 \mathrm{E}+00 & 0.0000 \mathrm{E}+00\end{array}$

$232 \quad 1.1297 \mathrm{E}-01 \quad 1.8446 \mathrm{E}-05 \quad 0.0000 \mathrm{E}+00 \quad 0.0000 \mathrm{E}+00$

$128 \quad 6.2802 \mathrm{E}-02 \quad 8.2814 \mathrm{E}-04 \quad 0.0000 \mathrm{E}+00 \quad 0.0000 \mathrm{E}+00$

$2 \quad 9.7676 \mathrm{E}-04 \quad 3.3160 \mathrm{E}-07 \quad 0.0000 \mathrm{E}+00 \quad 0.0000 \mathrm{E}+00$

$111 \quad 5.1533 \mathrm{E}-02 \quad 3.9251 \mathrm{E}-06 \quad 0.0000 \mathrm{E}+00 \quad 0.0000 \mathrm{E}+00$ $\begin{array}{llllll}33 & 1.5373 \mathrm{E}-02 & 2.0884 \mathrm{E}-08 & 0.0000 \mathrm{E}+00 & 0.0000 \mathrm{E}+00\end{array}$ $40 \quad 2.1271 \mathrm{E}-02 \quad 0.0000 \mathrm{E}+00 \quad 0.0000 \mathrm{E}+00 \quad 0.0000 \mathrm{E}+00$

$313 \quad 1.5623 \mathrm{E}-01 \quad 3.1072 \mathrm{E}-03 \quad 0.0000 \mathrm{E}+00 \quad 0.0000 \mathrm{E}+00$ $4 \quad 1.8747 \mathrm{E}-03 \quad 4.2322 \mathrm{E}-06 \quad 0.0000 \mathrm{E}+00 \quad 0.0000 \mathrm{E}+00$

$257 \quad 1.2131 \mathrm{E}-01 \quad 4.3914 \mathrm{E}-06 \quad 0.0000 \mathrm{E}+00 \quad 0.0000 \mathrm{E}+00$

$86 \quad 4.6121 \mathrm{E}-02 \quad 6.8027 \mathrm{E}-08 \quad 0.0000 \mathrm{E}+00 \quad 6.2306 \mathrm{E}-04$

$98 \quad 5.1682 E-02 \quad 2.7495 E-05 \quad 0.0000 E+00 \quad 0.0000 E+00$

$4759 \quad 2.3227 \mathrm{E}+00 \quad 1.3244 \mathrm{E}-01 \quad 4.4247 \mathrm{E}-01 \quad 5.8410 \mathrm{E}-04$ $\begin{array}{llllll}1470 & 7.3870 \mathrm{E}-01 & 2.3272 \mathrm{E}-02 & 8.4162 \mathrm{E}-03 & 1.8748 \mathrm{E}-03\end{array}$ $2207 \quad 1.0775 \mathrm{E}+00 \quad 1.6682 \mathrm{E}-02 \quad 0.0000 \mathrm{E}+00 \quad 0.0000 \mathrm{E}+00$ 


\section{$1002.55 \mathrm{c} \quad 3.3333 \mathrm{E}-01$ $8016.40 \mathrm{c} \quad 3.3333 \mathrm{E}-01$

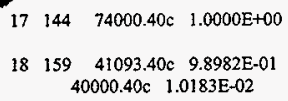

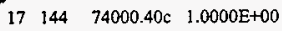

$18 \quad 159 \quad 41093.40 \mathrm{c} \quad 9.8982 \mathrm{E}-01$ $40000.40 \mathrm{c} \quad 1.0183 \mathrm{E}-02$

$\begin{array}{lllll}110 & 5.8433 \mathrm{E}-02 & 9.1921 \mathrm{E}-08 & 0.0000 \mathrm{E}+00 & 0.0000 \mathrm{E}+00\end{array}$

$125 \quad 6.3072 \mathrm{E}-02 \quad 3.1305 \mathrm{E}-05 \quad 0.0000 \mathrm{E}+00 \quad 0.0000 \mathrm{E}+00$
$19 \quad 141 \quad 1001.00$ c $3.3333 E-01$ $1002.55 \mathrm{c}$ 3.3333E-01 $8016.40 \mathrm{c}$ 3.3333E-0]

\author{
$885 \quad 4.2652 \mathrm{E}-01 \quad 1.4569 \mathrm{E}-02 \quad 0.0000 \mathrm{E}+00 \quad 0.0000 \mathrm{E}+00$ \\ $3965 \quad 2.1009 \mathrm{E}+00 \quad 4.9769 \mathrm{E}-02 \quad 0.0000 \mathrm{E}+00 \quad 0.0000 \mathrm{E}+00$ \\ $54 \quad 2.4590 \mathrm{E}-02 \quad 4.6175 \mathrm{E}-05 \quad 0.0000 \mathrm{E}+00 \quad 0.0000 \mathrm{E}+00$
}

$4029 \quad 1.9096 \mathrm{E}+00 \quad 7.9511 \mathrm{E}-05 \quad 0.0000 \mathrm{E}+00 \quad 0.0000 \mathrm{E}+00$ $\begin{array}{lllll}1122 & 5.8206 \mathrm{E}-01 & 9.0056 \mathrm{E}-07 & 0.0000 \mathrm{E}+00 & 0.0000 \mathrm{E}+00\end{array}$ $1372 \quad 7.3131 \mathrm{E}-01 \quad 3.7819 \mathrm{E}-04 \quad 0.0000 \mathrm{E}+00 \quad 0.0000 \mathrm{E}+00$

total $22764 \quad 1.1324 \mathrm{E}+01 \quad 3.9980 \mathrm{E}-01 \quad 4.5089 \mathrm{E}-01 \quad 3.0820 \mathrm{E}-03$

total over all cells for each nuclide total collisions weight lost weight loss weight gain coltisions weight to capture to fission by $(n, x n)$

$\begin{array}{ccccccc}1001.00 \mathrm{c} & 4742 & 2.2505 \mathrm{E}+00 & 9.2315 \mathrm{E}-05 & 0.0000 \mathrm{E}+00 & 0.0000 \mathrm{E}+00 \\ 1002.55 \mathrm{c} & 1351 & 7.0199 \mathrm{E}-01 & 1.0814 \mathrm{E}-06 & 0.0000 \mathrm{E}+00 & 6.2306 \mathrm{E}-04 \\ 5011.40 \mathrm{c} & 76 & 3.6073 \mathrm{E}-02 & 1.3591 \mathrm{E}-07 & 0.0000 \mathrm{E}+00 & 0.0000 \mathrm{E}+00 \\ 6012.40 \mathrm{c} & 232 & 1.1297 \mathrm{E}-01 & 1.8446 \mathrm{E}-05 & 0.0000 \mathrm{E}+00 & 0.0000 \mathrm{E}+00 \\ 7014.40 \mathrm{c} & 2207 & 1.0775 \mathrm{E}+00 & 1.6682 \mathrm{E}-02 & 0.0000 \mathrm{E}+00 & 0.0000 \mathrm{E}+00 \\ 8016.40 \mathrm{c} & 1635 & 8.6733 \mathrm{E}-01 & 4.3699 \mathrm{E}-04 & 0.0000 \mathrm{E}+00 & 0.0000 \mathrm{E}+00 \\ 40000.40 \mathrm{c} & 60 & 2.7441 \mathrm{E}-02 & 5.0739 \mathrm{E}-05 & 0.0000 \mathrm{E}+00 & 0.0000 \mathrm{E}+00 \\ 41093.40 \mathrm{c} & 4406 & 2.3199 \mathrm{E}+00 & 5.3704 \mathrm{E}-02 & 0.0000 \mathrm{E}+00 & 0.0000 \mathrm{E}+00 \\ 74000.40 \mathrm{c} & 885 & 4.2652 \mathrm{E}-01 & 1.4569 \mathrm{E}-02 & 0.0000 \mathrm{E}+00 & 0.0000 \mathrm{E}+00 \\ 92235.40 \mathrm{c} & 4759 & 2.3227 \mathrm{E}+00 & 1.3244 \mathrm{E}-01 & 4.4247 \mathrm{E}-01 & 5.8410 \mathrm{E}-04 \\ 92238.40 \mathrm{c} & 1470 & 7.3870 \mathrm{E}-01 & 2.3272 \mathrm{E}-02 & 8.4162 \mathrm{E}-03 & 1.8748 \mathrm{E}-03 \\ 5010.03 \mathrm{~d} & 94 \mathrm{I} & 4.4212 \mathrm{E}-01 & 1.5853 \mathrm{E}-01 & 0.0000 \mathrm{E}+00 & 0.0000 \mathrm{E}+00\end{array}$

the initial fission neutron source distribution used the 8 source points that were input on the ksre card.

he criticality problem was scheduled to skip 5 cycles and run a total of 6 cycles with nominally 200 neutrons per cycle problem has run 5 inactive cycles with 1027 neutron histories and 1 active cycles with 222 neutron histories.

this calculation has completed the requested number of keff cycles using a total of 1249 fission neuron source histories. all cells with fissionable material were sampled and had fission neutron source points.

there is no combined collision/absorption/track-length estimate for keff because only 1 active cycles were run. lindividual and average keff estimator results by cycle

keff neutron keff estimators by cycle average keff estimators and deviations average $k(\mathrm{c} / \mathrm{a} / \mathrm{t})$ cycle histories $k($ coll) $k(a b s) k(t r a c k) k($ coll $)$ st dev $k(a b s)$ st dev $k(t r a c k)$ st dev $k(c / a / t)$ st dev fom

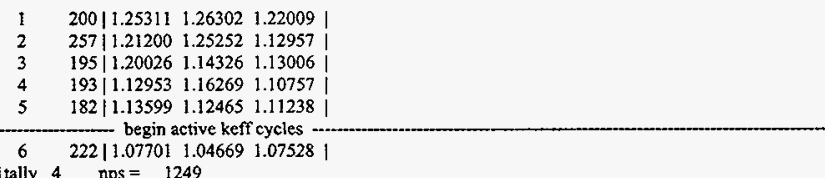

itally 4 nps $=1249$

$+\quad$ fuel rod flux in $5 y$ locations averaged over $5 x$ elements tally type 4 track length estimate of particle flux. units $1 / \mathrm{cm}^{* * 2}$ tally for neutrons

number of histories used for normalizing tallies $=200.00$ cell $a$ is $(154<42[-10-10] 42[-9-10] 42[-8-10] 42[-7-10] 42[-6-10])$ cell $b$ is $\left(154<42[-1030] 42\left[\begin{array}{lll}-9 & 3 & 0\end{array}\right) 42[-830] 42[-730] 42[-630]\right)$

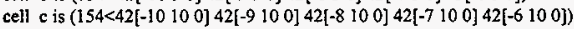
cell $d$ is $\left(154<42[-10210] 42[-9210] 42[-8210] 42\left[\begin{array}{llll}-7 & 21 & 0\end{array}\right] 42\left[\begin{array}{lll}-6 & 21 & 0\end{array}\right]\right)$ cell $e$ is $\left(154<42[-10290] 42[-9290] 42\left[\begin{array}{lll}-8 & 29 & 0\end{array} 42[-7290] 42\left[\begin{array}{lll}-6 & 29 & 0\end{array}\right)\right.\right.$

volumes
cell
$\begin{array}{cccccc}\mathrm{a} & \mathrm{b} & \mathrm{c} & \mathrm{d} & \mathrm{e} & \\ 1.04709 \mathrm{E}+02 & 1.04709 \mathrm{E}+02 & 1.04709 \mathrm{E}+02 & 1.04709 \mathrm{E}+02 & 1.04709 \mathrm{E}+02\end{array}$ 
HNF-SD-SNF-CSWD-005, Rev. 0

energy: $\quad 1.0000 \mathrm{E}-02 \quad 1.0000 \mathrm{E}-0 \mathrm{I} \quad 1.0000 \mathrm{E}+00 \quad 1.0000 \mathrm{E}+01 \quad$ total

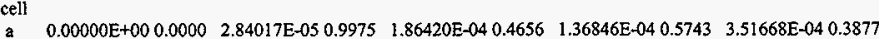

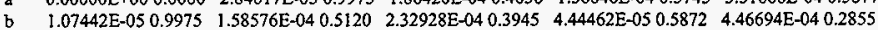

c $\quad 2.16778 \mathrm{E}-050.7232 \quad 9.40943 \mathrm{E}-050.5519 \quad 5.37835 \mathrm{E}-050.5876 \quad 1.51034 \mathrm{E}-04 \quad 0.7075 \quad 3.20589 \mathrm{E}-04 \quad 0.3937$

d $\quad 1.47903 \mathrm{E}-050.5831 \quad 0.00000 \mathrm{E}+000.0000 \quad 1.15515 \mathrm{E}-040.5838 \quad 1.69653 \mathrm{E}-040.5071 \quad 2.99958 \mathrm{E}-040.3718$

e $\quad 8.04943 \mathrm{E}-060.9975 \quad 0.00000 \mathrm{E}+000.0000 \quad 0.00000 \mathrm{E}+000.0000 \quad 6.81909 \mathrm{E}-050.7477 \quad 7.62403 \mathrm{E}-050.6763$

lanalysis of the results in the tally fluctuation chart bin (tfc) for tally 4 with nps $=1249$ print table 160

nomed average tally per history $=3.51668 \mathrm{E}-04$

estimated tally relative error $=0.3877$

relative entor from zero tallies $=0.3464$

number of nonzero history tallies $=\quad 8$

history number of largest tally $=\quad 1034$

(largest tally) $/$ (average tally) $=3.84476 E+01$

(confidence interval shift) $/$ mean $=0.0793$ unnormed average tally per history $=3.68228 \mathrm{E}-02$

estimated variance of the variance $=0.1752$

relative error from nonzero scores $=0.1740$

efficiency for the nonzero tallies $=0.0400$

largest unnormalized history tally $=1.41575 E+00$

(largest tally) $($ avg nonzero tally) $=1.53791 \mathrm{E}+00$

shifted confidence interval center $=3.79539 \mathrm{E}-04$

if the largest history score sampled so far were to occur on the very next history, the tfc bin quantities would change as follows: $n p s=222$ for this table because 5 keff cycles and 1027 histories were skipped before tally accumulation.

$\begin{array}{lccc}\text { estimated quantities } & \text { value at nps } & \text { value at nps+1 } & \text { value(nps+1)/value(nps } \\ \text { mean } & & & \\ \text { relative error } & 3.51668 \mathrm{E}-04 & 4.10723 \mathrm{E}-04 & 0.167927 \\ \text { variance of the variance } & 3.87653 \mathrm{E}-01 & 3.61597 \mathrm{E}-01 & -0.067213 \\ \text { shifted center } & 1.75161 \mathrm{E}-01 & 1.47665 \mathrm{E}-01 & -0.156976 \\ \text { figure of merit } & 3.79539 \mathrm{E}-04 & 3.77169 \mathrm{E}-04 & -0.006244 \\ \text { nat } & 0.00000 \mathrm{E}+00 & 0.00000 \mathrm{E}+00 & 0.000000\end{array}$

$e$ is not enough information in the largest history scores (usually less than 500 scores) for a reliable estimate of the slope.

history score probability density function appears to have an unsampled region at the largest history scores: please examine.

***** the nps-dependent tfc bin check results are suspect because there are only 1 nps tally values to analyze *****

results of 10 statistical checks for the estimated answer for the tally fluctuation chart (tfc) bin of tally 4

tfe bin -mean-- -.-----relative error-...-..- - - - variance of the variance--

behavior behavior value decrease decrease rate value decrease decrease rate value behavior slope

$\begin{array}{lcccccccc}\text { desired random } & <0.10 & \text { yes } & 1 / \text { sqrt(nps) } & <0.10 & \text { yes } & \text { linps } & \text { constant random }>3.00 \\ \text { observed random } & 0.39 & \text { yes } & \text { yes } & 0.18 \text { yes yes } & \text { yes } & 0.00 & \text { no }\end{array}$

waming. the tally in the tally fluctuation chart bin did not pass 3 of the 10 statistical checks.

lunnormed tally density for tally 4 nonzero tally mean $(m)=9.206 \mathrm{E}-01 \mathrm{nps}=1249$ print table 161

abscissa ordinate $\log$ plot of tally probability density function in tally fluctuation chart bin( $d=$ decade,slope $=0.0$ )

tally number num den log den:d--

$1.00-01 \quad 12.43-01 \quad-0.614 * * * * * * * * * * * * * * * * * * * * * * * * * * * * * * * * * * * * * * * * * * * * * * * * * * * * * * * * * * * * * * * * * * * * * * * * * * * * * * * * * * * * * * * * * * * * * * * * * * * * \mid *$

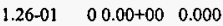

$\begin{array}{llll}1.58-01 & 0 & 0.00+00 & 0.000\end{array}$

$200-01 \quad 00.00+00 \quad 0.000$

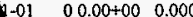

$6-01 \quad 00.00+00 \quad 0.000$ 
$\begin{array}{ccc}3.98-01 & 0.0 .00+00 & 0.000\end{array}$

$0.00+00 \quad 0.000$

$27.71-02-1.113 * * * * * * * * * * * * * * * * * * * * * * * * * * * * * * * * * * * * * * * * * * * * * * * * * *$

$0.00+00 \quad 0.000$

$.94-01$

$12.43-02-1.614$

$1.26+00 \quad 00.00+00 \quad 0.000$

$1.58+00$ 4 $6.14-02-1.212 * * * * * * * * * * * * * * * * * * * * * * * * * * * * * * * * * * * * * * * *$

total $84.00-02$

cumulative tally number for tally $4 \quad$ nonzero tally mean $(m)=9.206 \mathrm{E}-01 \quad \mathrm{nps}=1249$ print table 162

abscissa cum ordinate plot of the cumulative number of tallies in the tally fluctuation chart bin from 0 to 100 percent

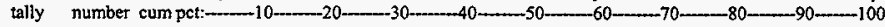

1.00000E-01

$1.25893 \mathrm{E}-01$

$1.58489 \mathrm{E}-01$

$1.99526 \mathrm{E}-01$

$2.51189 \mathrm{E}-0$

$3.16228 \mathrm{E}-01$

$3.98108 \mathrm{E}-01$

$5.01188 \mathrm{E}-01$

$6.30958 \mathrm{E}-01$

$7.94329 \mathrm{E}-01$

$1.00000 \mathrm{E}+00$

$1.25893 \mathrm{E}+00$

$1.58489 \mathrm{E}+00$

total

12.5001

1 $12.500 \mid * 4 * 4 * * * * ; * * *$

$1 \quad 12.500|* * * * * * * * *| * * *$

1 12.500|********|***

$\left.112.500\right|^{* * * * * * * * * \mid * * *}$

l $12.500|* * * * * * * * * ;| * * *$

$\left.1 \quad 12.500\right|^{\left.* * * * * * * * *\right|^{* * *}}$

112.500

I $\left.12.500\right|^{* * * * * * * * * \mid+* *}|||||| \mid$

$\left.337.500\right|^{* * * * * * * * *|* * * * * * * *| * * * * * * * * *|* * * * * * *|}$

$40.000 \mid \mathrm{mmmmmmmm}$ mmmmmmmmm|mmmmmmmmm|

$450.000|* * * * * * * * *| * * * * * * * * *|* * * * * * * * *| * * * * * * * * *|* * * * * * * *|$

$8100.000 * * * * 0$

8100.000

$-10--20$

20-

$60 \ldots+70+80$

$-100$

cumulative unnormed tally for tally $4 \quad$ nonzero tally mean $(m)=9.206 \mathrm{E}-01 \quad \mathrm{nps}=1249$ print table 162

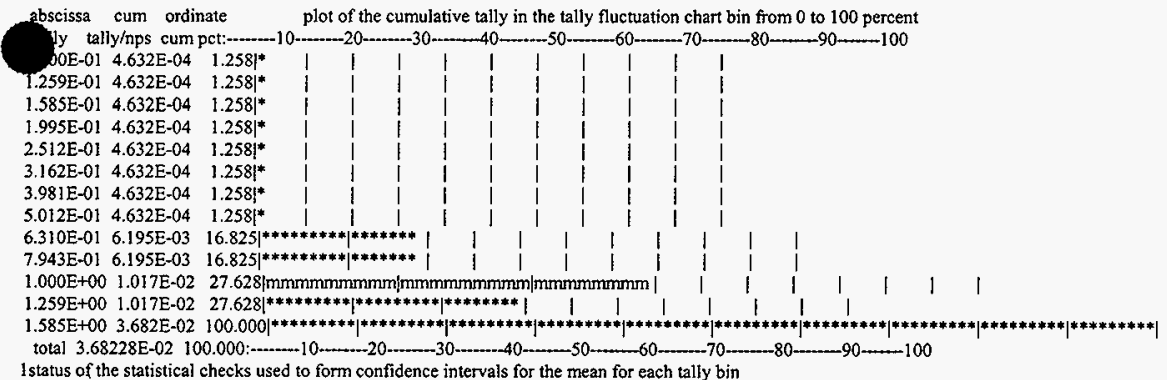

1status of the statistical checks used to form confidence intervals for the mean for each tally bin

tally result of statistical checks for the tfc bin (the first check not passed is listed) and error magnitude check for all bins

4 missed 3 of 10 tic bin checks: the relative error exceeds the recommended value of 0.1 for nonpoint detector tallies missed all bin error check: 25 tally bins had 4 bins with zeros and 21 bins with relative errors exceeding 0.10

the 10 statistical checks are only for the tally fluctuation chart bin and do not apply to other tally bins.

the tally bins with zeros may or may not be correct: compare the source, cutoffs, multipliers, et cetera with the tally bins

waming. 1 of the 1 tally fluctuation chart bins did not pass all 10 statistical checks

waming. 1 of the 1 tallies had bins with relative enors greater than recommended.

I tally fluctuation charts

tally 4

hps mean error vov slope fom 
$y$ data written to file inp $18 \mathrm{~m}$

ascii file inpl $8 \mathrm{p}$ written with

5 events from 5 histories.

6 warning messages so far.

run terminated when 6 kcode cycles were done.

\section{File: Outp18}

1- prob18 - kcode in a hexagonal prism lattice.

2- c three half control rods and five whole control rods.

3. $30 \quad 0-905-1929$ l fillml

4- $31 \quad 0-906-1929 !$ fill $=1(16.711300)$

5. $37 \quad 0-907-19291$ fill $=1(-16.711300)$

6- $\quad 34 \quad 0-913-1929$ fill $=1(011.91850)$

7. $32 \quad 0-914-1929$ fill $=1(10.32175 .95920)$

8. $\quad 33 \quad 0-915-1929$ fil $l=1(8.3557 \quad 14.47240)$

9. $35 \quad 0-916-1929 \quad$ fill $=\mathrm{I}(-8.3557 \quad 14.47240)$

10. $36 \quad 0-917-1929$ fill $=1(-10.32175 .95920)$

11- $c$ universe 1: structure of control rod.

12- $\quad \begin{array}{llllll}38 & 11 & -2.02 & -880 & u=1 & \$ \\ \text { control rod core }\end{array}$

13- $39 \quad 6 \quad-8.4 \quad 880-881 \quad u=1$ \$ control rod cladding

14- $\quad 40 \quad 12 \quad-1.00 \quad 881 \quad-882 \quad u=1 \quad \$$ control rod gap

15. $4 \mathrm{I} \quad 6 \quad-8.4 \quad 882 \quad u=1 \quad \$$ control rod sheath

16- - $c$ the space between the control rods, filled with lattice.

17- $\quad 140 \quad 0-17129-19905906907913914915916917$ fill $=2$

18- c universe 2: lattice of fuel rods with water in between.

19. $42 \quad 12-1.00 \quad-301 \quad 302-303 \quad 304-305306 \quad y=2$ lat $=2$ fill $=$

20- $\quad-37: 27-1: 330: 0$ \&

$24 r 39 r 24 r 311 r 24 r 311 r 24 r 39 r 2$

$24 \mathrm{r} 39 \mathrm{r} 23 \mathrm{r} 312 \mathrm{r} 23 \mathrm{r} 312 \mathrm{r} 23 \mathrm{r} 39 \mathrm{r} 2 \mathrm{Ir}$

$23 r 310 r 22 r 313 r 22 r 313 r 22 r 310 r 21 r$

$23 r 357 r 22 r \&$

$22 \mathrm{r} 358 \mathrm{r} 22 \mathrm{r}$

$22 r 316 r 22 r 317 r 22 r 316 r 23 r$

$22 r 315 r 23 r 316 r 23 r 315 r 24 r$

$21 r 315 r 24 r 315 r 24 r 315 r 24 r$

$21+315 r 23+316 r 23 r 315 r 25 r$

$21 \mathrm{r} 315 \mathrm{r} 22 \mathrm{r} 317 \mathrm{r} 22 \mathrm{r} 315 \mathrm{r} 26 \mathrm{r}$

$215354 \mathrm{r} 27 \mathrm{~T} \&$

c can code remember \& thru comment?

$2355 \mathrm{r} 27 \mathrm{r}$

$2325 r 2$ 2r $325 r 28 r$

$2324 \mathrm{r} 23 \mathrm{r} 324 \mathrm{r} 29 \mathrm{r}$

$2323 r 24$ r $323 r 210 r$

$2315 r 22 r 34 r 23 r 34 r 22 r 315 r 211 \mathrm{r}$

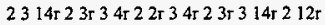

$2313 \mathrm{r} 24 \mathrm{r} 311 \mathrm{r} 24 \mathrm{r} 313 \mathrm{r} 213 \mathrm{r}$

$2313 r 23 r 312 r 23 r 313 r 214 \mathrm{r}$

$2313 r 22 r 313 r 22 r 313 r 215 r$

$2346 r 216 \mathrm{r}$

$2345 \mathrm{r} 217 \mathrm{r}$

$2344 \mathrm{r} 218 \mathrm{r}$

$21+341+220 r$

2 1r $340 \mathrm{r} 221 \mathrm{r}$

2 Ir $339 \mathrm{r} 222 \mathrm{r}$

2 2r $336 r 224 r$

$22 \mathrm{r} 335 \mathrm{r} 225 \mathrm{r}$

$23 \mathrm{r} 332 \mathrm{r} 227 \mathrm{r}$

$24 \mathrm{r} 329 \mathrm{r} 229 \mathrm{r}$

2 5r $326 r 231 r$

$26 r 323 r 233 r$

$28 \mathrm{r} 318 \mathrm{r} 236 \mathrm{r}$

$211 \mathrm{r} 111 \mathrm{r} 240 \mathrm{r}$ 
$60-144 \quad 7-19.66 \quad 268-478 \quad u=3 \quad$ Sliner

61- $\quad \begin{array}{lllllll}159 & 6 & -8.4 & 478 & -698 \quad u=3 & \$ & \text { cladding }\end{array}$

62- $141 \quad 12 \quad-1.00 \quad 698 \quad \mathrm{u}=3 \quad \$$ water between the fuel rods

63- $162 \quad 017:-29: 19:-1$ \$outside world

$64-$

65- $\quad 1$ py $0 \quad \$ \times-z$ plane, reflective

66. 17 cz 29.135

67- $\quad 19 \mathrm{pz} 31.75 \quad \$$ top of reactor

68. $29 \mathrm{pz}-31.75$ \$ bottom of reactor

69- $\quad \begin{array}{llllll}58 & \mathrm{c} / \mathrm{z} & 3.4414 & .8515 & .3240\end{array}$

70. $\begin{array}{llllll}268 & \mathrm{c} / \mathrm{z} & 3.4414 & .8515 & .3345\end{array}$

$\begin{array}{llllll}71- & 478 & \mathrm{c} / \mathrm{z} & 3.4414 & .8515 & .3475\end{array}$

$\begin{array}{llllll}72- & 698 & \mathrm{c} / \mathrm{z} & 3.4414 & .8515 & .4318\end{array}$

73- $\quad 880 \mathrm{cz} \quad 1.7251$

$\begin{array}{llll}74- & 881 & \mathrm{cz} & 1.8051\end{array}$

75- $882 \mathrm{cz} \quad 1.9051$

76- $905 \mathrm{cz} 2.1055$

77. $906 \mathrm{c} / \mathrm{z} \quad 16.711302 .1055$

78- $\quad 907 \mathrm{c} / 2-16.711302 .1055$

79. $\quad 913 \mathrm{c} / 2011.91852 .1055$

80. $\quad 914 \quad c / 2 \quad 10.32175 .95922 .1055$

81. $\quad 915 \mathrm{c} / \mathrm{z} \quad 8.355714 .47242 .1055$

82- $\quad 916 \mathrm{c} / \mathrm{z}+8.355714 .47242 .1055$

83- $\quad 917 \quad c / z-10.32175 .95922 .1055$

84- $\quad 301$ px 3.9330

85- $\quad 302$ px 2.9498

86- $\quad 303$ p $111.73205080760 \quad 5.8994$

87. $\quad 304$ p $11.73205080760 \quad 3.9330$

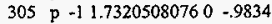

306 p $-11.73205080760-2.9498$

91- imp:n 1 18r 0

92- $\quad \mathrm{m}^{2} \quad 92235.40 \mathrm{c}-.70573 \quad 92238.40 \mathrm{c} \cdot-.23821 \quad 7014.40 \mathrm{c} \cdot .05605$

93- m6 $41093.40 \mathrm{c}-.99000 \quad 40000.40 \mathrm{c}-.01000$

94- $\mathrm{m} 7 \quad 74000.40 \mathrm{c}-.74000$

95- $\quad \mathrm{mll} \quad 5010.03 \mathrm{~d}-.6870 \quad 5011.40 \mathrm{c}-.0840 \quad 6012.40 \mathrm{c}+.2290$

96- $\mathrm{ml} 21001.00 \mathrm{cl} 1002.55 \mathrm{cl} \quad 8016.40 \mathrm{cl}$

97. mtl2 hwtr.01 lwtr.01

98- kcode 200 \& 56

waming. tallies are normed per fission neutron for one generation.

99- kssc $3.2 .2 .23 .2-3.2 .2 .23 .243 .2-43.243 .1-43.2$

100 - e .01 .11 .10 .

101- fq $\mathrm{fe}$

102- fc 4 fuel rod flux in 5 y locations averaged over $5 x$ elements

103- f4:n $(154<(42[-10:-6-10]))$ \$average $5 \times$ elements at $j=-1$

104- $\quad(154<(42[-10:-630])) \&$ \& average $5 x$ elements at $j=3$

105- $\quad(154<(42[-10:-6100])) \quad \$$ average $5 x$ elements at $j=10$ \&

106- $\quad(154<(42[-10:-6210])) \quad \$$ average $5 x$ elements at $j=21$

$107-\quad(154<(42[-10:-6290]))$ \$ average $5 \times$ elements at $j=29$

108- sd4 $104.70890624 \tau \quad \$ 5$ times the volume of cell 154

109- print -98

110- prdmp $2 j-1$

111- ptrac buffer $=20$ file $=$ asc write $=$ all event $=b n k$

1 initial source from $\mathrm{ksrc}$ card.

print table 90

original number of points points not in any cell points in cells of zero importance points in void cells points in ambiguous cells 1 points rejected its remaining 


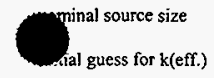

cycles to skip before tallying

number of keff cycles that can be stored
1.000000

5

201

total fission nubar data are being used.

itally 4

print table 30

fuel rod flux in 5 y locations averaged over $5 \times$ elements

tally type 4 track length estimate of particle flux.

tally for neutrons

order of printing: $f e$

cells $(154<(42[-10:-6-10]))(154<(42[-10:-630]))(154<(42[-10:-6100]))(154<(42[-10:-6210]))(154<(42[-10:-6$

$$
2901) \text { ) }
$$

energy bins

$0.00000 \mathrm{E}+00$ to $1.00000 \mathrm{E}-02 \mathrm{meV}$

$1.00000 \mathrm{E}-02$ to $1.00000 \mathrm{E}-01 \mathrm{mev}$

$1.00000 \mathrm{E}-01$ to $1.00000 \mathrm{E}+00 \mathrm{mev}$

$1.00000 \mathrm{E}+00$ to $1.00000 \mathrm{E}+01 \mathrm{mev}$

total bin

Imaterial composition

print table 40

the sum of the fractions of material 2 was $9.999900 \mathrm{E}-01$

the sum of the fractions of material 7 was $7.400000 \mathrm{E}-01$

the sum of the fractions of material 12 was $3.000000 \mathrm{E}+00$

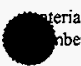

component nuclide, atom fraction

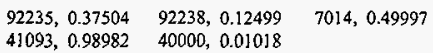

$5010,0.71977 \quad 5011,0.08004 \quad 6012,0.20019$

$1001,0.33333 \quad 1002,0.33333 \quad 8016,0.33333$

associated thermal s(a,b) data sets: hwtr.01t Iwtr.01t

material

number component nuclide, mass fraction

$\begin{array}{rrrr}2 & 92235,0.70574 & 92238,0.23821 & 7014,0.05605 \\ 6 & 41093,0.99000 & 40000,0.01000 & \\ 7 & 74000,1.00000 & & \\ 11 & 5010,0.68700 & 5011,0.08400 & 6012,0.22900 \\ 12 & 1001,0.05300 & 1002,0.10591 & 8016,0.84109\end{array}$

warning. 3 of the materials had unnormalized fractions. lcell volumes and masses

print table 50

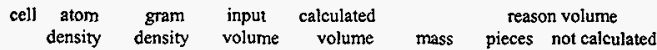

$\begin{array}{llllllll}130 & 0.00000 \mathrm{E}+00 & 0.00000 \mathrm{E}+00 & 0.00000 \mathrm{E}+00 & 0.00000 \mathrm{E}+00 & 0.00000 \mathrm{E}+00 & 0\end{array}$

$\begin{array}{llllllll}2 & 31 & 0.00000 \mathrm{E}+00 & 0.00000 \mathrm{E}+00 & 0.00000 \mathrm{E}+00 & 0.00000 \mathrm{E}+00 & 0.00000 \mathrm{E}+00 & 0\end{array}$

$\begin{array}{llllllll}3 & 37 & 0.00000 \mathrm{E}+00 & 0.00000 \mathrm{E}+00 & 0.00000 \mathrm{E}+00 & 0.00000 \mathrm{E}+00 & 0.00000 \mathrm{E}+00 & 0\end{array}$

$\begin{array}{llllll}34 & 0.00000 \mathrm{E}+00 & 0.00000 \mathrm{E}+00 & 0.00000 \mathrm{E}+00 & 8.84370 \mathrm{E}+02 & 0.00000 \mathrm{E}+00\end{array}$

$\begin{array}{lllllll}32 & 0.00000 \mathrm{E}+00 & 0.00000 \mathrm{E}+00 & 0.00000 \mathrm{E}+00 & 8.84370 \mathrm{E}+02 & 0.00000 \mathrm{E}+00 & 1\end{array}$

$\begin{array}{lllllll}33 & 0.00000 \mathrm{E}+00 & 0.00000 \mathrm{E}+00 & 0.00000 \mathrm{E}+00 & 8.84370 \mathrm{E}+02 & 0.00000 \mathrm{E}+00 & 1\end{array}$

asymmetric

asymmetric

asymmetric 


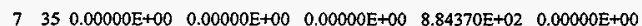
$\begin{array}{llllll}36 & 0.00000 \mathrm{E}+00 & 0.00000 \mathrm{E}+00 & 0.00000 \mathrm{E}+00 & 8.84370 \mathrm{E}+02 & 0.00000 \mathrm{E}+00\end{array}$ $\begin{array}{llllllll}38 & 1.15958 \mathrm{E}-01 & 2.02000 \mathrm{E}+00 & 0.00000 \mathrm{E}+00 & 0.00000 \mathrm{E}+00 & 0.00000 \mathrm{E}+00\end{array}$

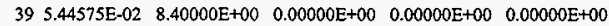
$\begin{array}{llllllll}11 & 40 & 9.50007 \mathrm{E}-02 & 1.00000 \mathrm{E}+00 & 0.00000 \mathrm{E}+00 & 0.00000 \mathrm{E}+00 & 0.00000 \mathrm{E}+00\end{array}$

$1241 \quad 5.44575 \mathrm{E}-02 \quad 8.40000 \mathrm{E}+00 \quad 0.00000 \mathrm{E}+00 \quad 0.00000 \mathrm{E}+00 \quad 0.00000 \mathrm{E}+00$

$13 \quad 140 \quad 0.00000 \mathrm{E}+00 \quad 0.00000 \mathrm{E}+00 \quad 0.00000 \mathrm{E}+00 \quad 0.00000 \mathrm{E}+00 \quad 0.00000 \mathrm{E}+00$

$\begin{array}{lllllll}14 & 42 & 9.50007 \mathrm{E}-02 & 1.00000 \mathrm{E}+00 & 0.00000 \mathrm{E}+00 & 0.00000 \mathrm{E}+00 & 0.00000 \mathrm{E}+00\end{array}$

$\begin{array}{llllllll}15 & 154 & 6.62920 \mathrm{E}-02 & 1.37500 \mathrm{E}+01 & 0.00000 \mathrm{E}+00 & 0.00000 \mathrm{E}+00 & 0.00000 \mathrm{E}+00\end{array}$

$\begin{array}{llllllll}16 & 149 & 9.50007 \mathrm{E}-02 & 1.00000 \mathrm{E}+00 & 0.00000 \mathrm{E}+00 & 0.00000 \mathrm{E}+00 & 0.00000 \mathrm{E}+00\end{array}$

$\begin{array}{llllllll}17 & 144 & 6.43969 \mathrm{E}-02 & 1.96600 \mathrm{E}+01 & 0.00000 \mathrm{E}+00 & 0.00000 \mathrm{E}+00 & 0.00000 \mathrm{E}+00\end{array}$

$\begin{array}{llllllll}18 & 159 & 5.44575 \mathrm{E}-02 & 8.40000 \mathrm{E}+00 & 0.00000 \mathrm{E}+00 & 0.00000 \mathrm{E}+00 & 0.00000 \mathrm{E}+00\end{array}$

$\begin{array}{lllllllll}19 & 141 & 9.50007 \mathrm{E}-02 & 1.00000 \mathrm{E}+00 & 0.00000 \mathrm{E}+00 & 0.00000 \mathrm{E}+00 & 0.00000 \mathrm{E}+00\end{array}$

$\begin{array}{llllllll}20 & 162 & 0.00000 \mathrm{E}+00 & 0.00000 \mathrm{E}+00 & 0.00000 \mathrm{E}+00 & 0.00000 \mathrm{E}+00 & 0.00000 \mathrm{E}+00\end{array}$ 1surface areas print table 50
HNF-SD-SNF-CSWD-005, Rev. 0

infinite

infinite

infinite

infinite

asymmetric

infinite

infinite

infinite

infinite

infinite

infinite

asymmetric

surface input calculated reason area area area not calculated
$10.00000 \mathrm{E}+00 \quad 0.00000 \mathrm{E}+00$ asymmetric

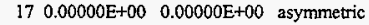
$190.00000 \mathrm{E}+00 \quad 0.00000 \mathrm{E}+00$ asymmetric
$29 \quad 0.00000 \mathrm{E}+00 \quad 0.00000 \mathrm{E}+00$ asymmetric
$\begin{array}{lll}58 & 0.00000 \mathrm{E}+00 & 0.00000 \mathrm{E}+00 \text { infinite }\end{array}$
$\begin{array}{lll}268 & 0.00000 \mathrm{E}+00 \quad 0.00000 \mathrm{E}+00 \text { infinite }\end{array}$
$478 \quad 0.00000 \mathrm{E}+00 \quad 0.00000 \mathrm{E} \div 00$ infinite
$\begin{array}{lll}698 & 0.00000 \mathrm{E}+00 & 0.00000 \mathrm{E}+00 \text { infinite }\end{array}$
$880 \quad 0.00000 \mathrm{E}+\infty \quad 0.00000 \mathrm{E}+00$ infinite
$108810.00000 \mathrm{E}+00 \quad 0.00000 \mathrm{E}+00$ infinite
$11 \quad 882 \quad 0.00000 \mathrm{E}+00 \quad 0.00000 \mathrm{E}+00$ infinite
$12905 \quad 0.00000 \mathrm{E}+00 \quad 0.00000 \mathrm{E}+00$ asymmetric
$139060.00000 \mathrm{E}+00 \quad 0.00000 \mathrm{E}+00$ asymmetric
$14907 \quad 0.00000 \mathrm{E}+00 \quad 0.00000 \mathrm{E}+00$ asymmetric
$913 \quad 0.00000 \mathrm{E}+00 \quad 8.40057 \mathrm{E}+02$
$914 \quad 0.00000 \mathrm{E}+00 \quad 8.40057 \mathrm{E}+02$
$9150.00000 \mathrm{E}+00 \quad 8.40057 \mathrm{E}+02$
$18 \quad 916 \quad 0.00000 \mathrm{E}+00 \quad 8.40057 \mathrm{E}+02$
$19917 \quad 0.00000 \mathrm{E}+00 \quad 8.40057 \mathrm{E}+02$
$203010.00000 \mathrm{E}+00 \quad 0.00000 \mathrm{E}+00$ infinite
$21302 \quad 0.00000 \mathrm{E}+00 \quad 0.00000 \mathrm{E}+00$ infinite
$22303 \quad 0.00000 \mathrm{E}+00 \quad 0.00000 \mathrm{E}+00$ infinite
$23 \quad 304 \quad 0.00000 \mathrm{E}+00 \quad 0.00000 \mathrm{E}+00$
$24305 \quad 0.00000 \mathrm{E}+00 \quad 0.00000 \mathrm{E}+00$
$25306 \quad 0.00000 \mathrm{E}+00 \quad 0.00000 \mathrm{E}+00$

Icells

cell mat density $\stackrel{2}{2}$ density volume mass pieces importance

I $30 \quad 0 \quad 0.00000 \mathrm{E}+00 \quad 0.00000 \mathrm{E}+000.00000 \mathrm{E}+000.00000 \mathrm{E}+00$

2 3I $00.00000 \mathrm{E}+00 \quad 0.00000 \mathrm{E}+000.00000 \mathrm{E}+000.00000 \mathrm{E}+00$

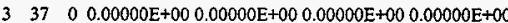

$434 \quad 0 \quad 0.00000 \mathrm{E}+00 \quad 0.00000 \mathrm{E}+00 \quad 8.84370 \mathrm{E}+02 \quad 0.00000 \mathrm{E}+00$

$53200.00000 \mathrm{E}+00 \quad 0.00000 \mathrm{E}+008.84370 \mathrm{E}+020.00000 \mathrm{E}+00$

$63300.00000 \mathrm{E}+000.00000 \mathrm{E}+008.84370 \mathrm{E}+02 \quad 0.00000 \mathrm{E}+00$

$735 \quad 0 \quad 0.00000 \mathrm{E}+00 \quad 0.00000 \mathrm{E}+008.84370 \mathrm{E}+020.00000 \mathrm{E}+00$

$836 \quad 0 \quad 0.00000 \mathrm{E}+00 \quad 0.00000 \mathrm{E}+008.84370 \mathrm{E}+02 \quad 0.00000 \mathrm{E}+00$

$938 \quad 11 \quad 1.15958 \mathrm{E}-012.02000 \mathrm{E}+00 \quad 0.00000 \mathrm{E}+000.00000 \mathrm{E}+00$

$10 \quad 39 \quad 6 \quad 5.44575 \mathrm{E}-02 \quad 8.40000 \mathrm{E}+000.00000 \mathrm{E}+00 \quad 0.00000 \mathrm{E}+00$

$11 \quad 40 \quad 12 \mathrm{~s} 9.50007 \mathrm{E}-021.00000 \mathrm{E}+000.00000 \mathrm{E}+000.00000 \mathrm{E}+00$

$1241 \quad 6 \quad 5.44575 \mathrm{E}-028.40000 \mathrm{E}+000.00000 \mathrm{E}+000.00000 \mathrm{E}+00$

$13 \quad 140 \quad 0 \quad 0.00000 \mathrm{E}+00 \quad 0.00000 \mathrm{E}+000.00000 \mathrm{E}+000.00000 \mathrm{E}+00$

$14 \quad 42$ i2s $9.50007 \mathrm{E}-02 \quad 1.00000 \mathrm{E}+000.00000 \mathrm{E}+000.00000 \mathrm{E}+00$

$15 \quad 15426.62920 \mathrm{E}-021.37500 \mathrm{E}+01 \quad 0.00000 \mathrm{E}+000.00000 \mathrm{E}+00$

$1614912 s 9.50007 \mathrm{E}-021.00000 \mathrm{E}+000.00000 \mathrm{E}+000.00000 \mathrm{E}+00$

$14476.43969 \mathrm{E}-02 \quad 1.96600 \mathrm{E}+010.00000 \mathrm{E}+000.00000 \mathrm{E}+00$

$15965.44575 \mathrm{E}-028.40000 \mathrm{E}+000.00000 \mathrm{E}+000.00000 \mathrm{E}+00$

$01.0000 \mathrm{E}+00$

$01.0000 \mathrm{E}+00$

$01.0000 \mathrm{E}+00$

$11.0000 \mathrm{E}+00$

$11.0000 \mathrm{E}+00$

$11.0000 \mathrm{E}+00$

$11.0000 E+00$

$11.0000 \mathrm{E}+00$

$0 \quad 1.0000 \mathrm{E}+00$

$01.0000 \mathrm{E}+00$

$01.0000 \mathrm{E}+00$

$0 \quad 1.0000 \mathrm{E}+00$

$0 \quad 1.0000 \mathrm{E}+00$

$0 \quad 1.0000 \mathrm{E}+00$

$0 \quad 1.0000 \mathrm{E}+00$

$01.0000 \mathrm{E}+00$

o $1.0000 \mathrm{E}+00$

$0 \quad 1.0000 E+00$ 
$141 \quad 125950007 \mathrm{E}-021.00000 \mathrm{E}+000.00000 \mathrm{E}+000.00000 \mathrm{E}+00 \quad 0 \quad 1.0000 \mathrm{E}+00$

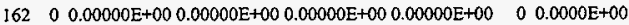

total

lsurfaces

\subsection{E+03 0.00000E+00}

print table 70

surface trans type surface coefficients

11 refl. py $0.0000000 \mathrm{E}+00$

217 cz $2.9135000 \mathrm{E}+01$

$3 \quad 19 \quad \mathrm{pz} 3.1750000 \mathrm{E}+01$

$429 \quad \mathrm{pz}-3.1750000 \mathrm{E}+0 \mathrm{I}$

$558 \quad \mathrm{c} / 2 \quad 3.4414000 \mathrm{E}+00 \quad 8.5150000 \mathrm{E}-01 \quad 3.2400000 \mathrm{E}-01$

$6268 \quad c / z \quad 3.4414000 \mathrm{E}+00 \quad 8.5150000 \mathrm{E}-01 \quad 3.3450000 \mathrm{E}-01$

$7 \quad 478 \quad c / z \quad 3.4414000 \mathrm{E}+00 \quad 8.5150000 \mathrm{E}-01 \quad 3.4750000 \mathrm{E}-01$

$\begin{array}{lllllll}8 & 698 & c & 3.4414000 \mathrm{E}+00 & 8.5150000 \mathrm{E}-01 & 4.3180000 \mathrm{E}-01\end{array}$

9880 cz $1.7251000 \mathrm{E}+00$

$\begin{array}{llll}10 & 881 \quad \text { cz } & 1.8051000 \mathrm{E}+00\end{array}$

$11882 \quad \mathrm{CZ} \quad 1.9051000 \mathrm{E}+00$

$12905 \quad \mathrm{cz} 2.1055000 \mathrm{E}+00$

$13906 \quad c / z \quad 1.6711300 E+01 \quad 0.0000000 E+00 \quad 2.1055000 E+00$

$14907 \quad c / z-1.6711300 \mathrm{E}+01 \quad 0.0000000 \mathrm{E}+00 \quad 2.1055000 \mathrm{E}+00$

$\begin{array}{llllll}15 & 913 \quad \mathrm{c} / \mathrm{z} & 0.0000000 \mathrm{E}+00 & 1.1918500 \mathrm{E}+01 & 2.1055000 \mathrm{E}+00\end{array}$

$\begin{array}{llllll}16 & 914 \quad \mathrm{c} / \mathrm{z} & 1.0321700 \mathrm{E}+01 & 5.9592000 \mathrm{E}+00 & 2.1055000 \mathrm{E}+00\end{array}$

$17915 \quad \mathrm{c} / \mathrm{z} \quad 8.3557000 \mathrm{E}+00 \quad 1.4472400 \mathrm{E}+01 \quad 2.1055000 \mathrm{E}+00$

$\begin{array}{lllllll}18 & 916 & c / z & -8.3557000 \mathrm{E}+00 & 1.4472400 \mathrm{E}+01 & 2.1055000 \mathrm{E}+00\end{array}$

$\begin{array}{llllll}19 & 917 \quad \mathrm{c} / z & -1.0321700 \mathrm{E}+01 & 5.9592000 \mathrm{E}+00 & 2.1055000 \mathrm{E}+00\end{array}$

$20301 \quad$ px $3.9330000 \mathrm{E}+00$

$21302 \quad \mathrm{px} 2.9498000 \mathrm{E}+00$

$\begin{array}{llllllll}22 & 303 & \text { p } & 1.0000000 \mathrm{E}+00 & 1.7320508 \mathrm{E}+00 & 0.0000000 \mathrm{E}+00 & 5.8994000 \mathrm{E}+00\end{array}$

$\begin{array}{llllllll}23 & 304 & \text { p } & 1.0000000 E+00 & 1.7320508 E+00 & 0.0000000 E+00 & 3.9330000 E+00\end{array}$

$\begin{array}{llllllll}24 & 305 & p & -1.0000000 \mathrm{E}+00 & 1.7320508 \mathrm{E}+00 & 0.0000000 \mathrm{E}+00 & -9.8340000 \mathrm{E}-01\end{array}$

p $-1.0000000 \mathrm{E}+00 \quad 1.7320508 \mathrm{E}+00 \quad 0.0000000 \mathrm{E}+00 \quad-2.9498000 \mathrm{E}+00$

ell temperatures in mev for the free-gas thermal neutron treatment. print table 72

all non-zero importance cells with materials have a temperature for thermal neutrons of $2.5300 \mathrm{E}-08 \mathrm{mev}$.

2 waming messages so far.

lcross-section tables

print table 100

table length

tables from file testlibl

$1001.00 \mathrm{c} \quad 1782$ 1-h-1 from endf-vi.l

mat $12505 / 26 / 93$

$1002.55 \mathrm{c} 4102$ njoy

( 120 ) $04 / 26 / 82$

$5011.40 \mathrm{c} 3229$ ENDL library name: nd900719 MCNP translation: 900831 14:10:05

$6012.40 \mathrm{C} 4858$ ENDL library name: nd900719 MCNP tanslation: 900831 14:10:05

$7014.40 \mathrm{c} 8535$ ENDL library name: nd900719 MCNP transiation: 900831 14:10:05

8016.40 5358 ENDL library name: nd900719 MCNP transiation: 900831 14:10:05

40000.40c 93372 ENDL library name: nd900719 MCNP translation: 900831 14:10:05

41093.40c 42746 ENDL library name: nd900719 MCNP translation: 900831 14:10:05

74000.40c 91244 ENDL library name: nd900719 MCNP translation: 900831 14:10:05

92235.40c 34772 ENDL library name: nd900719 MCNP translation: 9totai nu 10:05

92238.40c 40834 ENDL library name: no901118 MCNP translation: 9total nu 56:49

900207

900207

900207

900207

900323

900208

900208

900503

901119
$5010.03 \mathrm{~d} 3682$ b-10 endf/b-iv new gamma production format hwtr.01t 10193 deuterium in heavy water at 300 degrees kelvin lwtr.01t 10193 hydrogen in light water at 300 degrees kelvin

total 354900

warning. neutron energy cutoff is below some cross-section tables.
$10020010 / 22 / 85$

$1001 \quad 0 \quad 010 / 22 / 85$ 
3 waming messages so far.

I starting merun. field length $=0 \quad \mathrm{cp} 0=0.00$ print table 110

prob18 - kcode in a hexagonal prism lattice.

nps $x \quad y \quad z \quad$ cell lattice $(i, j, k)$ surface $u$

1 $3.000 \mathrm{E}+00 \quad 2.000 \mathrm{E}-01 \quad 2.000 \mathrm{E}-01 \quad 140$ $3.492 \mathrm{E}+00 \quad 1.051 \mathrm{E}+00 \quad 2.000 \mathrm{E}-01 \quad 42(0,-1,0)$ $3.492 \mathrm{E}+00 \quad 1.051 \mathrm{E}+00 \quad 2.000 \mathrm{E}-01 \quad 154$

$23.000 \mathrm{E}+00 \quad 2.000 \mathrm{E}-01 \quad 2.000 \mathrm{E}-01] \quad 140$ $3.492 \mathrm{E}+00 \quad 1.051 \mathrm{E}+00 \quad 2.000 \mathrm{E}-01 \quad 42(0,-1,0)$ $3.492 \mathrm{E}+00 \quad 1.051 \mathrm{E}+00 \quad 2.000 \mathrm{E}-01 \quad 154$ $33.000 \mathrm{E}+00 \quad 2.000 \mathrm{E}-01 \quad 2.000 \mathrm{E}-01 \quad 140$ $3.492 \mathrm{E}+00 \quad 1.051 \mathrm{E}+00 \quad 2.000 \mathrm{E}-01 \quad 42(0,-1,0)$ $3.492 \mathrm{E}+00 \quad 1.051 \mathrm{E}+00 \quad 2.000 \mathrm{E}-01 \quad 154$

$43.000 \mathrm{E}+00 \quad 2.000 \mathrm{E}-01 \quad 2.000 \mathrm{E}-01 \quad 140$ $3.492 \mathrm{E}+00 \quad 1.051 \mathrm{E}+00 \quad 2.000 \mathrm{E}-01 \quad 42(0,-1,0)$ $3.492 \mathrm{E}+00 \quad 1.051 \mathrm{E}+00 \quad 2.000 \mathrm{E}-01 \quad 154$

$53.000 \mathrm{E}+00 \quad 2.000 \mathrm{E}-01 \quad 2.000 \mathrm{E}-01 \quad 140$ $3.492 \mathrm{E}+00 \quad 1.051 \mathrm{E}+00 \quad 2.000 \mathrm{E}-01 \quad 42(0,-1,0)$ $3.492 \mathrm{E}+00 \quad 1.051 \mathrm{E}+00 \quad 2.000 \mathrm{E}-01 \quad 154$ $63.000 \mathrm{E}+00 \quad 2.000 \mathrm{E}-01 \quad 2.000 \mathrm{E}-01 \quad 140$ $3.492 \mathrm{E}+00 \quad 1.051 \mathrm{E}+00 \quad 2.000 \mathrm{E}-01 \quad 42(0,-1,0)$ $3.492 \mathrm{E}+00 \quad 1.051 \mathrm{E}+00 \quad 2.000 \mathrm{E}-01 \quad 154$

$73.000 \mathrm{E}+00 \quad 2.000 \mathrm{E}-01 \quad 2.000 \mathrm{E}-01 \quad 140$ $3.492 \mathrm{E}+00 \quad 1.051 \mathrm{E}+00 \quad 2.000 \mathrm{E}-01 \quad 42(0,-1,0)$ $3.492 \mathrm{E}+00 \quad 1.051 \mathrm{E}+00 \quad 2.000 \mathrm{E}-01 \quad 154$ $3.000 \mathrm{E}+00 \quad 2.000 \mathrm{E}-01 \quad 2.000 \mathrm{E}-01 \quad 140$ $3.492 \mathrm{E}+00 \quad 1.051 \mathrm{E}+00 \quad 2.000 \mathrm{E}-01 \quad 42($ $3.492 \mathrm{E}+00 \quad 1.051 \mathrm{E}+00 \quad 2.000 \mathrm{E}-01 \quad 154$

$93.000 \mathrm{E}+00 \quad 2.000 \mathrm{E}-01 \quad 2.000 \mathrm{E}-01140$ $3.492 \mathrm{E}+00 \quad 1.051 \mathrm{E}+00 \quad 2.000 \mathrm{E}-01 \quad 42($ $3.492 \mathrm{E}+00 \quad 1.051 \mathrm{E}+00 \quad 2.000 \mathrm{E}-01 \quad 154$

$103.000 \mathrm{E}+00 \quad 2.000 \mathrm{E}-01 \quad 2.000 \mathrm{E}-01 \quad 140$ $3.492 \mathrm{E}+00 \quad 1.051 \mathrm{E}+00 \quad 2.000 \mathrm{E}-01 \quad 42(0,-1,0)$ $3.492 \mathrm{E}+00 \quad 1.051 \mathrm{E}+00 \quad 2.000 \mathrm{E}-01 \quad 154$

$113.000 \mathrm{E}+00 \quad 2.000 \mathrm{E}-01 \quad 2.000 \mathrm{E}-01 \quad 140$ $3.492 \mathrm{E}+00 \quad 1.051 \mathrm{E}+00 \quad 2.000 \mathrm{E}-01 \quad 42(0,-1,0)$ $3.492 \mathrm{E}+00 \quad 1.051 \mathrm{E}+00 \quad 2.000 \mathrm{E}-01 \quad 154$

$123.000 \mathrm{E}+00 \quad 2.000 \mathrm{E}-01 \quad 2.000 \mathrm{E}-01 \quad 140$ $3.492 \mathrm{E}+00 \quad 1.051 \mathrm{E}+00 \quad 2.000 \mathrm{E}-01 \quad 42($ $3.492 \mathrm{E}+00 \quad 1.051 \mathrm{E}+00 \quad 2.000 \mathrm{E}-01 \quad 154$

I3 $3.000 \mathrm{E}+00 \quad 2.000 \mathrm{E}-01 \quad 2.000 \mathrm{E}-01 \quad 140$ $3.492 \mathrm{E}+00 \quad 1.051 \mathrm{E}+00 \quad 2.000 \mathrm{E}-01 \quad 42(0,-1,0)$ $3.492 \mathrm{E}+00 \quad 1.051 \mathrm{E}+00 \quad 2.000 \mathrm{E}-01 \quad 154$

$143.000 \mathrm{E}+00 \quad 2.000 \mathrm{E}-01 \quad 2.000 \mathrm{E}-01 \quad 140$ $3.492 \mathrm{E}+00 \quad 1.051 \mathrm{E}+00 \quad 2.000 \mathrm{E}-01 \quad 42(0,-1,0)$ $3.492 \mathrm{E}+00 \quad 1.051 \mathrm{E}+00 \quad 2.000 \mathrm{E}-01 \quad 154$

I5 $3.000 \mathrm{E}+00 \quad 2.000 \mathrm{E}-01 \quad 2.000 \mathrm{E}-01 \quad 140$ $3.492 \mathrm{E}+00 \quad 1.051 \mathrm{E}+00 \quad 2.000 \mathrm{E}-01 \quad 42(0,-1,0)$ $3.492 \mathrm{E}+00 \quad 1.051 \mathrm{E}+00 \quad 2.000 \mathrm{E}-01 \quad 154$

$16 \quad 3.000 \mathrm{E}+00 \quad 2.000 \mathrm{E}-01 \quad 2.000 \mathrm{E}-01 \quad 140$ $3.492 \mathrm{E}+00 \quad 1.051 \mathrm{E}+00 \quad 2.000 \mathrm{E}-01 \quad 42(0,-1,0)$ $3.492 \mathrm{E}+00 \quad 1.051 \mathrm{E}+00 \quad 2.000 \mathrm{E}-01 \quad 154$

$173.000 \mathrm{E}+00 \quad 2.000 \mathrm{E}-01 \quad 2.000 \mathrm{E}-01 \quad 140$ $3.492 \mathrm{E}+00 \quad 1.051 \mathrm{E}+00 \quad 2.000 \mathrm{E}-01 \quad 42(0,-1,0)$ $3.492 \mathrm{E}+00 \quad 1.051 \mathrm{E}+00 \quad 2.000 \mathrm{E}-01 \quad 154$ $183.000 \mathrm{E}+00 \quad 2.000 \mathrm{E}-01 \quad 2.000 \mathrm{E}-01 \quad 140$ $3.492 \mathrm{E}+00 \quad 1.051 \mathrm{E}+00 \quad 2.000 \mathrm{E}-01 \quad 42($ $.492 \mathrm{E}+00 \quad 1.051 \mathrm{E}+00 \quad 2.000 \mathrm{E}=01 \quad 154$ $3.000 \mathrm{E}+00 \quad 2.000 \mathrm{E}-01 \quad 2.000 \mathrm{E}-01 \quad 140$ $v$ w energy weight time

5.085E-01 4.733E-01 7.193E-01 $2.209 \mathrm{E}+00 \quad 1.000 \mathrm{E}+00 \quad 0.000 \mathrm{E}+00$ $5.085 \mathrm{E}-01 \quad 4.733 \mathrm{E}-017.193 \mathrm{E}-01$

0 5.085E-01 4.733E-01 7.193E-01

8.952E-01 -4.447E-01 -2.944E-02 4.904E+00 1.000E+00 $0.000 \mathrm{E}+00$ $8.952 \mathrm{E}-01-4.447 \mathrm{E}-01 \cdot 2.944 \mathrm{E}-02$

0 8.952E-01 -4.447E-01 -2.944E-02

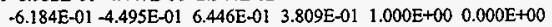
$-6.184 \mathrm{E}-01-4.495 \mathrm{E}-01 \quad 6.446 \mathrm{E}-01$

$0-6.184 \mathrm{E}-01-4.495 \mathrm{E}-01 \quad 6.446 \mathrm{E}-01$

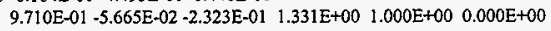
9.710E-01 -5.665E-02 -2.323E-01

0 9.710E-01 -5.665E-02 -2.323E-01

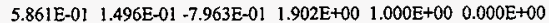
$5.861 \mathrm{E}-01 \quad 1.496 \mathrm{E}-01-7.963 \mathrm{E}-01$

$05.861 \mathrm{E}-01 \quad 1.496 \mathrm{E}-01-7.963 \mathrm{E}-01$

$-6.489 \mathrm{E}-02-1.626 \mathrm{E}-01 \quad 9.845 \mathrm{E}-01 \quad 4.410 \mathrm{E}-01 \quad 1.000 \mathrm{E}+00 \quad 0.000 \mathrm{E}+00$

$-6.489 \mathrm{E}-02-1.626 \mathrm{E}-01 \quad 9.845 \mathrm{E}-01$

$0-6.489 \mathrm{E}-02-1.626 \mathrm{E}-019.845 \mathrm{E}-01$

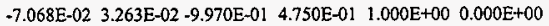
$-7.068 \mathrm{E}-02 \quad 3.263 \mathrm{E}-02-9.970 \mathrm{E}-01$

$0-7.068 \mathrm{E}-02 \quad 3.263 \mathrm{E}-02-9.970 \mathrm{E}-01$

$\begin{array}{lllll}-3.915 \mathrm{E}-01 & 4.664 \mathrm{E}-01-7.932 \mathrm{E}-01 & 4.136 \mathrm{E}+00 & 1.000 \mathrm{E}+00 & 0.000 \mathrm{E}+00\end{array}$ $-3.915 \mathrm{E}-01 \quad 4.664 \mathrm{E}-01-7.932 \mathrm{E}-01$

$0-3.915 \mathrm{E}-01 \quad 4.664 \mathrm{E}-01-7.932 \mathrm{E}-01$

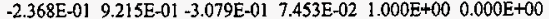

$0,-1,0) \quad-2.368 \mathrm{E}-01 \quad 9.215 \mathrm{E}-01-3.079 \mathrm{E}-01$

$0-2.368 \mathrm{E}-01 \quad 9.215 \mathrm{E}-01-3.079 \mathrm{E}-01$

$1.946 \mathrm{E}-01-3.204 \mathrm{E}-01 \quad 9.271 \mathrm{E}-01 \quad 3.128 \mathrm{E}+00 \quad 1.000 \mathrm{E}+00 \quad 0.000 \mathrm{E}+00$ $1.946 \mathrm{E}-01-3.204 \mathrm{E}-01 \quad 9.271 \mathrm{E}-01$

$0 \quad 1.946 \mathrm{E}-01-3.204 \mathrm{E}-01 \quad 9.271 \mathrm{E}-01$

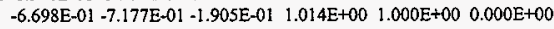
$-6.698 \mathrm{E}-01-7.177 \mathrm{E}-01-1.905 \mathrm{E}-01$

$0-6.698 \mathrm{E}-01-7.177 \mathrm{E}-01-1.905 \mathrm{E}-01$

$+8.398 \mathrm{E}-01-4.129 \mathrm{E}-01 \quad 3.524 \mathrm{E}-01 \quad 1.395 \mathrm{E}+00 \quad 1.000 \mathrm{E}+00 \quad 0.000 \mathrm{E}+00$

$-8.398 \mathrm{E}-01-4.129 \mathrm{E}-01 \quad 3.524 \mathrm{E}-01$

$0-8.398 \mathrm{E}-01-4.129 \mathrm{E}-01 \quad 3.524 \mathrm{E}-01$

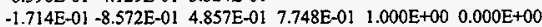

$-1.714 \mathrm{E}-01-8.572 \mathrm{E}-01 \quad 4.857 \mathrm{E}-01$

$0-1.714 \mathrm{E}-01-8.572 \mathrm{E}-01 \quad 4.857 \mathrm{E}-01$

$-2.489 \mathrm{E}-01-5.118 \mathrm{E}-01-8.222 \mathrm{E}-01 \quad 1.101 \mathrm{E}+00 \quad 1.000 \mathrm{E}+00 \quad 0.000 \mathrm{E}+00$ $-2.489 \mathrm{E}-01-5.118 \mathrm{E}-01-8.222 \mathrm{E}-01$

$0-2.489 \mathrm{E}-01-5.118 \mathrm{E}-01-8.222 \mathrm{E}-01$

$-2.959 \mathrm{E}-01 \quad 2.119 \mathrm{E}-01 \quad 9.314 \mathrm{E}-01 \quad 1.951 \mathrm{E}+00 \quad 1.000 \mathrm{E}+00 \quad 0.000 \mathrm{E}+00$ $-2.959 \mathrm{E}-01 \quad 2.119 \mathrm{E}-01 \quad 9.314 \mathrm{E}-01$

$0-2.959 \mathrm{E}-01 \quad 2.119 \mathrm{E}-01 \quad 9.314 \mathrm{E}-01$

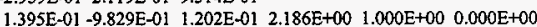
$1.395 \mathrm{E}-01-9.829 \mathrm{E}-01 \quad 1.202 \mathrm{E}-01$

$0 \quad 1.395 \mathrm{E}-01-9.829 \mathrm{E}-01 \quad 1.202 \mathrm{E}-01$

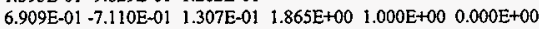
$6.909 \mathrm{E}-01+7.110 \mathrm{E}-01 \quad 1.307 \mathrm{E}-01$

$0 \quad 6.909 \mathrm{E}-01-7.110 \mathrm{E}-01 \quad 1.307 \mathrm{E}-01$

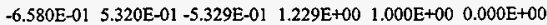
$-6.580 \mathrm{E}-015.320 \mathrm{E}-01-5.329 \mathrm{E}-01$

$0-6.580 \mathrm{E}-01 \quad 5.320 \mathrm{E}-01+5.329 \mathrm{E}-01$

$-9.903 \mathrm{E}-01-1.380 \mathrm{E}-01 \quad 1.353 \mathrm{E}-02 \quad 1.305 \mathrm{E}+00 \quad 1.000 \mathrm{E}+00 \quad 0.000 \mathrm{E}+00$ 


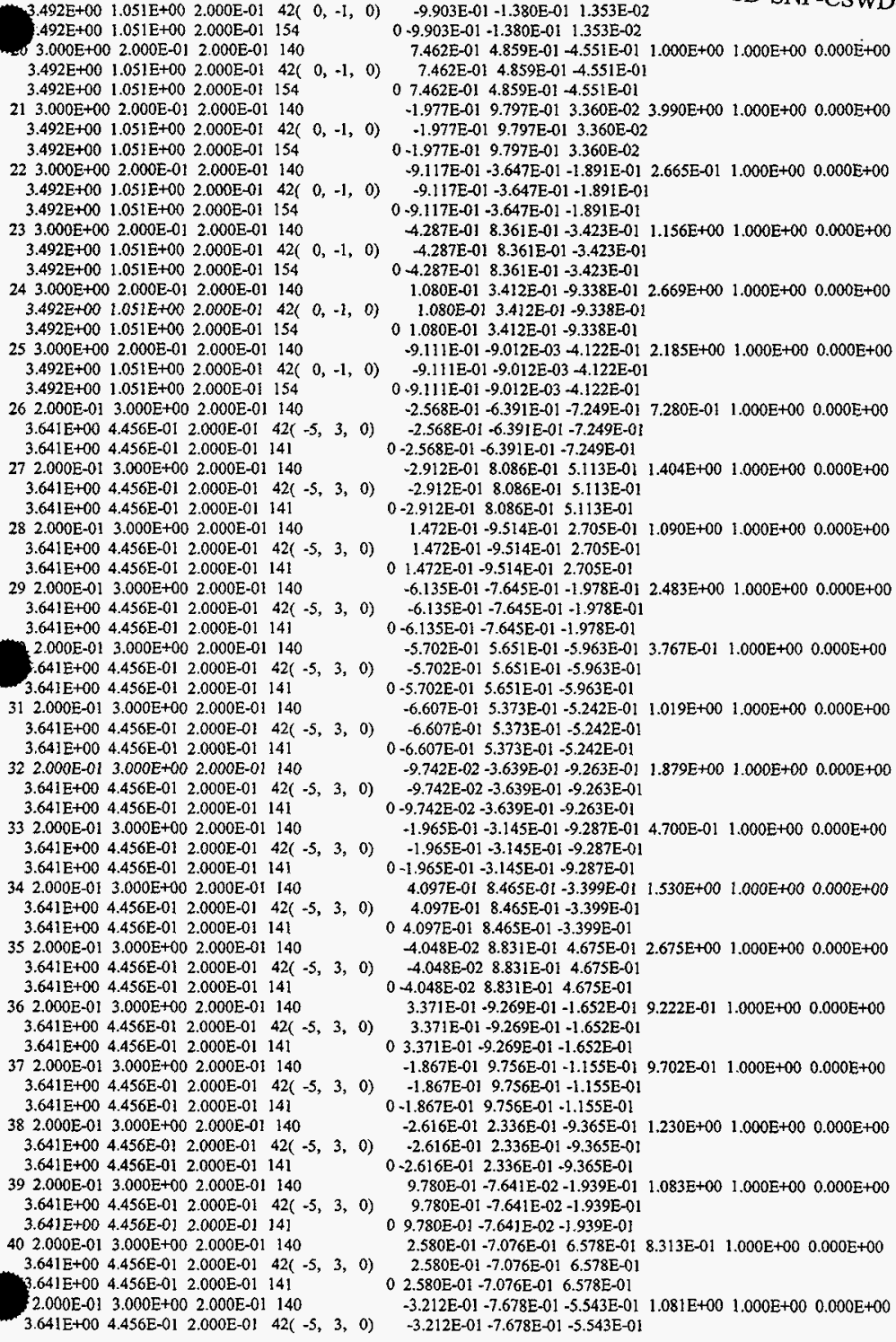




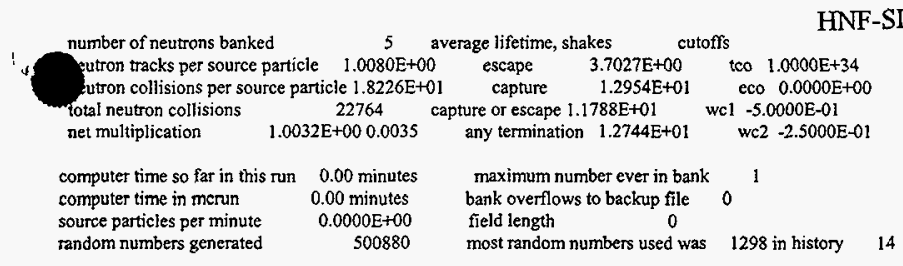

range of sampled source weights $=7.7821 \mathrm{E}-01$ to $1.0989 \mathrm{E}+00$

Ineutron activity in each cell

print table 126

tracks population collisions collisions number flux average average

cell entering * weight weighted weighted track weight track mfp (per history) energy energy (relative) (cm)

$\begin{array}{cccccccccccc}1 & 30 & 0 & 0 & 0 & 0.0000 \mathrm{E}+00 & 0.0000 \mathrm{E}+00 & 0.0000 \mathrm{E}+00 & 0.0000 \mathrm{E}+00 & 0.0000 \mathrm{E}+00 \\ 2 & 31 & 0 & 0 & 0 & 0.0000 \mathrm{E}+00 & 0.0000 \mathrm{E}+00 & 0.0000 \mathrm{E}+00 & 0.0000 \mathrm{E}+00 & 0.0000 \mathrm{E}+00 \\ 3 & 37 & 0 & 0 & 0 & 0.0000 \mathrm{E}+00 & 0.0000 \mathrm{E}+00 & 0.0000 \mathrm{E}+00 & 0.0000 \mathrm{E}+00 & 0.0000 \mathrm{E}+00 \\ 4 & 34 & 0 & 0 & 0 & 0.0000 \mathrm{E}+00 & 0.0000 \mathrm{E}+00 & 0.0000 \mathrm{E}+00 & 0.0000 \mathrm{E}+00 & 0.0000 \mathrm{E}+00 \\ 5 & 32 & 0 & 0 & 0 & 0.0000 \mathrm{E}+00 & 0.0000 \mathrm{E}+00 & 0.0000 \mathrm{E}+00 & 0.0000 \mathrm{E}+00 & 0.0000 \mathrm{E}+00 \\ 6 & 33 & 0 & 0 & 0 & 0.0000 \mathrm{E}+00 & 0.0000 \mathrm{E}+00 & 0.0000 \mathrm{E}+00 & 0.0000 \mathrm{E}+00 & 0.0000 \mathrm{E}+00 \\ 7 & 35 & 0 & 0 & 0 & 0.0000 \mathrm{E}+00 & 0.0000 \mathrm{E}+00 & 0.0000 \mathrm{E}+00 & 0.0000 \mathrm{E}+00 & 0.0000 \mathrm{E}+00 \\ 8 & 36 & 0 & 0 & 0 & 0.0000 \mathrm{E}+00 & 0.0000 \mathrm{E}+00 & 0.0000 \mathrm{E}+00 & 0.0000 \mathrm{E}+00 & 0.0000 \mathrm{E}+00 \\ 9 & 38 & 920 & 618 & 1249 & 5.9116 \mathrm{E}-01 & 3.0414 \mathrm{E}-01 & 8.9304 \mathrm{E}-01 & 6.4212 \mathrm{E}-01 & 2.6324 \mathrm{E}+00 \\ 10 & 39 & 1635 & 637 & 130 & 6.3779 \mathrm{E}-02 & 8.8236 \mathrm{E}-02 & 7.6766 \mathrm{E}-01 & 6.4931 \mathrm{E}-01 & 2.6947 \mathrm{E}+00 \\ 11 & 40 & 1728 & 663 & 184 & 8.8176 \mathrm{E}-02 & 7.6162 \mathrm{E}-02 & 7.6184 \mathrm{E}-01 & 6.4437 \mathrm{E}-01 & 2.5146 \mathrm{E}+00 \\ 12 & 41 & 1867 & 712 & 317 & 1.5811 \mathrm{E}-01 & 8.6238 \mathrm{E}-02 & 7.9306 \mathrm{E}-01 & 6.5030 \mathrm{E}-01 & 2.6940 \mathrm{E}+00 \\ 13 & 140 & 0 & 0 & 0 & 0.0000 \mathrm{E}+00 & 0.0000 \mathrm{E}+00 & 0.0000 \mathrm{E}+00 & 0.0000 \mathrm{E}+00 & 0.0000 \mathrm{E}+00 \\ 14 & 42 & 2069 & 867 & 441 & 2.1911 \mathrm{E}-01 & 8.9382 \mathrm{E}-02 & 7.8771 \mathrm{E}-01 & 6.6777 \mathrm{E}-01 & 2.5091 \mathrm{E}+00 \\ 15 & 154 & 25302 & 1253 & 8436 & 4.1390 \mathrm{E}+00 & 1.2606 \mathrm{E}-01 & 8.5005 \mathrm{E}-01 & 6.6920 \mathrm{E}-01 & 2.4712 \mathrm{E}+00 \\ 4 & 149 & 49542 & 1253 & 580 & 2.8963 \mathrm{E}-01 & 1.0795 \mathrm{E}-01 & 8.2361 \mathrm{E}-01 & 6.7129 \mathrm{E}-01 & 2.5408 \mathrm{E}+00 \\ 7 & 144 & 51224 & 1254 & 885 & 4.2652 \mathrm{E}-01 & 1.0541 \mathrm{E}-01 & 8.1599 \mathrm{E}-01 & 6.6971 \mathrm{E}-01 & 2.2391 \mathrm{E}+00 \\ 18 & 159 & 58650 & 1254 & 4019 & 2.1254 \mathrm{E}+00 & 9.8931 \mathrm{E}-02 & 8.1154 \mathrm{E}-01 & 6.6977 \mathrm{E}-01 & 2.7541 \mathrm{E}+00 \\ 19 & 141 & 73122 & 1254 & 6523 & 3.2229 \mathrm{E}+00 & 9.4499 \mathrm{E}-02 & 8.0639 \mathrm{E}-01 & 6.6810 \mathrm{E}-01 & 2.5056 \mathrm{E}+00\end{array}$

total $266059 \quad 9765 \quad 22764 \quad 1.1324 \mathrm{E}+01$

Ineutron weight balance in each celI - external events print table 130

cell entering source energy time exiting total
cutoff cutoff

I $30 \quad 0.0000 \mathrm{E}+00 \quad 0.0000 \mathrm{E}+00 \quad 0.0000 \mathrm{E}+00 \quad 0.0000 \mathrm{E}+00 \quad 0.0000 \mathrm{E}+00 \quad 0.0000 \mathrm{E}+00$

$\begin{array}{lllllllll}2 & 31 & 0.0000 \mathrm{E}+00 & 0.0000 \mathrm{E}+00 & 0.0000 \mathrm{E}+00 & 0.0000 \mathrm{E}+00 & 0.0000 \mathrm{E}+00 & 0.0000 \mathrm{E}+00\end{array}$

$337 \quad 0.0000 \mathrm{E}+00 \quad 0.0000 \mathrm{E}+00 \quad 0.0000 \mathrm{E}+00 \quad 0.0000 \mathrm{E}+00 \quad 0.0000 \mathrm{E}+00 \quad 0.0000 \mathrm{E}+00$

$\begin{array}{lllllllll}4 & 34 & 0.0000 \mathrm{E}+00 & 0.0000 \mathrm{E}+00 & 0.0000 \mathrm{E}+00 & 0.0000 \mathrm{E}+00 & 0.0000 \mathrm{E}+00 & 0.0000 \mathrm{E}+00\end{array}$

$\begin{array}{lllllllll}5 & 32 & 0.0000 \mathrm{E}+00 & 0.0000 \mathrm{E}+00 & 0.0000 \mathrm{E}+00 & 0.0000 \mathrm{E}+00 & 0.0000 \mathrm{E}+00 & 0.0000 \mathrm{E}+00\end{array}$

$\begin{array}{lllllllll}6 & 33 & 0.0000 \mathrm{E}+00 & 0.0000 \mathrm{E}+00 & 0.0000 \mathrm{E}+00 & 0.0000 \mathrm{E}+00 & 0.0000 \mathrm{E}+00 & 0.0000 \mathrm{E}+00\end{array}$

$\begin{array}{llllllllll}7 & 35 & 0.0000 \mathrm{E}+00 & 0.0000 \mathrm{E}+00 & 0.0000 \mathrm{E}+00 & 0.0000 \mathrm{E}+00 & 0.0000 \mathrm{E}+00 & 0.0000 \mathrm{E}+00\end{array}$

$\begin{array}{lllllllll}8 & 36 & 0.0000 \mathrm{E}+00 & 0.0000 \mathrm{E}+00 & 0.0000 \mathrm{E}+00 & 0.0000 \mathrm{E}+00 & 0.0000 \mathrm{E}+00 & 0.0000 \mathrm{E}+00\end{array}$

$\begin{array}{lllllllll}9 & 38 & 4.8822 \mathrm{E}-0 \mathrm{l} & 0.0000 \mathrm{E}+00 & 0.0000 \mathrm{E}+00 & 0.0000 \mathrm{E}+00 & -3.3091 \mathrm{E}-01 & 1.5731 \mathrm{E}-01\end{array}$

$\begin{array}{llllllll}10 & 39 & 8.4996 \mathrm{E}-01 & 0.0000 \mathrm{E}+00 & 0.0000 \mathrm{E}+00 & 0.0000 \mathrm{E}+00 & -8.4933 \mathrm{E}-01 & 6.2750 \mathrm{E}-04\end{array}$

$11 \quad 40 \quad 8.9904 \mathrm{E}-01 \quad 0.0000 \mathrm{E}+00 \quad 0.0000 \mathrm{E}+00 \quad 0.0000 \mathrm{E}+00 \quad-8.9904 \mathrm{E}-01 \quad 3.9460 \mathrm{E}-06$

$\begin{array}{llllllll}12 & 41 & 9.7260 \mathrm{E}-01 & 0.0000 \mathrm{E}+00 & 0.0000 \mathrm{E}+00 & 0.0000 \mathrm{E}+00 & -9.6978 \mathrm{E}-01 & 2.8204 \mathrm{E}-03\end{array}$

$13 \quad 140 \quad 0.0000 \mathrm{E}+00 \quad 0.0000 \mathrm{E}+00 \quad 0.0000 \mathrm{E}+00 \quad 0.0000 \mathrm{E}+00 \quad 0.0000 \mathrm{E}+00 \quad 0.0000 \mathrm{E}+00$

$14 \quad 42 \quad 1.0907 \mathrm{E}+00 \quad 0.0000 \mathrm{E}+00 \quad 0.0000 \mathrm{E}+00 \quad 0.0000 \mathrm{E}+00 \quad-1.0913 \mathrm{E}+00 \quad-5.9111 \mathrm{E}-04$

$\begin{array}{llllllll}15 & 154 & 1.2943 \mathrm{E}+01 & 8.4067 \mathrm{E}-01 & 0.0000 \mathrm{E}+00 & 0.0000 \mathrm{E}+00 & -1.3171 \mathrm{E}+01 & 6.1278 \mathrm{E}-01\end{array}$

$\begin{array}{llllllll}16 & 149 & 2.6508 \mathrm{E}+01 & 0.0000 \mathrm{E}+00 & 0.0000 \mathrm{E}+00 & 0.0000 \mathrm{E}+00 & -2.6508 \mathrm{E}+01 & 3.5884 \mathrm{E}-05\end{array}$

$\begin{array}{llllllll}17 & 144 & 2.7419 \mathrm{E}+01 & 0.0000 \mathrm{E}+00 & 0.0000 \mathrm{E}+00 & 0.0000 \mathrm{E}+00 & -2.7405 \mathrm{E}+01 & 1.4572 \mathrm{E}-02\end{array}$

$\begin{array}{llllllll}18 & 159 & 3.1331 \mathrm{E}+01 & 8.0064 \mathrm{E}-02 & 0.0000 \mathrm{E}+00 & 0.0000 \mathrm{E}+00 & -3.1361 \mathrm{E}+01 \quad 5.0067 \mathrm{E}-02\end{array}$

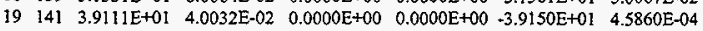

total $1.4161 \mathrm{E}+02 \quad 9.6077 \mathrm{E}-01 \quad 0.0000 \mathrm{E}+00 \quad 0.0000 \mathrm{E}+00 \quad-1.4174 \mathrm{E}+02 \quad 8.3809 \mathrm{E}-01$

tron weight balance in each cell - variance reduction events print table 130 
cell weight cell weight energy dxtran forced exponential total window importance cutoff importance collision transform

$\begin{array}{lllllllll}30 & 0.0000 \mathrm{E}+00 & 0.0000 \mathrm{E}+00 & 0.0000 \mathrm{E}+00 & 0.0000 \mathrm{E}+00 & 0.0000 \mathrm{E}+00 & 0.0000 \mathrm{E}+00 & 0.0000 \mathrm{E}+00 & 0.0000 \mathrm{E}+00\end{array}$

$\begin{array}{llllllllll}31 & 0.0000 \mathrm{E}+00 & 0.0000 \mathrm{E}+00 & 0.0000 \mathrm{E}+00 & 0.0000 \mathrm{E}+00 & 0.0000 \mathrm{E}+00 & 0.0000 \mathrm{E}+00 & 0.0000 \mathrm{E}+00 & 0.0000 \mathrm{E}+00\end{array}$

$\begin{array}{llllllllll}3 & 37 & 0.0000 \mathrm{E}+00 & 0.0000 \mathrm{E}+00 & 0.0000 \mathrm{E}+00 & 0.0000 \mathrm{E}+00 & 0.0000 \mathrm{E}+00 & 0.0000 \mathrm{E}+00 & 0.0000 \mathrm{E}+00 & 0.0000 \mathrm{E}+00\end{array}$

$\begin{array}{lllllllllll}4 & 34 & 0.0000 \mathrm{E}+00 & 0.0000 \mathrm{E}+00 & 0.0000 \mathrm{E}+00 & 0.0000 \mathrm{E}+00 & 0.0000 \mathrm{E}+00 & 0.0000 \mathrm{E}+00 & 0.0000 \mathrm{E}+00 & 0.0000 \mathrm{E}+00\end{array}$

$\begin{array}{lllllllllll}5 & 32 & 0.0000 \mathrm{E}+00 & 0.0000 \mathrm{E}+00 & 0.0000 \mathrm{E}+00 & 0.0000 \mathrm{E}+00 & 0.0000 \mathrm{E}+00 & 0.0000 \mathrm{E}+00 & 0.0000 \mathrm{E}+00 & 0.0000 \mathrm{E}+00\end{array}$

$\begin{array}{lllllllllll}6 & 33 & 0.0000 \mathrm{E}+00 & 0.0000 \mathrm{E}+00 & 0.0000 \mathrm{E}+00 & 0.0000 \mathrm{E}+00 & 0.0000 \mathrm{E}+00 & 0.0000 \mathrm{E}+00 & 0.0000 \mathrm{E}+00 & 0.0000 \mathrm{E}+00\end{array}$

$\begin{array}{llllllllllll}7 & 35 & 0.0000 \mathrm{E}+00 & 0.0000 \mathrm{E}+00 & 0.0000 \mathrm{E}+00 & 0.0000 \mathrm{E}+00 & 0.0000 \mathrm{E}+00 & 0.0000 \mathrm{E}+00 & 0.0000 \mathrm{E}+00 & 0.0000 \mathrm{E}+00\end{array}$

$\begin{array}{llllllllllll}8 & 36 & 0.0000 \mathrm{E}+00 & 0.0000 \mathrm{E}+00 & 0.0000 \mathrm{E}+00 & 0.0000 \mathrm{E}+00 & 0.0000 \mathrm{E}+00 & 0.0000 \mathrm{E}+00 & 0.0000 \mathrm{E}+00 & 0.0000 \mathrm{E}+00\end{array}$

$\begin{array}{llllllllll}9 & 38 & 0.0000 \mathrm{E}+00 & 0.0000 \mathrm{E}+00 & 1.2447 \mathrm{E}-03 & 0.0000 \mathrm{E}+00 & 0.0000 \mathrm{E}+00 & 0.0000 \mathrm{E}+00 & 0.0000 \mathrm{E}+00 & 1.2447 \mathrm{E}-03\end{array}$

$\begin{array}{lllllllllllll}10 & 39 & 0.0000 \mathrm{E}+00 & 0.0000 \mathrm{E}+00 & 2.0097 \mathrm{E}-04 & 0.0000 \mathrm{E}+00 & 0.0000 \mathrm{E}+00 & 0.0000 \mathrm{E}+00 & 0.0000 \mathrm{E}+00 & 2.0097 \mathrm{E}-04\end{array}$

$\begin{array}{lllllllllll}11 & 40 & 0.0000 \mathrm{E}+00 & 0.0000 \mathrm{E}+00 & 0.0000 \mathrm{E}+00 & 0.0000 \mathrm{E}+00 & 0.0000 \mathrm{E}+00 & 0.0000 \mathrm{E}+00 & 0.0000 \mathrm{E}+00 & 0.0000 \mathrm{E}+00\end{array}$

$\begin{array}{llllllllllll}12 & 41 & 0.0000 \mathrm{E}+00 & 0.0000 \mathrm{E}+00 & 2.9100 \mathrm{E}-04 & 0.0000 \mathrm{E}+00 & 0.0000 \mathrm{E}+00 & 0.0000 \mathrm{E}+00 & 0.0000 \mathrm{E}+00 & 2.9100 \mathrm{E}-04\end{array}$

$\begin{array}{lllllllllll}13 & 140 & 0.0000 \mathrm{E}+00 & 0.0000 \mathrm{E}+00 & 0.0000 \mathrm{E}+00 & 0.0000 \mathrm{E}+00 & 0.0000 \mathrm{E}+00 & 0.0000 \mathrm{E}+00 & 0.0000 \mathrm{E}+00 & 0.0000 \mathrm{E}+00\end{array}$

$\begin{array}{llllllllllll}14 & 42 & 0.0000 \mathrm{E}+00 & 0.0000 \mathrm{E}+00 & 0.0000 \mathrm{E}+00 & 0.0000 \mathrm{E}+00 & 0.0000 \mathrm{E}+00 & 0.0000 \mathrm{E}+00 & 0.0000 \mathrm{E}+00 & 0.0000 \mathrm{E}+00\end{array}$

$\begin{array}{llllllllllll}15 & 154 & 0.0000 \mathrm{E}+00 & 0.0000 \mathrm{E}+00 & 8.0354 \mathrm{E}-03 & 0.0000 \mathrm{E}+00 & 0.0000 \mathrm{E}+00 & 0.0000 \mathrm{E}+00 & 0.0000 \mathrm{E}+00 & 8.0354 \mathrm{E}-03\end{array}$

$\begin{array}{lllllllllll}16 & 149 & 0.0000 \mathrm{E}+00 & 0.0000 \mathrm{E}+00 & 0.0000 \mathrm{E}+00 & 0.0000 \mathrm{E}+00 & 0.0000 \mathrm{E}+00 & 0.0000 \mathrm{E}+00 & 0.0000 \mathrm{E}+00 & 0.0000 \mathrm{E}+00\end{array}$

$17 \quad 144 \quad 0.0000 \mathrm{E}+00 \quad 0.0000 \mathrm{E}+00 \quad-3.0848 \mathrm{E}-06 \quad 0.0000 \mathrm{E}+00 \quad 0.0000 \mathrm{E}+00 \quad 0.0000 \mathrm{E}+00 \quad 0.0000 \mathrm{E}+00 \quad-3.0848 \mathrm{E}-06$

$\begin{array}{llllllllll}18 & 159 & 0.0000 \mathrm{E}+00 & 0.0000 \mathrm{E}+00 & -2.5216 \mathrm{E}-04 & 0.0000 \mathrm{E}+00 & 0.0000 \mathrm{E}+00 & 0.0000 \mathrm{E}+00 & 0.0000 \mathrm{E}+00 & -2.5216 \mathrm{E}-04\end{array}$

$\begin{array}{llllllllll}19 & 141 & 0.0000 \mathrm{E}+00 & 0.0000 \mathrm{E}+00 & 0.0000 \mathrm{E}+00 & 0.0000 \mathrm{E}+00 & 0.0000 \mathrm{E}+00 & 0.0000 \mathrm{E}+00 & 0.0000 \mathrm{E}+00 & 0.0000 \mathrm{E}+00\end{array}$

total $\quad 0.0000 \mathrm{E}+00 \quad 0.0000 \mathrm{E}+00 \quad 9.5169 \mathrm{E}-03 \quad 0.0000 \mathrm{E}+00 \quad 0.0000 \mathrm{E}+00 \quad 0.0000 \mathrm{E}+00 \quad 0.0000 \mathrm{E}+00 \quad 9.5169 \mathrm{E}-03$ Ineutron weight balance in each cell -- physical events print table 130

cell $(\mathrm{n}, \mathrm{xn})$ fission capture loss to loss to total $(\mathrm{n}, \mathrm{xn})$ fission

$\begin{array}{llllllll}30 & 0.0000 \mathrm{E}+00 & 0.0000 \mathrm{E}+00 & 0.0000 \mathrm{E}+00 & 0.0000 \mathrm{E}+00 & 0.0000 \mathrm{E}+00 & 0.0000 \mathrm{E}+00\end{array}$

$\begin{array}{lllllllll}2 & 31 & 0.0000 \mathrm{E}+00 & 0.0000 \mathrm{E}+00 & 0.0000 \mathrm{E}+00 & 0.0000 \mathrm{E}+00 & 0.0000 \mathrm{E}+00 & 0.0000 \mathrm{E}+00\end{array}$

$\begin{array}{lllllllll}3 & 37 & 0.0000 \mathrm{E}+00 & 0.0000 \mathrm{E}+00 & 0.0000 \mathrm{E}+00 & 0.0000 \mathrm{E}+00 & 0.0000 \mathrm{E}+00 & 0.0000 \mathrm{E}+00\end{array}$

$\begin{array}{lllllllll}4 & 34 & 0.0000 \mathrm{E}+00 & 0.0000 \mathrm{E}+00 & 0.0000 \mathrm{E}+00 & 0.0000 \mathrm{E}+00 & 0.0000 \mathrm{E}+00 & 0.0000 \mathrm{E}+00\end{array}$ $\begin{array}{llllllll}32 & 0.0000 \mathrm{E}+00 & 0.0000 \mathrm{E}+00 & 0.0000 \mathrm{E}+00 & 0.0000 \mathrm{E}+00 & 0.0000 \mathrm{E}+00 & 0.0000 \mathrm{E}+00\end{array}$ $\begin{array}{llllllll}33 & 0.0000 \mathrm{E}+00 & 0.0000 \mathrm{E}+00 & 0.0000 \mathrm{E}+00 & 0.0000 \mathrm{E}+00 & 0.0000 \mathrm{E}+00 & 0.0000 \mathrm{E}+00\end{array}$ $\begin{array}{llllllll}35 & 0.0000 \mathrm{E}+00 & 0.0000 \mathrm{E}+00 & 0.0000 \mathrm{E}+00 & 0.0000 \mathrm{E}+00 & 0.0000 \mathrm{E}+00 & 0.0000 \mathrm{E}+00\end{array}$

$\begin{array}{lllllllll}8 & 36 & 0.0000 \mathrm{E}+00 & 0.0000 \mathrm{E}+00 & 0.0000 \mathrm{E}+00 & 0.0000 \mathrm{E}+00 & 0.0000 \mathrm{E}+00 & 0.0000 \mathrm{E}+00\end{array}$

$938 \quad 0.0000 \mathrm{E}+00 \quad 0.0000 \mathrm{E}+00-1.5855 \mathrm{E}-01 \quad 0.0000 \mathrm{E}+00 \quad 0.0000 \mathrm{E}+00+1.5855 \mathrm{E}-01$

$10 \quad 39 \quad 0.0000 \mathrm{E}+00 \quad 0.0000 \mathrm{E}+00 \quad-8.2847 \mathrm{E}-04 \quad 0.0000 \mathrm{E}+00 \quad 0.0000 \mathrm{E}+00 \quad-8.2847 \mathrm{E}-04$

i) $40 \quad 0.0000 \mathrm{E}+00 \quad 0.0000 \mathrm{E}+00 \quad+3.9460 \mathrm{E}-06 \quad 0.0000 \mathrm{E}+00 \quad 0.0000 \mathrm{E}+00 \quad-3.9460 \mathrm{E}-06$

$\begin{array}{lllllllll}12 & 41 & 0.0000 \mathrm{E}+00 & 0.0000 \mathrm{E}+00 & -3.1114 \mathrm{E}-03 & 0.0000 \mathrm{E}+00 & 0.0000 \mathrm{E}+00 & -3.1114 \mathrm{E}-03\end{array}$

$\begin{array}{llllllllll}13 & 140 & 0.0000 \mathrm{E}+00 & 0.0000 \mathrm{E}+00 & 0.0000 \mathrm{E}+00 & 0.0000 \mathrm{E}+00 & 0.0000 \mathrm{E}+00 & 0.0000 \mathrm{E}+00\end{array}$

$\begin{array}{llllllll}14 & 42 & 1.2461 \mathrm{E}-03 & 0.0000 \mathrm{E}+00 & -3.1955 \mathrm{E}-05 & -6.2306 \mathrm{E}-04 & 0.0000 \mathrm{E}+00 & 5.9111 \mathrm{E}-04\end{array}$

$15 \quad 154 \quad 4.9179 \mathrm{E}-03 \quad 0.0000 \mathrm{E}+00-1.7239 \mathrm{E}-01 \quad-2.4589 \mathrm{E}-03 \quad-4.5089 \mathrm{E}-01 \quad-6.2082 \mathrm{E}-01$

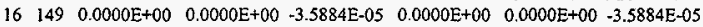

$\begin{array}{llllllll}17 & 144 & 0.0000 \mathrm{E}+00 & 0.0000 \mathrm{E}+00 & -1.4569 \mathrm{E}-02 & 0.0000 \mathrm{E}+00 & 0.0000 \mathrm{E}+00 & -1.4569 \mathrm{E}-02\end{array}$

$\begin{array}{llllllll}18 & 159 & 0.0000 \mathrm{E}+00 & 0.0000 \mathrm{E}+00 & -4.9815 \mathrm{E}-02 & 0.0000 \mathrm{E}+00 & 0.0000 \mathrm{E}+00 & -4.9815 \mathrm{E}-02\end{array}$

$\begin{array}{llllllll}19 & 141 & 0.0000 \mathrm{E}+00 & 0.0000 \mathrm{E}+00 & -4.5860 \mathrm{E}-04 & 0.0000 \mathrm{E}+00 & 0.0000 \mathrm{E}+00 & -4.5860 \mathrm{E}-04\end{array}$

total $\quad 6.1640 \mathrm{E}-03 \quad 0.0000 \mathrm{E}+00 \quad-3.9980 \mathrm{E}-01 \quad-3.0820 \mathrm{E}-03 \quad-4.5089 \mathrm{E}-01 \quad-8.4760 \mathrm{E}-01$

Ineutton activity of each nuclide in each cell, per source particle

print table 140

cell nuclides atom total collisions weight lost weight loss weight gain fraction collisions * weight to capture to fission by $(n, x n)$

$\begin{array}{lllll}9 & 38 & 5010.03 \mathrm{~d} & 7.1977 \mathrm{E}-01\end{array}$ $5011.40 \mathrm{c} \quad 8.0041 \mathrm{E}-02$ $6012.40 \mathrm{c} \quad 2.0019 \mathrm{E}-01$

$10 \quad 39 \quad 41093.40 \mathrm{c} \quad 9.8982 \mathrm{E}-01$ $40000.40 \mathrm{c} \quad 1.0183 \mathrm{E}-02$

$11 \quad 40 \quad 1001.00 \mathrm{c} \quad 3.3333 \mathrm{E}-01$ $1002.55 \mathrm{c} \quad 3.3333 \mathrm{E}-01$ $8016.40 \mathrm{c} \quad 3.3333 \mathrm{E}-01$

$4 ! \quad 41093.40 \mathrm{c} \quad 9.8982 \mathrm{E}-01$
$941 \quad 4.4212 \mathrm{E}-0] \quad 1.5853 \mathrm{E}-01 \quad 0.0000 \mathrm{E}+00 \quad 0.0000 \mathrm{E}+00$

$\begin{array}{lllll}76 & 3.6073 \mathrm{E}-02 & 1.3591 \mathrm{E}-07 & 0.0000 \mathrm{E}+00 & 0.0000 \mathrm{E}+00\end{array}$

$232 \quad 1.1297 \mathrm{E}-01 \quad 1.8446 \mathrm{E}-05 \quad 0.0000 \mathrm{E}+00 \quad 0.0000 \mathrm{E}+00$

$\begin{array}{llllll}128 & 6.2802 \mathrm{E}-02 & 8.2814 \mathrm{E}-04 & 0.0000 \mathrm{E}+00 & 0.0000 \mathrm{E}+00\end{array}$

$2 \quad 9.7676 \mathrm{E}-04 \quad 3.3160 \mathrm{E}-07 \quad 0.0000 \mathrm{E}+00 \quad 0.0000 \mathrm{E}+00$

$111 \quad 5.1533 \mathrm{E}-02 \quad 3.9251 \mathrm{E}-06 \quad 0.0000 \mathrm{E}+00 \quad 0.0000 \mathrm{E}+00$

$33 \quad 1.5373 \mathrm{E}-02 \quad 2.0884 \mathrm{E}-08 \quad 0.0000 \mathrm{E}+00 \quad 0.0000 \mathrm{E}+00$

$\begin{array}{lllll}40 & 2.1271 \mathrm{E}-02 & 0.0000 \mathrm{E}+00 & 0.0000 \mathrm{E}+00 & 0.0000 \mathrm{E}+00\end{array}$ 


\section{$40000.40 \mathrm{C} \quad$ 1.0183E-02 \\ $42 \quad 1001.00 \mathrm{c} \quad 3.3333 \mathrm{E}-01$ \\ $1002.55 \mathrm{c} \quad 3.3333 \mathrm{E}-01$ \\ $8016.40 \mathrm{c} \quad 3.3333 \mathrm{E}-01$}
$15 \quad 154 \quad 92235.40 \mathrm{c} \quad 3.7504 \mathrm{E}-01$ $92238.40 \mathrm{C} \quad 1.2499 \mathrm{E}-01$ 7014.40c 4.9997E-01

I6 $149 \quad 1001.00 \mathrm{c} \quad 3.3333 \mathrm{E}-01$ $1002.55 \mathrm{c} \quad 3.3333 \mathrm{E}-01$ $8016.40 \mathrm{c} \quad 3.3333 \mathrm{E}-01$
$4 \quad 1.8747 \mathrm{E}-03 \quad 4.2322 \mathrm{E}-06 \quad 0.0000 \mathrm{E}+00 \quad 0.0000 \mathrm{E}+00$

$257 \quad 1.2131 \mathrm{E}-01 \quad 4.3914 \mathrm{E}-06 \quad 0.0000 \mathrm{E}+00 \quad 0.0000 \mathrm{E}+00$

$\begin{array}{lllll}86 & 4.6121 \mathrm{E}-02 & 6.8027 \mathrm{E}-08 & 0.0000 \mathrm{E}+00 & 6.2306 \mathrm{E}-04\end{array}$

$\begin{array}{lllll}98 & 5.1682 \mathrm{E}-02 & 2.7495 \mathrm{E}-05 & 0.0000 \mathrm{E}+00 & 0.0000 \mathrm{E}+00\end{array}$ $\begin{array}{cccccc}4759 & 2.3227 \mathrm{E}+00 & 1.3244 \mathrm{E}-01 & 4.4247 \mathrm{E}-01 & 5.8410 \mathrm{E}-04 \\ 1470 & 7.3870 \mathrm{E}-01 & 2.3272 \mathrm{E}-02 & 8.4162 \mathrm{E}-03 & 1.8748 \mathrm{E}-03 \\ 2207 & 1.0775 \mathrm{E}+00 & 1.6682 \mathrm{E}-02 & 0.0000 \mathrm{E}+00 & 0.0000 \mathrm{E}+00\end{array}$

\begin{abstract}
$345 \quad 1.6812 \mathrm{E}-01 \quad 4.4875 \mathrm{E}-06 \quad 0.0000 \mathrm{E}+00 \quad 0.0000 \mathrm{E}+00$
$110 \quad 5.8433 \mathrm{E}-02 \quad 9.1921 \mathrm{E}-08 \quad 0.0000 \mathrm{E}+00 \quad 0.0000 \mathrm{E}+00$

$125 \quad 6.3072 \mathrm{E}-02 \quad 3.1305 \mathrm{E}-05 \quad 0.0000 \mathrm{E}+00 \quad 0.0000 \mathrm{E}+00$
\end{abstract}

\begin{abstract}
$885 \quad 4.2652 \mathrm{E}-01 \quad 1.4569 \mathrm{E}-02 \quad 0.0000 \mathrm{E}+00 \quad 0.0000 \mathrm{E}+00$
$3965 \quad 2.1009 \mathrm{E}+00 \quad 4.9769 \mathrm{E}-02 \quad 0.0000 \mathrm{E}+00 \quad 0.0000 \mathrm{E}+00$ $\begin{array}{lllll}54 & 2.4590 \mathrm{E}-02 & 4.6175 \mathrm{E}-05 & 0.0000 \mathrm{E}+00 & 0.0000 \mathrm{E}+00\end{array}$
\end{abstract}

$4029 \quad 1.9096 \mathrm{E}+00 \quad 7.9511 \mathrm{E}-05 \quad 0.0000 \mathrm{E}+00 \quad 0.0000 \mathrm{E}+00$

$\begin{array}{llllll}1122 & 5.8206 \mathrm{E}-01 & 9.0056 \mathrm{E}-07 & 0.0000 \mathrm{E}+00 & 0.0000 \mathrm{E}+00\end{array}$

$\begin{array}{lllll}1372 & 7.3131 \mathrm{E}-01 & 3.7819 \mathrm{E}-04 & 0.0000 \mathrm{E}+00 & 0.0000 \mathrm{E}+00\end{array}$

total $\begin{array}{llllll}22764 & 1.1324 \mathrm{E}+01 & 3.9980 \mathrm{E}-01 & 4.5089 \mathrm{E}-01 & 3.0820 \mathrm{E}-03\end{array}$

total over all cells for each nuclide total collisions weight lost weight loss weight gain collisions - weight to capture to fission by $(n, x n)$
$1001.00 \mathrm{c}$
$1002.55 \mathrm{c}$
$\begin{array}{lllll}4742 & 2.2505 \mathrm{E}+00 & 9.2315 \mathrm{E}-05 & 0.0000 \mathrm{E}+00 & 0.0000 \mathrm{E}+00\end{array}$
$5011.40 \mathrm{c}$
$1351 \quad 7.0199 \mathrm{E}-01 \quad 1.0814 \mathrm{E}-06 \quad 0.0000 \mathrm{E}+00 \quad 6.2306 \mathrm{E}-04$
$6012.40 \mathrm{c}$
$\begin{array}{llllll}76 & 3.6073 \mathrm{E}-02 & 1.3591 \mathrm{E}-07 & 0.0000 \mathrm{E}+00 & 0.0000 \mathrm{E}+00\end{array}$
$7014.40 \mathrm{c}$
$8016.40 \mathrm{c}$
$40000.40 c$
$41093.40 \mathrm{c}$
$74000.40 \mathrm{c}$
$92235.40 \mathrm{c}$
$92238.40 \mathrm{c}$
$232 \quad 1.1297 \mathrm{E}-01 \quad 1.8446 \mathrm{E}-05 \quad 0.0000 \mathrm{E}+00 \quad 0.0000 \mathrm{E}+00$
$\begin{array}{llllll}2207 & 1.0775 \mathrm{E}+00 & 1.6682 \mathrm{E}-02 & 0.0000 \mathrm{E}+00 & 0.0000 \mathrm{E}+00\end{array}$
$1635 \quad 8.6733 \mathrm{E}-01 \quad 4.3699 \mathrm{E}-04 \quad 0.0000 \mathrm{E}+00 \quad 0.0000 \mathrm{E}+00$
$\begin{array}{lllll}60 & 2.7441 \mathrm{E}-02 & 5.0739 \mathrm{E}-05 & 0.0000 \mathrm{E}+00 & 0.0000 \mathrm{E}+00\end{array}$
$\begin{array}{lllll}4406 & 2.3199 \mathrm{E}+00 \quad 5.3704 \mathrm{E}-02 & 0.0000 \mathrm{E}+00 & 0.0000 \mathrm{E}+00\end{array}$
$\begin{array}{lllll}885 & 4.2652 \mathrm{E}-01 & 1.4569 \mathrm{E}-02 & 0.0000 \mathrm{E}+00 & 0.0000 \mathrm{E}+00\end{array}$
$\begin{array}{lllll}4759 & 2.3227 \mathrm{E}+00 & 1.3244 \mathrm{E}-01 & 4.4247 \mathrm{E}-01 & 5.8410 \mathrm{E}-04\end{array}$
$1470 \quad 7.3870 \mathrm{E}-01 \quad 2.3272 \mathrm{E}-02 \quad 8.4162 \mathrm{E}-03 \quad 1.8748 \mathrm{E}-03$
$5010.03 \mathrm{~d}$
$941 \quad 4.4212 \mathrm{E}-01 \quad 1.5853 \mathrm{E}-01 \quad 0.0000 \mathrm{E}+00 \quad 0.0000 \mathrm{E}+00$

the initial fission neutron source distribution used the 8 source points that were input on the ksic card. the criticality problem was scheduled to skip 5 cycles and run a total of 6 cycles with nominally 200 neutrons per cycle. this problem has run 5 inactive cycles with 1027 neutron histories and 1 active cycles with 222 neutron histories.

this calculation has completed the requested number of keff cycles using a total of 1249 fission neutron source histories. all cells with fissionable material were sampled and had fission neutron source points.

there is no combined collision/absorption/track-length estimate for keff because only 1 active cycles were run.

lindividual and average keff estimator results by cycle

keff neutron keff estimators by cycle average keff estimators and deviations average $k(c / a / t)$

cycle histories $k$ (coll) $k$ (abs) $k$ (track) $k$ (coll) st dev $k$ (abs) st dev $k$ (track) st dev $k(c / a / t)$ st dev fom

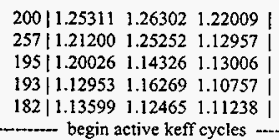


tally for neutrons

ber of histories used for normalizing tallies $=200.00$

HNF-SD-SNF-CSWD-005, Rev. 0

cell $a$ is $(154<42[-10-10] 42[-9-10] 42[-8-10] 42[-7-10] 42[-6-10])$

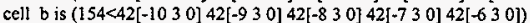

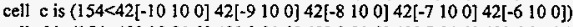

cell $d$ is $\left(154<42[-10210) 42[-9210] 42\left[\begin{array}{lll}-8 & 21 & 0\end{array}\right) 42[-7210] 42\left[\begin{array}{lll}-6 & 21 & 0\end{array}\right]\right)$

cell $e$ is $(154<42[-10290] 42[-9290] 42[-8290] 42[-72900] 42[-6290])$

volumes

cell: $a$ b $\quad c \quad d \quad e$

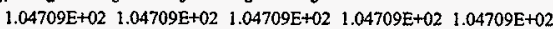

energy: $\quad 1.0000 \mathrm{E}-02 \quad 1.0000 \mathrm{E}-01 \quad 1.0000 \mathrm{E}+00 \quad 1.0000 \mathrm{E}+01$ total

cell

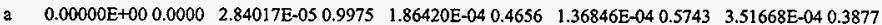

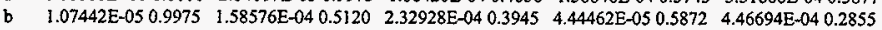

c $\quad 2.16778 \mathrm{E}-05 \quad 0.7232 \quad 9.40943 \mathrm{E}-05 \quad 0.5519 \quad 5.37835 \mathrm{E}-05 \quad 0.5876 \quad 1.51034 \mathrm{E}-040.7075 \quad 3.20589 \mathrm{E}-040.3937$

d $\quad 1.47903 \mathrm{E}-05 \quad 0.5831 \quad 0.00000 \mathrm{E}+00 \quad 0.0000 \quad 1.15515 \mathrm{E}-040.5838 \quad 1.69653 \mathrm{E}-04 \quad 0.5071 \quad 2.99958 \mathrm{E}-040.3718$

e $\quad 8.04943 \mathrm{E}-060.9975 \quad 0.00000 \mathrm{E}+000.0000 \quad 0.00000 \mathrm{E}+000.0000 \quad 6.81909 \mathrm{E}-05 \quad 0.7477 \quad 7.62403 \mathrm{E}-05 \quad 0.6763$

lanalysis of the results in the tally fluctuation chart bin (tfc) for tally 4 with nps $=1249$ print table 160

normed average tally per history $=3.51668 \mathrm{E}-04$

estimated tally relative error $=0.3877$

relative error from zero tallies $=0.3464$

number of nonzero history tallies $=\quad 8$

history number of largest tally $=\quad 1034$

(largest tally) $/($ average tally) $=3.84476 \mathrm{E}+01$

(confidence interval shift)/mean $=0.0793$ unnormed average tally per history $=3.68228 \mathrm{E}-02$

estimated variance of the variance $=0.1752$

relative error from nonzero scores $=0.1740$

efficiency for the nonzero tallies $=0.0400$

largest unnormalized history tally $=1.41575 \mathrm{E}+00$

(largest tally $)($ avg nonzero tally $)=1.53791 \mathrm{E}+00$

shifted confidence interval center $=3.79539 \mathrm{E}-04$

he largest history score sampled so far were to occur on the very next history, the tfe bin quantities would change as follows: 222 for this table because 5 keff cycles and 1027 histories were skipped before tally accumulation.

estimated quantities value at nps value at nps+1 value(nps+1)/value(nps)-1.

$\begin{array}{lccc}\text { mean } & 3.51668 \mathrm{E}-04 & 4.10723 \mathrm{E}-04 & 0.167927 \\ \text { relative error } & 3.87653 \mathrm{E}-01 & 3.61597 \mathrm{E}-01 & -0.067213 \\ \text { variance of the variance } & 1.75161 \mathrm{E}-01 & 1.47665 \mathrm{E}-01 & -0.156976 \\ \text { shifted center } & 3.79539 \mathrm{E}-04 & 3.77169 \mathrm{E}-04 & -0.006244 \\ \text { figure of merit } & 0.00000 \mathrm{E}+00 & 0.00000 \mathrm{E}+00 & 0.000000\end{array}$

there is not enough information in the largest history scores (usually less than 500 scores) for a reliable estimate of the slope. the history score probability density function appears to have an unsampled region at the largest history scores: please examine.

***** the nps-dependent tfc bin check results are suspect because there are only I nps tally values to analyze *****

results of 10 statistical checks for the estimated answer for the tally flucruation chart (tfc) bin of tally 4

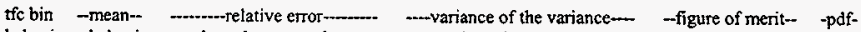

behavior behavior value decrease decrease mate value decrease decrease rate value behavior slope

desired random $<0.10$ yes $1 /$ sqrt(nps) $<0.10$ yes $1 / \mathrm{nps}$ constant random $>3.00$

observed random 0.39 yes yes 0.18 yes yes 0.00

passed? yes no yes yes no yes yes no 
waming. the tally in the tally fluctuation chart bin did not pass 3 of the 10 statistical checks.

HNF-SD-SNF-CSWD-005, Rev. 0

nonzero tally $\operatorname{mean}(\mathrm{m})=9.206 \mathrm{E}-01$ nps $=1249$ print table 161

ordinate $\log$ plot of tally probability density function in tally fluctuation chart bin(d=decade,slope $=0.0)$

tally number num den log den:d-1-

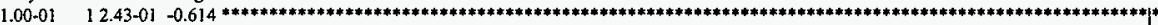

$1.26-0100.00+00 \quad 0.000$

$1.58-01 \quad 00.00+00 \quad 0.000$

$2.00-01 \quad 00.00+00 \quad 0.000$

$2.51-01 \quad 0.0 .00+00 \quad 0.000$

3.16-01 $00.00+00 \quad 0.000$

$\begin{array}{lll}3.98-01 & 0.000+00 & 0.000\end{array}$

$5.01-01 \quad 0.000+00 \quad 0.000$

$6.31-01 \quad 27.71-02-1.113 * * * * * * * * * * * * * * * * * * * * * * * * * * * * * * * * * * * * * * * * * * * * * * *$

$7.94-01 \quad 0.0 .00+00 \quad 0.000$

$1.00+00 \quad 12.43-02-1.614 \mathrm{~m}$

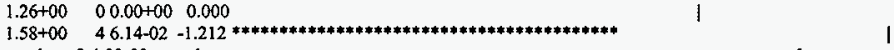

total $84.00-02$

cumulative tally number for tally $4 \quad$ nonzero tally mean $(m)=9.206 \mathrm{E}-01 \quad$ nps $=1249$ print table 162

abscissa cum ordinate plot of the cumulative number of tallies in the tally fluctuation chart bin from 0 to 100 percent

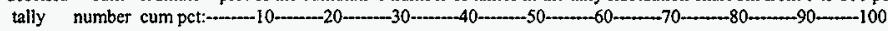

$1.00000 \mathrm{E}-01 \quad 112.500\} * * * * * * * * * ; * *$

$1.25893 \mathrm{E}-01$ i $\left.12.500\right|^{* * * * * * * * * * * * *}$

$1.58489 \mathrm{E}-01$ I 12.500$\}^{* * * * * * * * * * * * *}$

$1.99526 \mathrm{E}-01 \quad 1 \quad 12.500 \mid * * * * * * * * * * *$

$2.51189 \mathrm{E}-01$ I $\left.12.500\right|^{* * * * * * * * * * * * *}$

$3.16228 \mathrm{E}-01$

$3.98108 \mathrm{E}-01$

$5.01188 \mathrm{E}-01$

Q958E-01

329E-01

$00000 \mathrm{E}+00$

$1.25893 \mathrm{E}+00$

$1.58489 \mathrm{E}+00$

totat

$\left.112.500\right|^{\left.* * * * * * * * *\right|^{* * *}}$

$\left.12.500\right|^{* * * * * * * * * * * * *}$

$12.500|* * * * * * * * *| * * *$

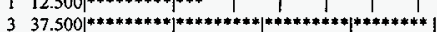

$337.500|* * * * * * * * *| * * * * * * * * *|* * * * * * * * *| * * * * * * * * \mid$

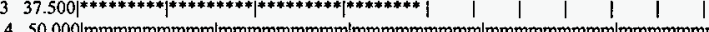

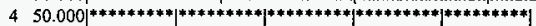

$8100.000]^{* * * * * * * * *|* * * * * * * * *| * * * * * * * * * * * * * * * * * * *|* * * 0|}$

$0-20-30-30-30$

$* * *$

8 100.000:-_- 10

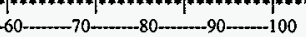

cumulative unnormed tally for tally $4 \quad$ nonzero tally $\operatorname{mean}(\mathrm{m})=9.206 \mathrm{E}-01 \quad \mathrm{nps}=1249$ print table 162

abscissa cum ordinate plot of the cumulative tally in the tally fluctuation chart bin from 0 to 100 percent

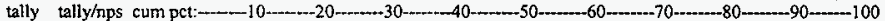

$1.000 \mathrm{E}-01 \quad 4.632 \mathrm{E}-\left.04 \quad 1.258\right|^{*}$

$1.259 \mathrm{E}-01 \quad 4.632 \mathrm{E}-04 \quad 1.2588^{*}$

$1.585 \mathrm{E}-01 \quad 4.632 \mathrm{E}-\left.04 \quad 1.258\right|^{*}$

$1.995 \mathrm{E}-01 \quad 4.632 \mathrm{E}-04 \quad 1.2588^{*}$

$2.512 \mathrm{E}-01 \quad 4.632 \mathrm{E}-04 \quad 1.2588^{*}$

$3.162 \mathrm{E}-01 \quad 4.632 \mathrm{E}-\left.04 \quad 1.258\right|^{*}$

$3.981 \mathrm{E}-01 \quad 4.632 \mathrm{E}-04 \quad 1.2588^{*}$

$5.012 \mathrm{E}-01 \quad 4.632 \mathrm{E}-\left.04 \quad 1.258\right|^{*}$

6.310E-01 $6.195 \mathrm{E}-03 \quad 16.825 *$

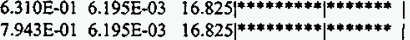

$1.000 \mathrm{E}+00 \quad 1.017 \mathrm{E}-02 \quad 27.628 / \mathrm{mmmmmmmmm}$ immmmmmmmm[mmmmmmmm |

1.259E+00 $1.017 \mathrm{E}-02 \quad 27.628]^{* * * * * * * * *|* * * * * * * * *| * * * * * * * * \mid}$

total $3.68228 \mathrm{E}-02100000$ -

-10 -

20

30 .

$40-30$

$-60$
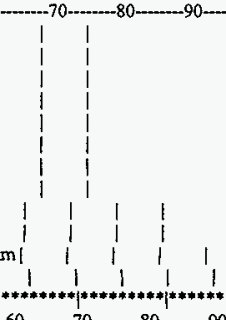

1

1status of the statistical checks used to form confidence intervals for the mean for each tally bin

tally result of statistical checks for the tfe bin (the first check not passed is listed) and error magnitude check for all bins

4 missed 3 of 10 tf bin checks: the relative error exceeds the recommended value of 0.1 for nonpoint detector tallies missed all bin error check: 25 tally bins had 4 bins with zeros and 21 bins with relative errors exceeding 0.10 
the 10 statistical checks are only for the tally fluctuation char bin and do not apply to other tally bins. HNF-SD-SNF-CSWD-005, Rev, 0

fally bins with zeros may or may not be correct: compare the source, cutoffs, multipliers, et cetera with the tally bins.

warning. 1 of the 1 tally fluctuation chart bins did not pass all 10 statistical checks.

warning. $\quad 1$ of the 1 tallies had bins with relative emors greater than recommended.

1 tally fluctuation charts

tally 4

nps mean emor vov slope fom

$10000.0000 \mathrm{E}+000.00000 .0000 \quad 0.0$

$12493.5167 \mathrm{E}-040.38770 .1752 \quad 0.0$

tally data written to file inp $18 \mathrm{~m}$

ascii file inp 18p written with 5 events from 5 histories.

6 warning messages so far.

num terminated when 6 kcode cycles were done.

\section{File: Inp200}

1- prob20 - continuous energy electron version of problem prob19.

2- 1 1 $1-19.31-23-45-6$

3. $20 \% 1$

4-

5. 1202

$+$

6- 2 pz.00635

plane

7. $3 \mathrm{px}-10$

8. $4 \times 100$

$+$

9. 5 y-103

6 p $0100 ! 1000101$

plane

plane

$\mathrm{ml} 74184.1$

13- sdef erg $=1$ sur $=1$ vec $=001$ par $=3$

14- imp:p,e 10

15- mode $\mathrm{pe}$

16- *fl:p 12

17- $\quad$ e 0 . $1.191 .282 .373 .464 .555 .645 .736 .827 .918 \quad 1.1$

18. *f1l:e 12

19. tr9 0001000100001

waming. non-orthogonality of surface transformation $9>2 . e-6$

20. fq ef

21- f21:e 12

22- *f28:p 1

23- e28 $\quad-.00101 . e-6.1 .191 .282 .373 .464 .555 .645 .736 .827 .9181 .1$

24. e58 -.001 0 l.e-6.1.191 .282.373 .464.555.645.736 .827.918 1.1

25- f58:e 1

26- f6:p 1

27. B $1:$ : I 2

28- ft31 elc 1

29. f4l:e 12

30- fill elc 2

31- f $51: \mathrm{e} 12$

32- ft51 elc 3

33- e8 $1000 \mathrm{nt}$

34- *f8:p,e 1

35- nps 10000

36- ctme 30

37- prdmp $2 \mathrm{j}-1$

38- cut:p $1 \mathrm{j} .1$

cut:e 1 j -1

print 11070 
hing. to 9 card unused.

warning. I energy bins of tally $I$ are below energy cutoff.

warning. tally 8 needs zero energy bin for negative $\mathbf{8} 8$ scores. lcells

print table 60

cell mat density density volume mass pieces importance importance

$1116.31828 \mathrm{E}-021.93000 \mathrm{E}+012.54000 \mathrm{E}+004.90220 \mathrm{E}+01 \quad 01.0000 \mathrm{E}+001.0000 \mathrm{E}+00$

$2200.00000 \mathrm{E}+000.00000 \mathrm{E}+00 \quad 0.00000 \mathrm{E}+000.00000 \mathrm{E}+00 \quad 0 \quad 0.0000 \mathrm{E}+000.0000 \mathrm{E}+00$

total

1surfaces

$2.54000 \mathrm{E}+004.90220 \mathrm{E}+01$

surface trans type surface coefficients

1 I p2 $0.0000000 \mathrm{E}+00$

2 pz $6.3500000 \mathrm{E}-03$

$3 \quad \mathrm{px}-1.0000000 \mathrm{E}+01$

$4 \quad \mathrm{px} \quad 1.0000000 \mathrm{E}+01$

5 py $-1.0000000 \mathrm{E}+01$

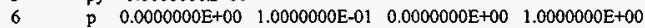

minimum source weight $=1.0000 \mathrm{E}+00$ maximum source weight $=1.0000 \mathrm{E}+00$

4 waming messages so far.

lcross-section tables

print table 100

tables from file testlib:

$74000.02 \mathrm{p} \quad 755$

$01 / 15 / 93$

total $\quad 755$

maximum photon energy set to 100.0 mev (maximum electron energy)

tables from file testlib 1

$74000.01 \mathrm{e} \quad 478$

$11 / 16 / 88$

decimal words of dynamically allocated storage

4 warning messages so far.

1 starting monun. field length $=0 \quad \mathrm{cp} 0=0.00 \quad$ print table 110

prob20 - continuous energy electron version of problem prob19.

nps $x \quad y \quad z \quad$ cell surf $y \quad v \quad w$ energy weight time

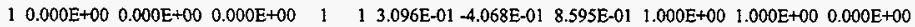

$20.000 \mathrm{E}+00 \quad 0.000 \mathrm{E}+00 \quad 0.000 \mathrm{E}+00$

$30.000 \mathrm{E}+00 \quad 0.000 \mathrm{E}+00 \quad 0.000 \mathrm{E}+00$

$40.000 \mathrm{E}+00 \quad 0.000 \mathrm{E}+00 \quad 0.000 \mathrm{E}+00$

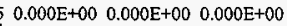

$0.000 \mathrm{E}+00 \quad 0.000 \mathrm{E}+00 \quad 0.000 \mathrm{E}+00$

$0.000 \mathrm{E}+00 \quad 0.000 \mathrm{E}+00 \quad 0.000 \mathrm{E}+00$

$1 \quad 1 \quad 9.871 \mathrm{E}-01 \quad 1.062 \mathrm{E}-01 \quad 1.197 \mathrm{E}-01 \quad 1.000 \mathrm{E}+00 \quad 1.000 \mathrm{E}+00 \quad 0.000 \mathrm{E}+00$

$1 \quad 1-1.347 \mathrm{E}-01 \quad 5.198 \mathrm{E}-01 \quad 8.436 \mathrm{E}-01 \quad 1.000 \mathrm{E}+00 \quad 1.000 \mathrm{E}+00 \quad 0.000 \mathrm{E}+00$

$1 \quad 1-6.548 \mathrm{E}-02 \quad 7.832 \mathrm{E}-01 \quad 6.184 \mathrm{E}-01 \quad 1.000 \mathrm{E}+00 \quad 1.000 \mathrm{E}+00 \quad 0.000 \mathrm{E}+00$

$1 \quad 1 \quad 1.554 \mathrm{E}-01-9.204 \mathrm{E}-01 \quad 3.588 \mathrm{E}-01 \quad 1.000 \mathrm{E}+00 \quad 1.000 \mathrm{E}+00 \quad 0.000 \mathrm{E}+00$

$1 \quad 12.608 \mathrm{E}-01-6.984 \mathrm{E}-01 \quad 6.665 \mathrm{E}-01 \quad 1.000 \mathrm{E}+00 \quad 1.000 \mathrm{E}+00 \quad 0.000 \mathrm{E}+00$

$1 \quad 16.931 \mathrm{E}-01-9.170 \mathrm{E}-02 \quad 7.149 \mathrm{E}-01 \quad 1.000 \mathrm{E}+00 \quad 1.000 \mathrm{E}+00 \quad 0.000 \mathrm{E}+00$ 


\section{File: Outp20}

prob 20 - continuous energy electron version of problem prob 19 .

I $1-19.31-23-45-6$

3- $2 \quad 0$ 2

5- 1202

6- $2 \mathrm{pz} .00635$

plane

7. $3 \mathrm{px}-10$

8. $4 \times 100$

$+$

9- 5 y -103

$+$

10- 6 p 010011000101

HNF-SD-SNF-CSWD-005, Rev. 0

11. $\mathrm{ml} \mathrm{74184.1}$

13- sdef erg=1 sur=1 vec=001 par $=3$

14- imp:p,e 10

15- mode $p$ e

16- *f1:p 12

17. $\quad \mathrm{e} 0 \quad .1 .191 .282 .373 .464 .555 .645 .736 .827 .918 \quad 1.1$

18. *f11:e 12

19. tr 00001000100001

waming. non-orthogonality of surface transformation $\quad 9>2 . e-6$

20. fo ef

21- f21:e 12

22- * $28: \mathrm{p} \quad 1$

23- e28 $-.00101 . e-6.1 .191 .282 .373 .464 .555 .645 .736 .827 .9181 .1$

24- e58 $\quad-.00101 . e-6.1 .191 .282 .373 .464 .555 .645 .736 .827 .9181 .1$

25- f58:e 1

26- $16: p 1$

(31:e 12

fth 31 elc 1

f $41: e 12$

ft4l elc 2

f5l:e 12

ft5l elc 3

e8 $1000 \mathrm{nt}$

*f8:p,e 1

nps 10000

ctme 30

prdmp $2 \mathrm{j}-1$

cut:p $1 j$.1

cut:e $\mathrm{lj} .1$

print 11070

41 -

warning. \& 9 card unused.

warning. 1 energy bins of tally 1 are below energy cutoff.

waming. tally 8 needs zero energy bin for negative 18 scores. lcells

print table 60

atom gram
cell mat density density volume mass pieces importance importance

$1116.31828 \mathrm{E}-021.93000 \mathrm{E}+012.54000 \mathrm{E}+004.90220 \mathrm{E}+01 \quad 01.0000 \mathrm{E}+001.0000 \mathrm{E}+00$

$220 \quad 0.00000 \mathrm{E}+00 \quad 0.00000 \mathrm{E}+00 \quad 0.00000 \mathrm{E}+00 \quad 0.00000 \mathrm{E}+00 \quad 0 \quad 0.0000 \mathrm{E}+00 \quad 0.0000 \mathrm{E}+00$

total

Isurfaces

$2.54000 \mathrm{E}+004.90220 \mathrm{E}+01$

print table 70

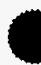

urface trans type surface coefficients 


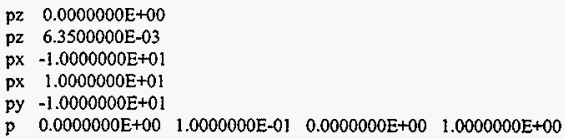

FINF-SD-SNF-CSWD-005, Rev. 0

minimum source weight $=1.0000 \mathrm{E}+00$ maximum source weight $=1.0000 \mathrm{E}+00$

4 waming messages so far.

icross-section tables

print table 100

table. length

tables from file testlibl

$74000.02 \mathrm{p} \quad 755$

$01 / 15 / 93$

total 755

maximum photon energy set to 100.0 mev (maximum electron energy)

tables from file testlib!

$74000.01 \mathrm{e} \quad 478$

$11 / 16 / 88$

decimal words of dynamically allocated storage

4 warning messages so far.

arting morun. field length $=0 \quad \mathrm{cp} 0=0.00$

print table 110

prob20 - continuous energy electron version of problem probl9

nps $x \quad y \quad z$ cell surf $u \quad v \quad w$ energy weight time

1 $0.000 \mathrm{E}+00 \quad 0.000 \mathrm{E}+00 \quad 0.000 \mathrm{E}+00$ 2: $0.000 \mathrm{E}+00 \quad 0.000 \mathrm{E}+00 \quad 0.000 \mathrm{E}+00$ $30.000 \mathrm{E}+00 \quad 0.000 \mathrm{E}+00 \quad 0.000 \mathrm{E}+00$ $4 \quad 0.000 \mathrm{E}+00 \quad 0.000 \mathrm{E}+00 \quad 0.000 \mathrm{E}+00$ $50.000 \mathrm{E}+00 \quad 0.000 \mathrm{E}+00 \quad 0.000 \mathrm{E}+00$ $6 \quad 0.000 \mathrm{E}+00 \quad 0.000 \mathrm{E}+00 \quad 0.000 \mathrm{E}+00$

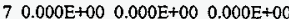

$8 \quad 0.000 \mathrm{E}+00 \quad 0.000 \mathrm{E}+00 \quad 0.000 \mathrm{E}+00$ $90.000 \mathrm{E}+00 \quad 0.000 \mathrm{E}+00 \quad 0.000 \mathrm{E}+00$ $10 \quad 0.000 \mathrm{E}+00 \quad 0.000 \mathrm{E}+00 \quad 0.000 \mathrm{E}+00$ $110.000 \mathrm{E}+00 \quad 0.000 \mathrm{E}+00 \quad 0.000 \mathrm{E}+00$ $12 \quad 0.000 \mathrm{E}+00 \quad 0.000 \mathrm{E}+00 \quad 0.000 \mathrm{E}+00$ $13 \quad 0.000 \mathrm{E}+00 \quad 0.000 \mathrm{E}+00 \quad 0.000 \mathrm{E}+00$ $140.000 \mathrm{E}+00 \quad 0.000 \mathrm{E}+00 \quad 0.000 \mathrm{E}+00$ $15 \quad 0.000 \mathrm{E}+00 \quad 0.000 \mathrm{E}+00 \quad 0.000 \mathrm{E}+00$ $\begin{array}{llll}16 & 0.000 \mathrm{E}+00 & 0.000 \mathrm{E}+00 & 0.000 \mathrm{E}+00\end{array}$ $17 \quad 0.000 \mathrm{E}+00 \quad 0.000 \mathrm{E}+00 \quad 0.000 \mathrm{E}+00$ $18 \quad 0.000 \mathrm{E}+00 \quad 0.000 \mathrm{E}+00 \quad 0.000 \mathrm{E}+00$ $190.000 \mathrm{E}+00 \quad 0.000 \mathrm{E}+00 \quad 0.000 \mathrm{E}+00$ $20 \quad 0.000 \mathrm{E}+00 \quad 0.000 \mathrm{E}+00 \quad 0.000 \mathrm{E}+00$ $210.000 \mathrm{E}+00 \quad 0.000 \mathrm{E}+00 \quad 0.000 \mathrm{E}+00$ $22 \quad 0.000 \mathrm{E}+00 \quad 0.000 \mathrm{E}+00 \quad 0.000 \mathrm{E}+00$ $23 \quad 0.000 \mathrm{E}+00 \quad 0.000 \mathrm{E}+00 \quad 0.000 \mathrm{E}+00$ $240.000 \mathrm{E}+00 \quad 0.000 \mathrm{E}+00 \quad 0.000 \mathrm{E}+00$ $\begin{array}{lll}0.000 \mathrm{E}+00 \quad 0.000 \mathrm{E}+00 \quad 0.000 \mathrm{E}+00 & 0.000 \mathrm{E}+00 \quad 0.000 \mathrm{E}+00 \quad 0.000 \mathrm{E}+00\end{array}$ $0.000 \mathrm{E}+00 \quad 0.000 \mathrm{E}+00 \quad 0.000 \mathrm{E}+00$ $\begin{array}{lll}0.000 E+00 & 0.000 E+00 & 0.000 E+00\end{array}$
$13.096 \mathrm{E}-0]-4.068 \mathrm{E}-01 \quad 8.595 \mathrm{E}-01 \quad 1.000 \mathrm{E}+00 \quad 1.000 \mathrm{E}+00 \quad 0.000 \mathrm{E}+00$

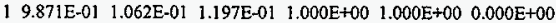
$1-1.347 \mathrm{E}-01 \quad 5.198 \mathrm{E}-01 \quad 8.436 \mathrm{E}-01 \quad 1.000 \mathrm{E}+00 \quad 1.000 \mathrm{E}+00 \quad 0.000 \mathrm{E}+00$ $\begin{array}{lllllll}1 & -6.548 \mathrm{E}-02 & 7.832 \mathrm{E}-01 & 6.184 \mathrm{E}-01 & 1.000 \mathrm{E}+00 & 1.000 \mathrm{E}+00 & 0.000 \mathrm{E}+00\end{array}$ $\begin{array}{lllllll}1 & 1.554 \mathrm{E}-01 & -9.204 \mathrm{E}-01 & 3.588 \mathrm{E}-01 & 1.000 \mathrm{E}+00 & 1.000 \mathrm{E}+00 & 0.000 \mathrm{E}+00\end{array}$ $\begin{array}{lllllll}1 & 2.608 \mathrm{E}-01 & -6.984 \mathrm{E}-01 & 6.665 \mathrm{E}-01 & 1.000 \mathrm{E}+00 & 1.000 \mathrm{E}+00 & 0.000 \mathrm{E}+00\end{array}$ $16.931 \mathrm{E}-01-9.170 \mathrm{E}-02 \quad 7.149 \mathrm{E}-01 \quad 1.000 \mathrm{E}+00 \quad 1.000 \mathrm{E}+00 \quad 0.000 \mathrm{E}+00$

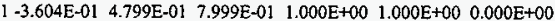

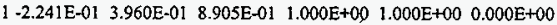
$1-2.928 \mathrm{E}-01-7.352 \mathrm{E}-01 \quad 6.114 \mathrm{E}-01 \quad 1.000 \mathrm{E}+00 \quad 1.000 \mathrm{E}+00 \quad 0.000 \mathrm{E}+00$ $18.260 \mathrm{E}-01 \quad 1.184 \mathrm{E}-01 \quad 5.510 \mathrm{E}-01 \quad 1.000 \mathrm{E}+00 \quad 1.000 \mathrm{E}+00 \quad 0.000 \mathrm{E}+00$ I 7.540E-01 $-1.976 \mathrm{E}-01 \quad 6.264 \mathrm{E}-01 \quad 1.000 \mathrm{E}+00 \quad 1.000 \mathrm{E}+00 \quad 0.000 \mathrm{E}+00$ $18.140 \mathrm{E}-01-3.427 \mathrm{E}-01 \quad 4.690 \mathrm{E}-01 \quad 1.000 \mathrm{E}+00 \quad 1.000 \mathrm{E}+00 \quad 0.000 \mathrm{E}+00$

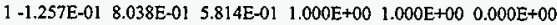
$1-1.464 \mathrm{E}-01-1.070 \mathrm{E}-01 \quad 9.834 \mathrm{E}-01 \quad 1.000 \mathrm{E}+00 \quad 1.000 \mathrm{E}+00 \quad 0.000 \mathrm{E}+00$ $1 \quad 1.310 \mathrm{E}-02-1.617 \mathrm{E}-02 \quad 9.998 \mathrm{E}-01 \quad 1.000 \mathrm{E}+00 \quad 1.000 \mathrm{E}+00 \quad 0.000 \mathrm{E}+00$

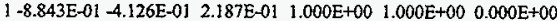

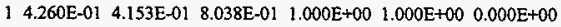
$1-7.311 \mathrm{E}-01-1.136 \mathrm{E}-02 \quad 6.822 \mathrm{E}-01 \quad 1.000 \mathrm{E}+00 \quad 1.000 \mathrm{E}+00 \quad 0.000 \mathrm{E}+00$

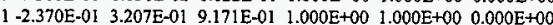
$14.276 \mathrm{E}-01-2.485 \mathrm{E}-02 \quad 9.036 \mathrm{E}-01 \quad 1.000 \mathrm{E}+00 \quad 1.000 \mathrm{E}+00 \quad 0.000 \mathrm{E}+00$

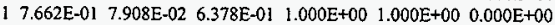
$\begin{array}{llllll}1-4.247 \mathrm{E}-01 & 2.688 \mathrm{E}-01 & 8.645 \mathrm{E}-01 & 1.000 \mathrm{E}+00 & 1.000 \mathrm{E}+00 & 0.000 \mathrm{E}+00\end{array}$ $12.096 \mathrm{E}-01 \quad 5.730 \mathrm{E}-01 \quad 7.923 \mathrm{E}-01 \quad 1.000 \mathrm{E}+00 \quad 1.000 \mathrm{E}+00 \quad 0.000 \mathrm{E}+00$ $1-2.665 \mathrm{E}-03-5.270 \mathrm{E}-01 \quad 8.499 \mathrm{E}-01 \quad 1.000 \mathrm{E}+00 \quad 1.000 \mathrm{E}+00 \quad 0.000 \mathrm{E}+00$ $1 \quad 7.228 \mathrm{E}-01 \quad 4.233 \mathrm{E}-01 \quad 5.463 \mathrm{E}-01 \quad 1.000 \mathrm{E}+00 \quad 1.000 \mathrm{E}+00 \quad 0.000 \mathrm{E}+00$ $13.484 \mathrm{E}-0 \mathrm{I}-3.564 \mathrm{E}-01 \quad 8.669 \mathrm{E}-01 \quad 1.000 \mathrm{E}+00 \quad 1.000 \mathrm{E}+00 \quad 0.000 \mathrm{E}+00$ 
$28 \quad 0.000 \mathrm{E}+00 \quad 0.000 \mathrm{E}+00 \quad 0.000 \mathrm{E}+00$ $\begin{array}{llll}9.000 \mathrm{E}+00 & 0.000 \mathrm{E}+00 & 0.000 \mathrm{E}+00\end{array}$ $0.000 \mathrm{E}+00 \quad 0.000 \mathrm{E}+00 \quad 0.000 \mathrm{E}+00$ $310.000 \mathrm{E}+00 \quad 0.000 \mathrm{E}+00 \quad 0.000 \mathrm{E}+00$ $32 \quad 0.000 \mathrm{E}+00 \quad 0.000 \mathrm{E}+00 \quad 0.000 \mathrm{E} \div 00$ $330.000 \mathrm{E} \div 00 \quad 0.000 \mathrm{E}+00 \quad 0.000 \mathrm{E}+00$ $340.000 \mathrm{E}+00 \quad 0.000 \mathrm{E}+00 \quad 0.000 \mathrm{E}+00$ $350.000 \mathrm{E}+00 \quad 0.000 \mathrm{E}+00 \quad 0.000 \mathrm{E}+00$ $\begin{array}{llll}36 & 0.000 \mathrm{E}+00 & 0.000 \mathrm{E}+00 & 0.000 \mathrm{E}+00\end{array}$ $37 \quad 0.000 \mathrm{E}+00 \quad 0.000 \mathrm{E}+00 \quad 0.000 \mathrm{E}+00$ $\begin{array}{llll}38 & 0.000 \mathrm{E}+00 & 0.000 \mathrm{E}+00 & 0.000 \mathrm{E}+00\end{array}$ $39 \quad 0.000 \mathrm{E}+00 \quad 0.000 \mathrm{E}+00 \quad 0.000 \mathrm{E}+00$ $40 \quad 0.000 \mathrm{E}+00 \quad 0.000 \mathrm{E}+00 \quad 0.000 \mathrm{E}+00$ $41 \quad 0.000 \mathrm{E}+00 \quad 0.000 \mathrm{E}+00 \quad 0.000 \mathrm{E}+00$ $42 \quad 0.000 \mathrm{E}+00 \quad 0.000 \mathrm{E}+00 \quad 0.000 \mathrm{E}+00$ $\begin{array}{llll}43 & 0.000 \mathrm{E}+00 & 0.000 \mathrm{E}+00 & 0.000 \mathrm{E}+00\end{array}$ $44 \quad 0.000 \mathrm{E}+00 \quad 0.000 \mathrm{E}+00 \quad 0.000 \mathrm{E}+00$ $45 \quad 0.000 \mathrm{E}+00 \quad 0.000 \mathrm{E}+00 \quad 0.000 \mathrm{E}+00$ $46 \quad 0.000 \mathrm{E}+00 \quad 0.000 \mathrm{E}+00 \quad 0.000 \mathrm{E}+00$ $470.000 \mathrm{E}+00 \quad 0.000 \mathrm{E}+00 \quad 0.000 \mathrm{E}+00$ $48 \quad 0.000 \mathrm{E}+00 \quad 0.000 \mathrm{E}+00 \quad 0.000 \mathrm{E}+00$ $49 \quad 0.000 \mathrm{E}+00 \quad 0.000 \mathrm{E}+00 \quad 0.000 \mathrm{E}+00$ So $0.000 \mathrm{E}+00 \quad 0.000 \mathrm{E}+00 \quad 0.000 \mathrm{E}+00$ 1 problem summary
I 8.706E-01 -3.198E-01 3.738E-01 1.000E+00 1.000E+00 $0.000 \mathrm{E}+00$

$\begin{array}{llllll}1-8.188 \mathrm{E}-01 & 2.059 \mathrm{E}-01 & 5.359 \mathrm{E}-01 & 1.000 \mathrm{E}+00 & 1.000 \mathrm{E}+00 & 0.000 \mathrm{E}+00\end{array}$

$15.727 \mathrm{E}-01 \quad 8.124 \mathrm{E}-01 \quad 1.100 \mathrm{E}-01 \quad 1.000 \mathrm{E}+00 \quad 1.000 \mathrm{E}+00 \quad 0.000 \mathrm{E}+00$

$15.390 \mathrm{E}-02$ 5.914E-01 $8.046 \mathrm{E}-0] \quad 1.000 \mathrm{E}+00 \quad 1.000 \mathrm{E}+00 \quad 0.000 \mathrm{E}+00$

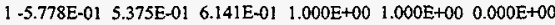

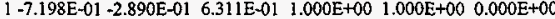

I $2.210 \mathrm{E}-01 \quad 2.482 \mathrm{E}-01 \quad 9.432 \mathrm{E}-01 \quad 1.000 \mathrm{E}+00 \quad 1.000 \mathrm{E}+00 \quad 0.000 \mathrm{E}+00$

I $4.339 \mathrm{E}-01$ 7.515E-02 8.978E-0] $1.000 \mathrm{E}+00 \quad 1.000 \mathrm{E}+00 \quad 0.000 \mathrm{E}+00$

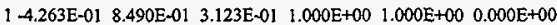

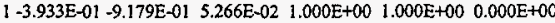

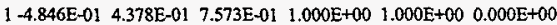

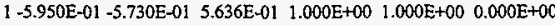

$\begin{array}{llll}1-7.252 \mathrm{E}-01 & -5.143 \mathrm{E}-01 & 4.578 \mathrm{E}-01 & 1.000 \mathrm{E}+00 \quad 1.000 \mathrm{E}+00 \quad 0.000 \mathrm{E}+00\end{array}$

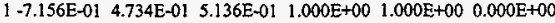
$1-1.771 \mathrm{E}-01$ 9.229E-01 3.417E-01 1.000E+00 1.000E+00 $0.000 \mathrm{E}+00$

$1-1.149 \mathrm{E}-01-4.206 \mathrm{E}-01 \quad 8.999 \mathrm{E}-01 \quad 1.000 \mathrm{E}+00 \quad 1.000 \mathrm{E}+00 \quad 0.000 \mathrm{E}+00$

$1 \quad 4.509 \mathrm{E}-01 \quad 8.630 \mathrm{E}-02 \quad 8.884 \mathrm{E}-01 \quad 1.000 \mathrm{E}+00 \quad 1.000 \mathrm{E}+00 \quad 0.000 \mathrm{E}+00$

$\begin{array}{llllll}1-7.358 \mathrm{E}-01 & 2.500 \mathrm{E}-01 & 6.294 \mathrm{E}-01 & 1.000 \mathrm{E}+00 & 1.000 \mathrm{E}+00 & 0.000 \mathrm{E}+00\end{array}$

$\begin{array}{lllllll}1-8.448 \mathrm{E}-01 & 3.645 \mathrm{E}-01 & 3.917 \mathrm{E}-01 & 1.000 \mathrm{E}+00 & 1.000 \mathrm{E}+00 & 0.000 \mathrm{E}+00\end{array}$

$1 \quad 1.958 \mathrm{E}-01-2.769 \mathrm{E}-01 \quad 9.407 \mathrm{E}-01 \quad 1.000 \mathrm{E}+00 \quad 1.000 \mathrm{E}+00 \quad 0.000 \mathrm{E}+00$

$12.788 \mathrm{E}-01-7.325 \mathrm{E}-03 \quad 9.603 \mathrm{E}-01 \quad 1.000 \mathrm{E}+00 \quad 1.000 \mathrm{E}+00 \quad 0.000 \mathrm{E}+00$

$\begin{array}{lllllll}1 & -3.201 \mathrm{E}-01 & 4.174 \mathrm{E}-01 & 8.505 \mathrm{E}-01 & 1.000 \mathrm{E}+00 & 1.000 \mathrm{E}+00 & 0.000 \mathrm{E}+00\end{array}$

1 $8.960 \mathrm{E}-01-1.641 \mathrm{E}-01 \quad 4.126 \mathrm{E}-01 \quad 1.000 \mathrm{E}+00 \quad 1.000 \mathrm{E}+00 \quad 0.000 \mathrm{E}+00$ 0

run terminated when 10000 particle histories were done.

photon creation tracks weight energy photon loss tracks weight energy
(per source particle)

$\begin{array}{cccccccc}\text { source } & 0 & 0 . & 0 . & \begin{array}{c}\text { escape } \\ \text { energy cutoff }\end{array} & 0 & 0.7500 \mathrm{E}-02 & 1.5476 \mathrm{E}-02\end{array}$

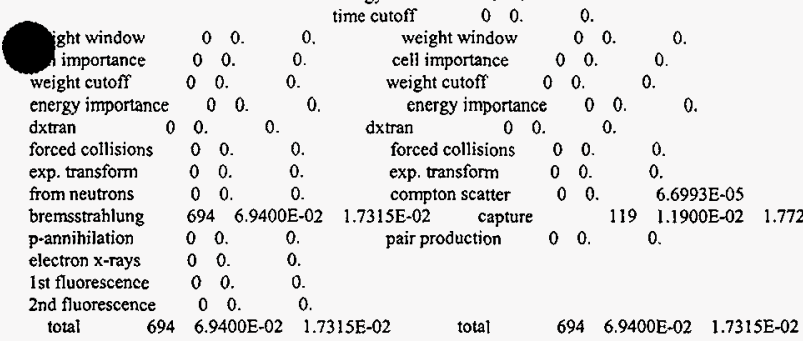

number of photons banked

694 average lifetime, shakes cutoffs photon tracks per source particle 6.9400E-02 escape 6.7786E-05 tco $1.0000 \mathrm{E}+34$ photon collisions per source particle $1.3000 \mathrm{E}-02$ capture $8.3325 \mathrm{E}-05$ eco $1.0000 \mathrm{E}-01$ total photon collisions $\quad 130$ capture or escape $7.0450 \mathrm{E}-05$ wc] $0.0000 \mathrm{E}+00$

\section{0} any termination $7.0450 \mathrm{E}-05 \quad$ wc2 $0.0000 \mathrm{E}+00$ electron creation tracks weight energy electron loss tracks weight energy (per source particle) (per source particle)

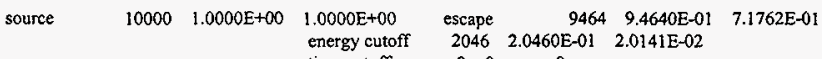

weight window $\quad \begin{array}{lllllll}0 & 0 . & 0 . & \text { weight window } & 0 & 0 . & 0 .\end{array}$

cell importance $\quad \begin{array}{llllllll}0 & 0 . & 0 . & \text { cell importance } & 0 & 0 . & 0 .\end{array}$

$\begin{array}{lllllllll}\text { weight cutoff } & 0 & 0 . & 0 . & \text { weight cutof } & 0 & 0 . & 0 .\end{array}$

energy importance $\quad \begin{array}{llllllll}0 & 0 . & 0 . & \text { energy importance } & 0 & 0 . & 0 .\end{array}$

$\begin{array}{llllllll}\text { mogir production } & 0 & 0 & 0 . & \text { scattering } & 0 & 0 . & 2.6560 \mathrm{E}-01\end{array}$

$\begin{array}{llllllll}\text { mpton recoil } & 2 & 2.0000 \mathrm{E}-04 & 3.7815 \mathrm{E}-05 & \text { bremsstrahlung } & 0 & 0 & 2.4158 \mathrm{E}-02\end{array}$

oto-electric

$29 \quad 2.9000 E-03 \quad 4.7387 E-04$ 


\section{$\begin{array}{rr}4.6400 \mathrm{E}-01 & 1.76412 \mathrm{E}-060.4116 \\ 5500 \mathrm{E}-01 & 6.34259 \mathrm{E}-070.4736\end{array}$ 5. $4500 \mathrm{E}-01 \quad 2.47457 \mathrm{E}-070.3129$ $7.3600 \mathrm{E}-01 \quad 2.58011 \mathrm{E}-070.6571$ $8.2700 \mathrm{E}-01 \quad 1.16245 \mathrm{E}-070.3470$ $9.1800 \mathrm{E}-01 \quad 4.46610 \mathrm{E}-080.6403$ $1.1000 \mathrm{E}+00 \quad 0.00000 \mathrm{E}+000.0000$ total 2.75512E-05 0.0722}

1 tally 28 nps $=10000$ tally type $8 *$ energy deposition tally for photons electrons

cell:

energy

$-1.0000 \mathrm{E}-03-8.72550 \mathrm{E}-040.1508$ $0.0000 \mathrm{E}+00 \quad 0.00000 \mathrm{E}+000.0000$ $1.0000 \mathrm{E}-06 \quad 0.00000 \mathrm{E}+000.0000$ $1.0000 \mathrm{E}-01 \quad 1.08193 \mathrm{E}-020.0227$ $1.9100 \mathrm{E}-01 \quad 3.91016 \mathrm{E}-020.0170$ $2.8200 \mathrm{E}-01 \quad 4.36100 \mathrm{E}-020.0210$ $3.7300 \mathrm{E}-01 \quad 3.53763 \mathrm{E}-02 \quad 0.0286$ 4.6400E-01 2.66881E-02 0.0382 $5.5500 \mathrm{E}-01 \quad 2.21833 \mathrm{E}-020.0468$ $6.4500 \mathrm{E}-01 \quad 1.58947 \mathrm{E}-020.0606$ $7.3600 \mathrm{E}-01 \quad 1.08330 \mathrm{E}-02 \quad 0.0790$ 8.2700E-01 $8.71177 \mathrm{E}-03 \quad 0.0940$ $9.1800 \mathrm{E}-01 \quad 6.65495 \mathrm{E}-030.1136$ 1. $1000 \mathrm{E}+00 \quad 4.79000 \mathrm{E}-020.0446$ total $2.66900 \mathrm{E}-010.0090$

Itally 11 nps $=10000$

tally type 1* energy crossing a surface. units mev tally for electrons units mev
HNF-SD-SNF-CSWD-005, Rev. 0

$1.0000 \mathrm{E}-01 \quad 0.00000 \mathrm{E}+00 \quad 0.0000 \quad 0.00000 \mathrm{E}+00 \quad 0.0000$ $1.9100 \mathrm{E}-01 \quad 1.08698 \mathrm{E}-03 \quad 0.1176 \quad 6.53601 \mathrm{E}-040.1495$ $2.8200 \mathrm{E}-01 \quad 2.19643 \mathrm{E}-03 \quad 0.1039 \quad 1.83325 \mathrm{E}-030.1165$ $3.7300 \mathrm{E}-01 \quad 3.60063 \mathrm{E}-03 \quad 0.0956 \quad 3.37231 \mathrm{E}-030.0988$ $\begin{array}{lllll}4.6400 \mathrm{E}-01 & 6.94424 \mathrm{E}-03 & 0.0774 & 6.18557 \mathrm{E}-03 & 0.0823\end{array}$

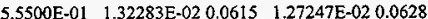
$6.4500 \mathrm{E}-01 \quad 2.19441 \mathrm{E}-020.0516 \quad 1.93787 \mathrm{E}-020.0550$ $\begin{array}{lllll}7.3600 \mathrm{E}-01 & 3.87821 \mathrm{E}-02 & 0.0411 & 4.62743 \mathrm{E}-02 & 0.0374\end{array}$

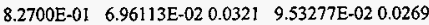
9.1800E-01 1.17955E-01 $0.0253 \quad 9.53443 \mathrm{E}-020.0284$

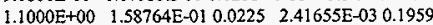
total $4.34113 \mathrm{E}-010.0097 \quad 2.83511 \mathrm{E}-010.0130$

1 tally $21 \quad$ nps $=10000$

tally type 1 number of particles crossing a surface. tally for electrons

surface: $\quad 1 \quad 2$ energy

$1.0000 \mathrm{E}-01 \quad 0.00000 \mathrm{E}+000.0000 \quad 0.00000 \mathrm{E}+000.0000$ $1.9100 \mathrm{E}-01 \quad 7.70000 \mathrm{E}-03 \quad 0.1165 \quad 4.60000 \mathrm{E}-03 \quad 0.1471$ $2.8200 \mathrm{E}-01 \quad 9.30000 \mathrm{E}-03 \quad 0.1032 \quad 7.60000 \mathrm{E}-03 \quad 0.1158$ $3.7300 \mathrm{E}-01 \quad 1.09000 \mathrm{E}-02 \quad 0.0953 \quad 1.02000 \mathrm{E}-020.0985$ 4.6400E-01 $1.65000 \mathrm{E}-02 \quad 0.0772 \quad 1.46000 \mathrm{E}-020.0822$

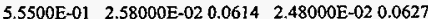
6.4500E-01 3.63000E-02 0.0515 $3.21000 \mathrm{E}-020.0549$ $\begin{array}{llllll}7.3600 \mathrm{E}-01 & 5.59000 \mathrm{E}-02 & 0.0411 & 6.67000 \mathrm{E}-02 & 0.0374\end{array}$

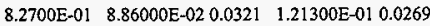
$\begin{array}{llllll}9.1800 \mathrm{E}-01 & 1.34800 \mathrm{E}-01 & 0.0253 & 1.10600 \mathrm{E}-01 & 0.0284\end{array}$

$1.1000 \mathrm{E}+00 \quad 1.65500 \mathrm{E}-010.0225 \quad 2.60000 \mathrm{E}-03 \quad 0.1959$ total $5.51300 \mathrm{E}-010.0094 \quad 3.95100 \mathrm{E}-010.0126$ ly $31 \quad \mathrm{nps}=10000$

tally type 1 number of particles crossing a surface. 
$1.0000 \mathrm{E}-01 \quad 0.00000 \mathrm{E}+00 \quad 0.0000 \quad 0.00000 \mathrm{E}+000.0000$ $1.9100 \mathrm{E}-01-7.70000 \mathrm{E}-03 \quad 0.1165-4.60000 \mathrm{E}-03 \quad 0.1471$ $2.8200 \mathrm{E}-01-9.30000 \mathrm{E}-03 \quad 0.1032-7.60000 \mathrm{E}-030.1158$ $3.7300 \mathrm{E}-01-1.09000 \mathrm{E}-020.0953-1.02000 \mathrm{E}-020.0985$ $4.6400 \mathrm{E}-01-1.65000 \mathrm{E}-020.0772-1.46000 \mathrm{E}-020.0822$ $5.5500 \mathrm{E}-01 \quad-2.58000 \mathrm{E}-02 \quad 0.0614-2.48000 \mathrm{E}-020.0627$ $6.4500 \mathrm{E}-01-3.63000 \mathrm{E}-020.0515-3.21000 \mathrm{E}-020.0549$ $7.3600 \mathrm{E}-01 \quad-5.59000 \mathrm{E}-02 \quad 0.04 ! 1-6.67000 \mathrm{E}-02 \quad 0.0374$ $8.2700 \mathrm{E}-01-8.86000 \mathrm{E}-020.0321-1.21300 \mathrm{E}-010.0269$ $9.1800 \mathrm{E}-01-1.34800 \mathrm{E}-010.0253-1.10600 \mathrm{E}-010.0284$ $1.1000 \mathrm{E}+00-1.65500 \mathrm{E}-010.0225-2.60000 \mathrm{E}-030.1959$ total $-5.51300 \mathrm{E}-010.0094-3.95100 \mathrm{E}-010.0126$ Itally $41 \quad \mathrm{nps}=10000$

tally type 1 number of particles crossing a surface. tally for electrons this tally is modified by fi elc

surface: 1

energy

$1.0000 \mathrm{E}-01 \quad 0.00000 \mathrm{E}+000.0000 \quad 0.00000 \mathrm{E}+000.0000$

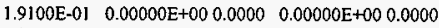
$2.8200 \mathrm{E}-01 \quad 0.00000 \mathrm{E}+00 \quad 0.0000 \quad 0.00000 \mathrm{E}+000.0000$ $3.7300 \mathrm{E}-01 \quad 0.00000 \mathrm{E}+000.0000 \quad 0.00000 \mathrm{E}+000.0000$ $4.6400 \mathrm{E}-01 \quad 0.00000 \mathrm{E}+00 \quad 0.0000 \quad 0.00000 \mathrm{E}+00 \quad 0.0000$

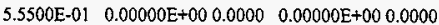

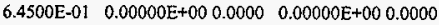

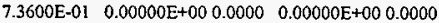
$8.2700 \mathrm{E}-01 \quad 0.00000 \mathrm{E}+00 \quad 0.0000 \quad 0.00000 \mathrm{E}+00 \quad 0.0000$ $1800 \mathrm{E}-01 \quad 0.00000 \mathrm{E}+000.0000 \quad 0.00000 \mathrm{E}+000.0000$ $1000 \mathrm{E}+00 \quad 0.00000 \mathrm{E}+00 \quad 0.0000 \quad 0.00000 \mathrm{E}+00 \quad 0.0000$ total $\quad 0.00000 \mathrm{E}+000.0000 \quad 0.00000 \mathrm{E}+000.0000$
surface:
1
2

energy

I.0000E-01 $0.00000 \mathrm{E}+00 \quad 0.0000 \quad 0.00000 \mathrm{E}+000.0000$

$1.9100 \mathrm{E}-01 \quad 7.70000 \mathrm{E}-03 \quad 0.1165 \quad 4.60000 \mathrm{E}-03 \quad 0.1471$

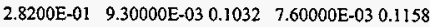

$3.7300 \mathrm{E}-01 \quad 1.09000 \mathrm{E}-02 \quad 0.0953 \quad 1.02000 \mathrm{E}-020.0985$

$4.6400 \mathrm{E}-01 \quad 1.65000 \mathrm{E}-02 \quad 0.0772 \quad 1.46000 \mathrm{E}-02 \quad 0.0822$

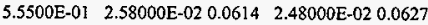

6.4500E-01 3.63000E-02 0.0515 3.21000E-02 0.0549

$7.3600 \mathrm{E}-01 \quad 5.59000 \mathrm{E}-020.0411 \quad 6.67000 \mathrm{E}-02 \quad 0.0374$

$8.2700 \mathrm{E}-01 \quad 8.86000 \mathrm{E}-02 \quad 0.0321 \quad 1.21300 \mathrm{E}-010.0269$

$9.1800 \mathrm{E}-01 \quad 1.34800 \mathrm{E}-010.0253 \quad 1.10600 \mathrm{E}-010.0284$

$1.1000 \mathrm{E}+00 \quad$ I. $.65500 \mathrm{E}-010.0225 \quad 2.60000 \mathrm{E}-03 \quad 0.1959$

total $5.51300 \mathrm{E}-010.0094 \quad 3.95100 \mathrm{E}-010.0126$

$\begin{array}{lll}\text { surface: } & 1 & 2 \\ \text { energy } & & \end{array}$

$1.0000 \mathrm{E}-01 \quad 0.00000 \mathrm{E}+00 \quad 0.0000 \quad 0.00000 \mathrm{E}+000.0000$ $\begin{array}{lllll}1.9100 \mathrm{E}-01 & 7.70000 \mathrm{E}-03 & 0.1165 & 4.60000 \mathrm{E}-03 & 0.1471\end{array}$ $2.8200 \mathrm{E}-01 \quad 9.30000 \mathrm{E}-03 \quad 0.1032 \quad 7.60000 \mathrm{E}-03 \quad 0.1158$ $3.7300 \mathrm{E}-01 \quad 1.09000 \mathrm{E}-02 \quad 0.0953 \quad 1.02000 \mathrm{E}-020.0985$ $4.6400 \mathrm{E}-01 \quad 1.65000 \mathrm{E}-02 \quad 0.0772 \quad 1.46000 \mathrm{E}-02 \quad 0.0822$

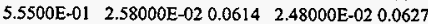

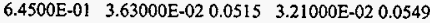

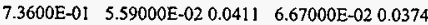
$\begin{array}{llllll}8.2700 \mathrm{E}-01 & 8.86000 \mathrm{E}-02 & 0.0321 & 1.21300 \mathrm{E}-01 & 0.0269\end{array}$

$9.1800 \mathrm{E}-01 \quad 1.34800 \mathrm{E}-01 \quad 0.0253 \quad 1.10600 \mathrm{E}-010.0284$ cml. $1000 \mathrm{E}+00 \quad 1.65500 \mathrm{E}-010.0225 \quad 2.60000 \mathrm{E}-03 \quad 0.1959$ tal $5.51300 \mathrm{E}-010.0094 \quad 3.95100 \mathrm{E}-010.0126$ nps $=10000$ 
tally type 1 number of particles crossing a surface. tally for electrons

this tally is modified by $\mathrm{ft}$ elc
surface: 1
energy
1.0000E-01 $\quad 0.00000 \mathrm{E}+00 \quad 0.0000 \quad 0.00000 \mathrm{E}+000.0000$
$1.9100 \mathrm{E}-01 \quad 0.00000 \mathrm{E}+000.0000 \quad 0.00000 \mathrm{E}+000.0000$
$2.8200 \mathrm{E}-01 \quad 0.00000 \mathrm{E}+00 \quad 0.0000 \quad 0.00000 \mathrm{E}+00 \quad 0.0000$
$3.7300 \mathrm{E}-01 \quad 0.00000 \mathrm{E}+00 \quad 0.0000 \quad 0.00000 \mathrm{E}+000.0000$
4.6400E-01 $0.00000 \mathrm{E}+00 \quad 0.0000 \quad 0.00000 \mathrm{E}+00 \quad 0.0000$

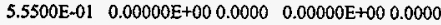
6.4500E-0] $0.00000 \mathrm{E}+000.0000 \quad 0.00000 \mathrm{E}+00 \quad 0.0000$

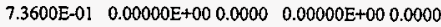

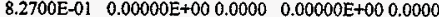
$9.1800 \mathrm{E}-01 \quad 0.00000 \mathrm{E}+00 \quad 0.0000 \quad 0.00000 \mathrm{E}+00 \quad 0.0000$
$1.1000 \mathrm{E}+00 \quad 0.00000 \mathrm{E}+00 \quad 0.0000 \quad 0.00000 \mathrm{E}+00 \quad 0.0000$
total $\quad 0.00000 \mathrm{E}+000.0000 \quad 0.00000 \mathrm{E}+000.0000$

surface: $\quad 1 \quad 2$

energy

$1.0000 \mathrm{E}-01 \quad 0.00000 \mathrm{E}+000.0000 \quad 0.00000 \mathrm{E}+000.0000$

$1.9100 \mathrm{E}-01-7.70000 \mathrm{E}-030.1165-4.60000 \mathrm{E}-030.1471$

$2.8200 \mathrm{E}-01-9.30000 \mathrm{E}-03 \quad 0.1032-7.60000 \mathrm{E}-030.1158$

$3.7300 \mathrm{E}-01-1.09000 \mathrm{E}-020.0953-1.02000 \mathrm{E}-020.0985$

$4.6400 \mathrm{E}-01-1.65000 \mathrm{E}-020.0772-1.46000 \mathrm{E}-020.0822$

$5.5500 \mathrm{E}-01-2.58000 \mathrm{E}-02 \quad 0.0614-2.48000 \mathrm{E}-020.0627$

$6.4500 \mathrm{E}-01-3.63000 \mathrm{E}-020.0515-3.21000 \mathrm{E}-020.0549$

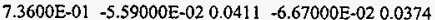

$8.2700 \mathrm{E}-01-8.86000 \mathrm{E}-020.0321-1.21300 \mathrm{E}-010.0269$

$9.1800 \mathrm{E}-01-1.34800 \mathrm{E}-010.0253-1.10600 \mathrm{E}-010.0284$

$1.1000 \mathrm{E}+00-1.65500 \mathrm{E}-010.0225-2.60000 \mathrm{E}-030.1959$

total $-5.51300 \mathrm{E}-010.0094-3.95100 \mathrm{E}-010.0126$

surface:

I

energy

$1.0000 \mathrm{E}-01 \quad 0.00000 \mathrm{E}+00 \quad 0.0000 \quad 0.00000 \mathrm{E}+000.0000$

$1.9100 \mathrm{E}-01-7.70000 \mathrm{E}-03 \quad 0.1165-4.60000 \mathrm{E}-03 \quad 0.1471$

$2.8200 \mathrm{E}-01-9.30000 \mathrm{E}-030.1032-7.60000 \mathrm{E}-030.1158$

$3.7300 \mathrm{E}-01-1.09000 \mathrm{E}-020.0953+1.02000 \mathrm{E}-020.0985$

$4.6400 \mathrm{E}-01-1.65000 \mathrm{E}-020.0772-1.46000 \mathrm{E}-020.0822$

$5.5500 \mathrm{E}-01 \quad-2.58000 \mathrm{E}-020.0614-2.48000 \mathrm{E}-02 \quad 0.0627$

$\begin{array}{lllll}6.4500 \mathrm{E}-01 & -3.63000 \mathrm{E}-02 & 0.0515 & -3.21000 \mathrm{E}-02 & 0.0549\end{array}$

$7.3600 \mathrm{E}-01-5.59000 \mathrm{E}-020.0411-6.67000 \mathrm{E}-020.0374$

$8.2700 \mathrm{E}-01-8.86000 \mathrm{E}-020.0321-1.21300 \mathrm{E}-010.0269$

$9.1800 \mathrm{E}-01-1.34800 \mathrm{E}-010.0253-1.10600 \mathrm{E}-010.0284$

$1.1000 \mathrm{E}+00-1.65500 \mathrm{E}-010.0225-2.60000 \mathrm{E}-030.1959$

total $-5.51300 \mathrm{E}-010.0094-3.95100 \mathrm{E}-010.0126$

Itally $58 \quad \mathrm{nps}=10000$

tally type 8 pulse height distribution. units number

tally for photons electrons

celt: $\quad$ t

energy

$-1.0000 \mathrm{E}-03 \quad 7.40000 \mathrm{E}-03 \quad 0.1158$

$0.0000 E+00 \quad 0.00000 E+000.0000$

$1.0000 \mathrm{E}-06 \quad 0.00000 \mathrm{E}+000.0000$

$1.0000 \mathrm{E}-01 \quad 2.12600 \mathrm{E}-010.0192$

$1.9100 \mathrm{E}-01 \quad 2.65500 \mathrm{E}-010.0166$

$2.8200 \mathrm{E}-01 \quad 1.87700 \mathrm{E}-010.0208$

$3.7300 \mathrm{E}-01 \quad 1.09500 \mathrm{E}-01 \quad 0.0285$

4.6400E-01 6.44000E-02 0.0381

5.5500 E-01 4.37000 E- 020.0468

$6.4500 \mathrm{E}-01 \quad 2.66000 \mathrm{E}-020.0605$

$7.3600 \mathrm{E}-01 \quad 1.58000 \mathrm{E}-02 \quad 0.0789$

$700 \mathrm{E}-01 \quad 1.12000 \mathrm{E}-020.0940$

$800 \mathrm{E}-01 \quad 7.70000 \mathrm{E}-030.1135$ 
$1.1000 \mathrm{E}+00 \quad 4.79000 \mathrm{E}-020.0446$

total $1.00000 \mathrm{E}+000.0000$

y $8 \mathrm{nps}=10000$

tally type $8^{*}$ energy deposition

HNF-SD-SNF-CSWD-005, Rev. 0

tally for photons electrons

cell:

energy

$1.0000 \mathrm{E}+03 \quad 2.66900 \mathrm{E}-01 \quad 0.0090$

1status of the statistical checks used to form confidence intervals for the mean for each tally bin

tally result of statistical checks for the tic bin (the first check not passed is listed) and error magnitude check for all bins

1 missed 1 of $10 \mathrm{tfc}$ bin checks: there is insufficient tfe bin tally information to estimate the large tally slope reliably missed all bin error check: 24 tally bins had 4 bins with zeros and 17 bins with relative errors exceeding 0.10

6 missed 1 of $10 \mathrm{tfc}$ bin checks: the variance of the variance appears not to decrease as $1 / n p s$ for the last half of problem missed all bin error check: 12 tally bins had 2 bins with zeros and 8 bins with relative errors exceeding 0.10

28 passed the 10 statistical checks for the tally fluctuation chart bin result missed all bin error check: 15 tally bins had 2 bins with zeros and 2 bins with relative errors exceeding 0.10

11 missed 1 of 10 tf bin checks: the slope of decrease of largest tallies is less than the minimum acceptable value of 3.0 missed all bin error check: 24 tally bins had 2 bins with zeros and 5 bins with relative errors exceeding 0.10

21 missed 1 of 10 tfc bin checks: the estimated mean has a trend during the last half of the problem missed all bin error check: 24 tally bins had 2 bins with zeros and 5 bins with relative errors exceeding 0.10

31 missed 2 of $10 \mathrm{tfc}$ bin checks: the estimated mean has a trend during the last half of the problem missed all bin error check: 24 tally bins had 2 bins with zeros and 5 bins with relative errors exceeding 0.10

41 missed 1 of $10 \mathrm{tfc}$ bin checks: the estimated mean has a trend during the last half of the problem missed all bin error check: 72 tally bins had 28 bins with zeros and 10 bins with relative enors exceeding 0.10

1 missed 2 of $10 \mathrm{tfc}$ bin checks: the estimated mean has a trend during the last half of the problem missed all bin error check: 72 tally bins had 28 bins with zeros and 10 bins with relative errors exceeding 0.10

58 passed the 10 statistical checks for the tally fluctuation chart bin result missed all bin error check: 15 tally bins had 2 bins with zeros and 2 bins with relative erors exceeding 0.10

8 passed the 10 statistical checks for the tally fluctuation chart bin result passed all bin enor check: I tally bins all have relative errors less than 0.10 with no zero bins

the 10 statistical checks are only for the tally fluctuation chart bin and do not apply to other tally bins.

the tally bins with zeros may or may not be correct: compare the source, cutofs, multipliers, et cetera with the tally bins.

waming. 7 of the 10 tally fluctuation chart bins did not pass all 10 statistical checks.

waming. 9 of the 10 tallies had bins with relative errors greater than recommended.

itally fluctuation charts

tally 1 tally $6 \quad$ tally 28

nps mean error vov slope fom mean error vov slope fom $10006.7975 E-03 \quad 0.23990 .08350 .0 \quad 2.0857$ E-05 $0.15890 .0525 \quad 0.0$ $20005.5780 \mathrm{E}-030.18840 .0641 \quad 0.0 \quad 2.4397 \mathrm{E}-050.17010 .1693 \quad 0.0$ $30005.8779 \mathrm{E}-03 \quad 0.14550 .03730 .0 \quad 2.6650 \mathrm{E}-050.13430 .10600 .0$

$\begin{array}{lllll}40005.7880 \mathrm{E}-03 & 0.13170 .0311 & 0.0 & 2.7894 \mathrm{E}-050.11450 .0656 & 0.0\end{array}$ $50005.9924 \mathrm{E}-03 \quad 0.11680 .02390 .0$ $60005.9423 \mathrm{E}-03 \quad 0.10550 .0195 \quad 0.0$ $70005.9749 \mathrm{E}-03 \quad 0.09680 .0170 \quad 0.0$ $80005.7670 \mathrm{E}-03 \quad 0.09130 .01540 .0$ $90005.7398 \mathrm{E}-030.08640 .01350 .0$ $100005.7777 \mathrm{E}-03 \quad 0.08110 .0120 \quad 0.0$
$2.7855 \mathrm{E}-050.10200 .0485 \quad 0.0$

$2.7793 \mathrm{E}-050.09310 .0418 \quad 0.0$

$2.8647 \mathrm{E}-050.09190 .0581 \quad 0.0$

$2.8249 \mathrm{E}-050.08380 .052410 .0$

$2.7485 E-050.07790 .049110 .0$ $2.7551 \mathrm{E}-050.07220 .043610 .0$ mean error vov slope fom 2.6993E-01 0.02790 .004410 .0 $2.6475 \mathrm{E}-010.01990 .002210 .0$ $2.6433 \mathrm{E}-010.01610 .001510 .0$ $2.6224 \mathrm{E}-01 \quad 0.01400 .001110 .0$

$2.6175 \mathrm{E}-010.01260 .000910 .0$ $2.6496 \mathrm{E}-010.01160 .000710 .0$

$2.6691 \mathrm{E}-010.01070 .000610 .0$

$2.6739 \mathrm{E}-010.01000 .000510 .0$

$2.6699 \mathrm{E}-010.00950 .000510 .0$

$2.6690 \mathrm{E}-010.00900 .000410 .0$ 
tally 11 nps mean error vov slope fom $10004.4504 E-010.02960 .0002 \quad 2.1$ $20004.4489 \mathrm{E}-010.02110 .00012 .0$ $30004.4226 E-01 \quad 0.01730 .0001 \quad 1.8$ $40004.4011 \mathrm{E}-010.01510 .00001 .8$ $50004.4004 \mathrm{E}-01 \quad 0.01350 .0000 \quad 1.9$ $60004.3549 \mathrm{E}-010.01250 .00001 .8$ $70004.3477 \mathrm{E}-010.01150 .0000 \quad 1.9$ $80004.3339 \mathrm{E}-010.01080 .00001 .8$ $90004.3428 E-010.01020 .0000 \quad 1.7$ $100004.3411 \mathrm{E}-010.00970 .0000 \quad 1.6$

tally 41

nps mean error vov slope fom $10005.6500 \mathrm{E}-010.02860 .00044 .4$ $20005.6500 \mathrm{E}-010.02040 .00034 .4$ $30005.6400 \mathrm{E} \cdot 01 \quad 0.0168 \quad 0.000210 .0$ $40005.6100 \mathrm{E}-010.01470 .000210 .0$ $50005.5860 \mathrm{E}-010.01310 .000110 .0$ $60005.5433 E-010.01210 .000110 .0$ $70005.5386 \mathrm{E}-010.01120 .000110 .0$ $80005.5238 \mathrm{E}-010.01050 .000110 .0$ $90005.5200 \mathrm{E}-010.00990 .000110 .0$ $100005.5130 E-010.00940 .000110 .0$

tally 8

nps mean error vov slope fom $10002.6993 \mathrm{E}-010.02790 .004410 .0$ $20002.6475 E-010.01990 .002210 .0$ $30002.6433 E-010.01610 .001510 .0$ $40002.6224 E-01 \quad 0.01400 .001110 .0$ $50002.6175 \mathrm{E}-010.01260 .000910 .0$ $60002.6496 E-010.01160 .000710 .0$ $70002.6691 \mathrm{E}-010.01070 .000610 .0$ $80002.6739 \mathrm{E}-010.01000 .000510 .0$ $90002.6699 \mathrm{E}-010.00950 .000510 .0$ $100002.6690 \mathrm{E}-010.00900 .000410 .0$ tally data written to file inp $20 \mathrm{~m}$

6 waming messages so far. tally 21

tally 31 mean error vov slope fom $5.6500 \mathrm{E}-010.02860 .00044 .4$ $5.6500 \mathrm{E}-010.02040 .0003 \quad 4.4$ $5.6400 \mathrm{E}-01 \quad 0.01680 .000210 .0$ $5.6100 \mathrm{E}-010.01470 .000210 .0$ $5.5860 \mathrm{E}-010.01310 .000110 .0$ $5.5433 \mathrm{E}-010.01210 .000110 .0$ $5.5386 \mathrm{E}-010.01120 .000110 .0$ $5.5238 \mathrm{E}-010.01050 .000110 .0$ $5.5200 \mathrm{E}-010.00990 .000110 .0$ $5.5130 \mathrm{E}-010.00940 .000110 .0$

tally 51 tally 58 mean etror vov slope fom

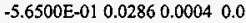
$\begin{array}{rlll}-5.6500 \mathrm{E}-01 & 0.02040 .0003 \quad 0.0\end{array}$

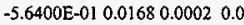
$\begin{array}{llll}-5.6100 \mathrm{E}-01 & 0.01470 .0002 & 0.0\end{array}$ $\begin{array}{llll}-5.5860 \mathrm{E}-01 & 0.0131 & 0.0001 & 0.0\end{array}$ $\begin{array}{llll}-5.5433 \mathrm{E}-01 & 0.0121 & 0.0001 & 0.0\end{array}$ $\begin{array}{llll}-5.5386 \mathrm{E}-01 & 0.01120 .0001 & 0.0\end{array}$

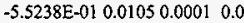
$-5.5200 \mathrm{E}-010.00990 .00010 .0$
0.0 .000 $\begin{array}{lll}-5.5130 \mathrm{E}-01 & 0.00940 .0001 & 0.0\end{array}$
HNF-SD-SNF-CSWD-005, Rev. 0 mean error vov slope fom $-5.6500 \mathrm{E}-010.02860 .0004 \quad 0.0$
$-5.6500 \mathrm{E}-010.02040 .00030 .0$ $-5.6500 \mathrm{E}-010.02040 .0003 \quad 0.0$
0.640 .000 $\begin{array}{llll}-5.6400 \mathrm{E}-01 & 0.0168 & 0.0002 & 0.0\end{array}$ $\begin{array}{llll}-5.6100 \mathrm{E}-01 & 0.0147 & 0.0002 & 0.0\end{array}$ $\begin{array}{llll}-5.5860 \mathrm{E}-01 & 0.0131 & 0.0001 & 0.0\end{array}$ $\begin{array}{lllll}-5.5433 \mathrm{E}-01 & 0.0121 & 0.0001 & 0.0\end{array}$

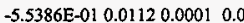
$\begin{array}{llll}-5.5238 \mathrm{E}-01 & 0.01050 .0001 & 0.0\end{array}$ $-5.5200 \mathrm{E}-010.00990 .00010 .0$
0.5 $\begin{array}{rl}-5.5130 \mathrm{E}-01 & 0.00940 .0001 \quad 0.0\end{array}$

mean error vov slope fom $1.0000 \mathrm{E}+000.00000 .000010 .0$ $1.0000 \mathrm{E}+000.00000 .000010 .0$ $1.0000 \mathrm{E}+000.00000 .000010 .0$ $1.0000 \mathrm{E}+00 \quad 0.0000 \quad 0.000010 .0$ $1.0000 \mathrm{E}+000.00000 .000010 .0$ $1.0000 \mathrm{E}+00 \quad 0.00000 .000010 .0$ $1.0000 \mathrm{E}+000.00000 .000010 .0$ $1.0000 \mathrm{E}+000.00000 .000010 .0$ $1.0000 \mathrm{E}+000.00000 .000010 .0$ $1.0000 \mathrm{E}+000.00000 .000010 .0$

תun terminated when 10000 particle histories were done.

\section{File: Inp240}

I- prob 24 - reflecting lattice. $15 \times 15$ at 3.75 w/o u-235 enrichment.

2- $11-10.182-1 \mathrm{u}=2$

3. 2 2-.001 $1-2 \mathrm{u}=2$

4- $3 \quad 3-6.552-3 u=2$

5- 4 4-1.03u=2

6- 5 - $4-1.0-14: 15 u=3$

7- $6 \quad 3-6.5514-15 \mathrm{u}=3$

8- $\quad 7 \quad 4-1.0-4+5-6+7 u=1$ lat $=1$ fill $=-8: 8-8: 80: 0$

9. $\quad 117 r 214 \mathrm{r} 11214 \mathrm{r} 1122322322+322$

10- $\quad 3221126 \mathrm{r} 326 \mathrm{r} 1123 \mathrm{r3}$

11. $\quad 24 r 323 r 1122328 \mathrm{r} 322$

12- $\quad 11214 \mathrm{r} 1122 \mathrm{r} 322 \mathrm{r} 322 \mathrm{r}$

13. $\quad 322 \mathrm{rl} 12 \mathrm{l} 4 \mathrm{rl} 122328 \mathrm{r} 3$

14- $\quad 221123 r 324 r 323 r 11$

15- $\quad 26 \mathrm{r} 326 \mathrm{r} 11223223$

$22 \mathrm{r} 32232211214 \mathrm{r} 1$ I $214 \mathrm{r} 117 \mathrm{r}$

$8 \quad 0-8 \quad-10 \quad-12 u=4$ fill $=1$

$95-7.98: 10 u=4$ 


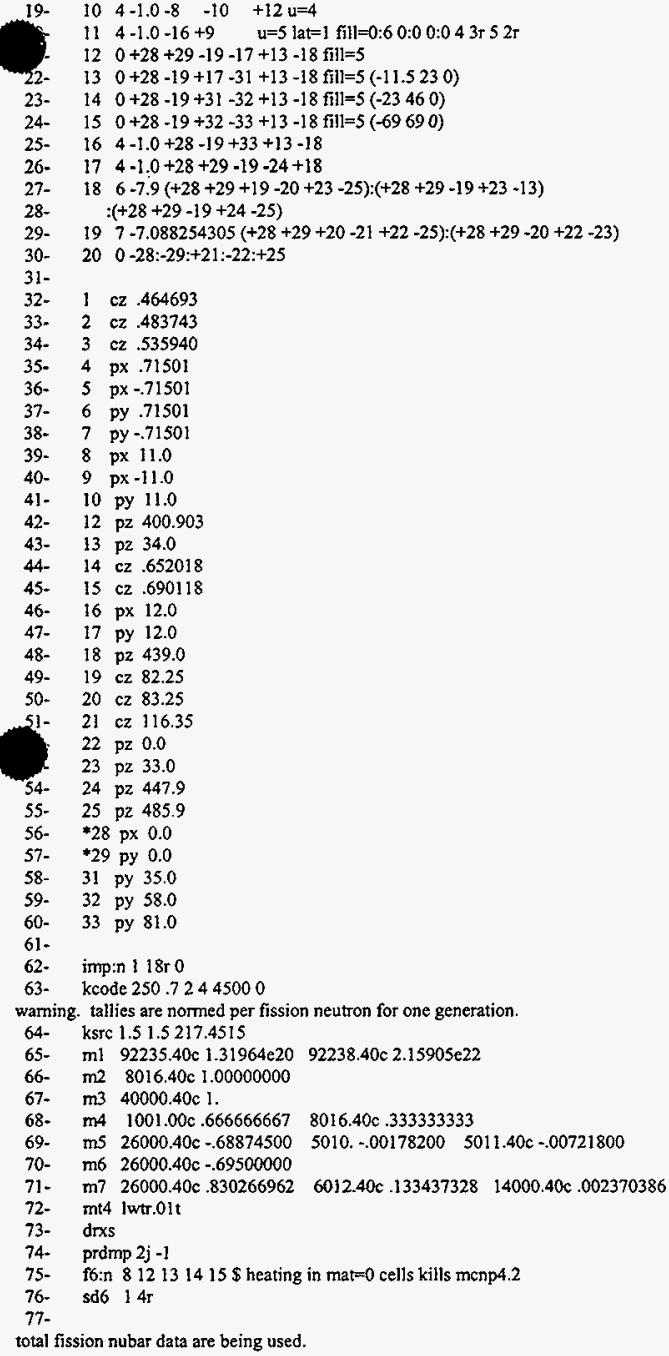




$$
\text { cell mat density density volume mass pieces importance }
$$

$\begin{array}{ccccccccc}1 & 1 & 1 & 2.57597 \mathrm{E}-02 & 1.01820 \mathrm{E}+01 & 0.00000 \mathrm{E}+00 & 0.00000 \mathrm{E}+00 & 0 & 1.0000 \mathrm{E}+00 \\ 2 & 2 & 2 & 3.76497 \mathrm{E}-05 & 1.00000 \mathrm{E}-03 & 0.00000 \mathrm{E}+00 & 0.00000 \mathrm{E}+00 & 0 & 1.0000 \mathrm{E}+00 \\ 3 & 3 & 3 & 4.32392 \mathrm{E}-02 & 6.55000 \mathrm{E}+00 & 0.000000 \mathrm{E}+00 & 0.00000 \mathrm{E}+00 & 0 & 1.0000 \mathrm{E}+00 \\ 4 & 4 & 4 \mathrm{~s} & 1.00309 \mathrm{E}-01 & 1.00000 \mathrm{E}+00 & 0.00000 \mathrm{E}+00 & 0.00000 \mathrm{E}+00 & 0 & 1.0000 \mathrm{E}+00 \\ 5 & 5 & 45 & 1.00309 \mathrm{E}-01 & 1.00000 \mathrm{E}+00 & 0.00000 \mathrm{E}+00 & 0.00000 \mathrm{E}+00 & 0 & 1.0000 \mathrm{E}+00 \\ 6 & 6 & 3 & 4.32392 \mathrm{E}+02 & 6.55000 \mathrm{E}+00 & 0.00000 \mathrm{E}+00 & 0.00000 \mathrm{E}+00 & 0 & 1.0000 \mathrm{E}+00 \\ 7 & 7 & 45 & 1.00309 \mathrm{E}-01 & 1.00000 \mathrm{E}+00 & 0.00000 \mathrm{E}+00 & 0.00000 \mathrm{E}+00 & 0 & 1.0000 \mathrm{E}+00 \\ 8 & 8 & 0 & 0.00000 \mathrm{E}+00 & 0.00000 \mathrm{E}+00 & 0.00000 \mathrm{E}+00 & 0.00000 \mathrm{E}+00 & 0 & 1.0000 \mathrm{E}+00 \\ 9 & 9 & 5 & 8.97738 \mathrm{E}-02 & 7.90000 \mathrm{E}+00 & 0.000000 \mathrm{E}+00 & 0.00000 \mathrm{E}+00 & 0 & 1.0000 \mathrm{E}+00 \\ 10 & 10 & 4 \mathrm{~s} & 1.00309 \mathrm{E}-01 & 1.00000 \mathrm{E}+00 & 0.00000 \mathrm{E}+00 & 0.00000 \mathrm{E}+00 & 0 & 1.0000 \mathrm{E}+00 \\ 11 & 11 & 4 \mathrm{~s} & 1.00309 \mathrm{E}-01 & 1.00000 \mathrm{E}+00 & 0.00000 \mathrm{E}+00 & 0.00000 \mathrm{E}+00 & 0 & 1.0000 \mathrm{E}+00 \\ 12 & 12 & 0 & 0.00000 \mathrm{E}+00 & 0.00000 \mathrm{E}+00 & 0.00000 \mathrm{E}+00 & 0.00000 \mathrm{E}+00 & 0 & 1.0000 \mathrm{E}+00 \\ 13 & 13 & 0 & 0.00000 \mathrm{E}+00 & 0.00000 \mathrm{E}+00 & 0.00000 \mathrm{E}+00 & 0.00000 \mathrm{E}+00 & 0 & 1.0000 \mathrm{E}+00 \\ 14 & 14 & 0 & 0.00000 \mathrm{E}+00 & 0.00000 \mathrm{E}+00 & 0.00000 \mathrm{E}+00 & 0.00000 \mathrm{E}+00 & 0 & 1.0000 \mathrm{E}+00 \\ 5 & 15 & 0 & 0.00000 \mathrm{E}+00 & 0.00000 \mathrm{E}+00 & 0.00000 \mathrm{E}+00 & 0.00000 \mathrm{E}+00 & 0 & 1.0000 \mathrm{E}+00 \\ 16 & 16 & 4 \mathrm{~s} & 1.00309 \mathrm{E}-01 & 1.00000 \mathrm{E}+00 & 0.00000 \mathrm{E}+00 & 0.00000 \mathrm{E}+00 & 0 & 1.0000 \mathrm{E}+00 \\ 17 & 17 & 4 \mathrm{~s} & 1.00309 \mathrm{E}-01 & 1.00000 \mathrm{E}+00 & 0.00000 \mathrm{E}+00 & 0.00000 \mathrm{E}+00 & 0 & 1.0000 \mathrm{E}+00 \\ 18 & 18 & 6 & 8.51890 \mathrm{E}-02 & 7.90000 \mathrm{E}+00 & 0.00000 \mathrm{E}+00 & 0.00000 \mathrm{E}+00 & 0 & 1.0000 \mathrm{E}+00 \\ 19 & 19 & 7 & 8.58502 \mathrm{E}-02 & 7.08825 \mathrm{E}+00 & 0.000000 \mathrm{E}+00 & 0.00000 \mathrm{E}+00 & 0 & 1.0000 \mathrm{E}+00 \\ 20 & 20 & 0 & 0.00000 \mathrm{E}+00 & 0.00000 \mathrm{E}+00 & 0.00000 \mathrm{E}+00 & 0.00000 \mathrm{E}+00 & 0 & 0.0000 \mathrm{E}+00\end{array}$

total

\section{$0.00000 \mathrm{E}+000.00000 \mathrm{E}+00$}

11 warning messages so far.

lcross-section tables

print table 100

table length

tables from file testlib]

$100100 \mathrm{c} 2139$ 1-h-1 from endf-vi.1

$5010.03 d \quad 3945$ b-10 end f/b-iv new gamma production format

mat $12505 / 26 / 93$ $(1273) \quad 14$ oct 75

5011.40c 3473 ENDL library name: nd900719 MCNP translation: 900831 14:10:05 $6012.40 \mathrm{C} 5049$ ENDL library name: nd900719 MCNP translation: 900831 14:10:05 8016.40c 5693 ENDL library name: nd900719 MCNP translation: 900831 14:10:05 14000.40 8732 ENDL library name: nd900719 MCNP translation: 900831 14:10:05 26000.40c 21846 ENDL library name: nd900719 MCNP translation: 900831 14:10:05 40000.40c 111279 ENDL library name: nd900719 MCNP translation: 900831 14:10:05 92235.40 40506 ENDL library name: nd900719 MCNP translation: 9total nu 10:05 92238.40c 48310 ENDL library name: nd901118 MCNP translation: 9total nu 56:49 lwtr.01t 10193 hydrogen in light water at 300 degrees kelvin 1001

900207 900207 900207 900207 900207 900323 900503 901119

total 261165

waming. neutron energy cutoff is below some cross-section tables. 
12 waming messages so far. lestimated keff results by cycle

print table 175

cycle 1 k(collision) 0.658210 removal lifetime(abs) $5.1517 \mathrm{E}+03$ source points generated 228

cycle $2 \mathrm{k}$ (collision) 0.549318 removal lifetime(abs) $3.7645 \mathrm{E}+03$ source points generated 209

cycle $3 \mathrm{k}$ (collision) 0.551862 removal lifetime(abs) $3.8422 \mathrm{E}+03$ source points generated 253

estimator cycle 4 ave of 2 cycles combination simple average combined average cort

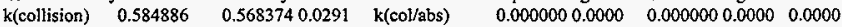
$\begin{array}{lllllll}\text { k(absorption) } & 0.583788 & 0.5911690 .0125 & \mathrm{k}(\mathrm{abs} / \mathrm{tk} \mathrm{ln}) & 0.0000000 .0000 & 0.0000000 .0000 & 0.0000\end{array}$

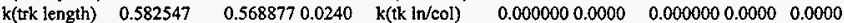
rem life(col) $3.9485 \mathrm{E}+03 \quad 3.8951 \mathrm{E}+030.0137$ rem life(abs) $4.0658 \mathrm{E}+03 \quad 3.9540 \mathrm{E}+03 \quad 0.0283 \quad$ life(col/abs) $\quad 0.0000 \mathrm{E}+000.0000 \quad 0.0000 \mathrm{E}+000.0000 \quad 0.0000$ source points generated 264

source distribution written to file inp $24 \mathrm{~s} \quad$ cycle $=4$ Iproblem summary run terminated when 4 kcode cycles were done.

neutron creation tracks weight energy neutron loss tracks weight energy (per source particle) $\quad$ (per source particle)

source $940 \quad 1.0638 \mathrm{E}+00 \quad 1.9$

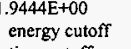

range of sampled source weights $=9.8814 \mathrm{E}-01$ to $1.1962 \mathrm{E}+00$

ineutron activity in each cell

print table 126

tracks population collisions collisions number flux average average cell entering weight weighted weighted track weight track mfp (per history) energy energy (relative) (cm)

$\begin{array}{ccccccccccc}1 & 15187 & 944 & 4054 & 3.5039 \mathrm{E}+00 & 3.6399 \mathrm{E}-04 & 6.5723 \mathrm{E}-01 & 8.4316 \mathrm{E}-01 & 3.8971 \mathrm{E}+00 \\ 2 & 29440 & 944 & 0 & 0.0000 \mathrm{E}+00 & 3.3819 \mathrm{E}-04 & 6.3029 \mathrm{E}-01 & 8.4006 \mathrm{E}-01 & 9.6437 \mathrm{E}+03 \\ 3 & 31610 & 944 & 1028 & 9.2538 \mathrm{E}-01 & 3.1626 \mathrm{E}-04 & 6.0475 \mathrm{E}-01 & 8.3961 \mathrm{E}-01 & 3.6368 \mathrm{E}+00\end{array}$




$\begin{array}{cccc}4 & 4 & 44969 & 944 \\ 5 & 5 & 8213 & 845 \\ 5 & 6 & 4924 & 791 \\ 7 & 7 & 2832 & 638 \\ 8 & 8 & 0 & 0 \\ 9 & 9 & 1333 & 607 \\ 10 & 10 & 0 & 0 \\ 11 & 11 & 7 & 1 \\ 12 & 12 & 0 & 0 \\ 13 & 13 & 0 & 0 \\ 14 & 14 & 0 & 0 \\ 15 & 15 & 0 & 0 \\ 16 & 16 & 0 & 0 \\ 17 & 17 & 0 & 0 \\ 18 & 18 & 0 & 0 \\ 19 & 19 & 0 & 0\end{array}$

$\begin{array}{llllll}40660 & 3.0973 \mathrm{E}+01 & 3.0008 \mathrm{E}-04 & 5.9877 \mathrm{E}-01 & 8.3882 \mathrm{E}-01 & 1.4858 \mathrm{E}+00\end{array}$

HNF-SD-SNF-CSWD-005, Rev. 0

$8775 \quad 6.4600 \mathrm{E}+00 \quad 2.0190 \mathrm{E}-04 \quad 5.0976 \mathrm{E}-01 \quad 8.1000 \mathrm{E}-01 \quad 1.3256 \mathrm{E}+00$

$\begin{array}{lllllll}109 & 9.6195 \mathrm{E}-02 & 2.0789 \mathrm{E}-04 & 5.2658 \mathrm{E}-01 & 8.2057 \mathrm{E}-01 & 3.6398 \mathrm{E}+00\end{array}$

$1607 \quad 1.4612 \mathrm{E}+00 \quad 8.5166 \mathrm{E}-04 \quad 7.1930 \mathrm{E}-01 \quad 9.4163 \mathrm{E}-01 \quad 1.7232 \mathrm{E}+00$

$\begin{array}{lllll}0 & 0.0000 \mathrm{E}+00 \quad 0.0000 \mathrm{E}+00 & 0.0000 \mathrm{E}+00 \quad 0.0000 \mathrm{E}+00 \quad 0.0000 \mathrm{E}+00\end{array}$

$\begin{array}{lllll}1347 \quad 1.2836 \mathrm{E}+00 \quad 3.8320 \mathrm{E}-03 & 8.0017 \mathrm{E}-01 & 9.7093 \mathrm{E}-01 & 3.1777 \mathrm{E}+00\end{array}$

$\begin{array}{lllllll}0 & 0.0000 \mathrm{E}+00 & 0.0000 \mathrm{E}+00 & 0.0000 \mathrm{E}+00 & 0.0000 \mathrm{E}+00 & 0.0000 \mathrm{E}+00\end{array}$

$126 \quad 9.7846 \mathrm{E}-02 \quad 9.0288 \mathrm{E}-05 \quad 3.2866 \mathrm{E}-01 \quad 7.7027 \mathrm{E}-01 \quad 1.0087 \mathrm{E}+00$

$\begin{array}{lllllll}0 & 0.0000 \mathrm{E}+00 & 0.0000 \mathrm{E}+00 & 0.0000 \mathrm{E}+00 & 0.0000 \mathrm{E}+00 & 0.0000 \mathrm{E}+00\end{array}$

$\begin{array}{llllllllllllll}0 & 0.0000 \mathrm{E}+00 & 0.0000 \mathrm{E}+00 & 0.0000 \mathrm{E}+00 & 0.0000 \mathrm{E}+00 & 0.0000 \mathrm{E}+00\end{array}$

$\begin{array}{llllllll}0 & 0.0000 \mathrm{E}+00 & 0.0000 \mathrm{E}+00 & 0.0000 \mathrm{E}+00 & 0.0000 \mathrm{E}+00 & 0.0000 \mathrm{E}+00\end{array}$

$\begin{array}{llllllllllll}0 & 0.0000 \mathrm{E}+00 & 0.0000 \mathrm{E}+00 & 0.0000 \mathrm{E}+00 & 0.0000 \mathrm{E}+00 & 0.0000 \mathrm{E}+00\end{array}$

$\begin{array}{lllllll}0 & 0.0000 \mathrm{E}+00 & 0.0000 \mathrm{E}+00 & 0.0000 \mathrm{E}+00 & 0.0000 \mathrm{E}+00 & 0.0000 \mathrm{E}+00\end{array}$

$\begin{array}{llllllllll}0 & 0.0000 \mathrm{E}+00 & 0.0000 \mathrm{E}+00 & 0.0000 \mathrm{E}+00 & 0.0000 \mathrm{E}+00 & 0.0000 \mathrm{E}+00\end{array}$

$\begin{array}{lllllllllllll}0 & 0.0000 \mathrm{E}+00 & 0.0000 \mathrm{E}+00 & 0.0000 \mathrm{E}+00 & 0.0000 \mathrm{E}+00 & 0.0000 \mathrm{E}+00\end{array}$

$\begin{array}{lllllllllllllll}0 & 0.0000 \mathrm{E}+00 & 0.0000 \mathrm{E}+00 & 0.0000 \mathrm{E}+00 & 0.0000 \mathrm{E}+00 & 0.0000 \mathrm{E}+00\end{array}$

total $\quad 138515 \quad 6658 \quad 57706 \quad 4.4801 \mathrm{E}+01$

the initial fission neutron source distribution used the 1 source points that were input on the kste card.

the criticality problem was scheduled to skip 2 cycles and run a total of 4 cycles with nominally 250 neutrons per cycle.

this problem has run 2 inactive cycles with 478 neutron histories and 2 active cycles with 462 neutron histories.

this calculation has completed the requested number of keff cycles using a total of 940 fission neutron source histories. all cells with fissionable material were sampled and had fission neutron source points.

there is no combined collision/absorption/track-length estimate for keff because only 2 active cycles were run.

the estimated average keffs, one standard deviations, and 68,95 , and 99 percent confidence intervals are:

\begin{tabular}{|cccccccc} 
keff estimator & keff & standard deviation & $68 \%$ confidence & $95 \%$ confidence & $99 \%$ confidence & corr \\
collision & 0.56837 & 0.01651 & 0.53834 to 0.59841 & 0.35851 to 0.77824 & 0.00000 to 1.61951 \\
absorption & 0.59117 & 0.00738 & 0.57774 to 0.60460 & 0.49735 to 0.68498 & 0.12129 to 1.06105 \\
track length & 0.56888 & 0.01367 & 0.54401 to 0.59374 & 0.39513 to 0.74263 & 0.00000 to 1.43912
\end{tabular}

lindividual and average keff estimator results by cycle

keff neutron keff estimators by cycle average keff estimators and deviations average $\mathrm{k}(\mathrm{c} / \mathrm{a} / \mathrm{t})$ cycle histories $k$ (coll) $k(a b s) k($ track) $k$ (coll) st dev $k(a b s)$ st dev $k($ track) st dev $k(c / a / t)$ st dev fom

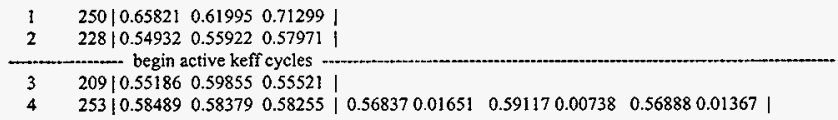

the largest active cycle keffs by estimator are:

collision 0.58489 on cycle 4 absorption 0.59855 on cycle 3 track length 0.58255 on cycle 4

ltally $6 \mathrm{nps}=940$

tally type 6 track length estimate of heating. units mev/gram

tally for neutrons

number of histories used for normalizing tallies $=\quad 500.00$

masses

cell: $\begin{array}{lllll}8 & 12 & 13 & 14 & 15\end{array}$

$1.00000 \mathrm{E}+00 \quad 1.00000 \mathrm{E}+00 \quad 1.00000 \mathrm{E}+00 \quad 1.00000 \mathrm{E}+00 \quad 1.00000 \mathrm{E}+00$ the smallest active cycle keffs by estimator are:

collision 0.55186 on cycle 3

absorption 0.58379 on cycle 4

track length 0.55521 on cycle 3

$4.02566 E+010.0434$ 
cell 14

\section{$9.78180 \mathrm{E}-010.4014$}

cell 15 1.20234E-01 0.9710

lanalysis of the results in the tally fluctuation chart bin (tfe) for tally 6 with nps $=940$ print table 160

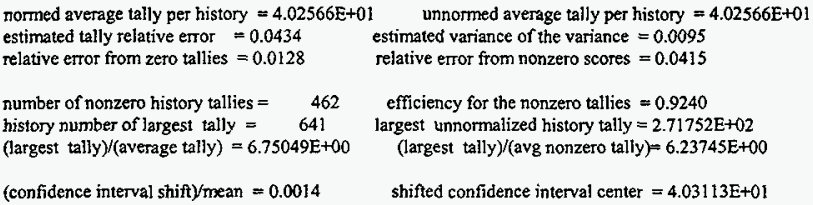

if the largest, history score sampled so far were to occur on the very next history, the tfc bin quantities would change as follows: nps $=462$ for this table because 2 keff cycles and 478 histories were skipped before tally accumulation.

$\begin{array}{lccc}\text { estimated quantities } & \text { value at nps } & \text { value at nps+1 } & \text { value(nps+1)/value(nps } \\ \text { mean } & & & \\ \text { relative error } & 4.02566 \mathrm{E}+01 & 4.07566 \mathrm{E}+01 & 0.012420 \\ \text { variance of the variance } & 4.34431 \mathrm{E}-02 & 4.24506 \mathrm{E}-02 & -0.022846 \\ \text { hifted center } & 9.52674 \mathrm{E}-03 & 1.33327 \mathrm{E}-02 & 0.399508 \\ \text { ligure of merit } & 4.03113 \mathrm{E}+01 & 4.03457 \mathrm{E}+01 & 0.000853 \\ \text { dit } & 0.00000 \mathrm{E}+00 & 0.00000 \mathrm{E}+00 & 0.000000\end{array}$

there is not enough information in the largest history scores (usually less than 500 scores) for a reliable estimate of the slope. the history score probability density function appears to have an unsampled region at the largest history scores: please examine.

the nps-dependent tic bin check results are suspect because there are only $1 \mathrm{nps}$ tally values to analyze *****

results of 10 statistical checks for the estimated answer for the tally fluctuation chart (tfc) bin of tally 6

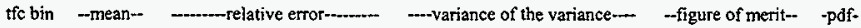

behavior behavior value decrease decrease rate value decrease decrease rate value behavior slope

$\begin{array}{llllllll}\text { desired random } & <0.10 & \text { yes } & 1 / \text { sqrit(nps) } & <0.10 \text { yes } 1 / \text { mps } & \text { constant random }>3.00 \\ \text { observed random } & 0.04 \text { yes } & \text { yes } 0.01 \text { yes yes } & 0.00 & \text { no }\end{array}$

passed? yes yes yes yes yes yes yes no

waming. the tally in the tally fluctuation chart bin did not pass 1 of the 10 statistical checks.

lunnormed tally density for tally 6 nonzero tally mean $(\mathrm{m})=4.357 \mathrm{E}+01$ nps $=940$ print table 161

abscissa ordinate log plot of taliy probability density function in tally fluctuation chart bin( $d=$ decade, slope $=0.0$ )

tally number num den $\log$ den:d-..._-

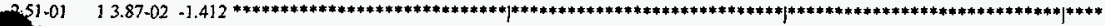

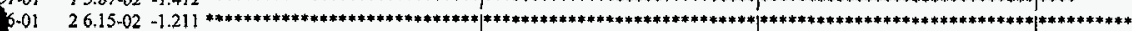

$88-01 \quad 12.44-02-1.612 * * * * * * * * * * * * * * * * * * * * * * * * * * * * *|* * * * * * * * * * * * * * * * * * * * * * * * * * * * * *| * * * * * * * * * * * * * * * * * * * * * * * * * * * * \mid$ 


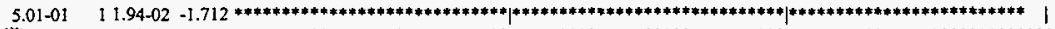

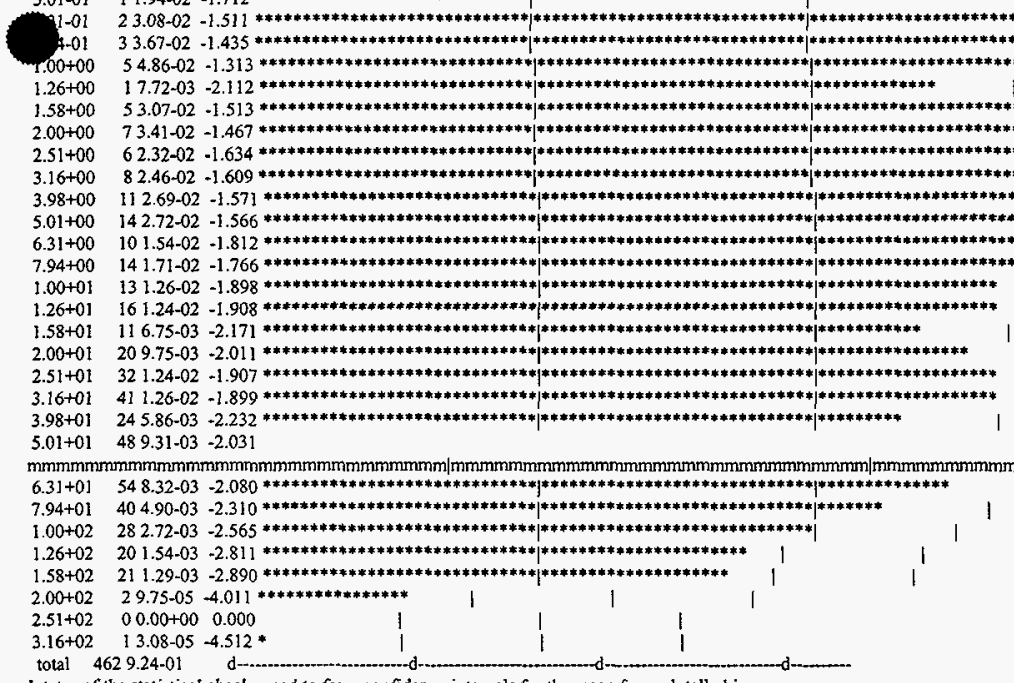

total $4629.24-01$
Istatus of the statistical checks used to form confidence intervals for the mean for each tally bin

result of statistical checks for the tfe bin (the first check not passed is listed) and error magnitude check for all bins

6 missed 1 of $10 \mathrm{tfc}$ bin checks: there is insufficient tfc bin tally information to estimate the large tally slope reliably missed all bin error check: 5 tally bins had 0 bins with zeros and 2 bins with relative errors exceeding 0.10

the 10 statistical checks are only for the tally fluctuation chart bin and do not apply to other tally bins.

waming. I of the 1 tally fluctuation chart bins did not pass all 10 statistical checks.

waming. I of the 1 tallies had bins with relative errors greater than recommended.

1tally fluctuation charts

tally 6

nps mean error vov slope fom

$9404.0257 \mathrm{E}+010.04340 .0095 \quad 0.0$

tally data written to file inp $24 \mathrm{~m}$

15 waming messages so far.

run terminated when 4 kcode cycles were done.

\section{File: Outp24}

1. prob 24 -- reflecting lattice. $15 \times 15$ at 3.75 w/o u-235 enrichment.

2- $1 \quad 1-10.182-1 \mathrm{u}=2$

3. 2 2. 2.001$]-2 \mathrm{u}=2$

4- $3 \quad 3-6.552-3 \mathrm{u}=2$

5. $4 \quad 4-1.03 u=2$

6- 5 - $4-1.0-14: 15 \mathrm{u}=3$

$6 \quad 3-6.5514-15 u=3$

$7 \quad 4-1.0-4+5-6+7 \mathrm{u}=1$ lat $=1$ fill $=-8: 8-8: 80: 0$

$117 r 214 r 11214 r 1122322322 r 322$ 
$3221126532651123 r 3$

$24 r 323 r 1122328 r 322$

$11214 \mathrm{r} 1122 \mathrm{r} 322 \mathrm{r} 32 \mathrm{2r}$

HNF-SD-SNF-CSWD-005, Rev. 0

$322 \mathrm{r} 11214 \mathrm{r} 1122328 \mathrm{r} 3$

$221123 r 324 r 323 r 1$

26r326r11223223

$22 r 32232211214 \mathrm{r} 11214 \mathrm{r} 117 \mathrm{r}$

$\begin{array}{lllll}8 & 0 & -8 & -10 & -12 \mathrm{u}=4 \mathrm{fill}=1\end{array}$

$9 \quad 5-7.98: 10 u=4$

$104-1.0-8+10 \quad+12 y=4$

$11 \quad 4+1.0-16+9 \quad v=5$ lat $=1$ fill $=0: 60: 00: 043 \mathrm{r} 52 \mathrm{r}$

$120+28+29-19-17+13-18$ fill $=5$

$130+28-19+17-31+13-18$ f fll $=5(-11.5230)$

$140+28-19+31-32+13-18$ fill $=5(-23460)$

$150+28-19+32-33+13-18$ fil $]=5(-69690)$

$164-1.0+28-19+33+13-18$

$17 \quad 4-1.0+28+29-19-24+18$

$186-7.9(+28+29+19-20+23-25):(+28+29-19+23-13)$ $:(+28+29-19+24-25)$

$197-7.088254305(+28+29+20-21+22-25):(+28+29-20+22-23)$

20 o $-28:-29:+21:-22:+25$

1 cz .464693

2 cz .483743

3 cz .535940

$4 \mathrm{px} .71501$

$5 \mathrm{px}-.71501$

6 py .71501

7 py -.71501

$8 \mathrm{px} 11.0$

$9 \mathrm{px}-11.0$

10 py 11.0

12 pz 400.903

13 pz 34.0

14 cz .652018

$15 \mathrm{cz} .690118$

$16 \mathrm{px} 12.0$

17 py 12.0

$18 \mathrm{pz} 439.0$

19 cz 82.25

$20 \mathrm{cz} 83.25$

21 cz 116.35

$22 \mathrm{pz} 0.0$

23 pz 33.0

24 pz 447.9

$25 \mathrm{pz} 485.9$

*28 px 0.0

*29 py 0.0

31 py 35.0

32 py 58.0

33 py 81.0

$61-$

62- imp:n $118 \mathrm{r} 0$

63- kcode 250.72445000

warning. tallies are normed per fission neutron for one generation.

64- $\quad \mathrm{kstc} 1.51 .5217 .4515$

65- $\mathrm{ml} \quad 92235.40 \mathrm{c} 1.31964 \mathrm{e} 20 \quad 92238.40 \mathrm{c} 2.15905 \mathrm{e} 22$

66. $\mathrm{m} 28016.40 \mathrm{c} 1.00000000$

67. $\mathrm{m} 3 \quad 40000.40 \mathrm{cl}$.

68- $\quad \mathrm{mA} \quad 1001.00 \mathrm{c} .666666667 \quad 8016.40 \mathrm{c} .333333333$

69- m5 $26000.40 \mathrm{c}-.68874500 \quad 5010 .-.00178200 \quad 5011.40 \mathrm{c}-.00721800$

70 m6 26000.40c-.69500000

$\begin{array}{lllll}71- & \mathrm{m} 7 & 26000.40 \mathrm{c} .830266962 & 6012.40 \mathrm{c} .133437328 & 14000.40 \mathrm{c} .002370386\end{array}$

72- $\mathrm{mt} 4$ Iwtr.01:

drxs

prdmp 2j -

f6:n $812131415 \$$ heating in mat $=0$ cells kills menp 4.2 
fission nubar data are being used.

warning. continuous-energy cross-section table used for $1001.00 \mathrm{~d}$

waning. continuous-energy cross-section table used for $5011.40 \mathrm{~d}$

warning. continuous-energy cross-section table used for $6012.40 \mathrm{~d}$

waming. continuous-energy cross-section table used for 8016.400

waming. continuous-energy cross-section table used for $14000.40 \mathrm{~d}$

warning. continuous-energy cross-section table used for $26000.40 \mathrm{~d}$

warning. continuous-energy cross-section table used for $40000.40 \mathrm{~d}$

warning. continuous-energy cross-section table used for $92235.40 d$

waming. continuous-energy cross-section table used for $92238.40 \mathrm{~d}$

waming. 4 of the materials had unnormalized fractions.

tcells

print table 60

atom gram neutron

cell mat density density volume mass pieces importance

1 1 2.57597E-02 $1.01820 \mathrm{E} \div 010.00000 \mathrm{E}+000.00000 \mathrm{E}+00$

$2223.76497 \mathrm{E}-05 \quad 1.00000 \mathrm{E}-030.00000 \mathrm{E}+000.00000 \mathrm{E}+00$

333 4.32392E-02 $6.55000 \mathrm{E}+000.00000 \mathrm{E}+000.00000 \mathrm{E}+00$

44 4s $1.00309 \mathrm{E}-01 \quad 1.00000 \mathrm{E}+000.00000 \mathrm{E}+000.00000 \mathrm{E}+00$

5 4s 1.00309E-01 1.00000E $+000.00000 \mathrm{E}+000.00000 \mathrm{E}+00$

$6 \quad 3$ 4.32392E-02 6.55000E $+000.00000 \mathrm{E}+000.00000 \mathrm{E}+00$

7 4s $1.00309 \mathrm{E}-011.00000 \mathrm{E}+000.00000 \mathrm{E}+000.00000 \mathrm{E}+00$

$800.00000 \mathrm{E}+000.00000 \mathrm{E}+00 \quad 0.00000 \mathrm{E}+000.00000 \mathrm{E}+00$

$958.97738 \mathrm{E}-027.90000 \mathrm{E}+000.00000 \mathrm{E}+000.00000 \mathrm{E}+00$

10 4s $1.00309 \mathrm{E}-011.00000 \mathrm{E}+000.00000 \mathrm{E}+000.00000 \mathrm{E}+00$

$11 \quad 11 \quad 4 s \quad 1.00309 \mathrm{E}-01 \quad 1.00000 \mathrm{E}+000.00000 \mathrm{E}+00 \quad 0.00000 \mathrm{E}+00$

$121200.00000 \mathrm{E}+00 \quad 0.00000 \mathrm{E}+00 \quad 0.00000 \mathrm{E}+00 \quad 0.00000 \mathrm{E}+00$

$13 \quad 13 \quad 0 \quad 0.00000 \mathrm{E}+000.00000 \mathrm{E}+00 \quad 0.00000 \mathrm{E}+000.00000 \mathrm{E}+00$

$14 \quad 14 \quad 0 \quad 0.00000 \mathrm{E}+00 \quad 0.00000 \mathrm{E}+00 \quad 0.00000 \mathrm{E}+00 \quad 0.00000 \mathrm{E}+00$

Is $15 \quad 0 \quad 0.00000 \mathrm{E}+000.00000 \mathrm{E}+000.00000 \mathrm{E}+000.00000 \mathrm{E}+00$

1616 4s $1.00309 \mathrm{E}-01 \quad 1.00000 \mathrm{E}+000.00000 \mathrm{E}+000.00000 \mathrm{E}+00$

$17 \quad 17 \quad 4$ s $1.00309 \mathrm{E}-01 \quad 1.00000 \mathrm{E}+000.00000 \mathrm{E}+000.00000 \mathrm{E}+00$

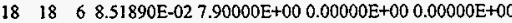

$191978.58502 \mathrm{E}-027.08825 \mathrm{E}+000.00000 \mathrm{E}+000.00000 \mathrm{E} \div 00$

$20 \quad 20 \quad 0 \quad 0.00000 \mathrm{E}+00 \quad 0.00000 \mathrm{E}+000.00000 \mathrm{E}+00 \quad 0.00000 \mathrm{E}+00$

$0 \quad 1.0000 \mathrm{E}+00$

$0 \quad 1.0000 \mathrm{E}+00$

$0 \quad 1.0000 E+00$

$0 \quad 1.0000 \mathrm{E}+00$

$01.0000 \mathrm{E}+00$

$0 \quad 1.0000 \mathrm{E}+00$

$01.0000 \mathrm{E}+00$

$01.0000 \mathrm{E}+00$

$01.0000 \mathrm{E}+00$

$0 \quad 1.0000 \mathrm{E}+00$

$01.0000 \mathrm{E}+00$

$01,0000 \mathrm{E}+00$

$0 \quad 1.0000 \mathrm{E}+00$

$01.0000 E+00$

$0 \quad 1.0000 \mathrm{E}+00$

$01.0000 \mathrm{E}+00$

$01.0000 \mathrm{E}+00$

$01.0000 \mathrm{E}+00$

$01.0000 \mathrm{E}+00$

$0 \quad 0.0000 \mathrm{E}+00$

\section{total $\quad 0.00000 \mathrm{E}+000.00000 \mathrm{E}+00$}

I1 warning messages so far

fcross-section tables

print table 100

table length

tables from file testlibl

1001.00c 2139 1-h-1 from endf-vi. I

mat $125 \quad 05 / 26 / 93$

5010.03d 3945 b-10 endf/b-iv new gamma production format

( 1273) 14 oct 75

$5011.40 \mathrm{c} 3473$ ENDL library name: nd900719 MCNP translation: 900831 14:10:05

$6012.40 \mathrm{C} 5049$ ENDL library name: nd900719 MCNP translation: 900831 14:10:05

$8016.40 \mathrm{c} 5693$ ENDL library name: nd900719 MCNP translation: 900831 14:10:05

$14000.40 \mathrm{C} 8732$ ENDL library name: nd900719 MCNP translation: 900831 14:10:05

900207

900207

900207

900207

900207

900323

0.40c 111279 ENDL library name: nd900719 MCNP translation: 900831 14:10:05

900503 
92238.40c 48310 ENDL library name: nd901118 MCNP translation: 9total nu 56:49 tr.01t 10193 hyodrogen in light water at 300 degrees kelvin

total 261165

waming. neutron energy cutoff is below some cross-section tables.

decimal words of dynamically allocated storage

source distribution written to file inp24s cycle $=0$

12 warning messages so far.

lestimated keff results by cycle

print table 175

cycle $1 \mathrm{k}$ (collision) 0.658210 temoval lifetime(abs) $5.1517 \mathrm{E}+03$ source points generated 228

cycle $2 \mathrm{k}$ (collision) 0.549318 removal lifetime(abs) $3.7645 \mathrm{E}+03$ source points generated 209

cycle $3 \mathrm{k}$ (collision) 0.551862 removal lifetime(abs) $3.8422 \mathrm{E}+03$ source points generated 253

source distribution written to file inp24s cycle $=3$

estimator cycle 4 ave of 2 cycies combination simple average combined average cort

$\begin{array}{lllllll}k \text { (collision) } & 0.584886 & 0.5683740 .0291 & k \text { (col/abs) } & 0.0000000 .0000 & 0.0000000 .0000 & 0.0000\end{array}$

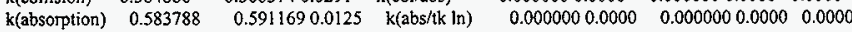

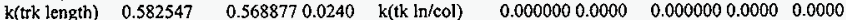

rem life(col) $3.9485 \mathrm{E}+03 \quad 3.8951 \mathrm{E}+030.0137$

rem life(abs) $4.0658 \mathrm{E}+03 \quad 3.9540 \mathrm{E}+03 \quad 0.0283$ life(col/abs) $\quad 0.0000 \mathrm{E}+000.0000 \quad 0.0000 \mathrm{E}+000.0000 \quad 0.0000$

rource points generated 264

rce distribution written to file inp24s cycle $=4$

lproblem summary

0

run terminated when 4 kcode cycles were done.

neutron creation tracks weight energy neutron loss tracks weight energy (per source particle) (per source particle)

\begin{tabular}{|c|c|c|c|c|c|c|c|c|c|}
\hline source & & $1.0638 \mathrm{E}+00$ & $\begin{array}{l}1.9444 E+00 \\
\text { energy cutoff } \\
\text { time cutoff }\end{array}$ & 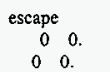 & & $0^{0}$ & 0. & 0. & \\
\hline weight window & & 00. & weigh & t window & & & & 0. & \\
\hline cell importance & 0 & 0 & cell im & ortance & 0 & 0. & & 0 & \\
\hline weight cutoff & 0 & $5.6409 \mathrm{E}-02$ & $1.1284 \mathrm{E}-06$ & weight cutoff & & & 944 & $5.2413 \mathrm{E}-02$ & $7.1769 \mathrm{E}-07$ \\
\hline energy importance & & 00 & o. energ & $y$ importance & & 0 & 0 & 0. & \\
\hline
\end{tabular}

$\begin{array}{lccccccccc}\text { dxtran } & 0 & 0 . & 0 . & d x t r a n & 0 & 0 . & 0 . & \\ \text { forced collisions } & 0 & 0 . & & 0 . & \text { forced collisions } & 0 & 0 . & 0 .\end{array}$

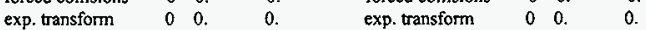

upscattering $\quad 0 \quad 0 . \quad 1.4710 \mathrm{E}-07$ downscattering $\quad 0$ o.

$\begin{array}{rrrr}\text { capture } & 0 & 8.1698 \mathrm{E}-01 & 1.8384 \mathrm{E}-02\end{array}$

$(n, x n) \quad 8 \quad 6.9970 \mathrm{E}-03 \quad 3.6428 \mathrm{E}-03 \quad$ loss to $(n, x n) \quad 4 \quad 3.4985 \mathrm{E}-03 \quad 2.8025 \mathrm{E}-02$

$\begin{array}{lllllllll}\text { fission } & 0 & 0 & 0 & 0 & \text { loss to fission } & 0 & 2.5434 \mathrm{E}-01 & 9.1864 \mathrm{E}-02\end{array}$

$\begin{array}{lllllllll}\text { total } & 948 & 1.1272 \mathrm{E}+00 & 1.9481 \mathrm{E}+00 & \text { total } & 948 & 1.1272 \mathrm{E}+00 & 1.9481 \mathrm{E}+00\end{array}$

number of neutrons banked 4 average lifetime, shakes cutoffs

neutron tracks per source particle $\quad 1.0085 \mathrm{E}+00$ escape $\quad 0.0000 \mathrm{E}+00 \quad$ tco $\quad 1.0000 \mathrm{E}+34$

neutron collisions per source particle $6.1389 \mathrm{E}+01$ capture $\quad 4.1766 \mathrm{E}+03$ eco $0.0000 \mathrm{E}+00$

total neutron collisions $\quad 57706$ capture or escape 4.1766E+03 wcl $-5.0000 \mathrm{E}-01$

net multiplication $\quad 1.0033 \mathrm{E}+000.0030$ any termination $4.4650 \mathrm{E}+03 \quad \mathrm{wc} 2-2.5000 \mathrm{E}-01$

mputer time so far in this run 0.00 minutes

puter time in mcrun $\quad 0.00$ minutes

0.00 minutes

maximum number ever in bank

bank overflows to backup file 0

field length 
tracks population collisions collisions number flux average average

cell entering

$\begin{array}{cccc}1 & 1 & 15187 & 944 \\ 2 & 2 & 29440 & 944 \\ 3 & 3 & 31610 & 944 \\ 4 & 4 & 44969 & 944 \\ 5 & 5 & 8213 & 845 \\ 6 & 6 & 4924 & 791 \\ 7 & 7 & 2832 & 638 \\ 8 & 8 & 0 & 0 \\ 9 & 9 & 1333 & 607 \\ 10 & 10 & 0 & 0 \\ 11 & 11 & 7 & 1 \\ 12 & 12 & 0 & 0 \\ 13 & 13 & 0 & 0 \\ 14 & 14 & 0 & 0 \\ 15 & 15 & 0 & 0 \\ 16 & 16 & 0 & 0 \\ 17 & 17 & 0 & 0 \\ 18 & 18 & 0 & 0 \\ 19 & 19 & 0 & 0\end{array}$

- weight weighted weighted track weight track mfp (per history) energy energy (relative) (cm)

\section{total $\quad 138515 \quad 6658 \quad 57706 \quad 4.4801 E+01$}

the initial fission neutron source distribution used the 1 source points that were input on the ksrc card.

the criticality problem was scheduled to skip 2 cycles and run a total of 4 cycles with nominally 250 neutrons per cycle. problem has run 2 inactive cycles with 478 neutron histories and 2 active cycles with 462 neutron histories.

this calculation has completed the requested number of keff cycles using a total of 940 fission neutron source histories. all cells with fissionable material were sampled and had fission neutron source points.

there is no combined collision/absorption/track-length estimate for keff because only 2 active cycles were run.

the estimated average keffs, one standard deviations, and 68,95 , and 99 percent confidence intervals are:

$\begin{array}{cccccccc}\text { keff estimator } & \text { keff } & \text { standard deviation } & 68 \% \text { confidence } & 95 \% \text { confidence } & 99 \% \text { confidence } \\ & & & & & & \\ \text { collision } & 0.56837 & 0.01651 & 0.53834 \text { to } 0.59841 & 0.35851 \text { to } 0.77824 & 0.00000 \text { to } 1.61951 \\ \text { absorption } & 0.59117 & 0.00738 & 0.57774 \text { to } 0.60460 & 0.49735 \text { to } 0.68498 & 0.12129 \text { to } 1.06105 \\ \text { track length } & 0.56888 & 0.01367 & 0.54401 \text { to } 0.59374 & 0.39513 \text { to } 0.74263 & 0.00000 \text { to } 1.43912\end{array}$

I individual and average keff estimator results by cycle

keff neutron keff estimators by cycle average keff estimators and deviations average $k(c / a / t)$ cycle histories $k$ (coll) $k$ (abs) $k$ (track) $k$ (coll) st dev $k$ (abs) st dev $k$ (track) st dev $k$ (c/a't) st dev fom

I 25010.658210 .619950 .71299

$2 \quad 228 \mid 0.549320 .559220 .57971$

begin active keff cycles

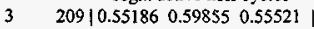

$\begin{array}{llllllllll}4 & 253 \mid 0.58489 & 0.58379 & 0.58255 & 0.56837 & 0.01651 & 0.59117 & 0.00738 & 0.56888 & 0.01367 \mid\end{array}$

the largest active cycle keffs by estimator are:

collision 0.58489 on cycle 4 absorption 0.59855 on cycle 3 track length 0.58255 on cycle 4 the smallest active cycle keffs by estimator are:

collision 0.55186 on cycle 3 absorption 0.58379 on cycle 4 track length 0.55521 on cycle 3 
1 tally 6 nps $=940$

tally type 6 track length estimate of heating. units mev/gram

tally for neutrons

HNF-SD-SNF-CSWD-005, Rev. 0

number of histories used for normalizing tallies $=\quad 500.00$

masses
cell: $\quad 8 \quad 12 \quad 13 \quad 14 \quad 15$
$1.00000 \mathrm{E}+00 \quad 1.00000 \mathrm{E}+00 \quad 1.00000 \mathrm{E}+00 \quad 1.00000 \mathrm{E}+00 \quad 1.00000 \mathrm{E}+00$

cell 8

$4.02566 E+010.0434$

cell 12

$2.65224 \mathrm{E}+010.0629$

cell 13

$1.32494 \mathrm{E}+010.0904$

cell 14

$9.78180 \mathrm{E}-010.4014$

cell 15

$1.20234 \mathrm{E}-010.9710$

1 analysis of the results in the tally fluctuation chart bin (tfe) for tally 6 with nps $=940$ print table 160

normed average tally per history $=4.02566 \mathrm{E}+01$ unnormed average tally per history $=4.02566 \mathrm{E}+01$

estimated tally relative error $=0.0434$

estimated variance of the variance $=0.0095$

relative error from zero tallies $=0.0128$

relative error from nonzero scores $=0.0415$

number of nonzero history tallies $=\quad 462$ efficiency for the nonzero tallies $=0.9240$

history number of largest tally $=\quad 641$

largest unnormalized history tally $=2.71752 E+02$

(largest tally)/(avg nonzero tally) $=6.23745 \mathrm{E}+00$

nfidence interval shift $/$ mean $=0.0014$

shifted confidence interval center $=4.03113 \mathrm{E}+01$

if the largest history score sampled so far were to occur on the very next history, the tfe bin quantities would change as follows: nps $=\quad 462$ for this table because 2 keff cycles and 478 histories were skipped before tally accumulation.

$\begin{array}{lccc}\text { estimated quantities } & \text { value at nps } & \text { value at nps+1 } & \text { value(nps+1)/value } \\ & & & \\ \text { mean } & 4.02566 \mathrm{E} \div 01 & 4.07566 \mathrm{E}+01 & 0.012420 \\ \text { relative entor } & 4.34431 \mathrm{E}-02 & 4.24506 \mathrm{E}-02 & -0.022846 \\ \text { variance of the variance } & 9.52674 \mathrm{E}-03 & 1.33327 \mathrm{E}-02 & 0.399508 \\ \text { shifted center } & 4.03113 \mathrm{E}+01 & 4.03457 \mathrm{E}+01 & 0.000853 \\ \text { figure of merit } & 0.00000 \mathrm{E}+00 & 0.00000 \mathrm{E}+00 & 0.000000\end{array}$

there is not enough information in the largest history scores (usually less than 500 scores) for a reliable estimate of the slope. the history score probability density function appears to have an unsampled region at the largest history scores: please examine.

***** the nps-dependent tfc bin check results are suspect because there are only I nps tally values to analyze *****

results of 10 statistical checks for the estimated answer for the tally fluctuation chart (tfe) bin of tally 6

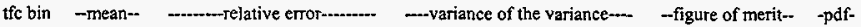

behavior behavior value decrease decrease rate value decrease decrease rate value behavior slope

$\begin{array}{ccccccccc}\text { nesired random } & <0.10 & \text { yes } & 1 / \text { sqrt(nps) } & <0.10 & \text { yes } & 1 / \mathrm{nps} & \text { constant random }>3.00 \\ \text { erved random } & 0.04 & \text { yes } & \text { yes } & 0.01 \text { yes yes } & \text { yes } & 0.00 & \text { no }\end{array}$


warning. the tally in the tally fluctuation chart bin did not pass 1 of the 10 statistical checks.

lunnormed tally density for tally $6 \quad$ nonzero tally mean $(m)=4.357 \mathrm{E}+01$ nps $=940$ print table 161

abscissa ordinate log plot of tally probability density function in tally fluctuation chart bin(d $=$ decade,slope $=0.0$ )

tally number num den $\log d e n: d-\ldots \ldots$ -

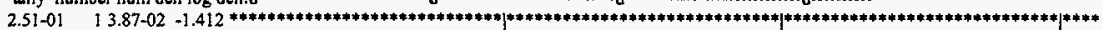

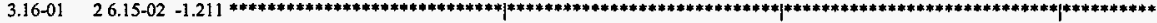

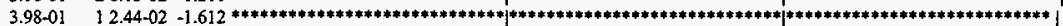

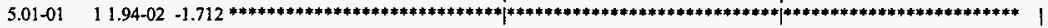

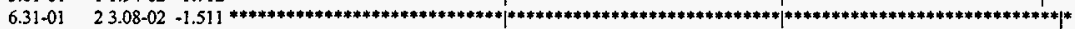

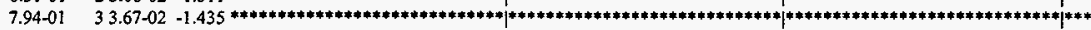

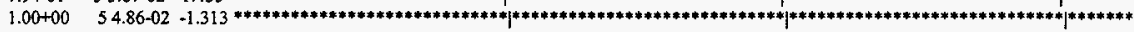

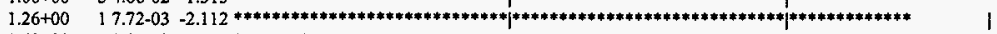

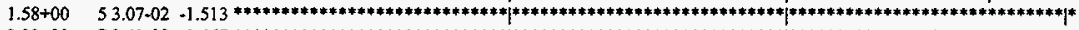

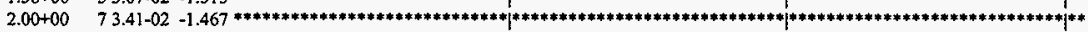

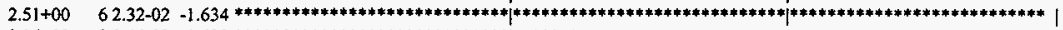

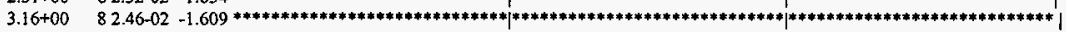

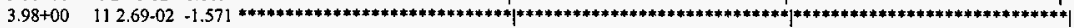

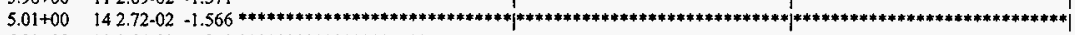

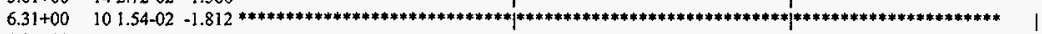

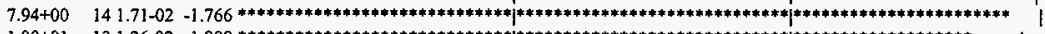

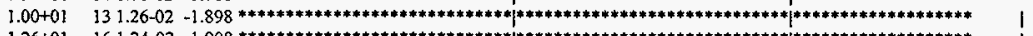

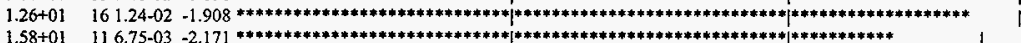

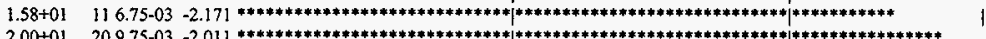

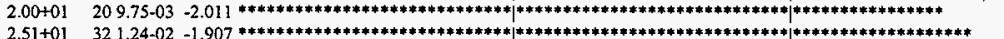

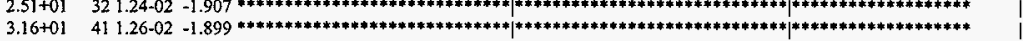

$3.98+01 \quad 245.86-03-2.232+4$

$5.01+01 \quad 489.31-03 \quad-2.031$

mmmmmmmmmmmmmmmmmmmmmmmm|

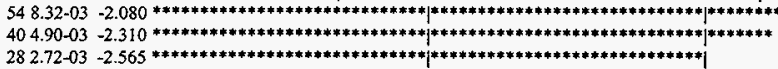

$1.26+02 \quad 201.54-03-2.811 * * * * * * * * * * * * * * * * * * * * * * * * * * * *|* * * * * * * * * * * * * * * * * * * * *|$

$1.58+02 \quad 211.29-03-2.890 * * * * * * * * * * * * * * * * * * * * * * * * * * * * * * * * * * * * * * * * * * * * * * * * *|| \mid$

$2.00+02 \quad 29.75-05-4.011 * * * * * * * * * * * * * * *$

$2.51+02 \quad 00.00+00 \quad 0.000$

$3.16+02 \quad 13.08-05-4.512$ *

total $4629.24-0$

1status of the statistical checks used to form confidence intervals for the mean for each tally bits

tally result of statistical checks for the tfe bin (the first check not passed is listed) and error magnitude check for all bins

6 missed 1 of $10 \mathrm{tfc}$ bin checks: there is insufficient tfc bin tally information to estimate the large tally slope reliably missed all bin error check: 5 tally bins had 0 bins with zeros and 2 bins with relative errors exceeding 0.10

the 10 statistical checks are only for the tally fluctuation chart bin and do not apply to other tally bins.

waming. 1 of the 1 tally fluctuation chart bins did not pass all 10 statistical checks.

waming. I of the 1 tallies had bins with relative errors greater thar recommended.

I tally fluctuation charts

$$
\text { tally } 6
$$

nps mean etror vov slope fom

$9404.0257 \mathrm{E}+010.04340 .00950 .0$

tally data written to file inp $24 \mathrm{~m}$

15 warning messages so far.

germinated when 4 kcode cycles were done. 


\section{Aomputer System ID : 501981-801 056AVO000041 (DOE 6239600)}

\section{File: Inp180}

1- probl8 - kcode in a hexagonal prism lattice.

2- c three half control rods and five whole control rods.

3. $30 \quad 0-905-19291 \mathrm{fill}=1$

4- $31 \quad 0-906-19291$ fill $=1(16.711300)$

5- $37 \quad 0-907-19291$ fill $=1(-16.711300)$

6- $34 \quad 0.913-1929$ fill $=1(011.91850)$

7- $32 \quad 0-914-1929$ fill $=1(10.32175 .95920)$

8- $33 \quad 0-915-1929$ fill $=1(8.3557 \quad 14.47240)$

9. $35 \quad 0-916-1929$ fill $=1(-8.3557 \quad 14.47240)$

10- $36 \quad 0-917-1929$ fill $=1(-10.32175 .95920)$

11. c universe 1: structure of control rod.

12- $\quad 38 \quad 11 \quad-2.02 \quad-880 u=1 \quad \$$ control rod core

13- $39 \quad 6 \quad-8.4 \quad 880-881 \quad u=1 \quad \$$ control rod cladding

14- $40 \quad 12-1.00 \quad 881-882$ u=1 $\$$ control rod gap

15. $41 \quad 6-8.4 \quad 882 \quad u=1 \quad \$$ control tod sheath

16- c the space between the control rods, filled with lattice.

17- $\quad 140 \quad 0-17129-19905906907913914915916917$ fill=2

18- $c$ universe 2: lattice of fuel rods with water in between.

19. $42 \quad 12-1.00 \quad-301302+303 \quad 304-305306 \quad u=2$ lat=2 fill=

20- $\quad-37: 27-1: 330: 0$ \&

$24 r 39 r 24 r 311 r 24 r 311 r 24 r 39 r 2$

$24 r 39 r 23 r 312 r 23 r 312 r 23 r 39 r 2 \mathrm{lr}$

$23 \mathrm{r} 310 \mathrm{rr} 2 \mathrm{r} 313 \mathrm{r} 2 \mathrm{2r} 313 \mathrm{r} 2 \mathrm{2r} 310 \mathrm{r} 2 \mathrm{lr}$

$23 r 357 \mathrm{r} 22 \mathrm{r} \&$

$22+358+22 r$

$22 r 316 r 22 r 317 r 22 r 316 r 23 r$

2 2r $315 r 23 r 316 r 23 r 315 r 24 r$

2 ir $315 r 24 r 315 r 24 r 315 r 24 r$

$21 r 315 r 23 r 316 r 23 r 315 r 25 r$

$21+315 r 22 r 317 r 22 r 315 r 26 r$

$21 r 354 \pi 27 \pi$ \&

c can code remember \& thru comment?

$2355 \mathrm{r} 27 \mathrm{r}$

$2325 \mathrm{r} 22 \mathrm{r} 325 \mathrm{r} 28 \mathrm{r}$

$2324 \mathrm{r} 23 \times 324 \mathrm{r} 29 \mathrm{r}$

$2323 \mathrm{r} 24 \mathrm{r} 323 \mathrm{r} 210 \mathrm{r}$

$2315 \mathrm{r} 22 \mathrm{r} 34 \mathrm{r} 23 \mathrm{r} 34 \mathrm{r} 22 \mathrm{r} 315 \mathrm{r} 21 \mathrm{tr}$

$2314 \mathrm{r} 23 \mathrm{r} 34 \mathrm{r} 2 \mathrm{2r} 34 \mathrm{r} 23 \mathrm{r} 314 \mathrm{r} 212 \mathrm{r}$

$2313 r 24 r 311 r 24 r 313 r 213 r$

$2313 \mathrm{r} 23 \mathrm{r} 312 \mathrm{r} 23 \mathrm{r} 313 \mathrm{r} 214 \mathrm{r}$

$2313 r 22 r 313 r 22 r 313 r 215 r$

$2346 r 216 r$

$2345 \mathrm{r} 217 \mathrm{r}$

$2344 \mathrm{r} 218 \mathrm{r}$

$21 \mathrm{r} 341 \mathrm{r} 220 \mathrm{r}$

2 1r $340 \mathrm{r} 221 \mathrm{r}$

$21 \mathrm{r} 339 \mathrm{r} 222 \mathrm{r}$

$22 \mathrm{r} 336 \mathrm{r} 224 \mathrm{r}$

$22 \mathrm{r} 335 \mathrm{r} 225 \mathrm{r}$

$23 \mathrm{r} 332 \mathrm{r} 227 \mathrm{r}$

$24 \mathrm{r} 329 \mathrm{r} 229 \mathrm{r}$

$25 \mathrm{r} 326 \mathrm{r} 231 \mathrm{r}$

2 Gr $323+233 \mathrm{r}$

$28 \mathrm{r} 318 \mathrm{r} 236 \mathrm{r}$

211 r $311+240 \mathrm{r}$

$264 \mathrm{r}$

c universe 3: structure of fuel rod lattice elements.

$154 \quad 2-13.75 \quad-58 \quad u=3 \quad$ \& fuel element

$\begin{array}{llllll}149 & 12 & -1.00 & 58-268 \quad u=3 & \$ & \text { gap }\end{array}$

$144 \quad 7-19.66 \quad 268-478 \quad u=3 \quad \$$ liner

$\begin{array}{lllllll}159 & 6 & -8.4 & 478 & -698 & u=3 & \$ \text { cladding }\end{array}$

$141 \quad 12-1.00698 \quad u=3 \quad \$$ water between the fuel rods 


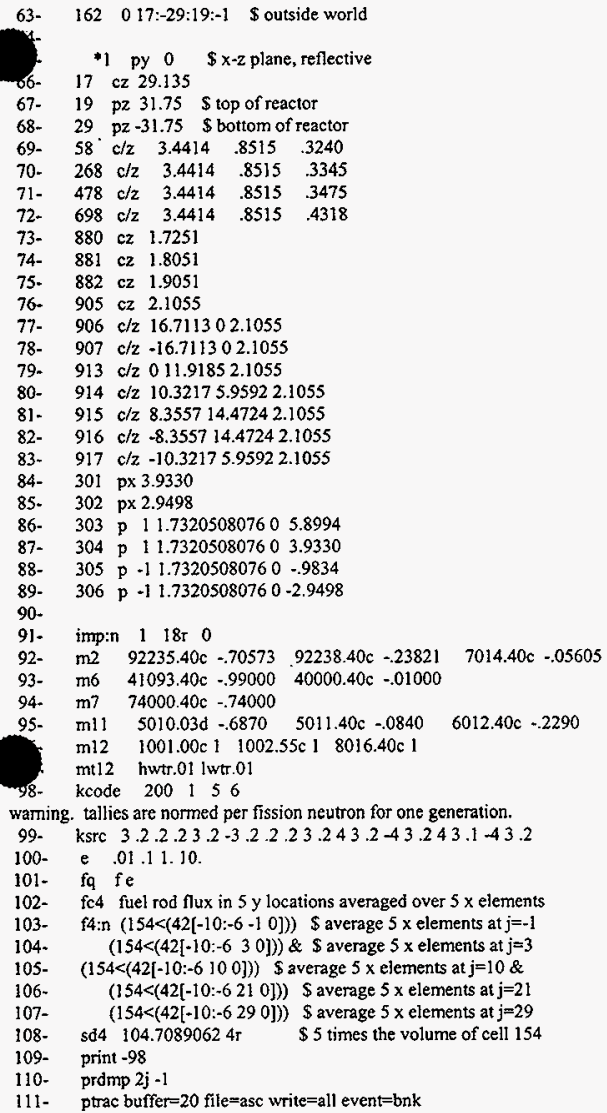

1 py $0 \quad \$ x-z$ plane, reflective

$17 \mathrm{cz} 29.135$

67- $\quad 19 \mathrm{pz} 31.75$ \$ top of reactor

68- $29 \mathrm{pz}-31.75$ \$ bottom of reactor

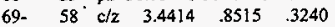

70. $\quad \begin{array}{lllll}268 & \mathrm{c} / 2 & 3.4414 & .8515 & .3345\end{array}$

$\begin{array}{llllll}71- & 478 & \mathrm{c} / \mathrm{z} & 3.4414 & .8515 & .3475\end{array}$

$\begin{array}{llllll}72 . & 698 & \mathrm{c} / \mathrm{z} & 3.4414 & .8515 & .4318\end{array}$

73- $\quad 880 \mathrm{cz} \quad 1.7251$

$\begin{array}{llll}74- & 881 & \mathrm{cz} & 1.8051\end{array}$

75. $882 \mathrm{cz} 1.9051$

76. $905 \mathrm{cz} 2.1055$

77- $\quad 906 \mathrm{c} / \mathrm{z} \quad 16.711302 .1055$

78- $\quad 907 \mathrm{c} / \mathrm{z}-16.711302 .1055$

79. $913 \mathrm{c} / 2 \quad 011.91852 .1055$

80- $\quad 914 \mathrm{c} / 2 \quad 10.32175 .95922 .1055$

81. $\quad 915 \mathrm{c} / \mathrm{z} \quad 8.355714 .47242 .1055$

82- $\quad 916 \quad c / 2-8.3557 \quad 14.47242 .1055$

83. $\quad 917 \mathrm{c} / \mathrm{z}-10.32175 .95922 .1055$

84- 301 px 3.9330

85. $\quad 302 \mathrm{px} 2.9498$

86- $\quad 303 \quad p \quad l l .73205080760 \quad 5.8994$

$87-304 \quad p \quad 11.73205080760 \quad 3.9330$

88- $\quad 305 \quad p-1 \quad 1.73205080760-.9834$

89- $306 \mathrm{p}-11.73205080760-2.9498$

90.

91. imp:n 1 18 0

92- $\quad \mathrm{m} 2 \quad 92235.40 \mathrm{c}-.70573 \quad 92238.40 \mathrm{c}-.23821 \quad 7014.40 \mathrm{c} \quad-.05605$

93- m6 $41093.40 \mathrm{c}-.99000 \quad 40000.40 \mathrm{c}-.01000$

94. $\mathrm{m} 7 \quad 74000.40 \mathrm{c}-.74000$

95- $\mathrm{mll} \quad 5010.03 \mathrm{~d} \quad-.6870 \quad 5011.40 \mathrm{c}-.0840 \quad 6012.40 \mathrm{c} \quad-.2290$

$\mathrm{m} 12 \quad 1001.00 \mathrm{c} \perp \quad 1002.55 \mathrm{cl} \quad 8016.40 \mathrm{cl}$

$\mathrm{mt} 12$ hwtr.01 lwtr.01

kcode 200 I 56

warning. tallies are normed per fission neutron for one generation.

99. $\quad k s r c \quad 3.2 .2 .23 .2-3.2 .2 .23 .243 .2-43.243 .1-43.2$

$100-\mathrm{e} .01 .11 .10$.

101. fq $f e$

102- fc4 fuel rod flux in $5 y$ locations averaged over $5 x$ elements

103- f4:n $(154<(42[-10:-6-10]))$ \$ average $5 x$ elements at $j=-1$

104. $\quad(154<(42[\cdot] 0:-630])) \&$ average $5 x$ elements at $j=3$

105- $\quad(154<(42[-10:-6100]))$ \$average $5 \times$ elements at $j=10$ \&

106. $\quad(154<(42[-10:-6210]))$ \$average $5 \times$ elements at $j=21$

107- $\quad(154<(42[-10:-6290])) \quad \$$ average $5 \times$ elements at $j=29$

108- $s d 4 \quad 104.70890624 \mathrm{r} \quad \$ 5$ times the volume of cell 154

109- print -98

110- prdmp $2 \mathrm{j}-1$

111- ptrac buffer $=20$ file $=a s c$ write $=$ all event $=$ bnk

1 initial source from $k s t c$ card.

print table 90

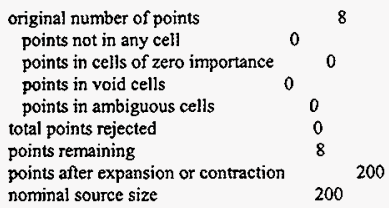

initial guess for $k$ (eff.) $\quad 1.000000$

cycles to skip before tallying 5 ber of keff cycles that can be stored $\quad 201$ 
total fission nubar datz are being used.

print table 30

fuel rod flux in $5 \mathrm{y}$ locations averaged over $5 \mathrm{x}$ elements

tally type 4 track length estimate of particle flux.

tally for neutrons

order of printing: $\mathrm{fe}$

cetls $(154<(42[-10:-6-10]))(154<(42[-10:-630]))(154<(42[-10:-6100]))(154<(42[-10:-6210]))(154<(42[-10 ;-6$ $2901)$ )

energy bins

$0.00000 \mathrm{E}+00$ to $1.00000 \mathrm{E}-02 \mathrm{mev}$

$1.00000 \mathrm{E}-02$ to $1.00000 \mathrm{E}-01 \mathrm{mev}$

$1.00000 \mathrm{E}-01$ to $1.00000 \mathrm{E}+00 \mathrm{mev}$

$1.00000 \mathrm{E}+00$ to $1.00000 \mathrm{E}+01 \mathrm{mev}$

total bin

Imaterial composition

print table 40

the sum of the fractions of material 2 was $9.999900 \mathrm{E}-01$

the sum of the fractions of material 7 was $7.400000 \mathrm{E}-01$

the sum of the fractions of material 12 was $3.000000 \mathrm{E}+00$

material

number component nuclide, atom fraction
$92235,0.37504$
$92238,0.12499$
$7014,0.49997$
$41093,0.98982$
$40000,0.01018$
$74000,1.00000$
$5010,0.71977$
$5011,0.08004$
$6012,0.20019$
$8016,0.33333$

$\begin{array}{cccr}12 & 1001,0.33333 \quad 1002,0.33333 & 8016, \\ \text { associated thermal } s(a, b) \text { data sets: } & \text { hwtr.01t } & \text { iwtr. } 01 \mathrm{t}\end{array}$

$\begin{array}{cccr}12 & 1001,0.33333 \quad 1002,0.33333 & 8016, \\ \text { associated thermal } s(a, b) \text { data sets: } & \text { hwtr.01t } & \text { iwtr. } 01 \mathrm{t}\end{array}$

material

number component nuclide, mass fraction
$92235,0.70574$
$92238,0.23821$
$7014,0.05605$
$41093,0.99000$
$74000,1.00000$
$5010,0.68700$
$1001,0.05300$
$40000,0.01000$
$5011,0.08400$
$6012,0.22900$
$1002,0.10591$
$8016,0.84109$

waming. 3 of the materials had unnormalized fractions.

1 cell volumes and masses

print table 50

cell atom gram input calculated reason volume density density volume volume mass picces not calculated
$130 \quad 0.00000 \mathrm{E}+00 \quad 0.00000 \mathrm{E}+00 \quad 0.00000 \mathrm{E}+00 \quad 0.00000 \mathrm{E}+00 \quad 0.00000 \mathrm{E}+00$

$\begin{array}{lllllllll}2 & 31 & 0.00000 \mathrm{E}+00 & 0.00000 \mathrm{E}+00 & 0.00000 \mathrm{E}+00 & 0.00000 \mathrm{E}+00 & 0.00000 \mathrm{E}+00\end{array}$

$\begin{array}{lllllllll}3 & 37 & 0.00000 \mathrm{E}+00 & 0.00000 \mathrm{E}+00 & 0.00000 \mathrm{E}+00 & 0.00000 \mathrm{E}+00 & 0.00000 \mathrm{E}+00\end{array}$

$\begin{array}{lllllll}4 & 34 & 0.00000 \mathrm{E}+00 & 0.00000 \mathrm{E}+00 & 0.00000 \mathrm{E}+00 & 8.84370 \mathrm{E}+02 & 0.00000 \mathrm{E}+00\end{array}$

$\begin{array}{llllllll}5 & 32 & 0.00000 \mathrm{E}+00 & 0.00000 \mathrm{E}+00 & 0.00000 \mathrm{E}+00 & 8.84370 \mathrm{E}+02 & 0.00000 \mathrm{E}+00\end{array}$

$\begin{array}{llllllll}6 & 33 & 0.00000 \mathrm{E}+00 & 0.00000 \mathrm{E}+00 & 0.00000 \mathrm{E}+00 & 8.84370 \mathrm{E}+02 & 0.00000 \mathrm{E}+00\end{array}$

$\begin{array}{llllllll}7 & 35 & 0.00000 \mathrm{E}+00 & 0.00000 \mathrm{E}+00 & 0.00000 \mathrm{E}+00 & 8.84370 \mathrm{E}+02 & 0.00000 \mathrm{E}+00\end{array}$

$836 \quad 0.00000 \mathrm{E}+00 \quad 0.00000 \mathrm{E}+00 \quad 0.00000 \mathrm{E}+00 \quad 8.84370 \mathrm{E}+02 \quad 0.00000 \mathrm{E}+00$

$\begin{array}{lllllll}9 & 38 & 1.15958 \mathrm{E}-01 & 2.02000 \mathrm{E}+00 & 0.00000 \mathrm{E}+00 & 0.00000 \mathrm{E}+00 & 0.00000 \mathrm{E}+00\end{array}$

$\begin{array}{lllllll}10 & 39 & 5.44575 \mathrm{E}-02 & 8.40000 \mathrm{E}+00 & 0.00000 \mathrm{E}+00 & 0.00000 \mathrm{E}+00 & 0.00000 \mathrm{E}+00\end{array}$

$\begin{array}{lllllllll}11 & 40 & 9.50007 \mathrm{E}-02 & 1.00000 \mathrm{E}+00 & 0.00000 \mathrm{E}+00 & 0.00000 \mathrm{E}+00 & 0.00000 \mathrm{E}+00\end{array}$

$12 \quad 41 \quad 5.44575 \mathrm{E}-02 \quad 8.40000 \mathrm{E}+00 \quad 0.00000 \mathrm{E}+00 \quad 0.00000 \mathrm{E}+00 \quad 0.00000 \mathrm{E}+00$

$140 \quad 0.00000 \mathrm{E}+00 \quad 0.00000 \mathrm{E}+00 \quad 0.00000 \mathrm{E}+00 \quad 0.00000 \mathrm{E}+00 \quad 0.00000 \mathrm{E}+00$

$42 \quad 9.50007 \mathrm{E}-02 \quad 1.00000 \mathrm{E}+00 \quad 0.00000 \mathrm{E}+00 \quad 0.00000 \mathrm{E}+00 \quad 0.00000 \mathrm{E}+00$

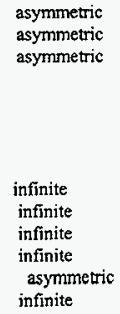

asymmetric asymmetric asymmetric

infinite

infinite

infinite

infinite

asymmetric

infinite 
$\begin{array}{llllll}154 & 6.62920 \mathrm{E}-02 & 1.37500 \mathrm{E}+01 & 0.00000 \mathrm{E}+00 & 0.00000 \mathrm{E}+00 & 0.00000 \mathrm{E}+00\end{array}$ $1499.50007 \mathrm{E}-02 \quad 1.00000 \mathrm{E}+00 \quad 0.00000 \mathrm{E}+00 \quad 0.00000 \mathrm{E}+00 \quad 0.00000 \mathrm{E}+00$ $\begin{array}{llllll}144 & 6.43969 \mathrm{E}-02 & 1.96600 \mathrm{E}+01 & 0.00000 \mathrm{E}+00 & 0.00000 \mathrm{E}+00 & 0.00000 \mathrm{E}+00\end{array}$ $\begin{array}{llllllll}18 & 159 & 5.44575 \mathrm{E}-02 & 8.40000 \mathrm{E}+00 & 0.00000 \mathrm{E}+00 & 0.00000 \mathrm{E}+00 & 0.00000 \mathrm{E}+00\end{array}$ $\begin{array}{lllllllll}19 & 141 & 9.50007 \mathrm{E}-02 & 1.00000 \mathrm{E}+00 & 0.00000 \mathrm{E}+00 & 0.00000 \mathrm{E}+00 & 0.00000 \mathrm{E}+00\end{array}$ $\begin{array}{lllllll}20 & 162 & 0.00000 \mathrm{E}+00 & 0.00000 \mathrm{E}+00 & 0.00000 \mathrm{E}+00 & 0.00000 \mathrm{E}+00 & 0.00000 \mathrm{E}+00\end{array}$ Isurface areas

print table 50 infinite

infinite infinite infinite infinite asymmetric

surface input calculated reason area area area not calculated

1 I $0.00000 \mathrm{E}+00 \quad 0.00000 \mathrm{E}+00$ asymmetric

$2170.00000 \mathrm{E}+00 \quad 0.00000 \mathrm{E}+00$ asymmetric

$319 \quad 0.00000 \mathrm{E}+00 \quad 0.00000 \mathrm{E}+00$ asymmetric

$4290.00000 \mathrm{E}+00 \quad 0.00000 \mathrm{E}+00$ asymmetric

$5 \quad 580.00000 \mathrm{E}+00 \quad 0.00000 \mathrm{E}+00$ infinite

$6268 \quad 0.00000 \mathrm{E}+00 \quad 0.00000 \mathrm{E}+00$ infinite

$7 \quad 4780.00000 \mathrm{E}+00 \quad 0.00000 \mathrm{E}+00$ infinite

$86980.00000 \mathrm{E}+00 \quad 0.00000 \mathrm{E}+00$ infinite

$9880 \quad 0.00000 \mathrm{E}+00 \quad 0.00000 \mathrm{E}+00$ infinite

$108810.00000 \mathrm{E}+00 \quad 0.00000 \mathrm{E}+00$ infinite

$118820.00000 \mathrm{E}+00 \quad 0.00000 \mathrm{E}+00$ infinite

$129050.00000 \mathrm{E}+00 \quad 0.00000 \mathrm{E} \div 00$ asymmetric

$139060.00000 \mathrm{E}+0.00 .00000 \mathrm{E}+00$ asymmetric

$14907 \quad 0.00000 \mathrm{E}+00 \quad 0.00000 \mathrm{E}+00$ asymmetric

$159130.00000 \mathrm{E}+00 \quad 8.40057 \mathrm{E}+02$

$16914 \quad 0.00000 \mathrm{E}+00 \quad 8.40057 \mathrm{E}+02$

$17 \quad 915 \quad 0.00000 \mathrm{E}+00 \quad 8.40057 \mathrm{E}+02$

$18916 \quad 0.00000 \mathrm{E}+00 \quad 8.40057 \mathrm{E}+02$

$19917 \quad 0.00000 \mathrm{E}+00 \quad 8.40057 \mathrm{E}+02$

$20301 \quad 0.00000 \mathrm{E} \div 00 \quad 0.00000 \mathrm{E}+00$ infinite

$213020.00000 \mathrm{E}+00 \quad 0.00000 \mathrm{E}+00$ infinite

$\begin{array}{lll}22 & 3030.00000 \mathrm{E}+00 \quad 0.00000 \mathrm{E}+00 \text { infinite }\end{array}$

$304 \quad 0.00000 \mathrm{E}+00 \quad 0.00000 \mathrm{E}+00$

$3050.00000 \mathrm{E}+00 \quad 0.00000 \mathrm{E}+00$

$25306 \quad 0.00000 \mathrm{E}+00 \quad 0.00000 \mathrm{E}+00$

lcells

print table 60

cell mat density density volume mass pieces importance

$130 \quad 0 \quad 0.00000 \mathrm{E}+00 \quad 0.00000 \mathrm{E}+00 \quad 0.00000 \mathrm{E}+000.00000 \mathrm{E}+00$

$23100.00000 \mathrm{E}+000.00000 \mathrm{E}+00 \quad 0.00000 \mathrm{E}+000.00000 \mathrm{E}+00$

$337 \quad 0 \quad 0.00000 \mathrm{E}+000.00000 \mathrm{E}+000.00000 \mathrm{E}+000.00000 \mathrm{E}+00$

$434 \quad 0 \quad 0.00000 \mathrm{E}+000.00000 \mathrm{E}+00 \quad 8.84370 \mathrm{E}+020.00000 \mathrm{E}+00$

$53200.00000 \mathrm{E}+000.00000 \mathrm{E}+008.84370 \mathrm{E}+020.00000 \mathrm{E}+00$

6 33 $0 \quad 0.00000 \mathrm{E}+00 \quad 0.00000 \mathrm{E}+008.84370 \mathrm{E}+02 \quad 0.00000 \mathrm{E}+00$

$735 \quad 0 \quad 0.00000 \mathrm{E}+00 \quad 0.00000 \mathrm{E}+008.84370 \mathrm{E}+020.00000 \mathrm{E}+0.0$

$836 \quad 0 \quad 0.00000 \mathrm{E}+00 \quad 0.00000 \mathrm{E}+00 \quad 8.84370 \mathrm{E}+020.00000 \mathrm{E}+00$

$9 \quad 38 \quad 11 \quad 1.15958 \mathrm{E}-01 \quad 2.02000 \mathrm{E}+00 \quad 0.00000 \mathrm{E}+000.00000 \mathrm{E}+00$

$10 \quad 39 \quad 6 \quad 5.44575 \mathrm{E}-028.40000 \mathrm{E}+00 \quad 0.00000 \mathrm{E}+00 \quad 0.00000 \mathrm{E}+00$

$11 \quad 40 \quad 12 \mathrm{~s} 9.50007 \mathrm{E}-021.00000 \mathrm{E}+000.00000 \mathrm{E}+000.00000 \mathrm{E}+00$

$124165.44575 \mathrm{E}-028.40000 \mathrm{E}+000.00000 \mathrm{E}+00 \quad 0.00000 \mathrm{E}+00$

$13140 \quad 0 \quad 0.00000 \mathrm{E}+00 \quad 0.00000 \mathrm{E}+00 \quad 0.00000 \mathrm{E}+000.00000 \mathrm{E}+00$

$14 \quad 4212 \mathrm{~s} 9.50007 \mathrm{E}-021.00000 \mathrm{E}+000.00000 \mathrm{E}+000.00000 \mathrm{E}+\infty$

is $1542 \quad 6.62920 \mathrm{E}-02 \quad 1.37500 \mathrm{E}+010.00000 \mathrm{E}+000.00000 \mathrm{E}+00$

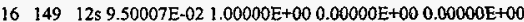

$17 \quad 14476.43969 \mathrm{E}-021.96600 \mathrm{E}+010.00000 \mathrm{E}+000.00000 \mathrm{E}+00$

$18 \quad 159 \quad 6 \quad 5.44575 \mathrm{E}-02 \quad 8.40000 \mathrm{E}+00 \quad 0.00000 \mathrm{E}+00 \quad 0.00000 \mathrm{E}+00$

$19 \quad 141 \quad 12 \mathrm{~s} 9.50007 \mathrm{E}-02 \quad 1.00000 \mathrm{E}+000.00000 \mathrm{E}+000.00000 \mathrm{E}+00$

$2016200.00000 \mathrm{E}+00 \quad 0.00000 \mathrm{E}+000.00000 \mathrm{E}+000.00000 \mathrm{E}+00$

total

lsurfaces

$4.42185 \mathrm{E}+030.00000 \mathrm{E}+00$

surface trans type surface coefficients
$01.0000 E+00$

$0 \quad 1.0000 \mathrm{E}+00$

$01.0000 \mathrm{E}+00$

$1.0000 \mathrm{E}+00$

$1.0000 \mathrm{E}+00$

$11.0000 \mathrm{E}+00$

$11.0000 \mathrm{E}+00$

$11.0000 \mathrm{E}+00$

o $1.0000 \mathrm{E}+00$

$01.0000 \mathrm{E}+00$

$0 \quad 1.0000 \mathrm{E}+00$

$01.0000 \mathrm{E}+00$

$0 \quad 1.0000 \mathrm{E}+00$

$0 \quad 1.0000 \mathrm{E}+00$

$01.0000 \mathrm{E}+00$

$03.0000 \mathrm{E}+00$

o $1.0000 \mathrm{E}+00$

o $1.0000 \mathrm{E}+00$

$0 \quad 1.0000 \mathrm{E}+00$

$0 \quad 0.0000 \mathrm{E}+00$

print table 70 
py $0.0000000 \mathrm{E}+00$

cz $2.9135000 \mathrm{E}+01$

pz $3.1750000 \mathrm{E}+01$

pz $-3.1750000 E+01$

c/z $3.4414000 \mathrm{E}+00 \quad 8.5150000 \mathrm{E}-01 \quad 3.2400000 \mathrm{E}-01$

$\begin{array}{cccc}c / 2 & 3.4414000 \mathrm{E}+00 & 8.5150000 \mathrm{E}-01 & 3.3450000 \mathrm{E}-01\end{array}$

c/z 3.4414000E +00 8.5150000E-01 3.4750000E-01

c/z $3.4414000 \mathrm{E}+00 \quad 8.5150000 \mathrm{E}-01 \quad 4.3180000 \mathrm{E}-01$

cz $1.7251000 \mathrm{E}+00$

cz $1.8051000 \mathrm{E}+00$

cz $1.9051000 \mathrm{E}+00$

cz 2.1055000E+00

c/z $\quad 1.6711300 \mathrm{E}+01 \quad 0.0000000 \mathrm{E}+00 \quad 2.1055000 \mathrm{E}+00$

$\mathrm{c} / \mathrm{z}-1.6711300 \mathrm{E}+01 \quad 0.0000000 \mathrm{E}+00 \quad 2.1055000 \mathrm{E}+00$

c/2 $\quad 0.0000000 E+00 \quad 1.1918500 E+01 \quad 2.1055000 E+00$

$c / z \quad 1.0321700 \mathrm{E}+01 \quad 5.9592000 \mathrm{E}+00 \quad 2.1055000 \mathrm{E}+00$

$c / z \quad 8.3557000 \mathrm{E}+00 \quad 1.4472400 \mathrm{E}+01 \quad 2.1055000 \mathrm{E}+00$

$c / 2-8.3557000 \mathrm{E}+00 \quad 1.4472400 \mathrm{E}+01 \quad 2.1055000 \mathrm{E}+00$

$\mathrm{c} / \mathrm{z} \cdot 1.0321700 \mathrm{E}+01 \quad 5.9592000 \mathrm{E}+00 \quad 2.1055000 \mathrm{E}+00$

px $3.9330000 \mathrm{E}+00$

px $2.9498000 \mathrm{E}+00$

p $\quad 1.0000000 \mathrm{E}+00 \quad 1.7320508 \mathrm{E}+00 \quad 0.0000000 \mathrm{E}+00 \quad 5.8994000 \mathrm{E}+00$

p $\quad 1.0000000 \mathrm{E}+00 \quad 1.7320508 \mathrm{E}+00 \quad 0.0000000 \mathrm{E}+00 \quad 3.9330000 \mathrm{E}+00$

p $-1.0000000 \mathrm{E}+00 \quad 1.7320508 \mathrm{E}+00 \quad 0.0000000 \mathrm{E}+00 \quad-9.8340000 \mathrm{E}-01$

p $-1.0000000 \mathrm{E}+00 \quad 1.7320508 \mathrm{E}+00 \quad 0.0000000 \mathrm{E}+00-2.9498000 \mathrm{E}+00$

1 cell temperatures in mev for the free-gas thermat neutron treatment. print table 72

all non-zero importance cells with materials have a temperature for thermal neutrons of $2.5300 \mathrm{E}-08$ mev.

2 waming messages so far.

Icross-section tables

print table 100

HNF-SD-SNF-CSWD-005, Rev. 0

table length

tables from file testlibl

$1001.00 \mathrm{c} 1782$ l.h.l from endf-vi.1 mat 125 $05 / 26 / 93$

$1002.55 \mathrm{c} 4102$ njoy $\quad(120) 04 / 26 / 82$

5011.40c 3229 ENDL library name: nd900719 MCNP translation: 900831 14:10:05 900207

$6012.40 \mathrm{c} 4858$ ENDL library name: nd900719 MCNP translation: 900831 14:10:05 900207

$7014.40 \mathrm{c} 8535$ ENDL library name: nd900719 MCNP translation: $90083114: 10: 05 \quad 900207$

$8016.40 \mathrm{C} 5358$ ENDL library name: nd900719 MCNP translation: 900831 14:10:05 900207

$40000.40 \mathrm{c} 93372$ ENDL library name: nd900719 MCNP tanslation: 900831 14:10:05 900323

41093.40c 42746 ENDL library name: nd900719 MCNP tanslation: 900831 14:10:05 900208

74000.40c 91244 ENDL library name: nd900719 MCNP translation: 900831 14:10:05 900208

92235.40c 34772 ENDL library name: nd900719 MCNP translation: 9total nu 10:05 900503

92238.40c 40834 ENDL library name: nd901118 MCNP translation: 9total nu 56:49

$5010.03 d \quad 3682$ b-10 endf/b-iv new gamma production format

901119

hwtr.01t 10193 deuterium in heavy water at 300 degrees kelvin

( 1273) $14 \operatorname{oct} 75$

lwtr.0lt 10193 hydrogen in light water at 300 degrees kelvin

$\begin{array}{lll}1002 & 0 & 010 / 22 / 85\end{array}$

$1001 \quad 0 \quad 010 / 22 / 85$

total 354900

warning. neutron energy cutoff is below some cross-section tables.

decimal words of dynamically allocated storage

source distribution written to file inp $18 \mathrm{~s} \quad$ cycle $=0$

3 waming messages so far.

starting merun. field length $=0 \quad \mathrm{cp} 0=0.00$ print table 110
prob $18-\mathrm{kcode}$ in a hexagonal prism lattice.




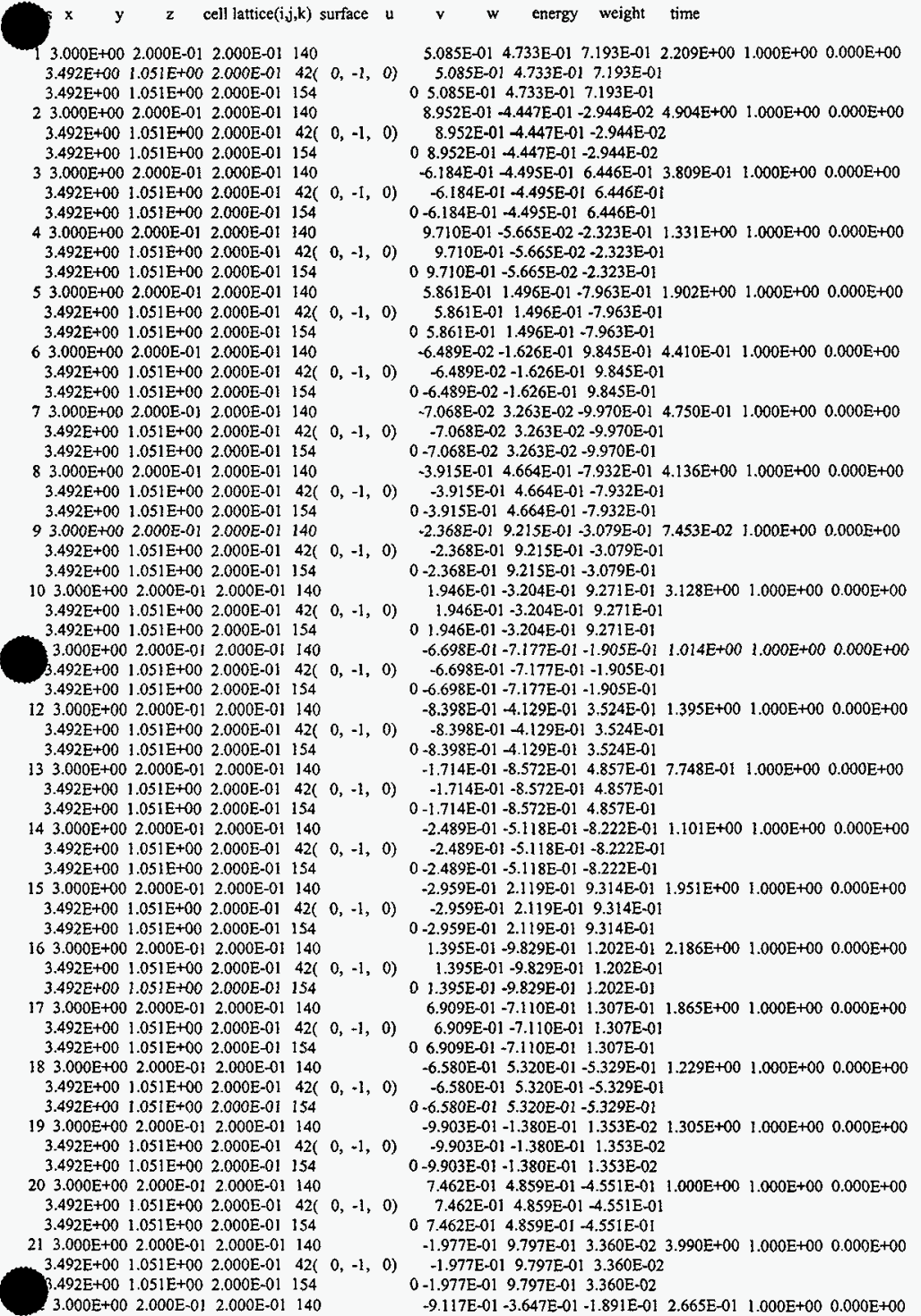


$3.492 \mathrm{E}+00 \quad 1.051 \mathrm{E}+00 \quad 2.000 \mathrm{E}-01 \quad 42(0,-1,0)$ $3.492 \mathrm{E}+00 \quad 1.051 \mathrm{E}+00 \quad 2.000 \mathrm{E}-01 \quad 154$ $3.000 \mathrm{E}+00 \quad 2.000 \mathrm{E}-01 \quad 2.000 \mathrm{E}-01 \quad 140$ $3.492 \mathrm{E}+00 \quad 1.051 \mathrm{E}+00 \quad 2.000 \mathrm{E}-01 \quad 42(0,-1,0)$ $3.492 \mathrm{E}+00 \quad 1.051 \mathrm{E}+00 \quad 2.000 \mathrm{E}-01 \quad 154$ $243.000 \mathrm{E}+00 \quad 2.000 \mathrm{E}-012.000 \mathrm{E}-01 \quad 140$ $3.492 \mathrm{E}+00 \quad 1.051 \mathrm{E}+00 \quad 2.000 \mathrm{E}-01 \quad 42(0,-1,0)$ $3.492 \mathrm{E}+00 \quad 1.051 \mathrm{E}+00 \quad 2.000 \mathrm{E}-01154$

$253.000 \mathrm{E}+00 \quad 2.000 \mathrm{E}-01 \quad 2.000 \mathrm{E}-01 \quad 140$ $3.492 \mathrm{E}+00 \quad 1.051 \mathrm{E}+00 \quad 2.000 \mathrm{E}-01 \quad 42(0,-1,0)$ $3.492 \mathrm{E}+00 \quad 1.051 \mathrm{E}+00 \quad 2.000 \mathrm{E}-01 \quad 154$

$262.000 \mathrm{E}-01 \quad 3.000 \mathrm{E}+00 \quad 2.000 \mathrm{E}-01 \quad 140$ $3.641 \mathrm{E}+00 \quad 4.456 \mathrm{E}-01 \quad 2.000 \mathrm{E}-01 \quad 42(-5,3,0)$ $3.64 \mathrm{IE}+00 \quad 4.456 \mathrm{E}-01 \quad 2.000 \mathrm{E}-01 \quad 141$

$272.000 \mathrm{E}-01 \quad 3.000 \mathrm{E}+00 \quad 2.000 \mathrm{E}-01 \quad 140$ $3.641 \mathrm{E}+00 \quad 4.456 \mathrm{E}-01 \quad 2.000 \mathrm{E}-01 \quad 42(-5,3,0)$ $3.641 \mathrm{E}+00 \quad 4.456 \mathrm{E}-01 \quad 2.000 \mathrm{E}-01 \quad 141$

28 2.000E-01 3.000E+00 2.000E-01 140 $3.641 \mathrm{E}+00 \quad 4.456 \mathrm{E}-01 \quad 2.000 \mathrm{E}-01 \quad 42(-5,3,0)$ $3.641 \mathrm{E}+00 \quad 4.456 \mathrm{E}-01 \quad 2.000 \mathrm{E}-01 \quad 141$

$292.000 \mathrm{E}-01 \quad 3.000 \mathrm{E}+00 \quad 2.000 \mathrm{E}-01 \quad 140$ $3.641 \mathrm{E}+00 \quad 4.456 \mathrm{E}-01 \quad 2.000 \mathrm{E}-01 \quad 42(-5,3,0)$ $3.641 \mathrm{E}+00 \quad 4.456 \mathrm{E}-01 \quad 2.000 \mathrm{E}-01 \quad 141$

$302.000 \mathrm{E}-01 \quad 3.000 \mathrm{E}+00 \quad 2.000 \mathrm{E}-01 \quad 140$ $3.641 \mathrm{E}+00 \quad 4.456 \mathrm{E}-01 \quad 2.000 \mathrm{E}-01 \quad 42(-5,3,0)$ $3.641 \mathrm{E}+00 \quad 4.456 \mathrm{E}-01 \quad 2.000 \mathrm{E}-01 \quad 141$

$312.000 \mathrm{E}-013.000 \mathrm{E}+00 \quad 2.000 \mathrm{E}-01 \quad 140$ $3.641 \mathrm{E}+00 \quad 4.456 \mathrm{E}-01 \quad 2.000 \mathrm{E}-01 \quad 42(-5,3,0)$ $3.641 \mathrm{E}+00 \quad 4.456 \mathrm{E}-01 \quad 2.000 \mathrm{E}-01 \quad 141$

32 2.000E-01 $3.000 \mathrm{E}+00 \quad 2.000 \mathrm{E}-01 \quad 140$ $3.641 \mathrm{E}+00 \quad 4.456 \mathrm{E}-01 \quad 2.000 \mathrm{E}-01 \quad 42(-5,3,0)$ $3.641 \mathrm{E}+00 \quad 4.456 \mathrm{E}-01 \quad 2.000 \mathrm{E}-01 \quad 141$

33 2.000E-01 3.000E+00 2.000E-01 140 $3.641 \mathrm{E}+00 \quad 4.456 \mathrm{E}-01 \quad 2.000 \mathrm{E}-01 \quad 42(-5,3,0)$ $3.641 \mathrm{E}+00 \quad 4.456 \mathrm{E}-01 \quad 2.000 \mathrm{E}-01 \quad 141$

$342.000 \mathrm{E}-01 \quad 3.000 \mathrm{E}+00 \quad 2.000 \mathrm{E}-01 \quad 140$ $3.641 \mathrm{E}+00 \quad 4.456 \mathrm{E}-01 \quad 2.000 \mathrm{E}-01 \quad 42(-5,3,0)$ $3.641 \mathrm{E}+00 \quad 4.456 \mathrm{E}-01 \quad 2.000 \mathrm{E}-01 \quad 141$

$35 \quad 2.000 \mathrm{E}-01 \quad 3.000 \mathrm{E}+00 \quad 2.000 \mathrm{E}-01 \quad 140$ $3.641 \mathrm{E}+00 \quad 4.456 \mathrm{E}-01 \quad 2.000 \mathrm{E}-01 \quad 42(-5,3,0)$ $3.641 \mathrm{E}+00 \quad 4.456 \mathrm{E}-01 \quad 2.000 \mathrm{E}-01 \quad 141$

$362.000 \mathrm{E}-01 \quad 3.000 \mathrm{E}+00 \quad 2.000 \mathrm{E}-01 \quad 140$ $3.641 \mathrm{E}+00 \quad 4.456 \mathrm{E}-01 \quad 2.000 \mathrm{E}-01 \quad 42(-5,3,0)$ $3.641 \mathrm{E}+00 \quad 4.456 \mathrm{E}-01 \quad 2.000 \mathrm{E}-01 \quad 141$ $372.000 \mathrm{E}-01 \quad 3.000 \mathrm{E}+00 \quad 2.000 \mathrm{E}-01 \quad 140$ $3.641 \mathrm{E}+00 \quad 4.456 \mathrm{E}-01 \quad 2.000 \mathrm{E}-01 \quad 42(-5,3,0)$ $3.641 \mathrm{E}+00 \quad 4.456 \mathrm{E}-01 \quad 2.000 \mathrm{E}-01 \quad 141$

$382.000 \mathrm{E}-013.000 \mathrm{E}+00 \quad 2.000 \mathrm{E}-01 \quad 140$ $3.641 \mathrm{E}+00 \quad 4.456 \mathrm{E}-01 \quad 2.000 \mathrm{E}-01 \quad 42(-5,3,0)$ $3.641 \mathrm{E}+00 \quad 4.456 \mathrm{E}-01 \quad 2.000 \mathrm{E}-01 \quad 141$

$392.000 \mathrm{E}-013.000 \mathrm{E}+00 \quad 2.000 \mathrm{E}-01 \quad 140$ $3.641 \mathrm{E}+00 \quad 4.456 \mathrm{E}-01 \quad 2.000 \mathrm{E}-01 \quad 42(-5,3,0)$ $3.641 \mathrm{E}+00 \quad 4.456 \mathrm{E}-01 \quad 2.000 \mathrm{E}-01 \quad 141$ $402.000 \mathrm{E}-013.000 \mathrm{E}+00 \quad 2.000 \mathrm{E}-01 \quad 140$ $3.641 \mathrm{E}+00 \quad 4.456 \mathrm{E}-01 \quad 2.000 \mathrm{E}-01 \quad 42(-5,3,0)$ $3.641 \mathrm{E}+00 \quad 4.456 \mathrm{E}-01 \quad 2.000 \mathrm{E}-01 \quad 141$

$412.000 \mathrm{E}-013.000 \mathrm{E}+00 \quad 2.000 \mathrm{E}-01 \quad 140$ $3.641 \mathrm{E}+00 \quad 4.456 \mathrm{E}-01 \quad 2.000 \mathrm{E}-01 \quad 42(-5,3,0)$ $3.641 \mathrm{E}+00 \quad 4.456 \mathrm{E}-01 \quad 2.000 \mathrm{E}-01 \quad 141$

$42 \quad 2.000 \mathrm{E}-01 \quad 3.000 \mathrm{E}+00 \quad 2.000 \mathrm{E}-01 \quad \mathrm{l} 40$ $3.641 \mathrm{E}+00 \quad 4.456 \mathrm{E}-01 \quad 2.000 \mathrm{E}-01 \quad 42(-5,3,0)$ $3.641 \mathrm{E}+00 \quad 4.456 \mathrm{E}-01 \quad 2.000 \mathrm{E}-01 \quad 141$

$432.000 \mathrm{E}-01 \quad 3.000 \mathrm{E}+00 \quad 2.000 \mathrm{E}-01 \quad 140$ $3.641 \mathrm{E}+00 \quad 4.456 \mathrm{E}-01 \quad 2.000 \mathrm{E}-01 \quad 42(-5,3,0)$ $3.641 \mathrm{E}+00 \quad 4.456 \mathrm{E}-01 \quad 2.000 \mathrm{E}-01 \quad 141$ $2.000 \mathrm{E}-01 \quad 3.000 \mathrm{E}+00 \quad 2.000 \mathrm{E}-01 \quad 140$ $3.641 \mathrm{E}+00 \quad 4.456 \mathrm{E}-01 \quad 2.000 \mathrm{E}-01 \quad 42(-5,3,0)$
$-9.117 \mathrm{E}-01-3.647 \mathrm{E}-01-1.891 \mathrm{E}-01$

$0-9.117 \mathrm{E}-01-3.647 \mathrm{E}-01-1.891 \mathrm{E}-01$

$-4.287 \mathrm{E}-01 \quad 8.361 \mathrm{E}-01-3.423 \mathrm{E}-01 \quad 1.156 \mathrm{E}+00 \quad 1.000 \mathrm{E}+00 \quad 0.000 \mathrm{E}+00$

$-4.287 \mathrm{E}-01 \quad 8.361 \mathrm{E}-01-3.423 \mathrm{E}-01$

$0-4.287 \mathrm{E}-01 \quad 8.361 \mathrm{E}-01 \quad-3.423 \mathrm{E}-01$

$\begin{array}{lllll}1.080 \mathrm{E}-01 & 3.412 \mathrm{E}-01 & -9.338 \mathrm{E}-01 & 2.669 \mathrm{E}+00 \quad 1.000 \mathrm{E}+00 & 0.000 \mathrm{E}+00\end{array}$ $1.080 \mathrm{E}-01 \quad 3.412 \mathrm{E}-01-9.338 \mathrm{E}-01$

0 1.080E-01 3.412E-01 -9.338E-01

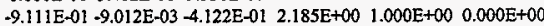

$-9.111 \mathrm{E}-01-9.012 \mathrm{E}-03-4.122 \mathrm{E}-01$

$0-9.111 \mathrm{E}-01-9.012 \mathrm{E}-03-4.122 \mathrm{E}-01$

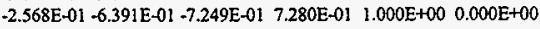

$-2.568 \mathrm{E}-01-6.391 \mathrm{E}-01-7.249 \mathrm{E}-01$

$0-2.568 \mathrm{E}-01-6.391 \mathrm{E}-01-7.249 \mathrm{E}-01$

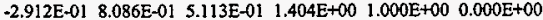

$-2.912 \mathrm{E}-01 \quad 8.086 \mathrm{E}-01$ 5.113E-01

$0-2.912 \mathrm{E}-01 \quad 8.086 \mathrm{E}-01 \quad 5.113 \mathrm{E}-01$

$1.472 \mathrm{E}-01-9.514 \mathrm{E}-01 \quad 2.70 \mathrm{SE}-01 \quad 1.090 \mathrm{E}+00 \quad 1.000 \mathrm{E}+00 \quad 0.000 \mathrm{E}+00$ $1.472 \mathrm{E}-01-9.514 \mathrm{E}-01 \quad 2.705 \mathrm{E}-01$

$0 \quad 1.472 \mathrm{E}-01 \cdot 9.514 \mathrm{E}-01 \quad 2.705 \mathrm{E}-01$

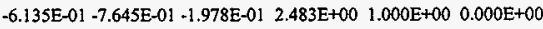
$-6.135 \mathrm{E}-01-7.645 \mathrm{E}-01-1.978 \mathrm{E}-01$

$0-6.135 \mathrm{E}-01-7.645 \mathrm{E}-0 !-1.978 \mathrm{E}-01$

$\begin{aligned} & -5.702 \mathrm{E}-01 \\ & 5.651 \mathrm{E}-01\end{aligned}-5.963 \mathrm{E}-01 \quad 3.767 \mathrm{E}-01 \quad 1.000 \mathrm{E}+00 \quad 0.000 \mathrm{E}+00$

$-5.702 \mathrm{E}-01 \quad 5.651 \mathrm{E}-01-5.963 \mathrm{E}-01$

$0-5.702 \mathrm{E}-01 \quad 5.651 \mathrm{E}-01-5.963 \mathrm{E}-01$

$-6.607 \mathrm{E}-01 \quad 5.373 \mathrm{E}-01-5.242 \mathrm{E}-01 \quad 1.019 \mathrm{E}+00 \quad 1.000 \mathrm{E}+00 \quad 0.000 \mathrm{E}+00$

$-6.607 \mathrm{E}-01 \quad 5.373 \mathrm{E}-01-5.242 \mathrm{E}-01$

$0-6.607 \mathrm{E}-01 \quad 5.373 \mathrm{E}-01-5.242 \mathrm{E}-01$

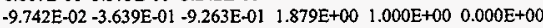
$-9.742 \mathrm{E}-02-3.639 \mathrm{E}-01-9.263 \mathrm{E}-01$

$0-9.742 \mathrm{E}-02-3.639 \mathrm{E}-01-9.263 \mathrm{E}-01$

$-1.965 \mathrm{E}-0 \mathrm{I}-3.145 \mathrm{E}-01-9.287 \mathrm{E}-01 \quad 4.700 \mathrm{E}-01 \quad 1.000 \mathrm{E}+00 \quad 0.000 \mathrm{E}+00$

$-1.965 \mathrm{E}-01-3.145 \mathrm{E}-01+9.287 \mathrm{E}-01$

$0-1.965 \mathrm{E}-01+3.145 \mathrm{E}-01-9.287 \mathrm{E}-01$

4.097E-01 8.465E-01 -3.399E-01 $\quad 1.530 \mathrm{E}+00 \quad 1.000 \mathrm{E}+00 \quad 0.000 \mathrm{E}+00$ $4.097 \mathrm{E}-01 \quad 8.465 \mathrm{E}-01-3.399 \mathrm{E}-01$

0 4.097E-01 8.465E-01 -3.399E-01

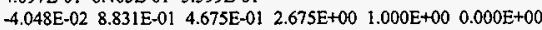
$-4.048 \mathrm{E}-02 \quad 8.831 \mathrm{E}-01 \quad 4.675 \mathrm{E}-01$

$0-4.048 \mathrm{E}-02 \quad 8.831 \mathrm{E}-01 \quad 4.675 \mathrm{E}-01$

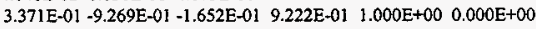

$3.371 \mathrm{E}-01-9.269 \mathrm{E}-01-1.652 \mathrm{E}-01$

$0 \quad 3.371 \mathrm{E}-01+9.269 \mathrm{E}-01 \cdot 1.652 \mathrm{E}-01$

$-1.867 \mathrm{E}-01 \quad 9.756 \mathrm{E}-01-1.155 \mathrm{E}-01 \quad 9.702 \mathrm{E}-01 \quad 1.000 \mathrm{E}+00 \quad 0.000 \mathrm{E}+00$

$-1.867 \mathrm{E}-01 \quad 9.756 \mathrm{E}-01-1.155 \mathrm{E}-01$

$0-1.867 \mathrm{E}-01 \quad 9.756 \mathrm{E}-01-1.155 \mathrm{E}-01$

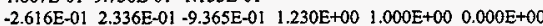
$-2.616 \mathrm{E}-01 \quad 2.336 \mathrm{E}-01-9.365 \mathrm{E}-01$

$0-2.616 \mathrm{E}-01 \quad 2.336 \mathrm{E}-01-9.365 \mathrm{E}-01$ $9.780 \mathrm{E}-01-7.641 \mathrm{E}-02-1.939 \mathrm{E}-01 \quad 1.083 \mathrm{E}+00 \quad 1.000 \mathrm{E}+00 \quad 0.000 \mathrm{E}+00$ $9.780 \mathrm{E}-01-7.641 \mathrm{E}-02-1.939 \mathrm{E}-01$

$09.780 \mathrm{E}-01-7.641 \mathrm{E}-02-1.939 \mathrm{E}-01$ $2.580 \mathrm{E}-01-7.076 \mathrm{E}-01 \quad 6.578 \mathrm{E}-01 \quad 8.313 \mathrm{E}-01 \quad 1.000 \mathrm{E}+00 \quad 0.000 \mathrm{E}+00$ $2.580 \mathrm{E}-01-7.076 \mathrm{E}-01 \quad 6.578 \mathrm{E}-01$

$0 \quad 2.580 \mathrm{E}-01-7.076 \mathrm{E}-01 \quad 6.578 \mathrm{E}-01$

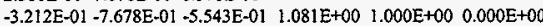
$-3.212 \mathrm{E}-01-7.678 \mathrm{E}-01-5.543 \mathrm{E}-01$

$0-3.212 \mathrm{E}-01-7.678 \mathrm{E}-01-5.543 \mathrm{E}-01$ $5.039 \mathrm{E}-01-1.460 \mathrm{E}-01 \quad 8.513 \mathrm{E}-01 \quad 3.341 \mathrm{E}+00 \quad 1.000 \mathrm{E}+00 \quad 0.000 \mathrm{E}+00$ $5.039 \mathrm{E}-01-1.460 \mathrm{E}-01 \quad 8.513 \mathrm{E}-01$

0 5.039E-01 -1.460E-01 8.513E-01 $\begin{array}{llllll}6.080 \mathrm{E}-01 & 5.487 \mathrm{E}-01 & 5.738 \mathrm{E}-01 & 4.097 \mathrm{E}-01 & 1.000 \mathrm{E}+00 \quad 0.000 \mathrm{E}+00\end{array}$ $6.080 \mathrm{E}-01 \quad 5.487 \mathrm{E}-01 \quad 5.738 \mathrm{E}-01$

$06.080 \mathrm{E}-01 \quad 5.487 \mathrm{E}-01 \quad 5.738 \mathrm{E}-01$

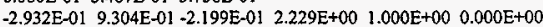
$-2.932 \mathrm{E}-01 \quad 9.304 \mathrm{E}-01-2.199 \mathrm{E}-01$ 
$3.641 \mathrm{E}+00 \quad 4.456 \mathrm{E}-0$ I $2.000 \mathrm{E}-01141$ $2.000 \mathrm{E}-01 \quad 3.000 \mathrm{E}+00 \quad 2.000 \mathrm{E}-01 \quad 140$ $.641 \mathrm{E}+00 \quad 4.456 \mathrm{E}-0) \quad 2.000 \mathrm{E}-01 \quad 42(-5,3,0)$ $3.641 \mathrm{E}+00 \quad 4.456 \mathrm{E}-012.000 \mathrm{E}-01 \quad 141$

46 2.000E-01 3.000E +00 2.000E-01 140 $3.641 \mathrm{E}+00 \quad 4.456 \mathrm{E}-012.000 \mathrm{E}-01 \quad 42(-5,3,0)$ $3.641 \mathrm{E}+00 \quad 4.456 \mathrm{E}-01 \quad 2.000 \mathrm{E}-01 \quad 141$ $472.000 \mathrm{E}-01 \quad 3.000 \mathrm{E}+00 \quad 2.000 \mathrm{E}-01 \quad 140$ $3.641 \mathrm{E}+00 \quad 4.456 \mathrm{E}-01 \quad 2.000 \mathrm{E}-01 \quad 42(-5,3,0)$ $3.641 \mathrm{E}+00 \quad 4.456 \mathrm{E}-01 \quad 2.000 \mathrm{E}-01 \quad 141$ 48 2.000E-01 3.000E+00 2.000E-01 140 $3.641 \mathrm{E}+00 \quad 4.456 \mathrm{E}-01 \quad 2.000 \mathrm{E}-01 \quad 42(-5,3,0)$ $3.641 \mathrm{E}+00 \quad 4.456 \mathrm{E}-01 \quad 2.000 \mathrm{E}-01 \quad 141$

$492.000 \mathrm{E}-01 \quad 3.000 \mathrm{E}+00 \quad 2.000 \mathrm{E}-01 \quad 140$ $3.641 \mathrm{E}+00 \quad 4.456 \mathrm{E}-01 \quad 2.000 \mathrm{E}-01 \quad 42(-5,3,0)$ $3.641 \mathrm{E}+00 \quad 4.456 \mathrm{E}-01 \quad 2.000 \mathrm{E}-01 \quad 141$ $50 \quad 2.000 \mathrm{E}-01 \quad 3.000 \mathrm{E}+00 \quad 2.000 \mathrm{E}-01 \quad 140$ $3.641 \mathrm{E}+00 \quad 4.456 \mathrm{E}-01 \quad 2.000 \mathrm{E}-01 \quad 42(-5,3,0)$ $3.641 \mathrm{E}+00 \quad 4.456 \mathrm{E}-01 \quad 2.000 \mathrm{E}-01 \quad 141$ lestimated keff results by cycle
$0-2.932 \mathrm{E}-01 \quad 9.304 \mathrm{E}-01 \quad-2.199 \mathrm{E}-01$ $-8.475 \mathrm{E}-01-3.993 \mathrm{E}-01-3.497 \mathrm{E}-01 \quad 1.362 \mathrm{E}+00 \quad 1.000 \mathrm{E}+00 \quad 0.000 \mathrm{E}+00$ $-8.475 \mathrm{E}-01-3.993 \mathrm{E}-01-3.497 \mathrm{E}-01$

$0-8.475 \mathrm{E}-01-3.993 \mathrm{E}-01-3.497 \mathrm{E}-01$

$1.200 \mathrm{E}-01-9.195 \mathrm{E}-01-3.743 \mathrm{E}-01 \quad 1.010 \mathrm{E}+00 \quad 1.000 \mathrm{E}+00 \quad 0.000 \mathrm{E}+00$ $1.200 \mathrm{E}-01-9.195 \mathrm{E}-01-3.743 \mathrm{E}-01$

0 1.200E-01 -9.195E-01 -3.743E-01 $7.085 \mathrm{E}-01 \quad 5.879 \mathrm{E}-01 \quad 3.904 \mathrm{E}-01 \quad 7.068 \mathrm{E}+00 \quad 1.000 \mathrm{E}+00 \quad 0.000 \mathrm{E}+00$ $7.085 \mathrm{E}-01 \quad 5.879 \mathrm{E}-01 \quad 3.904 \mathrm{E}-01$

0 7.085E-01 5.879E-01 3.904E-01 4.261E-01 9.046E-01 9.254E-03 3.122E-01 1.000E+00 $0.000 \mathrm{E}+00$ $4.26 ! \mathrm{E}+01 \quad 9.046 \mathrm{E}-01 \quad 9.254 \mathrm{E}-03$

0 4.261E-01 9.046E-01 9.254E-03 5.431E-01 4.270E-01 -7.230E-01 5.484E-01 1.000E+00 $0.000 \mathrm{E}+00$ $5.431 \mathrm{E}-01 \quad 4.270 \mathrm{E}-01-7.230 \mathrm{E}-01$

0 5.431E-01 4.270E-01 -7.230E-01

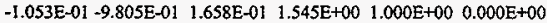
$-1.053 \mathrm{E}-01-9.805 \mathrm{E}-01 \quad 1.658 \mathrm{E}-01$ $0-1.053 \mathrm{E}-01-9.805 \mathrm{E}-01 \quad 1.658 \mathrm{E}-01$ print table 175

cycle $\quad 1 \mathrm{k}$ (collision) 1.253108 removal lifetime(abs) $1.7557 \mathrm{E}+01$ source points generated 257

cycle $2 \mathrm{k}$ (collision) 1.211997 removal lifetime(abs) $1.1671 \mathrm{E}+01$ source points generated 195

cycle $3 \mathrm{k}$ (collision) 1.200265 removal lifetime(abs) $9.6992 \mathrm{E}+00$ source points generated 193

cycle 4 k(collision) 1.129530 removal lifetime(abs) $9.3720 \mathrm{E}+00$ source points generated 182

cycle $5 \mathrm{k}$ (collision) 1.135994 removal lifetime(abs) $1.1136 \mathrm{E}+01$ source points generated 222

cycle $6 \mathrm{k}$ (collision) 1.077014 removal lifetime(abs) $1.2222 \mathrm{E}+01$ source points generated 204

rce distribution written to file inp $18 \mathrm{~s} \quad$ cycle $=6$ blem summary

run terminated when 6 kcode cycles were done.

neutron creation tracks weight energy neutron loss tracks weight energy
(per source particle)

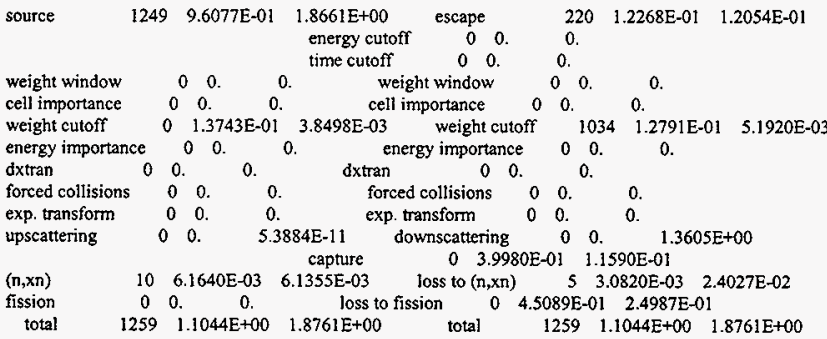

number of neutrons banked 5 average lifetime, shakes cutoffs

neutron tracks per source particle $1.0080 \mathrm{E}+00 \quad$ escape $\quad 3.7027 \mathrm{E}+00 \quad$ tco $\quad 1.0000 \mathrm{E}+34$

neutron collisions per source particle $1.8226 \mathrm{E}+0 \mathrm{l}$ capture $\quad 1.2954 \mathrm{E}+01$ eco $0.0000 \mathrm{E}+00$

total neutron collisions $\quad 22764 \quad$ capture or escape $1.1788 \mathrm{E}+01 \quad$ wcl $-5.0000 \mathrm{E}-01$

net multiplication $\quad 1.0032 \mathrm{E}+000.0035$ any termination $1.2744 \mathrm{E}+01 \quad \mathrm{wc2}-2.5000 \mathrm{E}-01$

computer time so far in this run 0.00 minutes computer time in morun se particles per minute om numbers generated 0.00 minutes $0.0000 \mathrm{E}+00$ 500880 maximum number ever in bank

bank overflows to backup file 0

field length

most random numbers used was 1298 in history 
range of sampled source weights $=7.7821 \mathrm{E}-01$ to $1.0989 \mathrm{E}+00$

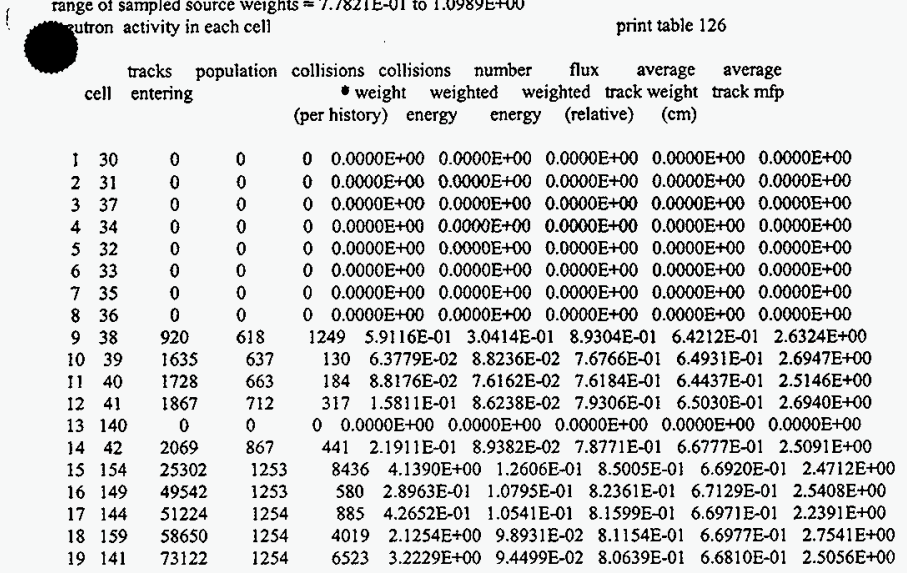

total $266059 \quad 9765 \quad 22764 \quad 1.1324 \mathrm{E}+01$

Ineutron weight balance in each cell -- external events

print table 130 cell entering source energy time exiting total
cutoff cutoff

$\begin{array}{lllllll}30 & 0.0000 \mathrm{E}+00 & 0.0000 \mathrm{E}+00 & 0.0000 \mathrm{E}+00 & 0.0000 \mathrm{E}+00 & 0.0000 \mathrm{E}+00 & 0.0000 \mathrm{E}+00\end{array}$ $31 \quad 0.0000 \mathrm{E}+00 \quad 0.0000 \mathrm{E}+00 \quad 0.0000 \mathrm{E}+00 \quad 0.0000 \mathrm{E}+00 \quad 0.0000 \mathrm{E}+00 \quad 0.0000 \mathrm{E}+00$ $\begin{array}{lllllll}37 & 0.0000 \mathrm{E}+00 & 0.0000 \mathrm{E}+00 & 0.0000 \mathrm{E}+00 & 0.0000 \mathrm{E}+00 & 0.0000 \mathrm{E}+00 & 0.0000 \mathrm{E}+00\end{array}$ $\begin{array}{lllllllll}34 & 0.0000 \mathrm{E}+00 & 0.0000 \mathrm{E}+00 & 0.0000 \mathrm{E}+00 & 0.0000 \mathrm{E}+00 & 0.0000 \mathrm{E}+00 & 0.0000 \mathrm{E}+00\end{array}$ $\begin{array}{llllllll}5 & 32 & 0.0000 \mathrm{E}+00 & 0.0000 \mathrm{E}+00 & 0.0000 \mathrm{E}+00 & 0.0000 \mathrm{E}+00 & 0.0000 \mathrm{E}+00 & 0.0000 \mathrm{E}+00\end{array}$ $\begin{array}{lllllllll}6 & 33 & 0.0000 \mathrm{E}+00 & 0.0000 \mathrm{E}+00 & 0.0000 \mathrm{E}+00 & 0.0000 \mathrm{E}+00 & 0.0000 \mathrm{E}+00 & 0.0000 \mathrm{E}+00\end{array}$ $\begin{array}{lllllllll}7 & 35 & 0.0000 \mathrm{E}+00 & 0.0000 \mathrm{E}+00 & 0.0000 \mathrm{E}+00 & 0.0000 \mathrm{E}+00 & 0.0000 \mathrm{E}+00 & 0.0000 \mathrm{E}+00\end{array}$ $\begin{array}{lllllllll}8 & 36 & 0.0000 \mathrm{E}+00 & 0.0000 \mathrm{E}+00 & 0.0000 \mathrm{E}+00 & 0.0000 \mathrm{E}+00 & 0.0000 \mathrm{E}+00 & 0.0000 \mathrm{E}+00\end{array}$ $938 \quad 4.8822 \mathrm{E}-01 \quad 0.0000 \mathrm{E}+00 \quad 0.0000 \mathrm{E}+00 \quad 0.0000 \mathrm{E}+00 \quad-3.3091 \mathrm{E}-01 \quad 1.5731 \mathrm{E}-01$

$\begin{array}{llllllllll}10 & 39 & 8.4996 \mathrm{E}-01 & 0.0000 \mathrm{E}+00 & 0.0000 \mathrm{E}+00 & 0.0000 \mathrm{E}+00 & -8.4933 \mathrm{E}-01 & 6.2750 \mathrm{E}-04\end{array}$

$11 \quad 40 \quad 8.9904 \mathrm{E}-01 \quad 0.0000 \mathrm{E}+00 \quad 0.0000 \mathrm{E}+00 \quad 0.0000 \mathrm{E}+00 \quad-8.9904 \mathrm{E}-01 \quad 3.9460 \mathrm{E}-06$

$\begin{array}{lllllllll}12 & 41 & 9.7260 \mathrm{E}-01 & 0.0000 \mathrm{E}+00 & 0.0000 \mathrm{E}+00 & 0.0000 \mathrm{E}+00 & -9.6978 \mathrm{E}-01 & 2.8204 \mathrm{E}-03\end{array}$

$13 \quad 140 \quad 0.0000 \mathrm{E}+00 \quad 0.0000 \mathrm{E}+00 \quad 0.0000 \mathrm{E}+00 \quad 0.0000 \mathrm{E}+00 \quad 0.0000 \mathrm{E}+00 \quad 0.0000 \mathrm{E}+00$

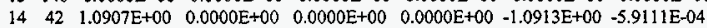

$\begin{array}{llllllll}15 & 154 & 1.2943 \mathrm{E}+01 & 8.4067 \mathrm{E}-01 & 0.0000 \mathrm{E}+00 & 0.0000 \mathrm{E}+00 & -1.3171 \mathrm{E}+01 & 6.1278 \mathrm{E}-01\end{array}$

$\begin{array}{llllllll}16 & 149 & 2.6508 \mathrm{E}+01 & 0.0000 \mathrm{E}+00 & 0.0000 \mathrm{E}+00 & 0.0000 \mathrm{E}+00 & -2.6508 \mathrm{E}+01 & 3.5884 \mathrm{E}-05\end{array}$

$\begin{array}{llllllll}17 & 144 & 2.7419 \mathrm{E}+01 & 0.0000 \mathrm{E}+00 & 0.0000 \mathrm{E}+00 & 0.0000 \mathrm{E}+00 & -2.7405 \mathrm{E}+01 & 1.4572 \mathrm{E}-02\end{array}$

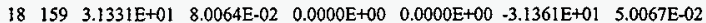

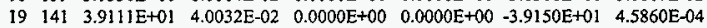

total $\quad 1.4161 \mathrm{E}+02 \quad 9.6077 \mathrm{E}-01 \quad 0.0000 \mathrm{E}+00 \quad 0.0000 \mathrm{E}+00 \quad-1.4174 \mathrm{E}+02 \quad 8.3809 \mathrm{E}-01$ Ineutron weight balance in each cell - variance reduction events print table 130

cell weight cell weight energy dxtran forced exponential total window importance cutof importance collision transform

$\begin{array}{llllllllll}1 & 30 & 0.0000 \mathrm{E}+00 & 0.0000 \mathrm{E}+00 & 0.0000 \mathrm{E}+00 & 0.0000 \mathrm{E}+0 & 0.0000 \mathrm{E}+00 & 0.0000 \mathrm{E}+00 & 0.0000 \mathrm{E}+00 & 0.0000 \mathrm{E}+00\end{array}$

$\begin{array}{lllllllllllll}2 & 31 & 0.0000 \mathrm{E}+00 & 0.0000 \mathrm{E}+00 & 0.0000 \mathrm{E}+00 & 0.0000 \mathrm{E}+00 & 0.0000 \mathrm{E}+00 & 0.0000 \mathrm{E}+00 & 0.0000 \mathrm{E}+00 & 0.0000 \mathrm{E}+00\end{array}$

$\begin{array}{lllllllllllll}3 & 37 & 0.0000 \mathrm{E}+00 & 0.0000 \mathrm{E}+00 & 0.0000 \mathrm{E}+00 & 0.0000 \mathrm{E}+00 & 0.0000 \mathrm{E}+00 & 0.0000 \mathrm{E}+00 & 0.0000 \mathrm{E}+00 & 0.0000 \mathrm{E}+00\end{array}$

$\begin{array}{llllllllll}4 & 34 & 0.0000 \mathrm{E}+00 & 0.0000 \mathrm{E}+00 & 0.0000 \mathrm{E}+00 & 0.0000 \mathrm{E}+00 & 0.0000 \mathrm{E}+00 & 0.0000 \mathrm{E}+00 & 0.0000 \mathrm{E}+00 & 0.0000 \mathrm{E}+00\end{array}$

$\begin{array}{llllllllllll}5 & 32 & 0.0000 \mathrm{E}+00 & 0.0000 \mathrm{E}+00 & 0.0000 \mathrm{E}+00 & 0.0000 \mathrm{E}+00 & 0.0000 \mathrm{E}+00 & 0.0000 \mathrm{E}+00 & 0.0000 \mathrm{E}+00 & 0.0000 \mathrm{E}+00\end{array}$

$\begin{array}{llllllllllll}6 & 33 & 0.0000 \mathrm{E}+00 & 0.0000 \mathrm{E}+00 & 0.0000 \mathrm{E}+00 & 0.0000 \mathrm{E}+00 & 0.0000 \mathrm{E}+00 & 0.0000 \mathrm{E}+00 & 0.0000 \mathrm{E}+00 & 0.0000 \mathrm{E}+00\end{array}$ $\begin{array}{lllllllllll}35 & 0.0000 \mathrm{E}+00 & 0.0000 \mathrm{E}+00 & 0.0000 \mathrm{E}+00 & 0.0000 \mathrm{E}+00 & 0.0000 \mathrm{E}+00 & 0.0000 \mathrm{E}+00 & 0.0000 \mathrm{E}+00 & 0.0000 \mathrm{E}+00\end{array}$ $\begin{array}{lllllllllll}36 & 0.0000 \mathrm{E}+00 & 0.0000 \mathrm{E}+00 & 0.0000 \mathrm{E}+00 & 0.0000 \mathrm{E}+00 & 0.0000 \mathrm{E}+00 & 0.0000 \mathrm{E}+00 & 0.0000 \mathrm{E}+00 & 0.0000 \mathrm{E}+00\end{array}$ 


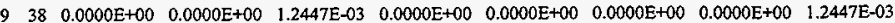
$\begin{array}{llllllllll}39 & 0.0000 \mathrm{E}+00 & 0.0000 \mathrm{E}+00 & 2.0097 \mathrm{E}-04 & 0.0000 \mathrm{E}+00 & 0.0000 \mathrm{E}+00 & 0.0000 \mathrm{E}+00 & 0.0000 \mathrm{E}+00 & 2.0097 \mathrm{E}-04\end{array}$ $\begin{array}{lllllllllll}40 & 0.0000 \mathrm{E}+00 & 0.0000 \mathrm{E}+00 & 0.0000 \mathrm{E}+00 & 0.0000 \mathrm{E}+00 & 0.0000 \mathrm{E}+00 & 0.0000 \mathrm{E}+00 & 0.0000 \mathrm{E}+00 & 0.0000 \mathrm{E}+00\end{array}$ $\begin{array}{lllllllllll}41 & 0.0000 \mathrm{E}+00 & 0.0000 \mathrm{E}+00 & 2.9100 \mathrm{E}-04 & 0.0000 \mathrm{E}+00 & 0.0000 \mathrm{E}+00 & 0.0000 \mathrm{E}+00 & 0.0000 \mathrm{E}+00 & 2.9100 \mathrm{E}-04\end{array}$ $\begin{array}{llllllllllll}13 & 140 & 0.0000 \mathrm{E}+00 & 0.0000 \mathrm{E}+00 & 0.0000 \mathrm{E}+00 & 0.0000 \mathrm{E}+00 & 0.0000 \mathrm{E}+00 & 0.0000 \mathrm{E}+00 & 0.0000 \mathrm{E}+00 & 0.0000 \mathrm{E}+00\end{array}$ $\begin{array}{llllllllll}14 & 42 & 0.0000 \mathrm{E}+00 & 0.0000 \mathrm{E}+00 & 0.0000 \mathrm{E}+00 & 0.0000 \mathrm{E}+00 & 0.0000 \mathrm{E}+00 & 0.0000 \mathrm{E}+00 & 0.0000 \mathrm{E}+00 & 0.0000 \mathrm{E}+00\end{array}$ $\begin{array}{llllllllllll}15 & 154 & 0.0000 \mathrm{E}+00 & 0.0000 \mathrm{E}+00 & 8.0354 \mathrm{E}-03 & 0.0000 \mathrm{E}+00 & 0.0000 \mathrm{E}+00 & 0.0000 \mathrm{E}+00 & 0.0000 \mathrm{E}+00 & 8.0354 \mathrm{E}-03\end{array}$ $\begin{array}{llllllllllllll}16 & 149 & 0.0000 \mathrm{E}+00 & 0.0000 \mathrm{E}+00 & 0.0000 \mathrm{E}+00 & 0.0000 \mathrm{E}+00 & 0.0000 \mathrm{E}+00 & 0.0000 \mathrm{E}+00 & 0.0000 \mathrm{E}+0.0 & 0.0000 \mathrm{E}+00\end{array}$ $\begin{array}{lllllllllll}17 & 144 & 0.0000 \mathrm{E}+00 & 0.0000 \mathrm{E}+00 & -3.0848 \mathrm{E}-06 & 0.0000 \mathrm{E}+00 & 0.0000 \mathrm{E}+00 & 0.0000 \mathrm{E}+00 & 0.0000 \mathrm{E}+00 & -3.0848 \mathrm{E}-06\end{array}$ $\begin{array}{lllllllllll}18 & 159 & 0.0000 \mathrm{E}+00 & 0.0000 \mathrm{E}+00 & -2.5216 \mathrm{E}-04 & 0.0000 \mathrm{E}+00 & 0.0000 \mathrm{E}+00 & 0.0000 \mathrm{E}+00 & 0.0000 \mathrm{E}+00 & -2.5216 \mathrm{E}-04\end{array}$ $\begin{array}{lllllllllll}19 & 141 & 0.0000 \mathrm{E}+00 & 0.0000 \mathrm{E}+00 & 0.0000 \mathrm{E}+00 & 0.0000 \mathrm{E}+00 & 0.0000 \mathrm{E}+00 & 0.0000 \mathrm{E}+00 & 0.0000 \mathrm{E}+00 & 0.0000 \mathrm{E}+00\end{array}$

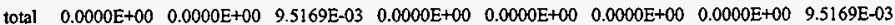
ineutron weight balance in each cell -- physical events print table 130

cell ( $n, x n)$ fission capture loss to loss to total $(\mathrm{n}, \mathrm{xn})$ fission

$\begin{array}{llllllll}30 & 0.0000 \mathrm{E}+00 & 0.0000 \mathrm{E}+00 & 0.0000 \mathrm{E}+00 & 0.0000 \mathrm{E}+00 & 0.0000 \mathrm{E}+00 & 0.0000 \mathrm{E}+00\end{array}$ $\begin{array}{llllllll}31 & 0.0000 \mathrm{E}+00 & 0.0000 \mathrm{E}+00 & 0.0000 \mathrm{E}+00 & 0.0000 \mathrm{E}+00 & 0.0000 \mathrm{E}+00 & 0.0000 \mathrm{E}+00\end{array}$ $337 \quad 0.0000 \mathrm{E}+00 \quad 0.0000 \mathrm{E}+00 \quad 0.0000 \mathrm{E}+00 \quad 0.0000 \mathrm{E}+00 \quad 0.0000 \mathrm{E}+00 \quad 0.0000 \mathrm{E}+00$ $\begin{array}{llllllll}34 & 0.0000 \mathrm{E}+00 & 0.0000 \mathrm{E}+00 & 0.0000 \mathrm{E}+00 & 0.0000 \mathrm{E}+00 & 0.0000 \mathrm{E}+00 & 0.0000 \mathrm{E}+00\end{array}$ $\begin{array}{llllllll}5 & 32 & 0.0000 \mathrm{E}+00 & 0.0000 \mathrm{E}+00 & 0.0000 \mathrm{E}+00 & 0.0000 \mathrm{E}+00 & 0.0000 \mathrm{E}+00 & 0.0000 \mathrm{E}+00\end{array}$ $\begin{array}{llllllll}33 & 0.0000 \mathrm{E}+00 & 0.0000 \mathrm{E}+00 & 0.0000 \mathrm{E}+00 & 0.0000 \mathrm{E}+00 & 0.0000 \mathrm{E}+00 & 0.0000 \mathrm{E}+00\end{array}$ $\begin{array}{lllllll}75 & 0.0000 \mathrm{E}+00 & 0.0000 \mathrm{E}+00 & 0.0000 \mathrm{E}+00 & 0.0000 \mathrm{E}+00 & 0.0000 \mathrm{E}+00 & 0.0000 \mathrm{E}+00\end{array}$ $\begin{array}{lllllllll}8 & 36 & 0.0000 \mathrm{E}+00 & 0.0000 \mathrm{E}+00 & 0.0000 \mathrm{E}+00 & 0.0000 \mathrm{E}+00 & 0.0000 \mathrm{E}+00 & 0.0000 \mathrm{E}+00\end{array}$ $938 \quad 0.0000 \mathrm{E}+00 \quad 0.0000 \mathrm{E}+00 \quad-1.5855 \mathrm{E}-01 \quad 0.0000 \mathrm{E}+00 \quad 0.0000 \mathrm{E}+00 \quad-1.5855 \mathrm{E}-01$ $\begin{array}{llllllll}10 & 39 & 0.0000 \mathrm{E}+00 & 0.0000 \mathrm{E}+00 & -8.2847 \mathrm{E}-04 & 0.0000 \mathrm{E}+00 & 0.0000 \mathrm{E}+00 & -8.2847 \mathrm{E}-04\end{array}$ $11 \quad 40 \quad 0.0000 \mathrm{E}+00 \quad 0.0000 \mathrm{E}+00 \quad-3.9460 \mathrm{E}-06 \quad 0.0000 \mathrm{E}+00 \quad 0.0000 \mathrm{E}+00 \quad-3.9460 \mathrm{E}-06$

$12 \quad 41 \quad 0.0000 \mathrm{E}+00 \quad 0.0000 \mathrm{E}+00 \quad-3.1114 \mathrm{E}-03 \quad 0.0000 \mathrm{E}+00 \quad 0.0000 \mathrm{E}+00 \quad-3.1114 \mathrm{E}-03$

$13 \quad 140 \quad 0.0000 \mathrm{E}+00 \quad 0.0000 \mathrm{E}+00 \quad 0.0000 \mathrm{E}+00 \quad 0.0000 \mathrm{E}+00 \quad 0.0000 \mathrm{E}+00 \quad 0.0000 \mathrm{E}+00$

$14 \quad 42 \quad 1.2461 \mathrm{E}-03 \quad 0.0000 \mathrm{E}+00 \quad-3.1955 \mathrm{E}-05 \quad-6.2306 \mathrm{E}-04 \quad 0.0000 \mathrm{E}+00 \quad 5.9111 \mathrm{E}-04$

$\begin{array}{lllllllll}15 & 154 & 4.9179 \mathrm{E}-03 & 0.0000 \mathrm{E}+00 & -1.7239 \mathrm{E}-01 & -2.4589 \mathrm{E}-03 & -4.5089 \mathrm{E}-01 & -6.2082 \mathrm{E}-01\end{array}$

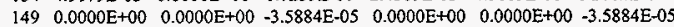

$144 \quad 0.0000 \mathrm{E}+00 \quad 0.0000 \mathrm{E}+00-1.4569 \mathrm{E}-02 \quad 0.0000 \mathrm{E}+00 \quad 0.0000 \mathrm{E}+00 \quad-1.4569 \mathrm{E}-02$

$\begin{array}{llllllll}18 & 159 & 0.0000 \mathrm{E}+00 & 0.0000 \mathrm{E}+00 & -4.9815 \mathrm{E}-02 & 0.0000 \mathrm{E}+00 & 0.0000 \mathrm{E}+00 & -4.9815 \mathrm{E}-02\end{array}$

$\begin{array}{llllllll}19 & 141 & 0.0000 \mathrm{E}+00 & 0.0000 \mathrm{E}+00 & -4.5860 \mathrm{E}-04 & 0.0000 \mathrm{E}+00 & 0.0000 \mathrm{E}+00 & -4.5860 \mathrm{E}-04\end{array}$

total $\quad 6.1640 \mathrm{E}-03 \quad 0.0000 \mathrm{E}+00 \quad-3.9980 \mathrm{E}-01 \quad-3.0820 \mathrm{E}-03 \quad-4.5089 \mathrm{E}-01 \quad-8.4760 \mathrm{E}-01$ Ineutron activity of each ruclide in each cell, per source particle print table 140

cell nuclides atom total collisions weight lost weight loss weight gain fraction collisions * weight to capture to fission by $(n, x n)$

$9385010.03 \mathrm{~d} \quad 7.1977 \mathrm{E}-0$ $5011.40 \mathrm{c} \quad 8.0041 \mathrm{E}-02$ $6012.40 \mathrm{c} \quad 2.0019 \mathrm{E}-01$

$10 \quad 39 \quad 41093.40 \mathrm{c} \quad 9.8982 \mathrm{E}-01$ $40000.40 \mathrm{c} \quad 1.0183 \mathrm{E}-02$

$11 \quad 40 \quad 1001.00 \mathrm{c} \quad 3.3333 \mathrm{E}-01$ $1002.55 \mathrm{c} \quad 3.3333 \mathrm{E}-01$ $8016.40 \mathrm{C} \quad 3.3333 \mathrm{E}-01$

$\begin{array}{llll}2 & 41 \quad 41093.40 \mathrm{c} & 9.8982 \mathrm{E}-01\end{array}$ $40000.40 \mathrm{c} \quad 1.0183 \mathrm{E}-02$

$14 \quad 42 \quad 1001.00 \mathrm{c} \quad 3.3333 \mathrm{E}-01$ 1002.55 c $3.3333 \mathrm{E}-01$ $8016.40 \mathrm{C} \quad 3.3333 \mathrm{E}-01$

$15 \quad 154 \quad 92235.40 \mathrm{c} \quad 3.7504 \mathrm{E}-01$ $92238.40 \mathrm{C} \quad 1.2499 \mathrm{E}-01$ $7014.40 \mathrm{c} \quad 4.9997 \mathrm{E}-01$

$149 \quad 1001.00 \mathrm{c} \quad 3.3333 \mathrm{E}-01$
941 $\quad 4.4212 \mathrm{E}-01 \quad 1.5853 \mathrm{E}-01 \quad 0.0000 \mathrm{E}+00 \quad 0.0000 \mathrm{E}+00$

$\begin{array}{lllll}76 & 3.6073 \mathrm{E}-02 & 1.3591 \mathrm{E}-07 & 0.0000 \mathrm{E}+00 & 0.0000 \mathrm{E}+00\end{array}$

$232 \quad$ l.1297E-01 $1.8446 \mathrm{E}-05 \quad 0.0000 \mathrm{E}+00 \quad 0.0000 \mathrm{E}+00$

$128 \quad 6.2802 \mathrm{E}-02 \quad 8.2814 \mathrm{E}-04 \quad 0.0000 \mathrm{E}+00 \quad 0.0000 \mathrm{E}+00$

$2 \quad 9.7676 \mathrm{E}-04 \quad 3.3160 \mathrm{E}-07 \quad 0.0000 \mathrm{E}+00 \quad 0.0000 \mathrm{E}+00$

$111 \quad 5.1533 \mathrm{E}+02 \quad 3.9251 \mathrm{E}-06 \quad 0.0000 \mathrm{E}+00 \quad 0.0000 \mathrm{E}+00$ $33 \quad 1.5373 \mathrm{E}-02 \quad 2.0884 \mathrm{E}-08 \quad 0.0000 \mathrm{E}+00 \quad 0.0000 \mathrm{E}+00$ $40 \quad 2.1271 \mathrm{E}-02 \quad 0.0000 \mathrm{E}+00 \quad 0.0000 \mathrm{E}+00 \quad 0.0000 \mathrm{E}+00$

$313 \quad 1.5623 \mathrm{E}-01 \quad 3.1072 \mathrm{E}-03 \quad 0.0000 \mathrm{E}+00 \quad 0.0000 \mathrm{E}+00$ $4 \quad 1.8747 \mathrm{E}-03 \quad 4.2322 \mathrm{E}-06 \quad 0.0000 \mathrm{E}+00 \quad 0.0000 \mathrm{E}+00$

$257 \quad 1.2131 \mathrm{E}-01 \quad 4.3914 \mathrm{E}-06 \quad 0.0000 \mathrm{E}+00 \quad 0.0000 \mathrm{E}+00$ $86 \quad 4.6121 \mathrm{E}-02 \quad 6.8027 \mathrm{E}-08 \quad 0.0000 \mathrm{E}+00 \quad 6.2306 \mathrm{E}-04$

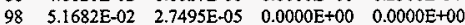

$4759 \quad 2.3227 \mathrm{E}+00 \quad 1.3244 \mathrm{E}-01 \quad 4.4247 \mathrm{E}-01 \quad 5.8410 \mathrm{E}-04$ $\begin{array}{lllll}1470 & 7.3870 \mathrm{E}-01 & 2.3272 \mathrm{E}-02 & 8.4162 \mathrm{E}-03 & 1.8748 \mathrm{E}-03\end{array}$ $2207 \quad 1.0775 \mathrm{E}+00 \quad 1.6682 \mathrm{E}-02 \quad 0.0000 \mathrm{E}+00 \quad 0.0000 \mathrm{E}+00$

$345 \quad 1.6812 \mathrm{E}-01 \quad 4.4875 \mathrm{E}-06 \quad 0.0000 \mathrm{E}+00 \quad 0.0000 \mathrm{E}+00$ 


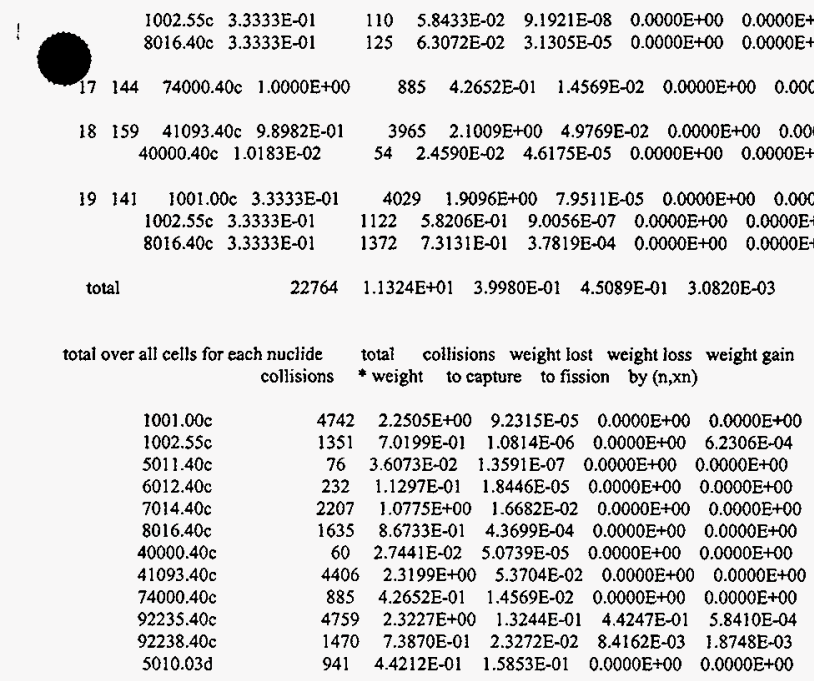

the initial fission neutron source distribution used the 8 source points that were input on the ksrc card.

the criticality problem was scheduled to skip 5 cycles and run a total of 6 cycles with nominally 200 neutrons per cycle.

problem has run 5 inactive cycles with 1027 neutron histories and 1 active cycles with 222 neutron histories.

this calculation has completed the requested number of keff cycles using a total of 1249 fission neutron source histories.

all cells with fissionable material were sampled and had fission neutron source points.

there is no combined collision/absorption/rack-length estimate for keff because only 1 active cycles were run.

lindividual and average keff estimator results by cycle

keff neutron keff estimators by cycte average keff estimators and deviations average $k(\mathrm{c} / \mathrm{a} / \mathrm{t})$

cycle histories $k$ (coll) $k$ (abs) $k$ (track) $k$ (coll) st dev $k$ (abs) st dev $k$ (track) st dev $k(c / a / t)$ st dev fom

\begin{tabular}{ccc|cc|}
1 & $200 \mid 1.25311$ & 1.26302 & 1.22009 \\
2 & $257 \mid 1.21200$ & 1.25252 & 1.12957 \\
3 & $195 \mid 1.20026$ & 1.14326 & 1.13006 \\
4 & $193 \mid 1.12953$ & 1.16269 & 1.10757 \\
5 & $182 \mid 1.13599$ & 1.12465 & 1.11238 \\
\hline 6 & $222 \mid 1.07701$ & 1.04669 & 1.07528 | \\
\hline Itally 4 & nps $=\quad 1249$
\end{tabular}

$+\quad$ nps $=$ fuel rod flux in 5 y locations averaged over $5 \mathrm{x}$ elements

tally type 4 track lergth estimate of particle flux. units $1 / \mathrm{cm}^{* * 2}$

tally for neutrons

number of histories used for nomalizing tallies $=200.00$

cell $a$ is $(154<42[-10-10] 42[-9-10] 42[-8-10] 42[-7-1 \quad 0] 42[-6-10])$

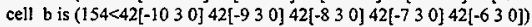

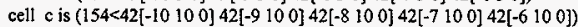

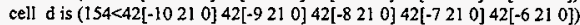

cell $e$ is $\left(154<42[-10290] 42[-9290) 42[-8290] 42\left[\begin{array}{lll}-7 & 29 & 0\end{array}\right] 42[-6290]\right)$

volumes

cell: a b a $\quad d \quad e$

$\begin{array}{lll}1.04709 E+02 & 1.04709 E+02 \quad 1.04709 E+02 \quad 1.04709 E+02 \quad 1.04709 E+02\end{array}$ 
normed average tally per history $=3.51668 \mathrm{E}-04$

estimated tally relative error $=0.3877$

relative error from zero tallies $=0.3464$

number of nonzero history tallies $=8$ efficiency for the nonzero tallies $=0.0400$

history number of largest tally $=1034$

(largest tally) $/$ (average tally) $=3.84476 \mathrm{E}+01$

(confidence interval shift) $/$ mean $=0.0793$ unnormed average tally per history $=3.68228 \mathrm{E}-02$

estimated variance of the variance $=0.1752$

relative error from nonzero scores $=0.1740$

largest unnormalized history tally $=1.41575 \mathrm{E}+00$

(largest tally) $/$ (avg nonzero tally $)=1.53791 \mathrm{E}+00$

shifted confidence interval center $=3.79539 \mathrm{E}-04$

if the largest history score sampled so far were to occur on the very next history, the tfic bin quantities would change as follows: nps = 222 for this table because 5 keff cycles and 1027 histories were skipped before tally accumulation.

\begin{tabular}{lccc} 
estimated quantities & \multicolumn{1}{c}{ value at nps } & value at nps+1 & value(nps+1)/value(nps)-1. \\
mean & $3.51668 \mathrm{E}-04$ & $4.10723 \mathrm{E}-04$ & 0.167927 \\
relative error & $3.87653 \mathrm{E}-01$ & $3.61597 \mathrm{E}-01$ & -0.067213 \\
variance of the variance & $1.75161 \mathrm{E}-01$ & $1.47665 \mathrm{E}-01$ & -0.156976 \\
shifted center & $3.79539 \mathrm{E}-04$ & $3.77169 \mathrm{E}-04$ & -0.006244 \\
figure of merit & $0.00000 \mathrm{E}+00$ & $0.00000 \mathrm{E}+00$ & 0.000000
\end{tabular}

is not enough information in the largest history scores (usually less than 500 scores) for a reliable estimate of the slope. fistory score probability density function appears to have an unsampled region at the largest history scores: please examine.

****** the nps-dependent tfc bin check results are suspect because there are only 1 nps tally values to analyze *****

results of 10 statistical checks for the estimated answer for the tally fluctuation chart (tfc) bin of tally 4

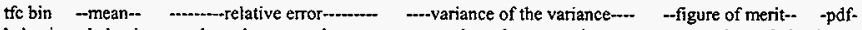

behavior behavior value decrease decrease rate value decrease decrease rate value behavior slope

$\begin{array}{lllllllll}\text { desired random } & <0.10 & \text { yes } & 1 / \text { sqrt(nps) } & <0.10 & \text { yes } & 1 / \text { nps } & \text { constant random }>3.00 \\ \text { observed random } & 0.39 \text { yes } & \text { yes } & 0.18 \text { yes yes } & \text { yes } & 0.00 & \text { no }\end{array}$

waming. the tally in the tally fluctuation chart bin did not pass 3 of the 10 statistical checks.

I unnormed tally density for tally $4 \quad$ nonzero tally mean $(\mathrm{m})=9.206 \mathrm{E}-0 \mathrm{l} \quad \mathrm{nps}=1249$ print table 161

abscissa ordinate $\log$ plot of tally probability density function in tally fluctuation chart bin( $d=$ decade, slope $=0.0$ )

tally number num den $\log$ den:d-

1.00-01 $12.43-01=0.614 * * * * * * * * * * * * 0$

$\begin{array}{llll}1.26-01 & 0.0 .00+00 & 0.000\end{array}$

$\begin{array}{llll}1.58-01 & 0 & 0.00+00 & 0.000\end{array}$

$\begin{array}{llll}2.00-01 & 00.00+00 & 0.000\end{array}$

$00.00+00 \quad 0.000$

$00.00+00 \quad 0.000$ 
$10000.0000 E+000.00000 .0000 \quad 0.0$

$12493.5167 \mathrm{E}-040.38770 .1752 \quad 0.0$

$y$ data written to file inp $18 \mathrm{~m}$

ascii file inp $18 \mathrm{p}$ written with

5 events from 5 histories.

HNF-SD-SNF-CSWD-005, Rev. 0

6 waming messages so far.

run terminated when 6 kcode cycles were done.

\section{File: Outp18}

1- prob18 -- kcode in a hexagonal prism lattice.

2. c three half control rods and five whole control rods.

3- $\quad 30 \quad 0-905-1929$ I fill $=1$

4. $31 \quad 0-906-1929$ l fill $=1(16.711300)$

5- $37 \quad 0-907-1929$ l fill $=1(-16.711300)$

6. $\quad 34 \quad 0-913-1929$ fill $=1(011.91850)$

$7-\quad 32 \quad 0-914-1929$ fill $=1(10.32175 .95920)$

8- $\quad 33 \quad 0.915-1929$ fill $=1(8.3557$ 14.4724 0)

9. $35 \quad 0-916-1929$ fill $=1(-8.3557 \quad 14.47240)$

10- $36 \quad 0-917-1929 \quad$ fill $=1(-10.32175 .95920)$

11- c universe 1: structure of control rod.

12- $\quad \begin{array}{lllll}38 & 11 & -2.02 & -880 \quad u=1 & \$ \text { control rod core }\end{array}$

13. $39 \quad 6 \quad-8.4 \quad 880-881 \quad u=1$ \$ control rod cladding

14- $\quad 40 \quad 12 \quad-1.00 \quad 881-882 u=1 \quad S$ control rod gap

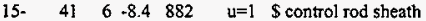

16- $c$ the space between the control rods, filled with lattice.

17. $140 \quad 0 \quad-17129-19905906907913914915916917$ fili $=2$

18- c universe 2: lattice of fuel rods with water in between.

19- $42 \quad 12-1.00 \quad-301302-303 \quad 304-305306 \quad u=2$ lat $=2 \quad$ fill $=$

20. $\quad-37: 27-1: 330: 0$ \&

$24 \mathrm{r} 39 \mathrm{r} 24 \mathrm{r} 311 \mathrm{r} 24 \mathrm{r} 311 \mathrm{r} 24 \mathrm{r} 39 \mathrm{r} 2$

$24 r 39 r 23 r 312 r 23 r 3$ r 2 r 23 3r $39 r 2$ lr

$23 r 310 r 22 r 313 r 22 r 313 r 22 r 310 r 2$ ir

$23 r 357+22 r \&$

$22 \mathrm{r} 358 \mathrm{r} 22 \mathrm{r}$

$22 r 316+22 r 317 r 22 r 316 r 23 r$ 2 2r $315 \mathrm{r} 23 \mathrm{r} 316 \mathrm{r} 23 \mathrm{r} 315 \mathrm{r} 24 \mathrm{r}$ 2 Ir $315 r 24 r 315 r 24 r 315 r 24 r$ $2 \operatorname{lr} 315 r 23 r 316 r 23 r 315 r 25 r$ $2 \operatorname{lr} 315 \mathrm{r} 22 \mathrm{r} 317 \mathrm{r} 22 \mathrm{r} 315 \mathrm{r} 26 \mathrm{r}$ $2 \operatorname{lr} 354 \mathrm{r} 27 \mathrm{r} \&$

c can code remember \& thru comment?

$2355 \mathrm{r} 27 \mathrm{r}$

$2325 r 22 r 325 r 28 r$

$2324 \mathrm{r} 23 \mathrm{r} 324 \mathrm{r} 29 \mathrm{r}$

$2323 r 24 r 323 r 210 r$

$2315 \mathrm{r} 22 \mathrm{r} 34 \mathrm{rr} 23 \mathrm{r} 34 \mathrm{r} 2 \mathrm{2r} 315 \mathrm{r} 211 \mathrm{r}$

$2314 r 23 r 34 r 22 r 34 r 23 r 314 r 212 r$

$2313 \mathrm{r} 24 \mathrm{r} 31 \mathrm{rr} 24 \mathrm{r} 313 \mathrm{r} 213 \mathrm{r}$

$2313 \mathrm{r} 23 \mathrm{r} 312 \mathrm{r} 23 \mathrm{r} 313 \mathrm{r} 2 \mathrm{i4r}$

$2313 r 22 r 313+22 r 313 r 215 r$

$2346 \mathrm{r} 216 \mathrm{r}$

$2345 \mathrm{r} 217 \mathrm{r}$

$2344 \mathrm{r} 218 \mathrm{r}$

$2 \operatorname{lr} 341 \mathrm{r} 220 \mathrm{r}$

$21+340 \mathrm{r} 221 \mathrm{r}$

2 Ir $339 \mathrm{r} 222 \mathrm{r}$

$22 \mathrm{r} 336 \mathrm{r} 224 \mathrm{r}$

$22 r 335 r 225 r$

$23 r 332 r 227 r$

$24 r 329 \pi 229 \mathrm{r}$

$25 \times 326 r 231 r$

$26 \mathrm{r} 323 \mathrm{r} 233 \mathrm{r}$

$28 \mathrm{r} 318 \mathrm{r} 236 \mathrm{r}$

$211 \times 311+240 \mathrm{r}$ 
$264 \mathrm{~T}$

c universe 3: structure of fuel rod lattice elements

$\begin{array}{lllll}154 & 2 & -13.75 \quad-58 \quad u=3 \quad \$ \text { fuel element }\end{array}$

$149 \quad 12 \quad-1.00 \quad 58-268 \quad$ u $=3 \quad \$$ gap

$\begin{array}{llllll}144 & 7 & -19.66 & 268 & -478 \quad \mathrm{u}=3 & \text { S liner }\end{array}$

$\begin{array}{llllll}159 & 6 & -8.4 & 478 & -698 \quad u=3 & \$ \text { cladding }\end{array}$

52- $\quad 141 \quad 12+1.00698 \quad \mathrm{u}=3$ \$ water between the fuel rods

63- $\quad 162 \quad 017:-29: 19:-1$ Soutside world

64.

$65-\quad * 1$ py $0 \quad \$ x-z$ plane, reflective

66. $17 \mathrm{cz} 29.135$

67- $\quad 19$ pz 31.75 \$ top of reactor

68- $29 \mathrm{pz}-31.75$ \$ bottom of reactor

69- $\quad \begin{array}{llllll}58 & \mathrm{c} / \mathrm{z} & 3.4414 & .8515 & .3240\end{array}$

70. $\quad \begin{array}{llllll}268 & \mathrm{c} / \mathrm{z} & 3.4414 & .8515 & .3345\end{array}$

$\begin{array}{llllll}71- & 478 & \mathrm{c} / z & 3.4414 & .8515 & .3475\end{array}$

$\begin{array}{llllll}72- & 698 & \mathrm{c} / \mathrm{z} & 3.4414 & .8515 & .4318\end{array}$

$\begin{array}{llll}73- & 880 & \mathrm{cz} & 1.7251\end{array}$

74- $881 \mathrm{cz} \quad 1.805 !$

75- $882 \mathrm{cz} 1.9051$

76- $\quad 905$ cz 2.1055

77. $\quad 906 \quad c / z \quad 16.711302 .1055$

$78-\quad 907 \quad c / z-16.711302 .1055$

79- $\quad 913 \mathrm{c} / \mathrm{z} \quad 011.91852 .1055$

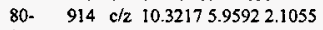

81- $\quad 915 \mathrm{c} / \mathrm{z} \quad 8.3557 \quad 14.47242 .1055$

82- $\quad 916 \quad c / 2 \quad-8.3557 \quad 14.47242 .1055$

83- $\quad 917 \mathrm{c} / \mathrm{z}-10.32175 .95922 .1055$

84- $301 \mathrm{px} 3.9330$

85- 302 px 2.9498

86- $\quad 303$ p $11.73205080760 \quad 5.8994$

87- $\quad 304$ p 111.732050807603 .9330

88- $\quad 305$ p $-11.73205080760-.9834$

306 p $-11.73205080760-2.9498$

impin $118 \mathrm{r} \quad 0$

$\begin{array}{llllllll}\mathrm{m}^{2} & 92235.40 \mathrm{c} & -.70573 & 92238.40 \mathrm{c} & -.23821 & 7014.40 \mathrm{c} & -.05605\end{array}$

m6 $\quad 41093.40 \mathrm{c}-.99000 \quad 40000.40 \mathrm{c}-.01000$

$\mathrm{m} 7 \quad 74000.40 \mathrm{c}-.74000$

$\begin{array}{lllllll}\mathrm{mll} & 5010.03 \mathrm{~d} & -.6870 & 5011.40 \mathrm{c} & -.0840 & 6012.40 \mathrm{c} & -.2290\end{array}$

$\mathrm{m} / 2 \quad 1001.00 \mathrm{c} 1 \quad 1002.55 \mathrm{c} 18016.40 \mathrm{cl}$

mtl2 hwtr.01 lwts.01

kcode 200156

waming. tallies are normed per fission neutron for one generation.

99. $\quad \mathrm{ksrc} 3.2 .2 .23 .2-3.2 .2 .23 .243 .2-43.243 .1-43.2$

$100-\mathrm{e} .01 .11 .10$.

101- fo $\mathrm{fe}$

102- fc4 fuel rod flux in 5 y locations averaged over $5 x$ elements

103- f4:n $(154<(42[-10:-6-10])) \$$ average $5 \times$ elements at $j=-1$

104- $\quad(154<(42[-10 ;-630])) \& \&$ average $5 x$ elements at $j=3$

105- $\quad(154<(42[-10:-6100]))$ S average $5 \times$ elements at $j=10$ \&

106- $\quad(154<(42[-10:-6.210])) \$$ average $5 x$ elements at $j=21$

107- $\quad(154<(42[-10:-6290])) \$$ average $5 \times$ elements at $j=29$

108- sd4 $104.70890624 \pi \quad \$ 5$ times the volume of cell 154

109. print -98

110. prdmp $2 \mathbf{j}-1$

111. ptrac buffer $=20$ file $=$ asc write $=a l l$ event $=b n k$

1 initial source from $\mathrm{ksrc}$ card.

print table 90

original number of points points not in any cell

points in cells of zero importance points in void cells points in ambiguous cells total points rejected is remaining

0

0

0

0

8

200 


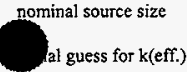

cycles to skip before tallying

number of keff cycles that can be stored
200

1.000000

5

201

total fission nubar data are being used.

Itally 4

$+$

tally type 4 track length estimate of particle flux.

tally for neutrons

order of printing: $\mathrm{fe}$

cells $(154<(42[-10:-6-10]))(154<(42[-10:-630]))(154<(42[-10:-6100]))(154<(42[-10 ;-6210]))(154<(42[-10:-6$ $290 \mathrm{j})$ )

energy bins

$0.00000 \mathrm{E}+00$ to $1.00000 \mathrm{E}-02 \mathrm{mev}$

$1.00000 \mathrm{E}-02$ to $1.00000 \mathrm{E}-01 \mathrm{mev}$

$1.00000 \mathrm{E}-01$ to $1.00000 \mathrm{E}+00 \mathrm{mev}$

$1.00000 \mathrm{E}+00$ to $1.00000 \mathrm{E}+01 \mathrm{mev}$

total bin

Imaterial composition

print table 40

the sum of the fractions of material 2 was $9.999900 \mathrm{E}-01$

the sum of the fractions of material 7 was $7.400000 \mathrm{E}-01$

the sum of the fractions of material 12 was $3.000000 \mathrm{E}+00$

print table 30

material

component nuclide, atom fraction

$92235,0.37504 \quad 92238,0.12499 \quad 7014,0.49997$

$41093,0.98982 \quad 40000,0.01018$

$74000,1.00000$

$5010,0.71977 \quad 5011,0.08004 \quad 6012,0.20019$

$1001,0.33333 \quad 1002,0.33333 \quad 8016,0.33333$

$\begin{array}{ccr}12 & 1001,0.33333 \quad 1002,0.33333 & 8016, \\ \text { associated thermal s(a,b) data sets: hwtr.01t iwtr.01t }\end{array}$

\section{material}

number component nuclide, mass fraction

$\begin{array}{crrr}2 & 92235,0.70574 & 92238,0.23821 & 7014,0.05605 \\ 6 & 41093,0.99000 & 40000,0.01000 & \\ 7 & 74000,1.00000 & & \\ 11 & 5010,0.68700 & 5011,0.08400 & 6012,0.22900 \\ 12 & 1001,0.05300 & 1002,0.10591 & 8016,0.84109\end{array}$

waming. 3 of the materials had unnormalized fractions. .

Icell volumes and masses

print table 50

$\begin{array}{ccc}\text { cell atom } & \text { gram } & \text { input calculated } \\ \text { density density volume } & \text { volume mass pieces not calculated }\end{array}$

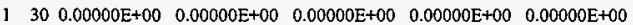

$\begin{array}{llllllll}2 & 31 & 0.00000 \mathrm{E}+00 & 0.00000 \mathrm{E}+00 & 0.00000 \mathrm{E}+00 & 0.00000 \mathrm{E}+00 & 0.00000 \mathrm{E}+00\end{array}$

$\begin{array}{lllllll}3 & 37 & 0.00000 \mathrm{E}+00 & 0.00000 \mathrm{E}+00 & 0.00000 \mathrm{E}+00 & 0.00000 \mathrm{E}+00 & 0.00000 \mathrm{E}+00\end{array}$

$\begin{array}{lllllll}34 & 0.00000 \mathrm{E}+00 & 0.00000 \mathrm{E}+00 & 0.00000 \mathrm{E}+00 & 8.84370 \mathrm{E}+02 & 0.00000 \mathrm{E}+00\end{array}$

$\begin{array}{lllllll}32 & 0.00000 \mathrm{E}+00 & 0.00000 \mathrm{E}+00 & 0.00000 \mathrm{E}+00 & 8.84370 \mathrm{E}+02 & 0.00000 \mathrm{E}+00 & 1\end{array}$

$\begin{array}{llllll}33 & 0.00000 \mathrm{E}+00 & 0.00000 \mathrm{E}+00 & 0.00000 \mathrm{E}+00 & 8.84370 \mathrm{E}+02 & 0.00000 \mathrm{E}+00\end{array}$ asymmetric asymmetric asymmetric 
$35 \quad 0.00000 \mathrm{E}+00 \quad 0.00000 \mathrm{E}+00 \quad 0.00000 \mathrm{E}+00 \quad 8.84370 \mathrm{E}+02 \quad 0.00000 \mathrm{E}+00$

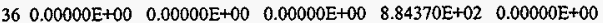
$\begin{array}{lllllll}38 & 1.15958 \mathrm{E}-01 & 2.02000 \mathrm{E}+00 & 0.00000 \mathrm{E}+00 & 0.00000 \mathrm{E}+00 & 0.00000 \mathrm{E}+00\end{array}$ $\begin{array}{lllllllll}0 & 39 & 5.44575 \mathrm{E}-02 & 8.40000 \mathrm{E}+00 & 0.00000 \mathrm{E}+00 & 0.00000 \mathrm{E}+00 & 0.00000 \mathrm{E}+00\end{array}$

$\begin{array}{llllllll}11 & 40 & 9.50007 \mathrm{E}-02 & 1.00000 \mathrm{E}+00 & 0.00000 \mathrm{E}+00 & 0.00000 \mathrm{E}+00 & 0.00000 \mathrm{E}+00\end{array}$

$\begin{array}{lllllll}12 & 41 & 5.44575 \mathrm{E}-02 & 8.40000 \mathrm{E}+00 & 0.00000 \mathrm{E}+00 & 0.00000 \mathrm{E}+00 & 0.00000 \mathrm{E}+00\end{array}$

$13 \quad 140 \quad 0.00000 \mathrm{E}+00 \quad 0.00000 \mathrm{E}+00 \quad 0.00000 \mathrm{E}+00 \quad 0.00000 \mathrm{E}+00 \quad 0.00000 \mathrm{E}+00$

$\begin{array}{llllllll}14 & 42 & 9.50007 \mathrm{E}-02 & 1.00000 \mathrm{E}+00 & 0.00000 \mathrm{E}+00 & 0.00000 \mathrm{E}+00 & 0.00000 \mathrm{E}+00\end{array}$

Is $154 \quad 6.62920 \mathrm{E}-02 \quad 1.37500 \mathrm{E}+01 \quad 0.00000 \mathrm{E}+00 \quad 0.00000 \mathrm{E}+00 \quad 0.00000 \mathrm{E}+00$

$\begin{array}{llllllll}16 & 149 & 9.50007 \mathrm{E}-02 & 1.00000 \mathrm{E}+00 & 0.00000 \mathrm{E}+00 & 0.00000 \mathrm{E}+00 & 0.00000 \mathrm{E}+00\end{array}$

$\begin{array}{llllllll}17 & 144 & 6.43969 \mathrm{E}-02 & 1.96600 \mathrm{E}+01 & 0.00000 \mathrm{E}+00 & 0.00000 \mathrm{E}+00 & 0.00000 \mathrm{E}+00\end{array}$

$\begin{array}{llllllll}18 & 159 & 5.44575 \mathrm{E}-02 & 8.40000 \mathrm{E}+00 & 0.00000 \mathrm{E}+00 & 0.00000 \mathrm{E}+00 & 0.00000 \mathrm{E}+00\end{array}$

$\begin{array}{lllllllll}19 & 141 & 9.50007 \mathrm{E}-02 & 1.00000 \mathrm{E}+00 & 0.00000 \mathrm{E}+00 & 0.00000 \mathrm{E}+00 & 0.00000 \mathrm{E}+00\end{array}$

$\begin{array}{lllllll}20 & 162 & 0.00000 \mathrm{E}+00 & 0.00000 \mathrm{E}+00 & 0.00000 \mathrm{E}+00 & 0.00000 \mathrm{E}+00 & 0.00000 \mathrm{E}+00\end{array}$ isurface areas print table 50

1

0 infinite

infinite

infinite

infinite

0 asymmetric

0 infinite

0 infinite

0 infinite

0 infinite

infinite

0 infinite

0 asymmetric surface input calculated reason area
area area not calculated

I I $0.00000 \mathrm{E}+00 \quad 0.00000 \mathrm{E}+00$ asymmetric

$2170.00000 \mathrm{E}+00 \quad 0.00000 \mathrm{E}+00$ asymmetric

$3190.00000 \mathrm{E}+00 \quad 0.00000 \mathrm{E}+00$ asymmetric

$4290.00000 \mathrm{E}+00 \quad 0.00000 \mathrm{E}+00$ asymmetric

$558 \quad 0.00000 \mathrm{E}+00 \quad 0.00000 \mathrm{E}+00$ infinite

$6268 \quad 0.00000 \mathrm{E}+00 \quad 0.00000 \mathrm{E}+00$ infinite

$7 \quad 478 \quad 0.00000 \mathrm{E}+00 \quad 0.00000 \mathrm{E}+00$ infinite

$8698 \quad 0.00000 \mathrm{E}+00 \quad 0.00000 \mathrm{E}+00$ infinite

$9880 \quad 0.00000 \mathrm{E}+00 \quad 0.00000 \mathrm{E}+00$ infinite

$108810.00000 \mathrm{E}+00 \quad 0.00000 \mathrm{E}+00$ infinite

$11882 \quad 0.00000 \mathrm{E}+00 \quad 0.00000 \mathrm{E}+00$ infinite

$129050.00000 \mathrm{E}+00 \quad 0.00000 \mathrm{E}+00$ asymmetric

$139060.00000 \mathrm{E}+00 \quad 0.00000 \mathrm{E}+00$ asymmetric

$4907 \quad 0.00000 \mathrm{E}+00 \quad 0.00000 \mathrm{E}+00$ asymmetic

$9130.00000 \mathrm{E}+00 \quad 8.40057 \mathrm{E}+02$

$9140.00000 \mathrm{E}+00 \quad 8.40057 \mathrm{E}+02$

$17915 \quad 0.00000 \mathrm{E}+00 \quad 8.40057 \mathrm{E}+02$

$18916 \quad 0.00000 \mathrm{E}+00 \quad 8.40057 \mathrm{E}+02$

$19917 \quad 0.00000 \mathrm{E}+00 \quad 8.40057 \mathrm{E}+02$

$20301 \quad 0.00000 \mathrm{E}+00 \quad 0.00000 \mathrm{E}+00$ infinite

$21302 \quad 0.00000 \mathrm{E}+00 \quad 0.00000 \mathrm{E}+00$ infinite

$22303 \quad 0.00000 \mathrm{E}+00 \quad 0.00000 \mathrm{E}+00$ infinite

$23 \quad 304 \quad 0.00000 \mathrm{E}+00 \quad 0.00000 \mathrm{E}+00$

$24305 \quad 0.00000 E+00 \quad 0.00000 E+00$

$25306 \quad 0.00000 \mathrm{E}+00 \quad 0.00000 \mathrm{E}+00$ 1 cells print table 60

$$
\text { cell mat density density volume mass pieces importance }
$$

$130 \quad 0 \quad 0.00000 \mathrm{E}+00 \quad 0.00000 \mathrm{E}+00 \quad 0.00000 \mathrm{E}+00 \quad 0.00000 \mathrm{E}+00$

31 $0 \quad 0.00000 \mathrm{E}+000.00000 \mathrm{E}+00 \quad 0.00000 \mathrm{E}+000.00000 \mathrm{E}+00$

$3700.00000 \mathrm{E}+000.00000 \mathrm{E}+000.00000 \mathrm{E}+00 \quad 0.00000 \mathrm{E}+00$

$3400.00000 E+000.00000 E+008.84370 E+020.00000 E+00$

$53200.00000 \mathrm{E}+00 \quad 0.00000 \mathrm{E}+00 \quad 8.84370 \mathrm{E}+020.00000 \mathrm{E}+00$

$63300.00000 \mathrm{E}+000.00000 \mathrm{E}+008.84370 \mathrm{E}+020.00000 \mathrm{E}+00$

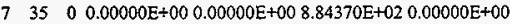

$83600.00000 \mathrm{E}+00 \quad 0.00000 \mathrm{E}+00 \quad 8.84370 \mathrm{E}+020.00000 \mathrm{E}+00$

$938 \quad 11 \quad 1.15958 \mathrm{E}-012.02000 \mathrm{E}+000.00000 \mathrm{E}+000.00000 \mathrm{E}+00$

$103965.44575 \mathrm{E}-02 \quad 8.40000 \mathrm{E} \div 000.00000 \mathrm{E}+000.00000 \mathrm{E}+00$

$1140 \quad 12 s 9.50007 \mathrm{E}-02 \quad 1.00000 \mathrm{E}+000.00000 \mathrm{E}+000.00000 \mathrm{E}+00$

$124165.44575 \mathrm{E}-028.40000 \mathrm{E}+000.00000 \mathrm{E}+000.00000 \mathrm{E}+00$

$3140 \quad 0 \quad 0.00000 \mathrm{E}+00 \quad 0.00000 \mathrm{E}+00 \quad 0.00000 \mathrm{E}+000.00000 \mathrm{E}+00$

$14 \quad 42 \quad 12 \mathrm{~s} 9.50007 \mathrm{E}-02 \quad 1.00000 \mathrm{E}+000.00000 \mathrm{E}+00 \quad 0.00000 \mathrm{E}+00$

$15 \quad 15422 \quad 6.62920 \mathrm{E}-021.37500 \mathrm{E}+010.00000 \mathrm{E}+000.00000 \mathrm{E}+00$

$14912 \mathrm{~s} 9.50007 \mathrm{E}-021.00000 \mathrm{E}+000.00000 \mathrm{E}+000.00000 \mathrm{E}+00$

$14476.43969 \mathrm{E}-021.96600 \mathrm{E}+010.00000 \mathrm{E}+000.00000 \mathrm{E}+00$

$15965.44575 \mathrm{E}-028.40000 \mathrm{E}+000.00000 \mathrm{E}+000.00000 \mathrm{E}+00$
$01.0000 \mathrm{E}+00$

$01.0000 \mathrm{E}+00$

$01.0000 \mathrm{E}+00$

$11.0000 \mathrm{E}+00$

$11.0000 \mathrm{E}+00$

$11.0000 \mathrm{E}+00$

$1.0000 \mathrm{E}+00$

$11.0000 \mathrm{E}+00$

$01.0000 \mathrm{E}+00$

O $1.0000 \mathrm{E}+00$

$01.0000 \mathrm{E}+00$

o $1.0000 \mathrm{E}+00$

$0 \quad 1.0000 \mathrm{E}+00$

$01.0000 \mathrm{E}+00$

o $1.0000 \mathrm{E}+00$

$01.0000 \mathrm{E}+00$

$0 \quad 1.0000 \mathrm{E}+00$

$01.0000 \mathrm{E}+00$ 
$19 \quad 141 \quad 12 \mathrm{~s} 9.50007 \mathrm{E}-021.00000 \mathrm{E}+000.00000 \mathrm{E}+000.00000 \mathrm{E}+00 \quad 0 \quad 1.0000 \mathrm{E}+00$

HNF-SD-SNF-CSWD-005, Rev, 0

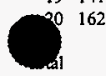

lsurfaces

\section{$4.42185 \mathrm{E}+030.00000 \mathrm{E}+00$}

surface trans type surface coefficients

1 I refl. py $0.0000000 E+00$

$\begin{array}{llll}2 & 17 & \mathrm{CZ} & 2.9135000 \mathrm{E}+01\end{array}$

19 pz $3.1750000 \mathrm{E}+01$

29 pz $-3.1750000 E+01$

$\begin{array}{llllll}5 & 58 & \mathrm{c} / 2 & 3.4414000 \mathrm{E}+00 & 8.5150000 \mathrm{E}-01 & 3.2400000 \mathrm{E}-01\end{array}$

$6 \quad 268 \quad c / z \quad 3.4414000 E+00 \quad 8.5150000 E-01 \quad 3.3450000 E-0 I$

$\begin{array}{lllllll}7 & 478 & c / z & 3.4414000 \mathrm{E}+00 & 8.5150000 \mathrm{E}-01 & 3.4750000 \mathrm{E}-01\end{array}$

$8698 \quad \mathrm{c} / \mathrm{z} \quad 3.4414000 \mathrm{E}+00 \quad 8.5150000 \mathrm{E}-01 \quad 4.3180000 \mathrm{E}-01$

9880 cz $1.7251000 \mathrm{E}+00$

10881 cz $1.8051000 \mathrm{E}+00$

$11 \quad 882 \quad \mathrm{CZ} \quad 1.9051000 \mathrm{E}+00$

2905 cz $2.1055000 \mathrm{E}+00$

$13906 \quad \mathrm{c} / \mathrm{z} \quad 1.6711300 \mathrm{E}+01 \quad 0.0000000 \mathrm{E}+00 \quad 2.1055000 \mathrm{E}+00$

$14907 \quad \mathrm{c} / 2-1.6711300 \mathrm{E}+01 \quad 0.0000000 \mathrm{E}+00 \quad 2.1055000 \mathrm{E}+00$

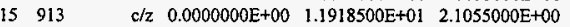

$\begin{array}{lllllll}16 & 914 & c & 2 & 1.0321700 \mathrm{E}+01 & 5.9592000 \mathrm{E}+00 & 2.1055000 \mathrm{E}+00\end{array}$

$17915 \quad \mathrm{c} / 2 \quad 8.3557000 \mathrm{E}+00 \quad 1.4472400 \mathrm{E}+01 \quad 2.1055000 \mathrm{E}+00$

$\begin{array}{lllllll}18 & 916 \quad c / z & -8.3557000 E+00 & 1.4472400 E+01 & 2.1055000 E+00\end{array}$

$\begin{array}{lllllll}19 & 917 & \mathrm{c} / \mathrm{z} & -1.0321700 \mathrm{E}+01 & 5.9592000 \mathrm{E}+00 & 2.1055000 \mathrm{E}+00\end{array}$

$20301 \quad \mathrm{px} 3.9330000 \mathrm{E}+00$

21302 px $2.9498000 \mathrm{E}+00$

$\begin{array}{llllllll}22 & 303 & \text { p } & 1.0000000 E+00 & 1.7320508 E+00 & 0.0000000 E+00 & 5.8994000 E+00\end{array}$

$\begin{array}{lllllll}23 & 304 & \text { p } & 1.0000000 \mathrm{E}+00 & 1.7320508 \mathrm{E}+00 & 0.0000000 \mathrm{E}+00 & 3.9330000 \mathrm{E}+00\end{array}$

$24305 \quad$ p $\quad-1.0000000 \mathrm{E}+00 \quad 1.7320508 \mathrm{E}+00 \quad 0.0000000 \mathrm{E}+00 \quad-9.8340000 \mathrm{E}-01$

$25306 \quad p \quad-1.0000000 \mathrm{E}+00 \quad 1.7320508 \mathrm{E}+00 \quad 0.0000000 \mathrm{E}+00 \quad-2.9498000 \mathrm{E}+00$

Il temperatures in mev for the free-gas thermal neutron treatment.

non-zero importance cells with materials have a temperature for thermal neutrons of $2.5300 \mathrm{E}-08 \mathrm{mev}$.

2 waming messages so far.

Icross-section tables

print table 100

table length

tables from file testlibl

$1001.00 \mathrm{c} \quad 1782$ 1-h-l from endf-vi.l

$1002.55 \mathrm{c} 4102$ mjoy

mat $12505 / 26 / 93$

5011.40 3229 ENDL jibrary name: nd900719 MCNP translation: 900831 14:10:05

$6012.40 \mathrm{c} 4858$ ENDL library name: nd900719 MCNP translation: 900831 14:10:05

7014.40c 8535 ENDL library name: nd900719 MCNP translation: 900831 14:10:05

8016.40c 5358 ENDL library name: nd900719 MCNP translation: 900831 14:10:05

40000.40c 93372 ENDL library name: nd900719 MCNP translation: 900831 14:10:05

41093.40c 42746 ENDL library name: nd900719 MCNP translation: 900831 14:10:05

74000.40c 91244 ENDL library name: nd900719 MCNP translation: 900831 14:10:05

92235.40c 34772 ENDL library name: nd900719 MCNP translation: 9total nu 10:05

92238.40c 40834 ENDL library name: nd901118 MCNP translation: 9total nu 56:49

900207

900207

900207

900207

900323

900208

900208

900503

901119
$5010.03 \mathrm{~d} 3682 \quad \mathrm{~b}-10$ endf/b-iv new gamma production format
(1273) $14 \operatorname{oct} 75$
hwtr.01t 10193 deuterium in heavy water at 300 degrees kelvin
$10020010 / 22 / 85$
lwtr.01t 10193 hydrogen in light water at 300 degrees kelvin
$10010010 / 22 / 85$

total 354900

waming. neutron energy cutoff is below some cross-section tables. 
HNF-SD-SNF-CSWD-00S, Rev. 0 rce distribution written to file inpl $8 \mathrm{~s} \quad$ cycle $=0$

3 warning messages so far.

1 starting mcrun. field length $=0 \quad$ cp $0=0.00$

print table 110

probl8 - kcode in a hexagonal prism lattice.

nps $x \quad y \quad z \quad$ cell lattice $(i, j, k)$ surface $u \quad v \quad w$ energy weight time

$13.000 \mathrm{E}+00 \quad 2.000 \mathrm{E}-01 \quad 2.000 \mathrm{E}-01 \quad 140$ $3.492 \mathrm{E}+00 \quad 1.051 \mathrm{E}+00 \quad 2.000 \mathrm{E}-01 \quad 42($ $3.492 \mathrm{E}+00 \quad 1.051 \mathrm{E}+00 \quad 2.000 \mathrm{E}-01 \quad 154$

$23.000 \mathrm{E}+00 \quad 2.000 \mathrm{E}-01 \quad 2.000 \mathrm{E}-01 \quad 140$ $3.492 \mathrm{E}+00 \quad 1.051 \mathrm{E}+00 \quad 2.000 \mathrm{E}-01 \quad 42(0,-1$, $3.492 \mathrm{E}+00 \quad 1.051 \mathrm{E}+00 \quad 2.000 \mathrm{E}-01 \quad 154$

$33.000 \mathrm{E}+00 \quad 2.000 \mathrm{E}-01 \quad 2.000 \mathrm{E}-01 \quad 140$ $3.492 \mathrm{E}+00 \quad 1.051 \mathrm{E}+00 \quad 2.000 \mathrm{E}-01 \quad 42(0,-1,0)$ $3.492 \mathrm{E}+00 \quad 1.051 \mathrm{E}+00 \quad 2.000 \mathrm{E}-01 \quad 154$

$43.000 \mathrm{E}+00 \quad 2.000 \mathrm{E}-01 \quad 2.000 \mathrm{E}-01 \quad 140$ $3.492 \mathrm{E}+00 \quad 1.051 \mathrm{E}+00 \quad 2.000 \mathrm{E}-01 \quad 42(0,-1,0)$ $3.492 \mathrm{E}+00 \quad 1.051 \mathrm{E}+00 \quad 2.000 \mathrm{E}-01154$ $53.000 \mathrm{E}+00 \quad 2.000 \mathrm{E}-01 \quad 2.000 \mathrm{E}-01 \quad 140$ $3.492 \mathrm{E}+00 \quad 1.051 \mathrm{E}+00 \quad 2.000 \mathrm{E}-01 \quad 42($ $3.492 \mathrm{E}+00 \quad 1.051 \mathrm{E}+00 \quad 2.000 \mathrm{E}-01 \quad 154$

$63.000 \mathrm{E}+00 \quad 2.000 \mathrm{E}-0 \mathrm{l} \quad 2.000 \mathrm{E}-01 \quad 140$ $3.492 \mathrm{E}+00 \quad 1.051 \mathrm{E}+00 \quad 2.000 \mathrm{E}-01 \quad 42(0,-1$, $3.492 \mathrm{E}+00 \quad 1.051 \mathrm{E}+00 \quad 2.000 \mathrm{E}-01 \quad 154$

$73.000 \mathrm{E}+00 \quad 2.000 \mathrm{E}-01 \quad 2.000 \mathrm{E}-01 \quad 140$ $3.492 \mathrm{E}+00 \quad 1.051 \mathrm{E}+00 \quad 2.000 \mathrm{E}-01 \quad 42($ $3.492 \mathrm{E}+001.051 \mathrm{E}+00 \quad 2.000 \mathrm{E}-01 \quad 154$ $3.000 E+00 \quad 2.000 E-01 \quad 2.000 E-01 \quad 140$ $.492 \mathrm{E}+00 \quad 1.051 \mathrm{E}+00 \quad 2.000 \mathrm{E}-01 \quad 42($ $3.492 \mathrm{E}+00 \quad 1.051 \mathrm{E}+00 \quad 2.000 \mathrm{E}-01 \quad 154$ $93.000 \mathrm{E}+00 \quad 2.000 \mathrm{E}-01 \quad 2.000 \mathrm{E}-01 \quad 140$ $3.492 \mathrm{E}+00 \quad 1.051 \mathrm{E}+00 \quad 2.000 \mathrm{E}-01 \quad 42(0,-1,0)$ $3.492 \mathrm{E}+00 \quad 1.051 \mathrm{E}+00 \quad 2.000 \mathrm{E}-01 \quad 154$

$10 \quad 3.000 \mathrm{E}+00 \quad 2.000 \mathrm{E}-01 \quad 2.000 \mathrm{E}-01 \quad 140$ $3.492 \mathrm{E}+00 \quad 1.051 \mathrm{E}+00 \quad 2.000 \mathrm{E}-01 \quad 42(0,-1,0)$ $3.492 \mathrm{E}+00 \quad 1.051 \mathrm{E}+00 \quad 2.000 \mathrm{E}-01 \quad 154$

I1 $3.000 \mathrm{E}+00 \quad 2.000 \mathrm{E}-01 \quad 2.000 \mathrm{E}-01 \quad 140$ $3.492 \mathrm{E}+00 \quad 1.051 \mathrm{E}+00 \quad 2.000 \mathrm{E}-01 \quad 42($ $3.492 \mathrm{E}+00 \quad 1.051 \mathrm{E}+00 \quad 2.000 \mathrm{E}-01 \quad 154$ $123.000 \mathrm{E}+00 \quad 2.000 \mathrm{E}-01 \quad 2.000 \mathrm{E}-01 \quad 140$ $3.492 \mathrm{E}+00 \quad 1.051 \mathrm{E}+00 \quad 2.000 \mathrm{E}-01 \quad 42(0,-1,0)$ $3.492 \mathrm{E}+00 \quad 1.051 \mathrm{E}+00 \quad 2.000 \mathrm{E}-01 \quad 154$

$133.000 \mathrm{E}+00 \quad 2.000 \mathrm{E}-01 \quad 2.000 \mathrm{E}-01 \quad 140$ $3.492 \mathrm{E}+00 \quad 1.051 \mathrm{E}+00 \quad 2.000 \mathrm{E}-01 \quad 42(0,-1,0)$ $3.492 \mathrm{E}+00 \quad 1.051 \mathrm{E}+00 \quad 2.000 \mathrm{E}-01 \quad 154$

$143.000 \mathrm{E}+00 \quad 2.000 \mathrm{E}-01 \quad 2.000 \mathrm{E}-01 \quad 140$ $3.492 \mathrm{E}+00 \quad 1.051 \mathrm{E}+00 \quad 2.000 \mathrm{E}-01 \quad 42(0,-1,0)$ $3.492 \mathrm{E}+00 \quad 1.051 \mathrm{E}+00 \quad 2.000 \mathrm{E}-01 \quad 154$ is 3.000E +00 2.000E-01 2.000E-01 140 $3.492 \mathrm{E}+00 \quad 1.051 \mathrm{E}+00 \quad 2.000 \mathrm{E}-01 \quad 42(0,-1,0)$ $3.492 \mathrm{E}+00 \quad 1.051 \mathrm{E}+00 \quad 2.000 \mathrm{E}-01154$

$163.000 \mathrm{E}+00 \quad 2.000 \mathrm{E}-01 \quad 2.000 \mathrm{E}-01 \quad 140$ $3.492 \mathrm{E}+00 \quad 1.051 \mathrm{E}+00 \quad 2.000 \mathrm{E}-01 \quad 42(0,-1,0)$ $3.492 \mathrm{E}+00 \quad 1.051 \mathrm{E}+00 \quad 2.000 \mathrm{E}-01 \quad 154$

$173.000 \mathrm{E}+00 \quad 2.000 \mathrm{E}-01 \quad 2.000 \mathrm{E}-01 \quad 140$ $3.492 \mathrm{E}+00 \quad 1.051 \mathrm{E}+00 \quad 2.000 \mathrm{E}-01 \quad 42(0,-1,0)$ $3.492 \mathrm{E}+00 \quad 1.051 \mathrm{E}+00 \quad 2.000 \mathrm{E}-01 \quad 154$ $18 \quad 3.000 \mathrm{E}+00 \quad 2.000 \mathrm{E}-01 \quad 2.000 \mathrm{E}-01 \quad 140$ $3.492 \mathrm{E}+00 \quad 1.051 \mathrm{E}+00 \quad 2.000 \mathrm{E}-01 \quad 42(0,-1,0)$ $.492 \mathrm{E}+00 \quad 1.051 \mathrm{E}+00 \quad 2.000 \mathrm{E}-0 ! 154$ $3.000 \mathrm{E}+00 \quad 2.000 \mathrm{E}-01 \quad 2.000 \mathrm{E}-01 \quad 140$

5.085E-01 4.733E-01 7.193E-01 2.209E+00 $1.000 \mathrm{E}+00 \quad 0.000 \mathrm{E}+00$ $5.085 \mathrm{E}-01 \quad 4.733 \mathrm{E}-01 \quad 7.193 \mathrm{E}-01$

0 5.085E-01 4.733E-01 7.193E-01 8.952E-01 - $4.447 \mathrm{E}-01-2.944 \mathrm{E}-02 \quad 4.904 \mathrm{E}+00 \quad 1.000 \mathrm{E}+00 \quad 0.000 \mathrm{E}+00$ $8.952 \mathrm{E}-01-4.447 \mathrm{E}-01 \cdot 2.944 \mathrm{E}-02$

0 8.952E-01 -4.447E-01 -2.944E-02

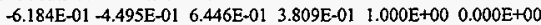
$-6.184 \mathrm{E}-01-4.495 \mathrm{E}-01 \quad 6.446 \mathrm{E}-0$ !

$0-6.184 \mathrm{E}-01-4.495 \mathrm{E}-01 \quad 6.446 \mathrm{E}-01$

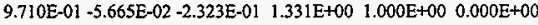
$9.710 \mathrm{E}-01-5.665 \mathrm{E}-02-2.323 \mathrm{E}-01$

$09.710 \mathrm{E}-01-5.665 \mathrm{E}-02-2.323 \mathrm{E}-0 \mathrm{l}$

$5.861 \mathrm{E}-01 \quad 1.496 \mathrm{E}-01-7.963 \mathrm{E}-01 \quad 1.902 \mathrm{E}+00 \quad 1.000 \mathrm{E}+00 \quad 0.000 \mathrm{E}+00$ $5.861 \mathrm{E}-01 \quad 1.496 \mathrm{E}-01+7.963 \mathrm{E}-01$

$05.861 \mathrm{E}-01 \quad 1.496 \mathrm{E}-01-7.963 \mathrm{E}-01$

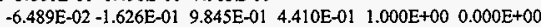
$-6.489 \mathrm{E}-02-1.626 \mathrm{E}-019.845 \mathrm{E}-01$

$0-6.489 \mathrm{E}-02-1.626 \mathrm{E}-01 \quad 9.845 \mathrm{E}-01$

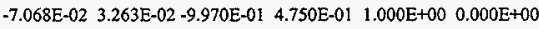
$-7.068 \mathrm{E}-02 \quad 3.263 \mathrm{E}-02-9.970 \mathrm{E}-01$

$0-7.068 \mathrm{E}-02 \quad 3.263 \mathrm{E}-02-9.970 \mathrm{E}-01$

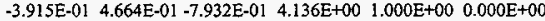
$-3.915 \mathrm{E}-01 \quad 4.664 \mathrm{E}-01-7.932 \mathrm{E}-01$

$0-3.915 \mathrm{E}-01 \quad 4.664 \mathrm{E}-01-7.932 \mathrm{E}-01$

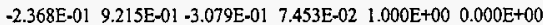

$-2.368 \mathrm{E}-019.215 \mathrm{E}-01-3.079 \mathrm{E}-01$

$0-2.368 \mathrm{E}-01 \quad 9.215 \mathrm{E}-01 \quad-3.079 \mathrm{E}-01$

$1.946 \mathrm{E}-01-3.204 \mathrm{E}-01 \quad 9.271 \mathrm{E}-01 \quad 3.128 \mathrm{E}+00 \quad 1.000 \mathrm{E}+00 \quad 0.000 \mathrm{E}+00$ $1.946 \mathrm{E}-01-3.204 \mathrm{E}-01 \quad 9.271 \mathrm{E}-01$

$01.946 \mathrm{E}-01-3.204 \mathrm{E}-01 \quad 9.271 \mathrm{E}-01$ $-6.698 \mathrm{E}-01-7.177 \mathrm{E}-01-1.905 \mathrm{E}-01 \quad 1.014 \mathrm{E}+00 \quad 1.000 \mathrm{E}+00 \quad 0.000 \mathrm{E}+00$ $-6.698 \mathrm{E}-01-7.177 \mathrm{E}-01-1.905 \mathrm{E}-01$

$0-6.698 \mathrm{E}-01-7.177 \mathrm{E}-01-1.905 \mathrm{E}-01$

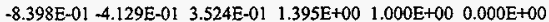
$-8.398 \mathrm{E}-01-4.129 \mathrm{E}-01$ 3.524E-01

$0-8.398 \mathrm{E}-01-4.129 \mathrm{E}-01 \quad 3.524 \mathrm{E}-01$

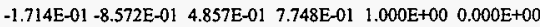
$-1.714 \mathrm{E}-01-8.572 \mathrm{E}-01$ 4.857E-01

$0-1.714 \mathrm{E}-01-8.572 \mathrm{E}-01 \quad 4.857 \mathrm{E}-01$ $-2.489 \mathrm{E}-01-5.118 \mathrm{E}-01-8.222 \mathrm{E}-01 \quad 1.101 \mathrm{E}+00 \quad 1.000 \mathrm{E}+00 \quad 0.000 \mathrm{E}+00$ $-2.489 \mathrm{E}-01-5.118 \mathrm{E}-01-8.222 \mathrm{E}-01$

$0-2.489 \mathrm{E}-0]-5.118 \mathrm{E}-01-8.222 \mathrm{E}-01$ $-2.959 \mathrm{E}-01 \quad 2.119 \mathrm{E}-01 \quad 9.314 \mathrm{E}-01 \quad 1.951 \mathrm{E}+00 \quad 1.000 \mathrm{E}+00 \quad 0.000 \mathrm{E}+00$ $-2.959 \mathrm{E}-01 \quad 2.119 \mathrm{E}-01 \quad 9.314 \mathrm{E}-01$

$0-2.959 \mathrm{E}-01 \quad 2.119 \mathrm{E}-01 \quad 9.314 \mathrm{E}-01$

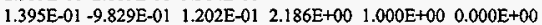
$1.395 \mathrm{E}-01-9.829 \mathrm{E}-01 \quad 1.202 \mathrm{E}-01$

0 1.395E-01 $-9.829 \mathrm{E}-01 \quad 1.202 \mathrm{E}-01$ $\begin{array}{llll}6.909 \mathrm{E}-01 & -7.110 \mathrm{E}-01 & 1.307 \mathrm{E}+01 & 1.865 \mathrm{E}+00 \quad 1.000 \mathrm{E}+00 \quad 0.000 \mathrm{E}+00\end{array}$ $6.909 \mathrm{E}-01-7.110 \mathrm{E}-01 \quad 1.307 \mathrm{E}-01$

0 6.909E-01 -7.110E-01 1.307E-01 $-6.580 \mathrm{E}-01 \quad 5.320 \mathrm{E}-01-5.329 \mathrm{E}-01 \quad 1.229 \mathrm{E}+00 \quad 1.000 \mathrm{E}+00 \quad 0.000 \mathrm{E}+00$ $-6.580 \mathrm{E}-015.320 \mathrm{E}-01-5.329 \mathrm{E}-01$

$0-6.580 \mathrm{E}-01 \quad 5.320 \mathrm{E}-01-5.329 \mathrm{E}-01$

$-9.903 \mathrm{E}-01-1.380 \mathrm{E}-01 \quad 1.353 \mathrm{E}-02 \quad 1.305 \mathrm{E}+00 \quad 1.000 \mathrm{E}+00 \quad 0.000 \mathrm{E}+00$ 
3.4 3.492E+00 $1.051 \mathrm{E}+00 \quad 2.000 \mathrm{E}-01 \quad 42(0,-1,0) \quad-9.903 \mathrm{E}-01-1.380 \mathrm{E}-01 \quad 1.353 \mathrm{E}-02$ $.492 \mathrm{E}+00 \quad 1.051 \mathrm{E}+00 \quad 2.000 \mathrm{E}-01 \quad 154$ $3.000 \mathrm{E}+00 \quad 2.000 \mathrm{E}-012.000 \mathrm{E}-01 \quad 140$ $3.492 \mathrm{E}+00 \quad 1.051 \mathrm{E}+00 \quad 2.000 \mathrm{E}-01 \quad 42(0,-1,0)$ $3.492 \mathrm{E}+00 \quad 1.051 \mathrm{E}+00 \quad 2.000 \mathrm{E}-01 \quad 154$ 21 $3.000 \mathrm{E}+00 \quad 2.000 \mathrm{E}-01 \quad 2.000 \mathrm{E}-01 \quad 140$ $3.492 \mathrm{E}+00 \quad 1.051 \mathrm{E}+00 \quad 2.000 \mathrm{E}-01 \quad 42(0,-1,0)$ $3.492 \mathrm{E}+00 \quad 1.051 \mathrm{E}+00 \quad 2.000 \mathrm{E}-01 \quad 154$ $223.000 \mathrm{E}+00 \quad 2.000 \mathrm{E}-01 \quad 2.000 \mathrm{E}-01 \quad 140$ $3.492 \mathrm{E}+00 \quad 1.051 \mathrm{E}+00 \quad 2.000 \mathrm{E}-01 \quad 42(0,-1,0)$ $3.492 \mathrm{E}+00 \quad 3.051 \mathrm{E}+00 \quad 2.000 \mathrm{E}-01 \quad 154$

$23 \quad 3.000 \mathrm{E}+00 \quad 2.000 \mathrm{E}-01 \quad 2.000 \mathrm{E}-01 \quad 140$ $3.492 \mathrm{E}+00 \quad 1.051 \mathrm{E}+00 \quad 2.000 \mathrm{E}-01 \quad 42(0,-1,0)$ $3.492 \mathrm{E}+00 \quad 1.051 \mathrm{E}+\infty \quad 2.000 \mathrm{E}-01 \quad 154$ $243.000 \mathrm{E}+00 \quad 2.000 \mathrm{E}-01 \quad 2.000 \mathrm{E}-01 \quad 140$ $3.492 \mathrm{E}+00 \quad 1.051 \mathrm{E}+00 \quad 2.000 \mathrm{E}-01 \quad 42(0,-1,0)$ $\begin{array}{lll}3.492 \mathrm{E}+00 & 1.051 \mathrm{E}+00 & 2.000 \mathrm{E}-01 \quad 154\end{array}$ $25 \quad 3.000 \mathrm{E}+00 \quad 2.000 \mathrm{E}-01 \quad 2.000 \mathrm{E}-01 \quad 140$ $3.492 \mathrm{E}+00 \quad 1.051 \mathrm{E}+00 \quad 2.000 \mathrm{E}-01 \quad 42(0,-1,0)$ $3.492 \mathrm{E}+00 \quad 1.051 \mathrm{E}+00 \quad 2.000 \mathrm{E}-01 \quad 154$ $262.000 \mathrm{E}-01 \quad 3.000 \mathrm{E}+00 \quad 2.000 \mathrm{E}-01 \quad 140$ $3.641 \mathrm{E}+00 \quad 4.456 \mathrm{E}-01 \quad 2.000 \mathrm{E}-01 \quad 42(-5,3,0)$ $3.641 \mathrm{E}+00 \quad 4.456 \mathrm{E}-01 \quad 2.000 \mathrm{E}-01 \quad 141$

$27 \quad 2.000 \mathrm{E}-01 \quad 3.000 \mathrm{E}+00 \quad 2.000 \mathrm{E}-01 \quad 140$ $3.641 \mathrm{E}+00 \quad 4.456 \mathrm{E}-01 \quad 2.000 \mathrm{E}-01 \quad 42(-5,3,0)$ $3.641 \mathrm{E}+00 \quad 4.456 \mathrm{E}-01 \quad 2.000 \mathrm{E}-01$ I 14$]$

$282.000 \mathrm{E}-01 \quad 3.000 \mathrm{E}+00 \quad 2.000 \mathrm{E}-01 \quad 140$ $3.641 \mathrm{E}+00 \quad 4.456 \mathrm{E}-01 \quad 2.000 \mathrm{E}-01 \quad 42(-5,3,0)$ 3.641E $\div 00 \quad 4.456 \mathrm{E}-01 \quad 2.000 \mathrm{E}-01 \quad 141$

$29 \quad 2.000 \mathrm{E}-01 \quad 3.000 \mathrm{E}+00 \quad 2.000 \mathrm{E}-01 \quad 140$ $3.641 \mathrm{E}+00 \quad 4.456 \mathrm{E}-01 \quad 2.000 \mathrm{E}-01 \quad 42(-5,3,0)$ $3.641 \mathrm{E}+00 \quad 4.456 \mathrm{E}-01 \quad 2.000 \mathrm{E}-01 \quad 141$

.30 2.000E-01 3.000E+00 2.000E-01 140 $641 \mathrm{E}+00 \quad 4.456 \mathrm{E}-01 \quad 2.000 \mathrm{E}-01 \quad 42(-5,3,0)$ $.641 \mathrm{E}+00 \quad 4.456 \mathrm{E}-01 \quad 2.000 \mathrm{E}-01 \quad 141$

$312.000 \mathrm{E}-01 \quad 3.000 \mathrm{E}+00 \quad 2.000 \mathrm{E}-01 \quad 140$ $3.641 \mathrm{E}+00 \quad 4.456 \mathrm{E}-01 \quad 2.000 \mathrm{E}-01 \quad 42(-5,3,0)$ $3.641 \mathrm{E}+00 \quad 4.456 \mathrm{E}-01 \quad 2.000 \mathrm{E}-01 \quad 141$

$32 \quad 2.000 \mathrm{E}-013.000 \mathrm{E}+00 \quad 2.000 \mathrm{E}-01 \quad 140$ $3.641 \mathrm{E}+00 \quad 4.456 \mathrm{E}-01 \quad 2.000 \mathrm{E}-01 \quad 42(-5,3,0)$ $3.641 \mathrm{E}+00 \quad 4.456 \mathrm{E}-01 \quad 2.000 \mathrm{E}-01 \quad 141$

$332.000 \mathrm{E}-013.000 \mathrm{E}+00 \quad 2.000 \mathrm{E}-01 \quad 140$ $3.64 \mathrm{IE}+00 \quad 4.456 \mathrm{E}+0$ ) $2.000 \mathrm{E}-01 \quad 42(-5,3,0)$ $3.641 \mathrm{E}+00 \quad 4.456 \mathrm{E}-01 \quad 2.000 \mathrm{E}-01 \quad 141$

34 2.000E-01 3.000E+00 2.000E-01 140 $3.641 \mathrm{E}+00 \quad 4.456 \mathrm{E}-01 \quad 2.000 \mathrm{E}-01 \quad 42(-5,3,0)$ $3.641 \mathrm{E}+00 \quad 4.456 \mathrm{E}-01 \quad 2.000 \mathrm{E}-01 \quad 141$

35 2.000E-01 3.000E $+00 \quad 2.000 \mathrm{E}-01 \quad 140$ $3.641 \mathrm{E}+00 \quad 4.456 \mathrm{E}-01 \quad 2.000 \mathrm{E}-01 \quad 42(-5,3,0)$ $3.641 \mathrm{E}+00 \quad 4.456 \mathrm{E}-01 \quad 2.000 \mathrm{E}-01 \quad 141$

$362.000 \mathrm{E}-01 \quad 3.000 \mathrm{E}+00 \quad 2.000 \mathrm{E}-01 \quad 140$ $3.641 \mathrm{E}+00 \quad 4.456 \mathrm{E}-01 \quad 2.000 \mathrm{E}-01 \quad 42(-5,3,0)$ $3.641 \mathrm{E}+00 \quad 4.456 \mathrm{E}-01 \quad 2.000 \mathrm{E}-01 \quad 141$

$372.000 \mathrm{E}-01 \quad 3.000 \mathrm{E}+00 \quad 2.000 \mathrm{E}-01 \quad 140$ $3.641 \mathrm{E}+00 \quad 4.456 \mathrm{E}-01 \quad 2.000 \mathrm{E}-01 \quad 42(-5,3,0)$ $3.641 \mathrm{E}+00 \quad 4.456 \mathrm{E}-01 \quad 2.000 \mathrm{E}-01 \quad 141$

$38 \quad 2.000 \mathrm{E}-01 \quad 3.000 \mathrm{E}+00 \quad 2.000 \mathrm{E}-01 \quad 140$ $3.641 \mathrm{E}+00 \quad 4.456 \mathrm{E}-01 \quad 2.000 \mathrm{E}-01 \quad 42(-5,3,0)$ 3.641E+00 4.456E-01 2.000E-01 14 !

$392.000 \mathrm{E}-01 \quad 3.000 \mathrm{E}+00 \quad 2.000 \mathrm{E}-01 \quad 140$ $3.641 \mathrm{E}+00 \quad 4.456 \mathrm{E}-01 \quad 2.000 \mathrm{E}-01 \quad 42(-5,3,0)$ $3.641 \mathrm{E}+00 \quad 4.456 \mathrm{E}-01 \quad 2.000 \mathrm{E}-01 \quad 141$

$40 \quad 2.000 \mathrm{E}-01 \quad 3.000 \mathrm{E}+00 \quad 2.000 \mathrm{E}-01 \quad 140$

$3.641 \mathrm{E}+00 \quad 4.456 \mathrm{E}-01 \quad 2.000 \mathrm{E}-01 \quad 42(-5,3,0)$ $3.641 \mathrm{E}+00 \quad 4.456 \mathrm{E}-01 \quad 2.000 \mathrm{E}-01 \quad 141$ 2.000E-01 3.000E+00 2.000E-01 140 $.641 \mathrm{E}+00 \quad 4.456 \mathrm{E}-01 \quad 2.000 \mathrm{E}-01 \quad 42(-5,3,0)$
$0-9.903 \mathrm{E}-01-1.380 \mathrm{E}-01$ 1.353E-02

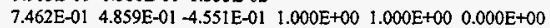

$7.462 \mathrm{E}-01 \quad 4.859 \mathrm{E}-01-4.551 \mathrm{E}-01$

$0 \quad 7.462 \mathrm{E}-01 \quad 4.859 \mathrm{E}-01-4.551 \mathrm{E}-01$

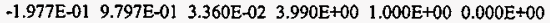

$-1.977 \mathrm{E}-01 \quad 9.797 \mathrm{E}-01 \quad 3.360 \mathrm{E}-02$

$0-1.977 \mathrm{E}-01 \quad 9.797 \mathrm{E}-01 \quad 3.360 \mathrm{E}-02$

$-9.117 \mathrm{E}-01-3.647 \mathrm{E}-01-1.891 \mathrm{E}-01 \quad 2.665 \mathrm{E}-01 \quad 1.000 \mathrm{E}+00 \quad 0.000 \mathrm{E}+00$ $-9.117 \mathrm{E}-01-3.647 \mathrm{E}-01-1.891 \mathrm{E}-01$

$0-9.117 \mathrm{E}-01-3.647 \mathrm{E}-01-1.891 \mathrm{E}-01$

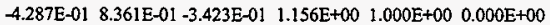
$4.287 \mathrm{E}-01 \quad 8.361 \mathrm{E}-01-3.423 \mathrm{E}-01$

$0-4.287 \mathrm{E}-01 \quad 8.361 \mathrm{E}-01 \quad-3.423 \mathrm{E}-01$

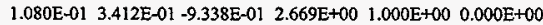
$1.080 \mathrm{E}-01 \quad 3.412 \mathrm{E}-01-9.338 \mathrm{E}-01$

0 1.080E-01 3.412E-01 -9.338E-01

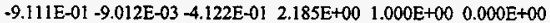
$-9.111 \mathrm{E}-01 \mathrm{-}-9.012 \mathrm{E}-03-4.122 \mathrm{E}-01$

$0-9.111 \mathrm{E}-01-9.012 \mathrm{E}-03-4.122 \mathrm{E}-01$

$-2.568 \mathrm{E}-01-6.391 \mathrm{E}-01-7.249 \mathrm{E}-01 \quad 7.280 \mathrm{E}-01 \quad 1.000 \mathrm{E}+00 \quad 0.000 \mathrm{E}+00$ $-2.568 \mathrm{E}-01-6.391 \mathrm{E}-01-7.249 \mathrm{E}-01$

$0-2.568 \mathrm{E}-01-6.391 \mathrm{E}-01-7.249 \mathrm{E}-01$

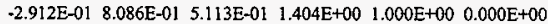
$-2.912 \mathrm{E}-01 \quad 8.086 \mathrm{E}-01 \quad 5.113 \mathrm{E}-01$

$0-2.912 \mathrm{E}-01 \quad 8.086 \mathrm{E}-01 \quad 5.113 \mathrm{E}-01$

1.472E-01 $-9.514 \mathrm{E}-01 \quad 2.705 \mathrm{E}-01 \quad 1.090 \mathrm{E}+00 \quad 1.000 \mathrm{E}+00 \quad 0.000 \mathrm{E}+00$ $1.472 \mathrm{E}-01-9.5[4 \mathrm{E}-01 \quad 2.705 \mathrm{E}-01$

$0 \quad 1.472 \mathrm{E}-01-9.514 \mathrm{E}-01 \quad 2.705 \mathrm{E}-01$

$\begin{array}{llll}-6.135 \mathrm{E}-01 & -7.645 \mathrm{E}-01-1.978 \mathrm{E}-01 & 2.483 \mathrm{E}+00 \quad 1.000 \mathrm{E}+00 & 0.000 \mathrm{E}+00\end{array}$ $-6.135 \mathrm{E}-01-7.645 \mathrm{E}-01-1.978 \mathrm{E}-01$

$0-6.135 \mathrm{E}-01-7.645 \mathrm{E}-01-1.978 \mathrm{E}-01$

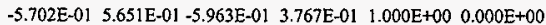
$-5.702 \mathrm{E}-01$ 5.651E-01 -5.963E-01

$0-5.702 \mathrm{E}-01 \quad 5.651 \mathrm{E}-01-5.963 \mathrm{E}-01$

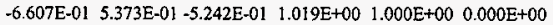
$-6.607 \mathrm{E}-015.373 \mathrm{E}-01-5.242 \mathrm{E}-01$

$0-6.607 \mathrm{E}-01 \quad 5.373 \mathrm{E}-01-5.242 \mathrm{E}-01$

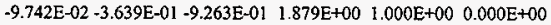
$-9.742 \mathrm{E}-02-3.639 \mathrm{E}-01-9.263 \mathrm{E}-01$

$0-9.742 \mathrm{E}-02-3.639 \mathrm{E}-01-9.263 \mathrm{E}-01$

$-1.965 \mathrm{E}-01-3.145 \mathrm{E}-01-9.287 \mathrm{E}-01 \quad 4.700 \mathrm{E}-01 \quad 1.000 \mathrm{E}+00 \quad 0.000 \mathrm{E}+00$ $-1.965 \mathrm{E}-01-3.145 \mathrm{E}-01-9.287 \mathrm{E}-01$

$0-1.965 \mathrm{E}-01-3.145 \mathrm{E}-01-9.287 \mathrm{E}-01$

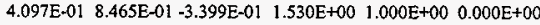
$4.097 \mathrm{E}-01 \quad 8.465 \mathrm{E}-01-3.399 \mathrm{E}-01$

0 4.097E-01 8.465E-01 -3.399E-01

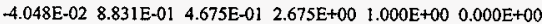
$-4.048 \mathrm{E}-02 \quad 8.831 \mathrm{E}-01 \quad 4.675 \mathrm{E}-01$

$0-4.048 \mathrm{E}-02 \quad 8.831 \mathrm{E}-0 \mathrm{I}$ 4.675E-01 $\begin{array}{llll}3.371 \mathrm{E}-01 & -9.269 \mathrm{E}-0 \mathrm{l}-1.652 \mathrm{E}-01 & 9.222 \mathrm{E}-01 & 1.000 \mathrm{E}+00 \quad 0.000 \mathrm{E}+00\end{array}$ 3.371E-01 -9.269E-01 -1.652E-01

0 3.371E-01 -9.269E-01 -1.652E-01

$-1.867 \mathrm{E}-01 \quad 9.756 \mathrm{E}-01-1.155 \mathrm{E}-01 \quad 9.702 \mathrm{E}-01 \quad 1.000 \mathrm{E}+00 \quad 0.000 \mathrm{E}+00$ $-1.867 \mathrm{E}-019.756 \mathrm{E}-01-1.155 \mathrm{E}-01$

$0-1.867 \mathrm{E}-01 \quad 9.756 \mathrm{E}-0 \mathrm{l}-1.155 \mathrm{E}-01$ $\begin{array}{lllll}-2.616 \mathrm{E}-01 & 2.336 \mathrm{E}-01-9.365 \mathrm{E}-01 & 1.230 \mathrm{E}+00 & 1.000 \mathrm{E}+00 & 0.000 \mathrm{E}+00\end{array}$ $-2.616 \mathrm{E}-01 \quad 2.336 \mathrm{E}-01-9.365 \mathrm{E}-01$

$0-2.616 \mathrm{E}-012.336 \mathrm{E}-01-9.365 \mathrm{E}-01$ $9.780 \mathrm{E}-01-7.641 \mathrm{E}-02-1.939 \mathrm{E}-01 \quad 1.083 \mathrm{E}+00 \quad 1.000 \mathrm{E}+00 \quad 0.000 \mathrm{E}+00$ $9.780 \mathrm{E}-01-7.641 \mathrm{E}-02-1.939 \mathrm{E}-01$

$0 \quad 9.780 \mathrm{E}-01-7.641 \mathrm{E}-02-1.939 \mathrm{E}-01$

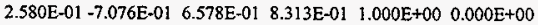
$2.580 \mathrm{E}-01-7.076 \mathrm{E}-01 \quad 6.578 \mathrm{E}-01$

0 2.580E-01 -7.076E-01 $6.578 \mathrm{E}-01$

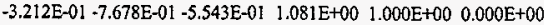
$-3.212 \mathrm{E}-01-7.678 \mathrm{E}-01-5.543 \mathrm{E}-01$ 


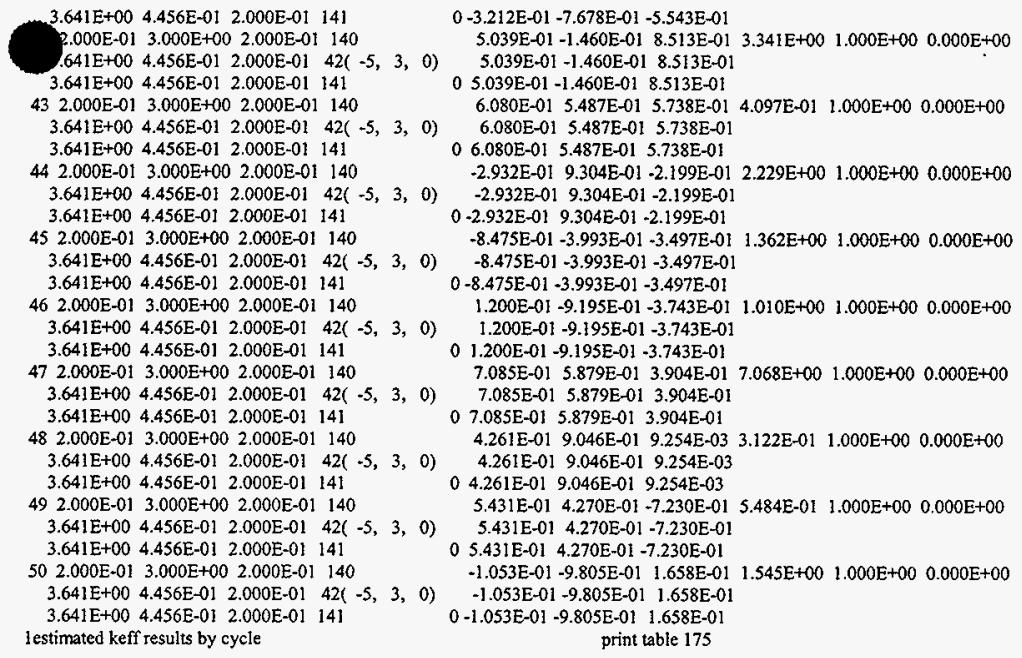

cycle $1 \mathrm{k}$ (collision) 1.253108 removal lifetime(abs) $1.7557 \mathrm{E}+01$ source points generated 257

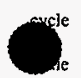

$2 \mathrm{k}$ (collision) 1.211997 temoval lifetime(abs) $1.1671 \mathrm{E}+01$ source points generated 195

$3 \mathrm{k}$ (collision) 1.200265 removal lifetime(abs) $9.6992 \mathrm{E}+00$ source points generated 193

cycle $4 \mathrm{k}$ (collision) 1.129530 removal lifetime(abs) $9.3720 \mathrm{E}+00$ source points generated 182

cycle $5 \mathrm{k}$ (collision) 1.135994 removal lifetime(abs) $1.1136 \mathrm{E}+01$ source points generated 222

source distribution written to file inpl $8 \mathrm{~s} \quad$ cycle $=5$

cycle $6 \mathrm{k}$ (collision) 1.077014 removal lifetime(abs) $1.2222 \mathrm{E}+01$ source points generated 204

source distribution written to file inpl $8 \mathrm{~s} \quad$ cycle $=6$

lproblem summary

0

nun terminated when 6 kcode cycles were done.

neutron creation tracks weight energy neutron loss tracks weight energy (per source particle) (per source particle)

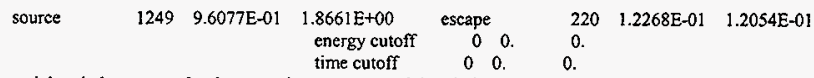

weight window $\quad \begin{array}{lllllll}0 & 0 . & 0 . & \text { weight window } & 0 & 0 . & 0 .\end{array}$

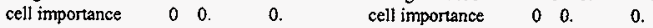

$\begin{array}{llllllll}\text { weight cutoff } & 0 & 1.3743 \mathrm{E}-01 & 3.8498 \mathrm{E}-03 & \text { weight cutoff } & 1034 & 1.2791 \mathrm{E}-01 & 5.1920 \mathrm{E}-03\end{array}$

energy importance $\begin{array}{llllllll}0 & 0 . & 0 . & \text { energy importance } & 0 & 0 & 0 .\end{array}$

$\begin{array}{lllllllllll}\text { dxtran } & 0 & 0 . & 0 . & & \text { dxtan } & 0 & 0 . & 0 . & \\ \text { forced collisions } & 0 & 0 . & 0 . & \text { forced collisions } & 0 & 0 . & 0 .\end{array}$

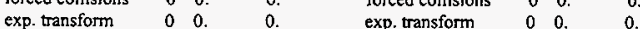

$\begin{array}{lllllllll}\text { upscattering } & 0 & 0 . & 5.3884 \mathrm{E}-11 & \text { downscattering } & 0 & 0 . & 1.3605 \mathrm{E}+00\end{array}$

capture $\quad 0 \quad 3.9980 \mathrm{E}-01 \quad 1.15 \% \mathrm{E}-01$

$\begin{array}{lllllll}10 & 6.1640 \mathrm{E}-03 & 6.1355 \mathrm{E}-03 & \text { loss to }(\mathrm{n}, \mathrm{xn}) & 5 & 3.0820 \mathrm{E}-03 & 2.4027 \mathrm{E}-02\end{array}$

$\begin{array}{llllllll}0 & 0 . & 0 . & \text { loss to fission } & 0 & 4.5089 \mathrm{E}-01 & 2.4987 \mathrm{E}-01\end{array}$

$\begin{array}{llllllll}1259 & 1.1044 \mathrm{E}+00 & 1.8761 \mathrm{E}+00 & \text { total } & 1259 & 1.1044 \mathrm{E}+00 & 1.8761 \mathrm{E}+00\end{array}$ 
number of neutrons banked 5 average lifetime, shakes cutoffs utron collisions per source particle $1.8226 \mathrm{E}+01$ capture $1.2954 \mathrm{E}+01$ eco $0.0000 \mathrm{E}+00$ total neutron collisions 22764 capture or escape 1.1788E+01 wcl $-5.0000 \mathrm{E}-01$ net multiplication $1.0032 E+000.0035$ any termination $1.2744 \mathrm{E}+01$ wc2 $-2.5000 \mathrm{E}-01$

$\begin{array}{lr}\text { computer time so far in this run } & 0.00 \text { minutes } \\ \text { computer time in morun } & 0.00 \text { minutes } \\ \text { source particles per minute } & 0.0000 \mathrm{E}+00 \\ \text { random numbers generated } & 500880\end{array}$

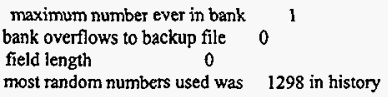

range of sampled source weights $=7.7821 \mathrm{E}-01$ to $1.0989 \mathrm{E}+00$

Ineutron activity in each cell

print table 126

tracks population collisions collisions number flux average average cell entering

- weight weighted weighted track weight track mfp (per history) energy energy (relative) (cm)

$\begin{array}{cccl}1 & 30 & 0 & 0 \\ 2 & 31 & 0 & 0 \\ 3 & 37 & 0 & 0 \\ 4 & 34 & 0 & 0 \\ 5 & 32 & 0 & 0 \\ 6 & 33 & 0 & 0 \\ 7 & 35 & 0 & 0 \\ 8 & 36 & 0 & 0 \\ 9 & 38 & 920 & 618 \\ 10 & 39 & 1635 & 637 \\ 11 & 40 & 1728 & 663 \\ 12 & 41 & 1867 & 712 \\ 13 & 140 & 0 & 0 \\ 14 & 42 & 2069 & 867 \\ 15 & 154 & 25302 & 1253 \\ 4 & 149 & 49542 & 1253 \\ 7 & 144 & 51224 & 1254 \\ 18 & 159 & 58650 & 1254 \\ 19 & 141 & 73122 & 1254\end{array}$

$\begin{array}{llllllll}0 & 0.0000 E+00 & 0.0000 E+00 & 0.0000 E+00 & 0.0000 E+00 & 0.0000 E+00\end{array}$

$\begin{array}{llllllll}0 & 0.0000 \mathrm{E}+00 & 0.0000 \mathrm{E}+00 & 0.0000 \mathrm{E}+00 & 0.0000 \mathrm{E}+00 & 0.0000 \mathrm{E}+00\end{array}$

$\begin{array}{llllllll}0 & 0.0000 \mathrm{E}+00 & 0.0000 \mathrm{E}+00 & 0.0000 \mathrm{E}+00 & 0.0000 \mathrm{E}+00 & 0.0000 \mathrm{E}+00\end{array}$

$\begin{array}{llllllll}0 & 0.0000 \mathrm{E}+00 & 0.0000 \mathrm{E}+00 & 0.0000 \mathrm{E}+00 & 0.0000 \mathrm{E}+00 & 0.0000 \mathrm{E}+00\end{array}$

$\begin{array}{llllllll}0 & 0.0000 \mathrm{E}+00 & 0.0000 \mathrm{E}+00 & 0.0000 \mathrm{E}+00 & 0.0000 \mathrm{E}+00 & 0.0000 \mathrm{E}+00\end{array}$

$\begin{array}{lllllll}0 & 0.0000 \mathrm{E}+00 & 0.0000 \mathrm{E}+00 & 0.0000 \mathrm{E}+00 & 0.0000 \mathrm{E}+00 & 0.0000 \mathrm{E}+00\end{array}$

$\begin{array}{lllllll}0 & 0.0000 E+00 & 0.0000 \mathrm{E}+00 & 0.0000 \mathrm{E}+00 & 0.0000 \mathrm{E}+00 & 0.0000 \mathrm{E}+00\end{array}$

$\begin{array}{lllllll}0 & 0.0000 \mathrm{E}+00 & 0.0000 \mathrm{E}+00 & 0.0000 \mathrm{E}+00 & 0.0000 \mathrm{E}+00 & 0.0000 \mathrm{E}+00\end{array}$

$\begin{array}{lllllll}1249 & 5.9116 \mathrm{E}-0 \mathrm{t} & 3.0414 \mathrm{E}-01 & 8.9304 \mathrm{E}-01 & 6.4212 \mathrm{E}-01 & 2.6324 \mathrm{E}+00\end{array}$

$\begin{array}{lllllll}130 & 6.3779 \mathrm{E}-02 & 8.8236 \mathrm{E}-02 & 7.6766 \mathrm{E}-01 & 6.4931 \mathrm{E}-01 & 2.6947 \mathrm{E}+00\end{array}$

$\begin{array}{lllllll}184 & 8.8176 \mathrm{E}-02 & 7.6162 \mathrm{E}-02 & 7.6184 \mathrm{E}-01 & 6.4437 \mathrm{E}-01 & 2.5146 \mathrm{E}+00\end{array}$

$\begin{array}{lllllll}317 & 1.5811 \mathrm{E}-01 & 8.6238 \mathrm{E}-02 & 7.9306 \mathrm{E}-01 & 6.5030 \mathrm{E}-01 & 2.6940 \mathrm{E}+00\end{array}$

$\begin{array}{llllllll}0 & 0.0000 \mathrm{E}+00 & 0.0000 \mathrm{E}+00 & 0.0000 \mathrm{E}+00 & 0.0000 \mathrm{E}+00 & 0.0000 \mathrm{E}+00\end{array}$

$441 \quad 2.1911 \mathrm{E}-01 \quad 8.9382 \mathrm{E}-02 \quad 7.8771 \mathrm{E}-01 \quad 6.6777 \mathrm{E}-01 \quad 2.5091 \mathrm{E}+00$

$8436 \quad 4.1390 \mathrm{E}+00 \quad 1.2606 \mathrm{E}-01 \quad 8.5005 \mathrm{E}-01 \quad 6.6920 \mathrm{E}-01 \quad 2.4712 \mathrm{E}+00$

$\begin{array}{lllllll}580 & 2.8963 \mathrm{E}-01 & 1.0795 \mathrm{E}-01 & 8.2361 \mathrm{E}-01 & 6.7129 \mathrm{E}-01 & 2.5408 \mathrm{E}+00\end{array}$

$885 \quad 4.2652 \mathrm{E}-01 \quad 1.0541 \mathrm{E}-01 \quad 8.1599 \mathrm{E}-01 \quad 6.6971 \mathrm{E}-01 \quad 2.2391 \mathrm{E}+00$

$\begin{array}{llllll}4019 & 2.1254 \mathrm{E}+00 & 9.8931 \mathrm{E}-02 & 8.1154 \mathrm{E}-01 & 6.6977 \mathrm{E}-01 & 2.7541 \mathrm{E}+00\end{array}$

$\begin{array}{lllll}6523 & 3.2229 \mathrm{E}+00 \quad 9.4499 \mathrm{E}-02 & 8.0639 \mathrm{E}-01 & 6.6810 \mathrm{E}-01 & 2.5056 \mathrm{E}+00\end{array}$

total $266059 \quad 9765 \quad 22764 \quad 1.1324 \mathrm{E}+01$

Ineutron weight balance in each cell -- external events print table 130

cell entering source energy time exiting total

cutoff cutoff

$\begin{array}{llllllll}30 & 0.0000 \mathrm{E}+00 & 0.0000 \mathrm{E}+00 & 0.0000 \mathrm{E}+00 & 0.0000 \mathrm{E}+00 & 0.0000 \mathrm{E}+00 & 0.0000 \mathrm{E}+00\end{array}$

2 3! $\quad 0.0000 \mathrm{E}+00 \quad 0.0000 \mathrm{E}+00 \quad 0.0000 \mathrm{E}+00 \quad 0.0000 \mathrm{E}+00 \quad 0.0000 \mathrm{E}+00 \quad 0.0000 \mathrm{E}+00$

$\begin{array}{llllllll}3 & 37 & 0.0000 E+00 & 0.0000 E+00 & 0.0000 E+00 & 0.0000 E+00 & 0.0000 E+00 & 0.0000 E+00\end{array}$

$\begin{array}{lllllllll}4 & 34 & 0.0000 \mathrm{E}+00 & 0.0000 \mathrm{E}+00 & 0.0000 \mathrm{E}+00 & 0.0000 \mathrm{E}+00 & 0.0000 \mathrm{E}+00 & 0.0000 \mathrm{E}+00\end{array}$

$\begin{array}{lllllllll}5 & 32 & 0.0000 \mathrm{E}+00 & 0.0000 \mathrm{E}+00 & 0.0000 \mathrm{E}+00 & 0.0000 \mathrm{E}+00 & 0.0000 \mathrm{E}+00 & 0.0000 \mathrm{E}+00\end{array}$

$\begin{array}{lllllllllll}6 & 33 & 0.0000 \mathrm{E}+00 & 0.0000 \mathrm{E}+00 & 0.0000 \mathrm{E}+00 & 0.0000 \mathrm{E}+00 & 0.0000 \mathrm{E}+00 & 0.0000 \mathrm{E}+00\end{array}$

$\begin{array}{lllllllll}7 & 35 & 0.0000 \mathrm{E}+00 & 0.0000 \mathrm{E}+00 & 0.0000 \mathrm{E}+00 & 0.0000 \mathrm{E}+00 & 0.0000 \mathrm{E}+00 & 0.0000 \mathrm{E}+00\end{array}$

$8 \quad 36 \quad 0.0000 \mathrm{E}+00 \quad 0.0000 \mathrm{E}+00 \quad 0.0000 \mathrm{E}+00 \quad 0.0000 \mathrm{E}+00 \quad 0.0000 \mathrm{E}+00 \quad 0.0000 \mathrm{E}+00$

$\begin{array}{lllllllll}9 & 38 & 4.8822 \mathrm{E}-01 & 0.0000 \mathrm{E}+00 & 0.0000 \mathrm{E}+00 & 0.0000 \mathrm{E}+00 & -3.3091 \mathrm{E}-01 & 1.5731 \mathrm{E}-01\end{array}$

$1039 \quad 8.4996 \mathrm{E}-01 \quad 0.0000 \mathrm{E}+00 \quad 0.0000 \mathrm{E}+00 \quad 0.0000 \mathrm{E}+00 \quad-8.4933 \mathrm{E}-01 \quad 6.2750 \mathrm{E}-04$

$11 \quad 40 \quad 8.9904 \mathrm{E}-01 \quad 0.0000 \mathrm{E}+00 \quad 0.0000 \mathrm{E}+00 \quad 0.0000 \mathrm{E}+00 \quad-8.9904 \mathrm{E}-01 \quad 3.9460 \mathrm{E}-06$

$\begin{array}{lllllllll}12 & 41 & 9.7260 \mathrm{E}-01 & 0.0000 \mathrm{E}+00 & 0.0000 \mathrm{E}+00 & 0.0000 \mathrm{E}+00 & -9.6978 \mathrm{E}-0 \mathrm{l} & 2.8204 \mathrm{E}-03\end{array}$

$\begin{array}{llllllll}13 & 140 & 0.0000 \mathrm{E}+00 & 0.0000 \mathrm{E}+00 & 0.0000 \mathrm{E}+00 & 0.0000 \mathrm{E}+00 & 0.0000 \mathrm{E}+00 & 0.0000 \mathrm{E}+00\end{array}$

$\begin{array}{lllllllll}14 & 42 & 1.0907 \mathrm{E}+00 & 0.0000 \mathrm{E}+00 & 0.0000 \mathrm{E}+00 & 0.0000 \mathrm{E}+00 & -1.0913 \mathrm{E}+00 & -5.9111 \mathrm{E}-04\end{array}$

$\begin{array}{lllllllll}15 & 154 & 1.2943 \mathrm{E}+01 & 8.4067 \mathrm{E}-01 & 0.0000 \mathrm{E}+00 & 0.0000 \mathrm{E}+00 & -1.3171 \mathrm{E}+01 & 6.1278 \mathrm{E}-01\end{array}$

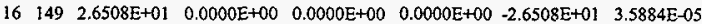

$\begin{array}{lllllllll}17 & 144 & 2.7419 E+01 & 0.0000 E+00 & 0.0000 E+00 & 0.0000 E+00 & -2.7405 E+01 & 1.4572 E-02\end{array}$

$\begin{array}{lllllllll}18 & 159 & 3.1331 \mathrm{E}+01 & 8.0064 \mathrm{E}-02 & 0.0000 \mathrm{E}+00 & 0.0000 \mathrm{E}+00 & -3.1361 \mathrm{E}+01 & 5.0067 \mathrm{E}-02\end{array}$

$\begin{array}{llllllll}19 & 141 & 3.911 \mathrm{EE}+01 & 4.0032 \mathrm{E}-02 & 0.0000 \mathrm{E}+00 & 0.0000 \mathrm{E}+00 & -3.9150 \mathrm{E}+01 \quad 4.5860 \mathrm{E}-04\end{array}$

tota] $\quad 1.4161 \mathrm{E}+02 \quad 9.6077 \mathrm{E}-01 \quad 0.0000 \mathrm{E}+00 \quad 0.0000 \mathrm{E}+00 \quad-1.4174 \mathrm{E}+02 \quad 8.3809 \mathrm{E}-01$

tron weight balance in each cell -- variance reduction events

print table 130 


\begin{tabular}{|c|c|c|c|c|c|c|c|c|c|}
\hline & & $\begin{array}{l}\text { weight } \\
\text { window }\end{array}$ & $\begin{array}{l}\text { ell weight } \\
\text { portance cuto }\end{array}$ & $\begin{array}{c}\text { energy } \\
\text { off importan }\end{array}$ & dxtran & $\begin{array}{l}\text { prced exponer } \\
\text { collision trans }\end{array}$ & $\begin{array}{l}\text { ntial total } \\
\text { isform }\end{array}$ & & \\
\hline & 30 & $0.0000 E+00$ & $0.0000 \mathrm{E}+00$ & $0.0000 \mathrm{E}+00$ & $0.0000 E+00$ & $0.0000 \mathrm{E}+00$ & $0.0000 \mathrm{E}+00$ & $0.0000 \mathrm{E}+00$ & $0.0000 \mathrm{E}+00$ \\
\hline 2 & 31 & $0.0000 \mathrm{E}+00$ & $0.0000 \mathrm{E}+00$ & $0.0000 \mathrm{E}+00$ & $0.0000 E+00$ & $0.0000 \mathrm{E}+00$ & $0.0000 \mathrm{E}+00$ & $0.0000 \mathrm{E}+00$ & $0.0000 \mathrm{E}+00$ \\
\hline 3 & 37 & $0.0000 \mathrm{E}+00$ & $0.0000 E+00$ & $0.0000 \mathrm{E}+00$ & $0.0000 \mathrm{E}+0.0$ & $0.0000 \mathrm{E}+00$ & $0.0000 \mathrm{E}+00$ & $0.0000 \mathrm{E}+00$ & $0.0000 \mathrm{E}+00$ \\
\hline 4 & 34 & $0.0000 \mathrm{E}+00$ & $0.0000 E+00$ & $0.0000 E+00$ & $0.0000 \mathrm{E}+00$ & $0.0000 E+00$ & $0.0000 \mathrm{E}+00$ & $0.0000 E+00$ & $0.0000 \mathrm{E}+00$ \\
\hline 5 & 32 & $0.0000 \mathrm{E}+00$ & $0.0000 \mathrm{E}+00$ & $0.0000 E+00$ & $0.0000 \mathrm{E}+00$ & $0.0000 \mathrm{E}+00$ & $0.0000 \mathrm{E}+00$ & $0.0000 \mathrm{E}+00$ & $0.0000 \mathrm{E}+00$ \\
\hline 6 & 33 & $0.0000 \mathrm{E}+00$ & $0.0000 \mathrm{E}+00$ & $0.0000 E+00$ & $0.0000 E+00$ & $0.0000 E+00$ & $0.0000 \mathrm{E}+00$ & $0.0000 \mathrm{E}+00$ & $0.0000 E+00$ \\
\hline 7 & 35 & $0.0000 \mathrm{E}+00$ & $0.0000 \mathrm{E}+00$ & $0.0000 \mathrm{E}+00$ & $0.0000 E+00$ & $0.0000 \mathrm{E}+00$ & $0.0000 \mathrm{E}+00$ & $0.0000 E+00$ & $0.0000 E+00$ \\
\hline 8 & 36 & $0.0000 \mathrm{E}+00$ & $0.0000 \mathrm{E}+00$ & $0.0000 \mathrm{E}+00$ & $0.0000 \mathrm{E}+00$ & $0.0000 \mathrm{E}+00$ & $0.0000 \mathrm{E}+00$ & $0.0000 \mathrm{E}+00$ & $0.0000 \mathrm{E}+00$ \\
\hline 9 & 38 & $0.0000 \mathrm{E}+00$ & $0.0000 \mathrm{E}+00$ & $1.2447 \mathrm{E}-03$ & $0.0000 E+00$ & $0.0000 \mathrm{E}+00$ & $0.0000 E+00$ & $0.0000 E+00$ & $1.2447 \mathrm{E}-03$ \\
\hline 10 & 39 & $0.0000 E+00$ & $0.0000 \mathrm{E}+00$ & $2.0097 \mathrm{E}-04$ & $0.0000 \mathrm{E}+00$ & $0.0000 \mathrm{E}+00$ & $0.0000 \mathrm{E}+00$ & $0.0000 \mathrm{E}+00$ & $2.0097 \mathrm{E}-04$ \\
\hline 11 & 40 & $0.0000 \mathrm{E}+00$ & $0.0000 E+00$ & $0.0000 \mathrm{E}+00$ & $0.0000 E+00$ & $0.0000 \mathrm{E}+00$ & $0.0000 \mathrm{E}+00$ & $0.0000 E+00$ & $0.0000 \mathrm{E}+00$ \\
\hline 12 & 41 & $0.0000 \mathrm{E}+00$ & $0.0000 \mathrm{E}+00$ & $2.9100 \mathrm{E}-04$ & $0.0000 \mathrm{E}+00$ & $0.0000 \mathrm{E}+00$ & $0.0000 E+00$ & $0.0000 \mathrm{E}+00$ & $2.9100 \mathrm{E}-04$ \\
\hline 13 & 140 & $0.0000 E+00$ & $0.0000 \mathrm{E}+00$ & $0.0000 \mathrm{E}+00$ & $0.0000 E+00$ & $0 \quad 0.0000 \mathrm{E}+00$ & $0.0000 \mathrm{E}+00$ & $0.0000 \mathrm{E}+00$ & $0.0000 \mathrm{E}+00$ \\
\hline 14 & 42 & $0.0000 \mathrm{E}+00$ & $0.0000 \mathrm{E}+00$ & $0.0000 E+00$ & $0.0000 \mathrm{E}+00$ & $0.0000 \mathrm{E}+00$ & $0.0000 \mathrm{E}+00$ & $0.0000 E+00$ & $0.0000 \mathrm{E}+00$ \\
\hline 15 & 154 & $0.0000 E+00$ & $0.0000 E+00$ & $8.0354 E-03$ & $0.0000 E+00$ & $0.0000 \mathrm{E}+00$ & $0.0000 \mathrm{E}+00$ & $0.0000 \mathrm{E}+00$ & $8.0354 \mathrm{E}-03$ \\
\hline 16 & 149 & $0.0000 \mathrm{E}+00$ & $0.0000 \mathrm{E}+00$ & $0.0000 \mathrm{E}+00$ & $0.0000 E+00$ & $0.0000 \mathrm{E}+00$ & $0.0000 \mathrm{E}+00$ & $0.0000 \mathrm{E}+00$ & $0.0000 E+00$ \\
\hline 17 & 144 & $0.0000 E+00$ & $0.0000 \mathrm{E}+00$ & $-3.0848 \mathrm{E}-06$ & $0.0000 \mathrm{E}+00$ & $0.0000 \mathrm{E}+00$ & $0.0000 \mathrm{E}+00$ & $0.0000 \mathrm{E}+00$ & $-3.0848 \mathrm{E}-06$ \\
\hline 18 & 159 & $0.0000 \mathrm{E}+00$ & $0.0000 \mathrm{E}+00$ & $-2.5216 E-04$ & $0.0000 \mathrm{E}+00$ & $0.0000 \mathrm{E}+00$ & $0.0000 \mathrm{E}+00$ & $0.0000 \mathrm{E}+00$ & $-2.5216 \mathrm{E}-04$ \\
\hline 19 & 141 & $0.0000 \mathrm{E}+00$ & $0.0000 \mathrm{E}+\infty 0$ & $0.0000 \mathrm{E}+00$ & $0.0000 \mathrm{E}+00$ & $0.0000 \mathrm{E}+00$ & $0.0000 \mathrm{E}+00$ & $0.0000 \mathrm{E}+\infty 0$ & $0.0000 \mathrm{E}+00$ \\
\hline
\end{tabular}

$\begin{array}{lllllllll}\text { total } & 0.0000 \mathrm{E}+00 & 0.0000 \mathrm{E}+00 & 9.5169 \mathrm{E}-03 & 0.0000 \mathrm{E}+00 & 0.0000 \mathrm{E}+00 & 0.0000 \mathrm{E}+00 & 0.0000 \mathrm{E}+00 & 9.5169 \mathrm{E}-03\end{array}$ Ineutron weight balance in each cell -- physical events print table 130

cell $(n, x n)$ fission capture loss to loss to total $(n, x n)$ fission

I $30 \quad 0.0000 \mathrm{E}+00 \quad 0.0000 \mathrm{E}+00 \quad 0.0000 \mathrm{E}+00 \quad 0.0000 \mathrm{E}+00 \quad 0.0000 \mathrm{E}+00 \quad 0.0000 \mathrm{E}+00$

$\begin{array}{lllllllll}2 & 31 & 0.0000 \mathrm{E}+00 & 0.0000 \mathrm{E}+00 & 0.0000 \mathrm{E}+00 & 0.0000 \mathrm{E}+00 & 0.0000 \mathrm{E}+00 & 0.0000 \mathrm{E}+00\end{array}$

$\begin{array}{lllllllll}3 & 37 & 0.0000 \mathrm{E}+00 & 0.0000 \mathrm{E}+00 & 0.0000 \mathrm{E}+00 & 0.0000 \mathrm{E}+00 & 0.0000 \mathrm{E}+00 & 0.0000 \mathrm{E}+00\end{array}$

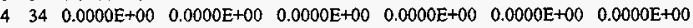
$\begin{array}{llllllll}32 & 0.0000 \mathrm{E}+00 & 0.0000 \mathrm{E}+00 & 0.0000 \mathrm{E}+00 & 0.0000 \mathrm{E}+00 & 0.0000 \mathrm{E}+00 & 0.0000 \mathrm{E}+00\end{array}$ $\begin{array}{lllllll}33 & 0.0000 \mathrm{E}+00 & 0.0000 \mathrm{E}+00 & 0.0000 \mathrm{E}+00 & 0.0000 \mathrm{E}+00 & 0.0000 \mathrm{E}+00 & 0.0000 \mathrm{E}+00\end{array}$ $\begin{array}{lllllllll}735 & 0.0000 \mathrm{E}+00 & 0.0000 \mathrm{E}+00 & 0.0000 \mathrm{E}+00 & 0.0000 \mathrm{E}+00 & 0.0000 \mathrm{E}+00 & 0.0000 \mathrm{E}+00\end{array}$

$\begin{array}{lllllllll}8 & 36 & 0.0000 \mathrm{E}+00 & 0.0000 \mathrm{E}+00 & 0.0000 \mathrm{E}+00 & 0.0000 \mathrm{E}+00 & 0.0000 \mathrm{E}+00 & 0.0000 \mathrm{E}+00\end{array}$

$938 \quad 0.0000 \mathrm{E}+00 \quad 0.0000 \mathrm{E}+00-1.5855 \mathrm{E}-01 \quad 0.0000 \mathrm{E}+00 \quad 0.0000 \mathrm{E}+00-1.5855 \mathrm{E}-01$

$10 \quad 39 \quad 0.0000 \mathrm{E}+00 \quad 0.0000 \mathrm{E}+00 \quad-8.2847 \mathrm{E}-04 \quad 0.0000 \mathrm{E}+00 \quad 0.0000 \mathrm{E}+00+8.2847 \mathrm{E}-04$

$11 \quad 40 \quad 0.0000 \mathrm{E}+00 \quad 0.0000 \mathrm{E}+00 \quad-3.9460 \mathrm{E}-06 \quad 0.0000 \mathrm{E}+00 \quad 0.0000 \mathrm{E}+00 \quad-3.9460 \mathrm{E}-06$

$\begin{array}{lllllllll}12 & 41 & 0.0000 \mathrm{E}+00 & 0.0000 \mathrm{E}+00 & -3.1114 \mathrm{E}-03 & 0.0000 \mathrm{E}+00 & 0.0000 \mathrm{E}+00 & -3.1114 \mathrm{E}-03\end{array}$

$\begin{array}{lllllllll}13 & 140 & 0.0000 \mathrm{E}+00 & 0.0000 \mathrm{E}+00 & 0.0000 \mathrm{E}+00 & 0.0000 \mathrm{E}+00 & 0.0000 \mathrm{E}+00 & 0.0000 \mathrm{E}+00\end{array}$

$\begin{array}{llllllll}14 & 42 & 1.2461 \mathrm{E}-03 & 0.0000 \mathrm{E}+00 & -3.1955 \mathrm{E}-05 & -6.2306 \mathrm{E}-04 & 0.0000 \mathrm{E}+00 & 5.9111 \mathrm{E}-04\end{array}$

$\begin{array}{lllllllll}15 & 154 & 4.9179 \mathrm{E}-03 & 0.0000 \mathrm{E}+00 & -1.7239 \mathrm{E}-01 & -2.4589 \mathrm{E}-03 & -4.5089 \mathrm{E}-01 & -6.2082 \mathrm{E}-01\end{array}$

$16 \quad 149 \quad 0.0000 \mathrm{E}+00 \quad 0.0000 \mathrm{E}+00 \quad-3.5884 \mathrm{E}-05 \quad 0.0000 \mathrm{E}+00 \quad 0.0000 \mathrm{E}+00 \quad-3.5884 \mathrm{E}-05$

$17 \quad 144 \quad 0.0000 \mathrm{E}+00 \quad 0.0000 \mathrm{E}+00-1.4569 \mathrm{E}-02 \quad 0.0000 \mathrm{E}+00 \quad 0.0000 \mathrm{E}+00-1.4569 \mathrm{E}-02$

$\begin{array}{llllllll}18 & 159 & 0.0000 \mathrm{E}+00 & 0.0000 \mathrm{E}+00 & -4.9815 \mathrm{E}-02 & 0.0000 \mathrm{E}+00 & 0.0000 \mathrm{E}+00 & -4.9815 \mathrm{E}-02\end{array}$

$19 \quad 141 \quad 0.0000 \mathrm{E}+00 \quad 0.0000 \mathrm{E}+00 \quad-4.5860 \mathrm{E}-04 \quad 0.0000 \mathrm{E}+00 \quad 0.0000 \mathrm{E}+00 \quad-4.5860 \mathrm{E}-04$

total $\quad 6.1640 \mathrm{E}-03 \quad 0.0000 \mathrm{E}+00 \quad-3.9980 \mathrm{E}-01 \quad-3.0820 \mathrm{E}-03 \quad-4.5089 \mathrm{E}-01 \quad-8.4760 \mathrm{E}-01$

ineutron activity of each nuclide in each cell, per source particle

print table 140

cell nuclides atom total collisions weight lost weight loss weight gain fraction collisions * weight to capture to fission by $(n, x n)$

$938 \quad 5010.03 \mathrm{~d} \quad 7.1977 \mathrm{E}-01$ $5011.40 \mathrm{c} \quad 8.0041 \mathrm{E}-02$ $6012.40 \mathrm{c} \quad 2.0019 \mathrm{E}-01$

$10 \quad 39 \quad 41093.40 c \quad 9.8982 \mathrm{E}-01$ $40000.40 \mathrm{c} \quad 1.0183 \mathrm{E}-02$

$11 \quad 40 \quad 1001.00 \mathrm{c} \quad 3.3333 \mathrm{E}-01$ $1002.55 \mathrm{c} \quad 3.3333 \mathrm{E}-01$ $8016.40 \mathrm{c} \quad 3.3333 \mathrm{E}-01$

$41 \quad 41093.40 \mathrm{c} \quad 9.8982 \mathrm{E}-01$
$941 \quad 4.4212 \mathrm{E}-01 \quad 1.5853 \mathrm{E}-01 \quad 0.0000 \mathrm{E}+00 \quad 0.0000 \mathrm{E}+00$ $\begin{array}{lllll}76 & 3.6073 \mathrm{E}-02 & 1.3591 \mathrm{E}-07 & 0.0000 \mathrm{E}+00 & 0.0000 \mathrm{E}+00\end{array}$ $232 \quad 1.1297 \mathrm{E}-01 \quad 1.8446 \mathrm{E}-05 \quad 0.0000 \mathrm{E}+00 \quad 0.0000 \mathrm{E}+00$

$128 \quad 6.2802 \mathrm{E}-02 \quad 8.2814 \mathrm{E}-04 \quad 0.0000 \mathrm{E}+00 \quad 0.0000 \mathrm{E}+00$ $\begin{array}{lllll}2 & 9.7676 \mathrm{E}-04 & 3.3160 \mathrm{E}-07 & 0.0000 \mathrm{E}+00 \quad 0.0000 \mathrm{E}+00\end{array}$

$111 \quad 5.1533 \mathrm{E}-02 \quad 3.9251 \mathrm{E}-06 \quad 0.0000 \mathrm{E}+00 \quad 0.0000 \mathrm{E}+00$ $33 \quad 1.5373 \mathrm{E}-02 \quad 2.0884 \mathrm{E}-08 \quad 0.0000 \mathrm{E}+00 \quad 0.0000 \mathrm{E}+00$ $\begin{array}{lllll}40 & 2.1271 \mathrm{E}-02 & 0.0000 \mathrm{E}+00 & 0.0000 \mathrm{E}+00 & 0.0000 \mathrm{E}+00\end{array}$ 
15 $154 \quad 92235.40 \mathrm{c} \quad 3.7504 \mathrm{E}-01$ $92238.40 \mathrm{c} \quad 1.2499 \mathrm{E}-01$ $7014.40 \mathrm{c}$ 4.9997E-01

$16 \quad 149 \quad 1001.00 \mathrm{c} \quad 3.3333 \mathrm{E}-01$ $1002.55 \mathrm{C} \quad 3.3333 \mathrm{E}-0$ ! $8016.40 \mathrm{c}$ 3.3333E-01

\section{$17 \quad 144 \quad 74000.40 \mathrm{c} \quad 1.0000 \mathrm{E}+00$ \\ $\begin{array}{lll}18 & 159 & 41093.40 \mathrm{c} \quad 9.8982 \mathrm{E}-01\end{array}$ $40000.40 \mathrm{C} \quad 1.0183 \mathrm{E}-02$}

$19 \quad 141 \quad 1001.00 \mathrm{c} \quad 3.3333 \mathrm{E}-01$ $1002.55 \mathrm{c} \quad 3.3333 \mathrm{E}-01$ $8016.40 \mathrm{c} \quad 3.3333 \mathrm{E}-01$

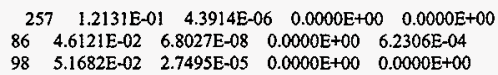

$4759 \quad 2.3227 \mathrm{E}+00 \quad 1.3244 \mathrm{E}-01 \quad 4.4247 \mathrm{E}-01 \quad 5.8410 \mathrm{E}-04$ $1470 \quad 7.3870 \mathrm{E}-01 \quad 2.3272 \mathrm{E}-02 \quad 8.4162 \mathrm{E}-03 \quad 1.8748 \mathrm{E}-03$ $\begin{array}{lllll}2207 & 1.0775 \mathrm{E}+00 & 1.6682 \mathrm{E}-02 & 0.0000 \mathrm{E}+00 & 0.0000 \mathrm{E}+00\end{array}$

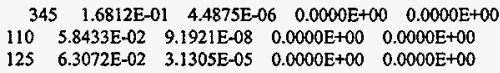

total $\quad 22764 \quad 1.1324 \mathrm{E}+01 \quad 3.9980 \mathrm{E}-01 \quad 4.5089 \mathrm{E}-01 \quad 3.0820 \mathrm{E}-03$

total over all cells for each nuclide total collisions weight lost weight loss weight gain collisions - weight to capture to fission by $(n, x n)$

$\begin{array}{ccccccc}1001.00 \mathrm{c} & 4742 & 2.2505 \mathrm{E}+00 & 9.2315 \mathrm{E}-05 & 0.0000 \mathrm{E}+00 & 0.0000 \mathrm{E}+00 \\ 1002.55 \mathrm{c} & 135 \mathrm{I} & 7.0199 \mathrm{E}-01 & 1.0814 \mathrm{E}-06 & 0.0000 \mathrm{E}+00 & 6.2306 \mathrm{E}-04 \\ 5011.40 \mathrm{c} & 76 & 3.6073 \mathrm{E}-02 & 1.3591 \mathrm{E}-07 & 0.0000 \mathrm{E}+00 & 0.0000 \mathrm{E}+00 \\ 6012.40 \mathrm{c} & 232 & 1.1297 \mathrm{E}-0 \mathrm{I} & 1.8446 \mathrm{E}-05 & 0.0000 \mathrm{E}+00 & 0.0000 \mathrm{E}+00 \\ 7014.40 \mathrm{c} & 2207 & 1.0775 \mathrm{E}+00 & 1.6682 \mathrm{E}-02 & 0.0000 \mathrm{E}+00 & 0.0000 \mathrm{E}+00 \\ 8016.40 \mathrm{c} & 1635 & 8.6733 \mathrm{E}-01 & 4.3699 \mathrm{E}-04 & 0.0000 \mathrm{E}+00 & 0.0000 \mathrm{E}+00 \\ 40000.40 \mathrm{c} & 60 & 2.7441 \mathrm{E}-02 & 5.0739 \mathrm{E}-05 & 0.0000 \mathrm{E}+00 & 0.0000 \mathrm{E}+00 \\ 41093.40 \mathrm{c} & 4406 & 2.3199 \mathrm{E}+00 & 5.3704 \mathrm{E}-02 & 0.0000 \mathrm{E}+00 & 0.0000 \mathrm{E}+00 \\ 74000.40 \mathrm{c} & 885 & 4.2652 \mathrm{E}-01 & 1.4569 \mathrm{E}-02 & 0.0000 \mathrm{E}+00 & 0.0000 \mathrm{E}+00 \\ 92235.40 \mathrm{c} & 4759 & 2.3227 \mathrm{E}+00 & 1.3244 \mathrm{E}-01 & 4.4247 \mathrm{E}-01 & 5.8410 \mathrm{E}-04 \\ 92238.40 \mathrm{c} & 1470 & 7.3870 \mathrm{E}-01 & 2.3272 \mathrm{E}-02 & 8.4162 \mathrm{E}-03 & 1.8748 \mathrm{E}-03 \\ 5010.03 \mathrm{~d} & 941 & 4.4212 \mathrm{E}-01 & 1.5853 \mathrm{E}-01 & 0.0000 \mathrm{E}+00 & 0.0000 \mathrm{E}+00\end{array}$

the initial fission neutron source distribution used the 8 source points that were input on the ksre card.

the criticality problem was scheduled to skip 5 cycles and run a total of 6 cycles with nominally 200 neutrons per cycle. this problem has run 5 inactive cycles with 1027 neutron histories and 1 active cycles with 222 neutron histories.

this calculation has completed the requested number of keff cycles using a total of 1249 fission neutron source histories. all cells with fissionable material were sampled and had fission neutron source points.

there is no combined collision/absorption/track-length estimate for keff because only 1 active cycles were run. lindividual and average keff estimator results by cycle

keff neutron keff estimators by cycle average keff estimators and deviations average $k(c / a / t)$ cycle histories $k$ (coll) $k$ (abs) $k$ (track) $k$ (coll) st dev $k(a b s)$ st dev $k$ (track) st dev $k(c / a / t)$ st dev fom

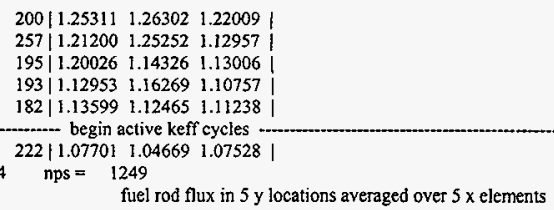


tally for neutrons

HNF-SD-SNF-CSWD-005, Rev. 0

ber of histories used for normalizing tallies $=200.00$

cell $a$ is $\left(154<42[-10-10] 42[-9-10] 42[-8-10] 42[-7-10] 42\left[\begin{array}{lll}-6 & -1 & 0\end{array}\right]\right)$

cell $b$ is $(154<42[-1030] 42[-930] 42[-830] 42[-730] 42[-630])$

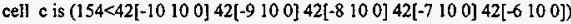

cell $d$ is $(154<42[-10210] 42[-9210] 42[-8210] 42[-7210] 42[-6210])$

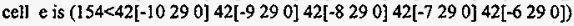

volumes

cell: $\quad$ a $\quad b \quad c \quad d \quad$ e

$1.04709 \mathrm{E}+02 \quad 1.04709 \mathrm{E}+02 \quad 1.04709 \mathrm{E}+02 \quad 1.04709 \mathrm{E}+02 \quad 1.04709 \mathrm{E}+02$

energy: $\quad 1.0000 \mathrm{E}-02 \quad 1.0000 \mathrm{E}-01 \quad 1.0000 \mathrm{E}+00 \quad 1.0000 \mathrm{E}+01 \quad$ total

cell

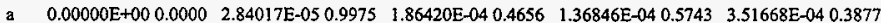

b $\quad 1.07442 \mathrm{E}-05 \quad 0.9975 \quad 1.58576 \mathrm{E}-04 \quad 0.5120 \quad 2.32928 \mathrm{E}-04 \quad 0.3945 \quad 4.44462 \mathrm{E}-05 \quad 0.5872 \quad 4.46694 \mathrm{E}-04 \quad 0.2855$

c $\quad 2.16778 \mathrm{E}-050.7232 \quad 9.40943 \mathrm{E}-050.5519 \quad 5.37835 \mathrm{E}-050.5876 \quad 1.51034 \mathrm{E}-04 \quad 0.7075 \quad 3.20589 \mathrm{E}-040.3937$

d $\quad 1.47903 \mathrm{E}-050.5831 \quad 0.00000 \mathrm{E}+000.0000 \quad 1.15515 \mathrm{E}-040.5838 \quad 1.69653 \mathrm{E}-040.5071 \quad 2.99958 \mathrm{E}-040.3718$

e $\quad 8.04943 \mathrm{E}-060.9975 \quad 0.00000 \mathrm{E}+000.0000 \quad 0.00000 \mathrm{E}+00 \quad 0.0000 \quad 6.81909 \mathrm{E}-05 \quad 0.7477 \quad 7.62403 \mathrm{E}-05 \quad 0.6763$ lanalysis of the results in the tally fluctuation chat bin (tfc) for tally 4 with nps $=1249$ print table 160

normed average tally per history $=3.51668 \mathrm{E}-04$

estimated tally relative error $=0.3877$

relative errot from zero tallies $=0.3464$

number of nonzero history tallies $=\quad 8$

history number of largest tally $=\quad 1034$

(largest tally $) /($ average tally $)=3.84476 \mathrm{E}+0 \mathrm{l}$

(confidence interval shift) $/$ mean $=0.0793$ unnormed average tally per history $=3.68228 \mathrm{E}-02$

estimated variance of the variance $=0.1752$

relative error from nonzero scores $\approx 0.1740$

efficiency for the nonzero tallies $=0.0400$

largest unnormalized history tally $=1.41575 \mathrm{E}+00$

(largest tally)/(avg nonzero tally) $=1.53791 \mathrm{E}+00$

shifted confidence interval center $=3.79539 \mathrm{E}-04$

he largest history score sampled so far were to occur on the very next history, the tfe bin quantities would change as follows: $s=222$ for this table because 5 keff cycles and 1027 histories were skipped before tally accumulation.

\begin{tabular}{lccc} 
estimated quantities & \multicolumn{1}{c}{ value at nps } & value at nps+1 & value(nps+1)/value \\
& & & \\
mean & $3.51668 \mathrm{E}-04$ & $4.10723 \mathrm{E}-04$ & 0.167927 \\
relative error & $3.87653 \mathrm{E}-01$ & $3.61597 \mathrm{E}-01$ & -0.067213 \\
variance of the variance & $1.75161 \mathrm{E}-01$ & $1.47665 \mathrm{E}-01$ & -0.156976 \\
shifted center & $3.79539 \mathrm{E}-04$ & $3.77169 \mathrm{E}-04$ & -0.006244 \\
figure of merit & $0.00000 \mathrm{E}+00$ & $0.00000 \mathrm{E}+00$ & 0.000000
\end{tabular}

there is not enough information in the largest history scores (usually less than 500 scores) for a reliable estimate of the slope. the history score probability density function appears to have an unsampled region at the largest history scores: please examine.

***** the nps-dependent $t \mathrm{fc}$ bin check results ate suspect because there are only 1 nps tally values to analyze *****

results of 10 statistical checks for the estimated answer for the tally fluctuation chart (tfe) bin of tally 4

tfe bin -mean-- -.--melative error--variance of the variance- - - figure of merit-- -pof-

behavior behavior value decrease decrease rate value decrease decrease rate value behavior slope

desired random $<0.10$ yes $1 /$ sqrt(nps) $<0.10$ yes $1 / n p s$ constant random $>3.00$

$\begin{array}{llllll}\text { observed randorn } 0.39 & \text { yes } & \text { yes } & 0.18 \text { yes yes } & \text { yes } & 0.00\end{array}$

passed? yes no yes yes no yes yes no 


\section{HNF-SD-SNF-CSWD-005, Rev. 0}

wning. the tally in the tally fluctuation chart bin did not pass 3 of the 10 statistical checks.

ormed tally density for tally $4 \quad$ nonzero tally mean $(m)=9.206 \mathrm{E}-01$ nps $=1249$ print table 161

abscissa ordinate log plot of tally probability density function in tally fluctuation chart bin( $d=$ decade,slope $=0.0$ )

tally number num den $\log$ den:d-

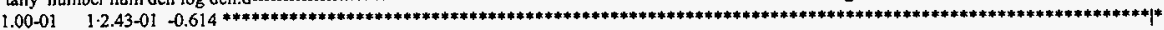

$1.26-01 \quad 00.00+00 \quad 0.000$

$1.58-01 \quad 00.00+00 \quad 0.000$

$2.00-01 \quad 00.00+00 \quad 0.000$

$2.51-01 \quad 0.0 .00+00 \quad 0.000$

$3.16-01 \quad 00.00+00 \quad 0.000$

$3.98-01 \quad 00.00+00 \quad 0.000$

$\begin{array}{lll}5.01-01 & 00.00+00 \quad 0.000\end{array}$

6.31-01 27.71-02-1.113

7.94-01 $0.0 .00+00 \quad 0.000$

$1.00+00 \quad 12.43-02-1.614 \mathrm{~m}$

$1.26+00 \quad 00.00+00 \quad 0.000$

$1.58+00 \quad 46.14-02 \cdot 1.212 * * * * * * * * * * * * * * * * * * * * * * * * * * * * * * * * * * * * * * * * * *$
0

total $84.00-02$

cumulative tally number for tally $4 \quad$ nonzero tally mean $(m)=9.206 \mathrm{E}-01 \quad \mathrm{nps}=1249$ print table 162

abscissa cum ordinate plot of the cumulative number of tallies in the tally fluctuation chart bin from 0 to 100 percent

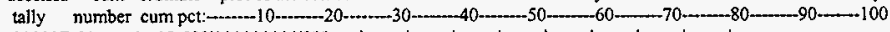

$1.00000 \mathrm{E}-01$ i $\left.12.500\right|^{* * * * * * * * * * * * *}$

$1.25893 \mathrm{E}-01 ;\left.12.500\right|^{* * * * * * * * * \mid * * *}$

$1.58489 \mathrm{E}-01 \quad 1 \quad 12.500|* * * * * * * *| * * *$

$1.99526 \mathrm{E}-01 ; 12.500|* * * * * * * *| * * *$

2.51189E-01

$3.16228 \mathrm{E}-01$

$3.98108 \mathrm{E}-01$

11 188E-01

$958 \mathrm{E}-01$

$12.500|* * * * * * * *| * * *$

$\left.12.500\right|^{* * * * * * * * * \mid * * *}$

1 $12.500 ; * * * * * * * * \mid * * *$

$\left.12.500\right|^{* * * * * * * * * \mid * * *}$

3 37.500| $\left.\right|^{* * * * * * * * * * ; * * * * * * * * *|* * * * * * * * *|^{* * * * * * * *} \mid}$

$\left.33.500\right|^{* * * * * * * * * * * * * * * * * * *|* * * * * * * * *| * * * * * * * \mid}$

$\begin{array}{llll}1 & 1 & 1 \\ 1 & 1 & 1 \\ 1 & 1 & 1 \\ 1 & 1 & 1 \\ 1 & 1 & 1 & 1 \\ 1 & & 1 & 1 \\ 1 & 1 & 1\end{array}$

$1.00000 \mathrm{E}+00$

$1.25893 \mathrm{E}+00$

$1.58489 \mathrm{E}+00$

total

$450.000 \mid \mathrm{mmmmmmmm}$ |mmmmmmmm|mmmmmmmm/mmmmmmmm| mmmmmmmmm

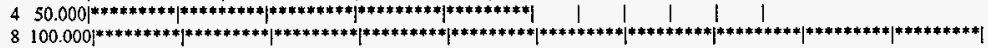
8 100.000:-10

cumulative unnormed tally for tally $4 \quad$ nonzero taliy mean $(m)=9.206 \mathrm{E}-01$ nps $=1249$ print table 162

abscissa cum ordinate plot of the cumulative tally in the tally fluctuation chart bin from 0 to 100 percent

tally tally/nps cum pet:-

$1.000 \mathrm{E}-01 \quad 4.632 \mathrm{E}-\left.04 \quad 1.258\right|^{*}$

$1.259 \mathrm{E}-01 \quad 4.632 \mathrm{E}-\left.04 \quad 1.258\right|^{*}$

$1.585 \mathrm{E}-01 \quad 4.632 \mathrm{E}-\left.0.4 \quad 1.258\right|^{*}$

$1.995 \mathrm{E}-01 \quad 4.632 \mathrm{E}-\left.04 \quad 1.258\right|^{*}$

2.512E-01 $4.632 \mathrm{E}-04 \quad 1.258]^{*}$

$3.162 \mathrm{E}-01 \quad 4.632 \mathrm{E}-\left.04 \quad 1.258\right|^{*}$

$3.981 \mathrm{E}-01 \quad 4.632 \mathrm{E}-\left.04 \quad 1.258\right|^{*}$

$5.012 \mathrm{E}-01 \quad 4.632 \mathrm{E}-\left.0.4 \quad 1.258\right|^{*}$

$6.310 \mathrm{E}-01 \quad 6.195 \mathrm{E}-03 \quad 16.825 \mathrm{j}^{* *}$

7.943E-01 $6.195 \mathrm{E}-\left.03 \quad 16.825\right|^{* * * 4}$

$10+20-30-40-50-60-70-30-90-90-100$

$1.000 \mathrm{E}+00 \quad 1.017 \mathrm{E}-02 \quad 27.628 \mid \mathrm{mmmmmmmmm} / \mathrm{mmmmmmmm} / \mathrm{mmmmmmmm}$ |

$1.259 \mathrm{E}+00$ 1.017E-02 $\left.27.628\right|^{* * * * * * * * *|* * * * * * * * *| * * * * * * * * \mid}$

$1.585 \mathrm{E}+003.682 \mathrm{E}-02 \quad 100.000 \mid * * 4$

total $3.68228 \mathrm{E}-02100.000$ :

$10-20$

$-30-40-50$

$\begin{array}{llll}1 & 1 & 1 \\ 1 & 1 & 1 \\ 1 & 1 & 1 \\ 1 & 1 & 1 \\ 1 & 1 & 1 \\ 1 & 1 & 1\end{array}$

1status of the statistical checks used to form confidence intervals for the mean for each tally bin

tally result of statistical checks for the tic bin (the first check not passed is listed) and error magnitude check for all bins

missed 3 of 10 tf $\mathrm{c}$ bin checks: the relative error exceeds the recommended value of 0.1 for nonpoint detector tallies

missed all bin error check: 25 tally bins had 4 bins with zeros and 21 bins with relative errors exceeding 0.10 
10 statistical checks are only for the tally fluctuation chart bin and do not apply to other tally bins.

tally bins with zeros may or may not be correct: compare the source, cutoffs, multipliers, et cetera with the tally bins.

waming. 1 of the 1 tally fluctuation chart bins did not pass all 10 statistical checks.

warning. I f the $I$ tallies had bins with relative errors greater than recommended.

itally fluctuation charts

$$
\text { tally } 4
$$

nps mean error vov slope fom

$10000.0000 \mathrm{E}+000.00000 .0000 \quad 0.0$

$12493.5167 \mathrm{E}-040.38770 .1752 \quad 0.0$

tally data written to file inp $18 \mathrm{~m}$

ascii file inp $18 \mathrm{p}$ written with 5 events from 5 histories

6 waming messages so far.

run terminated when 6 kcode cycles were done.

\section{File: Inp200}

1. prob20 - continuous energy electron version of problem prob19.

2- $\quad 1 \quad 1-19.3 \quad 1-23-45-6$

3. $20 \# 1$

4 -

5- $\quad 1 \quad 202$

$+$

6- $2 \mathrm{pz} .00635$

plane

7- 3 px -10

8- $4 \times 100$

$+$

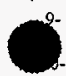

$5 y-103$

$6 p 010011000101$

plane

plane

II-

ml 74184. 1

sdef erg=l sus $=1$ vec $=001$ par $=3$

imp:p,e 10

mode $\mathrm{p} e$

16- *f1:p 12

17. $\quad$ e $0.1 .191 .282 .373 .464 .555 .645 .736 .827 .918 \quad 1.1$

18. *f11:e 12

19. 090001000100001

waming. non-orthogonality of surface transformation $9>2 . e-6$

20- $\mathrm{fq}$ ef

21- f21:e 12

22- *28:p 1

23- e28 $\quad-.00101 . e-6.1 .191 .282 .373 .464 \quad 555.645 .736 .827 .918 \quad 1.1$

24- es8 $\quad-.00101 . e-6.1 .191 .282 .373 .464 .555 .645 .736 .827 .918 \quad 1.1$

25- f58:e 1

26- f6:p 1

27- f31:e 12

28- ft3l elc !

29. f4l:e 12

30- ft41 elc 2

31- f51:e 12

32- $\mathrm{tt} 51$ elc 3

33- e8 $1000 \mathrm{nt}$

34 *f8:p,e 1

35- nps 10000

36- ctme 30

37- prdmp $2 \mathrm{j}-1$

cut:p $1 \mathrm{j} .1$

cut:e $1 \mathrm{j} .1$

print 11070 
ming. tr 9 card unused.

warning. I energy bins of tally 1 are below energy cutoff.

warning. tally 8 needs zero energy bin for negative fo scores.

lcells

print table 60

cell mat density density volume mass pieces importance importance

I I I $6.31828 \mathrm{E}-021.93000 \mathrm{E}+012.54000 \mathrm{E}+004.90220 \mathrm{E}+01 \quad 0 \quad 1.0000 \mathrm{E}+001.0000 \mathrm{E}+00$

$2200.00000 \mathrm{E}+000.00000 \mathrm{E}+000.00000 \mathrm{E}+000.00000 \mathrm{E}+00 \quad 0 \quad 0.0000 \mathrm{E}+000.0000 \mathrm{E}+00$

total

$2.54000 \mathrm{E}+004.90220 \mathrm{E}+01$

1surfaces

print table 70

surface trans type surface coefficients

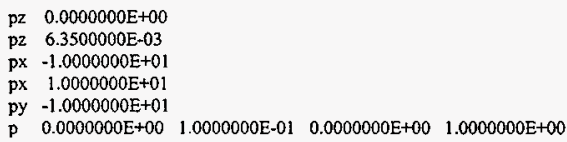

minimum source weight $=1.0000 \mathrm{E}+00$ maximum source weight $=1.0000 \mathrm{E}+00$

4 warning messages so far.

lcross-section tables

print table 100

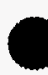

table length

tables from file testlib

$74000.02 p \quad 755$

$01 / 15 / 93$

total 755

maximum photon energy set to 100.0 mev (maximum electron energy)

tables from file testlib1

$74000.01 \mathrm{e} \quad 478$

$11 / 16 / 88$

decimal words of dynamically allocated storage

4 waming messages so far.

1 starting morun. field length $=0 \quad \mathrm{cp} 0=0.00 \quad$ print table 110

prob 20 - continuous energy electron version of problern probl9.

nps $x \quad y \quad z$ cell surf $u$ v $w$ energy weight time

$10.000 \mathrm{E}+00 \quad 0.000 \mathrm{E}+00 \quad 0.000 \mathrm{E}+00 \quad 1 \quad 1 \quad 3.096 \mathrm{E}-01-4.068 \mathrm{E}-01 \quad 8.595 \mathrm{E}-01 \quad 1.000 \mathrm{E}+00 \quad 1.000 \mathrm{E}+00 \quad 0.000 \mathrm{E}+00$

$20.000 \mathrm{E}+00 \quad 0.000 \mathrm{E}+00 \quad 0.000 \mathrm{E}+00 \quad 1 \quad 1 \quad 9.871 \mathrm{E}-01 \quad 1.062 \mathrm{E}-01 \quad 1.197 \mathrm{E}-01 \quad 1.000 \mathrm{E}+00 \quad 1.000 \mathrm{E}+00 \quad 0.000 \mathrm{E}+00$

$\begin{array}{lllllllllll}3 & 0.000 \mathrm{E}+00 & 0.000 \mathrm{E}+00 & 0.000 \mathrm{E}+00 & 1 & 1-1.347 \mathrm{E}-01 & 5.198 \mathrm{E}-01 & 8.436 \mathrm{E}-01 & 1.000 \mathrm{E}+00 & 1.000 \mathrm{E}+00 \quad 0.000 \mathrm{E}+00\end{array}$

$40.000 \mathrm{E}+00 \quad 0.000 \mathrm{E}+00 \quad 0.000 \mathrm{E}+00 \quad 1 \quad 1-6.548 \mathrm{E}-02 \quad 7.832 \mathrm{E}-01 \quad 6.184 \mathrm{E}-01 \quad 1.000 \mathrm{E}+00 \quad 1.000 \mathrm{E}+00 \quad 0.000 \mathrm{E}+00$

$\begin{array}{llllllllllll}5 & 0.000 \mathrm{E}+00 & 0.000 \mathrm{E}+00 & 0.000 \mathrm{E}+00 \quad 1 \quad+1.554 \mathrm{E}-01 & -9.204 \mathrm{E}-01 & 3.588 \mathrm{E}-01 & 1.000 \mathrm{E}+00 & 1.000 \mathrm{E}+00 \quad 0.000 \mathrm{E}+00\end{array}$

$6.000 \mathrm{E}+00 \quad 0.000 \mathrm{E}+00 \quad 0.000 \mathrm{E}+00 \quad 1 \quad 1 \quad 2.608 \mathrm{E}-01 \quad-6.984 \mathrm{E}-01 \quad 6.665 \mathrm{E}-01 \quad 1.000 \mathrm{E}+00 \quad 1.000 \mathrm{E}+00 \quad 0.000 \mathrm{E}+00$

$7 \quad 0.000 \mathrm{E}+00 \quad 0.000 \mathrm{E}+00 \quad 0.000 \mathrm{E}+00 \quad 1 \quad 1 \quad 6.931 \mathrm{E}-01-9.170 \mathrm{E}-02 \quad 7.149 \mathrm{E}-01 \quad 1.000 \mathrm{E}+00 \quad 1.000 \mathrm{E}+00 \quad 0.000 \mathrm{E}+00$ 


\section{File: Outp20}

prob 20 - continuous energy electron version of problem prob19.

2- 1 1 $1-19.31-23-45-6$

3- $20 \# 1$

5- 1202

$+$

6- 2 pz .00635

plane

7. $3 \mathrm{px}-10$

8. $4 \times 100$

9. 5 y -103

$+\quad$ plane

10. $6 \mathrm{p} 010011000101$

plane

11-

12- $\mathrm{ml} \mathrm{74184.1}$

13- sdef erg $=1$ sur $=1$ vec $=001$ par $=3$

14. imp:p,e 10

15- mode $\mathrm{p} \mathrm{e}$

16- *f1:p 12

17. $\quad \mathrm{e} 0 \quad .1 .191 \quad 282 \quad 373.464 .555 .645 .736 .827 .9181 .1$

18. *โ1 $1: 812$

19- 90001000100001

waming. non-orthogonality of surface transformation $9>2 . e-6$

20- fq ef

21- f21:e 12

22. *f28:p 1

23- e28 $\quad$-.001 $01 . e-6.1 .191 .282 .373 .464 .555 .645 .736 .827 .918 \quad 1.1$

24- es8 $\quad-.00101 . e-6.1 .191 .282 .373 .464 .555 .645 .736 .827 .9181 .1$

25. f58:e 1

26- f6:p 1

f3l:e 12

$\mathrm{ft} 3 \mathrm{l}$ elc 1

f4l:e 12

ft4l elc 2

151:e 12

fisl elc 3

e8 $1000 \mathrm{nt}$

*f8:p,e I

nps 10000

ctme 30

prdmp $2 \mathrm{j}-1$

cut:p $[\mathrm{j}$.I

cut:e $1 \mathbf{j} .1$

print 11070

41.

warning. to 9 card unused.

waming. I energy bins of tally l are below energy cutoff.

warning. tally 8 needs zero energy bin for negative $f 8$ scores.

Icelis

print table 60

atom gram
cell mat density density volume mass pieces importance importance

11 1 $6.31828 \mathrm{E}-021.93000 \mathrm{E}+012.54000 \mathrm{E}+004.90220 \mathrm{E}+01 \quad 01.0000 \mathrm{E}+001.0000 \mathrm{E}+00$

$220 \quad 0.00000 \mathrm{E}+000.00000 \mathrm{E}+00 \quad 0.00000 \mathrm{E}+000.00000 \mathrm{E}+00 \quad 0 \quad 0.0000 \mathrm{E}+000.0000 \mathrm{E}+00$

total

Isurfaces

$2.54000 \mathrm{E}+004.90220 \mathrm{E}+01$

print table 70

surface trans type surface coefficients 


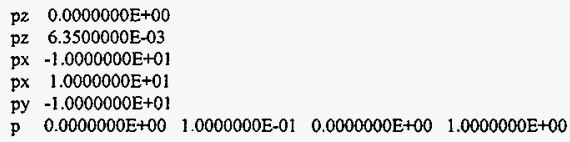

minimum source weight $=1.0000 \mathrm{E}+00$ maximum source weight $=1.0000 \mathrm{E}+00$

4 waming messages so far.

Icross-section tables

print table 100

table length

tables from file testlibl

$74000.02 p \quad 755$

$01 / 15 / 93$

total 755

maximum photon energy set to $100.0 \mathrm{mev}$ (maximum electron energy)

tables from file testlib]

74000.01 e 478

$11 / 16 / 88$

decimal words of dynamicalty allocated storage

4 warning messages so far.

tarting morun. field length $=0 \quad \mathrm{cp} 0=0.00 \quad$ print table 110

prob20 - continuous energy electron version of problem prob19.

nps $x \quad y \quad z \quad$ cell surf $u \quad v \quad w$ energy weight time

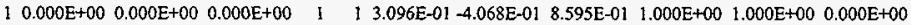
$20.000 \mathrm{E}+00 \quad 0.000 \mathrm{E}+00 \quad 0.000 \mathrm{E}+00 \quad 1 \quad 19.871 \mathrm{E}-01 \quad 1.062 \mathrm{E}-01 \quad 1.197 \mathrm{E}-01 \quad 1.000 \mathrm{E}+00 \quad 1.000 \mathrm{E}+00 \quad 0.000 \mathrm{E}+00$

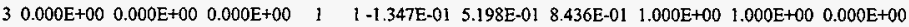

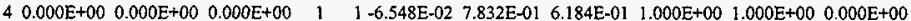
$50.000 \mathrm{E}+00 \quad 0.000 \mathrm{E}+00 \quad 0.000 \mathrm{E}+00 \quad 1 \quad$, $1.554 \mathrm{E}-01-9.204 \mathrm{E}-013.588 \mathrm{E}-01 \quad 1.000 \mathrm{E}+00 \quad 1.000 \mathrm{E}+00 \quad 0.000 \mathrm{E}+00$ $60.000 \mathrm{E}+00 \quad 0.000 \mathrm{E}+00 \quad 0.000 \mathrm{E}+00 \quad 1 \quad 12.608 \mathrm{E}-01-6.984 \mathrm{E}-01 \quad 6.665 \mathrm{E}-01 \quad 1.000 \mathrm{E}+00 \quad 1.000 \mathrm{E}+00 \quad 0.000 \mathrm{E}+00$ $\begin{array}{llllllllll}7 & 0.000 \mathrm{E}+00 & 0.000 \mathrm{E}+00 & 0.000 \mathrm{E}+00 \quad 1 \quad 1 & 6.931 \mathrm{E}-01 & -9.170 \mathrm{E}-02 & 7.149 \mathrm{E}-01 & 1.000 \mathrm{E}+00 & 1.000 \mathrm{E}+00 & 0.000 \mathrm{E}+00\end{array}$

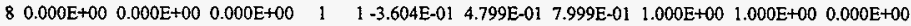
$9 \quad 0.000 \mathrm{E}+00 \quad 0.000 \mathrm{E}+00 \quad 0.000 \mathrm{E}+00 \quad 1 \quad 1-2.241 \mathrm{E}-01 \quad 3.960 \mathrm{E}-01 \quad 8.905 \mathrm{E}-01 \quad 1.000 \mathrm{E}+00 \quad 1.000 \mathrm{E}+00 \quad 0.000 \mathrm{E}+00$ $10 \quad 0.000 \mathrm{E}+00 \quad 0.000 \mathrm{E}+00 \quad 0.000 \mathrm{E}+00 \quad 1 \quad 1-2.928 \mathrm{E}-01-7.352 \mathrm{E}-01 \quad 6.114 \mathrm{E}-01 \quad 1.000 \mathrm{E}+00 \quad 1.000 \mathrm{E}+00 \quad 0.000 \mathrm{E}+00$ $110.000 \mathrm{E}+00 \quad 0.000 \mathrm{E}+00 \quad 0.000 \mathrm{E}+00 \quad 1 \quad 1 \quad 8.260 \mathrm{E}-01 \quad 1.184 \mathrm{E}-01 \quad 5.510 \mathrm{E}-01 \quad 1.000 \mathrm{E}+00 \quad 1.000 \mathrm{E}+00 \quad 0.000 \mathrm{E}+00$ $120.000 \mathrm{E}+00 \quad 0.000 \mathrm{E}+00 \quad 0.000 \mathrm{E}+00 \quad 1 \quad 1 \quad 7.540 \mathrm{E}-01-1.976 \mathrm{E}-01 \quad 6.264 \mathrm{E}-01 \quad 1.000 \mathrm{E}+00 \quad 1.000 \mathrm{E}+00 \quad 0.000 \mathrm{E}+00$ $130.000 \mathrm{E}+00 \quad 0.000 \mathrm{E}+00 \quad 0.000 \mathrm{E}+00 \quad 1 \quad 1 \quad 8.140 \mathrm{E}-01-3.427 \mathrm{E}-01 \quad 4.690 \mathrm{E}-01 \quad 1.000 \mathrm{E}+00 \quad 1.000 \mathrm{E}+00 \quad 0.000 \mathrm{E}+00$ $14 \quad 0.000 \mathrm{E}+00 \quad 0.000 \mathrm{E}+00 \quad 0.000 \mathrm{E}+00 \quad 1 \quad 1-1.257 \mathrm{E}-01 \quad 8.038 \mathrm{E}-01 \quad 5.814 \mathrm{E}-01 \quad 1.000 \mathrm{E}+00 \quad 1.000 \mathrm{E}+00 \quad 0.000 \mathrm{E}+00$ $150.000 \mathrm{E}+00 \quad 0.000 \mathrm{E}+00 \quad 0.000 \mathrm{E}+00 \quad 1 \quad 1-1.464 \mathrm{E}-01-1.070 \mathrm{E}-01 \quad 9.834 \mathrm{E}-01 \quad 1.000 \mathrm{E}+00 \quad 1.000 \mathrm{E}+00 \quad 0.000 \mathrm{E}+00$ $16 \quad 0.000 \mathrm{E}+00 \quad 0.000 \mathrm{E}+00 \quad 0.000 \mathrm{E}+00 \quad 1 \quad 1 \quad 1.310 \mathrm{E}-02-1.617 \mathrm{E}-02 \quad 9.998 \mathrm{E}-01 \quad 1.000 \mathrm{E}+00 \quad 1.000 \mathrm{E}+00 \quad 0.000 \mathrm{E}+00$

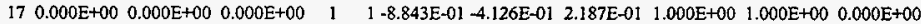
$\begin{array}{lllllllllllll}18 & 0.000 \mathrm{E}+00 & 0.000 \mathrm{E}+00 & 0.000 \mathrm{E}+00 & 1 & 1 & 4.260 \mathrm{E}-01 & 4.153 \mathrm{E}-01 & 8.038 \mathrm{E}-01 & 1.000 \mathrm{E}+00 & 1.000 \mathrm{E}+00 & 0.000 \mathrm{E}+00\end{array}$ $19 \quad 0.000 \mathrm{E}+00 \quad 0.000 \mathrm{E}+00 \quad 0.000 \mathrm{E}+00 \quad 1 \quad 1-7.311 \mathrm{E}-01+1.136 \mathrm{E}-02 \quad 6.822 \mathrm{E}-01 \quad 1.000 \mathrm{E}+00 \quad 1.000 \mathrm{E}+00 \quad 0.000 \mathrm{E}+00$ $\begin{array}{lllllllllll}20 & 0.000 \mathrm{E}+00 & 0.000 \mathrm{E}+00 & 0.000 \mathrm{E}+00 & 1 & 1-2.370 \mathrm{E}-01 & 3.207 \mathrm{E}-01 & 9.171 \mathrm{E}-01 & 1.000 \mathrm{E}+00 & 1.000 \mathrm{E}+00 & 0.000 \mathrm{E}+00\end{array}$

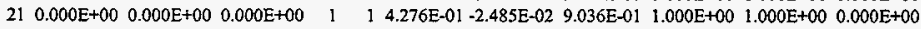
$22 \quad 0.000 \mathrm{E}+00 \quad 0.000 \mathrm{E}+00 \quad 0.000 \mathrm{E}+00 \quad 1 \quad 1 \quad 7.662 \mathrm{E}-01 \quad 7.908 \mathrm{E}-02 \quad 6.378 \mathrm{E}-01 \quad 1.000 \mathrm{E}+00 \quad 1.000 \mathrm{E}+00 \quad 0.000 \mathrm{E}+00$ $230.000 \mathrm{E}+00 \quad 0.000 \mathrm{E}+00 \quad 0.000 \mathrm{E}+00 \quad 1 \quad 1-4.247 \mathrm{E}-01 \quad 2.688 \mathrm{E}-01 \quad 8.645 \mathrm{E}-01 \quad 1.000 \mathrm{E}+00 \quad 1.000 \mathrm{E}+00 \quad 0.000 \mathrm{E}+00$ $24 \quad 0.000 \mathrm{E}+00 \quad 0.000 \mathrm{E}+00 \quad 0.000 \mathrm{E}+00 \quad 1 \quad 1 \quad 2.096 \mathrm{E}-01 \quad 5.730 \mathrm{E}-01 \quad 7.923 \mathrm{E}-01 \quad 1.000 \mathrm{E}+00 \quad 1.000 \mathrm{E}+00 \quad 0.000 \mathrm{E}+00$ $250.000 \mathrm{E}+00 \quad 0.000 \mathrm{E}+00 \quad 0.000 \mathrm{E}+00 \quad 1 \quad 1-2.665 \mathrm{E}-03-5.270 \mathrm{E}-01 \quad 8.499 \mathrm{E}-01 \quad 1.000 \mathrm{E}+00 \quad 1.000 \mathrm{E}+00 \quad 0.000 \mathrm{E}+00$ $60.000 \mathrm{E}+00 \quad 0.000 \mathrm{E}+00 \quad 0.000 \mathrm{E}+00 \quad$ I $\quad$ । $7.228 \mathrm{E}-01 \quad 4.233 \mathrm{E}-01 \quad 5.463 \mathrm{E}-01 \quad 1.000 \mathrm{E}+00 \quad 1.000 \mathrm{E}+00 \quad 0.000 \mathrm{E}+00$

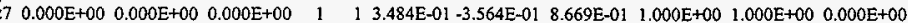




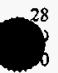
$0.000 \mathrm{E}+00 \quad 0.000 \mathrm{E}+00 \quad 0.000 \mathrm{E}+00$ $0.000 E+00 \quad 0.000 E+00 \quad 0.000 E+00$ $0.000 \mathrm{E}+00 \quad 0.000 \mathrm{E}+00 \quad 0.000 \mathrm{E}+00$ $310.000 \mathrm{E}+00 \quad 0.000 \mathrm{E}+00 \quad 0.000 \mathrm{E}+00$ $320.000 \mathrm{E}+00 \quad 0.000 \mathrm{E}+00 \quad 0.000 \mathrm{E}+00$ $33 \quad 0.000 \mathrm{E}+00 \quad 0.000 \mathrm{E}+00 \quad 0.000 \mathrm{E}+00$ $34 \quad 0.000 \mathrm{E}+00 \quad 0.000 \mathrm{E}+00 \quad 0.000 \mathrm{E}+00$ $350.000 \mathrm{E}+00 \quad 0.000 \mathrm{E}+00 \quad 0.000 \mathrm{E}+00$ $360.000 \mathrm{E}+00 \quad 0.000 \mathrm{E}+00 \quad 0.000 \mathrm{E}+00$ $37 \quad 0.000 \mathrm{E}+00 \quad 0.000 \mathrm{E}+00 \quad 0.000 \mathrm{E}+00$ $38 \quad 0.000 \mathrm{E}+00 \quad 0.000 \mathrm{E}+00 \quad 0.000 \mathrm{E}+00$ $390.000 \mathrm{E}+00 \quad 0.000 \mathrm{E}+00 \quad 0.000 \mathrm{E}+00$ $40 \quad 0.000 \mathrm{E}+00 \quad 0.000 \mathrm{E}+00 \quad 0.000 \mathrm{E}+00$ 41 $0.000 \mathrm{E}+00 \quad 0.000 \mathrm{E}+00 \quad 0.000 \mathrm{E}+00$ $42 \quad 0.000 E+00 \quad 0.000 \mathrm{E}+00 \quad 0.000 \mathrm{E}+00$ $430.000 \mathrm{E}+00 \quad 0.000 \mathrm{E}+00 \quad 0.000 \mathrm{E}+00$ $44 \quad 0.000 \mathrm{E}+00 \quad 0.000 \mathrm{E}+00 \quad 0.000 \mathrm{E}+00$ $45 \quad 0.000 \mathrm{E}+00 \quad 0.000 \mathrm{E}+00 \quad 0.000 \mathrm{E}+00$

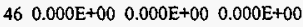
$47 \quad 0.000 \mathrm{E}+00 \quad 0.000 \mathrm{E}+00 \quad 0.000 \mathrm{E}+00$

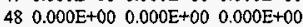
$490.000 \mathrm{E}+00 \quad 0.000 \mathrm{E}+00 \quad 0.000 \mathrm{E}+00$ so $0.000 \mathrm{E}+00 \quad 0.000 \mathrm{E}+00 \quad 0.000 \mathrm{E}+00$ Iproblem summary
$1 \quad 8.706 \mathrm{E}-01-3.198 \mathrm{E}-01 \quad 3.738 \mathrm{E}-01 \quad 1.000 \mathrm{E}+00 \quad 1.000 \mathrm{E}+00 \quad 0.000 \mathrm{E}+00$ $\begin{array}{lllllll}1 & -8.188 \mathrm{E}-01 & 2.059 \mathrm{E}-01 & 5.359 \mathrm{E}-01 & 1.000 \mathrm{E}+00 & 1.000 \mathrm{E}+00 & 0.000 \mathrm{E}+00\end{array}$ i $5.727 \mathrm{E}-01 \quad 8.124 \mathrm{E}-01 \quad 1.100 \mathrm{E}-01 \quad 1.000 \mathrm{E}+00 \quad 1.000 \mathrm{E}+00 \quad 0.000 \mathrm{E}+00$

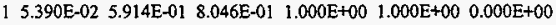

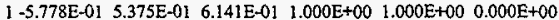
$1-7.198 \mathrm{E}-01 \quad-2.890 \mathrm{E}-01 \quad 6.31 \mathrm{IE}-01 \quad 1.000 \mathrm{E}+00 \quad 1.000 \mathrm{E}+00 \quad 0.000 \mathrm{E}+00$ $\begin{array}{lllllll}1 & 2.210 \mathrm{E}-01 & 2.482 \mathrm{E}-01 & 9.432 \mathrm{E}-01 & 1.000 \mathrm{E}+00 & 1.000 \mathrm{E}+00 & 0.000 \mathrm{E}+00\end{array}$ $14.339 \mathrm{E}-01$ 7.515E-02 $8.978 \mathrm{E}-01 \quad 1.000 \mathrm{E}+00 \quad 1.000 \mathrm{E}+00 \quad 0.000 \mathrm{E}+00$ $\begin{array}{lllllll}1-4263 \mathrm{E}-01 & 8.490 \mathrm{E}-01 & 3.123 \mathrm{E}-01 & 1.000 \mathrm{E}+00 & 1.000 \mathrm{E}+00 & 0.000 \mathrm{E}+00\end{array}$

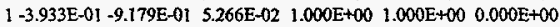

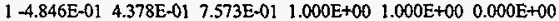

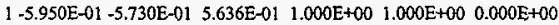
$1-7.252 \mathrm{E}-01+5.143 \mathrm{E}-01 \quad 4.578 \mathrm{E}-01 \quad 1.000 \mathrm{E}+00 \quad 1.000 \mathrm{E}+00 \quad 0.000 \mathrm{E}+00$ $\begin{array}{lllllll}1 & -7.156 \mathrm{E}-01 & 4.734 \mathrm{E}-01 & 5.136 \mathrm{E}-01 & 1.000 \mathrm{E}+00 & 1.000 \mathrm{E}+00 & 0.000 \mathrm{E}+00\end{array}$

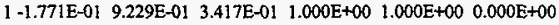

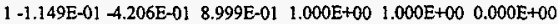

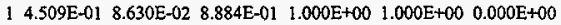
$\begin{array}{lllllll}1 & -7.358 \mathrm{E}-01 & 2.500 \mathrm{E}-01 & 6.294 \mathrm{E}-01 & 1.000 \mathrm{E}+00 & 1.000 \mathrm{E}+00 & 0.000 \mathrm{E}+00\end{array}$

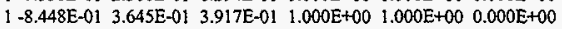
1 1 $1.958 \mathrm{E}-01-2.769 \mathrm{E}-01$ 9.407E-01 $1.000 \mathrm{E}+00 \quad 1.000 \mathrm{E}+00 \quad 0.000 \mathrm{E}+00$ $12.788 \mathrm{E}-01-7.325 \mathrm{E}-03 \quad 9.603 \mathrm{E}-01 \quad 1.000 \mathrm{E}+00 \quad 1.000 \mathrm{E}+00 \quad 0.000 \mathrm{E}+00$ $\begin{array}{llllll}1-3.201 \mathrm{E}-01 & 4.174 \mathrm{E}-01 & 8.505 \mathrm{E}-01 & 1.000 \mathrm{E}+00 & 1.000 \mathrm{E}+00 & 0.000 \mathrm{E}+00\end{array}$ 1 $8.960 \mathrm{E}-01-1.641 \mathrm{E}-01 \quad 4.126 \mathrm{E}-01 \quad 1.000 \mathrm{E}+00 \quad 1.000 \mathrm{E}+00 \quad 0.000 \mathrm{E}+00$ 0

run terminated when 10000 particle histories were done.

photon creation tracks weight energy photon loss tracks weight energy
(per source particle)

$\begin{array}{llllllll}\text { source } & 0 & 0 . & 0 . & \begin{array}{c}\text { escape } \\ \text { energy cutoff }\end{array} & 0 & 0.7500 \mathrm{E}-02 & 1.5476 \mathrm{E}-02 \\ & & & & 0 . & 0.75\end{array}$

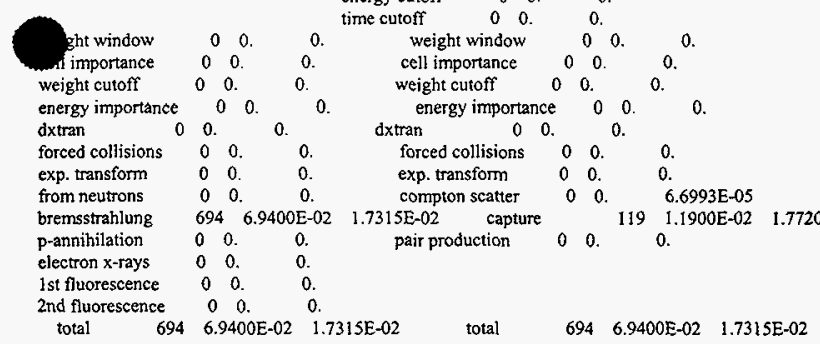

number of photons banked

694 average lifetime, shakes cutoffs photon tracks per source particle 6.9400E-02 escape $6.7786 \mathrm{E}-05$ tco $1.0000 \mathrm{E}+34$ photon collisions per source particle $1.3000 \mathrm{E}-02$ capture $8.3325 \mathrm{E}-05$ eco $1.0000 \mathrm{E}-01$ total photon collisions 130 capture or escape $7.0450 \mathrm{E}-05$ wcl $0.0000 \mathrm{E}+00$

0 any termination $7.0450 \mathrm{E}-05 \quad$ wc2 $0.0000 \mathrm{E}+00$ electron creation tracks weight energy electron loss tracks weight energy (per source particle) (per source particle)

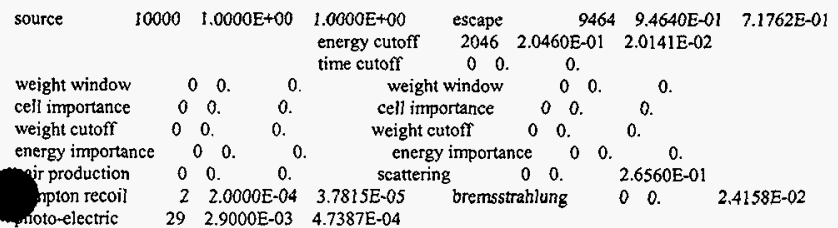




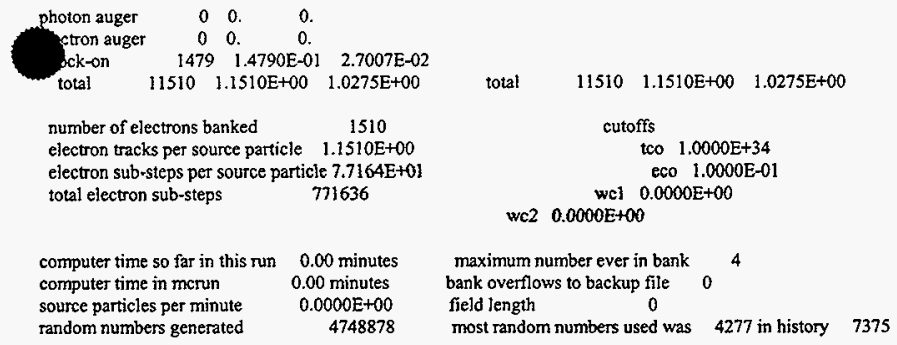

range of sampled source weights $=1.0000 \mathrm{E}+00$ to $1.0000 \mathrm{E}+00$

lphoton activity in each cell

print table 126

tracks population collisions collisions number flux average average

cell entering - weight weighted weighted track weight track mfp (per history) energy energy (relative) (cm)

$\begin{array}{llllllllll}1 & 1 & 0 & 694 & 130 & 1.3000 \mathrm{E}-02 & 2.8286 \mathrm{E}-01 & 2.8286 \mathrm{E}-01 & 1.0000 \mathrm{E}+00 & 1.6113 \mathrm{E}-01\end{array}$

total $\quad \begin{array}{llll}6 & 694 & 130 & 1.3000 \mathrm{E}-02\end{array}$

lelectron activity in each cell

print table 126

tracks population substeps substeps number flux average average

cell entering - weight weighted weighted track weight track mfp

(per history) energy energy (relative) (cm)

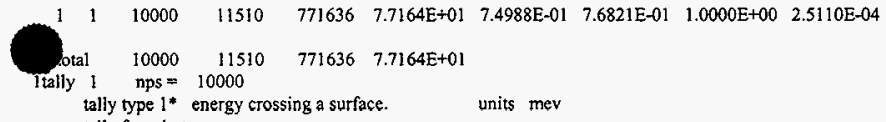

tally for photons

surface: $\quad 1 \quad 2$

energy

$1.0000 \mathrm{E}-01 \quad 0.00000 \mathrm{E}+000.0000 \quad 0.00000 \mathrm{E}+000.0000$

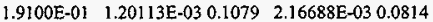

$2.8200 \mathrm{E}-01 \quad 8.17217 \mathrm{E}-040.1673 \quad 2.04350 \mathrm{E}-03 \quad 0.1062$

3.7300E-01 9.52191E-04 0.1829 $1.82060 \mathrm{E}-030.1357$

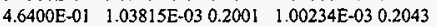

$\begin{array}{llll}5.5500 \mathrm{E}-01 & 5.66295 \mathrm{E}-04 & 0.3018 & 8.02842 \mathrm{E}-040.2503\end{array}$

6.4500E-01 6.09365E-04 0.3162 6.54966E-04 0.3016

7.3600 E-01 3.45345E-04 0.4472 4.20131E-04 0.4085

8.2700E-01 7.79347E-05 $0.9999 \quad 7.03770 \mathrm{E}-040.3334$

9.1800E-01 $\quad 1.70096 \mathrm{E}-040.7070 \quad 8.29418 \mathrm{E}-050.9999$

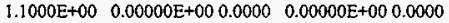

total $5.77773 \mathrm{E}-030.0811 \quad 9.69798 \mathrm{E}-03 \quad 0.0608$

1.ally 6 nps $=10000$

tally type 6 track length estimate of heating. units mev/gram

tally for photons

masses

$$
\text { cell: } \frac{1}{4.90220 \mathrm{E}+01}
$$

cell: 1

energy

$1.0000 \mathrm{E}-01 \quad 0.00000 \mathrm{E}+000.0000$

$1.9100 \mathrm{E}-01 \quad 1.80395 \mathrm{E}-050.0935$

$8200 \mathrm{E}-01 \quad 4.79830 \mathrm{E}-060.1242$

$.7300 \mathrm{E}-01 \quad 1.64870 \mathrm{E}-060.2061$ 
4.6400E-0I $\quad 1.76412 \mathrm{E}-060.4116$ $5500 \mathrm{E}-01 \quad 6.34259 \mathrm{E}-07 \quad 0.4736$ 4500E-01 2.47457E-07 0.3129 $7.3600 \mathrm{E}-01 \quad 2.58011 \mathrm{E}-070.6571$ 8.2700E-01 $1.16245 \mathrm{E}-07 \quad 0.3470$ $9.1800 \mathrm{E}-01 \quad 4.46610 \mathrm{E}-080.6403$ $1.1000 \mathrm{E}+00 \quad 0.00000 \mathrm{E}+0000000$ total $2.75512 \mathrm{E}-050.0722$

Itally 28 nps $=10000$

tally type 8 * energy deposition units mev tally for photons electrons

ceil: I

energy

$-1.0000 \mathrm{E}-03-8.72550 \mathrm{E}-040.1508$

$0.0000 \mathrm{E}+00 \quad 0.00000 \mathrm{E}+000.0000$

1.0000E-06 $0.00000 \mathrm{E}+000.0000$

$1.0000 \mathrm{E}-01 \quad 1.08193 \mathrm{E}-02 \quad 0.0227$

$1.9100 \mathrm{E}-01 \quad 3.91016 \mathrm{E}-02 \quad 0.0170$

$2.8200 \mathrm{E}-01 \quad 4.36100 \mathrm{E}-02 \quad 0.0210$

$3.7300 \mathrm{E}-01 \quad 3.53763 \mathrm{E}-020.0286$

$\begin{array}{lll}4.6400 \mathrm{E}-01 & 2.66881 \mathrm{E}-02 & 0.0382\end{array}$

$5.5500 \mathrm{E}-01 \quad 2.21833 \mathrm{E}-020.0468$

$6.4500 \mathrm{E}-0 \mathrm{I} \quad 1.58947 \mathrm{E}-02 \quad 0.0606$

$7.3600 \mathrm{E}-01 \quad 1.08330 \mathrm{E}-02 \quad 0.0790$

8.2700E-01 8.71177E-03 0.0940

$9.1800 \mathrm{E}-01 \quad 6.65495 \mathrm{E}-03 \quad 0.1136$

$1.1000 \mathrm{E}+00 \quad 4.79000 \mathrm{E}-020.0446$

total $2.66900 \mathrm{E}-010.0090$

jtally il nps $=10000$

tally type I* energy crossing a surface. units mev tally for electrons

\begin{tabular}{|c|c|c|}
\hline urface: & 1 & \\
\hline $1.0000 \mathrm{E}-01$ & $0.00000 \mathrm{E}+000.0000$ & $0.00000 \mathrm{E}+00 \quad 0.0000$ \\
\hline $1.9100 \mathrm{E}-01$ & $1.08698 \mathrm{E}-030.1176$ & $6.53601 \mathrm{E}-040.1495$ \\
\hline $2.8200 \mathrm{E}-01$ & $2.19643 \mathrm{E}-030.1039$ & $1.83325 \mathrm{E}-030.1165$ \\
\hline $3.7300 \mathrm{E}-01$ & $3.60063 \mathrm{E}-030.0956$ & 3.3723 IE-03 0.0988 \\
\hline $4.6400 \mathrm{E}-01$ & $6.94424 \mathrm{E}+030.0774$ & $6.18557 E-030.0823$ \\
\hline $5.5500 \mathrm{E}-01$ & $1.32283 \mathrm{E}-020.0615$ & $1.27247 \mathrm{E}-020.0628$ \\
\hline $6.4500 \mathrm{E}-01$ & $2.19441 \mathrm{E}-020.0516$ & $1.93787 \mathrm{E}-020.0550$ \\
\hline $7.3600 \mathrm{E}-01$ & $3.87821 \mathrm{E}-020.0411$ & $4.62743 \mathrm{E}-020.0374$ \\
\hline $8.2700 \mathrm{E}-0 \mathrm{t}$ & $6.96113 \mathrm{E}-020.0321$ & $9.53277 \mathrm{E}-020.0269$ \\
\hline $9.1800 \mathrm{E}-01$ & $1.17955 \mathrm{E}-010.0253$ & $9.53443 E-020.0284$ \\
\hline $1.1000 \mathrm{E}+00$ & $1.58764 \mathrm{E}-010.0225$ & $2.41655 \mathrm{E}-030.1959$ \\
\hline total $\quad 4.3$ & $4113 \mathrm{E}-010.0097 \quad 2.8$ & $3511 \mathrm{E}-010.0130$ \\
\hline ally $2 !$ & $\mathrm{nps}=10000$ & \\
\hline
\end{tabular}

tally type 1 number of particles crossing a surface. tally for electrons

surface: 12

energy

$1.0000 \mathrm{E}-01 \quad 0.00000 \mathrm{E}+00 \quad 0.0000 \quad 0.00000 \mathrm{E}+000.0000$

$1.9100 \mathrm{E}-01 \quad 7.70000 \mathrm{E}-03 \quad 0.1165 \quad 4.60000 \mathrm{E}-03 \quad 0.1471$

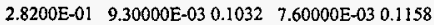

$3.7300 \mathrm{E}-01 \quad 1.09000 \mathrm{E}-02 \quad 0.0953 \quad 1.02000 \mathrm{E}-020.0985$

$\begin{array}{llll}4.6400 \mathrm{E}-01 & 1.65000 \mathrm{E}-02 & 0.0772 & \text { I.46000E-02 } 0.0822\end{array}$

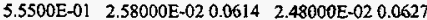

$6.4500 \mathrm{E}-01 \quad 3.63000 \mathrm{E}-020.0515 \quad 3.21000 \mathrm{E}-020.0549$

$\begin{array}{lllll}7.3600 \mathrm{E}-01 & 5.59000 \mathrm{E}-02 & 0.0411 & 6.67000 \mathrm{E}-02 & 0.0374\end{array}$

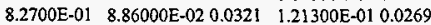

$9.1800 \mathrm{E}-01 \quad 1.34800 \mathrm{E}-01 \quad 0.0253 \quad 1.10600 \mathrm{E}-010.0284$

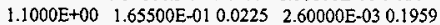

total $5.51300 \mathrm{E}-010.0094 \quad 3.95100 \mathrm{E}-010.0126$

תps $=10000$

tally type 1 number of particles crossing a surface 
tally for electrons

HNF-SD-SNF-CSWD-005, Rev. 0

this tally is modified by $\mathrm{ft}$ elc

surface: $\quad 1 \quad 2$

energy

$1.0000 \mathrm{E}-01 \quad 0.00000 \mathrm{E}+00 \quad 0.0000 \quad 0.00000 \mathrm{E}+00 \quad 0.0000$

$1.9100 \mathrm{E}-01-7.70000 \mathrm{E}-03 \quad 0.1165-4.60000 \mathrm{E}-030.1471$

$2.8200 \mathrm{E}-0$ ? $-9.30000 \mathrm{E}-03 \quad 0.1032-7.60000 \mathrm{E}-030.1158$

$3.7300 \mathrm{E}-01-1.09000 \mathrm{E}-020.0953-1.02000 \mathrm{E}-020.0985$

4.6400E-01 -1.65000E-02 $0.0772-1.46000 \mathrm{E}-020.0822$

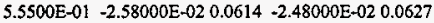

$6.4500 \mathrm{E}-01-3.63000 \mathrm{E}-020.0515-3.21000 \mathrm{E}-020.0549$

$\begin{array}{lllll}7.3600 \mathrm{E}-01 & -5.59000 \mathrm{E}-02 & 0.0411 & -6.67000 \mathrm{E}-02 & 0.0374\end{array}$

$8.2700 \mathrm{E}-01-8.86000 \mathrm{E}-020.0321-1.21300 \mathrm{E}-010.0269$

$9.1800 \mathrm{E}-01-1.34800 \mathrm{E}-0 \mathrm{I} 0.0253-1.10600 \mathrm{E}-010.0284$

$1.1000 \mathrm{E}+00-1.65500 \mathrm{E}-010.0225-2.60000 \mathrm{E}-030.1959$

total $-5.51300 \mathrm{E}-010.0094-3.95100 \mathrm{E}-010.0126$

Itally 41 nps $=10000$

tally type 1 number of particles crossing a surface.

tally for electrons

this tally is modified by $\mathrm{ft}$ elc

surface: $\quad 1$

energy

$1.0000 \mathrm{E}-01 \quad 0.00000 \mathrm{E}+000.0000 \quad 0.00000 \mathrm{E}+000.0000$

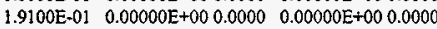

$2.8200 \mathrm{E}-01 \quad 0.00000 \mathrm{E}+00 \quad 0.0000 \quad 0.00000 \mathrm{E}+00 \quad 0.0000$

$3.7300 \mathrm{E}-01 \quad 0.00000 \mathrm{E}+00 \quad 0.0000 \quad 0.00000 \mathrm{E}+000.0000$

$4.6400 \mathrm{E}-01 \quad 0.00000 \mathrm{E}+00 \quad 0.0000 \quad 0.00000 \mathrm{E}+00 \quad 0.0000$

$5.5500 \mathrm{E}-01 \quad 0.00000 \mathrm{E}+00 \quad 0.0000 \quad 0.00000 \mathrm{E}+00 \quad 0.0000$

6.4500E-01 $\quad 0.00000 \mathrm{E}+00 \quad 0.0000 \quad 0.00000 \mathrm{E}+000.0000$

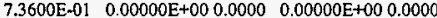

$8.2700 \mathrm{E}-01 \quad 0.00000 \mathrm{E}+00 \quad 0.0000 \quad 0.00000 \mathrm{E}+000.0000$

$1800 \mathrm{E}-01 \quad 0.00000 \mathrm{E}+00 \quad 0.0000 \quad 0.00000 \mathrm{E}+000.0000$

$1000 \mathrm{E}+00 \quad 0.00000 \mathrm{E}+00 \quad 0.0000 \quad 0.00000 \mathrm{E}+00 \quad 0.0000$ total $0.00000 E+000.0000 \quad 0.00000 E+000.0000$

surface: $\quad 1 \quad 2$

energy

$1.0000 \mathrm{E}-01 \quad 0.00000 \mathrm{E}+00 \quad 0.0000 \quad 0.00000 \mathrm{E}+00 \quad 0.0000$

$1.9100 \mathrm{E}-01 \quad 7.70000 \mathrm{E}-03 \quad 0.1165 \quad 4.60000 \mathrm{E}-03 \quad 0.1471$

$2.8200 \mathrm{E}-01 \quad 9.30000 \mathrm{E}-03 \quad 0.1032 \quad 7.60000 \mathrm{E}-03 \quad 0.1158$

$3.7300 E-01 \quad 1.09000 E-020.0953 \quad 1.02000$ E- $02 \quad 0.0985$

$4.6400 \mathrm{E}-01 \quad 1.65000 \mathrm{E}-02 \quad 0.0772 \quad 1.46000 \mathrm{E}-02 \quad 0.0822$

$5.5500 \mathrm{E}-01 \quad 2.58000 \mathrm{E}-02 \quad 0.0614 \quad 2.48000 \mathrm{E}-02 \quad 0.0627$

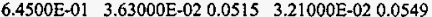

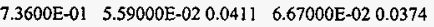

$8.2700 \mathrm{E}-01 \quad 8.86000 \mathrm{E}-020.0321 \quad 1.21300 \mathrm{E}-01 \quad 0.0269$

$9.1800 \mathrm{E}-01 \quad 1.34800 \mathrm{E}-01 \quad 0.0253 \quad 1.10600 \mathrm{E}-010.0284$

$1.1000 \mathrm{E}+00 \quad 1.65500 \mathrm{E}-01 \quad 0.0225 \quad 2.60000 \mathrm{E}-03 \quad 0.1959$

total $5.51300 \mathrm{E}-010.0094 \quad 3.95100 \mathrm{E}-010.0126$

surface: $\quad 1 \quad 2$

energy

$1.0000 \mathrm{E}-01 \quad 0.00000 \mathrm{E}+000.0000 \quad 0.00000 \mathrm{E}+000.0000$

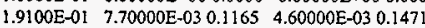

$2.8200 \mathrm{E}-01 \quad 9.30000 \mathrm{E}-03 \quad 0.1032 \quad 7.60000 \mathrm{E}-03 \quad 0.1158$

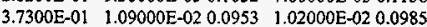

4.6400E-01 $1.65000 \mathrm{E}-02 \quad 0.0772 \quad 1.46000 \mathrm{E}-02 \quad 0.0822$

$5.5500 \mathrm{E}-0 \mathrm{I} \quad 2.58000 \mathrm{E}-02 \quad 0.0614 \quad 2.48000 \mathrm{E}-02 \quad 0.0627$

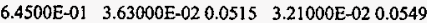

$\begin{array}{lllll}7.3600 \mathrm{E}-01 & 5.59000 \mathrm{E}-02 & 0.0411 & 6.67000 \mathrm{E}-02 & 0.0374\end{array}$

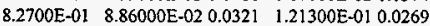

$9.1800 \mathrm{E}-01 \quad 1.34800 \mathrm{E}-01 \quad 0.0253 \quad 1.10600 \mathrm{E}-010.0284$

$1.1000 \mathrm{E}+00 \quad 1.65500 \mathrm{E}-010.0225 \quad 2.60000 \mathrm{E}-030.1959$

total $5.51300 \mathrm{E}-010.0094 \quad 3.95100 \mathrm{E}-010.0126$

$\mathrm{nps}=10000$ 
tally type 1 number of particles crossing a surface. tally for electrons

this tally is modified by $\mathrm{ft}$ elc

surface: $\quad 2$

energy

$1.0000 \mathrm{E}-01 \quad 0.00000 \mathrm{E}+000.0000 \quad 0.00000 \mathrm{E}+000.0000$

$1.9100 \mathrm{E}-0 \mathrm{I} \quad 0.00000 \mathrm{E}+000.0000 \quad 0.00000 \mathrm{E}+000.0000$

$2.8200 \mathrm{E}-01 \quad 0.00000 \mathrm{E}+000.0000 \quad 0.00000 \mathrm{E}+000.0000$

$3.7300 \mathrm{E}-01 \quad 0.00000 \mathrm{E}+000.0000 \quad 0.00000 \mathrm{E}+000.0000$

$\begin{array}{llll}4.6400 \mathrm{E}+01 & 0.00000 \mathrm{E}+00 & 0.0000 & 0.00000 \mathrm{E}+000.0000\end{array}$

$\begin{array}{llll}5.5500 \mathrm{E}-01 & 0.00000 \mathrm{E}+000.0000 & 0.00000 \mathrm{E}+000.0000\end{array}$

$6.4500 \mathrm{E}-01 \quad 0.00000 \mathrm{E}+000.0000 \quad 0.00000 \mathrm{E}+000.0000$

$\begin{array}{llll}7.3600 \mathrm{E}-01 & 0.00000 \mathrm{E}+00 & 0.0000 & 0.00000 \mathrm{E}+000.0000\end{array}$

$8.2700 \mathrm{E}-01 \quad 0.00000 \mathrm{E}+00 \quad 0.0000 \quad 0.00000 \mathrm{E}+000.0000$

$9.1800 \mathrm{E}-01 \quad 0.00000 \mathrm{E}+000.0000 \quad 0.00000 \mathrm{E}+000.0000$

$1.1000 \mathrm{E}+00 \quad 0.00000 \mathrm{E}+000.0000 \quad 0.00000 \mathrm{E}+000.0000$

total $0.00000 \mathrm{E}+00 \quad 0.0000 \quad 0.00000 \mathrm{E}+000.0000$

surface: $\quad 1 \quad 2$

energy

$1.0000 \mathrm{E}-01 \quad 0.00000 \mathrm{E}+00 \quad 0.0000 \quad 0.00000 \mathrm{E}+000.0000$

$1.9100 \mathrm{E}-01-7.70000 \mathrm{E}-030.1165-4.60000 \mathrm{E}-030.1471$

$2.8200 \mathrm{E}-01 \quad-9.30000 \mathrm{E}-030.1032-7.60000 \mathrm{E}-030.1158$

$3.7300 \mathrm{E}-01-1.09000 \mathrm{E}-020.0953-1.02000 \mathrm{E}-020.0985$

$4.6400 \mathrm{E}-01-1.65000 \mathrm{E}-020.0772-1.46000 \mathrm{E}-020.0822$

$5.5500 \mathrm{E}-01-2.58000 \mathrm{E}-020.0614-2.48000 \mathrm{E}-020.0627$

$6.4500 \mathrm{E}-01-3.63000 \mathrm{E}-020.0515-3.21000 \mathrm{E}-020.0549$

$7.3600 \mathrm{E}-01-5.59000 \mathrm{E}-020.0411-6.67000 \mathrm{E}-020.0374$

$8.2700 \mathrm{E}-01-8.86000 \mathrm{E}-020.0321-1.21300 \mathrm{E}-010.0269$

$9.1800 \mathrm{E}-01-1.34800 \mathrm{E}-010.0253-1.10600 \mathrm{E}-010.0284$

$1.1000 \mathrm{E}+00-1.65500 \mathrm{E}-010.0225-2.60000 \mathrm{E}-030.1959$

total $-5.51300 \mathrm{E}-010.0094-3.95100 \mathrm{E}-010.0126$

surface:

12

energy

$1.0000 E-01 \quad 0.00000 E+00 \quad 0.0000 \quad 0.00000 E+000.0000$

i.9100E-01 $-7.70000 \mathrm{E}-030.1165-4.60000 \mathrm{E}-030.1471$

$2.8200 \mathrm{E}-01-9.30000 \mathrm{E}-030.1032-7.60000 \mathrm{E}-030.1158$

$3.7300 \mathrm{E}-01-1.09000 \mathrm{E}-020.0953-1.02000 \mathrm{E}-020.0985$

$4.6400 \mathrm{E}-01-1.65000 \mathrm{E}-020.0772 \cdot 1.46000 \mathrm{E}-020.0822$

$5.5500 \mathrm{E}-01 \quad-2.58000 \mathrm{E}-02 \quad 0.0614-2.48000 \mathrm{E}-020.0627$

$6.4500 \mathrm{E}-01-3.63000 \mathrm{E}-020.0515-3.21000 \mathrm{E}-020.0549$

7.3600 E-01 -5.59000 E-02 $0.0411-6.67000$ E-02 0.0374

$8.2700 \mathrm{E}-01-8.86000 \mathrm{E}-020.0321-1.21300 \mathrm{E}-010.0269$

$9.1800 \mathrm{E}-01-1.34800 \mathrm{E}-010.0253-1.10600 \mathrm{E}-010.0284$

$1.1000 \mathrm{E}+00-1.65500 \mathrm{E}-010.0225-2.60000 \mathrm{E}-030.1959$

total $-5.51300 \mathrm{E}-010.0094-3.95100 \mathrm{E}-010.0126$

1 tally $58 \quad \mathrm{nps}=10000$

tally type 8 pulse height distribution. units number tally for photons electrons

celt: 1

energy

$-1.0000 \mathrm{E}-03 \quad 7.40000 \mathrm{E}-03 \quad 0.1158$

$0.0000 \mathrm{E}+00 \quad 0.00000 \mathrm{E}+000.0000$

$1.0000 \mathrm{E}-06 \quad 0.00000 \mathrm{E}+000.0000$

$\begin{array}{lll}1.0000 \mathrm{E}-01 & 2.12600 \mathrm{E}-01 & 0.0192\end{array}$

$1.9100 \mathrm{E}-01 \quad 2.65500 \mathrm{E}-010.0166$

2.8200E-01 1.87700E-01 0.0208

$3.7300 \mathrm{E}-01 \quad 1.09500 \mathrm{E}-010.0285$

4.6400E-01 6.44000E-02 0.0381

$5.5500 \mathrm{E}-01 \quad 4.37000 \mathrm{E}-02 \quad 0.0468$

$6.4500 \mathrm{E}-01 \quad 2.66000 \mathrm{E}-020.0605$

$7.3600 \mathrm{E}-01 \quad 1.58000 \mathrm{E}-02 \quad 0.0789$

2700E-01 1.12000E-02 0.0940

4.1800E-01 $\quad 7.70000 \mathrm{E}-03 \quad 0.1135$ 
cell: 1

energy

$1.0000 \mathrm{E}+03 \quad 2.66900 \mathrm{E}-010.0090$

Istatus of the statistical checks used to form confidence intervals for the mean for each tally bin

tally result of statistical checks for the tf bin (the first check not passed is listed) and enror magnitude check for all bins

1 missed I of $10 \mathrm{tfc}$ bin checks: there is insufficient tfc bin tally information to estimate the large tally slope reliably missed all bin error check: 24 tally bins had 4 bins with zeros and 17 bins with relative errors exceeding 0.10

6 missed 1 of $10 \mathrm{ffc}$ bin checks: the variance of the variance appears not to decrease as $1 / n p s$ for the last half of problem missed all bin error check: 12 tally bins had 2 bins with zeros and 8 bins with relative errors exceeding 0.10

28 passed the 10 statistical checks for the tally fluctuation chart bin result missed all bin error check: 15 tally bins had 2 bins with zeros and 2 bins with relative errors exceeding 0.10

11 missed 1 of 10 tfe bin checks: the slope of decrease of largest tallies is less than the minimum acceptable value of 3.0 missed all bin error check: 24 tally bins had 2 bins with zeros and 5 bins with relative errors exceeding 0.10

21 missed 1 of $10 \mathrm{tfc}$ bin checks: the estimated mean has a trend during the last half of the problem missed all bin eтror check: 24 tally bins had 2 bins with zeros and 5 bins with relative errors exceeding 0.10

31 missed 2 of 10 tfe bin checks: the estimated mean has a trend during the last half of the problem missed all bin error check: 24 tally bins had 2 bins with zeros and 5 bins with relative errors exceeding 0.10

41 missed 1 of 10 tf $\mathrm{c}$ bin checks: the estimated mean has a trend during the last half of the problem missed all bin eror check: 72 tally bins had 28 bins with zeros and 10 bins with relative errors exceeding 0.10

] missed 2 of 10 tf $\mathrm{c}$ bin checks: the estimated mean has a trend during the last half of the problem missed all bin error check: 72 tally bins had 28 bins with zeros and 10 bins with relative errors exceeding 0.10

58 passed the 10 statistical checks for the tally fluctuation chart bin result missed all bin error check: 15 tally bins had 2 bins with zeros and 2 bins with relative errors exceeding 0.10

8 passed the 10 statistical checks for the tally fluctuation chart bin result passed all bin error check: 1 tally bins all have relative errors less than 0.10 with no zero bins

the 10 statistical checks are only for the tally fluctuation chart bin and do not apply to other tally bins.

the tally bins with zeros may or may not be correct: compare the source, cutoffs, multipliers, et cetera with the tally bins.

waming. 7 of the 10 tally fluctuation chart bins did not pass all 10 statistical checks. waming. 9 of the 10 tallies had bins with relative errors greater than recommended. 1tally fluctuation charts

$$
\text { tally I tally } 6 \text { tally } 28
$$

nps mean enror vov slope fom mean error vov slope fom $10006.7975 \mathrm{E}-03 \quad 0.23990 .0835 \quad 0.0$ 20005.5780 E- $030.18840 .0641 \quad 0.0$ $30005.8779 \mathrm{E}-03 \quad 0.14550 .0373 \quad 0.0$ $40005.7880 \mathrm{E}-030.13170 .03110 .0$ $50005.9924 \mathrm{E}-030.11680 .02390 .0$ $60005.9423 \mathrm{E}-030.10550 .0195 \quad 0.0$ $70005.9749 \mathrm{E}-03 \quad 0.09680 .0170 \quad 0.0$ $80005.7670 \mathrm{E}-030.09130 .0154 \quad 0.0$ $90005.7398 \mathrm{E}-03 \quad 0.08640 .0135 \quad 0.0$ $100005.7777 \mathrm{E}-030.08110 .0120 \quad 0.0$

\section{$2.0857 \mathrm{E}-05 \quad 0.15890 .0525 \quad 0.0$} $\begin{array}{llll}2.4397 \mathrm{E}-05 & 0.1701 & 0.1693 & 0.0\end{array}$ $2.6650 \mathrm{E}-05 \quad 0.1343 \quad 0.1060 \quad 0.0$ $2.7894 \mathrm{E}-050.11450 .0656 \quad 0.0$ $2.7855 \mathrm{E}-050.10200 .0485 \quad 0.0$ $2.7793 \mathrm{E}-050.09310 .0418 \quad 0.0$ $2.8647 \mathrm{E}-05 \quad 0.09190 .0581 \quad 0.0$ $2.8249 \mathrm{E}-05 \quad 0.0838 \quad 0.052410 .0$ $2.7485 \mathrm{E}-050.07790 .049110 .0$ $2.7551 \mathrm{E}-050.07220 .043610 .0$ mean etror vov slope fom $\begin{array}{lll}2.6993 \mathrm{E}-01 & 0.0279 & 0.004410 .0\end{array}$ 2.6475E-01 0.01990 .002210 .0 2.6433E-01 0.01610 .001510 .0 2.6224E-01 0.01400 .001110 .0 2.6175E-01 0.01260 .000910 .0 $2.6496 \mathrm{E}-010.01160 .000710 .0$ 2.6691E-01 0.01070 .000610 .0 $2.6739 \mathrm{E}-01 \quad 0.01000 .000510 .0$ $2.6699 \mathrm{E}-010.00950 .000510 .0$ $2.6690 \mathrm{E}-010.00900 .000410 .0$ 
tally 11 tally 21 tally 31

ops mean error vov slope fom $10004.4504 \mathrm{E}-010.02960 .00022 .1$ $20004.4489 E-010.02110 .00012 .0$ $30004.4226 \mathrm{E}-010.01730 .0001 \quad 1.8$ 4000 4.4011E-01 $0.01510 .0000 \quad 1.8$ $50004.4004 \mathrm{E}-010.01350 .00001 .9$ $60004.3549 E-010.01250 .0000 \quad 1.8$ $70004.3477 \mathrm{E}-010.01150 .0000 \quad 1.9$ $80004.3339 E-010.01080 .0000 \quad 1.8$ $90004.3428 \mathrm{E}-010.01020 .0000 \quad 1.7$ $100004.3411 \mathrm{E}-010.00970 .0000 \quad 1.6$

$$
\text { tally } 41
$$

nps mean error vov slope fom $10005.6500 \mathrm{E}-010.02860 .00044 .4$ $20005.6500 \mathrm{E}-010.02040 .0003 \quad 4.4$ $30005.6400 \mathrm{E}-010.01680 .000210 .0$ $40005.6100 \mathrm{E}-01 \quad 0.01470 .000210 .0$ $50005.5860 \mathrm{E}-010.01310 .000110 .0$ $60005.5433 \mathrm{E}-01 \quad 0.01210 .000110 .0$ $70005.5386 \mathrm{E}-01 \quad 0.01120 .000110 .0$ $80005.5238 \mathrm{E}-010.01050 .000110 .0$ $90005.5200 \mathrm{E}-01 \quad 0.00990 .000110 .0$ $100005.5130 \mathrm{E}-010.00940 .000110 .0$

tally 51

mean error vov slope fom $\begin{array}{lll}-5.6500 \mathrm{E}-01 & 0.02860 .0004 & 0.0\end{array}$ $\begin{array}{llll}-5.6500 \mathrm{E}+01 & 0.0204 & 0.0003 & 0.0\end{array}$ $-5.6400 \mathrm{E}-010.01680 .0002 \quad 0.0$ $\begin{array}{llll}-5.6100 \mathrm{E}-01 & 0.0147 & 0.0002 & 0.0\end{array}$ $\begin{array}{llll}-5.5860 \mathrm{E}-01 & 0.0131 & 0.0001 & 0.0\end{array}$ $\begin{array}{llll}-5.5433 \mathrm{E}-01 & 0.0121 & 0.0001 & 0.0\end{array}$ $\begin{array}{llll}-5.5386 \mathrm{E}-01 & 0.0112 & 0.0001 & 0.0\end{array}$ $\begin{array}{llll}-5.5238 \mathrm{E}-01 & 0.01050 .0001 & 0.0\end{array}$

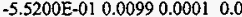
$-5.5130 \mathrm{E}-010.00940 .00010 .0$ mean error vov slope fom $-5.6500 \mathrm{E}-01$
$0.02860 .0004 \quad 0.0$ $\begin{array}{llll}-5.6500 E-01 & 0.02040 .0003 & 0.0\end{array}$ $-5.6400 \mathrm{E}-010.01680 .0002 \quad 0.0$ $-5.6100 \mathrm{E}-010.01470 .00020 .0$ $\begin{array}{ll}-5.5860 \mathrm{E}-01 & 0.01310 .00010 .0\end{array}$ $-5.5433 \mathrm{E}-010.01210 .00010 .0$
$0.5386 \mathrm{E}$ $\begin{array}{llll}-5.5386 \mathrm{E}-01 & 0.0112 & 0.0001 & 0.0\end{array}$ $\begin{array}{llll}-5.5238 \mathrm{E}-01 & 0.01050 .0001 & 0.0\end{array}$ $-5.5200 \mathrm{E}-010.00990 .00010 .0$ $\begin{array}{llll}-5.5130 \mathrm{E}-01 & 0.0094 & 0.0001 & 0.0\end{array}$

mean error vov slope fom $1.0000 \mathrm{E}+000.00000 .000010 .0$ $1.0000 \mathrm{E}+000.00000 .000010 .0$ $1.0000 E+000.00000 .000010 .0$ $1.0000 \mathrm{E}+000.00000 .000010 .0$ $1.0000 \mathrm{E}+000.00000 .000010 .0$ $1.0000 \mathrm{E}+00 \quad 0.00000 .000010 .0$ $1.0000 \mathrm{E}+000.00000 .000010 .0$ $1.0000 \mathrm{E}+000.00000 .000010 .0$ $1.0000 \mathrm{E}+000.00000 .000010 .0$ $1.0000 \mathrm{E}+000.00000 .000010 .0$ tally 8

nps mean error vov slope fom $10002.6993 \mathrm{E}-010.02790 .004410 .0$ $20002.6475 E-010.01990 .002210 .0$ $30002.6433 \mathrm{E}-010.01610 .001510 .0$ $40002.6224 E-010.01400 .001110 .0$ $\$ 0002.6175 E-010.01260 .000910 .0$ $60002.6496 \mathrm{E}-010.01160 .000710 .0$ $70002.6691 E-010.01070 .000610 .0$ $80002.6739 E-010.01000 .000510 .0$ $90002.6699 \mathrm{E}-010.00950 .000510 .0$ $100002.6690 \mathrm{E}-010.00900 .000410 .0$ tally data written to file inp $20 \mathrm{~m}$

6 waming messages so far. run terminated when $\quad 10000$ particle histories were done.

\section{File: Inp240}

1- prob 24 -- reflecting lattice. $15 \times 15$ at 3.75 w/o u-235 enrichment.

2. $11-10.182+1 u=2$

3- $22-.0011-2 \mathrm{u}=2$

4. $3 \quad 3-6.552-3 \mathrm{u}=2$

5. 4 4-1.03u=2

6. 5 - $4-1.0-14: 15 u=3$

7. $6 \quad 3-6.5514-15 u=3$

8. $74-1.0-4+5-6+7 u=1 \quad l a t=1 \quad f m=-8: 8-8: 80: 0$

9- $\quad 117 r 214 \pi 11214 \mathrm{r} 1122322322 r 322$

10- $\quad 3221126 \times 326 r 1123 r 3$

11. $\quad 24 r 323 r 1122328 \mathrm{r} 322$

12. $\quad 11214 \mathrm{r} 1122 \mathrm{r} 32 \mathrm{rr} 322 \mathrm{r}$

13- $\quad 322 \mathrm{r} 11214 \mathrm{r} 1122328 \mathrm{r} 3$

14. $\quad 221123 r_{324} 323 r_{11}$

15- $\quad 26 \mathrm{r326r11223223}$

$22 r 32232211214 r 11214 r 117 r$

$8 \quad 0-8 \quad-10 \quad-12 u=4$ fill=1

$95-7.98: 10 u=4$ 


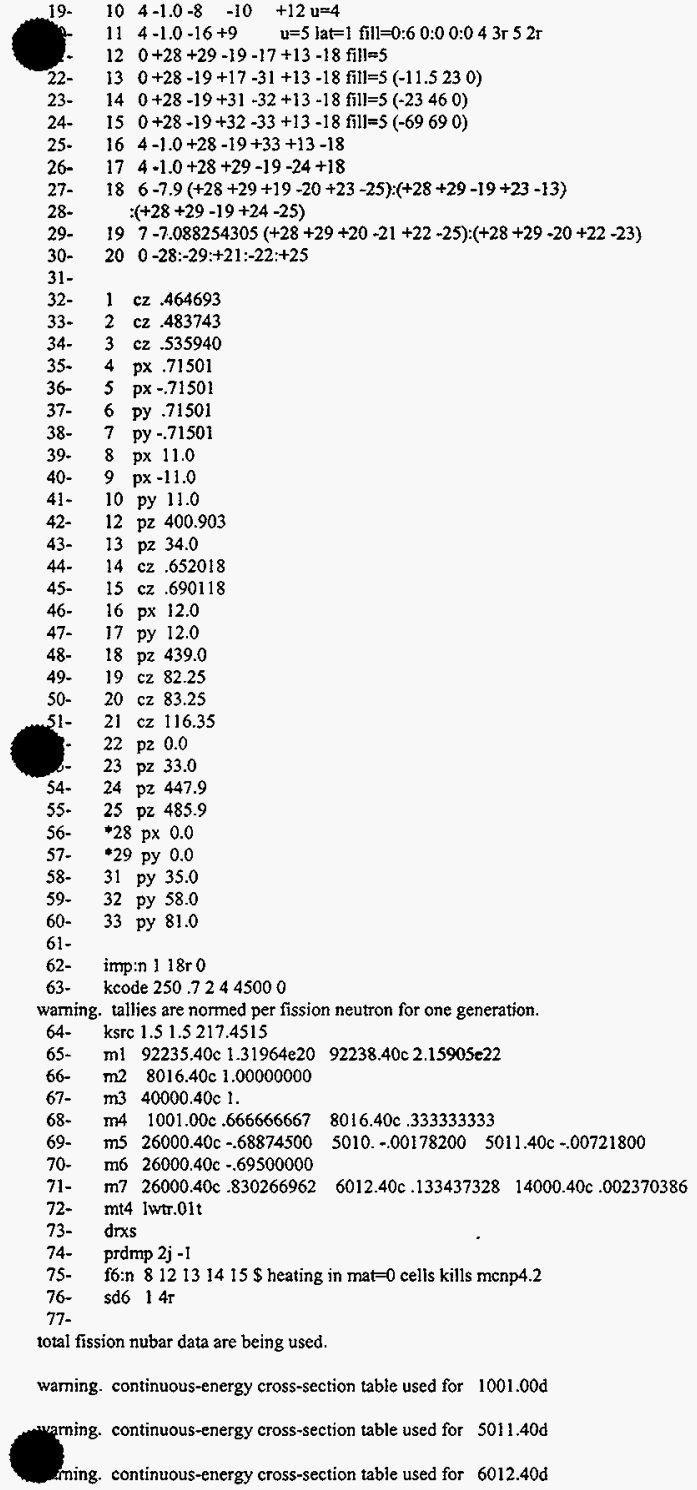


warning. continuous-energy cross-section table used for $14000.40 \mathrm{~d}$

waming, continuous-energy cross-section table used for $26000.40 \mathrm{~d}$

waming. continuous-energy cross-section table used for $40000.40 d$

waming. continuous-energy cross-section table used for $92235.40 \mathrm{~d}$

waming. continuous-energy cross-section table used for $92238.40 \mathrm{~d}$

warning. 4 of the materials had unnormalized fractions.

lcells

print table 60

$$
\text { cell mat density density volume mass pieces importance }
$$

$\begin{array}{llllllllll}1 & 1 & 1 & 2.57597 \mathrm{E}-02 & 1.01820 \mathrm{E}+01 & 0.00000 \mathrm{E}+00 & 0.00000 \mathrm{E}+00 & 0 & 1.0000 \mathrm{E}+00 \\ 2 & 2 & 2 & 3.76497 \mathrm{E}-05 & 1.00000 \mathrm{E}-03 & 0.00000 \mathrm{E}+00 & 0.00000 \mathrm{E}+00 & 0 & 1.0000 \mathrm{E}+00 \\ 3 & 3 & 3 & 4.32392 \mathrm{E}-02 & 6.55000 \mathrm{E}+00 & 0.00000 \mathrm{E}+00 & 0.00000 \mathrm{E}+00 & 0 & 1.0000 \mathrm{E}+00 \\ 4 & 4 & 4 \mathrm{~s} & 1.00309 \mathrm{E}-01 & 1.00000 \mathrm{E}+00 & 0.00000 \mathrm{E}+00 & 0.00000 \mathrm{E}+00 & 0 & 1.0000 \mathrm{E}+00 \\ 5 & 5 & 4 s & 1.00309 \mathrm{E}-01 & 1.00000 \mathrm{E}+00 & 0.00000 \mathrm{E}+00 & 0.00000 \mathrm{E}+00 & 0 & 1.0000 \mathrm{E}+00 \\ 6 & 6 & 3 & 4.32392 \mathrm{E}-02 & 6.55000 \mathrm{E}+00 & 0.00000 \mathrm{E}+00 & 0.00000 \mathrm{E}+00 & 0 & 1.0000 \mathrm{E}+00 \\ 7 & 7 & 4 \mathrm{~s} & 1.00309 \mathrm{E}-01 & 1.00000 \mathrm{E}+00 & 0.00000 \mathrm{E}+00 & 0.00000 \mathrm{E}+00 & 0 & 1.0000 \mathrm{E}+00 \\ 8 & 8 & 0 & 0.00000 \mathrm{E}+00 & 0.00000 \mathrm{E}+00 & 0.00000 \mathrm{E}+00 & 0.00000 \mathrm{E}+00 & 0 & 1.0000 \mathrm{E}+00 \\ 9 & 9 & 5 & 8.97738 \mathrm{E}-02 & 7.90000 \mathrm{E}+00 & 0.00000 \mathrm{E}+00 & 0.00000 \mathrm{E}+00 & 0 & 1.0000 \mathrm{E}+00 \\ 10 & 10 & 4 \mathrm{~s} & 1.00309 \mathrm{E}-01 & 1.00000 \mathrm{E}+00 & 0.00000 \mathrm{E}+00 & 0.00000 \mathrm{E}+00 & 0 & 1.0000 \mathrm{E}+00 \\ 11 & 11 & 4 \mathrm{~s} & 1.00309 \mathrm{E}-01 & 1.00000 \mathrm{E}+00 & 0.00000 \mathrm{E}+00 & 0.00000 \mathrm{E}+00 & 0 & 1.0000 \mathrm{E}+00 \\ 12 & 12 & 0 & 0.00000 \mathrm{E}+00 & 0.00000 \mathrm{E}+00 & 0.00000 \mathrm{E}+00 & 0.00000 \mathrm{E}+00 & 0 & 1.0000 \mathrm{E}+00 \\ 13 & 13 & 0 & 0.00000 \mathrm{E}+00 & 0.00000 \mathrm{E}+00 & 0.00000 \mathrm{E}+00 & 0.00000 \mathrm{E}+00 & 0 & 1.0000 \mathrm{E}+00 \\ 14 & 14 & 0 & 0.00000 \mathrm{E}+00 & 0.00000 \mathrm{E}+00 & 0.00000 \mathrm{E}+00 & 0.00000 \mathrm{E}+00 & 0 & 1.0000 \mathrm{E}+00 \\ 5 & 15 & 0 & 0.00000 \mathrm{E}+00 & 0.00000 \mathrm{E}+00 & 0.00000 \mathrm{E}+00 & 0.00000 \mathrm{E}+00 & 0 & 1.0000 \mathrm{E}+00 \\ 6 & 16 & 4 \mathrm{~s} & 1.00309 \mathrm{E}-01 & 1.00000 \mathrm{E}+00 & 0.00000 \mathrm{E}+00 & 0.00000 \mathrm{E}+00 & 0 & 1.0000 \mathrm{E}+00 \\ 17 & 17 & 4 \mathrm{~s} & 1.00309 \mathrm{E}-01 & 1.00000 \mathrm{E}+00 & 0.00000 \mathrm{E}+00 & 0.00000 \mathrm{E}+00 & 0 & 1.0000 \mathrm{E}+00 \\ 18 & 18 & 6 & 8.51890 \mathrm{E}-02 & 7.90000 \mathrm{E}+00 & 0.00000 \mathrm{E}+00 & 0.00000 \mathrm{E}+00 & 0 & 1.0000 \mathrm{E}+00 \\ 19 & 19 & 7 & 8.58502 \mathrm{E}-02 & 7.08825 \mathrm{E}+00 & 0.00000 \mathrm{E}+00 & 0.00000 \mathrm{E}+00 & 0 & 1.0000 \mathrm{E}+00 \\ 20 & 20 & 0 & 0.00000 \mathrm{E}+00 & 0.00000 \mathrm{E}+00 & 0.00000 \mathrm{E}+00 & 0.00000 \mathrm{E}+00 & 0 & 0.0000 \mathrm{E}+00\end{array}$

total

\section{$0.00000 \mathrm{E}+000.00000 \mathrm{E}+00$}

11 warning messages so far.

lcross-section tables

print table 100

table length

tables from file testlibl

I001.00c 2139 l-h-l from endf-vi. 1

mat $125 \quad 05 / 26 / 93$

$5010.03 d \quad 3945$ b-10 endf/b-iv new gamma production format $\quad$ ( 1273) 14 oct 75

5011.40c 3473 ENDL library name: nd900719 MCNP translation: 900831 14:10:05 900207

$6012.40 \mathrm{c} 5049$ ENDL library name: nd900719 MCNP translation: 900831 14:10:05

8016.40c 5693 ENDL library name: nd900719 MCNP translation: 900831 14:10:05 900207

14000.40 C 8732 ENDL library name: nd900719 MCNP translation: 900831 14:10:05 900207

26000.40c 21846 ENDL library name: nd900719 MCNP translation: 900831 14:10:05 900207

40000.40c 111279 ENDL library name: nd900719 MCNP translation: 900831 14:10:05 900323

92235.40c 40506 ENDL library name: nd900719 MCNP translation: 9total nu 10:05 900503

$92238.40 \mathrm{c} 48310$ ENDL library name: nd901118 MCNP translation: 9total nu 56:49 901119

$\begin{array}{lllll}\text { fwtr.01t } 10193 \text { hydrogen in light water at } 300 \text { degrees kelvin } & 1001 & 0 & 010 / 22 / 85\end{array}$

total 261165

warning. neutron energy cutoff is below some cross-section tables. 
source distribution written to file inp24s cycle $=0$

12 waming messages so far.

lestimated keff results by cycle

print table 175

cycle $1 \mathrm{k}$ (collision) 0.658210 removal lifetime(abs) $5.1517 \mathrm{E}+03$ source points generated 228

cycle $2 \mathrm{k}$ (collision) 0.549318 removal lifetime(abs) $3.7645 \mathrm{E}+03$ source points generated 209

cycle $3 \mathrm{k}$ (collision) 0.551862 removal lifetime(abs) $3.8422 \mathrm{E}+03$ source points generated 253

estimator cycle 4 ave of 2 cycles combination simple average combined average corr

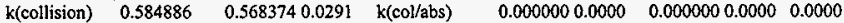

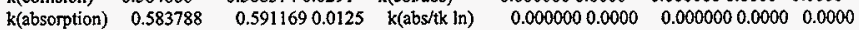

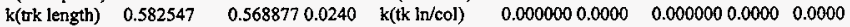

rem life(col) $3.9485 \mathrm{E}+03 \quad 3.8951 \mathrm{E}+030.0137$

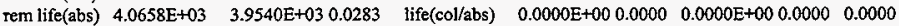

source points generated 264

source distribution written to file inp24s $\quad$ cycle $=4$

lproblem summary

0

run terminated when 4 kcode cycles were done

neutron creation tracks weight energy neutron loss tracks weight energy (per source particle) (per source particle)

$\begin{array}{llllllll}\text { source } & 940 & 1.0638 \mathrm{E}+00 & 1.9444 \mathrm{E}+00 & \text { escape } & 0 & 0 . & 0 .\end{array}$ energy cutoff $\quad 0 \quad 0 . \quad 0$. time cutoff $\quad 0 \quad 0$. 0 .

ght window $\quad \begin{array}{llllllll}0 & 0 . & 0 . & \text { weight window } & 0 & 0 . & 0 .\end{array}$

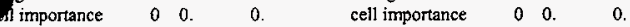

$\begin{array}{llllllllll}\text { weight cutoff } & 0 & 5.6409 \mathrm{E}-02 & 1.1284 \mathrm{E}-06 & \text { weight cutoff } & 944 & 5.2413 \mathrm{E}-02 & 7.1769 \mathrm{E}-07\end{array}$

energy importance $\quad 0 \quad 0 . \quad 0 . \quad 0 \quad$ energy importance $\begin{array}{lllll}0 & 0 . & 0 .\end{array}$

$\begin{array}{lllllllllll}\text { dxtran } & 0 & 0 . & 0 . & d x t r a n & 0 & 0 . & 0 . & \\ \text { forced collisions } & 0 & 0 . & 0 . & \text { forced collisions } & 0 & 0 . & 0 .\end{array}$

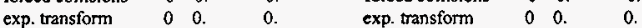

upscattering $\quad 0 \quad 0 . \quad 1.4710 \mathrm{E}-07 \quad$ downscattering $\quad 0.0 . \quad 1.8098 \mathrm{E}+00$

capture $\quad 0 \quad 8.1698 \mathrm{E}-01 \quad 1.8384 \mathrm{E}-02$

$(n, x n) \quad \begin{array}{llllllll}8 & 6.9970 \mathrm{E}-03 & 3.6428 \mathrm{E}-03 & \text { loss to }(\mathrm{n}, \mathrm{xn}) & 4 & 3.4985 \mathrm{E}-03 & 2.8025 \mathrm{E}-02\end{array}$

fission $\quad 0 \quad 0.00$ loss to fission $0 \begin{array}{llll}2.5434 \mathrm{E}-01 & 9.1864 \mathrm{E}-02\end{array}$

$\begin{array}{lllllllll}\text { total } & 948 & 1.1272 \mathrm{E}+00 & 1.9481 \mathrm{E}+00 \quad \text { total } & 948 & 1.1272 \mathrm{E}+00 & 1.9481 \mathrm{E}+00\end{array}$

number of neutrons banked 4 average lifetime, shakes cutoffs

neutron tracks per source particle $1.0085 \mathrm{E}+00$ escape $0.0000 \mathrm{E}+00$ tco $1.0000 \mathrm{E}+34$

reutron collisions per source particle $6.1389 \mathrm{E}+01$ capture $4.1766 \mathrm{E}+03$ eco $0.0000 \mathrm{E}+00$

total neutron collisions $\quad 57706$ capture or escape $4.1766 \mathrm{E}+03$ wcl $-5.0000 \mathrm{E}-01$

net multiplication $\quad 1.0033 \mathrm{E}+000.0030$ any termination $4.4650 \mathrm{E}+03$ wc2 $+2.5000 \mathrm{E}+01$

computer time so far in this run 0.00 minutes maximum number ever in bank 1

computer time in merun 0.00 minutes bank overflows to backup file 0

source particles per minute $\quad 0.0000 \mathrm{E}+00$

random numbers generated $\quad 690539$

field length

most random numbers used was 3677 in history $\quad 21$

range of sampled source weights $=9.8814 \mathrm{E}-01$ to $1.1962 \mathrm{E}+00$

Ineutron activity in each celt

print table 126

tracks population collisions collisions number flux average average

cell entering - weight weighted weighted track weight track mfp (per history) energy energy (relative) (cm) 
$40660 \quad 3.0973 \mathrm{E}+01 \quad 3.0008 \mathrm{E}-04 \quad 5.9877 \mathrm{E}-01 \quad 8.3882 \mathrm{E}-01 \quad 1.4858 \mathrm{E}+00$

$\begin{array}{llllll}8775 & 6.4600 \mathrm{E}+00 & 2.0190 \mathrm{E}-04 & 5.0976 \mathrm{E}-01 & 8.1000 \mathrm{E}-01 & 1.3256 \mathrm{E}+00\end{array}$

$\begin{array}{lllllll}109 & 9.6195 \mathrm{E}-02 & 2.0789 \mathrm{E}-04 & 5.2658 \mathrm{E}-01 & 8.2057 \mathrm{E}-01 & 3.6398 \mathrm{E}+00\end{array}$

$\begin{array}{llllll}1607 & 1.4612 \mathrm{E}+00 & 8.5166 \mathrm{E}-04 & 7.1930 \mathrm{E}-01 & 9.4163 \mathrm{E}-01 & 1.7232 \mathrm{E}+00\end{array}$

$\begin{array}{lllllll}0 & 0.0000 \mathrm{E}+00 & 0.0000 \mathrm{E}+00 & 0.0000 \mathrm{E}+00 & 0.0000 \mathrm{E}+00 & 0.0000 \mathrm{E}+00\end{array}$

$1347 \quad 1.2836 \mathrm{E}+00 \quad 3.8320 \mathrm{E}-03 \quad 8.0017 \mathrm{E}-01 \quad 9.7093 \mathrm{E}-01 \quad 3.1777 \mathrm{E}+00$

$\begin{array}{lllllll}0 & 0.0000 \mathrm{E}+00 & 0.0000 \mathrm{E}+00 & 0.0000 \mathrm{E}+00 & 0.0000 \mathrm{E}+00 & 0.0000 \mathrm{E}+00\end{array}$

$126 \quad 9.7846 \mathrm{E}-02 \quad 9.0288 \mathrm{E}-05 \quad 3.2866 \mathrm{E}-01 \quad 7.7027 \mathrm{E}-01 \quad 1.0087 \mathrm{E}+00$

$\begin{array}{lllllllll}0 & 0.0000 \mathrm{E}+00 & 0.0000 \mathrm{E}+00 & 0.0000 \mathrm{E}+00 & 0.0000 \mathrm{E}+0 & 0.0000 \mathrm{E}+00\end{array}$

$\begin{array}{llllllllllllll}0 & 0.0000 \mathrm{E}+00 & 0.0000 \mathrm{E}+00 & 0.0000 \mathrm{E}+00 & 0.0000 \mathrm{E}+00 & 0.0000 \mathrm{E}+00\end{array}$

$\begin{array}{llllllll}0 & 0.0000 \mathrm{E}+00 & 0.0000 \mathrm{E}+00 & 0.0000 \mathrm{E}+00 & 0.0000 \mathrm{E}+00 & 0.0000 \mathrm{E}+00\end{array}$

$\begin{array}{lllllllllllll}0 & 0.0000 \mathrm{E}+00 & 0.0000 \mathrm{E}+00 & 0.0000 \mathrm{E}+00 & 0.0000 \mathrm{E}+0 & 0.0000 \mathrm{E}+00\end{array}$

$\begin{array}{llllllllll}0 & 0.0000 \mathrm{E}+00 & 0.0000 \mathrm{E}+00 & 0.0000 \mathrm{E}+00 & 0.0000 \mathrm{E}+00 & 0.0000 \mathrm{E}+00\end{array}$

$\begin{array}{lllllll}0 & 0.0000 \mathrm{E}+00 & 0.0000 \mathrm{E}+00 & 0.0000 \mathrm{E}+00 & 0.0000 \mathrm{E}+00 & 0.0000 \mathrm{E}+00\end{array}$

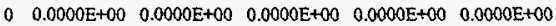

$\begin{array}{lllllllll}0 & 0.0000 \mathrm{E}+00 & 0.0000 \mathrm{E}+00 & 0.0000 \mathrm{E}+00 & 0.0000 \mathrm{E}+00 & 0.0000 \mathrm{E}+00\end{array}$

total $138515 \quad 6658 \quad 57706 \quad 4.4801 \mathrm{~L}+01$

the initial fission neutron source distribution used the 1 source points that were input on the ksre card.

the criticality problem was scheduled to skip 2 cycles and run a total of 4 cycles with nominally 250 neutrons per cycle.

this problem has run 2 inactive cycles with 478 neutron histories and 2 active cycles with 462 neutron histories.

this calculation has completed the requested number of keff cycles using a total of 940 fission neutron source histories. all cells with fissionable material were sampled and had fission neutron source points.

there is no combined collision/absorption/track-length estimate for keff because only 2 active cycles were run.

the estimated average keffs, one standard deviations, and 68,95 , and 99 percent confidence intervals are:

$\begin{array}{cccccccc}\text { keff estimator } & \text { keff } & \text { standard deviation } & 68 \% \text { confidence } & 95 \% \text { confidence } & 99 \% \text { confidence } \\ \text { collision } & 0.56837 & 0.01651 & 0.53834 \text { to } 0.59841 & 0.35851 \text { to } 0.77824 & 0.00000 \text { to } 1.61951 \\ \text { absorption } & 0.59117 & 0.00738 & 0.57774 \text { to } 0.60460 & 0.49735 \text { to } 0.68498 & 0.12129 \text { to } 1.06105 \\ \text { track length } & 0.56888 & 0.01367 & 0.54401 \text { to } 0.59374 & 0.39513 \text { to } 0.74263 & 0.00000 \text { to } 1.43912\end{array}$

lindividual and average keff estimator results by cycle

keff neutron keff estimators by cycle average keff estimators and deviations average $k(c / a / t)$ cycle histories $k$ (coll) $k$ (abs) $k$ (track) $k$ (coll) st dev $k(a b s)$ st dev $k$ (track) st dev $k(c / a / t)$ st dev forn

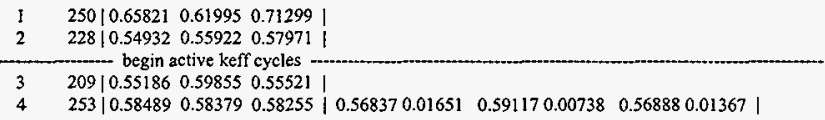

the largest active cycle keffs by estimator are:

collision 0.58489 on cycle 4

absorption 0.59855 on cycle 3

track length 0.58255 on cycle 4

1 tally 6 nps $=940$

tally type 6 track length estimate of heating. units mev/gram

tally for neutrons

number of histories used for normalizing tallies $=\quad 500.00$

masses
cell: $\begin{array}{lllll}8 & 12 & 13 & 14 & 15\end{array}$
$1.00000 \mathrm{E}+00 \quad 1.00000 \mathrm{E}+00 \quad 1.00000 \mathrm{E}+00 \quad 1.00000 \mathrm{E}+00 \quad 1.00000 \mathrm{E}+00$ 
cell 13

$1.32494 \mathrm{E}+010.0904$

cell 14

$9.78180 \mathrm{E}-010.4014$

cell 15

$1.20234 \mathrm{E}-010.9710$

lanalysis of the results in the tally fluctuation chart bin (tfc) for tally 6 with nps $=940$ print table 160

normed average tally per history $=4.02566 \mathrm{E}+03$ estimated tally relative error $=0.0434$ relative error from zero tallies $=0.0128$ number of nonzero history tallies $=\quad 462$
history number of largest tally $=\quad 64 !$ (largest tally)/(average tally) $=6.75049 \mathrm{E}+00$

(confidence interval shift)/mean $=0.0014$ unnormed average tally per history $=4.02566 \mathrm{E}+01$ estimated variance of the variance $=0.0095$ relative enor from nonzero scores $=0.0415$

efficiency for the nonzero tallies $=0.9240$

largest unnormalized history tally $=2.71752 \mathrm{E}+02$

(largest tally) $/($ avg nonzero tally $)=6.23745 \mathrm{E}+00$

shifted confidence interval center $=4.03113 E+01$

if the largest history score sampled so far were to occur on the very next history, the tfe bin quantities would change as follows: $\mathrm{nps}=462$ for this table because 2 keff cycles and 478 histories were skipped before tally accumulation.

$\begin{array}{lccc}\text { estimated quantities } & \text { value at nps } & \text { value at nps+1 } & \text { value(nps+1)/value(nps } \\ & & & \\ \text { mean } & 4.02566 \mathrm{E}+01 & 4.07566 \mathrm{E}+01 & 0.012420 \\ \text { relative error } & 4.34431 \mathrm{E}-02 & 4.24506 \mathrm{E}-02 & -0.022846 \\ \text { variance of the variance } & 9.52674 \mathrm{E}-03 & 1.33327 \mathrm{E}-02 & 0.399508 \\ \text { shifted center } & 4.03113 \mathrm{E}+01 & 4.03457 \mathrm{E}+01 & 0.000853 \\ \text { figure of merit } & 0.00000 \mathrm{E}+00 & 0.00000 \mathrm{E}+00 & 0.000000\end{array}$

there is not enough information in the largest history scores (usually less than 500 scores) for a reliable estimate of the slope. the history score probability density function appears to have an unsampled region at the largest history scores: please examine.

the nps-dependent tfe bin check results are suspect because there are only 1 nps tally values to analyze *****

results of 10 statistical checks for the estimated answer for the tally fluctuation chart (tfe) bin of tally 6

tfc bin -mean-- -...--relative error-.....- - -variance of the variance-- -figure of merit- -pdf-

behavior behavior value decrease decrease rate value decrease decrease rate value behavior slope

$\begin{array}{lccccccccc}\text { desired random } & <0.10 & \text { yes } & 1 / \text { sqrt(nps) } & <0.10 \text { yes } 1 / \text { nps } & \text { constant random }>3.00 \\ \text { observed random } & 0.04 \text { yes } & \text { yes } & 0.01 \text { yes yes } & & 0.00 & \text { no } \\ \text { passed? yes } & \text { yes yes } & \text { yes yes yes yes } & & \end{array}$

passed? yes yes yes yes yes yes yes no

waming. the tally in the tally fluctuation chart bin did not pass 1 of the 10 statistical checks.

lunnormed tally density for tally $6 \quad$ nonzero tally mean $(m)=4.357 \mathrm{E}+01$ nps $=940$ print table 161

abscissa ordinate $\log$ plot of tally probability density function in tally fluctuation chart bin(d=decade,slope $=0.0$ )

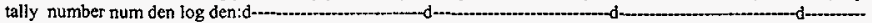

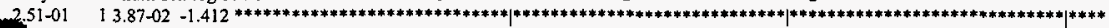

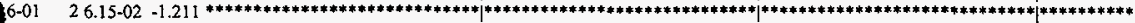

$38-01 \quad|2.44-02-1.612 * * * * * * * * * * * * * * * * * * * * * * * * * * * * *| * * * * * * * * * * * * * * * * * * * * * * * * * * * * *|* * * * * * * * * * * * * * * * * * * * * * * * * * * *|$ 


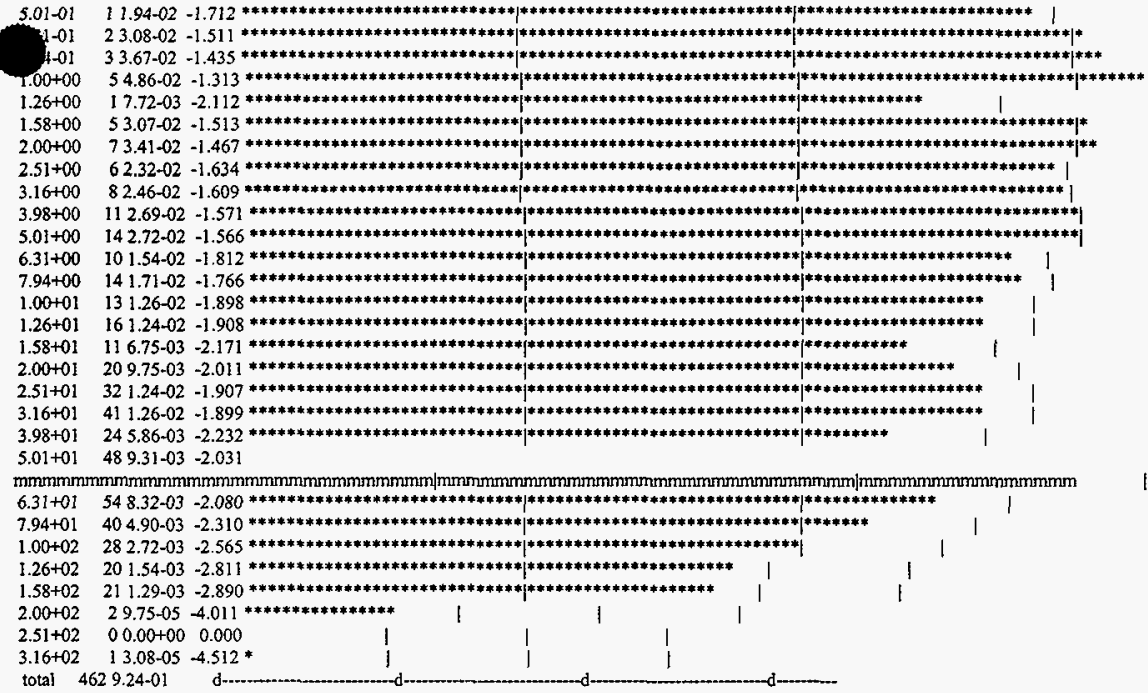

1status of the statistical checks used to form confidence intervals for the mean for each tally bin

result of statistical checks for the $t \mathrm{f}$ bin (the first check not passed is listed) and error magnitude check for all bins

6 missed 1 of 10 tf $\mathrm{c}$ bin checks: there is insufficient tfe bin tally information to estimate the large tally slope reliably missed all bin error check: 5 tally bins had 0 bins with zeros and 2 bins with relative errors exceeding 0.10

the 10 statistical checks are only for the tally fluctuation chart bin and do not apply to other tally bins.

waming. I of the 1 tally fluctuation chart bins did not pass all 10 statistical checks. warning. I of the 1 tallies had bins with relative errors greater than recommended. lally fuctuation charts

tally 6

nps mean ertor vov slope fom

$9404.0257 \mathrm{E}+010.04340 .00950 .0$

tally data written to file inp $24 \mathrm{~m}$

15 warning messages so far.

run terminated when 4 kcode cycles were done.

\section{File: Outp24}

1. prob 24 - reflecting lattice. $15 \times 15$ at 3.75 w/o u-235 enrichment.

2- $1 \quad 1+10.182-1 \mathrm{u}=2$

3- $\quad 2 \quad 2-0011-2 u=2$

$4.3 \quad 3-6.552-3 u=2$

5- 4 4-1.03 $u=2$

6- 5 - $4-1.0-14: 15 u=3$

7. $6 \quad 3-6.55 \quad 14-15 u=3$

$7 \quad 4-1,0-4+5-6+7 u=1$ lak $=1$ fill $=-8: 8-8: 80: 0$

$117 r 214 r 11214 r 1122322322 r 322$ 
fission nubar data are being used.

waming. continuous-energy cross-section table used for $1001.00 \mathrm{~d}$

warning. continuous-energy cross-section table used for $5011.40 \mathrm{~d}$

warning. continuous-energy cross-section table used for $6012.40 \mathrm{~d}$

waming. continuous-energy cross-section table used for $8016.40 \mathrm{~d}$

waming. continuous-energy cross-section table used for $14000.40 \mathrm{~d}$

waming. continuous-energy cross-section table used for $26000.40 \mathrm{~d}$

warning. continuous-energy cross-section table used for $40000.40 \mathrm{~d}$

warning. continuous-energy cross-section table used for $92235.40 \mathrm{~d}$

waming. continuous-energy cross-section tabje used for $92238.40 \mathrm{~d}$

waming. 4 of the materials had unnomalized fractions.

lcells

print table 60

$$
\text { cell mat density } \stackrel{\text { gram }}{\text { atomsity volume mass pieces importance }}
$$
$1 \quad 2.57597 \mathrm{E}+021.01820 \mathrm{E}+010.00000 \mathrm{E}+000.00000 \mathrm{E}+00$
$01.0000 \mathrm{E}+00$
$2223.76497 \mathrm{E}-05$ 1.00000E-03 0.00000E $+000.00000 \mathrm{E}+00$
$0 \quad 1.0000 \mathrm{E}+00$
33 4.32392E-02 6.55000E+00 0.00000E+00 0.00000E+00
$0 \backslash .0000 \mathrm{E}+00$
4 4s $1.00309 \mathrm{E}-01 \quad 1.00000 \mathrm{E}+000.00000 \mathrm{E}+000.00000 \mathrm{E}+00$
$01.0000 \mathrm{E}+00$
5 4s $1.00309 \mathrm{E}-01 \quad 1.00000 \mathrm{E}+000.00000 \mathrm{E}+000.00000 \mathrm{E}+00 \quad 0 \quad 1.0000 \mathrm{E}+00$
$63 \quad 3.32392 \mathrm{E}-02 \quad 6.55000 \mathrm{E}+000.00000 \mathrm{E}+000.00000 \mathrm{E}+00 \quad 0 \quad 1.0000 \mathrm{E}+00$
7 4s $1.00309 \mathrm{E}-01 \quad 1.00000 \mathrm{E}+000.00000 \mathrm{E}+000.00000 \mathrm{E}+00 \quad 0 \quad 1.0000 \mathrm{E}+00$

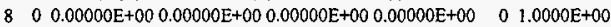
$958.97738 \mathrm{E}-027.90000 \mathrm{E}+000.00000 \mathrm{E}+000.00000 \mathrm{E}+00 \quad 0 \quad 1.0000 \mathrm{E}+00$
$10 \quad 10 \quad 4 \mathrm{~s} 1.00309 \mathrm{E}-01 \quad 1.00000 \mathrm{E}+00 \quad 0.00000 \mathrm{E}+00 \quad 0.00000 \mathrm{E}+00 \quad 0 \quad 1.0000 \mathrm{E}+00$
$11 \quad 11$ 4s $1.00309 \mathrm{E}-011.00000 \mathrm{E}+000.00000 \mathrm{E}+000.00000 \mathrm{E}+00 \quad 0 \quad 1.0000 \mathrm{E}+00$
$12 \quad 12 \quad 0 \quad 0.00000 \mathrm{E}+000.00000 \mathrm{E}+000.00000 \mathrm{E}+000.00000 \mathrm{E}+00 \quad 01.0000 \mathrm{E}+00$
$131300.00000 \mathrm{E}+000.00000 \mathrm{E}+000.00000 \mathrm{E}+000.00000 \mathrm{E}+00 \quad 0 \quad 1.0000 \mathrm{E}+00$
$14 \quad 14 \quad 0 \quad 0.00000 \mathrm{E}+00 \quad 0.00000 \mathrm{E}+00 \quad 0.00000 \mathrm{E}+000.00000 \mathrm{E}+00 \quad 0 \quad 1.0000 \mathrm{E}+00$
is is $0 \quad 0.00000 \mathrm{E}+000.00000 \mathrm{E}+000.00000 \mathrm{E}+00 \quad 0.00000 \mathrm{E}+00 \quad 0 \quad 1.0000 \mathrm{E}+00$
$16 \quad 16 \quad 4 \mathrm{~s} \quad 1.00309 \mathrm{E}+01 \quad 1.00000 \mathrm{E}+00 \quad 0.00000 \mathrm{E}+000.00000 \mathrm{E}+00 \quad 0 \quad 1.0000 \mathrm{E}+00$
$17 \quad 17 \quad 4 \mathrm{~s} \quad 1.00309 \mathrm{E}-011.00000 \mathrm{E}+000.00000 \mathrm{E}+000.00000 \mathrm{E}+00 \quad 0 \quad 1.0000 \mathrm{E}+00$
$18 \quad 18 \quad 6 \quad 8.51890 \mathrm{E}-027.90000 \mathrm{E}+000.00000 \mathrm{E}+000.00000 \mathrm{E}+00 \quad 0 \quad 1.0000 \mathrm{E}+00$
$19 \quad 19 \quad 7 \quad 8.58502 \mathrm{E}-027.08825 \mathrm{E}+000.00000 \mathrm{E}+000.00000 \mathrm{E}+00 \quad 0 \quad 1.0000 \mathrm{E}+00$
$20 \quad 20 \quad 0 \quad 0.00000 \mathrm{E}+00 \quad 0.00000 \mathrm{E}+000.00000 \mathrm{E}+000.00000 \mathrm{E}+00 \quad 0 \quad 0.0000 \mathrm{E}+00$

total

$0.00000 \mathrm{E}+000.00000 \mathrm{E}+00$

11 warning messages so far.

1cross-section tables

print table 100

table length

tables from file testlibi

$1001.00 \mathrm{c} 2139$ 1-h-l from endf-vi.1

mat $125 \quad 05 / 26 / 93$

$5010.03 d 3945$ b-10 endf/b-iv new gamma production format $\quad$ (1273) 14 oct 75

$5011.40 \mathrm{c} 3473$ ENDL library name: nd900719 MCNP translation: 900831 14:10:05 900207

6012.40c 5049 ENDL library name: nd900719 MCNP translation: 900831 14:10:05

8016.40c 5693 ENDL library name: nd900719 MCNP tansiation: 900831 14:10:05 900207

14000.40 8732 ENDL library name: nd900719 MCNP translation: 900831 14:10:05

26000.40c 21846 ENDL library name: nd900719 MCNP translation: $90083114: 10: 05 \quad 900207$

900.40c 111279 ENDL library name: nd900719 MCNP translation: 900831 14:10:05

35.40c 40506 ENDL library name: nd900719 MCNP translation: 9total nu 10:05

900503 
92238.40c 48310 ENDL library name: nd901118 MCNP translation: 9total nu $56: 49$

HNF-SD-SNF-CSWD-005, Rev. 0

$10010010 / 22 / 85$

901119

261165

waming. neutron energy cutoff is below some cross-section tables.

decimal words of dynamically allocated storage

source distribution written to file inp24s cycle $=0$

12 waming messages so far.

lestimated keff results by cycle

print table 175

cycle $1 \mathrm{k}$ (collision) 0.658210 removal lifetime(abs) $5.1517 \mathrm{E}+03$ source points generated 228

cycle $2 \mathrm{k}$ (callision) 0.549318 removal lifetime(abs) $3.7645 \mathrm{E}+03$ source points generated 209

cycle $3 \mathrm{k}$ (collision) 0.551862 removal lifetime(abs) $3.8422 \mathrm{E}+03$ source points generated 253

source distribution written to file inp24s cycle $=3$

estimator cycle 4 ave of 2 cycles combination simple average combined average corr

$\begin{array}{lllllll}\text { k(collision) } & 0.584886 & 0.5683740 .0291 & \mathrm{k} \text { (col/abs) } & 0.0000000 .0000 & 0.0000000 .0000 & 0.0000\end{array}$

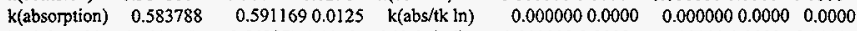

$\begin{array}{lllllll}\mathrm{k}(\mathrm{trk} \text { length }) & 0.582547 & 0.568877 & 0.0240 & \mathrm{k} \text { (tk } \ln / \mathrm{col}) & 0.0000000 .0000 & 0.0000000 .0000 \quad 0.0000\end{array}$

rem life(col) $3.9485 \mathrm{E}+03 \quad 3.8951 \mathrm{E}+030.0137$

$\begin{array}{lllllll}\text { rem life(abs) } & 4.0658 \mathrm{E}+03 & 3.9540 \mathrm{E}+03 & 0.0283 \quad \text { life(col/abs) } & 0.0000 \mathrm{E}+00 & 0.0000 & 0.0000 \mathrm{E}+000.0000 \quad 0.0000\end{array}$

source points generated 264

rce distribution written to file inp $24 \mathrm{~s} \quad$ cycle $=4$

problem summary

run terminated when 4 kcode cycles were done.

neutron creation tracks weight energy neutron loss tracks weight energy

(per source particle)

(per source particle)

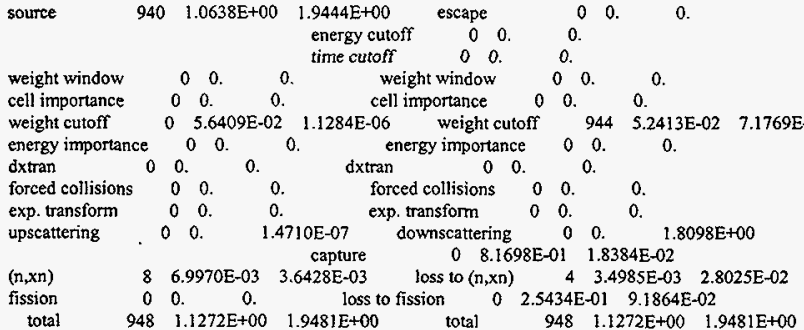

number of neutrons banked 4 average lifetime, shakes cutoffs

neutron tracks per source particle $\quad 1.0085 \mathrm{E}+00 \quad$ escape $0.0000 \mathrm{E}+00$ tco $1.0000 \mathrm{E}+34$

neutron collisions per source particle 6.1389E+01 capture 4.1766E+03 eco $0.0000 \mathrm{E}+00$

total neutron collisions $\quad 57706 \quad$ capture or escape 4.1766E+03 wcl $\quad-5.0000 \mathrm{E}-01$

net multiplication $\quad 1.0033 \mathrm{E}+000.0030$ any termination $4.4650 \mathrm{E}+03 \quad$ wc2 $-2.5000 \mathrm{E}-01$

$\begin{array}{llll}\text { computer time so far in this run } & 0.00 \text { minutes } & \text { maximum number ever in bank } & 1 \\ \text { pputer time in morun } & 0.00 \text { minutes } & \text { bank overflows to backup file } & 0\end{array}$

$0.0000 E+00$

field length

0 


$\begin{array}{cccccccccccc}1 & 1 & 15187 & 944 & 4054 & 3.5039 \mathrm{E}+00 & 3.6399 \mathrm{E}-04 & 6.5723 \mathrm{E}-01 & 8.4316 \mathrm{E}-01 & 3.8971 \mathrm{E}+00 \\ 2 & 2 & 29440 & 944 & 0 & 0.0000 \mathrm{E}+00 & 3.3819 \mathrm{E}-04 & 6.3029 \mathrm{E}-01 & 8.4006 \mathrm{E}-01 & 9.6437 \mathrm{E}+03 \\ 3 & 3 & 31610 & 944 & 1028 & 9.2538 \mathrm{E}-01 & 3.1626 \mathrm{E}-04 & 6.0475 \mathrm{E}-01 & 8.3961 \mathrm{E}-01 & 3.6368 \mathrm{E}+00 \\ 4 & 4 & 44969 & 944 & 40660 & 3.0973 \mathrm{E}+01 & 3.0008 \mathrm{E}-04 & 5.9877 \mathrm{E}-01 & 8.3882 \mathrm{E}-01 & 1.4858 \mathrm{E}+00 \\ 5 & 5 & 8213 & 845 & 8775 & 6.4600 \mathrm{E}+00 & 2.0190 \mathrm{E}-04 & 5.0976 \mathrm{E}-01 & 8.1000 \mathrm{E}-01 & 1.3256 \mathrm{E}+00 \\ 6 & 6 & 4924 & 791 & 109 & 9.6195 \mathrm{E}-02 & 2.0789 \mathrm{E}-04 & 5.2658 \mathrm{E}-01 & 8.2057 \mathrm{E}-01 & 3.6398 \mathrm{E}+00 \\ 7 & 7 & 2832 & 638 & 1607 & 1.4612 \mathrm{E}+00 & 8.5166 \mathrm{E}-04 & 7.1930 \mathrm{E}-01 & 9.4163 \mathrm{E}-01 & 1.7232 \mathrm{E}+00 \\ 8 & 8 & 0 & 0 & 0 & 0.0000 \mathrm{E}+00 & 0.0000 \mathrm{E}+00 & 0.0000 \mathrm{E}+00 & 0.0000 \mathrm{E}+00 & 0.0000 \mathrm{E}+00 \\ 9 & 9 & 1333 & 607 & 1347 & 1.2836 \mathrm{E}+00 & 3.8320 \mathrm{E}-03 & 8.0017 \mathrm{E}+01 & 9.7093 \mathrm{E}-01 & 3.1777 \mathrm{E}+00 \\ 10 & 10 & 0 & 0 & 0 & 0.0000 \mathrm{E}+00 & 0.0000 \mathrm{E}+00 & 0.0000 \mathrm{E}+00 & 0.0000 \mathrm{E}+00 & 0.0000 \mathrm{E}+00 \\ 11 & 11 & 7 & 1 & 126 & 9.7846 \mathrm{E}-02 & 9.0288 \mathrm{E}-05 & 3.2866 \mathrm{E}-01 & 7.7027 \mathrm{E}-01 & 1.0087 \mathrm{E}+00 \\ 12 & 12 & 0 & 0 & 0 & 0.0000 \mathrm{E}+00 & 0.0000 \mathrm{E}+00 & 0.0000 \mathrm{E}+00 & 0.0000 \mathrm{E}+00 & 0.0000 \mathrm{E}+00 \\ 13 & 13 & 0 & 0 & 0 & 0.0000 \mathrm{E}+00 & 0.0000 \mathrm{E}+00 & 0.0000 \mathrm{E}+00 & 0.0000 \mathrm{E}+00 & 0.0000 \mathrm{E}+00 \\ 14 & 14 & 0 & 0 & 0 & 0.0000 \mathrm{E}+00 & 0.0000 \mathrm{E}+00 & 0.0000 \mathrm{E}+00 & 0.0000 \mathrm{E}+00 & 0.0000 \mathrm{E}+00 \\ 15 & 15 & 0 & 0 & 0 & 0.0000 \mathrm{E}+00 & 0.0000 \mathrm{E}+00 & 0.0000 \mathrm{E}+00 & 0.0000 \mathrm{E}+00 & 0.0000 \mathrm{E}+00 \\ 16 & 16 & 0 & 0 & 0 & 0.0000 \mathrm{E}+00 & 0.0000 \mathrm{E}+00 & 0.0000 \mathrm{E}+00 & 0.0000 \mathrm{E}+00 & 0.0000 \mathrm{E}+00 \\ 17 & 17 & 0 & 0 & 0 & 0.0000 \mathrm{E}+00 & 0.0000 \mathrm{E}+00 & 0.0000 \mathrm{E}+00 & 0.0000 \mathrm{E}+00 & 0.0000 \mathrm{E}+00 \\ 18 & 18 & 0 & 0 & 0 & 0.0000 \mathrm{E}+00 & 0.0000 \mathrm{E}+00 & 0.0000 \mathrm{E}+00 & 0.0000 \mathrm{E}+00 & 0.0000 \mathrm{E}+00 \\ 19 & 19 & 0 & 0 & 0 & 0.0000 \mathrm{E}+00 & 0.0000 \mathrm{E}+00 & 0.0000 \mathrm{E}+00 & 0.0000 \mathrm{E}+00 & 0.0000 \mathrm{E}+00\end{array}$

total $\quad 138515 \quad 6658 \quad 57706 \quad 4.4801 E+01$

the initial fission neutron source distribution used the 1 source points that were input on the ksrc card.

the criticality problem was scheduled to skip 2 cycles and run a total of 4 cycles with nominally 250 neutrons per cycle. problem has run 2 inactive cycles with 478 neutron histories and 2 active cycles with 462 neutron histories.

this calculation has completed the requested number of keff cycles using a total of 940 fission neutron source histories. all cells with fissionable material were sampled and had fission neutron source points.

there is no combined collision/absorption/track-length estimate for keff because only 2 active cycles were run.

the estimated average keffs, one standard deviations, and 68,95 , and 99 percent confidence intervals are:

$\begin{array}{cccccccc}\text { keff estimator } & \text { keff } & \text { standard deviation } & 68 \% \text { confidence } & 95 \% \text { confidence } & 99 \% \text { confidence } & \text { cort } \\ \text { collision } & 0.56837 & 0.01651 & 0.53834 \text { to } 0.59841 & 0.35851 \text { to } 0.77824 & 0.00000 \text { to } 1.61951 \\ \text { absorption } & 0.59117 & 0.00738 & 0.57774 \text { to } 0.60460 & 0.49735 \text { to } 0.68498 & 0.12129 \text { to } 1.06105 \\ \text { track length } & 0.56888 & 0.01367 & 0.54401 \text { to } 0.59374 & 0.39513 \text { to } 0.74263 & 0.00000 \text { to } 1.43912 \\ \text { dividual and average keff estimator results by cycle } & & & \end{array}$

lindividual and average keff estimator results by cycle

keff neutron keff estimators by cycle average keff estimators and deviations average $k(c / a / t)$ cycle histories $k$ (coll) $k$ (abs) $k$ (track) $k$ (coll) st dev $k$ (abs) st dev $k$ (track) st dev $k$ (c/a/t) st dev fom

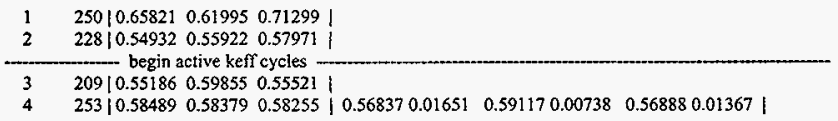

the largest active cycle keffs by estimator are:

collision 0.58489 on cycle 4 absorption 0.59855 on cycle 3 track length 0.58255 on cycle 4 the smatlest active cycle keffs by estimator are:

collision 0.55186 on cycle 3 absorption 0.58379 on cycle 4 track length 0.55521 on cycle 3 
1 taily $6 \quad \mathrm{nps}=940$

HNF-SD-SNF-CSWD-005, Rev. 0

tally type 6 track length estimate of heating. units mev/gram

tally for neutrons

number of histories used for nomalizing tallies $=500.00$

masses

cell: $\begin{array}{lllll}8 & 12 & 13 & 14 & 15\end{array}$

$1.00000 \mathrm{E}+00 \quad 1.00000 \mathrm{E}+00 \quad 1.00000 \mathrm{E}+00 \quad 1.00000 \mathrm{E}+00 \quad 1.00000 \mathrm{E}+00$

cell 8

$4.02566 \mathrm{E}+010.0434$

cell 12

$2.65224 E+010.0629$

cell 13

\section{$1.32494 \mathrm{E}+010.0904$}

cell 14

$9.78180 \mathrm{E}-010.4014$

cell 15

1.20234E-01 0.9710

lanalysis of the results in the tally fluctuation chart bin (tfe) for tally 6 with nps $=940$ print table 160

normed average tally per history $=4.02566 \mathrm{E}+01$ estimated tally relative error $=0.0434$

relative error from zero tallies $=0.0128$

number of nonzero history tallies $=\quad 462$

history number of largest tally $=\quad 641$

(largest tally)/(average tally) $=6.75049 \mathrm{E}+00$

fidence interval shift $/$ mean $=0.0014$ unnormed average tally per history $=4.02566 \mathrm{E}+0$.

estimated variance of the variance $=0.0095$

relative error from nonzero scores $=0.0415$

efficiency for the nonzero tallies $=0.9240$

largest unnormalized history tally $=2.71752 \mathrm{E}+02$

(largest tally) $/$ (avg nonzero tally) $=6.23745 \mathrm{E}+00$

shifted confidence interval center $=4.03113 \mathrm{E}+01$

if the largest history score sampled so far were to occur on the very next history, the tfc bin quantities would change as follows: nps $=462$ for this table because 2 keff cycles and 478 histories were skipped before tally accumulation.

$\begin{array}{lccc}\text { estimated quantities } & \text { value at nps } & \text { value at nps+1 } & \text { value(nps+1)/value(nps)-1. } \\ \text { mean } & & & \\ \text { relative error } & 4.02566 \mathrm{E}+01 & 4.07566 \mathrm{E}+01 & 0.012420 \\ \text { variance of the variance } & 4.34431 \mathrm{E}-02 & 4.24506 \mathrm{E}-02 & -0.022846 \\ \text { shifted center } & 9.52674 \mathrm{E}-03 & 1.33327 \mathrm{E}-02 & 0.399508 \\ \text { figure of merit } & 4.03113 \mathrm{E}+01 & 4.03457 \mathrm{E}+01 & 0.000853 \\ & 0.00000 \mathrm{E}+00 & 0.00000 \mathrm{E}+00 & 0.000000\end{array}$

there is not enough information in the largest history scores (usually less than 500 scores) for a reliable estimate of the slope. the history score probability density function appears to have an unsampled region at the largest history scores: please examine.

****** the nps-dependent tfc bin check results are suspect because there are only 1 nps tally values to analyze *****

results of 10 statistical checks for the estimated answer for the tally fluctuation chart (tfe) bin of tally 6

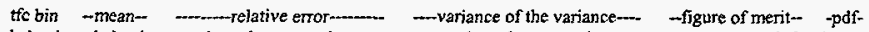

behavior behavior value decrease decrease rate value decrease decrease rate value behavior slope

$\begin{array}{llllll}\text { Aesired random } & <0.10 \text { yes } 1 / \text { sqrt(nps) }<0.10 \text { yes } 1 / \text { mps } & \text { constant random }>3.00 \\ \text { ferved random } & 0.04 \text { yes yes } 0.01 \text { yes yes } & 0.00 & \text { no } & \text { yes yes yes } & \end{array}$


waming. the tally in the tally fluctuation chart bin did not pass 1 of the 10 statistical checks.

lunnomed tally density for tally $6 \quad$ nonzero tally mean $(m)=4.357 \mathrm{E}+01 \mathrm{nps}=940$ print table 161

abscissa ordinate log plot of tally probability density function in tally fluctuation chart bin $(d=$ decade,slope $=0.0$ )

tally number num den log den:d-_-

$2.51-01 \quad 13.87-02-1.412 * * * * * * * * * * * * * * * * * * * * * * * * * * * *|* * * * * * * * * * * * * * * * * * * * * * * * * * *| * * * * * * * * * * * * * * * * * * * * * * * * * * * * * \mid * * * *$

$3.16-01 \quad 26.15-02-1.211 * * * * * * * * * * * * * * * * * * * * * * * * * * * * *|* * * * * * * * * * * * * * * * * * * * * * * * * * * * *| * * * * * * * * * * * * * * * * * * * * * * * * * k * * * * \mid * * * * * * * * * * *$

3.98-01 $12.44-02-1.612 * * * * * * * * * * * * * * * * * * * * * * * * * * *|* * * * * * * * * * * * * * * * * * * * * * * * * * *| * * * * * * * * * * * * * * * * * * * * * * * * * * * * \mid$

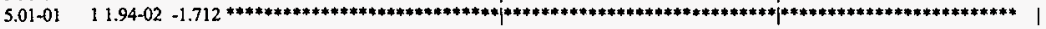

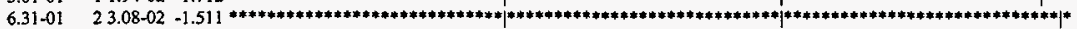

7.94-01 $33.67-02-1.435 * * * * * * * * * * * * * * * * * * * * * * * * * * * *|* * * * * * * * * * * * * * * * * * * * * * * * * * * *|+* * * * * * * * * * * * * * * * * * * * * * * * * * * * \mid * * *$

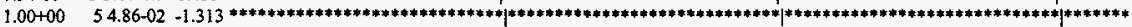

$1.26+00 \quad 17.72-03-2.112 * * * * * * * * * * * * * * * * * * * * * * * * * * * *|* * * * * * * * * * * * * * * * * * * * * * * * * * * * *| * * * * * * * * * * * * \mid$

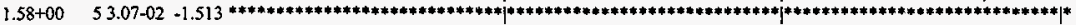

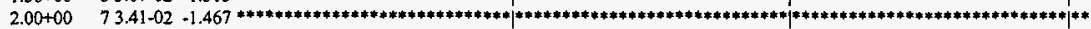

$2.51+00 \quad 62.32-02-1.634 * * * * * * * * * * * * * * * * * * * * * * * * * * * * * ; * * * * * * * * * * * * * * * * * * * * * * * * * * * * j * * * * * * * * * * * * * * * * * * * * * * * * * * * \mid$

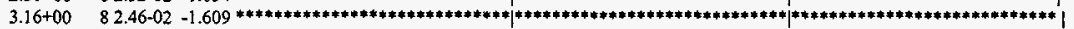

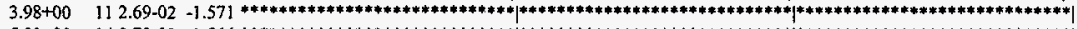

$5.01+00 \quad 142.72-02-1.566 * * * * * * * * * * * * * * * * * * * * * * * * * * * * * *|* * * * * * * * * * * * * * * * * * * * * * * * * * * * * *| * * * * * * * * * * * * * * * * * * * * * * * * * * * * * * * \mid$

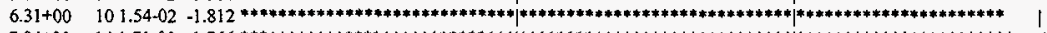

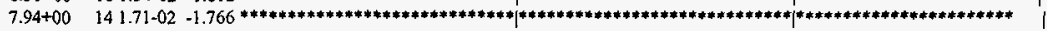

$1.00+01 \quad 131.26-02-1.898 * * * * * * * * * * * * * * * * * * * * * * * * * * *|* * * * * * * * * * * * * * * * * * * * * * * * * * * *| * * * * * * * * * * * * * * * * * * * \mid$

$1.26+01 \quad 161.24-02-1.908 * * * * * * * * * * * * * * * * * * * * * * * * * * * *|* * * * * * * * * * * * * * * * * * * * * * * * * * * *| * * * * * * * * * * * * * * * * * * *$

$1.58+01$ II $6.75-03-2.171 * * * * * * * * * * * * * * * * * * * * * * * * * * * *|* * * * * * * * * * * * * * * * * * * * * * * * * * * *| * * * * * * * * * * *$

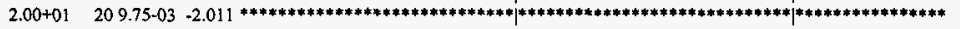

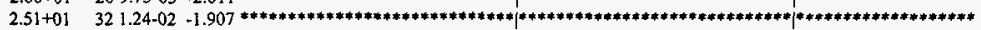

$3.16+01$ 41 $1.26-02-1.899 * * * * * * * * * * * * * * * * * * * * * * * * * * * * *|* * * * * * * * * * * * * * * * * * * * * * * * * * * * *| * * * * * * * * * * * * * * * * * * * *$

$3.98+01 \quad 245.86-03 \quad-2.232 * * * * * * * * * * * * * * * * * * * * * * * * * * * * * *|* * * * * * * * * * * * * * * * * * * * * * * * * * * * *| * * * * * * * * *$

$5.01+01 \quad 489.31-03 \quad-2.031$

nmmmmmmmmmmmmmmmmmmmmmmmmmmm| nmmmmmmmmmmmmmmmmmmmmmmmmmmmm/mmmmmmmmmmmmmmm

$+01548.32-03-2.080 * * * * * * * * * * * * * * * * * * * * * * * * * * * * * * \mid * * * * * * * * * * * * * * * * * * * * * * * * * * * * * j * * * * * * * * * * * * * *$

$4+01 \quad 404.90-03 \quad-2.310 * * * * * * * * * * * * * * * * * * * * * * * * * * * * *|* * * * * * * * * * * * * * * * * * * * * * * * * * * * *| * * * * * * *$

$1.00+02 \quad 282.72-03 \quad-2.565 * * * * * * * * * * * * * * * * * * * * * * * * * * * * *|* * * * * * * * * * * * * * * * * * * * * * * * * * * * *|$

$1.26+02201.54-03-2.811 * * * * * * * * * * * * * * * * * * * * * * * * * * * * *|* * * * * * * * * * * * * * * * * * * * *|$

$1.58+02 \quad 211.29-03-2.890 * * * * * * * * * * * * * * * * * * * * * * * * * * * * *|* * * * * * * * * * * * * * * * * * * *|$

$2.00+02 \quad 29.75-05-4.011 * * * * * * * * * * * * * * * *$

$2.51+02 \quad 00.00+00 \quad 0.000$

$3.16+02 \quad 13.08-05-4.512 *$

total 462 9.24-01 d-a.n.

1status of the statistical checks used to form confidence intervals for the mean for each tally bin

tally result of statistical checks for the tfc bin (the first check not passed is listed) and error magnitude check for all bins

6 missed 1 of 10 tf $\mathrm{c}$ bin checks: there is insufficient tfc bin tally information to estimate the large tally slope reliably missed all bin error check: 5 tally bins had 0 bins with zeros and 2 bins with relative errors exceeding 0.10

the 10 statistical checks are only for the tally fluctuation chart bin and do not apply to other tally bins.

waming. I f the 1 tally fluctuation chart bins did not pass all 10 statistical checks.

warning. $\mathrm{i}$ of the 1 tallies had bins with relative errors greater than recommended.

Itally fluctuation charts

$$
\text { tally } 6
$$

nps mean error vov slope fom

$9404.0257 \mathrm{E}+010.04340 .00950 .0$

tally data written to file inp $24 \mathrm{~m}$

15 waming messages so far.

terminated when 4 ksode cycles were done. 


\section{File: Inp180}

1. probl 8 - kcode in a hexagonal prism lattice.

2- c three half control rods and five whole cortrol rods.

3. $\quad 30 \quad 0-905-19291$ fill $=1$

4- 31 $0-906-19291$ fill=1 $(16.711300)$

5- $37 \quad 0-907-19291$ fill $=1(-16.711300)$

6- $\quad 34 \quad 0-913-1929$ fill $=1$ ( 011.91850$)$

$7-\quad 32 \quad 0-914-1929$ fill $=1(10.32175 .95920)$

8. $33 \quad 0-915-1929$ fill $=1(8.355714 .47240)$

9- $35 \quad 0-916-1929$ fill $=1(-8.355714 .47240)$

10- $36 \quad 0-917-1929$ fill $=1(-10.32175 .95920)$

11- c universe I: structure of control rod.

12. $38 \quad 11-2.02 \quad-880 \quad u=1 \quad \$$ control rod core

13- $39 \quad 6 \quad-8.4 \quad 880-881 \quad u=1 \quad \$$ control rod cladding

14- $40 \quad 12-1.00 \quad 881-882 \quad \mathrm{u}=1 \quad \$$ control rod gap

15- $41 \quad 6 \quad-8.4 \quad 882 \quad u=1 \quad \$$ control rod sheath

16- $\mathrm{c}$ the space between the control rods, filled with lattice.

17. $\quad 140 \quad 0-17129-19905906907913914915916917$ fill=2

18- c universe 2: lattice of fuel rods with water in between.

19- $42 \quad 12-1.00 \quad-301 \quad 302 \quad-303 \quad 304-305306 \quad \mathrm{u}=2 \quad \mathrm{lat}=2 \quad \mathrm{fill}=$

20- $-37: 27-1: 33$ 0:0 \&

21- $\quad 24 r 39 r 24 r 311 r 24 r 311 r 24 r 39+2$

22- $\quad 24 r 39 r^{2} 3 r 312 r 23 r 312 r 23 r 39 r 21 r$

23. $\quad 23 r 310+22 r 313 r 22 r 313 r 22 r 310 r 2$ ir

24. $\quad 23 r 35722 \mathrm{r} \&$

25. $\quad 22 \mathrm{r} 358 \mathrm{r} 22 \mathrm{r}$

26- $\quad 22 r 316 r 22 r 317 r 22 r 316 r 23 r$

27. $\quad 22 \mathrm{r} 315+23 \mathrm{r} 316 \mathrm{r} 3 \mathrm{r} 315 \mathrm{r} 24 \mathrm{r}$

28- $\quad 21 r 315 r 24 r 315 r 24 r 315 r 24 r$

2 r $315 r 23 r 316 r 23 r 315 r 25 r$

2 ir $315 r 22+317 r 22 r 315 r 26 r$ $21 r 354 \pi 27 r \&$

32- \& can code remember \& thru comment?

33- $\quad 2355 \mathrm{r} 27 \mathrm{r}$

$34-$

35.

$36-$

37.

38.

39.

$40-$

$41-$

42-

$43-$

44.

45 -

$46-$

$47-$

$2325 r 22$ r $325 \pi 28 \mathrm{r}$

$2324 \mathrm{r} 23 \mathrm{r} 324 \mathrm{r} 29 \mathrm{r}$

$2323 r 24 r 323 r 2$ 10r

$2315 r 22 \mathrm{r} 34 \mathrm{r} 23 \mathrm{r} 34 \mathrm{rr} 2 \mathrm{r} 315 \mathrm{r} 211 \mathrm{r}$

$2314 r 23 r 34 r 22 r 34 r 23 r 314 r 212 r$

$2313 r 24 r 311 r_{2} 4 r_{3} 13 r 213 r$

$2313 r 23 r 312 r 23 r 313 r 214 r$

$2313 \mathrm{r} 22 \mathrm{r} 313 \mathrm{r} 22 \mathrm{r} 313 \mathrm{r} 215 \mathrm{r}$

$2346 \mathrm{r} 216 \mathrm{r}$

$2345 \mathrm{r} 217 \mathrm{r}$

$2344 \mathrm{r} 218 \mathrm{r}$

$2 \operatorname{lr} 341 \mathrm{r} 20 \mathrm{r}$

2 ir $340 \mathrm{r} 221 \mathrm{r}$

2 ir $339 \mathrm{r} 222 \mathrm{r}$

$22 \mathrm{r} 336 \mathrm{r} 224 \mathrm{r}$

$22 r 335 r 225 r$

2 3r 332 r $227 \mathrm{r}$

$24 \mathrm{r} 329 \mathrm{r} 229 \mathrm{r}$

$25 \mathrm{r} 326 \mathrm{r} 231 \mathrm{r}$

$26 r 323 r 233 r$

$28 \mathrm{r} 318 \mathrm{r} 236 \mathrm{r}$

$211 \mathrm{r} 311 \mathrm{rr} 40 \mathrm{r}$

$264 \mathrm{~T}$

c universe 3: structure of fuel rod lattice elements

$\begin{array}{lllll}154 & 2 & -13.75 \quad-58 \quad u=3 & \$\end{array}$ fuel eiement

$149 \quad 12 \cdot 1.00 \quad 58-268 \quad \mathrm{u}=3 \quad \$$ gap

$144 \quad 7-19.66 \quad 268-478 \quad u=3$ Sliner

$\begin{array}{llllll}159 & 6 & -8.4 & 478 & -698 & u=3\end{array}$ s cladding

$\begin{array}{lllll}141 & 12 & -1.00 & 698 & u=3\end{array} \mathrm{~S}$ water between the fuel rods 
*1 py $0 \quad \$ \times-2$ plane, reflective

17 cz 29.135

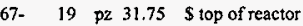

68- $29 \mathrm{pz}-31.75$ \$bottom of reactor

$\begin{array}{llllll}69 . & 58 & \mathrm{c} / z & 3.4414 & .8515 & .3240\end{array}$

70- $\quad 268 \mathrm{c} / \mathrm{z} \quad 3.4414 \quad .8515 \quad .3345$

$\begin{array}{llllll}71 . & 478 & \mathrm{c} / z & 3.4414 & .8515 & .3475\end{array}$

$\begin{array}{llllll}72- & 698 & \mathrm{c} / \mathrm{z} & 3.4414 & .8515 & .4318\end{array}$

73- $\quad 880 \mathrm{cz} \quad 1.7251$

74- $\quad 881 \quad \mathrm{cz} \quad 1.8051$

75- $\quad 882 \mathrm{cz} 1.9051$

76- $905 \mathrm{cz} 2.1055$

77. $906 \quad \mathrm{c} / 2 \quad 16.711302 .1055$

78- $\quad 907 \mathrm{c} / \mathrm{z}-16.711302 .1055$

79. $\quad 913 \mathrm{c} / 2011.91852 .1055$

80- $\quad 914 \quad c / z \quad 10.32175 .95922 .1055$

81- $\quad 915 \quad c / z \quad 8.3557 \quad 14.47242 .1055$

82- $\quad 916 \mathrm{c} / \mathrm{z}-8.3557$ [4.4724 2.1055

$83-\quad 917 \quad c / z-10.32175 .95922 .1055$

84- 301 px 3.9330

85- $\quad 302$ px 2.9498

86- $\quad 303$ p $11.73205080760 \quad 5.8994$

87. $\quad 304$ p $11.73205080760 \quad 3.9330$

88- $\quad 305$ p $-11.73205080760 \quad-.9834$

89- 306 p $-11.73205080760-2.9498$

90.

91- imp:n 1 18: 0

$\begin{array}{lllllllll}92- & \mathrm{m} 2 & 92235.40 \mathrm{c} & -.70573 & 92238.40 \mathrm{c} & -.23821 & 7014.40 \mathrm{c} & -.05605\end{array}$

93- m6 41093.40c $-.99000 \quad 40000.40 \mathrm{c}-.01000$

94- $\mathrm{m} 7 \quad 74000.40 \mathrm{c} \cdot .74000$

95- $\mathrm{mll} \quad 5010.03 \mathrm{~d}-.6870 \quad 5011.40 \mathrm{c}-.0840 \quad 6012.40 \mathrm{c}-.2290$

$\mathrm{ml2} \quad 1001.00 \mathrm{c}$ ] $1002.55 \mathrm{c} 1 \quad 8016.40 \mathrm{c} 1$

mt12 hwtr.01 Jwtr.01

kcode $200 \quad 156$

waming. tallies are normed per fission neutron for one generation.

99- kstc $3.2 .2 .23 .2-3.2 .2 .23 .243 .2-43.243 .1-43.2$

$100-$ e .01 .11 .10 .

101- fq $\mathrm{fe}$

102- fc4 fuel rod flux in 5 y locations averaged over $5 x$ elements

103- f4:n $(154<(42[-10:-6-10]))$ average $5 x$ elements at $j=-1$

104- $\quad(154<(42[-10:-630])) \&$ \& average $5 x$ elements at $j=3$

105- $\quad(154<(42[-10:-6100])) \$$ average $5 x$ elements at $j=10$ \&

106- $\quad(154<(42[-10:-6210])) \quad \$$ average $5 \times$ elements at $j=21$

$107-\quad(154<(42[-10:-6290])) \quad \$$ average $5 \times$ elements at $j=29$

108- sd4 $104.70890624 \Gamma \quad \$ 5$ times the volume of cell 154

109. print -98

110- prdmp $2 \mathrm{j}-1$

111- ptrac buffer $=20$ file $=$ asc write $=$ all event $=$ bnk

1 initial source from ksre card.

print table 90

$\begin{array}{ll}\text { original number of points } & \\ \begin{array}{l}\text { points not in any cell } \\ \text { points in cells of zero importance }\end{array} & 0 \\ \text { points in void cells } & 0 \\ \text { points in ambiguous cells } & 0 \\ \text { total points rejected } & 0 \\ \text { points remaining } & 8 \\ \text { points after expansion or contraction } & \end{array}$

nominal source size

initial guess for $k$ (eff.)

1.000000

cxcles to skip before tallying

8

0

0

0

200

5 
total fission nubar data are being used.

HNF-SD-SNF-CSWD-005, Rev. 0

\section{Mrint table 30}

fuel rod flux in $5 y$ locations averaged over $5 x$ elements

tally type 4 track length estimate of particle flux.

tally for neutrons

order of printing: $\mathrm{fe}$

cells $(154<(42[-10:-6-10]))(154<(42[-10:-630]))(154<(42[-10:-6100]))(154<(42[-10 ;-6210]))(154<(42[-10:-6$

$290])$ )

\section{energy bins \\ $0.00000 \mathrm{E}+00$ to $1.00000 \mathrm{E}-02 \mathrm{mev}$ \\ $1.00000 \mathrm{E}-02$ to $1.00000 \mathrm{E}-01 \mathrm{mev}$ \\ $1.00000 \mathrm{E}-01$ to $1.00000 \mathrm{E}+00 \mathrm{mev}$ \\ $1.00000 \mathrm{E}+00$ to $1.00000 \mathrm{E}+01 \mathrm{mev}$ \\ total bin}

Imaterial composition

print table 40

\begin{abstract}
the sum of the fractions of material 2 was 9.999900E-01
\end{abstract}
the sum of the fractions of material 7 was $7.400000 \mathrm{E}-01$

the sum of the fractions of material 12 was $3.000000 \mathrm{E}+00$

\section{material}

number component nuclide, atom fraction

$\begin{array}{crrr}2 & 92235,0.37504 & 92238,0.12499 & 7014,0.49997 \\ 6 & 41093,0.98982 & 40000,0.01018 & \\ 7 & 74000,1.00000 & & \\ 11 & 5010,0.71977 & 5011,0.08004 & 6012,0.20019 \\ 12 & 1001,0.33333 & 1002,0.33333 & 8016,0.33333\end{array}$

associated thermal s $(a, b)$ data sets: hwtr.01t lwtr.01t material

number component nuclide, mass fraction

$\begin{array}{rrrr}2 & 92235,0.70574 & 92238,0.23821 & 7014,0.05605 \\ 6 & 41093,0.99000 & 40000,0.01000 & \\ 7 & 74000,1.00000 & & \\ 11 & 5010,0.68700 & 5011,0.08400 & 6012,0.22900 \\ 12 & 1001,0.05300 & 1002,0.10591 & 8016,0.84109\end{array}$

waming. 3 of the materials had unnormalized fractions.

lcell volumes and masses

print table 50

$\begin{array}{rcccc}\text { cell atom } & \text { gram } & \text { input } & \text { calculated } \\ \text { density } & \text { density } & \text { volume } & \text { volume }\end{array}$ mass $\quad \begin{gathered}\text { reason volume } \\ \text { pieces not calculated }\end{gathered}$

$\begin{array}{lllllllll}1 & 30 & 0.00000 \mathrm{E}+00 & 0.00000 \mathrm{E}+00 & 0.00000 \mathrm{E}+00 & 0.00000 \mathrm{E}+00 & 0.00000 \mathrm{E}+00 & 0\end{array}$

$\begin{array}{lllllllll}2 & 31 & 0.00000 \mathrm{E}+00 & 0.00000 \mathrm{E}+00 & 0.00000 \mathrm{E}+00 & 0.00000 \mathrm{E}+00 & 0.00000 \mathrm{E}+00\end{array}$

$\begin{array}{llllllll}3 & 37 & 0.00000 \mathrm{E}+00 & 0.00000 \mathrm{E}+00 & 0.00000 \mathrm{E}+00 & 0.00000 \mathrm{E}+00 & 0.00000 \mathrm{E}+00 & 0\end{array}$

$\begin{array}{llllllll}434 & 0.00000 \mathrm{E}+00 & 0.00000 \mathrm{E}+00 & 0.00000 \mathrm{E}+00 & 8.84370 \mathrm{E}+02 & 0.00000 \mathrm{E}+00 & 1\end{array}$

$\begin{array}{lllllllll}5 & 32 & 0.00000 \mathrm{E}+00 & 0.00000 \mathrm{E}+00 & 0.00000 \mathrm{E}+00 & 8.84370 \mathrm{E}+02 & 0.00000 \mathrm{E}+00 & 1\end{array}$

$\begin{array}{lllllllll}6 & 33 & 0.00000 \mathrm{E}+00 & 0.00000 \mathrm{E}+00 & 0.00000 \mathrm{E}+00 & 8.84370 \mathrm{E}+02 & 0.00000 \mathrm{E}+00 & 1\end{array}$

$\begin{array}{llllllll}7 & 35 & 0.00000 \mathrm{E}+00 & 0.00000 \mathrm{E}+00 & 0.00000 \mathrm{E}+00 & 8.84370 \mathrm{E}+02 & 0.00000 \mathrm{E}+00 & 1\end{array}$

$\begin{array}{lllllllll}8 & 36 & 0.00000 \mathrm{E}+00 & 0.00000 \mathrm{E}+00 & 0.00000 \mathrm{E}+00 & 8.84370 \mathrm{E}+02 & 0.00000 \mathrm{E}+00 & 1\end{array}$

$\begin{array}{llllllll}9 & 38 & 1.15958 \mathrm{E}-01 & 2.02000 \mathrm{E}+00 & 0.00000 \mathrm{E}+00 & 0.00000 \mathrm{E}+00 & 0.00000 \mathrm{E}+00 & 0\end{array}$

$\begin{array}{lllllllll}10 & 39 & 5.44575 \mathrm{E}-02 & 8.40000 \mathrm{E}+00 & 0.00000 \mathrm{E}+00 & 0.00000 \mathrm{E}+00 & 0.00000 \mathrm{E}+00 & 0\end{array}$

$\begin{array}{llllllll}11 & 40 & 9.50007 \mathrm{E}-02 & 1.00000 \mathrm{E}+00 & 0.00000 \mathrm{E}+00 & 0.00000 \mathrm{E}+00 & 0.00000 \mathrm{E}+00 & 0\end{array}$

$\begin{array}{llllllll}12 & 41 & 5.44575 \mathrm{E}-02 & 8.40000 \mathrm{E}+00 & 0.00000 \mathrm{E}+00 & 0.00000 \mathrm{E}+00 & 0.00000 \mathrm{E}+00 & 0\end{array}$ $\begin{array}{lllllll}140 & 0.00000 \mathrm{E}+00 & 0.00000 \mathrm{E}+00 & 0.00000 \mathrm{E}+00 & 0.00000 \mathrm{E}+00 & 0.00000 \mathrm{E}+00 & 0\end{array}$ $\begin{array}{llllll}42 & 9.50007 \mathrm{E}-02 & 1.00000 \mathrm{E}+00 & 0.00000 \mathrm{E}+00 & 0.00000 \mathrm{E}+00 & 0.00000 \mathrm{E}+00\end{array}$

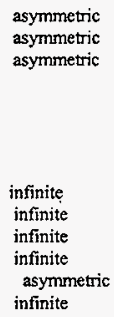


$\begin{array}{lllllll}5 & 154 & 6.62920 \mathrm{E}-02 & 1.37500 \mathrm{E}+01 & 0.00000 \mathrm{E}+00 & 0.00000 \mathrm{E}+00 & 0.00000 \mathrm{E}+00\end{array}$ $\begin{array}{llllll}149 & 9.50007 \mathrm{E}-02 & 1.00000 \mathrm{E}+00 & 0.00000 \mathrm{E}+00 & 0.00000 \mathrm{E}+00 & 0.00000 \mathrm{E}+00\end{array}$ $\begin{array}{lllllllll}7 & 144 & 6.43969 \mathrm{E}-02 & 1.96600 \mathrm{E}+01 & 0.00000 \mathrm{E}+00 & 0.00000 \mathrm{E}+00 & 0.00000 \mathrm{E}+00\end{array}$ $\begin{array}{lllllllll}18 & 159 & 5.44575 \mathrm{E}-02 & 8.40000 \mathrm{E}+00 & 0.00000 \mathrm{E}+00 & 0.00000 \mathrm{E}+00 & 0.00000 \mathrm{E}+00\end{array}$ $\begin{array}{llllllllll}19 & 141 & 9.50007 \mathrm{E}-02 & 1.00000 \mathrm{E}+00 & 0.00000 \mathrm{E}+00 & 0.00000 \mathrm{E}+00 & 0.00000 \mathrm{E}+00\end{array}$ $20 \quad 162 \quad 0.00000 \mathrm{E}+00 \quad 0.00000 \mathrm{E}+00 \quad 0.00000 \mathrm{E}+00 \quad 0.00000 \mathrm{E}+00 \quad 0.00000 \mathrm{E}+00$ Isurface areas print table 50 surface input calculated reason area
area area not calculated

$1 \quad 10.00000 \mathrm{E}+00 \quad 0.00000 \mathrm{E}+00$ asymmetric $170.00000 \mathrm{E}+00 \quad 0.00000 \mathrm{E}+00$ asymmetric $190.00000 \mathrm{E}+00 \quad 0.00000 \mathrm{E}+00$ asymmetric

$290.00000 \mathrm{E}+00 \quad 0.00000 \mathrm{E}+00$ asymmetric

$5 \quad 580.00000 \mathrm{E}+00 \quad 0.00000 \mathrm{E}+00$ infinite

$62680.00000 \mathrm{E}+00 \quad 0.00000 \mathrm{E}+00$ infinite

$7 \quad 478 \quad 0.00000 \mathrm{E}+00 \quad 0.00000 \mathrm{E}+00$ infinite

$86980.00000 \mathrm{E}+00 \quad 0.00000 \mathrm{E}+00$ infinite

$9880 \quad 0.00000 \mathrm{E}+00 \quad 0.00000 \mathrm{E}+00$ infinite

$10881 \quad 0.00000 \mathrm{E}+00 \quad 0.00000 \mathrm{E}+00$ infinite

$118820.00000 \mathrm{E}+00 \quad 0.00000 \mathrm{E}+00$ infinite

$129050.00000 \mathrm{E}+00 \quad 0.00000 \mathrm{E}+00$ asymmetric

$13 \quad 906 \quad 0.00000 \mathrm{E}+00 \quad 0.00000 \mathrm{E}+00$ asymmetric

$149070.00000 \mathrm{E}+00 \quad 0.00000 \mathrm{E}+00$ asymmetric

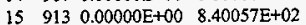

$\begin{array}{llll}16 & 914 & 0.00000 \mathrm{E}+00 & 8.40057 \mathrm{E}+02\end{array}$

$17915 \quad 0.00000 \mathrm{E}+00 \quad 8.40057 \mathrm{E}+02$

$18916 \quad 0.00000 \mathrm{E}+00 \quad 8.40057 \mathrm{E}+02$

$19917 \quad 0.00000 \mathrm{E}+00 \quad 8.40057 \mathrm{E}+02$

$203010.00000 \mathrm{E}+00 \quad 0.00000 \mathrm{E}+00$ infinite

$213020.00000 \mathrm{E}+00 \quad 0.00000 \mathrm{E}+00$ infinite

$22303 \quad 0.00000 \mathrm{E}+00 \quad 0.00000 \mathrm{E}+00$ infinite

$304 \quad 0.00000 \mathrm{E}+00 \quad 0.00000 \mathrm{E}+00$

$305 \quad 0.00000 \mathrm{E}+00 \quad 0.00000 \mathrm{E}+00$

$25306 \quad 0.00000 \mathrm{E}+00 \quad 0.00000 \mathrm{E}+00$

Icells

cell mat density density volume mass pieces importance

$130 \quad 0 \quad 0.00000 \mathrm{E}+00 \quad 0.00000 \mathrm{E}+000.00000 \mathrm{E}+00 \quad 0.00000 \mathrm{E}+00$

$23100.00000 \mathrm{E}+000.00000 \mathrm{E}+000.00000 \mathrm{E}+000.00000 \mathrm{E}+00$

$337 \quad 0 \quad 0.00000 \mathrm{E}+00 \quad 0.00000 \mathrm{E}+000.00000 \mathrm{E}+000.00000 \mathrm{E}+00$

$434 \quad 0 \quad 0.00000 \mathrm{E}+000.00000 \mathrm{E}+008.84370 \mathrm{E}+020.00000 \mathrm{E}+00$

$53200.00000 \mathrm{E}+000.00000 \mathrm{E}+008.84370 \mathrm{E}+02 \quad 0.00000 \mathrm{E}+00$

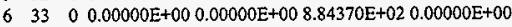

$735 \quad 0 \quad 0.00000 \mathrm{E}+000.00000 \mathrm{E}+008.84370 \mathrm{E}+020.00000 \mathrm{E}+00$

$8 \quad 36 \quad 0 \quad 0.00000 \mathrm{E}+000.00000 \mathrm{E}+008.84370 \mathrm{E}+02 \quad 0.00000 \mathrm{E}+00$

$938 \quad 11 \quad 1.15958 \mathrm{E}-012.02000 \mathrm{E}+000.00000 \mathrm{E}+000.00000 \mathrm{E}+00$

$10 \quad 39 \quad 6 \quad 5.44575 \mathrm{E}-02 \quad 8.40000 \mathrm{E}+000.00000 \mathrm{E}+00 \quad 0.00000 \mathrm{E}+00$

$11 \quad 40 \quad 12 \mathrm{~s} 9.50007 \mathrm{E}-021.00000 \mathrm{E}+000.00000 \mathrm{E}+00 \quad 0.00000 \mathrm{E}+00$

$124165.44575 \mathrm{E}-028.40000 \mathrm{E}+000.00000 \mathrm{E}+000.00000 \mathrm{E}+00$

$13 \quad 140 \quad 0 \quad 0.00000 \mathrm{E}+00 \quad 0.00000 \mathrm{E}+000.00000 \mathrm{E}+000.00000 \mathrm{E}+00$

$144212 \mathrm{~s} 9.50007 \mathrm{E}-021.00000 \mathrm{E}+000.00000 \mathrm{E}+000.00000 \mathrm{E}+00$

$15 \quad 15426.62920 \mathrm{E}-02 \quad 1.37500 \mathrm{E}+01 \quad 0.00000 \mathrm{E}+000.00000 \mathrm{E}+00$

$16 \quad 149 \quad 12 \mathrm{~s} 9.50007 \mathrm{E}-02 \quad 1.00000 \mathrm{E}+00 \quad 0.00000 \mathrm{E}+000.00000 \mathrm{E}+00$

$1714476.43969 \mathrm{E}-021.96600 \mathrm{E}+010.00000 \mathrm{E}+000.00000 \mathrm{E}+00$

$18 \quad 159 \quad 6 \quad 5.44575 \mathrm{E}-028.40000 \mathrm{E}+000.00000 \mathrm{E}+000.00000 \mathrm{E}+00$

$19141 \quad 12 s 9.50007 \mathrm{E}-021.00000 \mathrm{E}+000.00000 \mathrm{E}+000.00000 \mathrm{E}+0.0$

$20 \quad 162 \quad 0 \quad 0.00000 \mathrm{E}+000.00000 \mathrm{E}+000.00000 \mathrm{E}+000.00000 \mathrm{E}+00$

total

isurfaces infinite

infinite

infinite

infinite

infinite

asymmetric pint table 60

\section{O $1.0000 \mathrm{E}+00$}

o $1.0000 \mathrm{E}+00$

$01.0000 \mathrm{E}+00$

1 $1.0000 \mathrm{E}+00$

$11.0000 \mathrm{E}+00$

I $1.0000 \mathrm{E}+00$

$11.0000 \mathrm{E}+00$

I $1.0000 \mathrm{E}+00$

o $1.0000 \mathrm{E}+00$

$0 \quad 1.0000 \mathrm{E}+00$

$0 \quad 1.0000 \mathrm{E}+00$

0 $1.0000 \mathrm{E}+00$

$0 \quad 1.0000 E+00$

o $1.0000 \mathrm{E}+00$

$0 \quad 1.0000 \mathrm{E}+00$

$01.0000 \mathrm{E}+00$

$0 \quad 1.0000 \mathrm{E}+00$

$0 \quad 1.0000 \mathrm{E}+00$

$01.0000 E+00$

$0 \quad 0.0000 \mathrm{E}+00$ print table 70

surface trans type surface coefficients 
py $0.0000000 \mathrm{E}+00$

Cz $2.9135000 \mathrm{E}+01$

pz $3.1750000 \mathrm{E}+01$

pz $-3.1750000 \mathrm{E}+01$

$\mathrm{c} / 2 \quad 3.4414000 \mathrm{E}+00 \quad 8.5150000 \mathrm{E}-01 \quad 3.2400000 \mathrm{E}-0 \mathrm{I}$

$\mathrm{c} / \mathrm{z} \quad 3.4414000 \mathrm{E}+00 \quad 8.5150000 \mathrm{E}-01 \quad 3.3450000 \mathrm{E}-01$

$\mathrm{c} / \mathrm{z} \quad 3.4414000 \mathrm{E}+00 \quad 8.5150000 \mathrm{E}-0 \mathrm{I} \quad 3.4750000 \mathrm{E}-01$

$c / 2 \quad 3.4414000 \mathrm{E}+\infty 0 \quad 8.5150000 \mathrm{E}-0 \mathrm{I} \quad 4.3180000 \mathrm{E}-01$

cz $1.7251000 \mathrm{E}+00$

cz $1.8051000 \mathrm{E}+00$

CZ $1.9051000 \mathrm{E}+00$

cz $2.1055000 \mathrm{E}+00$

c/z $1.6711300 \mathrm{E}+01 \quad 0.0000000 \mathrm{E}+00 \quad 2.1055000 \mathrm{E}+00$

$c / z-1.6711300 \mathrm{E}+01 \quad 0.0000000 \mathrm{E}+00 \quad 2.1055000 \mathrm{E}+00$

$\begin{array}{llll}c / z & 0.0000000 \mathrm{E}+00 & 1.1918500 \mathrm{E}+01 & 2.1055000 \mathrm{E}+00\end{array}$

$c / z \quad 1.0321700 \mathrm{E}+01 \quad 5.9592000 \mathrm{E}+00 \quad 2.1055000 \mathrm{E}+00$

c/z $\quad 8.3557000 \mathrm{E}+00 \quad 1.4472400 \mathrm{E}+01 \quad 2.1055000 \mathrm{E}+00$

$\mathrm{c} / \mathbf{z}+8.3557000 \mathrm{E}+00 \quad 1.4472400 \mathrm{E}+01 \quad 2.1055000 \mathrm{E}+00$

c/z $-1.0321700 \mathrm{E}+01 \quad 5.9592000 \mathrm{E}+00 \quad 2.1055000 \mathrm{E}+00$

px $3.9330000 \mathrm{E}+00$

px $2.9498000 \mathrm{E}+00$

$\begin{array}{lllll}\text { p } & 1.0000000 \mathrm{E}+00 & 1.7320508 \mathrm{E}+00 & 0.0000000 \mathrm{E}+00 \quad 5.8994000 \mathrm{E}+00\end{array}$

$\begin{array}{lllll}\text { p } & 1.0000000 \mathrm{E}+00 & 1.7320508 \mathrm{E}+00 & 0.0000000 \mathrm{E}+00 & 3.9330000 \mathrm{E}+00\end{array}$

p $-1.0000000 \mathrm{E}+00 \quad 1.7320508 \mathrm{E}+00 \quad 0.0000000 \mathrm{E}+00 \quad-9.8340000 \mathrm{E}-01$

p $-1.0000000 \mathrm{E}+00 \quad 1.7320508 \mathrm{E}+00 \quad 0.0000000 \mathrm{E}+00 \quad-2.9498000 \mathrm{E}+00$

1 cell temperatures in mev for the free-gas thermal neutron treatment.

print table 72

all non-zero importance cells with materials have a temperature for thermal neutrons of $2.5300 \mathrm{E}-08 \mathrm{mev}$.

2 warning messages so far.

lcross-section tables

print table 100

table length

tables from file testlibl

$1001.00 \mathrm{c} 1782$ 1-h-1 fromendf-vi.I

$1002.55 \mathrm{c} 4102$ njoy

mat $125 \quad 05 / 26 / 93$

5011.40c 3229 ENDL library name: nd900719 MCNP translation: 900831 14:10:05

MCNP translation: $90083114: 10: 05$

7014.40c 8535 ENDL library name: nd900719 MCNP translation: 900831 14:10:05

8016.40c 5358 ENDL library name: nd900719 MCNP translation: 900831 14:10:05

40000.40c 93372 ENDL library name: nd900719 MCNP transiation: 900831 14:10:05

$41093.40 \mathrm{C} 42746$ ENDL library name: nd900719 MCNP translation: 900831 14:10:05

$74000.40 \mathrm{C}$ 9!244 ENDL library name: nd900719 MCNP translation: 900831 14:10:05

92235.40c 34772 ENDL library name: nd900719 MCNP translation: 9total nu 10:05

$92238.40 \mathrm{c} 40834$ ENDL library name: nd901118 MCNP translation: 9total nu 56:49

$5010.03 d 3682$ b-10 endf/b-iv new gamma production format

hwtr.01t 10193 deuterium in heavy water at 300 degrees kelvin

lwtr.01t 10193 hydrogen in light water at 300 degrees kelvin

$\begin{array}{lll}1002 & 0 & 010 / 22 / 85\end{array}$

total 354900

warning. neutron energy cutoff is below some cross-section tables.

decimal words of dymamically allocated storage

source distribution written to file inp $18 \mathrm{~s} \quad$ cycle $=0$

3 warning messages so far.

1 starting morun. field length $=0 \quad \mathrm{cp} 0=0.00$

print table 110

rob18 - kcode in a hexagonal prism lattice.

900207

900207

900207

900207

900323

900208

900208

900503

901119 
$\begin{array}{cccc}x & y & z & \text { cell lattice }(i, j, k) \\ 3.000 E+00 & 2.000 E-01 & 2.000 E-01 & 140\end{array}$ $3.492 \mathrm{E}+00 \quad 1.051 \mathrm{E}+00 \quad 2.000 \mathrm{E}-01 \quad 42($ $\begin{array}{llll}3.492 \mathrm{E}+00 & 1.051 \mathrm{E}+00 \quad 2.000 \mathrm{E}-01 \quad 154\end{array}$ $23.000 \mathrm{E}+00 \quad 2.000 \mathrm{E}-01 \quad 2.000 \mathrm{E}-01 \quad 140$ $3.492 \mathrm{E}+00 \quad 1.051 \mathrm{E}+00 \quad 2.000 \mathrm{E}-01 \quad 42(0,-1,0)$ $3.492 \mathrm{E}+00 \quad 1.051 \mathrm{E}+00 \quad 2.000 \mathrm{E}-01 \quad 154$

$33.000 \mathrm{E}+00 \quad 2.000 \mathrm{E}-01 \quad 2.000 \mathrm{E}-01 \quad 140$ $3.492 \mathrm{E}+00 \quad 1.051 \mathrm{E}+00 \quad 2.000 \mathrm{E}-01 \quad 42(0,-1,0)$ $3.492 \mathrm{E}+00 \quad 1.051 \mathrm{E}+00 \quad 2.000 \mathrm{E}-01 \quad 154$

$43.000 \mathrm{E}+00 \quad 2.000 \mathrm{E}-012.000 \mathrm{E}-01 \quad 140$ $3.492 \mathrm{E}+00 \quad 1.051 \mathrm{E}+00 \quad 2.000 \mathrm{E}-01 \quad 42(0,-1,0)$ $3.492 \mathrm{E}+00 \quad 1.051 \mathrm{E}+00 \quad 2.000 \mathrm{E}-01 \quad 154$

$53.000 \mathrm{E}+00 \quad 2.000 \mathrm{E}-01 \quad 2.000 \mathrm{E}-01 \quad 140$ $3.492 \mathrm{E}+00 \quad 1.051 \mathrm{E}+00 \quad 2.000 \mathrm{E}-01 \quad 42(0,-1,0)$ $3.492 \mathrm{E}+00 \quad 1.051 \mathrm{E}+00 \quad 2.000 \mathrm{E}-01154$

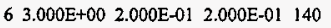
$3.492 \mathrm{E}+00 \quad 1.051 \mathrm{E}+00 \quad 2.000 \mathrm{E}-01 \quad 42(0,-1,0)$ $3.492 \mathrm{E}+00 \quad 1.051 \mathrm{E}+00 \quad 2.000 \mathrm{E}-01 \quad 154$

$73.000 \mathrm{E}+002.000 \mathrm{E}-012.000 \mathrm{E}-01140$ $3.492 \mathrm{E}+00 \quad 1.051 \mathrm{E}+00 \quad 2.000 \mathrm{E}-01 \quad 42(0,-1,0)$ $3.492 \mathrm{E}+00 \quad 1.051 \mathrm{E}+00 \quad 2.000 \mathrm{E}-01 \quad 154$ $83.000 \mathrm{E}+00 \quad 2.000 \mathrm{E}-01 \quad 2.000 \mathrm{E}-01 \quad 140$

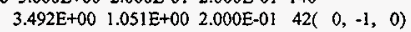
$3.492 \mathrm{E}+00 \quad 1.051 \mathrm{E}+00 \quad 2.000 \mathrm{E}-01154$ $93.000 \mathrm{E}+00 \quad 2.000 \mathrm{E}-01 \quad 2.000 \mathrm{E}-01 \quad 140$ $3.492 \mathrm{E}+00 \quad 1.051 \mathrm{E}+00 \quad 2.000 \mathrm{E}-01 \quad 42(0,-1,0)$ $\begin{array}{ll}3.492 \mathrm{E}+00 & 1.051 \mathrm{E}+00 \quad 2.000 \mathrm{E}-01 \quad 154\end{array}$

$\begin{array}{lll}10 & 3.000 \mathrm{E}+00 & 2.000 \mathrm{E}-01 \\ 2.000 \mathrm{E}-01 & 140\end{array}$ $3.492 \mathrm{E}+00 \quad 1.051 \mathrm{E}+00 \quad 2.000 \mathrm{E}-01 \quad 42(0,-1,0)$ $3.492 \mathrm{E}+00 \quad 1.051 \mathrm{E}+00 \quad 2.000 \mathrm{E}-01 \quad 154$ $3.000 \mathrm{E}+00 \quad 2.000 \mathrm{E}-01 \quad 2.000 \mathrm{E}-01 \quad 140$ $.492 \mathrm{E}+00 \quad 1.051 \mathrm{E}+00 \quad 2.000 \mathrm{E}-01 \quad 42(0,-1,0)$ $3.492 \mathrm{E}+00 \quad 1.051 \mathrm{E}+00 \quad 2.000 \mathrm{E}-01 \quad 154$ $123.000 \mathrm{E}+00 \quad 2.000 \mathrm{E}-012.000 \mathrm{E}-01 \quad 140$ $3.492 \mathrm{E}+00 \quad 1.051 \mathrm{E}+00 \quad 2.000 \mathrm{E}-01 \quad 42(0,-1,0)$ $\begin{array}{ll}3.492 \mathrm{E}+00 & 1.051 \mathrm{E}+00 \quad 2.000 \mathrm{E}-01 \quad 154\end{array}$

$133.000 \mathrm{E}+00 \quad 2.000 \mathrm{E}-012.000 \mathrm{E}-01 \quad 140$ $3.492 \mathrm{E}+00 \quad 1.051 \mathrm{E}+00 \quad 2.000 \mathrm{E}-01 \quad 42(0,-1,0)$ $3.492 \mathrm{E}+00 \quad 1.051 \mathrm{E}+00 \quad 2.000 \mathrm{E}-01 \quad 154$

$143.000 \mathrm{E}+00 \quad 2.000 \mathrm{E}-01 \quad 2.000 \mathrm{E}-01 \quad 140$ $3.492 \mathrm{E}+00 \quad 1.051 \mathrm{E}+00 \quad 2.000 \mathrm{E}-01 \quad 42(0,-1,0)$ $3.492 \mathrm{E}+00 \quad 1.051 \mathrm{E}+00 \quad 2.000 \mathrm{E}-01 \quad 154$

is $3.000 \mathrm{E}+00 \quad 2.000 \mathrm{E}-01 \quad 2.000 \mathrm{E}-01 \quad 140$ $3.492 \mathrm{E}+00 \quad 1.051 \mathrm{E}+00 \quad 2.000 \mathrm{E}-01 \quad 42(0,-1,0)$ $3.492 \mathrm{E}+00 \quad 1.051 \mathrm{E}+00 \quad 2.000 \mathrm{E}-01 \quad 154$

$163.000 \mathrm{E}+00 \quad 2.000 \mathrm{E}-01 \quad 2.000 \mathrm{E}-01 \quad 140$ $3.492 \mathrm{E}+00 \quad 1.051 \mathrm{E}+00 \quad 2.000 \mathrm{E}-01 \quad 42(0,-1,0)$ $\begin{array}{lll}3.492 \mathrm{E}+00 \quad 1.051 \mathrm{E}+00 \quad 2.000 \mathrm{E}-01 \quad 154 & 15\end{array}$

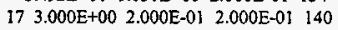
$3.492 \mathrm{E}+00 \quad 1.051 \mathrm{E}+00 \quad 2.000 \mathrm{E}-01 \quad 42(0,-1,0)$ $3.492 \mathrm{E}+00 \quad 1.051 \mathrm{E}+00 \quad 2.000 \mathrm{E}-01 \quad 154$

$183.000 \mathrm{E}+00 \quad 2.000 \mathrm{E}-01 \quad 2.000 \mathrm{E}-01 \quad 140$ $3.492 \mathrm{E}+00 \quad 1.051 \mathrm{E}+00 \quad 2.000 \mathrm{E}-01 \quad 42(0,-1,0)$ $\begin{array}{llll}3.492 \mathrm{E}+00 & 1.051 \mathrm{E}+00 & 2.000 \mathrm{E}-01 \quad 154\end{array}$

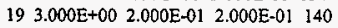
$3.492 \mathrm{E}+00 \quad 1.051 \mathrm{E}+00 \quad 2.000 \mathrm{E}-01 \quad 42(0,-1,0)$ $3.492 \mathrm{E}+00 \quad 1.051 \mathrm{E}+00 \quad 2.000 \mathrm{E}-01 \quad 154$

$20 \quad 3.000 \mathrm{E}+00 \quad 2.000 \mathrm{E}-01 \quad 2.000 \mathrm{E}-01 \quad 140$ $3.492 \mathrm{E}+00 \quad 1.051 \mathrm{E}+00 \quad 2.000 \mathrm{E}-01 \quad 42(0,-1,0)$ $3.492 \mathrm{E}+00 \quad 1.051 \mathrm{E}+00 \quad 2.000 \mathrm{E}-01 \quad 154$

$213.000 \mathrm{E}+00 \quad 2.000 \mathrm{E}+01 \quad 2.000 \mathrm{E}-01 \quad 140$ $\begin{array}{llll}3.492 \mathrm{E}+00 & 1.051 \mathrm{E}+00 \quad 2.000 \mathrm{E}-01 & 42( & 0,-1,0)\end{array}$ $492 \mathrm{E}+00 \quad 1.051 \mathrm{E}+00 \quad 2.000 \mathrm{E}-01 \quad 154$ B. $000 \mathrm{E}+00 \quad 2.000 \mathrm{E}-01 \quad 2.000 \mathrm{E}-01 \quad 140$ $\checkmark \quad w$ energy weight time

$\begin{array}{llllll}5.085 \mathrm{E}-01 & 4.733 \mathrm{E}-01 & 7.193 \mathrm{E}-01 & 2.209 \mathrm{E}+00 \quad 1.000 \mathrm{E}+00 & 0.000 \mathrm{E}+00\end{array}$ $5.085 \mathrm{E}-01 \quad 4.733 \mathrm{E}-01$ 7.193E-01

0 5.085E-01 4.733E-01 7.193E-01

$8.952 \mathrm{E}-01-4.447 \mathrm{E}-01-2.944 \mathrm{E}-02 \quad 4.904 \mathrm{E}+00 \quad 1.000 \mathrm{E}+00 \quad 0.000 \mathrm{E}+00$ 8.952E-01 -4.447E-01 -2.944E-02

0 8.952E-01 -4.447E-01 -2.944E-02

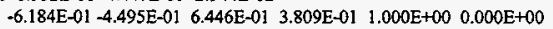

$-6.184 \mathrm{E}-01-4.495 \mathrm{E}-01 \quad 6.446 \mathrm{E}-01$

$0-6.184 \mathrm{E}-01-4.495 \mathrm{E}-01 \quad 6.446 \mathrm{E}-01$

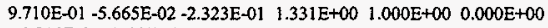

9.710E-01 -5.665E-02 - 2.323E-01

0 9.710E-01 -5.665E-02 -2.323E-01

$5.861 \mathrm{E}-01 \quad 1.496 \mathrm{E}-01-7.963 \mathrm{E}-01 \quad 1.902 \mathrm{E}+00 \quad 1.000 \mathrm{E}+00 \quad 0.000 \mathrm{E}+00$

$5.861 \mathrm{E}-011.496 \mathrm{E}-01-7.963 \mathrm{E}-01$

0 5.861E-01 1.496E-01 -7.963E-01

$-6.489 \mathrm{E}-02-1.626 \mathrm{E}-01 \quad 9.845 \mathrm{E}-01 \quad 4.410 \mathrm{E}-01 \quad 1.000 \mathrm{E}+00 \quad 0.000 \mathrm{E}+00$

$-6.489 \mathrm{E}-02-1.626 \mathrm{E}-019.845 \mathrm{E}-01$

$0-6.489 \mathrm{E}-02-1.626 \mathrm{E}-01 \quad 9.845 \mathrm{E}-01$

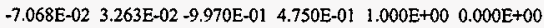
$-7.068 \mathrm{E}-02 \quad 3.263 \mathrm{E}-02-9.970 \mathrm{E}-01$

$0-7.068 \mathrm{E}-02 \quad 3.263 \mathrm{E}-02-9.970 \mathrm{E}-01$

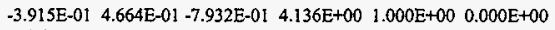

$-3.915 \mathrm{E}-01$ 4.664E-01 -7.932E-01

0 -3.915E-01 4.664E-01 -7.932E-01

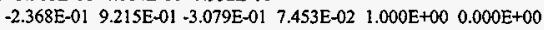

$-2.368 \mathrm{E}-019.215 \mathrm{E}-01-3.079 \mathrm{E}-01$

$0-2.368 \mathrm{E}-01 \quad 9.215 \mathrm{E}-01-3.079 \mathrm{E}-01$

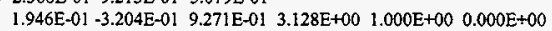

$1.946 \mathrm{E}-01+3.204 \mathrm{E}-019.271 \mathrm{E}-01$

$0 \quad 1.946 \mathrm{E}-01-3.204 \mathrm{E}-01 \quad 9.271 \mathrm{E}-01$

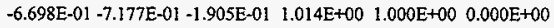

$-6.698 \mathrm{E}-01-7.177 \mathrm{E}-01+1.905 \mathrm{E}-01$

$0-6.698 \mathrm{E}-01-7.177 \mathrm{E}-01-1.905 \mathrm{E}-01$

$\begin{array}{llll}-8.398 \mathrm{E}-01 & -4.129 \mathrm{E}-01 & 3.524 \mathrm{E}-01 & 1.395 \mathrm{E}+00 \quad 1.000 \mathrm{E}+00 \quad 0.000 \mathrm{E}+00\end{array}$

$-8.398 \mathrm{E}-01-4.129 \mathrm{E}-01 \quad 3.524 \mathrm{E}-01$

$0-8.398 \mathrm{E}-01-4.129 \mathrm{E}-01 \quad 3.524 \mathrm{E}-01$

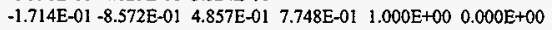

$-1.714 \mathrm{E}-01-8.572 \mathrm{E}-01 \quad 4.857 \mathrm{E}-01$

$0-1.714 \mathrm{E}-01-8.572 \mathrm{E}-01 \quad 4.857 \mathrm{E}-01$

$-2.489 \mathrm{E}-01-5.118 \mathrm{E}-01-8.222 \mathrm{E}-01 \quad 1.101 \mathrm{E}+00 \quad 1.000 \mathrm{E}+00 \quad 0.000 \mathrm{E}+00$

$-2.489 \mathrm{E}-01-5.118 \mathrm{E}-01-8.222 \mathrm{E}-01$

$0-2.489 \mathrm{E}-01-5.118 \mathrm{E}-01-8.222 \mathrm{E}-01$

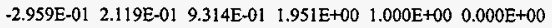
$-2.959 \mathrm{E}-01 \quad 2.119 \mathrm{E}-01 \quad 9.314 \mathrm{E}-01$

$0-2.959 \mathrm{E}-01 \quad 2.119 \mathrm{E}-01 \quad 9.314 \mathrm{E}-01$

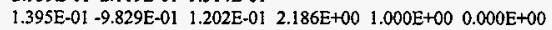

1.395E-01 $-9.829 \mathrm{E}-01 \quad 1.202 \mathrm{E}-01$

0 1.395E-01 -9.829E-01 1.202E-01

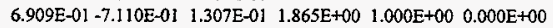

$6.909 \mathrm{E}-01-7.110 \mathrm{E}-01$ 1.307E-01

$0 \quad 6.909 \mathrm{E}-0 \mathrm{i}-7.110 \mathrm{E}-01 \quad 1.307 \mathrm{E}-01$

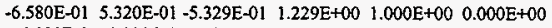

$-6.580 \mathrm{E}-01 \quad 5.320 \mathrm{E}-01-5.329 \mathrm{E}-01$

$0-6.580 \mathrm{E}-01 \quad 5.320 \mathrm{E}-01-5.329 \mathrm{E}-01$

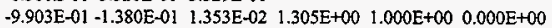

$-9.903 \mathrm{E}-01-1.380 \mathrm{E}-01 \quad 1.353 \mathrm{E}-02$

$0-9.903 \mathrm{E}-01-1.380 \mathrm{E}-01 \quad 1.353 \mathrm{E}-02$

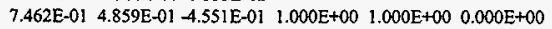

7.462E-01 4.859E-01 -4.551E-01

0 7.462E-01 4.859E-01 -4.551E-01

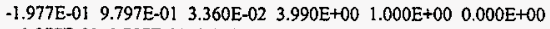

$-1.977 \mathrm{E}-01 \quad 9.797 \mathrm{E}-01 \quad 3.360 \mathrm{E}-02$

$0-1.977 \mathrm{E}-01 \quad 9.797 \mathrm{E}-01 \quad 3.360 \mathrm{E}-02$

$-9.117 \mathrm{E}-01-3.647 \mathrm{E}-01 \mathrm{l}-1.891 \mathrm{E}-01 \quad 2.665 \mathrm{E}-01 \quad 1.000 \mathrm{E}+00 \quad 0.000 \mathrm{E}+00$ 
$3.492 \mathrm{E}+00 \quad 1.051 \mathrm{E}+00 \quad 2.000 \mathrm{E}-01 \quad 42(0,-1,0)$ $-492 \mathrm{E}+00 \quad 1.051 \mathrm{E}+00 \quad 2.000 \mathrm{E}-01 \quad 154$ $3.000 \mathrm{E}+00 \quad 2.000 \mathrm{E}-01 \quad 2.000 \mathrm{E}-01 \quad 140$ $3.492 \mathrm{E}+00 \quad 1.051 \mathrm{E}+002.000 \mathrm{E}-01 \quad 42(0,-1,0)$ $3.492 \mathrm{E}+00 \quad 1.05 ! \mathrm{E}+00 \quad 2.000 \mathrm{E}-01 \quad 154$

$243.000 \mathrm{E}+00 \quad 2.000 \mathrm{E}-012.000 \mathrm{E}-01 \quad 140$ $3.492 \mathrm{E}+00 \quad 1.051 \mathrm{E}+00 \quad 2.000 \mathrm{E}-01 \quad 42(0,-1,0)$ $3.492 \mathrm{E}+00 \quad 1.051 \mathrm{E}+00 \quad 2.000 \mathrm{E}-01 \quad 154$

$253.000 \mathrm{E}+002.000 \mathrm{E}-012.000 \mathrm{E}-01 \quad 140$ $3.492 \mathrm{E}+00 \quad 1.051 \mathrm{E}+00 \quad 2.000 \mathrm{E}-01 \quad 42(0,-1,0)$ $3.492 \mathrm{E}+001.051 \mathrm{E}+002.000 \mathrm{E}-01154$

$262.000 \mathrm{E}-01 \quad 3.000 \mathrm{E}+00 \quad 2.000 \mathrm{E}-01 \quad 140$ $3.641 \mathrm{E}+00 \quad 4.456 \mathrm{E}-01 \quad 2.000 \mathrm{E}-01 \quad 42(-5,3,0)$ $3.641 \mathrm{E}+00 \quad 4.456 \mathrm{E}-01 \quad 2.000 \mathrm{E}-01 \quad 141$

$272.000 \mathrm{E}-013.000 \mathrm{E}+00 \quad 2.000 \mathrm{E}-01 \quad 140$ $3.641 \mathrm{E}+00 \quad 4.456 \mathrm{E}-01 \quad 2.000 \mathrm{E}-01 \quad 42(-5,3,0)$ $3.641 \mathrm{E}+00 \quad 4.456 \mathrm{E}-01 \quad 2.000 \mathrm{E}-01 \quad 141$

$28 \quad 2.000 \mathrm{E}-01 \quad 3.000 \mathrm{E}+00 \quad 2.000 \mathrm{E}-01 \quad 140$ $3.641 \mathrm{E}+00 \quad 4.456 \mathrm{E}-01 \quad 2.000 \mathrm{E}-01 \quad 42(-5,3,0)$ $3.641 \mathrm{E}+00 \quad 4.456 \mathrm{E}-01 \quad 2.000 \mathrm{E}-01 \quad 141$

$292.000 \mathrm{E}-013.000 \mathrm{E}+00 \quad 2.000 \mathrm{E}-01140$ $3.641 \mathrm{E}+00 \quad 4.456 \mathrm{E}-01 \quad 2.000 \mathrm{E}-01 \quad 42(-5,3,0)$ $3.641 \mathrm{E}+00 \quad 4.456 \mathrm{E}-01 \quad 2.000 \mathrm{E}-01 \quad 141$

$302.000 \mathrm{E}-013.000 \mathrm{E}+00 \quad 2.000 \mathrm{E}-01 \quad 140$ $3.641 \mathrm{E}+00 \quad 4.456 \mathrm{E}-01 \quad 2.000 \mathrm{E}-01 \quad 42(-5,3,0)$ $3.641 \mathrm{E}+00 \quad 4.456 \mathrm{E}-01 \quad 2.000 \mathrm{E}-01 \quad 141$

3) 2.000E-01 3.000E+00 2.000E-01 140 $3.641 \mathrm{E}+00 \quad 4.456 \mathrm{E}-012.000 \mathrm{E}-01 \quad 42(-5,3,0)$ $3.641 \mathrm{E}+00 \quad 4.456 \mathrm{E}-01 \quad 2.000 \mathrm{E}-01141$

$322.000 \mathrm{E}-01 \quad 3.000 \mathrm{E}+00 \quad 2.000 \mathrm{E}-01 \quad 140$ $3.641 \mathrm{E}+00 \quad 4.456 \mathrm{E}-01 \quad 2.000 \mathrm{E}-01 \quad 42(-5,3,0)$ $3.641 \mathrm{E}+00 \quad 4.456 \mathrm{E}-01 \quad 2.000 \mathrm{E}-01 \quad 141$

$332.000 \mathrm{E}-013.000 \mathrm{E}+00 \quad 2.000 \mathrm{E}-01 \quad 140$ $.641 \mathrm{E}+00 \quad 4.456 \mathrm{E}-01 \quad 2.000 \mathrm{E}-01 \quad 42(-5,3,0)$ $.641 \mathrm{E}+00 \quad 4.456 \mathrm{E}-01 \quad 2.000 \mathrm{E}-01 \quad 141$ $342.000 \mathrm{E}-01 \quad 3.000 \mathrm{E}+00 \quad 2.000 \mathrm{E}-01 \quad 140$ $3.641 \mathrm{E}+00 \quad 4.456 \mathrm{E}-01 \quad 2.000 \mathrm{E}-01 \quad 42(-5,3,0)$ $3.641 \mathrm{E}+00 \quad 4.456 \mathrm{E}-01 \quad 2.000 \mathrm{E}-01 \quad 141$

$352.000 \mathrm{E}-01 \quad 3.000 \mathrm{E}+00 \quad 2.000 \mathrm{E}-01 \quad 140$ $3.641 \mathrm{E}+00 \quad 4.456 \mathrm{E}-01 \quad 2.000 \mathrm{E}-01 \quad 42(-5,3,0)$ $3.641 \mathrm{E}+00 \quad 4.456 \mathrm{E}-01 \quad 2.000 \mathrm{E}-01 \quad 141$

$36 \quad 2.000 \mathrm{E}-01 \quad 3.000 \mathrm{E}+00 \quad 2.000 \mathrm{E}-01 \quad 140$ $3.64 \mathrm{IE}+00 \quad 4.456 \mathrm{E}-01 \quad 2.000 \mathrm{E}-01 \quad 42(-5,3,0)$ $3.641 \mathrm{E}+00 \quad 4.456 \mathrm{E}-01 \quad 2.000 \mathrm{E}-01 \quad 141$

$37 \quad 2.000 \mathrm{E}-01 \quad 3.000 \mathrm{E}+00 \quad 2.000 \mathrm{E}-01 \quad 140$ $3.641 \mathrm{E}+00 \quad 4.456 \mathrm{E}-01 \quad 2.000 \mathrm{E}-01 \quad 42(-5,3,0)$ $3.641 E+00 \quad 4.456 E-01 \quad 2.000 E-01 \quad 141$

$382.000 \mathrm{E}=013.000 \mathrm{E}+00 \quad 2.000 \mathrm{E}-01140$ $3.641 \mathrm{E}+00 \quad 4.456 \mathrm{E}-01 \quad 2.000 \mathrm{E}-01 \quad 42(-5,3,0)$ $3.641 \mathrm{E}+00 \quad 4.456 \mathrm{E}-01 \quad 2.000 \mathrm{E}-01 \quad 141$

$392.000 \mathrm{E}-01 \quad 3.000 \mathrm{E}+00 \quad 2.000 \mathrm{E}-01 \quad 140$ $3.641 \mathrm{E}+00 \quad 4.456 \mathrm{E}-01 \quad 2.000 \mathrm{E}-01 \quad 42(-5,3,0)$ $3.641 \mathrm{E}+00 \quad 4.456 \mathrm{E}-01 \quad 2.000 \mathrm{E}-01 \quad 141$

$402.000 \mathrm{E}-01 \quad 3.000 \mathrm{E}+00 \quad 2.000 \mathrm{E}-01 \quad 140$ $3.641 \mathrm{E}+00 \quad 4.456 \mathrm{E}-01 \quad 2.000 \mathrm{E}-01 \quad 42(-5,3,0)$ $3.641 \mathrm{E}+00 \quad 4.456 \mathrm{E}-01 \quad 2.000 \mathrm{E}-01 \quad 141$

$412.000 \mathrm{E}-01 \quad 3.000 \mathrm{E}+00 \quad 2.000 \mathrm{E}-01 \quad 140$ $3.641 \mathrm{E}+00 \quad 4.456 \mathrm{E}-01 \quad 2.000 \mathrm{E}-01 \quad 42(-5,3,0)$ $3.641 \mathrm{E}+00 \quad 4.456 \mathrm{E}-01 \quad 2.000 \mathrm{E}-01 \quad 141$

$42 \quad 2.000 \mathrm{E}-01 \quad 3.000 \mathrm{E}+00 \quad 2.000 \mathrm{E}-01 \quad 140$ $3.641 \mathrm{E}+00 \quad 4.456 \mathrm{E}-01 \quad 2.000 \mathrm{E}-01 \quad 42(-5,3,0)$ $3.641 \mathrm{E}+00 \quad 4.456 \mathrm{E}-01 \quad 2.000 \mathrm{E}-01 \quad 141$

$432.000 \mathrm{E}-013.000 \mathrm{E}+00 \quad 2.000 \mathrm{E}-01 \quad 140$ $3.641 \mathrm{E}+00 \quad 4.456 \mathrm{E}-01 \quad 2.000 \mathrm{E}-01 \quad 42(-5,3,0)$ $3.641 \mathrm{E}+00 \quad 4.456 \mathrm{E}-01 \quad 2.000 \mathrm{E}-01 \quad 141$ $2.000 \mathrm{E}-01 \quad 3.000 \mathrm{E}+00 \quad 2.000 \mathrm{E}-01 \quad 140$ $3.641 \mathrm{E}+00 \quad 4.456 \mathrm{E}-01 \quad 2.000 \mathrm{E}-01 \quad 42(-5,3,0)$
$-9.117 \mathrm{E}-01-3.647 \mathrm{E}-01-1.891 \mathrm{E}-01$

$0-9.117 \mathrm{E}-01-3.647 \mathrm{E}-01-1.891 \mathrm{E}-01$

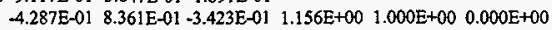
$-4.287 \mathrm{E}-01 \quad 8.361 \mathrm{E}-01 \mathrm{1}-3.423 \mathrm{E}-01$

$0-4.287 \mathrm{E}-01 \quad 8.361 \mathrm{E}-01-3.423 \mathrm{E}-01$

$1.080 \mathrm{E}-01 \quad 3.412 \mathrm{E}-01-9.338 \mathrm{E}-01 \quad 2.669 \mathrm{E}+00 \quad 1.000 \mathrm{E}+00 \quad 0.000 \mathrm{E}+00$ 1.080E-01 3.412E-01 -9.338E-01

0 1.080E-01 3.412E-01 -9.338E-01

$-9.111 \mathrm{E}-01-9.012 \mathrm{E}-03-4.122 \mathrm{E}-012.185 \mathrm{E}+00 \quad 1.000 \mathrm{E}+00 \quad 0.000 \mathrm{E}+00$

$-9.111 \mathrm{E}-01-9.012 \mathrm{E}-03-4.122 \mathrm{E}-0$ ?

$0-9.311 E-01-9.012 E-03-4.122 E-01$

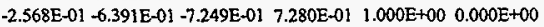

$-2.568 \mathrm{E}-01-6.391 \mathrm{E}-01-7.249 \mathrm{E}-01$

$0-2.568 \mathrm{E}-01-6.391 \mathrm{E}-01-7.249 \mathrm{E}-01$

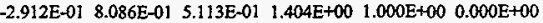

$-2.912 \mathrm{E}-01 \quad 8.086 \mathrm{E}-01$ 5.113E-01

0 -2.912E-01 8.086E-01 5.113E-01

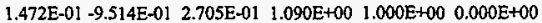

$1.472 \mathrm{E}-01-9.514 \mathrm{E}-012.705 \mathrm{E}-01$

0 1.472E-01 -9.514E-01 2.705E-01

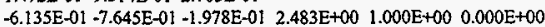
$-6.135 \mathrm{E}-01-7.645 \mathrm{E}-01-1.978 \mathrm{E}-01$

$0-6.135 \mathrm{E}-01-7.645 \mathrm{E}-01-1.978 \mathrm{E}-01$

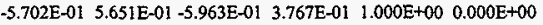
$-5.702 \mathrm{E}-015.651 \mathrm{E}-01-5.963 \mathrm{E}-01$

$0-5.702 \mathrm{E}-01 \quad 5.651 \mathrm{E}-01-5.963 \mathrm{E}-01$

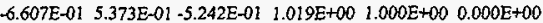
$-6.607 \mathrm{E}-015.373 \mathrm{E}-01-5.242 \mathrm{E}-01$

$0-6.607 \mathrm{E}-01 \quad 5.373 \mathrm{E}-01+5.242 \mathrm{E}-01$

$-9.742 \mathrm{E}-02-3.639 \mathrm{E}-01-9.263 \mathrm{E}-01 \quad 1.879 \mathrm{E}+00 \quad 1.000 \mathrm{E}+00 \quad 0.000 \mathrm{E}+00$ $-9.742 \mathrm{E}-02-3.639 \mathrm{E}-01-9.263 \mathrm{E}-01$

$0-9.742 \mathrm{E}-02-3.639 \mathrm{E}-01-9.263 \mathrm{E}-01$

$-1.965 \mathrm{E}-01-3.145 \mathrm{E}-01-9.287 \mathrm{E}-01 \quad 4.700 \mathrm{E}-01 \quad 1.000 \mathrm{E}+00 \quad 0.000 \mathrm{E}+00$

$-1.965 \mathrm{E}-01+3.145 \mathrm{E}-01-9.287 \mathrm{E}-01$

$0-1.965 \mathrm{E}-01-3.145 \mathrm{E}-01-9.287 \mathrm{E}-01$

$4.097 \mathrm{E}-01 \quad 8.465 \mathrm{E}-01-3.399 \mathrm{E}-01 \quad 1.530 \mathrm{E}+00 \quad 1.000 \mathrm{E}+00 \quad 0.000 \mathrm{E}+00$ 4.097E-01 8.465E-01 +3.399E-01

0 4.097E-01 8.465E-01 -3.399E-01

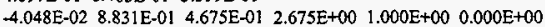
$-4.048 \mathrm{E}-02 \quad 8.831 \mathrm{E}-01 \quad 4.675 \mathrm{E}-01$

$0-4.048 \mathrm{E}-02 \quad 8.831 \mathrm{E}-01 \quad 4.675 \mathrm{E}-01$

$3.371 \mathrm{E}-01-9.269 \mathrm{E}-01-1.652 \mathrm{E}-01 \quad 9.222 \mathrm{E}-01 \quad 1.000 \mathrm{E}+00 \quad 0.000 \mathrm{E}+00$ $3.371 \mathrm{E}-01-9.269 \mathrm{E}-01-1.652 \mathrm{E}-01$

0 3.371E-01 -9.269E-01 -1.652E-01

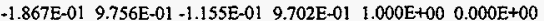
$-1.867 \mathrm{E}-019.756 \mathrm{E}-01-1.155 \mathrm{E}-01$

$0-1.867 \mathrm{E}-01 \quad 9.756 \mathrm{E}-01-1.155 \mathrm{E}-01$

$\begin{array}{lllll}-2.616 \mathrm{E}-01 & 2.336 \mathrm{E}-0 \mathrm{~L} & -9.365 \mathrm{E}-01 & 1.230 \mathrm{E}+00 & 1.000 \mathrm{E}+00 \quad 0.000 \mathrm{E}+00\end{array}$ $-2.616 \mathrm{E}-01 \quad 2.336 \mathrm{E}-01-9.365 \mathrm{E}-01$

$0-2.616 \mathrm{E}-01 \quad 2.336 \mathrm{E}-01-9.365 \mathrm{E}-01$

$9.780 \mathrm{E}-0 \mathrm{I}-7.641 \mathrm{E}-02-1.939 \mathrm{E}-01 \quad 1.083 \mathrm{E}+00 \quad 1.000 \mathrm{E}+00 \quad 0.000 \mathrm{E}+00$ $9.780 \mathrm{E}-01-7.641 \mathrm{E}-02-1.939 \mathrm{E}-01$

0 9.780E-01 -7.641E-02-1.939E-01 $2.580 \mathrm{E}-01-7.076 \mathrm{E}-01 \quad 6.578 \mathrm{E}-01 \quad 8.313 \mathrm{E}-01 \quad 1.000 \mathrm{E}+00 \quad 0.000 \mathrm{E}+00$ $2.580 \mathrm{E}-01-7.076 \mathrm{E}-01 \quad 6.578 \mathrm{E}-01$

$02.580 \mathrm{E}-01-7.076 \mathrm{E}-01 \quad 6.578 \mathrm{E}-01$

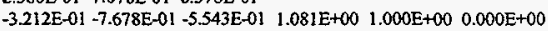
$-3.212 \mathrm{E}-01-7.678 \mathrm{E}-01+5.543 \mathrm{E}-01$

$0-3.212 \mathrm{E}-01-7.678 \mathrm{E}-01-5.543 \mathrm{E}-01$ 5.039E-01 -1.460E-01 8.513E-01 3.341E+00 $1.000 \mathrm{E}+00 \quad 0.000 \mathrm{E}+00$ $5.039 \mathrm{E}-01-1.460 \mathrm{E}-018.513 \mathrm{E}-01$

0 5.039E-01 -1.460E-01 8.513E-01

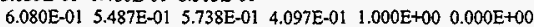
$6.080 \mathrm{E}-01 \quad 5.487 \mathrm{E}-01 \quad 5.738 \mathrm{E}-01$

$0 \quad 6.080 \mathrm{E}-01 \quad 5.487 \mathrm{E}-01 \quad 5.738 \mathrm{E}-01$

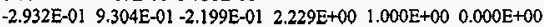
$-2.932 \mathrm{E}-01 \quad 9.304 \mathrm{E}-01-2.199 \mathrm{E}-01$ 
$3.641 \mathrm{E}+00 \quad 4.456 \mathrm{E}-01 \quad 2.000 \mathrm{E}-01 \quad 141$ $2.000 \mathrm{E}-01 \quad 3.000 \mathrm{E}+00 \quad 2.000 \mathrm{E}-01 \quad 140$ $.641 \mathrm{E}+00 \quad 4.456 \mathrm{E}-01 \quad 2.000 \mathrm{E}-01 \quad 42(-5,3,0)$ $3.641 \mathrm{E}+00 \quad 4.456 \mathrm{E}-01 \quad 2.000 \mathrm{E}-01 \quad 141$ $462.000 \mathrm{E}-013.000 \mathrm{E}+00 \quad 2.000 \mathrm{E}-01 \quad 140$ $3.641 \mathrm{E}+00 \quad 4.456 \mathrm{E}-01 \quad 2.000 \mathrm{E}-01 \quad 42(-5,3,0)$ $3.641 \mathrm{E}+00 \quad 4.456 \mathrm{E}-01 \quad 2.000 \mathrm{E}-01 \quad 141$

$47 \quad 2.000 \mathrm{E}-01 \quad 3.000 \mathrm{E}+00 \quad 2.000 \mathrm{E} \div 01 \quad 140$ $3.641 \mathrm{E}+00 \quad 4.456 \mathrm{E}-01 \quad 2.000 \mathrm{E}-01 \quad 42(-5,3,0)$ $3.641 \mathrm{E}+00 \quad 4.456 \mathrm{E}-01 \quad 2.000 \mathrm{E}-01 \quad 141$ $48 \quad 2.000 \mathrm{E}-01 \quad 3.000 \mathrm{E}+00 \quad 2.000 \mathrm{E}-01 \quad 140$ $3.641 \mathrm{E}+00 \quad 4.456 \mathrm{E}-01 \quad 2.000 \mathrm{E}-01 \quad 42(-5,3,0)$ $3.641 \mathrm{E}+00 \quad 4.456 \mathrm{E}-01 \quad 2.000 \mathrm{E}-01$ [41

$492.000 \mathrm{E}-01 \quad 3.000 \mathrm{E}+00 \quad 2.000 \mathrm{E}-01 \quad 140$ $3.641 \mathrm{E}+00 \quad 4.456 \mathrm{E}-01 \quad 2.000 \mathrm{E}-01 \quad 42(-5,3,0)$ $3.641 \mathrm{E}+00 \quad 4.456 \mathrm{E}-012.000 \mathrm{E}-01 \quad 141$ So $2.000 \mathrm{E}-01 \quad 3.000 \mathrm{E}+00 \quad 2.000 \mathrm{E}-01 \quad 140$ $3.641 \mathrm{E}+00 \quad 4.456 \mathrm{E}-01 \quad 2.000 \mathrm{E}-01 \quad 42(-5,3,0)$ $3.641 \mathrm{E}+00 \quad 4.456 \mathrm{E}-01 \quad 2.000 \mathrm{E}-01 \quad 141$ lestimated keff results by cycle
$0-2.932 \mathrm{E}-01 \quad 9.304 \mathrm{E}-01-2.199 \mathrm{E}-01$ $-8.475 \mathrm{E}-01-3.993 \mathrm{E}-01-3.497 \mathrm{E}-01 \quad 1.362 \mathrm{E}+00 \quad 1.000 \mathrm{E}+00 \quad 0.000 \mathrm{E}+00$ $-8.475 \mathrm{E}-0 \mathrm{I}-3.993 \mathrm{E}-01-3.497 \mathrm{E}-01$

$0-8.475 \mathrm{E}-01-3.993 \mathrm{E}-01-3.497 \mathrm{E}-01$ $1.200 \mathrm{E}-0 \mathrm{I}-9.195 \mathrm{E}-01-3.743 \mathrm{E}-01 \quad 1.010 \mathrm{E}+00 \quad 1.000 \mathrm{E}+00 \quad 0.000 \mathrm{E}+00$ $1.200 \mathrm{E}-01-9.195 \mathrm{E}-01-3.743 \mathrm{E}-01$

$0 \quad 1.200 \mathrm{E}-01-9.195 \mathrm{E}-01-3.743 \mathrm{E}-01$ $7.085 \mathrm{E}-01 \quad 5.879 \mathrm{E}-01 \quad 3.904 \mathrm{E}-01 \quad 7.068 \mathrm{E}+00 \quad 1.000 \mathrm{E}+00 \quad 0.000 \mathrm{E}+00$ 7.085E-01 5.879E-01 3.904E-01

0 7.085E-01 5.879E-01 3.904E-01 4.261E-01 $9.046 \mathrm{E}-01 \quad 9.254 \mathrm{E}-03 \quad 3.122 \mathrm{E}-01 \quad 1.000 \mathrm{E}+00 \quad 0.000 \mathrm{E}+00$ $4.261 \mathrm{E}-01 \quad 9.046 \mathrm{E}-01 \quad 9.254 \mathrm{E}-03$

$0 \quad 4.261 \mathrm{E}-01 \quad 9.046 \mathrm{E}-01 \quad 9.254 \mathrm{E}-03$ $\begin{array}{llll}5.431 \mathrm{E}-01 & 4.270 \mathrm{E}-01-7.230 \mathrm{E}-01 & 5.484 \mathrm{E}-01 & 1.000 \mathrm{E}+00 \quad 0.000 \mathrm{E}+00\end{array}$ $5.431 \mathrm{E}-01 \quad 4.270 \mathrm{E}-01-7.230 \mathrm{E}-01$

0 5.431E-01 4.270E-01 -7.230E-01 $-1.053 \mathrm{E}-01-9.805 \mathrm{E}-01 \quad 1.658 \mathrm{E}-01 \quad 1.545 \mathrm{E}+00 \quad 1.000 \mathrm{E}+00 \quad 0.000 \mathrm{E}+00$ $-1.053 \mathrm{E}-01-9.805 \mathrm{E}-01 \quad 1.658 \mathrm{E}-01$

$0-1.053 \mathrm{E}-01+9.805 \mathrm{E}-01 \quad 1.658 \mathrm{E}-01$ print table 175

cycle $1 \mathrm{k}$ (collision) 1.253108 removal lifetime(abs) $1.7557 \mathrm{E}+01$ source points generated 257

cycle $2 \mathrm{k}$ (collision) 1.211997 removal lifetime(abs) $1.1671 \mathrm{E}+01$ source points generated 195

cycle $3 \mathrm{k}$ (collision) 1.200265 removal lifetime(abs) $9.6992 \mathrm{E}+00$ source points generated 193

cycle $4 \mathrm{k}$ (collision) 1.129530 removal lifetime(abs) $9.3720 \mathrm{E}+00$ source points generated 182

cycle $5 \mathrm{k}$ (collision) 1.135994 removal lifetime(abs) $1.1136 \mathrm{E}+01$ source points generated 222

cycle $6 \mathrm{k}$ (collision) 1.077014 removal lifetime(abs) $1.2222 \mathrm{E}+01$ source points generated 204

ce distribution written to file inp $18 \mathrm{~s} \quad$ cycle $=6$

blem summary

0

run terminated when 6 kcode cycles were done.

neutron creation tracks weight energy neutron loss tracks weight energy (per source particle) (per source particle)

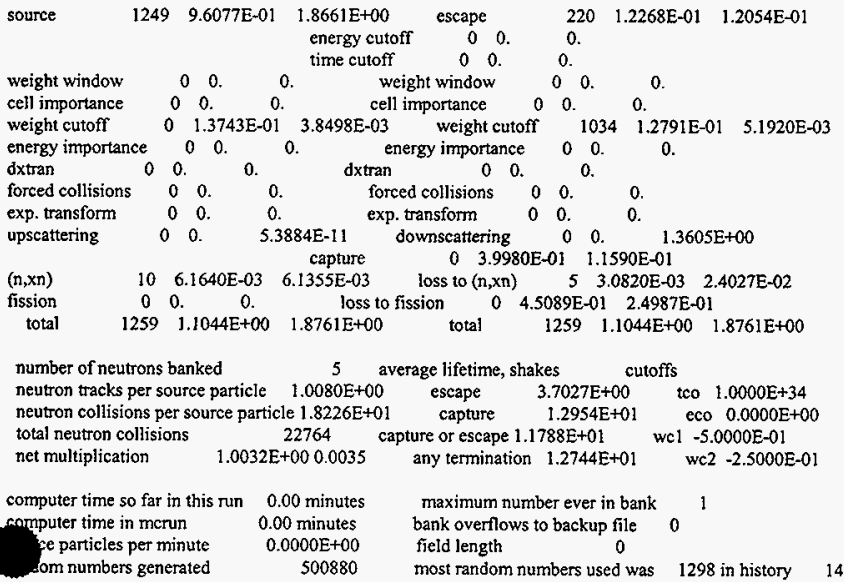


tracks population collisions collisions number flux average average cell entering * weight weighted weighted track weight track mfp (per history) energy energy (relative) (cm)

$\begin{array}{cccccccccccc}1 & 30 & 0 & 0 & 0 & 0.0000 \mathrm{E}+00 & 0.0000 \mathrm{E}+00 & 0.0000 \mathrm{E}+00 & 0.0000 \mathrm{E}+00 & 0.0000 \mathrm{E}+00 \\ 2 & 31 & 0 & 0 & 0 & 0.0000 \mathrm{E}+00 & 0.0000 \mathrm{E}+00 & 0.0000 \mathrm{E}+00 & 0.0000 \mathrm{E}+00 & 0.0000 \mathrm{E}+00 \\ 3 & 37 & 0 & 0 & 0 & 0.0000 \mathrm{E}+00 & 0.0000 \mathrm{E}+00 & 0.0000 \mathrm{E}+00 & 0.0000 \mathrm{E}+00 & 0.0000 \mathrm{E}+00 \\ 4 & 34 & 0 & 0 & 0 & 0.0000 \mathrm{E}+00 & 0.0000 \mathrm{E}+00 & 0.0000 \mathrm{E}+00 & 0.0000 \mathrm{E}+00 & 0.0000 \mathrm{E}+00 \\ 5 & 32 & 0 & 0 & 0 & 0.0000 \mathrm{E}+00 & 0.0000 \mathrm{E}+00 & 0.0000 \mathrm{E}+00 & 0.0000 \mathrm{E}+00 & 0.0000 \mathrm{E}+00 \\ 6 & 33 & 0 & 0 & 0 & 0.0000 \mathrm{E}+00 & 0.0000 \mathrm{E}+00 & 0.0000 \mathrm{E}+00 & 0.0000 \mathrm{E}+00 & 0.0000 \mathrm{E}+00 \\ 7 & 35 & 0 & 0 & 0 & 0.0000 \mathrm{E}+00 & 0.0000 \mathrm{E}+00 & 0.0000 \mathrm{E}+00 & 0.0000 \mathrm{E}+00 & 0.0000 \mathrm{E}+00 \\ 8 & 36 & 0 & 0 & 0 & 0.0000 \mathrm{E}+00 & 0.0000 \mathrm{E}+00 & 0.0000 \mathrm{E}+00 & 0.0000 \mathrm{E}+00 & 0.0000 \mathrm{E}+00 \\ 9 & 38 & 920 & 618 & 1249 & 5.9116 \mathrm{E}-01 & 3.0414 \mathrm{E}-01 & 8.9304 \mathrm{E}-01 & 6.4212 \mathrm{E}-01 & 2.6324 \mathrm{E}+00 \\ 10 & 39 & 1635 & 637 & 130 & 6.3779 \mathrm{E}-02 & 8.8236 \mathrm{E}-02 & 7.6766 \mathrm{E}-01 & 6.4931 \mathrm{E}-01 & 2.6947 \mathrm{E}+00 \\ 11 & 40 & 1728 & 663 & 184 & 8.8176 \mathrm{E}-02 & 7.6162 \mathrm{E}-02 & 7.6184 \mathrm{E}-01 & 6.4437 \mathrm{E}-01 & 2.5146 \mathrm{E}+00 \\ 12 & 41 & 1867 & 712 & 317 & 1.5811 \mathrm{E}-01 & 8.6238 \mathrm{E}-02 & 7.9306 \mathrm{E}-01 & 6.5030 \mathrm{E}-01 & 2.6940 \mathrm{E}+00 \\ 13 & 140 & 0 & 0 & 0 & 0.0000 \mathrm{E}+00 & 0.0000 \mathrm{E}+00 & 0.0000 \mathrm{E}+00 & 0.0000 \mathrm{E}+00 & 0.0000 \mathrm{E}+00 \\ 14 & 42 & 2069 & 867 & 441 & 2.1911 \mathrm{E}-01 & 8.9382 \mathrm{E}-02 & 7.8771 \mathrm{E}-01 & 6.6777 \mathrm{E}-01 & 2.5091 \mathrm{E}+00 \\ 15 & 154 & 25302 & 1253 & 8436 & 4.1390 \mathrm{E}+00 & 1.2606 \mathrm{E}-01 & 8.5005 \mathrm{E}-01 & 6.6920 \mathrm{E}-01 & 2.4712 \mathrm{E}+00 \\ 16 & 149 & 49542 & 1253 & 580 & 2.8963 \mathrm{E}-01 & 1.0795 \mathrm{E}-01 & 8.2361 \mathrm{E}-01 & 6.7129 \mathrm{E}-01 & 2.5408 \mathrm{E}+00 \\ 17 & 144 & 51224 & 1254 & 885 & 4.2652 \mathrm{E}-01 & 1.0541 \mathrm{E}-01 & 8.1599 \mathrm{E}-01 & 6.6971 \mathrm{E}-01 & 2.2391 \mathrm{E}+00 \\ 18 & 159 & 58650 & 1254 & 4019 & 2.1254 \mathrm{E}+00 & 9.8931 \mathrm{E}-02 & 8.1154 \mathrm{E}-01 & 6.6977 \mathrm{E}-01 & 2.7541 \mathrm{E}+00 \\ 19 & 141 & 73122 & 1254 & 6523 & 3.2229 \mathrm{E}+00 & 9.4499 \mathrm{E}-02 & 8.0639 \mathrm{E}-01 & 6.6810 \mathrm{E}-01 & 2.5056 \mathrm{E}+00\end{array}$

total $\quad 266059 \quad 9765 \quad 22764 \quad 1.1324 \mathrm{E}+01$

Ineutron weight balance in each cell - external events

print table 130

cell entering source energy time exiting total

$\begin{array}{llllllll}30 & 0.0000 \mathrm{E}+00 & 0.0000 \mathrm{E}+00 & 0.0000 \mathrm{E}+00 & 0.0000 \mathrm{E}+00 & 0.0000 \mathrm{E}+00 & 0.0000 \mathrm{E}+00\end{array}$

$31 \quad 0.0000 \mathrm{E}+00 \quad 0.0000 \mathrm{E}+00 \quad 0.0000 \mathrm{E}+00 \quad 0.0000 \mathrm{E}+00 \quad 0.0000 \mathrm{E}+00 \quad 0.0000 \mathrm{E}+00$

$\begin{array}{llllllll}37 & 0.0000 \mathrm{E}+00 & 0.0000 \mathrm{E}+00 & 0.0000 \mathrm{E}+00 & 0.0000 \mathrm{E}+00 & 0.0000 \mathrm{E}+00 & 0.0000 \mathrm{E}+00\end{array}$

$\begin{array}{lllllllll}4 & 34 & 0.0000 \mathrm{E}+00 & 0.0000 \mathrm{E}+00 & 0.0000 \mathrm{E}+00 & 0.0000 \mathrm{E}+00 & 0.0000 \mathrm{E}+00 & 0.0000 \mathrm{E}+00\end{array}$

$\begin{array}{llllllllll}5 & 32 & 0.0000 \mathrm{E}+00 & 0.0000 \mathrm{E}+00 & 0.0000 \mathrm{E}+00 & 0.0000 \mathrm{E}+00 & 0.0000 \mathrm{E}+00 & 0.0000 \mathrm{E}+00\end{array}$

$\begin{array}{lllllllll}6 & 33 & 0.0000 \mathrm{E}+00 & 0.0000 \mathrm{E}+00 & 0.0000 \mathrm{E}+00 & 0.0000 \mathrm{E}+00 & 0.0000 \mathrm{E}+00 & 0.0000 \mathrm{E}+00\end{array}$

$\begin{array}{lllllllllll}7 & 35 & 0.0000 \mathrm{E}+00 & 0.0000 \mathrm{E}+00 & 0.0000 \mathrm{E}+00 & 0.0000 \mathrm{E}+00 & 0.0000 \mathrm{E}+00 & 0.0000 \mathrm{E}+00\end{array}$

$\begin{array}{llllllllll}8 & 36 & 0.0000 \mathrm{E}+00 & 0.0000 \mathrm{E}+00 & 0.0000 \mathrm{E}+00 & 0.0000 \mathrm{E}+00 & 0.0000 \mathrm{E}+00 & 0.0000 \mathrm{E}+00\end{array}$

$938 \quad 4.8822 \mathrm{E}-01 \quad 0.0000 \mathrm{E}+00 \quad 0.0000 \mathrm{E}+00 \quad 0.0000 \mathrm{E}+00 \quad-3.3091 \mathrm{E}-01 \quad 1.5731 \mathrm{E}-01$

$\begin{array}{lllllllll}10 & 39 & 8.4996 \mathrm{E}-01 & 0.0000 \mathrm{E}+00 & 0.0000 \mathrm{E}+00 & 0.0000 \mathrm{E}+00 & -8.4933 \mathrm{E}-01 & 6.2750 \mathrm{E}-04\end{array}$

$\begin{array}{lllllllll}11 & 40 & 8.9904 \mathrm{E}-01 & 0.0000 \mathrm{E}+00 & 0.0000 \mathrm{E}+00 & 0.0000 \mathrm{E}+00 & -8.9904 \mathrm{E}-01 & 3.9460 \mathrm{E}-06\end{array}$

$\begin{array}{llllllll}12 & 41 & 9.7260 \mathrm{E}-0 \mathrm{I} & 0.0000 \mathrm{E}+00 & 0.0000 \mathrm{E}+00 & 0.0000 \mathrm{E}+00 & -9.6978 \mathrm{E}-01 & 2.8204 \mathrm{E}-03\end{array}$

$\begin{array}{lllllllll}13 & 140 & 0.0000 \mathrm{E}+00 & 0.0000 \mathrm{E}+00 & 0.0000 \mathrm{E}+00 & 0.0000 \mathrm{E}+00 & 0.0000 \mathrm{E}+00 & 0.0000 \mathrm{E}+00\end{array}$

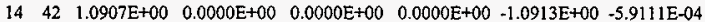

$\begin{array}{lllllllll}15 & 154 & 1.2943 \mathrm{E}+01 & 8.4067 \mathrm{E}-01 & 0.0000 \mathrm{E}+00 & 0.0000 \mathrm{E}+00 & -1.3171 \mathrm{E}+01 & 6.1278 \mathrm{E}-01\end{array}$

$\begin{array}{lllllllll}16 & 149 & 2.6508 \mathrm{E}+01 & 0.0000 \mathrm{E}+00 & 0.0000 \mathrm{E}+00 & 0.0000 \mathrm{E}+00 & -2.6508 \mathrm{E}+01 & 3.5884 \mathrm{E}-05\end{array}$

$\begin{array}{lllllllll}17 & 144 & 2.7419 \mathrm{E}+01 & 0.0000 \mathrm{E}+00 & 0.0000 \mathrm{E}+00 & 0.0000 \mathrm{E}+00 & -2.7405 \mathrm{E}+01 & 1.4572 \mathrm{E}-02\end{array}$

$\begin{array}{lllllllll}18 & 159 & 3.1331 \mathrm{E}+01 & 8.0064 \mathrm{E}-02 & 0.0000 \mathrm{E}+00 & 0.0000 \mathrm{E}+00 & -3.1361 \mathrm{E}+01 & 5.0067 \mathrm{E}-02\end{array}$

$191413.9111 \mathrm{E}+01 \quad 4.0032 \mathrm{E}-02 \quad 0.0000 \mathrm{E}+00 \quad 0.0000 \mathrm{E}+00-3.9150 \mathrm{E}+01 \quad 4.5860 \mathrm{E}-04$

total $\quad 1.4161 \mathrm{E}+02 \quad 9.6077 \mathrm{E}-01 \quad 0.0000 \mathrm{E}+00 \quad 0.0000 \mathrm{E}+00 \quad-1.4174 \mathrm{E}+02 \quad 8.3809 \mathrm{E}-01$

Ineutron weight balance in each cell - variance reduction events

print table 130

cell weight cell weight energy dxtran forced exponential total window importance cutoff importance collision transform

1 $30 \quad 0.0000 \mathrm{E}+00 \quad 0.0000 \mathrm{E}+00 \quad 0.0000 \mathrm{E}+00 \quad 0.0000 \mathrm{E}+00 \quad 0.0000 \mathrm{E}+00 \quad 0.0000 \mathrm{E}+00 \quad 0.0000 \mathrm{E}+00 \quad 0.0000 \mathrm{E}+00$

$\begin{array}{lllllllllll}2 & 31 & 0.0000 \mathrm{E}+00 & 0.0000 \mathrm{E}+00 & 0.0000 \mathrm{E}+00 & 0.0000 \mathrm{E}+00 & 0.0000 \mathrm{E}+00 & 0.0000 \mathrm{E}+00 & 0.0000 \mathrm{E}+00 & 0.0000 \mathrm{E}+00\end{array}$

$\begin{array}{lllllllllllll}3 & 37 & 0.0000 \mathrm{E}+00 & 0.0000 \mathrm{E}+00 & 0.0000 \mathrm{E}+00 & 0.0000 \mathrm{E}+00 & 0.0000 \mathrm{E}+00 & 0.0000 \mathrm{E}+00 & 0.0000 \mathrm{E}+00 & 0.0000 \mathrm{E}+00\end{array}$

$\begin{array}{llllllllll}4 & 34 & 0.0000 \mathrm{E}+00 & 0.0000 \mathrm{E}+00 & 0.0000 \mathrm{E}+00 & 0.0000 \mathrm{E}+00 & 0.0000 \mathrm{E}+00 & 0.0000 \mathrm{E}+00 & 0.0000 \mathrm{E}+00 & 0.0000 \mathrm{E}+00\end{array}$

$\begin{array}{llllllllll}5 & 32 & 0.0000 \mathrm{E}+00 & 0.0000 \mathrm{E}+00 & 0.0000 \mathrm{E}+00 & 0.0000 \mathrm{E}+00 & 0.0000 \mathrm{E}+00 & 0.0000 \mathrm{E}+00 & 0.0000 \mathrm{E}+00 & 0.0000 \mathrm{E}+00\end{array}$

$\begin{array}{llllllllll}6 & 33 & 0.0000 \mathrm{E}+00 & 0.0000 \mathrm{E}+00 & 0.0000 \mathrm{E}+00 & 0.0000 \mathrm{E}+00 & 0.0000 \mathrm{E}+00 & 0.0000 \mathrm{E}+00 & 0.0000 \mathrm{E}+00 & 0.0000 \mathrm{E}+00\end{array}$ $\begin{array}{llllllllll}35 & 0.0000 \mathrm{E}+00 & 0.0000 \mathrm{E}+00 & 0.0000 \mathrm{E}+00 & 0.0000 \mathrm{E}+00 & 0.0000 \mathrm{E}+00 & 0.0000 \mathrm{E}+00 & 0.0000 \mathrm{E}+00 & 0.0000 \mathrm{E}+00\end{array}$ $\begin{array}{llllllllll}36 & 0.0000 \mathrm{E}+00 & 0.0000 \mathrm{E}+00 & 0.0000 \mathrm{E}+00 & 0.0000 \mathrm{E}+00 & 0.0000 \mathrm{E}+00 & 0.0000 \mathrm{E}+00 & 0.0000 \mathrm{E}+00 & 0.0000 \mathrm{E}+00\end{array}$ 


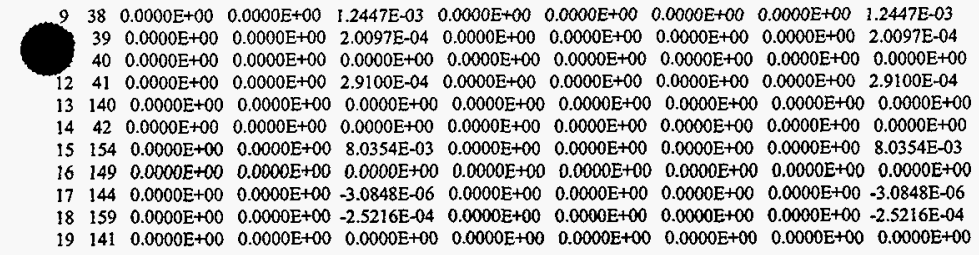

total $\quad 0.0000 \mathrm{E}+00 \quad 0.0000 \mathrm{E}+00 \quad 9.5169 \mathrm{E}-03 \quad 0.0000 \mathrm{E}+00 \quad 0.0000 \mathrm{E}+00 \quad 0.0000 \mathrm{E}+00 \quad 0.0000 \mathrm{E}+00 \quad 9.5169 \mathrm{E}-03$ Ineutron weight balance in each cell - physical events print table 130

cell $(n, x n)$ fission capture loss to loss to total $(n, x n)$ fission

$\begin{array}{llllllll}1 & 30 & 0.0000 \mathrm{E}+00 & 0.0000 \mathrm{E}+00 & 0.0000 \mathrm{E}+00 & 0.0000 \mathrm{E}+00 & 0.0000 \mathrm{E}+00 & 0.0000 \mathrm{E}+00\end{array}$

$\begin{array}{llllllllll}2 & 31 & 0.0000 \mathrm{E}+00 & 0.0000 \mathrm{E}+00 & 0.0000 \mathrm{E}+00 & 0.0000 \mathrm{E}+00 & 0.0000 \mathrm{E}+00 & 0.0000 \mathrm{E}+00\end{array}$

$\begin{array}{llllllll}3 & 37 & 0.0000 \mathrm{E}+00 & 0.0000 \mathrm{E}+00 & 0.0000 \mathrm{E}+00 & 0.0000 \mathrm{E}+00 & 0.0000 \mathrm{E}+00 & 0.0000 \mathrm{E}+00\end{array}$

$\begin{array}{lllllllll}4 & 34 & 0.0000 \mathrm{E}+00 & 0.0000 \mathrm{E}+00 & 0.0000 \mathrm{E}+00 & 0.0000 \mathrm{E}+00 & 0.0000 \mathrm{E}+00 & 0.0000 \mathrm{E}+00\end{array}$

$\begin{array}{lllllllll}5 & 32 & 0.0000 E+00 & 0.0000 E+00 & 0.0000 E+00 & 0.0000 E+00 & 0.0000 E+00 & 0.0000 E+00\end{array}$

$\begin{array}{lllllllll}6 & 33 & 0.0000 \mathrm{E}+00 & 0.0000 \mathrm{E}+00 & 0.0000 \mathrm{E}+00 & 0.0000 \mathrm{E}+00 & 0.0000 \mathrm{E}+00 & 0.0000 \mathrm{E}+00\end{array}$

$\begin{array}{llllllllll}7 & 35 & 0.0000 \mathrm{E}+00 & 0.0000 \mathrm{E}+00 & 0.0000 \mathrm{E}+00 & 0.0000 \mathrm{E}+00 & 0.0000 \mathrm{E}+00 & 0.0000 \mathrm{E}+00\end{array}$

$\begin{array}{lllllllll}8 & 36 & 0.0000 \mathrm{E}+00 & 0.0000 \mathrm{E}+00 & 0.0000 \mathrm{E}+00 & 0.0000 \mathrm{E}+00 & 0.0000 \mathrm{E}+00 & 0.0000 \mathrm{E}+00\end{array}$

$938 \quad 0.0000 \mathrm{E}+00 \quad 0.0000 \mathrm{E}+00-1.5855 \mathrm{E}-01 \quad 0.0000 \mathrm{E}+00 \quad 0.0000 \mathrm{E}+00-1.5855 \mathrm{E}-01$

$1039 \quad 0.0000 \mathrm{E}+00 \quad 0.0000 \mathrm{E}+00 \quad-8.2847 \mathrm{E}-04 \quad 0.0000 \mathrm{E}+00 \quad 0.0000 \mathrm{E}+00 \quad-8.2847 \mathrm{E}-04$

$\begin{array}{llllllll}11 & 40 & 0.0000 \mathrm{E}+00 & 0.0000 \mathrm{E}+00 & -3.9460 \mathrm{E}-06 & 0.0000 \mathrm{E}+00 & 0.0000 \mathrm{E}+00 & -3.9460 \mathrm{E}-06\end{array}$

$1241 \quad 0.0000 \mathrm{E}+00 \quad 0.0000 \mathrm{E}+00 \quad-3.1114 \mathrm{E}-03 \quad 0.0000 \mathrm{E}+00 \quad 0.0000 \mathrm{E}+00 \quad-3.1114 \mathrm{E}-03$

$\begin{array}{llllllllll}13 & 140 & 0.0000 \mathrm{E}+00 & 0.0000 \mathrm{E}+00 & 0.0000 \mathrm{E}+00 & 0.0000 \mathrm{E}+00 & 0.0000 \mathrm{E}+00 & 0.0000 \mathrm{E}+00\end{array}$

$\begin{array}{llllllll}14 & 42 & 1.2461 \mathrm{E}-03 & 0.0000 \mathrm{E}+00 & -3.1955 \mathrm{E}-05 & -6.2306 \mathrm{E}-04 & 0.0000 \mathrm{E}+00 & 5.9111 \mathrm{E}-04\end{array}$

$\begin{array}{llllllll}15 & 154 & 4.9179 \mathrm{E}-03 & 0.0000 \mathrm{E}+00 & -1.7239 \mathrm{E}-01 & -2.4589 \mathrm{E}-03 & -4.5089 \mathrm{E}-01 & -6.2082 \mathrm{E}-01\end{array}$

$\begin{array}{lllllll}149 & 0.0000 \mathrm{E}+00 & 0.0000 \mathrm{E}+00 & -3.5884 \mathrm{E}-05 & 0.0000 \mathrm{E}+00 & 0.0000 \mathrm{E}+00 & -3.5884 \mathrm{E}-05\end{array}$

$144 \quad 0.0000 \mathrm{E}+00 \quad 0.0000 \mathrm{E}+00 \quad-1.4569 \mathrm{E}-02 \quad 0.0000 \mathrm{E}+00 \quad 0.0000 \mathrm{E}+00 \quad-1.4569 \mathrm{E}-02$

$\begin{array}{lllllll}18 & 159 & 0.0000 \mathrm{E}+00 & 0.0000 \mathrm{E}+00 & -4.9815 \mathrm{E}-02 & 0.0000 \mathrm{E}+00 & 0.0000 \mathrm{E}+00-4.9815 \mathrm{E}-02\end{array}$

$\begin{array}{lllllllll}19 & 141 & 0.0000 \mathrm{E}+00 & 0.0000 \mathrm{E}+00 & -4.5860 \mathrm{E}-04 & 0.0000 \mathrm{E}+00 & 0.0000 \mathrm{E}+00 & -4.5860 \mathrm{E}-04\end{array}$

total $\quad 6.1640 \mathrm{E}-03 \quad 0.0000 \mathrm{E}+00 \quad-3.9980 \mathrm{E}-01 \quad-3.0820 \mathrm{E}-03-4.5089 \mathrm{E}-01 \quad-8.4760 \mathrm{E}-01$

ineutron activity of each nuclide in each cell, per source particle

print table 140

cell nuclides atom total collisions weight lost weight loss weight gain fraction collisions * weight to capture to fission by $(n, x n)$

$\begin{array}{rrccccccc}938 & 5010.03 \mathrm{~d} & 7.1977 \mathrm{E}-01 & 941 & 4.4212 \mathrm{E}-01 & 1.5853 \mathrm{E}-01 & 0.0000 \mathrm{E}+00 & 0.0000 \mathrm{E}+00 \\ 5011.40 \mathrm{c} & 8.0041 \mathrm{E}-02 & 76 & 3.6073 \mathrm{E}-02 & 1.3591 \mathrm{E}-07 & 0.0000 \mathrm{E}+00 & 0.0000 \mathrm{E}+00 \\ 6012.40 \mathrm{C} & 2.0019 \mathrm{E}-01 & 232 & 1.1297 \mathrm{E}-01 & 1.8446 \mathrm{E}-05 & 0.0000 \mathrm{E}+00 & 0.0000 \mathrm{E}+00\end{array}$

$\begin{array}{lllllllll}10 & 39 & 41093.40 \mathrm{c} & 9.8982 \mathrm{E}-0 ! & 128 & 6.2802 \mathrm{E}-02 & 8.2814 \mathrm{E}-04 & 0.0000 \mathrm{E}+00 & 0.0000 \mathrm{E}+00\end{array}$ $\begin{array}{lllllll}40000.40 \mathrm{c} & 1.0183 \mathrm{E}-02 & 2 & 9.7676 \mathrm{E}-04 & 3.3160 \mathrm{E}-07 & 0.0000 \mathrm{E}+00 & 0.0000 \mathrm{E}+00\end{array}$

$11 \quad 40 \quad 1001.00 \mathrm{c} \quad 3.3333 \mathrm{E}-01 \quad 111 \quad 5.1533 \mathrm{E}-02 \quad 3.9251 \mathrm{E}-06 \quad 0.0000 \mathrm{E}+00 \quad 0.0000 \mathrm{E}+00$ $\begin{array}{llllllll}1002.55 \mathrm{c} & 3.3333 \mathrm{E}-0 \mathrm{I} & 33 & 1.5373 \mathrm{E}-02 & 2.0884 \mathrm{E}-08 & 0.0000 \mathrm{E}+00 & 0.0000 \mathrm{E}+00\end{array}$ $\begin{array}{lllllll}8016.40 c & 3.3333 \mathrm{E}-01 & 40 & 2.1271 \mathrm{E}-02 & 0.0000 \mathrm{E}+00 & 0.0000 \mathrm{E}+00 & 0.0000 \mathrm{E}+0\end{array}$

$\begin{array}{lllllllll}12 & 41 & 41093.40 c & 9.8982 \mathrm{E}-01 & 313 & 1.5623 \mathrm{E}-01 & 3.1072 \mathrm{E}-03 & 0.0000 \mathrm{E}+00 & 0.0000 \mathrm{E}+00\end{array}$ $\begin{array}{llllllll}40000.40 \mathrm{c} & 1.0183 \mathrm{E}-02 & 4 & 1.8747 \mathrm{E}-03 & 4.2322 \mathrm{E}-06 & 0.0000 \mathrm{E}+00 & 0.0000 \mathrm{E}+00\end{array}$

$\begin{array}{lllllllll}14 & 42 & 1001.00 \mathrm{c} & 3.3333 \mathrm{E}-01 & 257 & 1.2131 \mathrm{E}-01 & 4.3914 \mathrm{E}-06 & 0.0000 \mathrm{E}+00 & 0.0000 \mathrm{E}+00\end{array}$ $\begin{array}{llllllll}1002.55 \mathrm{C} & 3.3333 \mathrm{E}-01 & 86 & 4.6121 \mathrm{E}-02 & 6.8027 \mathrm{E}-08 & 0.0000 \mathrm{E}+00 & 6.2306 \mathrm{E}-04\end{array}$ $\begin{array}{lllllll}8016.40 c & 3.3333 \mathrm{E}-01 & 98 & 5.1682 \mathrm{E}-02 & 2.7495 \mathrm{E}-05 & 0.0000 \mathrm{E}+00 & 0.0000 \mathrm{E}+00\end{array}$

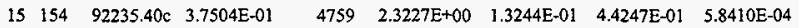
$\begin{array}{llllllll}92238.40 \mathrm{c} & 1.2499 \mathrm{E}-0 \mathrm{I} & 1470 & 7.3870 \mathrm{E}-01 & 2.3272 \mathrm{E}-02 & 8.4162 \mathrm{E}-03 & 1.8748 \mathrm{E}-03\end{array}$ $\begin{array}{lllllll}7014.40 \mathrm{c} & 4.9997 \mathrm{E}-01 & 2207 & 1.0775 \mathrm{E}+00 & 1.6682 \mathrm{E}-02 & 0.0000 \mathrm{E}+00 & 0.0000 \mathrm{E}+00\end{array}$

$149 \quad 1001.00 \mathrm{c} \quad 3.3333 E-01 \quad 345 \quad 1.6812 E-01 \quad 4.4875 E-06 \quad 0.0000 E+00 \quad 0.0000 E+00$ 
$\begin{array}{lllllll}1002.55 \mathrm{c} & 3.3333 \mathrm{E}-01 & 110 & 5.8433 \mathrm{E}-02 & 9.1921 \mathrm{E}-08 & 0.0000 \mathrm{E}+00 & 0.0000 \mathrm{E}+00\end{array}$

$\begin{array}{lllllll}8016.40 \mathrm{c} & 3.3333 \mathrm{E}-01 & 125 & 6.3072 \mathrm{E}-02 & 3.1305 \mathrm{E}-05 & 0.0000 \mathrm{E}+00 & 0.0000 \mathrm{E}+00\end{array}$

$\begin{array}{llllllll}144 & 74000.40 \mathrm{c} & 1.0000 \mathrm{E}+00 & 885 & 4.2652 \mathrm{E}-01 & 1.4569 \mathrm{E}-02 & 0.0000 \mathrm{E}+00 & 0.0000 \mathrm{E}+00\end{array}$

$\begin{array}{lllllllll}18 & 159 & 41093.40 \mathrm{c} & 9.8982 \mathrm{E}-01 & 3965 & 2.1009 \mathrm{E}+00 & 4.9769 \mathrm{E}-02 & 0.0000 \mathrm{E}+00 & 0.0000 \mathrm{E}+00\end{array}$ $\begin{array}{lllllll}40000.40 \mathrm{c} & 1.0183 \mathrm{E}-02 & 54 & 2.4590 \mathrm{E}-02 & 4.6175 \mathrm{E}-05 & 0.0000 \mathrm{E}+00 & 0.0000 \mathrm{E}+00\end{array}$

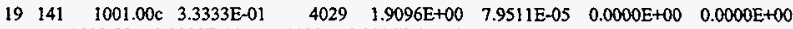
$\begin{array}{llllllll}1002.55 \mathrm{c} & 3.3333 \mathrm{E}-01 & 1122 & 5.8206 \mathrm{E}-01 & 9.0056 \mathrm{E}-07 & 0.0000 \mathrm{E}+00 & 0.0000 \mathrm{E}+00\end{array}$

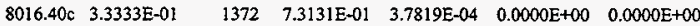

total $22764 \quad 1.1324 \mathrm{E}+01 \quad 3.9980 \mathrm{E}-01 \quad 4.5089 \mathrm{E}-01 \quad 3.0820 \mathrm{E}-03$

total over all cells for each nuelide total collisions weight lost weight loss weight gain collisions * weight to capture to fission by $(n, x n)$

$\begin{array}{ccccccc}1001.00 \mathrm{c} & 4742 & 2.2505 \mathrm{E}+00 & 9.2315 \mathrm{E}-05 & 0.0000 \mathrm{E}+00 & 0.0000 \mathrm{E}+00 \\ 1002.55 \mathrm{c} & 135 \mathrm{1} & 7.0199 \mathrm{E}-01 & 1.0814 \mathrm{E}-06 & 0.0000 \mathrm{E}+00 & 6.2306 \mathrm{E}-04 \\ 5011.40 \mathrm{c} & 76 & 3.6073 \mathrm{E}-02 & 1.3591 \mathrm{E}-07 & 0.0000 \mathrm{E}+00 & 0.0000 \mathrm{E}+00 \\ 6012.40 \mathrm{c} & 232 & 1.1297 \mathrm{E}-01 & 1.8446 \mathrm{E}-05 & 0.0000 \mathrm{E}+00 & 0.0000 \mathrm{E}+00 \\ 7014.40 \mathrm{c} & 2207 & 1.0775 \mathrm{E}+00 & 1.6682 \mathrm{E}-02 & 0.0000 \mathrm{E}+00 & 0.0000 \mathrm{E}+00 \\ 8016.40 \mathrm{c} & 1635 & 8.6733 \mathrm{E}-01 & 4.3699 \mathrm{E}-04 & 0.0000 \mathrm{E}+00 & 0.0000 \mathrm{E}+00 \\ 40000.40 \mathrm{c} & 60 & 2.7441 \mathrm{E}-02 & 5.0739 \mathrm{E}-05 & 0.0000 \mathrm{E}+00 & 0.0000 \mathrm{E}+00 \\ 41093.40 \mathrm{c} & 4406 & 2.3199 \mathrm{E}+00 & 5.3704 \mathrm{E}-02 & 0.0000 \mathrm{E}+00 & 0.0000 \mathrm{E}+00 \\ 74000.40 \mathrm{c} & 885 & 4.2652 \mathrm{E}-01 & 1.4569 \mathrm{E}-02 & 0.0000 \mathrm{E}+00 & 0.0000 \mathrm{E}+00 \\ 92235.40 \mathrm{c} & 4759 & 2.3227 \mathrm{E}+00 & 1.3244 \mathrm{E}-01 & 4.4247 \mathrm{E}-01 & 5.8410 \mathrm{E}-04 \\ 92238.40 \mathrm{c} & 1470 & 7.3870 \mathrm{E}-01 & 2.3272 \mathrm{E}-02 & 8.4162 \mathrm{E}-03 & 1.8748 \mathrm{E}-03 \\ 5010.03 \mathrm{~d} & 941 & 4.4212 \mathrm{E}-01 & 1.5853 \mathrm{E}-01 & 0.0000 \mathrm{E}+00 & 0.0000 \mathrm{E}+00\end{array}$

the initial fission neutron source distribution used the 8 source points that were input on the ksre card.

the criticality problem was scheduled to skip 5 cycles and run a total of 6 cycles with nominally 200 neutrons per cycle. problem has run 5 inactive cycles with 1027 neutron histories and 1 active cycles with 222 neutron histories.

this calculation has completed the requested number of keff cycles using a total of 1249 fission neutron source histories. all cells with fissionable material were sampled and had fission neutron source points.

there is no combined collision/absorption/track-length estimate for keff because only 1 active cycles were run. lindividual and average keff estimator results by cycle

keff neutron keff estimators by cycle average keff estimators and deviations average $k(\mathrm{c} / \mathrm{a} / \mathrm{t})$ cycle histories $k$ (coll) $k$ (abs) $k$ (track) $k$ (coll) st dev $k(a b s)$ st dev $k$ (track) st dev $k(c / a / t)$ st dev fom

\begin{tabular}{lllll|}
1 & $200 \mid 1.25311$ & 1.26302 & 1.22009 \\
2 & $257 \mid 1.21200$ & 1.25252 & 1.12957 \\
3 & $195 \mid 1.20026$ & 1.14326 & 1.13006 \\
4 & $193 \mid 1.12953$ & 1.16269 & 1.10757 \\
5 & $182 \mid 1.13599$ & 1.12465 & 1.11238 \\
\hline
\end{tabular}

$6222|1.07701 \quad 1.04669 \quad 1.07528|$

Itally $4 \quad \mathrm{nps}=1249$

+ fuel rod flux in $5 y$ locations averaged over $5 x$ elements

tally type 4 track length estimate of particle flux. units $1 / \mathrm{cm}^{* * 2}$

tally for neutrons

number of histories used for normalizing tallies $=200.00$

cell $a$ is $(154<42[-10-10] 42[-9-10] 42[-8-10] 42[-7-10] 42[-6-10])$

cell $b$ is $(154<42[-1030] 42[-930] 42[-830] 42[-730] 42[-630])$

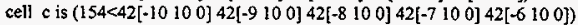

cell $d$ is $\left(154<42[-10210] 42[-9210] 42\left[\begin{array}{lll}-8 & 21 & 0\end{array}\right] 42\left[\begin{array}{lll}-7 & 21 & 0\end{array}\right] 42[-6210]\right)$

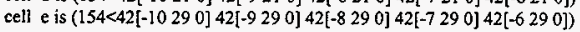

volumes

cell: $a$ a $\quad b \quad c \quad d \quad$ e

$1.04709 \mathrm{E}+02 \quad 1.04709 \mathrm{E}+02 \quad 1.04709 \mathrm{E}+02 \quad 1.04709 \mathrm{E}+02 \quad 1.04709 \mathrm{E}+02$ 
a $\quad 0.00000 \mathrm{E}+000.0000 \quad 2.84017 \mathrm{E}-050.9975 \quad 1.86420 \mathrm{E}-04 \quad 0.4656 \quad 1.36846 \mathrm{E}-04 \quad 0.5743 \quad 3.51668 \mathrm{E}-04 \quad 0.3877$

b $\quad 1.07442 \mathrm{E}-050.9975 \quad 1.58576 \mathrm{E}-04 \quad 0.5120 \quad 2.32928 \mathrm{E}-04 \quad 0.3945 \quad 4.44462 \mathrm{E}-050.5872 \quad 4.46694 \mathrm{E}-040.2855$

c $\quad 2.16778 \mathrm{E}-050.7232 \quad 9.40943 \mathrm{E}-050.5519 \quad 5.37835 \mathrm{E}-050.5876 \quad 1.51034 \mathrm{E}-040.7075 \quad 3.20589 \mathrm{E}-04 \quad 0.3937$

d $\quad 1.47903 \mathrm{E}-050.5831 \quad 0.00000 \mathrm{E}+00 \quad 0.0000 \quad 1.15515 \mathrm{E}-04 \quad 0.5838 \quad 1.69653 \mathrm{E}-040.5071 \quad 2.99958 \mathrm{E}-04 \quad 0.3718$

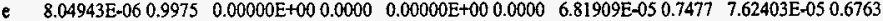
lanalysis of the results in the tally fluctuation chart bin (tfc) for tally 4 with nps $=1249$ print table 160

normed average tally per history $=3.51668 \mathrm{E}-04$

estimated tally relative error $=0.3877$

relative error from zero tallies $=0.3464$

number of nonzero history tallies $=\quad 8$

history number of largest tally $=\quad 1034$

(largest tally)/(average tally) $=3.84476 \mathrm{E}+01$

(confidence interval shif) $/$ mean $=0.0793$ unnormed average tally per history $=3.68228 \mathrm{E}-02$

estimated variance of the variance $=0.1752$

relative error from nonzero scores $=0.1740$

efficiency for the nonzero tallies $=0.0400$

largest unnormalized history tally $=1.41575 \mathrm{E}+00$

(largest tally)/(avg nonzero tally) $=1.53791 \mathrm{E}+00$

shifted confidence interval center $=3.79539 \mathrm{E}-04$

if the largest history score sampled so far were to occur on the very next history, the tfe bin quantities would change as follows: $n p s=222$ for this table because 5 keff cycles and 1027 histories were skipped before tally accumulation.

$\begin{array}{lccc}\text { estimated quantities } & \text { value at nps } & \text { value at nps }+1 & \text { value(nps+1)/value(n } \\ \text { mean } & 3.51668 \mathrm{E}-04 & 4.10723 \mathrm{E}-04 & 0.167927 \\ \text { relative error } & 3.87653 \mathrm{E}-01 & 3.61597 \mathrm{E}-01 & -0.067213 \\ \text { variance of the variance } & 1.75161 \mathrm{E}-01 & 1.47665 \mathrm{E}-01 & -0.156976 \\ \text { shifted center } & 3.79539 \mathrm{E}-04 & 3.77169 \mathrm{E}-04 & -0.006244 \\ \text { figure of merit } & 0.00000 \mathrm{E}+00 & 0.00000 \mathrm{E}+00 & 0.000000\end{array}$

re is not enough information in the largest history scores (usually less than 500 scores) for a reliable estimate of the slope.

history score probability density function appears to have an unsampled region at the largest history scores: please examine.

the nps-dependent tfo bin check results are suspect because there are only I nps tally values to analyze *****

results of 10 statistical checks for the estimated answer for the tally fluctuation chart (tfe) bin of tally 4

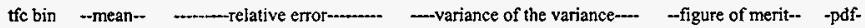

behavior behavior value decrease decrease rate value decrease decrease rate value behavior slope

desired random $<0.10$ yes $1 /$ sqrt(nps) $<0.10$ yes $1 /$ nps constant random $>3.00$

observed random 0.39 yes yes 0.18 yes yes 0.00

passed? yes no yes yes no yes yes no

warning. the tally in the tally fluctuation chart bin did not pass 3 of the 10 statistical checks.

lunnormed tally density for tally $4 \quad$ nonzero tally mean $(m)=9.206 \mathrm{E}-01 \mathrm{nps}=1249$ print table 161

abscissa ordinate $\log$ plot of tally probability density function in tally fluctuation chart bin( $d=$ decade,slope $=0.0$ )

tally number num den log den:d-..._-

$1.00-01 \quad 12.43-01-\left.0.614 * * * * * * * * * * * * * * * * * * * * * * * * * * * * * * * * * * * * * * * * * * * * * * * * * * * * * * * * * * * * * * * * * * * * * * * * * * * * * * * * * * * * * * * * * * * * * * * * * * * * * *\right|^{*}$

$1.26-01 \quad 00.00+00 \quad 0.000$

$1.58-01 \quad 00.00+00 \quad 0.000$

$00.00+00 \quad 0.000$

$00.00+00 \quad 0.000$

$0.00+00 \quad 0.000$ 
$00.00+00 \quad 0.000$

$00.00+00 \quad 0.000$

$27.71-02-1.113 * * * * * * * * * * * * * * * * * * * * * * * * * * * * * * * * * * * * * * * * * * * * * * * * * * *$

$00.00+00 \quad 0.000$

$12.43-02-1.614 \mathrm{~m}$

$00.00+00 \quad 0.000$

$1.00+00$

$1.26+00$

$46.14-02-1.212$

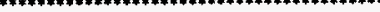

total $84.00-02$

cumulative tally number for tally 4

nonzero tally mean $(\mathrm{m})=9.206 \mathrm{E}-01$ nps $=$

1249 print table 162

abscissa cum ordinate plot of the cumulative number of tallies in the tally fluctuation chan bin from 0 to 100 percent

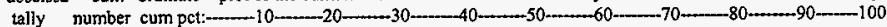

$1.00000 \mathrm{E}-01$ i $\left.12.500\right|^{* * * * * * * * * \mid * * *}$

$1.25893 \mathrm{E}-\left.01 \quad 1 \quad 12.500\right|^{\left.* * * * * * * * *\right|^{* * *}}$

$1.58489 \mathrm{E}-01 \quad$ l $\left.12.500\right|^{* * * * * * * * * \mid * * *}$

$1.99526 \mathrm{E}-01 \quad \mathrm{l} 12.500\}^{* * * * * * * * *[* * *}$

$2.51189 \mathrm{E}-01 \quad 112.500]^{* * * * * * * * * \mid * * *}$

$3.16228 \mathrm{E}-01 \quad 1 \quad 12.500 \mid * * * * * * * * * ; * * *$

$3.98108 \mathrm{E}-01$

$5.01188 \mathrm{E}-0 \mathrm{]}$

$6.30958 \mathrm{E}-01$

$7.94329 \mathrm{E}-01$

$1.00000 \mathrm{E}+00$

$1.25893 \mathrm{E}+00$

$1.58489 \mathrm{E}+00$

tota]

$\left.112.500\right|^{* * * * * * * * * \mid * * *}$

$\left.1 \quad 12.500\right|^{* * * * * * * * * * * * *}$

$37.500|* * * * * * * * *| * * * * * * * * *|* * * * * * * * *| * * * * * * * * \mid$

$337.500|* * * * * * * *| * * * * * * * * *|* * * * * * * *| * * * * * * * * \mid$

$450.000 \mid$ mmmmmmmmm/nmmmmmmm/mmmmmmmmm/mmmmmmmm/mmmmmmmm]

$\left.450.000\right|^{* * * * * * * * *|* * * * * * * * *| * * * * * * * * *|* * * * * * * * * * * * * * * * * * *|}$

8100.000

10

$-20$

$30+-40$

$50+\ldots+\ldots+\ldots 0$

$70-80-90-100$

cumulative unnomed tally for tally $4 \quad$ nonzero tally mean $(m)=9.206 \mathrm{E}-01 \quad \mathrm{nps}=1249$ print table 162

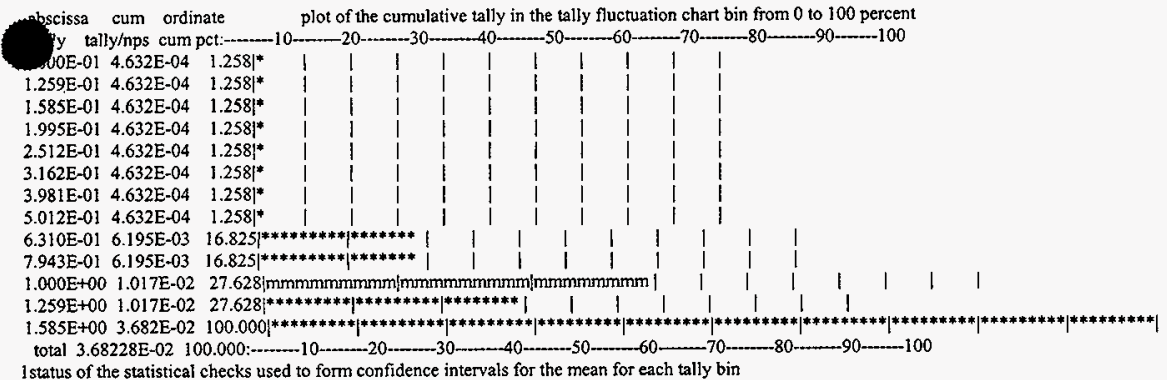

tally result of statistical checks for the tfe bin (the first check not passed is listed) and enror magnitude check for all bins

4 missed 3 of 10 tfc bin checks: the relative error exceeds the recommended value of 0.1 for nonpoint detector tallies missed all bin error check: 25 tally bins had 4 bins with zeros and 21 bins with relative errors exceeding 0.10

the 10 statistical checks are only for the tally fluctuation chart bin and do not apply to other tally bins.

the tally bins with zeros may or may not be correct: compare the source, cutoffs, multipliess, et cetera with the tally bins.

waming. 1 of the 1 tally fluctuation chart bins did not pass all 10 statistical checks.

waming. 1 of the 1 tallies had bins with relative errors greater than recommended.

1tally fluctuation charts

tally 4

nps mean etror vov slope fom 
$10000.0000 \mathrm{E}+00 \quad 0.00000 .0000 \quad 0.0$

$2493.5167 \mathrm{E}-040.38770 .1752 \quad 0.0$

data written to file inp $18 \mathrm{~m}$

ascii file inp $18 \mathrm{p}$ written with

5 events from 5 histories.

6 waming messages so far.

nn terminated when 6 kcode cycles were done.

\section{File: Outp18}

1- prob18 - kcode in a hexagonal prism lattice.

2- c three half control rods and five whole control rods.

3- $30^{\circ} 0-905-19291$ fill $=1$

$4-\quad 31 \quad 0-906-19291$ fill $=1(16.711300)$

5- $\quad 37 \quad 0-907-19291$ fill $=1(-16.711300)$

6- $34 \quad 0-913-1929$ fill $=1(0-11.91850)$

7 - $32 \quad 0-914-1929$ fill $=1(10.32175 .95920)$

8- $\quad 33 \quad 0-915-1929$ fill $=1(8.3557 \quad 14.47240)$

9- $35 \quad 0-916 \cdot 1929$ fill $=1(-8.3557 \quad 14.47240)$

10- $36 \quad 0-917-1929$ fill $=1(-10.32175 .95920)$

11- c universe 1: structure of control rod.

12. $38 \quad 11-2.02 \quad-880 u=1 \quad \$$ control rod core

13- $\quad 39 \quad 6 \quad-8.4 \quad 880-881 \quad u=1 \quad \$$ control rod cladding

14- $40 \quad 12 \quad-1.00 \quad 881-882 \quad u=1 \quad S$ control tod gap

15. $\quad 41 \quad 6-8.4 \quad 882 \quad u=1 \quad \$$ control tod sheath

16- c the space between the control rods, filled with lattice.

17. $140 \quad 0 \quad-17129-19905906907913914915916917$ fill $=2$

18- $c$ universe 2: lattice of fuel rods with water in between.

19. $42 \quad 12-1.00-301302-303 \quad 304-305306 \quad u=2$ lat $=2$ fill $=$

20. $\quad-37: 27-1: 330: 0$ \&

$24 r 39 \mathrm{r} 24 \mathrm{r} 311 \mathrm{r} 24 \mathrm{r} 311 \mathrm{r} 24 \mathrm{r} 39 \mathrm{r} 2$

$24 r 39 r 23 r 312 r 23 r 312 r 23 r 39 r 21 r$

$23 r 310 r 22 r 313 r 22 r 313 r 22 r 310 r 2$ ir

$23+357 r 22 r \&$

2 2r $358 \mathrm{r} 22 \mathrm{r}$

$22 \mathrm{r} 316 \mathrm{r} 22 \mathrm{r} 317 \mathrm{r} 22 \mathrm{r} 316 \mathrm{r} 23 \mathrm{r}$

2 2r $315 r 23 r 316 r 23 r 315 r 24 r$

$21 \mathrm{r} 315 \mathrm{r} 24 \mathrm{r} 315 \mathrm{r} 24 \mathrm{r} 315 \mathrm{r} 24 \mathrm{r}$

$21+315 r 23 r 316 r 23 r 315 r 25 r$

$21+315 r 22 \times 317 \times 22 \times 315 \times 26 r$

$2 \operatorname{lr} 354 \mathrm{r} 27 \mathrm{r} \&$

c can code temember \& thru comment?

$2355 \mathrm{r} 27 \mathrm{r}$

$2325 r 22 r 325 r 28 r$

$2324 \mathrm{r} 23 \mathrm{r} 324 \mathrm{r} 29 \mathrm{r}$

$2323 r 24 r 323 r 210 r$

$2315 \mathrm{r} 22 \mathrm{r} 34 \mathrm{r} 23 \mathrm{r} 34 \mathrm{r} 22 \mathrm{r} 31 \mathrm{st} 21 \mathrm{lr}$

$2314 \mathrm{r}_{2} 3 \mathrm{r} 34 \mathrm{r} 2 \mathrm{rr} 34 \mathrm{r} 23 \mathrm{r} 314 \mathrm{r} 2 \mathrm{r} 2 \mathrm{r}$

$2313 \mathrm{r} 24 \mathrm{r} 3 \mathrm{ll} 2 \mathrm{r} 2 \mathrm{r} 313 \mathrm{r} 213 \mathrm{r}$

$2313 \mathrm{r} 23 \mathrm{r} 312 \mathrm{r} 23 \mathrm{r} 313 \mathrm{r} 214 \mathrm{r}$

$2313 r 22 r 313 r 22 r 313 r 215 r$

$2346 \mathrm{r} 216 \mathrm{r}$

$2345 \mathrm{r} 217 \mathrm{r}$

$2344 \mathrm{r} 218 \mathrm{r}$

$2 \operatorname{lr} 341 \pi 220 r$

$2 \operatorname{lr} 340 \mathrm{r} 22 \mathrm{lr}$

$21+339 r 222 \mathrm{r}$

2 2r $336 r 224 r$

$2 \mathrm{rr} 335 \mathrm{r} 225 \mathrm{r}$

$23 \mathrm{r} 332 \mathrm{r} 227 \mathrm{r}$

$24 \mathrm{r} 329 \mathrm{r} 229 \mathrm{r}$

$25 \mathrm{r} 326 \mathrm{r} 231 \mathrm{r}$

$26 \mathrm{r} 323 \mathrm{r} 233 \mathrm{r}$

$28 \mathrm{r} 318 \mathrm{r} 236 \mathrm{r}$

$211 r 311+240 \mathrm{r}$ 
$264 \mathrm{r}$

c universe 3: structure of fuel rod lattice elements.

$154 \quad 2-13.75 \quad-58 \quad u=3 \quad \$$ fuel element

$149 \quad 12 \quad-1.00 \quad 58-268 \quad u=3 \quad \$$ gap

$144 \quad 7-19.66 \quad 268-478 \quad u=3 \quad$ S liner

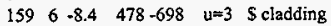

$141 \quad 12-1.00698 \quad u=3 \quad \$$ water between the fuel rods

162 0 17:*29:19:-1 \$outside world

* 1 py $0 \quad \$ x-z$ plane, reflective

17 cz 29.135

$19 \mathrm{pz} 31.75 \mathrm{~S}$ top of reactor

$29 \mathrm{pz}-31.75 \quad \$$ bottom of reactor

$\begin{array}{lllll}58 & \mathrm{c} / 2 & 3.4414 & .8515 & .3240\end{array}$

$\begin{array}{lllll}268 & \mathrm{c} / \mathrm{z} & 3.4414 & .8515 & .3345\end{array}$

$\begin{array}{lllll}478 & \mathrm{c} / \mathrm{z} & 3.4414 & .8515 & .3475\end{array}$

$\begin{array}{lllll}698 & \mathrm{c} / \mathrm{z} & 3.4414 & .8515 & .4318\end{array}$

$880 \mathrm{cz} \quad 1.7251$

$881 \mathrm{cz} 1.805$

$882 \mathrm{cz} 1.9051$

905 cz 2.1055

$906 \mathrm{c} / \mathrm{z} \quad 16.711302 .1055$

$907 \mathrm{c} / 2-16.711302 .1055$

$913 \mathrm{c} / \mathrm{z} \quad 011.91852 .1055$

$914 \mathrm{c} / \mathrm{z} \quad 10.32175 .95922 .1055$

$915 \mathrm{c} / \mathrm{z} \quad 8.3557 \quad 14.47242 .1055$

82- $\quad 916 \mathrm{c} / z-8.355714 .47242 .1055$

83- $\quad 917 \mathrm{c} / \mathrm{z}-10.32175 .95922 .1055$

84- 301 px 3.9330

85- $\quad 302$ px 2.9498

86- 303 p 11.732050807605 .8994

87- $\quad 304$ p $11.73205080760 \quad 3.9330$

305 p $-1 ! 1.73205080760-.9834$

306 p $-11.73205080760-2.9498$

impin $118 \mathrm{r} 0$

m2 $92235.40 \mathrm{c} \cdot .70573 \quad 92238.40 \mathrm{c} \cdot .23821 \quad 7014.40 \mathrm{c}-.05605$

m6 $\quad 41093.40 \mathrm{c}-.99000 \quad 40000.40 \mathrm{c}-.01000$

m7 $74000.40 \mathrm{c}-.74000$

$\mathrm{ml} 15010.03 \mathrm{~d}-.6870 \quad 5011.40 \mathrm{c} \quad-.0840 \quad 6012.40 \mathrm{c} \quad-.2290$

$\mathrm{m} 12 \quad 1001.00 \mathrm{cl} \quad 1002.55 \mathrm{c} 1 \quad 8016.40 \mathrm{c} 1$

$\mathrm{mt12}$ hwtr.01 lwtr.01

kcode 200156

warning. tallies are normed per fission neutron for one generation.

99- kste $3.2 .2 .23 .2-3.2 .2 .23 .243 .243 .243 .1-43.2$

$100-\mathrm{e} .01 .11 .10$.

101- fq fe

102- fo4 fuel rod flux in 5 y locations averaged over $5 x$ elements

103- f4: $n(154<(42[-10:-6-10])) \$$ average $5 \times$ elements at $j=-1$

104- $\quad(154<(42[-10:-630])) \&$ S average $5 x$ elements at $j=3$

105- $\quad(154<(42[-10:-6100]))$ \$ average $5 \times$ elements at $j=10$ \&

106- $\quad(154<(42[-10:-6210])) \quad \$$ average $5 \times$ elements at $j=21$

107. $\quad(154<(42[-10:-6290])) \quad \$$ average $5 \times$ elements at $j=29$

108- \$d4 $104.70890624 \mathrm{r} \quad \$ 5$ times the volume of cell 154

109- print -98

110- prdmp $2 \mathrm{j}-1$

111- ptrac buffer=20 file=asc write $=$ all event=bnk

1 initial source from ksrc card.

print table 90

original number of points points not in any cell points in cells of zero importance points in void cells points in ambiguous cells rotal points rejected nts remaining

0

0

8 


cycles to skip before tallying
number of keff cycles that can be stored
total fission nubar data are being used.
1tally 4
$+\quad$ fuel rod flux in 5
tally type 4 track length estimate
tally for neutrons
order of printing: fe
cells (154<(42[-10:-6 -10$]))(154<(42$
$290]))$
energy bins
$0.00000 \mathrm{E}+00$ to $1.00000 \mathrm{E}-02$ mev
$1.00000 \mathrm{E}-02$ to $1.00000 \mathrm{E}-01$ mev
$1.00000 \mathrm{E}-01$ to $1.00000 \mathrm{E}+00$ mev
$1.00000 \mathrm{E}+00$ to $1.00000 \mathrm{E}+01 \mathrm{mev}$
total bin

Imaterial composition

the sum of the fractions of material 2 was $9.999900 \mathrm{E}-01$

the sum of the fractions of material 7 was $7.400000 \mathrm{E}-01$

the sum of the fractions of material 12 was $3.000000 \mathrm{E}+00$

material

$\begin{array}{crrr}\text { material } & & \\ \text { mber } & \text { component nuclide, atom fraction } & \\ & & & \\ 2 & 92235,0.37504 & 92238,0.12499 & 7014,0.49997 \\ 6 & 41093,0.98982 & 40000,0.01018 & \\ 7 & 74000,1.00000 & & \\ 11 & 5010,0.71977 & 5011,0.08004 & 6012,0.20019 \\ 12 & 1001,0.33333 & 1002,0.33333 & 8016,0.33333 \\ \text { associated thermal s(a,b) data sets: hwtr.01t } & \text { lwtt.01t }\end{array}$

print table 30

ever $5 x$ elements

HNF-SD-SNF-CSWD-005, Rev. 0 
$\begin{array}{lllllll}7 & 35 & 0.00000 \mathrm{E}+00 & 0.00000 \mathrm{E}+00 & 0.00000 \mathrm{E}+00 & 8.84370 \mathrm{E}+02 & 0.00000 \mathrm{E}+00\end{array}$ $\begin{array}{lllll}36 & 0.00000 \mathrm{E}+00 & 0.00000 \mathrm{E}+00 & 0.00000 \mathrm{E}+00 & 8.84370 \mathrm{E}+02 \quad 0.00000 \mathrm{E}+00\end{array}$ $\begin{array}{llllll}38 & 1.15958 \mathrm{E}-01 & 2.02000 \mathrm{E}+00 & 0.00000 \mathrm{E}+00 & 0.00000 \mathrm{E}+00 & 0.00000 \mathrm{E}+00\end{array}$ $\begin{array}{llllll}39 & 5.44575 \mathrm{E}-02 & 8.40000 \mathrm{E}+00 & 0.00000 \mathrm{E}+00 & 0.00000 \mathrm{E}+00 & 0.00000 \mathrm{E}+00\end{array}$ $11 \quad 40 \quad 9.50007 \mathrm{E}-02 \quad 1.00000 \mathrm{E}+00 \quad 0.00000 \mathrm{E}+00 \quad 0.00000 \mathrm{E}+00 \quad 0.00000 \mathrm{E}+00$

$\begin{array}{llllllll}12 & 41 & 5.44575 \mathrm{E}-02 & 8.40000 \mathrm{E}+00 & 0.00000 \mathrm{E}+00 & 0.00000 \mathrm{E}+00 & 0.00000 \mathrm{E}+00\end{array}$

$13 \quad 140 \quad 0.00000 \mathrm{E}+00 \quad 0.00000 \mathrm{E}+00 \quad 0.00000 \mathrm{E}+00 \quad 0.00000 \mathrm{E}+00 \quad 0.00000 \mathrm{E}+00$

$\begin{array}{lllllll}14 & 42 & 9.50007 \mathrm{E}-02 & 1.00000 \mathrm{E}+00 & 0.00000 \mathrm{E}+00 & 0.00000 \mathrm{E}+00 & 0.00000 \mathrm{E}+00\end{array}$

$\begin{array}{lllllllll}15 & 154 & 6.62920 \mathrm{E}-02 & 1.37500 \mathrm{E}+01 & 0.00000 \mathrm{E}+00 & 0.00000 \mathrm{E}+00 & 0.00000 \mathrm{E}+00\end{array}$

$\begin{array}{lllllllll}16 & 149 & 9.50007 \mathrm{E}-02 & 1.00000 \mathrm{E}+00 & 0.00000 \mathrm{E}+00 & 0.00000 \mathrm{E}+00 & 0.00000 \mathrm{E}+00\end{array}$

$\begin{array}{llllllll}17 & 144 & 6.43969 \mathrm{E}-02 & 1.96600 \mathrm{E}+01 & 0.00000 \mathrm{E}+00 & 0.00000 \mathrm{E}+00 & 0.00000 \mathrm{E}+00\end{array}$

$\begin{array}{lllllllll}18 & 159 & 5.44575 \mathrm{E}-02 & 8.40000 \mathrm{E}+00 & 0.00000 \mathrm{E}+00 & 0.00000 \mathrm{E}+00 & 0.00000 \mathrm{E}+00\end{array}$

$\begin{array}{lllllllllll}19 & 141 & 9.50007 \mathrm{E}-02 & 1.00000 \mathrm{E}+00 & 0.00000 \mathrm{E}+00 & 0.00000 \mathrm{E}+0.0 .00000 \mathrm{E}+00\end{array}$

$\begin{array}{lllllll}20 & 162 & 0.00000 \mathrm{E}+00 & 0.00000 \mathrm{E}+00 & 0.00000 \mathrm{E}+00 & 0.00000 \mathrm{E}+00 & 0.00000 \mathrm{E}+00\end{array}$ 1surface areas print table 50
1

1

0 infinite

0 infinite

0 infinite

0 infinite

0 asymmetric

0 infinite

infinite

infinite

infinite

infinite

infinite

asymmetric

surface input calculated reason area area area not calculated

1 i $0.00000 \mathrm{E}+00 \quad 0.00000 \mathrm{E}+00$ asymmetric

$2 \quad 170.00000 \mathrm{E}+00 \quad 0.00000 \mathrm{E}+00$ asymmetric

$3 \quad 190.00000 \mathrm{E}+00 \quad 0.00000 \mathrm{E}+00$ asymmetric

$4290.00000 \mathrm{E}+00 \quad 0.00000 \mathrm{E}+00$ asymmetric

$558 \quad 0.00000 \mathrm{E}+00 \quad 0.00000 \mathrm{E}+00$ infinite

$6268 \quad 0.00000 \mathrm{E}+00 \quad 0.00000 \mathrm{E}+00$ infinite

$74780.00000 \mathrm{E}+00 \quad 0.00000 \mathrm{E}+00$ infinite

$8698 \quad 0.00000 \mathrm{E}+00 \quad 0.00000 \mathrm{E}+00$ infinite

$9880 \quad 0.00000 \mathrm{E}+00 \quad 0.00000 \mathrm{E}+00$ infinite

$10881 \quad 0.00000 \mathrm{E}+00 \quad 0.00000 \mathrm{E}+00$ infinite

$118820.00000 \mathrm{E}+00 \quad 0.00000 \mathrm{E}+00$ infinite

$12905 \quad 0.00000 \mathrm{E}+00 \quad 0.00000 \mathrm{E}+00$ asymmetric

$139060.00000 \mathrm{E} \div 00 \quad 0.00000 \mathrm{E}+00$ asymmetric

$149070.00000 \mathrm{E}+00 \quad 0.00000 \mathrm{E}+00$ asymmetric

$913 \quad 0.00000 \mathrm{E}+00 \quad 8.40057 \mathrm{E}+02$

$914 \quad 0.00000 \mathrm{E}+00 \quad 8.40057 \mathrm{E}+02$

$915 \quad 0.00000 \mathrm{E}+00 \quad 8.40057 \mathrm{E}+02$

$18 \quad 916 \quad 0.00000 \mathrm{E}+00 \quad 8.40057 \mathrm{E}+02$

$19 \quad 917 \quad 0.00000 \mathrm{E}+00 \quad 8.40057 \mathrm{E}+02$

$20301 \quad 0.00000 \mathrm{E} \div 00 \quad 0.00000 \mathrm{E}+00$ infinite

$21302 \quad 0.00000 \mathrm{E} \div 00 \quad 0.00000 \mathrm{E}+00$ infinite

$22303 \quad 0.00000 \mathrm{E}+00 \quad 0.00000 \mathrm{E}+00$ infinite

$233040.00000 E+00 \quad 0.00000 E+00$

$24305 \quad 0.00000 \mathrm{E}+00 \quad 0.00000 \mathrm{E}+00$

$25306 \quad 0.00000 \mathrm{E}+00 \quad 0.00000 \mathrm{E}+00$

lcells

print table 60

atom gram

cell mat density density volume mass pieces importance

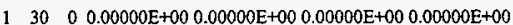

$23100.00000 \mathrm{E}+000.00000 \mathrm{E}+000.00000 \mathrm{E}+000.00000 \mathrm{E}+00$

$3 \quad 37 \quad 0 \quad 0.00000 \mathrm{E}+00 \quad 0.00000 \mathrm{E}+00 \quad 0.00000 \mathrm{E}+000.00000 \mathrm{E}+00$

$434 \quad 0 \quad 0.00000 \mathrm{E}+00 \quad 0.00000 \mathrm{E}+008.84370 \mathrm{E}+02 \quad 0.00000 \mathrm{E}+00$

$53200.00000 \mathrm{E}+000.00000 \mathrm{E}+008.84370 \mathrm{E}+020.00000 \mathrm{E}+00$

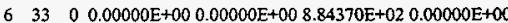

$735 \quad 0 \quad 0.00000 \mathrm{E}+00 \quad 0.00000 \mathrm{E}+008.84370 \mathrm{E}+02 \quad 0.00000 \mathrm{E}+00$

$8 \quad 36 \quad 0 \quad 0.00000 \mathrm{E}+000.00000 \mathrm{E}+00 \quad 8.84370 \mathrm{E}+02 \quad 0.00000 \mathrm{E}+00$

$938 \quad 111.15958 \mathrm{E}-012.02000 \mathrm{E}+000.00000 \mathrm{E}+00 \quad 0.00000 \mathrm{E}+00$

$10 \quad 39 \quad 6 \quad 5.44575 \mathrm{E}-02 \quad 8.40000 \mathrm{E}+00 \quad 0.00000 \mathrm{E}+00 \quad 0.00000 \mathrm{E}+00$

$11 \quad 40 \quad 12 \mathrm{~s} 9.50007 \mathrm{E}-02 \quad 1.00000 \mathrm{E}+00 \quad 0.00000 \mathrm{E}+000.00000 \mathrm{E}+00$

$12 \quad 41 \quad 6 \quad 5.44575 \mathrm{E}-02 \quad 8.40000 \mathrm{E}+00 \quad 0.00000 \mathrm{E}+000.00000 \mathrm{E}+00$

$13140 \quad 0 \quad 0.00000 \mathrm{E}+00 \quad 0.00000 \mathrm{E}+000.00000 \mathrm{E}+000.00000 \mathrm{E}+00$

$14 \quad 42 \quad 12 s 9.50007 \mathrm{E}-021.00000 \mathrm{E}+000.00000 \mathrm{E}+000.00000 \mathrm{E}+00$

$15 \quad 15422 \quad 6.62920 \mathrm{E}-021.37500 \mathrm{E}+010.00000 \mathrm{E}+000.00000 \mathrm{E}+00$

$16 \quad 149 \quad 12 \mathrm{~s} 9.50007 \mathrm{E}-02 \quad 1.00000 \mathrm{E}+000.00000 \mathrm{E}+000.00000 \mathrm{E}+00$

$14476.43969 \mathrm{E}-021.96600 \mathrm{E}+010.00000 \mathrm{E}+000.00000 \mathrm{E}+00$

$15965.44575 \mathrm{E}-028.40000 \mathrm{E}+000.00000 \mathrm{E}+000.00000 \mathrm{E}+00$
$0 \quad 1.0000 \mathrm{E}+00$ $01.0000 \mathrm{E}+00$

$0 \quad 1.0000 \mathrm{E}+00$

$11.0000 \mathrm{E}+00$

$11.0000 \mathrm{E}+00$

$11.0000 \mathrm{E}+00$

$11.0000 \mathrm{E}+00$

$1.0000 E+00$

- $1.0000 \mathrm{E}+00$

$01.0000 \mathrm{E}+00$

$01.0000 \mathrm{E}+00$

$0 \quad 1.0000 \mathrm{E}+00$

$01.0000 \mathrm{E}+00$

$01.0000 E+00$

$0 \quad 1.0000 \mathrm{E}+00$

$0 \quad 1.0000 E+00$

$01.0000 \mathrm{E}+00$

$0 \quad 1.0000 E+00$ 
$19 \quad 141 \quad 12 \mathrm{~s} 9.50007 \mathrm{E}-021.00000 \mathrm{E}+000.00000 \mathrm{E}+000.00000 \mathrm{E}+00 \quad 0 \quad 1.0000 \mathrm{E}+00$

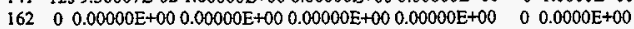

$4.42185 \mathrm{E}+030.00000 \mathrm{E}+00$

lsurfaces

print table 70

surface trans type surface coefficients

11 refl. py $0.0000000 \mathrm{E}+00$

$\begin{array}{llll}2 & 17 & \mathrm{Cz} & 2.9135000 \mathrm{E}+01\end{array}$

$\begin{array}{llll}3 & 19 & \text { pz } & 3.1750000 \mathrm{E}+01\end{array}$

429 pz $-3.1750000 \mathrm{E}+01$

$558 \quad \mathrm{c} / \mathrm{z} \quad 3.4414000 \mathrm{E}+00 \quad 8.5150000 \mathrm{E}-01 \quad 3.2400000 \mathrm{E}-01$

$\begin{array}{llllll}6 & 268 \quad \mathrm{c} / \mathrm{z} & 3.4414000 \mathrm{E}+00 & 8.5150000 \mathrm{E}-01 & 3.3450000 \mathrm{E}-0 \text { l }\end{array}$

$\begin{array}{lllllll}7 & 478 & \mathrm{c} / \mathrm{z} & 3.4414000 \mathrm{E}+00 & 8.5150000 \mathrm{E}-01 & 3.4750000 \mathrm{E}-0 !\end{array}$

$\begin{array}{llllll}8 & 698 & c / z & 3.4414000 \mathrm{E}+00 & 8.5150000 \mathrm{E}-01 & 4.3180000 \mathrm{E}-01\end{array}$

9880 cz $1.7251000 \mathrm{E}+00$

$10 \quad 881 \quad$ cz $1.8051000 \mathrm{E}+00$

$\begin{array}{llll}11 & 882 \quad \mathrm{CZ} & 1.9051000 \mathrm{E}+00\end{array}$

$12905 \quad \mathrm{cz} 2.1055000 \mathrm{E}+00$

$13906 \quad \mathrm{~d} / \mathrm{z} \quad 1.6711300 \mathrm{E}+01 \quad 0.0000000 \mathrm{E}+00 \quad 2.1055000 \mathrm{E}+00$

$14907 \quad c / z=1.6711300 \mathrm{E}+01 \quad 0.0000000 \mathrm{E}+00 \quad 2.1055000 \mathrm{E}+00$

$\begin{array}{lllll}15913 \quad c / 2 & 0.0000000 \mathrm{E}+00 & 1.1918500 \mathrm{E}+01 & 2.1055000 \mathrm{E}+00\end{array}$

$\begin{array}{llllll}16 & 914 & \mathrm{c} / \mathrm{z} & 1.0321700 \mathrm{E}+01 & 5.9592000 \mathrm{E}+00 & 2.1055000 \mathrm{E}+00\end{array}$

$\begin{array}{lllll}17915 \quad c / z & 8.3557000 \mathrm{E}+00 & 1.4472400 \mathrm{E}+01 & 2.1055000 \mathrm{E}+00\end{array}$

$\begin{array}{llllll}18916 \quad \mathrm{c} / \mathrm{z} & -8.3557000 \mathrm{E}+00 & 1.4472400 \mathrm{E}+01 & 2.1055000 \mathrm{E}+00\end{array}$

$\begin{array}{llllll}19 & 917 \quad c / z & -1.0321700 \mathrm{E}+01 & 5.9592000 \mathrm{E}+00 & 2.1055000 \mathrm{E}+00\end{array}$

$\begin{array}{llll}20 & 301 & \mathrm{px} & 3.9330000 \mathrm{E}+00\end{array}$

$21302 \quad \mathrm{px} \quad 2.9498000 \mathrm{E}+00$

$\begin{array}{lllllll}22 & 303 & \text { p } & 1.0000000 \mathrm{E}+00 & 1.7320508 \mathrm{E}+00 & 0.0000000 \mathrm{E}+00 & 5.8994000 \mathrm{E}+00\end{array}$

$\begin{array}{llllllll}23 & 304 & p & 1.0000000 \mathrm{E}+00 & 1.7320508 \mathrm{E}+00 & 0.0000000 \mathrm{E}+00 & 3.9330000 \mathrm{E}+00\end{array}$

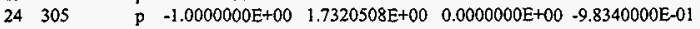

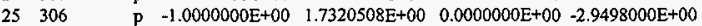

vil temperatures in mev for the free-gas themal neutron treatment. print table 72

all non-zero importance cells with materials have a temperature for themal neutrons of $2.5300 \mathrm{E}-08 \mathrm{mev}$.

2 warning messages so far.

Icross-section tables

print table 100

table length

tables from file testlib1

$1001.00 \mathrm{c} \quad 1782$ 1-h-1 from endf-vi.1

mat $125 \quad 05 / 26 / 93$

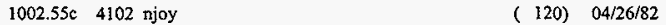

5011.40c 3229 ENDL library name: nd900719 MCNP translation: 900831 14:10:05 $\quad 900207$

$6012.40 \mathrm{c} 4858$ ENDL library name: nd900719 MCNP translation: 900831 14:10:05 900207

$7014.40 \mathrm{c} 8535$ ENDL library name: nd900719 MCNP translation: 900831 14:10:05 900207

$8016.40 \mathrm{c} 5358$ ENDL library name: nd900719 MCNP translation: 900831 14:10:05 900207

40000.40c 93372 ENDL library name: nd900719 MCNP translation: 900831 14:10:05 900323

41093.40c 42746 ENDL library name: nd900719 MCNP translation: 900831 14:10:05 900208

74000.40 91244 ENDL library name: nd900719 MCNP translation: 900831 14:10:05 900208

92235.40c 34772 ENDL library name: nd900719 MCNP translation: 9total nu 10:05 900503

92238.40c 40834 ENDL library name: nd901118 MCNP translation: 9total nu 56:49

$5010.03 d 3682$ b-10 endf/b-iv new gamma production format

901119

hwtr.01t 10193 deuterium in heavy water at 300 degrees kelvin

( 1273) 14 oct 75

lwtr.01t 10193 hydrogen in light water at 300 degrees kelvin

$10020010 / 22 / 85$

$\begin{array}{lll}1001 & 0 & 010 / 22 / 85\end{array}$

total 354900

warning, neutron energy cutoff is below some cross-section tables. 
HNF-SD-SNF-CSWD-005, Rev. 0

3.492E+00 1.051E+00 2.000E-01 42( $0,-1,0) \quad-9.903 \mathrm{E}-01-1.380 \mathrm{E}-01 \quad 1.353 \mathrm{E}-02$ $3.492 \mathrm{E}+00 \quad 1.051 \mathrm{E}+00 \quad 2.000 \mathrm{E}-01 \quad 154 \quad 0-9.903 \mathrm{E}-01-1.380 \mathrm{E}-01 \quad 1.353 \mathrm{E}-02$ $3.000 \mathrm{E}+00 \quad 2.000 \mathrm{E}-01 \quad 2.000 \mathrm{E}-01 \quad 140 \quad 7.462 \mathrm{E}-01 \quad 4.859 \mathrm{E}-01-4.551 \mathrm{E}-01 \quad 1.000 \mathrm{E}+00 \quad 1.000 \mathrm{E}+00 \quad 0.000 \mathrm{E}+00$ $3.492 \mathrm{E}+00 \quad 1.051 \mathrm{E}+00 \quad 2.000 \mathrm{E}-01 \quad 42(0,-1,0) \quad 7.462 \mathrm{E}-01 \quad 4.859 \mathrm{E}-01-4.551 \mathrm{E}-01$ $3.492 \mathrm{E}+00 \quad 1.051 \mathrm{E}+00 \quad 2.000 \mathrm{E}-01 \quad 154$

$213.000 \mathrm{E}+00 \quad 2.000 \mathrm{E}-012.000 \mathrm{E}-01 \quad 140$ $3.492 \mathrm{E}+00 \quad 1.051 \mathrm{E}+00 \quad 2.000 \mathrm{E}-01 \quad 42(0,-1,0)$ $3.492 \mathrm{E}+00 \quad 1.051 \mathrm{E}+00 \quad 2.000 \mathrm{E}-01 \quad 154$

$223.000 \mathrm{E}+\infty 02.000 \mathrm{E}-01 \quad 2.000 \mathrm{E}-01 \quad 140$ $3.492 \mathrm{E}+00 \quad 1.051 \mathrm{E}+00 \quad 2.000 \mathrm{E}-01 \quad 42(0,+1,0)$ $3.492 \mathrm{E}+00 \quad 1.051 \mathrm{E}+00 \quad 2.000 \mathrm{E}-01 \quad 154$

$23 \quad 3.000 \mathrm{E}+00 \quad 2.000 \mathrm{E}-01 \quad 2.000 \mathrm{E}-01 \quad 140$ $3.492 \mathrm{E}+00 \quad 1.051 \mathrm{E}+00 \quad 2.000 \mathrm{E}-01 \quad 42(0,-1,0)$ $3.492 \mathrm{E}+00 \quad 1.051 \mathrm{E}+00 \quad 2.000 \mathrm{E}-01 \quad 154$

$243.000 \mathrm{E}+00 \quad 2.000 \mathrm{E}-01 \quad 2.000 \mathrm{E}-01 \quad 140$ $3.492 \mathrm{E}+00 \quad 1.05 \mathrm{IE}+00 \quad 2.000 \mathrm{E}-01 \quad 42(0,-1,0)$ $3.492 \mathrm{E}+00 \quad 1.051 \mathrm{E}+00 \quad 2.000 \mathrm{E}-01 \quad 154$

$253.000 \mathrm{E}+00 \quad 2.000 \mathrm{E}-012.000 \mathrm{E}-01 \quad 140$ $3.492 \mathrm{E}+00 \quad 1.051 \mathrm{E}+00 \quad 2.000 \mathrm{E}-01 \quad 42(0,-1,0)$ $3.492 \mathrm{E}+00 \quad 1.051 \mathrm{E}+00 \quad 2.000 \mathrm{E}-01 \quad 154$

$262.000 \mathrm{E}-01 \quad 3.000 \mathrm{E}+00 \quad 2.000 \mathrm{E}-01 \quad 140$ $3.641 \mathrm{E}+00 \quad 4.456 \mathrm{E}-01 \quad 2.000 \mathrm{E}-01 \quad 42(-5,3,0)$ $3.641 \mathrm{E}+00 \quad 4.456 \mathrm{E}-012.000 \mathrm{E}-01 \quad 14 \mathrm{I}$

$27 \quad 2.000 \mathrm{E}-01 \quad 3.000 \mathrm{E}+00 \quad 2.000 \mathrm{E}-01 \quad 140$ $3.641 \mathrm{E}+00 \quad 4.456 \mathrm{E}-01 \quad 2.000 \mathrm{E}-01 \quad 42(-5,3,0)$ $3.641 \mathrm{E}+00 \quad 4.456 \mathrm{E}-01 \quad 2.000 \mathrm{E}-01 \quad 141$

$28 \quad 2.000 \mathrm{E}-01 \quad 3.000 \mathrm{E}+00 \quad 2.000 \mathrm{E}-01 \quad 140$ $3.641 \mathrm{E}+00 \quad 4.456 \mathrm{E}-01 \quad 2.000 \mathrm{E}-01 \quad 42(-5,3,0)$ $3.641 \mathrm{E}+00 \quad 4.456 \mathrm{E}-01 \quad 2.000 \mathrm{E}-01 \quad 141$

$292.000 \mathrm{E}-01 \quad 3.000 \mathrm{E}+00 \quad 2.000 \mathrm{E}-01 \quad 140$ $3.641 \mathrm{E}+00 \quad 4.456 \mathrm{E}-01 \quad 2.000 \mathrm{E}-01 \quad 42(-5,3,0)$ $3.641 \mathrm{E}+00 \quad 4.456 \mathrm{E}-01 \quad 2.000 \mathrm{E}-01 \quad 141$

$302.000 \mathrm{E}-013.000 \mathrm{E}+00 \quad 2.000 \mathrm{E}-01 \quad 140$ $641 \mathrm{E}+00 \quad 4.456 \mathrm{E}-01 \quad 2.000 \mathrm{E}-01 \quad 42(-5,3$, $.641 \mathrm{E}+00 \quad 4.456 \mathrm{E}-01 \quad 2.000 \mathrm{E}-01 \quad 141$

$312.000 \mathrm{E}-01 \quad 3.000 \mathrm{E}+00 \quad 2.000 \mathrm{E}-01 \quad 140$ $3.641 \mathrm{E}+00 \quad 4.456 \mathrm{E}-01 \quad 2.000 \mathrm{E}-01 \quad 42(-5,3,0)$ $3.641 \mathrm{E}+00 \quad 4.456 \mathrm{E}-01 \quad 2.000 \mathrm{E}-01 \quad 141$

32 2.000E-01 3.000E $+002.000 \mathrm{E}-01 \quad 140$ $3.641 \mathrm{E}+00 \quad 4.456 \mathrm{E}-01 \quad 2.000 \mathrm{E}-01 \quad 42(-5,3,0)$ $3.641 \mathrm{E}+00 \quad 4.456 \mathrm{E}-01 \quad 2.000 \mathrm{E}-01 \quad 141$

33 2.000E-01 3.000E+00 2.000E-01 140 $3.641 \mathrm{E}+00 \quad 4.456 \mathrm{E}-01 \quad 2.000 \mathrm{E}-01 \quad 42(-5,3,0)$ $3.641 \mathrm{E}+00 \quad 4.456 \mathrm{E}-01 \quad 2.000 \mathrm{E}-01 \quad 141$

$342.000 \mathrm{E}-01 \quad 3.000 \mathrm{E}+00 \quad 2.000 \mathrm{E}-01 \quad 140$ 3.641E+00 4.456E-01 2.000E-01 42( $-5,3,0)$ $3.641 \mathrm{E}+00 \quad 4.456 \mathrm{E}-01 \quad 2.000 \mathrm{E}-01 \quad 141$

$352.000 \mathrm{E}-01 \quad 3.000 \mathrm{E}+00 \quad 2.000 \mathrm{E}-01 \quad 140$ $3.641 \mathrm{E}+00 \quad 4.456 \mathrm{E}-01 \quad 2.000 \mathrm{E}-01 \quad 42(-5,3,0)$ $3.641 \mathrm{E}+00$ 4.456E-0! 2.000E-01 141

$36 \quad 2.000 \mathrm{E}-01 \quad 3.000 \mathrm{E}+00 \quad 2.000 \mathrm{E}-01 \quad 140$ $3.641 \mathrm{E}+00 \quad 4.456 \mathrm{E}-01 \quad 2.000 \mathrm{E}-01 \quad 42(-5,3,0)$ $3.641 \mathrm{E}+00 \quad 4.456 \mathrm{E}-01 \quad 2.000 \mathrm{E}-01 \quad 141$

$37 \quad 2.000 \mathrm{E}-01 \quad 3.000 \mathrm{E}+00 \quad 2.000 \mathrm{E}-01 \quad 140$ $3.641 \mathrm{E}+00 \quad 4.456 \mathrm{E}-01 \quad 2.000 \mathrm{E}-01 \quad 42(-5,3,0)$ $3.641 \mathrm{E}+00 \quad 4.456 \mathrm{E}-01 \quad 2.000 \mathrm{E}-01 \quad 141$

$38 \quad 2.000 \mathrm{E}-01 \quad 3.000 \mathrm{E}+00 \quad 2.000 \mathrm{E}-01 \quad 140$ $3.641 \mathrm{E}+00 \quad 4.456 \mathrm{E}-01 \quad 2.000 \mathrm{E}-01 \quad 42(-5,3,0)$ $3.641 \mathrm{E}+00 \quad 4.456 \mathrm{E}-01 \quad 2.000 \mathrm{E}-01 \quad 141$

$392.000 \mathrm{E}-013.000 \mathrm{E}+00 \quad 2.000 \mathrm{E}-01 \quad 140$ $3.641 \mathrm{E}+00 \quad 4.456 \mathrm{E}-01 \quad 2.000 \mathrm{E}-01 \quad 42(\cdot 5,3,0)$ $3.641 \mathrm{E}+00 \quad 4.456 \mathrm{E}-01 \quad 2.000 \mathrm{E}-01 \quad 141$

$40 \quad 2.000 \mathrm{E}-01 \quad 3.000 \mathrm{E}+00 \quad 2.000 \mathrm{E}-01 \quad 140$ $3.641 \mathrm{E}+00 \quad 4.456 \mathrm{E}-01 \quad 2.000 \mathrm{E}-01 \quad 42(-5,3,0)$ 3.641E+00 4.456E-0! 2.000E-01 141 $.000 \mathrm{E}-01 \quad 3.000 \mathrm{E}+00 \quad 2.000 \mathrm{E}-01 \quad 140$ $641 \mathrm{E}+00 \quad 4.456 \mathrm{E}-01 \quad 2.000 \mathrm{E}-01 \quad 42(-5,3,0)$

0 7.462E-01 4.859E-01 -4.551E-01

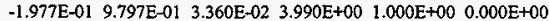
$-1.977 \mathrm{E}-019.797 \mathrm{E}-01 \quad 3.360 \mathrm{E}-02$

$0-1.977 \mathrm{E}-019.797 \mathrm{E}-013.360 \mathrm{E}-02$

$-9.117 \mathrm{E}-01 \quad-3.647 \mathrm{E}-01-1.891 \mathrm{E}-01 \quad 2.665 \mathrm{E}-01 \quad 1.000 \mathrm{E}+00 \quad 0.000 \mathrm{E}+00$ $-9.117 \mathrm{E}-01-3.647 \mathrm{E}-01-1.891 \mathrm{E}-01$

$0-9.117 \mathrm{E}-01-3.647 \mathrm{E}-01-1.891 \mathrm{E}-01$

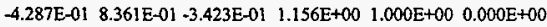
$-4.287 \mathrm{E}-01 \quad 8.361 \mathrm{E}-01-3.423 \mathrm{E}-0 \mathrm{t}$

$0-4.287 \mathrm{E}-01 \quad 8.361 \mathrm{E}-01-3.423 \mathrm{E}-01$ $1.080 \mathrm{E}-01 \quad 3.412 \mathrm{E}-01-9.338 \mathrm{E}-01 \quad 2.669 \mathrm{E}+00 \quad 1.000 \mathrm{E}+00 \quad 0.000 \mathrm{E}+00$ $1.080 \mathrm{E}-01 \quad 3.412 \mathrm{E}-01-9.338 \mathrm{E}-01$

$0 \quad 1.080 \mathrm{E}-01 \quad 3.412 \mathrm{E}-01-9.338 \mathrm{E}-01$

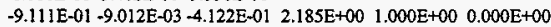
$-9.111 \mathrm{E}-01-9.012 \mathrm{E}-03-4.122 \mathrm{E}-01$

$0-9.111 \mathrm{E}-01-9.012 \mathrm{E}-03-4.122 \mathrm{E}-01$

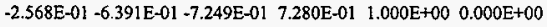
$-2.568 \mathrm{E}-01-6.391 \mathrm{E}-01-7.249 \mathrm{E}-01$

$0-2.568 \mathrm{E}-01-6.391 \mathrm{E}-01-7.249 \mathrm{E}-01$ $-2.912 \mathrm{E}-01 \quad 8.086 \mathrm{E}-01 \quad 5.113 \mathrm{E}-01 \quad 1.404 \mathrm{E}+00 \quad 1.000 \mathrm{E}+00 \quad 0.000 \mathrm{E}+00$ $-2.912 \mathrm{E}-01 \quad 8.086 \mathrm{E}-015.113 \mathrm{E}-01$

$0-2.912 \mathrm{E}-01 \quad 8.086 \mathrm{E}-01 \quad 5.113 \mathrm{E}-01$

$1.472 \mathrm{E}-01-9.514 \mathrm{E}-01 \quad 2.705 \mathrm{E}-01 \quad 1.090 \mathrm{E}+00 \quad 1.000 \mathrm{E}+00 \quad 0.000 \mathrm{E}+00$ 1.472E-01 -9.514E-01 2.705E-01

$0 \quad 1.472 \mathrm{E}-01-9.514 \mathrm{E}-01 \quad 2.705 \mathrm{E}-01$

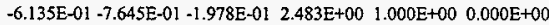
$-6.135 \mathrm{E}-01-7.645 \mathrm{E}-01-1.978 \mathrm{E}-01$

$0-6.135 \mathrm{E}-01-7.645 \mathrm{E}-01+1.978 \mathrm{E}-01$ $\begin{array}{llllll}-5.702 \mathrm{E}-01 & 5.651 \mathrm{E}-01 & -5.963 \mathrm{E}-01 & 3.767 \mathrm{E}-01 & 1.000 \mathrm{E}+00 & 0.000 \mathrm{E}+00\end{array}$ $-5.702 \mathrm{E}-01$ 5.651E-01 -5.963E-01

$0-5.702 \mathrm{E}-01 \quad 5.651 \mathrm{E}-01-5.963 \mathrm{E}-01$

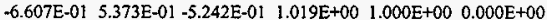
$-6.607 \mathrm{E}-01 \quad 5.373 \mathrm{E}-01-5.242 \mathrm{E}-01$

$0-6.607 \mathrm{E}-01 \quad 5.373 \mathrm{E}-01-5.242 \mathrm{E}-01$ $-9.742 \mathrm{E}-02-3.639 \mathrm{E}-01-9.263 \mathrm{E}-01 \quad 1.879 \mathrm{E}+00 \quad 1.000 \mathrm{E}+00 \quad 0.000 \mathrm{E}+00$ $-9.742 \mathrm{E}-02-3.639 \mathrm{E}-01-9.263 \mathrm{E}-01$

$0-9.742 \mathrm{E}-02-3.639 \mathrm{E}-01-9.263 \mathrm{E}-01$ $-1.965 \mathrm{E}-01-3.145 \mathrm{E}-01-9.287 \mathrm{E}-01 \quad 4.700 \mathrm{E}-01 \quad 1.000 \mathrm{E}+00 \quad 0.000 \mathrm{E}+00$ $-1.965 \mathrm{E}-01-3.145 \mathrm{E}-01-9.287 \mathrm{E}-01$

$0-1.965 \mathrm{E}-01-3.145 \mathrm{E}-01+9.287 \mathrm{E}-01$ 4.097E-01 8.465E-01 -3.399E-01 $1.530 \mathrm{E}+00 \quad 1.000 \mathrm{E}+00 \quad 0.000 \mathrm{E}+00$ 4.097E-01 8.465E-01 -3.399E-01

0 4.097E-01 8.465E-01 -3.399E-01

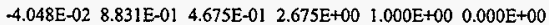
$4.048 \mathrm{E}-02 \quad 8.831 \mathrm{E}-01 \quad 4.675 \mathrm{E}-01$

$0-4.048 \mathrm{E}-02 \quad 8.831 \mathrm{E}-01 \quad 4.675 \mathrm{E}-01$ $3.371 \mathrm{E}-01-9.269 \mathrm{E}-01-1.652 \mathrm{E}-01 \quad 9.222 \mathrm{E}-01 \quad 1.000 \mathrm{E}+00 \quad 0.000 \mathrm{E}+00$ $3.371 \mathrm{E}-01-9.269 \mathrm{E}-01-1.652 \mathrm{E}-01$

$0 \quad 3.371 \mathrm{E}-01-9.269 \mathrm{E}-01-1.652 \mathrm{E}-01$

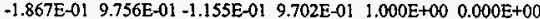
$-1.867 \mathrm{E}-01 \quad 9.756 \mathrm{E}-01-1.155 \mathrm{E}-01$

$0-1.867 \mathrm{E}-01 \quad 9.756 \mathrm{E}-01-1.155 \mathrm{E}-01$

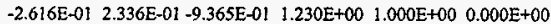
$-2.616 \mathrm{E}-01 \quad 2.336 \mathrm{E}-01-9.365 \mathrm{E}-01$

$0-2.616 E-01 \quad 2.336 \mathrm{E}-01-9.365 \mathrm{E}-01$ $9.780 \mathrm{E}-01-7.641 \mathrm{E}-02-1.939 \mathrm{E}-01 \quad 1.083 \mathrm{E}+00 \quad 1.000 \mathrm{E}+00 \quad 0.000 \mathrm{E}+00$ $9.780 \mathrm{E}-0 \mathrm{~L}-7.641 \mathrm{E}-02-1.939 \mathrm{E}-01$

$0 \quad 9.780 \mathrm{E}-01-7.641 \mathrm{E}-02-1.939 \mathrm{E}-01$ 2.580E-01 -7.076E-01 6.578E-01 8.313E-01 $1.000 \mathrm{E}+00 \quad 0.000 \mathrm{E}+00$ $2.580 \mathrm{E}-01-7.076 \mathrm{E}-01 \quad 6.578 \mathrm{E}-01$

$02.580 \mathrm{E}-01-7.076 \mathrm{E}-01 \quad 6.578 \mathrm{E}-01$

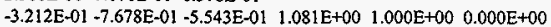
$-3.212 \mathrm{E}-01-7.678 \mathrm{E}-01-5.543 \mathrm{E}-01$ 
$3.641 \mathrm{E}+00 \quad 4.456 \mathrm{E}-01 \quad 2.000 \mathrm{E}-01 \quad 141$

$2.000 \mathrm{E}-01 \quad 3.000 \mathrm{E}+00 \quad 2.000 \mathrm{E}-01 \quad 140$

$3.641 \mathrm{E}+00 \quad 4.456 \mathrm{E}-01 \quad 2.000 \mathrm{E}-01 \quad 42(-5,3,0)$

$3.641 \mathrm{E}+00 \quad 4.456 \mathrm{E}-01 \quad 2.000 \mathrm{E}-01 \quad 14 \mathrm{I}$

$43 \quad 2.000 \mathrm{E}-01 \quad 3.000 \mathrm{E}+00 \quad 2.000 \mathrm{E}-01 \quad 140$

$3.641 \mathrm{E}+00 \quad 4.456 \mathrm{E}-012.000 \mathrm{E}-01 \quad 42(-5,3,0)$

$3.641 \mathrm{E}+00 \quad 4.456 \mathrm{E}-012.000 \mathrm{E}-0$ ? 141

$442.000 \mathrm{E}-013.000 \mathrm{E}+00 \quad 2.000 \mathrm{E}-01 \quad 140$

$3.641 \mathrm{E}+00 \quad 4.456 \mathrm{E}-01 \quad 2.000 \mathrm{E}-01 \quad 42(-5,3,0)$

$3.641 \mathrm{E}+00 \quad 4.456 \mathrm{E}-01 \quad 2.000 \mathrm{E}-01 \quad 14 \mathrm{l}$

$452.000 \mathrm{E}-01 \quad 3.000 \mathrm{E}+00 \quad 2.000 \mathrm{E}-01 \quad 140$ $3.641 \mathrm{E}+00 \quad 4.456 \mathrm{E}-01 \quad 2.000 \mathrm{E}-01 \quad 42(-5,3,0)$

$3.641 \mathrm{E}+00 \quad 4.456 \mathrm{E}-01 \quad 2.000 \mathrm{E}-01 \quad 141$

$46 \quad 2.000 \mathrm{E}-01 \quad 3.000 \mathrm{E}+00 \quad 2.000 \mathrm{E}-01 \quad 140$

$3.641 \mathrm{E}+00 \quad 4.456 \mathrm{E}-01 \quad 2.000 \mathrm{E}-01 \quad 42(-5,3,0)$

$3.641 \mathrm{E}+00 \quad 4.456 \mathrm{E}-01 \quad 2.000 \mathrm{E}-01 \quad 14 \mathrm{l}$

$47 \quad 2.000 \mathrm{E}-01 \quad 3.000 \mathrm{E}+00 \quad 2.000 \mathrm{E}-01 \quad 140$

$3.641 \mathrm{E}+00 \quad 4.456 \mathrm{E}-01 \quad 2.000 \mathrm{E}-01 \quad 42(-5,3,0)$

$3.641 \mathrm{E}+00 \quad 4.456 \mathrm{E}-01 \quad 2.000 \mathrm{E}-01 \quad 141$

$\begin{array}{ll}48 & 2.000 \mathrm{E}-01 \quad 3.000 \mathrm{E}+00 \quad 2.000 \mathrm{E}-01 \quad 140\end{array}$

$3.641 \mathrm{E}+00 \quad 4.456 \mathrm{E}-01 \quad 2.000 \mathrm{E}-01 \quad 42(-5,3,0)$

$3.641 \mathrm{E}+00 \quad 4.456 \mathrm{E}-01 \quad 2.000 \mathrm{E}-01 \quad 141$

$492.000 \mathrm{E}-01 \quad 3.000 \mathrm{E}+00 \quad 2.000 \mathrm{E}-01 \quad 140$

$3.641 \mathrm{E}+00 \quad 4.456 \mathrm{E}-01 \quad 2.000 \mathrm{E}-01 \quad 42(-5,3,0)$

$3.641 \mathrm{E}+00 \quad 4.456 \mathrm{E}-01 \quad 2.000 \mathrm{E}-01 \quad 141$

so $2.000 \mathrm{E}-01 \quad 3.000 \mathrm{E}+00 \quad 2.000 \mathrm{E}-01 \quad 140$

$3.641 \mathrm{E}+00 \quad 4.456 \mathrm{E}-01 \quad 2.000 \mathrm{E}-01 \quad 42(-5,3,0)$

$3.641 \mathrm{E}+00 \quad 4.456 \mathrm{E}-01 \quad 2.000 \mathrm{E}-01 \quad 141$

lestimated keff results by cycle
HNF-SD-SNF-CSWD-005, Rev. 0

$0-3.212 \mathrm{E}-01-7.678 \mathrm{E}-01-5.543 \mathrm{E}-0 \mathrm{I}$

$5.039 \mathrm{E}-01-1.460 \mathrm{E}-01 \quad 8.513 \mathrm{E}-01 \quad 3.341 \mathrm{E}+00 \quad 1.000 \mathrm{E}+00 \quad 0.000 \mathrm{E}+00$

$5.039 \mathrm{E}-01-1.460 \mathrm{E}-01 \quad 8.513 \mathrm{E}-01$

0 5.039E-01 -1.460E-01 8.513E-01

6.080E-01 5.487E-01 5.738E-01 $4.097 \mathrm{E}-01 \quad 1.000 \mathrm{E}+00 \quad 0.000 \mathrm{E}+00$

$6.080 \mathrm{E}-01 \quad 5.487 \mathrm{E}-01 \quad 5.738 \mathrm{E}-01$

$0 \quad 6.080 \mathrm{E}-01 \quad 5.487 \mathrm{E}-01 \quad 5.738 \mathrm{E}-01$

$\begin{array}{lllll}-2.932 \mathrm{E}-01 & 9.304 \mathrm{E}-01 & -2.199 \mathrm{E}-01 & 2.229 \mathrm{E}+00 \quad 1.000 \mathrm{E}+00 \quad 0.000 \mathrm{E}+00\end{array}$

$-2.932 \mathrm{E}-01 \quad 9.304 \mathrm{E}-01-2.199 \mathrm{E}-01$

$0-2.932 \mathrm{E}-01$ 9.304E-01 -2.199E-01

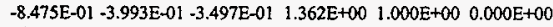

-8.475E-01 -3.993E-01 -3.497E-01

$0-8.475 \mathrm{E}-01-3.993 \mathrm{E}-01-3.497 \mathrm{E}-01$

$1.200 \mathrm{E}-01-9.195 \mathrm{E}-01-3.743 \mathrm{E}-01 \quad 1.010 \mathrm{E}+00 \quad 1.000 \mathrm{E}+00 \quad 0.000 \mathrm{E}+00$

$1.200 \mathrm{E}-01-9.195 \mathrm{E}-01-3.743 \mathrm{E}-01$

$0 \quad 1.200 \mathrm{E}-01-9.195 \mathrm{E}-01-3.743 \mathrm{E}-01$

$\begin{array}{llll}7.085 \mathrm{E}-01 & 5.879 \mathrm{E}-01 \quad 3.904 \mathrm{E}-01 & 7.068 \mathrm{E}+00 \quad 1.000 \mathrm{E}+00 & 0.000 \mathrm{E}+00\end{array}$

$7.085 \mathrm{E}-01 \quad 5.879 \mathrm{E}-01 \quad 3.904 \mathrm{E}-01$

$0 \quad 7.085 \mathrm{E}-01 \quad 5.879 \mathrm{E}-01 \quad 3.904 \mathrm{E}-01$

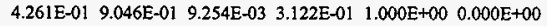

4.261E-01 $9.046 \mathrm{E}-01 \quad 9.254 \mathrm{E}-03$

$0 \quad 4.261 \mathrm{E}-01 \quad 9.046 \mathrm{E}-01 \quad 9.254 \mathrm{E}-03$

$\begin{array}{lllll}5.431 \mathrm{E}-01 & 4.270 \mathrm{E}-01-7.230 \mathrm{E}-01 & 5.484 \mathrm{E}-01 & 1.000 \mathrm{E}+00 & 0.000 \mathrm{E}+00\end{array}$

5.431E-01 4.270E-01 -7.230E-01

$0 \quad 5.431 \mathrm{E}-01 \quad 4.270 \mathrm{E}-01+7.230 \mathrm{E}-01$

$-1.053 \mathrm{E}-01 \cdot 9.805 \mathrm{E}-01 \quad 1.658 \mathrm{E}-01 \quad 1.545 \mathrm{E}+00 \quad 1.000 \mathrm{E}+00 \quad 0.000 \mathrm{E}+00$

$-1.053 \mathrm{E}-01-9.805 \mathrm{E}-01 \quad 1.658 \mathrm{E}-01$

$0-1.053 \mathrm{E}-01-9.805 \mathrm{E}-01 \quad 1.658 \mathrm{E}-01$

print table 175

cycle $1 \mathrm{k}$ (collision) 1.253108 removal lifetime(abs) $1.7557 \mathrm{E}+01$ source points generated 257

cycle $2 \mathrm{k}$ (collision) 1.211997 removal lifetime(abs) $1.1671 \mathrm{E}+01$ source points generated 195

$3 \mathrm{k}$ (collision) 1.200265 removal lifetime(abs) $9.6992 \mathrm{E}+00$ source points generated 193

cycle $4 \mathrm{k}$ (collision) 1.129530 removal lifetime(abs) $9.3720 \mathrm{E}+00$ source points generated 182

cycle $5 \mathrm{k}$ (collision) 1.135994 removal lifetime(abs) $1.1136 \mathrm{E}+01$ source points generated 222

source distribution written to fite inp $18 \mathrm{~s} \quad$ cycle $=5$

cycle $6 \mathrm{k}$ (collision) 1.077014 removal lifetime(abs) $1.2222 \mathrm{E}+01$ source points generated 204

source distribution written to file inp $18 \mathrm{~s} \quad$ cycle $=6$

lproblem summary

0

run terminated when 6 kcode cycles were done.

neutron creation tracks weight energy neutron loss tracks weight energy (per source particle) (per source particle)

source

$1249 \quad 9.6077 \mathrm{E}-01 \quad 1.8661 \mathrm{E}+00 \quad$ escape $\quad 220 \quad 1.2268 \mathrm{E}-01 \quad 1.2054 \mathrm{E}-01$

energy cutoff $\quad 00 . \quad 0$.

$\begin{array}{llll}\text { time cutoff } \quad 0 & 0 . & 0\end{array}$

weight window $\quad \begin{array}{lllllll}0 & 0 . & 0 . & \text { weight window } & 0 & 0 . & 0 .\end{array}$

cell importance $\quad 0 \quad 0 . \quad 0 . \quad 0 . l l$ importance $\quad 0 \quad 0 . \quad 0$.

$\begin{array}{llllllllll}\text { weight cutof } & 0 & 1.3743 \mathrm{E}-01 & 3.8498 \mathrm{E}-03 & \text { weight cutoff } & 1034 & 1.2791 \mathrm{E}-01 & 5.1920 \mathrm{E}-03\end{array}$

energy importance $\begin{array}{llllllll}0 & 0 & 0 & 0 & \text { energy importance } & 0 & 0 . & 0 .\end{array}$

dxtran 0 o. 0.

forced collisions 00 . 0 .

$\begin{array}{llll}\text { exp. transform } & 0 & 0 . & 0 .\end{array}$

upscattering

00 .

$5.3884 \mathrm{E}-11$

xtran $\quad 0 \quad 0$.

0.

0.

forced collisions 0 . $0 . \quad 0$.

exp. transform $\quad 0 \quad 0 . \quad 0$.

$\begin{array}{ccccc}\text { capture } & \text { downscattering } & 0 & 0 . & 1.360 \\ 0 & 0 & 3.9980 \mathrm{E}-01 & 1.1590 \mathrm{E}-01\end{array}$

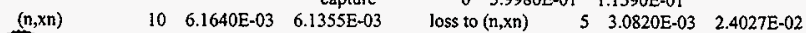

$\begin{array}{llllllll}0 & 0 . & 0 & \text { loss to fission } & 0 & 4.5089 \mathrm{E}-01 & 2.4987 \mathrm{E}-01\end{array}$

$\begin{array}{lllllllll}1259 & 1.1044 \mathrm{E}+00 & 1.8761 \mathrm{E}+00 & \text { total } & 1259 & 1.1044 \mathrm{E}+00 & 1.8761 \mathrm{E}+00\end{array}$ 
number of neutrons banked

5 average lifetime, shakes cutoffs

per source particle $1.0080 \mathrm{E}+00$ escape

Atron collisions per source particle $1.8226 \mathrm{E}+01$

capture $\quad 1.2954 \mathrm{E}+01$

eco $0.0000 E+00$

tal neutron collisions

$1.0032 \mathrm{E}+000.0035$

capture or escape $1.1788 \mathrm{E}+0 \mathrm{l}$

wel $-5.0000 \mathrm{E}-01$

net multiplication

any termination $1.2744 E+01$

wc2 $-2.5000 \mathrm{E}-01$

computer time so far in this run 0.00 minutes computer time in merun $\quad 0.00$ minutes source particles per minute $\quad 0.0000 \mathrm{E}+00$ random numbers generated $\quad 500880$ maximum numbet ever in bank 1 bank overflows to backup file 0 field length most random numbers used was 1298 in history

14

range of sampled source weights $=7.7821 \mathrm{E}-01$ to $1.0989 \mathrm{E}+00$

Ineutron activity in each cell

print table 126

tracks population collisions collisions number flux average average cell entering - weight weighted weighted track weight track mfp (perhistory) energy energy (relative) (cm)

I 30

$2 \quad 31$

$3 \quad 37$

$4 \quad 34$

$5 \quad 32$

$6 \quad 33$

735

836

938

$10 \quad 39$

$11 \quad 40 \quad 1728$

$\begin{array}{lll}12 & 41 & 1867\end{array}$

13140

$\begin{array}{ll}14 & 42\end{array}$

$2069 \quad 867$

$25302 \quad 1253$

$49542 \quad 1253$

$51224 \quad 1254$

$58650 \quad 1254$

$\begin{array}{lllllll}0 & 0.0000 \mathrm{E}+00 & 0.0000 \mathrm{E}+00 & 0.0000 \mathrm{E}+00 & 0.0000 \mathrm{E}+00 & 0.0000 \mathrm{E}+00\end{array}$

$\begin{array}{llllllll}0 & 0.0000 \mathrm{E}+00 & 0.0000 \mathrm{E}+00 & 0.0000 \mathrm{E}+00 & 0.0000 \mathrm{E}+00 & 0.0000 \mathrm{E}+00\end{array}$

$\begin{array}{lllllll}0 & 0.0000 \mathrm{E}+00 & 0.0000 \mathrm{E}+00 & 0.0000 \mathrm{E}+00 & 0.0000 \mathrm{E}+00 & 0.0000 \mathrm{E}+00\end{array}$

$\begin{array}{lllllll}0 & 0.0000 E+00 & 0.0000 E+00 & 0.0000 E+00 & 0.0000 E+00 & 0.0000 E+00\end{array}$

$\begin{array}{llllllll}0 & 0.0000 \mathrm{E}+00 & 0.0000 \mathrm{E}+00 & 0.0000 \mathrm{E}+00 & 0.0000 \mathrm{E}+00 & 0.0000 \mathrm{E}+00\end{array}$

$\begin{array}{lllllll}0 & 0.0000 \mathrm{E}+00 & 0.0000 \mathrm{E}+00 & 0.0000 \mathrm{E}+00 & 0.0000 \mathrm{E}+00 & 0.0000 \mathrm{E}+00\end{array}$

$\begin{array}{llllllll}0 & 0.0000 \mathrm{E}+00 & 0.0000 \mathrm{E}+00 & 0.0000 \mathrm{E}+00 & 0.0000 \mathrm{E}+00 & 0.0000 \mathrm{E}+00\end{array}$

$\begin{array}{llllllll}0 & 0.0000 \mathrm{E}+00 & 0.0000 \mathrm{E}+00 & 0.0000 \mathrm{E}+00 & 0.0000 \mathrm{E}+00 & 0.0000 \mathrm{E}+00\end{array}$

144

$18 \quad 159 \quad 58650$

$\begin{array}{llllllllll}18 & 159 & 58650 & 1254 & 4019 & 2.1254 \mathrm{E}+00 & 9.8931 \mathrm{E}-02 & 8.1154 \mathrm{E}-01 & 6.6977 \mathrm{E}-01 & 2.7541 \mathrm{E}+00 \\ 19 & 141 & 73122 & 1254 & 6523 & 3.2229 \mathrm{E}+00 & 9.4499 \mathrm{E}-02 & 8.0639 \mathrm{E}-01 & 6.6810 \mathrm{E}-01 & 2.5056 \mathrm{E}+00\end{array}$

$\begin{array}{lllllll}1249 & 5.9116 \mathrm{E}-01 & 3.0414 \mathrm{E}-01 & 8.9304 \mathrm{E}-01 & 6.4212 \mathrm{E}-01 & 2.6324 \mathrm{E}+00\end{array}$

$\begin{array}{lllllll}130 & 6.3779 \mathrm{E}-02 & 8.8236 \mathrm{E}-02 & 7.6766 \mathrm{E}-01 & 6.4931 \mathrm{E}-01 & 2.6947 \mathrm{E}+00\end{array}$

$\begin{array}{lllllll}184 & 8.8176 \mathrm{E}-02 & 7.6162 \mathrm{E}-02 & 7.6184 \mathrm{E}-01 & 6.4437 \mathrm{E}-01 & 2.5146 \mathrm{E}+00\end{array}$

$317 \quad 1.5811 \mathrm{E}-01 \quad 8.6238 \mathrm{E}-02 \quad 7.9306 \mathrm{E}-01 \quad 6.5030 \mathrm{E}-01 \quad 2.6940 \mathrm{E}+00$

$\begin{array}{lllllllll}0 & 0.0000 \mathrm{E}+00 & 0.0000 \mathrm{E}+00 & 0.0000 \mathrm{E}+00 & 0.0000 \mathrm{E}+00 & 0.0000 \mathrm{E}+00\end{array}$

$441 \quad 2.1911 \mathrm{E}-01 \quad 8.9382 \mathrm{E}-02 \quad 7.8771 \mathrm{E}-01 \quad 6.6777 \mathrm{E}-01 \quad 2.5091 \mathrm{E}+00$

$8436 \quad 4.1390 \mathrm{E}+00 \quad 1.2606 \mathrm{E}-01 \quad 8.5005 \mathrm{E}-01 \quad 6.6920 \mathrm{E}-01 \quad 2.4712 \mathrm{E}+00$

$\begin{array}{lllllll}580 & 2.8963 \mathrm{E}-01 & 1.0795 \mathrm{E}-01 & 8.2361 \mathrm{E}-01 & 6.7129 \mathrm{E}-01 & 2.5408 \mathrm{E}+00\end{array}$

$885 \quad 4.2652 \mathrm{E}-01 \quad 1.0541 \mathrm{E}-01 \quad 8.1599 \mathrm{E}-01 \quad 6.6971 \mathrm{E}-01 \quad 2.2391 \mathrm{E}+00$

$4019 \quad 2.1254 \mathrm{E}+00 \quad 9.8931 \mathrm{E}-02 \quad 8.1154 \mathrm{E}-01 \quad 6.6977 \mathrm{E}-01 \quad 2.7541 \mathrm{E}+00$

total $266059 \quad 9765 \quad 22764 \quad 1.1324 E+01$

Ineutron weight balance in each cell - extemal events

print table 130

cell entering source energy time exiting total
cutoff cutoff

$\begin{array}{llllllll}30 & 0.0000 \mathrm{E}+00 & 0.0000 \mathrm{E}+00 & 0.0000 \mathrm{E}+00 & 0.0000 \mathrm{E}+00 & 0.0000 \mathrm{E}+00 & 0.0000 \mathrm{E}+00\end{array}$

$\begin{array}{lllllllll}2 & 31 & 0.0000 \mathrm{E}+00 & 0.0000 \mathrm{E}+00 & 0.0000 \mathrm{E}+00 & 0.0000 \mathrm{E}+00 & 0.0000 \mathrm{E}+00 & 0.0000 \mathrm{E}+00\end{array}$

$\begin{array}{llllllll}3 & 37 & 0.0000 E+00 & 0.0000 E+00 & 0.0000 E+00 & 0.0000 E+00 & 0.0000 E+00 & 0.0000 E+00\end{array}$

$\begin{array}{lllllllll}4 & 34 & 0.0000 \mathrm{E}+00 & 0.0000 \mathrm{E}+00 & 0.0000 \mathrm{E}+00 & 0.0000 \mathrm{E}+00 & 0.0000 \mathrm{E}+00 & 0.0000 \mathrm{E}+00\end{array}$

$\begin{array}{lllllllll}5 & 32 & 0.0000 \mathrm{E}+00 & 0.0000 \mathrm{E}+00 & 0.0000 \mathrm{E}+00 & 0.0000 \mathrm{E}+00 & 0.0000 \mathrm{E}+00 & 0.0000 \mathrm{E}+00\end{array}$

$\begin{array}{lllllllll}6 & 33 & 0.0000 \mathrm{E}+00 & 0.0000 \mathrm{E}+00 & 0.0000 \mathrm{E}+00 & 0.0000 \mathrm{E}+00 & 0.0000 \mathrm{E}+00 & 0.0000 \mathrm{E}+00\end{array}$

$7 \quad 35 \quad 0.0000 \mathrm{E}+00 \quad 0.0000 \mathrm{E}+00 \quad 0.0000 \mathrm{E}+00 \quad 0.0000 \mathrm{E}+00 \quad 0.0000 \mathrm{E}+00 \quad 0.0000 \mathrm{E}+00$

$\begin{array}{lllllllll}8 & 36 & 0.0000 \mathrm{E}+00 & 0.0000 \mathrm{E}+00 & 0.0000 \mathrm{E}+00 & 0.0000 \mathrm{E}+00 & 0.0000 \mathrm{E}+00 & 0.0000 \mathrm{E}+00\end{array}$

$938 \quad 4.8822 \mathrm{E}-01 \quad 0.0000 \mathrm{E}+00 \quad 0.0000 \mathrm{E}+00 \quad 0.0000 \mathrm{E}+00 \quad-3.3091 \mathrm{E}-01 \quad 1.5731 \mathrm{E}-01$

$\begin{array}{lllllllll}10 & 39 & 8.4996 \mathrm{E}-01 & 0.0000 \mathrm{E}+00 & 0.0000 \mathrm{E}+00 & 0.0000 \mathrm{E}+00 & -8.4933 \mathrm{E}-01 & 6.2750 \mathrm{E}-04\end{array}$

$11 \quad 40 \quad 8.9904 \mathrm{E}-01 \quad 0.0000 \mathrm{E}+00 \quad 0.0000 \mathrm{E}+00 \quad 0.0000 \mathrm{E}+00 \quad-8.9904 \mathrm{E}-01 \quad 3.9460 \mathrm{E}-06$

$\begin{array}{llllllll}12 & 41 & 9.7260 \mathrm{E}-01 & 0.0000 \mathrm{E}+00 & 0.0000 \mathrm{E}+00 & 0.0000 \mathrm{E}+00 & -9.6978 \mathrm{E}-01 & 2.8204 \mathrm{E}-03\end{array}$

$\begin{array}{llllllllll}13 & 140 & 0.0000 \mathrm{E}+00 & 0.0000 \mathrm{E}+00 & 0.0000 \mathrm{E}+00 & 0.0000 \mathrm{E}+00 & 0.0000 \mathrm{E}+00 & 0.0000 \mathrm{E}+00\end{array}$

$\begin{array}{llllllll}14 & 42 & 1.0907 \mathrm{E}+00 & 0.0000 \mathrm{E}+00 & 0.0000 \mathrm{E}+00 & 0.0000 \mathrm{E}+00 & -1.0913 \mathrm{E}+00 & -5.9111 \mathrm{E}-04\end{array}$

$\begin{array}{llllllll}15 & 154 & 1.2943 \mathrm{E}+01 & 8.4067 \mathrm{E}-01 & 0.0000 \mathrm{E}+00 & 0.0000 \mathrm{E}+00 & -1.3171 \mathrm{E}+01 & 6.1278 \mathrm{E}-01\end{array}$

$\begin{array}{llllllllll}16 & 149 & 2.6508 \mathrm{E}+01 & 0.0000 \mathrm{E}+00 & 0.0000 \mathrm{E}+00 & 0.0000 \mathrm{E}+00 & -2.6508 \mathrm{E}+01 & 3.5884 \mathrm{E}-05\end{array}$

$\begin{array}{llllllll}17 & 144 & 2.7419 \mathrm{E}+01 & 0.0000 \mathrm{E}+00 & 0.0000 \mathrm{E}+00 & 0.0000 \mathrm{E}+00 & -2.7405 \mathrm{E}+01 & 1.4572 \mathrm{E}-02\end{array}$

$\begin{array}{lllllllll}18 & 159 & 3.1331 \mathrm{E}+01 & 8.0064 \mathrm{E}-02 & 0.0000 \mathrm{E}+00 & 0.0000 \mathrm{E}+00 & -3.1361 \mathrm{E}+01 & 5.0067 \mathrm{E}-02\end{array}$

$19 \quad 141 \quad 3.9111 \mathrm{E}+01 \quad 4.0032 \mathrm{E}-02 \quad 0.0000 \mathrm{E}+00 \quad 0.0000 \mathrm{E}+00 \quad-3.9150 \mathrm{E}+01 \quad 4.5860 \mathrm{E}-04$

total $\quad 1.4161 \mathrm{E}+02 \quad 9.6077 \mathrm{E}-01 \quad 0.0000 \mathrm{E}+00 \quad 0.0000 \mathrm{E}+00-1.4174 \mathrm{E}+02 \quad 8.3809 \mathrm{E}-01$

tron weight balance in each cell -- varjance reduction events

print table 130 
cell weight cell weight energy dxtran forced exponential total window importance cutof importance collision transform

$\begin{array}{llllllllllll}30 & 0.0000 \mathrm{E}+00 & 0.0000 \mathrm{E}+00 & 0.0000 \mathrm{E}+00 & 0.0000 \mathrm{E}+00 & 0.0000 \mathrm{E}+00 & 0.0000 \mathrm{E}+00 & 0.0000 \mathrm{E}+00 & 0.0000 \mathrm{E}+00\end{array}$ $\begin{array}{lllllllllll}31 & 0.0000 \mathrm{E}+00 & 0.0000 \mathrm{E}+00 & 0.0000 \mathrm{E}+00 & 0.0000 \mathrm{E}+00 & 0.0000 \mathrm{E}+0 & 0.0000 \mathrm{E}+00 & 0.0000 \mathrm{E}+00 & 0.0000 \mathrm{E}+00\end{array}$ $\begin{array}{lllllllllll}37 & 0.0000 \mathrm{E}+00 & 0.0000 \mathrm{E}+00 & 0.0000 \mathrm{E}+00 & 0.0000 \mathrm{E}+00 & 0.0000 \mathrm{E}+00 & 0.0000 \mathrm{E}+00 & 0.0000 \mathrm{E}+00 & 0.0000 \mathrm{E}+00\end{array}$ $\begin{array}{llllllllll}34 & 0.0000 \mathrm{E}+00 & 0.0000 \mathrm{E}+00 & 0.0000 \mathrm{E}+00 & 0.0000 \mathrm{E}+00 & 0.0000 \mathrm{E}+00 & 0.0000 \mathrm{E}+00 & 0.0000 \mathrm{E}+00 & 0.0000 \mathrm{E}+00\end{array}$ $\begin{array}{lllllllllll}32 & 0.0000 \mathrm{E}+00 & 0.0000 \mathrm{E}+00 & 0.0000 \mathrm{E}+00 & 0.0000 \mathrm{E}+00 & 0.0000 \mathrm{E}+00 & 0.0000 \mathrm{E}+00 & 0.0000 \mathrm{E}+00 & 0.0000 \mathrm{E}+00\end{array}$ $\begin{array}{lllllllllll}33 & 0.0000 \mathrm{E}+00 & 0.0000 \mathrm{E}+00 & 0.0000 \mathrm{E}+00 & 0.0000 \mathrm{E}+00 & 0.0000 \mathrm{E}+00 & 0.0000 \mathrm{E}+00 & 0.0000 \mathrm{E}+00 & 0.0000 \mathrm{E}+00\end{array}$ $\begin{array}{llllllllll}7 & 35 & 0.0000 \mathrm{E}+00 & 0.0000 \mathrm{E}+00 & 0.0000 \mathrm{E}+00 & 0.0000 \mathrm{E}+00 & 0.0000 \mathrm{E}+00 & 0.0000 \mathrm{E}+00 & 0.0000 \mathrm{E}+00 & 0.0000 \mathrm{E}+00\end{array}$ $\begin{array}{llllllllllllll}8 & 36 & 0.0000 \mathrm{E}+00 & 0.0000 \mathrm{E}+00 & 0.0000 \mathrm{E}+00 & 0.0000 \mathrm{E}+00 & 0.0000 \mathrm{E}+00 & 0.0000 \mathrm{E}+00 & 0.0000 \mathrm{E}+00 & 0.0000 \mathrm{E}+00\end{array}$ $\begin{array}{llllllllll}9 & 38 & 0.0000 \mathrm{E}+00 & 0.0000 \mathrm{E}+00 & 1.2447 \mathrm{E}-03 & 0.0000 \mathrm{E}+00 & 0.0000 \mathrm{E}+00 & 0.0000 \mathrm{E}+00 & 0.0000 \mathrm{E}+00 & 1.2447 \mathrm{E}-03\end{array}$

$\begin{array}{llllllllll}10 & 39 & 0.0000 \mathrm{E}+00 & 0.0000 \mathrm{E}+00 & 2.0097 \mathrm{E}-04 & 0.0000 \mathrm{E}+00 & 0.0000 \mathrm{E}+00 & 0.0000 \mathrm{E}+00 & 0.0000 \mathrm{E}+00 & 2.0097 \mathrm{E}-04\end{array}$

$11 \quad 40 \quad 0.0000 \mathrm{E}+00 \quad 0.0000 \mathrm{E}+00 \quad 0.0000 \mathrm{E}+0.00 .0000 \mathrm{E}+00 \quad 0.0000 \mathrm{E}+00 \quad 0.0000 \mathrm{E}+00 \quad 0.0000 \mathrm{E}+00 \quad 0.0000 \mathrm{E}+00$ $\begin{array}{lllllllllll}12 & 41 & 0.0000 \mathrm{E}+00 & 0.0000 \mathrm{E}+00 & 2.9100 \mathrm{E}-04 & 0.0000 \mathrm{E}+00 & 0.0000 \mathrm{E}+0 & 0.0000 \mathrm{E}+00 & 0.0000 \mathrm{E}+00 & 2.9100 \mathrm{E}-04\end{array}$

$\begin{array}{lllllllllllll}13 & 140 & 0.0000 \mathrm{E}+00 & 0.0000 \mathrm{E}+00 & 0.0000 \mathrm{E}+00 & 0.0000 \mathrm{E}+0 & 0.0000 \mathrm{E}+00 & 0.0000 \mathrm{E}+00 & 0.0000 \mathrm{E}+00 & 0.0000 \mathrm{E}+00\end{array}$

$\begin{array}{llllllllll}14 & 42 & 0.0000 \mathrm{E}+00 & 0.0000 \mathrm{E}+00 & 0.0000 \mathrm{E}+00 & 0.0000 \mathrm{E}+00 & 0.0000 \mathrm{E}+00 & 0.0000 \mathrm{E}+00 & 0.0000 \mathrm{E}+00 & 0.0000 \mathrm{E}+00\end{array}$ $\begin{array}{llllllllllll} & 15 & 154 & 0.0000 \mathrm{E}+00 & 0.0000 \mathrm{E}+00 & 8.0354 \mathrm{E}-03 & 0.0000 \mathrm{E}+00 & 0.0000 \mathrm{E}+00 & 0.0000 \mathrm{E}+00 & 0.0000 \mathrm{E}+00 & 8.0354 \mathrm{E}-03\end{array}$

$\begin{array}{lllllllllll}16 & 149 & 0.0000 \mathrm{E}+00 & 0.0000 \mathrm{E}+00 & 0.0000 \mathrm{E}+00 & 0.0000 \mathrm{E}+00 & 0.0000 \mathrm{E}+00 & 0.0000 \mathrm{E}+00 & 0.0000 \mathrm{E}+00 & 0.0000 \mathrm{E}+00\end{array}$

$\begin{array}{llllllllll}17 & 144 & 0.0000 \mathrm{E}+00 & 0.0000 \mathrm{E}+00 & -3.0848 \mathrm{E}-06 & 0.0000 \mathrm{E}+00 & 0.0000 \mathrm{E}+00 & 0.0000 \mathrm{E}+00 & 0.0000 \mathrm{E}+00 & -3.0848 \mathrm{E}-06\end{array}$

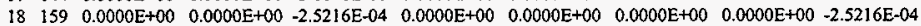

$\begin{array}{lllllllllll}19 & 141 & 0.0000 \mathrm{E}+00 & 0.0000 \mathrm{E}+00 & 0.0000 \mathrm{E}+00 & 0.0000 \mathrm{E}+00 & 0.0000 \mathrm{E}+00 & 0.0000 \mathrm{E}+00 & 0.0000 \mathrm{E}+00 & 0.0000 \mathrm{E}+00\end{array}$

$\begin{array}{lllllllll}\text { total } & 0.0000 \mathrm{E}+00 & 0.0000 \mathrm{E}+00 & 9.5169 \mathrm{E}-03 & 0.0000 \mathrm{E}+00 & 0.0000 \mathrm{E}+00 & 0.0000 \mathrm{E}+00 & 0.0000 \mathrm{E}+00 & 9.5169 \mathrm{E}-03\end{array}$ ineutron weight balance in each cell -- physical events print table 130

cell (n,xn) fission capture loss to loss to total

\begin{abstract}
$\begin{array}{llllllll}30 & 0.0000 \mathrm{E}+00 & 0.0000 \mathrm{E}+00 & 0.0000 \mathrm{E}+00 & 0.0000 \mathrm{E}+00 & 0.0000 \mathrm{E}+00 & 0.0000 \mathrm{E}+00\end{array}$
$\begin{array}{lllllllll}2 & 31 & 0.0000 \mathrm{E}+00 & 0.0000 \mathrm{E}+00 & 0.0000 \mathrm{E}+00 & 0.0000 \mathrm{E}+00 & 0.0000 \mathrm{E}+00 & 0.0000 \mathrm{E}+00\end{array}$

$\begin{array}{lllllllll}3 & 37 & 0.0000 \mathrm{E}+00 & 0.0000 \mathrm{E}+00 & 0.0000 \mathrm{E}+00 & 0.0000 \mathrm{E}+00 & 0.0000 \mathrm{E}+00 & 0.0000 \mathrm{E}+00\end{array}$

$\begin{array}{llllllllll}4 & 34 & 0.0000 \mathrm{E}+00 & 0.0000 \mathrm{E}+00 & 0.0000 \mathrm{E}+00 & 0.0000 \mathrm{E}+00 & 0.0000 \mathrm{E}+00 & 0.0000 \mathrm{E}+00\end{array}$ $\begin{array}{llllllll}32 & 0.0000 \mathrm{E}+00 & 0.0000 \mathrm{E}+00 & 0.0000 \mathrm{E}+00 & 0.0000 \mathrm{E}+00 & 0.0000 \mathrm{E}+00 & 0.0000 \mathrm{E}+00\end{array}$ $\begin{array}{lllllll}33 & 0.0000 \mathrm{E}+00 & 0.0000 \mathrm{E}+00 & 0.0000 \mathrm{E}+00 & 0.0000 \mathrm{E}+00 & 0.0000 \mathrm{E}+00 & 0.0000 \mathrm{E}+00\end{array}$ $\begin{array}{llllllll}35 & 0.0000 \mathrm{E}+00 & 0.0000 \mathrm{E}+00 & 0.0000 \mathrm{E}+00 & 0.0000 \mathrm{E}+00 & 0.0000 \mathrm{E}+00 & 0.0000 \mathrm{E}+00\end{array}$ $\begin{array}{llllllllll}8 & 36 & 0.0000 \mathrm{E}+00 & 0.0000 \mathrm{E}+00 & 0.0000 \mathrm{E}+00 & 0.0000 \mathrm{E}+00 & 0.0000 \mathrm{E}+00 & 0.0000 \mathrm{E}+00\end{array}$

$\begin{array}{lllllllll}9 & 38 & 0.0000 \mathrm{E}+00 & 0.0000 \mathrm{E}+00 & -1.5855 \mathrm{E}-01 & 0.0000 \mathrm{E}+00 & 0.0000 \mathrm{E}+00 & +1.5855 \mathrm{E}-01\end{array}$

$\begin{array}{llllllll}10 & 39 & 0.0000 \mathrm{E}+00 & 0.0000 \mathrm{E}+00 & -8.2847 \mathrm{E}-04 & 0.0000 \mathrm{E}+00 & 0.0000 \mathrm{E}+00 & -8.2847 \mathrm{E}-04\end{array}$

$11 \quad 40 \quad 0.0000 \mathrm{E}+00 \quad 0.0000 \mathrm{E}+00 \quad-3.9460 \mathrm{E}-06 \quad 0.0000 \mathrm{E}+00 \quad 0.0000 \mathrm{E}+00 \quad-3.9460 \mathrm{E}-06$

$\begin{array}{lllllllll}12 & 41 & 0.0000 \mathrm{E}+00 & 0.0000 \mathrm{E}+00 & -3.1114 \mathrm{E}-03 & 0.0000 \mathrm{E}+00 & 0.0000 \mathrm{E}+00 & -3.1114 \mathrm{E}-03\end{array}$

$\begin{array}{llllllll}13 & 140 & 0.0000 \mathrm{E}+00 & 0.0000 \mathrm{E}+00 & 0.0000 \mathrm{E}+00 & 0.0000 \mathrm{E}+00 & 0.0000 \mathrm{E}+00 & 0.0000 \mathrm{E}+00\end{array}$

$14 \quad 42 \quad 1.2461 \mathrm{E}-03 \quad 0.0000 \mathrm{E}+00 \quad-3.1955 \mathrm{E}-05 \quad-6.2306 \mathrm{E}-04 \quad 0.0000 \mathrm{E}+00 \quad 5.9111 \mathrm{E}-04$

$\begin{array}{lllllllll}15 & 154 & 4.9179 \mathrm{E}-03 & 0.0000 \mathrm{E}+00 & -1.7239 \mathrm{E}-01 & -2.4589 \mathrm{E}-03 & -4.5089 \mathrm{E}-01 & -6.2082 \mathrm{E}-01\end{array}$

$\begin{array}{lllllllll}16 & 149 & 0.0000 \mathrm{E}+00 & 0.0000 \mathrm{E}+00 & -3.5884 \mathrm{E}-05 & 0.0000 \mathrm{E}+00 & 0.0000 \mathrm{E}+00 & -3.5884 \mathrm{E}-05\end{array}$

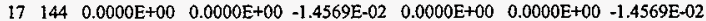

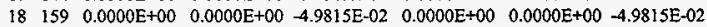

$19 \quad 14 ! \quad 0.0000 \mathrm{E}+00 \quad 0.0000 \mathrm{E}+00-4.5860 \mathrm{E}-04 \quad 0.0000 \mathrm{E}+00 \quad 0.0000 \mathrm{E}+00-4.5860 \mathrm{E}-04$
\end{abstract}

total $\quad 6.1640 \mathrm{E}-03 \quad 0.0000 \mathrm{E}+00 \quad-3.9980 \mathrm{E}-01 \quad-3.0820 \mathrm{E}-03 \quad-4.5089 \mathrm{E}-01 \quad-8.4760 \mathrm{E}-01$

ineutron activity of each nuclide in each cell, per source particle

print table 140

cell nuclides atom total collisions weight lost weight loss weight gain fraction collisions * weight to capture to fission by $(n, x n)$

$9 \quad 38 \quad 5010.03 \mathrm{~d} \quad 7.1977 \mathrm{E}-01$ $5011.40 \mathrm{c} \quad 8.0041 \mathrm{E}-02$ $6012.40 \mathrm{c} 2.0019 \mathrm{E}-01$

$941 \quad 4.4212 \mathrm{E}-01 \quad 1.5853 \mathrm{E}-01 \quad 0.0000 \mathrm{E}+00 \quad 0.0000 \mathrm{E}+00$ $\begin{array}{lllll}76 & 3.6073 \mathrm{E}-02 & 1.3591 \mathrm{E}-07 & 0.0000 \mathrm{E}+00 & 0.0000 \mathrm{E}+00\end{array}$ $232 \quad 1.1297 \mathrm{E}-01 \quad 1.8446 \mathrm{E}-05 \quad 0.0000 \mathrm{E}+00 \quad 0.0000 \mathrm{E}+00$

$\begin{array}{llll}10 & 39 & 41093.40 \mathrm{c} \quad 9.8982 \mathrm{E}-01\end{array}$ $40000.40 \mathrm{c} \quad 1.0183 \mathrm{E}-02$

$128 \quad 6.2802 \mathrm{E}-02 \quad 8.2814 \mathrm{E}-04 \quad 0.0000 \mathrm{E}+00 \quad 0.0000 \mathrm{E}+00$ $\begin{array}{lllll}2 & 9.7676 \mathrm{E}-04 & 3.3160 \mathrm{E}-07 & 0.0000 \mathrm{E}+00 & 0.0000 \mathrm{E}+00\end{array}$

$1140 \quad 1001.00$ e $3.3333 \mathrm{E}-01$ $1002.55 \mathrm{c} \quad 3.3333 \mathrm{E}-01$ $8016.40 \mathrm{c} 3.3333 \mathrm{E}-01$

$111 \quad 5.1533 \mathrm{E}-02 \quad 3.9251 \mathrm{E}-06 \quad 0.0000 \mathrm{E}+00 \quad 0.0000 \mathrm{E}+00$ $33 \quad 1.5373 \mathrm{E}-02 \quad 2.0884 \mathrm{E}-08 \quad 0.0000 \mathrm{E}+00 \quad 0.0000 \mathrm{E}+00$ $\begin{array}{lllll}40 & 2.1271 \mathrm{E}-02 & 0.0000 \mathrm{E}+00 & 0.0000 \mathrm{E}+00 & 0.0000 \mathrm{E}+00\end{array}$

$41 \quad 41093.40 \mathrm{c} \quad 9.8982 \mathrm{E}-0 \mathrm{I} \quad 313 \quad 1.5623 \mathrm{E}-01 \quad 3.1072 \mathrm{E}-03 \quad 0.0000 \mathrm{E}+00 \quad 0.0000 \mathrm{E}+00$ 
$40000.40 \mathrm{c} \quad 1.0183 \mathrm{E}-02$

$4 \quad 1.8747 \mathrm{E}-03 \quad 4.2322 \mathrm{E}-06 \quad 0.0000 \mathrm{E}+00 \quad 0.0000 \mathrm{E}+00$

$42 \quad 1001.00 \mathrm{c} \quad 3.3333 \mathrm{E}-01$

$1002.55 \mathrm{c} \quad 3.3333 \mathrm{E}-01$

$257 \quad 1.2131 \mathrm{E}-01 \quad 4.3914 \mathrm{E}-06 \quad 0.0000 \mathrm{E}+00 \quad 0.0000 \mathrm{E}+00$

$8016.40 \mathrm{c} 3.3333 \mathrm{E}-01$

$86 \quad 4.6121 \mathrm{E}-02 \quad 6.8027 \mathrm{E}-08 \quad 0.0000 \mathrm{E}+00 \quad 6.2306 \mathrm{E}-04$

$\begin{array}{lllll}98 & 5.1682 \mathrm{E}-02 & 2.7495 \mathrm{E}-05 & 0.0000 \mathrm{E}+00 & 0.0000 \mathrm{E}+00\end{array}$

$\begin{array}{lll}15 & 154 & 92235.40 \mathrm{c} \quad 3.7504 \mathrm{E}-01\end{array}$ $92238.40 \mathrm{c} \quad 1.2499 \mathrm{E}-01$

$4759 \quad 2.3227 \mathrm{E}+00 \quad 1.3244 \mathrm{E}-01 \quad 4.4247 \mathrm{E}-01 \quad 5.8410 \mathrm{E}-04$ $7014.40 \mathrm{c} \quad 4.9997 \mathrm{E}-01$

$\begin{array}{llllll}1470 & 7.3870 \mathrm{E}-01 & 2.3272 \mathrm{E}-02 & 8.4162 \mathrm{E}-03 & 1.8748 \mathrm{E}-03\end{array}$

$2207 \quad 1.0775 \mathrm{E}+00 \quad 1.6682 \mathrm{E}-02 \quad 0.0000 \mathrm{E}+00 \quad 0.0000 \mathrm{E}+00$

$16 \quad 149 \quad 1001.00 \mathrm{c} \quad 3.3333 \mathrm{E}-01$ $1002.55 \mathrm{c} \quad 3.3333 \mathrm{E}-01$

$345 \quad 1.6812 \mathrm{E}-01 \quad 4.4875 \mathrm{E}-06 \quad 0.0000 \mathrm{E}+00 \quad 0.0000 \mathrm{E}+00$

$8016.40 \mathrm{c}$ 3.3333E-01

$110 \quad 5.8433 \mathrm{E}-02 \quad 9.1921 \mathrm{E}-08 \quad 0.0000 \mathrm{E}+00 \quad 0.0000 \mathrm{E}+00$

$125 \quad 6.3072 \mathrm{E}-02 \quad 3.1305 \mathrm{E}-05 \quad 0.0000 \mathrm{E}+00 \quad 0.0000 \mathrm{E}+00$

$\begin{array}{lll}17 \quad 144 & 74000.40 \mathrm{c} & 1.0000 \mathrm{E}+00\end{array}$

$885 \quad 4.2652 \mathrm{E}-01 \quad 1.4569 \mathrm{E}-02 \quad 0.0000 \mathrm{E}+00 \quad 0.0000 \mathrm{E}+00$

$\begin{array}{lll}18 & 159 \quad 41093.40 \mathrm{C} \quad 9.8982 \mathrm{E}-01\end{array}$ $40000.40 \mathrm{C} \quad 1.0183 \mathrm{E}-02$

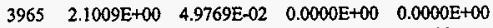
$54 \quad 2.4590 \mathrm{E}-02 \quad 4.6175 \mathrm{E}-05 \quad 0.0000 \mathrm{E}+00 \quad 0.0000 \mathrm{E}+00$

$\begin{array}{llllllll}19 & 141 & 1001.00 \mathrm{c} & 3.3333 \mathrm{E}-01 \quad 4029 & 1.9096 \mathrm{E}+00 & 7.9511 \mathrm{E}-05 & 0.0000 \mathrm{E}+00 & 0.0000 \mathrm{E}+00\end{array}$ $\begin{array}{llllllll}1002.55 \mathrm{c} & 3.3333 \mathrm{E}-0 \mathrm{I} & 1122 & 5.8206 \mathrm{E}-01 & 9.0056 \mathrm{E}-07 & 0.0000 \mathrm{E}+00 & 0.0000 \mathrm{E}+00\end{array}$ $\begin{array}{lllllll}8016.40 \mathrm{c} & 3.3333 \mathrm{E}-01 & 1372 & 7.3131 \mathrm{E}-01 & 3.7819 \mathrm{E}-04 & 0.0000 \mathrm{E}+00 & 0.0000 \mathrm{E}+00\end{array}$

total $\quad 22764 \quad 1.1324 \mathrm{E}+01 \quad 3.9980 \mathrm{E}-01 \quad 4.5089 \mathrm{E}-01 \quad 3.0820 \mathrm{E}-03$

total over all cells for each nuclide total collisions weight lost weight loss weight gain collisions * weight to capture to fission by $(n, x n)$

$\begin{array}{ccccccc}1001.00 \mathrm{c} & 4742 & 2.2505 \mathrm{E}+00 & 9.2315 \mathrm{E}-05 & 0.0000 \mathrm{E}+00 & 0.0000 \mathrm{E}+00 \\ 1002.55 \mathrm{c} & 1351 & 7.0199 \mathrm{E}-01 & 1.0814 \mathrm{E}-06 & 0.0000 \mathrm{E}+00 & 6.2306 \mathrm{E}-04 \\ 5011.40 \mathrm{c} & 76 & 3.6073 \mathrm{E}-02 & 1.3591 \mathrm{E}-07 & 0.0000 \mathrm{E}+00 & 0.0000 \mathrm{E}+00 \\ 6012.40 \mathrm{c} & 232 & 1.1297 \mathrm{E}-01 & 1.8446 \mathrm{E}-05 & 0.0000 \mathrm{E}+00 & 0.0000 \mathrm{E}+00 \\ 7014.40 \mathrm{c} & 2207 & 1.0775 \mathrm{E}+00 & 1.6682 \mathrm{E}-02 & 0.0000 \mathrm{E}+00 & 0.0000 \mathrm{E}+00 \\ 8016.40 \mathrm{c} & 1635 & 8.6733 \mathrm{E}-01 & 4.3699 \mathrm{E}-04 & 0.0000 \mathrm{E}+00 & 0.0000 \mathrm{E}+00 \\ 40000.40 \mathrm{c} & 60 & 2.7441 \mathrm{E}-02 & 5.0739 \mathrm{E}-05 & 0.0000 \mathrm{E}+00 & 0.0000 \mathrm{E}+00 \\ 41093.40 \mathrm{c} & 4406 & 2.3199 \mathrm{E}+00 & 5.3704 \mathrm{E}-02 & 0.0000 \mathrm{E}+00 & 0.0000 \mathrm{E}+00 \\ 74000.40 \mathrm{c} & 885 & 4.2652 \mathrm{E}-01 & 1.4569 \mathrm{E}-02 & 0.0000 \mathrm{E}+00 & 0.0000 \mathrm{E}+00 \\ 92235.40 \mathrm{c} & 4759 & 2.3227 \mathrm{E}+00 & 1.3244 \mathrm{E}-01 & 4.4247 \mathrm{E}-01 & 5.8410 \mathrm{E}-04 \\ 92238.40 \mathrm{c} & 1470 & 7.3870 \mathrm{E}-01 & 2.3272 \mathrm{E}-02 & 8.4162 \mathrm{E}-03 & 1.8748 \mathrm{E}-03 \\ 5010.03 \mathrm{~d} & 941 & 4.4212 \mathrm{E}-01 & 1.5853 \mathrm{E}-01 & 0.0000 \mathrm{E}+00 & 0.0000 \mathrm{E}+00\end{array}$

the initial fission neutron source distribution used the 8 source points that were input on the ksre card.

the criticality problem was scheduled to skip 5 cycles and run a total of 6 cycles with nominally 200 neutrons per cycle. this problem has run 5 inactive cycles with 1027 neutron histories and 1 active cycles with 222 neutron histories.

this calculation has completed the requested number of keff cycles using a total of 1249 fission neutron source histories. all cells with fissionable material were sampled and had fission neutron source points.

there is no combined collision/absorption/track-length estimate for keff because only 1 active cycles were run. lindividual and average keff estimator results by cycle

keff neutron keff estimators by cycle average keff estimators and deviations average $k(\mathrm{c} / \mathrm{a} / \mathrm{t})$ cycle histories $k$ (coll) $k$ (abs) $k$ (track) $k$ (coll) st dev $k(a b s)$ st dev $k$ (track) st dev $k(c / a / t)$ st dev fom

$200|1.25311 \quad 1.26302 \quad 1.22009|$

$257 \mid \begin{array}{llll}1.21200 & 1.25252 & 1.12957\end{array}$

$195|1.20026 \quad 1.14326 \quad 1.13006|$

$193|1.12953 \quad 1.16269 \quad 1.10757|$

$182\left[\begin{array}{ll}1.13599 & 1.12465 \quad 1.11238 \mid\end{array}\right.$

begin active keff cycles

$6 \quad 222$ |1.07701 1.046691 .07528 |

tally 4 nps $=1249$

fuel rod flux in $5 y$ locations averaged over $5 x$ elements

tally type 4 track length estimate of particle flux. units $1 / \mathrm{cm}^{* *} 2$ 
tally for neutrons

bet of histories used for normalizing tallies $=200.00$

cell $a$ is $(154<42[-10-10] 42[-9-10] 42[-8-10] 42[-7-10] 42[-6-10])$

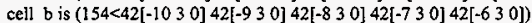

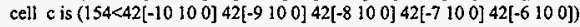

cell $d$ is $\left(154<42[-10210] 42[-9210] 42[-8210] 42\left[\begin{array}{lll}-7 & 21 & 0] \\ 42[-6 & 210 & 0\end{array}\right)\right.$

cell $\mathrm{e}$ is $\left(154<42\left[-1029\right.\right.$ 0] $\left.42[-9290] 42[-8290] 42\left[\begin{array}{lll}-7 & 29 & 0\end{array}\right] 42\left[\begin{array}{lll}-6 & 29 & 0\end{array}\right]\right)$

volumes

cell: a b c d e

$1.04709 \mathrm{E}+02 \quad 1.04709 \mathrm{E}+02 \quad 1.04709 \mathrm{E}+02 \quad 1.04709 \mathrm{E}+02 \quad 1.04709 \mathrm{E}+02$

energy: $\quad 1.0000 \mathrm{E}-02 \quad 1.0000 \mathrm{E}-01 \quad 1.0000 \mathrm{E}+00 \quad 1.0000 \mathrm{E}+01 \quad$ total

cell

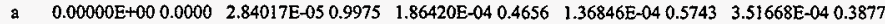

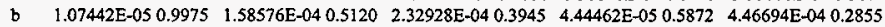

c $\quad 2.16778 \mathrm{E}-050.7232 \quad 9.40943 \mathrm{E}-050.5519 \quad 5.37835 \mathrm{E}-050.5876 \quad 1.51034 \mathrm{E}-040.7075 \quad 3.20589 \mathrm{E}-040.3937$

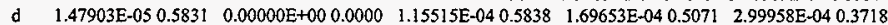

e $\quad 8.04943 \mathrm{E}-060.9975 \quad 0.00000 \mathrm{E}+000.0000 \quad 0.00000 \mathrm{E}+00 \quad 0.0000 \quad 6.81909 \mathrm{E}-050.7477 \quad 7.62403 \mathrm{E}-050.6763$

lanalysis of the results in the tally fluctuation chart bin (tfc) for tally 4 with nps $=1249$ print table 160

normed average tally per history $=3.51668 \mathrm{E}-04$

estimated tally relative error $=0.3877$

relative error from zero tallies $=0.3464$

number of nonzero history tallies $=8$ efficiency for the nonzero tallies $=0.0400$

history number of largest tally $=\quad 1034$

(largest tally)/(average tally) $=3.84476 \mathrm{E}+01$

(confidence interval shift $) /$ mean $=0.0793$ unnormed average tally per history $=3.68228 \mathrm{E}-02$

estimated variance of the variance $=0.1752$

relative error from nonzero scores $=0.1740$

largest unnormalized history tally $=1.41575 \mathrm{E}+00$

(largest tally)/(avg nonzero tally) $=1.53791 \mathrm{E}+00$

shifted confidence interval center $=3.79539 \mathrm{E}-04$

largest history score sampled so far were to occur on the very next history, the tfc bin quantities would change as follows: 222 for this table because 5 keff cycles and 1027 histories were skipped before tally accumulation.

$\begin{array}{lccc}\text { estimated quantities } & \text { value at nps } & \text { value at nps+1 } & \text { value(nps+1)/value(nps } \\ \text { mean } & 3.51668 \mathrm{E}-04 & 4.10723 \mathrm{E}-04 & 0.167927 \\ \text { relative error } & 3.87653 \mathrm{E}-01 & 3.61597 \mathrm{E}-01 & -0.067213 \\ \text { variance of the variance } & 1.75161 \mathrm{E}-01 & 1.47665 \mathrm{E}-01 & -0.156976 \\ \text { shifted center } & 3.79539 \mathrm{E}-04 & 3.77169 \mathrm{E}-04 & -0.006244 \\ \text { figure of merit } & 0.00000 \mathrm{E}+00 & 0.00000 \mathrm{E}+00 & 0.000000\end{array}$

there is not enough information in the largest history scores (usually less than 500 scores) for a reliable estimate of the slope. the history score probability density function appears to have an unsampled region at the largest history scores: please examine.

***** the nps-dependent tfe bin check results are suspect because there are only 1 nps tally values to analyze *****

results of 10 statistical checks for the estimated answer for the tally fluctuation chart (ffc) bin of tally 4

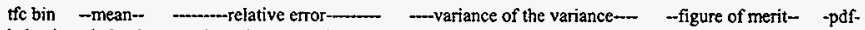

behavior behavior value decrease decrease rate value decrease decrease rate value behavior slope

desired random $<0.10$ yes $1 /$ sqrt(nps) $<0.10$ yes $1 /$ nps constant random $>3.00$

observed random 0.39 yes yes 0.18 yes yes 0.00

passed? yes no yes yes no yes yes no 
warning. the tally in the tally fluctuation chatt bin did not pass 3 of the 10 statistical checks.

normed tally density for tally 4 nonzero tally mean $(\mathrm{m})=9.206 \mathrm{E}-01 \mathrm{nps}=1249$ print table 161

abscissa ordinate log plot of tally probability density function in tally fluctuation chart bin $(d=d e c a d e, s l o p e=0.0)$

tally number num den $\log$ den:d-.....

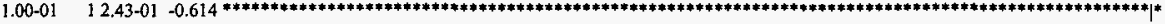

$1.26-01 \quad 00.00+00 \quad 0.000$

$\begin{array}{lll}1.58-01 & 0.000+00 & 0.000\end{array}$

$2.00-01 \quad 0.0 .00+00 \quad 0.000$

$2.51-01 \quad 00.00+00 \quad 0.000$

$3.16-01 \quad 0.000+00 \quad 0.000$

$\begin{array}{llll}3.98-01 & 0 & 0.00+00 & 0.000\end{array}$

$\begin{array}{llll}5.01-01 & 00.00+00 & 0.000\end{array}$

$6.31-01 \quad 27.71-02-1.113 * * * * * * * * * * * * * * * * * * * * * * * * * * * * * * * * * * * * * * * * * * * * * * * * * * *$

7.94-01 $00.00+00 \quad 0.000$

$1.00+00 \quad 12.43-02-1.614 \mathrm{~m}$

$1.26+00 \quad 00.00+00 \quad 0.000$

$1.58+00 \quad 46.14-02-1.212 * * * * * * * * * * * * * * * * * * * * * * * * * * * * * * * * * * * * * * * * *$

total $84.00-02$

cumulative tally number for tally $4 \quad$ nonzero tally mean $(\mathrm{m})=9.206 \mathrm{E}-01 \mathrm{nps}=1249$ print table 162

abscissa cum ordinate plot of the cumulative number of tallies in the tally fluctuation chart bin from 0 to 100 percent tally number cum pet:--. 10 -

$1.00000 \mathrm{E}-0$

$1.25893 \mathrm{E}-01$

$1.58489 \mathrm{E}-01$

$1.99526 \mathrm{E}-01$

2.51189E-01

3.16228E-01

$3.98108 \mathrm{E}-01$

$5.01188 \mathrm{E}-01$

0958E-01

3329E-01

$1.00000 \mathrm{E}+00$

$1.25893 \mathrm{E}+00$

$1.58489 E+00$

total
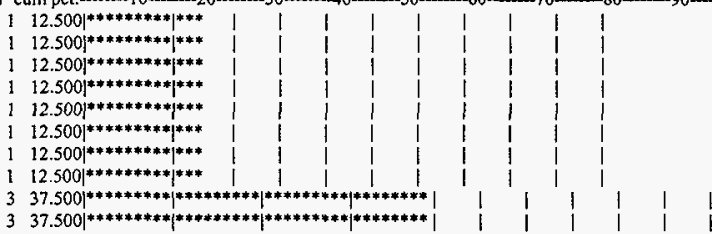

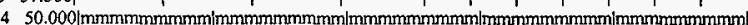

$450.000]^{* * * * * * * * *|* * * * * * * * *| * * * * * * * *|* * * * * * * * *| * * * * * * * * \mid}$

$8100.000 \mid$

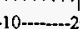

$20-30-10$

$-50$

-60 -

$70 \ldots-80 \ldots-100$

cumulative unnormed tally for tally $4 \quad$ nonzero tally mean $(\mathrm{m})=9.206 \mathrm{E}-01$ nps $=1249$ print table 162

abscissa cum ordinate

tally tally/nps cum pct:

1.000E-0] 4.632E-04 1.258!*

$1.259 \mathrm{E}-01 \quad 4.632 \mathrm{E}-04 \quad 1.258$ |

1.585E-01 4.632E-04 $\left.1.258\right|^{*}$

$1.995 \mathrm{E}-01 \quad 4.632 \mathrm{E}-\left.04 \quad 1.258\right|^{*}$

2.512E-01 4.632E-04 $\left.1.258\right|^{*}$

$3.162 \mathrm{E}-01$ 4.632E-04 1.258|*

$3.981 \mathrm{E}-01$ 4.632E-04 $\left.1.258\right|^{*}$

$5.012 \mathrm{E}-01 \quad 4.632 \mathrm{E}-04 \quad 1.258{ }^{*}$

$6.310 \mathrm{E}-01 \quad 6.195 \mathrm{E}-03 \quad 16.825$

$7.943 \mathrm{E}-01 \quad 6.195 \mathrm{E}-\left.03 \quad 16.825\right|^{* * * * * * * * * \mid * * * * * * *}$

$1.000 \mathrm{E}+00 \quad 1.017 \mathrm{E}-02 \quad 27.628 \mid \mathrm{mmmmmmmm} / \mathrm{mmmmmmmm} / \mathrm{mmmmmmm}$ ।

$1.259 \mathrm{E}+00 \quad 1.017 \mathrm{E}-\left.02 \quad 27.628\right|^{* * * * * * * * * j * * * * * * * * *|* * * * * * * *|}$

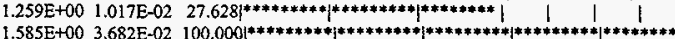

total $3.68228 \mathrm{E}-02$ 100.000:

plot of the cumulative tally in the tally fluctuation chart bin from 0 to 100 percent

status of the statistical checks used to form confidence intervals for the mean for each tally bin

tally result of statistical checks for the tfc bin (the first check not passed is listed) and error magnitude check for all bins

4 missed 3 of $10 \mathrm{tfc}$ bin checks: the relative error exceeds the recommended value of 0.1 for nonpoint detector tallies missed all bin ertor check: 25 tally bins had 4 bins with zeros and 21 bins with relative errors exceeding 0.10 
the 10 statistical checks are only for the tally fluctuation chart bin and do not apply to other tally bins.

tally bins with zeros may or may not be correct: compare the source, cutoffs, multipliers, et cetera with the tally bins.

waming. I f the I tally fluctuation chart bins did not pass all 10 statistical checks.

warning. 1 of the 1 tallies had bins with relative errors greater than recommended.

ltally fluctuation charts

$$
\text { tally } 4
$$

nps mean error vov slope fom

$10000.0000 \mathrm{E}+000.00000 .0000 \quad 0.0$

1249 3.5167E-04 $0.38770 .1752 \quad 0.0$

tally data written to file inp $18 \mathrm{~m}$

ascii file inpl $8 \mathrm{p}$ written with 5 events from 5 histories.

6 waming messages so far.

run terminated when 6 kcode cycles were done.

\section{File: Inp200}

1- prob20 - continuous energy electron version of problem prob19.

2- $\quad 1 \quad 1+19.3 \quad 1-23-45-6$

3- $20 \# 1$

4-

5- I 202

$+$

6- $2 \mathrm{pz} .00635$

$7-3 \mathrm{px}+10$

8. $4 \times 100$

$+$

9. 5 y-103

6 p 010011000101

plane

plane

$\mathrm{ml} 74184.1$

sdef erg=1 sur=1 vec $=001$ par=3

impip,e 10

mode $p$ e

*f $1: p 12$

17. e $\quad .1 .191 .282 .373 .464 .555 .645 .736 .827 .918 \quad 1.1$

18- *f11:e 12

19. t9 0001000100001

waming. non-orthogonality of surface transformation $9>2 . e-6$

20. fq ef

21- f21:e 12

22- *f28:p 1

23- $\quad$ e28 $\quad-.00101 . e-6.1 .191 .282 .373 .464 .555 .645 .736 .827 .9181 .1$

24- e58 $\quad-.001 \quad 01 . e-6.1 .191 .282 .373 .464 .555 .645 .736 .827 .918 \quad 1.1$

25- f58:e 1

26- f6:p 1

27- f31:e 12

28- f31 eic 1

29. f4l:e 12

30. ff4l elc 2

31- f5l:e 12

32. fisl elc 3

33- e8 $1000 \mathrm{nt}$

34- *f8:p,e 1

35- nps 10000

36- ctme 30

37. prdmp $2 \mathrm{j}+\mathrm{l}$

cut:p $1 \mathrm{j}$.]

cut:e $1 \mathbf{j} .1$

print 11070 
jing. to 9 card unused.

waming. I energy bins of tally $I$ are below energy cutoff.

warning. tally 8 needs zero energy bin for negative $\mathrm{f} 8$ scores. lcells

print table 60

atom gram
cell mat density density volume mass pieces importance importance

I $116.31828 \mathrm{E}-021.93000 \mathrm{E}+012.54000 \mathrm{E}+004.90220 \mathrm{E}+01 \quad 0 \quad 1.0000 \mathrm{E}+001.0000 \mathrm{E}+00$

$2200.00000 \mathrm{E}+000.00000 \mathrm{E}+00 \quad 0.00000 \mathrm{E}+000.00000 \mathrm{E}+00 \quad 0 \quad 0.0000 \mathrm{E}+000.0000 \mathrm{E}+00$

total

$2.54000 \mathrm{E}+004.90220 \mathrm{E}+01$

1surfaces

print table 70

surface trans type surface coefficients

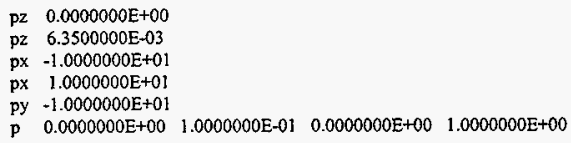

minimum source weight $=1.0000 \mathrm{E}+00$ maximum source weight $=1.0000 \mathrm{E}+00$

4 warning messages so far.

leross-section tables

print table 100

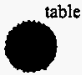

length

tables from file testlib1

$74000.02 p \quad 755$

$01 / 15 / 93$

total 755

maximum photon energy set to 100.0 mev (maximum electron energy)

tables from file testlib

$74000.01 \mathrm{e} \quad 478$

$11 / 16 / 88$

decimal words of dynamically allocated storage

4 warming messages so far.

1 starting morun. field length $=0 \quad \mathrm{cp} 0=0.00 \quad$ print table 110

prob20 - continuous energy electron version of problem prob19.

Jps $x \quad y \quad z$ cell surf $u \quad v$ w energy weight time

I $0.000 \mathrm{E}+00 \quad 0.000 \mathrm{E}+00 \quad 0.000 \mathrm{E}+00 \quad 1 \quad 1 \quad 3.096 \mathrm{E}-01-4.068 \mathrm{E}-01 \quad 8.595 \mathrm{E}-01 \quad 1.000 \mathrm{E}+00 \quad 1.000 \mathrm{E}+00 \quad 0.000 \mathrm{E}+00$

$20.000 \mathrm{E}+00 \quad 0.000 \mathrm{E}+00 \quad 0.000 \mathrm{E}+00 \quad 1 \quad 19.871 \mathrm{E}-01 \quad 1.062 \mathrm{E}-01 \quad 3.197 \mathrm{E}-01 \quad 1.000 \mathrm{E}+00 \quad 1.000 \mathrm{E}+00 \quad 0.000 \mathrm{E}+00$

$30.000 \mathrm{E}+00 \quad 0.000 \mathrm{E}+00 \quad 0.000 \mathrm{E}+00 \quad 1 \quad 1-1.347 \mathrm{E}-01 \quad 5.198 \mathrm{E}-01 \quad 8.436 \mathrm{E}-01 \quad 1.000 \mathrm{E}+00 \quad 1.000 \mathrm{E}+00 \quad 0.000 \mathrm{E}+00$

$40.000 \mathrm{E}+00 \quad 0.000 \mathrm{E}+00 \quad 0.000 \mathrm{E}+00 \quad 1 \quad 1-6.548 \mathrm{E}-02 \quad 7.832 \mathrm{E}-01 \quad 6.184 \mathrm{E}-01 \quad 1.000 \mathrm{E}+00 \quad 1.000 \mathrm{E}+00 \quad 0.000 \mathrm{E}+00$

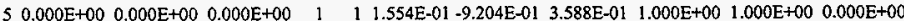

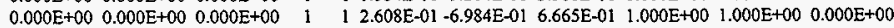

$\begin{array}{lllllllll}0.000 \mathrm{E}+00 & 0.000 \mathrm{E}+00 & 0.000 \mathrm{E}+00 \text { I } 1 & 6.931 \mathrm{E}-01 & -9.170 \mathrm{E}-02 & 7.149 \mathrm{E}-01 & 1.000 \mathrm{E}+00 & 1.000 \mathrm{E}+00 & 0.000 \mathrm{E}+00\end{array}$ 


\section{File: Outp20}

prob20 - continuous energy electron version of problem prob19.

1 1 $1-19.3 \quad 1-23-45-6$

3- $20 \# 1$

4. $1 ; 02$

6. $2 \mathrm{pz} .00635$

plane

7- 3 px -10

8. $4 \times 100$

+9. 5 y -103

$+$

10. 6 p 010011000101

11 -

12- $\mathrm{ml} 74184.1$

13- sdef erg $=1$ sur $=1$ vec $=001$ par $=3$

14. imp:p,e 10

15- mode $\mathrm{pe}$

16- *fl:p 12

17- $\quad$ e0 $\quad .1 .191 .282 .373 .464 .555 \quad 645.736 .827 .918 \quad 1.1$

18- *f11:e 12

19. t9 0001000100001

waming. non-orthogonality of surface transformation $9>2 . e-6$

20- fq ef

21- $21: e 12$

22- *28:p 1

23- e28 $-.00101 . e-6.1 .191 .282 .373 .464 .555 .645 .736 .827 .918 \quad 1.1$

24- es8 $-.00101 . e-6.1 .191 .282 .373 .464 .555 .645 .736 .827 .9181 .1$

25- f58:e I

26- $66: \mathrm{p} 1$

Bi:e 12

fi3l elc 1

f4l:e 12

ft4l elc 2

f51:e 12

ft5l elc 3

e8 $1000 \mathrm{nt}$

*f8:p,e 1

nps 10000

ctrme 30

prdmp $2 \mathrm{j}-1$

cut:p $1 \mathrm{j}, 1$

cut:e $1 \mathrm{j} .1$

print 11070

41 -

waming. to 9 card unused.

waming. I energy bins of tally 1 are below energy cutoff.

waming. tally 8 needs zero energy bin for negative $f 8$ scores.

lcells

print table 60

$$
\begin{aligned}
& \text { atom gram } \\
& \text { cell mat density density volume mass pieces importance importance }
\end{aligned}
$$

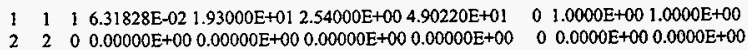

total

1 surfaces

$2.54000 \mathrm{E}+004.90220 \mathrm{E}+01$

print table 70

surface trans type surface coefficients 


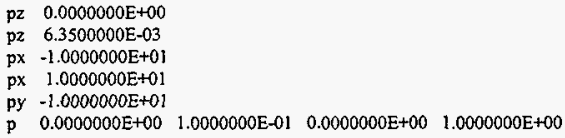

HNF-SD-SNF-CSWD-005, Rev. 0

minimum source weight $=1.0000 \mathrm{E}+00$ maximum source weight $=1.0000 \mathrm{E}+00$

4 waming messages so far.

lcross-section tables $\quad$ print table 100

table length

tables from file testlib]

$74000.02 \mathrm{p} \quad 755$

$01 / 15 / 93$

total $\quad 755$

maximum photon energy set to 100.0 mev (maximum electron energy)

tables from file testlibl

74000.01 e $\quad 478$

$11 / 16 / 88$

decimal words of dynamically allocated storage

4 warning messages so far.

tarting mcrun. field length $=0 \quad \mathrm{cp} 0=0.00 \quad$ print table 110

prob20 - continuous energy electron version of problem prob19.

nps

$y \quad z \quad$ cell surf $u$

$10.000 \mathrm{E}+00 \quad 0.000 \mathrm{E}+00 \quad 0.000 \mathrm{E}+00$ $20.000 \mathrm{E}+00 \quad 0.000 \mathrm{E}+00 \quad 0.000 \mathrm{E}+00$ $3 \quad 0.000 \mathrm{E}+00 \quad 0.000 \mathrm{E}+00 \quad 0.000 \mathrm{E}+00$ $40.000 \mathrm{E}+00 \quad 0.000 \mathrm{E}+00 \quad 0.000 \mathrm{E}+00$

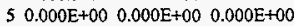
$60.000 \mathrm{E}+00 \quad 0.000 \mathrm{E}+00 \quad 0.000 \mathrm{E}+00$ $7 \quad 0.000 \mathrm{E}+00 \quad 0.000 \mathrm{E}+00 \quad 0.000 \mathrm{E}+00$ $80.000 \mathrm{E}+00 \quad 0.000 \mathrm{E}+00 \quad 0.000 \mathrm{E}+00$ $90.000 \mathrm{E}+00 \quad 0.000 \mathrm{E}+00 \quad 0.000 \mathrm{E}+00$ $10 \quad 0.000 \mathrm{E}+00 \quad 0.000 \mathrm{E}+00 \quad 0.000 \mathrm{E}+00$ $110.000 E+00 \quad 0.000 E+00 \quad 0.000 \mathrm{E}+00$ $12 \quad 0.000 \mathrm{E}+00 \quad 0.000 \mathrm{E}+00 \quad 0.000 \mathrm{E}+00$ $130.000 \mathrm{E}+00 \quad 0.000 \mathrm{E}+00 \quad 0.000 \mathrm{E}+00$ $14 \quad 0.000 \mathrm{E}+00 \quad 0.000 \mathrm{E}+00 \quad 0.000 \mathrm{E}+00$ $15 \quad 0.000 \mathrm{E}+00 \quad 0.000 \mathrm{E}+00 \quad 0.000 \mathrm{E}+00$ $16 \quad 0.000 \mathrm{E}+00 \quad 0.000 \mathrm{E}+00 \quad 0.000 \mathrm{E}+00$ $17 \quad 0.000 E+00 \quad 0.000 E+00 \quad 0.000 E+00$ $18 \quad 0.000 \mathrm{E}+00 \quad 0.000 \mathrm{E}+00 \quad 0.000 \mathrm{E}+00$ $190.000 \mathrm{E}+00 \quad 0.000 \mathrm{E}+00 \quad 0.000 \mathrm{E}+00$ $20 \quad 0.000 \mathrm{E}+00 \quad 0.000 \mathrm{E}+00 \quad 0.000 \mathrm{E}+00$ $21 \quad 0.000 \mathrm{E}+00 \quad 0.000 \mathrm{E}+00 \quad 0.000 \mathrm{E}+00$ $22 \quad 0.000 \mathrm{E}+00 \quad 0.000 \mathrm{E}+00 \quad 0.000 \mathrm{E}+00$ $230.000 \mathrm{E}+00 \quad 0.000 \mathrm{E}+00 \quad 0.000 \mathrm{E}+00$ $24 \quad 0.000 \mathrm{E}+00 \quad 0.000 \mathrm{E}+00 \quad 0.000 \mathrm{E}+00$ $25 \quad 0.000 \mathrm{E}+00 \quad 0.000 \mathrm{E}+00 \quad 0.000 \mathrm{E}+00$ $\checkmark 6.000 \mathrm{E}+00 \quad 0.000 \mathrm{E}+00 \quad 0.000 \mathrm{E}+00$ $270.000 \mathrm{E}+00 \quad 0.000 \mathrm{E}+00 \quad 0.000 \mathrm{E}+00$ $v$ w energy weight time

$13.096 \mathrm{E}-01-4.068 \mathrm{E}-01 \quad 8.595 \mathrm{E}-01 \quad 1.000 \mathrm{E}+00 \quad 1.000 \mathrm{E}+00 \quad 0.000 \mathrm{E}+00$ $1 \quad 9.871 \mathrm{E}-01 \quad 1.062 \mathrm{E}-01 \quad 1.197 \mathrm{E}-01 \quad 1.000 \mathrm{E}+00 \quad 1.000 \mathrm{E}+00 \quad 0.000 \mathrm{E}+00$ $\begin{array}{llllll}1-1.347 \mathrm{E}-01 & 5.198 \mathrm{E}-01 & 8.436 \mathrm{E}-01 & 1.000 \mathrm{E}+00 & 1.000 \mathrm{E}+00 & 0.000 \mathrm{E}+00\end{array}$ $1-6.548 \mathrm{E}-02 \quad 7.832 \mathrm{E}-01 \quad 6.184 \mathrm{E}-01 \quad 1.000 \mathrm{E}+00 \quad 1.000 \mathrm{E}+00 \quad 0.000 \mathrm{E}+00$ $1 \quad 1.554 \mathrm{E}-01-9.204 \mathrm{E}-01 \quad 3.588 \mathrm{E}-01 \quad 1.000 \mathrm{E}+00 \quad 1.000 \mathrm{E}+00 \quad 0.000 \mathrm{E}+00$ $12.608 \mathrm{E}-01-6.984 \mathrm{E}-01 \quad 6.665 \mathrm{E}-01 \quad 1.000 \mathrm{E}+00 \quad 1.000 \mathrm{E}+00 \quad 0.000 \mathrm{E}+00$ $1 \quad 6.931 \mathrm{E}-01-9.170 \mathrm{E}-02 \quad 7.149 \mathrm{E}-01 \quad 1.000 \mathrm{E}+00 \quad 1.000 \mathrm{E}+00 \quad 0.000 \mathrm{E}+00$ 1 -3.604E-01 4.799E-0l 7.999E-01 $1.000 \mathrm{E}+00 \quad 1.000 \mathrm{E}+00 \quad 0.000 \mathrm{E}+00$ $\begin{array}{llllllll}1-2.241 \mathrm{E}-01 & 3.960 \mathrm{E}-01 & 8.905 \mathrm{E}-01 & 1.000 \mathrm{E}+00 & 1.000 \mathrm{E}+00 & 0.000 \mathrm{E}+00\end{array}$ $\begin{array}{lllllll}1 & -2.928 \mathrm{E}-01 & -7.352 \mathrm{E}-01 & 6.114 \mathrm{E}-01 & 1.000 \mathrm{E}+00 & 1.000 \mathrm{E}+00 & 0.000 \mathrm{E}+00\end{array}$ ] $8.260 \mathrm{E}-0] \quad 1.184 \mathrm{E}-015.510 \mathrm{E}-01 \quad 1.000 \mathrm{E}+00 \quad 1.000 \mathrm{E}+00 \quad 0.000 \mathrm{E}+00$ I $7.540 \mathrm{E}-01-1.976 \mathrm{E}-01 \quad 6.264 \mathrm{E}-01 \quad 1.000 \mathrm{E}+00 \quad 1.000 \mathrm{E}+00 \quad 0.000 \mathrm{E}+00$ 1 8.140E-01 -3.427E-01 4.690E-01 1.000E +00 1.000E+00 $0.000 \mathrm{E}+00$ $\begin{array}{llllll}1-1.257 \mathrm{E}-01 & 8.038 \mathrm{E}-01 & 5.814 \mathrm{E}-01 & 1.000 \mathrm{E}+00 & 1.000 \mathrm{E}+00 & 0.000 \mathrm{E}+00\end{array}$ $1-1.464 \mathrm{E}-01-1.070 \mathrm{E}-01 \quad 9.834 \mathrm{E}-01 \quad 1.000 \mathrm{E}+00 \quad 1.000 \mathrm{E}+00 \quad 0.000 \mathrm{E}+00$ $11.310 \mathrm{E}-02 \cdot 1.617 \mathrm{E}-02 \quad 9.998 \mathrm{E}-01 \quad 1.000 \mathrm{E}+00 \quad 1.000 \mathrm{E}+00 \quad 0.000 \mathrm{E}+00$ $1-8.843 E-01-4.126 E-01 \quad 2.187 E-01 \quad 1.000 E+00 \quad 1.000 E+00 \quad 0.000 E+00$ $14.260 \mathrm{E}-01 \quad 4.153 \mathrm{E}-01 \quad 8.038 \mathrm{E}-01 \quad 1.000 \mathrm{E}+00 \quad 1.000 \mathrm{E}+00 \quad 0.000 \mathrm{E}+00$

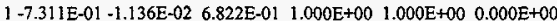

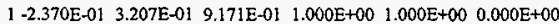
$14.276 \mathrm{E}-01-2.485 \mathrm{E}-02 \quad 9.036 \mathrm{E}-01 \quad 1.000 \mathrm{E}+00 \quad 1.000 \mathrm{E}+00 \quad 0.000 \mathrm{E}+00$ $17.662 \mathrm{E}-01 \quad 7.908 \mathrm{E}-02 \quad 6.378 \mathrm{E}-0$ ! $1.000 \mathrm{E}+00 \quad 1.000 \mathrm{E}+00 \quad 0.000 \mathrm{E}+00$ $1-4.247 \mathrm{E}-0 \mathrm{I} \quad 2.688 \mathrm{E}-01 \quad 8.645 \mathrm{E}-0 \mathrm{~L} \quad 1.000 \mathrm{E}+00$ 1.000E $+00 \quad 0.000 \mathrm{E}+00$ $12.096 \mathrm{E}-01 \quad 5.730 \mathrm{E}-01 \quad 7.923 \mathrm{E}-01 \quad 1.000 \mathrm{E}+00 \quad 1.000 \mathrm{E}+00 \quad 0.000 \mathrm{E}+00$

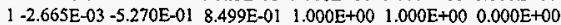
$1 \quad 7.228 \mathrm{E}-01 \quad 4.233 \mathrm{E}-01 \quad 5.463 \mathrm{E}-01 \quad 1.000 \mathrm{E}+00 \quad 1.000 \mathrm{E}+00 \quad 0.000 \mathrm{E}+00$ $\begin{array}{lllllll}3.484 \mathrm{E}-01 & -3.564 \mathrm{E}-01 & 8.669 \mathrm{E}-01 & 1.000 \mathrm{E}+00 & 1.000 \mathrm{E}+00 & 0.000 \mathrm{E}+00\end{array}$ 
$280.000 E+000.000 \mathrm{E}+00 \quad 0.000 \mathrm{E}+00$ $90.000 \mathrm{E}+00 \quad 0.000 \mathrm{E}+00 \quad 0.000 \mathrm{E}+00$ $0.000 \mathrm{E}+00 \quad 0.000 \mathrm{E}+00 \quad 0.000 \mathrm{E}+00$ $31 \quad 0.000 \mathrm{E}+00 \quad 0.000 \mathrm{E}+00 \quad 0.000 \mathrm{E}+00$ $32 \quad 0.000 \mathrm{E}+00 \quad 0.000 \mathrm{E}+00 \quad 0.000 \mathrm{E}+00$ $33 \quad 0.000 \mathrm{E}+00 \quad 0.000 \mathrm{E}+00 \quad 0.000 \mathrm{E}+00$ $34 \quad 0.000 \mathrm{E}+00 \quad 0.000 \mathrm{E}+00 \quad 0.000 \mathrm{E}+00$ $35 \quad 0.000 \mathrm{E}+00 \quad 0.000 \mathrm{E}+00 \quad 0.000 \mathrm{E}+00$ $360.000 \mathrm{E}+00 \quad 0.000 \mathrm{E}+00 \quad 0.000 \mathrm{E}+00$ $370.000 \mathrm{E}+00 \quad 0.000 \mathrm{E}+00 \quad 0.000 \mathrm{E}+00$ $\begin{array}{llllll}38 & 0.000 \mathrm{E}+00 & 0.000 \mathrm{E}+00 & 0.000 \mathrm{E}+00\end{array}$ $390.000 \mathrm{E}+00 \quad 0.000 \mathrm{E}+00 \quad 0.000 \mathrm{E}+00$ $40 \quad 0.000 \mathrm{E}+00 \quad 0.000 \mathrm{E}+00 \quad 0.000 \mathrm{E}+00$ $\begin{array}{lllll}41 & 0.000 \mathrm{E}+00 & 0.000 \mathrm{E}+00 & 0.000 \mathrm{E}+00\end{array}$ $420.000 \mathrm{E}+00 \quad 0.000 \mathrm{E}+00 \quad 0.000 \mathrm{E}+00$ $\begin{array}{llll}43 & 0.000 \mathrm{E}+00 & 0.000 \mathrm{E}+00 & 0.000 \mathrm{E}+00\end{array}$ $440.000 \mathrm{E}+00 \quad 0.000 \mathrm{E}+00 \quad 0.000 \mathrm{E}+00$ $45 \quad 0.000 \mathrm{E}+00 \quad 0.000 \mathrm{E}+00 \quad 0.000 \mathrm{E}+00$ $\begin{array}{llll}46 & 0.000 \mathrm{E}+00 & 0.000 \mathrm{E}+00 \quad 0.000 \mathrm{E}+00\end{array}$ $47 \quad 0.000 \mathrm{E}+00 \quad 0.000 \mathrm{E}+00 \quad 0.000 \mathrm{E}+00$ $\begin{array}{llll}48 & 0.000 \mathrm{E}+00 & 0.000 \mathrm{E}+00 & 0.000 \mathrm{E}+00\end{array}$ $490.000 \mathrm{E}+00 \quad 0.000 \mathrm{E}+00 \quad 0.000 \mathrm{E}+00$ $50 \quad 0.000 \mathrm{E}+00 \quad 0.000 \mathrm{E}+00 \quad 0.000 \mathrm{E}+00$ lproblem summary

תn terminated when 10000 particle histories were done

photon creation tracks weight energy photon loss tracks weight energy

source

00.

0 .

escape energy cutoff

$575 \quad 5.7500 \mathrm{E}-02 \quad 1.5476 \mathrm{E}-02$ $\begin{array}{llll}\text { energy cutoff } & 0 & 0 . & 0 . \\ \text { time cutoff } & 0 & 0 . & 0 .\end{array}$

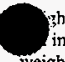

$18.706 \mathrm{E}-01-3.198 \mathrm{E}-01 \quad 3.738 \mathrm{E}-01+000 \mathrm{E}+00 \quad 1.000 \mathrm{E}+000.000 \mathrm{E}+00$ $\begin{array}{llllll}-8.188 \mathrm{E}-01 & 2.059 \mathrm{E}-01 & 5.359 \mathrm{E}-01 & 1.000 \mathrm{E}+00 & 1.000 \mathrm{E}+00 & 0.000 \mathrm{E}+00\end{array}$ $15.727 \mathrm{E}-01 \quad 8.124 \mathrm{E}-01 \quad 1.100 \mathrm{E}-01 \quad 1.000 \mathrm{E}+00 \quad 1.000 \mathrm{E}+00 \quad 0.000 \mathrm{E}+00$ $\begin{array}{llllll}5.390 \mathrm{E}-02 & 5.914 \mathrm{E}-01 & 8.046 \mathrm{E}-01 & 1.000 \mathrm{E}+00 & 1.000 \mathrm{E}+00 & 0.000 \mathrm{E}+00\end{array}$ $\begin{array}{lllllllll}1 & -5.778 \mathrm{E}-01 & 5.375 \mathrm{E}-01 & 6.141 \mathrm{E}-01 & 1.000 \mathrm{E}+00 & 1.000 \mathrm{E}+00 & 0.000 \mathrm{E}+00\end{array}$ $-7.198 \mathrm{E}-01-2.890 \mathrm{E}-01 \quad 6.311 \mathrm{E}-01 \quad 1.000 \mathrm{E}+00 \quad 1.000 \mathrm{E}+00 \quad 0.000 \mathrm{E}+00$ $12.210 \mathrm{E}-012.482 \mathrm{E}-01 \quad 9.432 \mathrm{E}-01 \quad 1.000 \mathrm{E}+00 \quad 1.000 \mathrm{E}+00 \quad 0.000 \mathrm{E}+00$ $14.339 \mathrm{E}-01 \quad 7.515 \mathrm{E}-02 \quad 8.978 \mathrm{E}-01 \quad 1.000 \mathrm{E}+00 \quad 1.000 \mathrm{E}+00 \quad 0.000 \mathrm{E}+00$

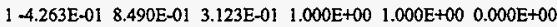
$1-3.933 \mathrm{E}-01-9.179 \mathrm{E}-01 \quad 5.266 \mathrm{E}-02 \quad 1.000 \mathrm{E}+00 \quad 1.000 \mathrm{E}+00 \quad 0.000 \mathrm{E}+00$ I $-4.846 \mathrm{E}-01 \quad 4.378 \mathrm{E}-01 \quad 7.573 \mathrm{E}-01 \quad 1.000 \mathrm{E}+00 \quad 1.000 \mathrm{E}+00 \quad 0.000 \mathrm{E}+00$

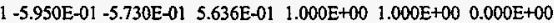
$1-7.252 \mathrm{E}+01+5.143 \mathrm{E}-01 \quad 4.578 \mathrm{E}-01 \quad 1.000 \mathrm{E}+00 \quad 1.000 \mathrm{E}+00 \quad 0.000 \mathrm{E}+00$ $\begin{array}{lllllll}1 & -7.156 \mathrm{E}-01 & 4.734 \mathrm{E}-01 & 5.136 \mathrm{E}-01 & 1.000 \mathrm{E}+00 & 1.000 \mathrm{E}+00 & 0.000 \mathrm{E}+00\end{array}$ $1-1.771 \mathrm{E}-01 \quad 9.229 \mathrm{E}-01 \quad 3.417 \mathrm{E}-01 \quad 1.000 \mathrm{E}+00 \quad 1.000 \mathrm{E}+00 \quad 0.000 \mathrm{E}+00$

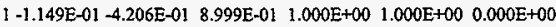
$14.509 \mathrm{E}-01 \quad 8.630 \mathrm{E}-02 \quad 8.884 \mathrm{E}-01 \quad 1.000 \mathrm{E}+00 \quad 1.000 \mathrm{E}+00 \quad 0.000 \mathrm{E}+00$

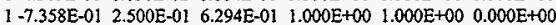
$\begin{array}{llllll}1-8.448 \mathrm{E}-01 & 3.645 \mathrm{E}-01 & 3.917 \mathrm{E}-01 & 1.000 \mathrm{E}+00 & 1.000 \mathrm{E}+00 & 0.000 \mathrm{E}+00\end{array}$

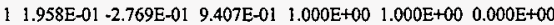
$12.788 \mathrm{E}-01-7.325 \mathrm{E}-03 \quad 9.603 \mathrm{E}-01 \quad 1.000 \mathrm{E}+00 \quad 1.000 \mathrm{E}+00 \quad 0.000 \mathrm{E}+00$

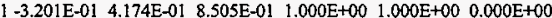
$18.960 \mathrm{E}-01-1.641 \mathrm{E}-01 \quad 4.126 \mathrm{E}-01 \quad 1.000 \mathrm{E}+00 \quad 1.000 \mathrm{E}+00 \quad 0.000 \mathrm{E}+00$ ght window

importance

weight cutoff

energy importance

dxtran

forced collisions

exp. transform

from neutrons

bremsstrahlung

p-annihilation

electron $x$-rays

Ist fluorescence

2nd fluorescence

total 694

number of photons banked weight window cell importance 00 . 00. weight cutoff 00 . energy importance $0 \quad 0$.

0. 00.0 photon tracks per source particle photon collisions per source particle total photon collisions 0.0 0. 0. $\begin{array}{lll}0 & 0 . & 0 .\end{array}$ $\begin{array}{lll}0 & 0 . & 0 .\end{array}$ $\begin{array}{lll}0 & 0 . & 0 .\end{array}$ $6946.9400 \mathrm{E}-02$ $\begin{array}{lll}0 & 0 . & 0 .\end{array}$ 0 o. 0. $\begin{array}{lll}0 & 0 . & 0 .\end{array}$ $\begin{array}{lll}0 & 0 . & 0 .\end{array}$ electron creation tracks weight energy (per source particle)

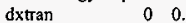
forced collisions $0 \quad 0$. exp. transform $\quad 0 \quad 0$ compton scatter 1.7315E-02 capture pair production $\begin{array}{ll}0 & 0 .\end{array}$ 0. 0. $119 \quad 1.1900 \mathrm{E}-02 \quad 1.7720 \mathrm{E}-03$ 00.0 0. 0. $\begin{array}{lllll}1.7315 \mathrm{E}-02 & \text { total } & 694 & 6.9400 \mathrm{E}-02 & 1.7315 \mathrm{E}-02\end{array}$

694 average lifetime, shakes cutoffs $6.9400 \mathrm{E}-02$ escape $6.7786 \mathrm{E}-05$ tco $1.0000 \mathrm{E}+34$ $1.3000 \mathrm{E}-02$ capture $8.3325 \mathrm{E}-05$ eco $1.0000 \mathrm{E}-0$ ? 130 capture or escape 7.0450E-05 wc1 $0.0000 \mathrm{E}+00$ any termination $7.0450 \mathrm{E}-05$ WC2 $0.0000 \mathrm{E}+00$$$
000 \text { 1 } 00005+00
$$

$10000 \mathrm{E}+00$ energy cutoff electron loss tracks weight energy

source $10000 \quad 1.0000 \mathrm{E}+00$ time cutoff

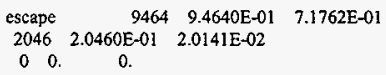

weight window cell importance weight cutoff energy importance $\begin{array}{ll}0 & 0 .\end{array}$ 0 00. 0. $\begin{array}{lll}0 & 0 .\end{array}$ 0. 00. 0 $\begin{array}{lll}0 & 0 . & 0 .\end{array}$

0. $\begin{array}{cc}2 & 2.0000 \mathrm{E}-04 \\ 2.9000 \mathrm{E}-03\end{array}$

scattering

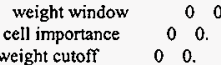

$0 \quad 0$.

0. 0. $\begin{array}{lrrrrr}\text { weight cutoff } & 0 & 0 . & & 0 . \\ \text { energy importance } & 0 & 0 . & & 0 .\end{array}$ $\begin{array}{llll}\text { scattering } & 0 & 0 . & 2.6560 \mathrm{E}-0]\end{array}$ pton recoil to-electric $29 \quad 2.9000 \mathrm{E}-03 \quad 4.7387 \mathrm{E}-04$ bremsstrahlung 00 . $2.4158 \mathrm{E}-02$ 
photon auger
tron auger
total

number of electrons banked

00.

$0 \quad 0$. $14791.4790 \mathrm{E}-0$

$1510 \quad 1.1510 \mathrm{E}+00 \quad 1.0275 \mathrm{E}+00$

electron sub-steps per source particle 7.7164E+Ol

total electron sub-steps $\quad 771636$

computer time so far in this run 0.00 minutes computer time in morun $\quad 0.00$ minutes source particles per minute $\quad 0.0000 \mathrm{E}+00$ random numbers generated $\quad 4748878$ total $\quad 11510 \quad \mathrm{I} .1510 \mathrm{E}+00 \quad 1.0275 \mathrm{E}+00$

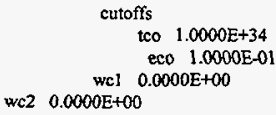

range of sampled source weights $=1.0000 \mathrm{E}+00$ to $1.0000 \mathrm{E}+00$

Iphoton activity in each cell

print table 126

tracks population collisions collisions number flux average average cell entering

* weight weighted weighted track weight track mfp (per history) energy energy (relative) (cm)

$\begin{array}{llllllllll}1 & 1 & 0 & 694 & 130 & 1.3000 \mathrm{E}-02 & 2.8286 \mathrm{E}-01 & 2.8286 \mathrm{E}-01 & 1.0000 \mathrm{E}+00 & 1.6113 \mathrm{E}-01\end{array}$

$\begin{array}{lllll}\text { total } & 0 & 694 & 130 & 1.3000 \mathrm{E}-02\end{array}$

lelectron activity in each cell

print table 126

tracks population substeps substeps number flux average average

cell entering

- weight weighted weighted track weight track mfp (per history) energy energy (relative) (cm)

\footnotetext{
$\begin{array}{llllllllll}1 & 1 & 10000 & 11510 & 771636 & 7.7164 \mathrm{E}+01 & 7.4988 \mathrm{E}-01 & 7.6821 \mathrm{E}-01 & 1.0000 \mathrm{E}+00 & 2.5110 \mathrm{E}-04\end{array}$

tal $\quad 10000 \quad 11510 \quad 771636 \quad 7.7164 \mathrm{E}+01$

Itally $1 \quad$ nps $=10000$

tally type 1* energy crossing a surface. units mev tally for photons
}

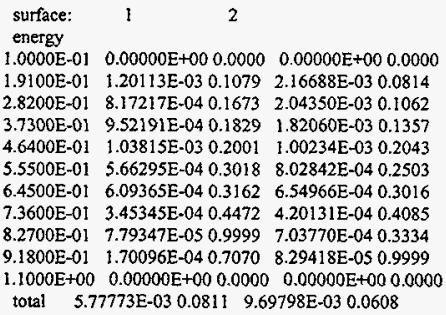

Itally 6 nps $=10000$

tally type 6 track length estimate of heating. units mev/gram tally for photons

masses

cell: $\quad 1$

$4.90220 \mathrm{E}+01$

\footnotetext{
cell: $\quad$ I

energy

$1.0000 \mathrm{E}-01 \quad 0.00000 \mathrm{E}+000.0000$

1.9100E-01 $1.80395 \mathrm{E}-050.0935$

$3200 \mathrm{E}-01 \quad 4.79830 \mathrm{E}-060.1242$

/300E-01 $1.64870 \mathrm{E}-060.2061$
} 

$5500 \mathrm{E}-01 \quad 6.34259 \mathrm{E}-07 \quad 0.4736$ $4500 \mathrm{E}-01 \quad 2.47457 \mathrm{E}-07 \quad 0.3129$ $7.3600 \mathrm{E}-01 \quad 2.58011 \mathrm{E}-07 \quad 0.6571$

8.2700E-01 $\quad 1.16245 \mathrm{E}-070.3470$

$9.1800 \mathrm{E}-01 \quad 4.46610 \mathrm{E}-080.6403$

$1.1000 \mathrm{E}+00 \quad 0.00000 \mathrm{E}+00 \quad 0.0000$ total 2.75512E-05 0.0722

Itally 28 nps $=10000$

tally type 8* energy deposition units mev tally for photons electrons

$$
\text { cell: } 1
$$

energy

$-1.0000 \mathrm{E}-03-8.72550 \mathrm{E}-040.1508$

$0.0000 \mathrm{E}+00 \quad 0.00000 \mathrm{E}+000.0000$

$1.0000 \mathrm{E}-06 \quad 0.00000 \mathrm{E}+000.0000$

$1.0000 \mathrm{E}-01 \quad 1.08193 \mathrm{E}-020.0227$

I.9100E-01 3.91016E-02 0.0170

$2.8200 \mathrm{E}-01 \quad 4.36100 \mathrm{E}-02 \quad 0.0210$

$3.7300 \mathrm{E}-01 \quad 3.53763 \mathrm{E}-02 \quad 0.0286$

$4.6400 \mathrm{E}-01 \quad 2.66881 \mathrm{E}-02 \quad 0.0382$

$5.5500 \mathrm{E}-01 \quad 2.21833 \mathrm{E}-020.0468$

$6.4500 \mathrm{E}-01 \quad 1.58947 \mathrm{E}-020.0606$

$7.3600 \mathrm{E}-01 \quad 1.08330 \mathrm{E}-02 \quad 0.0790$

8.2700E-01 8.71177E-03 0.0940

$9.1800 \mathrm{E}-0 \mathrm{O} \quad 6.65495 \mathrm{E}-030.1136$

1.1000E+00 4.79000E-02 0.0446

total $2.66900 \mathrm{E}-010.0090$

1 tally $11 \quad$ nps $=10000$

tally type $1^{*}$ energy crossing a surface. units mev tally for electrons

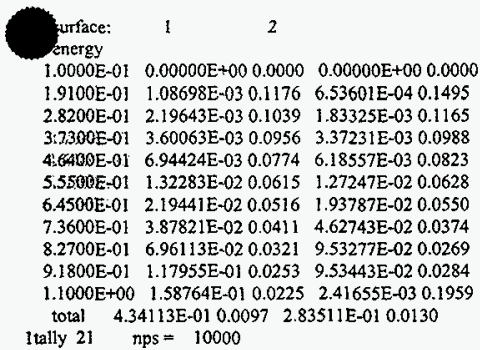

tally type 1 number of particles crossing a surface. tally for electrons

surface: 112

energy

$1.0000 \mathrm{E}-01 \quad 0.00000 \mathrm{E}+00 \quad 0.0000 \quad 0.00000 \mathrm{E}+000.0000$

$\begin{array}{lllll}1.9100 \mathrm{E}-01 & 7.70000 \mathrm{E}-03 & 0.1165 & 4.60000 \mathrm{E}-03 & 0.1471\end{array}$

$2.8200 \mathrm{E}-01 \quad 9.30000 \mathrm{E}-03 \quad 0.1032 \quad 7.60000 \mathrm{E}-03 \quad 0.1158$

$\begin{array}{lllll}3.7300 \mathrm{E}-01 & 1.09000 \mathrm{E}-02 & 0.0953 & 1.02000 \mathrm{E}-02 & 0.0985\end{array}$

$4.6400 \mathrm{E}-01 \quad 1.65000 \mathrm{E}-02 \quad 0.0772 \quad 1.46000 \mathrm{E}-020.0822$

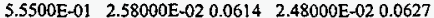

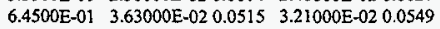

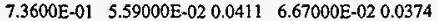

$\begin{array}{lllll}8.2700 E-01 & 8.86000 E-02 & 0.0321 & 1.21300 E-01 & 0.0269\end{array}$

$9.1800 \mathrm{E}-01 \quad 1.34800 \mathrm{E}-01 \quad 0.0253 \quad 1.10600 \mathrm{E}-010.0284$

$1.1000 \mathrm{E}+00 \quad 1.65500 \mathrm{E}-01 \quad 0.0225 \quad 2.60000 \mathrm{E}-03 \quad 0.1959$ total $5.51300 \mathrm{E}-010.0094 \quad 3.95100 \mathrm{E}-010.0126$ nps $=10000$

tally type 1 number of particles crossing a surface. 
문

this tally is modified by ft elc

surface:

energy

$1.0000 \mathrm{E}-01 \quad 0.00000 \mathrm{E}+00 \quad 0.0000 \quad 0.00000 \mathrm{E}+000.0000$

$1.9100 \mathrm{E}-01-7.70000 \mathrm{E}-030.1165-4.60000 \mathrm{E}-03 \quad 0.1471$

$2.8200 \mathrm{E}-01-9.30000 \mathrm{E}-03 \quad 0.1032-7.60000 \mathrm{E}-030.1158$

$3.7300 \mathrm{E}-01-1.09000 \mathrm{E}-020.0953+1.02000 \mathrm{E}-020.0985$

$4.6400 \mathrm{E}-01-1.65000 \mathrm{E}-020.0772-1.46000 \mathrm{E}-020.0822$

$5.5500 \mathrm{E}-01-2.58000 \mathrm{E}-020.0614-2.48000 \mathrm{E}-020.0627$

$6.4500 \mathrm{E}-01-3.63000 \mathrm{E}-020.0515-3.21000 \mathrm{E}-020.0549$

$7.3600 \mathrm{E}-01-5.59000 \mathrm{E}-020.0411-6.67000 \mathrm{E}-020.0374$

$8.2700 \mathrm{E}-01-8.86000 \mathrm{E}-02 \quad 0.0321-1.21300 \mathrm{E}-010.0269$

$9.1800 \mathrm{E}-01-1.34800 \mathrm{E}-010.0253-1.10600 \mathrm{E}-010.0284$

$1.1000 \mathrm{E}+00-1.65500 \mathrm{E}-010.0225-2.60000 \mathrm{E}-030.1959$

total $-5.51300 \mathrm{E}-010.0094-3.95100 \mathrm{E}-010.0126$

J tally 41 nps $=10000$

tally type 1 number of particles crossing a surface. tally for electrons

this tally is modified by ft elc

surface: $\quad 1 \quad 2$

energy

1.0000E-0I $\quad 0.00000 \mathrm{E}+000.0000 \quad 0.00000 \mathrm{E}+000.0000$

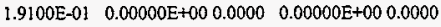

$2.8200 \mathrm{E}-01 \quad 0.00000 \mathrm{E}+00 \quad 0.0000 \quad 0.00000 \mathrm{E}+000.0000$

$3.7300 \mathrm{E}-01 \quad 0.00000 \mathrm{E}+00 \quad 0.0000 \quad 0.00000 \mathrm{E}+00 \quad 0.0000$

$4.6400 \mathrm{E}-01 \quad 0.00000 \mathrm{E}+00 \quad 0.0000 \quad 0.00000 \mathrm{E}+00 \quad 0.0000$

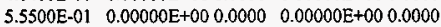

$6.4500 \mathrm{E}-01 \quad 0.00000 \mathrm{E}+00 \quad 0.0000 \quad 0.00000 \mathrm{E}+00 \quad 0.0000$

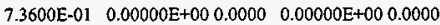

$8.2700 \mathrm{E}-01 \quad 0.00000 \mathrm{E}+000.0000 \quad 0.00000 \mathrm{E}+000.0000$

$1800 \mathrm{E}-01 \quad 0.00000 \mathrm{E}+00 \quad 0.0000 \quad 0.00000 \mathrm{E}+000.0000$

$1000 \mathrm{E}+00 \quad 0.00000 \mathrm{E}+00 \quad 0.0000 \quad 0.00000 \mathrm{E}+00 \quad 0.0000$

total $0.00000 \mathrm{E}+000.0000 \quad 0.00000 \mathrm{E}+000.0000$

surface: $\quad 1 \quad 2$

energy

$1.0000 \mathrm{E}-0 \mathrm{t} \quad 0.00000 \mathrm{E}+00 \quad 0.0000 \quad 0.00000 \mathrm{E}+00 \quad 0.0000$

$1.9100 \mathrm{E}-01 \quad 7.70000 \mathrm{E}-03 \quad 0.1165 \quad 4.60000 \mathrm{E}-03 \quad 0.1471$

$2.8200 \mathrm{E}-01 \quad 9.30000 \mathrm{E}-03 \quad 0.1032 \quad 7.60000 \mathrm{E}-03 \quad 0.1158$

$3.7300 \mathrm{E}-01 \quad 1.09000 \mathrm{E}-02 \quad 0.0953 \quad 1.02000 \mathrm{E}-020.0985$

$4.6400 \mathrm{E}-01 \quad 1.65000 \mathrm{E}-02 \quad 0.0772 \quad 1.46000 \mathrm{E}-02 \quad 0.0822$

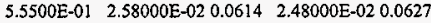

$6.4500 \mathrm{E}-01 \quad 3.63000 \mathrm{E}-02 \quad 0.0515 \quad 3.21000 \mathrm{E}-020.0549$

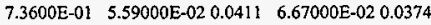

$8.2700 \mathrm{E}-01 \quad 8.86000 \mathrm{E}-020.0321 \quad 1.21300 \mathrm{E}-010.0269$

$9.1800 \mathrm{E}-0 \mathrm{I} \quad 1.34800 \mathrm{E}-01 \quad 0.0253 \quad 1.10600 \mathrm{E}-010.0284$

$1.1000 \mathrm{E}+00 \quad 1.65500 \mathrm{E}-01 \quad 0.0225 \quad 2.60000 \mathrm{E}-030.1959$

total $\quad 5.51300 \mathrm{E}-010.0094 \quad 3.95100 \mathrm{E}-01 \quad 0.0126$

surface: $\quad 1 \quad 2$

energy

$1.0000 \mathrm{E}-0 \mathrm{I} \quad 0.00000 \mathrm{E}+000.0000 \quad 0.00000 \mathrm{E}+000.0000$

$1.9100 \mathrm{E}-01 \quad 7.70000 \mathrm{E}-03 \quad 0.1165 \quad 4.60000 \mathrm{E}-03 \quad 0.1471$

$2.8200 \mathrm{E}-01 \quad 9.30000 \mathrm{E}-03 \quad 0.1032 \quad 7.60000 \mathrm{E}-03 \quad 0.1158$

$3.7300 \mathrm{E}-01 \quad 1.09000 \mathrm{E}-02 \quad 0.0953 \quad 1.02000 \mathrm{E}-020.0985$

$4.6400 \mathrm{E}-01 \quad 1.65000 \mathrm{E}-02 \quad 0.0772 \quad 1.46000 \mathrm{E}-02 \quad 0.0822$

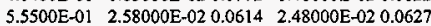

$6.4500 \mathrm{E}-01 \quad 3.63000 \mathrm{E}-02 \quad 0.0515 \quad 3.21000 \mathrm{E}-020.0549$

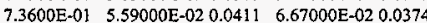

$\begin{array}{lllll}8.2700 \mathrm{E}-01 & 8.86000 \mathrm{E}-02 & 0.0321 & 1.21300 \mathrm{E}-01 & 0.0269\end{array}$

$9.1800 \mathrm{E}-01 \quad 1.34800 \mathrm{E}-01 \quad 0.0253 \quad 1.10600 \mathrm{E}-01 \quad 0.0284$

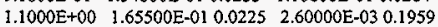

yotal $5.51300 \mathrm{E}-010.0094 \quad 3.95100 \mathrm{E}-010.0126$

y $51 \quad n p s=10000$
HNF-SD-SNF-CSWD-005, Rev. 0 
tally type 1 number of particles crossing a surface.

HNF-SD-SNF-CSWD-005, Rev. 0

for electrons

this tally is modified by $\mathrm{ft}$ elc

surface: $1 \quad 2$

energy

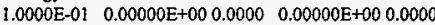

$1.9100 \mathrm{E}-01 \quad 0.00000 \mathrm{E}+00 \quad 0.0000 \quad 0.00000 \mathrm{E}+000.0000$

$2.8200 E-01 \quad 0.00000 E+000.0000 \quad 0.00000 E+000.0000$

$3.7300 \mathrm{E}-01 \quad 0.00000 \mathrm{E}+00 \quad 0.0000 \quad 0.00000 \mathrm{E}+000.0000$

$4.6400 \mathrm{E}-01 \quad 0.00000 \mathrm{E}+00 \quad 0.0000 \quad 0.00000 \mathrm{E}+00 \quad 0.0000$

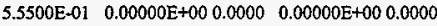

6.4500E-01 $\quad 0.00000 \mathrm{E}+00 \quad 0.0000 \quad 0.00000 \mathrm{E}+00 \quad 0.0000$

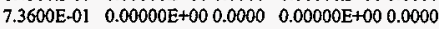

$8.2700 \mathrm{E}-0 \mathrm{I} \quad 0.00000 \mathrm{E}+000.0000 \quad 0.00000 \mathrm{E}+000.0000$

$9.1800 \mathrm{E}-01 \quad 0.00000 \mathrm{E}+00 \quad 0.0000 \quad 0.00000 \mathrm{E}+00 \quad 0.0000$

$1.1000 \mathrm{E}+00 \quad 0.00000 \mathrm{E}+00 \quad 0.0000 \quad 0.00000 \mathrm{E}+00 \quad 0.0000$

total $0.00000 \mathrm{E}+000.0000 \quad 0.00000 \mathrm{E}+000.0000$

surface: $\quad 1 \quad 2$

energy

$1.0000 \mathrm{E}-01 \quad 0.00000 \mathrm{E}+00 \quad 0.0000 \quad 0.00000 \mathrm{E}+00 \quad 0.0000$

1.9100E-01 -7.70000E-03 $0.1165-4.60000 \mathrm{E}-03 \quad 0.147$

2.8200E-01 $-9.30000 \mathrm{E}-030.1032-7.60000 \mathrm{E}-030.1158$

$3.7300 \mathrm{E}-01-1.09000 \mathrm{E}-020.0953-1.02000 \mathrm{E}-020.0985$

4.6400E-01 - $1.65000 \mathrm{E}-020.0772-1.46000 \mathrm{E}-020.0822$

$5.5500 \mathrm{E}-01-2.58000 \mathrm{E}-020.0614-2.48000 \mathrm{E}-020.0627$

$6.4500 \mathrm{E}-01-3.63000 \mathrm{E}-020.0515-3.21000 \mathrm{E}-020.0549$ $7.3600 \mathrm{E}-01-5.59000 \mathrm{E}-02 \quad 0.0411-6.67000 \mathrm{E}-020.0374$

8.2700E-01 $-8.86000 \mathrm{E}-020.0321-1.21300 \mathrm{E}-010.0269$

$9.1800 \mathrm{E}-01-1.34800 \mathrm{E}-010.0253-1.10600 \mathrm{E}-010.0284$

$1.1000 \mathrm{E}+00-1.65500 \mathrm{E}-010.0225-2.60000 \mathrm{E}-03 \quad 0.1959$

total $-5.51300 \mathrm{E}-010.0094-3.95100 \mathrm{E}-010.0126$

surface: $\quad 1 \quad 2$

energy $0.00000 \mathrm{E}+000.0000 \quad 0.00000 \mathrm{E}+000.0000$ $1.9100 \mathrm{E}-01-7.70000 \mathrm{E}-030.1165-4.60000 \mathrm{E}-030.1471$

$2.8200 \mathrm{E}-01-9.30000 \mathrm{E}-03 \quad 0.1032-7.60000 \mathrm{E}-030.1158$

$3.7300 \mathrm{E}-01-1.09000 \mathrm{E}-02 \quad 0.0953-1.02000 \mathrm{E}-020.0985$

$4.6400 \mathrm{E}-01-1.65000 \mathrm{E}-020.0772-1.46000 \mathrm{E}-020.0822$

$5.5500 \mathrm{E}-01 \quad-2.58000 \mathrm{E}-02 \quad 0.0614 \quad-2.48000 \mathrm{E}-020.0627$

$6.4500 \mathrm{E}-01-3.63000 \mathrm{E}-02 \quad 0.0515-3.21000 \mathrm{E}-020.0549$

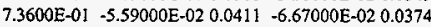

$8.2700 E-01-8.86000 E-02 \quad 0.0321-1.21300 E-010.0269$

$9.1800 \mathrm{E}-01-1.34800 \mathrm{E}-010.0253+1.10600 \mathrm{E}-010.0284$

$1.1000 \mathrm{E}+00-1.65500 \mathrm{E}-010.0225-2.60000 \mathrm{E}-03 \quad 0.1959$

total $-5.51300 \mathrm{E}-010.0094-3.95100 \mathrm{E}-010.0126$

1tally 58 nps $=10000$

tally type 8 pulse height distribution. units number

tally for photons electrons

cell:

energy

$-1.0000 \mathrm{E}-03 \quad 7.40000 \mathrm{E}-03 \quad 0.1158$

$0.0000 \mathrm{E}+00 \quad 0.00000 \mathrm{E}+000.0000$

$1.0000 \mathrm{E}-06 \quad 0.00000 \mathrm{E}+000.0000$

$1.0000 \mathrm{E}-01 \quad 2.12600 \mathrm{E}-010.0192$

$1.9100 \mathrm{E}-01 \quad 2.65500 \mathrm{E}-010.0166$

$2.8200 \mathrm{E}-01 \quad 1.87700 \mathrm{E}-010.0208$

$3.7300 \mathrm{E}-01 \quad 1.09500 \mathrm{E}-01 \quad 0.0285$

$4.6400 \mathrm{E}-01 \quad 6.44000 \mathrm{E}-020.0381$

$5.5500 \mathrm{E}-01 \quad 4.37000 \mathrm{E}-020.0468$

$6.4500 \mathrm{E}-01 \quad 2.66000 \mathrm{E}-02 \quad 0.0605$

$7.3600 \mathrm{E}-01 \quad 1.58000 \mathrm{E}-020.0789$

$2700 \mathrm{E}-01 \quad 1.12000 \mathrm{E}-020.0940$

$1800 \mathrm{E}-01 \quad 7.70000 \mathrm{E}-03 \quad 0.1135$ 

tally for photons electrons

units mev

cell: - 1

energy

$1.0000 \mathrm{E}+03 \quad 2.66900 \mathrm{E}-01 \quad 0.0000$

1status of the statistical checks used to form confidence intervals for the mean for each tally bin

tally result of statistical checks for the tfe bin (the first check not passed is listed) and error magnitude check for all bins

1 missed 1 of $10 \mathrm{tfc}$ bin checks: there is insufficient tfe bin tally information to estimate the large tally slope reliably missed all bin error check: 24 tally bins had 4 bins with zeros and 17 bins with relative errors exceeding 0.10

6 missed 1 of $10 \mathrm{tfc}$ bin checks: the variance of the variance appears not to decrease as $1 / \mathrm{nps}$ for the last half of problem missed all bin error check: 12 tally bins had 2 bins with zeros and 8 bins with relative errors exceeding 0.10

28 passed the 10 statistical checks for the tally fluctuation chart bin result missed all bin etror check: 15 tally bins had 2 bins with zeros and 2 bins with relative errors exceeding 0.10

11 missed 1 of 10 tic bin checks: the slope of decrease of largest tallies is less than the minimum acceptable value of 3.0 missed all bin error check: 24 tally bins had 2 bins with zeros and 5 bins with relative errors exceeding 0.10

21 missed 1 of 10 tf $\mathrm{c}$ bin checks: the estimated mean has a trend during the last half of the problem missed all bin error check: 24 tally bins had 2 bins with zeros and 5 bins with relative errors exceeding 0.10

31 missed 2 of $10 \mathrm{tfc}$ bin checks: the estimated mean has a trend during the last half of the problem missed all bin error check: 24 tally bins had 2 bins with zeros and 5 bins with relative errors exceeding 0.10

41 missed 1 of 10 tf bin checks: the estimated mean has a trend during the last half of the problem missed all bin error check: 72 tally bins had 28 bins with zeros and 10 bins with relative errors exceeding 0.10

missed 2 of $10 \mathrm{tfc}$ bin checks: the estimated mean has a trend during the last half of the problem missed all bin error check: 72 tally bins had 28 bins with zeros and 10 bins with relative enrors exceeding 0.10

58 passed the 10 statistical checks for the tally fluctuation chart bin result missed all bin error check: 15 tally bins had 2 bins with zeros and 2 bins with telative errors exceeding 0.10

8 passed the 10 statistical checks for the tally fluctuation chart bin result passed all bin error check: 1 tally bins all have relative errors less than 0.10 with no zero bins

the 10 statistical checks are only for the tally fluctuation chart bin and do not apply to other tally bins.

the tally bins with zeros may or may not be correct: compare the source, cutoffs, multipliers, et cetera with the tally bins.

warning. 7 of the 10 tally fluctuation chart bins did not pass all 10 statistical checks. warning. 9 of the 10 tallies had bins with relative errors greater than recommended. J tally fluctuation charts

tally 1 tally $6 \quad$ tally 28

nps mean error vov slope fom mean error vov slope fom $10006.7975 \mathrm{E}-030.23990 .0835 \quad 0.0$ $20005.5780 \mathrm{E}-030.18840 .06410 .0$ $30005.8779 \mathrm{E}-030.14550 .0373 \quad 0.0$ $40005.7880 \mathrm{E}-030.13170 .03110 .0$ $50005.9924 \mathrm{E}-030.11680 .02390 .0$ $60005.9423 \mathrm{E}-030.10550 .01950 .0$ $70005.9749 \mathrm{E}-030.09680 .01700 .0$ 8000 5.7670E-03 $0.09130 .0154 \quad 0.0$ $90005.7398 \mathrm{E}-030.08640 .0135 \quad 0.0$ $100005.7777 \mathrm{E}-030.08110 .0120 \quad 0.0$
$2.0857 \mathrm{E}-050.15890 .0525 \quad 0.0$ $2.4397 \mathrm{E}-050.17010 .1693 \quad 0.0$ $\begin{array}{llll}2.6650 \mathrm{E}-05 & 0.1343 & 0.1060 & 0.0\end{array}$ $2.7894 \mathrm{E}-050.11450 .0656 \quad 0.0$ $2.7855 \mathrm{E}-050.10200 .0485 \quad 0.0$ $2.7793 \mathrm{E}-0.50 .09310 .0418 \quad 0.0$ $2.8647 \mathrm{E}-05 \quad 0.09190 .0581 \quad 0.0$ $2.8249 \mathrm{E}-050.08380 .052410 .0$ $2.7485 \mathrm{E}-050.07790 .049110 .0$ $2.7551 \mathrm{E}-050.07220 .043610 .0$ mean error voy slope fom 2.6993E-01 0.02790 .004410 .0 $2.6475 \mathrm{E}-010.01990 .002210 .0$ $2.6433 \mathrm{E}-010.01610 .001510 .0$ 2.6224E-01 0.01400 .001110 .0 $2.6175 \mathrm{E}-010.01260 .000910 .0$ $2.6496 \mathrm{E}-010.01160 .000710 .0$ 2.6691E-01 0.01070 .000610 .0 $2.6739 \mathrm{E}-010.01000 .000510 .0$ $2.6699 \mathrm{E}-010.00950 .000510 .0$ $2.6690 \mathrm{E}-010.00900 .000410 .0$ 
tally 11 nps mean error vov slope fom $10004.4504 \mathrm{E}-01 \quad 0.02960 .00022 .1$ $20004.4489 \mathrm{E}-010.02110 .00012 .0$ $30004.4226 \mathrm{E}-010.01730 .0001 \quad 1.8$ $40004.4011 \mathrm{E}-010.01510 .0000 \quad 1.8$ $50004.4004 \mathrm{E}-010.01350 .0000 \quad 1.9$ $60004.3549 \mathrm{E}-01 \quad 0.01250 .0000 \quad 1.8$ $70004.3477 \mathrm{E}-010.01150 .0000 \quad 1.9$ $80004.3339 E-010.01080 .0000 \quad 1.8$ $90004.3428 \mathrm{E}-01 \quad 0.0102 \quad 0.0000 \quad 1.7$ $100004.3411 \mathrm{E}-010.00970 .00001 .6$ tally 21 $5.6500 \mathrm{E}+010.02860 .0004 \quad 4.4$ $5.6500 \mathrm{E}-010.02040 .0003 \quad 4.4$ $5.6400 \mathrm{E}-010.01680 .000210 .0$ $5.6100 \mathrm{E}-0 ! 0.01470 .000210 .0$ $5.5860 \mathrm{E}-010.01310 .000110 .0$ $5.5433 \mathrm{E}-010.01210 .000110 .0$ $5.5386 \mathrm{E}-010.01120 .000110 .0$ $5.5238 \mathrm{E}-010.01050 .000110 .0$ $5.5200 \mathrm{E}-01 \quad 0.00990 .000110 .0$ $5.5130 \mathrm{E}-010.00940 .000110 .0$ mean error vov slope fom $\begin{array}{llll}-5.6500 \mathrm{E}-01 & 0.02860 .0004 & 0.0\end{array}$ $\begin{array}{llll}-5.6500 \mathrm{E}-01 & 0.02040 .0003 & 0.0\end{array}$ $\begin{array}{lll}-5.6400 \mathrm{E}-01 & 0.01680 .0002 & 0.0\end{array}$ $-5.6100 \mathrm{E}-010.01470 .0002 \quad 0.0$
$-5860 \mathrm{E}-010.01310 .00010 .0$ $\begin{array}{llllll}-5.5860 \mathrm{E}-01 & 0.01310 .0001 & 0.0\end{array}$ $\begin{array}{llllll}-5.5433 E-01 & 0.0121 & 0.0001 & 0.0\end{array}$

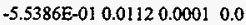
$-5.5238 \mathrm{E}-010.01050 .00010 .0$
$0.5200 \mathrm{E}-010.00990 .0001$ $-5.5200 \mathrm{E}-010.00990 .00010 .0$
$-5.5130 \mathrm{E}-010.00940 .0001$ $\begin{array}{llll}-5.5130 E-01 & 0.00940 .0001 & 0.0\end{array}$ tally 41

nps mean error vov slope fom $10005.6500 \mathrm{E}-010.02860 .0004 \quad 4.4$ $20005.6500 \mathrm{E}-010.02040 .00034 .4$ $30005.6400 \mathrm{E}-010.01680 .000210 .0$ $40005.6100 \mathrm{E}-01 \quad 0.01470 .000210 .0$ $50005.5860 \mathrm{E}-010.01310 .000110 .0$ $60005.5433 \mathrm{E}-010.01210 .000110 .0$ $70005.5386 \mathrm{E}-01 \quad 0.01120 .000110 .0$ $80005.5238 \mathrm{E}-010.01050 .000110 .0$ $90005.5200 \mathrm{E}-010.00990 .000110 .0$ $100005.5130 \mathrm{E}-010.00940 .000110 .0$

tally 8

nps mean error vov slope fom $10002.6993 \mathrm{E}-010.02790 .004410 .0$ $20002.6475 \mathrm{E}-01 \quad 0.01990 .002210 .0$ $30002.6433 \mathrm{E}-010.01610 .001510 .0$ $40002.6224 \mathrm{E}-01 \quad 0.01400 .001110 .0$ $50002.6175 \mathrm{E}-01 \quad 0.01260 .000910 .0$ $60002.6496 \mathrm{E}-010.01160 .000710 .0$ $70002.6691 \mathrm{E}-010.01070 .000610 .0$ $80002.6739 \mathrm{E}-01 \quad 0.01000 .000510 .0$ $90002.6699 \mathrm{E}-01 \quad 0.00950 .000510 .0$ $100002.6690 \mathrm{E}-010.00900 .000410 .0$ tally data written to file inp $20 \mathrm{~m}$

6 warning messages so far. tally 51

tally 58 mean error vov slope fom $-5.6500 \mathrm{E}-010.02860 .00040 .0$
$0.6500 \mathrm{E}-010.02040 .0030$

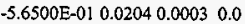
$-5.6400 \mathrm{E}-010.01680 .00020 .0$
$0.6100 \mathrm{E}-010.01470 .0002$ $\begin{array}{llll}-5.6100 E-01 & 0.0147 & 0.0002 & 0.0\end{array}$ $\begin{array}{lllllll}-5.5860 E-01 & 0.0131 & 0.0001 & 0.0\end{array}$ $\begin{array}{llllllll}-5.5433 \mathrm{E}-01 & 0.0121 & 0.0001 & 0.0\end{array}$ $\begin{array}{llll}-5.5386 \mathrm{E}-01 & 0.01120 .0001 & 0.0\end{array}$

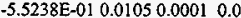
$-5.5200 \mathrm{E}-010.00990 .00010 .0$
0.50 $-5.5130 \mathrm{E}-010.00940 .0001 \quad 0.0$
0.000 mean enror vov slope fom $1.0000 \mathrm{E}+000.00000 .000010 .0$ $1.0000 \mathrm{E}+000.00000 .000010 .0$ $1.0000 \mathrm{E}+000.00000 .000010 .0$ $1.0000 \mathrm{E}+000.0000 \quad 0.000010 .0$ $1.0000 E+000.00000 .000010 .0$ $1.0000 \mathrm{E}+00 \quad 0.00000 .000010 .0$ $1.0000 \mathrm{E}+000.00000 .000010 .0$ $1.0000 \mathrm{E}+000.00000 .000010 .0$ $1.0000 \mathrm{E}+000.00000 .000010 .0$ $1.0000 \mathrm{E}+000.00000 .000010 .0$

run terminated when 10000 particle histories were done.

\section{File: Inp240}

1- prob 24 - reflecting lattice. $15 x 15$ at 3.75 w/o $u-235$ enrichment.

2- 1 1 $-10.182-1 u=2$

3. 2 2-.001 $1-2 \mathrm{u}=2$

4. $3 \quad 3-6.552-3 u=2$

5- 4 4-1.03u=2

6- 5 - $4-1.0-14: 15 u=3$

7- $6 \quad 3-6.5514-15 u=3$

8- $7 \quad 4-1.0-4+5-6+7 u=1$ lat $=1$ finl $=-8: 8-8: 80: 0$

9- $\quad$ ] $17 \mathrm{r}_{2} 14 \mathrm{r} 11214 \mathrm{r} 1122322322 \mathrm{r} 322$

10- $\quad 3221126 r 326 r 1123 r 3$

11- $\quad 24+323 r 1122328 \mathrm{r} 322$

12- $\quad 11214 \mathrm{r} 1122 \mathrm{r} 322 \mathrm{r} 322 \mathrm{r}$

13- $\quad 322 r 11214 r_{1122328}$

14. $\quad 221123 r 324532311$

15- $\quad 26 r 326 r 11223223$

$22 \times 32232211214 \mathrm{r} 11214 \mathrm{r} 117 \mathrm{r}$

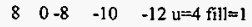

$9 \quad 5-7.98: 10 u=4$ 
19- $10 \quad 4-1.0-8 \quad-10 \quad+12 u=4$

$11 \quad 4-1.0-16+9 \quad u=5$ lat $=1$ fill $=0: 60: 00: 043 r 52 \mathrm{r}$

$120+28+29-19-17+13-18$ fill $=5$

$130+28-19+17-31+13-18$ fill $=5(-11.5230)$

23- $140+28-19+31-32+13-18$ fill $=5(-23460)$

24- $150+28-19+32-33+13-18$ fill $=5(-69690)$

25. $\quad 164-1.0+28-19+33+13-18$

26- $17 \quad 4-1.0+28+29-19-24+18$

27- $18 \quad 6-7.9(+28+29+19-20+23-25):(+28+29-19+23-13)$

28. $:(+28+29-19+24-25)$

29. $197-7.088254305(+28+29+20-21+22-25):(+28+29-20+22-23)$

30- $20 \quad 0-28:-29:+21:-22:+25$

31.

32. $\quad 1$ cz .464693

33- $\quad 2$ cz $\quad .483743$

34- $3 \mathrm{cz} .535940$

35. 4 px $\quad .71501$

36- 5 px -.71501

37- 6 py .71501

38- 7 py -.71501

39. 8 px 11.0

40. $9 \mathrm{px}-11.0$

41. 10 py 11.0

42- $\quad 12$ pz 400.903

43. $13 \mathrm{pz} 34.0$

44- $\quad 14 \quad \mathrm{cz} .652018$

45- $\quad 15 \quad \mathrm{cz} .690118$

46- $16 \quad \mathrm{px} 12.0$

47. 17 py 12.0

$48-\quad 18$ pz 439.0

49. 19 cz 82.25

50. $\quad 20 \mathrm{cz} 83.25$

51. 21 cz 116.35

$22 \mathrm{pz} 0.0$

23 pz 33.0

$24 \mathrm{pz} 447.9$

55. $\quad 25 \mathrm{pz} 485.9$

56. $\quad 28 \mathrm{px} 0.0$

57- $\quad 29$ py 0.0

$58-\quad 31$ py 35.0

$59-\quad 32$ py 58.0

60. 33 py 81.0

61.

62- imp:n 1 18r 0

63- kcode 250.72445000

waming. tallies are normed per fission neutron for one generation.

64. kstc 1.51 .5217 .4515

65- $\quad \mathrm{ml} 92235.40 \mathrm{c} 1.31964 \mathrm{e} 20 \quad 92238.40 \mathrm{c} 2.15905 \mathrm{e} 22$

66- $\mathrm{m} 2 \quad 8016.40 \mathrm{c} 1.00000000$

67. $\mathrm{m} 340000.40 \mathrm{c} 1$.

68- $\quad \mathrm{m} 4 \quad 1001.00 \mathrm{c} .666666667 \quad 8016.40 \mathrm{c} .3333333333$

69- $\mathrm{m} 5 \quad 26000.40 \mathrm{c}-.68874500 \quad 5010 .-.00178200 \quad 5011.40 \mathrm{c}-.00721800$

70- $\mathrm{m} 6 \quad 26000.40 \mathrm{c}-.69500000$

71- $\quad \mathrm{m} 7 \quad 26000.40 \mathrm{c} .830266962 \quad 6012.40 \mathrm{c} .133437328 \quad 14000.40 \mathrm{c} .002370386$

72- $\mathrm{mt} 4 \mathrm{lwtr} .01 \mathrm{t}$

73- dixs

74- prdmp $2 \mathrm{j}-1$

75- $\quad$ f6:n $812131415 \$$ heating in mat $=0$ cells kills menp4.2

76. $\operatorname{sd} 6 \quad 14 \mathrm{r}$

77 -

total fission nubar data are being used.

waming continuous-energy cross-section table used for $1001.00 d$

warning. continuous-energy cross-section table used for $5011.40 \mathrm{~d}$

hing. continuous-energy cross-section table used for $6012.40 \mathrm{~d}$ 
warning. continuous-energy cross-section table used for $26000.40 \mathrm{~d}$

warning. continuous-energy cross-section table used for $40000.40 \mathrm{~d}$

waming. continuous-energy cross-section table used for $92235.40 \mathrm{~d}$

waming. continuous-energy cross-section table used for $92238.40 \mathrm{~d}$

waming. 4 of the materials had unnormalized fractions.

lcells

print table 60

cell mat density density volume mass pieces importance

$1112.57597 \mathrm{E}-02 \quad 1.01820 \mathrm{E} \div 010.00000 \mathrm{E}+000.00000 \mathrm{E}+00$

$222 \quad 3.76497 \mathrm{E}-0.51 .00000 \mathrm{E}-030.00000 \mathrm{E}+000.00000 \mathrm{E}+00$

$333 \quad 4.32392 \mathrm{E}-02 \quad 6.55000 \mathrm{E}+000.00000 \mathrm{E}+000.00000 \mathrm{E}+00$

44 4s $1.00309 \mathrm{E}-011.00000 \mathrm{E}+000.00000 \mathrm{E}+000.00000 \mathrm{E}+00$

55 4s $1.00309 \mathrm{E}-011.00000 \mathrm{E}+000.00000 \mathrm{E}+000.00000 \mathrm{E}+00$

$6 \quad 6 \quad 3 \quad 4.32392 E-026.55000 E+000.00000 E+000.00000 E+00$

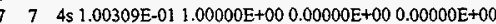

$88 \quad 0 \quad 0.00000 \mathrm{E}+000.00000 \mathrm{E}+000.00000 \mathrm{E}+000.00000 \mathrm{E}+00$

$9958.97738 \mathrm{E}-027.90000 \mathrm{E}+000.00000 \mathrm{E}+000.00000 \mathrm{E}+00$

$10 \quad 10 \quad$ is $1.00309 \mathrm{E}-01 \quad 1.00000 \mathrm{E}+000.00000 \mathrm{E}+000.00000 \mathrm{E}+00$

11 11 4s 1.00309E-01 1.00000E+00 0.00000E+00 0.00000E+00

$12 \quad 12 \quad 0 \quad 0.00000 \mathrm{E}+00 \quad 0.00000 \mathrm{E}+00 \quad 0.00000 \mathrm{E}+000.00000 \mathrm{E}+00$

$13 \quad 13 \quad 0 \quad 0.00000 \mathrm{E}+000.00000 \mathrm{E}+00 \quad 0.00000 \mathrm{E}+000.00000 \mathrm{E}+00$

$14 \quad 1400.00000 \mathrm{E}+00 \quad 0.00000 \mathrm{E}+00 \quad 0.00000 \mathrm{E}+000.00000 \mathrm{E}+00$

is $0 \quad 0.00000 E+000.00000 E+000.00000 E+000.00000 E+00$

I6 4s $1.00309 \mathrm{E}-01 \quad 1.00000 \mathrm{E}+000.00000 \mathrm{E}+000.00000 \mathrm{E}+00$

$174 \mathrm{~s} 1.00309 \mathrm{E}-01 \quad 1.00000 \mathrm{E}+000.00000 \mathrm{E}+000.00000 \mathrm{E}+00$

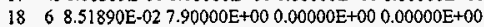

$1978.58502 \mathrm{E}-027.08825 \mathrm{E}+00 \quad 0.00000 \mathrm{E}+000.00000 \mathrm{E}+00$

$20 \quad 0 \quad 0.00000 \mathrm{E}+000.00000 \mathrm{E}+000.00000 \mathrm{E}+000.00000 \mathrm{E}+00$

total

$0.00000 \mathrm{E}+000.00000 \mathrm{E}+00$

Il waming messages so far.

1cross-section tables

print table 100

table length

tables from file testlib 1

$1001.00 \mathrm{c} 21391-\mathrm{h}+1$ from endf-vi. 1

$5010.03 d 3945$ b-10 endf/b-iv new gamma production format

mat $125 \quad 05 / 26 / 93$ ( 1273) 14 oct 75

$5011.40 \mathrm{c} 3473$ ENDL library name: nd900719 MCNP translation: 900831 14:10:05

$6012.40 \mathrm{c} 5049$ ENDL library name: nd900719 MCNP translation: 900831 14:10:05

8016.40c 5693 ENDL library name: nd900719 MCNP translation: 900831 14:10:05

$14000.40 \mathrm{c} 8732$ ENDL library name: nd900719 MCNP translation: 900831 14:10:05

26000.40c 21846 ENDL library name: nd900719 MCNP translation: 900831 14:10:05

$01.0000 \mathrm{E}+00$

$0 \quad 1.0000 \mathrm{E}+00$

$1.0000 \mathrm{E}+00$

$000 \mathrm{E}+00$

$01.0000 E+00$

$01.0000 \mathrm{E}+00$

$1.0000 \mathrm{E}+00$

$0 \quad 1.0000 \mathrm{E}+00$

$1.0000 \mathrm{E}+00$

$01.0000 \mathrm{E}+00$

$1.0000 \mathrm{E}+0.0$

$01.0000 \mathrm{E}+00$

$1.0000 \mathrm{E}+00$

$01.0000 \mathrm{E}+00$

40000.40c 111279 ENDL library name: nd900719 MCNP transiation: 900831 14:10:05

92235.40c 40506 ENDL library name: no900719 MCNP transiation: 9total nu 10:05

92238.40c 48310 ENDL library name: nd901118 MCNP translation: 9total nu 56:49

lwt.01t 10193 hydrogen in light water at 300 degrees kelvin

$10010010 / 22 / 85$

900207

900207

900207

900207

900207

900323

900503

901119

\section{total 261165}

warning. neutron energy cutoff is below some cross-section tables. 
12 warning messages so far.

lestimated keff results by cycle

print table 175

cycle $1 \mathrm{k}$ (collision) 0.658210 renoval lifetime(abs) $5.1517 \mathrm{E}+03$ source points generated 228

cycle $2 \mathrm{k}$ (collision) 0.549318 removal lifetimefabs) $3.7645 \mathrm{E}+03$ source points generated 209

cycle $3 \mathrm{k}$ (collision) 0.551862 removal lifetime(abs) $3.8422 \mathrm{E}+03$ source points generated 253

estimator cycle 4 ave of 2 cycles combination simple average combined average cort

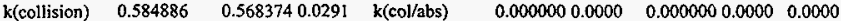

$\begin{array}{lllllllll}\text { k(absorption) } & 0.583788 & 0.5911690 .0125 & \mathrm{k}(\mathrm{abs} / \mathrm{k} \mathrm{k} \text { in) } & 0.000000 & 0.0000 & 0.000000 & 0.0000 & 0.0000\end{array}$

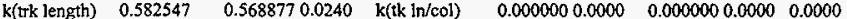

rem life(col) $3.9485 \mathrm{E}+03 \quad 3.8951 \mathrm{E}+030.0137$

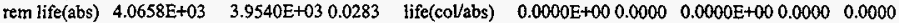

source points generated 264

source distribution written to file inp24s cycle $=4$

1problem summary

run terminated when 4 kcode cycles were done.

neutron creation tracks weight energy neutron loss tracks weight energy (per source particle) (per source particle)

$\begin{array}{llllllll}\text { source } & 940 & 1.0638 \mathrm{E}+00 & 1.9444 \mathrm{E}+00 & \text { escape } & 0 & 0 . & 0 .\end{array}$ energy cutoff $\quad 0 \quad 0$. 0 time cutoff $00 . \quad 0$.

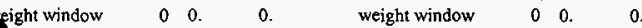

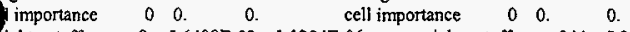
$\begin{array}{lllllllll}\text { sight cutoff } & 0 & 5.6409 \mathrm{E}-02 & 1.1284 \mathrm{E}-06 & \text { weight cutoff } & 944 & 5.2413 \mathrm{E}-02 & 7.1769 \mathrm{E}-07\end{array}$ energy importance $\quad \begin{array}{lllllll}0 & 0 . & 0 . & \text { energy impottance } & 0 & 0 . & 0 .\end{array}$ $\begin{array}{lllllllllll}\text { dxtran } & 0 & 0 . & & 0 . & & \text { dxtran } & 0 & 0 . & 0 . & \\ \text { forced collisions } & 0 & 0 . & & 0 . & \text { forced collisions } & 0 & 0 . & 0 .\end{array}$

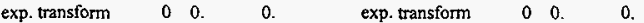
$\begin{array}{llllllll}\text { upscattering } & 0 & 0 . & 1.4710 \mathrm{E}-07 & \text { downscattering } & 0 & 0 . & 1.8098 \mathrm{E}+00\end{array}$

$\begin{array}{llllll} & \text { capture } & 0 & 8.1698 \mathrm{E}-0] & 1.8384 \mathrm{E}-02\end{array}$

$\begin{array}{llllllll}(n, x n) & 8 & 6.9970 E-03 & 3.6428 \mathrm{E}-03 & \text { loss to }(n, x n) & 4 & 3.4985 \mathrm{E}-03 & 2.8025 \mathrm{E}-02\end{array}$

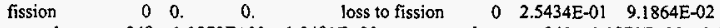

$\begin{array}{llllllllll}\text { total } & 948 & 1.1272 \mathrm{E}+00 & 1.9481 \mathrm{E}+00 & \text { total } & 948 & 1.1272 \mathrm{E}+00 & 1.9481 \mathrm{E}+00\end{array}$

number of neutrons banked 4 average lifetime, shakes cutoffs

neutron tracks per source particle $1.0085 \mathrm{E}+00$ escape $0.0000 \mathrm{E}+00$ tco $1.0000 \mathrm{E}+34$

neutron collisions per source particle $6.1389 \mathrm{E}+01$ capture $4.1766 \mathrm{E}+03$ eco $0.0000 \mathrm{E}+00$

total neutron collisions 57706 capture or escape $4.1766 \mathrm{E}+03$ wcl $-5.0000 \mathrm{E}-01$

net multiplication $\quad 1.0033 \mathrm{E}+000.0030$ any termination $4.4650 \mathrm{E}+03$ wc2 $-2.5000 \mathrm{E}-01$

$\begin{array}{lll}\text { computer time so far in this run } & 0.00 \text { minutes } & \text { maximum number ever in bank } \\ \text { computer time in merm } & 0.00 \text { minutes } & 1\end{array}$

source particles per minute $\quad 0.0000 \mathrm{E}+00$

random numbers generated $\quad 690539$

field length

most random numbers used was 3677 in history

21

range of sampled source weights $=9.8814 \mathrm{E}-01$ to $1.1962 \mathrm{E}+00$

Ineutron activity in each cell

print table 126

tracks population collisions collisions number flux average average

cell entering * weight weighted weighted track weight track mfp (per history) energy energy (relative) (cm)

$\begin{array}{cccccccccccc}1 & 1 & 15187 & 944 & 4054 & 3.5039 \mathrm{E}+00 & 3.6399 \mathrm{E}-04 & 6.5723 \mathrm{E}-01 & 8.4316 \mathrm{E}-01 & 3.8971 \mathrm{E}+00 \\ 2 & 2 & 29440 & 944 & 0 & 0.0000 \mathrm{E}+00 & 3.3819 \mathrm{E}-04 & 6.3029 \mathrm{E}-01 & 8.4006 \mathrm{E}-01 & 9.6437 \mathrm{E}+03 \\ 3 & 3 & 31610 & 944 & 1028 & 9.2538 \mathrm{E}-01 & 3.1626 \mathrm{E}-04 & 6.0475 \mathrm{E}-01 & 8.3961 \mathrm{E}-01 & 3.6368 \mathrm{E}+00\end{array}$




$\begin{array}{cccc}4 & 4 & 44969 & 944 \\ 5 & 5 & 8213 & 845 \\ 6 & 4924 & 791 \\ 7 & 7 & 2832 & 638 \\ 8 & 8 & 0 & 0 \\ 9 & 9 & 1333 & 607 \\ 10 & 10 & 0 & 0 \\ 11 & 11 & 7 & 1 \\ 12 & 12 & 0 & 0 \\ 13 & 13 & 0 & 0 \\ 14 & 14 & 0 & 0 \\ 15 & 15 & 0 & 0 \\ 16 & 16 & 0 & 0 \\ 17 & 17 & 0 & 0 \\ 18 & 18 & 0 & 0 \\ 19 & 19 & 0 & 0\end{array}$

$\begin{array}{llllll}40660 & 3.0973 \mathrm{E}+01 & 3.0008 \mathrm{E}-04 & 5.9877 \mathrm{E}-01 & 8.3882 \mathrm{E}-01 & 1.4858 \mathrm{E}+00\end{array}$

$\begin{array}{llllll}8775 & 6.4600 \mathrm{E}+00 & 2.0190 \mathrm{E}-04 & 5.0976 \mathrm{E}-01 & 8.1000 \mathrm{E}-01 & 1.3256 \mathrm{E}+00\end{array}$

$109 \quad 9.6195 \mathrm{E}-02 \quad 2.0789 \mathrm{E}-04 \quad 5.2658 \mathrm{E}-01 \quad 8.2057 \mathrm{E}-01 \quad 3.6398 \mathrm{E}+00$

$\begin{array}{lllllll}1607 & 1.4612 \mathrm{E}+00 & 8.5166 \mathrm{E}-04 & 7.1930 \mathrm{E}-01 & 9.4163 \mathrm{E}-01 & 1.7232 \mathrm{E}+00\end{array}$

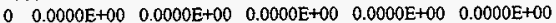

$\begin{array}{lllllll}1347 & 1.2836 \mathrm{E}+00 & 3.8320 \mathrm{E}-03 & 8.0017 \mathrm{E}-01 & 9.7093 \mathrm{E}-01 & 3.1777 \mathrm{E}+00\end{array}$

$\begin{array}{lllllll}0 & 0.0000 \mathrm{E}+00 & 0.0000 \mathrm{E}+00 & 0.0000 \mathrm{E}+00 & 0.0000 \mathrm{E}+00 & 0.0000 \mathrm{E}+00\end{array}$

$\begin{array}{llllll}126 & 9.7846 \mathrm{E}-02 & 9.0288 \mathrm{E}-05 & 3.2866 \mathrm{E}-01 & 7.7027 \mathrm{E}-01 & 1.0087 \mathrm{E}+00\end{array}$

$\begin{array}{lllllll}0 & 0.0000 \mathrm{E}+00 & 0.0000 \mathrm{E}+00 & 0.0000 \mathrm{E}+00 & 0.0000 \mathrm{E}+00 & 0.0000 \mathrm{E}+00\end{array}$

$\begin{array}{lllllllllll}0 & 0.0000 \mathrm{E}+00 & 0.0000 \mathrm{E}+00 & 0.0000 \mathrm{E}+00 & 0.0000 \mathrm{E}+00 & 0.0000 \mathrm{E}+00\end{array}$

$\begin{array}{lllllll}0 & 0.0000 \mathrm{E}+00 & 0.0000 \mathrm{E}+00 & 0.0000 \mathrm{E}+00 & 0.0000 \mathrm{E}+00 & 0.0000 \mathrm{E}+00\end{array}$

$\begin{array}{lllllllllll}0 & 0.0000 E+00 & 0.0000 E+00 & 0.0000 E+00 & 0.0000 E+00 & 0.0000 E+00\end{array}$

$\begin{array}{llllllllllllll}0 & 0.0000 \mathrm{E}+00 & 0.0000 \mathrm{E}+00 & 0.0000 \mathrm{E}+00 & 0.0000 \mathrm{E}+00 & 0.0000 \mathrm{E}+00\end{array}$

$\begin{array}{llllllllllll}0 & 0.0000 \mathrm{E}+00 & 0.0000 \mathrm{E}+00 & 0.0000 \mathrm{E}+00 & 0.0000 \mathrm{E}+00 & 0.0000 \mathrm{E}+00\end{array}$

$\begin{array}{lllllllll}0 & 0.0000 \mathrm{E}+00 & 0.0000 \mathrm{E}+00 & 0.0000 \mathrm{E}+00 & 0.0000 \mathrm{E}+00 & 0.0000 \mathrm{E}+00\end{array}$

$\begin{array}{lllllllll}0 & 0.0000 \mathrm{E}+00 & 0.0000 \mathrm{E}+00 & 0.0000 \mathrm{E}+00 & 0.0000 \mathrm{E}+00 & 0.0000 \mathrm{E}+00\end{array}$

total $\quad 138515 \quad 6658 \quad 57706 \quad 4.4801 E+01$

the initial fission neutron source distribution used the 1 source points that were input on the ksrc card.

the criticality problem was scheduled to skip 2 cycles and run a total of 4 cycles with nominally 250 neutrons per cycle. this problem has run 2 inactive cycles with 478 neutron histories and 2 active cycles with 462 neutron histories.

this calculation has completed the requested number of keff cycles using a total of 940 fission neutron source histories. all cells with fissionable material were sampled and had fission neutron source points.

there is no combined collision/absorption/track-length estimate for keff because only 2 active cycles were run.

the estimated average keffs, one standard deviations, and 68,95 , and 99 percent confidence intervals are:

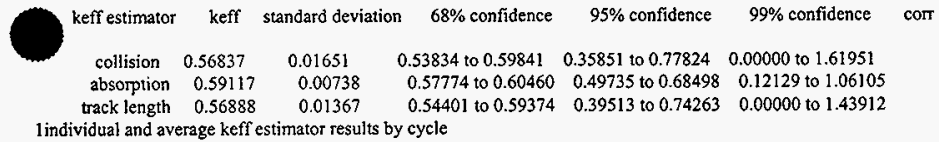

keff neutron keff estimators by cycle average keff estimators and deviations average $k(\mathrm{c} / \mathrm{a} / \mathrm{t})$ cycle histories $k$ (coll) $k$ (abs) $k$ (track) $k$ (coll) st dev $k$ (abs) st dev $k$ (track) st dev $k$ (c/a/t) st dev fom

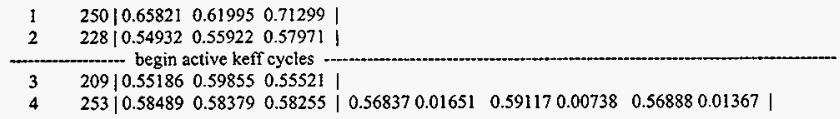

the largest active cycle keffs by estimator are:

collision 0.58489 on cycle 4 dibsorption 0.59855 an cycle 3 maks length 0.58255 on cycle 4

Iraipy if $\mathrm{nps}=940$

tally type 6 track length estimate of heating. units mev/gram

tally for neutrons

number of histories used for normalizing tallies $=\quad 500.00$

masses

cell: $\begin{array}{lllll}8 & 12 & 13 & 14 & 15\end{array}$

$1.00000 \mathrm{E}+00 \quad 1.00000 \mathrm{E}+00 \quad 1.00000 \mathrm{E}+00 \quad 1.00000 \mathrm{E}+00 \quad 1.00000 \mathrm{E}+00$ the smallest active cycle keffs by estimator are:

collision 0.55186 on cycle 3

absorption 0.58379 on cycle 4

track length 0.55521 on cycle 3

cell 8

$4.02566 \mathrm{E}+010.0434$ 
cell 14 .

$9.78180 \mathrm{E}-010.4014$

cell 15

$1.20234 E-010.9710$

lanalysis of the results in the tally fluctuation chart bin (tfe) for tally 6 with nps $=940$ print table 160

normed average tally per history $=4.02566 \mathrm{E}+01$ estimated tally relative error $=0.0434$ relative entor from zero tallies $=0.0128$

number of nonzero history tallies $=\quad 462$

history number of largest tally = $\quad 641$ (largest tally)/(average taily) $=6.75049 \mathrm{E}+00$

(confidence interval shift)/mean $=0.0014$ unnormed average tally per history $=4.02566 \mathrm{E}+01$ estimated variance of the variance $=0.0095$ relative error from nonzero scores $=0.0415$

efficiency for the nonzero tallies $=0.9240$ largest unnormalized history tally $=2.71752 \mathrm{E}+02$ (largest tally)/(avg nonzero tally) $=6.23745 \mathrm{E}+00$

shifted confidence interval center $=4.03113 \mathrm{E}+01$

if the largest history score sampled so far were to occur on the very next history, the tfe bin quantities would change as follows: $n p s=462$ for this table because 2 keff cycles and 478 histories were skipped before tally accumulation.

estimated quantities value at nps value at nps+1 value(nps+1)/value(nps)-1

mean $\quad 4.02566 \mathrm{E}+01 \quad 4.07566 \mathrm{E}+01 \quad 0.012420$

relative error $\quad 4.34431 \mathrm{E}-02 \quad 4.24506 \mathrm{E}-02 \quad-0.022846$

variance of the variance $\quad 9.52674 \mathrm{E}-03 \quad 1.33327 \mathrm{E}-02 \quad 0.399508$

$\begin{array}{llll}\text { shifted center } & 4.03113 \mathrm{E}+01 & 4.03457 \mathrm{E}+01 & 0.000853\end{array}$

figure of merit $\quad 0.00000 \mathrm{E}+00 \quad 0.00000 \mathrm{E}+00 \quad 0.000000$

there is not enough information in the largest history scores (usually less than 500 scores) for a reliable estimate of the slope. the history score probability density function appears to have an unsampled region at the largest history scores: please examine.

***** the nps-dependent tfc bin check results are suspect because there are only 1 nps tally values to analyze *****

results of $\mathrm{I} 0$ statistical checks for the estimated answer for the tally fluctuation chart (tfc) bin of tally 6

tfc bin -mean-- _-..--relative error-_- -variance of the variance-- -figure of merit- pdf-

behavior behavior value decrease decrease rate value decrease decrease rate value behavior slope

$\begin{array}{lcccccccc}\text { desired random } & <0.10 & \text { yes } & 1 / \mathrm{sqrt}(\mathrm{nps}) & <0.10 \text { yes } 1 / \mathrm{nps} & \text { constant random }>3.00 \\ \text { observed random } & 0.04 \text { yes } & 0.01 \text { yes yes yes } & 0.00 & \text { no } \\ \text { passed? yes } & \text { yes yes yes yes yes yes } & & \end{array}$

waming. the tally in the tally fluctuation chart bin did not pass 1 of the 10 statistical checks.

lunnormed tally density for tally $6 \quad$ nonzero tally mean $(m)=4.357 \mathrm{E}+01$ nps $=940$ print table 161

abscissa ordinate log plot of tally probability density function in tally fluctuation chart bin(d=decade, slope $=0.0$ )

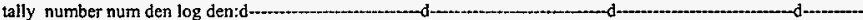

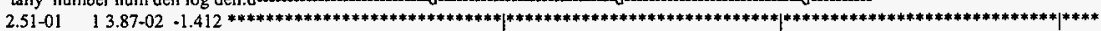

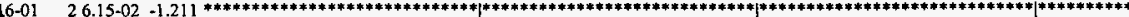

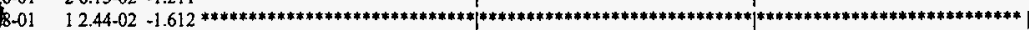


$11.94-02-1.712 * * * * * * * * * * * * * * * * * * * * * * * * * * * \mid * * * * * * * * * * * * * * * * * * * * * * * * * * * *)$ HNF-SD-SNF-CSWD-005, ReV. 0

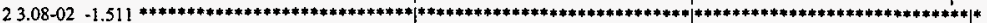

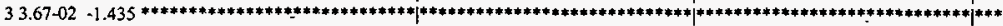
$54.86-02-1.313$ *****************************;|****************************|******************************|******* $1.26+00 \quad 17.72-03-2.112 * * * * * * * * * * * * * * * * * * * * * * * * * * * * * * ; * * * * * * * * * * * * * * * * * * * * * * * * * * * * * * ; * * * * * * * * * * * * * *)$

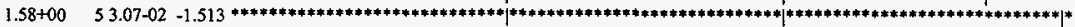

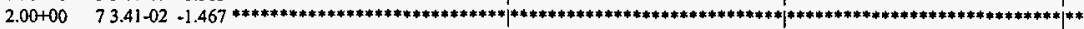
$2.51+00 \quad 62.32-02-1.634 * * * * * * * * * * * * * * * * * * * * * * * * * * * *|* * * * * * * * * * * * * * * * * * * * * * * * * * *| * * * * * * * * * * * * * * * * * * * * * * * * * * * \mid$

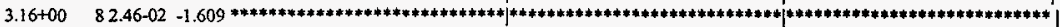
$3.98+00 \quad 112.69-02-1.571 * * * * * * * * * * * * * * * * * * * * * * * * * * * * *|* * * * * * * * * * * * * * * * * * * * * * * * * * * * *| * * * * * * * * * * * * * * * * * * * * * * * * * * * * * \mid$

$5.01+00 \quad 142.72-02-1.566 * * * * * * * * * * * * * * * * * * * * * * * * * * *|* * * * * * * * * * * * * * * * * * * * * * * * * * * * *| * * * * * * * * * * * * * * * * * * * * * * * * * * * * * \mid$

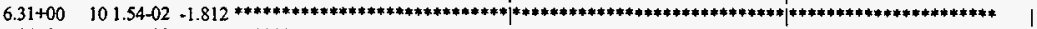

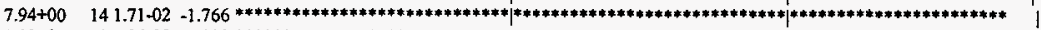

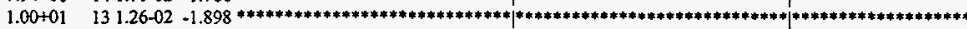

$1.26+01 \quad 161.24-02-1.908 * * * * * * * * * * * * * * * * * * * * * * * * * * * * * * * * * * * * * * * * * * * * * * * * * * * * * * * * \mid * * * * * * * * * * * * * * * * * * * *)$

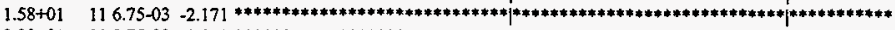

$2.00+0$

$209.75-03-2.011$

$321.24-02-1.907 * 4$

$* * * * * * * * * * * * * * *+* * * * * * * * * * * * * \mid * * *$

$2.51+01$

$3.16+01$

$3.98+01$

41 1.26-02 -1.899

$245.86-03-2.232$

$5.01+01 \quad 489.31-03-2.031$

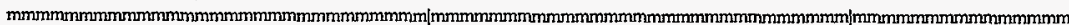

$6.31+01548.32-03 \quad-2.080 * * * * * * * * * * * * * * * * * * * * * * * * * * * * * * \mid * * * * * * * * * * * * * * * * * * * * * * * * * * * * * * * * * * * * * * * * * * * * *$

$7.94+01 \quad 404.90-03-2.310 * * * * * * * * * * * * * * * * * * * * * * * * * * * * *|* * * * * * * * * * * * * * * * * * * * * * * * * * * * *| * * * * * * *$

$1.00+02 \quad 282.72-03 \quad-2.565 * * * * * * * * * * * * * * * * * * * * * * * * * * * *|* * * * * * * * * * * * * * * * * * * * * * * * * * * *|$

$1.26+02$

$201.54-03-2.811$

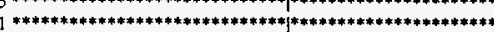

21 1.29-03 $-2.890 * * * * * * * * * * * * * * * * * * * * * * * * * * * * * \mid ; * * * * * * * * * * * * * * * * * * * \pi$

$29.75-05-4.011 * * * * * * * * * * * * * * * *$

$2.00+02$

$2.51+02$

$00.00+00 \quad 0.000$

$3.16+02 \quad 13.08-05-4.512 *$

total $4629.24-01$

d-

I

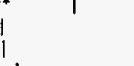

1status of the statistical checks used to form confidence intervals for the mean for each tally bin

result of statistical checks for the tfc bin (the first check not passed is listed) and error magnitude check for all bins

6 missed l of lo tfe bin checks: there is insufficient tfc bin tally information to estimate the large tally slope reliably missed all bin enor check: 5 tally bins had 0 bins with zeros and 2 bins with relative errors exceeding 0.10

the 10 statistical checks are only for the tally fluctuation chart bin and do not apply to other tally bins.

warning. I of the 1 tally fluctuation chart bins did not pass all 10 statistical checks.

warning. I of the I tallies had bins with relative errors greater than recommended.

1 tally fluctuation charts

tally 6

nps mean error vov slope fom

$9404.0257 \mathrm{E}+010.04340 .0095 \quad 0.0$

tally data written to file inp $24 \mathrm{~m}$

15 warning messages so far.

run teminated when 4 kcode cycles were done.

\section{File: Outp24}

1- prob $24-$ reflecting lattice. $15 \times 15$ at 3.75 w/o u-235 enrichment.

2- 1 1-10.182-1 u=2

3. $2 \quad 2 \cdot .0011-2 u=2$

4- $3 \quad 3-6.552-3 u=2$

5- $4 \quad 4-1.03 \mathrm{u}=2$

6- 5 - $4-1.0-14: 15 u=3$

7- $\quad 6 \quad 3+6.5514-15 u=3$

$7 \quad 4-1.0-4+5-6+7 u=1$ lat $=1$ fill $=-8: 8-8: 80: 0$

1 17r214r11214r1122322322r322 


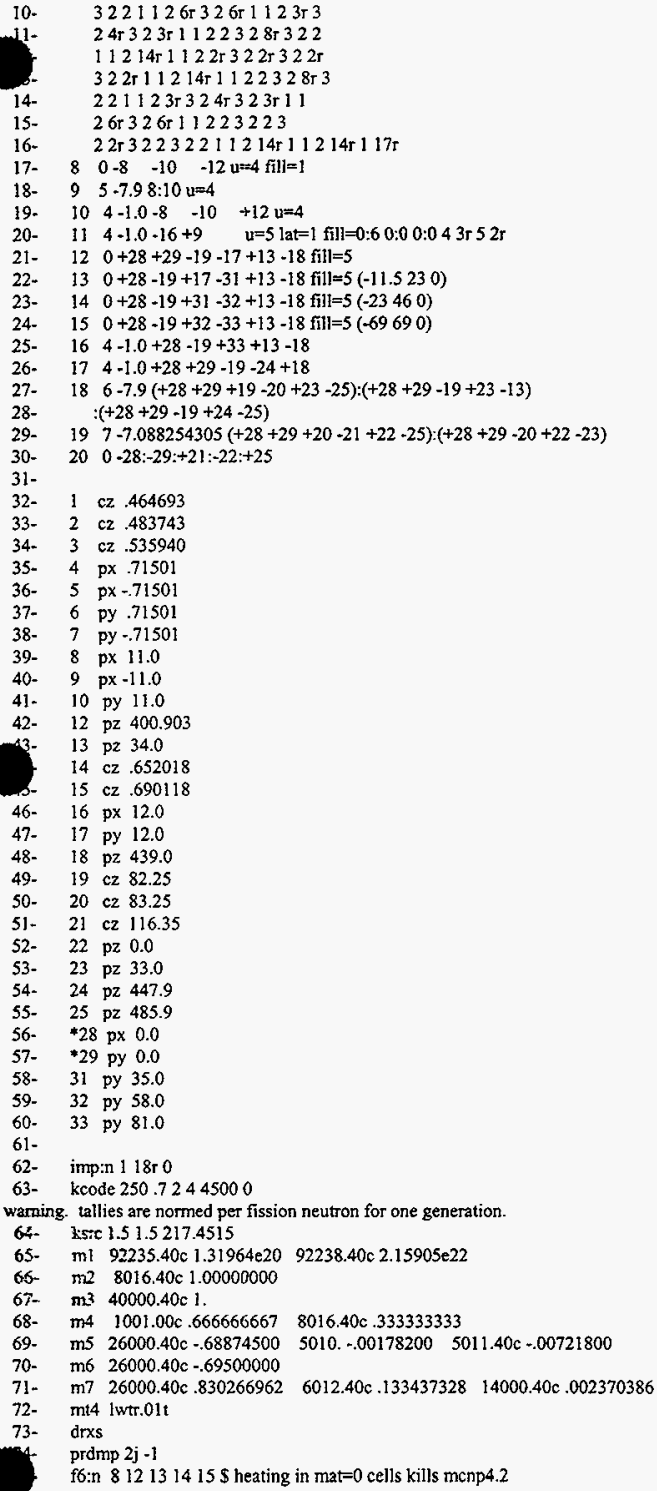


fission nubar data are being used

warning. continuous-energy cross-section table used for $1001.00 \mathrm{~d}$

waming. continuous-energy cross-section table used for $5011.40 \mathrm{~d}$

waming. continuous-energy cross-section table used for $6012.40 \mathrm{~d}$

waming. continuous-energy cross-section table used for $8016.40 \mathrm{~d}$

waming. continuous-energy cross-section table used for $14000.40 \mathrm{~d}$

warning. continuous-energy cross-section table used for $26000.40 \mathrm{~d}$

waming. continuous-energy cross-section table used for $40000.40 d$

waming. continuous-energy cross-section table used for $92235.40 \mathrm{~d}$

waming. continuous-energy cross-section table used for $92238.40 \mathrm{~d}$

waming. 4 of the materials had unnomalized fractions.

icells

print table 60

cell mat density density volume mass pieces importance

$1112.57597 \mathrm{E}-021.01820 \mathrm{E}+010.00000 \mathrm{E}+000.00000 \mathrm{E}+00$

$22 \quad 2 \quad 3.76497 \mathrm{E}-051.00000 \mathrm{E}-030.00000 \mathrm{E}+000.00000 \mathrm{E}+00$

$333 \quad 4.32392 \mathrm{E}-02 \quad 6.55000 \mathrm{E}+00 \quad 0.00000 \mathrm{E}+000.00000 \mathrm{E}+00$

4 4 4s $1.00309 \mathrm{E}-011.00000 \mathrm{E}+000.00000 \mathrm{E}+000.00000 \mathrm{E}+00$

5 5 $4 s \quad 1.00309 \mathrm{E}-01 \quad 1.00000 \mathrm{E}+000.00000 \mathrm{E}+000.00000 \mathrm{E}+00$

$6 \quad 3 \quad 4.32392 \mathrm{E}-02 \quad 6.55000 \mathrm{E}+000.00000 \mathrm{E}+000.00000 \mathrm{E}+00$

7 4s $1.00309 \mathrm{E}-011.00000 \mathrm{E}+000.00000 \mathrm{E}+000.00000 \mathrm{E}+00$

$800.00000 \mathrm{E}+000.00000 \mathrm{E}+000.00000 \mathrm{E}+000.00000 \mathrm{E}+00$

$958.97738 \mathrm{E}-027.90000 \mathrm{E}+000.00000 \mathrm{E}+000.00000 \mathrm{E}+00$

10 I0 4s $1.00309 \mathrm{E}-011.00000 \mathrm{E}+000.00000 \mathrm{E}+000.00000 \mathrm{E} \div 00$

$11 \quad 11$ 4s $1.00309 \mathrm{E}-011.00000 \mathrm{E}+00 \quad 0.00000 \mathrm{E}+000.00000 \mathrm{E}+00$

$12 \quad 12 \quad 0 \quad 0.00000 \mathrm{E}+000.00000 \mathrm{E}+000.00000 \mathrm{E}+000.00000 \mathrm{E}+00$

$13 \quad 13 \quad 0 \quad 0.00000 \mathrm{E}+000.00000 \mathrm{E}+000.00000 \mathrm{E}+000.00000 \mathrm{E}+00$

$14 \quad 1400.00000 \mathrm{E}+00 \quad 0.00000 \mathrm{E}+00 \quad 0.00000 \mathrm{E}+00 \quad 0.00000 \mathrm{E}+00$

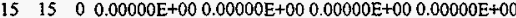

$16 \quad 16 \quad 451.00309 \mathrm{E}-01 \quad 1.00000 \mathrm{E}+000.00000 \mathrm{E}+000.00000 \mathrm{E}+00$

$17 \quad 17$ 4s $1.00309 \mathrm{E}-011.00000 \mathrm{E}+000.00000 \mathrm{E}+000.00000 \mathrm{E}+00$

$18 \quad 18 \quad 6 \quad 8.51890 \mathrm{E}-02 \quad 7.90000 \mathrm{E}+000.00000 \mathrm{E}+000.00000 \mathrm{E}+00$

$191978.58502 \mathrm{E}-027.08825 \mathrm{E}+000.00000 \mathrm{E}+000.00000 \mathrm{E}+00$

$2020 \quad 0 \quad 0.00000 \mathrm{E}+000.00000 \mathrm{E}+00 \quad 0.00000 \mathrm{E}+00 \quad 0.00000 \mathrm{E}+00$

$01.0000 \mathrm{E}+00$

$01.0000 \mathrm{E}+00$

$0 \quad 1.0000 \mathrm{E}+00$

$0 \quad 1.0000 \mathrm{E}+00$

$01.0000 \mathrm{E}+00$

$01.0000 \mathrm{E}+00$

$0 \quad 1.0000 \mathrm{E}+00$

$01.0000 \mathrm{E}+00$

$01.0000 \mathrm{E}+00$

$01.0000 E+00$

$0 \quad 1.0000 \mathrm{E}+00$

$0 \quad 1.0000 \mathrm{E}+00$

$01.0000 \mathrm{E}+00$

$01.0000 \mathrm{E}+00$

$01.0000 \mathrm{E}+00$

$01.0000 \mathrm{E}+00$

$0 \quad 1.0000 \mathrm{E}+00$

$0 \quad 1.0000 \mathrm{E}+00$

$01.0000 \mathrm{E}+00$

$00.0000 \mathrm{E}+00$

total $\quad 0.00000 \mathrm{E}+000.00000 \mathrm{E}+00$

11 waming messages so far.

1 cross-section tables

print table 100

table length

tables from file testlibl

$1001.00 \mathrm{c} 2139 \mathrm{l}$-h-1 from endf-vi.1

mat $125 \quad 05 / 26 / 93$

$5010.03 d 3945$ b-10 endf 3 -iv new gamma production format ( 1273 ) $\quad 44$ oct 75

$5011.40 \mathrm{c} 3473$ ENDL library name: nd900719 MCNP tanslation: 900831 14:10:05

6012.40c 5049 ENDL library name: nd900719 MCNP translation: 900831 14:10:05

900207

$8016.40 \mathrm{c} 5693$ ENDL library name: nd900719 MCNP translation: 900831 14:10:05

900207

$14000.40 \mathrm{c} 8732$ ENDL library name: nd900719 MCNP translation: 900831 14:10:0

900207

26000.40c 21846 ENDL library name: nd900719 MCNP translation: 900831 14:10:05 
92238.40c 48310 ENDL library name: nd901118 MCNP translation: 9total nu 56:49

901119

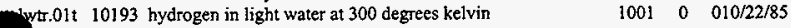

261165

waming. neutron energy cutoff is below some cross-section tables.

decimal words of dynamically allocated storage

source distribution written to file inp24s cycle $=0$

12 warning messages so far.

1estimated keff results by cycle

print table 175

cycle $1 \mathrm{k}$ (collision) 0.658210 removal lifetime(abs) $5.1517 \mathrm{E}+03$ source points generated 228

cycle $2 \mathrm{k}$ (collision) 0.549318 removal lifetime(abs) $3.7645 \mathrm{E}+03$ source points generated 209

cycle $3 \mathrm{k}$ (collision) 0.551862 removal lifetime(abs) $3.8422 \mathrm{E}+03$ source points generated 253

source distribution written to file inp $24 \mathrm{~s} \quad$ cycle $=3$

estimator cycle 4 ave of 2 cycles combination simple average combined average corr

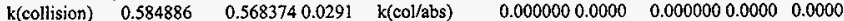

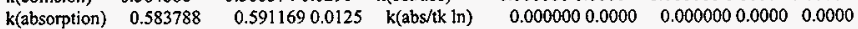

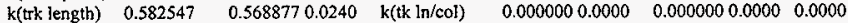

rem life(col) $3.9485 \mathrm{E}+03 \quad 3.8951 \mathrm{E}+030.0137$

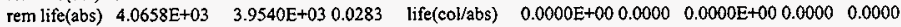

source points generated 264

rce distribution written to file inp $24 \mathrm{~s} \quad$ cycle $=4$

problem sumnary

0

run terminated when 4 kcode cycles were done.

neutron creation tracks weight energy neutron loss tracks weight energy (per source particle) (per source particle)

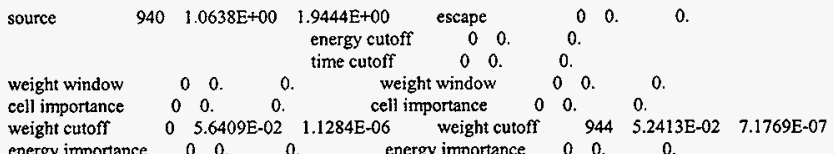

energy importance $\begin{array}{llllllll}0 & 0 & 0 . & \text { energy importance } & 0 & 0 & 0\end{array}$

$\begin{array}{lllllllllll}\text { dxtran } & 0 & 0 . & 0 . & & \text { dxtran } & 0 & 0 . & 0 . & \\ \text { forced collisions } & 0 & 0 . & & 0 . & \text { forced collisions } & 0 & 0 . & 0 .\end{array}$

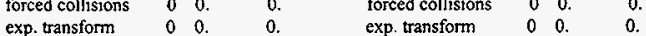

$\begin{array}{llllllll}\text { upscattering } & 0 & 0 & 1.4710 \mathrm{E}-07 & \text { downscattering } & 0 & 0 . & 1.8098 \mathrm{E}+00\end{array}$

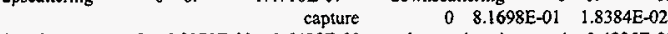

$\begin{array}{llllllll}(n, x n) & 8 & 6.9970 \mathrm{E}-03 & 3.6428 \mathrm{E}-03 & \text { loss to }(n, x n) & 4 & 3.4985 \mathrm{E}-03 & 2.8025 \mathrm{E}-02\end{array}$

fission $\quad 0 \quad 0.00$ loss to fission 0 . 0 . $0434 \mathrm{E}-01 \quad 9.1864 \mathrm{E}-02$

$\begin{array}{llllllllll}\text { total } & 948 & 1.1272 \mathrm{E}+00 & 1.9481 \mathrm{E}+00 \quad \text { total } & 948 & 1.1272 \mathrm{E}+00 & 1.9481 \mathrm{E}+00\end{array}$

number of neutrons banked 4 average lifetime, shakes cutofts

neutron tracks per source particle $1.0085 \mathrm{E}+00$ escape $0.0000 \mathrm{E}+00$ tco $1.0000 \mathrm{E}+34$

neutron collisions per source particle $6.1389 \mathrm{E}+01$ capture $4.1766 \mathrm{E}+03$ eco $0.0000 \mathrm{E}+00$

total neutron collisions $\quad 57706$ capture or escape $4.1766 \mathrm{E}+03$ wcl $-5.0000 \mathrm{E}-0 \mathrm{I}$

net multiplication $\quad 1.0033 \mathrm{E}+000.0030$ any termination $4.4650 \mathrm{E}+03$ wc2 $-2.5000 \mathrm{E}-01$

computer time so far in this run 0.00 minutes maximum number ever in bank

mputer time in merun $\quad 0.00$ minutes bank overflows to backup file 0

Tce particles per minute $\quad 0.0000 \mathrm{E}+00$

field length 0 
tracks population collisions collisions number flux average average cell entering

- weight weighted weighted track weight track mfp (per history) energy cnergy (relative) (cm)

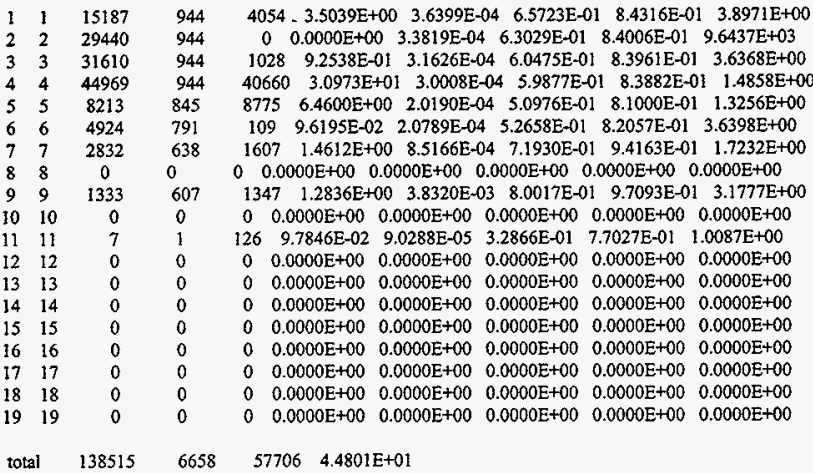

the initial fission neutron source distribution used the 1 source points that were input on the ksrc card.

the criticality problem was scheduled to skip 2 cycles and run a total of 4 cycles with nominally 250 neutrons per cycle. is problem has run 2 inactive cycles with 478 neutron histories and 2 active cycles with 462 neutron histories.

this calculation has completed the requested number of keff cycles using a total of 940 fission neutron source histories. all cells with fissionable material were sampled and had fission neutron source points.

there is no combined collision/absorption/track-length estimate for keff because only 2 active cycles were run.

the estimated average keffs, one standard deviations, and 68,95 , and 99 percent confidence intervals are:

keff estimator keff standard deviation $68 \%$ confidence $95 \%$ confidence $99 \%$ confidence corr

$\begin{array}{ccccccc}\text { collision } & 0.56837 & 0.01651 & 0.53834 \text { to } 0.59841 & 0.35851 \text { to } 0.77824 & 0.00000 \text { to } 1.6195 ! \\ \text { absorption } & 0.59117 & 0.00738 & 0.57774 \text { to } 0.60460 & 0.49735 \text { to } 0.68498 & 0.12129 \text { to } 1.06105 \\ \text { track length } & 0.56888 & 0.01367 & 0.54401 \text { to } 0.59374 & 0.39513 \text { to } 0.74263 & 0.00000 \text { to } 1.43912\end{array}$

Iindividual and average keff estimator results by cycle

keff neutron keff estimators by cycle average keffestimators and deviations average $k$ (c/a/t) cycle histories $k$ (coll) $k$ (abs) $k$ (track) $k$ (coll) st dev $k$ (abs) st dev $k(t r a c k) s t d e v \quad k(c / a / t) s t d e v \quad$ fom

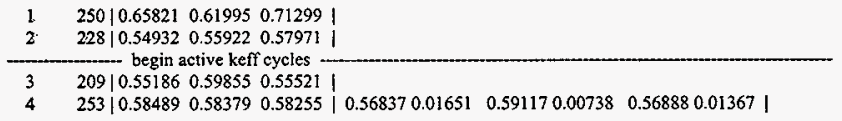

the largest active cycle keffs by estimator are:

collision 0.58489 on cycle 4 absorption 0.59855 on cycle 3 track length 0.58255 on cycle 4 the smallest active cycle keffs by estimator are

collision 0.55186 on cycle 3 absorption 0.58379 on cycle 4 track length 0.55521 on cycle 3 
itally 6 nps $=940$

tally type 6 track length estimate of heating. units mev/gram

tally for neutrons

mber of histories used for normalizing tallies $=\quad 500.00$

masses

cell: $\quad \begin{array}{lllll}8 & 12 & 13 & 14 & 15\end{array}$

$1.00000 \mathrm{E}+00 \quad 1.00000 \mathrm{E}+00 \quad 1.00000 \mathrm{E}+00 \quad 1.00000 \mathrm{E}+00 \quad 1.00000 \mathrm{E}+00$

cell 8

4.02566E+01 0.0434

cell 12

\author{
$2.65224 E+010.0629$
}

cell 13

$1.32494 E+010.0904$

cell 14

9.78180E-01 0.4014

cell 15

$1.20234 \mathrm{E}-010.9710$

Lanalysis of the results in the tally fluctuation chart bin (tfc) for tally 6 with nps $=940$ print table 160

normed average tally per history $=4.02566 \mathrm{E}+01$ unnormed average tally per history $=4.02566 \mathrm{E}+01$

estimated tally relative error $=0.0434$ estimated variance of the variance $=0.0095$

relative error from zero tallies $=0.0128 \quad$ relative error from nonzero scores $=0.0415$

number of nonzero history tallies $=\quad 462$ efficiency for the nonzero tallies $=0.9240$

history number of largest tally $=641$

largest unnormalized history tally $=2.71752 \mathrm{E}+02$

(largest tally)/(average tally) $=6.75049 \mathrm{E}+00$

(largest tally)/(avg nonzero tally) $=6.23745 \mathrm{E}+00$

nfidence interval shift)/mean $=0.0014$

shifted confidence interval center $=4.03113 \mathrm{E}+01$

if the largest history score sampled so far were to occur on the very next history, the tfc bin quantities would change as follows: nps $=462$ for this table because 2 keff cycles and 478 histories were skipped before tally accumulation.

$\begin{array}{lccc}\text { estimated quantities } & \text { value at nps } & \text { value at nps+1 } & \text { value(nps+1)/value(nps)-1. } \\ \text { mean } & & & \\ \text { relative error } & 4.02566 \mathrm{E}+01 & 4.07566 \mathrm{E}+01 & 0.012420 \\ \text { variance of the variance } & 4.34431 \mathrm{E}-02 & 4.24506 \mathrm{E}-02 & -0.022846 \\ \text { shifted center } & 9.52674 \mathrm{E}-03 & 1.33327 \mathrm{E}-02 & 0.399508 \\ \text { figure of merit } & 4.03113 \mathrm{E}+01 & 4.03457 \mathrm{E}+01 & 0.000853 \\ & 0.00000 \mathrm{E}+00 & 0.00000 \mathrm{E}+00 & 0.000000\end{array}$

there is not enough information in the largest history scores (usually less than 500 scores) for a reliable estimate of the slope. the history score probability density function appears to have an unsampled region at the largest history scores: please examine.

***** the nps-dependent tfc bin check results are suspect because there are only 1 nps tally values to analyze *****

results of 10 statistical checks for the estimated answer for the tally fluctuation chart (tfc) bin of tally 6

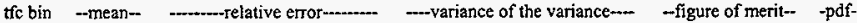

behavior behavior value decrease decrease rate value decrease decrease rate value behavior slope

$\begin{array}{ccccccccc}\text { desired random } & <0.10 & \text { yes } & 1 / \text { sqrt(nps) } & <0.10 \text { yes } 1 / n p s & \text { constant random } & >3.00 \\ \text { served random } & 0.04 \text { yes } & \text { yes } & 0.01 \text { yes yes } & \text { yes } & 0.00 & \text { no }\end{array}$


aming. the tally in the tally fluctuation chart bin did not pass 1 of the 10 statistical checks.

lunnormed tally density for tally $6 \quad$ nonzero tally $\operatorname{mean}(\mathrm{m})=4.357 \mathrm{E}+01 \mathrm{nps}=940$ print table 161

abscissa ordinate $\log$ plot of tally probability density function in tally fluctuation chart bin $(d=$ decade,slope $=0.0)$

tally number num den $\log$ den:d--._-

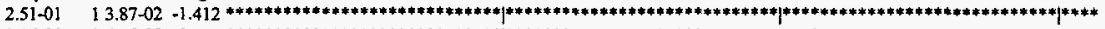

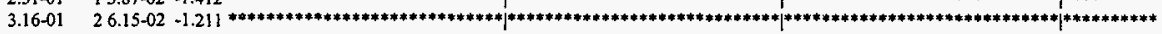

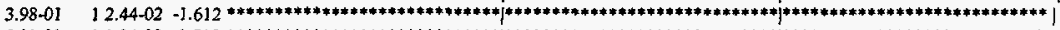

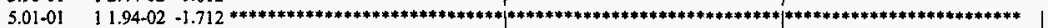

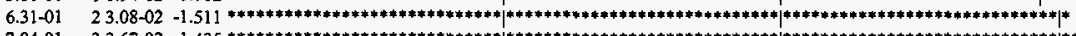

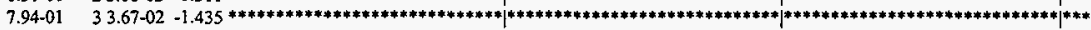

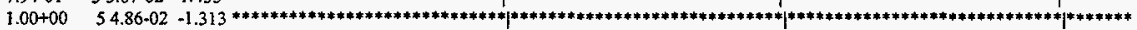

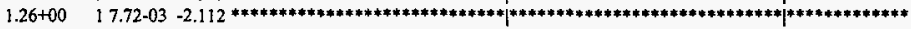

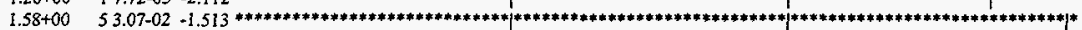

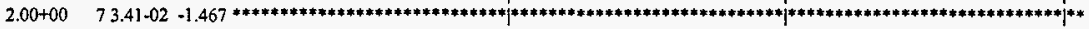

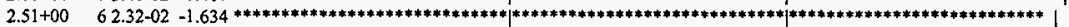

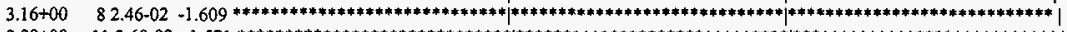

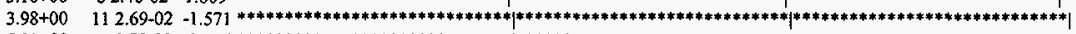

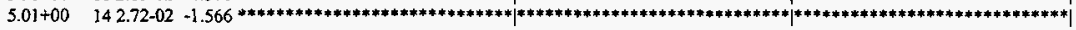

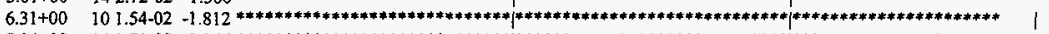

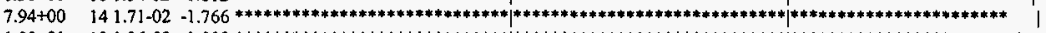

$1.00+01 \quad 131.26-02-1.898 * * * * * * * * * * * * * * * * * * * * * * * * * * * * *|* * * * * * * * * * * * * * * * * * * * * * * * * * * *| * * * * * * * * * * * * * * * * * * *$

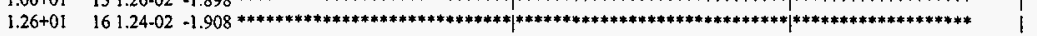

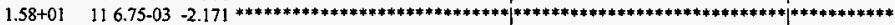

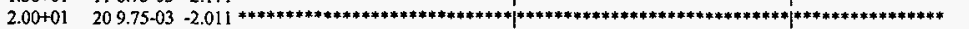

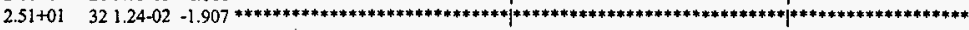

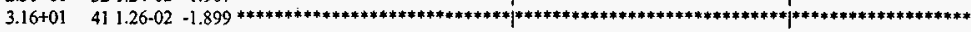

$3.98+01 \quad 245.86-03-2.232 * * * * * * * * * * * * * * * * * * * * * * * * * * * * * j * * * * * * * * * * * * * * * * * * * * * * * * * * * * * j * * * * * * * * *$

$5.01+01 \quad 489.31+03-2.031$

nnmmmmmmmmmmmmmmmmmmmmmmmmminmmmmmmmmmmmmmmmmmmmmmmmmmmm|mmmmmmmmmmmmm

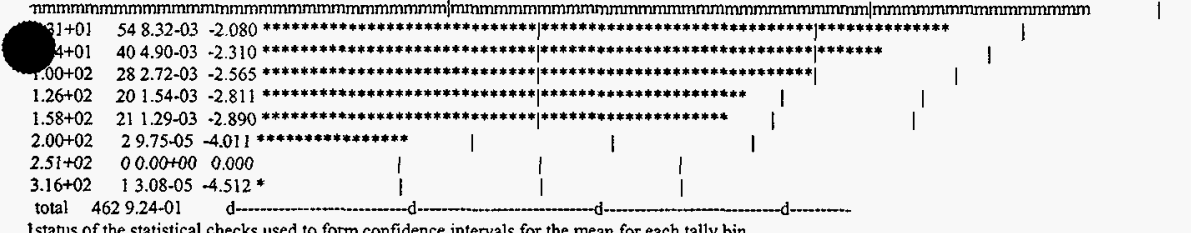

istatus of the statistical checks used to form confidence intervals for the mean for each tally bin

tally result of statistical checks for the tfe bin (the first check not passed is listed) and error magnitude check for all bins

6 missed 1 of 10 tfe bin checks: there is insufficient tfe bin tally information to estimate the large tally slope reliably missed all bin error check: 5 tally bins had 0 bins with zeros and 2 bins with relative errors exceeding 0.10

the 10 statistical checks are only for the tally fluctuation chart bin and do not apply to other tally bins.

warning. I f the 1 tally fluctuation chart bins did not pass all 10 statistical checks. waming. 1 of the 1 tallies had bins with relative errors greater than recommended. Itally fluctuation charts

tally 6

nps mean error vov slope fom

9404.0257 E +010.04340 .00950 .0$

tally data written to file inp $24 \mathrm{~m}$

15 waming messages so far.

terminated when 4 kcode cycles were done. 


\section{Computer System ID : 0012358}

\section{Tile: Inp180}

1. probl 8 - kcode in a hexagonal prism lattice.

2- c three half control rods and five whole control rods.

3. $30 \quad 0-905-19291$ fill $=1$

4- $31 \quad 0-906-19291$ fill $=1(16.711300)$

5. $37 \quad 0-907-19291$ fill $=1(-16.711300)$

6- $34 \quad 0-913-1929$ fill $=1(0.11 .91850)$

$7-\quad 32 \quad 0-914-1929$ fill $=1(10.32175 .95920)$

8. $33 \quad 0-915-1929$ fill $=1(8.355714 .47240)$

9. $35 \quad 0-916-1929$ fill $=1 \quad(-8.3557 \quad 14.47240)$

10. $36 \quad 0-917-1929$ fill $=1(-10.32175 .95920)$

11- c universe 1: structure of control rod.

12- $\quad 38 \quad 11-2.02 \quad-880 u=1 \quad S$ control rod core

13. $39 \quad 6 \quad-8.4 \quad 880-881$ u=1 $\$$ control rod cladding

14. $40 \quad 12 \quad-1.00 \quad 881-882 u=1 \quad \$$ control rod gap

15- $41 \quad 6 \quad-8.4 \quad 882 \quad u=1 \quad \$$ control rod sheath

16- c the space between the control rods, filled with lattice.

17- $\quad 140 \quad 0 \quad-17129-19905906907913914915916917$ fill $=2$

18- $c$ universe 2: lattice of fuel rods with water in between.

19- $42 \quad 12-1.00 \quad-301302-303 \quad 304-305306 \quad u=2 \quad$ lat $=2$ fill= $-37: 27-1: 330: 0$ \&

$24 \mathrm{r} 39 \mathrm{r} 24 \mathrm{r} 311 \mathrm{r} 24 \mathrm{r} 311 \mathrm{r} 24 \mathrm{r} 39 \mathrm{r} 2$

$24 \mathrm{r} 3$ 9r $23 \mathrm{r} 312 \mathrm{r} 23 \mathrm{r} 312 \mathrm{r} 23 \mathrm{r} 39 \mathrm{~T} 2 \mathrm{lr}$

23 r 310 r 2 2r3 13 r 22 r 313 r 2 2r 310 r $2 \mathrm{IT}$

$23 \mathrm{r} 357 \mathrm{r} 22 \pi \&$

2 2r $358 \mathrm{r} 22 \mathrm{r}$

$22 r 316 r 22 r 317 r 22 r 316 r 23 r$

2 2r3 $15 \mathrm{r} 23 \mathrm{r} 316 \mathrm{r} 23 \mathrm{r} 315 \mathrm{r} 24 \mathrm{r}$

2 ir $315 \mathrm{r} 24 \mathrm{r} 315 \mathrm{r} 24 \mathrm{r} 315 \mathrm{r} 24 \mathrm{r}$

$2 \operatorname{lr} 315 r 23 r 316 r 23 r 315 r 25 r$

2 1r $315 r 22 r 317 r 22 r 315 r 26 r$

$21,354 \mathrm{r} 27 \mathrm{r} \&$

c can code remember \& thru comment? $2355+27 \mathrm{r}$

$2325 \mathrm{r} 22 \mathrm{r} 325 \mathrm{rr} 2 \mathrm{r}$

$2324 \mathrm{r} 23 \mathrm{r} 324 \mathrm{r} 29 \mathrm{r}$

$2323+24 r 323+210 r$

$2315 \mathrm{r} 2$ 2r $34 \mathrm{r} 23 \mathrm{r} 34 \mathrm{r} 22 \mathrm{r} 315 \mathrm{r} 211 \mathrm{r}$

$2314 \mathrm{r} 23 \mathrm{r} 3$ 4r 2 2r $34 \mathrm{r} 23 \mathrm{r} 314 \mathrm{r} 2$ 12r

$2313 \mathrm{r} 24 \mathrm{r} 31 \mathrm{lr} 24 \mathrm{r} 313 \mathrm{r} 213 \mathrm{r}$

$2313 r 23 r 312 r 23 r 313 r 214 r$

2313 r 22 r $313 r 22 r 313 r 215 r$

$2346 \mathrm{r} 216 \mathrm{r}$

$2345 \mathrm{r} 217 \mathrm{r}$

$2344 \mathrm{r} 218 \mathrm{r}$

$21+341+220 \mathrm{r}$

$2 \operatorname{lr} 340 \mathrm{r} 221 \mathrm{r}$

2 ir $339 \mathrm{r} 222 \mathrm{r}$

2 2r $336 r 224 \mathrm{r}$

2 2r $335 \mathrm{r} 225 \mathrm{r}$

$23 \mathrm{r} 332 \mathrm{r} 227 \mathrm{r}$

$24 \mathrm{r} 329 \mathrm{r} 229 \mathrm{r}$

$25 r 326 r 231 \mathrm{r}$

$26 r 323 r 233 r$

$28 \mathrm{r} 318 \mathrm{r} 236 \mathrm{r}$

211 r $311+240 \tau$

\section{$264 \mathrm{r}$}

c universe 3: structure of fuel rod lattice elements.

$154 \quad 2-13.75 \quad-58 \quad u=3 \quad \$$ fuel element

$\begin{array}{lllll}149 & 12 & -1.00 & 58+268 \quad u=3 & \$\end{array}$ gap

$144 \quad 7-19.66 \quad 268-478 \quad u=3$ Sliner

$\begin{array}{llll}159 & 6 & -8.4 & 478-698 \quad u=3 \\ \text { S cladding }\end{array}$

$141 \quad 12-1.00 \quad 698 \quad u=3 \quad \$$ water between the fuel rods 
* 1 py $0 \quad \$ x-z$ plane, reflective

17 cz 29.135

67. $19 \mathrm{pz} 31.75 \quad \$$ top of reactor

$68-29 \mathrm{pz}-31.75 \quad \$$ bottom of reactor

$\begin{array}{llllll}69- & 58 & \mathrm{c} / \mathrm{z} & 3.4414 & .8515 & .3240\end{array}$

$\begin{array}{llllll}70- & 268 & \mathrm{c} / \mathrm{z} & 3.4414 & .8515 & .3345\end{array}$

$\begin{array}{llllll}71- & 478 & \mathrm{c} / \mathrm{z} & 3.4414 & .8515 & .3475\end{array}$

$\begin{array}{llllll}72- & 698 & \mathrm{c} / \mathrm{z} & 3.4414 & .8515 & .4318\end{array}$

73- $880 \mathrm{cz} \quad 1.7251$

74- $\quad 881 \quad \mathrm{cz} \quad 1.8051$

75- $\quad 882$ cz 1.9051

76- $905 \mathrm{cz} 2.1055$

77- $\quad 906 \mathrm{c} / \mathrm{z} \quad 16.711302 .1055$

78- $\quad 907 \mathrm{c} / \mathrm{z}-16.711302 .1055$

79- $\quad 913 \mathrm{c} / z \quad 011.91852 .1055$

80- $\quad 914 \mathrm{c} / \mathrm{z} \quad 10.32175 .95922 .1055$

81- $\quad 915 \mathrm{c} / \mathrm{z} \quad 8.355714 .47242 .1055$

82- $\quad 916 \quad \mathrm{c} / 2-8.3557 \quad 14.47242 .1055$

83- $\quad 917 \quad c / 2 r-10.32175 .95922 .1055$

84- $\quad 301$ px 3.9330

85- $\quad 302$ px 2.9498

86- $\quad 303$ p 11.73205080760 \begin{tabular}{l}
5.8994 \\
\hline
\end{tabular}

87- $\quad 304$ p 111.732050807603 .9330

88- $\quad 305$ p $-11.73205080760-.9834$

89- $306 \quad p-11.73205080760-2.9498$

90 -

91- imp:n 1 18 0

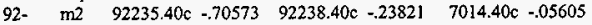

93- $\mathrm{m} 6 \quad 41093.40 \mathrm{c}-.99000 \quad 40000.40 \mathrm{c}+.01000$

94- $\mathrm{m} 7 \quad 74000.40 \mathrm{c}-.74000$

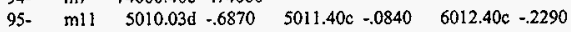

$\mathrm{m} 12 \quad 1001.00 \mathrm{c} / 1002.55 \mathrm{c} 18016.40 \mathrm{cl}$

$\mathrm{mt} 12$ hwtr.01 lwtr.01

kcode 200 i 56

warning. tallies are normed per fission neutron for one generation.

99- ksrc $3.2 .2 .23 .2-3.2 .2 .23 .243 .2-43.243 .1-43.2$

100- e .01.11.10.

101- $\mathrm{fq} \mathrm{fe}$

102- fo4 fuel rod flux in $5 y$ locations averaged over $5 x$ elements

103. f4:n $(154<(42[-10 ;-6-10]))$ S average $5 x$ e]ements at $j=-1$

104- $\quad(154<(42[-10:-630])) \&$ s average $5 x$ elements at $j=3$

105- $\quad(154<(42[-10:-6100])\}$ average $5 \times$ elements at $j=10$ \&

106- $\quad(154<(42[-10:-621$ 0]) $\$$ average $5 \times$ elements at $j=21$

107- $\quad(154<(42[-10:-6290]))$ S average $5 \times$ elements at $j=29$

108- sd4 $104.70890624 \mathrm{r} \quad \$ 5$ times the volume of cell 154

109. print -98

110. prdmp $2 \mathrm{j}-1$

111- ptrac buffer $=20$ file $=a$ sc write $=a l l$ event $=$ bnk

1 initial source from ksre card.

print table 90

$\begin{array}{lll}\begin{array}{lll}\text { original number of points } \\ \text { points not in any cell }\end{array} & & \\ \text { points in cells of zero importance } & & 0 \\ \text { points in void cells } & 0 & \\ \text { points in ambiguous cells } & 0 \\ \text { total points rejected } & 0 \\ \text { points remaining } & 8 \\ \text { points after expansion or contraction } & \end{array}$

nominal source size

200

initial guess for $\mathrm{k}(\mathrm{eff}$ )

1.000000

cycles to skip before tallying

5

hber of keff cycles that can be stored

200

print table 90 
total fission nubar data are being used.

jtally 4

print table 30

fuel rod flux in $5 y$ locations averaged over $5 x$ elements

tally type 4 track length estimate of particle flux.

tally for neutrons

order of printing: $\mathrm{fe}$

cells $(154<(42[-10:-6-10]))(154<(42[-10:-630]))(154<(42[-10:-6100]))(154<(42[-10 ;-6210]))(154<(42[-10:-6$

$2907)$ )

energy bins

$0.00000 \mathrm{E}+00$ to $1.00000 \mathrm{E}-02 \mathrm{mev}$

$1.00000 \mathrm{E}-02$ to $1.00000 \mathrm{E}-01 \mathrm{mev}$

$1.00000 \mathrm{E}-01$ to $1.00000 \mathrm{E}+00 \mathrm{mev}$

$1.00000 \mathrm{E}+00$ to $1.00000 \mathrm{E}+01 \mathrm{mev}$

total bin

Imaterial composition

print table 40

the sum of the fractions of material 2 was $9.999900 \mathrm{E}-01$

the sum of the fractions of material 7 was $7.400000 \mathrm{E}-01$

the sum of the fractions of material 12 was $3.000000 \mathrm{E}+00$

material

number component nuclide, atom fraction

$292235,0.37504 \quad 92238,0.12499 \quad 7014,0.49997$

$6 \quad 41093,0.98982 \quad 40000,0.01018$

$7 \quad 74000,1.00000$

$11 \quad 5010,0.71977 \quad 5011,0.08004 \quad 6012,0.20019$

$12 \quad 1001,0.33333 \quad 1002,0.33333 \quad 8016,0.33333$

associated thermal $\mathrm{s}(\mathrm{a}, \mathrm{b})$ data sets: hwtr.01t lwtr.01t

material

number component nuclide, mass fraction

$\begin{array}{rrrr}2 & 92235,0.70574 & 92238,0.23821 & 7014,0.05605 \\ 6 & 41093,0.99000 & 40000,0.01000 & \\ 7 & 74000,1.00000 & & \\ 11 & 5010,0.68700 & 5011,0.08400 & 6012,0.22900 \\ 12 & 1001,0.05300 & 1002,0.10591 & 8016,0.84109\end{array}$

waming. 3 of the materials had unnormalized fractions.

1 cell volumes and masses

print table 50

cell atom gram input calculated reason volume density density volume volume mass pieces not calculated

$130 \quad 0.00000 \mathrm{E}+00 \quad 0.00000 \mathrm{E}+00 \quad 0.00000 \mathrm{E}+00 \quad 0.00000 \mathrm{E}+00 \quad 0.00000 \mathrm{E}+00$ 2 31 $0.00000 \mathrm{E}+00 \quad 0.00000 \mathrm{E}+00 \quad 0.00000 \mathrm{E}+00 \quad 0.00000 \mathrm{E}+00 \quad 0.00000 \mathrm{E}+00$

$\begin{array}{llllllll}3 & 37 & 0.00000 \mathrm{E}+00 & 0.00000 \mathrm{E}+00 & 0.00000 \mathrm{E}+00 & 0.00000 \mathrm{E}+00 & 0.00000 \mathrm{E}+00\end{array}$

$\begin{array}{llllllll}4 & 34 & 0.00000 \mathrm{E}+00 & 0.00000 \mathrm{E}+00 & 0.00000 \mathrm{E}+00 & 8.84370 \mathrm{E}+02 & 0.00000 \mathrm{E}+00\end{array}$

$\begin{array}{lllllll}5 & 32 & 0.00000 \mathrm{E}+00 & 0.00000 \mathrm{E}+00 & 0.00000 \mathrm{E}+00 & 8.84370 \mathrm{E}+02 & 0.00000 \mathrm{E}+00\end{array}$

$\begin{array}{llllllll}6 & 33 & 0.00000 \mathrm{E}+00 & 0.00000 \mathrm{E}+00 & 0.00000 \mathrm{E}+00 & 8.84370 \mathrm{E}+02 & 0.00000 \mathrm{E}+00\end{array}$

$\begin{array}{llllllll}7 & 35 & 0.00000 E+00 & 0.00000 E+00 & 0.00000 E+00 & 8.84370 E+02 & 0.00000 E+00\end{array}$

$\begin{array}{llllllll}8 & 36 & 0.00000 \mathrm{E}+00 & 0.00000 \mathrm{E}+00 & 0.00000 \mathrm{E}+00 & 8.84370 \mathrm{E}+02 & 0.00000 \mathrm{E}+00\end{array}$

$\begin{array}{llllllll}9 & 38 & 1.15958 \mathrm{E}-01 & 2.02000 \mathrm{E}+00 & 0.00000 \mathrm{E}+00 & 0.00000 \mathrm{E}+00 & 0.00000 \mathrm{E}+00\end{array}$

$\begin{array}{lllllllll}10 & 39 & 5.44575 \mathrm{E}-02 & 8.40000 \mathrm{E}+00 & 0.00000 \mathrm{E}+00 & 0.00000 \mathrm{E}+00 & 0.00000 \mathrm{E}+00\end{array}$

$11 \quad 40 \quad 9.50007 \mathrm{E}-02 \quad 1.00000 \mathrm{E}+00 \quad 0.00000 \mathrm{E}+00 \quad 0.00000 \mathrm{E}+00 \quad 0.00000 \mathrm{E}+00$

$12 \quad 41 \quad 5.44575 \mathrm{E}-02 \quad 8.40000 \mathrm{E}+00 \quad 0.00000 \mathrm{E}+00 \quad 0.00000 \mathrm{E}+00 \quad 0.00000 \mathrm{E}+00$

$\begin{array}{llllll}140 & 0.00000 \mathrm{E}+00 & 0.00000 \mathrm{E} \div 00 & 0.00000 \mathrm{E}+00 & 0.00000 \mathrm{E}+00 & 0.00000 \mathrm{E}+00\end{array}$

$42 \quad 9.50007 \mathrm{E}-02 \quad 1.00000 \mathrm{E}+00 \quad 0.00000 \mathrm{E}+00 \quad 0.00000 \mathrm{E}+00 \quad 0.00000 \mathrm{E}+00$

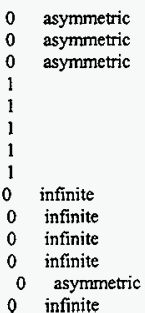


HNF-SD-SNF-CSWD-005, Rev. 0

$15 \quad 154 \quad 6.62920 \mathrm{E}-02 \quad 1.37500 \mathrm{E}+01 \quad 0.00000 \mathrm{E}+00 \quad 0.00000 \mathrm{E}+00 \quad 0.00000 \mathrm{E}+00$ ( -16

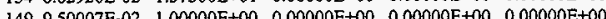
$\begin{array}{llllllll}144 & 6.43969 \mathrm{E}-02 & 1.96600 \mathrm{E}+01 & 0.00000 \mathrm{E}+00 & 0.00000 \mathrm{E}+00 & 0.00000 \mathrm{E}+00\end{array}$ $\begin{array}{llllllll}159 & 5.44575 \mathrm{E}-02 & 8.40000 \mathrm{E}+00 & 0.00000 \mathrm{E}+00 & 0.00000 \mathrm{E}+00 & 0.00000 \mathrm{E}+00\end{array}$ $\begin{array}{llllllll}19 & 141 & 9.50007 \mathrm{E}-02 & 1.00000 \mathrm{E}+00 & 0.00000 \mathrm{E}+00 & 0.00000 \mathrm{E}+00 & 0.00000 \mathrm{E}+00\end{array}$ $\begin{array}{llllllll}20 & 162 & 0.00000 \mathrm{E}+00 & 0.00000 \mathrm{E}+00 & 0.00000 \mathrm{E}+00 & 0.00000 \mathrm{E}+00 & 0.00000 \mathrm{E}+00\end{array}$ 1surface areas print table 50 infinite infinite infinite infinite infinite asymmetric

surface input calculated reason area area area not calculated

$1 \quad 10.00000 \mathrm{E}+00 \quad 0.00000 \mathrm{E}+00$ asymmetric

$2170.00000 \mathrm{E}+00 \quad 0.00000 \mathrm{E}+00$ asymmetric

$3190.00000 \mathrm{E}+00 \quad 0.00000 \mathrm{E}+00$ asymmetric

$429 \quad 0.00000 \mathrm{E}+00 \quad 0.00000 \mathrm{E}+00$ asymmetric

$558 \quad 0.00000 \mathrm{E}+00 \quad 0.00000 \mathrm{E}+00$ ininite

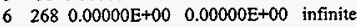

$7478 \quad 0.00000 \mathrm{E}+00 \quad 0.00000 \mathrm{E}+00$ infinite

$8698 \quad 0.00000 \mathrm{E}+00 \quad 0.00000 \mathrm{E}+00$ infinite

$9880 \quad 0.00000 \mathrm{E}+00 \quad 0.00000 \mathrm{E}+00$ infinite

$10 \quad 881 \quad 0.00000 \mathrm{E}+00 \quad 0.00000 \mathrm{E}+00$ infinite

$18820.00000 \mathrm{E}+00 \quad 0.00000 \mathrm{E}+00$ infinite

$129050.00000 \mathrm{E}+00 \quad 0.00000 \mathrm{E}+00$ asymmetric

$13906 \quad 0.00000 \mathrm{E}+00 \quad 0.00000 \mathrm{E}+00$ asymmetric

$14 \quad 907 \quad 0.00000 \mathrm{E}+00 \quad 0.00000 \mathrm{E}+00$ asymmetric

$15913 \quad 0.00000 \mathrm{E}+00 \quad 8.40057 \mathrm{E}+02$

$\begin{array}{lll}16 & 914 & 0.00000 E+00 \quad 8.40057 E+02\end{array}$

$\begin{array}{llll}17 & 915 & 0.00000 \mathrm{E}+00 \quad 8.40057 \mathrm{E}+02\end{array}$

$\begin{array}{ll}18 & 916 \quad 0.00000 \mathrm{E}+00 \quad 8.40057 \mathrm{E} \div 02\end{array}$

$19917 \quad 0.00000 \mathrm{E}+00 \quad 8.40057 \mathrm{E}+02$

$203010.00000 \mathrm{E}+00 \quad 0.00000 \mathrm{E}+00$ infinite

$21302 \quad 0.00000 \mathrm{E}+00 \quad 0.00000 \mathrm{E}+00$ infinite

$22 \quad 303 \quad 0.00000 \mathrm{E}+00 \quad 0.00000 \mathrm{E}+00$ infinite

$3040.00000 \mathrm{E}+00 \quad 0.00000 \mathrm{E}+00$

$305 \quad 0.00000 \mathrm{E}+00 \quad 0.00000 \mathrm{E}+00$

$3060.00000 \mathrm{E}+00 \quad 0.00000 \mathrm{E}+00$

1cells

print table 60

cell mat density density volume mass pieces importance

$130 \quad 0 \quad 0.00000 \mathrm{E}+00 \quad 0.00000 \mathrm{E}+00 \quad 0.00000 \mathrm{E}+00 \quad 0.00000 \mathrm{E}+00$

$231 \quad 0 \quad 0.00000 \mathrm{E}+000.00000 \mathrm{E}+00 \quad 0.00000 \mathrm{E}+000.00000 \mathrm{E}+00$

$3 \quad 37 \quad 0 \quad 0.00000 \mathrm{E}+00 \quad 0.00000 \mathrm{E}+00 \quad 0.00000 \mathrm{E}+000.00000 \mathrm{E}+00$

$434 \quad 0 \quad 0.00000 \mathrm{E}+000.00000 \mathrm{E}+008.84370 \mathrm{E}+02 \quad 0.00000 \mathrm{E}+00$

$53200.00000 \mathrm{E}+000.00000 \mathrm{E}+008.84370 \mathrm{E}+020.00000 \mathrm{E}+00$

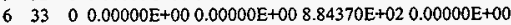

$7 \quad 35 \quad 0 \quad 0.00000 \mathrm{E}+000.00000 \mathrm{E}+008.84370 \mathrm{E}+020.00000 \mathrm{E}+00$

$836 \quad 0 \quad 0.00000 \mathrm{E}+00 \quad 0.00000 \mathrm{E}+00 \quad 8.84370 \mathrm{E}+020.00000 \mathrm{E}+00$

$938 \quad 11 \quad 1.15958 \mathrm{E}-012.02000 \mathrm{E}+000.00000 \mathrm{E}+000.00000 \mathrm{E}+00$

$10 \quad 39 \quad 6 \quad 5.44575 \mathrm{E}-02 \quad 8.40000 \mathrm{E}+000.00000 \mathrm{E}+000.00000 \mathrm{E}+00$

$1140 \quad 12 s 9.50007 \mathrm{E}-021.00000 \mathrm{E}+000.00000 \mathrm{E}+000.00000 \mathrm{E}+00 \quad 01.0000 \mathrm{E}+00$

$1241 \quad 6 \quad 5.44575 \mathrm{E}-02 \quad 8.40000 \mathrm{E}+00 \quad 0.00000 \mathrm{E}+000.00000 \mathrm{E}+00 \quad 0 \quad 1.0000 \mathrm{E}+00$

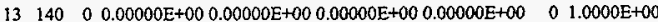

$14 \quad 4212 \$ 9.50007 \mathrm{E}-021.00000 \mathrm{E}+000.00000 \mathrm{E}+000.00000 \mathrm{E}+00 \quad 0 \quad 1.0000 \mathrm{E}+00$

$15 \quad 15426.62920 \mathrm{E}-02 \quad 1.37500 \mathrm{E}+01 \quad 0.00000 \mathrm{E}+000.00000 \mathrm{E}+00 \quad 0 \quad 1.0000 \mathrm{E}+00$

$16 \quad 14912 s 9.50007 \mathrm{E}-021.00000 \mathrm{E}+000.00000 \mathrm{E}+000.00000 \mathrm{E}+00 \quad 0 \quad 1.0000 \mathrm{E}+00$

$17 \quad 14476.43969 \mathrm{E}-021.96600 \mathrm{E}+010.00000 \mathrm{E}+000.00000 \mathrm{E}+00 \quad 0 \quad 1.0000 \mathrm{E}+00$

$18 \quad 159 \quad 6 \quad 5.44575 \mathrm{E}-028.40000 \mathrm{E}+000.00000 \mathrm{E}+00 \quad 0.00000 \mathrm{E}+00 \quad 0 \quad 1.0000 \mathrm{E}+00$

$19141 \quad 12 s 9.50007 \mathrm{E}-021.00000 \mathrm{E}+000.00000 \mathrm{E}+000.00000 \mathrm{E}+00 \quad 01.0000 \mathrm{E}+00$

$20 \quad 16200.00000 \mathrm{E}+00 \quad 0.00000 \mathrm{E}+00 \quad 0.00000 \mathrm{E}+00 \quad 0.00000 \mathrm{E}+00 \quad 0 \quad 0.0000 \mathrm{E}+00$

total

isurfaces
$4.42185 E+030.00000 E+00$

print table 70

surface trans type surface coefficients 
all non-zero importance cells with materials have a temperature for thermal neutrons of $2.5300 \mathrm{E}-08$ mev.

2 warning messages so far.

icross-section tables print table 100

table length

tables from file testlibi

$1001.00 \mathrm{c} 1782 \mathrm{l}$-h-1 from endf-vi.l

mat $12505 / 26 / 93$

$1002.55 \mathrm{c} \quad 4102$ njoy (120) $04 / 26 / 82$

$5011.40 \mathrm{c} 3229$ ENDL library name: nd900719 MCNP translation: 900831 14:10:05

$6012.40 \mathrm{c} 4858$ ENDL library name: nd900719 MCNP translation: 900831 14:10:05

$7014.40 \mathrm{c} 8535$ ENDL library name: nd900719 MCNP translation: 900831 14:10:05

8016.40c 5358 ENDL library name: nd900719 MCNP translation: 900831 14:10:05

40000:40c 93372 ENDL library name: nd900719 MCNP translation: 900831 14:10:05

41093.40c 42746 ENDL library name: nd900719 MCNP translation: 900831 14:10:05

$74000.40 \mathrm{c} 91244$ ENDL library name: nd900719 MCNP translation: 900831 14:10:05

92235.40c 34772 ENDL library name: nd900719 MCNP translation: 9total nu 10:05

900207

900207

900207

900207

900323

900208

900208

900503

901119

$\begin{array}{lll}92238.40 \mathrm{C} & 40834 & \text { ENDL library name: nd901118 MCNP translation: 9total nu 56:49 } \\ 5010.03 \mathrm{~d} & 3682 \text { b-10 endf/b-iv new gamma production format }\end{array}$

(1273) $\quad 14 \operatorname{oct} 75$

hwt $01 \mathrm{t} 10193$ deuterium in heavy water at 300 degtees kelvin

$1002 \quad 0 \quad 010 / 22 / 85$

lwtr.01t 10193 hydrogen in light water at 300 degrees kelvin

$\begin{array}{lll}1001 & 0 & 010 / 22 / 85\end{array}$

total 354900

waming. neutron energy cutoff is below some cross-section tables.

decimal words of dynamically allocated storage

source distribution written to file inp $18 \mathrm{~s} \quad$ cycle $=0$

3 warning messages so far.

starting merun. field length $=0 \quad$ cp $0=0.00$

print table 110

probl 8 -- kcode in a hexagonal prism lattice. 
$\begin{array}{ccccc}x & y & z & \text { cell lattice }(i, j, k) \\ 3.000 E+00 & 2.000 E+0 I & 2.000 E-01 & 140 \\ 3.492 E+00 & 1.051 E+00 & 2.000 E-01 & 42(\end{array}$ $\begin{array}{llll}3.492 \mathrm{E}+00 & 1.051 \mathrm{E}+00 & 2.000 \mathrm{E}-01 & 42 \\ 3.492 \mathrm{E}+00 & 1.051 \mathrm{E}+00 & 2.000 \mathrm{E}-01 & 154\end{array}$ $23.000 \mathrm{E}+00 \quad 2.000 \mathrm{E}-01 \quad 2.000 \mathrm{E}-01 \quad 140$ $3.492 \mathrm{E}+00 \quad 1.051 \mathrm{E}+00 \quad 2.000 \mathrm{E}-01 \quad 42(0,-1,0)$ $3.492 \mathrm{E}+00 \quad 1.051 \mathrm{E}+00 \quad 2.000 \mathrm{E}-01 \quad 154$ $33.000 \mathrm{E}+002.000 \mathrm{E}-01 \quad 2.000 \mathrm{E}-01 \quad 140$ $3.492 E+00$ 1.051E+00 2.000E-01 42( $0,-1,0)$ $3.492 \mathrm{E}+00 \quad 1.051 \mathrm{E}+00 \quad 2.000 \mathrm{E}-01 \quad 154$

$43.000 \mathrm{E}+00$ 2.000E-01 2.000E-01 140 $3.492 \mathrm{E}+00 \quad 1.051 \mathrm{E}+00 \quad 2.000 \mathrm{E}-01 \quad 42(0,-1,0)$ $3.492 \mathrm{E}+00 \quad 1.051 \mathrm{E}+00 \quad 2.000 \mathrm{E}-01 \quad 154$ $53.000 \mathrm{E}+00 \quad 2.000 \mathrm{E}-01 \quad 2.000 \mathrm{E}-01 \quad 140$ $3.492 \mathrm{E}+00 \quad 1.051 \mathrm{E}+00 \quad 2.000 \mathrm{E}-01 \quad 42(0,-1,0)$ $3.492 \mathrm{E}+00 \quad 1.051 \mathrm{E}+00 \quad 2.000 \mathrm{E}-01 \quad 154$ $63.000 \mathrm{E}+00 \quad 2.000 \mathrm{E}-012.000 \mathrm{E}-01 \quad 140$ $3.492 \mathrm{E}+00 \quad 1.051 \mathrm{E}+00 \quad 2.000 \mathrm{E}-01 \quad 42(0,-1,0)$ $3.492 \mathrm{E}+00 \quad 1.051 \mathrm{E}+00 \quad 2.000 \mathrm{E}-01 \quad 154$

$73.000 \mathrm{E}+00 \quad 2.000 \mathrm{E}-012.000 \mathrm{E}-01 \quad 140$ $3.492 E+001.051 E+00 \quad 2.000 E-01 \quad 42(0,-1,0)$ $3.492 \mathrm{E}+00 \quad 1.051 \mathrm{E}+00 \quad 2.000 \mathrm{E}-01 \quad 154$ $83.000 E+00 \quad 2.000 E+01 \quad 2.000 E-01 \quad 140$ $3.492 \mathrm{E}+00 \quad 1.051 \mathrm{E}+00 \quad 2.000 \mathrm{E}-01 \quad 42(0,-1,0)$ $3.492 \mathrm{E}+00 \quad 1.051 \mathrm{E}+00 \quad 2.000 \mathrm{E}-01 \quad 154$ $93.000 E+00$ 2.000E-01 2.000E-01 140 $3.492 \mathrm{E}+00 \quad 1.051 \mathrm{E}+00 \quad 2.000 \mathrm{E}-01 \quad 42(0,-1,0)$ $3.492 \mathrm{E}+00 \quad 1.051 \mathrm{E}+00 \quad 2.000 \mathrm{E}-01 \quad 154$ $103.000 \mathrm{E}+00 \quad 2.000 \mathrm{E}-01 \quad 2.000 \mathrm{E}-01 \quad 140$ $3.492 \mathrm{E}+001.051 \mathrm{E}+002.000 \mathrm{E}-01 \quad 42(0,-1,0)$ $3.492 \mathrm{E}+00 \quad 1.051 \mathrm{E}+00 \quad 2.000 \mathrm{E}-01 \quad 154$ $3.000 \mathrm{E}+00 \quad 2.000 \mathrm{E}-01 \quad 2.000 \mathrm{E}-01 \quad 140$ $3.492 \mathrm{E}+001.051 \mathrm{E}+00 \quad 2.000 \mathrm{E}-01 \quad 42(0,-1,0)$ $3.492 \mathrm{E}+00 \quad 1.051 \mathrm{E}+00 \quad 2.000 \mathrm{E}-01 \quad 154$ $123.000 \mathrm{E}+00 \quad 2.000 \mathrm{E}-01 \quad 2.000 \mathrm{E}-01 \quad 140$ $3.492 \mathrm{E}+001.051 \mathrm{E}+00 \quad 2.000 \mathrm{E}-01 \quad 42(0,-1,0)$ $3.492 \mathrm{E}+00 \quad 1.051 \mathrm{E}+00 \quad 2.000 \mathrm{E}-01 \quad 154$ $133.000 \mathrm{E}+00 \quad 2.000 \mathrm{E}-012.000 \mathrm{E}-01 \quad 140$ $3.492 \mathrm{E}+00 \quad 1.051 \mathrm{E}+00 \quad 2.000 \mathrm{E}-01 \quad 42(0,-1,0)$ $3.492 \mathrm{E}+00 \quad 1.051 \mathrm{E}+00 \quad 2.000 \mathrm{E}-01 \quad 154$ $143.000 \mathrm{E}+00$ 2.000E-01 2.000E-01 140 $3.492 \mathrm{E}+00 \quad 1.051 \mathrm{E}+00 \quad 2.000 \mathrm{E}-01 \quad 42(0,-1,0)$ $3.492 \mathrm{E}+00 \quad 1.051 \mathrm{E}+00 \quad 2.000 \mathrm{E}-01154$ $153.000 \mathrm{E}+00 \quad 2.000 \mathrm{E}-01 \quad 2.000 \mathrm{E}-01 \quad 140$ $3.492 \mathrm{E}+00$ 1.051 E+00 2.000E-01 42 ( $3.492 \mathrm{E}+00 \quad 1.051 \mathrm{E}+00 \quad 2.000 \mathrm{E}-01154$ $16 \quad 3.000 \mathrm{E} \div 00 \quad 2.000 \mathrm{E}-01 \quad 2.000 \mathrm{E}+01 \quad 140$ $3.492 \mathrm{E}+00 \quad 1.051 \mathrm{E}+00 \quad 2.000 \mathrm{E}-01 \quad 42(0,-1$, $3.492 \mathrm{E}+00 \quad 1.051 \mathrm{E}+00 \quad 2.000 \mathrm{E}-01 \quad 154$

$173.000 \mathrm{E}+00 \quad 2.000 \mathrm{E}-01 \quad 2.000 \mathrm{E}-01 \quad 140$ $3.492 \mathrm{E}+00 \quad 1.051 \mathrm{E}+00 \quad 2.000 \mathrm{E}-0 \mathrm{I} \quad 42(0,-1,0)$ $3.492 \mathrm{E}+00 \quad 1.051 \mathrm{E}+00 \quad 2.000 \mathrm{E}-01 \quad 154$

$18 \quad 3.000 \mathrm{E}+00 \quad 2.000 \mathrm{E}-01 \quad 2.000 \mathrm{E}-01 \quad 140$ $3.492 \mathrm{E}+00 \quad 1.051 \mathrm{E}+00 \quad 2.000 \mathrm{E}-01 \quad 42(0,-1,0)$ $3.492 \mathrm{E}+00 \quad 1.051 \mathrm{E}+00 \quad 2.000 \mathrm{E}-01 \quad 154$

$193.000 \mathrm{E}+00 \quad 2.000 \mathrm{E}-01 \quad 2.000 \mathrm{E}-01140$ $3.492 \mathrm{E}+00 \quad 3.051 \mathrm{E}+002.000 \mathrm{E}-01 \quad 42(0,-1,0)$ $3.492 \mathrm{E}+00 \quad 1.05 \mathrm{E}+00 \quad 2.000 \mathrm{E}-01 \quad 154$

$203.000 \mathrm{E}+00 \quad 2.000 \mathrm{E}-01 \quad 2.000 \mathrm{E}-01 \quad 140$ $3.492 \mathrm{E}+00 \quad 1.051 \mathrm{E}+00 \quad 2.000 \mathrm{E}-01 \quad 42(0,-1,0)$ $3.492 \mathrm{E}+00 \quad 1.051 \mathrm{E}+00 \quad 2.000 \mathrm{E}-01 \quad 154$

$21 \quad 3.000 \mathrm{E}+00 \quad 2.000 \mathrm{E}-01 \quad 2.000 \mathrm{E}-01 \quad 140$ $3.492 \mathrm{E}+00 \quad 1.051 \mathrm{E}+00 \quad 2.000 \mathrm{E}-01 \quad 42(0,-1,0)$ $3.492 \mathrm{E}+00 \quad 1.051 \mathrm{E}+00 \quad 2.000 \mathrm{E}-01 \quad 154$ $3.000 \mathrm{E}+00 \quad 2.000 \mathrm{E}-01 \quad 2.000 \mathrm{E}-01 \quad 140$

$v$ w energy weight time

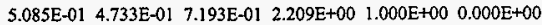
$5.085 \mathrm{E}-01 \quad 4.733 \mathrm{E}-017.193 \mathrm{E}-01$

$0 \quad 5.085 \mathrm{E}-01 \quad 4.733 \mathrm{E}-01 \quad 7.193 \mathrm{E}-01$

$8.952 \mathrm{E}-01-4.447 \mathrm{E}-01-2.944 \mathrm{E}-02 \quad 4.904 \mathrm{E}+00 \quad 1.000 \mathrm{E}+00 \quad 0.000 \mathrm{E}+00$ 8.952E-01 -4.447E-01 -2.944E-02

$0 \quad 8.952 \mathrm{E}-01-4.447 \mathrm{E}-01-2.944 \mathrm{E}-02$

$-6.184 \mathrm{E}-01-4.495 \mathrm{E}-01 \quad 6.446 \mathrm{E}-01 \quad 3.809 \mathrm{E}-01 \quad 1.000 \mathrm{E}+00 \quad 0.000 \mathrm{E}+00$ $-6.184 \mathrm{E}-01-4.495 \mathrm{E}-01 \quad 6.446 \mathrm{E}-01$

$0-6.184 \mathrm{E}-01-4.495 \mathrm{E}-01 \quad 6.446 \mathrm{E}-01$

$9.710 \mathrm{E}-01-5.665 \mathrm{E}-02-2.323 \mathrm{E}-01 \quad 1.331 \mathrm{E}+00 \quad 1.000 \mathrm{E}+00 \quad 0.000 \mathrm{E}+00$ $9.710 \mathrm{E}-01-5.665 \mathrm{E}-02-2.323 \mathrm{E}-01$

0 9.710E-01 -5.665E-02 -2.323E-01

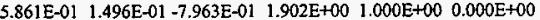
$5.861 \mathrm{E}-01 \quad 1.496 \mathrm{E}-01-7.963 \mathrm{E}-01$

$0 \quad 5.861 \mathrm{E}-01 \quad 1.496 \mathrm{E}-01-7.963 \mathrm{E}-01$

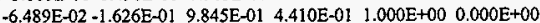
$-6.489 \mathrm{E}-02-1.626 \mathrm{E}-019.845 \mathrm{E}-01$

$0-6.489 \mathrm{E}-02-1.626 \mathrm{E}-01 \quad 9.845 \mathrm{E}-01$

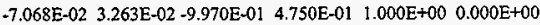
$-7.068 \mathrm{E}-02 \quad 3.263 \mathrm{E}-02-9.970 \mathrm{E}-01$

$0-7.068 \mathrm{E}-02 \quad 3.263 \mathrm{E}-02-9.970 \mathrm{E}-01$

$-3.915 \mathrm{E}-01 \quad 4.664 \mathrm{E}-01-7.932 \mathrm{E}-01 \quad 4.136 \mathrm{E}+00 \quad 1.000 \mathrm{E}+00 \quad 0.000 \mathrm{E}+00$

$-3.915 \mathrm{E}-01 \quad 4.664 \mathrm{E}-01-7.932 \mathrm{E}-01$

$0-3.915 \mathrm{E}-01 \quad 4.664 \mathrm{E}-01-7.932 \mathrm{E}-01$

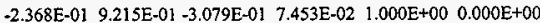

$-2.368 \mathrm{E}-01$ 9.215E-01 $-3.079 \mathrm{E}-01$

$0-2.368 \mathrm{E}-01 \quad 9.215 \mathrm{E}-01-3.079 \mathrm{E}-01$

$1.946 \mathrm{E}-01 \cdot 3.204 \mathrm{E}-01 \quad 9.271 \mathrm{E}-01 \quad 3.128 \mathrm{E}+00 \quad 1.000 \mathrm{E}+00 \quad 0.000 \mathrm{E}+00$ $1.946 \mathrm{E}-01-3.204 \mathrm{E}-01 \quad 9.271 \mathrm{E}-01$

$0 \quad 1.946 \mathrm{E}-01-3.204 \mathrm{E}-01 \quad 9.271 \mathrm{E}-01$

$-6.698 \mathrm{E}-01-7.177 \mathrm{E}-01-1.905 \mathrm{E}-01 \quad 1.014 \mathrm{E}+00 \quad 1.000 \mathrm{E}+00 \quad 0.000 \mathrm{E}+00$ $-6.698 \mathrm{E}-01-7.177 \mathrm{E}-01-1.905 \mathrm{E}-01$

$0-6.698 \mathrm{E}-01-7.177 \mathrm{E}-01-1.905 \mathrm{E}-01$

$\begin{array}{llll}-8.398 \mathrm{E}-01 & -4.129 \mathrm{E}-01 \quad 3.524 \mathrm{E}-01 & 1.395 \mathrm{E}+00 \quad 1.000 \mathrm{E}+00 & 0.000 \mathrm{E}+00\end{array}$

$-8.398 \mathrm{E}-01-4.129 \mathrm{E}-01 \quad 3.524 \mathrm{E}-01$

$0-8.398 \mathrm{E}-01-4.129 \mathrm{E}-01 \quad 3.524 \mathrm{E}-01$

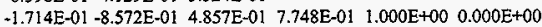
$-1.714 \mathrm{E}-01-8.572 \mathrm{E}-01 \quad 4.857 \mathrm{E}-01$

$0-1.714 \mathrm{E}-01-8.572 \mathrm{E}-01 \quad 4.857 \mathrm{E}-01$

$\begin{aligned} & -2.489 \mathrm{E}-01 \\ & -5.118 \mathrm{E}-01\end{aligned}-8.222 \mathrm{E}-01 \quad 1.101 \mathrm{E}+00 \quad 1.000 \mathrm{E}+00 \quad 0.000 \mathrm{E}+00$ $-2.489 \mathrm{E}-01-5.118 \mathrm{E}-01-8.222 \mathrm{E}-01$

$0-2.489 \mathrm{E}-01-5.118 \mathrm{E}-01-8.222 \mathrm{E}-01$ $\begin{array}{lllll}-2.959 \mathrm{E}-01 & 2.119 \mathrm{E}-01 & 9.314 \mathrm{E}-01 & 1.951 \mathrm{E}+00 \quad 1.000 \mathrm{E}+00 & 0.000 \mathrm{E}+00\end{array}$

$-2.959 \mathrm{E}-01 \quad 2.119 \mathrm{E}-01 \quad 9.314 \mathrm{E}-01$

$0-2.959 \mathrm{E}-01 \quad 2.119 \mathrm{E}-01 \quad 9.314 \mathrm{E}-01$

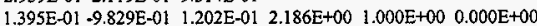
$1.395 \mathrm{E}-01-9.829 \mathrm{E}-01 \quad 1.202 \mathrm{E}-01$

0 1.395E-01 -9.829E-01 1.202E-01

6.909E-01 -7.110E-01 $1.307 \mathrm{E}-01 \quad 1.865 \mathrm{E}+00 \quad 1.000 \mathrm{E}+00 \quad 0.000 \mathrm{E}+00$ $6.909 \mathrm{E}-01-7.110 \mathrm{E}-01 \quad 1.307 \mathrm{E}-01$

0 6.909E-01 -7.110E-01 1.307E-01

$-6.580 \mathrm{E}-01 \quad 5.320 \mathrm{E}-01-5.329 \mathrm{E}-01 \quad 1.229 \mathrm{E}+00 \quad 1.000 \mathrm{E}+00 \quad 0.000 \mathrm{E}+00$ $-6.580 \mathrm{E}-01 \quad 5.320 \mathrm{E}-01-5.329 \mathrm{E}-01$

$0-6.580 \mathrm{E}-01 \quad 5.320 \mathrm{E}-01-5.329 \mathrm{E}-01$

$-9.903 \mathrm{E}-01-1.380 \mathrm{E}-01 \quad 1.353 \mathrm{E}-02 \quad 1.305 \mathrm{E}+00 \quad 1.000 \mathrm{E}+00 \quad 0.000 \mathrm{E}+00$ $-9.903 \mathrm{E}-01-1.380 \mathrm{E}-01 \quad 1.353 \mathrm{E}-02$

$0-9.903 \mathrm{E}-01-1.380 \mathrm{E}-01 \quad 1.353 \mathrm{E}-02$

7.462E-01 4.859E-01 $-4.551 \mathrm{E}-01 \quad 1.000 \mathrm{E}+00 \quad 1.000 \mathrm{E}+00 \quad 0.000 \mathrm{E}+00$ $7.462 \mathrm{E}-01 \quad 4.859 \mathrm{E}-01-4.551 \mathrm{E}-01$

$07.462 \mathrm{E}-01 \quad 4.859 \mathrm{E}-01-4.551 \mathrm{E}-01$

$\begin{array}{llll}-1.977 \mathrm{E}-01 & 9.797 \mathrm{E}-01 \quad 3.360 \mathrm{E}-02 \quad 3.990 \mathrm{E}+00 \quad 1.000 \mathrm{E}+00 \quad 0.000 \mathrm{E}+00\end{array}$ $-1.977 \mathrm{E}-019.797 \mathrm{E}-013.360 \mathrm{E}-02$

$0-1.977 \mathrm{E}-01 \quad 9.797 \mathrm{E}-01 \quad 3.360 \mathrm{E}-02$

$-9.117 \mathrm{E}-01-3.647 \mathrm{E}-01-1.891 \mathrm{E}-01 \quad 2.665 \mathrm{E}-01 \quad 1.000 \mathrm{E}+00 \quad 0.000 \mathrm{E}+00$ 
HNF-SD-SNF-CSWD-005, Rev. 0

3.492E+00 1.051E+00 2.000E-01 42( $0,-1,0) \quad-9.117 \mathrm{E}-01-3.647 \mathrm{E}-01-1.891 \mathrm{E}-01$

$3.492 \mathrm{E}+00 \quad 1.051 \mathrm{E}+00 \quad 2.000 \mathrm{E}-01 \quad 154 \quad 0-9.117 \mathrm{E}-01-3.647 \mathrm{E}-01-1.891 \mathrm{E}-01$

$\begin{array}{llllllllllll}3.000 \mathrm{E}+00 & 2.000 \mathrm{E}-01 & 2.000 \mathrm{E}-01 & 140 & -4.287 \mathrm{E}-01 & 8.36 \mathrm{E}-01 & -3.423 \mathrm{E}-01 & 1.156 \mathrm{E}+00 & 1.000 \mathrm{E}+00 & 0.000 \mathrm{E}+00\end{array}$

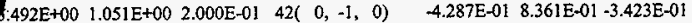

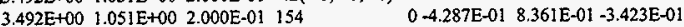

$243.000 \mathrm{E}+00 \quad 2.000 \mathrm{E}-01 \quad 2.000 \mathrm{E}-01 \quad 140 \quad 1.080 \mathrm{E}-01 \quad 3.412 \mathrm{E}-01-9.338 \mathrm{E}-01 \quad 2.669 \mathrm{E}+00 \quad 1.000 \mathrm{E}+00 \quad 0.000 \mathrm{E}+00$ $3.492 \mathrm{E}+00 \quad 1.051 \mathrm{E}+00 \quad 2.000 \mathrm{E}-01 \quad 42(0,-1,0) \quad 1.080 \mathrm{E}-01 \quad 3.412 \mathrm{E}-01-9.338 \mathrm{E}-01$

$3.492 \mathrm{E}+00 \quad 1.051 \mathrm{E}+00 \quad 2.000 \mathrm{E}-01 \quad 154 \quad 0 \quad 1.080 \mathrm{E}-01 \quad 3.412 \mathrm{E}-01-9.338 \mathrm{E}-01$

$253.000 \mathrm{E}+00 \quad 2.000 \mathrm{E}-01 \quad 2.000 \mathrm{E}-01 \quad 140 \quad-9.111 \mathrm{E}-01-9.012 \mathrm{E}-03-4.122 \mathrm{E}-01 \quad 2.185 \mathrm{E}+00 \quad 1.000 \mathrm{E}+00 \quad 0.000 \mathrm{E}+00$

$3.492 \mathrm{E}+00 \quad 1.051 \mathrm{E}+00 \quad 2.000 \mathrm{E}-01 \quad 42(0,-1,0) \quad-9.111 \mathrm{E}-01-9.012 \mathrm{E}-03-4.122 \mathrm{E}-01$

$3.492 \mathrm{E}+00 \quad 1.051 \mathrm{E}+00 \quad 2.000 \mathrm{E}-01 \quad 154 \quad 0-9.111 \mathrm{E}-01-9.012 \mathrm{E}-03-4.122 \mathrm{E}-01$

$262.000 \mathrm{E}-01 \quad 3.000 \mathrm{E}+00 \quad 2.000 \mathrm{E}-01 \quad 140$

$3.641 \mathrm{E}+00 \quad 4.456 \mathrm{E}-01 \quad 2.000 \mathrm{E}-01 \quad 42(-5,3,0) \quad-2.568 \mathrm{E}-01-6.391 \mathrm{E}-01-7.249 \mathrm{E}-01$

$3.641 \mathrm{E}+00 \quad 4.456 \mathrm{E}-01 \quad 2.000 \mathrm{E}-01141,0.2 .568 \mathrm{E}-01-6.391 \mathrm{E}-01-7.249 \mathrm{E}-01$

$272.000 \mathrm{E}-01 \quad 3.000 \mathrm{E}+00 \quad 2.000 \mathrm{E}-01 \quad 140$ $3.641 \mathrm{E}+00 \quad 4.456 \mathrm{E}-01 \quad 2.000 \mathrm{E}-01 \quad 42(-5,3,0)$ $3.641 \mathrm{E}+00 \quad 4.456 \mathrm{E}-01 \quad 2.000 \mathrm{E}-01 \quad 141$

$28 \quad 2.000 \mathrm{E}-0] \quad 3.000 \mathrm{E}+00 \quad 2.000 \mathrm{E}-01 \quad 140$ $3.64] \mathrm{E}+00 \quad 4.456 \mathrm{E}-01 \quad 2.000 \mathrm{E}-01 \quad 42(-5,3,0)$ $3.641 \mathrm{E}+00 \quad 4.456 \mathrm{E}-01 \quad 2.000 \mathrm{E}-01 \quad 141$

$292.000 \mathrm{E}-01 \quad 3.000 \mathrm{E}+00 \quad 2.000 \mathrm{E}-01 \quad 140$ $3.641 \mathrm{E}+00 \quad 4.456 \mathrm{E}-01 \quad 2.000 \mathrm{E}-01 \quad 42(-5,3,0)$ $3.641 \mathrm{E}+00 \quad 4.456 \mathrm{E}-01 \quad 2.000 \mathrm{E}-01 \quad 141$

$30 \quad 2.000 \mathrm{E}-01 \quad 3.000 \mathrm{E}+00 \quad 2.000 \mathrm{E}-01 \quad 140$ $3.641 \mathrm{E}+00 \quad 4.456 \mathrm{E}-01 \quad 2.000 \mathrm{E}-01 \quad 42(-5,3,0)$ $3.641 \mathrm{E}+00 \quad 4.456 \mathrm{E}-01 \quad 2.000 \mathrm{E}-01 \quad 141$

31 2.000E-01 $3.000 \mathrm{E}+002.000 \mathrm{E}-01 \quad 140$ $3.641 \mathrm{E}+00 \quad 4.456 \mathrm{E}-01 \quad 2.000 \mathrm{E}-01 \quad 42(-5,3,0)$ $3.641 \mathrm{E}+00 \quad 4.456 \mathrm{E}-01 \quad 2.000 \mathrm{E}-01 \quad 141$

$322.000 \mathrm{E}-01 \quad 3.000 \mathrm{E}+00 \quad 2.000 \mathrm{E}-01 \quad 140$ $3.641 \mathrm{E}+00 \quad 4.456 \mathrm{E}-01 \quad 2.000 \mathrm{E}-01 \quad 42(-5,3,0)$ $3.641 \mathrm{E}+00 \quad 4.456 \mathrm{E}+01 \quad 2.000 \mathrm{E}-01 \quad 141$

$33 \quad 2.000 \mathrm{E}-01 \quad 3.000 \mathrm{E}+00 \quad 2.000 \mathrm{E}-01 \quad 140$

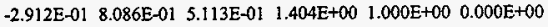
-2.912E-01 8.086E-01 5.113E-01

$0-2.912 \mathrm{E}-01 \quad 8.086 \mathrm{E}-01 \quad 5.113 \mathrm{E}-0$ l $1.472 \mathrm{E}-01 \quad-9.514 \mathrm{E}-01 \quad 2.705 \mathrm{E}-01 \quad 1.090 \mathrm{E}+00 \quad 1.000 \mathrm{E}+00 \quad 0.000 \mathrm{E}+00$ $1.472 \mathrm{E}-01-9.514 \mathrm{E}-012.705 \mathrm{E}-0$ ]

0 1.472E-01 -9.514E-01 2.705E-01

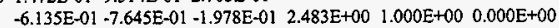
$-6.135 \mathrm{E}-01-7.645 \mathrm{E}-01-1.978 \mathrm{E}-01$

$0-6.135 \mathrm{E}-01-7.645 \mathrm{E}-01-1.978 \mathrm{E}-01$

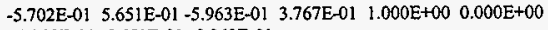
$-5.702 \mathrm{E}-01$ 5.651E-01 -5.963E-01

$0-5.702 \mathrm{E}-01 \quad 5.651 \mathrm{E}-01-5.963 \mathrm{E}-01$

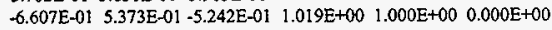
$-6.607 \mathrm{E}-01 \quad 5.373 \mathrm{E}-01-5.242 \mathrm{E}-01$

$0-6.607 \mathrm{E}-01 \quad 5.373 \mathrm{E}-01-5.242 \mathrm{E}-01$

$\begin{array}{llll}-9.742 \mathrm{E}-02 & -3.639 \mathrm{E}-01 & -9.263 \mathrm{E}-01 & 1.879 \mathrm{E}+00 \quad 1.000 \mathrm{E}+00 \quad 0.000 \mathrm{E}+00\end{array}$ $-9.742 \mathrm{E}-02-3.639 \mathrm{E}-01-9.263 \mathrm{E}-01$

$0-9.742 \mathrm{E}-02-3.639 \mathrm{E}-01-9.263 \mathrm{E}-01$

$-1.965 \mathrm{E}-0 \mathrm{l}-3.145 \mathrm{E}-01-9.287 \mathrm{E}-01 \quad 4.700 \mathrm{E}-01 \quad 1.000 \mathrm{E}+00 \quad 0.000 \mathrm{E}+00$ $-1.965 \mathrm{E}-01-3.145 \mathrm{E}-01-9.287 \mathrm{E}-01$

$3.641 \mathrm{E}+00 \quad 4.456 \mathrm{E}-01 \quad 2.000 \mathrm{E}-01 \quad 42(-5,3,0)$

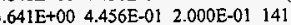
$2.000 \mathrm{E}-013.000 \mathrm{E}+00 \quad 2.000 \mathrm{E}-01 \quad 140$ $0-1.965 \mathrm{E}-01-3.145 \mathrm{E}-01-9.287 \mathrm{E}-01$ $4.097 \mathrm{E}-01 \quad 8.465 \mathrm{E}-01-3.399 \mathrm{E}-01 \quad 1.530 \mathrm{E}+00 \quad 1.000 \mathrm{E}+00 \quad 0.000 \mathrm{E}+00$ $3.641 \mathrm{E}+00 \quad 4.456 \mathrm{E}-01 \quad 2.000 \mathrm{E}-01 \quad 42(-5,3,0)$ $3.641 \mathrm{E}+00 \quad 4.456 \mathrm{E}-01 \quad 2.000 \mathrm{E}-01 \quad 141$

35 2.000E-01 $3.000 \mathrm{E}+00 \quad 2.000 \mathrm{E}-01 \quad 140$ $3.641 \mathrm{E}+00$ 4.456E-01 2.000E-01 $42(-5,3,0)$ $3.641 \mathrm{E}+00 \quad 4.456 \mathrm{E}-01 \quad 2.000 \mathrm{E}-01 \quad 141$

$362.000 \mathrm{E}-01 \quad 3.000 \mathrm{E}+00 \quad 2.000 \mathrm{E}-01 \quad 140$ $3.641 \mathrm{E}+00 \quad 4.456 \mathrm{E}-01 \quad 2.000 \mathrm{E}-01 \quad 42(-5,3,0)$ $3.641 \mathrm{E}+00 \quad 4.456 \mathrm{E}-01 \quad 2.000 \mathrm{E}-01 \quad 141$

$372.000 \mathrm{E}-01 \quad 3.000 \mathrm{E}+00 \quad 2.000 \mathrm{E}-01 \quad 140$ $3.641 \mathrm{E}+00 \quad 4.456 \mathrm{E}-01 \quad 2.000 \mathrm{E}-0 \mathrm{I} \quad 42(-5,3,0)$

$3.641 \mathrm{E}+00 \quad 4.456 \mathrm{E}-01 \quad 2.000 \mathrm{E}-01 \quad 141$

$38 \quad 2.000 \mathrm{E}-01 \quad 3.000 \mathrm{E}+00 \quad 2.000 \mathrm{E}-01 \quad 140$

$3.641 \mathrm{E}+00 \quad 4.456 \mathrm{E}-01 \quad 2.000 \mathrm{E}-01 \quad 42(-5,3,0)$ $3.641 \mathrm{E}+00 \quad 4.456 \mathrm{E}-01 \quad 2.000 \mathrm{E}-01 \quad 141$

$392.000 \mathrm{E}-01 \quad 3.000 \mathrm{E}+00 \quad 2.000 \mathrm{E}-01 \quad 140$ $3.641 \mathrm{E}+00 \quad 4.456 \mathrm{E}-01 \quad 2.000 \mathrm{E}-01 \quad 42(-5,3,0)$ $3.641 \mathrm{E}+00 \quad 4.456 \mathrm{E}-01 \quad 2.000 \mathrm{E}-01 \quad 141$

$40 \quad 2.000 \mathrm{E}-01 \quad 3.000 \mathrm{E}+00 \quad 2.000 \mathrm{E}-01 \quad 140$ $3.641 \mathrm{E}+00 \quad 4.456 \mathrm{E}-01 \quad 2.000 \mathrm{E}-01 \quad 42(-5,3,0)$

$3.641 \mathrm{E}+00 \quad 4.456 \mathrm{E}-01 \quad 2.000 \mathrm{E}-01 \quad 141$

$41 \quad 2.000 \mathrm{E}-01 \quad 3.000 \mathrm{E}+00 \quad 2.000 \mathrm{E}-01 \quad 140$

$3.641 \mathrm{E}+00 \quad 4.456 \mathrm{E}-01 \quad 2.000 \mathrm{E}-01 \quad 42(-5,3,0)$

$3.641 \mathrm{E}+00 \quad 4.456 \mathrm{E}-01 \quad 2.000 \mathrm{E}-01 \quad 141$

$42 \quad 2.000 \mathrm{E}-01 \quad 3.000 \mathrm{E}+00 \quad 2.000 \mathrm{E}-01 \quad 140$

$3.641 \mathrm{E}+00 \quad 4.456 \mathrm{E}-01 \quad 2.000 \mathrm{E}-01 \quad 42(-5,3,0)$

$3.641 \mathrm{E}+00 \quad 4.456 \mathrm{E}-01 \quad 2.000 \mathrm{E}-01 \quad 141$

$432.000 \mathrm{E}-01 \quad 3.000 \mathrm{E}+00 \quad 2.000 \mathrm{E}-01 \quad 140$

$3.641 \mathrm{E}+00 \quad 4.456 \mathrm{E}-01 \quad 2.000 \mathrm{E}-01 \quad 42(-5,3,0)$

$3.641 \mathrm{E}+00 \quad 4.456 \mathrm{E}-01 \quad 2.000 \mathrm{E}-01 \quad 141$ $2.000 \mathrm{E}-01 \quad 3.000 \mathrm{E}+00 \quad 2.000 \mathrm{E}-01 \quad 140$ 4.097E-01 8.465E-01 +3.399E-01

0 4.097E-01 8.465E-01 -3.399E-01

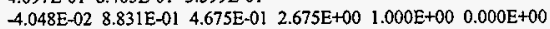
$-4.048 \mathrm{E}-02 \quad 8.831 \mathrm{E}-01 \quad 4.675 \mathrm{E}-01$

$0-4.048 \mathrm{E}-02 \quad 8.831 \mathrm{E}-01 \quad 4.675 \mathrm{E}-01$

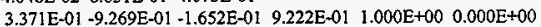
$3.371 \mathrm{E}-01-9.269 \mathrm{E}-01-1.652 \mathrm{E}-01$

$0 \quad 3.371 \mathrm{E}-01-9.269 \mathrm{E}-01-1.652 \mathrm{E}-01$

$-1.867 \mathrm{E}-01 \quad 9.756 \mathrm{E}-01+1.155 \mathrm{E}-01 \quad 9.702 \mathrm{E}-01 \quad 1.000 \mathrm{E}+00 \quad 0.000 \mathrm{E}+00$ $-1.867 \mathrm{E}-01 \quad 9.756 \mathrm{E}-01-1.155 \mathrm{E}-01$

$0-1.867 \mathrm{E}-019.756 \mathrm{E}-01+1.155 \mathrm{E}-01$ $\begin{array}{llll}-2.616 \mathrm{E}-01 & 2.336 \mathrm{E}-01-9.365 \mathrm{E}-01 & 1.230 \mathrm{E}+00 & 1.000 \mathrm{E}+00 \quad 0.000 \mathrm{E}+00\end{array}$ $-2.616 \mathrm{E}-01 \quad 2.336 \mathrm{E}-01-9.365 \mathrm{E}-01$

$0-2.616 \mathrm{E}-01 \quad 2.336 \mathrm{E}-01-9.365 \mathrm{E}-01$ $9.780 \mathrm{E}-01-7.641 \mathrm{E}-02-1.939 \mathrm{E}-01 \quad 1.083 \mathrm{E}+00 \quad 1.000 \mathrm{E}+00 \quad 0.000 \mathrm{E}+00$ $9.780 \mathrm{E}-01-7.641 \mathrm{E}-02-1.939 \mathrm{E}-01$

0 9.780E-01 -7.641E-02 +1.939E-01

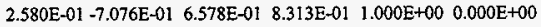
$2.580 \mathrm{E}-01-7.076 \mathrm{E}-01 \quad 6.578 \mathrm{E}-01$

0 $2.580 \mathrm{E}-01-7.076 \mathrm{E}-01 \quad 6.578 \mathrm{E}-01$

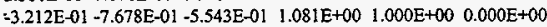
$-3.212 \mathrm{E}-01-7.678 \mathrm{E}-01-5.543 \mathrm{E}-01$

$0-3.212 \mathrm{E}-01-7.678 \mathrm{E}-01-5.543 \mathrm{E}-01$

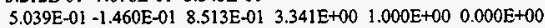
$5.039 \mathrm{E}-01-1.460 \mathrm{E}-01 \quad 8.513 \mathrm{E}-01$

0 5.039E-01 -1.460E-01 8.513E-01 $\begin{array}{llllll}6.080 \mathrm{E}-01 & 5.487 \mathrm{E}-01 & 5.738 \mathrm{E}-01 & 4.097 \mathrm{E}-01 & 1.000 \mathrm{E}+00 & 0.000 \mathrm{E}+00\end{array}$ $6.080 \mathrm{E}-01 \quad 5.487 \mathrm{E}-01 \quad 5.738 \mathrm{E}-01$

$0 \quad 6.080 \mathrm{E}-01 \quad 5.487 \mathrm{E}-015.738 \mathrm{E}-01$

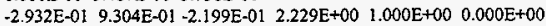
$641 \mathrm{E}+00 \quad 4.456 \mathrm{E}-01 \quad 2.000 \mathrm{E}-01 \quad 42(-5,3,0)$ $-2.932 E-01 \quad 9.304 E-01-2.199 E-01$ 
HNF-SD-SNF-CSWD-005, Rev. 0

3.641E+00 4.456E-01 2.000E-01 141

$2.000 \mathrm{E}-013.000 \mathrm{E}+00 \quad 2.000 \mathrm{E}-01 \quad 140$

$0-2.932 \mathrm{E}-01 \quad 9.304 \mathrm{E}-01-2.199 \mathrm{E}-01$

$\begin{aligned} & -8.475 \mathrm{E}-01 \\ & -3.993 \mathrm{E}-01\end{aligned}-3.497 \mathrm{E}-01 \quad 1.362 \mathrm{E}+00 \quad 1.000 \mathrm{E}+00 \quad 0.000 \mathrm{E}+00$

$.641 \mathrm{E}+00 \quad 4.456 \mathrm{E}-01 \quad 2.000 \mathrm{E}-01 \quad 42(-5,3,0) \quad-8.475 \mathrm{E}-01-3.993 \mathrm{E}-01-3.497 \mathrm{E}-01$

$3.641 \mathrm{E}+00 \quad 4.456 \mathrm{E}-01 \quad 2.000 \mathrm{E}-01 \quad 141,0-8.47 \mathrm{SE}-01-3.993 \mathrm{E}-01-3.497 \mathrm{E}-01$

$46 \quad 2.000 \mathrm{E}-01 \quad 3.000 \mathrm{E}+00 \quad 2.000 \mathrm{E}-01 \quad 140$

$3.641 \mathrm{E}+00 \quad 4.456 \mathrm{E}-01 \quad 2.000 \mathrm{E}-01 \quad 42(-5,3,0)$

$3.641 \mathrm{E}+00 \quad 4.456 \mathrm{E}-01 \quad 2.000 \mathrm{E}-01 \quad 141$

$472.000 \mathrm{E}-013.000 \mathrm{E}+00 \quad 2.000 \mathrm{E}-0 ! 140$

$1.200 \mathrm{E}-01-9.195 \mathrm{E}-01-3.743 \mathrm{E}-01 \quad 1.010 \mathrm{E}+00 \quad 1.000 \mathrm{E}+00 \quad 0.000 \mathrm{E}+00$

$1.200 \mathrm{E}-01-9.195 \mathrm{E}-01-3.743 \mathrm{E}-01$

0 1.200E-01 -9.195E-01 -3.743E-01

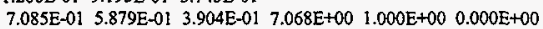

$3.641 \mathrm{E}+00 \quad 4.456 \mathrm{E}-01 \quad 2.000 \mathrm{E}-01 \quad 42(-5,3,0) \quad 7.085 \mathrm{E}-01 \quad 5.879 \mathrm{E}-01 \quad 3.904 \mathrm{E}-01$

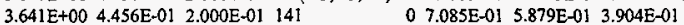

$48 \quad 2.000 \mathrm{E}-013.000 \mathrm{E}+00 \quad 2.000 \mathrm{E}-01 \quad 140$

$3.641 \mathrm{E}+00 \quad 4.456 \mathrm{E}-01 \quad 2.000 \mathrm{E}-01 \quad 42(-5,3,0)$

$3.641 \mathrm{E}+00 \quad 4.456 \mathrm{E}-01 \quad 2.000 \mathrm{E}-01 \quad 141$

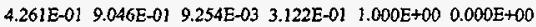

4.261E-01 9.046E-01 9.254E-03

$492.000 \mathrm{E}-01 \quad 3.000 \mathrm{E}+00 \quad 2.000 \mathrm{E}-01 \quad 140$

$3.641 \mathrm{E}+00 \quad 4.456 \mathrm{E}-012.000 \mathrm{E}-01 \quad 42(-5,3,0)$

$3.641 \mathrm{E}+00 \quad 4.456 \mathrm{E}-01 \quad 2.000 \mathrm{E}-01 \quad 141$

$50 \quad 2.000 \mathrm{E}-01 \quad 3.000 \mathrm{E}+00 \quad 2.000 \mathrm{E}-01 \quad 140$

$3.64 \mathrm{lE}+00 \quad 4.456 \mathrm{E}-01 \quad 2.000 \mathrm{E}-01 \quad 42(-5,3,0)$

$3.641 \mathrm{E}+00 \quad 4.456 \mathrm{E}-01 \quad 2.000 \mathrm{E}-01 \quad 141$

lestimated keff results by cycle

$4.261 \mathrm{E}-01 \quad 9.046 \mathrm{E}-01 \quad 9.254 \mathrm{E}-03$

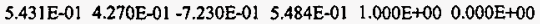

$5.431 \mathrm{E}-01 \quad 4.270 \mathrm{E}-01-7.230 \mathrm{E}-01$

0 5.431E-01 4.270E-01 -7.230E-01

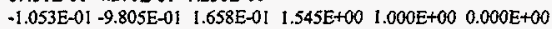

$-1.053 \mathrm{E}-01-9.805 \mathrm{E}-01 \quad 1.658 \mathrm{E}-01$

$0-1.053 \mathrm{E}-01-9.805 \mathrm{E}-01 \quad 1.658 \mathrm{E}-01$

print table 175

cycle $1 \mathrm{k}$ (collision) 1.253108 removal lifetime(abs) $1.7557 \mathrm{E}+01$ source points generated 257

cycle $2 \mathrm{k}$ (collision) 1.211997 removal lifetime(abs) $1.1671 \mathrm{E}+01$ source points generated 195

cycle $3 \mathrm{k}$ (collision) 1.200265 removal lifetime(abs) $9.6992 \mathrm{E}+00$ source points generated 193

$\therefore 4 \mathrm{k}$ (collision) 1.129530 removal lifetime(abs) $9.3720 \mathrm{E}+00$ source points generated 182

cycle 5 k(collision) 1.135994 removal lifetime(abs) $1.1136 \mathrm{E}+01$ source points generated 222

cycle $6 \mathrm{k}$ (collision) 1.077014 removal lifetime(abs) $1.2222 \mathrm{E}+01$ source points generated 204

yrce distribution written to file inpl $18 \mathrm{~s} \quad$ cycle $=6$

blem summary

run terminated when 6 kcode cycles were done. neutron creation tracks weight energy neutron loss tracks weight energy
(per source particle)

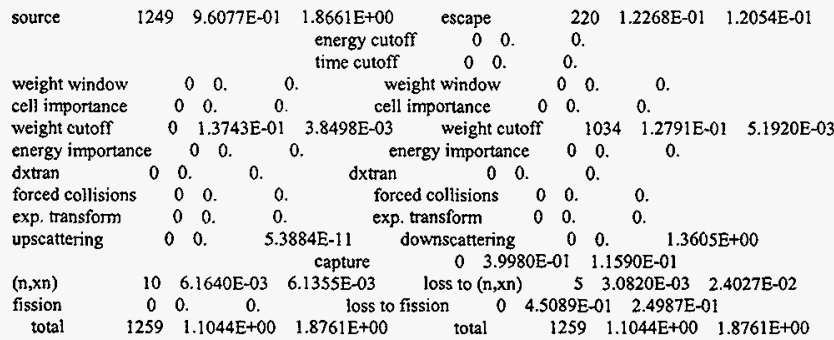

number of neutrons banked 5 average lifetime, shakes cutoffs

neutron tracks per source particle $1.0080 \mathrm{E}+00$ escape $3.7027 \mathrm{E}+00$ tco $1.0000 \mathrm{E}+34$

neutron collisions per source particle $1.8226 \mathrm{E}+01$ capture $1.2954 \mathrm{E}+01$ eco $0.0000 \mathrm{E}+00$

total neutron collisions 22764 capture or escape $1.1788 \mathrm{E}+01$ wcl $-5.0000 \mathrm{E}-01$

net multiplication $\quad 1.0032 \mathrm{E}+000.0035$ any termination $1.2744 \mathrm{E}+01$ wc2 $-2.5000 \mathrm{E}-01$

computer time so far in this run 0.00 minutes maximum number ever in bank

computer time in morm

urce particles per minute $\quad 0.0000 \mathrm{E}+00$

bank overflows to backup file 0

dom numbers generated $\quad 500880$

field length 0

most random numbers used was 1298 in history 
tracks population collisions collisions number flux average average cell entering

* weight weighted weighted track weight track mfp (per history) energy energy (relative) (cm)

$\begin{array}{cccccccccccc}1 & 30 & 0 & 0 & 0 & 0.0000 \mathrm{E}+00 & 0.0000 \mathrm{E}+00 & 0.0000 \mathrm{E}+00 & 0.0000 \mathrm{E}+00 & 0.0000 \mathrm{E}+00 \\ 2 & 31 & 0 & 0 & 0 & 0.0000 \mathrm{E}+00 & 0.0000 \mathrm{E}+00 & 0.0000 \mathrm{E}+00 & 0.0000 \mathrm{E}+00 & 0.0000 \mathrm{E}+00 \\ 3 & 37 & 0 & 0 & 0 & 0.0000 \mathrm{E}+00 & 0.0000 \mathrm{E}+00 & 0.0000 \mathrm{E}+00 & 0.0000 \mathrm{E}+00 & 0.0000 \mathrm{E}+00 \\ 4 & 34 & 0 & 0 & 0 & 0.0000 \mathrm{E}+00 & 0.0000 \mathrm{E}+00 & 0.0000 \mathrm{E}+00 & 0.0000 \mathrm{E}+00 & 0.0000 \mathrm{E}+00 \\ 5 & 32 & 0 & 0 & 0 & 0.0000 \mathrm{E}+00 & 0.0000 \mathrm{E}+00 & 0.0000 \mathrm{E}+00 & 0.0000 \mathrm{E}+00 & 0.0000 \mathrm{E}+00 \\ 6 & 33 & 0 & 0 & 0 & 0.0000 \mathrm{E}+00 & 0.0000 \mathrm{E}+00 & 0.0000 \mathrm{E}+00 & 0.0000 \mathrm{E}+00 & 0.0000 \mathrm{E}+00 \\ 7 & 35 & 0 & 0 & 0 & 0.0000 \mathrm{E}+00 & 0.0000 \mathrm{E}+00 & 0.0000 \mathrm{E}+00 & 0.0000 \mathrm{E}+00 & 0.0000 \mathrm{E}+00 \\ 8 & 36 & 0 & 0 & 0 & 0.0000 \mathrm{E}+00 & 0.0000 \mathrm{E}+00 & 0.0000 \mathrm{E}+00 & 0.0000 \mathrm{E}+00 & 0.0000 \mathrm{E}+00 \\ 9 & 38 & 920 & 618 & 1249 & 5.9116 \mathrm{E}-01 & 3.0414 \mathrm{E}-01 & 8.9304 \mathrm{E}-01 & 6.4212 \mathrm{E}-01 & 2.6324 \mathrm{E}+00 \\ 10 & 39 & 1635 & 637 & 130 & 6.3779 \mathrm{E}-02 & 8.8236 \mathrm{E}-02 & 7.6766 \mathrm{E}-01 & 6.4931 \mathrm{E}-01 & 2.6947 \mathrm{E}+00 \\ 11 & 40 & 1728 & 663 & 184 & 8.8176 \mathrm{E}-02 & 7.6162 \mathrm{E}-02 & 7.6184 \mathrm{E}-01 & 6.4437 \mathrm{E}-01 & 2.5146 \mathrm{E}+00 \\ 12 & 41 & 1867 & 712 & 317 & 1.5811 \mathrm{E}-01 & 8.6238 \mathrm{E}-02 & 7.9306 \mathrm{E}-01 & 6.5030 \mathrm{E}-01 & 2.6940 \mathrm{E}+00 \\ 13 & 140 & 0 & 0 & 0 & 0.0000 \mathrm{E}+00 & 0.0000 \mathrm{E}+00 & 0.0000 \mathrm{E}+00 & 0.0000 \mathrm{E}+00 & 0.0000 \mathrm{E}+00 \\ 14 & 42 & 2069 & 867 & 441 & 2.1911 \mathrm{E}-01 & 8.9382 \mathrm{E}-02 & 7.8771 \mathrm{E}-01 & 6.6777 \mathrm{E}-01 & 2.5091 \mathrm{E}+00 \\ 15 & 154 & 25302 & 1253 & 8436 & 4.1390 \mathrm{E}+00 & 1.2606 \mathrm{E}-01 & 8.5005 \mathrm{E}-01 & 6.6920 \mathrm{E}-01 & 2.4712 \mathrm{E}+00 \\ 16 & 149 & 49542 & 1253 & 580 & 2.8963 \mathrm{E}-01 & 1.0795 \mathrm{E}-01 & 8.2361 \mathrm{E}-01 & 6.7129 \mathrm{E}-01 & 2.5408 \mathrm{E}+00 \\ 17 & 144 & 51224 & 1254 & 885 & 4.2652 \mathrm{E}-01 & 1.0541 \mathrm{E}-01 & 8.1599 \mathrm{E}-01 & 6.6971 \mathrm{E}-01 & 2.2391 \mathrm{E}+00 \\ 18 & 159 & 58650 & 1254 & 4019 & 2.1254 \mathrm{E}+00 & 9.8931 \mathrm{E}-02 & 8.1154 \mathrm{E}-01 & 6.6977 \mathrm{E}-01 & 2.7541 \mathrm{E}+00 \\ 19 & 141 & 73122 & 1254 & 6523 & 3.2229 \mathrm{E}+00 & 9.4499 \mathrm{E}-02 & 8.0639 \mathrm{E}-01 & 6.6810 \mathrm{E}-01 & 2.5056 \mathrm{E}+00\end{array}$

total $266059 \quad 9765 \quad 22764 \quad 1.1324 \mathrm{E}+01$

Ineutron weight balance in each cell -- external events

print table 130

cell entering source energy time exiting total

$\begin{array}{lllllll}30 & 0.0000 E+00 & 0.0000 E+00 & 0.0000 E+00 & 0.0000 E+00 & 0.0000 E+00 & 0.0000 E+00\end{array}$

$\begin{array}{llllllll}31 & 0.0000 \mathrm{E}+00 & 0.0000 \mathrm{E}+00 & 0.0000 \mathrm{E}+00 & 0.0000 \mathrm{E}+00 & 0.0000 \mathrm{E}+00 & 0.0000 \mathrm{E}+00\end{array}$

$\begin{array}{llllllll}37 & 0.0000 E+00 & 0.0000 E+00 & 0.0000 E+00 & 0.0000 E+00 & 0.0000 E+00 & 0.0000 E+00\end{array}$

$\begin{array}{llllllll}4 & 34 & 0.0000 \mathrm{E}+00 & 0.0000 \mathrm{E}+00 & 0.0000 \mathrm{E}+00 & 0.0000 \mathrm{E}+00 & 0.0000 \mathrm{E}+00 & 0.0000 \mathrm{E}+00\end{array}$

$\begin{array}{lllllllll}5 & 32 & 0.0000 \mathrm{E}+00 & 0.0000 \mathrm{E}+00 & 0.0000 \mathrm{E}+00 & 0.0000 \mathrm{E}+00 & 0.0000 \mathrm{E}+00 & 0.0000 \mathrm{E}+00\end{array}$

$\begin{array}{lllllllll}6 & 33 & 0.0000 \mathrm{E}+00 & 0.0000 \mathrm{E}+00 & 0.0000 \mathrm{E}+00 & 0.0000 \mathrm{E}+00 & 0.0000 \mathrm{E}+00 & 0.0000 \mathrm{E}+00\end{array}$

$\begin{array}{lllllllll}7 & 35 & 0.0000 \mathrm{E}+00 & 0.0000 \mathrm{E}+00 & 0.0000 \mathrm{E}+00 & 0.0000 \mathrm{E}+00 & 0.0000 \mathrm{E}+00 & 0.0000 \mathrm{E}+00\end{array}$

$\begin{array}{lllllllll}8 & 36 & 0.0000 \mathrm{E}+00 & 0.0000 \mathrm{E}+00 & 0.0000 \mathrm{E}+00 & 0.0000 \mathrm{E}+00 & 0.0000 \mathrm{E}+00 & 0.0000 \mathrm{E}+00\end{array}$

$938 \quad 4.8822 \mathrm{E}-01 \quad 0.0000 \mathrm{E}+00 \quad 0.0000 \mathrm{E}+00 \quad 0.0000 \mathrm{E}+00 \quad-3.3091 \mathrm{E}-01 \quad 1.5731 \mathrm{E}-01$

$\begin{array}{lllllllll}10 & 39 & 8.4996 \mathrm{E}-01 & 0.0000 \mathrm{E}+00 & 0.0000 \mathrm{E}+00 & 0.0000 \mathrm{E}+00 & -8.4933 \mathrm{E}-01 & 6.2750 \mathrm{E}-04\end{array}$

$11 \quad 40 \quad 8.9904 \mathrm{E}-01 \quad 0.0000 \mathrm{E}+00 \quad 0.0000 \mathrm{E}+00 \quad 0.0000 \mathrm{E}+00 \quad-8.9904 \mathrm{E}-01 \quad 3.9460 \mathrm{E}-06$

$\begin{array}{lllllllll}12 & 41 & 9.7260 \mathrm{E}-01 & 0.0000 \mathrm{E}+00 & 0.0000 \mathrm{E}+00 & 0.0000 \mathrm{E}+00 & -9.6978 \mathrm{E}-01 & 2.8204 \mathrm{E}-03\end{array}$

$\begin{array}{lllllllll}13 & 140 & 0.0000 \mathrm{E}+00 & 0.0000 \mathrm{E}+00 & 0.0000 \mathrm{E}+00 & 0.0000 \mathrm{E}+00 & 0.0000 \mathrm{E}+00 & 0.0000 \mathrm{E}+00\end{array}$

$14 \quad 42 \quad 1.0907 \mathrm{E}+00 \quad 0.0000 \mathrm{E}+00 \quad 0.0000 \mathrm{E}+00 \quad 0.0000 \mathrm{E}+00-1.0913 \mathrm{E}+00-5.9111 \mathrm{E}-04$

$\begin{array}{llllllll}15 & 154 & 1.2943 \mathrm{E}+01 & 8.4067 \mathrm{E}-01 & 0.0000 \mathrm{E}+00 & 0.0000 \mathrm{E}+00 & -1.3171 \mathrm{E}+01 & 6.1278 \mathrm{E}-01\end{array}$

$\begin{array}{lllllllll}16 & 149 & 2.6508 \mathrm{E}+01 & 0.0000 \mathrm{E}+00 & 0.0000 \mathrm{E}+00 & 0.0000 \mathrm{E}+00 & -2.6508 \mathrm{E}+01 & 3.5884 \mathrm{E}-05\end{array}$

$\begin{array}{lllllllll}17 & 144 & 2.7419 \mathrm{E}+01 & 0.0000 \mathrm{E}+00 & 0.0000 \mathrm{E}+00 & 0.0000 \mathrm{E}+00 & -2.7405 \mathrm{E}+01 & 1.4572 \mathrm{E}-02\end{array}$

$\begin{array}{lllllllll}18 & 159 & 3.1331 \mathrm{E}+01 & 8.0064 \mathrm{E}-02 & 0.0000 \mathrm{E}+00 & 0.0000 \mathrm{E}+00 & -3.1361 \mathrm{E}+01 & 5.0067 \mathrm{E}-02\end{array}$

$\begin{array}{llllllll}19 & 141 & 3.9111 \mathrm{E}+01 & 4.0032 \mathrm{E}-02 & 0.0000 \mathrm{E}+00 & 0.0000 \mathrm{E}+00 & -3.9150 \mathrm{E}+01 & 4.5860 \mathrm{E}-04\end{array}$

total $\quad 1.4161 \mathrm{E}+02 \quad 9.6077 \mathrm{E}-01 \quad 0.0000 \mathrm{E}+00 \quad 0.0000 \mathrm{E}+00 \quad-1.4174 \mathrm{E}+02 \quad 8.3809 \mathrm{E}-01$

Ineutron weight balance in each cell - variance reduction events

print table 130

cel] wejght cell weight energy dxtran forced exponential total window importance cutoff importance collision transform

$\begin{array}{llllllllll}30 & 0.0000 \mathrm{E}+00 & 0.0000 \mathrm{E}+00 & 0.0000 \mathrm{E}+00 & 0.0000 \mathrm{E}+00 & 0.0000 \mathrm{E}+00 & 0.0000 \mathrm{E}+00 & 0.0000 \mathrm{E}+00 & 0.0000 \mathrm{E}+00\end{array}$

$\begin{array}{llllllllllll}2 & 31 & 0.0000 \mathrm{E}+00 & 0.0000 \mathrm{E}+00 & 0.0000 \mathrm{E}+00 & 0.0000 \mathrm{E}+00 & 0.0000 \mathrm{E}+00 & 0.0000 \mathrm{E}+00 & 0.0000 \mathrm{E}+00 & 0.0000 \mathrm{E}+00\end{array}$

$\begin{array}{llllllllllll}3 & 37 & 0.0000 \mathrm{E}+00 & 0.0000 \mathrm{E}+00 & 0.0000 \mathrm{E}+00 & 0.0000 \mathrm{E}+00 & 0.0000 \mathrm{E}+00 & 0.0000 \mathrm{E}+00 & 0.0000 \mathrm{E}+00 & 0.0000 \mathrm{E}+00\end{array}$

$\begin{array}{llllllllll}4 & 34 & 0.0000 \mathrm{E}+00 & 0.0000 \mathrm{E}+00 & 0.0000 \mathrm{E}+00 & 0.0000 \mathrm{E}+00 & 0.0000 \mathrm{E}+00 & 0.0000 \mathrm{E}+00 & 0.0000 \mathrm{E}+00 & 0.0000 \mathrm{E}+00\end{array}$

$\begin{array}{lllllllllll}5 & 32 & 0.0000 \mathrm{E}+00 & 0.0000 \mathrm{E}+00 & 0.0000 \mathrm{E}+00 & 0.0000 \mathrm{E}+00 & 0.0000 \mathrm{E}+00 & 0.0000 \mathrm{E}+00 & 0.0000 \mathrm{E}+00 & 0.0000 \mathrm{E}+00\end{array}$

$\begin{array}{lllllllllll}6 & 33 & 0.0000 \mathrm{E}+00 & 0.0000 \mathrm{E}+00 & 0.0000 \mathrm{E}+00 & 0.0000 \mathrm{E}+00 & 0.0000 \mathrm{E}+00 & 0.0000 \mathrm{E}+00 & 0.0000 \mathrm{E}+00 & 0.0000 \mathrm{E}+00\end{array}$

$\begin{array}{llllllllll}35 & 0.0000 E+00 & 0.0000 E+00 & 0.0000 E+00 & 0.0000 E+00 & 0.0000 E+00 & 0.0000 E+00 & 0.0000 E+00 & 0.0000 E+00\end{array}$

$\begin{array}{lllllllllll}36 & 0.0000 \mathrm{E}+00 & 0.0000 \mathrm{E}+00 & 0.0000 \mathrm{E}+00 & 0.0000 \mathrm{E}+00 & 0.0000 \mathrm{E}+00 & 0.0000 \mathrm{E}+00 & 0.0000 \mathrm{E}+00 & 0.0000 \mathrm{E}+00\end{array}$ 
HNF-SD-SNF-CSWD-005, Rev. 0

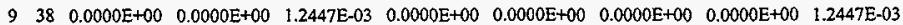
$\begin{array}{llllllllll}39 & 0.0000 \mathrm{E}+00 & 0.0000 \mathrm{E}+00 & 2.0097 \mathrm{E}-04 & 0.0000 \mathrm{E}+00 & 0.0000 \mathrm{E}+00 & 0.0000 \mathrm{E}+00 & 0.0000 \mathrm{E}+00 & 2.0097 \mathrm{E}-04\end{array}$ $\begin{array}{llllllllllll}40 & 0.0000 \mathrm{E}+00 & 0.0000 \mathrm{E}+00 & 0.0000 \mathrm{E}+00 & 0.0000 \mathrm{E}+00 & 0.0000 \mathrm{E}+00 & 0.0000 \mathrm{E}+00 & 0.0000 \mathrm{E}+00 & 0.0000 \mathrm{E}+00\end{array}$ $\begin{array}{llllllllll}41 & 0.0000 \mathrm{E}+00 & 0.0000 \mathrm{E}+00 & 2.9100 \mathrm{E}-04 & 0.0000 \mathrm{E}+00 & 0.0000 \mathrm{E}+00 & 0.0000 \mathrm{E}+00 & 0.0000 \mathrm{E}+00 & 2.9100 \mathrm{E}-04\end{array}$ $\begin{array}{lllllllllll}13 & 140 & 0.0000 \mathrm{E}+00 & 0.0000 \mathrm{E}+00 & 0.0000 \mathrm{E}+00 & 0.0000 \mathrm{E}+00 & 0.0000 \mathrm{E}+00 & 0.0000 \mathrm{E}+00 & 0.0000 \mathrm{E}+00 & 0.0000 \mathrm{E}+00\end{array}$ $\begin{array}{llllllllllll}14 & 42 & 0.0000 \mathrm{E}+00 & 0.0000 \mathrm{E}+00 & 0.0000 \mathrm{E}+00 & 0.0000 \mathrm{E}+00 & 0.0000 \mathrm{E}+00 & 0.0000 \mathrm{E}+00 & 0.0000 \mathrm{E}+00 & 0.0000 \mathrm{E}+00\end{array}$ $\begin{array}{llllllllllll}15 & 154 & 0.0000 \mathrm{E}+00 & 0.0000 \mathrm{E}+00 & 8.0354 \mathrm{E}-03 & 0.0000 \mathrm{E}+00 & 0.0000 \mathrm{E}+0 & 0.0000 \mathrm{E}+00 & 0.0000 \mathrm{E}+00 & 8.0354 \mathrm{E}-03\end{array}$ $\begin{array}{lllllllllllll}16 & 149 & 0.0000 \mathrm{E}+00 & 0.0000 \mathrm{E}+00 & 0.0000 \mathrm{E}+00 & 0.0000 \mathrm{E}+00 & 0.0000 \mathrm{E}+00 & 0.0000 \mathrm{E}+00 & 0.0000 \mathrm{E}+00 & 0.0000 \mathrm{E}+00\end{array}$

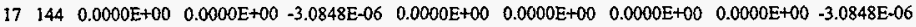
$\begin{array}{lllllllllll}18 & 159 & 0.0000 \mathrm{E}+00 & 0.0000 \mathrm{E}+00 & -2.5216 \mathrm{E}-04 & 0.0000 \mathrm{E}+00 & 0.0000 \mathrm{E}+00 & 0.0000 \mathrm{E}+00 & 0.0000 \mathrm{E}+00 & -2.5216 \mathrm{E}-04\end{array}$ $19 \quad 141 \quad 0.0000 E+00 \quad 0.0000 E+00 \quad 0.0000 E+00 \quad 0.0000 E+00 \quad 0.0000 E+00 \quad 0.0000 E+00 \quad 0.0000 E+00 \quad 0.0000 E+00$

$\begin{array}{lllllllll}\text { total } & 0.0000 \mathrm{E}+00 & 0.0000 \mathrm{E}+00 & 9.5169 \mathrm{E}-03 & 0.0000 \mathrm{E}+0 & 0.0000 \mathrm{E}+00 & 0.0000 \mathrm{E}+00 & 0.0000 \mathrm{E}+00 & 9.5169 \mathrm{E}-03\end{array}$ Ineutron weight balance in each cell - physical events print table 130

$$
\text { cell (n,xn) fission capture loss to loss to total }
$$

I $30 \quad 0.0000 \mathrm{E}+00 \quad 0.0000 \mathrm{E}+00 \quad 0.0000 \mathrm{E}+00 \quad 0.0000 \mathrm{E}+00 \quad 0.0000 \mathrm{E}+00 \quad 0.0000 \mathrm{E}+00$

$\begin{array}{llllllllll}2 & 31 & 0.0000 \mathrm{E}+00 & 0.0000 \mathrm{E}+00 & 0.0000 \mathrm{E}+00 & 0.0000 \mathrm{E}+00 & 0.0000 \mathrm{E}+00 & 0.0000 \mathrm{E}+00\end{array}$

$\begin{array}{llllllllll}3 & 37 & 0.0000 \mathrm{E}+00 & 0.0000 \mathrm{E}+00 & 0.0000 \mathrm{E}+00 & 0.0000 \mathrm{E}+00 & 0.0000 \mathrm{E}+00 & 0.0000 \mathrm{E}+00\end{array}$

$434 \quad 0.0000 \mathrm{E}+00 \quad 0.0000 \mathrm{E}+00 \quad 0.0000 \mathrm{E}+00 \quad 0.0000 \mathrm{E}+00 \quad 0.0000 \mathrm{E}+00 \quad 0.0000 \mathrm{E}+00$

$\begin{array}{lllllllll}5 & 32 & 0.0000 \mathrm{E}+00 & 0.0000 \mathrm{E}+00 & 0.0000 \mathrm{E}+00 & 0.0000 \mathrm{E}+00 & 0.0000 \mathrm{E}+00 & 0.0000 \mathrm{E}+00\end{array}$

$\begin{array}{llllllll}6 & 33 & 0.0000 \mathrm{E}+00 & 0.0000 \mathrm{E}+00 & 0.0000 \mathrm{E}+00 & 0.0000 \mathrm{E}+00 & 0.0000 \mathrm{E}+00 & 0.0000 \mathrm{E}+00\end{array}$

$\begin{array}{lllllllll}7 & 35 & 0.0000 \mathrm{E}+00 & 0.0000 \mathrm{E}+00 & 0.0000 \mathrm{E}+00 & 0.0000 \mathrm{E}+00 & 0.0000 \mathrm{E}+00 & 0.0000 \mathrm{E}+00\end{array}$

$\begin{array}{llllllllllll}8 & 36 & 0.0000 \mathrm{E}+00 & 0.0000 \mathrm{E}+00 & 0.0000 \mathrm{E}+00 & 0.0000 \mathrm{E}+00 & 0.0000 \mathrm{E}+00 & 0.0000 \mathrm{E}+00\end{array}$

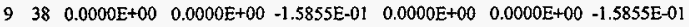

$10 \quad 39 \quad 0.0000 \mathrm{E}+00 \quad 0.0000 \mathrm{E}+00 \quad-8.2847 \mathrm{E}-04 \quad 0.0000 \mathrm{E}+00 \quad 0.0000 \mathrm{E}+00 \quad-8.2847 \mathrm{E}-04$

$1140 \quad 0.0000 \mathrm{E}+00 \quad 0.0000 \mathrm{E}+00 \quad-3.9460 \mathrm{E}-06 \quad 0.0000 \mathrm{E}+00 \quad 0.0000 \mathrm{E}+00 \quad-3.9460 \mathrm{E}-06$

$\begin{array}{lllllllll}12 & 41 & 0.0000 \mathrm{E}+00 & 0.0000 \mathrm{E}+00 & -3.1114 \mathrm{E}-03 & 0.0000 \mathrm{E}+00 & 0.0000 \mathrm{E}+00 & -3.1114 \mathrm{E}-03\end{array}$

$\begin{array}{lllllllllll}13 & 140 & 0.0000 \mathrm{E}+00 & 0.0000 \mathrm{E}+00 & 0.0000 \mathrm{E}+00 & 0.0000 \mathrm{E}+00 & 0.0000 \mathrm{E}+00 & 0.0000 \mathrm{E}+00\end{array}$

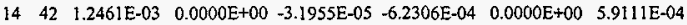

$\begin{array}{llllllll}15 & 154 & 4.9179 \mathrm{E}-03 & 0.0000 \mathrm{E}+00 & -1.7239 \mathrm{E}-01 & -2.4589 \mathrm{E}-03 & -4.5089 \mathrm{E}-01 & -6.2082 \mathrm{E}-01\end{array}$

$\begin{array}{llllllll}6 & 149 & 0.0000 E+00 & 0.0000 E+00 & -3.5884 E-05 & 0.0000 E+00 & 0.0000 E+00 & -3.5884 E-05\end{array}$

$144 \quad 0.0000 \mathrm{E}+00 \quad 0.0000 \mathrm{E}+00 \quad-1.4569 \mathrm{E}-02 \quad 0.0000 \mathrm{E}+00 \quad 0.0000 \mathrm{E}+00 \quad-1.4569 \mathrm{E}-02$

$\begin{array}{llllllll}18 & 159 & 0.0000 \mathrm{E}+00 & 0.0000 \mathrm{E}+00 & -4.9815 \mathrm{E}-02 & 0.0000 \mathrm{E}+00 & 0.0000 \mathrm{E}+00 & -4.9815 \mathrm{E}-02\end{array}$

$\begin{array}{lllllll}19 & 141 & 0.0000 \mathrm{E}+00 & 0.0000 \mathrm{E}+0 & -4.5860 \mathrm{E}-04 & 0.0000 \mathrm{E}+0.0 .0000 \mathrm{E}+00 & -4.5860 \mathrm{E}-04\end{array}$

total $\quad 6.1640 \mathrm{E}-03 \quad 0.0000 \mathrm{E}+00 \quad-3.9980 \mathrm{E}-01 \quad-3.0820 \mathrm{E}-03 \quad-4.5089 \mathrm{E}-01 \quad-8.4760 \mathrm{E}-01$

ineutron activity of each nuclide in each cell, per source particle

print table 140

cell nuclides atom total collisions weight lost weight loss weight gain fraction collisions * weight to capture to fission by $(n, x n)$

$\begin{array}{lllll}9 & 38 & 5010.03 d & 7.1977 \mathrm{E}-01\end{array}$ $5011.40 \mathrm{c} \quad 8.0041 \mathrm{E}-02$ $6012.40 \mathrm{c} \quad 2.0019 \mathrm{E}-01$

$10 \quad 39 \quad 41093.40 c \quad 9.8982 \mathrm{E}-01$ $40000.40 \mathrm{C} \quad 1.0183 \mathrm{E}-02$

$1140 \quad 1001.00 \mathrm{c} \quad 3.3333 \mathrm{E}-01$ $1002.55 \mathrm{c} \quad 3.3333 \mathrm{E}-0$ ] 8016.40 c 3.3333E-01

$1241 \quad 41093.40 c \quad 9.8982 \mathrm{E}-01$ $40000.40 \mathrm{C} \quad 1.0183 \mathrm{E}-02$

$14 \quad 42 \quad 1001.00 \mathrm{c} \quad 3.3333 \mathrm{E}-01$ $1002.55 \mathrm{c}$ 3.3333E-0] $8016.40 \mathrm{c} \quad 3.3333 \mathrm{E}-01$

I5 $154 \quad 92235.40 \mathrm{c} \quad 3.7504 \mathrm{E}-01$ $92238.40 \mathrm{c} \quad 1.2499 \mathrm{E}-01$ $7014.40 \mathrm{c} \quad 4.9997 \mathrm{E}-01$

$1001.00 \mathrm{c} \quad 3.3333 \mathrm{E}-01$
$941 \quad 4.4212 \mathrm{E}-01 \quad 1.5853 \mathrm{E}-01 \quad 0.0000 \mathrm{E}+00 \quad 0.0000 \mathrm{E}+00$ $\begin{array}{llllll}76 & 3.6073 \mathrm{E}-02 & 1.3591 \mathrm{E}-07 & 0.0000 \mathrm{E}+00 & 0.0000 \mathrm{E}+00\end{array}$ $232 \quad 1.1297 \mathrm{E}-01 \quad 1.8446 \mathrm{E}-05 \quad 0.0000 \mathrm{E}+00 \quad 0.0000 \mathrm{E}+00$

$128 \quad 6.2802 \mathrm{E}-02 \quad 8.2814 \mathrm{E}-04 \quad 0.0000 \mathrm{E}+00 \quad 0.0000 \mathrm{E}+00$ $2 \quad 9.7676 \mathrm{E}-04 \quad 3.3160 \mathrm{E}-07 \quad 0.0000 \mathrm{E}+00 \quad 0.0000 \mathrm{E}+00$

$111 \quad 5.1533 \mathrm{E}-02 \quad 3.9251 \mathrm{E}-06 \quad 0.0000 \mathrm{E}+00 \quad 0.0000 \mathrm{E}+00$ $33 \quad 1.5373 \mathrm{E}-02 \quad 2.0884 \mathrm{E}-08 \quad 0.0000 \mathrm{E}+00 \quad 0.0000 \mathrm{E}+00$ $\begin{array}{lllll}40 & 2.1271 E-02 & 0.0000 E+00 & 0.0000 E+00 & 0.0000 E+00\end{array}$

$313 \quad 1.5623 \mathrm{E}-01 \quad 3.1072 \mathrm{E}-03 \quad 0.0000 \mathrm{E}+00 \quad 0.0000 \mathrm{E}+00$ $4 \quad 1.8747 E-03 \quad 4.2322 E-06 \quad 0.0000 E+00 \quad 0.0000 E+00$

$257 \quad 1.2131 \mathrm{E}-01 \quad 4.3914 \mathrm{E}-06 \quad 0.0000 \mathrm{E}+00 \quad 0.0000 \mathrm{E}+00$ $\begin{array}{lllll}86 & 4.6121 \mathrm{E}-02 & 6.8027 \mathrm{E}-08 & 0.0000 \mathrm{E}+00 & 6.2306 \mathrm{E}-04\end{array}$ $\begin{array}{lllll}98 & 5.1682 \mathrm{E}-02 & 2.7495 \mathrm{E}-0.5 & 0.0000 \mathrm{E}+00 & 0.0000 \mathrm{E}+00\end{array}$

$4759 \quad 2.3227 \mathrm{E}+00 \quad 1.3244 \mathrm{E}-01 \quad 4.4247 \mathrm{E}-01 \quad 5.8410 \mathrm{E}-04$ $1470 \quad 7.3870 \mathrm{E}-01 \quad 2.3272 \mathrm{E}-02 \quad 8.4162 \mathrm{E}-03 \quad 1.8748 \mathrm{E}-03$ $\begin{array}{lllll}2207 & 1.0775 \mathrm{E}+00 & 1.6682 \mathrm{E}-02 & 0.0000 \mathrm{E}+00 & 0.0000 \mathrm{E}+00\end{array}$ 
$\begin{array}{lllllll}1002.55 \mathrm{c} & 3.3333 \mathrm{E}-01 & 110 & 5.8433 \mathrm{E}-02 & 9.1921 \mathrm{E}-08 & 0.0000 \mathrm{E}+00 & 0.0000 \mathrm{E}+00\end{array}$

$8016.40 \mathrm{c} \quad 3.3333 \mathrm{E}-01 \quad 125 \quad 6.3072 \mathrm{E}-02 \quad 3.1305 \mathrm{E}-05 \quad 0.0000 \mathrm{E}+00 \quad 0.0000 \mathrm{E}+00$

$144 \quad 74000.40 \mathrm{c} \quad 1.0000 \mathrm{E}+00 \quad 885 \quad 4.2652 \mathrm{E}-01 \quad 1.4569 \mathrm{~J}-02 \quad 0.0000 \mathrm{E}+00 \quad 0.0000 \mathrm{E}+00$

$18 \quad 159 \quad 41093.40 \mathrm{c} \quad 9.8982 \mathrm{E}-01$

$3965 \quad 2.1009 \mathrm{E}+00 \quad 4.9769 \mathrm{E}-02 \quad 0.0000 \mathrm{E}+00 \quad 0.0000 \mathrm{E}+00$ $40000.40 \mathrm{c} \quad 1.0183 \mathrm{E}-02$

$54 \quad 2.4590 \mathrm{E}-02 \quad 4.6175 \mathrm{E}-05 \quad 0.0000 \mathrm{E}+00 \quad 0.0000 \mathrm{E}+00$

$19 \quad 141 \quad 1001.00 \mathrm{c} \quad 3.3333 \mathrm{E}-01$ $1002.55 \mathrm{c} \quad 3.3333 \mathrm{E}-01$

$4029 \quad 1.9096 \mathrm{E}+00 \quad 7.9511 \mathrm{E}+05 \quad 0.0000 \mathrm{E}+00 \quad 0.0000 \mathrm{E}+00$ $1122 \quad 5.8206 \mathrm{E}-01 \quad 9.0056 \mathrm{E}-07 \quad 0.0000 \mathrm{E}+00 \quad 0.0000 \mathrm{E}+00$ $\begin{array}{lllllll}8016.40 \mathrm{c} & 3.3333 \mathrm{E}-01 & 1372 & 7.3131 \mathrm{E}-01 & 3.7819 \mathrm{E}-04 & 0.0000 \mathrm{E}+00 & 0.0000 \mathrm{E}+00\end{array}$

tota]

$22764 \quad 1.1324 \mathrm{E}+01 \quad 3.9980 \mathrm{E}-01 \quad 4.5089 \mathrm{E}-01 \quad 3.0820 \mathrm{E}-03$

total over all cells for each nuclide total collisions weight lost weight loss weight gain collisions * weight to capture to fission by $(n, x n)$

$\begin{array}{lcccccc}1001.00 c & 4742 & 2.2505 \mathrm{E}+00 & 9.2315 \mathrm{E}-05 & 0.0000 \mathrm{E}+00 & 0.0000 \mathrm{E}+00 \\ 1002.55 \mathrm{c} & 1351 & 7.0199 \mathrm{E}-01 & 1.0814 \mathrm{E}-06 & 0.0000 \mathrm{E}+00 & 6.2306 \mathrm{E}-04 \\ 5011.40 \mathrm{c} & 76 & 3.6073 \mathrm{E}-02 & 1.3591 \mathrm{E}-07 & 0.0000 \mathrm{E}+00 & 0.0000 \mathrm{E}+00 \\ 6012.40 \mathrm{c} & 232 & 1.1297 \mathrm{E}-01 & 1.8446 \mathrm{E}-05 & 0.0000 \mathrm{E}+00 & 0.0000 \mathrm{E}+00 \\ 7014.40 \mathrm{c} & 2207 & 1.0775 \mathrm{E}+00 & 1.6682 \mathrm{E}-02 & 0.0000 \mathrm{E}+00 & 0.0000 \mathrm{E}+00 \\ 8016.40 \mathrm{c} & 1635 & 8.6733 \mathrm{E}-01 & 4.3699 \mathrm{E}-04 & 0.0000 \mathrm{E}+00 & 0.0000 \mathrm{E}+00 \\ 40000.40 \mathrm{c} & 60 & 2.7441 \mathrm{E}-02 & 5.0739 \mathrm{E}-05 & 0.0000 \mathrm{E}+00 & 0.0000 \mathrm{E}+00 \\ 41093.40 \mathrm{c} & 4406 & 2.3199 \mathrm{E}+00 & 5.3704 \mathrm{E}-02 & 0.0000 \mathrm{E}+00 & 0.0000 \mathrm{E}+00 \\ 74000.40 \mathrm{c} & 885 & 4.2652 \mathrm{E}-01 & 1.4569 \mathrm{E}-02 & 0.0000 \mathrm{E}+00 & 0.0000 \mathrm{E}+00 \\ 92235.40 \mathrm{c} & 4759 & 2.3227 \mathrm{E}+00 & 1.3244 \mathrm{E}-01 & 4.4247 \mathrm{E}-01 & 5.8410 \mathrm{E}-04 \\ 92238.40 \mathrm{c} & 1470 & 7.3870 \mathrm{E}-01 & 2.3272 \mathrm{E}-02 & 8.4162 \mathrm{E}-03 & 1.8748 \mathrm{E}-03 \\ 5010.03 \mathrm{~d} & 941 & 4.4212 \mathrm{E}-01 & 1.5853 \mathrm{E}-01 & 0.0000 \mathrm{E}+00 & 0.0000 \mathrm{E}+00\end{array}$

the initial fission neutron source distribution used the 8 source points that were input on the kstc card. the criticality problem was scheduled to skip 5 cycles and run a total of 6 cycles with nominally 200 neutrons per cycle. s problem has run 5 inactive cycles with 1027 neutron histories and 1 active cycles with 222 neutron histories.

this calculation has completed the requested number of keff cycles using a total of 1249 fission neutron source histories. all cells with fissionable material were sampled and had fission neutron source points.

there is no combined collision/absorption/track-length estimate for keff because only 1 active cycles were run. lindividuat and average keff estimator results by cycle

keff neutron keff estimators by cycle average keff estimators and deviations average $k(\mathrm{c} / \mathrm{a} / \mathrm{t})$ cycle histories $k$ (coll) $k$ (abs) $k$ (track) $k$ (coll) st dev $k(a b s)$ st dev $k$ (track) st dev $k(c / a / t)$ st dev fom

\begin{tabular}{lllll|}
1 & $200 \mid 1.25311$ & 1.26302 & 1.22009 \\
2 & $257 \mid 1.21200$ & 1.25252 & 1.12957 \\
3 & $195 \mid 1.20026$ & 1.14326 & 1.13006 \\
4 & $193 \mid 1.12953$ & 1.16269 & 1.10757 \\
5 & $182 \mid 1.13599$ & 1.12465 & 1.11238 \\
\hline
\end{tabular}

$222|1.07701 \quad 1.046691 .07528|$

Itally 4 nps $=1249$

fuel rod fiux in $5 y$ locations averaged over $5 x$ elements

tally type 4 track length estimate of particie flux. units $1 / \mathrm{cm}^{* * 2}$

tally for neutrons

number of histories used for nomalizing tallies $=200.00$

cell $a$ is $(154<42[-10-10] 42[-9-10] 42[-8+10] 42[-7-10] 42[-6-10])$

cell $b$ is $(154<42[-1030] 42[-930] 42[-830] 42[-730] 42[-630])$

cell $c$ is $\left(\begin{array}{llll}154<42[-10 & 10 & 0\end{array}\right) 42\left[\begin{array}{lll}-9 & 10 & 0\end{array}\right) 42\left[\begin{array}{llll}-8 & 10 & 0\end{array}\right) 42\left[\begin{array}{lll}-7 & 10 & 0\end{array}\right] 42\left[\begin{array}{lll}-6 & 10 & 0\end{array}\right)$

cell $\mathrm{d}$ is $\left(154<42[-10210] 42[-9210] 42[-8210] 42[-7210] 42\left[\begin{array}{lll}-6 & 21 & 0\end{array}\right)\right.$

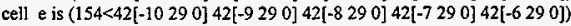

volumes

$$
\begin{array}{ccccc}
\text { cell: } \stackrel{a}{a} & b & c & d & e \\
1.04709 \mathrm{E}+02 & 1.04709 \mathrm{E}+02 & 1.04709 \mathrm{E}+02 & 1.04709 \mathrm{E}+02 & 1.04709 \mathrm{E}+02
\end{array}
$$




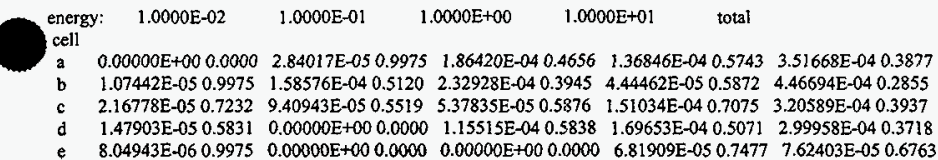

Ianalysis of the results in the tally fluctuation chart bin (tfc) for tally 4 with nps = 1249 print table 160

normed average tally per history $=3.51668 \mathrm{E}-04$

estimated tally relative errot $=0.3877$

relative error from zero tallies $=0.3464$

number of nonzero history tallies $=\quad 8$

fistory number of largest tally $=\quad 1034$

(largest tally) $/$ (average tally) $=3.84476 \mathrm{E}+01$

(confidence interval shift) $/$ mean $=0.0793$ unnormed average tally per history $=3.68228 \mathrm{E}-02$

estimated variance of the variance $=0.1752$

relative error from nonzero scores $=0.1740$

efficiency for the nonzero tallies $=0.0400$

largest unnormalized history tally $=1.41575 \mathrm{E}+00$

(largest tally) $($ avg nonzero tally) $=1.53791 \mathrm{E}+00$

shifted confidence interval center $=3.79539 \mathrm{E}-04$

if the largest history score sampled so far were to occur on the very next history, the tfc bin quantities would change as follows: nps $=\quad 222$ for this table because 5 keff cycles and $\quad 2027$ histories were skipped before tally accumulation.

$\begin{array}{lccc}\text { estimated quantities } & \text { value at nps } & \text { value at nps+1 } & \text { value(nps+1)/value(nps } \\ & & & \\ \text { mean } & 3.51668 \mathrm{E}-04 & 4.10723 \mathrm{E}-04 & 0.167927 \\ \text { relative eror } & 3.87653 \mathrm{E}-01 & 3.61597 \mathrm{E}-0 \mathrm{j} & -0.067213 \\ \text { variance of the variance } & 1.75161 \mathrm{E}-01 & 1.47665 \mathrm{E}-01 & -0.156976 \\ \text { shifted center } & 3.79539 \mathrm{E}-04 & 3.77169 \mathrm{E}-04 & -0.006244 \\ \text { figure of merit } & 0.00000 \mathrm{E}+00 & 0.00000 \mathrm{E}+00 & 0.000000\end{array}$

re is not enough information in the largest history scores (usually less than 500 scores) for a reliable estimate of the slope. history score probability density function appears to have an unsampled region at the largest history scores: please examine.

***** the nps-dependent tfe bin check results are suspect because there are only 1 nps tally values to analyze *****

results of 10 statistical checks for the estimated answer for the tally fluctuation chart (tfc) bin of tally 4

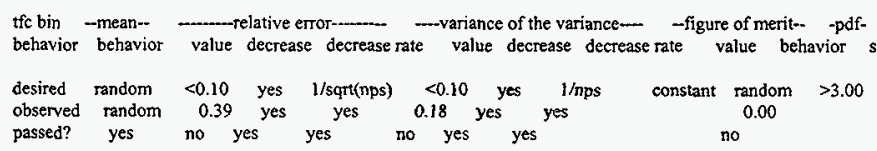

warning. the tally in the tally fluctuation chart bin did not pass 3 of the 10 statistical checks.

lunnormed tally density for tally 4 nonzero tally mean $(\mathrm{m})=9.206 \mathrm{E}-01$ nps $=1249$ print table 161

abscissa ordinate $\log$ plot of tally probability density function in tally fluctuation chart bin( $d=$ decade,slope $=0.0)$

tally number num den log den:d-...........

$1.00-01$ i $2.43-01-0.614^{* * * * * * * * * * * * * * * * * * * * * * * * * * * * * * * * * * * * * * * * * * * * * * * * * * * * * * * * * * * * * * * * * * * * * * * * * * * * * * * * * * * * * * * * * * * * * * * \mid *}$

$\begin{array}{lll}1.26-01 & 00.00+00 & 0.000\end{array}$

$\begin{array}{ccc}1.58-01 & 0.000 \div 00 & 0.000\end{array}$

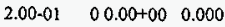

$00.00+00 \quad 0.000$

$00.00+00 \quad 0.000$ 


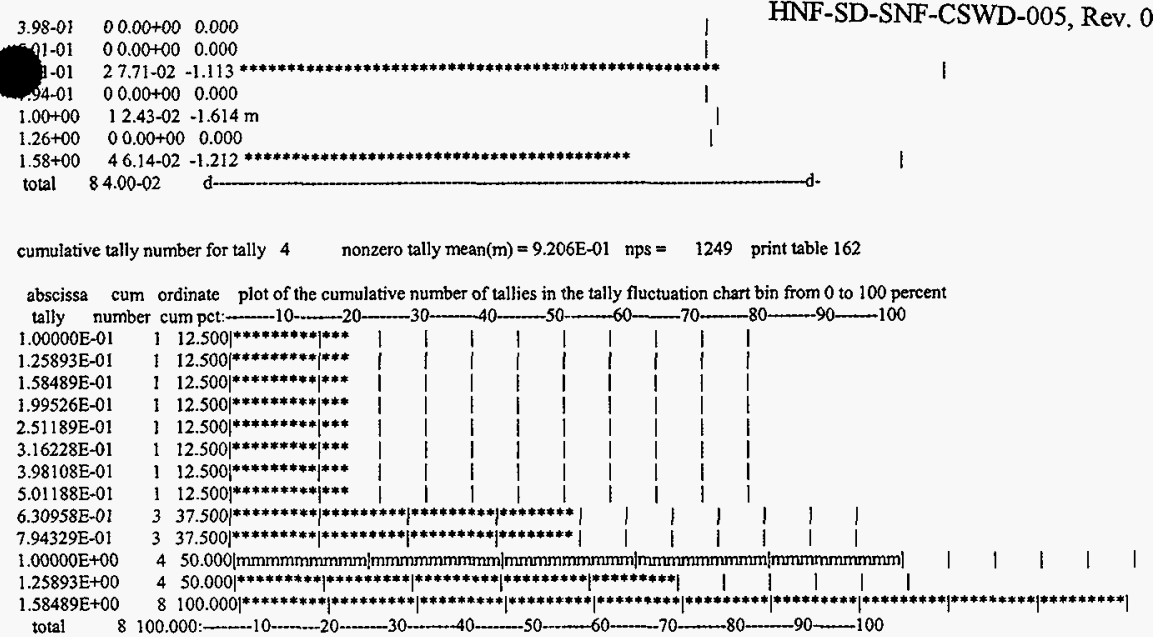

cumulative unnormed tally for tally 4 nonzero tally mean $(m)=9.206 \mathrm{E}-01$ nps $=1249$ print table 162

abscissa cum ordinate plot of the cumulative tally in the tally fluctuation chart bin from 0 to 100 percent

tally/nps cum pct:-

DOE-01 4.632E-04 $\left.1.258\right|^{*}$

$1.259 \mathrm{E}-01 \quad 4.632 \mathrm{E}-\left.04 \quad 1.258\right|^{*}$

$\begin{array}{lll}1.585 \mathrm{E}-01 & 4.632 \mathrm{E}-04 & \left.1.258\right|^{*}\end{array}$

$1.995 \mathrm{E}-01 \quad 4.632 \mathrm{E}-04 \quad 1.2581^{*}$

$2.512 \mathrm{E}-01 \quad 4.632 \mathrm{E}-\left.04 \quad 1.258\right|^{*}$

3.162E-01 4.632E-04 $\left.1.258\right|^{*}$

$3.981 \mathrm{E}-01 \quad 4.632 \mathrm{E}-\left.04 \quad 1.258\right|^{*}$

5.012E-01 4.632E-04 1.258$]^{*}$

$6.310 \mathrm{E}-016.195 \mathrm{E}-\left.03 \quad 16.825\right|^{* * * * * * * * * * * * * * * *}$

$7.943 \mathrm{E}-01$ 6.19SE-03 $\left.16.825\right|^{* * * * * * * * * * ; * * * * * * *}$

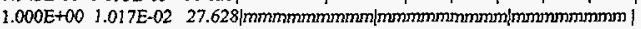

$1.259 \mathrm{E}+00 \quad 1.017 \mathrm{E}-\left.\left.\left.02 \quad 27.628\right|^{* * * * * * * * *}\right|^{* * * * * * * * *}\right|^{* * * * * * * *} \mid$

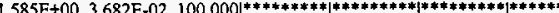

total $3.68228 \mathrm{E}-02100.000:-1$

$10-20$

$-30$

$40--50$
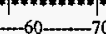

70.

Istatus of the statistical checks used to form confidence intervals for the mean for each tally bin

tally result of statistical checks for the tf $\mathrm{c}$ bin (the first check not passed is listed) and error magnitude check for all bins

4 missed 3 of $10 \mathrm{tfc}$ bin checks: the relative error exceeds the recommended value of 0.1 for nonpoint detector tallies missed all bin error check: 25 tally bins had 4 bins with zeros and 21 bins with relative errors exceeding 0.10

the 10 statistical checks are only for the tally fluctuation chart bin and do not apply to other tally bins.

the tally bins with zeros may or may not be correct: compare the source, cutoffs, multipliers, et cetera with the tally bins.

warning. 1 of the 1 tally fluctuation chart bins did not pass all 10 statistical checks.

waming. 1 of the 1 tallies had bins with relative errors greater than recommended.

Itally fluctuation charts

tally 4

nps mean error vov slope fom 
$10000.0000 \mathrm{E}+000.00000 .00000 .0$ $2493.5167 \mathrm{E}-040.38770 .1752 \quad 0.0$
0.000

$\checkmark$ data written to file inp $18 \mathrm{~m}$

cii file inp $18 \mathrm{p}$ written with 5 events from 5 histories.

6 warning messages so far.

run terminated when 6 kcode cycles were done.

\section{File: Outp18}

1- probit8 - kcode in a hexagonal prism lattice.

2- c three half control rods and five whole control rods.

3- $30 \quad 0-905-19291$ fill $=1$

4- $\quad 31 \quad 0-906-1929$, fill $=1(16.711300)$

5. $\quad 37 \quad 0.907-1929$ i fill $=1 \quad(-16.711300)$

6. $\quad 34 \quad 0-913-1929$ fill $=1(011.91850)$

7- $\quad 32 \quad 0-914-1929$ fill $=1(10.32175 .95920)$

8. $33 \quad 0-915-1929$ fill $=1(8.3557 \quad 14.47240)$

9- $35 \quad 0-916-1929$ fill $=1 \quad(-8.355714 .47240)$

10. $36 \quad 0-917-1929$ fill $=1(-10.32175 .95920)$

11- c universe 1: structure of control rod.

12- $\quad 38 \quad 11 \quad-2.02 \quad-880 \quad$ u=1 $\$$ control rod core

13- $39 \quad 6 \quad-8.4 \quad 880-881 \quad u=1 \quad \$$ control rod cladding

14- $40 \quad 12+1.00 \quad 88 !-882 \mathrm{u}=1$ \& control rod gap

15- $\quad 41 \quad 6 \quad-8.4 \quad 882 \quad u=1 \quad \$$ control rod sheath

16- $c$ the space between the control rods, filled with lattice.

17- $\quad 140 \quad 0 \quad-17129-19905906907913914915916917$ fill $=2$

18- c universe 2: lattice of fuel rods with water in between.

19- $42 \quad 12-1.00-301302-303 \quad 304-305306 \quad u=2$ lat $=2$ fill=

20- $\quad-37: 27-1: 330: 0 \&$

21- $\quad 24 r 39 r 24 r 311$ r 24 r 311 r 24 4r 39 r 2

$24 r 39 r 23 r 312 r 23 r 312 r 23 r 39 r 21 r$

$23 r 310 r 22 r 313 r 22 r 313 r 22 r 310 r 2 \mathrm{lr}$

$23 r 357 r 22 r \&$

$22 \mathrm{r} 358 \mathrm{r} 22 \mathrm{r}$

$22 \mathrm{r} 316 \mathrm{r} 22 \mathrm{r} 317 \mathrm{r} 22 \mathrm{r} 316 \mathrm{r} 23 \mathrm{r}$

2 2r $315 r 23 r 316 r 23 r 315 r 24 r$

2 Ir $315 r 24 r 315 r 24 r 315 r 24 r$

$2 \operatorname{lr} 315 r 23 r 316 r 23 r 315 r 25 r$

$21 r 315 r 22 \pi 317 \pi 22 r 315 r 26 r$

$2 \operatorname{lr} 354 r 27 r \&$

c can code remember \& thru comment?

$2355 \mathrm{r} 27 \mathrm{r}$

$2325 r 22 r 325 r 28 r$

$2324 r 23 r 324 \pi 29 t$

$2323 \mathrm{r} 24 \mathrm{r} 323 \mathrm{r} 210 \mathrm{r}$

$2315 \mathrm{r} 22 \mathrm{r} 34 \mathrm{r} 23 \mathrm{r} 34 \mathrm{r} 2 \mathrm{2r} 3 \mathrm{1Sr} 211 \mathrm{r}$

$2314 r 23 r 34 r 2$ 2r $34 r 2$ 3r $314 r 212 r$

$2313 r 24 r 311+24 r 313 r 213 r$

$2313 r 23 r 312 r 23 r 3+3 r 214 r$

$2313 r 22 r 313 r 22 r 313 \times 215 r$

$2346 r 216 r$

$2345 \mathrm{r} 217 \mathrm{r}$

$2344 \mathrm{r} 218 \mathrm{r}$

$21+341 \mathrm{r} 220 \mathrm{r}$

2 ir 340r $221 \mathrm{r}$

2 Ir 339r $222 \mathrm{r}$

$22 \mathrm{r} 336 \mathrm{r} 224 \mathrm{r}$

$22 r 335 r 225 r$

$23 \mathrm{r} 332 \mathrm{r} 227 \mathrm{r}$

$24 \mathrm{r} 329 \mathrm{r} 229 \mathrm{r}$

$25 \mathrm{r} 326 \mathrm{r} 231 \mathrm{r}$

$26 \mathrm{r} 323 \mathrm{r} 233 \mathrm{r}$

$28 \mathrm{r} 318 \mathrm{r} 236 \mathrm{r}$

$211 \times 311 \times 240 \mathrm{r}$ 


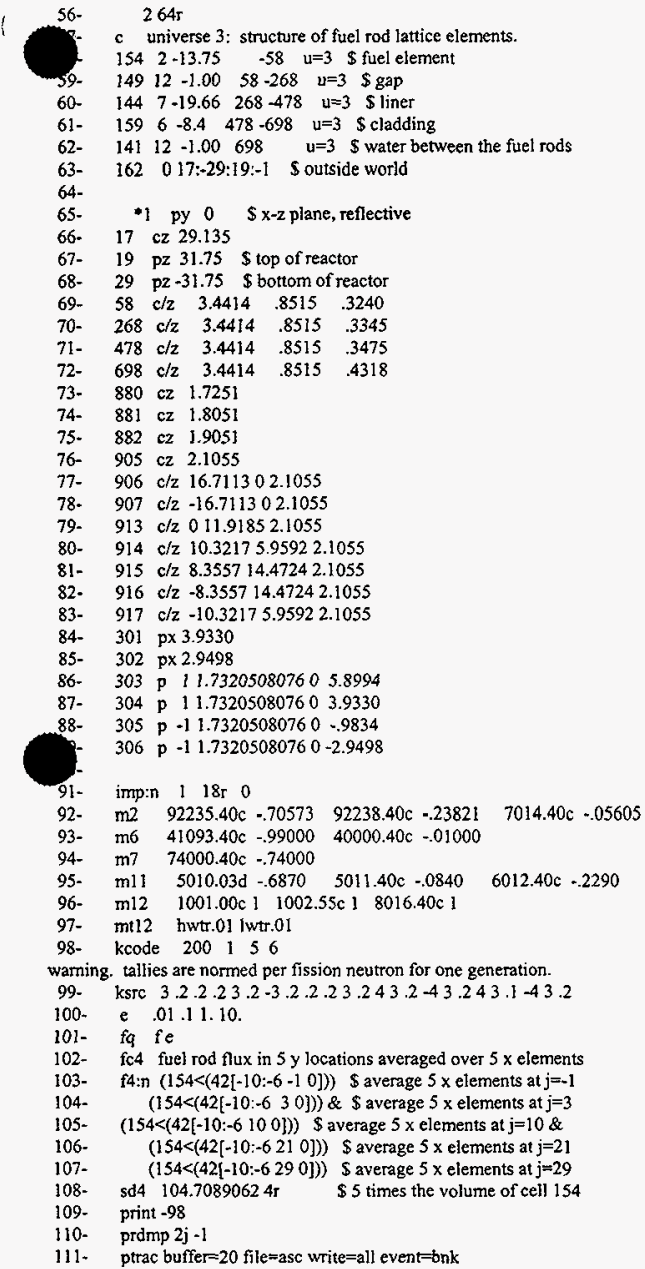

warning. tallies are normed per fission neutron for one generation.

99- ksrc $3.2 .2 .23 .2-3.2 .2 .23 .243 .243 .243 .143 .2$

100 e .01 .11 .10 .

101- fo fe

102- fc4 fuel rod flux in 5 y locations averaged over $5 x$ elements

103- f4:n $(154<(42[-10:-6-10]))$ average $5 \times$ elements at $j=-1$

104- $\quad(154<(42[-10:-630])) \&$ \& average $5 x$ elements at $j=3$

105- $\quad(154<(42[-10:-6100])) \$$ average $5 \times$ elements at $j=10$ \&

106- $\quad(154<(42[-10:-6210])) \$$ average $5 \times$ elements at $j=21$

107. $\quad(154<(42[-10:-6290]))$ S average $5 \times$ elements at $j=29$

108- so4 $104.70890624 \mathrm{~T} \quad \$ 5$ times the volume of cell 154

109. print -98

110. prdmp $2 \mathbf{j}-1$

111. ptrac buffer $=20$ file=asc write=all event=bnk

i intial source from $\mathrm{ksrc}$ card.

print table 90

\begin{tabular}{|c|c|}
\hline ral number of points & 8 \\
\hline points not in any cell & 0 \\
\hline oints in cells of zero importance & 0 \\
\hline points in void cells & 0 \\
\hline points in ambiguous cells & 0 \\
\hline tofal points rejected & 0 \\
\hline$s$ remaining & 8 \\
\hline
\end{tabular}

ts after expansion or contraction 
total fission nubar data are being used. 1tally 4

print table 30

tally type 4 track length estimate of particle flux.

tally for neutrons

order of printing: $f \mathrm{e}$

cells $(\mathrm{I} 54<(42[-10:-6-10]))(154<(42[-10:-630]))(154<(42[-10:-6100]))(154<(42[-10:-6210]))(154<(42[-10:-6$ $2901)$ )

energy bins

$0.00000 \mathrm{E}+00$ to $1.00000 \mathrm{E}-02 \mathrm{mev}$

$1.00000 \mathrm{E}-02$ to $1.00000 \mathrm{E}-01 \mathrm{mev}$

$1.00000 \mathrm{E}-01$ to $1.00000 \mathrm{E}+00 \mathrm{mev}$

$1.00000 \mathrm{E}+00$ to $1.00000 \mathrm{E}+01 \mathrm{mev}$

total bin

Imaterial composition

print table 40

the sum of the fractions of material 2 was $9.999900 \mathrm{E}-01$

the sum of the fractions of material 7 was $7.400000 \mathrm{E}-01$

the sum of the fractions of material 12 was $3.000000 \mathrm{E}+00$

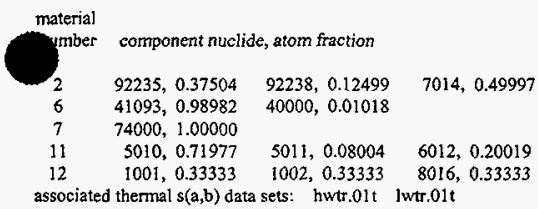

material

number component nuclide, mass fraction

$\begin{array}{rrrr}2 & 92235,0.70574 & 92238,0.23821 & 7014,0.05605 \\ 6 & 41093,0.99000 & 40000,0.01000 & \\ 7 & 74000,1.00000 & & \\ 11 & 5010,0.68700 & 5011,0.08400 & 6012,0.22900 \\ 12 & 1001,0.05300 & 1002,0.10591 & 8016,0.84109\end{array}$

waming. 3 of the materials had unnomalized fractions.

lcell volumes and masses

print table 50

cell atom gram input calculated reason volume density density volume volume mass pieces not calculated

$\begin{array}{llllllll}1 & 30 & 0.00000 \mathrm{E}+00 & 0.00000 \mathrm{E}+00 & 0.00000 \mathrm{E}+00 & 0.00000 \mathrm{E}+00 & 0.00000 \mathrm{E}+00\end{array}$

$2310.00000 E+00 \quad 0.00000 E+00 \quad 0.00000 E+00 \quad 0.00000 E+00 \quad 0.00000 E+00$

$\begin{array}{llllllll}37 & 0.00000 \mathrm{E}+00 & 0.00000 \mathrm{E}+00 & 0.00000 \mathrm{E}+00 & 0.00000 \mathrm{E}+00 & 0.00000 \mathrm{E}+00\end{array}$

$\begin{array}{llllllll}4 & 34 & 0.00000 \mathrm{E}+00 & 0.00000 \mathrm{E}+00 & 0.00000 \mathrm{E}+00 & 8.84370 \mathrm{E}+02 & 0.00000 \mathrm{E}+00\end{array}$

$\begin{array}{llllll}32 & 0.00000 \mathrm{E}+00 & 0.00000 \mathrm{E}+00 & 0.00000 \mathrm{E}+00 & 8.84370 \mathrm{E}+02 & 0.00000 \mathrm{E}+00\end{array}$

$\begin{array}{lllll}33 & 0.00000 \mathrm{E}+00 & 0.00000 \mathrm{E}+00 & 0.00000 \mathrm{E}+00 \quad 8.84370 \mathrm{E}+02 \quad 0.00000 \mathrm{E}+00\end{array}$

\footnotetext{
asymmetric

asymmetric

asymmetric
} 
$\begin{array}{llllll}75 & 0.00000 \mathrm{E}+00 & 0.00000 \mathrm{E}+00 & 0.00000 \mathrm{E}+00 & 8.84370 \mathrm{E}+02 & 0.00000 \mathrm{E}+00\end{array}$ $\begin{array}{llllll}36 & 0.00000 \mathrm{E}+00 & 0.00000 \mathrm{E}+00 & 0.00000 \mathrm{E}+00 \quad 8.84370 \mathrm{E}+02 & 0.00000 \mathrm{E}+00\end{array}$ $\begin{array}{llllllll}38 & 1.15958 \mathrm{E}-01 & 2.02000 \mathrm{E}+00 & 0.00000 \mathrm{E}+00 & 0.00000 \mathrm{E}+00 & 0.00000 \mathrm{E}+00\end{array}$ $\begin{array}{llllll}39 & 5.44575 \mathrm{E}-02 & 8.40000 \mathrm{E}+00 & 0.00000 \mathrm{E}+00 & 0.00000 \mathrm{E}+00 & 0.00000 \mathrm{E}+00\end{array}$ $1140 \quad 9.50007 \mathrm{E}-02 \quad 1.00000 \mathrm{E}+00 \quad 0.00000 \mathrm{E}+00 \quad 0.00000 \mathrm{E}+00 \quad 0.00000 \mathrm{E}+00$ 12 4! $5.44575 \mathrm{E}-02 \quad 8.40000 \mathrm{E}+00 \quad 0.00000 \mathrm{E}+00 \quad 0.00000 \mathrm{E}+00 \quad 0.00000 \mathrm{E}+00$ $13 \quad 140 \quad 0.00000 \mathrm{E}+00 \quad 0.00000 \mathrm{E}+00 \quad 0.00000 \mathrm{E}+00 \quad 0.00000 \mathrm{E}+00 \quad 0.00000 \mathrm{E}+00$ $\begin{array}{lllllll}14 & 42 & 9.50007 \mathrm{E}-02 & 1.00000 \mathrm{E}+00 & 0.00000 \mathrm{E}+00 & 0.00000 \mathrm{E}+00 & 0.00000 \mathrm{E}+00\end{array}$ $\begin{array}{lllllllll}15 & 154 & 6.62920 \mathrm{E}-02 & 1.37500 \mathrm{E}+01 & 0.00000 \mathrm{E}+00 & 0.00000 \mathrm{E}+00 & 0.00000 \mathrm{E}+00\end{array}$ $\begin{array}{lllllllll}16 & 149 & 9.50007 \mathrm{E}-02 & 1.00000 \mathrm{E}+00 & 0.00000 \mathrm{E}+00 & 0.00000 \mathrm{E}+00 & 0.00000 \mathrm{E}+00\end{array}$ $\begin{array}{llllllll}17 & 144 & 6.43969 \mathrm{E}-02 & 1.96600 \mathrm{E}+01 & 0.00000 \mathrm{E}+00 & 0.00000 \mathrm{E}+\infty & 0.00000 \mathrm{E}+00\end{array}$ $\begin{array}{lllllllll}18 & 159 & 5.44575 \mathrm{E}-02 & 8.40000 \mathrm{E}+00 & 0.00000 \mathrm{E}+00 & 0.00000 \mathrm{E}+00 & 0.00000 \mathrm{E}+00\end{array}$ $\begin{array}{llllllllll}19 & 141 & 9.50007 \mathrm{E}-02 & 1.00000 \mathrm{E}+00 & 0.00000 \mathrm{E}+00 & 0.00000 \mathrm{E}+00 & 0.00000 \mathrm{E}+00\end{array}$ $20 \quad 162 \quad 0.00000 \mathrm{E}+00 \quad 0.00000 \mathrm{E}+00 \quad 0.00000 \mathrm{E}+00 \quad 0.00000 \mathrm{E}+00 \quad 0.00000 \mathrm{E}+00$ 1 surface areas

print table 50
HNF-SD-SNF-CSWD-005, Rev. 0

infinite

infinite

infinite

infinite

asymmetric

infinite

infinite

infinite

infinite

infinite

infinite

asymmetric

surface input calculated reason area area area not calculated

I $10.00000 \mathrm{E}+00 \quad 0.00000 \mathrm{E}+00$ asymmetric

$217 \quad 0.00000 \mathrm{E}+00 \quad 0.00000 \mathrm{E}+00$ asymmetric

$3 \quad 19 \quad 0.00000 \mathrm{E}+00 \quad 0.00000 \mathrm{E}+00$ asymmetric

$4290.00000 \mathrm{E}+00 \quad 0.00000 \mathrm{E}+00$ asymmetric

$558 \quad 0.00000 \mathrm{E}+00 \quad 0.00000 \mathrm{E}+00$ infinite

$62680.00000 \mathrm{E}+00 \quad 0.00000 \mathrm{E}+00$ infinite

$7 \quad 478 \quad 0.00000 \mathrm{E}+00 \quad 0.00000 \mathrm{E}+00$ infinite

$86980.00000 \mathrm{E}+00 \quad 0.00000 \mathrm{E}+00$ infinite

$9880 \quad 0.00000 \mathrm{E}+00 \quad 0.00000 \mathrm{E}+00$ infinite

$10881 \quad 0.00000 \mathrm{E}+00 \quad 0.00000 \mathrm{E}+00$ infinite

11 $882 \quad 0.00000 \mathrm{E}+00 \quad 0.00000 \mathrm{E}+00$ infinite

$129050.00000 \mathrm{E}+00 \quad 0.00000 \mathrm{E}+00$ asymmetric

$139060.00000 \mathrm{E}+00 \quad 0.00000 \mathrm{E}+00$ asymmetric

$149070.00000 \mathrm{E}+00 \quad 0.00000 \mathrm{E}+00$ asymmetric

$913 \quad 0.00000 \mathrm{E}+00 \quad 8.40057 \mathrm{E}+02$

$9140.00000 \mathrm{E}+00 \quad 8.40057 \mathrm{E}+02$

$9150.00000 \mathrm{E}+00 \quad 8.40057 \mathrm{E}+02$

$18916 \quad 0.00000 \mathrm{E}+00 \quad 8.40057 \mathrm{E}+02$

$\begin{array}{lll}19 & 917 & 0.00000 \mathrm{E}+00 \quad 8.40057 \mathrm{E}+02\end{array}$

$203010.00000 \mathrm{E}+00 \quad 0.00000 \mathrm{E}+00$ infinite

$213020.00000 \mathrm{E}+00 \quad 0.00000 \mathrm{E}+00$ infinite

$22303 \quad 0.00000 \mathrm{E}+00 \quad 0.00000 \mathrm{E}+00$ infinite

$23304 \quad 0.00000 \mathrm{E}+00 \quad 0.00000 \mathrm{E}+00$

$24305 \quad 0.00000 \mathrm{E}+00 \quad 0.00000 \mathrm{E}+00$

$25306 \quad 0.00000 E+00 \quad 0.00000 E \div 00$

icells

print table 60

atom gram
cell mat density density volume mass pieces importance

$130 \quad 0 \quad 0.00000 \mathrm{E}+000.00000 \mathrm{E}+000.00000 \mathrm{E}+000.00000 \mathrm{E}+00$

2 31 $0 \quad 0.00000 \mathrm{E}+000.00000 \mathrm{E}+000.00000 \mathrm{E}+000.00000 \mathrm{E}+00$

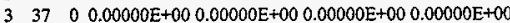

$434 \quad 0 \quad 0.00000 \mathrm{E}+000.00000 \mathrm{E}+00 \quad 8.84370 \mathrm{E}+020.00000 \mathrm{E}+00$

$53200.00000 \mathrm{E}+00 \quad 0.00000 \mathrm{E}+00 \quad 8.84370 \mathrm{E}+020.00000 \mathrm{E}+00$

$63300.00000 \mathrm{E}+000.00000 \mathrm{E}+00 \quad 8.84370 \mathrm{E}+020.00000 \mathrm{E}+00$

$735 \quad 0 \quad 0.00000 \mathrm{E}+00 \quad 0.00000 \mathrm{E}+00 \quad 8.84370 \mathrm{E}+02 \quad 0.00000 \mathrm{E}+00$

$8360.00000 \mathrm{E}+000.00000 \mathrm{E}+008.84370 \mathrm{E}+020.00000 \mathrm{E}+00$

$938 \quad 11 \quad 1.15958 \mathrm{E}-012.02000 \mathrm{E}+00 \quad 0.00000 \mathrm{E}+000.00000 \mathrm{E}+00$

$10 \quad 39 \quad 6 \quad 5.44575 \mathrm{E}-02 \quad 8.40000 \mathrm{E}+00 \quad 0.00000 \mathrm{E}+00 \quad 0.00000 \mathrm{E}+00$

$1140 \quad 12 s 9.50007 \mathrm{E}-02 \quad 1.00000 \mathrm{E}+00 \quad 0.00000 \mathrm{E}+000.00000 \mathrm{E}+00$

$1241 \quad 65.44575 \mathrm{E}-028.40000 \mathrm{E}+000.00000 \mathrm{E}+000.00000 \mathrm{E}+00$

$13 \quad 140 \quad 0 \quad 0.00000 \mathrm{E}+00 \quad 0.00000 \mathrm{E}+000.00000 \mathrm{E}+000.00000 \mathrm{E}+00$

$14 \quad 42 \quad 12 \$ 9.50007 \mathrm{E}-021.00000 \mathrm{E}+000.00000 \mathrm{E}+000.00000 \mathrm{E}+00$

$15 \quad 15426.62920 \mathrm{E}-02 \quad 1.37500 \mathrm{E}+010.00000 \mathrm{E}+000.00000 \mathrm{E}+00$

$16 \quad 14912 \mathrm{~s} 9.50007 \mathrm{E}-021.00000 \mathrm{E}+000.00000 \mathrm{E}+000.00000 \mathrm{E}+00$

$14476.43969 \mathrm{E}-02 \quad 1.96600 \mathrm{E}+01 \quad 0.00000 \mathrm{E}+000.00000 \mathrm{E} \div 00$

$159 \quad 6 \quad 5.44575 \mathrm{E}-028.40000 \mathrm{E}+00 \quad 0.00000 \mathrm{E}+000.00000 \mathrm{E}+00$
0 $1.0000 \mathrm{E}+00$

$01.0000 \mathrm{E}+00$

$01.0000 \mathrm{E}+00$

$11.0000 \mathrm{E}+00$

$11.0000 \mathrm{E}+00$

$11.0000 \mathrm{E}+00$

1 $1.0000 \mathrm{E}+00$

$11.0000 \mathrm{E}+00$

$0 \quad 1.0000 \mathrm{E}+00$

$0 \quad 1.0000 \mathrm{E}+00$

0 $1.0000 \mathrm{E}+00$

$0 \quad 1.0000 \mathrm{E}+00$

o $1.0000 \mathrm{E}+00$

$0 \quad 1.0000 \mathrm{E}+00$

$01.0000 \mathrm{E}+00$

o $1.0000 \mathrm{E}+00$

$01.0000 \mathrm{E}+00$

$0 \quad 1.0000 \mathrm{E}+00$ 
$19 \quad 141 \quad 12 s 9.50007 \mathrm{E}-02 \quad 1.00000 \mathrm{E}+000.00000 \mathrm{E}+00 \quad 0.00000 \mathrm{E}+00 \quad 0 \quad 1.0000 \mathrm{E}+00$

HNF-SD-SNF-CSWD-005, Rev. 0

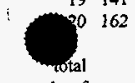

Isurfaces
$4.42185 \mathrm{E}+030.00000 \mathrm{E}+00$

$0.0000 E+00$

surface trans type surface coefficients

print table 70

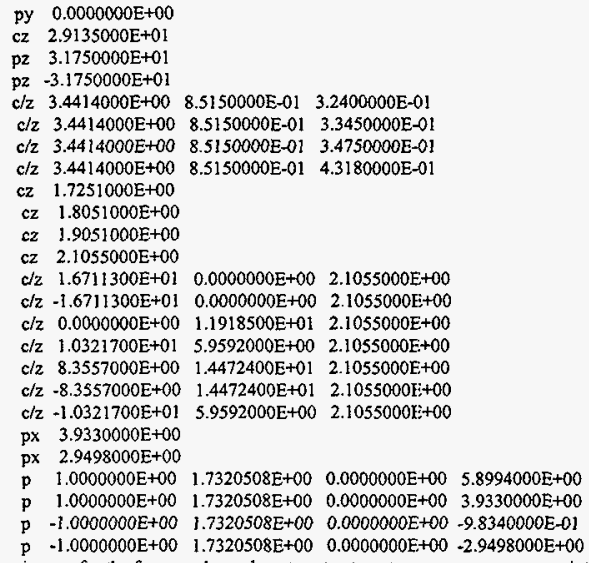

print table 72

all non-zero importance cells with materials have a temperature for themal neutrons of $2.5300 \mathrm{E}-08 \mathrm{mev}$.

2 warning messages so far.

1cross-section tables

print table 100

table length

tables from file testlib1

$1001.00 \mathrm{c} \quad \mathrm{I} 782 \mathrm{1}+\mathrm{h}-\mathrm{l}$ from endf-vi.

$1002.55 \mathrm{c} 4102$ njoy

mat $125 \quad 05 / 26 / 93$

$6012.40 \mathrm{c} 4858$ ENDL library name: nd900719 MCNP translation: 900831 14:10:05 900207

7014.40c 8535 ENDL library name: nd900719 MCNP translation: 900831 14:10:05 900207

8016.40c 5358 ENDL library name: nd900719 MCNP translation: 900831 14:10:05 900207

40000.40c 93372 ENDL library name: nd900719 MCNP translation: 900831 14:10:05 900323

41093.40c 42746 ENDL library name: nd900719 MCNP translation: 900831 14:10:05 900208

74000.40c 91244 ENDL library name: nd900719 MCNP translation: 900831 14:10:05 900208

92235.40c 34772 ENDL library name: nd900719 MCNP translation: 9total nu 10:05 900503

92238.40c 40834 ENDL library name: nd901118 MCNP translation: 9total nu 56:49 901119

$5010.03 \mathrm{~d} 3682$ b-10 endf/b-iv new gamma production format

hwtr.01t 10193 deuterium in heavy water at 300 degrees kelvin $\quad 1002 \quad 0 \quad 010 / 22 / 85$

$\begin{array}{llll}\text { Iwtr.01t } 10193 \text { hydrogen in light water at } 300 \text { degrees kelvin } & 1001 & 0 & 010 / 22 / 85\end{array}$

total 354900

waming. neutron energy cutoff is below some cross-section tables. 
3 warning messages so far.

1 starting merun. field length $=0 \quad \mathrm{cp} 0=0.00 \quad$ print table 110

probls - kcode in a hexagonal prism lattice.

nps $x \quad y \quad z \quad$ cell lattice $(i, j, k)$ surface $u \quad v \quad w \quad$ energy weight time

$13.000 \mathrm{E}+00 \quad 2.000 \mathrm{E}-01 \quad 2.000 \mathrm{E}-01 \quad 140$ $3.492 \mathrm{E}+00 \quad 1.051 \mathrm{E}+00 \quad 2.000 \mathrm{E}-01 \quad 42(0,-1,0)$ $3.492 \mathrm{E}+00 \quad 1.051 \mathrm{E}+00 \quad 2.000 \mathrm{E}-01 \quad 154$ $23.000 \mathrm{E}+00 \quad 2.000 \mathrm{E}-01 \quad 2.000 \mathrm{E}-01 \quad 140$ $3.492 \mathrm{E}+00 \quad 1.051 \mathrm{E}+00 \quad 2.000 \mathrm{E}-01 \quad 42($ $3.492 \mathrm{E}+00 \quad 1.051 \mathrm{E}+00 \quad 2.000 \mathrm{E}-01 \quad 154$

$33.000 \mathrm{E}+00 \quad 2.000 \mathrm{E}-01 \quad 2.000 \mathrm{E}-01 \quad 140$

$3.492 \mathrm{E}+00 \quad 1.051 \mathrm{E}+00 \quad 2.000 \mathrm{E}-0) \quad 42(0,-1,0)$

$3.492 \mathrm{E}+00 \quad 1.051 \mathrm{E}+002.000 \mathrm{E}-01154$

$43.000 \mathrm{E}+00 \quad 2.000 \mathrm{E}-01 \quad 2.000 \mathrm{E}-01 \quad 140$ $3.492 \mathrm{E}+00 \quad 1.05\lfloor\mathrm{E}+00 \quad 2.000 \mathrm{E}-01 \quad 42(0,-1,0)$ $3.492 \mathrm{E}+00 \quad 1.051 \mathrm{E}+00 \quad 2.000 \mathrm{E}-01 \quad 154$ $53.000 \mathrm{E}+00 \quad 2.000 \mathrm{E}-01 \quad 2.000 \mathrm{E}-01 \quad 140$ $3.492 \mathrm{E}+00 \quad 1.051 \mathrm{E}+00 \quad 2.000 \mathrm{E}-01 \quad 42($ $3.492 \mathrm{E}+00 \quad 1.051 \mathrm{E}+00 \quad 2.000 \mathrm{E}-01 \quad 154$

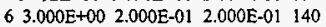
$3.492 \mathrm{E}+00 \quad 1.051 \mathrm{E}+00 \quad 2.000 \mathrm{E}-01 \quad 42(0,-1,0)$ $\begin{array}{llll}3.492 \mathrm{E}+00 & 1.051 \mathrm{E}+00 \quad 2.000 \mathrm{E}-01 \quad 154\end{array}$

$73.000 \mathrm{E}+00 \quad 2.000 \mathrm{E}-01 \quad 2.000 \mathrm{E}-01 \quad 140$ $3.492 \mathrm{E}+00 \quad 1.051 \mathrm{E}+00 \quad 2.000 \mathrm{E}-01 \quad 42(0,-1,0)$ $3.492 \mathrm{E}+00 \quad 1.051 \mathrm{E}+00 \quad 2.000 \mathrm{E}-01154$ $3.000 \mathrm{E}+00 \quad 2.000 \mathrm{E}-01 \quad 2.000 \mathrm{E}-01 \quad 140$ $.492 \mathrm{E}+00 \quad 1.051 \mathrm{E}+00 \quad 2.000 \mathrm{E}-01 \quad 42($ $3.492 \mathrm{E}+00 \quad 1.05 \mathrm{IE}+00 \quad 2.000 \mathrm{E}-01 \quad 154$

$93.000 \mathrm{E}+002.000 \mathrm{E}-01 \quad 2.000 \mathrm{E}-01 \quad 140$ $3.492 \mathrm{E}+00 \quad 1.051 \mathrm{E}+00 \quad 2.000 \mathrm{E}-01 \quad 42(0,-1,0)$ $3.492 \mathrm{E}+00 \quad 1.051 \mathrm{E}+00 \quad 2.000 \mathrm{E}-01 \quad 154$ $103.000 \mathrm{E}+00 \quad 2.000 \mathrm{E}-01 \quad 2.000 \mathrm{E}-01 \quad 140$ $3.492 \mathrm{E}+00 \quad 1.051 \mathrm{E}+00 \quad 2.000 \mathrm{E}-01 \quad 42(0,-1,0)$ $3.492 \mathrm{E}+00 \quad 1.051 \mathrm{E}+00 \quad 2.000 \mathrm{E}-01 \quad 154$ II $3.000 \mathrm{E}+002.000 \mathrm{E}-01 \quad 2.000 \mathrm{E}-0 \mathrm{l} \quad 140$ $3.492 \mathrm{E}+001.051 \mathrm{E}+00 \quad 2.000 \mathrm{E}-01 \quad 42(0,-1,0)$ $3.492 \mathrm{E}+00 \quad 1.051 \mathrm{E}+00 \quad 2.000 \mathrm{E}-01 \quad 154$

$123.000 \mathrm{E}+00 \quad 2.000 \mathrm{E}-01 \quad 2.000 \mathrm{E}-01 \quad 140$ $3.492 \mathrm{E}+00 \quad 1.051 \mathrm{E}+00 \quad 2.000 \mathrm{E}-01 \quad 42(0,-1,0)$ $3.492 \mathrm{E}+00 \quad 1.051 \mathrm{E}+00 \quad 2.000 \mathrm{E}-01 \quad 154$ $133.000 \mathrm{E}+00 \quad 2.000 \mathrm{E}-01 \quad 2.000 \mathrm{E}-01 \quad 140$ $3.492 \mathrm{E}+00 \quad 1.051 \mathrm{E}+00 \quad 2.000 \mathrm{E}-01 \quad 42(0,-1,0)$ $3.492 \mathrm{E}+00 \quad 1.05[\mathrm{E}+00 \quad 2.000 \mathrm{E}-01 \quad 154$

$143.000 \mathrm{E}+00 \quad 2.000 \mathrm{E}-01 \quad 2.000 \mathrm{E}-01 \quad 140$ $3.492 \mathrm{E}+00 \quad 1.051 \mathrm{E}+00 \quad 2.000 \mathrm{E}-01 \quad 42(0,-1,0)$ $3.492 \mathrm{E}+00 \quad 1.051 \mathrm{E}+00 \quad 2.000 \mathrm{E}-01 \quad 154$

is $3.000 \mathrm{E}+002.000 \mathrm{E}-01 \quad 2.000 \mathrm{E}-01 \quad 140$ $3.492 \mathrm{E}+00 \quad 1.051 \mathrm{E}+00 \quad 2.000 \mathrm{E}-01 \quad 42(0,-1,0)$ $3.492 \mathrm{E}+00 \quad 1.051 \mathrm{E}+00 \quad 2.000 \mathrm{E}-01 \quad 154$

$16 \quad 3.000 \mathrm{E}+00 \quad 2.000 \mathrm{E}-01 \quad 2.000 \mathrm{E}-01 \quad 140$ $3.492 \mathrm{E}+00 \quad 1.05 \mathrm{JE}+00 \quad 2.000 \mathrm{E}-01 \quad 42(0,-1,0)$ $3.492 \mathrm{E}+00 \quad 1.051 \mathrm{E}+00 \quad 2.000 \mathrm{E}-01 \quad 154$

$173.000 \mathrm{E}+00 \quad 2.000 \mathrm{E}-012.000 \mathrm{E}-01 \quad 140$ $3.492 \mathrm{E}+00 \quad 1.051 \mathrm{E}+00 \quad 2.000 \mathrm{E}-01 \quad 42(0,-1,0)$ $3.492 \mathrm{E}+00 \quad 1.051 \mathrm{E}+00 \quad 2.000 \mathrm{E}-01 \quad 154$

$183.000 \mathrm{E}+00 \quad 2.000 \mathrm{E}-01 \quad 2.000 \mathrm{E}-01 \quad 140$ $\begin{array}{lllll}3.492 \mathrm{E}+00 & 1.051 \mathrm{E}+00 \quad 2.000 \mathrm{E}-01 & 42( & 0,-1,0)\end{array}$ $3.492 \mathrm{E}+00 \quad 1.051 \mathrm{E}+00 \quad 2.000 \mathrm{E}-01 \quad 154$ $3.000 \mathrm{E}+00 \quad 2.000 \mathrm{E}-01 \quad 2.000 \mathrm{E}-01 \quad 140$

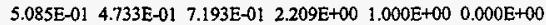
$5.085 E-01 \quad 4.733 E-01$ 7.193E-01

0 5.085E-01 4.733E-01 7.193E-01

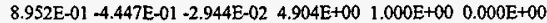
8.952E-01 -4.447E-01 -2.944E-02

0 8.952E-01 -4.447E-01 -2.944E-02

$-6.184 \mathrm{E}-01$ - $4.495 \mathrm{E}-01 \quad 6.446 \mathrm{E}-01 \quad 3.809 \mathrm{E}-01 \quad 1.000 \mathrm{E}+00 \quad 0.000 \mathrm{E}+00$ $+6.184 \mathrm{E}-01-4.495 \mathrm{E}-01 \quad 6.446 \mathrm{E}-01$

$0-6.184 \mathrm{E}-01 \mathrm{I}-4.495 \mathrm{E}-01 \quad 6.446 \mathrm{E}-01$

$9.710 \mathrm{E}-01-5.665 \mathrm{E}-02-2.323 \mathrm{E}-01 \quad 1.331 \mathrm{E}+00 \quad 1.000 \mathrm{E}+00 \quad 0.000 \mathrm{E}+00$ $9.710 \mathrm{E}-01-5.665 \mathrm{E}-02-2.323 \mathrm{E}-01$

0 9.710E-0! -5.665E-02 -2.323E-01

$5.861 \mathrm{E}-01 \quad 1.496 \mathrm{E}-01-7.963 \mathrm{E}-01 \quad 1.902 \mathrm{E}+00 \quad 1.000 \mathrm{E}+00 \quad 0.000 \mathrm{E}+00$ $5.861 \mathrm{E}-01 \quad 1.496 \mathrm{E}-01-7.963 \mathrm{E}-01$

0 5.861E-01 1.496E-01 -7.963E-01

$-6.489 \mathrm{E}-02-1.626 \mathrm{E}-01 \quad 9.845 \mathrm{E}-01 \quad 4.410 \mathrm{E}-01 \quad 1.000 \mathrm{E}+00 \quad 0.000 \mathrm{E}+00$ $-6.489 \mathrm{E}-02-1.626 \mathrm{E}-019.845 \mathrm{E}-01$

$0 \cdot 6.489 \mathrm{E}-02-1.626 \mathrm{E}-01 \quad 9.845 \mathrm{E}-01$

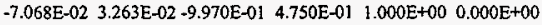

$-7.068 \mathrm{E}-02 \quad 3.263 \mathrm{E}-02-9.970 \mathrm{E}-01$

$0-7.068 \mathrm{E}-02 \quad 3.263 \mathrm{E}-02-9.970 \mathrm{E}-01$

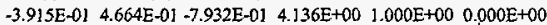

$-3.915 \mathrm{E}-01 \quad 4.664 \mathrm{E}-01-7.932 \mathrm{E}-01$

$0-3.915 \mathrm{E}-01 \quad 4.664 \mathrm{E}-01-7.932 \mathrm{E}-01$

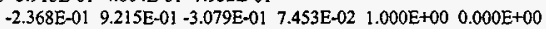

$-2.368 \mathrm{E}-019.215 \mathrm{E}-01-3.079 \mathrm{E}-01$

$0-2.368 \mathrm{E}-01 \quad 9.21 \mathrm{SE}-0]-3.079 \mathrm{E}-01$

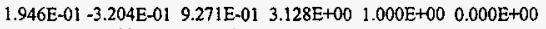
$1.946 \mathrm{E}-01 \cdot 3.204 \mathrm{E}-01 \quad 9.271 \mathrm{E}-01$

$0 \quad 1.946 \mathrm{E}-01-3.204 \mathrm{E}-01 \quad 9.271 \mathrm{E}-01$

$-6.698 \mathrm{E}-01-7.177 \mathrm{E}-01-1.905 \mathrm{E}-01 \quad 1.014 \mathrm{E}+00 \quad 1.000 \mathrm{E}+00 \quad 0.000 \mathrm{E}+00$

$-6.698 \mathrm{E}-01-7.177 \mathrm{E}-01-1.905 \mathrm{E}-01$

$0-6.698 \mathrm{E}-01-7.177 \mathrm{E}-01-1.905 \mathrm{E}-01$

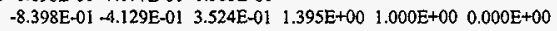
$-8.398 \mathrm{E}-01-4.129 \mathrm{E}-013.524 \mathrm{E}-01$

$0-8.398 \mathrm{E}-01-4.129 \mathrm{E}-01 \quad 3.524 \mathrm{E}-01$

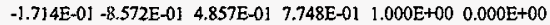

$-1.714 \mathrm{E}-01+8.572 \mathrm{E}-01 \quad 4.857 \mathrm{E}-01$

$0-1.714 \mathrm{E}-01-8.572 \mathrm{E}-01 \quad 4.857 \mathrm{E}-01$

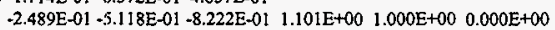

$-2.489 \mathrm{E}-01-5.118 \mathrm{E}-01-8.222 \mathrm{E}-01$

$0-2.489 \mathrm{E}-01-5.118 \mathrm{E}-01-8.222 \mathrm{E}-0$ !

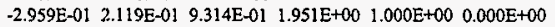

$-2.959 \mathrm{E}-01 \quad 2.119 \mathrm{E}-01 \quad 9.314 \mathrm{E}-01$

$0=2.959 \mathrm{E}-01 \quad 2.119 \mathrm{E}-01 \quad 9.314 \mathrm{E}-01$

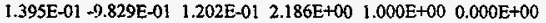
1.395E-01 $-9.829 \mathrm{E}-01 \quad 1.202 \mathrm{E}-01$

$0 \quad 1.395 \mathrm{E}-01-9.829 \mathrm{E}-01 \quad 1.202 \mathrm{E}-01$

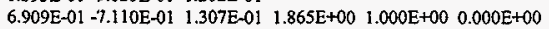
$6.909 \mathrm{E}-01-7.110 \mathrm{E}-01 \quad 1.307 \mathrm{E}-01$

$06.909 \mathrm{E}-01-7.110 \mathrm{E}-01 \quad 1.307 \mathrm{E}-01$

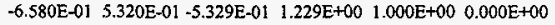

$-6.580 \mathrm{E}-015.320 \mathrm{E}-01-5.329 \mathrm{E}-01$

$0-6.580 \mathrm{E}-01 \quad 5.320 \mathrm{E}-01-5.329 \mathrm{E}-01$

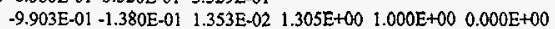


HNF-SD-SNF-CSWD-005, Rev. 0

$3.492 \mathrm{E}+001.051 \mathrm{E}+00 \quad 2.000 \mathrm{E}-01 \quad 42(0,-1,0) \quad-9.903 \mathrm{E}-01-1.380 \mathrm{E}-01 \quad 1.353 \mathrm{E}-02$ $\begin{array}{llll}3.492 \mathrm{E}+00 & 1.051 \mathrm{E}+00 & 2.000 \mathrm{E}-01 \quad 154 \quad 0-9.903 \mathrm{E}-01-1.380 \mathrm{E}-01 & 1.353 \mathrm{E}-02\end{array}$ $3.000 \mathrm{E}+002.000 \mathrm{E}-012.000 \mathrm{E}-01 \quad 140$ $3.492 \mathrm{E}+00 \quad 1.051 \mathrm{E}+00 \quad 2.000 \mathrm{E}-01 \quad 42(0,-1,0)$ $3.492 \mathrm{E}+00 \quad 1.051 \mathrm{E}+00 \quad 2.000 \mathrm{E}-01 \quad 154$

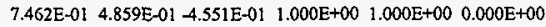
$7.462 \mathrm{E}-01 \quad 4.859 \mathrm{E}-01-4.551 \mathrm{E}-01$

$213.000 \mathrm{E}+00 \quad 2.000 \mathrm{E}-01 \quad 2.000 \mathrm{E}-01 \quad 140$ $3.492 \mathrm{E}+00 \quad 1.051 \mathrm{E}+00 \quad 2.000 \mathrm{E}-01 \quad 42(0,-1,0)$ $3.492 \mathrm{E}+00 \quad 1.051 \mathrm{E}+002.000 \mathrm{E}-01 \quad 154$ $07.462 \mathrm{E}-01 \quad 4.859 \mathrm{E}-01-4.551 \mathrm{E}-01$

$-1.977 \mathrm{E}-01 \quad 9.797 \mathrm{E}-01 \quad 3.360 \mathrm{E}-02 \quad 3.990 \mathrm{E}+00 \quad 1.000 \mathrm{E}+00 \quad 0.000 \mathrm{E}+00$ $-1.977 \mathrm{E}-01 \quad 9.797 \mathrm{E}-01 \quad 3.360 \mathrm{E}-02$

$223.000 \mathrm{E}+00 \quad 2.000 \mathrm{E}-01 \quad 2.000 \mathrm{E}-01 \quad 140$ $3.492 \mathrm{E}+00 \quad 1.051 \mathrm{E}+00 \quad 2.000 \mathrm{E}-01 \quad 42(0,-1,0)$ $3.492 \mathrm{E}+00 \quad 1.051 \mathrm{E}+00 \quad 2.000 \mathrm{E}-01 \quad 354$

$233.000 \mathrm{E}+00 \quad 2.000 \mathrm{E}-012.000 \mathrm{E}-01140$ $3.492 \mathrm{E}+00 \quad 1.051 \mathrm{E}+00 \quad 2.000 \mathrm{E}-01 \quad 42(0,-1,0)$ $3.492 \mathrm{E}+00 \quad 1.051 \mathrm{E}+00 \quad 2.000 \mathrm{E}-01 \quad 154$

$243.000 \mathrm{E}+00 \quad 2.000 \mathrm{E}-012.000 \mathrm{E}-01 \quad 140$ $3.492 \mathrm{E}+00 \quad 1.051 \mathrm{E}+00 \quad 2.000 \mathrm{E}-01 \quad 42(0,-1,0)$ $3.492 \mathrm{E}+00 \quad 1.051 \mathrm{E}+00 \quad 2.000 \mathrm{E}-01 \quad 154$

$253.000 \mathrm{E}+00 \quad 2.000 \mathrm{E}-01 \quad 2.000 \mathrm{E}-01 \quad 140$ $3.492 \mathrm{E}+00 \quad 1.051 \mathrm{E}+00 \quad 2.000 \mathrm{E}-01 \quad 42(0,-1,0)$ $\begin{array}{llll}3.492 \mathrm{E}+00 & 1.051 \mathrm{E}+00 & 2.000 \mathrm{E}-01 \quad 154\end{array}$

$262.000 \mathrm{E}-01 \quad 3.000 \mathrm{E}+00 \quad 2.000 \mathrm{E}-01 \quad 140$ $3.641 \mathrm{E}+00 \quad 4.456 \mathrm{E}-01 \quad 2.000 \mathrm{E}-01 \quad 42(-5,3,0)$ $3.641 \mathrm{E}+00 \quad 4.456 \mathrm{E}-01 \quad 2.000 \mathrm{E}-01 \quad 141$

$27 \quad 2.000 \mathrm{E}-01 \quad 3.000 \mathrm{E}+00 \quad 2.000 \mathrm{E}-01 \quad 140$ $3.641 \mathrm{E}+00 \quad 4.456 \mathrm{E}-01 \quad 2.000 \mathrm{E}+01 \quad 42(+5,3,0)$ $3.641 \mathrm{E}+00 \quad 4.456 \mathrm{E}-01 \quad 2.000 \mathrm{E}-01 \quad 141$

$282.000 \mathrm{E}-01 \quad 3.000 \mathrm{E}+00 \quad 2.000 \mathrm{E}-0) \quad 140$ $3.641 \mathrm{E}+00 \quad 4.456 \mathrm{E}-01 \quad 2.000 \mathrm{E}-01 \quad 42(-5,3,0)$ $3.641 \mathrm{E}+00 \quad 4.456 \mathrm{E}-01 \quad 2.000 \mathrm{E}-01 \quad 141$

$292.000 \mathrm{E}-013.000 \mathrm{E}+00 \quad 2.000 \mathrm{E}-01140$ $3.641 \mathrm{E}+00 \quad 4.456 \mathrm{E}-01 \quad 2.000 \mathrm{E}-01 \quad 42(-5,3,0)$ $3.641 \mathrm{E}+00 \quad 4.456 \mathrm{E}-01 \quad 2.000 \mathrm{E}-01 \quad 141$ $0-1.977 \mathrm{E}-01 \quad 9.797 \mathrm{E}-01 \quad 3.360 \mathrm{E}-02$

$-9.117 \mathrm{E}-01-3.647 \mathrm{E}-01+1.891 \mathrm{E}-01 \quad 2.665 \mathrm{E}-01 \quad 1.000 \mathrm{E}+00 \quad 0.000 \mathrm{E}+00$ $-9.137 \mathrm{E}-01-3.647 \mathrm{E}-01-1.891 \mathrm{E}-01$

0 -9.117E-01 -3.647E-01 -1.891E-01

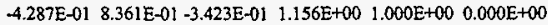
$-4.287 \mathrm{E}-01$ 8.36!E-01 -3.423E-01

$0-4.287 \mathrm{E}-01 \quad 8.361 \mathrm{E}-01+3.423 \mathrm{E}-01$

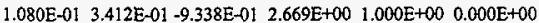
$1.080 \mathrm{E}-01 \quad 3.412 \mathrm{E}-01-9.338 \mathrm{E}-01$

$0 \quad 1.080 \mathrm{E}-01 \quad 3.412 \mathrm{E}-01-9.338 \mathrm{E}-01$

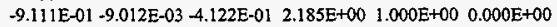
$-9.111 \mathrm{E}-01-9.012 \mathrm{E}-03-4.122 \mathrm{E}-01$

$0-9.111 \mathrm{E}-01-9.012 \mathrm{E}-03-4.122 \mathrm{E}-01$ $\begin{array}{lllll}-2.568 \mathrm{E}-01 & -6.391 \mathrm{E}-01-7.249 \mathrm{E}-01 & 7.280 \mathrm{E}-01 & 1.000 \mathrm{E}+00 \quad 0.000 \mathrm{E}+00\end{array}$ $-2.568 \mathrm{E}-01-6.391 \mathrm{E}-01-7.249 \mathrm{E}-01$

$0-2.568 \mathrm{E}-01-6.391 \mathrm{E}-01-7.249 \mathrm{E}-0$ ] $\begin{array}{lllll}-2.912 \mathrm{E}-01 & 8.086 \mathrm{E}-01 & 5.113 \mathrm{E}-0 ! & 1.404 \mathrm{E}+00 \quad 1.000 \mathrm{E}+00 & 0.000 \mathrm{E}+00\end{array}$ $-2.912 \mathrm{E}-01 \quad 8.086 \mathrm{E}-01 \quad 5.113 \mathrm{E}-01$

$0-2.912 \mathrm{E}-01 \quad 8.086 \mathrm{E}-01 \quad 5.113 \mathrm{E}-01$ $1.472 \mathrm{E}-01 \quad-9.514 \mathrm{E}-01 \quad 2.705 \mathrm{E}-01 \quad 1.090 \mathrm{E}+00 \quad 1.000 \mathrm{E}+00 \quad 0.000 \mathrm{E}+00$ $1.472 \mathrm{E}-01-9.514 \mathrm{E}-01 \quad 2.705 \mathrm{E}-01$

$0 \quad 1.472 \mathrm{E}-01-9.514 \mathrm{E}-012.705 \mathrm{E}-0 \mathrm{I}$

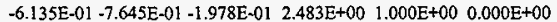
$-6.135 \mathrm{E}-01-7.645 \mathrm{E}-01-1.978 \mathrm{E}-01$

$0-6.135 \mathrm{E}-01-7.645 \mathrm{E}-01-1.978 \mathrm{E}-01$

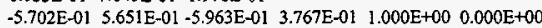

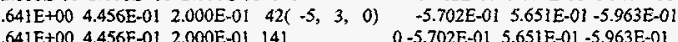
$.641 \mathrm{E}+00 \quad 4.456 \mathrm{E}-0$ ? 2.000E-0! 141 $2.000 \mathrm{E}-01 \quad 3.000 \mathrm{E}+00 \quad 2.000 \mathrm{E}-01 \quad 140$ $3.641 \mathrm{E}+00 \quad 4.456 \mathrm{E}-01 \quad 2.000 \mathrm{E}-01 \quad 42(-5,3,0)$ $3.641 \mathrm{E}+00 \quad 4.456 \mathrm{E}-01 \quad 2.000 \mathrm{E}-01 \quad 141$

$32 \quad 2.000 \mathrm{E}-01 \quad 3.000 \mathrm{E}+00 \quad 2.000 \mathrm{E}-01 \quad 140$ $3.641 \mathrm{E}+00 \quad 4.456 \mathrm{E}-01 \quad 2.000 \mathrm{E}-01 \quad 42(-5,3,0)$ $3.641 \mathrm{E}+00 \quad 4.456 \mathrm{E}-01 \quad 2.000 \mathrm{E}-01 \quad 141$

$332.000 \mathrm{E}-01 \quad 3.000 \mathrm{E}+00 \quad 2.000 \mathrm{E}-01 \quad 140$ $3.641 \mathrm{E}+00 \quad 4.456 \mathrm{E}-01 \quad 2.000 \mathrm{E}-01 \quad 42(-5,3,0)$ $3.641 \mathrm{E}+00 \quad 4.456 \mathrm{E}-01 \quad 2.000 \mathrm{E}-01 \quad 141$

34 2.000E-01 3.000E+00 2.000E-01 140 $3.641 \mathrm{E}+00 \quad 4.456 \mathrm{E}-01 \quad 2.000 \mathrm{E}-01 \quad 42(-5,3,0)$ $3.641 \mathrm{E}+00 \quad 4.456 \mathrm{E}-01 \quad 2.000 \mathrm{E}-01 \quad 141$

$352.000 \mathrm{E}-01 \quad 3.000 \mathrm{E}+00 \quad 2.000 \mathrm{E}-01 \quad 140$ $3.641 \mathrm{E}+00 \quad 4.456 \mathrm{E}-01 \quad 2.000 \mathrm{E}-0 ! \quad 42(-5,3,0)$ $3.641 \mathrm{E}+00 \quad 4.456 \mathrm{E}-01 \quad 2.000 \mathrm{E}-01 \quad 141$

$36 \quad 2.000 \mathrm{E}-01 \quad 3.000 \mathrm{E}+00 \quad 2.000 \mathrm{E}-01 \quad 140$ $3.641 \mathrm{E}+00 \quad 4.456 \mathrm{E}-01 \quad 2.000 \mathrm{E}-01 \quad 42(-5,3,0)$ $3.641 \mathrm{E}+00 \quad 4.456 \mathrm{E}-01 \quad 2.000 \mathrm{E}-01 \quad 141$

37 2.000E-01 3.000E +00 2.000E-01 140 $3.641 \mathrm{E}+00 \quad 4.456 \mathrm{E}-01 \quad 2.000 \mathrm{E}-01 \quad 42(-5,3,0)$ $3.641 \mathrm{E}+00 \quad 4.456 \mathrm{E}-01 \quad 2.000 \mathrm{E}-01 \quad 141$

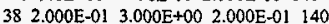
$3.641 \mathrm{E}+00 \quad 4.456 \mathrm{E}-01 \quad 2.000 \mathrm{E}-01 \quad 42(-5,3,0)$ $3.641 \mathrm{E}+00 \quad 4.456 \mathrm{E}-012.000 \mathrm{E}-01 \quad 141$

$\begin{array}{llll}39 & 2.000 \mathrm{E}-01 \quad 3.000 \mathrm{E}+00 & 2.000 \mathrm{E}-01 & 140\end{array}$ $3.641 \mathrm{E}+00 \quad 4.456 \mathrm{E}-01 \quad 2.000 \mathrm{E}-0 \mathrm{l} \quad 42(-5,3,0)$ $3.641 \mathrm{E}+00 \quad 4.456 \mathrm{E}-01 \quad 2.000 \mathrm{E}-01 \quad 141$

$402.000 \mathrm{E}-01 \quad 3.000 \mathrm{E}+00 \quad 2.000 \mathrm{E}-01 \quad 140$ $3.641 \mathrm{E}+00 \quad 4.456 \mathrm{E}-01 \quad 2.000 \mathrm{E}-01 \quad 42(-5,3,0)$ $3.641 \mathrm{E}+00 \quad 4.456 \mathrm{E}-01 \quad 2.000 \mathrm{E}-01 \quad 141$ $2.000 \mathrm{E}-01 \quad 3.000 \mathrm{E}+00 \quad 2.000 \mathrm{E}-01 \quad 140$

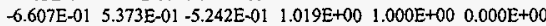
$-6.607 \mathrm{E}-015.373 \mathrm{E}-01-5.242 \mathrm{E}-01$

$0-6.607 \mathrm{E}-01 \quad 5.373 \mathrm{E}-01-5.242 \mathrm{E}-01$ $-9.742 \mathrm{E}-02+3.639 \mathrm{E}-01-9.263 \mathrm{E}-01 \quad 1.879 \mathrm{E}+00 \quad 1.000 \mathrm{E}+00 \quad 0.000 \mathrm{E}+00$ $-9.742 \mathrm{E}-02-3.639 \mathrm{E}-01-9.263 \mathrm{E}-01$

$0-9.742 \mathrm{E}-02-3.639 \mathrm{E}-01-9.263 \mathrm{E}-01$

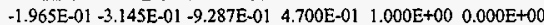
$-1.965 \mathrm{E}-01-3.145 \mathrm{E}-01-9.287 \mathrm{E}-01$

$0-1.965 E-01-3.145 E-0 !-9.287 E-01$

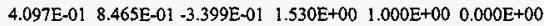
4.097E-01 8.465E-01 -3.399E-01

0 4.097E-01 8.465E-01 -3.399E-01

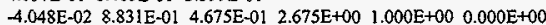
$-4.048 E-02 \quad 8.831 E-01 \quad 4.675 E-01$

$0-4.048 \mathrm{E}-02 \quad 8.831 \mathrm{E}-01 \quad 4.675 \mathrm{E}-01$ $3.371 \mathrm{E}-01-9.269 \mathrm{E}-01-1.652 \mathrm{E}-01 \quad 9.222 \mathrm{E}-01 \quad 1.000 \mathrm{E}+00 \quad 0.000 \mathrm{E}+00$ $3.371 \mathrm{E}-01-9.269 \mathrm{E}-01-1.652 \mathrm{E}-01$

0 3.371E-01 -9.269E-01-1.652E-01 $-1.867 \mathrm{E}-01 \quad 9.756 \mathrm{E}-01-1.155 \mathrm{E}-01 \quad 9.702 \mathrm{E}-01 \quad 1.000 \mathrm{E}+00 \quad 0.000 \mathrm{E}+00$ $-1.867 \mathrm{E}-019.756 \mathrm{E}-01 \mathrm{l}-1.155 \mathrm{E}-01$

$0+1.867 \mathrm{E}-01 \quad 9.756 \mathrm{E}-01-1.155 \mathrm{E}-01$

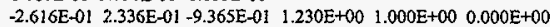
$-2.616 \mathrm{E}-01 \quad 2.336 \mathrm{E}-01 \quad-9.365 \mathrm{E}-01$

$0-2.616 \mathrm{E}-01 \quad 2.336 \mathrm{E}-01-9.365 \mathrm{E}-01$ $\begin{array}{lllll}9.780 \mathrm{E}-01 & -7.64] \mathrm{E}-02-1.939 \mathrm{E}-01 \quad 1.083 \mathrm{E}+00 \quad 1.000 \mathrm{E}+00 \quad 0.000 \mathrm{E}+00\end{array}$ $9.780 \mathrm{E}-01-7.641 \mathrm{E}-02+1.939 \mathrm{E}-01$

$09.780 \mathrm{E}-01-7.641 \mathrm{E}-02-1.939 \mathrm{E}-01$ $\begin{array}{llllll}2.580 \mathrm{E}-01 & -7.076 \mathrm{E}-01 & 6.578 \mathrm{E}-01 & 8.313 \mathrm{E}-01 & 1.000 \mathrm{E}+00 & 0.000 \mathrm{E}+00\end{array}$ $2.580 \mathrm{E}-01-7.076 \mathrm{E}-0] \quad 6.578 \mathrm{E}-01$

0 2.580E-01 -7.076E-01 6.578E-01

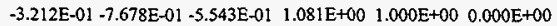
$-3.212 \mathrm{E}-01-7.678 \mathrm{E}-01+5.543 \mathrm{E}-01$ 
3.641E+00 4.456E-01 2.000E-01 141 $2.000 \mathrm{E}-01 \quad 3.000 \mathrm{E}+00 \quad 2.000 \mathrm{E}-01 \quad 140$ $.641 \mathrm{E}+00 \quad 4.456 \mathrm{E}-01 \quad 2.000 \mathrm{E}-01 \quad 42(-5,3,0)$ $641 \mathrm{E}+00 \quad 4.456 \mathrm{E}-01 \quad 2.000 \mathrm{E}-01 \quad 141$

$432.000 \mathrm{E}-01 \quad 3.000 \mathrm{E}+00 \quad 2.000 \mathrm{E}-01 \quad 140$ $3.641 \mathrm{E}+00 \quad 4.456 \mathrm{E}-01 \quad 2.000 \mathrm{E}-01 \quad 42(-5,3,0)$ $3.641 \mathrm{E}+00 \quad 4.456 \mathrm{E}-01 \quad 2.000 \mathrm{E}-01 \quad 141$ $442.000 \mathrm{E}-0 \mathrm{I} \quad 3.000 \mathrm{E}+00 \quad 2.000 \mathrm{E}-0 \mathrm{l} 140$ $3.641 \mathrm{E}+00 \quad 4.456 \mathrm{E}-01 \quad 2.000 \mathrm{E}-01 \quad 42(-5,3,0)$ $3.641 \mathrm{E}+00 \quad 4.456 \mathrm{E}-01 \quad 2.000 \mathrm{E}-01 \quad 141$ $452.000 \mathrm{E}-01 \quad 3.000 \mathrm{E}+00 \quad 2.000 \mathrm{E}-01 \quad 140$ $3.641 \mathrm{E}+00 \quad 4.456 \mathrm{E}-01 \quad 2.000 \mathrm{E}-01 \quad 42(-5,3,0)$ $3.641 \mathrm{E}+00 \quad 4.456 \mathrm{E}-01 \quad 2.000 \mathrm{E}-01 \quad 141$

$46 \quad 2.000 \mathrm{E}-01 \quad 3.000 \mathrm{E}+00 \quad 2.000 \mathrm{E}-01 \quad 140$ $3.641 \mathrm{E}+00 \quad 4.456 \mathrm{E}-01 \quad 2.000 \mathrm{E}-01 \quad 42(-5,3,0)$ $3.641 \mathrm{E}+00 \quad 4.456 \mathrm{E}-01 \quad 2.000 \mathrm{E}-01 \quad 141$

$472.000 \mathrm{E}-013.000 \mathrm{E}+00 \quad 2.000 \mathrm{E}-01140$ $3.641 \mathrm{E}+00 \quad 4.456 \mathrm{E}-01 \quad 2.000 \mathrm{E}-01 \quad 42(-5,3,0)$ $3.64 \mathrm{IE}+00 \quad 4.456 \mathrm{E}-01 \quad 2.000 \mathrm{E}-01 \quad 141$ $482.000 \mathrm{E}-01 \quad 3.000 \mathrm{E}+00 \quad 2.000 \mathrm{E}-01 \quad 140$ $3.641 \mathrm{E}+00 \quad 4.456 \mathrm{E}-01 \quad 2.000 \mathrm{E}-01 \quad 42(-5,3,0)$ $3.641 \mathrm{E}+00 \quad 4.456 \mathrm{E}-01 \quad 2.000 \mathrm{E}-01 \quad 141$

$492.000 \mathrm{E}-01 \quad 3.000 \mathrm{E}+00 \quad 2.000 \mathrm{E}-01 \quad 140$ $3.641 \mathrm{E}+00 \quad 4.456 \mathrm{E}-01 \quad 2.000 \mathrm{E}-01 \quad 42(-5,3,0)$ $3.641 \mathrm{E}+00 \quad 4.456 \mathrm{E}-01 \quad 2.000 \mathrm{E}-01 \quad 141$

$50 \quad 2.000 \mathrm{E}-01 \quad 3.000 \mathrm{E}+00 \quad 2.000 \mathrm{E}-01 \quad 140$ $3.641 \mathrm{E}+00 \quad 4.456 \mathrm{E}-01 \quad 2.000 \mathrm{E}-01 \quad 42(-5,3,0)$ $3.641 \mathrm{E}+00 \quad 4.456 \mathrm{E}-01 \quad 2.000 \mathrm{E}-01141$ lestimated keff results by cycle
$0-3.212 \mathrm{E}-01-7.678 \mathrm{E}-0 \mathrm{l}-5.543 \mathrm{E}-01$ $5.039 \mathrm{E}-01-1.460 \mathrm{E}-01 \quad 8.513 \mathrm{E}-01 \quad 3.341 \mathrm{E}+00 \quad 1.000 \mathrm{E}+00 \quad 0.000 \mathrm{E}+00$ $5.039 \mathrm{E}-01+1.460 \mathrm{E}-01 \quad 8.513 \mathrm{E}-01$

0 5.039E-01-1.460E-01 $8.513 \mathrm{E}-01$

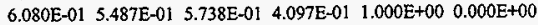
$6.080 \mathrm{E}-015.487 \mathrm{E}-015.738 \mathrm{E}-01$

$0 \quad 6.080 \mathrm{E}-01 \quad 5.487 \mathrm{E}-01 \quad 5.738 \mathrm{E}-01$ $-2.932 \mathrm{E}-01 \quad 9.304 \mathrm{E}-01 \quad-2.199 \mathrm{E}-01 \quad 2.229 \mathrm{E}+00 \quad 1.000 \mathrm{E}+00 \quad 0.000 \mathrm{E}+00$ $-2.932 \mathrm{E}-01 \quad 9.304 \mathrm{E}-01-2.199 \mathrm{E}-01$

$0-2.932 \mathrm{E}-01 \quad 9.304 \mathrm{E}-0 \mathrm{l}-2.199 \mathrm{E}-01$ $-8.475 \mathrm{E}-01-3.993 \mathrm{E}-01-3.497 \mathrm{E}-01 \quad 1.362 \mathrm{E}+00 \quad 1.000 \mathrm{E}+00 \quad 0.000 \mathrm{E}+00$ -8.475E-01 -3.993E-01 -3.497E-01

$0-8.475 \mathrm{E}-01-3.993 \mathrm{E}-01-3.497 \mathrm{E}-01$ $1.200 \mathrm{E}-01-9.195 \mathrm{E}-01-3.743 \mathrm{E}-01 \quad 1.010 \mathrm{E}+00 \quad 1.000 \mathrm{E}+00 \quad 0.000 \mathrm{E}+00$ $1.200 \mathrm{E}-01-9.195 \mathrm{E}-01-3.743 \mathrm{E}-01$

0 1.200E-01 -9.195E-01 -3.743E-01

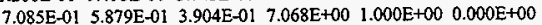
$7.085 \mathrm{E}-01 \quad 5.879 \mathrm{E}-01 \quad 3.904 \mathrm{E}-01$

$07.085 \mathrm{E}-01 \quad 5.879 \mathrm{E}-01 \quad 3.904 \mathrm{E}-01$

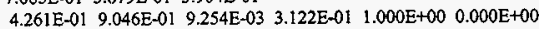
4.261E-01 9.046E-01 9.254E-03

$0 \quad 4.261 \mathrm{E}-019.046 \mathrm{E}-019.254 \mathrm{E}-03$

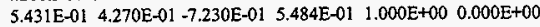
$5.431 \mathrm{E}-01 \quad 4.270 \mathrm{E}-01-7.230 \mathrm{E}-01$

0 5.431E-01 4.270E-01 -7.230E-01

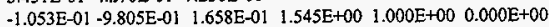

$-1.053 \mathrm{E}-01-9.805 \mathrm{E}-01$ 1.658E-01

$0-1.053 \mathrm{E}-01-9.805 \mathrm{E}-01 \quad 1.658 \mathrm{E}-01$ print table 175

cycle $1 \mathrm{k}$ (collision) 1.253108 removal lifetime(abs) $1.7557 \mathrm{E}+01$ source points generated 257

cycle 2 k(collision) 1.211997 removal lifetime(abs) $1.1671 \mathrm{E}+01$ source points generated 195

e $3 \mathrm{k}$ (collision) 1.200265 removal lifetime(abs) $9.6992 \mathrm{E}+00$ source points generated 193

cycle $4 \mathrm{k}$ (collision) 1.129530 removal lifetime(abs) $9.3720 \mathrm{E}+00$ source points generated 182

cycle $5 \mathrm{k}$ (collision) 1.135994 removal lifetime(abs) $1.1136 \mathrm{E}+01$ source points generated 222

source distribution written to file inp $18 \mathrm{~s} \quad$ cycle $=5$

cycle $6 \mathrm{k}$ (collision) 1.077014 temoval lifetime(abs) $1.2222 \mathrm{E}+01$ source points generated 204

source distribution written to file inp $18 \mathrm{~s} \quad$ cycle $=6$ Iproblem summary 0

run terminated when 6 kcode cycles were done.

neutron creation tracks weight energy neutron loss tracks weight energy (per source particle) (per source particle)

source

$1249 \quad 9.6077 \mathrm{E}-01 \quad 1.8661 \mathrm{E}+00$ energy cutoff

escape $220 \quad 1.2268 \mathrm{E}-01 \quad 1.2054 \mathrm{E}-01$ time cutoff

$\begin{array}{lll}0 & 0 . & 0 . \\ 0 & 0 . & 0 .\end{array}$

weight window cell importance $\begin{array}{lll}0 & 0\end{array}$ weight window 0 $\begin{array}{lllllllll}\text { weight cutoff } & 0 & 1.3743 \mathrm{E}-01 & 3.8498 \mathrm{E}-03 & \text { weight cutoff } & 1034 & 1.27 \\ \text { energy importance } & 0 & 0 . & 0 . & \text { energy importance } & 0 & 0 . & 0\end{array}$ $\begin{array}{lllllll}0 & 0 . & 0 . & \text { cell importance } & 0 & 0 . & 0 .\end{array}$ dxtran 0 o. 0 . forced collisions $\quad 0 \quad 0$ 0 . exp. transform 0 0. 0 $\begin{array}{lllllrrrr}\text { exp. transform } & 0 & 0 . & 0 . & \text { exp. transform } & 0 & 0 . & 0 . & \\ \text { upscattering } & 0 & 0 . & 5.3884 \mathrm{E}-11 & \text { downscattering } & 0 & 0 . & 1.3605 \mathrm{E}+00\end{array}$ $\begin{array}{llllllllll}\text { exp. transform } & 0 & 0 . & 0 . & \text { exp. transform } & 0 & 0 . & 0 . & \\ \text { upscattering } & 0 & 0 . & 5.3884 \mathrm{E}-11 & \text { downscattering } & 0 & 0 . & 1.3605 \mathrm{E}+00\end{array}$ 0. dxtran 0 . forced collisions $0.0 . \quad 0$.

$$
\text { capture } \quad 0 \quad 3.9980 \mathrm{E}-01 \quad 1.1590 \mathrm{E}-01
$$

$\begin{array}{lllllll}10 & 6.1640 \mathrm{E}-03 & 6.1355 \mathrm{E}-03 & \text { loss to }(\mathrm{n}, \mathrm{xn}) & 5 & 3.0820 \mathrm{E}-03 & 2.4027 \mathrm{E}-02\end{array}$

$\begin{array}{llllllll}0 & 0 . & 0 & \text { loss to fission } 0 & 4.5089 \mathrm{E}-01 & 2.4987 \mathrm{E}-01\end{array}$

$\begin{array}{lllllll}1259 & 1.1044 \mathrm{E}+00 & 1.8761 \mathrm{E}+00 \quad \text { total } & 1259 & 1.1044 \mathrm{E}+00 & 1.8761 \mathrm{E}+00\end{array}$ 
5 average lifetime, shakes

HNF-SD-SNF-CSWD-005, Rev. 0

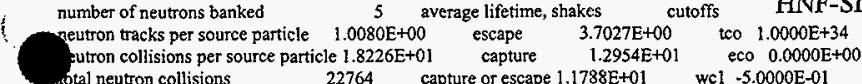

net multiplication

$1.0032 \mathrm{E}+000.0035$

any termination $1.2744 \mathrm{E}+01$

wc2 $-2.5000 \mathrm{E}-01$

computer time so far in this nom 0.00 minutes computer time in merum

source particles per minute

0.00 minutes

maximum number ever in bank 1

bank overfows to backup file 0

random numbers generated

$0.0000 \mathrm{E}+00$

500880

field length

most random numbers used was 1298 in history

range of sampled source weights $=7.7821 \mathrm{E}-01$ to $1.0989 \mathrm{E}+00$

Ineutron activity in each cell

print table 126

tracks population collisions collisions number flux average average cell entering

- weight weighted weighted track weight track mfp (per history) energy energy (relative) (cm)

$\begin{array}{llllllllllll}1 & 30 & 0 & 0 & 0 & 0.0000 \mathrm{E}+00 & 0.0000 \mathrm{E}+00 & 0.0000 \mathrm{E}+00 & 0.0000 \mathrm{E}+00 & 0.0000 \mathrm{E}+00 \\ 2 & 31 & 0 & 0 & 0 & 0.0000 \mathrm{E}+00 & 0.0000 \mathrm{E}+00 & 0.0000 \mathrm{E}+00 & 0.0000 \mathrm{E}+00 & 0.0000 \mathrm{E}+00 \\ 3 & 37 & 0 & 0 & 0 & 0.0000 \mathrm{E}+00 & 0.0000 \mathrm{E}+00 & 0.0000 \mathrm{E}+00 & 0.0000 \mathrm{E}+00 & 0.0000 \mathrm{E}+00 \\ 4 & 34 & 0 & 0 & 0 & 0.0000 \mathrm{E}+00 & 0.0000 \mathrm{E}+00 & 0.0000 \mathrm{E}+00 & 0.0000 \mathrm{E}+00 & 0.0000 \mathrm{E}+00 \\ 5 & 32 & 0 & 0 & 0 & 0.0000 \mathrm{E}+00 & 0.0000 \mathrm{E}+00 & 0.0000 \mathrm{E}+00 & 0.0000 \mathrm{E}+00 & 0.0000 \mathrm{E}+00 \\ 6 & 33 & 0 & 0 & 0 & 0.0000 \mathrm{E}+00 & 0.0000 \mathrm{E}+00 & 0.0000 \mathrm{E}+00 & 0.0000 \mathrm{E}+00 & 0.0000 \mathrm{E}+00 \\ 7 & 35 & 0 & 0 & 0 & 0.0000 \mathrm{E}+00 & 0.0000 \mathrm{E}+00 & 0.0000 \mathrm{E}+00 & 0.0000 \mathrm{E}+00 & 0.0000 \mathrm{E}+00 \\ 8 & 36 & 0 & 0 & 0 & 0.0000 \mathrm{E}+00 & 0.0000 \mathrm{E}+00 & 0.0000 \mathrm{E}+00 & 0.0000 \mathrm{E}+00 & 0.0000 \mathrm{E}+00 \\ 9 & 38 & 920 & 618 & 1249 & 5.9116 \mathrm{E}-01 & 3.0414 \mathrm{E}-01 & 8.9304 \mathrm{E}-01 & 6.4212 \mathrm{E}-01 & 2.6324 \mathrm{E}+00 \\ 10 & 39 & 1635 & 637 & 130 & 6.3779 \mathrm{E}-02 & 8.8236 \mathrm{E}-02 & 7.6766 \mathrm{E}+01 & 6.4931 \mathrm{E}-01 & 2.6947 \mathrm{E}+00 \\ 11 & 40 & 1728 & 663 & 184 & 8.8176 \mathrm{E}-02 & 7.6162 \mathrm{E}-02 & 7.6184 \mathrm{E}-01 & 6.4437 \mathrm{E}-01 & 2.5146 \mathrm{E}+00 \\ 12 & 41 & 1867 & 712 & 317 & 1.5811 \mathrm{E}-01 & 8.6238 \mathrm{E}-02 & 7.9306 \mathrm{E}-01 & 6.5030 \mathrm{E}-01 & 2.6940 \mathrm{E}+00 \\ 13 & 140 & 0 & 0 & 0 & 0.0000 \mathrm{E}+00 & 0.0000 \mathrm{E}+00 & 0.0000 \mathrm{E}+00 & 0.0000 \mathrm{E}+00 & 0.0000 \mathrm{E}+00 \\ 14 & 42 & 2069 & 867 & 441 & 2.1911 \mathrm{E}-01 & 8.9382 \mathrm{E}-02 & 7.8771 \mathrm{E}-01 & 6.6777 \mathrm{E}-01 & 2.5091 \mathrm{E}+00 \\ 15 & 154 & 25302 & 1253 & 8436 & 4.1390 \mathrm{E}+00 & 1.2606 \mathrm{E}-01 & 8.5005 \mathrm{E}-01 & 6.6920 \mathrm{E}-01 & 2.4712 \mathrm{E}+00 \\ 6 & 149 & 49542 & 1253 & 580 & 2.8963 \mathrm{E}-01 & 1.0795 \mathrm{E}-01 & 8.2361 \mathrm{E}-01 & 6.7129 \mathrm{E}-01 & 2.5408 \mathrm{E}+00 \\ 7 & 144 & 51224 & 1254 & 885 & 4.2652 \mathrm{E}-01 & 1.0541 \mathrm{E}-01 & 8.1599 \mathrm{E}-01 & 6.6971 \mathrm{E}-01 & 2.2391 \mathrm{E}+00 \\ 8 & 159 & 58650 & 1254 & 4019 & 2.1254 \mathrm{E}+00 & 9.8931 \mathrm{E}-02 & 8.1154 \mathrm{E}-01 & 6.6977 \mathrm{E}-01 & 2.7541 \mathrm{E}+00 \\ 19 & 141 & 73122 & 1254 & 6523 & 3.2229 \mathrm{E}+00 & 9.4499 \mathrm{E}-02 & 8.0639 \mathrm{E}-01 & 6.6810 \mathrm{E}-01 & 2.5056 \mathrm{E}+00\end{array}$

total $266059 \quad 9765 \quad 22764 \quad 1.1324 \mathrm{E}+01$

Ineutron weight balance in each cell - extemal events

print table 130

cell entering source energy time exiting total cutoff cutoff

$\begin{array}{lllllllll}1 & 30 & 0.0000 \mathrm{E}+00 & 0.0000 \mathrm{E}+00 & 0.0000 \mathrm{E}+00 & 0.0000 \mathrm{E}+00 & 0.0000 \mathrm{E}+00 & 0.0000 \mathrm{E}+00\end{array}$

$\begin{array}{lllllllll}2 & 31 & 0.0000 \mathrm{E}+00 & 0.0000 \mathrm{E}+00 & 0.0000 \mathrm{E}+00 & 0.0000 \mathrm{E}+00 & 0.0000 \mathrm{E}+00 & 0.0000 \mathrm{E}+00\end{array}$

$\begin{array}{lllllllll}3 & 37 & 0.0000 \mathrm{E}+00 & 0.0000 \mathrm{E}+00 & 0.0000 \mathrm{E}+00 & 0.0000 \mathrm{E}+00 & 0.0000 \mathrm{E}+00 & 0.0000 \mathrm{E}+00\end{array}$

$\begin{array}{llllllllll}4 & 34 & 0.0000 \mathrm{E}+00 & 0.0000 \mathrm{E}+00 & 0.0000 \mathrm{E}+00 & 0.0000 \mathrm{E}+00 & 0.0000 \mathrm{E}+00 & 0.0000 \mathrm{E}+00\end{array}$

$\begin{array}{llllllll}5 & 32 & 0.0000 \mathrm{E}+00 & 0.0000 \mathrm{E}+00 & 0.0000 \mathrm{E}+00 & 0.0000 \mathrm{E}+00 & 0.0000 \mathrm{E}+00 & 0.0000 \mathrm{E}+00\end{array}$

$\begin{array}{llllllllll}6 & 33 & 0.0000 \mathrm{E}+00 & 0.0000 \mathrm{E}+00 & 0.0000 \mathrm{E}+00 & 0.0000 \mathrm{E}+00 & 0.0000 \mathrm{E}+00 & 0.0000 \mathrm{E}+00\end{array}$

$\begin{array}{lllllllll}7 & 35 & 0.0000 \mathrm{E}+00 & 0.0000 \mathrm{E}+00 & 0.0000 \mathrm{E}+00 & 0.0000 \mathrm{E}+00 & 0.0000 \mathrm{E}+00 & 0.0000 \mathrm{E}+00\end{array}$

$\begin{array}{llllllllllll}8 & 36 & 0.0000 \mathrm{E}+00 & 0.0000 \mathrm{E}+00 & 0.0000 \mathrm{E}+00 & 0.0000 \mathrm{E}+00 & 0.0000 \mathrm{E}+00 & 0.0000 \mathrm{E}+00\end{array}$

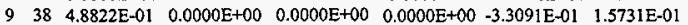

$\begin{array}{lllllllll}10 & 39 & 8.4996 \mathrm{E}-0 \mathrm{~J} & 0.0000 \mathrm{E}+00 & 0.0000 \mathrm{E}+00 & 0.0000 \mathrm{E}+00 & -8.4933 \mathrm{E}-01 & 6.2750 \mathrm{E}-04\end{array}$

$\begin{array}{llllllll}11 & 40 & 8.9904 \mathrm{E}-01 & 0.0000 \mathrm{E}+00 & 0.0000 \mathrm{E}+00 & 0.0000 \mathrm{E}+00 & -8.9904 \mathrm{E}-01 & 3.9460 \mathrm{E}-06\end{array}$

$\begin{array}{lllllllll}12 & 41 & 9.7260 \mathrm{E}-01 & 0.0000 \mathrm{E}+00 & 0.0000 \mathrm{E}+00 & 0.0000 \mathrm{E}+00 & -9.6978 \mathrm{E}-01 & 2.8204 \mathrm{E}-03\end{array}$

$\begin{array}{llllllll}13 & 140 & 0.0000 \mathrm{E}+00 & 0.0000 \mathrm{E}+00 & 0.0000 \mathrm{E}+00 & 0.0000 \mathrm{E}+00 & 0.0000 \mathrm{E}+00 & 0.0000 \mathrm{E}+00\end{array}$

I4 $42 \quad 1.0907 \mathrm{E}+00 \quad 0.0000 \mathrm{E}+00 \quad 0.0000 \mathrm{E}+00 \quad 0.0000 \mathrm{E}+00 \quad-1.0913 \mathrm{E}+00 \quad-5.9111 \mathrm{E}-0.4$

$\begin{array}{lllllllll}15 & 154 & 1.2943 \mathrm{E}+01 & 8.4067 \mathrm{E}-01 & 0.0000 \mathrm{E}+00 & 0.0000 \mathrm{E}+00 & -1.3171 \mathrm{E}+01 & 6.1278 \mathrm{E}-01\end{array}$

$\begin{array}{lllllllll}16 & 149 & 2.6508 \mathrm{E}+01 & 0.0000 \mathrm{E}+00 & 0.0000 \mathrm{E}+00 & 0.0000 \mathrm{E}+00 & -2.6508 \mathrm{E}+01 & 3.5884 \mathrm{E}-0.5\end{array}$

$\begin{array}{llllllllll}17 & 144 & 2.7419 \mathrm{E}+01 & 0.0000 \mathrm{E}+00 & 0.0000 \mathrm{E}+00 & 0.0000 \mathrm{E}+00 & -2.7405 \mathrm{E}+01 & 1.4572 \mathrm{E}-02\end{array}$

$\begin{array}{llllllll}18 & 159 & 3.1331 \mathrm{E}+01 & 8.0064 \mathrm{E}-02 & 0.0000 \mathrm{E}+00 & 0.0000 \mathrm{E}+00 & -3.1361 \mathrm{E}+01 & 5.0067 \mathrm{E}-02\end{array}$

$\begin{array}{lllllllll}19 & 1.41 & 3.9111 \mathrm{E}+01 & 4.0032 \mathrm{E}-02 & 0.0000 \mathrm{E}+00 & 0.0000 \mathrm{E}+00 & -3.9150 \mathrm{E}+01 & 4.5860 \mathrm{E}-04\end{array}$

total $\quad 1.4161 \mathrm{E}+02 \quad 9.6077 \mathrm{E}-01 \quad 0.0000 \mathrm{E}+00 \quad 0.0000 \mathrm{E}+00 \quad-1.4174 \mathrm{E}+02 \quad 8.3809 \mathrm{E}-01$

gutron weight balance in each cell - variance reduction events

print table 130 
cell weight cell weight energy dxtran forced exponential total window importance cutoff importance collision transform

$\begin{array}{llllllllll}30 & 0.0000 E+00 & 0.0000 E+00 & 0.0000 E+00 & 0.0000 E+00 & 0.0000 E+00 & 0.0000 E+00 & 0.0000 E+00 & 0.0000 E+00\end{array}$

$\begin{array}{lllllllllll}2 & 31 & 0.0000 E+00 & 0.0000 E+00 & 0.0000 E+00 & 0.0000 E+00 & 0.0000 E+00 & 0.0000 E+00 & 0.0000 E+00 & 0.0000 E+00\end{array}$

$\begin{array}{llllllllll}37 & 0.0000 E+00 & 0.0000 E+00 & 0.0000 E+00 & 0.0000 E+00 & 0.0000 E+00 & 0.0000 E+00 & 0.0000 E+00 & 0.000 & 0.0 E+00\end{array}$

$\begin{array}{llllllllllll}4 & 34 & 0.0000 \mathrm{E}+00 & 0.0000 \mathrm{E}+00 & 0.0000 \mathrm{E}+00 & 0.0000 \mathrm{E}+00 & 0.0000 \mathrm{E}+00 & 0.0000 \mathrm{E}+00 & 0.0000 \mathrm{E}+00 & 0.0000 \mathrm{E}+00\end{array}$

$\begin{array}{llllllllllll}5 & 32 & 0.0000 \mathrm{E}+00 & 0.0000 \mathrm{E}+00 & 0.0000 \mathrm{E}+00 & 0.0000 \mathrm{E}+00 & 0.0000 \mathrm{E}+00 & 0.0000 \mathrm{E}+00 & 0.0000 \mathrm{E}+00 & 0.0000 \mathrm{E}+00\end{array}$

$\begin{array}{llllllllllll}6 & 33 & 0.0000 \mathrm{E}+00 & 0.0000 \mathrm{E}+00 & 0.0000 \mathrm{E}+00 & 0.0000 \mathrm{E}+00 & 0.0000 \mathrm{E}+00 & 0.0000 \mathrm{E}+00 & 0.0000 \mathrm{E}+00 & 0.0000 \mathrm{E}+00\end{array}$

$\begin{array}{lllllllllllll}7 & 35 & 0.0000 \mathrm{E}+00 & 0.0000 \mathrm{E}+00 & 0.0000 \mathrm{E}+0 & 0.0000 \mathrm{E}+0 & 0.0000 \mathrm{E}+00 & 0.0000 \mathrm{E}+00 & 0.0000 \mathrm{E}+00 & 0.0000 \mathrm{E}+00\end{array}$

$\begin{array}{llllllllllll}8 & 36 & 0.0000 \mathrm{E}+00 & 0.0000 \mathrm{E}+00 & 0.0000 \mathrm{E}+00 & 0.0000 \mathrm{E}+00 & 0.0000 \mathrm{E}+00 & 0.0000 \mathrm{E}+00 & 0.0000 \mathrm{E}+00 & 0.0000 \mathrm{E}+00\end{array}$

$\begin{array}{lllllllllll}9 & 38 & 0.0000 \mathrm{E}+00 & 0.0000 \mathrm{E}+00 & 1.2447 \mathrm{E}-03 & 0.0000 \mathrm{E}+00 & 0.0000 \mathrm{E}+00 & 0.0000 \mathrm{E}+00 & 0.0000 \mathrm{E}+00 & 1.2447 \mathrm{E}-03\end{array}$

$\begin{array}{lllllllllllll}10 & 39 & 0.0000 \mathrm{E}+00 & 0.0000 \mathrm{E}+00 & 2.0097 \mathrm{E}-04 & 0.0000 \mathrm{E}+00 & 0.0000 \mathrm{E}+00 & 0.0000 \mathrm{E}+00 & 0.0000 \mathrm{E}+00 & 2.0097 \mathrm{E}-04\end{array}$

$\begin{array}{llllllllllllllll}11 & 40 & 0.0000 \mathrm{E}+00 & 0.0000 \mathrm{E}+00 & 0.0000 \mathrm{E}+00 & 0.0000 \mathrm{E}+00 & 0.0000 \mathrm{E}+00 & 0.0000 \mathrm{E}+00 & 0.0000 \mathrm{E}+00 & 0.0000 \mathrm{E}+00\end{array}$

$\begin{array}{llllllllllll}12 & 41 & 0.0000 \mathrm{E}+00 & 0.0000 \mathrm{E}+00 & 2.9100 \mathrm{E}-04 & 0.0000 \mathrm{E}+00 & 0.0000 \mathrm{E}+00 & 0.0000 \mathrm{E}+00 & 0.000 .0 \mathrm{E}+00 & 2.9100 \mathrm{E}-04\end{array}$

$\begin{array}{lllllllllllllll}13 & 140 & 0.0000 \mathrm{E}+00 & 0.0000 \mathrm{E}+00 & 0.0000 \mathrm{E}+00 & 0.0000 \mathrm{E}+00 & 0.0000 \mathrm{E}+00 & 0.0000 \mathrm{E}+00 & 0.0000 \mathrm{E}+00 & 0.0000 \mathrm{E}+00\end{array}$

$\begin{array}{lllllllllll}14 & 42 & 0.0000 \mathrm{E}+00 & 0.0000 \mathrm{E}+00 & 0.0000 \mathrm{E}+00 & 0.0000 \mathrm{E}+00 & 0.0000 \mathrm{E}+00 & 0.0000 \mathrm{E}+00 & 0.0000 \mathrm{E}+00 & 0.0000 \mathrm{E}+00\end{array}$

$\begin{array}{llllllllllll}15 & 154 & 0.0000 \mathrm{E}+00 & 0.0000 \mathrm{E}+00 & 8.0354 \mathrm{E}-03 & 0.0000 \mathrm{E}+00 & 0.0000 \mathrm{E}+00 & 0.0000 \mathrm{E}+00 & 0.0000 \mathrm{E}+00 & 8.0354 \mathrm{E}-03\end{array}$

$\begin{array}{lllllllllll}16 & 149 & 0.0000 \mathrm{E}+00 & 0.0000 \mathrm{E}+00 & 0.0000 \mathrm{E}+00 & 0.0000 \mathrm{E}+00 & 0.0000 \mathrm{E}+00 & 0.0000 \mathrm{E}+00 & 0.0000 \mathrm{E}+00 & 0.0000 \mathrm{E}+00\end{array}$

$17 \quad 144 \quad 0.0000 \mathrm{E}+00 \quad 0.0000 \mathrm{E}+00 \quad-3.0848 \mathrm{E}-06 \quad 0.0000 \mathrm{E}+00 \quad 0.0000 \mathrm{E}+00 \quad 0.0000 \mathrm{E}+00 \quad 0.0000 \mathrm{E}+00 \quad-3.0848 \mathrm{E}-06$

$\begin{array}{lllllllllll}18 & 159 & 0.0000 \mathrm{E}+00 & 0.0000 \mathrm{E}+0 & -2.52 \mathrm{I} 6 \mathrm{E}-04 & 0.0000 \mathrm{E}+00 & 0.0000 \mathrm{E}+00 & 0.0000 \mathrm{E}+00 & 0.0000 \mathrm{E}+00 & -2.5216 \mathrm{E}-04\end{array}$

$19 \quad 141 \quad 0.0000 \mathrm{E}+00 \quad 0.0000 \mathrm{E}+00 \quad 0.0000 \mathrm{E}+00 \quad 0.0000 \mathrm{E}+00 \quad 0.0000 \mathrm{E}+00 \quad 0.0000 \mathrm{E}+00 \quad 0.0000 \mathrm{E}+00 \quad 0.0000 \mathrm{E}+00$

total $\quad 0.0000 \mathrm{E}+00 \quad 0.0000 \mathrm{E}+00 \quad 9.5169 \mathrm{E}-03 \quad 0.0000 \mathrm{E}+00 \quad 0.0000 \mathrm{E}+00 \quad 0.0000 \mathrm{E}+00 \quad 0.0000 \mathrm{E}+00 \quad 9.5169 \mathrm{E}-03$

Ineutron weight balance in each cell - physical events print table 130

cell $(n, x n)$ fission capture loss to loss to total $(n, x n)$ fission

$\begin{array}{lllllllll}1 & 30 & 0.0000 \mathrm{E}+00 & 0.0000 \mathrm{E}+00 & 0.0000 \mathrm{E}+00 & 0.0000 \mathrm{E}+00 & 0.0000 \mathrm{E}+00 & 0.0000 \mathrm{E}+00\end{array}$

$\begin{array}{llllllllll}2 & 31 & 0.0000 \mathrm{E}+00 & 0.0000 \mathrm{E}+00 & 0.0000 \mathrm{E}+00 & 0.0000 \mathrm{E}+00 & 0.0000 \mathrm{E}+00 & 0.0000 \mathrm{E}+00\end{array}$

$\begin{array}{llllllllll}3 & 37 & 0.0000 \mathrm{E}+00 & 0.0000 \mathrm{E}+00 & 0.0000 \mathrm{E}+00 & 0.0000 \mathrm{E}+00 & 0.0000 \mathrm{E}+00 & 0.0000 \mathrm{E}+00\end{array}$

$\begin{array}{lllllllll}4 & 34 & 0.0000 \mathrm{E}+00 & 0.0000 \mathrm{E}+00 & 0.0000 \mathrm{E}+00 & 0.0000 \mathrm{E}+00 & 0.0000 \mathrm{E}+00 & 0.0000 \mathrm{E}+00\end{array}$

$\begin{array}{llllllll}32 & 0.0000 \mathrm{E}+00 & 0.0000 \mathrm{E}+00 & 0.0000 \mathrm{E}+00 & 0.0000 \mathrm{E}+00 & 0.0000 \mathrm{E}+00 & 0.0000 \mathrm{E}+00\end{array}$ $\begin{array}{llllllll}33 & 0.0000 \mathrm{E}+00 & 0.0000 \mathrm{E}+00 & 0.0000 \mathrm{E}+00 & 0.0000 \mathrm{E}+00 & 0.0000 \mathrm{E}+00 & 0.0000 \mathrm{E}+00\end{array}$ $\begin{array}{lllllllll}35 & 0.0000 E+00 & 0.0000 E+00 & 0.0000 E+00 & 0.0000 E+00 & 0.0000 E+00 & 0.0000 E+00\end{array}$

$\begin{array}{lllllllll}8 & 36 & 0.0000 \mathrm{E}+00 & 0.0000 \mathrm{E}+00 & 0.0000 \mathrm{E}+00 & 0.0000 \mathrm{E}+00 & 0.0000 \mathrm{E}+00 & 0.0000 \mathrm{E}+00\end{array}$

$938 \quad 0.0000 \mathrm{E}+00 \quad 0.0000 \mathrm{E}+00 \quad-1.5855 \mathrm{E}-01 \quad 0.0000 \mathrm{E}+00 \quad 0.0000 \mathrm{E}+00-1.5855 \mathrm{E}-01$

$\begin{array}{llllllll}10 & 39 & 0.0000 \mathrm{E}+00 & 0.0000 \mathrm{E}+00 & -8.2847 \mathrm{E}-04 & 0.0000 \mathrm{E}+00 & 0.0000 \mathrm{E}+00 & -8.2847 \mathrm{E}-04\end{array}$

$11 \quad 40 \quad 0.0000 \mathrm{E}+00 \quad 0.0000 \mathrm{E}+00 \quad-3.9460 \mathrm{E}-06 \quad 0.0000 \mathrm{E}+00 \quad 0.0000 \mathrm{E}+00 \quad-3.9460 \mathrm{E}-06$

$\begin{array}{llllllll}12 & 41 & 0.0000 \mathrm{E}+00 & 0.0000 \mathrm{E}+00 & -3.1114 \mathrm{E}-03 & 0.0000 \mathrm{E}+00 & 0.0000 \mathrm{E}+00 & -3.1114 \mathrm{E}-03\end{array}$

$\begin{array}{lllllllll}13 & 140 & 0.0000 \mathrm{E}+00 & 0.0000 \mathrm{E}+00 & 0.0000 \mathrm{E}+00 & 0.0000 \mathrm{E}+00 & 0.0000 \mathrm{E}+00 & 0.0000 \mathrm{E}+00\end{array}$

$\begin{array}{llllllll}14 & 42 & 1.2461 \mathrm{E}-03 & 0.0000 \mathrm{E}+00 & -3.1955 \mathrm{E}-05 & -6.2306 \mathrm{E}-04 & 0.0000 \mathrm{E}+00 & 5.9111 \mathrm{E}-04\end{array}$

$15 \quad 154 \quad 4.9179 \mathrm{E}-03 \quad 0.0000 \mathrm{E}+00 \quad-1.7239 \mathrm{E}-01 \quad-2.4589 \mathrm{E}-03 \quad-4.5089 \mathrm{E}-01 \quad-6.2082 \mathrm{E}-0 \mathrm{I}$

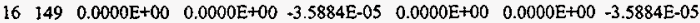

$17 \quad 144 \quad 0.0000 \mathrm{E}+00 \quad 0.0000 \mathrm{E}+00-1.4569 \mathrm{E}-02 \quad 0.0000 \mathrm{E}+00 \quad 0.0000 \mathrm{E}+00 \quad-1.4569 \mathrm{E}-02$

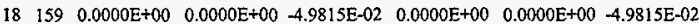

$19 \quad 141 \quad 0.0000 \mathrm{E}+00 \quad 0.0000 \mathrm{E}+00 \quad-4.5860 \mathrm{E}-04 \quad 0.0000 \mathrm{E}+00 \quad 0.0000 \mathrm{E}+00 \quad-4.5860 \mathrm{E}-04$

total $\quad 6.1640 \mathrm{E}-03 \quad 0.0000 \mathrm{E}+00 \quad-3.9980 \mathrm{E}-01 \quad-3.0820 \mathrm{E}-03 \quad-4.5089 \mathrm{E}-01 \quad-8.4760 \mathrm{E}-01$

lneutron activity of each nuclide in each cell, per source particle print table 140

cell nuclides atom total collisions weight lost weight loss weight gain fraction collisions * weight to capture to fission by $(n, x n)$

$9385010.03 \mathrm{~d} \quad 7.1977 \mathrm{E}-01$ $5011.40 \mathrm{c} 8.0041 \mathrm{E}-02$ $6012.40 \mathrm{c} \quad 2.0019 \mathrm{E}-01$

$10 \quad 39 \quad 41093.40 \mathrm{c} \quad 9.8982 \mathrm{E}-0 \mathrm{I}$ $40000.40 \mathrm{c} \quad 1.0183 \mathrm{E}-02$

$11 \quad 40 \quad 1001.00 \mathrm{c} \quad 3.3333 \mathrm{E}-01$ $1002.55 \mathrm{c} \quad 3.3333 \mathrm{E}-01$ $8016.40 \mathrm{c} \quad 3.3333 \mathrm{E}-01$

$41 \quad 41093.40 \mathrm{c} \quad 9.8982 \mathrm{E}-01$
$941 \quad 4.4212 \mathrm{E}-01 \quad 1.5853 \mathrm{E}-01 \quad 0.0000 \mathrm{E}+00 \quad 0.0000 \mathrm{E}+00$ $\begin{array}{llllll}76 & 3.6073 \mathrm{E}-02 & 1.3591 \mathrm{E}-07 & 0.0000 \mathrm{E}+00 & 0.0000 \mathrm{E}+00\end{array}$ $232 \quad 1.1297 \mathrm{E}-01 \quad 1.8446 \mathrm{E}-05 \quad 0.0000 \mathrm{E}+00 \quad 0.0000 \mathrm{E}+00$

$128 \quad 6.2802 \mathrm{E}-02 \quad 8.2814 \mathrm{l} \mathrm{E}-04 \quad 0.0000 \mathrm{E}+00 \quad 0.0000 \mathrm{E}+00$ $2 \quad \begin{array}{lllll}1.7676 \mathrm{E}-04 & 3.3160 \mathrm{E}-07 & 0.0000 \mathrm{E}+00 & 0.0000 \mathrm{E}+00\end{array}$

$1115.1533 \mathrm{E}-02 \quad 3.9251 \mathrm{E}-06 \quad 0.0000 \mathrm{E}+00 \quad 0.0000 \mathrm{E}+00$ $33 \quad 1.5373 \mathrm{E}-02 \quad 2.0884 \mathrm{E}-08 \quad 0.0000 \mathrm{E}+00 \quad 0.0000 \mathrm{E}+00$ $40 \quad 2.1271 \mathrm{E}-02 \quad 0.0000 \mathrm{E}+00 \quad 0.0000 \mathrm{E}+00 \quad 0.0000 \mathrm{E}+00$

$313 \quad 1.5623 E-01 \quad 3.1072 E-03 \quad 0.0000 E+00 \quad 0.0000 E+00$ 
$42 \quad 1001.00 \mathrm{c} \quad 3.3333 \mathrm{E}-01$ $1002.55 \mathrm{c} \quad 3.3333 \mathrm{E}-01$ $8016.40 \mathrm{C} \quad 3.3333 \mathrm{E}-01$
$257 \quad 1.2131 \mathrm{E}-01 \quad 4.3914 \mathrm{E}-06 \quad 0.0000 \mathrm{E}+00 \quad 0.0000 \mathrm{E}+00$

$\begin{array}{lllll}86 & 4.6121 \mathrm{E}-02 & 6.8027 \mathrm{E}-08 & 0.0000 \mathrm{E}+00 & 6.2306 \mathrm{E}-04\end{array}$

$98 \quad 5.1682 \mathrm{E}-02 \quad 2.7495 \mathrm{E}-05 \quad 0.0000 \mathrm{E}+00 \quad 0.0000 \mathrm{E}+00$

I5 $154 \quad 92235.40 \mathrm{c} \quad 3.7504 \mathrm{E}-01$ $92238.40 \mathrm{c} \quad 1.2499 \mathrm{E}-01$ $7014.40 \mathrm{C} \quad 4.9997 \mathrm{E}-01$

$4759 \quad 2.3227 \mathrm{E}+00 \quad 1.3244 \mathrm{E}-01 \quad 4.4247 \mathrm{E}-01 \quad 5.8410 \mathrm{E}-04$

$\begin{array}{llllll}1470 & 7.3870 \mathrm{E}-01 & 2.3272 \mathrm{E}-02 & 8.4162 \mathrm{E}-03 & 1.8748 \mathrm{E}-03\end{array}$

$2207 \quad 1.0775 \mathrm{E}+00 \quad 1.6682 \mathrm{E}-02 \quad 0.0000 \mathrm{E}+00 \quad 0.0000 \mathrm{E}+00$

$16149 \quad 1001.00 \mathrm{c} \quad 3.3333 \mathrm{E}-01$ $1002.55 \mathrm{c} \quad 3.3333 \mathrm{E}-01$ $8016.40 \mathrm{c} \quad 3.3333 \mathrm{E}-01$ $\begin{array}{cccccc}345 & 1.6812 \mathrm{E}-01 & 4.4875 \mathrm{E}-06 & 0.0000 \mathrm{E}+00 & 0.0000 \mathrm{E}+00 \\ 110 & 5.8433 \mathrm{E}-02 & 9.1921 \mathrm{E}-08 & 0.0000 \mathrm{E}+00 & 0.0000 \mathrm{E}+00 \\ 125 & 6.3072 \mathrm{E}-02 & 3.1305 \mathrm{E}-05 & 0.0000 \mathrm{E}+00 & 0.0000 \mathrm{E}+00\end{array}$

$17 \quad 144 \quad 74000.40 \mathrm{c} \quad 1.0000 \mathrm{E}+00$

$885 \quad 4.2652 \mathrm{E}-01 \quad 1.4569 \mathrm{E}-02 \quad 0.0000 \mathrm{E}+00 \quad 0.0000 \mathrm{E}+00$

$18 \quad 159 \quad 41093.40 \mathrm{c} \quad 9.8982 \mathrm{E}-01$

$3965 \quad 2.1009 \mathrm{E}+00 \quad 4.9769 \mathrm{E}-02 \quad 0.0000 \mathrm{E}+00 \quad 0.0000 \mathrm{E}+00$ $40000.40 \mathrm{c} \quad 1.0183 \mathrm{E}-02$ $\begin{array}{lllll}54 & 2.4590 \mathrm{E}-02 & 4.6175 \mathrm{E}-05 & 0.0000 \mathrm{E}+00 & 0.0000 \mathrm{E}+00\end{array}$

$19 \quad 141 \quad 1001.00 \mathrm{c} \quad 3.3333 \mathrm{E}-01$ $1002.55 \mathrm{c} \quad 3.3333 \mathrm{E}-01$ $4029 \quad 1.9096 \mathrm{E}+00 \quad 7.9511 \mathrm{E}-05 \quad 0.0000 \mathrm{E}+00 \quad 0.0000 \mathrm{E}+00$ $8016.40 \mathrm{c} \quad 3.3333 \mathrm{E}-01$ $\begin{array}{llllll}1122 & 5.8206 \mathrm{E}-01 & 9.0056 \mathrm{E}-07 & 0.0000 \mathrm{E}+00 & 0.0000 \mathrm{E}+00\end{array}$ $\begin{array}{lllll}1372 & 7.3131 \mathrm{E}-01 & 3.7819 \mathrm{E}-04 & 0.0000 \mathrm{E}+00 & 0.0000 \mathrm{E}+00\end{array}$

total $22764 \quad 1.1324 \mathrm{E}+0 \mathrm{l} \quad 3.9980 \mathrm{E}-0 \mathrm{I} \quad 4.5089 \mathrm{E}-01 \quad 3.0820 \mathrm{E}-03$

total over all cells for each nuclide total collisions weight lost weight loss weight gain collisions * weight to capture to fission by $(n, x n)$

$\begin{array}{ccccccc}1001.00 \mathrm{c} & 4742 & 2.2505 \mathrm{E}+00 & 9.2315 \mathrm{E}-05 & 0.0000 \mathrm{E}+00 & 0.0000 \mathrm{E}+00 \\ 1002.55 \mathrm{c} & 1351 & 7.0199 \mathrm{E}-01 & 1.0814 \mathrm{E}-06 & 0.0000 \mathrm{E}+00 & 6.2306 \mathrm{E}-04 \\ 5011.40 \mathrm{c} & 76 & 3.6073 \mathrm{E}-02 & 1.3591 \mathrm{E}-07 & 0.0000 \mathrm{E}+00 & 0.0000 \mathrm{E}+00 \\ 6012.40 \mathrm{c} & 232 & 1.1297 \mathrm{E}-01 & 1.8446 \mathrm{E}-05 & 0.0000 \mathrm{E}+00 & 0.0000 \mathrm{E}+00 \\ 7014.40 \mathrm{c} & 2207 & 1.0775 \mathrm{E}+00 & 1.6682 \mathrm{E}-02 & 0.0000 \mathrm{E}+00 & 0.0000 \mathrm{E}+00 \\ 8016.40 \mathrm{c} & 1635 & 8.6733 \mathrm{E}-01 & 4.3699 \mathrm{E}-04 & 0.0000 \mathrm{E}+00 & 0.0000 \mathrm{E}+00 \\ 40000.40 \mathrm{c} & 60 & 2.7441 \mathrm{E}-02 & 5.0739 \mathrm{E}-05 & 0.0000 \mathrm{E}+00 & 0.0000 \mathrm{E}+00 \\ 41093.40 \mathrm{c} & 4406 & 2.3199 \mathrm{E}+00 & 5.3704 \mathrm{E}-02 & 0.0000 \mathrm{E}+00 & 0.0000 \mathrm{E}+00 \\ 74000.40 \mathrm{c} & 885 & 4.2652 \mathrm{E}-01 & 1.4569 \mathrm{E}-02 & 0.0000 \mathrm{E}+00 & 0.0000 \mathrm{E}+00 \\ 92235.40 \mathrm{c} & 4759 & 2.3227 \mathrm{E}+00 & 1.3244 \mathrm{E}-01 & 4.4247 \mathrm{E}-01 & 5.8410 \mathrm{E}-04 \\ 92238.40 \mathrm{c} & 1470 & 7.3870 \mathrm{E}-01 & 2.3272 \mathrm{E}-02 & 8.4162 \mathrm{E}-03 & 1.8748 \mathrm{E}-03 \\ 5010.03 \mathrm{~d} & 941 & 4.4212 \mathrm{E}-01 & 1.5853 \mathrm{E}-01 & 0.0000 \mathrm{E}+00 & 0.0000 \mathrm{E}+00\end{array}$

the initial fission neutron source distribution used the 8 source points that were input on the ksrc card. the criticality problem was scheduled to skip 5 cycles and run a total of 6 cycles with nominally 200 neutrons per cycle. this problem has run 5 inactive cycles with 1027 neutron histories and 1 active cycles with 222 neutron histories.

this calculation has completed the requested number of keff cycles using a total of 1249 fission neutron source histories. all cells with fissionable material were sampled and had fission neutron source points.

there is no combined collision/absorption/track-length estimate for keff because only $l$ active cycles were run. lindividual and average keff estimator results by cycle

keff neutron keff estimators by cycje average keff estimators and deviations average $k(c / a / t)$ cycle histories $k$ (coll) $k(a b s) k(t r a c k) \quad k$ (coll) st dev $k(a b s)$ st dev $k$ (track) st dev $k(c / a / t)$ st dev fom

$200 \mid 1.25311 \quad 1.26302 \quad 1.22009$ |

$257|1.21200 \quad 1.25252 \quad 1.12957|$

$195|1.20026 \quad 1.14326 \quad 1.13006|$

$193 \mid$\begin{tabular}{lllll|}
1.12953 & 1.16269 & 1.10757
\end{tabular}

$182\} 1.135991 .12465 \quad 1.11238$ begin active keff cycles

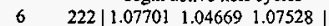

itally $4 \quad$ nps $=1249$

fuel rod flux in 5 y locations averaged over $5 \mathrm{x}$ elements

telly type 4 track length estimate of particle flux. units $1 / \mathrm{cm}^{* * 2}$ 
tally for neutrons

ter of histories used for normalizing tallies $=200.00$

cell $a$ is $\left(154<42[-10-10] 42\left[\begin{array}{lll}-9 & -1 & 0\end{array}\right] 42[-8-10] 42[-7-1 \quad 0] 42\left[\begin{array}{lll}-6 & -1 & 0\end{array}\right)\right.$

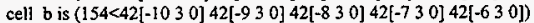

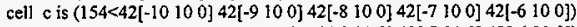

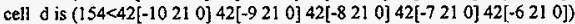

cell $e$ is $(154<42[-10290] 42[-9290] 42[-8290] 42[-7290] 42[-6290])$

volumes

cell: a b c d e

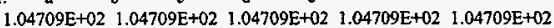

energy: $\quad \begin{array}{llll}1.0000 \mathrm{E}-02 & 1.0000 \mathrm{E}-01 \quad 1.0000 \mathrm{E}+00 & 1.0000 \mathrm{E}+01 \quad \text { total }\end{array}$

cell

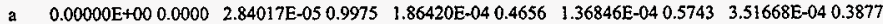

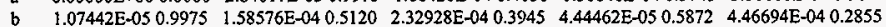

c $\quad 2.16778 \mathrm{E}-050.7232 \quad 9.40943 \mathrm{E}-050.5519 \quad 5.37835 \mathrm{E}-050.5876 \quad 1.51034 \mathrm{E}-04 \quad 0.7075 \quad 3.20589 \mathrm{E}-040.3937$

d $\quad 1.47903 \mathrm{E}-050.5831 \quad 0.00000 \mathrm{E}+000.0000 \quad 1.15515 \mathrm{E}-040.5838 \quad 1.69653 \mathrm{E}-040.5071 \quad 2.99958 \mathrm{E}-04 \quad 0.3718$

e $\quad 8.04943 \mathrm{E}-060.9975 \quad 0.00000 \mathrm{E}+000.0000 \quad 0.00000 \mathrm{E}+000.0000 \quad 6.81909 \mathrm{E}-050.7477 \quad 7.62403 \mathrm{E}-050.6763$

lanalysis of the results in the tally fluctuation chart bin (tfc) for tally 4 with nps $=1249$ print table 160

normed average tally per history $=3.51668 \mathrm{E}-04$

estimated tally relative error $=0.3877$

relative ertor from zero tallies $=0.3464$

number of nonzero history tallies $=8$ efficiency for the nonzero tallies $=0.0400$

history number of largest tally $=\quad 1034$

(largest tally) $/$ (average tally) $=3.84476 \mathrm{E}+01$

(confidence interval shift)/mean $=0.0793$ unnormed average tally per history $=3.68228 \mathrm{E}-02$

estimated variance of the variance $=0.1752$

relative error from nonzero scores $=0.1740$

largest unnormalized history tally $=1.41575 \mathrm{E}+00$

(largest tally) $)^{\prime}$ (avg nonzero tally) $=1.53791 \mathrm{E}+00$

shifted confidence interval center $=3.79539 \mathrm{E}-04$

gest history score sampled so far were to occur on the very next history, the tfe bin quantities would change as follows: 222 for this table because $S$ keff cycles and

1027 histories were skipped before tally accumulation.

$\begin{array}{lccc}\text { estimated quantities } & \text { value at nps } & \text { value at nps+1 } & \text { value(nps+1)/value } \\ & & & \\ \text { mean } & 3.51668 \mathrm{E}-04 & 4.10723 \mathrm{E}-04 & 0.167927 \\ \text { relative error } & 3.87653 \mathrm{E}-01 & 3.61597 \mathrm{E}-01 & -0.067213 \\ \text { variance of the variance } & 1.75161 \mathrm{E}-01 & 1.47665 \mathrm{E}-01 & -0.156976 \\ \text { shifted center } & 3.79539 \mathrm{E}-04 & 3.77169 \mathrm{E}-04 & -0.006244 \\ \text { figure of merit } & 0.00000 \mathrm{E}+00 & 0.00000 \mathrm{E}+00 & 0.000000\end{array}$

there is not enough information in the largest history scores (usually less than 500 scores) for a reliable estimate of the slope. the history score probability density function appears to have an unsampled region at the largest history scores: please examine.

***** the nps-dependent tfc bin check results are suspect because there are only 1 nps tally values to analyze *****

results of 10 statistical checks for the estimated answer for the tally fluctuation chart (tfe) bin of tally 4

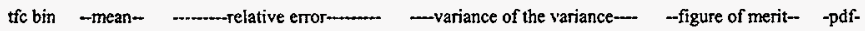

behavior behavior value decrease decrease rate value decrease decrease rate value behavior slope

desired random $<0.10$ yes $1 /$ sqrt(nps) $<0.10$ yes $1 / \mathrm{nps}$ constant random $>3.00$

$\begin{array}{llllll}\text { observed random } 0.39 \text { yes yes } 0.18 \text { yes yes } & \text { yes } & 0.00\end{array}$

passed? yes no yes yes no yes yes no 
waming. the tally in the tally fluctuation chart bin did not pass 3 of the 10 statistical checks.

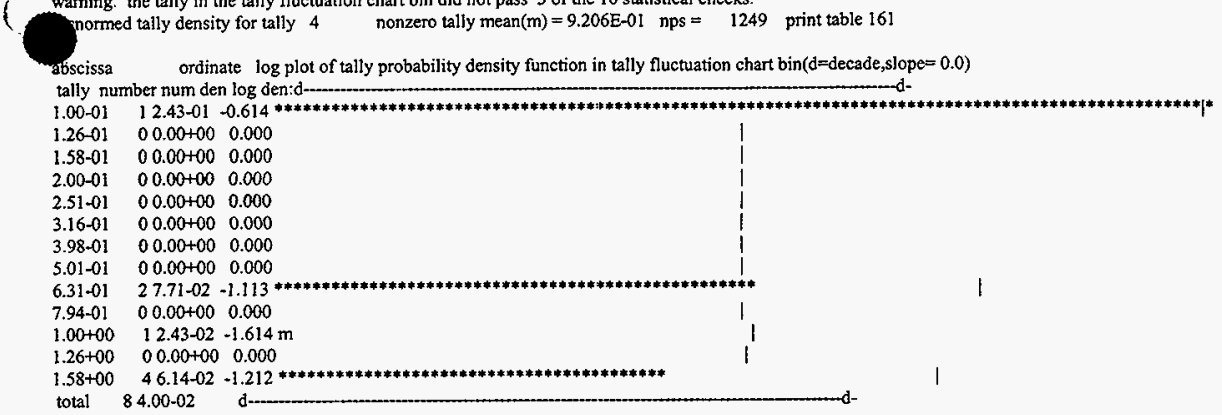

cumulative tally number for tally $4 \quad$ nonzero tally mean $(m)=9.206 \mathrm{E}-01$ nps $=1249$ print table 162

abscissa cum ordinate plot of the cumulative number of tallies in the tally fluctuation chart bin from 0 to 100 percent

tally number cum pet:-

$1.00000 \mathrm{E}-01$

$1.25893 \mathrm{E}-0 \mathrm{I}$

$1.58489 \mathrm{E}-01$

$1.99526 \mathrm{E}-0 \mathrm{I}$

$2.51189 \mathrm{E}-01$

$3.16228 \mathrm{E}-01$

$3.98108 \mathrm{E}-01$

$5.01188 \mathrm{E}-01$

$0958 \mathrm{E}-01$

$4329 \mathrm{E}-01$

$.00000 \mathrm{E}+00$

$1.25893 \mathrm{E}+00$

$1.58489 \mathrm{E}+00$

total

$112500 \mid * * * * * * * * * * * * *$

$\left.12.500\right|^{* * * * * * * * * * * * *}$

$\left.12.500\right|^{* * * * * * * * * * * * *}$

i $12.500 \mid * * * * * * * * * * * *$

$\left.12.500\right|^{\left.* * * * * * * * *\right|^{* * *}}$

I $\left.12.500\right|^{* * * * * * * * * \mid * * *}$

$12.500|* * * * * * * * *| * * *$

$12.500|* * * * * * * * *| * * *$

$\left.33.500\right|^{* * * * * * * * * * * * * * * * * * *|* * * * * * * * *| * * * * * * * * \mid}$

$337.500|* * * * * * * * *| * * * * * * * * *|* * * * * * * * *| * * * * * * * * \mid$

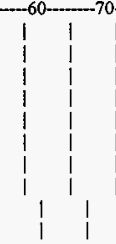

$450.000|\mathrm{mmmmmmmm} / \mathrm{mmmmmmmm} / \mathrm{mmmmmmmm}| \mathrm{mmmmmmmm}$ mmmmmmmmm

$\left.450.000\right|^{* * * * * * * * * *|* * * * * * * *| * * * * * * * * *|* * * * * * * * *| * * * * * * * * * \mid}$

$8100.000|* * * * * * * *| * * * * * * * * *|* * * * * * * * *| * * * * * * * * *|* * * * * * * * *| * * * * * * * * * *|* * * * * * * * * *| * * * * * * * * *|* * * * * * * * * *| * * * * * * * * * * \mid$

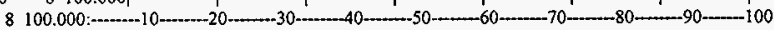

cumulative unnormed tally for tally 4 nonzero tally mean $(m)=9.206 \mathrm{E}-01$ nps $=1249$ print table 162

abscissa cum ordinate plot of the cumulative tally in the tally fluctuation chart bin from 0 to 100 percent tally tally/nps cum pet:-_-10-10 100

$1.000 \mathrm{E}-01 \quad 4.632 \mathrm{E}-04 \quad 1.258$ | $^{*}$

$1.259 \mathrm{E}-01 \quad 4.632 \mathrm{E}-\left.04 \quad 1.258\right|^{*}$

$\begin{array}{lll}1.585 \mathrm{E}-01 & 4.632 \mathrm{E}-04 & \left.1.258\right|^{*}\end{array}$

1.995E-01 4.632E-04 $\left.1.258\right|^{*}$

$2.512 \mathrm{E}-01 \quad 4.632 \mathrm{E}-\left.04 \quad 1.258\right|^{*}$

$3.162 \mathrm{E}-01 \quad 4.632 \mathrm{E}-\left.04 \quad 1.258\right|^{*}$

$3.981 \mathrm{E}-01 \quad 4.632 \mathrm{E}-\left.04 \quad 1.258\right|^{*}$

$5.012 \mathrm{E}-01 \quad 4.632 \mathrm{E}-\left.04 \quad 1.258\right|^{*}$

$6.310 \mathrm{E}-01 \quad 6.195 \mathrm{E}-\left.03 \quad 16.825\right|^{\left.* * * * * * * * *\right|^{* * * * * * * *} \mid}$

7.943E-01 $6.195 \mathrm{E}-\left.03 \quad 16.825\right|^{\left.* * * * * * * * *\right|^{* * * * * * *}}$

$1.000 \mathrm{E}+00 \quad 1.017 \mathrm{E}-02 \quad 27.628 \mid \mathrm{mmmmmmmm} / \mathrm{mmmmmmmm} / \mathrm{mmmmmmm}$ !

$1.259 \mathrm{E}+00 \quad 1.017 \mathrm{E}-\left.02 \quad 27.628\right|_{* * * * * * * * * j ; * * * * * * * * ; * * * * * * * *}$

$1.585 \mathrm{E}+00 \quad 3.682 \mathrm{E}-02 \quad 100.000$

$10 \ldots$

$-30$

$-40-50-60$

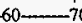

1

Istatus of the statistical checks used to form confidence intervals for the mean for each tally bin

tally result of statistical checks for the tfc bin (the first check not passed is listed) and error magnitude check for all bins

4 missed 3 of 10 tf $\mathrm{f}$ bin checks: the relative error exceeds the recommended value of 0.1 for nonpoint detector tallies missed all bin error check: 25 tally bins had 4 bins with zeros and 21 bins with relative errors exceeding 0.10 
the 10 statistical checks are only for the tally fluctuation chart bin and do not apply to other tally bins.

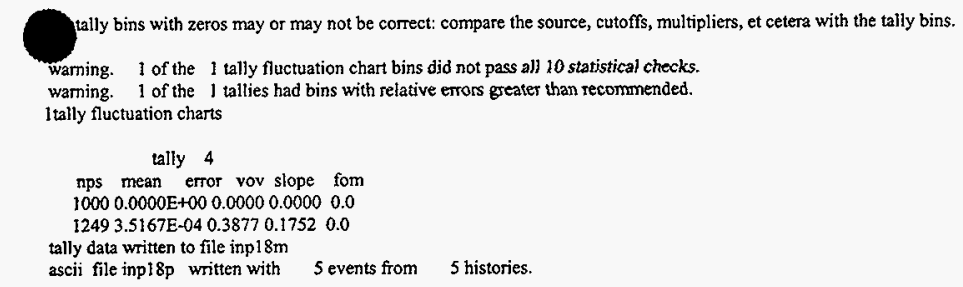

6 waming messages so far.

run terminated when 6 kcode cycles were done.

\section{File: Inp200}

1- prob20 - continuous energy electron version of problem probl9.

2- $\quad 1 \quad 1-19.31-23-45-6$

3. 20 2

4.

5- $1 \mathrm{zO}^{2}$

$+$

6- $2 \quad \mathrm{pz} .00635$

plane

7- $3 \mathrm{px}+10$

8. $4 \times 100$

$+$

9- $5 y-103$

6 p 010011000101

plane

ml 74184. 1

sdef erg=1 sur=1 vec=0 01 par $=3$

imp:p,e 10

mode $p$ e

*fl:p 12

e0 $\quad .1 .191 .282 .373 .464 .555 .645 .736 .827 .918 \quad 1.1$

* fll:e 12

$\operatorname{tr} 90001000100001$

waming. non-orthogonality of surface transformation $9>2 . e-6$

20- fq ef

21- f1:e 12

22- * $288: \mathrm{p} 1$

23- e28 $\quad$-.001 0 1.e-6 $\quad .1 .191 .282 .373 .464 .555 .645 .736 .827 .918 \quad 1.1$

24- es8 -.001 0 1.e-6.1.191 .282.373.464.555.645 .736.827.918 1.1

25- f58:e 1

26- f6:p 1

27- $\quad$ B $3: 0 \quad 12$

28- fl31 elc 1

29- $\quad$ f41:e 12

30. ft4l elc 2

31- f5l:e 12

32. fist elc 3

33- e8 $1000 \mathrm{nt}$

34- *f8:p,e 1

35- nps 10000

36- ctme 30

37. prdmp $2 \mathrm{j}-1$

38- cut:p $\mathrm{lj} .1$

cut:e $1 \mathrm{j} .1$

print 11070 
ming. to 9 card unused.

warning. 1 energy bins of tally 1 are below energy cutoff.

waming. tally 8 needs zero energy bin for negative fo scores. lcells

print table 60

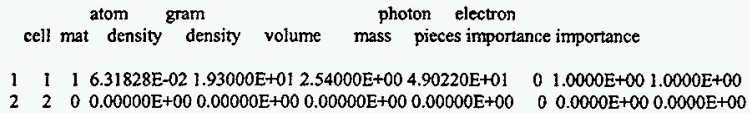

total

$2.54000 \mathrm{E}+004.90220 \mathrm{E}+01$

Isurfaces

print table 70

surface trans type surface coefficients

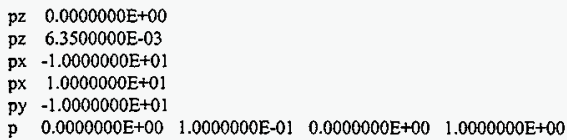

minimum source weight $=1.0000 \mathrm{E}+00$ maximum source weight $=1.0000 \mathrm{E}+00$

4 waming messages so far.

lcross-section tables

table length print table 100

tables from fite testlibl

$74000.02 p \quad 755$

$01 / 15 / 93$

total 755

maximum photon energy set to 100.0 mev (maximum electron energy)

tables from file testlibl

$74000.01 \mathrm{e} \quad 478$

$11 / 16 / 88$

decimal words of dynamically allocated storage

4 warning messages so far.

1 starting mcrun. field length $=0 \quad$ cp $0=0.00 \quad$ print table 110

prob20 - continuous energy electron version of problem prob19

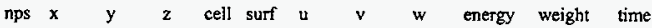

I $0.000 \mathrm{E}+00 \quad 0.000 \mathrm{E}+00 \quad 0.000 \mathrm{E}+00 \quad 1 \quad 1 \quad 3.096 \mathrm{E}-01-4.068 \mathrm{E}-01 \quad 8.595 \mathrm{E}-01 \quad 1.000 \mathrm{E}+00 \quad 1.000 \mathrm{E}+00 \quad 0.000 \mathrm{E}+00$

$20.000 \mathrm{E}+00 \quad 0.000 \mathrm{E}+00 \quad 0.000 \mathrm{E}+00119.871 \mathrm{E}-01 \quad 1.062 \mathrm{E}-01 \quad 1.197 \mathrm{E}-01 \quad 1.000 \mathrm{E}+00 \quad 1.000 \mathrm{E}+00 \quad 0.000 \mathrm{E}+00$

$\begin{array}{lllllllllll}3 & 0.000 \mathrm{E}+00 & 0.000 \mathrm{E}+00 & 0.000 \mathrm{E}+00 & 1 & 1-1.347 \mathrm{E}-01 & 5.198 \mathrm{E}-01 & 8.436 \mathrm{E}-01 & 1.000 \mathrm{E}+00 & 1.000 \mathrm{E}+00 & 0.000 \mathrm{E}+00\end{array}$

$40.000 \mathrm{E}+00 \quad 0.000 \mathrm{E}+00 \quad 0.000 \mathrm{E}+00$ i $1-6.548 \mathrm{E}-02 \quad 7.832 \mathrm{E}-01 \quad 6.184 \mathrm{E}-01 \quad 1.000 \mathrm{E}+00 \quad 1.000 \mathrm{E}+00 \quad 0.000 \mathrm{E}+00$

$\begin{array}{lllllllllllll}5 & 0.000 \mathrm{E}+00 & 0.000 \mathrm{E}+00 & 0.000 \mathrm{E}+00 & \mathrm{l} & 1 & 1.554 \mathrm{E}-01 & -9.204 \mathrm{E}-01 & 3.588 \mathrm{E}-01 & 1.000 \mathrm{E}+00 & 1.000 \mathrm{E}+00 & 0.000 \mathrm{E}+00\end{array}$

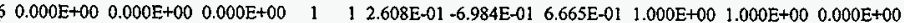

$\begin{array}{lllllllllll}0.000 \mathrm{E}+00 & 0.000 \mathrm{E}+00 & 0.000 \mathrm{E}+00 & 1 & 1 & 6.931 \mathrm{E}-01 & -9.170 \mathrm{E}+02 & 7.149 \mathrm{E}-01 & 1.000 \mathrm{E}+00 & 1.000 \mathrm{E}+00 & 0.000 \mathrm{E}+00\end{array}$ 


\section{File: Outp20}

prob20 - continuous energy electron version of problem probl9.

1 $1-19.31-23-45-6$

3. 20 \$

5. 1202

$+$

6. $2 \mathrm{pz} .00635$

plane

7. $3 \mathrm{px}-10$

8- $4 \times 100$

9. 5 y -103

$+$

10. 6 p 010011000101

plane

11.

12- $\mathrm{ml} 74184 . \mathrm{I}$

13. sdef erg $=1$ sur $=1$ vec $=001$ par $=3$

14- imp:p,e 10

15- mode $\mathrm{p} \mathrm{e}$

16- *fl:p I 2

17- $\quad$ e0 $1.1 .191 .282 .373 .464 .555 .645 .736 .827 .918 \quad 1.1$

18- *f11:e 12

19. 590001000100001

waming. non-orthogonality of surface transformation $9>2 . e-6$

20- fq ef

21- $21: e 12$

22- *f28:p 1

23- e28 $\quad$-.001 $01 . e-6.1 .191 \quad 282.373 .464$.555.645 $.736 .827 .918 \quad 1.1$

24- e58 $\quad-.00101 . \mathrm{e}-6.1 .191 .282 .373 .464 .555 .645 .736 .827 .918 \quad 1.1$

25- f58:e ?

26- f6:p 1

f31:e 12

$\mathrm{ft} 31$ elc 1

f $41: e$ : 2

30. $\mathrm{ft} 41$ elc 2

31- f51:e 12

32- $\mathrm{ft} 51$ elc 3

33- e8 $1000 \mathrm{nt}$

34- *f8:p,e 1

35- nps 10000

36. ctme 30

37- prdmp $2 \mathrm{j}-1$

38- cut:p $1 \mathrm{j} .1$

39- cut:e $1 \mathbf{j} .1$

40- print 11070

$41-$

waming. to 9 card unused.

warning. I energy bins of tally $l$ are below energy cutoff

warning. tally 8 needs zero energy bin for negative 8 scores. lcells

print fable 60

atom gram
cell mat density density volume mass pieces importance importance

I $116.31828 \mathrm{E}-021.93000 \mathrm{E}+012.54000 \mathrm{E}+004.90220 \mathrm{E}+01 \quad 01.0000 \mathrm{E}+001.0000 \mathrm{E}+00$

$220 \quad 0.00000 \mathrm{E}+00 \quad 0.00000 \mathrm{E}+000.00000 \mathrm{E}+00 \quad 0.00000 \mathrm{E}+00 \quad 0 \quad 0.0000 \mathrm{E}+000.0000 \mathrm{E}+00$

total

1 surfaces

$2.54000 \mathrm{E}+004.90220 \mathrm{E}+01$

print table 70

surface trans type surface coefficients 
HNF-SD-SNF-CSWD-005, Rev. 0

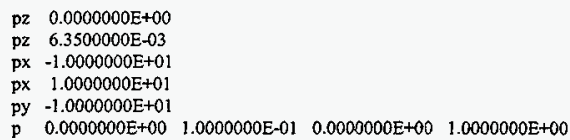

minimum source weight $=1.0000 \mathrm{E}+00 \quad$ maximum source weight $=1.0000 \mathrm{E}+00$

4 waming messages so far.

1cross-section tables

print table 100

table length

tables from file testlibl

$74000.02 p \quad 755$

$01 / 15 / 93$

total 755

maximum photon energy set to 100.0 mev (maximum electron energy)

tables from file testlibl

$74000.01 \mathrm{e} \quad 478$

$11 / 16 / 88$

decimal words of dynamically allocated storage

4 warning messages so far.

tatting morun. field length $=0 \quad \mathrm{cp} 0=0.00 \quad$ print table 110

prob20 - continuous energy electron version of problem prob19.

nps $x \quad y \quad z \quad$ cell surf $u \quad v \quad w$ energy weight time

$10.000 \mathrm{E}+00 \quad 0.000 \mathrm{E}+00 \quad 0.000 \mathrm{E}+00$ $20.000 \mathrm{E}+00 \quad 0.000 \mathrm{E}+00 \quad 0.000 \mathrm{E}+00$

$30.000 \mathrm{E}+000.000 \mathrm{E}+00 \quad 0.000 \mathrm{E}+00$

$40.000 \mathrm{E}+00 \quad 0.000 \mathrm{E}+00 \quad 0.000 \mathrm{E}+00$

$50.000 \mathrm{E}+00 \quad 0.000 \mathrm{E}+00 \quad 0.000 \mathrm{E}+00$

$60.000 \mathrm{E}+00 \quad 0.000 \mathrm{E}+00 \quad 0.000 \mathrm{E}+00$

$7 \quad 0.000 \mathrm{E}+00 \quad 0.000 \mathrm{E}+00 \quad 0.000 \mathrm{E}+00$

$80.000 \mathrm{E}+00 \quad 0.000 \mathrm{E}+00 \quad 0.000 \mathrm{E}+00$

$\begin{array}{llll}9 & 0.000 \mathrm{E}+00 & 0.000 \mathrm{E}+00 & 0.000 \mathrm{E}+00\end{array}$

$10 \quad 0.000 \mathrm{E}+00 \quad 0.000 \mathrm{E}+00 \quad 0.000 \mathrm{E}+00$

$110.000 \mathrm{E}+00 \quad 0.000 \mathrm{E}+00 \quad 0.000 \mathrm{E}+00$

$12 \quad 0.000 \mathrm{E}+00 \quad 0.000 \mathrm{E}+00 \quad 0.000 \mathrm{E}+00$

$130.000 \mathrm{E}+00 \quad 0.000 \mathrm{E}+00 \quad 0.000 \mathrm{E}+00$

$140.000 \mathrm{E}+00 \quad 0.000 \mathrm{E}+00 \quad 0.000 \mathrm{E}+00$

$15 \quad 0.000 \mathrm{E}+00 \quad 0.000 \mathrm{E}+00 \quad 0.000 \mathrm{E}+00$

$16 \quad 0.000 \mathrm{E}+00 \quad 0.000 \mathrm{E}+00 \quad 0.000 \mathrm{E}+00$

$170.000 \mathrm{E}+00 \quad 0.000 \mathrm{E}+00 \quad 0.000 \mathrm{E}+00$

$180.000 \mathrm{E}+00 \quad 0.000 \mathrm{E}+00 \quad 0.000 \mathrm{E}+00$

$190.000 \mathrm{E}+00 \quad 0.000 \mathrm{E}+00 \quad 0.000 \mathrm{E}+00$

$20 \quad 0.000 \mathrm{E}+00 \quad 0.000 \mathrm{E}+00 \quad 0.000 \mathrm{E}+00$

$210.000 \mathrm{E}+00 \quad 0.000 \mathrm{E}+00 \quad 0.000 \mathrm{E}+00$

$22 \quad 0.000 \mathrm{E}+00 \quad 0.000 \mathrm{E}+00 \quad 0.000 \mathrm{E}+00$

$23 \quad 0.000 \mathrm{E}+00 \quad 0.000 \mathrm{E}+00 \quad 0.000 \mathrm{E}+00$

$24 \quad 0.000 \mathrm{E}+00 \quad 0.000 \mathrm{E}+00 \quad 0.000 \mathrm{E}+00$

$25 \quad 0.000 \mathrm{E}+00 \quad 0.000 \mathrm{E}+00 \quad 0.000 \mathrm{E}+00$

$60.000 \mathrm{E}+00 \quad 0.000 \mathrm{E}+00 \quad 0.000 \mathrm{E}+00$

$70.000 \mathrm{E}+00 \quad 0.000 \mathrm{E}+00 \quad 0.000 \mathrm{E}+00$

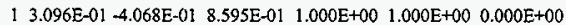
$19.871 \mathrm{E}-01 \quad 1.062 \mathrm{E}-01 \quad 1.197 \mathrm{E}-01 \quad 1.000 \mathrm{E}+00 \quad 1.000 \mathrm{E}+00 \quad 0.000 \mathrm{E}+00$ $\begin{array}{llllll}1-1.347 \mathrm{E}-01 & 5.198 \mathrm{E}-0 \mathrm{~L} & 8.436 \mathrm{E}-01 & 1.000 \mathrm{E}+00 & 1.000 \mathrm{E}+00 & 0.000 \mathrm{E}+00\end{array}$ $\begin{array}{lllllll}1 & -6.548 \mathrm{E}-02 & 7.832 \mathrm{E}-01 & 6.184 \mathrm{E}-01 & 1.000 \mathrm{E}+00 & 1.000 \mathrm{E}+00 & 0.000 \mathrm{E}+00\end{array}$ $1 \quad 1.554 \mathrm{E}-01-9.204 \mathrm{E}-01 \quad 3.588 \mathrm{E}-01 \quad 1.000 \mathrm{E}+00 \quad 1.000 \mathrm{E}+00 \quad 0.000 \mathrm{E}+00$

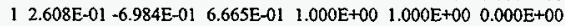
$16.931 \mathrm{E}-01-9.170 \mathrm{E}-02 \quad 7.149 \mathrm{E}-01 \quad 1.000 \mathrm{E}+00 \quad 1.000 \mathrm{E}+00 \quad 0.000 \mathrm{E}+00$

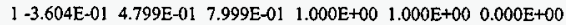

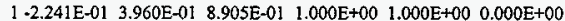

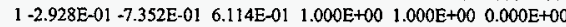
] $8.260 \mathrm{E}-0 \mathrm{I} \quad 1.184 \mathrm{E}-01 \quad 5.510 \mathrm{E}-01 \quad 1.000 \mathrm{E}+00 \quad 1.000 \mathrm{E}+00 \quad 0.000 \mathrm{E}+00$ $17.540 \mathrm{E}-01-1.976 \mathrm{E}-01 \quad 6.264 \mathrm{E}-01 \quad 1.000 \mathrm{E}+00 \quad 1.000 \mathrm{E}+00 \quad 0.000 \mathrm{E}+00$

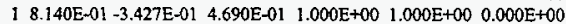
$1-1.257 \mathrm{E}-01 \quad 8.038 \mathrm{E}-01 \quad 5.814 \mathrm{E}-01 \quad 1.000 \mathrm{E}+00 \quad 1.000 \mathrm{E}+00 \quad 0.000 \mathrm{E}+00$ $1-1.464 \mathrm{E}-01-1.070 \mathrm{E}-01 \quad 9.834 \mathrm{E}-01 \quad 1.000 \mathrm{E}+00 \quad 1.000 \mathrm{E}+00 \quad 0.000 \mathrm{E}+00$ $1 \quad 1.310 \mathrm{E}-02-1.617 \mathrm{E}-02 \quad 9.998 \mathrm{E}-01 \quad 1.000 \mathrm{E}+00 \quad 1.000 \mathrm{E}+00 \quad 0.000 \mathrm{E}+00$ $1-8.843 \mathrm{E}-01-4.126 \mathrm{E}-01 \quad 2.187 \mathrm{E}-01 \quad 1.000 \mathrm{E}+00 \quad 1.000 \mathrm{E}+00 \quad 0.000 \mathrm{E}+00$

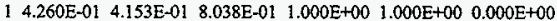
$1-7.311 \mathrm{E}-01-1.136 \mathrm{E}-02 \quad 6.822 \mathrm{E}-01 \quad 1.000 \mathrm{E}+00 \quad 1.000 \mathrm{E}+00 \quad 0.000 \mathrm{E}+00$ $1-2.370 \mathrm{E}-01 \quad 3.207 \mathrm{E}-01 \quad 9.171 \mathrm{E}-01 \quad 1.000 \mathrm{E}+00 \quad 1.000 \mathrm{E}+00 \quad 0.000 \mathrm{E}+00$

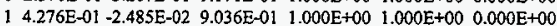

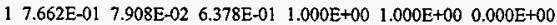

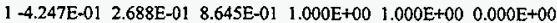
$12.096 \mathrm{E}-01 \quad 5.730 \mathrm{E}-01 \quad 7.923 \mathrm{E}-01 \quad 1.000 \mathrm{E}+00 \quad 1.000 \mathrm{E}+00 \quad 0.000 \mathrm{E}+00$

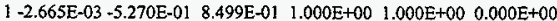
$\begin{array}{lllllll}1 & 7.228 \mathrm{E}-01 & 4.233 \mathrm{E}-01 & 5.463 \mathrm{E}-01 & 1.000 \mathrm{E}+00 & 1.000 \mathrm{E}+00 & 0.000 \mathrm{E}+00\end{array}$ $13.484 \mathrm{E}-01-3.564 \mathrm{E}-01 \quad 8.669 \mathrm{E}-01 \quad 1.000 \mathrm{E}+00 \quad 1.000 \mathrm{E}+00 \quad 0.000 \mathrm{E}+00$ 
$\begin{array}{llll}28 & 0.000 \mathrm{E}+00 & 0.000 \mathrm{E}+00 & 0.000 \mathrm{E}+00\end{array}$ $290.000 \mathrm{E}+00 \quad 0.000 \mathrm{E}+00 \quad 0.000 \mathrm{E}+00$ $0.000 \mathrm{E}+00 \quad 0.000 \mathrm{E}+00 \quad 0.000 \mathrm{E}+00$ $\begin{array}{llll}0.000 \mathrm{E}+00 & 0.000 \mathrm{E}+00 & 0.000 \mathrm{E}+00\end{array}$ $32 \quad 0.000 E \div 00 \quad 0.000 E \div 00 \quad 0.000 E+00$ $33 \quad 0.000 \mathrm{E}+00 \quad 0.000 \mathrm{E}+00 \quad 0.000 \mathrm{E}+00$ $34 \quad 0.000 E+00 \quad 0.000 E+00 \quad 0.000 E+00$ $\begin{array}{llll}35 & 0.000 \mathrm{E}+00 & 0.000 \mathrm{E}+00 & 0.000 \mathrm{E}+00\end{array}$ $\begin{array}{llll}36 & 0.000 \mathrm{E}+00 & 0.000 \mathrm{E}+00 & 0.000 \mathrm{E}+00\end{array}$ $370.000 \mathrm{E}+00 \quad 0.000 \mathrm{E}+00 \quad 0.000 \mathrm{E}+00$ $\begin{array}{llll}38 & 0.000 \mathrm{E}+00 & 0.000 \mathrm{E}+00 & 0.000 \mathrm{E}+00\end{array}$ $39 \quad 0.000 \mathrm{E}+00 \quad 0.000 \mathrm{E}+00 \quad 0.000 \mathrm{E}+00$ $40 \quad 0.000 \mathrm{E}+00 \quad 0.000 \mathrm{E}+00 \quad 0.000 \mathrm{E}+00$ $41 \quad 0.000 E+00 \quad 0.000 E+00 \quad 0.000 E+00$ $42 \quad 0.000 \mathrm{E}+00 \quad 0.000 \mathrm{E}+00 \quad 0.000 \mathrm{E}+00$ $430.000 \mathrm{E}+00 \quad 0.000 \mathrm{E}+00 \quad 0.000 \mathrm{E}+00$ $440.000 \mathrm{E}+00 \quad 0.000 \mathrm{E}+00 \quad 0.000 \mathrm{E}+00$ $450.000 \mathrm{E}+00 \quad 0.000 \mathrm{E}+00 \quad 0.000 \mathrm{E}+00$ $460.000 \mathrm{E}+00 \quad 0.000 \mathrm{E}+00 \quad 0.000 \mathrm{E}+00$ $47 \quad 0.000 \mathrm{E}+00 \quad 0.000 \mathrm{E}+00 \quad 0.000 \mathrm{E}+00$ $48 \quad 0.000 \mathrm{E}+00 \quad 0.000 \mathrm{E}+00 \quad 0.000 \mathrm{E}+00$ $\begin{array}{lllll}49 & 0.000 \mathrm{E}+00 & 0.000 \mathrm{E}+00 & 0.000 \mathrm{E}+00\end{array}$ $50 \quad 0.000 \mathrm{E}+00 \quad 0.000 \mathrm{E}+00 \quad 0.000 \mathrm{E}+00$ Iproblem summary

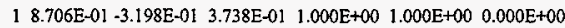
$1-8.188 \mathrm{E}-01 \quad 2.059 \mathrm{E}-01 \quad 5.359 \mathrm{E}-01 \quad 1.000 \mathrm{E}+00 \quad 1.000 \mathrm{E}+00 \quad 0.000 \mathrm{E}+00$

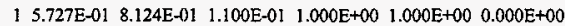
I $5.390 \mathrm{E}-02 \quad 5.914 \mathrm{E}-01 \quad 8.046 \mathrm{E}-01 \quad 1.000 \mathrm{E}+00 \quad 1.000 \mathrm{E}+00 \quad 0.000 \mathrm{E}+00$

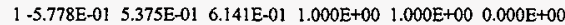
$\begin{array}{lllll}1-7.198 \mathrm{E}-01 & -2.890 \mathrm{E}-01 & 6.311 \mathrm{E}-01 \quad 1.000 \mathrm{E}+00 & 1.000 \mathrm{E}+00 \quad 0.000 \mathrm{E}+00\end{array}$ $12.210 \mathrm{E}-01 \quad 2.482 \mathrm{E}-01 \quad 9.432 \mathrm{E}-01 \quad 1.000 \mathrm{E}+00 \quad 1.000 \mathrm{E}+00 \quad 0.000 \mathrm{E}+00$

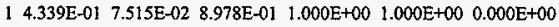
$\begin{array}{llllll}1-4.263 \mathrm{E}-01 & 8.490 \mathrm{E}-01 & 3.123 \mathrm{E}-01 & 1.000 \mathrm{E}+00 & 1.000 \mathrm{E}+00 & 0.000 \mathrm{E}+00\end{array}$

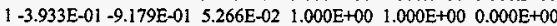

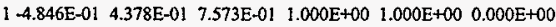
$1-5.950 \mathrm{E}-01+5.730 \mathrm{E}-01 \quad 5.636 \mathrm{E}-01 \quad 1.000 \mathrm{E}+00 \quad 1.000 \mathrm{E}+00 \quad 0.000 \mathrm{E}+00$ $1-7.252 \mathrm{E}-01-5.143 \mathrm{E}-01 \quad 4.578 \mathrm{E}-01 \quad 1.000 \mathrm{E}+00 \quad 1.000 \mathrm{E}+00 \quad 0.000 \mathrm{E}+00$

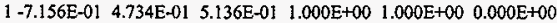
$\begin{array}{llllll}1-1.771 \mathrm{E}-01 & 9.229 \mathrm{E}-01 & 3.417 \mathrm{E}-01 & 1.000 \mathrm{E}+00 & 1.000 \mathrm{E}+00 & 0.000 \mathrm{E}+00\end{array}$ $1-1.149 \mathrm{E}-01-4.206 \mathrm{E}-01 \quad 8.999 \mathrm{E}-01 \quad 1.000 \mathrm{E}+00 \quad 1.000 \mathrm{E}+00 \quad 0.000 \mathrm{E}+00$ i $4.509 \mathrm{E}-01 \quad 8.630 \mathrm{E}-02 \quad 8.884 \mathrm{E}-01 \quad 1.000 \mathrm{E}+00 \quad 1.000 \mathrm{E}+00 \quad 0.000 \mathrm{E}+00$ $\begin{array}{llllllll}1-7.358 \mathrm{E}-01 & 2.500 \mathrm{E}-01 & 6.294 \mathrm{E}-01 & 1.000 \mathrm{E}+00 & 1.000 \mathrm{E}+00 & 0.000 \mathrm{E}+00\end{array}$

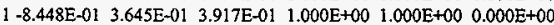
$1 \quad 1.958 \mathrm{E}-01-2.769 \mathrm{E}-01 \quad 9.407 \mathrm{E}-01 \quad 1.000 \mathrm{E}+00 \quad 1.000 \mathrm{E}+00 \quad 0.000 \mathrm{E}+00$ $12.788 \mathrm{E}-01-7.325 \mathrm{E}-03 \quad 9.603 \mathrm{E}-01 \quad 1.000 \mathrm{E}+00 \quad 1.000 \mathrm{E}+00 \quad 0.000 \mathrm{E}+00$

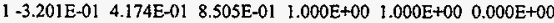
$18.960 \mathrm{E}-01-1.641 \mathrm{E}-01 \quad 4.126 \mathrm{E}-01 \quad 1.000 \mathrm{E}+00 \quad 1.000 \mathrm{E}+00 \quad 0.000 \mathrm{E}+00$ 0

run terminated when 10000 particle histories were done.

photon creation tracks weight energy photon loss tracks weight energy
(per source particle)

$\begin{array}{cccccccc}\text { source } & 0 & 0 . & 0 . & \text { escape } & 575 & 5.7500 \mathrm{E}-02 & 1.5476 \mathrm{E}-02 \\ & & & & \text { energy cutoff } & 0 & 0 . & 0 .\end{array}$

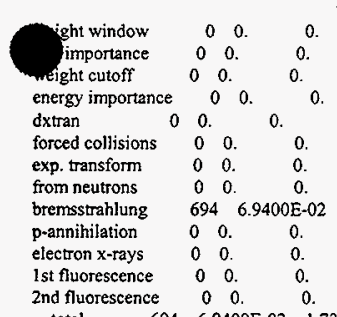

time cutoff $\quad 00$. 0 .

$\begin{array}{llllllll}\text { total } & 694 & 6.9400 \mathrm{E}-02 & 1.73 \mathrm{LE}-02 & \text { total } & 694 & 6.9400 \mathrm{E}-02 & 1.7315 \mathrm{E}-02\end{array}$

number of photons banked

weight window

00 . cell importance $\quad 00$. 0

weight cutoff $\begin{array}{lll}0 & 0 . & 0\end{array}$

forced collisions $\quad 0 \quad 0$. 0 .

exp. transform 00 . 0 .

compton scatter $\quad 00$. $\quad 6.6993 \mathrm{E}-05$

$\begin{array}{lllll}1.7315 \mathrm{E}-02 & \text { capture } & 119 & 1.1900 \mathrm{E}-02 & 1.7720 \mathrm{E}-03\end{array}$

pair production $\quad 0 \quad 0 . \quad 0$.

photon tracks per source particle $\quad 6.9400 \mathrm{E}-02 \quad$ escape $\quad 6.7786 \mathrm{E}-05$ tco $\quad 1.0000 \mathrm{E}+34$

photon collisions per source particle $1.3000 \mathrm{E}-02$ capture $8.3325 \mathrm{E}-05$ eco $1.0000 \mathrm{E}-01$

total photon collisions 130 capture or escape $7.0450 \mathrm{~J}-05$ wcl $0.0000 \mathrm{E}+00$

0

any termination $7.0450 \mathrm{E}-05 \quad$ wc2 $0.0000 \mathrm{E}+00$

electron creation tracks weight energy electron loss tracks weight energy (per source particle)

source

$10000 \quad 1.0000 \mathrm{E}+00$

$1.0000 \mathrm{E}+00$ energy cutoff $2046 \quad 2.0460 \mathrm{E}-01 \quad 2.0141 \mathrm{E}-02$ time cutoff 00.

weight window cell importance weight cutoff energy importance pair production 00. 0 weight window 00 $\begin{array}{lllllll}0 & 0 . & 0 . & 0 . l l & 0 . & 0 & 0 .\end{array}$ 00 . 0. 00. 0. 00. 0. 00 . weight cutoff $\begin{array}{lll}0 & 0 .\end{array}$ energy importance scattering 00.

$2 \quad 2.0000 \mathrm{E}-04 \quad 3.7815 \mathrm{E}-05$ bremsstrahlung $\begin{array}{lrcc}\text { pion recoil } & 2 & 2.0000 \mathrm{E}-04 & 3.7815 \mathrm{E}-05 \\ \text { lo-electric } & 29 & 2.9000 \mathrm{E}-03 & 4.7387 \mathrm{E}-04\end{array}$ 


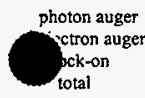

$\begin{array}{cccc}0 & 0 . & 0 . & \\ 0 & 0 . & 0 . & \\ 1479 & 1.4790 \mathrm{E}-01 & 2.7007 \mathrm{E}-02 \\ 11510 & 1.1510 \mathrm{E}+00 & 1.0275 \mathrm{E}+00\end{array}$

number of electrons banked

1510

electron tracks per source particle $1.1510 \mathrm{E}+00$

electron sub-steps per source particle $7.7164 \mathrm{E}+01$

total electron sub-steps

771636

\begin{abstract}
computer time so far in this run 0.00 minutes computer time in mcrun 0.00 minutes source particles per minute $0.0000 \mathrm{E}+00$

4748878
\end{abstract}

total $\quad 11510 \quad 1.1510 \mathrm{E}+00 \quad 1.0275 \mathrm{E}+00$

\author{
cutoffs \\ tco $1.0000 \mathrm{E}+34$ \\ eco $1.0000 \mathrm{E}-01$ \\ wel $0.0000 \mathrm{E}+00$ \\ wc2 $0.0000 E+00$
}

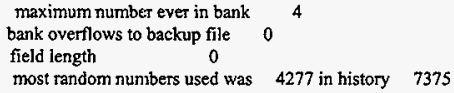

range of sampled source weights $=1.0000 \mathrm{E}+00$ to $1.0000 \mathrm{E}+00$

Iphoton activity in each cell

print table 126

tracks population collisions collisions number flux average average

cell entering * weight weighted weighted track weight track mfp (per history) energy energy (relative) (cm)

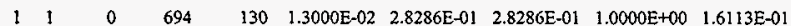

$\begin{array}{lllll}\text { total } & 0 & 694 & 130 & 1.3000 \mathrm{E}-02\end{array}$

lelectron activity in each cell $\quad$ print table 126

tracks population substeps substeps number flux average average

cell entering weight weighted weighted track weight track mfp (per history) energy energy (relative) (cm)

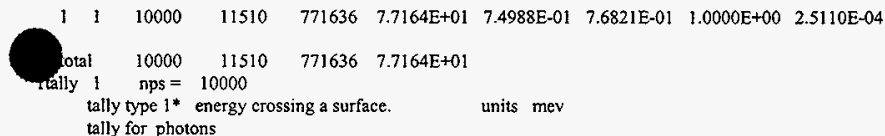

surface: 1

energy

$1.0000 \mathrm{E}-01 \quad 0.00000 \mathrm{E}+00 \quad 0.0000 \quad 0.00000 \mathrm{E}+00 \quad 0.0000$

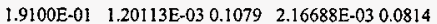

2.8200E-01 8.17217E-04 0.1673 2.04350E-03 0.1062

3.7300E-01 9.52191E-04 0.1829 $1.82060 \mathrm{E}-030.1357$

4.6400E-01 $\quad 1.03815 \mathrm{E}-03 \quad 0.2001 \quad 1.00234 \mathrm{E}-03 \quad 0.2043$

5.5500E-01 5.66295E-04 0.3018 $8.02842 \mathrm{E}-040.2503$

$\begin{array}{lllll}6.4500 \mathrm{E}-01 & 6.09365 \mathrm{E}-04 & 0.3162 & 6.54966 \mathrm{E}-04 & 0.3016\end{array}$

$7.3600 \mathrm{E}-01 \quad 3.45345 \mathrm{E}-04 \quad 0.4472 \quad 4.20131 \mathrm{E}-040.4085$

8.2700E-01 7.79347E-05 $0.9999 \quad 7.03770 \mathrm{E}-040.3334$

$9.1800 \mathrm{E}-01 \quad 1.70096 \mathrm{E}-040.7070 \quad 8.29418 \mathrm{E}-050.9999$

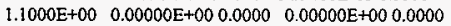

total $5.77773 \mathrm{E}-030.0811 \quad 9.69798 \mathrm{E}-030.0608$

Itally 6 nps $=10000$

tally type 6 track length estimate of heating. units mev/gram

tally for photons

masses

cell: 1

$4.90220 \mathrm{E}+01$

cell:

energy

$1.0000 \mathrm{E}-01 \quad 0.00000 \mathrm{E}+000.0000$

$1.9100 \mathrm{E}-01 \quad 1.80395 \mathrm{E}-050.0935$

$8200 \mathrm{E}-01 \quad 4.79830 \mathrm{E}-060.1242$

.7300 E-01 1.64870E-06 0.2061 
4.6400E-01 $1.76412 \mathrm{E}-060.4116$ 5500E-01 $6.34259 \mathrm{E}-07 \quad 0.4736$ -4500E-01 2.47457E-07 0.3129 3600E-01 2.58011E-07 0.6571

$8.2700 \mathrm{E}-01 \quad 1.16245 \mathrm{E}-07 \quad 0.3470$

$9.1800 \mathrm{E}-01 \quad 4.46610 \mathrm{E}-080.6403$

$1.1000 \mathrm{E}+00 \quad 0.00000 \mathrm{E}+000.0000$

total $2.75512 \mathrm{E}-050.0722$

t tally 28 nps $=10000$

tally type 8* energy deposition

units mev

tally for photons electrons

cell: 1

energy

$-1.0000 \mathrm{E}-03 \quad-8.72550 \mathrm{E}-040.1508$

$0.0000 \mathrm{E}+00 \quad 0.00000 \mathrm{E}+00 \quad 0.0000$

$1.0000 \mathrm{E}-06 \quad 0.00000 \mathrm{E}+000.0000$

$1.0000 \mathrm{E}-01 \quad 1.08193 \mathrm{E}-020.0227$

$1.9100 \mathrm{E}-01 \quad 3.91016 \mathrm{E}-020.0170$

$2.8200 \mathrm{E}-01 \quad 4.36100 \mathrm{E}-020.0210$

$3.7300 \mathrm{E}-01 \quad 3.53763 \mathrm{E}-02 \quad 0.0286$

4.6400E $-01 \quad 2.66881 \mathrm{E}-02 \quad 0.0382$

$5.5500 \mathrm{E}-01 \quad 2.21833 \mathrm{E}-02 \quad 0.0468$

6.4500E-01 $1.58947 \mathrm{E}-020.0606$

$7.3600 \mathrm{E}-01 \quad 1.08330 \mathrm{E}-02 \quad 0.0790$

$8.2700 \mathrm{E}-01 \quad 8.71177 \mathrm{E}-030.0940$

$9.1800 \mathrm{E}-01 \quad 6.65495 \mathrm{E}-030.1136$

$1.1000 \mathrm{E}+00 \quad 4.79000 \mathrm{E}-020.0446$

total 2.66900E-01 0.0090

itally II $\mathrm{nps}=10000$

tally type 1* energy crossing a surface. units mev tally for electrons

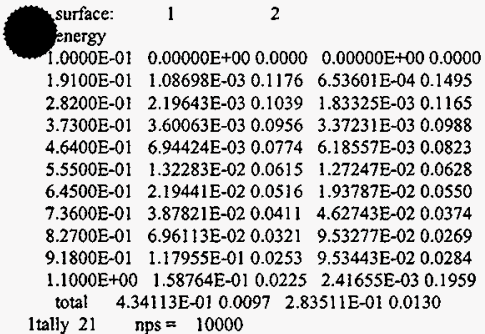

tally type 1 number of particles crossing a surface. tally for electrons

surface: $1 \quad 2$

$1.0000 \mathrm{E}-01 \quad 0.00000 \mathrm{E}+00 \quad 0.0000 \quad 0.00000 \mathrm{E}+00 \quad 0.0000$ $1.9100 \mathrm{E}-01 \quad 7.70000 \mathrm{E}-03 \quad 0.1165 \quad 4.60000 \mathrm{E}-03 \quad 0.1471$

$2.8200 \mathrm{E}-01 \quad 9.30000 \mathrm{E}-03 \quad 0.1032 \quad 7.60000 \mathrm{E}-03 \quad 0.1158$

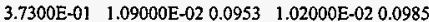
$\begin{array}{lllll}4.6400 \mathrm{E}-01 & 1.65000 \mathrm{E}-02 & 0.0772 & 1.46000 \mathrm{E}-02 & 0.0822\end{array}$ $\begin{array}{lllll}5.5500 \mathrm{E}-01 & 2.58000 \mathrm{E}-02 & 0.0614 & 2.48000 \mathrm{E}-02 & 0.0627\end{array}$ $6.4500 \mathrm{E}-0 \mathrm{I} \quad 3.63000 \mathrm{E}-020.0515 \quad 3.21000 \mathrm{E}-02 \quad 0.0549$ $\begin{array}{lllll}7.3600 \mathrm{E}-01 & 5.59000 \mathrm{E}-02 & 0.0411 & 6.67000 \mathrm{E}-02 & 0.0374\end{array}$

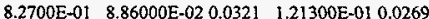
$9.1800 \mathrm{E}-01 \quad 1.34800 \mathrm{E}-010.0253 \quad 1.30600 \mathrm{E}-010.0284$ $1.1000 \mathrm{E}+00 \quad 1.65500 \mathrm{E}-01 \quad 0.0225 \quad 2.60000 \mathrm{E}-03 \quad 0.1959$ total $5.51300 \mathrm{E}-010.0094 \quad 3.95100 \mathrm{E}-010.0126$ ly $31 \quad \mathrm{nps}=10000$

tally type 1 number of particles crossing a surface. 
taily for electrons

HNF-SD-SNF-CSWD-005, Rev. 0

this tally is modified by ft elc

surface: $\quad 1 \quad 2$

energy

$1.0000 \mathrm{E}-01 \quad 0.00000 \mathrm{E}+000.0000 \quad 0.00000 \mathrm{E}+00 \quad 0.0000$

$1.9100 \mathrm{E}-01-7.70000 \mathrm{E}-03 \quad 0.1165-4.60000 \mathrm{E}-030.1471$

$2.8200 \mathrm{E}-01-9.30000 \mathrm{E}-03 \quad 0.1032-7.60000 \mathrm{E}-03 \quad 0.1158$

$3.7300 \mathrm{E}-01-1.09000 \mathrm{E}-020.0953-1.02000 \mathrm{E}-020.0985$

$4.6400 \mathrm{E}-01-1.65000 \mathrm{E}-02 \quad 0.0772-1.46000 \mathrm{E}-020.0822$

$\begin{array}{lllll}5.5500 \mathrm{E}-01 & -2.58000 \mathrm{E}-02 & 0.0614 & -2.48000 \mathrm{E}-02 & 0.0627\end{array}$

$6.4500 \mathrm{E}-01 \quad-3.63000 \mathrm{E}-02 \quad 0.0515-3.21000 \mathrm{E}-020.0549$

7.3600E-01 $-5.59000 \mathrm{E}-020.0411-6.67000 \mathrm{E}-020.0374$

8.2700E-01 $-8.86000 \mathrm{E}-020.0321-1.21300 \mathrm{E}-010.0269$

$9.1800 \mathrm{E}-01-1.34800 \mathrm{E}-010.0253-1.10600 \mathrm{E}-010.0284$

$1.1000 \mathrm{E}+00-1.65500 \mathrm{E}-010.0225-2.60000 \mathrm{E}-030.1959$

totaI $-5.51300 \mathrm{E}-010.0094 \cdot 3.95100 \mathrm{E}-010.0126$

itally $41 \quad \mathrm{nps}=10000$

tally type 1 number of particles crossing a surface.

tally for electrons

this tally is modified by $\mathrm{ft}$ elc

surface: $\quad 1 \quad 2$

energy

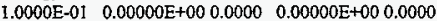

$1.9100 \mathrm{E}-01 \quad 0.00000 \mathrm{E}+00 \quad 0.0000 \quad 0.00000 \mathrm{E}+000.0000$

$2.8200 \mathrm{E}-01 \quad 0.00000 \mathrm{E}+00 \quad 0.0000 \quad 0.00000 \mathrm{E}+00 \quad 0.0000$

$3.7300 \mathrm{E}-01 \quad 0.00000 \mathrm{E}+00 \quad 0.0000 \quad 0.00000 \mathrm{E}+00 \quad 0.0000$

4.6400E-01 $\quad 0.00000 \mathrm{E}+00 \quad 0.0000 \quad 0.00000 \mathrm{E}+00 \quad 0.0000$

$5.5500 \mathrm{E}-01 \quad 0.00000 \mathrm{E}+00 \quad 0.0000 \quad 0.00000 \mathrm{E}+000.0000$

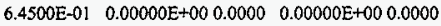
$7.3600 \mathrm{E}-01 \quad 0.00000 \mathrm{E}+00 \quad 0.0000 \quad 0.00000 \mathrm{E}+00 \quad 0.0000$

$8.2700 \mathrm{E}-01 \quad 0.00000 \mathrm{E}+00 \quad 0.0000 \quad 0.00000 \mathrm{E}+000.0000$

$1800 \mathrm{E}-01 \quad 0.00000 \mathrm{E} \div 00 \quad 0.0000 \quad 0.00000 \mathrm{E}+000.0000$

$1000 \mathrm{E}+00 \quad 0.00000 \mathrm{E}+00 \quad 0.0000 \quad 0.00000 \mathrm{E}+000.0000$

tota] $0.00000 \mathrm{E}+00 \quad 0.0000 \quad 0.00000 \mathrm{E}+000.0000$

surface: $1 \quad 2$

energy

$1.0000 \mathrm{E}-01 \quad 0.00000 \mathrm{E}+00 \quad 0.0000 \quad 0.00000 \mathrm{E}+000.0000$ $1.9100 \mathrm{E}-01 \quad 7.70000 \mathrm{E}-03 \quad 0.1165 \quad 4.60000 \mathrm{E}-03 \quad 0.1471$

$2.8200 \mathrm{E}-01 \quad 9.30000 \mathrm{E}-03 \quad 0.1032 \quad 7.60000 \mathrm{E}-03 \quad 0.1158$

$3.7300 \mathrm{E}-01 \quad 1.09000 \mathrm{E}-02 \quad 0.0953 \quad 1.02000 \mathrm{E}-02 \quad 0.0985$

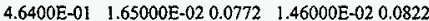

$5.5500 \mathrm{E}-01 \quad 2.58000 \mathrm{E}-02 \quad 0.0614 \quad 2.48000 \mathrm{E}-020.0627$

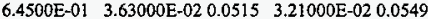

$7.3600 \mathrm{E}-01 \quad 5.59000 \mathrm{E}-020.0411 \quad 6.67000 \mathrm{E}-02 \quad 0.0374$

$\begin{array}{lllll}8.2700 \mathrm{E}-01 & 8.86000 \mathrm{E}-02 & 0.0321 & 1.21300 \mathrm{E}-01 & 0.0269\end{array}$

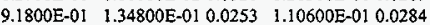

$1.1000 \mathrm{E}+00 \quad 1.65500 \mathrm{E}-01 \quad 0.0225 \quad 2.60000 \mathrm{E}-03 \quad 0.1959$

total $5.51300 \mathrm{E}-010.0094 \quad 3.95100 \mathrm{E}-010.0126$

surface: 1

energy

$1.0000 \mathrm{E}-0 \mathrm{I} \quad 0.00000 \mathrm{E}+000.0000 \quad 0.00000 \mathrm{E}+000.0000$ $1.9100 \mathrm{E}-01 \quad 7.70000 \mathrm{E}-03 \quad 0.1165 \quad 4.60000 \mathrm{E}-03 \quad 0.1471$

$2.8200 \mathrm{E}-01 \quad 9.30000 \mathrm{E}-03 \quad 0.1032 \quad 7.60000 \mathrm{E}-03 \quad 0.1158$

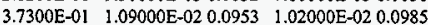

$4.6400 \mathrm{E}-01 \quad 1.65000 \mathrm{E}-02 \quad 0.0772 \quad 1.46000 \mathrm{E}-02 \quad 0.0822$

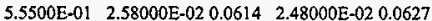

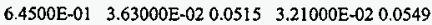

$7.3600 \mathrm{E}-01 \quad 5.59000 \mathrm{E}-02 \quad 0.0411 \quad 6.67000 \mathrm{E}-02 \quad 0.0374$

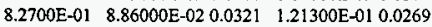

$9.1800 \mathrm{E}-01 \quad 1.34800 \mathrm{E}-01 \quad 0.0253 \quad 1.10600 \mathrm{E}-010.0284$

$1.1000 \mathrm{E}+00 \quad 1.65500 \mathrm{E}-01 \quad 0.0225 \quad 2.60000 \mathrm{E}-03 \quad 0.1959$

total $5.51300 \mathrm{E}-010.0094 \quad 3.95100 \mathrm{E}-010.0126$

y 51 nps $=10000$ 
tally type 1 number of particles crossing a surface. tally for electrons

this tally is modified by ft elc

$$
\text { surface: } 1 \quad 2
$$

energy

$1.0000 \mathrm{E}-01 \quad 0.00000 \mathrm{E}+00 \quad 0.0000 \quad 0.00000 \mathrm{E}+000.0000$

$1.9100 \mathrm{E}-01 \quad 0.00000 \mathrm{E}+00 \quad 0.0000 \quad 0.00000 \mathrm{E}+00 \quad 0.0000$

$2.8200 \mathrm{E}-01 \quad 0.00000 \mathrm{E}+00 \quad 0.0000 \quad 0.00000 \mathrm{E}+000.0000$

$3.7300 \mathrm{E}-01 \quad 0.00000 \mathrm{E}+00 \quad 0.0000 \quad 0.00000 \mathrm{E}+000.0000$

4.6400E-01 $\quad 0.00000 \mathrm{E}+00 \quad 0.0000 \quad 0.00000 \mathrm{E}+00 \quad 0.0000$

$5.5500 \mathrm{E}-01 \quad 0.00000 \mathrm{E}+00 \quad 0.0000 \quad 0.00000 \mathrm{E}+00 \quad 0.0000$

6.4500E-01 $\quad 0.00000 \mathrm{E}+00 \quad 0.0000 \quad 0.00000 \mathrm{E}+00 \quad 0.0000$

$7.3600 \mathrm{E}-01 \quad 0.00000 \mathrm{E}+00 \quad 0.0000 \quad 0.00000 \mathrm{E}+000.0000$

$8.2700 \mathrm{E}-01 \quad 0.00000 \mathrm{E}+000.0000 \quad 0.00000 \mathrm{E}+000.0000$

$9.1800 \mathrm{E}-01 \quad 0.00000 \mathrm{E}+00 \quad 0.0000 \quad 0.00000 \mathrm{E}+000.0000$

$1.1000 \mathrm{E}+00 \quad 0.00000 \mathrm{E}+000.0000 \quad 0.00000 \mathrm{E}+000.0000$ total $\quad 0.00000 \mathrm{E}+000.0000 \quad 0.00000 \mathrm{E}+000.0000$

surface: 112

energy

$1.0000 \mathrm{E}-01 \quad 0.00000 \mathrm{E}+000.0000 \quad 0.00000 \mathrm{E}+000.0000$ $1.9100 \mathrm{E}-01-7.70000 \mathrm{E}-03 \quad 0.1165-4.60000 \mathrm{E}-03 \quad 0.1471$

$2.8200 \mathrm{E}-01-9.30000 \mathrm{E}-030.1032-7.60000 \mathrm{E}-03 \quad 0.1158$ $3.7300 \mathrm{E}-01-1.09000 \mathrm{E}-020.0953-1.02000 \mathrm{E}-020.0985$ $4.6400 \mathrm{E}-01-1.65000 \mathrm{E}-020.0772-1.46000 \mathrm{E}-020.0822$ $5.5500 \mathrm{E}-01-2.58000 \mathrm{E}-020.0614-2.48000 \mathrm{E}-020.0627$ $6.4500 \mathrm{E}-01-3.63000 \mathrm{E}-020.0515-3.21000 \mathrm{E}-020.0549$ $7.3600 \mathrm{E}-01 \quad-5.59000 \mathrm{E}-020.0411-6.67000 \mathrm{E}-020.0374$ $8.2700 \mathrm{E}-01-8.86000 \mathrm{E}-020.0321-1.21300 \mathrm{E}-010.0269$ $9.1800 \mathrm{E}-01-1.34800 \mathrm{E}-010.0253+1.10600 \mathrm{E}-010.0284$ $1.1000 \mathrm{E}+00-1.65500 \mathrm{E}-01 \quad 0.0225-2.60000 \mathrm{E}-03 \quad 0.1959$ total $-5.51300 \mathrm{E}-010.0094-3.95100 \mathrm{E}-010.0126$

surface: $\quad 1 \quad 2$

energy

$1.0000 \mathrm{E}-01 \quad 0.00000 \mathrm{E}+00 \quad 0.0000 \quad 0.00000 \mathrm{E}+000.0000$

$1.9100 \mathrm{E}-01 \quad-7.70000 \mathrm{E}-03 \quad 0.1165-4.60000 \mathrm{E}-030.1471$

$2.8200 \mathrm{E}-01 \quad-9.30000 \mathrm{E}-03 \quad 0.1032-7.60000 \mathrm{E}-030.1158$

$3.7300 \mathrm{E}-01-1.09000 \mathrm{E}-020.0953-1.02000 \mathrm{E}-020.0985$

4.6400E-01 - $-1.65000 \mathrm{E}-020.0772-1.46000 \mathrm{E}-02 \quad 0.0822$

$5.5500 \mathrm{E}-0]-2.58000 \mathrm{E}-020.0614-2.48000 \mathrm{E}-020.0627$

$6.4500 \mathrm{E}-01-3.63000 \mathrm{E}-02 \quad 0.0515-3.21000 \mathrm{E}-02 \quad 0.0549$ $7.3600 \mathrm{E}-01-5.59000 \mathrm{E}-020.041]-6.67000 \mathrm{E}-020.0374$

$8.2700 \mathrm{E}-01-8.86000 \mathrm{E}-020.0321-1.21300 \mathrm{E}-010.0269$ $9.1800 \mathrm{E}-01-1.34800 \mathrm{E}-010.0253-1.10600 \mathrm{E}-010.0284$

$1.1000 \mathrm{E}+00-1.65500 \mathrm{E}-010.0225-2.60000 \mathrm{E}-030.1959$

total $-5.51300 \mathrm{E}-010.0094-3.95100 \mathrm{E}-010.0126$

1tally $58 \quad \mathrm{nps}=10000$

tally type 8 pulse height distribution. units number tally for photons electrons

cell: $\quad 1$

energy

-1.0000 E-03 7.40000E-03 0.1158

$0.0000 \mathrm{E}+00 \quad 0.00000 \mathrm{E}+00 \quad 0.0000$

$1.0000 \mathrm{E}-06 \quad 0.00000 \mathrm{E}+000.0000$

$1.0000 \mathrm{E}-01 \quad 2.12600 \mathrm{E}-010.0192$

$1.9100 \mathrm{E}-01 \quad 2.65500 \mathrm{E}-010.0166$

$2.8200 \mathrm{E}-01 \quad 1.87700 \mathrm{E}-010.0208$

$3.7300 \mathrm{E}-01 \quad 1.09500 \mathrm{E}-010.0285$

4.6400E-01 6.44000E-02 0.0381

$5.5500 \mathrm{E}-01 \quad 4.37000 \mathrm{E}-020.0468$

$6.4500 \mathrm{E}-01 \quad 2.66000 \mathrm{E}-020.0605$

\begin{tabular}{l}
$7.3600 \mathrm{E}-01 \quad 1.58000 \mathrm{E}-02 \quad 0.0789$ \\
\hline
\end{tabular}

$.2700 \mathrm{E}-01 \quad 1.12000 \mathrm{E}-020.0940$

$1800 \mathrm{E}-01 \quad 7.70000 \mathrm{E}-030.1135$ 
HNF-SD-SNF-CSWD-005, Rev. 0

$1.1000 \mathrm{E}+00 \quad 4.79000 \mathrm{E}-020.0446$

$1.00000 \mathrm{E}+000.0000$

ly $8 \quad n p s=10000$

tally type $8^{*}$ energy deposition

tally for photons electrons

units mev

cell: $\quad 1$

energy

$1.0000 \mathrm{E}+03 \quad 2.66900 \mathrm{E}-010.0090$

Istatus of the statistical checks used to form conffdence intervals for the mean for each tally bin

tally result of statistical checks for the tfe bin (the first check not passed is listed) and error magnitude check for all bins

1 missed 1 of $10 \mathrm{tfc}$ bin checks: there is insufficient tic bin taliy information to estimate the large tally slope reliably missed all bin error check: 24 tally bins had 4 bins with zeros and 17 bins with relative errors exceeding 0.10

6 missed 1 of 10 tf $\mathrm{f}$ bin checks: the variance of the variance appears not to decrease as $1 / \mathrm{mps}$ for the last half of problem missed all bin error check: 12 tally bins had 2 bins with zeros and 8 bins with relative errors exceeding 0.10

28 passed the 10 statistical checks for the tally fluctuation chart bin result missed all bin cror check: 15 tally bins had 2 bins with zeros and 2 bins with relative errors exceeding 0.10

11 missed 1 of 10 tfe bin checks: the slope of decrease of largest tallies is less than the minimum acceptable value of 3.0 missed all bin error check: 24 tally bins had 2 bins with zeros and 5 bins with relative etrors exceeding 0.10

21 missed 1 of $10 \mathrm{tfc}$ bin checks: the estimated mean has a trend during the last half of the problem missed all bin error check: 24 tally bins had 2 bins with zeros and 5 bins with relative errors exceeding 0.10

31 missed 2 of $10 \mathrm{tfc}$ bin checks: the estimated mean has a trend during the last half of the problem missed all bin error check: 24 tally bins had 2 bins with zeros and 5 bins with relative errors exceeding 0.10

41 missed 1 of $10 \mathrm{tfc}$ bin checks: the estimated mean has a trend during the last half of the problem missed all bin error check: 72 tally bins had 28 bins with zeros and 10 bins with relative errors exceeding 0.10

51 missed 2 of 10 tfe bin checks: the estimated mean has a trend during the last half of the problem missed all bin error check: 72 tally bins had 28 bins with zeros and 10 bins with relative errors exceeding 0.10

58 passed the 10 statistical checks for the tally fluctuation chart bin result missed all bin error check: 15 tally bins had 2 bins with zeros and 2 bins with relative errors exceeding 0.10

8 passed the 10 statistical checks for the tally fluctuation chart bin result passed all bin error check: 1 tally bins all have relative errors less than 0.10 with no zero bins

the 10 statistical checks are only for the tally fluctuation chart bin and do not apply to other tally bins.

the tally bins with zeros may or may not be correct: compare the source, cutofis, multipliers, et cetera with the tally bins.

warning. 7 of the 10 tally fuctuation chart bins did not pass all 10 statistical checks. warning. 9 of the 10 tallies had bins with telative errors greater than recommended. 1 tally fluctuation charts

tally 1 tally $6 \quad$ tally 28 nps mean exror vov slope fom mean error vov slope fom I000 6.7975E-03 $0.23990 .08350 .0 \quad 2.0857 \mathrm{E}-050.15890 .05250 .0$ $20005.5780 \mathrm{E}-030.18840 .0641 \quad 0.0 \quad 2.4397 E-050.17010 .16930 .0$ $30005.8779 \mathrm{E}-030.14550 .0373 \quad 0.0 \quad 2.6650 \mathrm{E}-050.13430 .1060 \quad 0.0$

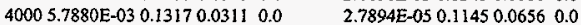
$50005.9924 \mathrm{E}-030.11680 .02390 .0 \quad 2.7855 \mathrm{E}-050.10200 .04850 .0$ $60005.9423 \mathrm{E}-030.10550 .0195 \quad 0.0 \quad 2.7793 \mathrm{E}-050.09310 .04180 .0$ $70005.9749 \mathrm{E}-030.09680 .0170 \quad 0.0 \quad 2.8647 \mathrm{E}-050.09190 .05810 .0$ $80005.7670 \mathrm{E}-030.09130 .01540 .0 \quad 2.8249 \mathrm{E}-050.08380 .052410 .0$ $90005.7398 \mathrm{E}-030.08640 .01350 .0 \quad 2.7485 \mathrm{E}-050.07790 .049110 .0$ $100005.7777 \mathrm{E}-03 \quad 0.08110 .0120 \quad 0.0 \quad 2.7551 \mathrm{E}-050.07220 .043610 .0$ mean error vov slope fom $2.6993 \mathrm{E}-010.02790 .004410 .0$ $2.6475 \mathrm{E}-010.01990 .002210 .0$ $2.6433 \mathrm{E}-010.01610 .001510 .0$ $2.6224 \mathrm{E}-010.0140 \quad 0.001110 .0$ 2.6175E-01 0.01260 .000910 .0 $2.6496 \mathrm{E}-010.01160 .000710 .0$ $2.6691 \mathrm{E}-010.01070 .000610 .0$

$2.6739 \mathrm{E}-010.01000 .000510 .0$ $2.6699 \mathrm{E}-010.0095 \quad 0.000510 .0$

$2.6690 \mathrm{E}-010.00900 .000410 .0$ 
taily 11 nps mean error vov slope fom $0004.4504 \mathrm{E}-010.02960 .00022 .1$ $20004.4489 \mathrm{E}-010.02110 .00012 .0$ $30004.4226 \mathrm{E}-010.01730 .0001 \quad 1.8$ $40004.4011 \mathrm{E}-010.01510 .00001 .8$ $50004.4004 \mathrm{E}-010.01350 .00001 .9$ $60004.3549 \mathrm{E}-01 \quad 0.01250 .0000 \quad 1.8$ $70004.3477 \mathrm{E}-010.01150 .0000 \quad 1.9$ $80004.3339 \mathrm{E}-010.01080 .00001 .8$ $90004.3428 \mathrm{E}-01 \quad 0.01020 .0000 \quad 1.7$ $100004.3411 \mathrm{E}-010.00970 .00001 .6$ tally 21 mean error vov slope fom 5.6500E-01 $0.02860 .0004 \quad 4.4$ $5.6500 \mathrm{E}-010.02040 .0003 \quad 4.4$ $5.6400 \mathrm{E}-010.01680 .000210 .0$ $5.6100 \mathrm{E}-010.01470 .000210 .0$ $5.5860 \mathrm{E}-010.01310 .000110 .0$ $5.5433 \mathrm{E}-010.01210 .000110 .0$ $5.5386 \mathrm{E}-0 \mathrm{I} \quad 0.01120 .000110 .0$ $5.5238 \mathrm{E}-010.01050 .000110 .0$ $5.5200 \mathrm{E}-010.00990 .000110 .0$ $5.5130 \mathrm{E}-010.00940 .000110 .0$ mean error vov slope fom $\begin{array}{llll}-5.6500 \mathrm{E}-01 & 0.0286 & 0.0004 & 0.0\end{array}$ $\begin{array}{llll}-5.6500 \mathrm{E}-01 & 0.02040 .0003 & 0.0\end{array}$ $\begin{array}{llll}-5.6400 \mathrm{E}-01 & 0.0168 & 0.0002 & 0.0\end{array}$

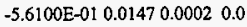
$\begin{array}{llll}-5.5860 \mathrm{E}-01 & 0.0131 & 0.0001 & 0.0\end{array}$ $\begin{array}{llll}-5.5433 \mathrm{E}-01 & 0.0121 & 0.0001 & 0.0\end{array}$ $-5.5386 \mathrm{E}-010.01120 .00010 .0$ $-5.5238 \mathrm{E}-010.01050 .00010 .0$ $-5.5200 \mathrm{E}-010.00990 .00010 .0$
$0.5130 \mathrm{E}-010.00940 .0001$ $-5.5130 \mathrm{E}-010.00940 .00010 .0$ tally 41

nps mean error vov slope fom $10005.6500 \mathrm{E}-010.02860 .00044 .4$ $20005.6500 \mathrm{E}-010.02040 .00034 .4$ $30005.6400 \mathrm{E}-01 \quad 0.0168 \quad 0.000210 .0$ $40005.6100 \mathrm{E}-01 \quad 0.01470 .000210 .0$ $50005.5860 \mathrm{E}-010.01310 .000110 .0$ $60005.5433 \mathrm{E}-01 \quad 0.01210 .000110 .0$ $70005.5386 \mathrm{E}-010.01120 .000110 .0$ $80005.5238 \mathrm{E}-010.01050 .000110 .0$ $90005.5200 \mathrm{E}-010.00990 .000110 .0$ $100005.5130 \mathrm{E}-010.00940 .000110 .0$

tally 8

nps mean error vov slope fom $10002.6993 \mathrm{E}-010.02790 .004410 .0$ $20002.6475 \mathrm{E}-01 \quad 0.01990 .002210 .0$ $30002.6433 \mathrm{E}-010.01610 .001510 .0$ $40002.6224 \mathrm{E}-01 \quad 0.01400 .001110 .0$ $50002.6175 \mathrm{E}-010.01260 .000910 .0$ $60002.6496 E-010.01160 .000710 .0$ $70002.6691 \mathrm{E}-01 \quad 0.01070 .000610 .0$ $80002.6739 \mathrm{E}-010.01000 .000510 .0$ 9000 2.6699E-01 $0.0095 \quad 0.000510 .0$ $100002.6690 \mathrm{E}-010.00900 .000410 .0$ tally data written to file inp $20 \mathrm{~m}$

6 warning messages so far tally 51

tally 58 mean error vov slope form $-5.6500 \mathrm{E}-010.02860 .00040 .0$ $\begin{array}{ll}-5.6500 \mathrm{E}-01 & 0.02040 .0003 \quad 0.0\end{array}$ $\begin{array}{llll}-5.6400 \mathrm{E}-01 & 0.0168 & 0.0002 & 0.0\end{array}$ $-5.6100 \mathrm{E}-010.01470 .0002 \quad 0.0$
$0.5860 \mathrm{E}-010.01310 .0001$ $\begin{array}{lllllll}-5.5860 \mathrm{E}-01 & 0.0131 & 0.0001 & 0.0\end{array}$ $\begin{array}{lllll}-5.5433 \mathrm{E}-01 & 0.0121 & 0.0001 & 0.0\end{array}$ $-5.5386 \mathrm{E}-010.01120 .00010 .0$
$0.5238 \mathrm{E}-010.01050 .0001$ $-5.5238 \mathrm{E}-010.01050 .00010 .0$
$0.5200 \mathrm{E}-010.009001$ $\begin{array}{llll}-5.5200 \mathrm{E}-01 & 0.0099 & 0.0001 & 0.0\end{array}$ $-5.5130 \mathrm{E}-010.00940 .0001 \quad 0.0$
0

\author{
mean eпror vov slope fom \\ $1.0000 \mathrm{E}+000.00000 .000010 .0$ \\ $1.0000 \mathrm{E}+000.00000 .000010 .0$ \\ $1.0000 \mathrm{E}+000.00000 .000010 .0$ \\ $1.0000 \mathrm{E}+000.00000 .000010 .0$ \\ $1.0000 \mathrm{E}+000.00000 .000010 .0$ \\ $1.0000 \mathrm{E}+000.00000 .000010 .0$ \\ $1.0000 \mathrm{E}+00 \quad 0.00000 .000010 .0$ \\ $1.0000 \mathrm{E}+000.00000 .000010 .0$ \\ $1.0000 \mathrm{E}+000.00000 .000010 .0$ \\ $1.0000 \mathrm{E}+000.0000 \quad 0.000010 .0$
}

run terminated when 10000 particle histories were done.

\section{File: Inp240}

1- prob 24 - reflecting lattice. $15 \times 15$ at 3.75 w/o u-235 enrichment.

2- $11-10.182-1 \mathrm{u}=2$

3. 2 2-.0011-2 $\mathrm{u}=2$

4- $33-6.552-3 u=2$

5- $4 \quad 4-1.03 \mathrm{u}=2$

6- 5 - $4-1.0-14: 15 \mathrm{u}=3$

7- $6 \quad 3-6.5514-15 \mathrm{u}=3$

8- $74-1.0-4+5-6+7$ u=1 lat $=1$ fill $=-8: 8-8: 80: 0$

9- $\quad 117 \mathrm{r} 214 \mathrm{r} 11214 \mathrm{r} 1122322322 \mathrm{r} 322$

10- $\quad 3221126 r 326 r 1123 r 3$

11- $\quad 24 r 323 r 1122328 \mathrm{r} 322$

12- $\quad 11214 \mathrm{r} 1122 \mathrm{r} 322 \mathrm{r} 22 \mathrm{r}$

13- $\quad 322 \pi 11214 \mathrm{r} 1122328 \mathrm{r} 3$

14- $\quad 221123 r 324 r 323 r 11$

15- $\quad 265326 \mathrm{rl1223223}$

16- $\quad 22 \mathrm{r} 32232211214 \pi 11214 \mathrm{~T} 117 \mathrm{r}$

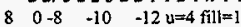

$95-7.98: 10 u=4$ 


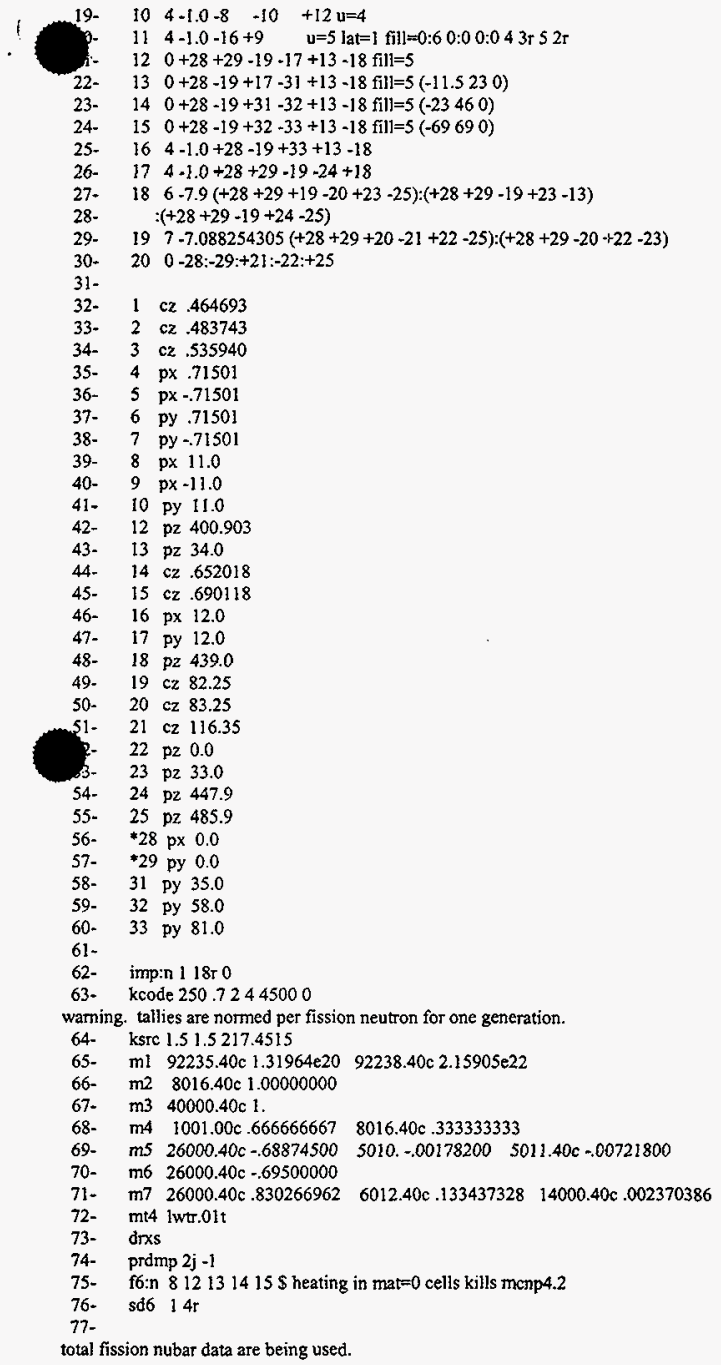

waming. continuous-energy cross-section table used for $1001.00 \mathrm{~d}$

ming. continuous-energy cross-section table used for $5011.40 \mathrm{~d}$ 
waming. continuous-energy cross-section table used for $26000.40 \mathrm{~d}$

waming continuous-energy cross-section table used for $40000.40 \mathrm{~d}$

waming. continuous-energy cross-section table used for $92235.40 \mathrm{~d}$

waming, continuous-energy cross-section table used for $92238.40 d$

waming. 4 of the materials had unnormalized fractions.

icells

print table 60

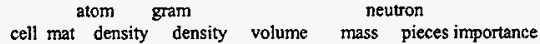

\begin{tabular}{|c|c|c|}
\hline 1 & $12.57597 \mathrm{E}-021.01820 \mathrm{E}+010.00000 \mathrm{E}+000.00000 \mathrm{E}+00$ & 01. \\
\hline 2 & $23.76497 \mathrm{E}-051.00000 \mathrm{E}-030.00000 \mathrm{E}+000.00000 \mathrm{E}+00$ & $01.0000 \mathrm{E}+00$ \\
\hline 3 & $34.32392 \mathrm{E}-026.55000 \mathrm{E}+000.00000 \mathrm{E}+000.00000 \mathrm{E}+00$ & $01.0000 \mathrm{E}+00$ \\
\hline 4 & 4s $1.00309 \mathrm{E}-011.00000 \mathrm{E}+000.00000 \mathrm{E}+000.00000 \mathrm{E}+00$ & 0 1.0000E+00 \\
\hline 5 & 4s $1.00309 \mathrm{E}-011.00000 \mathrm{E}+000.00000 \mathrm{E}+000.00000 \mathrm{E}+00$ & $01.0000 \mathrm{E}+00$ \\
\hline 6 & $34.32392 E-026.55000 E+000.00000 E+000.00000 E+00$ & $0 \quad 1.0000 \mathrm{E}+00$ \\
\hline 7 & 4s 1.00309E-01 1.00000E $+000.00000 \mathrm{E}+000.00000 \mathrm{E}+00$ & $01.0000 \mathrm{E}+00$ \\
\hline 8 & $00.00000 \mathrm{E}+000.00000 \mathrm{E}+000.00000 \mathrm{E}+000.00000 \mathrm{E}+00$ & $01.0000 \mathrm{E}+00$ \\
\hline 9 & $58.97738 \mathrm{E}-027.90000 \mathrm{E}+000.00000 \mathrm{E}+000.00000 \mathrm{E}+00$ & $01.0000 \mathrm{E}+00$ \\
\hline 10 & $4 s 1.00309 \mathrm{E}-011.00000 \mathrm{E}+000.00000 \mathrm{E}+000.00000 \mathrm{E}+00$ & $01.0000 \mathrm{E}+00$ \\
\hline 11 & 4s $1.00309 \mathrm{E}-011.00000 \mathrm{E}+000.00000 \mathrm{E}+000.00000 \mathrm{E}+00$ & $000 \mathrm{E}+00$ \\
\hline 12 & $00.00000 E+000.00000 E+000.00000 E+000.00000 E+00$ & 01. \\
\hline 13 & $00.00000 E+000.00000 E+000.00000 E+000.00000 E+00$ & $01.0000 E+00$ \\
\hline 14 & $00.00000 \mathrm{E}+000.00000 \mathrm{E}+000.00000 \mathrm{E}+00 \quad 0.00000 \mathrm{E}+00$ & $01.0000 E+00$ \\
\hline 15 & $00.00000 \mathrm{E}+000.00000 \mathrm{E}+000.00000 \mathrm{E}+000.00000 \mathrm{E}+00$ & $01.0000 \mathrm{E}+00$ \\
\hline 16 & 4s 1.00309E-0I 1.00000E+00 $0.00000 \mathrm{E}+000.00000 \mathrm{E}+00$ & $01.0000 E+00$ \\
\hline 17 & 4s $1.00309 \mathrm{E}-011.00000 \mathrm{E}+000.00000 \mathrm{E}+000.00000 \mathrm{E}+00$ & $01.0000 \mathrm{E}+00$ \\
\hline 18 & $68.51890 E-027.90000 E+000.00000 E+000.00000 E+00$ & $01.0000 E+00$ \\
\hline 19 & $78.58502 \mathrm{E}-027.08825 \mathrm{E}+000.00000 \mathrm{E}+00$ & $01.0000 \mathrm{E}+00$ \\
\hline & $0 \quad 0.00000 \mathrm{E}+00 \quad 0.00000 \mathrm{E}+000.00000 \mathrm{E}+000.00000 \mathrm{E}+00$ & $0 \quad 0.0000 \mathrm{E}+00$ \\
\hline
\end{tabular}

total $\quad 0.00000 E+000.00000 E+00$

11 warning messages so far.

Icross-section tables

print table 100

table length

tables from file testlibl

$1001.00 \mathrm{c} 2139$ 1-h-1 from endf-vi. 1

mat $12505 / 26 / 93$

$5010.03 d \quad 3945$ b-10 endf/o-iv new gamma production format

5011.40c 3473 ENDL library name: nd900719 MCNP translation: 900831 14:10:05 900207

$6012.40 \mathrm{c} 5049$ ENDL library name: nd900719 MCNP translation: 900831 14:10:05 900207

$8016.40 \mathrm{c} 5693$ ENDL library name: nd900719 MCNP translation: 900831 14:10:05 900207

14000.40c 8732 ENDL library name: nd900719 MCNP translation: 900831 14:10:05 900207

26000.40c 21846 ENDL library name: nd900719 MCNP translation: $90083114: 10: 05 \quad 900207$

40000.40c 111279 ENDL library name: nd900719 MCNP translation: 900831 14:10:05 900323

92235.40c 40506 ENDL library name: nd900719 MCNP translation: 9total nu 10:05 900503

92238.40c 48310 ENDL library name: nd901118 MCNP translation: 9total nu 56:49 901119

lwt.01t 10193 hydrogen in light water at 300 degrees kelvin $\quad 1001 \quad 0 \quad 010 / 22 / 85$

total 261165

waming. neutron energy cutoff is below some cross-section tables. 
cycle $1 \mathrm{k}$ (collision) 0.658210 removal lifetime(abs) $5.1517 \mathrm{E}+03$ source points generated 228

cycle $2 \mathrm{k}$ (collision) 0.549318 removal lifetime(abs) $3.7645 \mathrm{E}+03$ source points generated 209

cycle $3 \mathrm{k}$ (collision) 0.551862 removal lifetime(abs) $3.8422 \mathrm{E}+03$ source points generated 253

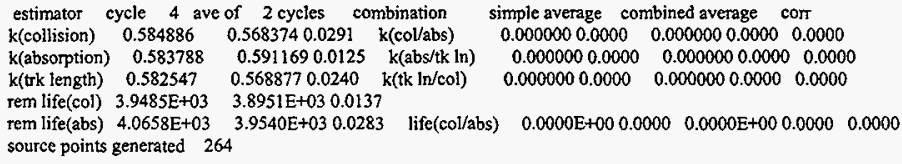


cell 12

$2.65224 E+010.0629$

13

$1.32494 \mathrm{E}+010.0904$

cell 14

\section{$9.78180 \mathrm{E}-01 \quad 0.4014$}

cell 15

$1.20234 \mathrm{E}-010.9710$

lanalysis of the results in the tally fluctuation chart bin (tfc) for tally 6 vith nps $=940$ print table 160

normed average tally per history $=4.02566 \mathrm{E}+01 \quad$ unnormed average tally per history $=4.02566 \mathrm{E}+01$

estimated tally relative error $=0.0434$

estimated variance of the variance $=0.0095$

relative error from zero tallies $=0.0128$

relative error from nonzero scores $=0.0415$

number of nonzero history tallies $=462$ efficiency for the nonzero tallies $=0.9240$

history number of largest tally $=\quad 641$ largest unnormalized history tally $=2.71752 E+02$

(largest tally $)($ average tally) $=6.75049 \mathrm{E}+00 \quad$ (largest tally)/(avg nonzero tally) $=6.23745 \mathrm{E}+00$

(confidence interval shift)/mean $=0.0014$

shifted confidence interval center $=4.03113 \mathrm{E}+01$

if the largest history score sampled so far were to occur on the very next history, the tfc bin quantities would change as follows:

$\mathrm{nps}=462$ for this table because 2 keff cycles and 478 histories were skipped before tally accumulation.

estimated quantities value at nps value at nps+1 value(nps+1)/value(nps)-1.

mean $\quad 4.02566 \mathrm{E}+01 \quad 4.07566 \mathrm{E}+01 \quad 0.012420$

$\begin{array}{lrr}\text { relative error } \quad 4.34431 \mathrm{E}-02 & 4.24506 \mathrm{E}-02 & -0.022846\end{array}$

variance of the variance $\quad 9.52674 \mathrm{E}-03 \quad 1.33327 \mathrm{E}-02 \quad 0.399508$

whifted center $\quad 4.03113 \mathrm{E}+01 \quad 4.03457 \mathrm{E}+01 \quad 0.000853$

igure of merit $\quad 0.00000 \mathrm{E}+00 \quad 0.00000 \mathrm{E}+00 \quad 0.000000$

there is not enough information in the largest history scores (usually less than 500 scores) for a reliable estimate of the slope.

the history score probability density function appears to have an unsampled region at the largest history scores: please examine.

***** the nps-dependent $t f c$ bin check results are suspect because there are only $1 \mathrm{nps}$ tally values to analyze *****

results of 10 statistical checks for the estimated answer for the tally fluctuation chart (tfc) bin of tally 6

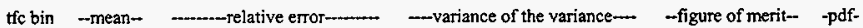

behavior behavior value decrease decrease rate value decrease decrease rate value behavior slope

desired random $<0.10$ yes $1 /$ sqrt(nps) $<0.10$ yes $1 / \mathrm{nps}$ constant random $>3.00$

observed random 0.04 yes yes 0.01 yes yes 0.00

passed? yes yes yes yes yes yes yes no

warning. the tally in the tally fluctuation chart bin did not pass 1 of the 10 statistical checks.

1 unnormed tally density for tally $6 \quad$ nonzero tally mean $(m)=4.357 \mathrm{E}+01 \quad \mathrm{nps}=940$ print table 161

abscissa ordinate $\log$ plot of tally probability density fumction in tally fluctuation chart bin( $d=$ decade,siope $=0.0$ )

tally number num den log den:d-_-

2.51-01 $13.87-02-1.412 * * * * * * * * * * * * * * * * * * * * * * * * * * * *|* * * * * * * * * * * * * * * * * * * * * * * * * * * * *| * * * * * * * * * * * * * * * * * * * * * * * * * * * * * \mid * * * *$

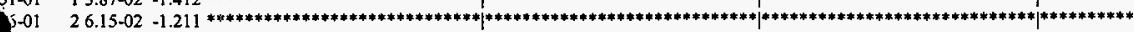

$12.44-02-1.612 * * * * * * * * * * * * * * * * * * * * * * * * * * * *|* * * * * * * * * * * * * * * * * * * * * * * * * * * * *| * * * * * * * * * * * * * * * * * * * * * * * * * * * * \mid$ 


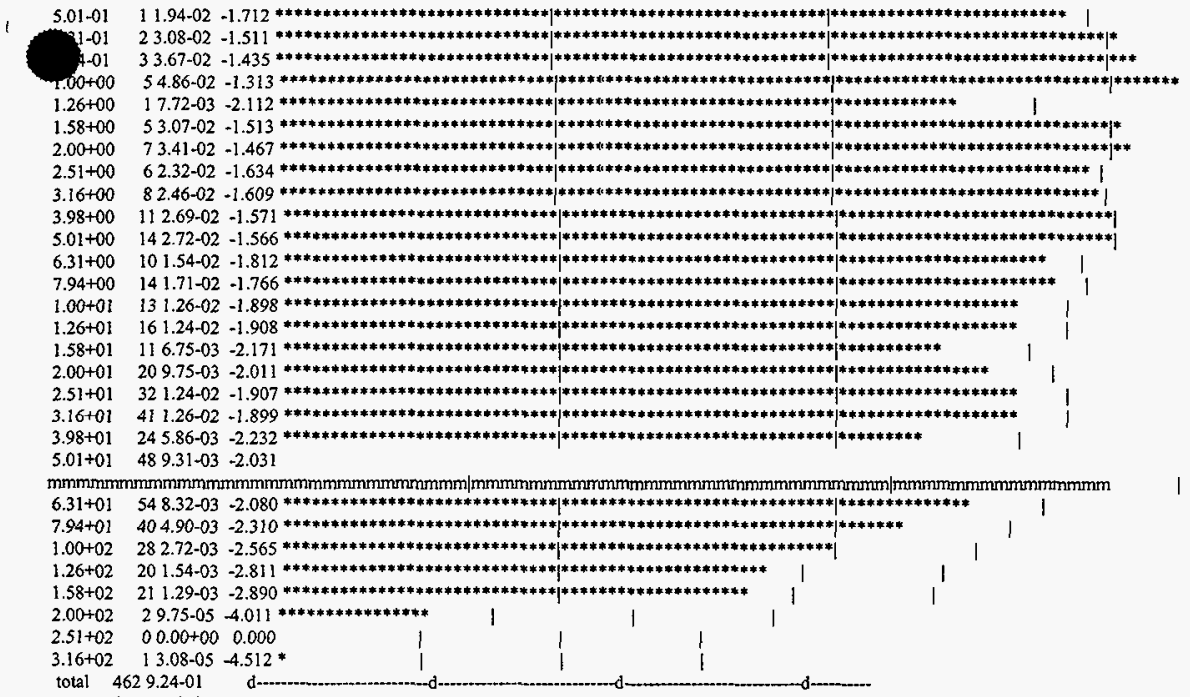

lstatus of the statistical checks used to form confidence intervals for the mean for each tally bin

result of statistical checks for the tfc bin (the first check not passed is listed) and error magnitude check for all bins

6 missed 1 of 10 tf bin checks: there is insufficient tfc bin tally information to estimate the large tally slope reliably missed all bin error check: 5 tally bins had 0 bins with zeros and 2 bins with relative errors exceeding 0.10

the 10 statistical checks are only for the tally fluctuation chart bin and do not apply to other tally bins.

warning. 1 of the 1 tally fluctuation chart bins did not pass all 10 statistical checks. warning. I f the 1 tallies had bins with relative errors greater than recommended. 1 tally fluctuation charts

tally 6

nos mean etror vov slope fom

$9404.0257 \mathrm{E}+010.04340 .00950 .0$

tally data written to file inp $24 \mathrm{~m}$

15 warning messages so far.

run terminated when 4 kcode cycles were done.

\section{File: Outp24}

1- prob 24 - reflecting lattice. $15 \times 15$ at 3.75 w/o u-235 enrichment.

2. $1 \quad 1-10.182-1 \mathrm{u}=2$

3. 2 2 $-.0011-2 \mathrm{u}=2$

4. 3 3 $-6.552-3 u=2$

5. 4 4-1.03 $u=2$

6- 5 - $4-1.0-14: 15 u=3$

$63-6.5514-15 u=3$

$7 \quad 4-1.0-4+5-6 \div 7 u=1$ lat $=1$ fill $=-8: 8-8: 80: 0$

$117 \mathrm{r} 214 \mathrm{r} 11214 \mathrm{r} 1122322322 \mathrm{r} 322$ 
$3221126 r 32651123 r 3$

$24 \pi 323 \mathrm{r} 1122328 \mathrm{r} 322$

$11214 \mathrm{r} 1122 \mathrm{r} 322 \mathrm{r} 322 \mathrm{~T}$

$322 \mathrm{r} 11214 \mathrm{r} 1122328 \mathrm{r} 3$

$221123 r 324 r 323 r 11$

$26 \% 326511223223$

$22 \mathrm{r} 2232211214 \mathrm{r} 11214 \mathrm{r} 117 \mathrm{r}$

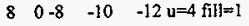

$9 \quad 5-7.98: 10 u=4$

$10 \quad 4-1.0-8 \quad-10 \quad+12 u=4$

$114-1.0-16+9 \quad u=5$ lat $=1$ fill $=0: 60: 00: 043 \mathrm{r} 52 \mathrm{r}$

$120+28+29-19-17+13-18$ fill $=5$

$130+28-19+17-31+13-18$ fill $=5(-11.5230)$

$140+28-19+31-32+13-18$ fill $=5(-23460)$

$150+28-19+32-33+13-18$ fill $=5(-69690)$

$164-1.0+28-19+33+13-18$

$17 \quad 4-1.0+28+29-19-24+18$

$186-7.9(+28+29+19-20+23-25):(+28+29-19+23-13)$

$:(+28+29-19+24-25)$

$197-7.088254305(+28+29+20-21+22-25):(+28+29-20+22-23)$

$20 \quad 0-28:-29:+21:-22:+25$

1 cz .464693

2 cz $\quad \mathbf{A 8 3 7 4 3}$

3 cz .535940

$4 \mathrm{px} .71501$

5 px -.71501

6 py .71501

7 py -.71501

$8 \mathrm{px} 11.0$

$9 \mathrm{px}-11.0$

10 py 11.0

$12 \mathrm{pz} 400.903$

$13 \mathrm{pz} 34.0$

14 cz .652018

$15 \mathrm{cz} .690118$

$\begin{array}{llll}46- & 16 & \mathrm{px} & 12.0\end{array}$

47- 17 py 12.0

48- $\quad 18$ pz 439.0

49: $\quad$ F. c2: 82.25

503. 20. cz: $\$ 3.25$

51:- 21 cz 116.35

52- $22 \mathrm{pz} 0.0$

53- 23 pz 33.0

54- 24 pz 447.9

$55-\quad 25 \mathrm{pz} 485.9$

56- $\quad 28 \mathrm{px} 0.0$

57- $\quad 29$ py 0.0

58- 31 py 35.0

59. 32 py 58.0

$60-\quad 33$ py 81.0

61-

62- imp:n $118 \mathrm{r} 0$

63- kcode 250.72445000

waming. tallies are normed per fission neutron for one generation.

64- kste 1.51 .5217 .4515

65- $\mathrm{ml} \quad 92235.40 \mathrm{c} 1.31964 \mathrm{e} 20 \quad 92238.40 \mathrm{c} 2.15905 \mathrm{e} 22$

66- $\mathrm{m} 2 \quad 8016.40 \mathrm{c} 1.00000000$

67. $\mathrm{m}^{3} \quad 40000.40 \mathrm{cl}$.

68- $m 4 \quad 1001.00 \mathrm{c} .666666667 \quad 8016.40 \mathrm{c} .333333333$

69- $\mathrm{m} 5 \quad 26000.40 \mathrm{c}-.68874500 \quad 5010 .-.00178200 \quad 5011.40 \mathrm{c}-.00721800$

$70-\quad \mathrm{m} 6 \quad 26000.40 \mathrm{c}-.69500000$

71- $\quad \begin{array}{lllll}\mathrm{m} 7 & 26000.40 \mathrm{c} .830266962 & 6012.40 \mathrm{c} .133437328 & 14000.40 \mathrm{c} .002370386\end{array}$

72- mt4 lwtr.01t

dixs

prdmp $2 \mathrm{j}-\mathrm{I}$

f6:n $812131415 \$$ heating in mat=0 cells kills menp4.2 
fission nubar data are being used.

waming. continuous-energy cross-section table used for $100 \mathrm{~L} .00 \mathrm{~d}$

waming. continuous-energy cross-section table used for $5011.40 \mathrm{~d}$

waming. continuous-energy cross-section table used for $6012.40 \mathrm{~d}$

waming. continuous-energy cross-section table used for $8016.40 \mathrm{~d}$

waming. continuous-energy cross-section table used for $14000.40 \mathrm{~d}$

warning. continuous-energy cross-section table used for $26000.40 \mathrm{~d}$

warning. continuous-energy cross-section table used for $40000.40 \mathrm{~d}$

warning. continuous-energy cross-section table used for 92235.40d

warning. continuous-energy cross-section table used for $92238.40 \mathrm{~d}$

waming. 4 of the materials had unnormalized fractions.

Icells

print table 60

cell mat density density volume mass pieces importance

$1 \quad 1 \quad 12.57597 \mathrm{E}-02 \quad 1.01820 \mathrm{E}+010.00000 \mathrm{E}+000.00000 \mathrm{E}+00$

$222 \quad 3.76497 \mathrm{E}-051.00000 \mathrm{E}-030.00000 \mathrm{E}+000.00000 \mathrm{E}+00$

$3334.32392 \mathrm{E}-026.55000 \mathrm{E}+000.00000 \mathrm{E}+000.00000 \mathrm{E}+00$

44 4s $1.00309 \mathrm{E}-011.00000 \mathrm{E}+000.00000 \mathrm{E}+000.00000 \mathrm{E}+00$

5 As $1.00309 \mathrm{E}-011.00000 \mathrm{E}+000.00000 \mathrm{E}+000.00000 \mathrm{E}+00$

$634.32392 \mathrm{E}-026.55000 \mathrm{E}+000.00000 \mathrm{E}+000.00000 \mathrm{E}+00$

7 4s $1.00309 \mathrm{E}-011.00000 \mathrm{E}+000.00000 \mathrm{E}+000.00000 \mathrm{E}+00$

$8800.00000 \mathrm{E}+000.00000 \mathrm{E}+000.00000 \mathrm{E}+000.00000 \mathrm{E}+00$

$9958.97738 \mathrm{E}-027.90000 \mathrm{E}+000.00000 \mathrm{E}+000.00000 \mathrm{E}+00$

$10 \quad 10$ 4s 1.00309E-01 $1.00000 \mathrm{E}+000.00000 \mathrm{E}+000.00000 \mathrm{E}+00$

$11 \quad 11$ 4s $1.00309 \mathrm{E}-011.00000 \mathrm{E}+000.00000 \mathrm{E}+000.00000 \mathrm{E}+00$

$12 \quad 12 \quad 0 \quad 0.00000 \mathrm{E}+00 \quad 0.00000 \mathrm{E}+000.00000 \mathrm{E}+00 \quad 0.00000 \mathrm{E}+00$

$13 \quad 13 \quad 0 \quad 0.00000 \mathrm{E}+00 \quad 0.00000 \mathrm{E}+00 \quad 0.00000 \mathrm{E}+000.00000 \mathrm{E}+00$

$14 \quad 14 \quad 0 \quad 0.00000 E+00 \quad 0.00000 E+00 \quad 0.00000 E+00 \quad 0.00000 E+00$

is is $00.00000 \mathrm{E}+000.00000 \mathrm{E}+000.00000 \mathrm{E}+00 \quad 0.00000 \mathrm{E}+00$

$16 \quad 16$ 4s $1.00309 \mathrm{E}-011.00000 \mathrm{E}+000.00000 \mathrm{E}+000.00000 \mathrm{E}+00$

$17 \quad 17 \quad$ 4s $1.00309 \mathrm{E}-011.00000 \mathrm{E}+000.00000 \mathrm{E}+000.00000 \mathrm{E}+00$

$18 \quad 18 \quad 6 \quad 8.51890 \mathrm{E}-02 \quad 7.90000 \mathrm{E}+000.00000 \mathrm{E}+000.00000 \mathrm{E}+00$

$19 \quad 1978.58502 \mathrm{E}-027.08825 \mathrm{E}+000.00000 \mathrm{E}+000.00000 \mathrm{E}+00$

$2020 \quad 0 \quad 0.00000 \mathrm{E}+000.00000 \mathrm{E}+000.00000 \mathrm{E}+000.00000 \mathrm{E}+00$

total

$0.00000 \mathrm{E}+000.00000 \mathrm{E}+00$
$01.0000 \mathrm{E}+00$

$0 \quad 1.0000 \mathrm{E}+00$

$0 \quad 1.0000 \mathrm{E}+00$

$01.0000 \mathrm{E}+00$

o $1.0000 \mathrm{E}+00$

$0 \quad 1.0000 \mathrm{E}+00$

$01.0000 \mathrm{E}+00$

$01.0000 \mathrm{E}+00$

$01.0000 \mathrm{E}+00$

$01.0000 \mathrm{E}+00$

$01.0000 \mathrm{E}+00$

$01.0000 E+00$

$01.0000 \mathrm{E}+00$

$01.0000 \mathrm{E}+00$

$01.0000 \mathrm{E}+00$

0 1.0000E+00

$01.0000 \mathrm{E}+00$

$0 \quad 1.0000 \mathrm{E}+00$

$0 \quad 1.0000 \mathrm{E}+00$

$00.0000 \mathrm{E}+00$

I1 waming messages so far.

icross-section tables

print table 100

table length

tables from file testlib1

$1001.00 \mathrm{c} 21391$-h-1 from endf-vi.1

mat $12505 / 26 / 93$

(1273) 14 oct 75

$5010.03 d 3945$ b-10 endf/b-iv new gamma production format

$\begin{array}{llll}5011.40 \mathrm{c} & 3473 & \text { ENDL library name: nd900719 MCNP translation: 900831 14:10:05 } \\ 6012.40 \mathrm{c} & 5049 & \text { ENDL library name: nd900719 MCNP translation: } 900831 \text { 14:10:05 }\end{array}$

900207

900207

8016.40c 5693 ENDL library name: nd900719 MCNP translation: 900831 14:10:05

900207

$14000.40 \mathrm{c} 8732$ ENDL library name: nd900719 MCNP translation: 900831 14:10:05

900207

900207

900323

600.40 111279 ENDL library name: nd900719 MCNP tanslation: 900831 14:10:05

$2235.40 \mathrm{c} 40506$ ENDL library name: nd900719 MCNP translation: 9total nu 10:05 
$2238.40 \mathrm{c} 48310$ ENDL library name: nd901118 MCNP translation: 9total nu 56:49

ar.01t 10193 hydrogen in light water at 300 degrees kelvin

$10010010 / 22 / 85$

901119

total 261165

warning. neutron energy cutoff is below some cross-section tables.

decimal words of dynamically allocated storage

source distribution written to file inp $24 \mathrm{~s} \quad$ cycle $=0$

12 waming messages so far. lestimated keff results by cycle

print table 175

cycle $1 \mathrm{k}$ (collision) 0.658210 removal lifetime(abs) $5.1517 \mathrm{E}+03$ source points generated 228

cycle $2 \mathrm{k}$ (collision) 0.549318 removal lifetime(abs) $3.7645 \mathrm{E}+03$ source points generated 209

cycle $3 \mathrm{k}$ (collision) 0.551862 removal lifetime(abs) $3.8422 \mathrm{E}+03$ source points generated 253

source distribution written to file inp $24 \mathrm{~s} \quad$ cycle $=3$

estimator cycle 4 ave of 2 cycles combination simple average combined average corr

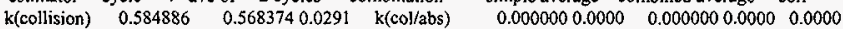

$\begin{array}{llllllll}\mathrm{k} \text { (absorption) } & 0.583788 & 0.5911590 .0125 & \mathrm{k}(\mathrm{abs} / \mathrm{tk} \mathrm{ln}) & 0.000000 & 0.0000 & 0.0000000 .0000 & 0.0000\end{array}$

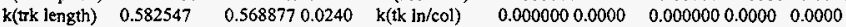

Tem life(col) $3.9485 \mathrm{E}+03 \quad 3.8951 \mathrm{E}+030.0137$

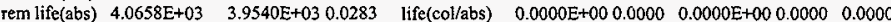

murce points generated 264

W.ource distribution written to file inp $24 \mathrm{~s} \quad$ cycle $=4$

Iproblem summary

0

run terminated when 4 kcode cycles were done.

neutron creation tracks weight energy neutron loss tracks weight energy
(per source particle)

$\begin{array}{llllllll}\text { source } & 940 & 1.0638 \mathrm{E}+00 & 1.9444 \mathrm{E}+00 & \text { escape } & 0 & 0 . & 0 .\end{array}$ energy cutoff $00 . \quad 0$. time cutoff $0 \quad 0.00$.

weight window

0.

cell importance $\quad \begin{array}{llllllll}0 & 0 . & 0 . & \text { cell importance } & 0 & 0 . & 0 .\end{array}$

$\begin{array}{lllllllll}\text { weight cutoff } & 0 & 5.6409 \mathrm{E}-02 & 1.1284 \mathrm{E}-06 & \text { weight cutoff } & 944 & 5.2413 \mathrm{E}-02 & 7.1769 \mathrm{E}-07\end{array}$

energy importance $\begin{array}{lllllllll}0 & 0 . & 0 . & \text { energy importance } & 0 & 0 . & 0 .\end{array}$

$\begin{array}{llllllllllll}\text { dxtran } & 0 & 0 . & 0 . & \text { dxtran } & 0 & 0 . & 0 . & \\ \text { forced collisions } & 0 & 0 . & & 0 . & \text { forced collisions } & 0 & 0 . & 0 .\end{array}$

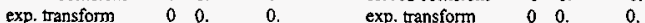

$\begin{array}{llllllll}\text { upscattering } & 0 & 0 & 1.4710 \mathrm{E}-07 & \text { downscattering } & 0 & 0 . & 1.8098 \mathrm{E}+00\end{array}$

capture $\quad 0 \quad 8.1698 \mathrm{E}-01 \quad 1.8384 \mathrm{E}-02$

$\begin{array}{llllllll}(n, x n) \quad & 8 & 6.9970 E-03 & 3.6428 E-03 & \text { loss to }(n, x n) & 4 & 3.4985 E-03 & 2.8025 E-02\end{array}$

$\begin{array}{llllllll}\text { fission } & 0 & 0 . & 0 . & \text { loss to fission } 0 & 2.5434 \mathrm{E}-01 & 9.1864 \mathrm{E}-02\end{array}$

$\begin{array}{llllllllll}\text { total } & 948 & 1.1272 \mathrm{E}+00 & 1.948 \mathrm{E}+00 & \text { total } & 948 & 1.1272 \mathrm{E}+00 & 1.9481 \mathrm{E}+00\end{array}$

number of neutrons banked $\quad 4$ average lifetime, shakes cutoffs

neutron tracks per source particle $1.0085 \mathrm{E}+00$ escape $0.0000 \mathrm{E}+00$ tco $1.0000 \mathrm{E}+34$

neutron collisions per source particle $6.1389 \mathrm{E}+01$ capture $4.1766 \mathrm{E}+03$ eco $0.0000 \mathrm{E}+00$

total neutron collisions

57706

net multiplication

$1.0033 E+000.0030$

any termination $4.4650 \mathrm{E}+03$

wcl $-5.0000 \mathrm{E}-01$

mputer time so far in this run 0.00 minutes

nputer time in mcrun

urce particles per minute
0.00 minutes

$0.0000 \mathrm{E}+00$ maximum number ever in bank

bank overflows to backup file 0

field length 
tracks population collisions collisions number flux average average cell entering $\quad$ weight weighted weighted track weight track mip (per history) energy energy (relative) (cm)

$\begin{array}{ccccccccccccc}1 & 1 & 15187 & 944 & 4054 & 3.5039 \mathrm{E}+00 & 3.6399 \mathrm{E}-04 & 6.5723 \mathrm{E}-01 & 8.4316 \mathrm{E}-01 & 3.8971 \mathrm{E}+00 \\ 2 & 2 & 29440 & 944 & 0 & 0.0000 \mathrm{E}+00 & 3.3819 \mathrm{E}-04 & 6.3029 \mathrm{E}-01 & 8.4006 \mathrm{E}-01 & 9.6437 \mathrm{E}+03 \\ 3 & 3 & 31610 & 944 & 1028 & 9.2538 \mathrm{E}-01 & 3.1626 \mathrm{E}-04 & 6.0475 \mathrm{E}-01 & 8.3961 \mathrm{E}-01 & 3.6368 \mathrm{E}+00 \\ 4 & 4 & 44969 & 944 & 40660 & 3.0973 \mathrm{E}+01 & 3.0008 \mathrm{E}-04 & 5.9877 \mathrm{E}-01 & 8.3882 \mathrm{E}-01 & 1.4858 \mathrm{E}+00 \\ 5 & 5 & 8213 & 845 & 8775 & 6.4600 \mathrm{E}+00 & 2.0190 \mathrm{E}-04 & 5.0976 \mathrm{E}-01 & 8.1000 \mathrm{E}-01 & 1.3256 \mathrm{E}+00 \\ 6 & 6 & 4924 & 791 & 109 & 9.6195 \mathrm{E}-02 & 2.0789 \mathrm{E}-04 & 5.2658 \mathrm{E}-01 & 8.2057 \mathrm{E}-01 & 3.6398 \mathrm{E}+00 \\ 7 & 7 & 2832 & 638 & 1607 & 1.4612 \mathrm{E}+00 & 8.5166 \mathrm{E}-04 & 7.1930 \mathrm{E}-01 & 9.4163 \mathrm{E}-01 & 1.7232 \mathrm{E}+00 \\ 8 & 8 & 0 & 0 & 0 & 0.0000 \mathrm{E}+00 & 0.0000 \mathrm{E}+00 & 0.0000 \mathrm{E}+00 & 0.0000 \mathrm{E}+00 & 0.0000 \mathrm{E}+00 \\ 9 & 9 & 1333 & 607 & 1347 & 1.2836 \mathrm{E}+00 & 3.8320 \mathrm{E}-03 & 8.0017 \mathrm{E}-01 & 9.7093 \mathrm{E}-01 & 3.1777 \mathrm{E}+00 \\ 10 & 10 & 0 & 0 & 0 & 0.0000 \mathrm{E}+00 & 0.0000 \mathrm{E}+00 & 0.0000 \mathrm{E}+00 & 0.0000 \mathrm{E}+00 & 0.0000 \mathrm{E}+00 \\ 11 & 11 & 7 & 1 & 126 & 9.7846 \mathrm{E}-02 & 9.0288 \mathrm{E}-05 & 3.2866 \mathrm{E}-01 & 7.7027 \mathrm{E}-01 & 1.0087 \mathrm{E}+00 \\ 12 & 12 & 0 & 0 & 0 & 0.0000 \mathrm{E}+00 & 0.0000 \mathrm{E}+00 & 0.0000 \mathrm{E}+00 & 0.0000 \mathrm{E}+00 & 0.0000 \mathrm{E}+00 \\ 13 & 13 & 0 & 0 & 0 & 0.0000 \mathrm{E}+00 & 0.0000 \mathrm{E}+00 & 0.0000 \mathrm{E}+00 & 0.0000 \mathrm{E}+00 & 0.0000 \mathrm{E}+00 \\ 14 & 14 & 0 & 0 & 0 & 0.0000 \mathrm{E}+00 & 0.0000 \mathrm{E}+00 & 0.0000 \mathrm{E}+00 & 0.0000 \mathrm{E}+00 & 0.0000 \mathrm{E}+00 \\ 15 & 15 & 0 & 0 & 0 & 0.0000 \mathrm{E}+00 & 0.0000 \mathrm{E}+00 & 0.0000 \mathrm{E}+00 & 0.0000 \mathrm{E}+00 & 0.0000 \mathrm{E}+00 \\ 16 & 16 & 0 & 0 & 0 & 0.0000 \mathrm{E}+00 & 0.0000 \mathrm{E}+00 & 0.0000 \mathrm{E}+00 & 0.0000 \mathrm{E}+00 & 0.0000 \mathrm{E}+00 \\ 17 & 17 & 0 & 0 & 0 & 0.0000 \mathrm{E}+00 & 0.0000 \mathrm{E}+00 & 0.0000 \mathrm{E}+00 & 0.0000 \mathrm{E}+00 & 0.0000 \mathrm{E}+00 \\ 18 & 18 & 0 & 0 & 0 & 0.0000 \mathrm{E}+00 & 0.0000 \mathrm{E}+00 & 0.0000 \mathrm{E}+00 & 0.0000 \mathrm{E}+00 & 0.0000 \mathrm{E}+00 \\ 19 & 19 & 0 & 0 & 0 & 0.0000 \mathrm{E}+00 & 0.0000 \mathrm{E}+00 & 0.0000 \mathrm{E}+00 & 0.0000 \mathrm{E}+00 & 0.0000 \mathrm{E}+00\end{array}$

total $\quad 138515 \quad 6658 \quad 57706 \quad 4.4801 \mathrm{E}+01$

the initial fission neutron source distribution used the 1 source points that were input on the kstc card.

the criticality problem was scheduled to skip 2 cycles and run a total of 4 cycles with nominally 250 neutrons per cycle. problem has run 2 inactive cycles with 478 neutron histories and 2 active cycles with 462 neutron histories.

this calculation has completed the requested number of keff cycles using a total of 940 fission neutron source histories. all cells with fissionable material were sampled and had fission neutron source points.

there is no combined collision/absorption/track-length estimate for keff because only 2 active cycles were run.

the estimated average keffs, one standard deviations, and 68,95 , and 99 percent confidence intervals are:

$\begin{array}{ccccccc}\text { keff estimator } & \text { keff } & \text { standaro deviation } & 68 \% \text { confidence } & 95 \% \text { confidence } & 99 \% \text { confidence } \\ \text { collision } & 0.56837 & 0.01651 & 0.53834 \text { to } 0.59841 & 0.35851 \text { to } 0.77824 & 0.00000 \text { to } 1.61951 \\ \text { absorption } & 0.59117 & 0.00738 & 0.57774 \text { to } 0.60460 & 0.49735 \text { to } 0.68498 & 0.12129 \text { to } 1.06105 \\ \text { track length } & 0.56888 & 0.01367 & 0.54401 \text { to } 0.59374 & 0.39513 \text { to } 0.74263 & 0.00000 \text { to } 1.43912\end{array}$

lindividual and average keff estimator results by cycle

keff neutron keff estimators by cycle average keff estimators and deviations average $k(\mathrm{c} / \mathrm{a} / \mathrm{t})$ cycle histories $k$ (coll) $k$ (abs) $k$ (track) $k$ (coll) st dev $k$ (abs) st dev $k$ (track) st dev $k(c / a / t)$ st dev fom

$\begin{array}{lllllll}1 & 250 & 0.65821 & 0.61995 & 0.71299\end{array}$

$2 \quad 228|0.549320 .559220 .57971|$

begin active keff cycles

$209|0.55186 \quad 0.59855 \quad 0.55521|$

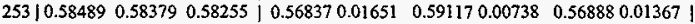

the largest active cycle keffs by estimator are:

collision 0.58489 on cycle 4 absorption 0.59855 on cycle 3 track length 0.58255 on cycle 4 the smallest active cycle keffs by estimator are:

collision 0.55186 on cycle 3 absorption 0.58379 on cycle 4 track length 0.55521 on cycle 3 
nps $=940$

tally type 6 track length estimate of heating. units mev/gram

tally for neutrons

number of histories used for normalizing tallies $=\quad 500.00$

masses
cell: $\begin{array}{lllll}8 & 12 & 13 & 14 & 15\end{array}$
$1.00000 \mathrm{E}+00 \quad 1.00000 \mathrm{E}+00 \quad 1.00000 \mathrm{E}+00 \quad 1.00000 \mathrm{E}+00 \quad 1.00000 \mathrm{E}+00$

cell 8

\section{$4.02566 \mathrm{E}+010.0434$}

ce)1 12

$2.65224 \mathrm{E}+010.0629$

cell 13

$1.32494 \mathrm{E}+010.0904$

cell 14

\section{$9.78180 E-010.4014$}

cell 15

$1.20234 \mathrm{E}-010.9710$

lanalysis of the results in the tally fluctuation chart bin (tfe) for tally 6 with nps $=940$ print table 160

normed average tally per history $=4.02566 \mathrm{E}+0 \mathrm{I}$ estimated tally relative error $=0.0434$

relative error from zero tallies $=0.0128$

number of nonzero history tallies = 462

history number of largest tally $=641$

nagest tally)/(average tally) $=6.75049 \mathrm{E}+00$

nfidence interval shift $/$ mean $=0.0014$ unnormed average tally per history $=4.02566 \mathrm{E}+01$

estimated variance of the variance $=0.0095$

relative erTor from nonzero scores $=0.0415$

efficiency for the nonzero tallies $=0.9240$

largest unnormalized history tally $=2.71752 \mathrm{E}+02$

$($ largest tally $) /($ avg nonzero tally $)=6.23745 \mathrm{E}+00$

shifted confidence interval center $=4.03113 \mathrm{E}+01$

if the largest history score sampled so far were to occur on the very next history, the tfe bin quantities would change as follows: $\mathrm{nps}=462$ for this table because 2 keff cycles and 478 histories were skipped before tally accumulation.

$\begin{array}{lccc}\text { estimated quantities } & \text { value at nps } & \text { value at nps+1 } & \text { value(nps+1)/value } \\ & & & \\ \text { mean } & 4.02566 \mathrm{E}+01 & 4.07566 \mathrm{E}+01 & 0.012420 \\ \text { relative error } & 4.34431 \mathrm{E}-02 & 4.24506 \mathrm{E}-02 & -0.022846 \\ \text { variance of the variance } & 9.52674 \mathrm{E}-03 & 1.33327 \mathrm{E}-02 & 0.399508 \\ \text { shifted center } & 4.03113 \mathrm{E}+01 & 4.03457 \mathrm{E}+01 & 0.000853 \\ \text { figure of merit } & 0.00000 \mathrm{E}+00 & 0.00000 \mathrm{E}+00 & 0.000000\end{array}$

there is not enough information in the largest history scores (usually less than 500 scores) for a reliable estimate of the slope. the history score probability density function appears to have an unsampled region at the largest history scores: please examine.

****** the nps-dependent tfc bin check results are suspect because there are only 1 nps tally values to analyze *****

results of 10 statistical checks for the estimated answer for the tally fluctuation chart (tfc) bin of tally 6

tfc bin -mean- --_-_-relative error-____ --variance of the variance-_ -figure of merit-- -pdf-

behavior behavior value decrease decrease rate value decrease decrease rate value behavior slope

$\begin{array}{ccccccccc}\text { rijed random } & <0.10 & \text { yes } & 1 / \text { sqrt(nps) } & <0.10 \text { yes } 1 / n p s & \text { constant random }>3.00 \\ \text { twed random } & 0.04 \text { yes yes } 0.01 \text { yes yes } & \text { no } & 0.00 & \text { no }\end{array}$


waming. the tally in the tally fluctuation chart bin did not pass 1 of the 10 statistical checks.

lunnormed tally density for tally $6 \quad$ nonzero tally mean $(\mathrm{m})=4.357 \mathrm{E}+01 \mathrm{nps}=940$ print table $16 \mathrm{I}$

abscissa ordinate log plot of tally probability density function in tally fluctuation chart bin(dindecade,slope $=0.0$ )

tally number num den log dend-

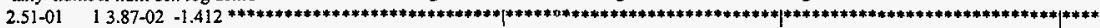

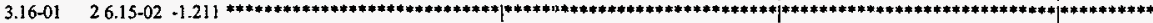

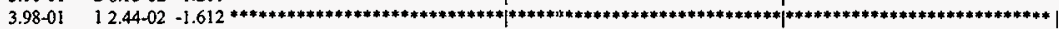

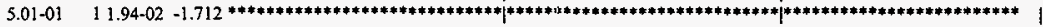

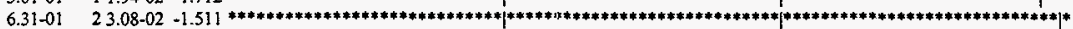

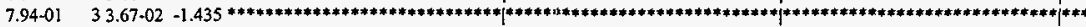

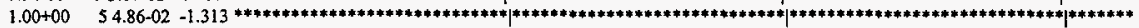

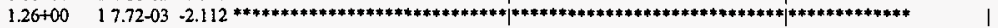

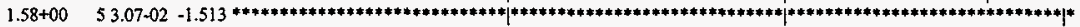

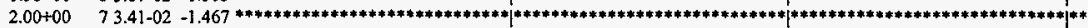

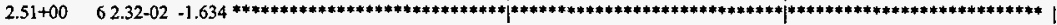

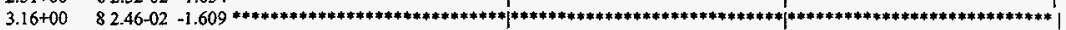

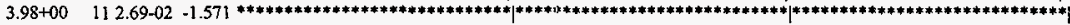

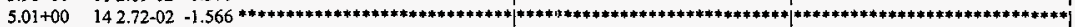

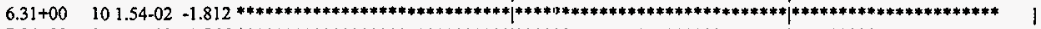

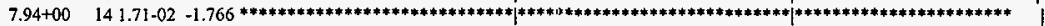

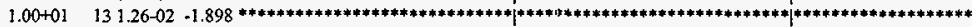

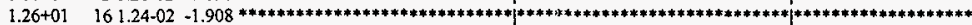

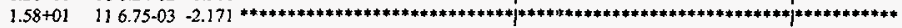

$200+01$

$2.51+01$

$3.16+01$

$3.98+01$

$5.01+01$

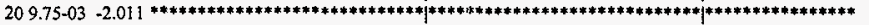

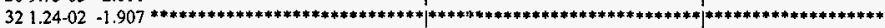

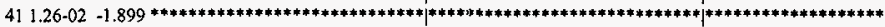

$245.86-03-2.232 * * * * * * * * * * * * * * * * * * * * * * * * * * * * * * * * * i * * * * * * * * * * * * * * * * * * * * * * * * * * * * * * * * *$

$489.31-03-2.031$

nmmmmmmmmmmmmmmmmmmmmmmmmm/mmmmmmmmmmmmmmmmmmmmmmmmmmm/mmmmmmmmmmmm

$1+0 t$

54 8.32-03 $-2.080 * * * * * * * * * * * * * * * * * * * * * * * * * * * * *+\left.\right|^{* * * * * * * * * * * * * * * * * * * * * * * * * * * * * * * \mid * * * * * * * * * * * * * * *}$

$404.90-03-2.310 * * * * * * * * * * * * * * * * * * * * * * * * * * * * * \mid * * * * ; 1 * * * * * * * * * * * * * * * * * * * * * * * * * * * * * * * * *$

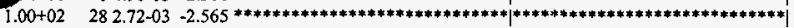

$1.26+02 \quad 201.54-03-2.811+* * * * * * * * * * * * * * * * * * * * * * * * * * * \mid * * * * * * * * * * * * * * * * * * * *$

J.58+02 21 1.29-03 $-2.890 * * * * * * * * * * * * * * * * * * * * * * * * * * * * *|* * * * * * * * * * * * * * * * * * * *|$

$2.00+02 \quad 29.75 \cdot 05-4.011 * * * * * * * * * * * * * * * *$

$2.51+02 \quad 00.00+00 \quad 0.000$

$3.16+02 \quad 13.08-05-4.512 *$

total $4629.24-01$

d-ann........................

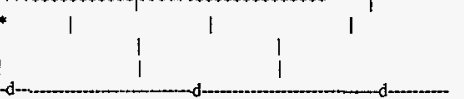

Istatus of the statistical checks used to form confidence intervais for the mean for each tally bin

tally result of statistical checks for the tfc bin (the first check not passed is listed) and error magnitude check for all bins

6 missed 1 of $10 \mathrm{tfc}$ bin checks: there is insufficient tfe bin tally information to estimate the large tally slope reliably missed all bin error check: 5 tally bins had 0 bins with zeros and 2 bins with relative errors exceeding 0.10

the 10 statistical checks are only for the tally fluctuation chart bin and do not apply to other tally bins.

waming. I of the 1 tally fluctuation char bins did not pass all 10 statistical checks.

waming. 1 of the 1 tallies had bins with relative errors greater than recommended.

I tally fluctuation charts

tally 6

nps mean error vov slope fom

9404.0257 E+01 0.04340 .00950 .0

tally data written to file inp $24 \mathrm{~m}$

15 waming messages so far.

terminated when $4 \mathrm{kcode}$ cycles were done. 


\section{MULTI-CANISTER OVERPACK PROJECT VERIFICATION AND VALIDATION ANSYS 5.0A}


HNF-SD-SNF-CSWD-005, Rev. 0

Document Number: QA040.216 (P9O)

Revision 0

File: QA040.216.00001

Software Verification and Validation Report

Program: ANSYS5.0A

Version: PC 386/486

Prepared By:

Wand hp les $10 / 3 / 95$

Approved By:

hand foch For Sos

Steve Sisley

Software Project Engineer
Checked By:

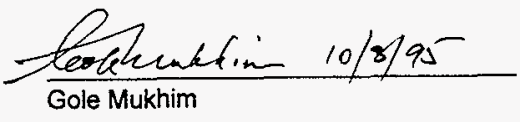

Date: $10 / 15 / 95$

278 
Software Verification and Validation Report ANSYS Revision 5.0A, Version: PC 386/486
HNF-SD-SNF-CSWD-005, Rev. 0 Document Number: QA040.216 (P90)

Revision 0

Page 2 of 95

\section{Table Of Contents}

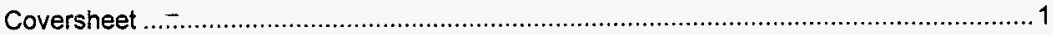

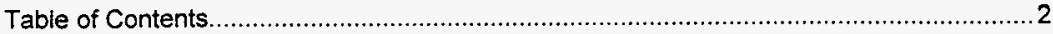

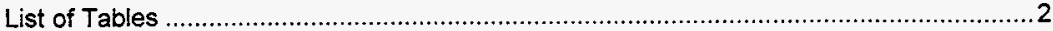

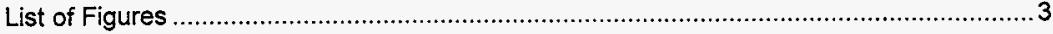

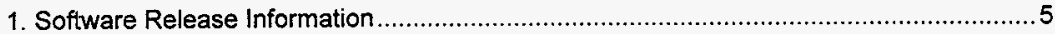

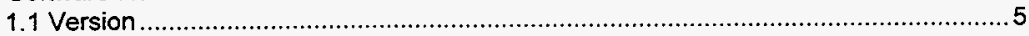

1.2 Computer System and File Creation Date ................................................ 5

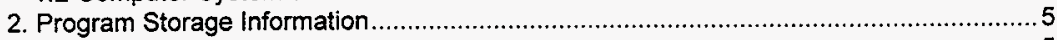

3. User Documentation ................................................................................... 5

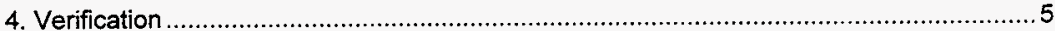

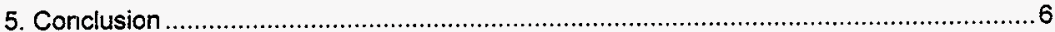

\section{List of Tables}

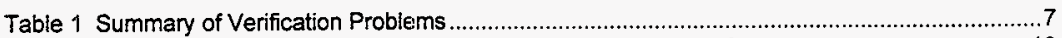

Table 2 Directory Structure - ANSYS Version PC 386/486 (Pentium PC) .....................................12

Table 3 Comparison of Calculated and Published Results ...................................................... 15 


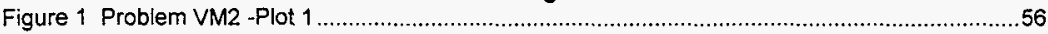

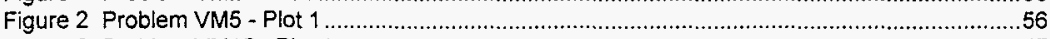

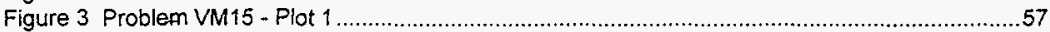

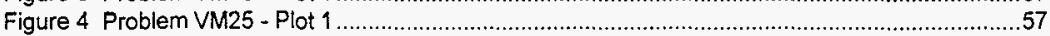

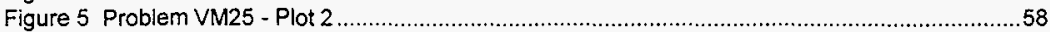

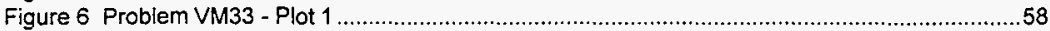

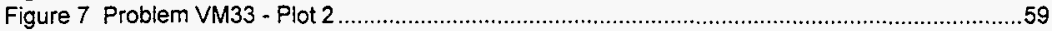

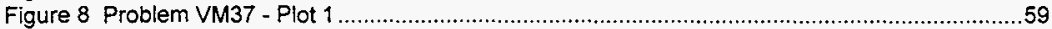

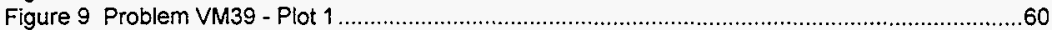

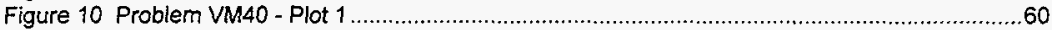

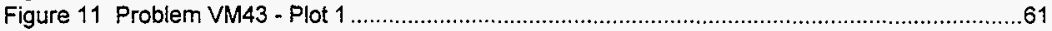

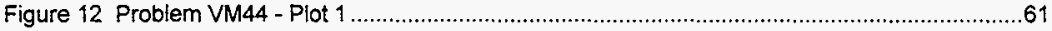

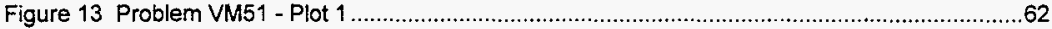

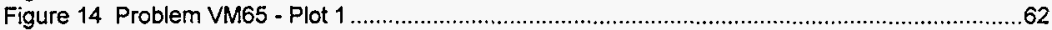

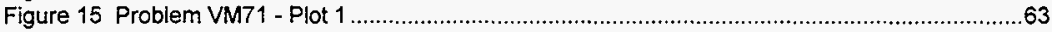

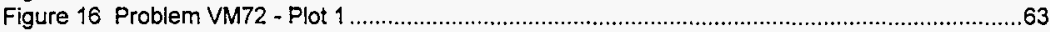

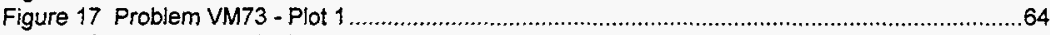

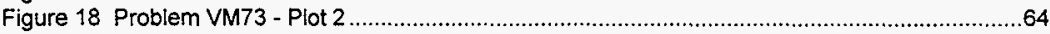

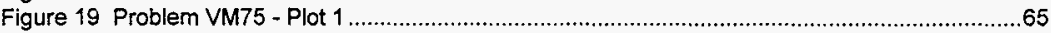

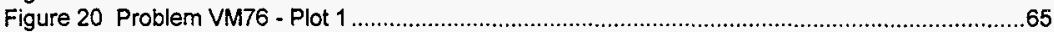

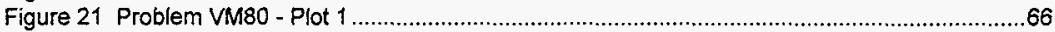

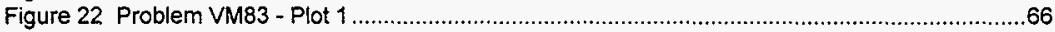

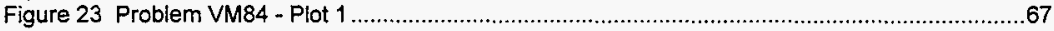

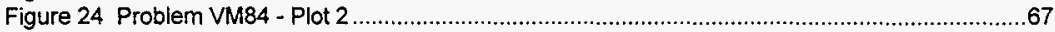

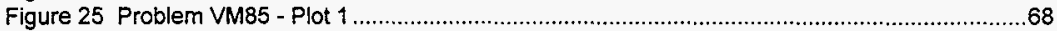

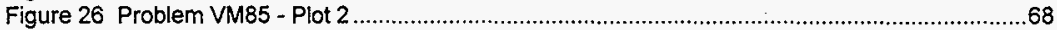

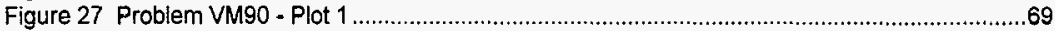

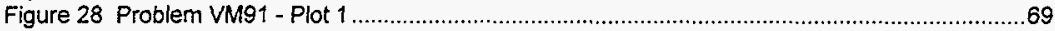

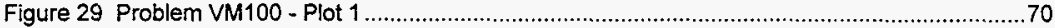

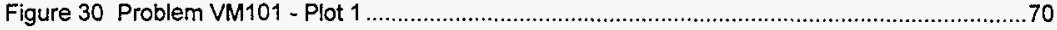

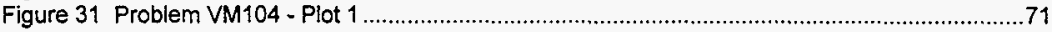

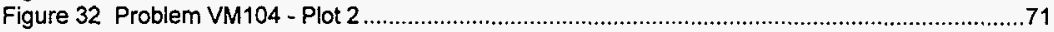

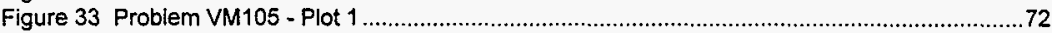

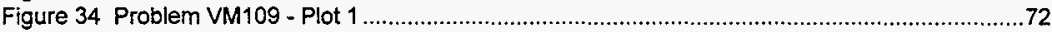

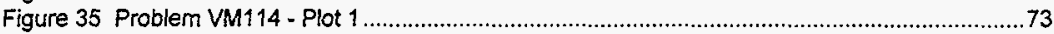

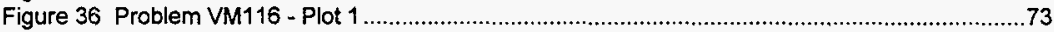

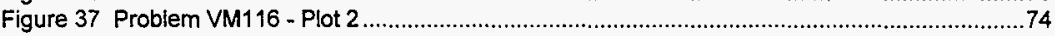

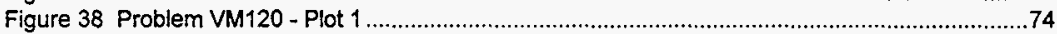

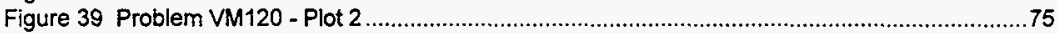

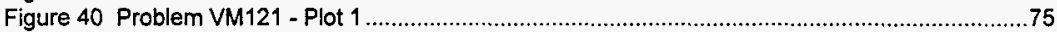

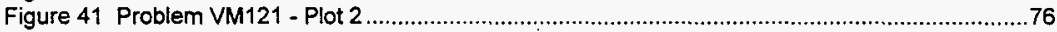

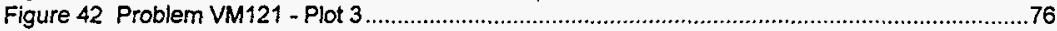

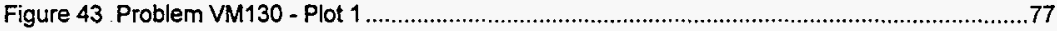

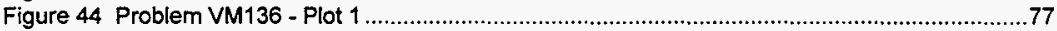

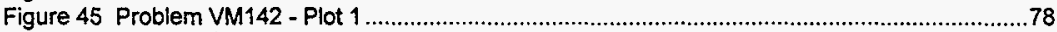

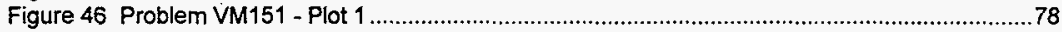

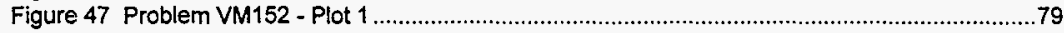

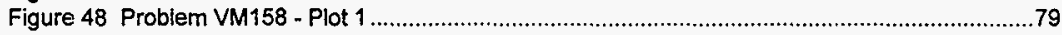

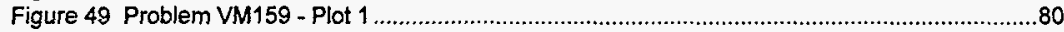

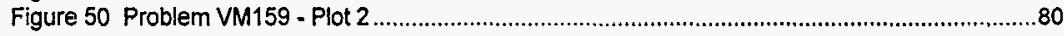




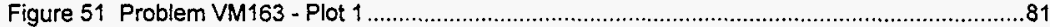

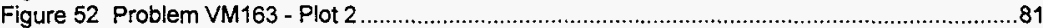

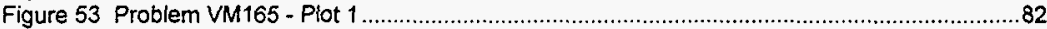

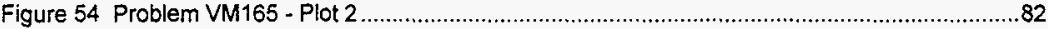

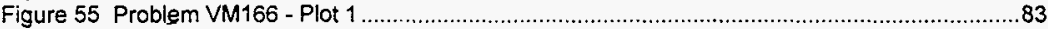

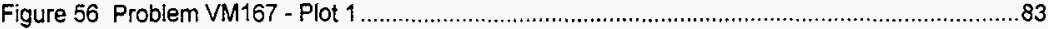

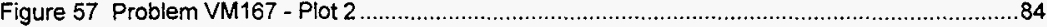

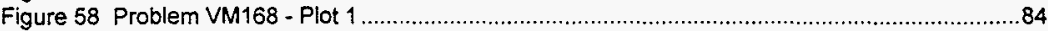

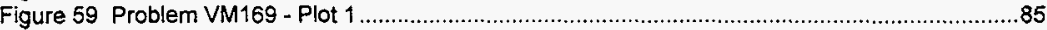

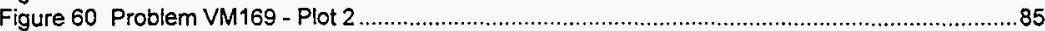

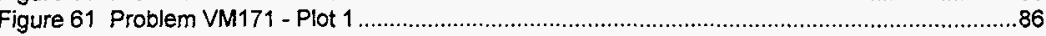

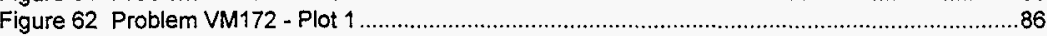

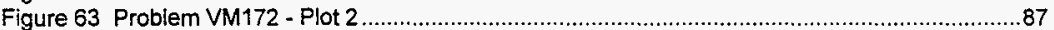

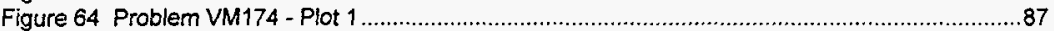

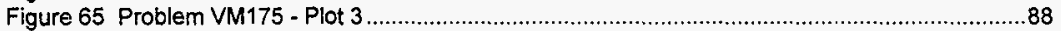

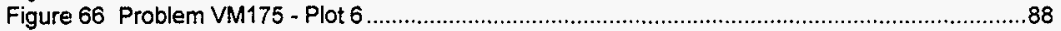

Figure 67 Probiem VM175 - Plot 15

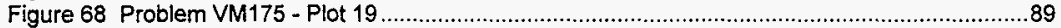

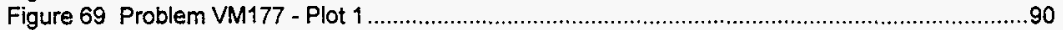

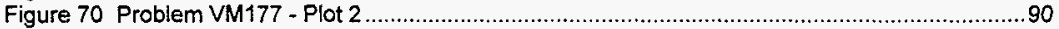

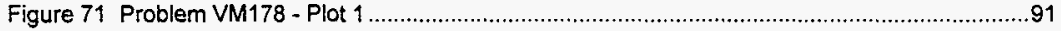

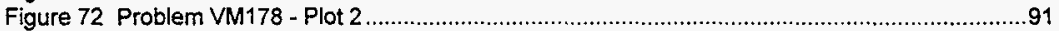

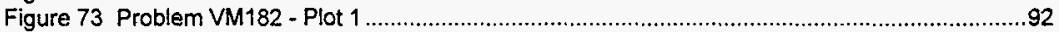

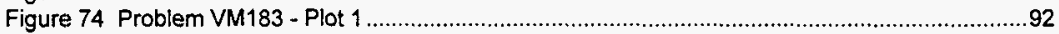

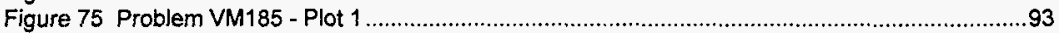

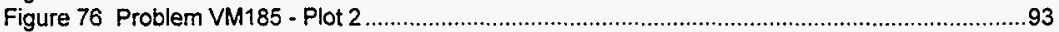

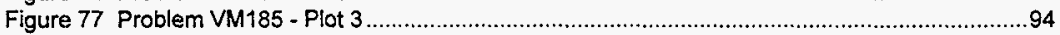

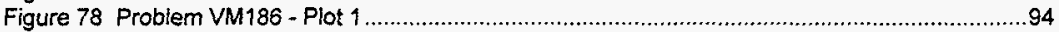

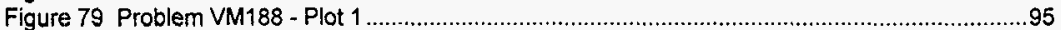




\section{Software Release Information}

\subsection{Version -}

Version PC $386 / 486$ of the ANSYS program (Revision 5.0A) was supplied by Swanson Analysis Systems (Houston, PA.) through their local representative, MCR Associates of Sunnyvale CA.).

\subsection{Computer System and File Creation Date}

ANSYS 5.OA, Version PC 386/486 an IBM compatable PC using the MS-DOS operating system. The verification in this documented used version 6.22 of MS-DOS running on a PC with a $90 \mathrm{Mhz}$ Pentium processor (the processor is an "updated" chip obtained from Intel as a result of the "floating point" error in the original Pentium processors). The directory structure of ANSYS on the PC is shown in Table 2.

\section{Program Storage Information}

ANSYS Version PC $386 / 486$ is con'ained on 9 (nine) $51 / 4$ floppy disks. The disks are stored in the San Jose Office of VECTRA.

\section{User Documentation}

Documentation for the subject Code is provided on-line.

\section{Verification}

Verification of the program on the Pentium PC was performed by execution of the 188 problems from the ANSYS Veritication Manual (UpdO DN-Q145:50, April 1, 1993). The element and analysis types for each problem are summarized in Table 1. Results generated by the Pentium PC were tabluated and compared to the ANSYS results published in the Verification Manual. Results of these comparisons are listed in Table 3 (In the table, the ratio column is equal to the published value divided by the analyzed value). In all cases differences between the published results and the results obtained from the Pentium PC are insignificant (differences are primarily due to round-oft).

Plots generated by the sample problems are included as Figures 1 through 79 of this document. The figures were compared to the plots in the Verification Manual. No significant deviations were noted. (Minor differences between the PC generated mesh and 
the published mesh were noted for Problem VM120 (Figures 38 and 39). No change in results was noted.)

\section{Conclusion}

As shown in Table 3, the Pentium PC results compare closely to the published results. Therefore ANSYS5.OA Version PC 386/486, running on a Pentium PC, is acceptable for production use. 
Software Verification and Validation Report ANSYS Revision 5.0A, Version: PC 386/486
HNF-SD-SNF-CSWD-005, Rev. 0 Document Number: QA040.216 (P90)

Revision 0

Page 7 of 95

Table 1

Summary of Verification Problems

\begin{tabular}{|c|c|l|}
\hline $\begin{array}{c}\text { Problem } \\
\text { Number }\end{array}$ & $\begin{array}{c}\text { Analysis } \\
\text { Type }\end{array}$ & \\
\hline VM1 & 0 & LINK1 \\
\hline VM2 & 0 & BEAM3 \\
\hline VM3 & 0 & LINK1 \\
\hline VM4 & 0 & LINK1 \\
\hline VM5 & 0 & PLANE42, PLANE 82 \\
\hline VM6 & 0 & SHELL93 \\
\hline VM7 & 0 & PIPE20, SOLID45, SHELL43 \\
\hline VM8 & & n/a \\
\hline VM9 & 4 & COMBIN14, COMBIN40 \\
\hline VM10 & 0 & BEAM54 \\
\hline VM11 & 0 & LINK1 \\
\hline VM12 & 0 & PIPE16 \\
\hline VM13 & 0 & SHELL51 \\
\hline VM14 & 0 & BEAM54 \\
\hline VM15 & 0 & SHELL51 \\
\hline VM16 & 0 & PLANE42 \\
\hline VM17 & 0 & SOLID45 \\
\hline VM18 & 0 & PIPE18 \\
\hline VM19 & 0 & BEAM3 \\
\hline VM20 & 0 & SHELL41 \\
\hline VM21 & 0 & BEAM4 \\
\hline VM22 & 0 & SHELL51 \\
\hline VM23 & 0 & SHELL63 \\
\hline VM24 & 0 & BEAM23 \\
\hline VM25 & 0 & PLANE42 \\
\hline VM26 & 0 & SHELL43 \\
\hline VM27 & 0 & LINK1, CONTAC12, LINK8, CONTAC52 \\
\hline VM28 & 4 & PLANE77 \\
\hline VM29 & 0 & CONTAC12 \\
\hline VM30 & 0 & LINK8 \\
\hline VM31 & 0 & LINK10 \\
\hline VM32 & 0 & PLANE55, PLANE42 \\
\hline VM33 & 4 & SOLID5 \\
\hline VM34 & 0 & SHELL63, BEAM44 \\
\hline VM35 & 0 & SHELL91 \\
\hline VM36 & 0 & COMBIN40, BEAM4 \\
\hline VM37 & 0 & SOLID45 \\
\hline VM38 & 0 & PLANE42, SOLID45 \\
\hline VM39 & 0 & SHELL63 \\
\hline VM40 & 4 & BEAM3 \\
\hline
\end{tabular}


Software Verification and Validation Report ANSYS Revision 5.0A, Version: PC 386/486
HNF-SD-SNF-CSWD-005, Rev. 0 Document Number: QA040.216 (P90)

Revision 0

Page 8 of 95

Table 1

Summary of Verification Problems

\begin{tabular}{|l|c|l|}
\hline $\begin{array}{c}\text { Problem } \\
\text { Number }\end{array}$ & $\begin{array}{c}\text { Analysis } \\
\text { Type }\end{array}$ & \\
\hline VM41 & 0 & MATRIX27, BEAM3 \\
\hline VM42 & 0 & SHELL93 \\
\hline VM43 & 0 & PLANE25 \\
\hline VM44 & 0 & SHELL61 \\
\hline VM45 & 2 & COMBIN14, MASS21 \\
\hline VM46 & 2 & BEAM3, MASS21 \\
\hline VM47 & 2 & COMBIN14, MASS21 \\
\hline VM48 & 2 & PIPE16, MASS21 \\
\hline VM49 & 2 & LINK1, MASS21 \\
\hline VM50 & 2 & BEAM3 \\
\hline VM51 & 2 & BEAM3 \\
\hline VM52 & 2 & BEAM3, COMBIN14, MASS21 \\
\hline VM53 & 0,2 & LINK10 \\
\hline VM54 & 0,2 & SHELL63 \\
\hline VM55 & 0,2 & SHELL51 \\
\hline VM56 & 0 & HYPER84, HYPER86 \\
\hline VM57 & 2 & PIPE16, BEAM4, MASS21 \\
\hline VM58 & 0 & PLANE35, SURF19 \\
\hline VM59 & 0,2 & BEAM4 \\
\hline VM60 & 2 & SHELL99 \\
\hline VM61 & 2 & BEAM3 \\
\hline VM62 & 2 & SHELL63 \\
\hline VM63 & 0 & PLANE82, PLANE2, CONTAC26 \\
\hline VM64 & 0 & CONTAC26, PLANE42 \\
\hline VM65 & 4 & CONTAC26, MASS21 \\
\hline VM66 & 2 & SHELL63 \\
\hline VM67 & 2 & PLANE25 \\
\hline VM68 & 2,8 & COMBIN40 \\
\hline VM69 & 2 & COMBIN40 \\
\hline VM70 & 2 & BEAM3 \\
\hline VM71 & 4 & COMBIN40 \\
\hline VM72 & 4 & COMBIN40 \\
\hline VM73 & 4 & COMBIN40 \\
\hline VM74 & 4 & COMBIN40 \\
\hline VM75 & 4 & COMBIN40 \\
\hline VM76 & $0,2,3$ & LINK1 \\
\hline VM77 & 4 & BEAM3, MASS21 \\
\hline VM78 & 0 & SHELL99 \\
\hline VM79 & 4 & COMBIN40, GP \\
\hline VM80 & 4 & LINK1, MASS21 \\
\hline
\end{tabular}


Software Verification and Validation Report ANSYS Revision 5.0A, Version: PC 386/486
HNF-SD-SNF-CSWD-005, Rev. 0

Document Number: QA040.216 (P9O)

Revision 0

Page 9 of 95

Table 1

Summary of Verification Problems

\begin{tabular}{|c|c|l|}
\hline Problem & $\begin{array}{c}\text { Analysis } \\
\text { Type }\end{array}$ & \\
\hline VM81 & 4 & COMBIN40, MASS21, GP \\
\hline VM82 & 0 & SHELL99, SOLID46 \\
\hline VM83 & 4 & COMBIN40 \\
\hline VM84 & 4 & LINK1 \\
\hline VM85 & 5 & LINK1, GP \\
\hline VM86 & 3 & COMBIN40 \\
\hline VM87 & 3 & COMBIN40 \\
\hline VM88 & 3 & COMBIN40 \\
\hline VM89 & 2 & COMBIN14, MASS21 \\
\hline VM90 & 3 & COMBIN14, MASS21 \\
\hline VM91 & 4 & LINK8, MASS21 \\
\hline VM92 & 0 & LINK34, LINK32 \\
\hline VM93 & 0 & LINK32 \\
\hline VM94 & 0 & LINK32, LINK34 \\
\hline VM95 & 0 & LINK33, LINK34, SOLID70 \\
\hline VM96 & 0 & SOLID87 \\
\hline VM97 & 0 & SHELL57, LINK34 \\
\hline VM98 & 0 & PLANE55 \\
\hline VM99 & 0 & PLANE55 \\
\hline VM100 & 0 & PLANE55 \\
\hline VM101 & 0 & SOLID70 \\
\hline VM102 & 0 & PLANE55 \\
\hline VM103 & 0 & SHELL57 \\
\hline VM104 & 4 & PLANE55 \\
\hline VM105 & 0 & PLANE55 \\
\hline VM106 & 0 & LINK31 \\
\hline VM107 & 0 & LINK31, LINK34 \\
\hline VM108 & 0 & PLANE75 \\
\hline VM109 & 4 & LINK34, MASS71 \\
\hline VM110 & 4 & LINK32, LINK34 \\
\hline VM111 & 4 & PLANE55 \\
\hline VM112 & 4 & PLANE77 \\
\hline VM113 & 4 & PLANE55 \\
\hline VM114 & 4 & LINK33 \\
\hline VM115 & 4 & LINK32 \\
\hline VM116 & 4 & LINK34, LINK32 \\
\hline VM117 & 0 & LINK68 \\
\hline VM118 & 0 & PLNE55, SOLID70 \\
\hline VM119 & 0 & PLANE67, SOLID69 \\
\hline VM120 & 0 & PLANE77 \\
\hline
\end{tabular}


Software Verification and Validation Report ANSYS Revision 5.OA, Version: PC 386/486
HNF-SD-SNF-CSWD-005, Rev. 0

Document Number: QA040.216 (P90)

Revision 0

Page 10 of 95

Table 1

Summary of Verification Problems

\begin{tabular}{|l|c|l|}
\hline Problem & $\begin{array}{c}\text { Analysis } \\
\text { Number }\end{array}$ & \\
\hline Type & \\
\hline VM121 & 0 & FLUID15 \\
\hline VM122 & 0 & FLUID66 \\
\hline VM123 & 0 & FLUID66 \\
\hline VM124 & 0 & FLUID66 \\
\hline VM125 & 0, AUX12 & LINK32, MATRIX50 \\
\hline VM126 & 0 & FLUID66 \\
\hline VM127 & 1,0 & BEAM3 \\
\hline VM128 & 1,0 & PLANE42 \\
\hline VM129 & & none \\
\hline VM130 & & nOne \\
\hline VM131 & 0 & MASS21 \\
\hline VM132 & 0 & LINK1 \\
\hline VM133 & 0 & BEAM23 \\
\hline VM134 & 0 & BEAM24 \\
\hline VM135 & 0 & BEAM54 \\
\hline VM136 & 0 & BEAM3 \\
\hline VM137 & 0 & SHELL51 \\
\hline VM138 & 0 & SHELL51 \\
\hline VM139 & 0 & SHELL63 \\
\hline VM140 & 0 & PLANE83 \\
\hline VM141 & 0,7 & PLANE82, PLANE2, SHELL93, MATRIX50 \\
\hline VM142 & 0 & PLANE2, PLANE42 \\
\hline VM143 & 0 & SOLID95, SOLID45, PLANE82 \\
\hline VM144 & 0 & SHELL99, SOLID46 \\
\hline VM145 & 0 & SOLID64 \\
\hline VM146 & 0 & SOLID65, LINK8, PIPE16 \\
\hline VM147 & 0 & SHELL93 \\
\hline VM148 & 0 & SOLID95 \\
\hline VM149 & 0 & FLUID79 \\
\hline VM150 & 0 & FLUID80 \\
\hline VM151 & 2 & SHELL61 \\
\hline VM152 & 2,0 & SHELL61 \\
\hline VM153 & $2,0, M a c r o$ & SHELL41 \\
\hline VM154 & 2 & FLUID38, COMBIN14, FLUID81 \\
\hline VM155 & $10 P T, 0$ & PLANE42 \\
\hline VM156 & 4 & COMBIN39, LINK1, MASS21 \\
\hline VM157 & $109 T, 0$ & BEAM3 \\
\hline VM158 & 4 & PIPE59 \\
\hline VM159 & 4 & COMBIN37, LINK34, MASS71 \\
\hline VM160 & 0 & PLANE78 \\
\hline
\end{tabular}


Software Verification and Validation Report ANSYS Revision 5.0A, Version: PC 386/486
HNF-SD-SNF-CSWD-005, Rev. 0 Document Number: QA040.216 (P90)

Revision 0

Page 11 of 95

Table 1

Summary of Verification Problems

\begin{tabular}{|l|c|l|}
\hline $\begin{array}{c}\text { Problem } \\
\text { Number }\end{array}$ & $\begin{array}{c}\text { Analysis } \\
\text { Type }\end{array}$ & \\
\hline VM161 & 0 & SOLID90 \\
\hline VM162 & 0 & SOLID90 \\
\hline VM163 & 0 & PLANE55 \\
\hline VM164 & 4 & LINK32 \\
\hline VM165 & 0 & PLANE13, INFIN9 \\
\hline VM166 & 3 & PLANE13 \\
\hline VM167 & 4 & PLANE13 \\
\hline VM168 & 0 & SOLID5, SOURC36 \\
\hline VM169 & 0 & SOLID98 \\
\hline VM170 & 0 & LINK68 \\
\hline VM171 & 0 & PLANE13, COMBIN14 \\
\hline VM172 & 0 & PLANE13 \\
\hline VM173 & 0 & SOLID5 \\
\hline VM174 & 0 & PLANE13 \\
\hline VM175 & 2 & SOLID5 \\
\hline VM176 & 3 & SOLID5 \\
\hline VM177 & 3 & FLUID30, SHELL63 \\
\hline VM178 & 0 & FLUID15 \\
\hline VM179 & 4 & COMBIN7, BEAM4 \\
\hline VM180 & 0 & PLANE2, BEAM3 \\
\hline VM181 & 2 & PLANE2 \\
\hline VM182 & 2,4 & COMBIN40 \\
\hline VM183 & 2,3 & COMBIN40 \\
\hline VM184 & 0 & SOLID5, SOLID92, SOLID98 \\
\hline VM185 & 3 & PLANE13 \\
\hline VM186 & 4 & PLANE13 \\
\hline VM187 & 0 & SOLID5, SOLID92, SOLID98 \\
\hline VM188 & 0 & PLANE13 \\
\hline & & \\
\hline
\end{tabular}

Note: Analysis Type Codes are as follows:

$\begin{array}{cl}\text { ANTYPE } & \text { Analysis Type } \\ 0 & \text { Static } \\ 1 & \text { Buckling } \\ 2 & \text { Modal } \\ 3 & \text { Harmonic } \\ 4 & \text { Transient } \\ 7 & \text { Substructure } \\ 8 & \text { Response Spectrum }\end{array}$


Software Verification and Validation Report ANSYS Revision 5.0A, Version: PC 386/486
HNF-SD-SNF-CSWD-005, Rev. 0 Document Number: QA040.216 (PSO)

Revision 0

Page 12 of 95

Table 2

Directory Structure - ANSYS Version PC 386/486

(Pentium PC)

$-$

Directory of C: \ANSYS50A

\begin{tabular}{|c|c|c|c|c|}
\hline BIN & & $\langle D I R\rangle$ & $03-08-95$ & $10: 16 \mathrm{a}$ \\
\hline DATA & & $\langle\mathrm{DIR}\rangle$ & $03-08-95$ & $10: 16 \mathrm{a}$ \\
\hline DEMO & & $\langle D I R\rangle$ & $03-08-95$ & $10: 16 a$ \\
\hline DOCU & & $\langle\mathrm{DIR}\rangle$ & $03-08-95$ & $10: 16 \mathrm{a}$ \\
\hline GRDRV & & $\langle D I R\rangle$ & $03-08-95$ & $9: 58 \mathrm{a}$ \\
\hline OBJECTS & & $\langle\mathrm{DIR}\rangle$ & $03-08-95$ & $10: 16 \mathrm{a}$ \\
\hline SOURCE & & $\langle D I R\rangle$ & $03-08-95$ & $10: 16 a$ \\
\hline PHARI_AP & 386 & 9,343 & $02-10-93$ & $4: 33 \mathrm{p}$ \\
\hline SYSINFO & $50 \mathrm{~A}$ & 9,887 & $03-08-95$ & $10: 05$ \\
\hline SYSINFO & $\mathrm{AP}$ & 10,696 & $06-15-95$ & $4: 25 p$ \\
\hline INSTALL & ERR & 3,073 & $03-08-95$ & $10: 17 a$ \\
\hline APROGRAM & EXE & 163,840 & $06-15-95$ & $4: 26 p$ \\
\hline TELLME & EXE & 65,389 & $12-11-92$ & $5: 34$ \\
\hline
\end{tabular}

Dixectory of C: \ANSYS5OA\BIN

$\begin{array}{llrll}\text { ANSSPD } & \text { EXE } & 278,820 & 09-30-93 & 4: 59 p \\ \text { ANSYS } & \text { EXE } & 12,473,250 & 01-19-95 & 4: 45 p \\ \text { CMAP } & \text { EXE } & 857,596 & 09-30-93 & 4: 59 p \\ \text { DISPLAY } & \text { EXE } & 961,383 & 05-25-94 & 4: 03 p \\ \text { GENAUTH } & \text { EXE } & 907,126 & 09-30-93 & 4: 59 p \\ \text { MKCNF } & \text { EXE } & 52,73209-30-93 & 4: 59 p \\ \text { QUERY } & \text { EXE } & 47,876 & 09-30-93 & 4: 59 p \\ \text { TRANS16 } & \text { EXE } & 1,886,979 & 09-30-93 & 4: 59 p \\ \text { VIEWS0A } & \text { EXE } & 937,32009-30-93 & 4: 59 p\end{array}$

Directory of $C: \backslash A N S Y S 50 A \backslash D A T A$

$\begin{array}{llll}\text { EXAMPLES } & \text { LDIR> } & 03-08-95 & 10: 17 a \\ \text { USERMAC } & \langle\text { DIR } & 03-08-95 & 10: 17 a \\ \text { VERIF } & \langle\text { DIR } & 03-08-95 & 10: 17 a\end{array}$

Directory of C: \ANSYS50A\DATA \EXAMPLES

PM EXE $\quad 50,92209-30-93 \quad 4: 59 p$ 
Software Verification and Validation Report ANSYS Revision 5.0A, Version: PC 386/486
HNF-SD-SNF-CSWD-005, Rev. 0 Document Number: QA040.216 (P90)

Revision 0

Page 13 of 95

Table 2

Directory Structure - ANSYS Version PC 386/486

(Pentium PC)

- Directory of C: \ANSYS50A\DATA \USERMAC

$\begin{array}{llrll}\text { UAANIM } & \text { MAC } & 2,411 & 09-30-93 & 4: 59 p \\ \text { UADAPT } & \text { MAC } & 30,494 & 09-30-93 & 4: 59 p \\ \text { UANCONT } & \text { MAC } & 1,425 & 09-30-93 & 4: 59 p \\ \text { UANDISP } & \text { MAC } & 1,224 & 09-30-93 & 4: 59 p \\ \text { UANERAM } & \text { MAC } & 273 & 09-30-93 & 4: 59 p \\ \text { UANMAC } & \text { MAC } & 9,676 & 09-30-93 & 4: 59 p \\ \text { UANPOST } & \text { MAC } & 1,168 & 09-30-93 & 4: 59 p \\ \text { UANUSER } & \text { MAC } & 434 & 09-30-93 & 4: 59 p \\ \text { UANWALK } & \text { MAC } & 2,244 & 09-30-93 & 4: 59 p \\ \text { UETEXP } & \text { MAC } & 10,250 & 09-30-93 & 4: 59 p \\ \text { USHEIL } & \text { MAC } & 564 & 09-30-93 & 4: 59 p\end{array}$

Directory of $\mathrm{C}: \backslash A N S Y S 50 A \backslash D A T A \backslash V E R I F$

VM EXE $\quad 155,93609-30-93 \quad 4: 59 p$

D1rectory of C: \ANSYS5OA \DEMO

$\begin{array}{llrlr}\text { RUNDEMO } & \text { BAT } & 1,168 & 09-30-93 & 4: 59 p \\ \text { DEMOFILE } & \text { EXE } & 1,728,619 & 09-30-93 & 4: 59 p\end{array}$

Directory of C: \ANSYSSOA \DOCU

$\begin{array}{llrlr}\text { AUTH } & 50 & 223 & 12-21-92 & 3: 13 p \\ \text { CMDIDX } & 50 A & 99,748 & 09-30-93 & 4: 59 p \\ \text { DTDOCU } & 50 A & 8,843,840 & 09-30-93 & 4: 59 p \\ \text { KORIDX } & 50 A & 127,488 & 09-30-93 & 4: 59 p \\ \text { START } & \text { ANS } & 770 & 09-30-93 & 4: 59 p \\ \text { F16CNVRT } & \text { DAT } & 542 & 09-30-93 & 4: 59 p \\ \text { AANIM } & \text { MAC } & 2,408 & 09-30-93 & 4: 59 p \\ \text { ADAPT } & \text { MAC } & 30,494 & 09-30-93 & 4: 59 p \\ \text { ANCONT } & \text { MAC } & 1,425 & 09-30-93 & 4: 59 p \\ \text { ANDISP } & \text { MAC } & 1,221 & 09-30-93 & 4: 59 p \\ \text { ANERAM } & \text { MAC } & 273 & 09-30-93 & 4: 59 p \\ \text { ANMAC } & \text { MAC } & 9,672 & 09-30-93 & 4: 59 p \\ \text { ANPOST } & \text { MAC } & 1,167 & 09-30-93 & 4: 59 p \\ \text { ANUSER } & \text { MAC } & 434 & 09-30-93 & 4: 59 p \\ \text { ANWALR } & \text { MAC } & 2,244 & 09-30-93 & 4: 59 p \\ \text { ETEXP } & \text { MAC } & 10,250 & 09-30-93 & 4: 59 p \\ \text { F16CNVRT } & \text { MAC } & 315 & 09-30-93 & 4: 59 p \\ \text { HYPER } & \text { MAC } & 24,048 & 09-30-93 & 4: 59 p\end{array}$


Software Verification and Validation Report ANSYS Revision 5.0A, Version: PC 386/486
HNF-SD-SNF-CSWD-005, Rev. 0

Document Number: QA040.216 (P9O)

Revision 0

Page 14 of 95

Table 2

Directory Structure - ANSYS Version PC $386 / 486$

(Pentium PC)

\begin{tabular}{|c|c|c|c|c|}
\hline \multicolumn{2}{|c|}{ Directory of } & \multicolumn{3}{|c|}{ C: \ANSYS50A \DOCD (continued) } \\
\hline LSSOLVE & MAC & 5,123 & $09-30-93$ & $4: 59 p$ \\
\hline MODCYC & MAC & 4,292 & $09-30-93$ & $4: 59 p$ \\
\hline MODFUL & MAC & 4,093 & $09-30-93$ & $4: 59 p$ \\
\hline SETSPEED & MAC & 35 & $03-08-95$ & $10: 17 \mathrm{a}$ \\
\hline SHELL & MAC & 564 & $09-30-93$ & $4: 59 p$ \\
\hline \multicolumn{5}{|c|}{ Directory of C: \ANSYS50A \GRDRV } \\
\hline HALO & BAK & 431 & $03-08-95$ & $10: 05 a$ \\
\hline HALO & $\mathrm{CNF}$ & 431 & $06-15-95$ & $4: 25 p$ \\
\hline KEEPDRVS & DAT & 223 & $06-15-95$ & $4: 25 p$ \\
\hline AHDATIL & DSP & 20,507 & $06-23-93$ & $4: 46 p$ \\
\hline AHDATIP & DSP & 23,419 & $06-23-93$ & $4: 46 p$ \\
\hline AHDATIU & DSP & 22,023 & $09-27-93$ & $2: 11 p$ \\
\hline AHDIBME & DSP & 23,387 & $09-27-93$ & $2: 11 p$ \\
\hline AHDIBMN & DSP & 23,083 & $01-11-93$ & $2: 11 \mathrm{p}$ \\
\hline AHDVRI & DSP & 26,619 & $06-23-93$ & $4: 46 p$ \\
\hline AHDHP & EXE & 103,164 & $01-29-93$ & $2: 11 p$ \\
\hline AHDO 10 & FNT & 3,584 & $06-23-93$ & $4: 46 p$ \\
\hline AHD011 & FNT & 3,072 & $06-23-93$ & $4: 46 \mathrm{p}$ \\
\hline AHD012 & FNT & 2,560 & $06-23-93$ & $4: 46 p$ \\
\hline AHDO 13 & FNT & 8,192 & $06-23-93$ & $4: 46 p$ \\
\hline AHDIO2 & FNT & 5,042 & $06-23-93$ & $4: 46 p$ \\
\hline BUILTIN & FNT & 2,068 & $06-23-93$ & $4: 46 p$ \\
\hline AHDWF 32 & KRN & 61,520 & $06-23-93$ & $4: 46 p$ \\
\hline AHDMOU & LOC & 360 & $06-23-93$ & $4: 46 p$ \\
\hline AHDBW8 & PRT & 6,580 & $06-23-93$ & $4: 46 p$ \\
\hline AHDLJT4 & RRT & 6,958 & $04-27-93$ & $2: 11 p$ \\
\hline AHDLJTP & PRT & 6,748 & $06-23-93$ & $4: 46 p$ \\
\hline \multicolumn{5}{|c|}{ Directory of $\mathrm{C}:$ IANSYS5OA \OBJECTS } \\
\hline BINLIB & A & 37,376 & $09-30-93$ & $4: 59 p$ \\
\hline BINTST & LNK & 378 & $09-30-93$ & $4: 59 p$ \\
\hline \multicolumn{5}{|c|}{ Directory of C: \ANSYS5OA\SOURCB } \\
\hline BINTRD & $F$ & 10,742 & $09-30-93$ & $4: 59 p$ \\
\hline BINTST & F & 4,592 & $09-30-93$ & $4: 59 p$ \\
\hline BINTWR & $F$ & 10,168 & $09-30-93$ & $4: 59 p$ \\
\hline
\end{tabular}


Software Verification and Validation Report ANSYS Revision 5.OA, Version: PC 386/486
HNF-SD-SNF-CSWD-005, Rev. 0 Document Number: QA040.216 (P90)

Revision 0

Page 15 of 95

Table 3

Comparison of Calculated and Published Results

VM1: Statically Indeterminate Reaction Force Analysis

\begin{tabular}{|c|c|c|c|c|}
\hline & Manual & Pentium 90 & Ratio & Notes \\
\hline$R 1, \mathrm{lb}$ & 900.0 & 900.0 & 1.000 & Reaction Forces, Node 4 \\
\hline $\mathrm{R} 2, \mathrm{lb}$ & 600.0 & 600.0 & 1.000 & Reaction Forces, Node 1 \\
\hline
\end{tabular}

VM2: Beam Stresses \& Deflections

\begin{tabular}{|c|c|c|c|c|}
\hline & Manual & Pentium 90 & Ratio & Notes \\
\hline$\sigma, \mathrm{psi}$ & $-11,404$ & $-11,404$ & 1.000 & EL $=2$, SBYB \\
\hline$\delta$, in & 0.182 & 0.182463 & 0.997 & Node 3, UY \\
\hline
\end{tabular}

VM3: Thermally Loaded Support Structure

\begin{tabular}{|c|c|c|c|c|}
\hline & Manual & Pentium 90 & Ratio & Notes \\
\hline$\sigma_{\text {steel, }}$ psi & 19,695 & 19,695 & 1.000 & EL=3, SAXL \\
\hline$\sigma_{\text {copper, }}$ psi & 10,152 & 10,152 & 1.000 & EL=1,2, SAXL \\
\hline
\end{tabular}

VM4: Deflection of a Hinged Support

\begin{tabular}{|c|c|c|c|c|}
\hline & Manual & Pentium 90 & Ratio & Notes \\
\hline$\sigma$, psi & 10,000 & 10,000 & 1.000 & EL=1,2, SAXL \\
\hline$\delta$, in & -0.120 & -0.120 & 1.000 & UY @ Node 2 \\
\hline
\end{tabular}

VM5: Laterally Loaded Tapered Support Structure

\begin{tabular}{|c|c|c|c|c|}
\hline Plane42 & Manual & Pentium 90 & Ratio & Notes \\
\hline$\sigma$, psi (El. 3, Face I-J) & 8,282 & 8281.59 & 1.000 & EL=3, S(PAR) Face I-J) \\
\hline$\sigma$, psi (Fixed End/POST1) & 7,086 & 7086.17 & 1.000 & Fixed End/POST1, SX6 \\
\hline
\end{tabular}

\begin{tabular}{|c|c|c|c|c|}
\hline Plane82 & Manual & Pentium 90 & Ratio & Notes \\
\hline$\sigma$, psi (El. 3, Face l-J) & 8,349 & 8348.6 & 1.000 & EL=3, S(PAR) Face 1-J) \\
\hline$\sigma$, psi & 7,410 & 7410.25 & 1.000 & Fixed End/POST1, SX6 \\
\hline
\end{tabular}

VM6: Pinched Cylinder

\begin{tabular}{|c|c|c|c|c|}
\hline & Manual & Pentium 90 & Ratio & Notes \\
\hline$\delta$, in & 0.1138 & 0.11375 & 1.000 & POST1, UY @ 34 \\
\hline
\end{tabular}

VM7: Plastic Compression of a Pipe Assembly

\begin{tabular}{|c|c|c|c|c|}
\hline PIPE20 & Manual & Pentium 90 & Ratio & Notes \\
\hline Load for $\delta=0.032$ in & $1,024,400$ & $1,024,400$ & 1.000 & $\begin{array}{c}\text { Nodes } 1 \text { \& 2, POST1 Macro, } \\
\text { (first) Value of FZ }\end{array}$ \\
\hline Load for $\delta=0.05$ in & $1,262,000$ & $1,262,000$ & 1.000 & \\
\hline Load for $\delta=0.10$ in & $1,262,000$ & $1,262,000$ & 1.000 & \\
\hline
\end{tabular}


Software Verification and Validation Report ANSYS Revision 5.0A, Version: PC 386/486
HNF-SD-SNF-CSWD-005, Rev 0

Document Number: QA040.216 (P9O)

Revision 0

Page 16 of 95

Table 3

Comparison of Calculated and Published Results

\begin{tabular}{|c|c|c|c|c|}
\hline SOLID45 & Manuai & Pentium 90 & Ratio & Notes \\
\hline Load for $\delta=0.032$ in & $1,022,500$ & $1,022,529$ & 1.000 & $\begin{array}{c}\text { Nodes 101 \& 118, POST1 } \\
\text { Macro Parameter LOAD }\end{array}$ \\
\hline Load for $\delta=0.05$ in & $1,259,700$ & $1,259,695$ & 1.000 & \\
\hline Load for $\delta=0.10$ in & $1,259,700$ & $1,259,695$ & 1.000 & \\
\hline
\end{tabular}

\begin{tabular}{|c|c|c|c|c|}
\hline SHELL43 & Manual & Pentium 90 & Ratio & Notes \\
\hline Load for $\delta=0.032$ in & $1,023,900$ & $1,023,932$ & 1.000 & \multirow{2}{*}{$\begin{array}{c}\text { Nodes 201 \& 212, POST1 } \\
\text { Macro Parameter LOAD }\end{array}$} \\
\hline Load for $\delta=0.05$ in & $1,261,400$ & $1,261,423$ & 1.000 & \\
\hline Load for $\delta=0.10$ in & $1,261,400$ & $1,261,423$ & 1.000 & \\
\hline
\end{tabular}

VM8: Parametric Calculation of Point to Point Distance

\begin{tabular}{|l|c|c|c|}
\hline & Manual & Pentium 90 & Ratio \\
\hline$N_{1}-N_{2}$ distance (LEN2) & 8.5849 & 8.5849 & 1.000 \\
\hline$K_{3}-K_{4}$ distance (LEN1) & 305.16 & 305.16 & 1.000 \\
\hline
\end{tabular}

VM9: Large Lateral Deflection of Unequal Stiffness Springs

\begin{tabular}{|c|c|c|c|c|}
\hline & Manual & Pentium 90 & Ratio & Notes \\
\hline Strain -Energy, N-cm & 24.02 & 24.02 & 1.000 & POST1 SENE \\
\hline$\delta_{x}, \mathrm{~cm}$ & 8.633 & 8.633 & 1.000 & UX @ Node 2 \\
\hline$\delta_{y,} \mathrm{~cm}$ & 4.532 & 4.533 & 1.000 & UY @ Node 2 \\
\hline
\end{tabular}

VM10: Bending of a Tee-Shaped Beam

\begin{tabular}{|c|c|c|c|c|}
\hline & Manual & Pentium 90 & Ratio & Notes \\
\hline$\sigma_{\text {bend, Bot, psi }}$ & 300. & 300.00. & 1.000 & Element Solution, EL=1, SBYB \\
\hline$\sigma_{\text {bend, Top, psi }}$ & -700. & -700.00. & 1.000 & Element Solution, EL=1, SBYT \\
\hline
\end{tabular}

VM11: Residual Stress Problem

\begin{tabular}{|c|c|c|c|c|}
\hline & Manual & Pentium 90 & Ratio & Notes \\
\hline$\delta$ at $F_{\uparrow}$, in & -0.07534 & -0.075338 & 1.000 & LS 1, UY @ 4 \\
\hline$\sigma_{r,}$ psi (SAXL, El 2) & $-5,650$. & $-5,650$ & 1.000 & LS 3, SAXL @ EL=2 \\
\hline
\end{tabular}

VM12: Combined Bending and Torsion

\begin{tabular}{|c|c|c|c|c|}
\hline & Manual & Pentium 90 & Ratio & Notes \\
\hline$\sigma_{\max }, \mathrm{psi}$ & 7,527 & 7526.9 & 1.000 & PIPE16 - S1MX \\
\hline \multirow{2}{*}{$\tau_{\max }, \mathrm{psi}$} & -- & 7553.8 & - & PIPE16 - SINTMX \\
\cline { 2 - 5 } & 3,777 & 3776.9 & 1.000 & PIPE16 - SINTMX/2 \\
\hline
\end{tabular}


Table 3

Comparison of Calculated and Published Results

VM13: Cylindrical Shell Under Pressure

\begin{tabular}{|c|c|c|c|c|}
\hline & Manual & Pentium 90 & Ratio & Notes \\
\hline$\sigma_{\mathrm{y},} \mathrm{psi}$ & 15,000 & 15,000 & 1.000 & $\mathrm{EL}=1, \mathrm{~T}(\mathrm{X})$ \\
\hline$\sigma_{\mathrm{z}}, \mathrm{psi}$ & 29,750 & 29,750 & 1.000 & $\mathrm{EL}=1, \mathrm{~T}(\mathrm{Z})$ \\
\hline
\end{tabular}

VM14: Large Deflection Eccentric Compression of a Slender Column

\begin{tabular}{|c|c|c|c|c|}
\hline & Manual & Pentium 90 & Ratio & Notes \\
\hline$\delta$, in & 0.1261 & 0.126061 & 1.000 & UX @ 5 \\
\hline$\sigma_{\text {tens, }}$ psi & 2,456 & 2456.4 & 1.000 & SMAX @ EL=1(I) \\
\hline$\sigma_{\text {comp }}$ psi & $-2,450$ & -2449.5 & 1.000 & SMIN @ EL=1(I) \\
\hline
\end{tabular}

VM15: Bending of a Circular Plate Using Axisymmetric Shell Elements

Case 1 (Fixed Edges w/Uniform L.oad)

\begin{tabular}{|c|c|c|c|c|}
\hline Case 1 & Manual & Pentium 90 & Ratio & Notes \\
\hline$\delta$, in & -0.08737 & -0.08737 & 1.000 & UY (Max) \\
\hline$\sigma_{\max }$, psi & 7,040 & -7040.4 & -1.000 & EL=10, SM @ BOT (Node 7) \\
\hline
\end{tabular}

Case 2 ( Fixed Edges w/Point Load)

\begin{tabular}{|c|c|c|c|c|}
\hline Case 2 & Manual & Pentium 90 & Ratio & Notes \\
\hline$\delta$, in & -0.08735 & -0.08735 & 1.000 & UY (Max) \\
\hline$\sigma_{\max }$, psi & 3,568 & 3568.3 & 1.000 & EL=10, SM @ BOT (Node 7) \\
\hline
\end{tabular}

Case 3 (Pinned Edges w/Uniform Load)

\begin{tabular}{|c|c|c|c|c|}
\hline Case 3 & Manual & Pentium 90 & Ratio & Notes \\
\hline$\delta$, in & -0.08903 & -0.08903 & 1.000 & UY (Max) \\
\hline$\sigma_{\text {max }}$, psi & $2,969$. & -2968.6 & -1.000 & EL=1, SM @ TOP \\
\hline
\end{tabular}

VM16: Bending of a Solid Beam (Plane Elements)

\begin{tabular}{|c|c|c|c|c|}
\hline Case 1 & Manual & Pentium 90 & Ratio & Notes \\
\hline$\delta$, in & 0.00500 & 0.00500 & 1.000 & UY @ 6, 16 \\
\hline$\sigma_{\text {Bend, psi }}$ & 3,000 & 3000.00 & 1.000 & S(PAR) @ EL=1, Face l-J \\
\hline Case 2 & Manual & Pentium 90 & Ratio & Notes \\
\hline$\delta$, in & 0.00505 & 0.00505 & 1.000 & UY @ 6, 16 \\
\hline$\sigma_{\text {Bend, psi }}$ & 4,050 & 4050.00 & 1.000 & S(PAR) @ EL=1, Face $1-J$ \\
\hline
\end{tabular}

VM17: Bending of a Solid Beam

\begin{tabular}{|c|c|c|c|c|}
\hline Case 1 & Manual & Pentium 90 & Ratio & Notes \\
\hline$\delta$, in & 0.00500 & 0.00500 & 1.000 & UZ Max. \\
\hline$\sigma_{\text {Bond, psi (SX, el 1) }}$ & $3,000$. & 3000.00 & 1.000 & EL=1, SX @ Face KLPO \\
\hline
\end{tabular}


Table 3

Comparison of Calculated and Published Results

VM17: Bending of a Solid Beam (continued)

\begin{tabular}{|c|c|c|c|c|}
\hline Case 2 & Manual & Pentium 90 & Ratio & Notes \\
\hline \hline$\delta$, in & 0.00505 & 0.00505 & 1.000 & UZ Max. \\
\hline$\sigma_{\text {Bend, }}$ Psi (SX, el 1) & $4,050$. & 4050.00 & 1.000 & EL=1, SX @ Face KLPO \\
\hline
\end{tabular}

VM18: Out-Of-Plane Bending of a Curve Bar

\begin{tabular}{|c|c|c|c|c|}
\hline & Manual & Pentium 90 & Ratio & Notes \\
\hline \hline$\delta$, in & -2.650 & -2.64973 & 1.000 & UZ @ 2 \\
\hline$\sigma_{\text {Bend, }}$ psi & $6,366$. & 6366.2 & 1.000 & SAXL O O O (Element Sol.) \\
\hline$\tau$, psi & $-3,183$. & -3183.1 & 1.000 & SXH Q 0 (Element Sol.) \\
\hline
\end{tabular}

Notes:

1. $\sigma_{\text {Bend }}$ from max. $S A X L$ at $0^{\circ}$ angle location in element solution.

2. $\tau$ from $S X H$ at $0^{\circ}$ angle location in element solution

VM19: Propped Cantilever Under Gravity Loading

\begin{tabular}{|c|c|c|c|c|}
\hline & Manual & Pentium 90 & Ratio & Notes \\
\hline$\delta$, in (Node 2) & -0.01528 & -0.015277 & 1.000 & UY @ 2 \\
\hline$\theta$, radians & $-5.877 \mathrm{E}-04$ & $-5.877 \mathrm{E}-04$ & 1.000 & ROTZ @ 1 \\
\hline$\sigma_{\text {Bend, }}, \mathrm{psi}$ & 594.92 & 594.92 & 1.000 & EL=1, SBYB (Max.) \\
\hline$M_{\mathrm{B}}$, in-lb & $-1,410.4$ & -1410.36 & 1.000 & MZ \& Node 3 (Reaction) \\
\hline$R_{\mathrm{A}}, \mathrm{b}$ & 42.311 & 42.3108 & 1.000 & FY @ Node 1 (Reaction) \\
\hline$R_{\mathrm{B}}, \mathrm{lb}$ & 70.518 & 70.5180 & 1.000 & FY @ Node 3 (Reaction) \\
\hline
\end{tabular}

VM20: Cylindrical Membrane Under Pressure

\begin{tabular}{|c|c|c|c|c|}
\hline & Manual & Pentium 90 & Ratio & Notes \\
\hline$\sigma_{1}, \mathrm{psi}$ & 15,000 & 15,000 & 1.000 & EL $=1, \mathrm{~S}(2)$ \\
\hline$\sigma_{2}, \mathrm{psi}$ & $29,886$. & 29,886 & 1.000 & EL=1, S(1) \\
\hline
\end{tabular}

VM21: Tie Rod with Lateral Loading

\begin{tabular}{|c|c|c|c|c|}
\hline $\mathrm{F} !=0$ (stiffened) & Manual & Pentium 90 & Ratio & LS=1, Substep=4 \\
\hline$Z_{\max }$ in & -0.19946 & -0.19946 & 1.000 & POST1, UZ Q 5 \\
\hline 0, rad & $3.235 E-03$ & 0.0032353 & 1.000 & POST1, ROTY Q 1 \\
\hline$M_{\max }$, in-16 & $-4,580.1$ & -4580.1 & 1.000 & POST1, MY Q 5 \\
\hline
\end{tabular}

\begin{tabular}{|c|c|c|c|c|}
\hline$F=0$ (unstiffened) & Manual & Pentium 90 & Ratio & LS=1, Substep $=1$ \\
\hline$Z_{\max }$ in & -0.38241 & -0.38241 & 1.000 & POST1 UZ @ 5 \\
\hline$\theta$, rad & $6.119 \mathrm{E}-03$ & $6.119 \mathrm{E}-03$ & 1.000 & POST1 ROTY @ 1 \\
\hline$M_{\max }$, in-lb & $-8,962.6$ & -8962.6 & 1.000 & POST1 MY @ 5 \\
\hline
\end{tabular}




\section{Table 3}

\section{Comparison of Calculated and Published Results}

VM22: Small Deflection of a Belleville Spring

\begin{tabular}{|c|c|c|c|c|}
\hline \hline & Manual & Pentium 90 & Ratio & Notes \\
\hline \hline$y$, in & -0.00284 & -0.00284 & 1.000 & UY Q 1 \\
\hline
\end{tabular}

VM23: Thermal Stresses in a Plate

\begin{tabular}{|c|c|c|c|c|}
\hline & Manual & Pentium 90 & Ratio & Notes \\
\hline \hline $\mathrm{M}, \mathrm{lb}-\mathrm{in} / \mathrm{in}$ & -625. & -625.00 & 1.000 & $\mathrm{Mx}, \mathrm{My}(\mathrm{EL}=1, \mathrm{El}$. Solution) \\
\hline$\sigma_{\max }, \mathrm{psi}$ & $-15,000$. & $-15,000$ & 1.000 & $\mathrm{EL}=1, \mathrm{~S}(\mathrm{X}) \& \mathrm{~S}(\mathrm{Y})$ TOP \\
\hline
\end{tabular}

VM24: Plastic Hinge in a Rectangular Beam

\begin{tabular}{|c|c|c|c|c|}
\hline $\mathrm{M}_{\mathrm{yp}}$ & Manual & Pentium 90 & Ratio & Notes \\
\hline \hline 1 & Fully Elastic & 0 & $\mathrm{~N} / \mathrm{A}$ & POST26, EPPLAXL \\
(EPPLAXL = Plastic Strain) \\
\hline 1.1666 & Elastic-Flastic & $3.20 \mathrm{E}-04$ & $\mathrm{~N} / \mathrm{A}$ & \\
\hline 1.3333 & Elastic-Plastic & $6.40 \mathrm{E}-04$ & $\mathrm{~N} / \mathrm{A}$ & \\
\hline $1.5^{* *}$ & Fully Plastic & 397 & $\mathrm{~N} / \mathrm{A}$ & \\
\hline
\end{tabular}

* Solution does not converge, indicates structure has collapsed

VM25: Stresses in a Long Cylinder Load Step 1

\begin{tabular}{|c|c|c|c|c|}
\hline$P=30,000$ psi & Manual & Pentium 90 & Ratio & Notes \\
\hline$\delta_{r}$, in $(r=4$ in $)$ & 0.0079111 & 0.007911 & 1.000 & $\mathrm{UX} @ 1 \& 2$, DOF Solution \\
\hline$s_{r} p s i(r=4$ in $)$ & $-30,000$ & $-30,000$ & 1.000 & Element Sol., EL=1, S(PER), Face |-J \\
\hline$s_{r}, p s i(r=6$ in $)$ & $-7,845$ & $-7,845$ & 1.000 & Element Sol., $E L=4, S(X)$ \\
\hline$s_{r}, p s i(r=8$ in $)$ & 0 & 0 & na & Element Sol., EL=7, S(PER), Face K-L \\
\hline$s_{t}, p s i(r=4$ in $)$ & 46,636 & 46,636 & 1.000 & Element Sol., EL=1, S(Z), Face l-J \\
\hline$s_{t}$ psi $(r=6$ in) & 27,869 & 27,869 & 1.000 & Element Sol., $E L=4, S(Z)$ \\
\hline$s_{t}$ psi $(r=8 \mathrm{in})$ & 19,690 & 19,690 & 1.000 & Element Sol., EL=7, S(Z), Face K-L \\
\hline
\end{tabular}

Load Step 2

\begin{tabular}{|c|c|c|c|c|}
\hline$w=1000 \mathrm{rad} / \mathrm{sec}$ & Manual & Pentium 90 & Ratio & Notes \\
\hline$s_{r}, p s i(r=4 \mathrm{in})$ & 0 & 0 & $n / a$ & Element Sol, EL=1, S(PER), Face lv \\
\hline$s_{t}, p s i(r=4$ in $)$ & 38,885 & 38,885 & 1.000 & Element Sol., EL=1. S(Z), Face IJ \\
\hline$s_{r}, p s i(r=5.43$ in $)$ & 4,846 & 4,846 & 1.000 & Element Sol., $E L=3, S(X)$ \\
\hline$s_{t}, p s i(r=5.43 \mathrm{in})$ & 29,778 & 29,778 & 1.000 & Element Sol., $E L=3, S(Z)$ \\
\hline
\end{tabular}


Software Verification and Validation Report

ANSYS Revision 5.0A, Version: PC 386/486
HNF-SD-SNF-CSWD-005, Rev. 0

Document Number: QA040.216 (P90)

Revision 0

Page 20 of 95

Table 3

Comparison of Calculated and Published Results

VM26: Large Deflection of a Cantilever

\begin{tabular}{|c|c|c|c|c|}
\hline (From POST1) & Manual & Pentium 90 & Ratio & Notes \\
\hline UX, node 4 & -2.9 & -2.8678 & 1.011 & POST1, UX @ 4 \\
\hline UZ, node 4 & -6.8 & -6.818 & 0.997 & POST1, UZ @ 4 \\
\hline ROTY, node 4 & 1.3 & 1.303 & 0.998 & POST1, ROTY @ 4 \\
\hline$\sigma_{x}$, node 1 & 95.56 & 95.56 & 1.000 & POST1, SX @ 1 \\
\hline
\end{tabular}

VM27: Thermal Expansion to Close a Gap

\begin{tabular}{|c|c|c|c|c|}
\hline 2-D Analysis (LINK1) & Manual & Pentium 90 & Ratio & Notes \\
\hline$\sigma$, psi & $-6,125$ & -6124.8 & 1.000 & EL=1, SAXL \\
\hline$\varepsilon_{\text {Thermat }}$ & 0.00125 & 0.001250 & 1.000 & EL=1, EPTHAXL \\
\hline
\end{tabular}

\begin{tabular}{|c|c|c|c|c|}
\hline 3-D Analysis (LINK8) & Manual & Pentium 90 & Ratio & \\
\hline$\sigma, \mathrm{psi}$ & $-6,125$. & -6124.8 & 1.000 & EL=1, SAXL \\
\hline$\varepsilon_{\text {Thermal }}$ & 0.00125 & 0.001250 & 1.000 & EL=1, EPTHAXL \\
\hline
\end{tabular}

VM28: Transient Heat Transfer in an Infinite Slab

\begin{tabular}{|c|c|c|c|c|}
\hline Temperature, ${ }^{\circ} \mathrm{C}$ & Manual & Pentium 90 & Ratio & Notes \\
\hline$Y=.9777$ (node 11) & 141 & 140.7 & 1.002 & POST26, T11 Max. \\
\hline$Y=.9141$ (node 9) & 99.1 & 99.10 & 1.000 & POST26, T9 Max. \\
\hline$Y=.8134$ (node 7) & 51.7 & 51.67 & 1.001 & POST26, T7 Max. \\
\hline$Y=.6538$ (node 5) & 14 & 14.03 & 0.998 & POST26, T5 Max. \\
\hline
\end{tabular}

VM29: Friction on a Support Block

\begin{tabular}{|c|c|c|c|c|}
\hline Load Step 1 & Manual & Pentium 90 & Ratio & Notes \\
\hline Status & Sticking & STAT $=1$ & $\mathrm{n} / \mathrm{a}$ & STAT=1, No Sliding \\
\hline$F_{n}, \mathrm{lb}$ & -95.942 & -95.942 & 1.000 & Element Solution \\
\hline$F_{\mathrm{s}}, \mathrm{lb}$ & 28.783 & 28.783 & 1.000 & Element Solution \\
\hline
\end{tabular}

\begin{tabular}{|c|c|c|c|c|}
\hline Load Step 2 & Manual & Pentium 90 & Ratio & Notes \\
\hline Status & Sliding & STAT $=2$ & $\mathrm{n} / \mathrm{a}$ & STAT=2, Sliding \\
\hline $\mathrm{F}_{\mathrm{n}} \mathrm{l} \mathrm{b}$ & -95.942 & -95.942 & 1.000 & Element Solution \\
\hline $\mathrm{F}_{\mathrm{s}} \mathrm{l} \mathrm{b}$ & 28.783 & 28.783 & 1.000 & Element Solution \\
\hline
\end{tabular}

VM30: Pin-Jointed Support Truss

\begin{tabular}{|c|c|c|c|c|}
\hline & Manual & Pentium 90 & Ratio & Notes \\
\hline$\sigma_{\text {element 1, psi }}$ & 10.4 & 10.393 & 1.001 & EL=1, MFORX \\
\hline$\sigma_{\text {element 2, psi }}$ & 31.2 & 31.180 & 1.001 & EL $=2$, MFORX \\
\hline$\sigma_{\text {element 3, psi }}$ & 22.9 & 22.906 & 1.000 & EL=3, MFORX \\
\hline
\end{tabular}


Software Verification and Validation Report ANSYS Revision 5.0A, Version: PC 386/486
HNF-SD-SNF-CSWD-005, Rey 0 Document Number: QA040.216 (P90)

Revision 0

Page 21 of 95

Table 3

Comparison of Calculated and Published Results

VM31: Cable Supporting Hanging Loads

\begin{tabular}{|c|c|c|c|c|}
\hline- & Manual & Pentium 90 & Ratio & Notes \\
\hline$A_{x}$ kips & -17.997 & -17.9966 & 1.000 & Reaction N Node 1 \\
\hline$A_{y}$, kips & 5.0009 & 5.00088 & 1.000 & Reaction @ Node 1 \\
\hline T, kips & 24.756 & 24.756 & 1.000 & EL =4, MFORX \\
\hline
\end{tabular}

VM32: Thermal Stresses in a Long Cylinder

\begin{tabular}{|c|c|c|c|c|}
\hline Thermal Analysis & Manual & Pentium 90 & Ratio & Notes \\
\hline $\mathrm{T},{ }^{\circ} \mathrm{C}$ at $\mathrm{X}=.1875 \mathrm{in}$ & -1.0000 & -1.0000 & 1.000 & L.S. 1 , Node 1 \\
\hline $\mathrm{T},{ }^{\circ} \mathrm{C}$ at $\mathrm{X}=.2788 \mathrm{in}$ & -0.67061 & -0.67061 & 1.000 & L.S. 1, Node 3 \\
\hline$T,{ }^{\circ} \mathrm{C}$ at $\mathrm{X}=.625 \mathrm{in}$ & 0.0000 & 0.0000 & $\mathrm{n} / \mathrm{a}$ & L.S. 1 , Node 18 \\
\hline \multicolumn{5}{|l|}{ Static Analysis } \\
\hline$\sigma_{2}$, psi $(x=.1875 \mathrm{in})$ & 419.59 & 419.594 & 1.000 & $E L=1, S(P A R)$ \\
\hline$\sigma_{t}, p s i(x=.1875 \mathrm{in})$ & 420.51 & 420.511 & 1.000 & $E L=1, S(Z)$ \\
\hline$\sigma_{a}, p s i(x=.625$ in $)$ & -193.76 & -193.763 & 1.000 & $E L=7, S(P A R)$ \\
\hline$\sigma_{\mathrm{t}}$, psi $(\mathrm{x}=.625 \mathrm{in})$ & -189.01 & -189.011 & 1.000 & $E L=7, S(Z)$ \\
\hline
\end{tabular}

VM33: Transient Thermal Stresses in a Cylinder

\begin{tabular}{|c|r|r|r|c|}
\hline \hline$T=430$ & Manual & Pentium 90 & Ratio & \multicolumn{1}{c|}{ Notes } \\
\hline$\sigma_{Y,}$ psi $(r=b)$ & $-13,086$ & $-13,086.4$ & 1.000 & POST26, SYC $\otimes$ Time $=430$ \\
\hline$\sigma_{Y}, p s i(r=a)$ & $10,416.2$ & $10,416.2$ & 1.000 & POST26, SYB $\otimes$ Time $=430$ \\
\hline
\end{tabular}

VM34: Bending of a Tapered Beam

\begin{tabular}{|c|c|c|c|c|}
\hline SHELL63 & Manual & Pentium 90 & Ratio & Notes \\
\hline \hline$\delta$, in & -0.042667 & 0.042667 & -1.000 & UZ @ Node 1, POST1 \\
\hline$\left(\sigma_{1}\right)_{\text {max }}$ psi & 1600.4 & 1600.4 & 1.000 & Element Sol., EL=3, S(1) TOP \\
\hline
\end{tabular}

\begin{tabular}{|c|c|r|c|c|}
\hline BEAM44 & Manual & Pentium 90 & Ratio & \multicolumn{1}{c|}{ Notes } \\
\hline \hline$\delta$, in & -0.043842 & -0.043842 & 1.000 & UZ Q Node 1, POST1 \\
\hline$\left(\sigma_{1}\right)_{\max }$, psi & 1600.0 & 1600.0 & 1.000 & Element Sol., EL=1-10, SMAX \\
\hline
\end{tabular}

VM35: Bimetallic Layered Cantilever Plate w/Thermal Loading

\begin{tabular}{|l|c|c|c|c|}
\hline & Manual & Pentium 90 & Patio & Notes \\
\hline free end deflection, $\Delta z$ & 0.750 & 0.7500 & 1.000 & POST1, UZ Node 15 \\
\hline free end deflection, $\Delta x$ & 0.015 & 0.0150 & 1.000 & POST1, UX Node 15 \\
\hline fixed end top stress, $\sigma x$ & 7500 & 7500.0 & 1.000 & POST1, S(X) Nodes 1-23 \\
\hline
\end{tabular}


Software Verification and Validation Report

ANSYS Revision 5.OA, Version: PC 386/486
HNF-SD-SNF-CSWD-005, Rev. 0 Document Number: QA040.216 (P9O)

Revision 0

Page 22 of 95

Table 3

Comparison of Calculated and Published Results

VM36: Limit Moment Analysis

Load Step 1

\begin{tabular}{|c|c|c|c|c|}
\hline$P=1000$ lbs (elastic) & Manual & Pentium 90 & Ratio & Notes \\
\hline Smax, in & -0.02829 & -0.028293 & 1.000 & UZ @ Nodes $2 \& 3$ \\
\hline$R_{a}, \mathrm{bb}$ & 148.15 & 148.150 & 1.000 & FZ @ Node 1 (Reactions) \\
\hline$M_{c}$, in-lb & 27,778 & $27,777.6$ & 1.000 & MY @ Node 5 (Reactions) \\
\hline
\end{tabular}

\section{Load Step 2}

\begin{tabular}{|l|c|c|c|c|}
\hline $\mathrm{P}=1388.8 \mathrm{lb}$. & Manual & Pentium 90 & Ratio & Notes \\
\hline Hinge @ B (STAT @ EL=3) & Elastic & STAT $=1$ & N.A. & COMBIN40, STAT $=1 \rightarrow$ No Sliding \\
\hline Hinge @ C (STAT @ EL =4) & Sliding & STAT $=2$ & N.A. & COMBIN40, STAT $=2 \rightarrow$ Sliding \\
\hline
\end{tabular}

\section{Load Step 3}

\begin{tabular}{|c|c|c|c|c|}
\hline $\mathrm{P}=1390 \mathrm{lb}$ & Manual & Pentium 90 & Ratio & Notes \\
\hline Hinge@B (STAT @EL=3) & Sliding & -STAT $=2$ & N.A. & COMBIN40, STAT $=2 \rightarrow$ Sliding \\
\hline Hinge @ C (STAT @EL=4) & Sliding & STAT=2 & N.A. & COMBIN40, STAT $=2 \rightarrow$ Sliding \\
\hline
\end{tabular}

\section{VM37: Elongation of a Solid Bar}

\begin{tabular}{|c|c|c|c|c|}
\hline & Manual & Pentium 90 & Ratio & Notes \\
\hline$\delta$, in & 0.004757 & 0.0047570 & 1.000 & UY @ Node 8 \\
\hline$\sigma_{y}$, psi (elem. 4) & 4,441 & 4441.1 & 1.000 & EL $=4, S(Y)$ \\
\hline
\end{tabular}

VM38: Plastic Loading of a Thick Cylinder Under Pressure

Fully Elastic, Load Step 1

\begin{tabular}{|c|c|c|c|c|}
\hline PLANE42 & Manual & Pentium 90 & Ratio & Notes \\
\hline$\sigma_{\mathrm{r}}, \mathrm{psi}(X=4.4 \mathrm{in})$ & $-9,900$ & -9900.4 & 1.000 & $\mathrm{EL}=1, \mathrm{~S}(\mathrm{X})$ \\
\hline$\sigma_{\mathrm{t}}, \mathrm{psi}(X=4.4 \mathrm{in})$ & 18,820 & 18,820 & 1.000 & $\mathrm{EL}=1, \mathrm{~S}(\mathrm{Z})$ \\
\hline$\sigma_{\mathrm{r}}, \mathrm{psi}(X=7.6 \mathrm{in})$ & -458 & -457.67 & 1.001 & $\mathrm{EL}=5, \mathrm{~S}(\mathrm{X})$ \\
\hline$\sigma_{\mathrm{t}}, \mathrm{psi}(X=7.6 \mathrm{in})$ & 9,116 & 9115.7 & 1.000 & $\mathrm{EL}=5, \mathrm{~S}(\mathrm{Z})$ \\
\hline
\end{tabular}

Fully Plastic, Load Step 2

\begin{tabular}{|c|c|c|c|c|}
\hline PLANE42 & Manual & Pentium 90 & Ratio & Notes \\
\hline$\sigma_{\text {eff. }}$ psi $(X=4.4$ in $)$ & 29,992 & 29,991 & 1.000 & EL=1, SEQV \\
\hline$\sigma_{\text {eff, }}$ psi $(X=7.6$ in $)$ & 30,000 & 30,000 & 1.000 & EL=5, SEQV \\
\hline
\end{tabular}


Software Verification and Validation Report ANSYS Revision 5.0A, Version: PC 386/486
HNF-SD-SNF-CSWD-005, Rev. 0 Document Number: QA040.216 (P9O)

Revision 0

Page 23 of 95

Table 3

Comparison of Calculated and Published Results

VM38: Plastic Loading of a Thick Cylinder Under Pressure (continued)

Fully Elastic -

\begin{tabular}{|c|c|c|c|c|}
\hline SOLID45 & Manual & Pentium 90 & Ratio & Notes \\
\hline$\sigma_{r}, p s i(X=4.4$ in $)$ & $-9,891$ & -9891.4 & 1.000 & $E L=1, S(X)$ \\
\hline$\sigma_{1}, p s i(X=4.4$ in $)$ & 18,811 & 18,811 & 1.000 & $E L=1, S(Y)$ \\
\hline$\sigma_{n}$, psi $(X=7.6 \mathrm{in})$ & -455 & -454.65 & 1.001 & $E L=5, S(X)$ \\
\hline$\sigma_{t}, p s i(X=7.6$ in $)$ & 9,113 & 9112.7 & 1.000 & $E L=5, S(Y)$ \\
\hline
\end{tabular}

Fully Plastic

\begin{tabular}{|c|c|c|c|c|}
\hline SOLID45 & Manual & Pentium 90 & Ratio & Notes \\
\hline$\sigma_{\text {eft, }}$ psi $(X=4.4 \mathrm{in})$ & 29,982 & 29,982 & 1.000 & $\mathrm{EL}=1, \mathrm{SEQV}$ \\
\hline$\sigma_{\text {eff }}, \mathrm{psi}(X=7.6 \mathrm{in})$ & 29,992 & 29,992 & 1.000 & $\mathrm{EL}=5, \mathrm{SEQV}$ \\
\hline
\end{tabular}

Note: $\sigma_{\text {eff }}$ from ouput quantity SEQV.

VM39: Bending of a Circular Plate w/Center Hole

\begin{tabular}{|c|c|c|c|c|}
\hline \hline & Manual & Pentium 90 & Ratio & Notes \\
\hline$\delta$, in & 0.049218 & 0.0492180 & 1.000 & UZ Q Nodes 7, 17 \\
\hline$\phi$, rad & -0.0045249 & -0.0045249 & 1.000 & ROTY @ Nodes 7, 17 \\
\hline
\end{tabular}

\begin{tabular}{|c|c|c|c|c|}
\hline (9) $\mathrm{X}=10.81$ in. & Manual & Pentium 90 & Ratio & Notes \\
\hline $\mathrm{M}, \mathrm{in}-\mathrm{b} / \mathrm{b} / \mathrm{in}$ & -13.675 & -13.675 & 1.000 & $\mathrm{EL}=1, \mathrm{M}(\mathrm{X})$ \\
\hline$\sigma_{\mathrm{x}}, \mathrm{psi}$ & -1312.8 & -1312.8 & 1.000 & $\mathrm{EL}=1, \mathrm{TOP}, \mathrm{S}(\mathrm{X})$ \\
\hline
\end{tabular}

\begin{tabular}{|c|c|c|c|c|}
\hline $\mathrm{X}=27.1$ in. & Manual & Pentium 90 & Ratio & Notes \\
\hline $\mathrm{M}$, in-tb/in & -10.133 & -10.133 & 1.000 & $E L=6, M(X)$ \\
\hline$\sigma_{\mathrm{x}}, \mathrm{psi}$ & -972.72 & -972.72 & 1.000 & EL $=6, \mathrm{TOP}, \mathrm{S}(\mathrm{X})$ \\
\hline
\end{tabular}

VM40: Large Deflection and Rotation of a Beam Pinned at 1 End

\begin{tabular}{|c|c|c|c|c|c|}
\hline \multicolumn{2}{|c|}{$\theta_{2}, \operatorname{deg}$} & Manual & Pentium 90 & Ratio & Notes / POST26 \\
\hline $60^{\circ}$ & $\delta_{x}$, in & -5.0 & -5.000 & 1.000 & $U X @ T=0.025(R O T Z=1.047)$ \\
\hline $90^{\circ}$ & $\delta_{y}$, in & 10.0 & 10.000 & 1.000 & UY $@ T=0.0375$ (ROTZ $=1.571)$ \\
\hline $180^{\circ}$ & $\delta_{x}$, in & -20.0 & 0.00 & 1.000 & $\mathrm{UX} @ \mathrm{~T}=0.0750(\mathrm{ROTZ}=3.142)$ \\
\hline $210^{\circ}$ & $\delta_{y}$, in & -5.0 & & 1.000 & UY Q T $=0.0875$ (ROTZ=3.665) \\
\hline $315^{\circ}$ & $\delta_{x,}$ in & -2.93 & -2.9289 & 1.000 & $U X \otimes T=0.13125(R O T Z=5.498)$ \\
\hline $360^{\circ}$ & $\delta$, in & 0.0 & $1.61 \mathrm{E}-08$ & $n / a$ & UY $\mathrm{T}=0.1500(\mathrm{ROTZ}=6.283)$ \\
\hline
\end{tabular}


Table 3

\section{Comparison of Calculated and Published Results}

VM41: Small Deflection of a Rigid Beam

\begin{tabular}{|c|c|c|c|c|}
\hline Thick Beam & Manual & Pentium 90 & Ratio & Notes \\
\hline$\delta_{x}$, in & 0.0 & 0.000 & $n / a$ & Node 3, UX \\
\hline$\delta$, in & -0.1 & -0.1000 & 1.000 & Node 3, UY \\
\hline$\theta, \mathrm{rad}$ & -0.01 & -0.0100 & 1.000 & Node 3, ROTZ \\
\hline$\sigma_{\text {bend, }}$, psi & -0.5 & -0.500 & 1.000 & $\mathrm{EL}=2, \mathrm{SBYB} B 1(\mathrm{l})$ \\
\hline
\end{tabular}

Note: $\sigma_{\text {bend }}$ considered negligibie

\begin{tabular}{|c|c|c|c|c|}
\hline Constraint Equation & Manual & Pentium 90 & Ratio & Notes \\
\hline \hline$\delta_{x}$, in & 0.0 & 0.0000 & n/a & Node 3, UX \\
\hline$\delta_{y}$, in & -0.1 & -0.1000 & 1.000 & Node 3, UY \\
\hline$\theta$, rad & -0.01 & -0.0100 & 1.000 & Node 3, ROTZ \\
\hline$\sigma_{\text {bend, }}$ psi & 0.0 & $1.56 \mathrm{E}-12$ & 0.000 & EL=2, SBYB \\
\hline
\end{tabular}

VM42: Barrel Vault Roof Under Self Weight

\begin{tabular}{|c|c|c|c|c|}
\hline & Manual & Pentium 90 & Ratio & Notes \\
\hline$U Y_{A}, m$ & -0.3053 & -0.30528 & 1.000 & POST1, Node 1, UY \\
\hline$U X_{A}, m$ & -0.1614 & -0.16141 & 1.000 & POST1, Node 1, UX \\
\hline
\end{tabular}

\begin{tabular}{|l|c|c|c|c|}
\hline$\sigma_{\mathrm{z}}$, Top $\mathrm{A}, \mathrm{Pa}$ & 215,980 & 215,980 & 1.000 & POST1, Node 1, SZ (Top) \\
\hline$\sigma_{\mathrm{z}}$, Bot $@ \mathrm{~A}, \mathrm{~Pa}$ & 342,480 & 342,480 & 1.000 & POST1, Node 1, SZ (Bot) \\
\hline$\sigma_{\theta}$, Bot $@ \mathrm{~B}, \mathrm{~Pa}$ & 190,710 & 190,710 & 1.000 & POST1, Node 2, SY (Top) \\
\hline$\sigma_{\theta}$, Top $8 \mathrm{~B}, \mathrm{~Pa}$ & $-219,410$ & $-219,410$ & 1.000 & POST1, Node 2, SY (Bot) \\
\hline
\end{tabular}

VM43: Bending of an Axisymmetric Thick Pipe

\begin{tabular}{|c|c|c|c|c|}
\hline & Manual & Pentium 90 & RATIO & Notes \\
\hline$\delta_{x}$, in $(\theta=0)$ & -0.12529 & -0.125295 & 1.000 & Node 15, UX (L.S. 1) \\
\hline$\delta_{z}$, in $(\theta=90)$ & 0.12530 & 0.125296 & 1.000 & Node 15, UZ (L.S. 1) \\
\hline$\sigma_{\max }$, psi $\left(\theta=0^{\circ}\right)$ & 2652.4 & 2652.4 & 1.000 & EL=1, S(PAR) \& Face I-J \\
\hline
\end{tabular}

VM44: Bending of an Axisymmetric Thin Pipe

\begin{tabular}{|c|c|c|c|c|}
\hline & Manual & Pentium 90 & RATIO & Notes \\
\hline$\delta_{x}$, in $(\theta=0)$ & -0.19079 & -0.19079 & 1.000 & POST1 (1st)UX @ Node 8 \\
\hline$\delta_{z}$ in $(\theta=90)$ & 0.19079 & 0.19079 & 1.000 & POST1 (2nd) UZ Node 8 \\
\hline$\sigma_{\max }$, psi $\left(\theta=0^{\circ}\right)$ & 3059.1 & 3059.1 & 1.000 & EL=1, S1 BOT (Sec. \& Node ) \\
\hline
\end{tabular}

Note: $\sigma_{\max }$ corresponds to $S 1$ BOT of element 1. 
Software Verification and Validation Report ANSYS Revision 5.0A, Version: PC 386/486
HNF-SD-SNF-CSWD-005, Rev, 0

Document Number: QA040.216 (P90)

Revision 0

Page 25 of 95

Table 3

Comparison of Calculated and Published Results

VM45: Natural Frequency of a Spring-Mass System

\begin{tabular}{|c|c|c|c|}
\hline & Manual & Pentium 90 & Ratio \\
\hline $\mathrm{f}, \mathrm{Hz}$ & 13.701 & 13.70105 & 1.000 \\
\hline
\end{tabular}

VM46: Natural Frequency of a Cantilevered Mass

\begin{tabular}{|c|c|c|c|}
\hline & Manual & Pentium 90 & Ratio \\
\hline$f, \mathrm{~Hz}$ & 33.553 & 33.5528 & 1.000 \\
\hline
\end{tabular}

VM47: Torsional Frequency of a Suspended Disk

\begin{tabular}{|c|c|c|c|}
\hline & Manual & Pentium 90 & Ratio \\
\hline $\mathrm{f}, \mathrm{Hz}$ & 0.63333 & 0.6333349 & 1.000 \\
\hline
\end{tabular}

VM48: Natural Frequency of a Motor Generator

\begin{tabular}{|c|c|c|c|}
\hline & Manual & Pentium 90 & Ratio \\
\hline $\mathrm{f}, \mathrm{Hz}$ & 48.781 & 48.7806 & 1.000 \\
\hline
\end{tabular}

VM49: Fundamental Frequency of a Bar-Mass System

\begin{tabular}{|c|c|c|c|c|}
\hline & Manual & Pentium 90 & Ratio & Notes \\
\hline Bar w/mass: $f, \mathrm{~Hz}$ & 12.508 & 12.5076 & 1.000 & \\
\hline Bar w/o mass: $\mathrm{f}, \mathrm{Hz}$ & 13.701 & 13.7011 & 1.000 & See Problem VM45 \\
\hline
\end{tabular}

VM50: Fundamental Frequency of a Simply Supported Beam

\begin{tabular}{|c|c|c|c|c|}
\hline & Manual & Pentium 90 & Ratio & Notes \\
\hline f. Hz & 28.767 & 28.7670 & 1.000 & Mode 1 \\
\hline
\end{tabular}

VM51: Natural Frequencies of a Cantilever Beam

\begin{tabular}{|c|c|c|c|c|}
\hline & Manual & Pentium 90 & Ratio & Notes \\
\hline$f_{1}, \mathrm{~Hz}$ & 10.246 & 10.2465 & 1.000 & \\
\hline $\mathrm{f}_{2,} \mathrm{~Hz}$ & 64.183 & 64.1827 & 1.000 & \\
\hline $\mathrm{f}_{3}, \mathrm{~Hz}$ & 179.79 & 179.787 & 1.000 & \\
\hline
\end{tabular}

VM52: Automobile Suspension System Vibration

\begin{tabular}{|c|c|c|c|}
\hline & Manual & Pentium 90 & Ratio \\
\hline $\mathrm{f}_{i}, \mathrm{~Hz}$ & 1.0981 & 1.09807 & 1.000 \\
\hline $\mathrm{f}_{2}, \mathrm{~Hz}$ & 1.4406 & 1.44056 & 1.000 \\
\hline
\end{tabular}


Table 3

Comparison of Calculated and Published Results

VM53: Vibration of a String Under Tension

\begin{tabular}{|c|c|c|c|c|}
\hline Static & Manual & Pentium 90 & Ratio & Notes \\
\hline \hline F, ib & 500 & 500.00 & 1.000 & MFORX (Element Sol.) \\
\hline$\sigma$, psi & 162,970 & $162,970$. & 1.000 & SAXL (Element Sol.) \\
\hline
\end{tabular}

\begin{tabular}{|c|c|c|c|}
\hline Modal & Manual & Pentium 90 & Ratio \\
\hline \hline$f_{1}, \mathrm{~Hz}$ & 75.094 & 75.0944 & 1.000 \\
\hline$f_{2}, \mathrm{~Hz}$ & 151.29 & 151.286 & 1.000 \\
\hline $\mathrm{f}_{3}, \mathrm{~Hz}$ & 229.68 & 229.684 & 1.000 \\
\hline
\end{tabular}

VM54: Vibration of a Rotating Cantilever Blade

\begin{tabular}{|c|c|c|c|}
\hline & Manual & Pentium 90 & Ratio \\
\hline $\mathrm{f}, \mathrm{Hz}$ & 52.01 & 52.01099 & 1.000 \\
\hline
\end{tabular}

VM55: Vibration of a Stretched Circular Membrane

\begin{tabular}{|c|c|c|c|c|}
\hline \hline Static & Manual & Pentium 90 & Ratio & Notes \\
\hline \hline$\sigma_{\mathrm{r}}, \mathrm{psi}$ & 10,000 & 10,000 & 1.000 & SM, EL=1 to 9 \\
\hline
\end{tabular}

\begin{tabular}{|c|c|c|c|}
\hline Modal & Manual & Pentium 90 & Ratio \\
\hline$f_{1}, \mathrm{~Hz}$ & 94.365 & 94.3649 & 1.000 \\
\hline$f_{2}, \mathrm{~Hz}$ & 217.11 & 217.106 & 1.000 \\
\hline$f_{3}, \mathrm{~Hz}$ & 342.46 & 342.461 & 1.000 \\
\hline
\end{tabular}

VM56: Hyperelastic Thick Cylinder Under Internal Pressure

\begin{tabular}{|c|c|c|c|c|}
\hline HYPER84 & Manual & Pentium 90 & Ratio & Note \\
\hline$u_{r}$ (inner radius), in & 7.388 & 7.38758 & 1.000 & L.S.2, UX @ 1, 14 \\
\hline$\sigma_{r}$ (element 1), psi & -122.5 & -122.48 & 1.000 & EL=1, $S(X)$ \\
\hline
\end{tabular}

\begin{tabular}{|c|c|c|c|c|}
\hline HYPER86 & Manual & Pentium 90 & Ratio & Notes \\
\hline$u_{r}$ (inner radius), in & 7.175 & 7.17475 & 1.000 & L.S.2, UX @ Nodes 1,2,3,4 \\
\hline$\sigma_{r}$ (element 1), psi & -122.1 & -122.13 & 1.000 & EL=1, $S(X)$ \\
\hline
\end{tabular}

VM57: Torsional Frequecies of a Drill Pipe

\begin{tabular}{|c|c|c|c|}
\hline Pipe Elements & Manual & Pentium 90 & Ratio \\
\hline $\mathrm{f}_{1}, \mathrm{~Hz}$ & 0.3834 & 0.383392 & 1.000 \\
\hline $\mathrm{f}_{2}, \mathrm{~Hz}$ & 1.264 & 1.26386 & 1.000 \\
\hline
\end{tabular}


Software Verification and Validation Report ANSYS Revision 5.0A, Version: PC 386/486
HNF-SD-SNF-CSWD-005, Rev. 0

Document Number: QA040.216 (P90)

Revision 0

Page 27 of 95

Table 3

Comparison of Calculated and Published Results

VM57: Torsional Frequecies of a Drill Pipe (continued)

\begin{tabular}{|c|c|c|c|}
\hline Beam Elements & Manual & Pentium 90 & Ratio \\
\hline$f_{1}, \mathrm{~Hz}$ & 0.3834 & 0.383383 & 1.000 \\
\hline$f_{2}, \mathrm{~Hz}$ & 1.264 & 1.26385 & 1.000 \\
\hline
\end{tabular}

VM58: Centerline Temperature of a Heat Generating Wire

\begin{tabular}{|c|c|c|c|c|}
\hline & Manual & Pentium 90 & Ratio & Notes \\
\hline $\mathrm{T}_{\mathrm{CL}},{ }^{\circ} \mathrm{F}$ & 419.9 & 419.94 & 1.000 & POST1, Node 1 \\
\hline $\mathrm{T}_{\mathrm{s}},{ }^{\circ} \mathrm{F}$ & 417.9 & 417.85 & 1.000 & POST1, Nodes 2, 10-17 \\
\hline $\mathrm{q}, \mathrm{BTU} / \mathrm{hr}$ & 341.5 & 341.5 & 1.000 & POST1, HFLOW \\
\hline
\end{tabular}

VM59: Lateral Vibration of an Axially Loaded Bar

\begin{tabular}{|c|c|c|c|c|}
\hline Static & Manual & Pentium 90 & Ratio & Notes \\
\hline$\delta$, in & -0.026667 & -0.026667 & 1.000 & UX Max. (Node 14) \\
\hline$\sigma_{r,}$ psi & $-10,000$ & $-10,000$ & 1.000 & EL=1 to 13, SDIR \\
\hline
\end{tabular}

$F=40,000 \mathrm{lb}$.

\begin{tabular}{|c|c|c|c|}
\hline Modal & Manual & Pentium 90 & Ratio \\
\hline$f_{1}, \mathrm{~Hz}$ & 17.052 & 17.0515 & 1.000 \\
\hline $\mathrm{f}_{2}, \mathrm{~Hz}$ & 105.22 & 105.2226 & 1.000 \\
\hline $\mathrm{f}_{3}, \mathrm{~Hz}$ & 248.88 & 248.877 & 1.000 \\
\hline
\end{tabular}

$F=0 \mathrm{lb}$.

\begin{tabular}{|c|c|c|c|c|}
\hline Modal & Manual & Pentium 90 & Ratio & Notes \\
\hline$f_{1}, \mathrm{~Hz}$ & 28.7670 & 28.7670 & 1.000 & See Problem VM50 \\
\hline
\end{tabular}

VM60: Natural Freq. of a Cross-F'ly Laminated Spherical Shell

\begin{tabular}{|c|c|c|c|}
\hline & Manual & Pentium 90 & Ratio \\
\hline$f_{1}, \mathrm{~Hz}$ & 0.73149 & 0.731493 & 1.000 \\
\hline
\end{tabular}

VM61: Longitudinal Vibration of a Free-Free Rod

\begin{tabular}{|c|c|c|c|}
\hline & Manual & Pentium 90 & Ratio \\
\hline$f_{1}, \mathrm{~Hz}$ (rigid body) & 0.0 & 0.000 & $\mathrm{n} / \mathrm{a}$ \\
\hline $\mathrm{f}_{2}, \mathrm{~Hz}$ & 127.13 & 127.132 & 1.000 \\
\hline $\mathrm{f}_{3}, \mathrm{~Hz}$ & 256.86 & 256.859 & 1.000 \\
\hline
\end{tabular}


Table 3

Comparison of Calculated and Published Results

VM62: Vibration of a Wedge

\begin{tabular}{|c|c|c|c|}
\hline Plate Elements & Manual & Pentium 90 & Ratio \\
\hline$f_{1}, \mathrm{~Hz}$ & 261.11 & 261.111 & 1.000 \\
\hline
\end{tabular}

\begin{tabular}{|c|c|c|c|}
\hline Shell Elements & Manual & Pentium 90 & Ratio \\
\hline$f_{1}, \mathrm{~Hz}$ & 261.11 & 261.111 & 1.000 \\
\hline
\end{tabular}

VM63: Static Hertz Contact Problem

\begin{tabular}{|c|c|c|c|c|}
\hline Plate Elements & Manual & Pentium 90 & Ratio & Notes \\
\hline a. mm & 1.0110 & 1.0109 & 1.000 & POST1 Parameter AACT \\
\hline
\end{tabular}

VM64: Thermal Expansion to Close a Gap

\begin{tabular}{|c|c|c|c|c|}
\hline Static & Manual & Pentium 90 & Ratio & Notes \\
\hline$\sigma_{x}$, psi & $-13,125$ & $-13,125$ & 1.000 & EL=3, SX \\
\hline$\sigma_{y}$, psi & $-6,125$ & $-6,125$ & 1.000 & EL=3, SY \\
\hline
\end{tabular}

VM65: Trans. Response of a Ball Impacting a Flexible Surface

\begin{tabular}{|c|c|c|c|c|}
\hline At Impact & Manual & Pentium 90 & Ratio & Notes \\
\hline Time, sec & 0.072 & 0.072 & n/a & \\
\hline y displacement, in & -0.9991 & -0.999137 & 1.000 & POST26 @ T=.072 sec. \\
\hline y velocity, in/sec & -27.76 & -27.7594 & 1.000 & POST26 @ T=.072 sec. \\
\hline kinetic energy, ib-in & 192.65 & 192.646 & 1.000 & POST26 @ T=.072 sec. \\
\hline
\end{tabular}

\begin{tabular}{|c|c|c|c|c|}
\hline At "zero" velocity & Manual & Pentium 90 & Ratio & Notes \\
\hline max. y displacement & -1.5503 & -1.55028 & 1.000 & Velocity Sign Change \\
POST 26 @ T=.100
\end{tabular}

VM66: Vibration of a Flat Plate

\begin{tabular}{|c|c|c|c|}
\hline & Manual & Pentium 90 & Ratio \\
\hline$f_{1}, \mathrm{~Hz}$ & 128.43 & 128.4255 & 1.000 \\
\hline
\end{tabular}

VM67: Radial Vibrations of a Circular Ring

\begin{tabular}{|c|c|c|c|c|}
\hline & Manual & Pentium 90 & Ratio & Notes \\
\hline$f_{0}, \mathrm{~Hz}$ & 3226.4 & 3226.408 & 1.000 & Run 1 \\
\hline $\mathrm{f}_{1}, \mathrm{~Hz}$ & 12.496 & 12.4957 & 1.000 & Run 2 \\
\hline
\end{tabular}


HNF-SD-SNF-CSWD-005, Rev. 0

Software Verification and Validation Report

Document Number: QA040.216 (P9O)

ANSYS Revision 5.0A, Version: PC 386/486

Revision 0

Page 29 of 95

Table 3

Comparison of Calculated and Published Results

VM68: PSD Response of a 2-DOF Spring-Mass System

\begin{tabular}{|c|c|c|c|c|}
\hline Modal & Manual & Pentium 90 & Ratio & Notes \\
\hline$f_{1}, \mathrm{~Hz}$ & 20.57 & 20.5721 & 1.000 & \\
\hline $\mathrm{f}_{2}, \mathrm{~Hz}$ & 64.88 & 64.8846 & 1.000 & \\
\hline Mass $1,1 \sigma$ Std Dev. & 9.059 & 9.0587 & 1.000 & POST1, UX@ Node 2 \\
\hline Mass $2,1 \sigma$ Std Dev. & 10.63 & 10.633 & 1.000 & POST1, UX@ Node 3 \\
\hline
\end{tabular}

VM69: Seismic Response

\begin{tabular}{|c|c|c|c|c|}
\hline Modal & Manual & Pentium 90 & Ratio & Notes \\
\hline$f, \mathrm{~Hz}$ & 0.5000 & 0.5 & 1.000 & \\
\hline$A_{\theta}$, in & 1.0200 & 1.0200 & 1.000 & POST1, UX @ Node 2 \\
\hline
\end{tabular}

VM70: Seismic Response of a Beam Structure

\begin{tabular}{|c|c|c|c|c|}
\hline Modal & Manual & Pentium 90 & Ratio & Notes \\
\hline $\mathrm{f}_{1}, \mathrm{~Hz}$ & 6.0974 & 6.097398 & 1.000 & Mode 1 \\
\hline$\delta$, in & 0.55301 & 0.55301 & 1.000 & POST1, UY @ Node 6 \\
\hline$\sigma_{\text {max }}$ psi & 20,156 & 20,156 & 1.000 & POST1, EL=4, SBYT(J) \\
\hline
\end{tabular}

VM71: Transient Response of a Spring-Mass-Damper System

\begin{tabular}{|c|c|c|c|c|}
\hline$t=0.09$ seconds & Manual & Pentium 90 & Ratio & Notes \\
\hline$u$, in (for $\zeta=2.0$ ) & 0.47637 & 0.476369 & 1.000 & POST26, Node 1 \\
\hline$u$, in (for $\zeta=1.0$ ) & 0.19245 & 0.192447 & 1.000 & POST26, Node 3 \\
\hline$u$, in (for $\zeta=0.2$ ) & -0.51951 & -0.519509 & 1.000 & POST26, Node 5 \\
\hline$u$, in (for $\zeta=0.0$ ) & -0.99498 & -0.994977 & 1.000 & POST26, Node 7 \\
\hline
\end{tabular}

VM72: Logarithmic Decrement

\begin{tabular}{|c|c|c|c|c|}
\hline Amplitude/Time & Manual & Pentium 90 & Ratio & Notes \\
\hline Peak 1-Amplitude & 1.00000 & 1.00000 & 1.000 & Load Step 1 - Initial \\
\hline Peak 1 - Time (Sec.) & 0.00000 & 0.00000 & $\mathrm{n} / \mathrm{a}$ & Conditions \\
\hline Peak 2 - Amplitude & 0.64981 & 0.649805 & 1.000 & Load Step 2 \\
\hline Peak 2 - Time (Sec.) & 0.18600 & 0.18600 & 1.000 & \\
\hline Peak 3-Amplitude & 0.42306 & 0.423056 & 1.000 & \\
\hline Peak 3-Time (Sec.) & 0.37200 & 0.37200 & 1.000 & \\
\hline Peak 4 - Amplitude & 0.27525 & 0.275246 & 1.000 & \\
\hline Peak 4 - Time (Sec.) & 0.55800 & 0.55800 & 1.000 & \\
\hline
\end{tabular}


Software Verification and Validation Report ANSYS Revision 5.0A, Version: PC $386 / 486$
HNF-SD-SNF-CSWD-005, Rev. 0 Document Number: QA040.216 (PSO)

Revision 0

Page 30 of 95

Table 3

Comparison of Calculated and Published Results

VM73: Free Vibration with Coulomb Damping

\begin{tabular}{|c|r|r|r|r|}
\hline & Manual & Pentium 90 & Ratio & Notes \\
\hline$u_{1}$ in $(t=0.09 \mathrm{sec})$ & 0.87204 & 0.872044 & 1.000 & POST26 \\
\hline$u_{\text {, in }}(t=0.102 \mathrm{sec})$ & 0.83019 & 0.830186 & 1.000 & POST26 \\
\hline$u$, in $(t=0.183 \mathrm{sec})$ & -0.74875 & -0.748749 & 1.000 & POST26 \\
\hline
\end{tabular}

VM74: Transient Response to an Impulsive Excitation

\begin{tabular}{|c|c|c|c|c|}
\hline Time $=0.08 \mathrm{~s} / \zeta=0.0$ & Manual & Pentium 90 & Ratio & Notes \\
\hline$y_{\max }$, in & 0.99896 & 0.998964 & 1.000 & UY @ Node 2 (Time $=.080$ ) \\
\hline
\end{tabular}

\begin{tabular}{|c|r|r|r|c|}
\hline Time $=0.1$ & Manual & Pentium 90 & Ratio & Notes \\
\hline$y$, in $($ for $\zeta=0.0)$ & 0.90857 & 0.908572 & 1.000 & UY @ Node 2 (Time $=.1025)$ \\
\hline$y$, in $($ for $\zeta=0.070)$ & 0.34185 & 0.341847 & 1.000 & UY @ Node 4 (Time $=.1025)$ \\
\hline
\end{tabular}

Based on time $=.1+.0025$ seconds to account for impulse duration

VM75: Transient response to a Step Excitiation

\begin{tabular}{|c|r|r|r|r|}
\hline Time $=0.1575 \mathrm{sec}$ & Manual & Pentium 90 & Ratio & Notes \\
\hline $\mathrm{u}_{\max }$ in & 1.9992 & 1.99915 & 1.000 & UY @ 2, T=.1575 \\
\hline
\end{tabular}

\begin{tabular}{|c|r|r|r|r|}
\hline Time $=0.20 \mathrm{sec}$ & Manual & Pentium 90 & Ratio & \\
\hline$u$, in $($ for $\zeta=0.0)$ & 1.6723 & 1.67233 & 1.000 & UY @ 2 (POST26, Time $=.20$ ) \\
\hline$u$, in $($ for $\zeta=0.5)$ & 1.1544 & 1.15439 & 1.000 & UY @ 4 (POST26, Time $=.20)$ \\
\hline
\end{tabular}

VM76: Harmonic response a a Guitar String

\begin{tabular}{|c|c|c|c|c|}
\hline MODAL & Manual & Pentium 90 & Ratio & \\
\hline $\mathrm{f}, \mathrm{Hz}$ & 322.3 & 322.335 & 1.000 & 2nd Analysis \\
\hline
\end{tabular}

\begin{tabular}{|c|c|c|c|c|}
\hline POST26 & \multicolumn{2}{|c|}{ Response Frequency } & Ratio & Pentium 90 Frequency \\
\hline$f_{1},(322.2 \mathrm{~Hz})$ & $320<f<328$ & 320 & N.A. & $322.335 \mathrm{~Hz}$ \\
\hline$f_{2},(644.4 \mathrm{~Hz})$ & No Response & - & N.A. & $645.554 \mathrm{~Hz}$ \\
\hline$f_{3},(966.6 \mathrm{~Hz})$ & $968<<976$ & 968 & N.A. & $970.543 \mathrm{~Hz}$ \\
\hline$f_{4},(1288.8 \mathrm{~Hz})$ & No Response & - & N.A. & $1298.19 \mathrm{~Hz}$ \\
\hline$f_{5},(1611.0 \mathrm{~Hz})$ & $1624<<1632$ & 1632 & N.A. & $1629.4 \mathrm{~Hz}$ \\
\hline$f_{6},(1933.2 \mathrm{~Hz})$ & No Response & - & N.A. & $1724.83 \mathrm{~Hz}$ \\
\hline
\end{tabular}

* Response Frequencies from peaks in |UY| (Real Displacement) in POST26 
Software Verification and Validation Report ANSYS Revision 5.OA, Version: PC $386 / 486$
HNF-SD-SNF-CSWD-005, Rev. 0

Document Number: QA040.216 (P90)

Revision 0

Page 31 of 95

Table 3

Comparison of Calculated and Published Results

VM77: Trans. Response to a Constant Force w/Finite Rise Time

\begin{tabular}{|c|r|r|r|c|}
\hline Transiēnt & Manual & Pentium 90 & Ratio & \multicolumn{1}{c|}{ Notes } \\
\hline$t\left(@ Y_{\max }\right)$, sec & 0.092 & 0.0920 & 1.000 & Time @ L.S. 3, Substep 5 \\
\hline$y_{\max }$ in & 0.335 & 0.335459 & 0.999 & UY @ Node 2 \\
\hline
\end{tabular}

\begin{tabular}{|c|c|c|c|c|}
\hline EXPANSION PASS & Manual & Pentium 90 & Ratio & \\
\hline S $_{\text {bend, }}$ ksi & -18.9 & -18.8700 & 1.002 & SBYB, EL $=1(\mathrm{~J})$ \& EL=2(I) \\
\hline
\end{tabular}

VM78: Transverse Shear Stresses in a Cantilever Beam

\begin{tabular}{|c|c|c|c|c|}
\hline & Manual & Pentium 90 & Ratio & \\
\hline$\sigma_{x z}$, psi $(z=h / 2)$ & 0.0 & 0.0 & $\mathrm{n} / \mathrm{a}$ & $\begin{array}{l}\text { SXZ, Layer } 1, \text { BOT or Layer } 4 \text {, } \\
\text { TOP (any element) }\end{array}$ \\
\hline$\sigma_{x z}$, psi $(z=h / 4)$ & 5625.0 & 5625.0 & 1.000 & IL.SXZ for Layers $1-2$ (or $3-4)$ \\
\hline$\sigma_{x z}, p s i(z=0)$ & 7500.0 & 7500.0 & 1.000 & fLSXZ for Layers $2-3$ (also lLMX) \\
\hline $\mathrm{FC}_{\max }(\mathrm{FCMX})$ & 225.0 & 225.0 & 1.000 & POST1 Parameter \\
\hline
\end{tabular}

VM79: Transient Response of a Bilinear Spring Assembly

\begin{tabular}{|c|c|c|c|c|}
\hline Time $=0.09 \mathrm{sec}$ & Manual & Pentium 90 & Ratio & Notes \\
\hline $\mathrm{y}_{\max }$ in & -1.0405 & -1.04047 & 1.000 & Load Step 3, Cum. Iter. 37 \\
\hline
\end{tabular}

\begin{tabular}{|c|c|c|c|c|}
\hline Displacements & Manual & Pentium 90 & Ratio & Notes \\
\hline Time $=.040$ & -0.68122 & -0.681218 & 1.00 & UY @ 2 (L.S. 3, Substep 15) \\
\hline Time $=.070$ & -0.98672 & -0.986716 & 1.00 & UY @ 2 (L.S. 3, Substep 27) \\
\hline Time $=.085$ & -1.0383 & -1.03828 & 1.00 & UY @ 2 (L.S. 3, Substep 33) \\
\hline Time $=.105$ & -1.0020 & -1.00203 & 1.00 & UY @ 2 (L.S. 3, Substep 41) \\
\hline
\end{tabular}

VM80: Plastic Response to a Sucidenly Applied Constant Force

\begin{tabular}{|c|r|r|r|l|}
\hline & Manual & Pentium 90 & Ratio & \multicolumn{1}{c|}{ Notes } \\
\hline$y_{\max }$ in & 0.804 & 0.804290 & 1.000 & POST26, UY \\
\hline Time, sec & 0.0680 & 0.0680 & 1.000 & POST26, UY \\
\hline
\end{tabular}

\begin{tabular}{|c|r|r|r|l|}
\hline & Manual & Pentium 90 & Ratio & \multicolumn{1}{c|}{ Notes } \\
\hline$y_{\min }$, in & 0.438 & 0.437734 & 1.001 & POST26, UY \\
\hline Time,sec & 0.122 & 0.1220 & 1.000 & POST26, UY \\
\hline
\end{tabular}


Table 3

\section{Comparison of Calculated and Published Results}

VM81: Transient Response of a Drop Container

At Impact:

\begin{tabular}{|c|c|c|c|c|}
\hline Full Transient Dynamic & Manual & Pentium 90 & Ratio & Notes \\
\hline time, sec & 0.072 & 0.072 & n/a & \\
\hline$y$, in & -1.0005 & -1.00051 & 1.000 & POST26, UY @ T=.072 \\
\hline vel, in/sec & -27.78 & -27.7767 & 1.000 & POST26, VEL_1UY @ T $=.072$ \\
\hline
\end{tabular}

At Zero Velocity (theoretical $Y_{\max }:=-1.5506 @ t=100366$ )

\begin{tabular}{|c|c|c|c|c|}
\hline Full Transient Dynamic & Manual & Pentium 90 & Ratio & Notes \\
\hline$t=0.100 \mathrm{sec} / \mathrm{y}$ (in) & -1.5503 & -1.55033 & 1.000 & POST26, UY @ T=.100 \\
\hline$t=0.101 \mathrm{sec} / \mathrm{y}$ (in) & -1.5502 & -1.55024 & 1.000 & POST26, UY @ T $=.101$ \\
\hline
\end{tabular}

\section{At Impact:}

\begin{tabular}{|c|c|c|c|c|}
\hline Reduced Transient Dynamic & Manual & Pentium 90 & Ratio & Notes \\
\hline time, sec & 0.072 & 0.072 & $\mathrm{n} / \mathrm{a}$ & \\
\hline $\mathrm{y}$, in & -0.987 & -0.98685 & 1.000 & POST26, UY @ T $=.072$ \\
\hline vel, in/sec & -27.59 & -27.5936 & 1.000 & POST26,VEL_1UY @ T $=.072$ \\
\hline
\end{tabular}

At Zero Velocity (theoretical $Y_{\operatorname{mex}}=-1.5506 @ \mathrm{t}=. \mathbf{1 0 0 3 6 6}$ )

\begin{tabular}{|c|r|r|r|r|}
\hline Reduced Transient Oynamic & Manual & Pentium 90 & Ratio & \multicolumn{1}{c|}{ Notes } \\
\hline$t=0.100 \mathrm{sec} / \mathrm{y}$ (in) & -1.5495 & -1.54947 & 1.000 & POST26, UY @ T $=.100$ \\
\hline$t=0.101 \mathrm{sec} / \mathrm{y}$ (in) & -1.5503 & -1.55027 & 1.000 & POST26, UY @ T $=.101$ \\
\hline
\end{tabular}

VM82: Simply Supported Laminated Plate Under Pressure

\begin{tabular}{|c|r|r|r|r|}
\hline & Manual & Pentium 90 & Ratio & \multicolumn{1}{c|}{ Notes } \\
\hline$\delta, m$ (SHELL99) & $* 0.0692$ & 0.0691919 & 1.000 & UZ @ Node 1 \\
\hline$\delta, m$ (SOLID46) & 0.0676 & 0.067594 & 1.000 & UZ @ Node 1,51 \\
\hline
\end{tabular}

\section{VM83: Impact of a Block on a Spring Scale}

\begin{tabular}{|c|r|r|r|r|}
\hline Time $=0.689 \mathrm{sec}$ & Manual & Pentium 90 & Ratio & \multicolumn{2}{|c|}{ Notes } \\
\hline$\delta$, in & -7.641 & -7.6410 & 1.000 & POST26, UY @ 2, T=.68897 \\
\hline$y_{\text {, in }}$ & -79.44 & -79.4417 & 1.000 & POST26, UY @ 3, T=.68897 \\
\hline
\end{tabular}

\section{VM84: Displacement Propagation Along a Bar w/Free Ends}

\begin{tabular}{|c|r|r|r|r|}
\hline Time $=0.24 \mathrm{sec}$ & Manual & Pentium 90 & Ratio & \multicolumn{1}{c|}{ Notes } \\
\hline$\delta$, in & 4.8404 & 4.84035 & 1.000 & POST26, UX @ 17 \\
\hline
\end{tabular}


Table 3

Comparison of Calculated and Published Results

VM85: Transient Disp. in a Suddenly Stopped Moving Bar

\begin{tabular}{|c|r|r|r|r|}
\hline Reduced Transient Dynamic & Manual & Pentium 90 & \multicolumn{1}{c|}{ Ratio } & \multicolumn{1}{c|}{ Notes } \\
\hline $\mathrm{d}$, in $(\mathrm{t}=.0544)$ & 4.7732 & 4.7733 & 1.000 & POST26, Variable 4, REL_DISP \\
\hline $\mathrm{d}$, in $(\mathrm{t}=.0557)$ & 4.7471 & 4.74715 & 1.000 & POST26, Variable 4, REL_DISP \\
\hline
\end{tabular}

\begin{tabular}{|c|r|r|r|r|}
\hline Expansion Pass & Manual & Pentium 90 & Ratio & \\
\hline$s_{x}, p s i(t=.0557 \mathrm{sec})$ & 14804 & 14805 & 1.000 & EL $=1, S A X L$ \\
\hline
\end{tabular}

VM86: Harmonic Response of a Dynamic System

\begin{tabular}{|c|r|r|r||c|}
\hline & Manual & Pentium 90 & Ratio & \multicolumn{1}{c|}{ Notes } \\
\hline \hline$A_{0}$, in & 0.0833 & 0.083333 & 1.000 & UZ @ 2, Amplitude \\
\hline$\phi$, deg & -90.0 & -90.00007 & 1.000 & UZ \& 2, Phase \\
\hline
\end{tabular}

VM87: Equivalent Structural Damping

\begin{tabular}{|c|c|c|c|c|}
\hline & Manual & Pentium 90 & Ratio & Notes \\
\hline \hline Amp, in & 0.083333 & 0.083333 & 1.000 & UZ @ 2, Amplitude \\
\hline$\phi$, deg & -90.000 & -90.00007 & 1.000 & UZ @ 2, Phase \\
\hline
\end{tabular}

\section{VM88: Response of an Eccentric Weight Exciter}

Load Step 1

\begin{tabular}{|c|c|c|c|c|}
\hline \hline$f=f_{n}$ & Manual & Pentium 90 & Ratio & Notes \\
\hline \hline Amp, in & 0.60000 & 0.6000005 & 1.000 & UX @ Node 2 - Amplitude \\
\hline$\phi$, deg & -90.000 & -89.99989 & 1.000 & UX @ Node 2 - Phase \\
\hline
\end{tabular}

\section{Load Step 2}

\begin{tabular}{|c|r|r|c|c|}
\hline$f=100 f_{n}$ & Manual & Pentium 90 & Ratio & Notes \\
\hline Amp, in & 0.080008 & 0.080008 & 1.000 & UX @ Node 2 - Amplitude \\
\hline$\phi$, deg & -179.920 & -179.9236 & 1.000 & UX @ Node 2 - Phase \\
\hline
\end{tabular}

VM89: Natural Frequencies of a Two-Mass-Spring System

\begin{tabular}{|c|c|c|c|c|}
\hline & Manual & Pentium 90 & Ratio & Notes \\
\hline $\mathrm{f}_{1}, \mathrm{~Hz}$ & 2.5814 & 2.581395 & 1.000 & Mode 1 \\
\hline $\mathrm{f}_{2}, \mathrm{~Hz}$ & 8.3263 & 8.32630 & 1.000 & Mode 2 \\
\hline Mode 1: UX & - & 0.771864 & - & $U X \otimes 2$ \\
\hline $\mathrm{UX}_{3}$ & -- & 0.837922 & -- & $U X @ 3$ \\
\hline$(\mathrm{A} 1 / \mathrm{A} 2)_{1}{ }^{* *}$ & 0.92116 & 0.92116 & 1.000 & $\mathrm{UX}_{2} / \mathrm{UX}_{3}$ \\
\hline Mode 2: $\mathrm{UX}_{2}$ & - & 1.18500 & -- & $U X @ 2$ \\
\hline $\mathrm{UX}_{3}$ & -- & -0.545790 & $\cdots$ & $U \times \otimes 3$ \\
\hline$\left.(\mathrm{A} 1 / \mathrm{A} 2)_{2}\right)^{\star *}$ & -2.1712 & -2.1712 & 1.000 & $\mathrm{UX}_{2} / \mathrm{UX}_{3}$ \\
\hline
\end{tabular}


HNF-SD-SNF-CSWD-005, Rev. 0

Software Verification and Validation Report ANSYS Revision 5.0A, Version: PC $386 / 486$
Document Number: QA040.216 (P90)

Revision 0

Page 34 of 95

Table 3

Comparison of Calculated and Published Results

VM90: Harmonic Response of a Two-Mass Spring System

\begin{tabular}{|c|r|r|c|c|}
\hline & Manual & Pentium 90 & Ratio & Notes \\
\hline$X_{1}$, in $(f=1.5 \mathrm{~Hz})$ & 0.82272 & 0.8227180 & 1.000 & UX @ Node 2 - Amplitude \\
\hline$\phi_{1}$, in $(f=1.5 \mathrm{~Hz})$ & 0.0000 & 0 & $\mathrm{n} / \mathrm{a}$ & \\
\hline$X_{2}$, in $(f=1.5 \mathrm{~Hz})$ & 0.46274 & 0.4627382 & 1.000 & UX @ Node 3 - Amplitude \\
\hline$\phi_{2}$, in $(f=1.5 \mathrm{~Hz})$ & 0.0000 & 0 & $n / a$ & Phase \\
\hline$X_{1}$, in $(f=4.0 \mathrm{~Hz})$ & 0.51146 & 0.5114553 & 1.000 & UX @ Node 2 - Amplitude \\
\hline$\phi_{1}$, in $(f=4.0 \mathrm{~Hz})$ & 180.00 & -180.0000 & -1.000 & Phase \\
\hline$X_{2}$, in $(f=4.0 \mathrm{~Hz})$ & 1.2153 & 1.215253 & 1.000 & UX @ Node 3 - Amplitude \\
\hline$\phi_{2}$, in $(f=4.0 \mathrm{~Hz})$ & 180.00 & 180.0000 & 1.000 & Phase \\
\hline$X_{1}$, in $(f=6.5 \mathrm{~Hz})$ & 0.58512 & 0.5851174 & 1.000 & UX @ Node 2 - Amplitude \\
\hline$\phi_{1}$, in $(f=6.5 \mathrm{~Hz})$ & 180.00 & -180.0000 & -1.000 & \\
\hline$X_{2}$, in $(f=6.5 \mathrm{~Hz})$ & 0.26965 & 0.2696508 & 1.000 & UX @ Node 3 - Amplitude \\
\hline$\phi_{2}$, in $(f=6.5 \mathrm{~Hz})$ & 0.0000 & 0 & $n / a$ & \\
\hline
\end{tabular}

VM91: Large Rotation of a Swinging Pendulum

\begin{tabular}{|l|r|r|r|r|}
\hline & Manual & Pentium 90 & Ratio & POST26, UX \& UY @ 2 \\
\hline$\delta_{x}$, in $(t=\tau / 4)$, & -59.334 & -59.3336 & 1.000 & UX @ 2 $(t=.8207)$ \\
\hline$\delta_{y,}$ in $(t=\tau / 4)$ & -20.004 & -20.0037 & 1.000 & UY @ 2 $(t=.8207)$ \\
\hline$\delta_{x}$, in $(t=\tau / 2)$ & -119.88 & -119.878 & 1.000 & UX @ 2 $(t=1.6414)$ \\
\hline$\delta_{y}$, in $(t=\tau / 2)$ & -0.0907 & -0.09067 & 1.000 & UY @ 2 $(t=1.6414)$ \\
\hline$\delta_{x}$, in $(t=3 \tau / 4)$ & -61.724 & -61.7236 & 1.000 & UX @ 2 $(t=2.4621)$ \\
\hline$\delta_{y}$, in $(t=3 \tau / 4)$ & -19.993 & -19.9925 & 1.000 & UY @ 2 $(t=2.4621)$ \\
\hline$\delta_{x}$ in $(t=\tau)$ & -0.2432 & -0.243156 & 1.000 & UX @ 2 $(t=3.2828)$ \\
\hline$\delta_{x}$, in $(t=\tau)$ & -0.1921 & -0.192104 & 1.000 & UY @2 $(t=3.2828)$ \\
\hline
\end{tabular}

VM92: Insulated Wall Temperature

\begin{tabular}{|c|r|r|r|c|}
\hline & Manual & Pentium 90 & Ratio & Notes \\
\hline $\mathrm{q}^{\mathrm{q}} \mathrm{BTU} / \mathrm{hr}$ & 513 & 513.41 & 0.999 & POST1 Heat @ 1 \& 5 \\
\hline $\mathrm{T}_{\mathrm{i},}{ }^{\circ} \mathrm{F}$ (node 2) & 2957 & 2957.2 & 1.000 & POST1, Temp @ 2 \\
\hline $\mathrm{T}_{0},{ }^{\circ} \mathrm{F}$ (node 4) & 337 & 336.7 & 1.001 & POST1, Temp @ 4 \\
\hline
\end{tabular}

VM93: Temperature Dependent Conductivity

\begin{tabular}{|c|r|r|r|r|}
\hline & Manual & Pentium 90 & Ratio & \multicolumn{1}{c|}{ Notes } \\
\hline q. BTU/hr & 29.76 & 29.76 & 1.000 & Reaction Sol./El. Solution \\
\hline
\end{tabular}


Table 3

Comparison of Calculated and Published Results

VM94: Heat Generating Plate

\begin{tabular}{|c|r|r|r|c|}
\hline & Manual & Pentium 90 & Ratio & \multicolumn{1}{c|}{ Notes } \\
\hline $\mathrm{q}, \mathrm{BTU} / \mathrm{hr}$ & 2083.3 & 2083.3 & 1.000 & POST1, Node 6 \\
\hline $\mathrm{T}_{c_{1}}{ }^{\mathrm{o}} \mathrm{F}$ (node 1) & 300.0 & 300 & 1.000 & POST1, Node 3 \\
\hline
\end{tabular}

VM95: Heat Transfer from a Cooling Spine

\begin{tabular}{|c|r|r|r|l|}
\hline LINK33 \& LINK34 & \multicolumn{1}{|c|}{ Manual } & Pentium 90 & Ratio & \multicolumn{1}{c|}{ Notes } \\
\hline $\mathrm{T}_{1}{ }^{\circ} \mathrm{F}$ (node 9) & 68.618 & 68.618 & 1.000 & POST1, Node 9 \\
\hline $\mathrm{q}, \mathrm{BTU} / \mathrm{hr}$ & 17.528 & -17.528 & -1.000 & POST1, Node 1 \\
\hline
\end{tabular}

\begin{tabular}{|c|c|r|c|c|}
\hline SOLID70 & Manual & Pentium 90 & Ratio & Notes \\
\hline$T_{1}$, F (el. 8) & 68.618 & 68.618 & 1.000 & POST1, Nodes $9,19,29,39$ \\
\hline q, BTU/hr & - & -4.3821 & - & POST1, Nodes $1,11,21,31$ \\
\cline { 2 - 5 } & 17.528 & -17.5284 & -1.000 & SUM Nodes $1,11,21,31$ \\
\hline
\end{tabular}

VM96: Temperature Distribution in a Short Solid Cylinder

\begin{tabular}{|c|r|r|c|c|}
\hline At Centerline & Manual & Pentium 90 & Ratio & Notes \\
\hline$z=0.0, T\left({ }^{\circ} \mathrm{F}\right)$ & 0.0 & 0.000 & $\mathrm{n} / \mathrm{a}$ & Node 1 \\
\hline$z=1.5, T\left({ }^{\circ} \mathrm{F}\right)$ & 6.9 & 6.8943 & 1.001 & Node 39 \\
\hline$\left.z=3.0, T{ }^{\circ} \mathrm{F}\right)$ & 15.4 & 15.391 & 1.001 & Node 37 \\
\hline$z=4.5, T\left({ }^{\circ} \mathrm{F}\right)$ & 26.7 & 26.740 & 0.999 & Node 35 \\
\hline$z=6.0, T\left({ }^{\circ} \mathrm{F}\right)$ & 40.0 & 40.000 & 1.000 & Node 30 \\
\hline
\end{tabular}

VM97: Temperature Distribution Along a Straight Fin

\begin{tabular}{|c|c|c|c|c|}
\hline & Manual & Pentium 90 & Ratio & Notes \\
\hline $\bar{T}, \mathrm{~F}($ at $x / L=0.0)$ & 1100.0 & 1100.000 & 1.000 & POST1, Nodes $1 \& 21$ \\
\hline$T,{ }^{\circ} \mathrm{F}(\mathrm{at} \times / \mathrm{L}=0.1)$ & 958.0 & 957.5900 & 1.000 & POST1, Nodes 2 \& 22 \\
\hline$T_{1}{ }^{\circ} \mathrm{F}($ at $X / L=0.2)$ & 838.0 & 838.050 & 1.000 & POST1, Nodes $3 \& 23$ \\
\hline$T^{\circ} F($ at $x / L=0.3)$ & 738.0 & 738.190 & 1.000 & POST1, Nodes $4 \& 24$ \\
\hline$T,{ }^{\circ} F($ at $x / L=0.4)$ & 655.0 & 655.360 & 0.999 & POST1, Nodes 5 \& 25 \\
\hline $\mathrm{T},{ }^{\circ} \mathrm{F}($ at $\times / \mathrm{L}=0.5)$ & 587.0 & 587.330 & 0.999 & POST1, Nodes $6 \& 26$ \\
\hline $\mathrm{T}, \mathrm{F}($ at $\mathrm{X} / \mathrm{L}=0.6)$ & 532.0 & 532.290 & 0.999 & POST1, Nodes $7 \& 27$ \\
\hline$T^{\circ} \mathrm{F}($ at $\times / \mathrm{L}=0.7)$ & 489.0 & 488.780 & 1.000 & POST1, Nodes $8 \& 28$ \\
\hline$T,{ }^{\circ} F$ (at $x / L=0.8$ ) & 456.0 & 455.650 & 1.001 & POST1, Nodes $9 \& 29$ \\
\hline $\mathrm{T}^{\circ}{ }^{\circ} \mathrm{F}($ at $x / L=0.9)$ & 432.0 & 431.990 & 1.000 & POST1, Nodes $10 \& 30$ \\
\hline$T{ }^{\circ} \mathrm{F}$ (at $\left.\mathrm{X} / \mathrm{L}=1.0\right)$ & 417 & 417.19 & 1.000 & POST1, Nodes $11 \& 31$ \\
\hline \multirow[t]{2}{*}{$q, B T U / h r$} & -- & 2920.2 & - & POST1, Nodes $1 \& 21$ \\
\hline & 5840 & 5840.4 & 1.000 & SUM, Nodes 1 \& 21 \\
\hline
\end{tabular}


Table 3

\section{Comparison of Calculated and Published Results}

VM98: Temperature Distribution Along a Tapered Fin

\begin{tabular}{|c|c|c|c|c|}
\hline & Manual & Pentium 90 & Ratio & Notes \\
\hline $\mathrm{T},{ }^{\circ} \mathrm{F}$ (at $\left.\mathrm{x} / \mathrm{L}=0.0\right)$ & 1100.0 & 1100.000 & 1.000 & POST1, Nodes $1 \& 21$ \\
\hline$T,{ }^{\circ} \mathrm{F}($ at $X / L=0.1)$ & 971.0 & 970.7400 & 1.000 & POST1, Nodes $2 \& 22$ \\
\hline$T^{\circ}{ }^{\circ} \mathrm{F}$ (at $X / L=0.2$ ) & 854.0 & 853.800 & 1.000 & POST1, Nodes $3 \& 23$ \\
\hline$T,{ }^{\circ} F($ at $x / L=0.3)$ & 748.0 & 748.280 & 1.000 & POST1, Nodes $4 \& 24$ \\
\hline$T,{ }^{\circ} \mathrm{F}($ at $x / L=0.4)$ & 653.0 & 653.330 & 0.999 & POST1, Nodes $5 \& 25$ \\
\hline $\mathrm{T},{ }^{\circ} \mathrm{F}$ (at $\left.x / \mathrm{L}=0.5\right)$ & 568.0 & 568.160 & 1.000 & POST1, Nodes $6 \& 26$ \\
\hline$T^{\circ}{ }^{\circ} F($ at $x / L=0.6)$ & 492.0 & 492.010 & 1.000 & POST1, Nodes $7 \& 27$ \\
\hline$T,{ }^{\circ} \mathrm{F}$ (at $\left.x / \mathrm{L}=0.7\right)$ & 424.0 & 424.210 & 1.000 & POST1, Nodes $8 \& 28$ \\
\hline$T,{ }^{\circ} F($ at $x / L=0.8)$ & 364.0 & 364.150 & 1.000 & POST1, Nodes $9 \& 29$ \\
\hline $\mathrm{T},{ }^{\circ} \mathrm{F}$ (at $\mathrm{x} / \mathrm{L}=0.9$ ) & 311.0 & 311.360 & 0.999 & POST1, Nodes $10 \& 30$ \\
\hline$T,{ }^{\circ} F($ at $x / L=1.0)$ & 267.0 & 266.590 & 1.002 & POST1, Node 11 \\
\hline \multirow[t]{2}{*}{ q, BTU/hr } & $\approx$ & -2554.4 & - & POST1, Nodes, $1 \& 21$ \\
\hline & 5109 & -5108.8 & -1.000 & SUM, Nodes $1 \& 21$ \\
\hline
\end{tabular}

VM99: Temperature Distribution in a Trapezoidal Fin

\begin{tabular}{|c|c|c|c|c|}
\hline & Manual & Pentium 90 & Ratio & Notes \\
\hline$T,{ }^{\circ} \mathrm{F}$ (at node 4) & 27.8 & 27.764 & 1.001 & POST1Temp. @ Node 4 \\
\hline $\mathrm{T},{ }^{\gamma} \mathrm{F}$ (at node 5$)$ & 32.8 & 32.8160 & 1.000 & POST1 Temp.@ Node 5 \\
\hline $\mathrm{T},{ }^{\circ} \mathrm{F}$ (at node 6$)$ & 9.5 & 9.541 & 0.996 & POST1 Temp.@Node 6 \\
\hline $\mathrm{T}_{1}{ }^{\circ} \mathrm{F}$ (at node 7 ) & 10.7 & 10.736 & 0.997 & POST1 Temp. @ Node 7 \\
\hline $\mathrm{q}, \mathrm{BTU} / \mathrm{hr}$ & - & 1122.100 & - & POST1 Heat @ Node 1 \\
\hline q, BTU/hr & - & 418.260 & - & POST 1 Heat @ Node 2 \\
\hline q, BTU/hr & $=$ & 200.640 & - & POST1 Heat@ Node 3 \\
\hline SUM q, BTU/hr & 3482.0 & 3482.00 & 1.000 & 2(SUM q) (See Note) \\
\hline
\end{tabular}

Note: Since symmetry is used to model only half of the fin, the sum of heat tranfered is factored by two. 
Software Verification and Validation Report ANSYS Revision 5.0A, Version: PC 386/486
Document Number: QA040.216 (P90)

Revision 0

Page 37 of 95

Table 3

Comparison of Calculated and Published Results

VM100: Heat Conduction Across a Chimney Section

\begin{tabular}{|c|c|c|c|c|}
\hline & Manual & Pentium 90 & Ratio & Notes \\
\hline$T,{ }^{\circ}$ (at node 1) & 93.6 & 93.629 & 1.000 & POST1 \\
\hline $\mathrm{T},{ }^{\circ} \mathrm{F}$ (at node 2 ) & 56.8 & 56.7670 & 1.001 & " \\
\hline T. $F$ (at node 3 ) & 22.1 & 22.086 & 1.001 & $"$ \\
\hline$T, F($ at node 4 ) & 93.2 & 93.189 & 1.000 & 4 \\
\hline$T,{ }^{\circ} \mathrm{F}$ (at node 5 ) & 54.9 & 54.945 & 0.999 & 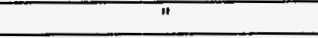 \\
\hline $\mathrm{T},{ }^{\circ} \mathrm{F}$ (at node 6 ) & 21.1 & 21.077 & 1.001 & $"$ \\
\hline $\mathrm{T}^{\circ} \mathrm{F}$ (at node 7 ) & 87.8 & 87.762 & 1.000 & $\pi$ \\
\hline $\mathrm{T}_{1}^{\circ} \mathrm{F}$ (at node 8 ) & 47.7 & 47.746 & 0.999 & $"$ \\
\hline$T,{ }^{\circ}$ (at node 9 ) & 17.3 & 17.302 & 1.000 & 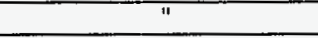 \\
\hline $\mathrm{T},{ }^{\circ} \mathrm{F}$ (at node 11 ) & 27.6 & 27.572 & 1.001 & 11 \\
\hline $\mathrm{T},{ }^{6} \mathrm{~F}$ (at node 12 ) & 12.5 & 12.533 & 0.997 & 11 \\
\hline $\mathrm{T}_{1}{ }^{\circ} \mathrm{F}$ (at node 15$)$ & 5 & 5.0131 & 0.997 & " \\
\hline q, BTU/hr & 773.5 & 773.533 & 1.000 & POST1 Parameter HEAT (HT13) \\
\hline
\end{tabular}

VM101: Temperature Distribution in a Short Solid Cylinder

\begin{tabular}{|c|c|c|c|c|}
\hline & Manual & Pentium 90 & Ratio & Notes \\
\hline$T^{8}{ }^{8} \mathrm{~F}$ (at node 11) & 7.4 & 7.4427 & 0.994 & POST1 \\
\hline $\mathrm{T},{ }^{\circ} \mathrm{F}$ (at node 21) & 16.4 & 16.361 & 1.002 & $"$ \\
\hline $\mathrm{T},{ }^{\circ} \mathrm{F}$ (at node 31) & 27.4 & 27.411 & 1.000 & $"$ \\
\hline $\mathrm{T},{ }^{\circ} \mathrm{F}$ (at node 13) & 5.3 & 5.2996 & 1.000 & $"$ \\
\hline $\mathrm{T},{ }^{\circ} \mathrm{F}$ (at node 23) & 13.0 & 13.033 & 0.997 & $"$ \\
\hline$T,{ }^{\circ} \mathrm{F}$ (at node 33) & 24.8 & 24.816 & 0.999 & $"$ \\
\hline
\end{tabular}

VM102: Cylinder w/Temperature Dependent Conductivity

\begin{tabular}{|c|c|c|c|c|}
\hline$k=$ constant, L.S. 1 & Manual & Pentium 90 & Ratio & Notes \\
\hline $\mathrm{T},{ }^{\mathrm{T}} \mathrm{F}($ at node 2$)$ & 73.7 & 73.722 & 1.000 & POST1 Results (L.S. 1) \\
\hline $\mathrm{T},{ }^{\circ} \mathrm{F}$ (at node 3 ) & 51.5 & 51.487 & 1.000 & 4 \\
\hline $\bar{T},{ }^{\circ} \mathrm{F}$ (at node 4$)$ & 32.2 & 32.217 & 0.899 & $"$ \\
\hline $\mathrm{T},{ }^{\circ} \mathrm{F}$ (at node 5) & 15.2 & 15.213 & $0 . \overline{999}$ & 7 \\
\hline \multicolumn{5}{|l|}{$k=k(T)$, L.S. 2} \\
\hline $\mathrm{T},{ }^{\circ} \mathrm{F}$ (at node 2 ) & 79.2 & 79.207 & 1.000 & POST1 Results (L.S. 2) \\
\hline$T,{ }^{\circ} \mathrm{F}$ (at node 3 ) & 59.5 & 59.513 & 1.000 & " \\
\hline $\mathrm{T},{ }^{\circ} \mathrm{F}$ (at node 4 ) & 40.2 & 40.230 & 0.999 & $"$ \\
\hline $\bar{T},{ }^{\circ} \mathrm{F}$ (at node 5 ) & 20.7 & 20.683 & 1.001 & $\overline{T i}$ \\
\hline
\end{tabular}


Software Verification and Validation Report ANSYS Revision 5.0A, Version: PC $386 / 486$
Document Number: QA040.216 (P90)

Revision 0

Page 38 of 95

Table 3

Comparison of Calculated and Published Results

VM103 - Thin Plate with Central Heat Source

\begin{tabular}{|c|c|c|c|c|}
\hline & Manual & Pentium 90 & Ratio & Notes \\
\hline$T^{\circ} \mathrm{F}$ (at node 1 ) & 227.0 & 226.97 & 1.000 & Nodal Solution \\
\hline$T_{1}{ }^{\circ} \mathrm{F}$ (at node 2 ) & 102.4 & 102.39 & 1.000 & $1 "$ \\
\hline T, ${ }^{\circ} \mathrm{F}$ (at node 3 ) & 73.6 & 73.586 & 1.000 & $"$ \\
\hline$T,{ }^{\circ} \mathrm{F}$ (at node 4$)$ & 64.6 & 64.568 & 1.000 & $\pi$ \\
\hline$\tau,{ }^{\circ} \mathrm{F}$ (at node 5) & 61.6 & 61.583 & 1.000 & $"$ \\
\hline$T,{ }^{\circ} \mathrm{F}$ (at node 6 ) & 60.6 & 60.569 & 1.001 & $"$ \\
\hline$T,{ }^{\circ} \mathrm{F}$ (at node 7 ) & 60.2 & 60.231 & 0.999 & $"$ \\
\hline $\mathrm{T},{ }^{\circ} \mathrm{F}$ (at node 8 ) & 60.2 & 60.154 & 1.001 & $"$ \\
\hline $\mathrm{T},{ }^{\circ} \mathrm{F}$ (at node 9 ) & 164.9 & 164.85 & 1.000 & 11 \\
\hline $\mathrm{T},{ }^{\circ} \mathrm{F}$ (at node 10$)$ & 126.8 & 126.81 & 1.000 & $"$ \\
\hline
\end{tabular}

VM104 - Liquid-Solid Phase Change

\begin{tabular}{|l|l|l|l|l|}
\hline & Manual & Pentium 90 & Ratio & Notes \\
\hline$t_{f}$ seconds & $798.0 \rightarrow 801.0$ & See Note & N.A. & Node $2,-1^{\circ} \mathrm{C} \rightarrow 0^{\circ} \mathrm{C}$ \\
\hline
\end{tabular}

Note: As executed by the P90 (using auto time stepping and the input stream provided by ANSYS), the time step for problem VM104 is 10 seconds. Using the 10 second time step, the temperature at Node 2 passes $-1^{\circ} \mathrm{F}$ between 814.92 seconds $\left(-.969^{\circ} \mathrm{F}\right)$ and 824.9 seconds $\left(-1.18^{\circ} \mathrm{F}\right)$. If the maximum time step is set to 3 seconds (corresponding to the step reported in the Verification Manual), $-1{ }^{\circ} \mathrm{F}$ is reached between 801 and 804 seconds $\left(-.95^{\circ} \mathrm{F}\right.$ and $-1.03^{\circ} \mathrm{F}$, respectively).

\begin{tabular}{|c|c|c|c|c|}
\hline At $t=500$ seconds & Manual & Pentium 90 & Ratio & Notes \\
\hline$T_{1}{ }^{\circ} \mathrm{C}(x=.002 \mathrm{~m})$ & -3.7 & -3.696 & 1.001 & POST1, Node 5 \\
\hline$T_{1}{ }^{\circ} \mathrm{C}(\mathrm{x}=.004 \mathrm{~m})$ & -2.43 & -2.4256 & 1.002 & POST1, Node 8 \\
\hline
\end{tabular}

VM105 - Heat Generating Coil w/Variable Conductivity

\begin{tabular}{|c|c|c|c|}
\hline Temp, $^{\circ} \mathrm{F}$ & Manual & Pentium 90 & Ratio \\
\hline Node 2 & 23.0 & 23.039 & 0.998 \\
\hline Node 3 & 35.5 & 35.526 & 0.999 \\
\hline Node 4 & 41.8 & 41.815 & 1.000 \\
\hline Node 5 & 43.7 & 43.659 & 1.001 \\
\hline Node 6 & 41.9 & 41.862 & 1.001 \\
\hline Node 7 & 36.8 & 36.756 & 1.001 \\
\hline Node 8 & 28.4 & 28.354 & 1.002 \\
\hline Node 9 & 16.4 & 16.357 & 1.003 \\
\hline
\end{tabular}

VM106 - Radient Energy Emission

\begin{tabular}{|c|c|c|c|c|}
\hline Node 2 & Manual & Pentium 90 & Ratio & Notes \\
\hline q BTU/hr & 245,520 & 245,515 & 1.000 & Reaction Solution, Nodes 1 \& 2 \\
\hline
\end{tabular}


Table 3

Comparison of Calculated and Published Results

VM107 - Thermocouple Radiation

\begin{tabular}{|c|r|r|c|c|}
\hline Node 2 & \multicolumn{1}{|c|}{ Manual } & Pentium 90 & Ratio & Notes \\
\hline $\mathrm{T}_{\mathrm{f}}{ }^{\mathrm{S}} \mathrm{F}$ & 999.95 & 999.95 & 1.000 & Node 2, DOF Solution \\
\hline $\mathrm{q}, \mathrm{BTU} / \mathrm{hr}$ & 3662.24 & 3662.24 & 1.000 & Element Solution/Reaction Solution \\
\hline
\end{tabular}

VM108 - Temperature Gradient Across Solid Cylinder

\begin{tabular}{|c|c|c|c|c|}
\hline Mode $=1\left(\Theta=0^{\circ}\right)$ & Manual & Pentium 90 & Ratio & Notes \\
\hline $\mathrm{T}^{\circ}{ }^{\circ} \mathrm{F}($ Node 1) & 0.0 & 0 & n/a & DOF Solution \\
\hline $\mathrm{T}_{1}{ }^{\circ} \mathrm{F}$ (Node 2) & 20.0 & 20.000 & 1.000 & $"$ \\
\hline $\mathrm{T}_{1}{ }^{\circ} \mathrm{F}$ (Node 3) & 40.0 & 40.000 & 1.000 & $"$ \\
\hline $\mathrm{T},{ }^{\circ} \mathrm{F}$ (Node 4) & 60.0 & 60.000 & 1.000 & \\
\hline
\end{tabular}

VM109 - Temperature Response of Cooled Wire

\begin{tabular}{|c|c|c|c|c|}
\hline Node 1 & Manual & Pentium 90 & Ratio & Notes \\
\hline$T_{1}{ }^{\circ} \mathrm{F} @ .0125 \mathrm{hr}$ & 197.03 & 197.026 & 1.000 & POST26, Node 1 \\
\hline $\mathrm{T}_{1}{ }^{\circ} \mathrm{F} @ .0325 \mathrm{hr}$ & 128.50 & 128.504 & 1.000 & $"$ \\
\hline $\mathrm{T},{ }^{\circ} \mathrm{F} @ .0500 \mathrm{hr}$ & 109.77 & 109.766 & 1.000 & $"$ \\
\hline
\end{tabular}

VM1 10 - Transient Temperature Distribution in a Slab

\begin{tabular}{|c|c|c|c|c|}
\hline Time $=14.5 \mathrm{hr}$ & Manual & Pentium 90 & Ratio & Notes \\
\hline $\mathrm{T},{ }^{\circ} \mathrm{F}$ (at node 1) & 503 & 503.01 & 1.000 & DOF Solution \\
\hline $\mathrm{T},{ }^{\circ} \mathrm{F}$ (at node 3) & 547 & 547.29 & 0.999 & $"$ \\
\hline $\mathrm{T},{ }^{\circ} \mathrm{F}$ (at node 5) & 676 & 676.33 & 1.000 & $"$ \\
\hline $\mathrm{T}_{1}{ }^{\circ} \mathrm{F}$ (at node 7) & 879 & 879.17 & 1.000 & $"$ \\
\hline $\mathrm{T},{ }^{\circ} \mathrm{F}$ (at node 9) & 1139 & 1139.0 & 1.000 & \\
\hline $\mathrm{T}_{1}{ }^{\circ} \mathrm{F}$ (at node 11) & 1435 & 1435.10 & 1.000 & \\
\hline $\mathrm{Q}, \mathrm{BTU} / \mathrm{ft}^{2}$ & $-20,717$ & $-20,717$ & 1.000 & POST26, TOTAL_HT @ T=14.50 \\
\hline
\end{tabular}

VM111 - Cooling of a Spherical Body

\begin{tabular}{|c|c|c|c|c|}
\hline Time $=6 \mathrm{hr}$ & Manual & Pentium 90 & Ratio & Notes \\
\hline $\mathrm{T}_{1}{ }^{\circ} \mathrm{F}$ & 28.3 & 28.349 & 0.998 & Node 1, DOF Solution \\
\hline
\end{tabular}

VM112 - Cooling of a Spherical Body

\begin{tabular}{|c|c|c|c|c|}
\hline Time $=6 \mathrm{hr}$ & Manual & Pentium 90 & Ratio & Notes \\
\hline $\mathrm{T},{ }^{\circ} \mathrm{F}$ & 28.7 & 28.748 & 0.998 & Node 1, DOF Solution \\
\hline
\end{tabular}


Table 3

\section{Comparison of Calculated and Published Results}

VM113 - Transient Temperature Distribution in an Orthotropic Metal Bar

\begin{tabular}{|c|c|c|c|c|}
\hline Tirne $=.000833 \mathrm{hr}$ & Manual & Pentium 90 & Ratio & Notes \\
\hline $\mathrm{T},{ }^{\circ} \mathrm{F}$ (node 1) & 463 & 463.26 & 0.999 & POST1 \\
\hline $\mathrm{T},{ }^{\circ} \mathrm{F}$ (node 7) & 154 & 153.63 & 1.002 & $"$ \\
\hline $\mathrm{T}_{1}{ }^{\circ} \mathrm{F}$ (node 13) & 285 & 284.97 & 1.000 & $"$ \\
\hline $\mathrm{T},{ }^{\circ} \mathrm{F}$ (node 2) & 200 & 199.98 & 1.000 & $"$ \\
\hline
\end{tabular}

VM114 - Temp. Response to a Rising Surface Temperature

\begin{tabular}{|c|c|c|c|c|}
\hline Time $=.03333 \mathrm{hr}$ & Manual & Pentium 90 & Ratio & Notes \\
\hline $\mathrm{T}^{\circ}{ }^{\circ} \mathrm{F}$ (node 1) & 120.00 & 120.0000 & 1.000 & POST 1 \& POST26 \\
\hline $\mathrm{T},{ }^{\circ} \mathrm{F}$ (node 2) & 0.03 & 0.0336 & 0.893 & POST1 \\
\hline $\mathrm{T}_{1}{ }^{\circ} \mathrm{F}$ (node 3) & 79.08 & 79.0748 & 1.000 & POST 1 \& POST26 \\
\hline $\mathrm{T}_{1}{ }^{\circ} \mathrm{F}$ (node 4) & 46.35 & 46.3470 & 1.000 & $"$ \\
\hline $\mathrm{T}_{1}{ }^{\circ} \mathrm{F}$ (node 5) & 23.25 & 23.2486 & 1.000 & $"$ \\
\hline $\mathrm{T}_{1}{ }^{\circ} \mathrm{F}$ (node 6) & 9.52 & 9.5217 & 1.000 & $"$ \\
\hline
\end{tabular}

VM115 - Thermal Response of a Heat Generating Slab

\begin{tabular}{|c|c|c|c|c|}
\hline Time $=.2 \mathrm{hr}$ & Manual & Pentium 90 & Ratio & Notes \\
\hline $\mathrm{T},{ }^{\circ} \mathrm{F}$ (node 1) & 32.00 & 32.000 & 1.000 & DOF Solution \\
\hline $\mathrm{T}^{\circ}{ }^{\circ} \mathrm{F}$ (node 3) & 75.10 & 75.101 & 1.000 & $"$ \\
\hline $\mathrm{T},{ }^{\circ} \mathrm{F}$ (node 4) & 102.74 & 102.74 & 1.000 & $"$ \\
\hline $\mathrm{T}^{\circ}{ }^{\circ} \mathrm{F}$ (node 5) & 119.10 & 119.10 & 1.000 & $"$ \\
\hline $\mathrm{T}^{\circ}{ }^{\circ} \mathrm{F}$ (node 6) & 127.52 & 127.52 & 1.000 & $"$ \\
\hline $\mathrm{T},{ }^{\circ} \mathrm{F}$ (node 2) & 130.07 & 130.07 & 1.000 & $"$ \\
\hline
\end{tabular}

VM116 - Heat Conducting Plate w/Sudden Cooling

\begin{tabular}{|c|c|c|c|c|}
\hline Time $=7 \mathrm{hr}$ & Manual & Pentium 90 & Ratio & Notes \\
\hline $\mathrm{T}^{\circ} \mathrm{F}$ (node 2) & 287.0 & 287.1 & 1.000 & POST1, POST26 \\
\hline
\end{tabular}

VM117 - Electric Current Flowing in a Network

\begin{tabular}{|c|c|c|c|c|}
\hline & Manual & Pentium 90 & Ratio & Notes \\
\hline $\mathrm{V}_{1}$, volts & 0.0 & 0.0000 & n/a & DOF Solution, Node 1 \\
\hline $\mathrm{V}_{2,}$, volts & 28.0 & 28.0000 & 1.000 & DOF Solution, Node 2 \\
\hline $\mathrm{V}_{3}$, volts & 19.0 & 19.0000 & 1.000 & DOF Solution, Node 3 \\
\hline $\mathrm{V}_{4}$, volts & 100.0 & 100.0000 & 1.000 & DOF Solution, Node 4 \\
\hline $\mathrm{I}_{2-1}$, amps & 1.4 & 1.4000 & 1.000 & POST1, EL $=1$ \\
\hline $\mathrm{I}_{3-1}$ amps & 1.9 & 1.9000 & 1.000 & POST1, EL $=2$ \\
\hline $\mathrm{I}_{2-3}$, amps & 1.0 & 1.0000 & 1.000 & POST1, EL $=3$ \\
\hline $\mathrm{I}_{4-2}$, amps & 2.4 & 2.4000 & 1.000 & POST1, EL $=4$ \\
\hline $\mathrm{I}_{4-3}$, amps & 0.9 & 0.9000 & 1.000 & POST1, EL $=5$ \\
\hline
\end{tabular}


Software Verification and Validation Report ANSYS Revision 5.0A, Version: PC 386/486
HNF-SD-SNF-CSWD-005, Rev. 0 Document Number: QA040.216 (P9O)

Revision 0

Page 41 of 95

Table 3

Comparison of Calculated and Published Results

\begin{tabular}{|l|l|l|l|l|}
\hline$l_{1-4}$, amps & 3.3 & 3.3000 & 1.000 & Reaction Solution, POST1,114 \\
\hline
\end{tabular}

VM118 - Centērline Temp. of Heat Generating Wire

\begin{tabular}{|c|c|c|c|c|}
\hline PLANE55 & Manual & Pentium 90 & Ratio & Notes \\
\hline$T^{\circ}, \mathrm{F}$ (node 1) & 418.7 & 418.65 & 1.000 & POST1 \\
\hline $\mathrm{T}_{\mathrm{s}},{ }^{\circ} \mathrm{F}$ (node 6, 16) & 416.5 & 416.53 & 1.000 & POST1 \\
\hline $\mathrm{q}$, BTU/hr & 339.8 & 339.77 & 1.000 & POST1 Parameter HRATE \\
\hline
\end{tabular}

VM119 - Centerline Temp. of an Electrical Wire

\begin{tabular}{|c|c|c|c|c|}
\hline PLANE67 & Manual & Pentium 90 & Ratio & Notes \\
\hline $\mathrm{T}_{1}{ }^{\circ} \mathrm{F}$ & 420.0 & 419.990 & 1.000 & POST1, Nodes 1 \& 11 \\
\hline $\mathrm{T}_{\mathrm{s},}{ }^{\circ} \mathrm{F}$ & 417.9 & 417.850 & 1.000 & POST1, Nodes 6 \& 16 \\
\hline $\mathrm{q}, \mathrm{BTU} / \mathrm{hr}$ & 341.5 & 341.500 & 1.000 & POST1 Parameter HRATE \\
\hline
\end{tabular}

\begin{tabular}{|c|c|c|c|c|}
\hline SOLID69 & Manual & Pentium 90 & Ratio & Notes \\
\hline$T_{\mathrm{CL}},{ }^{\circ} \mathrm{F}$ & 418.7 & 418.649 & 1.000 & DOF Solution, Nodes 1 \& 21 \\
\hline $\mathrm{T}_{\mathrm{S},}{ }^{\circ} \mathrm{F}$ & 416.5 & 416.525 & 1.000 & DOF Solution, Nodes 6,16,26,36 \\
\hline $\mathrm{q}, \mathrm{BTU} / \mathrm{hr}$ & 339.8 & 339.769 & 1.000 & POST1 Parameter HRATE \\
\hline
\end{tabular}

VM120 - Microstrip Transmission Line Capacitance

\begin{tabular}{|l|r|r|r|c|}
\hline & Manual & Pentium 90 & Ratio & Notes \\
\hline Capacitance, pF/m & 179.7 & 179.241 & 1.003 & POST1 Parameter C \\
\hline
\end{tabular}

VM121 - Flow Through a Pipe w/Uniform Heat Flux

\begin{tabular}{|c|c|c|c|c|}
\hline at $Y=6 \mathrm{~cm}$ & Manual & Pentium 90 & Ratio & Notes \\
\hline$V_{c},{ }^{\text {dnl }} /$ sec & $6.657 \mathrm{E}-03$ & $6.657 \mathrm{E}-03$ & 1.000 & POST1, VY @ 61 \\
\hline$T_{c,}{ }^{\circ} \mathrm{C}$ & 48.32 & 48.319 & 1.000 & POST1, T @ 61 \\
\hline$T_{w,}{ }^{\circ} \mathrm{C}$ & 49.53 & 49.533 & 1.000 & POST1, T @ 34 \\
\hline
\end{tabular}

VM122 - Pressure Drop in a Turbulent Flowing Fluid

\begin{tabular}{|c|c|c|c|c|}
\hline & Manual & Pentium 90 & Ratio & Notes \\
\hline$\Delta$ p, psi (node 1) & 4.69 & 4.69003 & 1.000 & PRES @ Node 1 \\
\hline
\end{tabular}


HNF-SD-SNF-CSWD-005, Rev. 0

Software Verification and Validation Report

Document Number: QA040.216 (P90) ANSYS Revision 5.0A, Version: PC 386/486

Revision 0

Page 42 of 95

Table 3

Comparison of Calculated and Published Results

VM123: Laminar Flow in a Piping System

\begin{tabular}{|c|c|c|c|c|}
\hline & Manual & Pentium 90 & Ratio & Notes \\
\hline$\Delta \mathrm{p}, \mathrm{Ib} / \mathrm{ft}^{2}$ (node 1) & 6165 & 6165.34 & 1.000 & PRES @ Node 1 \\
\hline $\operatorname{Re}$ & 708 & 708.02 & 1.000 & Element Solution, $\mathrm{EL}=1$ \\
\hline
\end{tabular}

VM124: Discharge of Water from a Reservoir

\begin{tabular}{|c|c|c|c|c|}
\hline & Manual & Pentium 90 & Ratio & Notes \\
\hline w, lb/sec (node 1) & 29.9 & 29.90 & 1.000 & Nodal Flow @ 1, (Reaction Sol.) \\
\hline$R e$ & 201000 & 200730.00 & 1.001 & Element Solution, EL=all \\
\hline
\end{tabular}

VM125: Radiation Heat Transfer between Concentric Cylinders

\begin{tabular}{|l|r|r|r|c|}
\hline & Manual & Pentium 90 & Ratio & Notes \\
\hline Q, Btu/hr-in & 36.4 & -36.397 & -1.000 & POST 1 Total \\
\hline
\end{tabular}

VM126: Heat Transferred to a Flowing Fluid

\begin{tabular}{|c|c|c|c|c|}
\hline & Manual & Pentium 90 & Ratio & Notes \\
\hline$T_{0,}{ }^{\circ} \mathrm{F}$ & 121.3 & 121.33 & 1.000 & POST1, Temp.@19 \\
\hline$q_{\text {in, }}, B T U / h r$ & 113.28 & 113.28 & 1.000 & POST1, Heat@EL =1 \\
\hline$q_{\text {out }}$, BTU/hr & 137.44 & 137.44 & 1.000 & POST1, Heat@EL $=9$ \\
\hline
\end{tabular}

VM127: Buckling of a Bar w/Hinged Ends (Line Elements)

\begin{tabular}{|l|c|c|c|c|}
\hline & Manual & Pentium 90 & Ratio & Notes \\
\hline$F_{c, 1}$ lb (1st mode) & 38.553 & 38.5529 & 1.000 & Eigenvalue Buckling Sol. \\
\hline
\end{tabular}

VM128: Buckling of a Bar w/Hinged Ends (Area Elements)

\begin{tabular}{|l|l|l|l|l|}
\hline & Manual & Pentium 90 & Ratio & Notes \\
\hline$F_{c r}, \mathrm{lb}$ (1st mode) & 38.714 & 38.7143 & 1.000 & Static Load Mult. for Bucking \\
\hline
\end{tabular}

VM129: Numerical Differentiation \& Integration

\begin{tabular}{|c|c|c|c|c|}
\hline & Manual & Pentium 90 & Ratio & Notes \\
\hline $\mathrm{df} / \mathrm{dt}$ (max) & $5.554 \mathrm{E}-02$ & $5.554 \mathrm{E}-02$ & 1.000 & Parameter DERIV1 \\
\hline $\mathrm{d}^{2} \mathrm{f} / \mathrm{d \textrm {d } ^ { 2 }}$ (max) & $2.422 \mathrm{E}-03$ & $2.422 \mathrm{E}-03$ & 1.000 & Parameter DERIV2 \\
\hline $\mathrm{I}_{1}$ & 29.18 & 29.1749 & 1.000 & Parameter Status F \\
\hline $\mathrm{I}_{2}$ & 381.7 & 381.656 & 1.000 & Parameter Status H \\
\hline
\end{tabular}


Table 3

Comparison of Calculated and Published Results

VM130: Fourier Series Generation for a Saw Tooth Wave

\begin{tabular}{|c|c|c|c|}
\hline & Manual & Pentium 90 & Ratio \\
\hline Mode 1 Coefficent & 0.811 & 0.8108 & 1.000 \\
\hline Mode 3 Coefficent & $-9.020 \mathrm{E}-02$ & -0.0902 & 1.000 \\
\hline Mode 5 Coefficent & $3.260 \mathrm{E}-02$ & 0.0326 & 1.000 \\
\hline Mode 7 Coefficent & $-1.670 \mathrm{E}-02$ & -0.0167 & 1.000 \\
\hline
\end{tabular}

VM131: Acceleration of Rotating Crane Boom

\begin{tabular}{|l|c|c|c|c|c|}
\hline & Manual & Pentium 90 & Ratio & \multicolumn{2}{|c|}{ Notes } \\
\hline$a_{\mathbf{x}}, \mathrm{ft} / \mathrm{sec}^{2}$ & -11.78 & -11.778 & 1.000 & Reaction Forces, FX @ 1 \\
\hline$a_{y}, f \mathrm{ftsec}$ & -5.00 & -5.000 & 1.000 & & "FY @ 1 \\
\hline$a_{\mathbf{z}}, \mathrm{ft} / \mathrm{sec}^{4}$ & 6.00 & 6.000 & 1.000 & & , FZ @ 1 \\
\hline
\end{tabular}

VM132: Stress Relaxation of Tightened Bolt due to Creep

\begin{tabular}{|c|c|c|c|c|}
\hline$\sigma$, psi & Manual & Pentium 90 & Ratio & Notes \\
\hline$@ \mathrm{t}=190$ hour & 976. & 976.093 & 1.000 & POST26, SIG \\
\hline$@ \mathrm{t}=420$ hour & 951. & 950.536 & 1.000 & $"$ \\
\hline$@ \mathrm{t}=690$ hour & 926. & 925.636 & 1.000 & $"$ \\
\hline$@ \mathrm{t}=880$ hour & 911. & 910.557 & 1.000 & $"$ \\
\hline$@ \mathrm{t}=950$ hour & 905. & 905.414 & 1.000 & \\
\hline
\end{tabular}

VM133: Motion of Rod due to Irradiation Induced Creep

\begin{tabular}{|c|c|c|c|c|}
\hline$\varepsilon_{C R}$ & Manual & Pentium 90 & Ratio & Notes \\
\hline$t=0.0 \mathrm{hr}$ & 0.00000 & 0.00000 & $\mathrm{n} / \mathrm{a}$ & POST26, EPCR \\
\hline$t=0.5 \mathrm{hr}$ & 0.00197 & 0.00197 & 1.002 & $"$ \\
\hline$t=1.0 \mathrm{hr}$ & 0.00316 & 0.00316 & 1.000 & $"$ \\
\hline$t=5.0 \mathrm{hr}$ & 0.00496 & 0.00496 & 0.999 & $"$ \\
\hline
\end{tabular}

\section{VM134: Plastic Bending of a Clamped I-Beam}

\section{Load Step 1}

\begin{tabular}{|c|c|c|c|c|c|}
\hline$w_{1}=2190 \mathrm{lb} / \mathrm{in}$ & Manual & & Pentium 90 & Ratio & Notes \\
\hline$\delta_{\text {mid, }}$ in (node 10) & -0.160 & & -0.160 & 1.001 & UZ, Node 10 \\
\hline End Moment, in-lb & $-3,784,000$ & $-3,784,320$ & 1.000 & Reaction, MY @ 1 \\
\hline Mid-Moment, in-lb & $-1,894,000$ & $-1,892,160$ & 1.001 & Reaction, MY @ 10 \\
\hline End Status & At Yield & 37,919 & At Yield & SAXL v. $\sigma_{y}$ (38 ksi) EL=1(l) \\
\hline Mid Status & Elastic & 18,959 & Elastic & SAXL v. $\sigma_{y}(38 \mathrm{ksi})$ EL $=9(\mathrm{~J})$ \\
\hline
\end{tabular}


Software Verification and Validation Report ANSYS Revision 5.0A, Version: PC 386/486
HNF-SD-SNF-CSWD-005, Rev. 0

Document Number: QA040.216 (PSO)

Revision 0

Page 44 of 95

Table 3

Comparison of Calculated and Published Results

VM134: Plastic Bending of a Clamped I-Beam (continued)

Load Step 2

\begin{tabular}{|c|c|c|c|c|}
\hline$w_{2}=3771 \mathrm{lb} / \mathrm{in}$ & Manual & Pentium 90 & Ratio & \\
\hline$\delta_{\text {mid }}$ in (node 10) & -0.360 & -0.35911 & 1.002 & UZ, Node 10 \\
\hline End Moment, in-lb & $-5,970,000$ & $-5,974,250$ & 0.999 & Reaction, MY@1 \\
\hline Mid-Moment, in-lb & $-3,800,000$ & $-3,799,970$ & 1.000 & Reaction, MY@10 \\
\hline End Status & Plastic & 59,862 & Plastic & SAXL v. $\sigma_{\mathrm{y}}(38 \mathrm{ksi}) \mathrm{EL}=1(1)$ \\
\hline Mid Status & At Yield & 38,075 & At Yield & SAXL v. $\sigma_{y}(38 \mathrm{ksi}) E L=9(\mathrm{~J})$ \\
\hline
\end{tabular}

\section{Load Step 3}

\begin{tabular}{|c|c|r|c|c|}
\hline$w_{3}=9039 \mathrm{lb} / \mathrm{in}$ & Manual & Pentium 90 & Ratio & Notes \\
\hline$\delta_{\text {mid, }}$ in (node 10) & -2.110 & -2.110 & 1.000 & UZ, Node 10 \\
\hline End Moment, in-lb (node 1) & $-15,000,000$ & $-14,972,300$ & 1.002 & Reaction, MY @ 1 \\
\hline Mid-Moment, in-1b (node 10) & $-8,450,000$ & $-8,456,810$ & 0.999 & Reaction, MY @ 10 \\
\hline EPELAXL & -- & 0.005173 & - & $E L=1$ (Elastic Strain) \\
\hline EPPLAXL & - & 0.015451 & - & $E L=1$ (Plastic Strain) \\
\hline Total End Strain & 0.0206 & 0.020624 & 0.999 & $E L=1$, Total Strain \\
\hline EPELAXL & - & 0.002922 & - & $E L=9$ (Elastic Strain) \\
\hline EPPLAXL & - & 0.006446 & - & $E L=9$ (Plastic Strain) \\
\hline Total Mid Strain & 0.0094 & $\mathbf{0 . 0 0 9 3 6 8}$ & 1.003 & $E L=9$, Total Strain \\
\hline
\end{tabular}

VM135: Bending of Beam on Elastic Foundation

\begin{tabular}{|l|l|l|l|}
\hline & Manual & Pentium 90 & Ratio \\
\hline$\delta_{\text {end, in (UY node 1) }}$ & -0.03761 & -0.03761 & 1.000 \\
\hline
\end{tabular}

VM136: Large Deflection of a BuCkled Bar

\begin{tabular}{|c|c|c|c|c|}
\hline & Manual & Pentium 90| & Ratio & \\
\hline \multicolumn{5}{|c|}{$F=44.413$, L.S. 3} \\
\hline \multirow[t]{2}{*}{$\theta, \operatorname{deg}$} & $\begin{array}{l}- \\
-\end{array}$ & -1.0363 & - & ROTZ@Node 11 \\
\hline & -59.4 & -59.38 & 1.000 & ROTZ $^{*} 180 / \pi$, Node 11 \\
\hline$\Delta x$, in & 58.9 & 58.8651 & 1.001 & UX@ Node 11 \\
\hline$\Delta y$, in & -25.3 & -25.3393 & 0.998 & UY@ Node 11 \\
\hline \multicolumn{5}{|c|}{$F=49.849$, L.S. 4} \\
\hline \multirow[t]{2}{*}{$\theta$, deg } & - & -1.3881 & - & ROTZ@ Node 11 \\
\hline & -79.5 & -79.53 & 1.000 & ROTZ $* 180 / \pi$, Node 11 \\
\hline$\Delta x$, in & 71.8 & 71.7503 & 1.001 & UX@Node 11 \\
\hline$\Delta y$, in & -43.5 & -43.5032 & 1.000 & UY@ Node 11 \\
\hline
\end{tabular}


Table 3

Comparison of Calculated and Published Results

VM136: Large Deflection of a Buckled Bar (continued)

\begin{tabular}{|c|c|c|c|c|}
\hline \multicolumn{5}{|c|}{$F=58.523$, L.S. 5} \\
\hline \multirow{2}{*}{$\theta, \operatorname{deg}$} & - & -1.7422 & - & ROTZ@Node 11 \\
\hline & -99.8 & -99.82 & 1.000 & ROTZ ${ }^{\star} 180 / \pi$, Node 11 \\
\hline$\Delta x$, in & 79.2 & 79.1886 & 1.000 & UX@ Node 11 \\
\hline$\Delta y$, in & -64.8 & -64.7569 & 1.001 & UY@ Node 11 \\
\hline \multicolumn{5}{|c|}{$F=72.634$, L.S. 6} \\
\hline \multirow[t]{2}{*}{$\theta, \operatorname{deg}$} & - & -2.0941 & - & ROTZ@ Node 11 \\
\hline & -120.0 & -119.99 & 1.000 & ROTZ $^{*} 180 / \pi$, Node 11 \\
\hline$\Delta x$, in & 80.4 & 80.4466 & 0.999 & UX@ Node 11 \\
\hline$\Delta y$, in & -87.5 & -87.4509 & 1.001 & UY@Node 11 \\
\hline
\end{tabular}

VM137: Large Deflection of a Circular Membrane

\begin{tabular}{|c|c|c|c|c|}
\hline Load Step 3 & Manual & Pentium 90 & Ratio & Notes \\
\hline$\delta$, in & -0.463 & -0.4629 & 1.000 & UY @ Node 1 \\
\hline$\sigma_{0}$, psi & 61,158 & 61,158 & 1.000 & SM @ Mid El 1 \\
\hline$\sigma_{\mathrm{a}}$, psi & 48,257 & 48,257 & 1.000 & SM @ Mid El 10 \\
\hline
\end{tabular}

VM138: Large Deflection Bending of Circular Plate

\begin{tabular}{|l|l|r|l|}
\hline & Manual & Pentium 90 & Ratio \\
\hline$\delta$, m (UY node 1) & -0.00124 & -0.00124 & 1.000 \\
\hline
\end{tabular}

VM139: Bending of a Long Uniformly Loaded Rectangular Plate

\begin{tabular}{|c|c|c|c|c|}
\hline Small $\delta$ Solution & Manual & Pentium 90 & Ratio & \multicolumn{2}{|c|}{ Notes } \\
\hline$\sigma_{x,}$ psi (MID) & 0.0 & 0.0 & $n / a$ & El. Sol., EL=1 to 4, S(X) Mid. \\
\hline$\sigma_{x}$, psi (BOT) & 107,490 & 107,490 & 1.000 & POST1 Max. nodal stress S(X) Bot \\
\hline Large $\delta$ Solution & \multicolumn{5}{|l}{} \\
\hline$\sigma_{x}$, psi (MID) & 10,962 & 10,962 & 1.000 & POST1 Max. nodal stress, SX \\
\hline$\sigma_{x}$, psi (BOT) & 25,000 & 25,000 & 1.000 & POST1 Max. nodal stress, SX \\
\hline
\end{tabular}

Note: Stresses are POST1 maximum nodal stresses

VM140: Stretching, Twisting, Bending of Long Shaft

\begin{tabular}{|c|c|c|c|c|}
\hline Max. Stresses (node 51) & Manual & Pentium 90 & Ratio & \multicolumn{2}{|c|}{ Notes } \\
\hline Torsion, $\tau$, psi & 1018.6 & 1018.6 & 1.000 & POST1 Stress, Node 51, SYZ \\
\hline Axial + Bending, $\sigma_{y}$ & 6239.9 & 6239.9 & 1.000 & " SY \\
\hline Combined, $\sigma_{1}$, psi & 6402.1 & 6402.1 & 1.000 & , S1 \\
\hline
\end{tabular}


Table 3

Comparison of Calculated and Published Results

VM141: Diametral Compression of a Disk

\begin{tabular}{|c|c|c|c|c|}
\hline PLANE82 \& PLANE2 & Manual & Pentium 90 & Ratio & Notes \\
\hline$\sigma_{y}$, psi $(x=0, y=0)$ & $-9,649$ & -9649.2 & 1.000 & POST1 Path Variable, SY \\
\hline$\sigma_{y}$, psi $(x=1, y=0)$ & $-9,140$ & -9139.5 & 1.000 & \\
\hline
\end{tabular}

\begin{tabular}{|c|c|c|c|c|}
\hline SHELL93 & Manual & Pentium 90 & Ratio & Notes \\
\hline$\sigma_{y}$, psi $(x=0, y=0)$ & $-9,649$ & -9649.2 & 1.000 & POST1 Path Variable, SY \\
\hline$\sigma_{y}$, psi $(x=.1, y=0)$ & $-9,140$ & -9139.5 & 1.000 & " \\
\hline
\end{tabular}

VM142: Stress Concentration at a Hole in a Plate

\begin{tabular}{|c|c|c|c|c|}
\hline & Manual & Pentium 90 & Ratio & Notes \\
\hline Coarse Model, $\sigma_{x} \max$ & 2417. & 2581.2 & 0.936 & POST1, SX Max. \\
\hline Submodel, $\sigma_{x} \max$ & 3004. & 3004.4 & 1.000 & " \\
\hline
\end{tabular}

VM143: Fracture Mechanics Stress Intensity for Crack in Finite Width Plate

\begin{tabular}{|c|c|c|c|c|}
\hline SOLID95 \& SOLID45 & Manual & Pentium 90 & Ratio & Notes \\
\hline K11 & 1.0620 & 1.0620 & 1.000 & POST1 Parameter Status \\
\hline K12 & 1.0458 & 1.0458 & 1.000 & $"$ \\
\hline
\end{tabular}

\begin{tabular}{|c|c|c|c|c|}
\hline PLANE82 & Manual & Pentium 90 & Ratio & Notes \\
\hline Kl1 (KCALC) & 1.0581 & 1.0548 & 1.003 & POST1 Parameter Status \\
\hline $\mathrm{Kl2}$ & 1.0552 & 1.0596 & 0.996 & $"$ \\
\hline
\end{tabular}

VM144: Bending of a Composite Beam

\begin{tabular}{|c|c|c|c|c|}
\hline SHELL99 & Manual & Pentium 90 & Ratio & Notes \\
\hline$\delta$, in (nodes 9,19,29) & 0.832 & 0.83181 & 1.000 & POST1, UZ @ 9,19,29 \\
\hline$\sigma_{x}{ }^{\text {IOP }}$, psi & 2258 & 2257.6 & 1.000 & POST1, SX @ Top \\
\hline$\sigma_{x}^{\text {Bण, }}$ psi & 1731 & 1730.6 & 1.000 & POST1, SX @ Bottom \\
\hline
\end{tabular}

\begin{tabular}{|c|c|c|c|c|}
\hline SOLID46 & Manual & Pentium 90 & Ratio & Notes \\
\hline $\begin{array}{c}\delta, \text { in (nodes } \\
9,19,29,39)\end{array}$ & 0.832 & 0.83181 & 1.000 & POST1, UZ @ 9,19,29,39 \\
\hline$\sigma_{x}{ }^{\text {IOP }}$, psi & 2258 & 2257.6 & 1.000 & POST1, SX @ Top (21-39) \\
\hline$\sigma_{x}{ }^{\text {BOT }}$, psi & 1731 & 1730.6 & 1.000 & POST1, SX @ Bottom (1-19) \\
\hline
\end{tabular}


HNF-SD-SNF-CSWD-005, Rev. 0

Software Verification and Validation Report ANSYS Revision 5.0A, Version: PC 386/486

Document Number: QA040.216 (P9O)

Revision 0

Page 47 of 95

Table 3

Comparison of Calculated and Published Results

VM145: Stretching of an Orthotropic Solid

\begin{tabular}{|c|c|c|c|c|}
\hline Nodes 8 \& 16 & Manual & Pentium 90 & Ratio & Notes \\
\hline$\Delta x$, in & $9.00 \mathrm{E}-06$ & $9.00 \mathrm{E}-06$ & 1.000 & UX @ Nodes 8 \& 16 \\
\hline$\Delta y$, in & $9.50 \mathrm{E}-06$ & $9.50 \mathrm{E}-06$ & 1.000 & UY @ Nodes 8 \& 16 \\
\hline$\Delta z$, in & $-1.75 \mathrm{E}-06$ & $-1.75 \mathrm{E}-06$ & 1.000 & UZ @ Nodes 8 \& 16 \\
\hline
\end{tabular}

VM146: Bending of Reinforced Concrete Beam

\begin{tabular}{|c|c|c|c|c|}
\hline & Manual & Pentium 90 & Ratio & Notes \\
\hline$\delta_{\mathrm{ck}}$, in & $3.32-4.18$ & $3.32-4.18$ & $\mathrm{n} / \mathrm{a}$ & See Following Note \\
\hline$\sigma_{\mathrm{t}}$ psi (SAXL, el 13,14) & 387.25 & 387.25 & 1.000 & EL $=13,14$, SAXL \\
\hline$\sigma_{\mathrm{c}}$, psi (SX, el 1) & -18.5 & -18.492 & 1.000 & EL $=1$, Nodes $9,10,19,20$ \\
\hline
\end{tabular}

NOTE: The range of crack depth provided in the Verification Manual corresponds to the area between the integration points of element 4 of the analysis model. (See Chapter 13 of the ANSYS Theory Manual for 8 node brick elements). In terms of the element output, there is cracking at all points of eiements 7 and 10, and at integration points $1,2,5$ and 6 of element 4 . All other points have no cracking.

VM147: Bending of a Curved Thick Beam

\begin{tabular}{|c|c|c|c|c|}
\hline & Manual & Pentium 90 & Ratio & Notes \\
\hline$\sigma_{\mathrm{t}}$, psi (S1 at TOP) & 656.2 & 656.16 & 1.000 & POST1, Nodes 1 - 21 \\
\hline$\sigma_{\mathrm{c}}$, psi (S3 at BOT) & -556.4 & -556.38 & 1.000 & \\
\hline
\end{tabular}

VM148: Bending of Parabolic Beam

\begin{tabular}{|c|c|c|c|c|}
\hline & Manual & Pentium 90 & Ratio & Notes \\
\hline$\delta$, in & -0.01062 & -0.010619 & 1.000 & UY @ Node 11 or 71 \\
\hline
\end{tabular}

VM149: Rotation of a Tank of Fluid

\begin{tabular}{|c|c|c|c|c|}
\hline POST1 Values & Manual & Pentium 90 & Ratio & Notes \\
\hline$\delta$, in (@X=12) & 0.197 & 0.197 & 1.002 & POST1 Parameter UY26 \\
\hline$\delta$, in (@X=24) & 0.756 & 0.756 & 1.000 & POST1 Parameter UY29 \\
\hline$\delta$, in (@X=40) & 2.081 & 2.081 & 1.000 & POST1 Parameter UY33 \\
\hline p, psi (PR60) & 0.695 & 0.695 & 1.000 & POST1 Parameter PR60 \\
\hline
\end{tabular}

VM150: Acceleration of a Tank of Fluid

\begin{tabular}{|c|c|c|c|c|}
\hline POSTI Values & Manual & Pentium 90 & Ratio & Notes \\
\hline$\delta$, in $(@ X=8)$ & 1.863 & 1.8627 & 1.000 & POST1 UZ @ 4 \\
\hline$\delta$, in $(@ X=24)$ & 0.000 & 0.0000 & 0.000 & POST1UZ@8 \\
\hline 8 , in $(@ X=40)$ & -1.863 & -1.8627 & 1.000 & POST1 UZ @ 12 \\
\hline$\Theta(\Delta Z / \Delta Y$ at $y=8 \& 40)$ & -0.1164 & -0.1164 & 1.000 & SLOPE in POST1 \\
\hline
\end{tabular}


HNF-SD-SNF-CSWD-005, Rev. 0

Software Verification and Validation Report

Document Number: QA040.216 (P90)

ANSYS Revision 5.0A, Version: PC 386/486

Revision 0

Page 48 of 95

Table 3

Comparison of Calculated and Published Results

\begin{tabular}{|c|c|c|c|c|c|}
\hline p, psi (PREL) & 0.7425 & 0.7425 & 1.000 & POST1, EL=5, PREL \\
\hline
\end{tabular}
VM151: Nonaxisymmetric Vibration of a Circular Plate
\begin{tabular}{|c|c|c|c|c|}
\hline & Manual & Pentium 90 & Ratio & Notes \\
\hline $\mathrm{f}_{0,1}, \mathrm{~Hz}$ & 267.67 & 267.6740 & 1.000 & Mode 1, Analysis 1 \\
\hline $\mathrm{f}_{1,1}, \mathrm{~Hz}$ & 753.74 & 753.7410 & 1.000 & Mode 1, Analysis 2 \\
\hline $\mathrm{f}_{2,1}, \mathrm{~Hz}$ & 1388.9 & 1388.8900 & 1.000 & Mode 1, Analysis 3 \\
\hline
\end{tabular}

VM152: Nonaxisymmetric Vibration of a Circular Membrane

\begin{tabular}{|c|c|c|c|c|}
\hline & Manual & Pentium 90 & Ratio & Notes \\
\hline$f_{0,1}, \mathrm{~Hz}$ & 211.2 & 211.225 & 1.000 & Analysis 1, Iteration 1 \\
\hline$f_{1,1}, \mathrm{~Hz}$ & 336.6 & 336.585 & 1.000 & Analysis 2 \\
\hline $\mathrm{f}_{2,1}, \mathrm{~Hz}$ & 451.3 & 451.278 & 1.000 & Analysis 3 \\
\hline $\mathrm{f}_{0.2,}, \mathrm{~Hz}$ & 486.2 & 486.176 & 1.000 & Analysis 1, Iteration 2 \\
\hline
\end{tabular}

VM153: Nonaxisymmetric Vibration of a Stretched Membrane

\begin{tabular}{|c|c|c|c|c|}
\hline & Manual & Pentium 90 & Ratio & Notes \\
\hline$f_{0,1}, \mathrm{~Hz}$ & 212.1 & 212.09 & 1.000 & POST1, F01_1 \\
\hline$f_{0,2}, \mathrm{~Hz}$ & 491.7 & 491.69 & 1.000 & POST1, F02_1 \\
\hline$f_{1,1}, \mathrm{~Hz}$ & 338.9 & 338.86 & 1.000 & POST1, F11_1 \\
\hline$f_{1,2,}, \mathrm{~Hz}$ & 629.0 & 629.00 & 1.000 & POST1, F12_1 \\
\hline
\end{tabular}

VM154: Vibration of a Fluid Coupling

\begin{tabular}{|c|c|c|c|}
\hline & Manual & Pentium 90 & Ratio \\
\hline $\mathrm{f}, \mathrm{Hz}$ (FLUID38) & $\mathbf{1 . 5 2 9 3}$ & 1.529256 & 1.000 \\
\hline $\mathrm{f}, \mathrm{Hz}$ (FLUID81) & $\mathbf{1 . 5 2 2 8}$ & 1.522833 & 1.000 \\
\hline
\end{tabular}

VM155: Shape Optimization of a Cantilever Beam

\begin{tabular}{|c|c|c|c|c|}
\hline & Manual & Pentium 90 & Ratio & \multicolumn{1}{c|}{ Notes } \\
\hline TVOL $\left(\right.$ in $\left.^{3}\right)$ & 3.64 & 3.6383 & 1.000 & POST1 Design Sensitivity \\
SEFL, $\left(\delta_{y}\right)_{\max }$ & 0.498 & 0.498 & 1.000 & Summary Table, Set 14 \\
\hline STRS, $\sigma_{\max }$, psi & $29,539$. & $29,539$. & 1.000 & \\
\hline
\end{tabular}

VM156: Natural Frequency of a Nonlinear Spring-Mass System

\begin{tabular}{|c|c|c|c|c|}
\hline & Manual & Pentium 90 & Ratio & Notes \\
\hline CoMBIN39, $\tau$, sec & 0.1440 & 0.1440 & 1.000 & POST26, Time @UY2 $\approx-1.0$ \\
\hline LINK1, $\tau$, sec & 0.1440 & 0.1440 & 1.000 & " \\
\hline
\end{tabular}


Software Verification and Validation Report

ANSYS Revision 5.0A, Version: PC 386/486
HNF-SD-SNF-CSWD-005, Rev, 0

Document Number: QA040.216 (P9O)

Revision 0

Page 49 of 95

Table 3

Comparison of Calculated and Published Results

VM157: Optimization of a Frame Structure

\begin{tabular}{|c|c|c|c|c|}
\hline & Manual & Pentium 90 & Ratio & Notes \\
\hline Volume, $\mathrm{m}^{3}$ (TVOL) & 0.0775 & 0.07750 & 1.000 & \multirow{3}{*}{$\begin{array}{c}\text { POST1 Design Sensitivity } \\
\text { Summary Table, Set } 9 \text { : } \\
\text { TVOL, D1, D2 }\end{array}$} \\
\hline$d_{1}, m$ & 0.120 & 0.11996 & 1.000 & \\
\hline$d_{2}, m$ & 0.129 & 0.12888 & 1.001 & \\
\hline
\end{tabular}

VM158: Motion of a Bobbing Bouy

\begin{tabular}{|c|c|c|c|c|}
\hline & Manual & Pentium 90 & Ratio & Notes \\
\hline$\delta, \mathrm{m}$ & -0.312 & -0.31202 & 1.000 & POST26, UZ @ 25.5 \\
\hline
\end{tabular}

\section{VM159: Temperature Controlled Heater}

\begin{tabular}{|l|c|c|c|c|}
\hline & Manual & Pentium 90 & Ratio & Notes \\
\hline First "off" Temp, ${ }^{\circ} \mathrm{F}$ & $124.842-125.003$ & 125.003 & n/a & POST26, 2TEMP @ Stat $1 \rightarrow 0$ \\
\hline First "on" Temp, ${ }^{\circ} \mathrm{F}$ & 100.854 .99 .564 & 99.564 & n/a & POST26, 2TEMP @ Stat 0 $\rightarrow 1$ \\
\hline
\end{tabular}

VM160: Solid Cylinder w/Harmonic Temperature Load

\begin{tabular}{|c|c|c|c|c|}
\hline Mode 2, $\Theta=0^{\circ}$ & Manual & Pentium 90 & Ratio & Notes \\
\hline T, ${ }^{\circ} \mathrm{F}$ (node 1$)$ & 0.0 & 0.0000 & $n / a$ & POST1 Temperatures \\
\hline $\mathrm{T},{ }^{\circ} \mathrm{F}$ (node 3 ) & 5.0 & 5.0000 & 1.000 & $"$ \\
\hline $\mathrm{T},{ }^{\circ} \mathrm{F}$ (node 5$)$ & 20.0 & 20.000 & 1.000 & $"$ \\
\hline $\mathrm{T}^{\circ} \mathrm{F}$ (node 7 ) & 45.0 & 45.000 & 1.000 & 7 \\
\hline \multicolumn{5}{|l|}{ Mode 2, $\Theta=90^{\circ}$} \\
\hline $\mathrm{T},{ }^{\circ} \mathrm{F}$ (node 1 ) & 0.0 & 0.0000 & $n / a$ & POST1 Temperatures \\
\hline T, ${ }^{\circ} \mathrm{F}$ (node 3$)$ & -5.0 & -5.0000 & 1.000 & " \\
\hline T, ${ }^{\circ} \mathrm{F}$ (node 5 ) & -20.0 & -20.000 & 1.000 & $"$ \\
\hline T, ${ }^{\circ} \mathrm{F}$ (node 7 ) & -45.0 & -45.000 & 1.000 & $\bar{"}$ \\
\hline
\end{tabular}

VM161: Heat Flow From an Insulated Pipe

\begin{tabular}{|c|c|c|c|c|}
\hline & Manual & Pentium 90 & Ratio & Notes \\
\hline \multirow{2}{*}{ q, BTU/hr } & - & 30.165 & - & EL. Sol., Convection Heat Rate \\
\cline { 2 - 5 } & 362.0 & 362.0 & 1.000 & Convection Heat Rate ${ }^{*} 360^{\circ} / 30^{\circ}$ \\
\hline
\end{tabular}

VM162: Cooling of a Circular Fin of Rectangular Profile

\begin{tabular}{|c|c|c|c|c|}
\hline & Manual & Pentium 90 & Ratio & Notes \\
\hline \multirow{3}{*}{$\mathrm{T}_{2},{ }^{\circ} \mathrm{F}$} & 52.37 & 52.37 & 1.000 & Nodes $9,19,29$ \\
\hline & 52.37 & 52.82 & 0.991 & Node 39,59 \\
\hline & 52.37 & 52.37 & 1.000 & Node $69,79,89$ \\
\hline \multirow{2}{*}{$\begin{array}{c}q, \text { BTU/hr } \\
\text { (Reaction Heat Flows) }\end{array}$} & - & 1.41246 & - & Total Reaction Heat Flow \\
\hline & 101.70 & 101.70 & 1.000 & Total $360^{\circ} / 5^{\circ}$ \\
\hline
\end{tabular}


HNF-SD-SNF-CSWD-005, Rev. 0

Software Verification and Validation Report Document Number: QAO40.216 (P9O) ANSYS Revision 5.0A, Version: PC $386 / 486$

Revision 0

Page 50 of 95

Table 3

Comparison of Calculated and Published Results

VM163: Grouñdwater Seepage

\begin{tabular}{|c|c|c|c|c|}
\hline & Manual & Pentium 90 & Ratio & Notes \\
\hline $\mathrm{q}, \mathrm{m} / \mathrm{day} / \mathrm{radian}$ & - & 54.085 & - & POST1 - TOTAL HEAT \\
\hline & 8.6 & 8.61 & 0.999 & POST $1 / 2 \pi$ \\
\hline
\end{tabular}

VM164: Drying of a Thick Wooden Slab

\begin{tabular}{|c|c|c|c|c|}
\hline & Manual & Pentium 90 & Ratio & Notes \\
\hline c, \% & 10.2 & 10.18 & 1.002 & EL=1, TEMP(I) \\
\hline
\end{tabular}

VM165: Current-Carrying Ferromagnetic Conductor

\begin{tabular}{|l|c|c|c|c|}
\hline & Manual & Pentium 90 & Ratio & POST1 \\
\hline $\mathrm{B}_{\Theta}, \mathrm{T} @ \mathrm{r}=.325$ in & 0.45 & 0.4516 & 0.996 & BY @ Nodes 15 \& 21 \\
\hline $\mathrm{B}_{\Theta}, \mathrm{T} @ \mathrm{r}=.375$ in & 1.02 & 1.017 & 1.003 & BY @ Nodes 17 \& 23 \\
\hline $\mathrm{B}_{\Theta}, \mathrm{T} @ \mathrm{r}=.425$ in & 1.21 & 1.2139 & 0.997 & BY @ Nodes 19 \& 25 \\
\hline
\end{tabular}

VM166: Long Cylinder in a Sinusoidal Magnetic Field

\begin{tabular}{|c|c|c|c|c|}
\hline & Manual & Pentium 90 & Ratio & Notes \\
\hline $\begin{array}{c}\text { Node } 1-B_{x}(0,0), T \\
\text { (Real) }\end{array}$ & 0 & $-2.24 \mathrm{E}-23$ & $n / a$ & POST1 Mag. Flux Density Listing \\
\hline (Imaginary) & 0j & $-2.09 E-22$ & $n / a$ & Complex Solution Key \\
\hline $\mathrm{B}_{y}(0,0), \mathrm{T}$ (Real) & -0.00192 & -0.00192 & 1.000 & POST1 Mag. Flux Density Listing \\
\hline (Imaginary) & $-0.0214 j$ & -0.021395 & 1.000 & Complex Solution Key \\
\hline Power Loss, W/m & 2340 & 2340.09 & 1.000 & POST1 Label P \\
\hline
\end{tabular}

VM167: Transient Eddy Currents in Semi-Infinite Solid

Vector Potential ( $\mathrm{Wb} / \mathrm{m})$

\begin{tabular}{|c|c|c|c|c|}
\hline$t=0.15 \mathrm{sec}$ & Manual & Pentium 90 & Ratio & Notes \\
\hline$@ x=.2517$ & 0.840 & -0.840492 & -0.999 & Node 4, AZ (POST26 @ T=.15) \\
\hline$@ x=.4547$ & 0.286 & -0.285974 & -1.000 & Node 6, AZ (POST26 @ T=.15) \\
\hline$@ x=.6914$ & 0.047 & -0.047361 & -0.992 & Node 8, AZ (POST26 @ T=.15) \\
\hline
\end{tabular}

Flux Density (T)

\begin{tabular}{|c|c|c|c|c|}
\hline$t=0.15 \mathrm{sec}$ & Manual & Pentium 90 & Ratio & Notes \\
\hline$@ x=.2517$ & 3.673 & 3.6731 & 1.000 & POST1, BY @ 4 \\
\hline$@ x=.4547$ & 1.813 & 1.8127 & 1.000 & POST1, BY @ 6 \\
\hline $\mathrm{x}=.6914$ & 0.472 & 0.47199 & 1.000 & POST1, BY @ 8 \\
\hline
\end{tabular}


HNF-SD-SNF-CSWD-005, Rev. 0

Software Verification and Validation Report

Document Number: QA040.216 (P90) ANSYS Revision 5.0A, Version: PC 386/486

Revision 0

Page 51 of 95

Table 3

Comparison of Calculated and Published Results

VM167: Transient Eddy Currents in Semi-Infinite Solid (continued)

Eddy Current Density $\left(\times 10^{7} \mathrm{Am}^{2}\right)$

\begin{tabular}{|c|c|c|c|c|}
\hline$t=0.15 \mathrm{sec}$ & Manual & Pentium 90 & Ratio & Notes \\
\hline$@ x=.2517$ & -0.762 & -0.7615 & 1.001 & POST26, JEDDY @ 4 \\
\hline$@ x=.4547$ & -0.670 & -0.6704 & 0.999 & POST26, JEDDY @6 \\
\hline$@ x=.6914$ & -0.250 & -0.2503 & 0.999 & POST26, JEDDY @ 8 \\
\hline
\end{tabular}

VM168: Magnetic Field in a Nonferrous Solenoid

\begin{tabular}{|c|c|c|c|c|}
\hline & Manual & Pentium 90 & Ratio & Notes \\
\hline $\mathrm{B}_{\mathrm{z}}\left(\times 10^{\circ}\right) \mathrm{T}, \mathrm{z}=0$ & 1121.000 & 1120.70 & 1.000 & POST1 LPATH, S $=0$ \\
\hline $\mathrm{B}_{\mathrm{z}}\left(\times 10^{\circ}\right) \mathrm{T}, \mathrm{z}=2.5$ & 569.000 & 568.59 & 1.001 & POST1 LPATH, S $=.0635$ \\
\hline $\mathrm{B}_{\mathrm{z}}\left(\times 10^{\circ}\right) \mathrm{T}, \mathrm{z}=7.5$ & 2.120 & 2.1232 & 0.998 & POST1 LPATH, S $=.1905$ \\
\hline
\end{tabular}

VM169: Permanent Magnet Circuit w/ Air Gap

\begin{tabular}{|c|c|c|c|c|}
\hline SOLID98 & Manual & Pentium 90 & Ratio & Notes \\
\hline$|\mathrm{B}|, \mathrm{G}$ (perm. mag.) & 7386 & 7386 & 1.000 & POST1, Nodes 1-28, BSUM \\
\hline$|\mathrm{H}|, \mathrm{O}_{\mathrm{e}}$ (perm. mag.) & 493 & 493 & 1.000 & POST1, Nodes 1-28, HSUM \\
\hline$|\mathrm{B}|, \mathrm{G}$ (air gap) & 7386 & 7386 & 1.000 & POST1, Nodes 53-107, BSUM \\
\hline$|\mathrm{H}|, \mathrm{O}_{\mathrm{e}}$ (air gap) & 7386 & 7386 & 1.000 & POST1, Nodes 53-107, HSUM \\
\hline
\end{tabular}

VM170: Magnetic Field from a Square Current Loop

\begin{tabular}{|c|c|c|c|c|}
\hline flux density & Manual & Pentium 90 & Ratio & Notes \\
\hline$B \times\left(\times 10^{6}\right.$ Tesla $)$ & 2.010 & 2.00960 & 1.000 & Parameters \\
\hline$B Y\left(\times 10^{6}\right.$ Tesla $)$ & -0.662 & -0.66165 & 1.001 & $"$ \\
\hline$B Z\left(\times 10^{6}\right.$ Tesla $)$ & 2.010 & 2.00961 & 1.000 & $"$ \\
\hline
\end{tabular}

VM171: Permanent Magnet w/Elastic Keeper

\begin{tabular}{|c|c|c|c|c|}
\hline & Manual & Pentium 90 & Ratio & Notes \\
\hline$\delta, \mathrm{cm}$ & 0.150 & 0.14962 & 1.003 & POST1, UY @ 25-30 \\
\hline B, Gauss & 2496 & 2495.2 & 1.000 & POST1, Mag. Flux Density, 8SUM \\
\hline
\end{tabular}

VM172: Stress Analysis of Long, Thick, Isotropic Solenoid

\begin{tabular}{|c|c|c|c|c|}
\hline & Manual & Pentium 90 & Ratio & Notes \\
\hline $\mathrm{B}_{\Theta} \mathrm{G} @ \mathrm{r}=1.0 \mathrm{~cm}$ & 122.6 & 122.57 & 1.000 & POST1, Nodes 2 \& 7 \\
\hline $\mathrm{B}_{\Theta} \mathrm{G} @ \mathrm{r}=1.3 \mathrm{~cm}$ & 87.97 & 87.97 & 1.000 & POST1, Nodes $19 \& 39$ \\
\hline $\mathrm{B}_{\Theta} \mathrm{G} @ \mathrm{r}=1.7 \mathrm{~cm}$ & 37.69 & 37.689 & 1.000 & POST1, Nodes $27 \& 47$ \\
\hline$\sigma_{0}$, Dyne $/ \mathrm{cm}^{2}, r=1.0 \mathrm{~cm}$ & 1442 & 1441.5 & 1.000 & POST1, SZ @ Nodes $2 \& 7$ \\
\hline$\sigma_{0}$, Dyne $/ \mathrm{cm}^{2}, r=1.3 \mathrm{~cm}$ & 977.0 & 976.91 & 1.000 & POST1, SZ @ Nodes $19 \& 39$ \\
\hline$\sigma_{0,}$ Dyne $/ \mathrm{cm}^{2}, r=1.7 \mathrm{~cm}$ & 626.1 & 626.14 & 1.000 & POST1, SZ @ Nodes $27 \& 47$ \\
\hline
\end{tabular}


Software Verification and Validation Report ANSYS Revision 5.0A, Version: PC $386 / 486$
HNF-SD-SNF-CSWD-005, Rev. 0 Document Number: QA040.216 (P90)

Revision 0

Page 52 of 95

Table 3

Comparison of Calculated and Published Results

VM173: Centerline Temp. of an Electric Wire

\begin{tabular}{|c|c|c|c|c|}
\hline & Manual & Pentium 90 & Ratio & Notes \\
\hline$T_{C L},{ }^{\circ} \mathrm{F}$ & 418.7 & 418.65 & 1.000 & POST1, Nodes 1 \& 21 \\
\hline $\mathrm{T}_{\mathrm{S},}{ }^{\circ} \mathrm{F}$ & 416.5 & 416.53 & 1.000 & POST1, Nodes 6,16,26,36 \\
\hline $\mathrm{q}, \mathrm{BTU} / \mathrm{hr} / \mathrm{ft}$ & 339.8 & 339.769 & 1.000 & POST1, Parameter HRATE \\
\hline
\end{tabular}

VM174: Bimetallic Beam Under Thermal Load

\begin{tabular}{|c|c|c|c|c|}
\hline & Manual & Pentium 90 & Ratio & Notes \\
\hline$y$, in & 0.886 & 0.8855 & 1.001 & POST1, UY @ 7 \\
\hline$T,{ }^{\circ} \mathrm{F}$ & 400.0 & 400.000 & 1.000 & POST1, Temp. @ 7 \\
\hline
\end{tabular}

VM175: Natural Frequency of Piezoelectric Transducer

\begin{tabular}{|c|c|c|c|c|}
\hline Short Circuit & Manual & Pentium 90 & Ratio & Notes \\
\hline $\mathrm{f}_{1}, \mathrm{kHz}$ & 66.38 & 66.3767 & 1.000 & Mode 3 \\
\hline $\mathrm{f}_{2}, \mathrm{kHz}$ & 89.97 & 89.969 & 1.000 & Mode 6 \\
\hline
\end{tabular}

\begin{tabular}{|c|c|c|c|c|}
\hline Open Circuit & Manual & Pentium 90 & Ratio & Notes \\
\hline$f_{1}, \mathrm{kHz}$ & 83.89 & 83.8917 & 1.000 & Mode 5 \\
\hline$f_{2}, \mathrm{kHz}$ & 96.46 & 96.458 & 1.000 & Mode 9 \\
\hline
\end{tabular}

VM176: Freq. Response of Electrical Input Admittance for a Piezoelectric Transducer

\begin{tabular}{|l|c|c|c|c|}
\hline & Manual & Pentium 90 & Ratio & Notes \\
\hline Y, mmhos @ 20 kHz & 0.43 & 0.429157 & 1.002 & POST26, MMHO @ Time \\
\hline Y, mmhos @ 35 kHz & 0.96 & 0.956626 & 1.004 & " \\
\hline Y, mmhos @ 42 kHz & 2.80 & 2.80449 & 0.998 & $"$ \\
\hline$Y$, mmhos @ 45 kHz & -1.7 & -1.73363 & 0.981 & $"$ \\
\hline$Y$, mmhos @ 50 kHz & 0.33 & 0.325265 & 1.015 & " \\
\hline$Y$, mmhos @ 54 kHz & 0.63 & 0.625656 & 1.007 & \\
\hline
\end{tabular}

VM177: Natural Frequency of a Submerged Ring

\begin{tabular}{|c|c|c|c|c|}
\hline & Manual & Pentium 90 & Ratio & Notes \\
\hline f, Hz & $10.56->10.57$ & 10.57 & - & POST26 Time @ Max. $8 @ 1.41,81$ \\
\hline
\end{tabular}

VM178 : Plane Poiseuille Flow

\begin{tabular}{|c|c|c|c|c|}
\hline & Manual & Pentium 90 & Ratio & Notes \\
\hline $\mathrm{VX} @ S=1.0$ & 0.0050 & 0.005000 & 1.000 & POST1, Path Summary \\
\hline $\mathrm{vX} @ \mathrm{~S}=1.33$ & 0.0044 & 0.004444 & 0.990 & " \\
\hline $\mathrm{VX} @ \mathrm{~S}=1.67$ & 0.0028 & 0.027778 & 0.101 & " \\
\hline
\end{tabular}


Software Verification and Validation Report ANSYS Revision 5.0A, Version: PC 386/486
HINF-SD-SNF-CSWD-005, Rev. 0

Document Number: QA040.216 (P90)

Revision 0

Page 53 of 95

Table 3

Comparison of Calculated and Published Results

VM179: Dynamic Double Rotation of a Jointed Beam

\begin{tabular}{||c|c|c|c|c|}
\hline & Manual & Pentium 90 & Ratio & Notes \\
\hline$\delta_{x,}$ in $(t=1.0)$ & -0.5749 & -0.5830 & 0.986 & UX @ Node 4 \\
\hline$\delta_{y}$, in $(t=1.0)$ & 1.4032 & 1.4115 & 0.994 & UY @ Node 4 \\
\hline$\Theta_{z}$, rad $(t=1.0)$ & 0.7777 & 0.7835 & 0.993 & ROTZ @ Node 4 \\
\hline$\delta_{x}$, in $(t=2.0)$ & -2.0000 & -2.0000 & 1.000 & UX @ Node 4 \\
\hline$\delta_{y}$, in $(t=2.0)$ & 2.0000 & 2.0000 & 1.000 & UY @ Node 4 \\
\hline$\Theta_{z}$, rad $(t=2.0)$ & 1.5707 & 1.5708 & 1.000 & ROTZ @ Node 4 \\
\hline$\delta_{x}$ in $(t=3.0)$ & -2.0000 & -2.0000 & 1.000 & UX @ Node 4 \\
\hline$\delta_{y}$, in $(t=3.0)$ & 1.9962 & 1.9962 & 1.000 & UY @ Node 4 \\
\hline$\delta_{z}$, in $(t=3.0)$ & 0.08716 & 0.08717 & 1.000 & UZ @ Node 4 \\
\hline$\Theta_{x}, \operatorname{rad}(t=3.0)$ & 0.08727 & 0.08728 & 1.000 & ROTX @ Node 4 \\
\hline$\Theta_{z}, \operatorname{rad}(t=3.0)$ & 1.5708 & 1.5708 & 1.000 & ROTZ @ Node 4 \\
\hline
\end{tabular}

VM180: Bending of a Curved Beam

\begin{tabular}{|c|c|c|c|c|}
\hline Maximum (+/-) & Manual & Pentium 90 & Ratio & Notes \\
\hline$\sigma_{1,}$ psi & 657.9 & 657.93 & 1.000 & POST1, SY Maximum, Nodes 6-54 \\
\hline$\sigma_{c}$, psi & -556.8 & -556.79 & 1.000 & POST1, SY Minimum, Nodes 66-104 \\
\hline
\end{tabular}

VM181: Natural Frequency of a Flat Circular Plate w/Clamped Edges

\begin{tabular}{|c|c|c|c|c|}
\hline & Manual & Pentium 90 & Ratio & Notes \\
\hline $\mathrm{f}_{0,0}, \mathrm{~Hz}$ & 172.82 & 172.817 & 1.000 & Eigenvalue Solution, Mode 1 \\
\hline $\mathrm{f}_{0,1}, \mathrm{~Hz}$ & 677.86 & 677.857 & 1.000 & Eigenvalue Solution, Mode 2 \\
\hline $\mathrm{f}_{0.2}, \mathrm{~Hz}$ & 1537.92 & 1537.92 & 1.000 & Eigenvalue Solution, Mode 3 \\
\hline
\end{tabular}

VM182: Transient Response of a Spring-Mass System

\begin{tabular}{|c|c|c|c|c|}
\hline & Manual & Pentium 90 & Ratio & \multicolumn{2}{|c|}{ Notes } \\
\hline$Y_{1},(@ t=1.3 s)$ & 14.40 & 14.4037 & 1.000 & POST26, UY1 \\
\hline$Y_{2},(@ t=1.3 s)$ & 3.95 & 3.95077 & 1.000 & , UY2 \\
\hline$Y_{1},(@ t=2.4 s)$ & 18.40 & 18.3984 & 1.000 & " UY1 \\
\hline$Y_{2},(@ t=2.4 s)$ & 6.16 & 6.16299 & 1.000 & , UY2 \\
\hline
\end{tabular}

VM183: Harmonic Response of a Spring-Mass System

\begin{tabular}{|c|c|c|c|c|}
\hline & Manual & Pentium 90 & Ratio & \multicolumn{2}{|c|}{ Notes } \\
\hline$Y_{1},(@ .226 \mathrm{~Hz})$ & -1371.7000 & -1371.73 & 1.000 & POST26, UY1 \\
\hline$Y_{2},(@ .226 \mathrm{~Hz})$ & -458.0800 & -458.078 & 1.000 & "UY2 \\
\hline$Y_{1},(@ .910 \mathrm{~Hz})$ & -0.8539 & -0.853907 & 1.000 & "UY1 \\
\hline$Y_{2},(@ .910 \mathrm{~Hz})$ & 0.1181 & 0.118095 & 1.000 & , UY2 \\
\hline
\end{tabular}


Software Verification and Validation Report ANSYS Revision 5.0A, Version: PC 386/486
HNF-SD-SNF-CSWD-005, Rev. 0 Document Number: QA040.216 (P90)

Revision 0

Page 54 of 95

Table 3

Comparison of Calculated and Published Results

VM184: Straight Cantilever Beam

\begin{tabular}{|c|c|c|c|c|}
\hline \hline SOLID5 & Manual & Pentium 90 & Ratio & Notes \\
\hline$\delta_{x}$, in & $2.978 \mathrm{E}-05$ & $2.978 \mathrm{E}-05$ & 1.000 & POST1, Nodes 2,12,23,34-LS1 \\
\hline$\delta_{\mathrm{r}, \text { in }}$ & 0.10683 & 0.10683 & 1.000 & "(LS2) \\
\hline$\delta_{2}$, in & 0.42554 & 0.42554 & 1.000 & "(LS3) \\
\hline
\end{tabular}

\begin{tabular}{|c|c|c|c|c|}
\hline SOLID92 & Manual & Pentium 90 & Ratio & Notes \\
\hline$\delta_{x}$, in & $2.990 \mathrm{E}-05$ & $2.988 \mathrm{E}-05$ & 1.001 & POST1, UX, LS1 (154-170) \\
\hline$\delta_{y}$, in & 0.10547 & 0.10546 & 1.000 & POST1, UY, LS2 \\
\hline$\delta_{2}$, in & 0.41988 & 0.41988 & 1.000 & POST1, UZ, LS3 \\
\hline
\end{tabular}

\begin{tabular}{|c|c|c|c|c|}
\hline \hline SOLID98 & Manual & Pentium 90 & Ratio & Notes \\
\hline$\delta_{x}$, in & 2.990 E-05 & $2.988 E-05$ & 1.001 & POST1, UX, LS1 (154-170) \\
\hline$\delta_{y}$, in & 0.10547 & 0.10546 & 1.000 & POST1, UY, LS2 \\
\hline$\delta_{z}$, in & 0.41988 & 0.41988 & 1.000 & POST1, UZ, LS3 \\
\hline
\end{tabular}

VM185: AC Analysis of a Slot Embedded Conductor

\begin{tabular}{|r|c|c|c|c|}
\hline & Manual & Pentium 90 & Ratio & POST1 Parameters \\
\hline$J_{s}^{\text {he }}$ & 10135 & 10135.04 & 1.000 & JSR \\
\hline$J_{s}^{l m}$ & 27320 & 27336.82 & 0.999 & JSI \\
\hline Impedance $(\Omega / m) \times 10^{6}$ & -- & -- & -- & \\
\hline Real & 174.7 & 174.728 & 1.000 & ACRE \\
\hline Imaginary & $j 471.4$ & $j 471.287$ & 1.000 & ACRA \\
\hline Loss Ratio & 2.39 & 2.38989 & 1.000 & RAT \\
\hline
\end{tabular}

VM186: Transient Analysis of a Slot Embedded Conductor

\begin{tabular}{|c|c|c|c|c|}
\hline Vector Potential & Manual & Pentium 90 & Ratio & Notes \\
\hline \multicolumn{5}{|l|}{ Load Step 61} \\
\hline node $1(t=3 \pi / 2)$ & -15.03 & -15.03 & 1.000 & DOF Solution, Node $1, T=4.71, \mathrm{AZ}$ \\
\hline node $4(t=3 \pi / 2)$ & -14.66 & -14.6556 & 1.000 & DOF Solution, Node 4, $T=4.71, A Z$ \\
\hline node $7(t=3 \pi / 2)$ & -4.00 & -4.00 & 1.000 & DOF Solution, Node 7, $T=4.71, A Z$ \\
\hline \multicolumn{5}{|l|}{ Load Step 81} \\
\hline node $1(t=2 \pi)$ & -3.21 & -3.21082 & 1.000 & DOF Solution, Node 1, $T=6.28, A Z$ \\
\hline node $4(t=2 \pi)$ & -0.91 & -0.914718 & 0.995 & DOF Solution, Node 4, $T=6.28, A Z$ \\
\hline node $7(t=2 \pi)$ & 0.0 & 0 & $\mathrm{n} / \mathrm{a}$ & DOF Solution, Node $7, T=6.28, A Z$ \\
\hline
\end{tabular}




\section{Table 3}

Comparison of Calculated and Published Results

VM187: Bending of a Curved Beam

\begin{tabular}{|c|c|c|c|c|}
\hline Maximum $\delta$ & Manual & Pentium 90 & Ratio & POST1 \\
\hline$\delta,($ SOLID5) & 0.08814 & 0.088136 & 1.000 & UY @ 5,25,45,65 \\
\hline$\delta$, (SOLID92) & 0.08811 & 0.088110 & 1.000 & UY @ 352-368 \\
\hline$\delta$, (SOLID98) & 0.08811 & 0.088110 & 1.000 & UY @ 352-368 \\
\hline
\end{tabular}

VM188: Force Calculation of a Current Carrying Conductor

\begin{tabular}{|c|c|c|c|c|}
\hline & Manual & Pentium 90 & Ratio & Notes \\
\hline$F$ (Lorentz) & -0.00974 & -0.009699 & 1.004 & POST1 Scalar FXL \\
\hline F (Maxwell) & -0.00964 & -0.009459 & 1.020 & POST1 Scalar FXM \\
\hline
\end{tabular}


HNF-SD-SNF-CSWD-005, Rev. 0

Software Verification and Validation Report

ANSYS Revision 5.0A, Version: PC 386/486

Document Number: QA040.216 (P9O)

Revision 0

Page 56 of 95

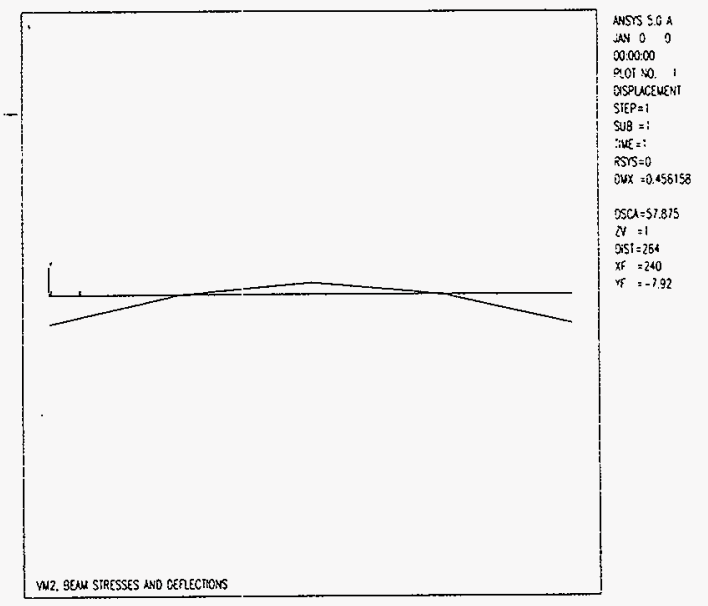

Figure 1

Problem VM2 -Plot 1

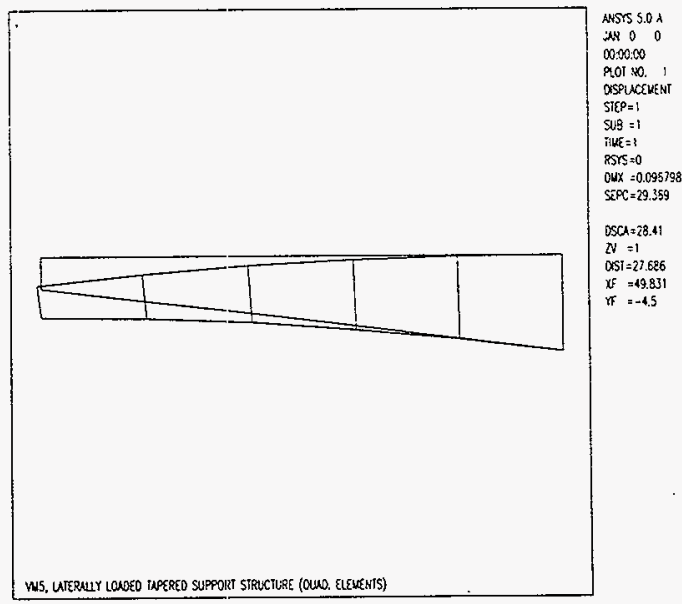

Figure 2

Problem VM5 - Plot 1 


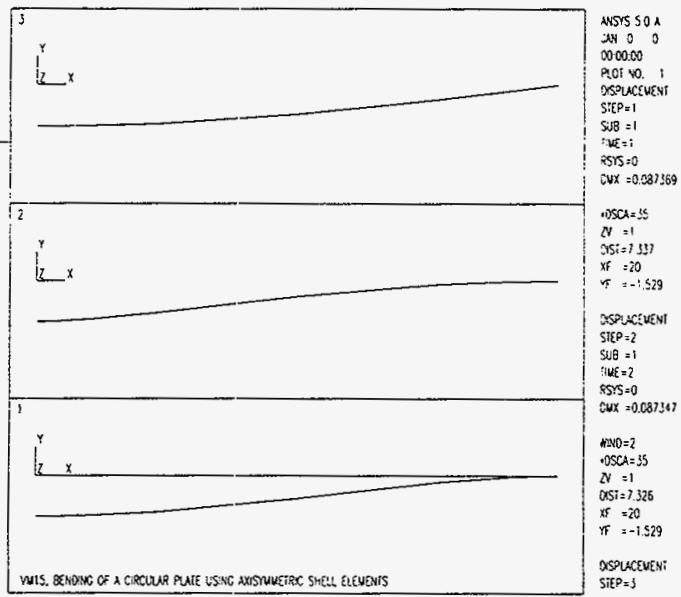

Figure 3

Problem VM15 - Plot 1

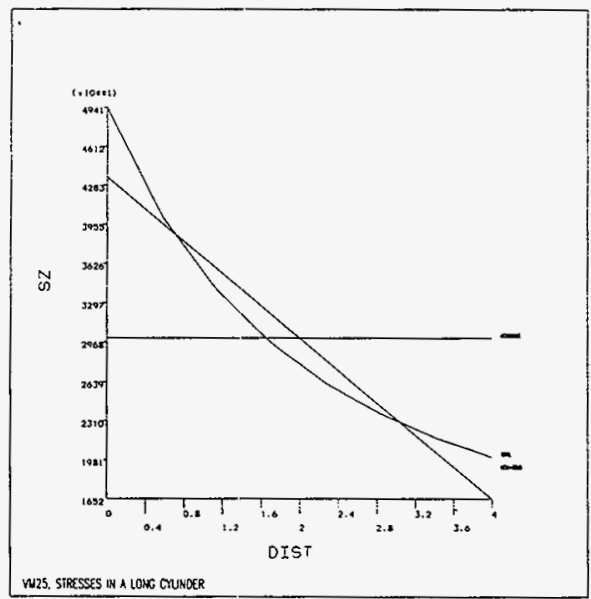

posil

$\operatorname{SIEP}=1$

Siv8 $=$ :

THUE $=1$

srcrow ror

Secrion $\operatorname{co0} 90$

$8001=2$

$3002=5$

$\$ 2$

STRESS SECT CS

$N=1$

OS $51=0.75$

$x:=0.5$

if $=0.5$

if $=0.5$

Figure 4

Problem VM25 - Plot 1 
HNF-SD-SNF-CSWD-005, Rev. 0

Software Verification and Validation Report ANSYS Revision 5.0A, Version: PC 386/486

Document Number: QA040.216 (P9O)

Revision 0

Page 58 of 95

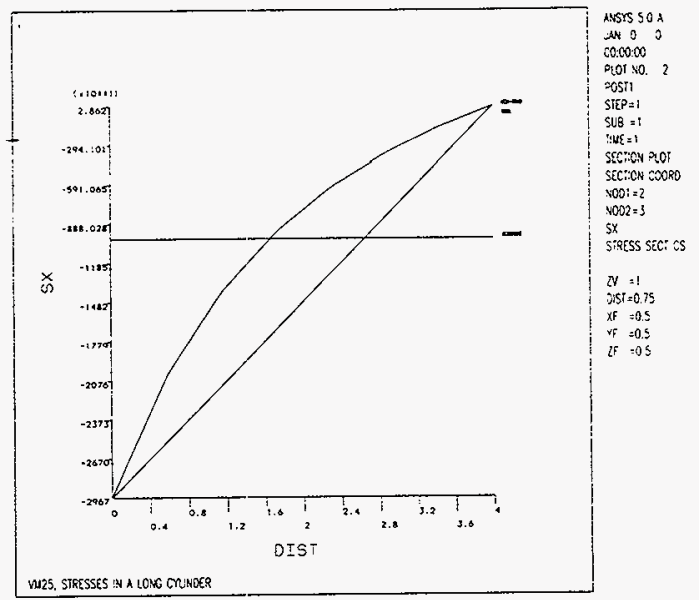

Figure 5

Problem VM25 - Plot 2

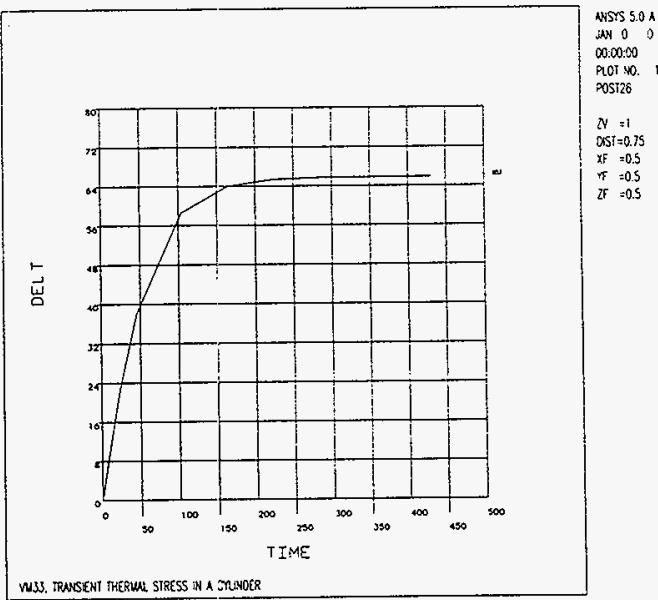

Figure 6

Problem VM33 - Plot 1 
HNF-SD-SNF-CSWD-005, Rev. 0

Software Verification and Validation Report ANSYS Revision 5.OA, Version: PC 386/486

Document Number: QA040.216 (P90)

Revision 0

Page 59 of 95

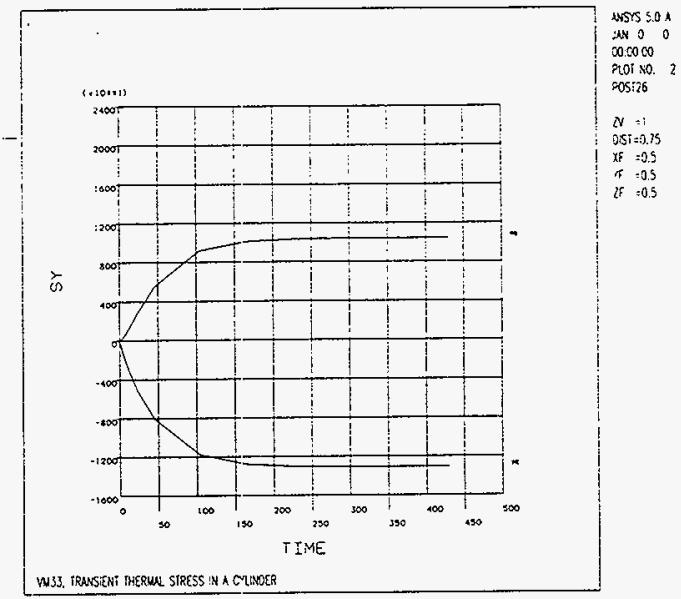

Figure 7

Problem VM33 - Plot 2

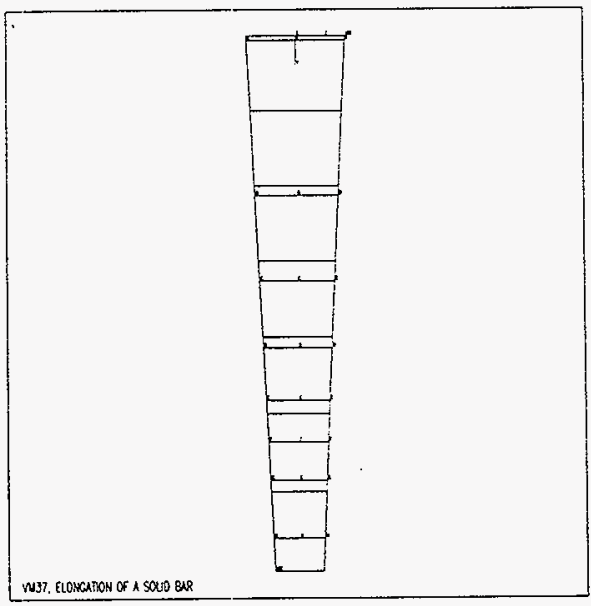

ANS 5.0

WN 0

+o: 00.60

Pot wo. 1

YOON SOLUtCN

STIP $=1$

SUB $=1$

TIUE: $=$ :

RSYS $=0$
(ANO)

OUx $=0.00476$

Solty $=2583$

SUNO $=2464$

SUX $=8598$

Sux $=9660$

$1=2700$

$8=3500$

$c=1300$

a $=5100$

c $=\$ 900$

$r=8700$

c $=7500$

h 78500

Figure 8

Problem VM37 - Plot 1 
ᄂ ว0Id - O๖WA WO|qOId

OL osnb!y

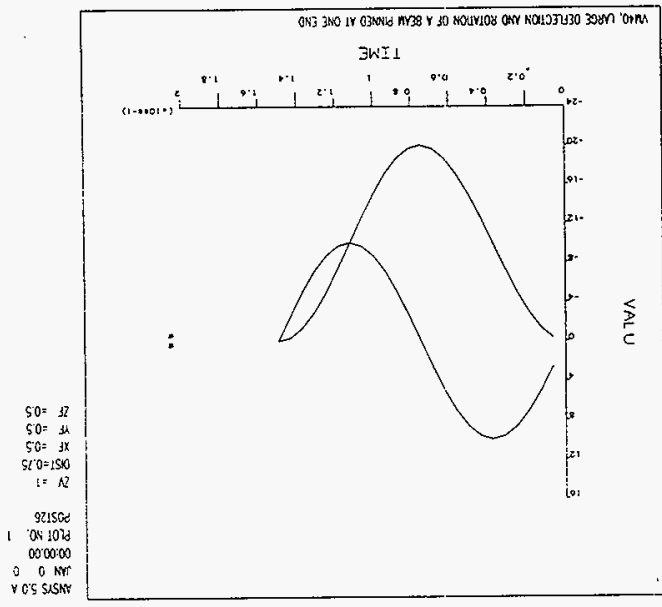

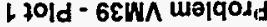 \\ 6 o.n6i!}

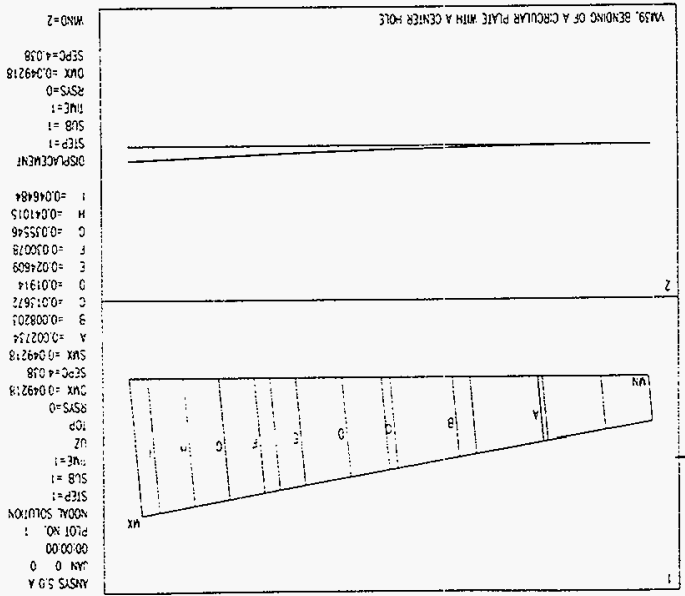

96 to 09 a6ed 0 uo!s!n^əy

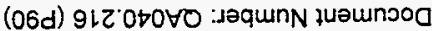

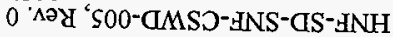

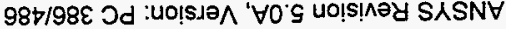

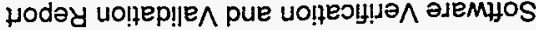


Software Verification and Validation Report ANSYS Revision 5.0A, Version: PC 386/486

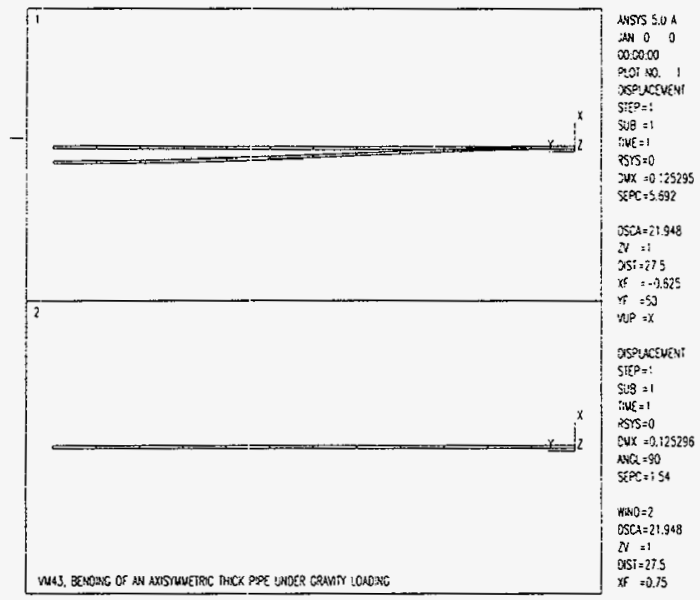

Figure 11

Problem VM43 - Plot 1

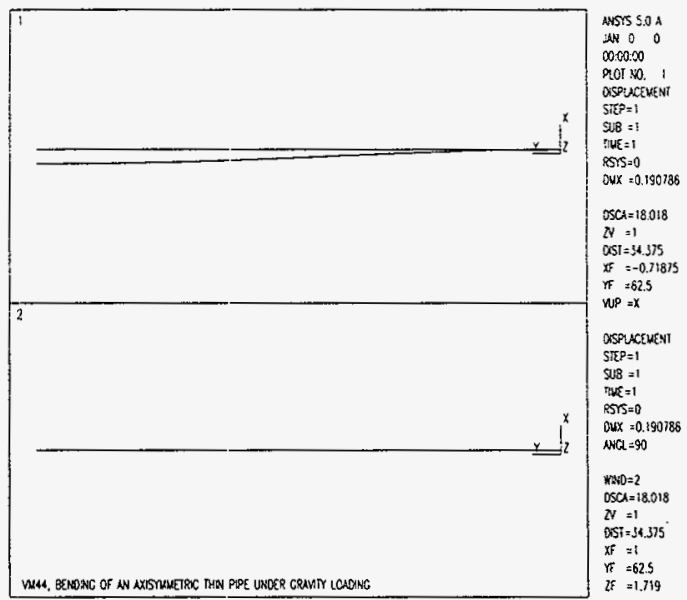

Figure 12

Problem VM44 - Plot 1
Revision 0

Page 61 of 95
HNF-SD-SNF-CSWD-005, Rev. 0 Document Number: QA040.216 (P9O) 


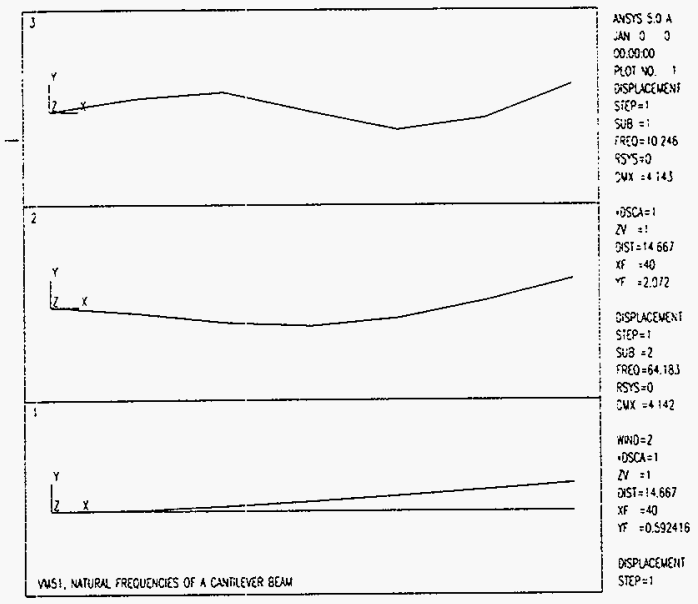

Figure 13

Problem VM51 - Plot 1

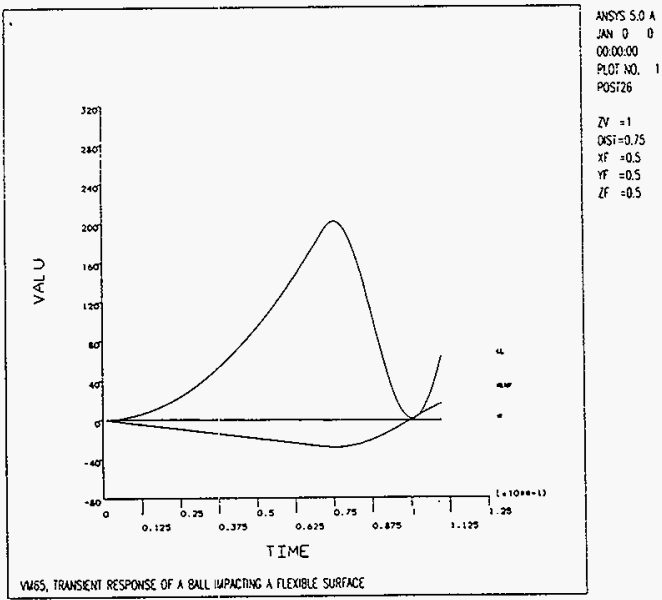

Figure 14

Problem VM65 - Plot 1 
HNF-SD-SNF-CSWD-005, Rev. 0

Software Verification and Validation Report ANSYS Revision 5.0A, Version: PC 386/486
Document Number: QA040.216 (P90)

Revision 0

Page 63 of 95

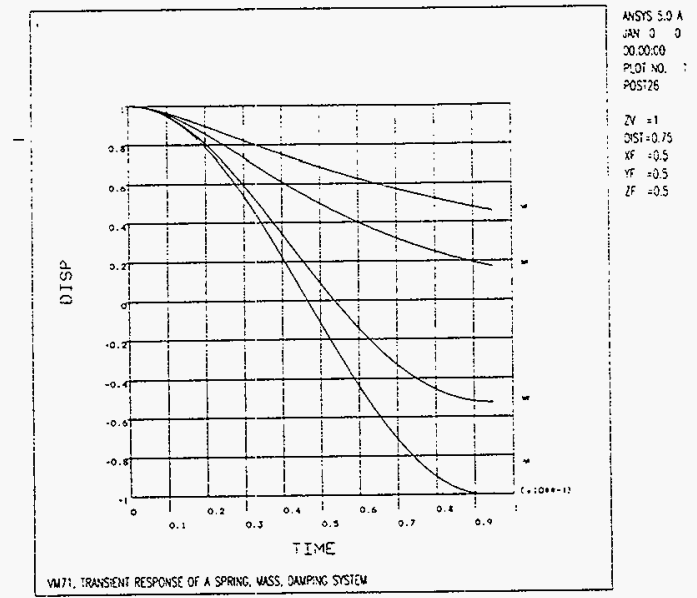

Figure 15

Froblem VM71 - Plot 1

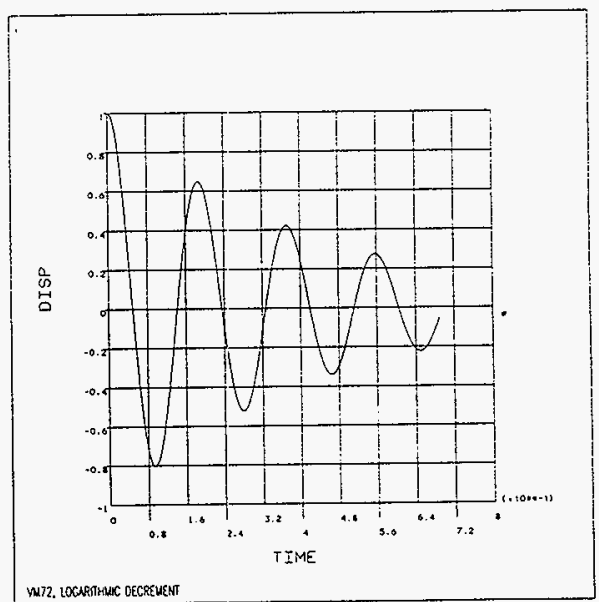




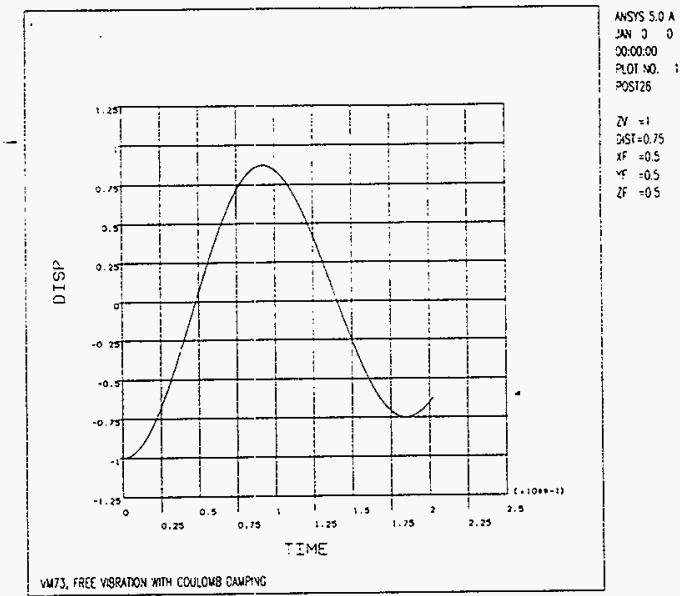

Figure 17

Problem VM73 - Plot 1

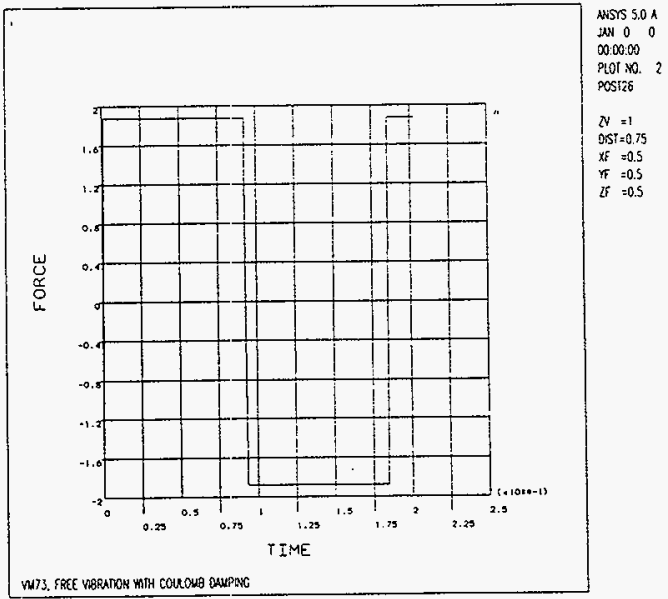

Figure 18

Froblem VM73 - Plot 2 


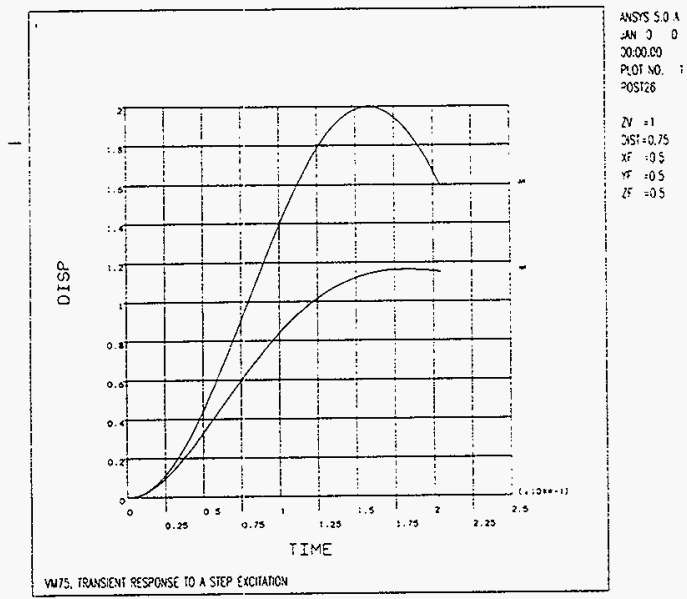

Figure 19

Problem VM75 - Plot 1

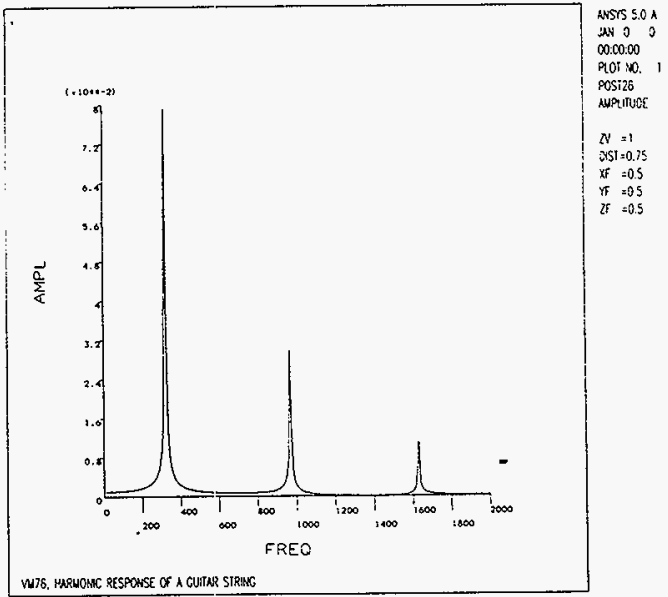

Figure 20

Problem VM76 - Plot 1 
Software Verification and Validation Report ANSYS Revision 5.0A, Version: PC 386/486
HNF-SD-SNF-CSWD-005, Rev. 0 Document Number: QA040.216 (P90)

Revision 0

Page 66 of 95

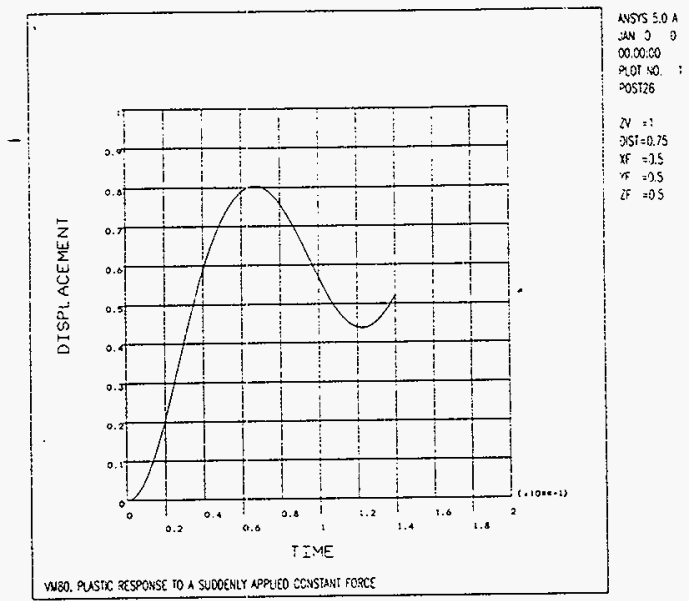

Figure 21

Problem VM80 - Plot 1

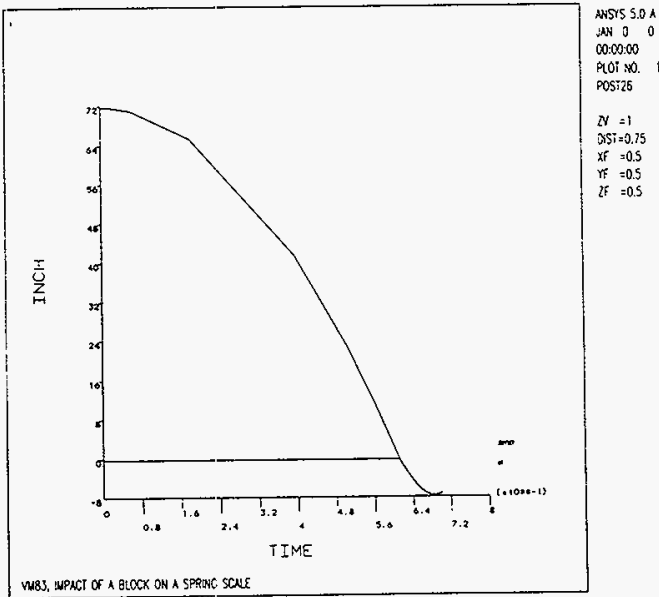

Figure 22

Problem VM83 - Plot 1 
Software Verification and Validation Report ANSYS Revision 5.0A, Version: PC 386/486
HNF-SD-SNF-CSWD-005, Rev. 0 Document Number: QA040.216 (P90)

Revision 0

Page 67 of 95

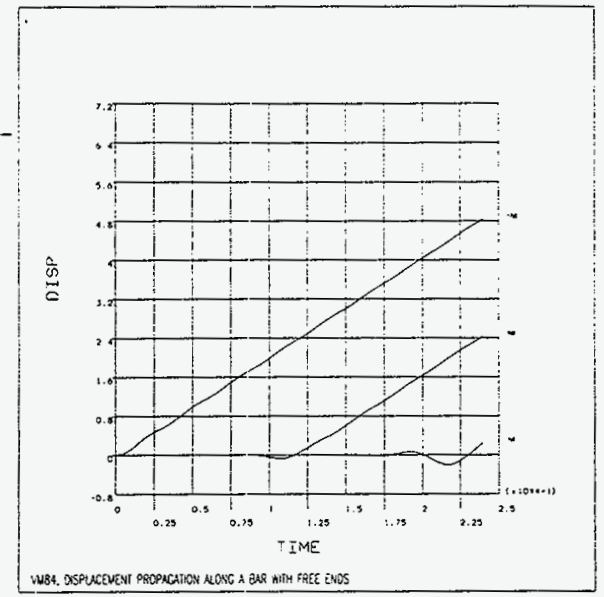

NWSS $5.0 A$

AN 30

9.0? vo

$\cos 26$

$N=1$

$\mathrm{MiST}=0 \pi_{\mathrm{S}}$

जि : क्रा

$x p=0.5$
$x=0.5$

Li, 05

Figure 23

Problem VM84 - Plot 1

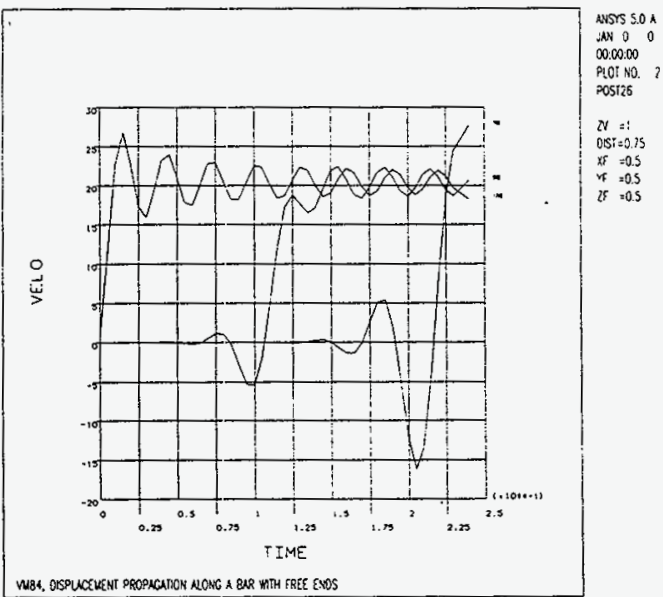

Figure 24

Problem VM84 - Plot 2 
HNF-SD-SNF-CSWD-005, Rev. 0

Software Verification and Validation Report ANSYS Revision 5.0A, Version: PC 386/486

Document Number: QA040.216 (P90)

Revision 0

Page 68 of 95

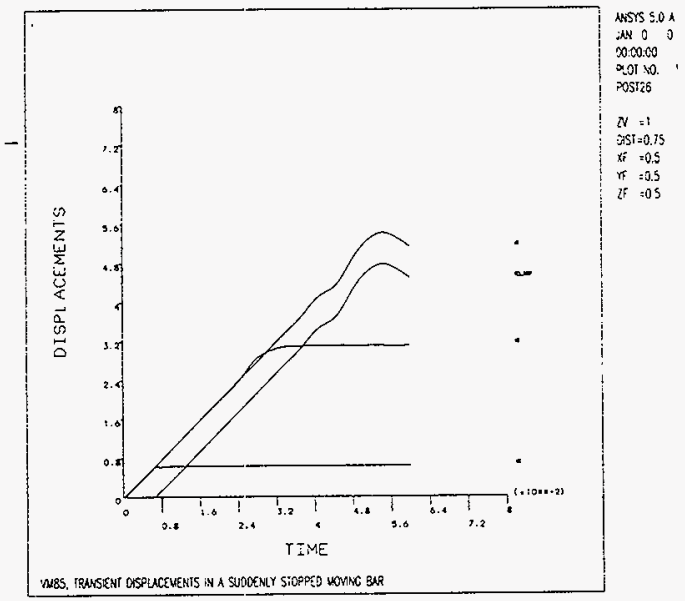

Figure 25

Problem VM85 - Plot 1

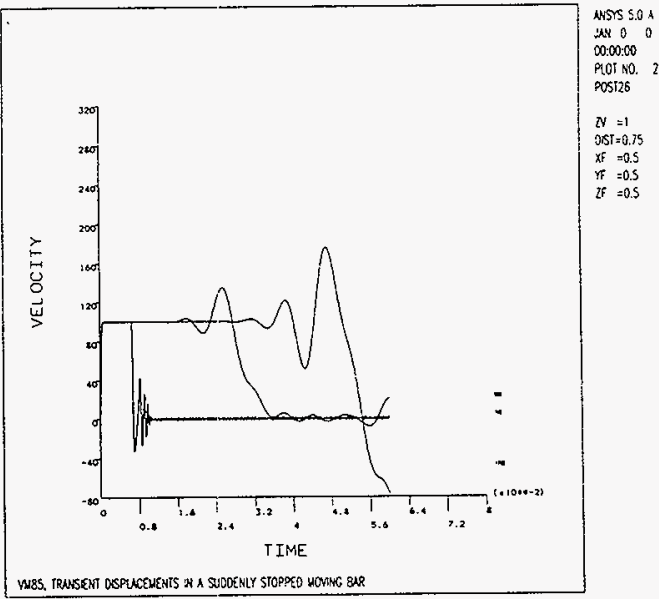

Figure 26

Problem VM85 - Plot 2 
Software Verification and Validation Report ANSYS Revision 5.OA, Version: PC 386/486
HNF-SD-SNF-CSWD-005, Rev. 0 Document Number: QA040.216 (P90) Revision 0 Page 69 of 95

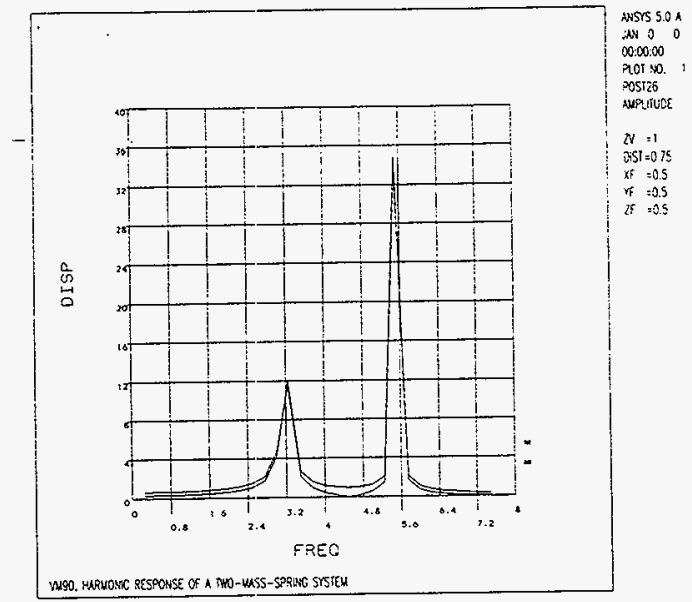

Figure 27

Problem VM90 - Plot 1

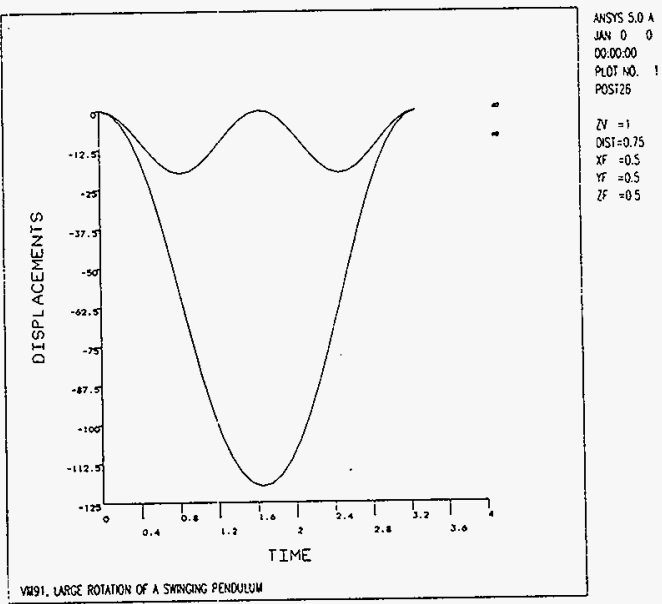

Figure 28

Problem VM91 - Plot 1 


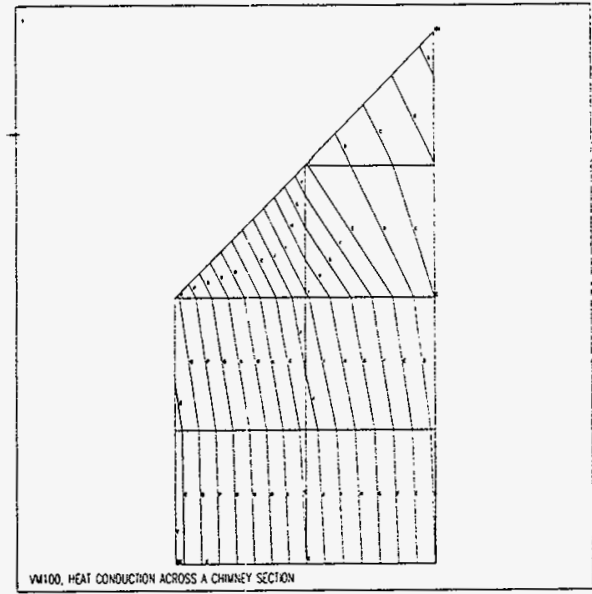

$+0, \div)$;

SOCN SCLTRON

SIEP $=$ :

$\$ \mathrm{~S} B \mathrm{~B}=\mathrm{:}$

TIUE: :

'६भP

$\sum P x=4,388$

SW $=500$ is

$510 x=9 \geq 629$

$A=7475$

9 51239

$c=1 \geqslant 521$

o $=22244$

$\varepsilon=27,67$

$i \quad=32.09$

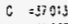

. $=419 ; 1$

i $=4636$

J $\quad \$ 5:, 783$

$x=56.706$

$\begin{aligned} & 1 \\ & 1\end{aligned} \quad-61529$

$4=065.52$

$\mathrm{N}=11.475$

$0=15.598$

$=81322$

$\checkmark=85.245$

$i=91.163$

Figure 29

Problem VM100 - Plot 1

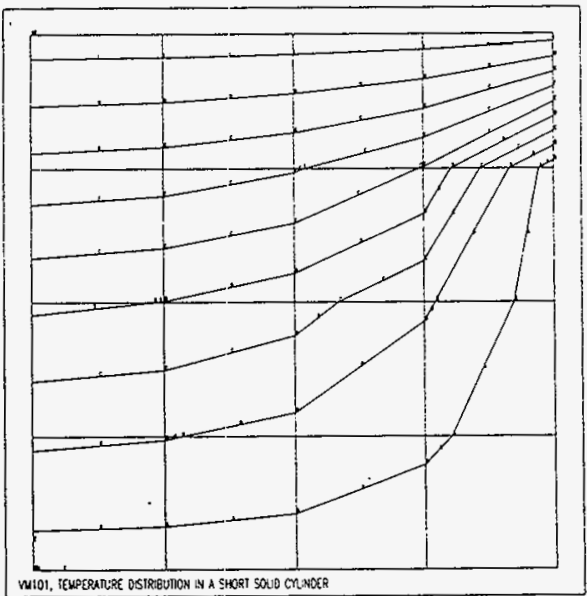

SIEP =!

$S L 3=i$

TIUE $=1$

ICUP

JEPC $=56.909$

$\operatorname{Sin}=40$

k $=2.222$

B $=6.657$

C $=11111$

D $=15.556$

$E=20$

$F=24.412$

$G=28.889$

$H=33.353$

$1=37.178$

Figure 30

Problem VM101 - Plot 1 
Software Verification and Validation Report ANSYS Revision 5.0A, Version: PC 386/486
HNF-SD-SNF-CSWD-005, Rev. 0 Document Number: QA040.216 (P90)

Revision 0

Page 71 of 95

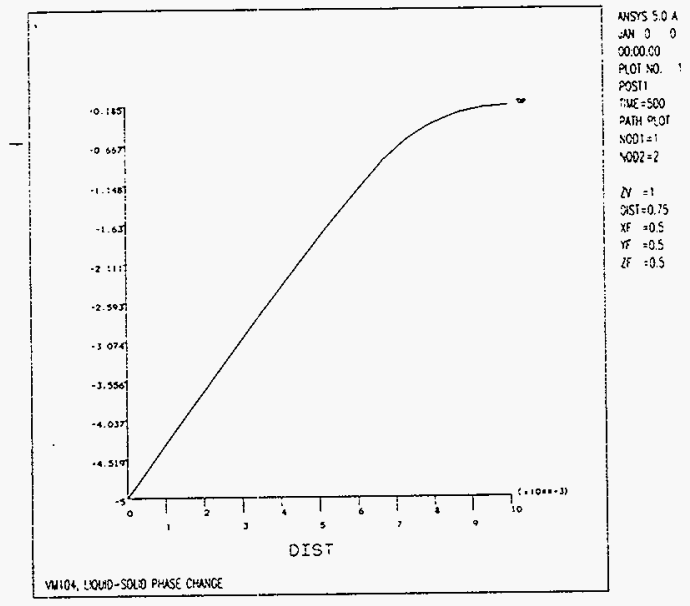

Figure 31

Problem VM104 - Plot 1

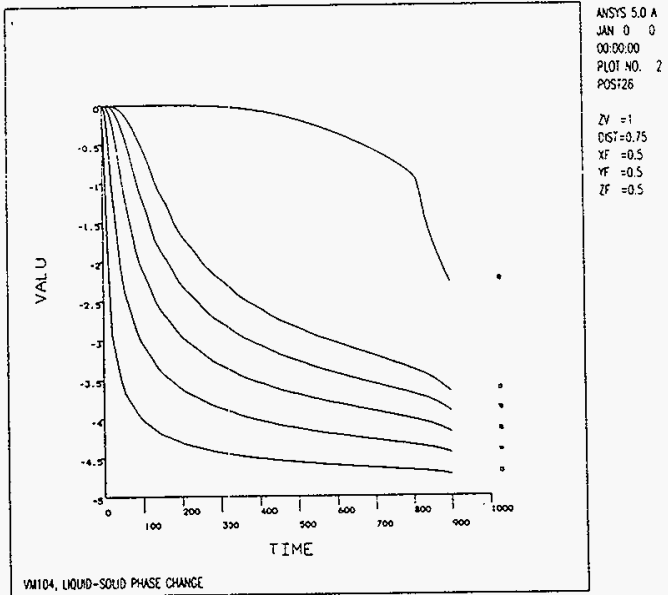

Figure 32

Problem VM104 - Plot 2 


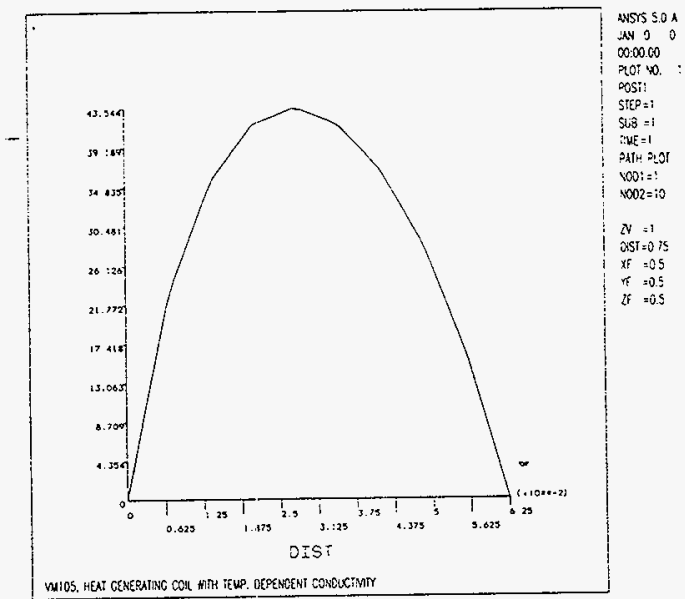

Figure 33

Problem VM105 - Plot 1

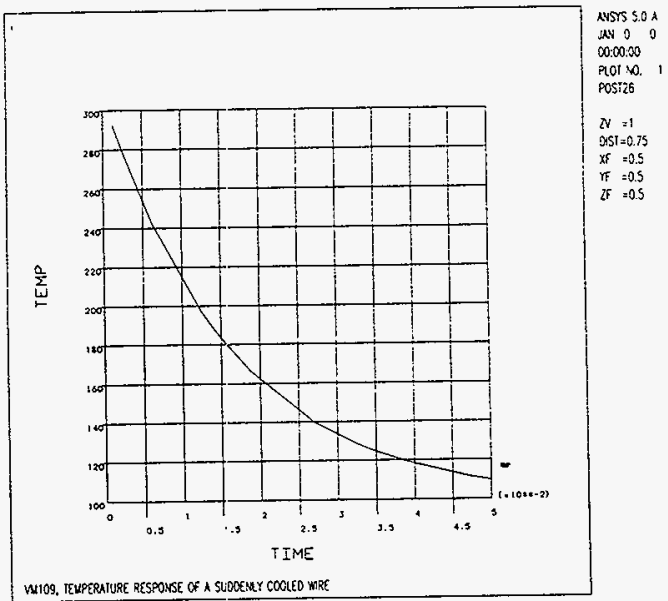

Figure 34

Problem VM109 - Plot 1 
Software Verification and Validation Report ANSYS Revision 5.0A, Version: PC 386/486
HNF-SD-SNF-CSWD-005, Rev. 0 Document Number: QA040.216 (P9O)

Revision 0

Page 73 of 95

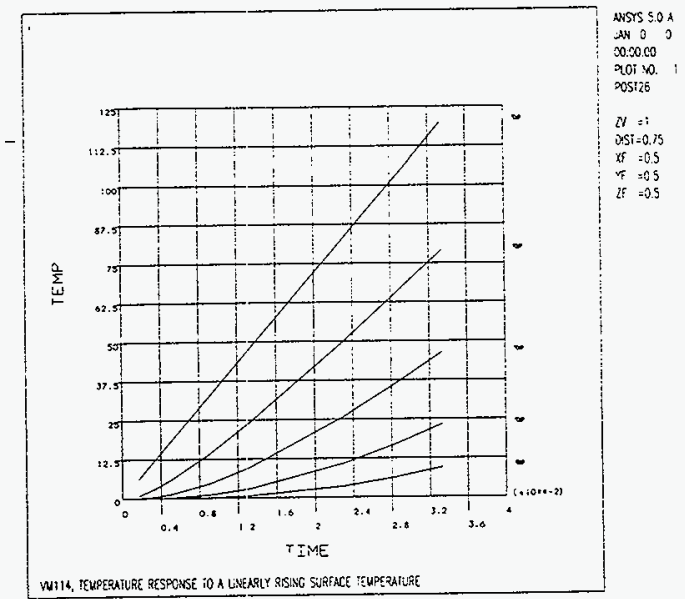

Figure 35

Problem VM114 - Plot 1

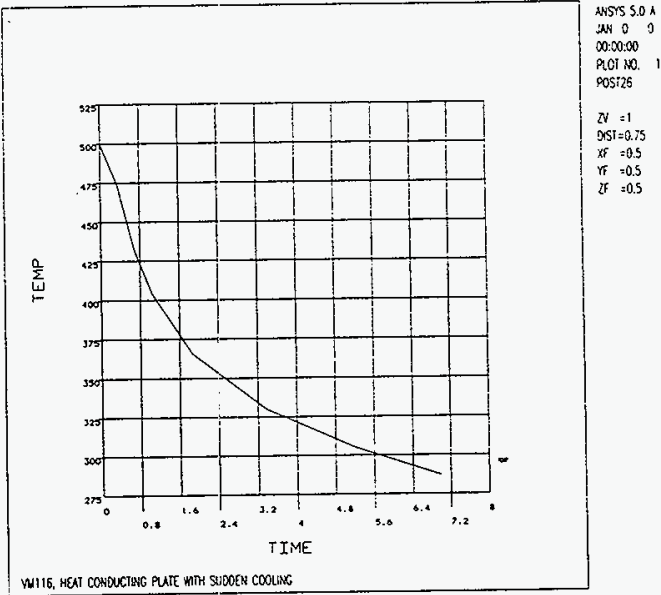

Figure 36

Problem VM116 - Plot 1 


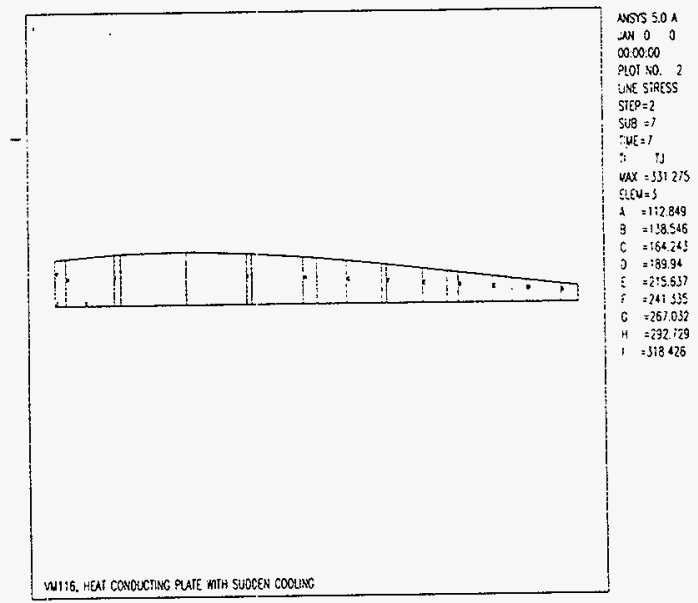

Figure 37

Problem VM116 - Plot 2

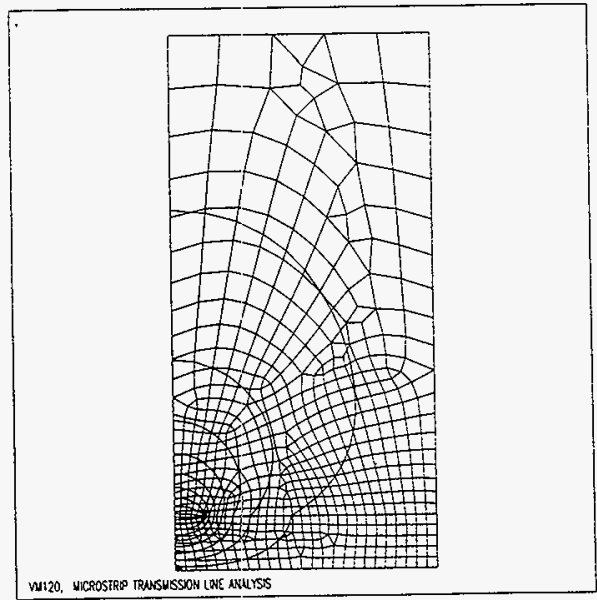

Plol wo. i

SiE $P=1$

Su8 $=1$

ine $=1$

TKS

TEPC $=50.24$

SWN $=0.5$

$s, x=1.5$

$=0.555556$

$8=0.666567$

C $=0.777758$

$0=0.883889$

$\varepsilon=1$

$=1.111$

G $=1.222$

$H=1.533$

$\quad \simeq 1444$

Figure 38

Problem VM120 - Plot 1 
HNF-SD-SNF-CSWD-005, Rev. 0

Software Verification and Validation Report ANSYS Revision 5.0A, Version: PC 386/486
Document Number: QA040.216 (P90)

Revision 0

Page 75 of 95

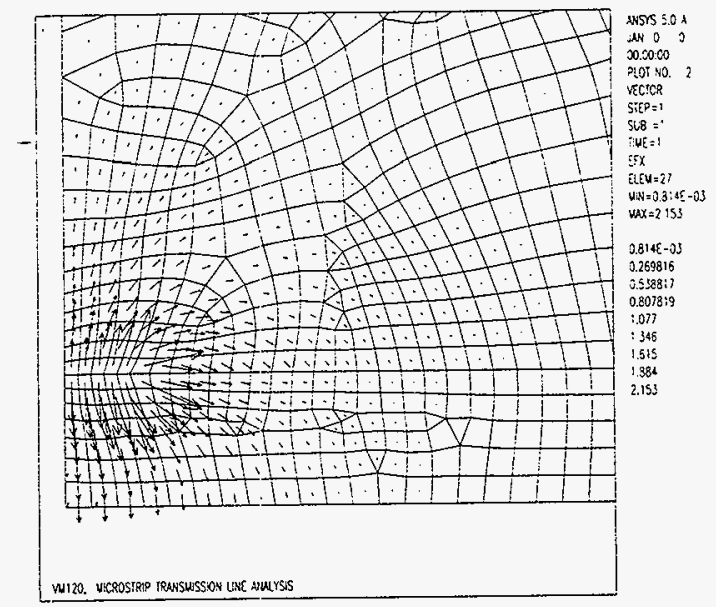

Figure 39

Problem VM120 - Plot 2

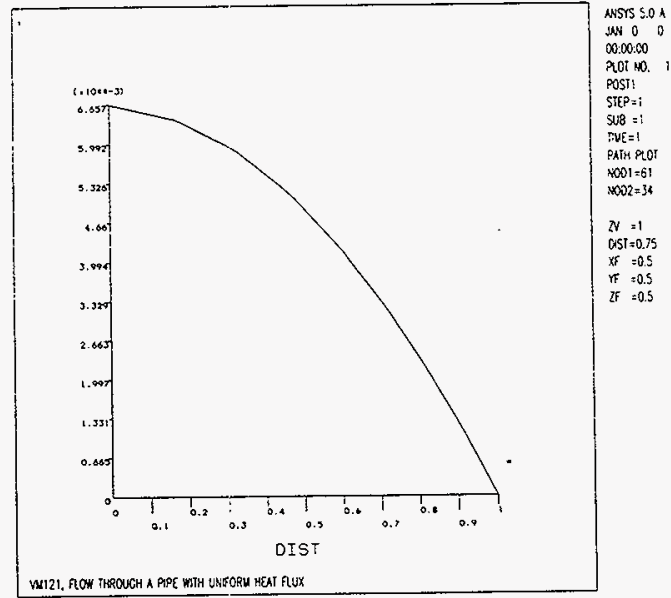

Figure 40

Problem VM121 - Plot 1 
Software Verification and Validation Report

ANSYS Revision 5.0A, Version: PC 386/486

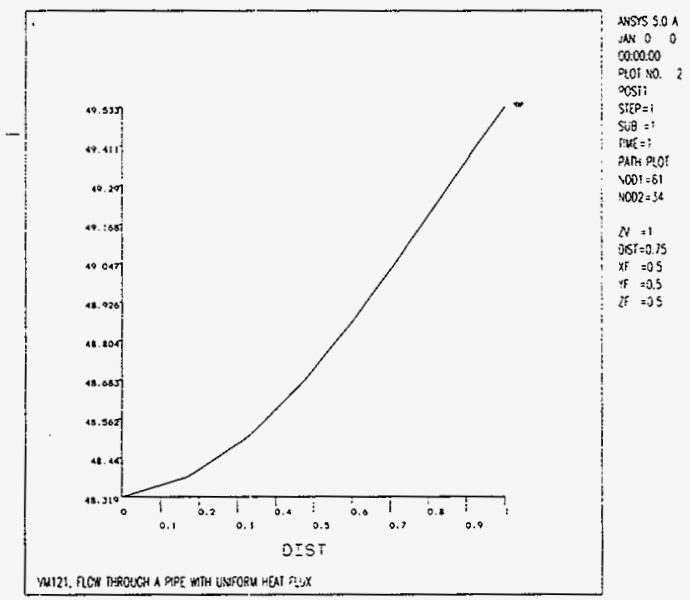

Figure 41

Problem VM121 - Plot 2

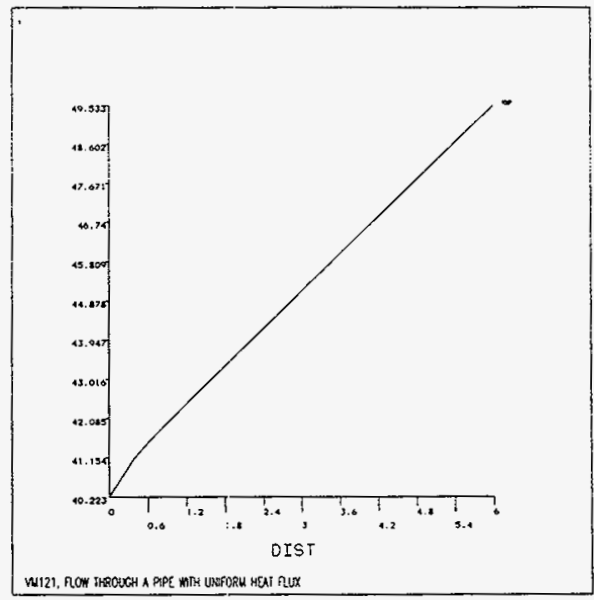

Figure 42

Problem VM121 - Plot 3
Revision 0

Page 76 of 95 


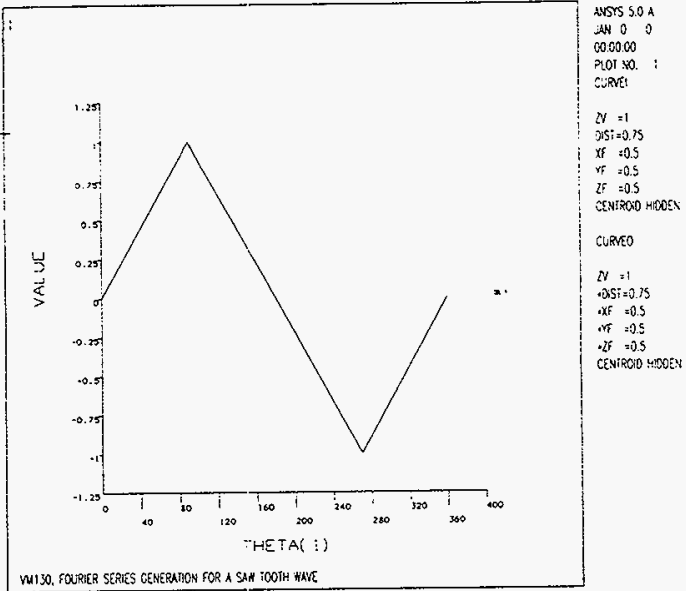

Figure 43

Problem VM130 - Plot 1

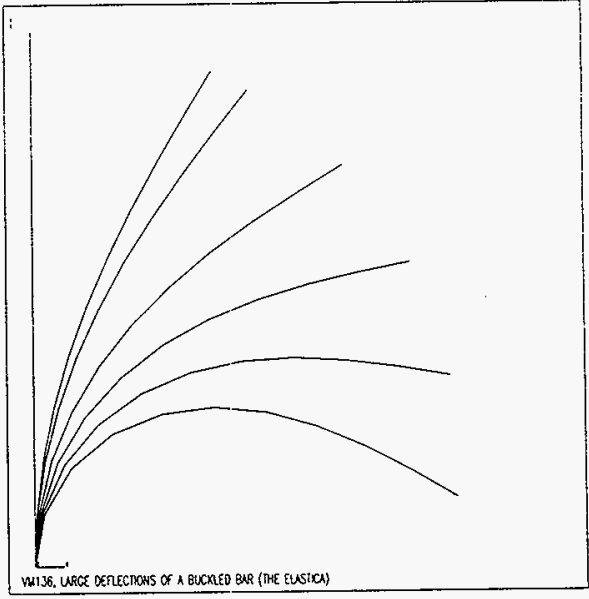

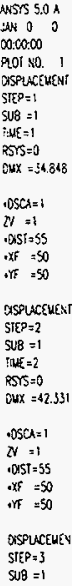

Figure 44

Problem VM136 - Plot 1 
HNF-SD-SNF-CSWD-005, Rev. 0

Software Verification and Validation Report ANSYS Revision 5.0A, Version: PC 386/486
Document Number: QA040.216 (P90)

Revision 0

Page 78 of 95

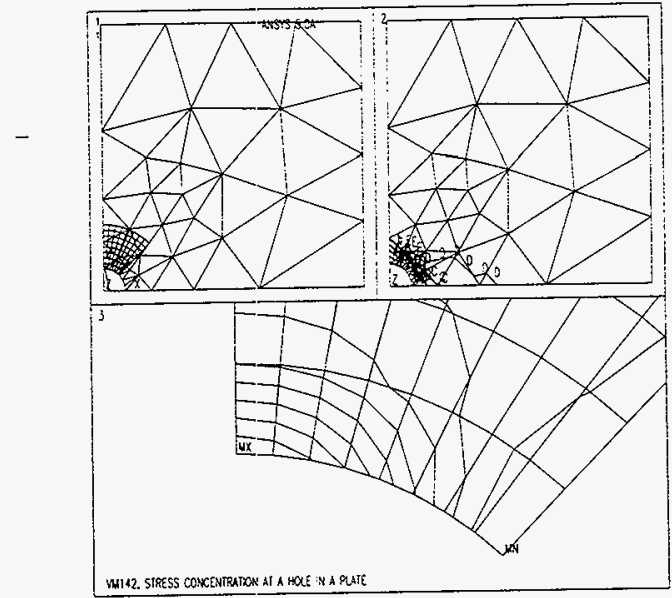

Figure 45

Problem VM142 - Plot 1

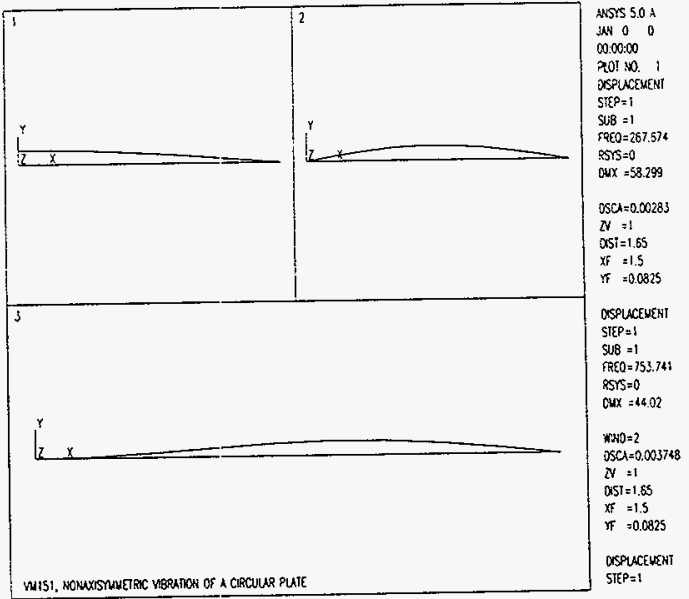

Figure 46

Problem VM151 - Plot 1 
HNF-SD-SNF-CSWD-005, Rev. 0

Software Verification and Validation Report ANSYS Revision 5.0A, Version: PC 386/486

Document Number: QA040.216 (P90)

Revision 0

Page 79 of 95

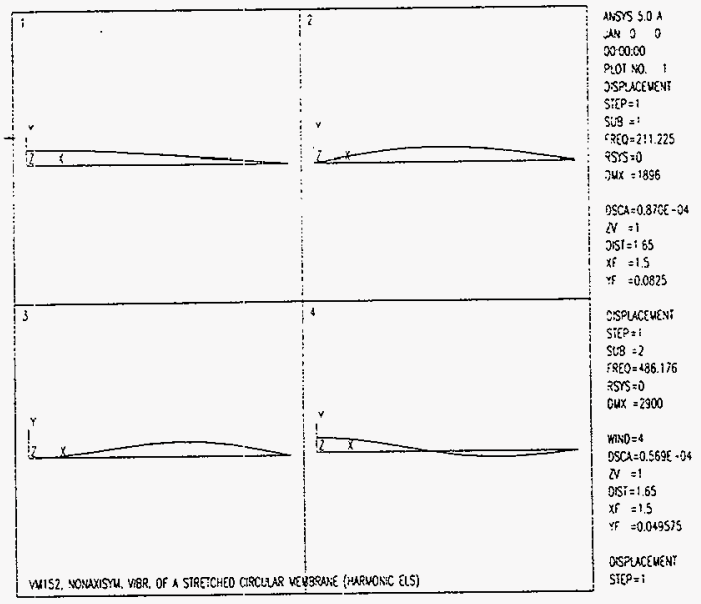

Figure 47

Problem VM152 - Plot 1

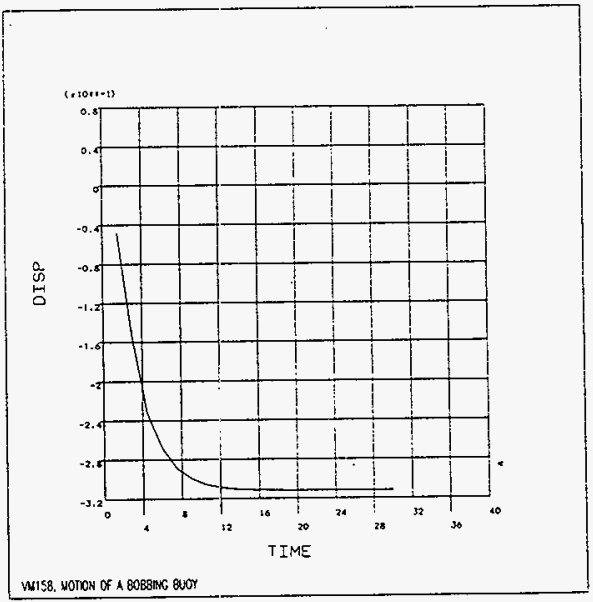

NSSS $5.0 \mathrm{~A}$

in: 00

00.0000

PloI 10.

posize

IV $=1$

NAST $=0.75$

$x_{\text {ff }}=0.5$

if $=0.5$

2f $=0.5$

Figure 48

Problem VM158 - Plot 1 


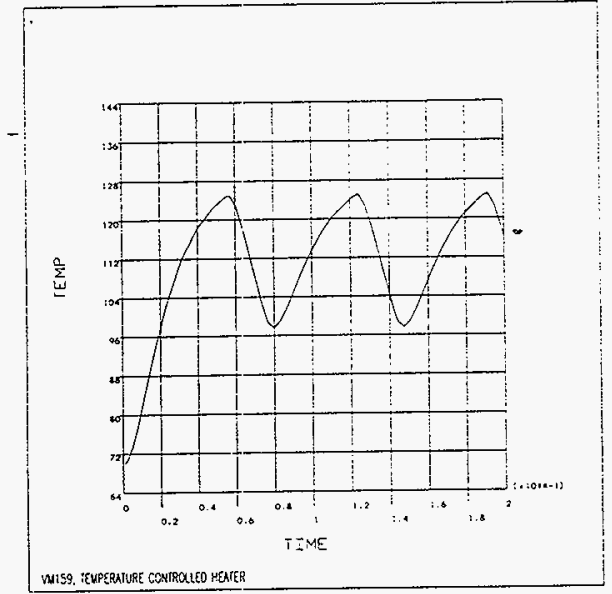

positis

$N=1$

Figure 49

Problem VM159 - Plot 1

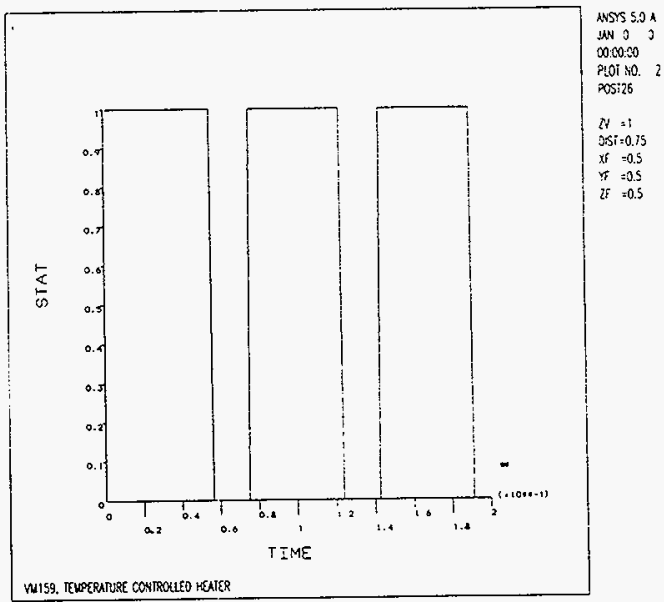

Figure 50

Problem VM159 - Plot 2 
Software Verification and Validation Report ANSYS Revision 5.0A, Version: PC 386/486
HNF-SD-SNF-CSWD-005, Rev. 0 Document Number: QA040.216 (P90)

Revision 0

Page 81 of 95

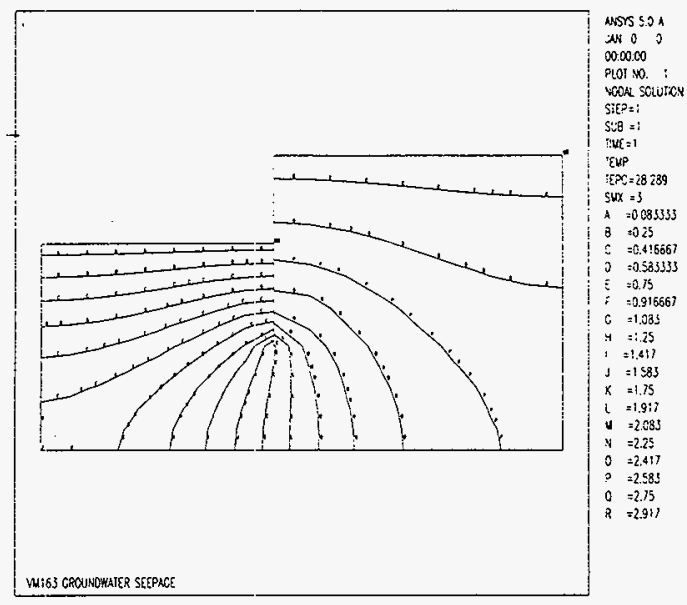

Figure 51

\section{Problem VM163 - Plot 1}

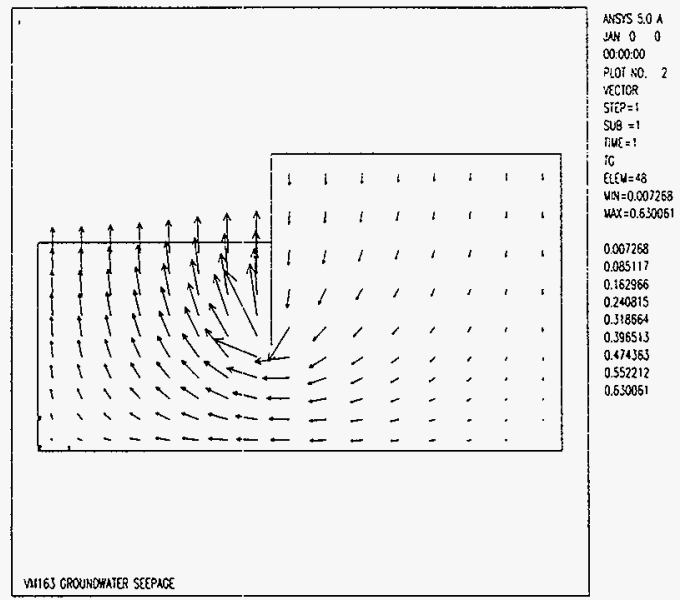

Figure 52

Problem VM163 - Plot 2 
HNF-SD-SNF-CSWD-005, Rev. 0

Software Verification and Validation Report ANSYS Revision 5.0A, Version: PC 386/486

Revision 0

Page 82 of 95

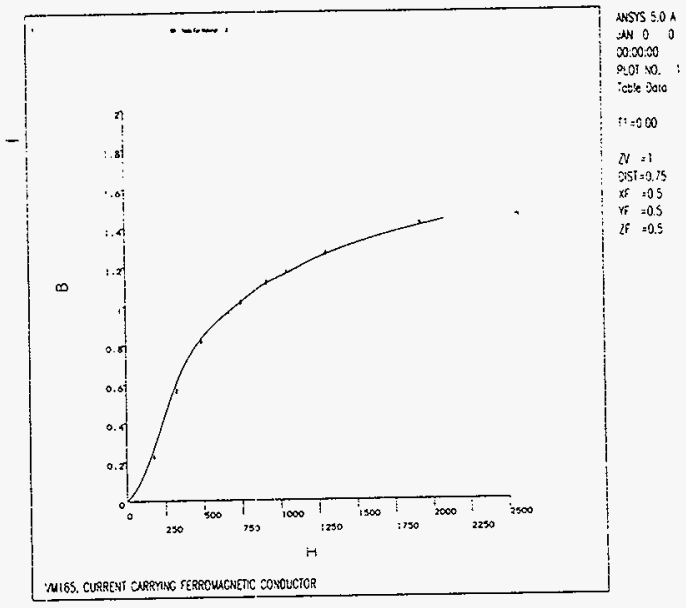

Figure 53

Problem VM165 - Plot 1

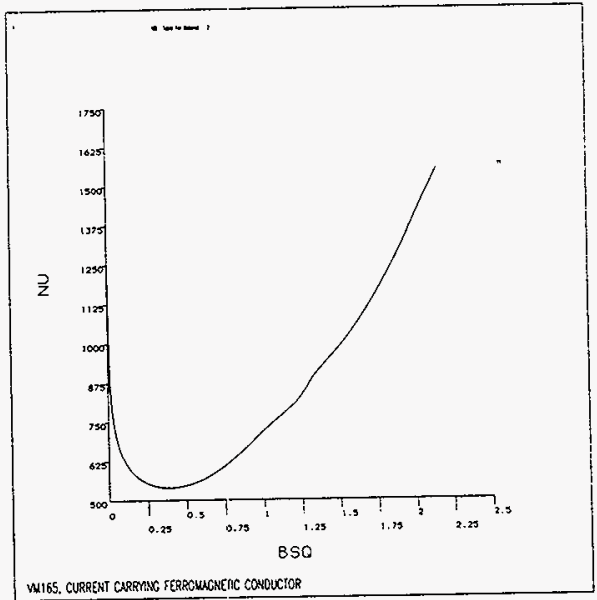

NWSYS $5.0 \mathrm{~A}$

IN 0 O 0

00.00 .00

91.0I :10. 2

ictle follo

$11=0.00$

$N=1$
$0.5 i=0.75$

$x=0.5$

$r=0.5$

$r=0.5$

Figure 54

Problem VM165 - Plot 2 
HNF-SD-SNF-CSWD-005, Rev. 0

Software Verification and Validation Report ANSYS Revision 5.0A, Version: PC 386/486

Document Number: QA040.216 (P9O)

Revision 0

Page 83 of 95

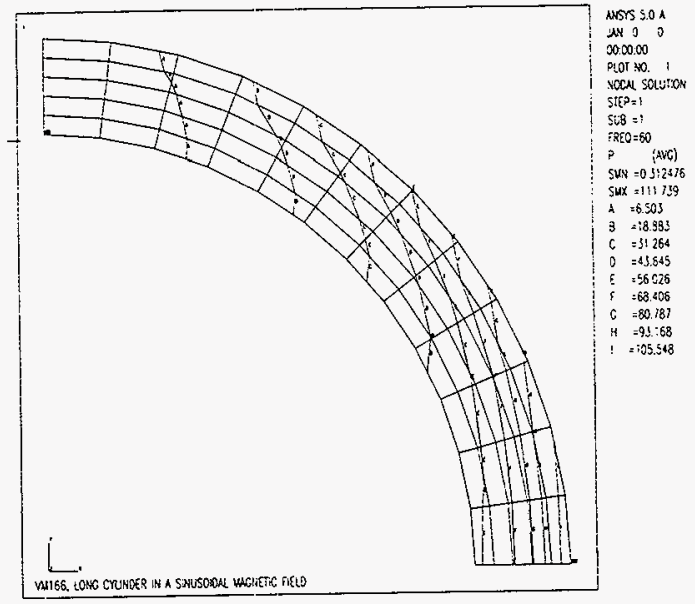

Figure 55

Problem VM166 - Plot 1

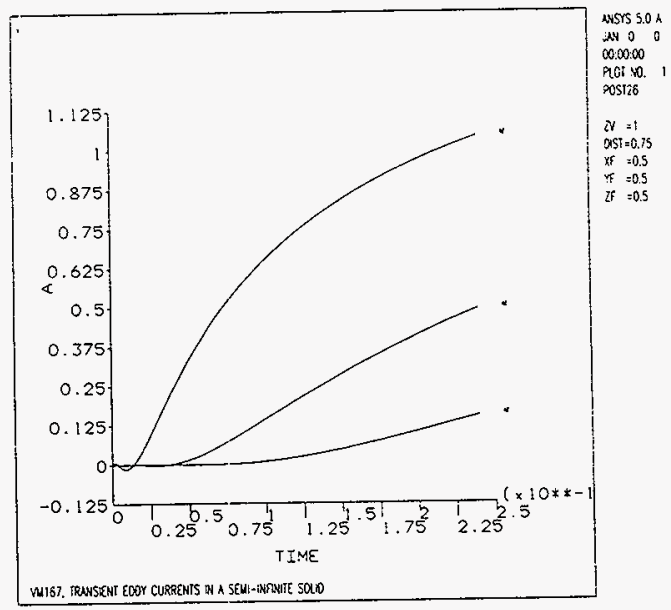

Figure 56

Problem VM167 - Plot 1 
Software Verification and Validation Report ANSYS Revision 5.0A, Version: PC 386/486
HNF-SD-SNF-CSWD-005, Rev. 0 Document Number: QA040.216 (P90)

Revision 0

Page 84 of 95

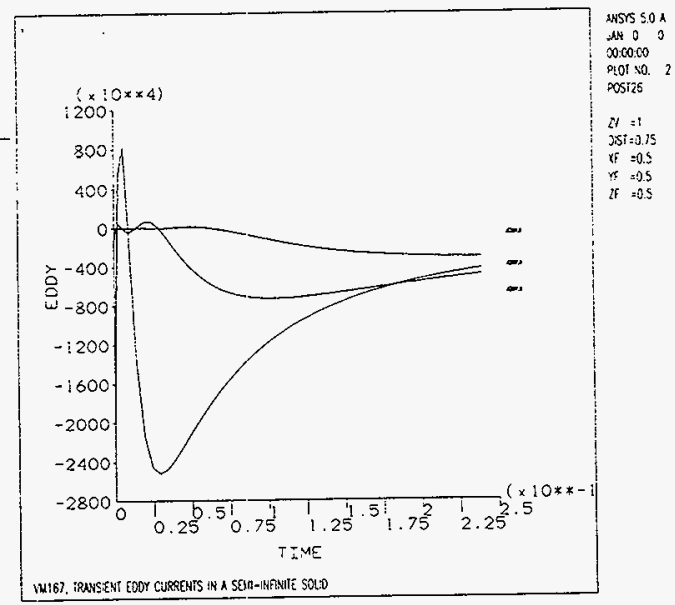

Figure 57

Ploblem VM167 - Plot 2

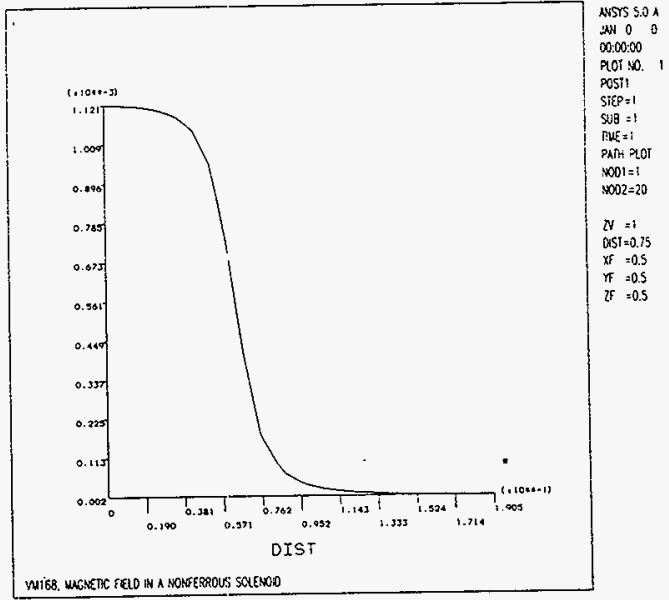

Figure 58

F'roblem VM168 - Plot 1 
HNF-SD-SNF-CSWD-005, Rev. 0

Software Verification and Validation Report ANSYS Revision 5.0A, Version: PC 386/486

Document Number: QA040.216 (P90)

Revision 0

Page 85 of 95

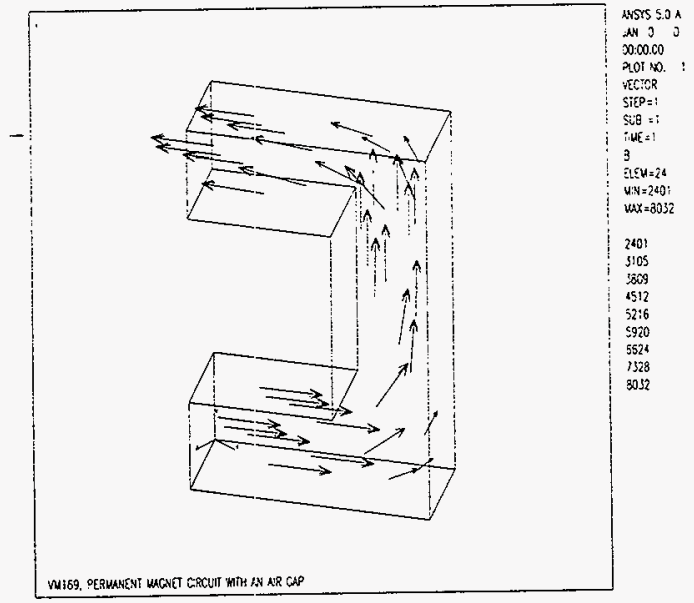

Figure 59

Problem VM169 - Plot 1

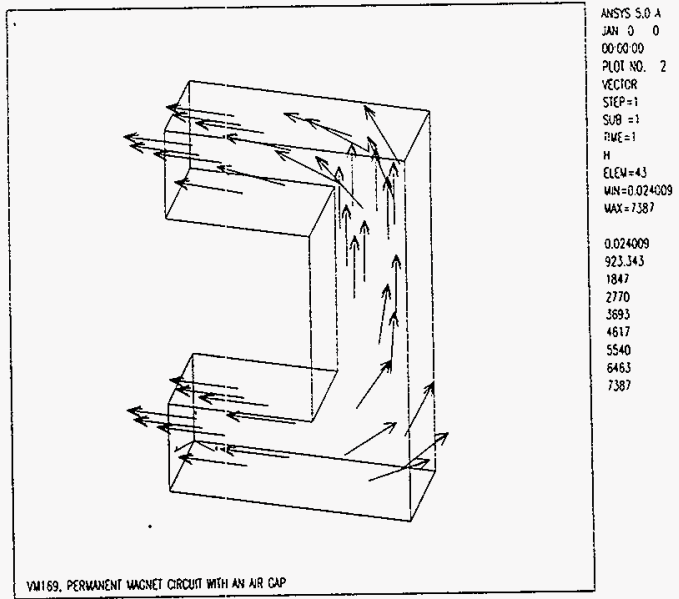

Figure 60

Problem VM169 - Plot 2 
Software Verification and Validation Report ANSYS Revision 5.OA, Version: PC 386/486
HNF-SD-SNF-CSWD-005, Rev. 0 Document Number: QA040.216 (P90)

Revision 0

Page 86 of 95

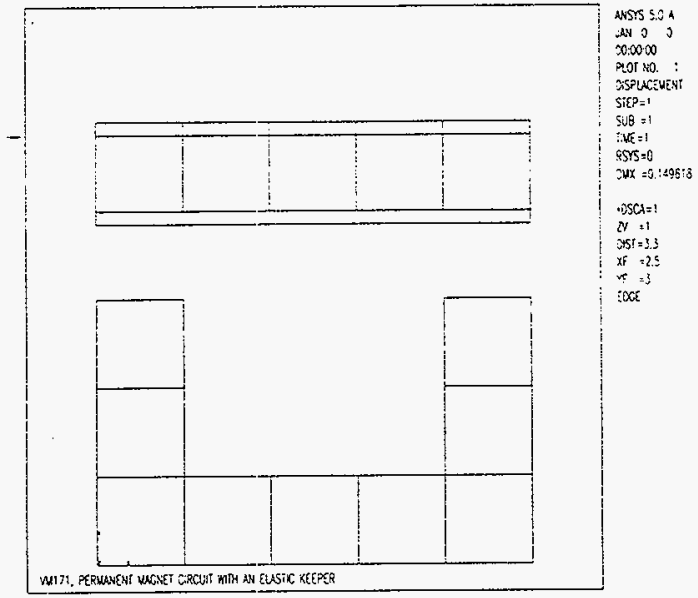

Figure 61

Problem VM171 - Plot 1

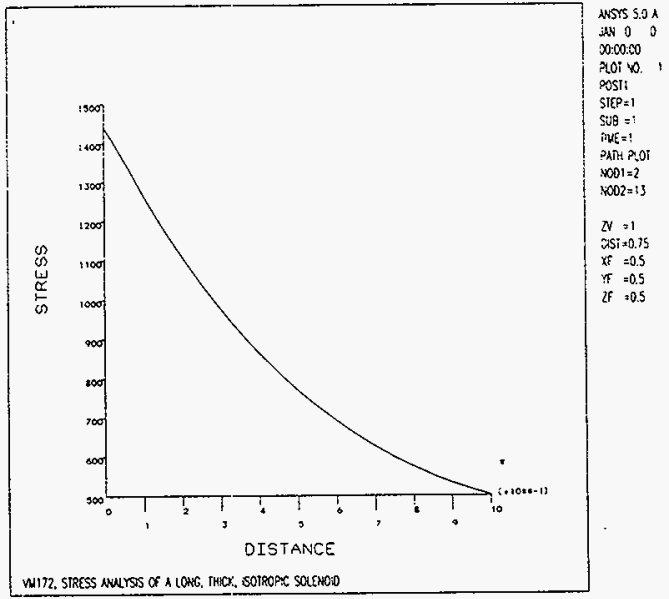

Figure 62

Problem VM172 - Plot 1 
Software Verification and Validation Report ANSYS Revision 5.0A, Version: PC 386/486

HNF-SD-SNF-CSWD-005, Rev. 0 Document Number: QA040.216 (P90)

Revision 0

Page 87 of 95

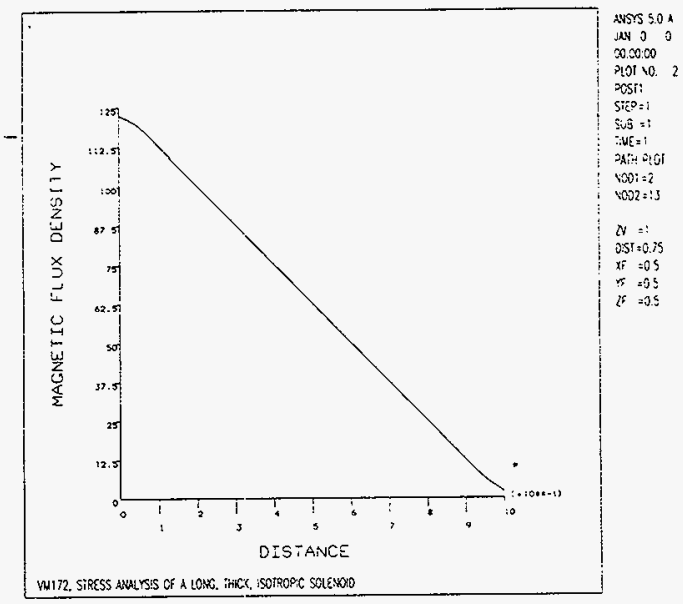

Figure 63

Problem VM172 - Plot 2

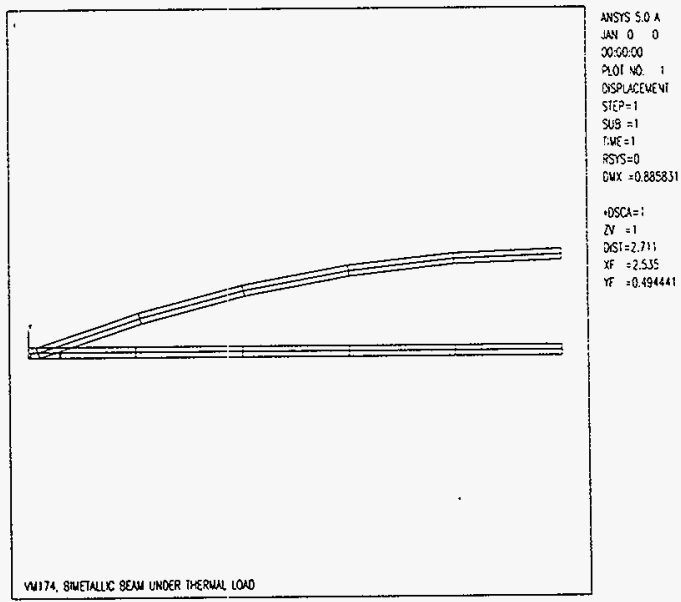

Figure 64

Problem VM174 - Plot 1 

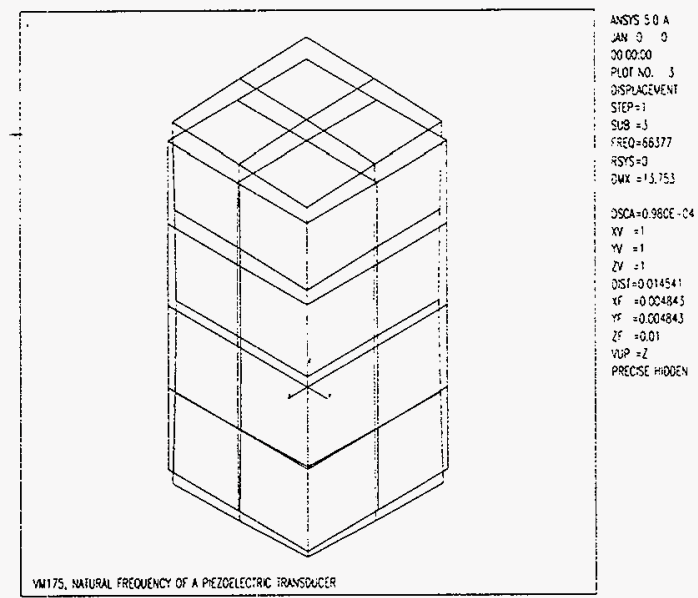

Figure 65

Problem VM175 - Plot 3

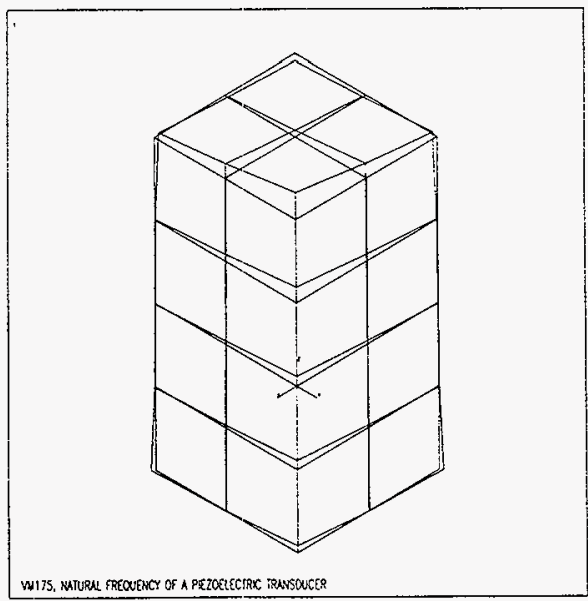

STEP=1

$S T E P=1$
$S 18=5$

FR[0 899969

TSTS $=0$

DHx $=37: 97$

$0 \sec =0.432 E-1.4$

WV $=1$

$w=1$

$N=1$

OAST $=0.014004$

$x=0.005307$

$\sqrt{5}=0.005 .307$

if $=$ i. 0105

une $=l$

PRCCSE HIDDEY

Figure 66

Problem VM175 - Plot 6 


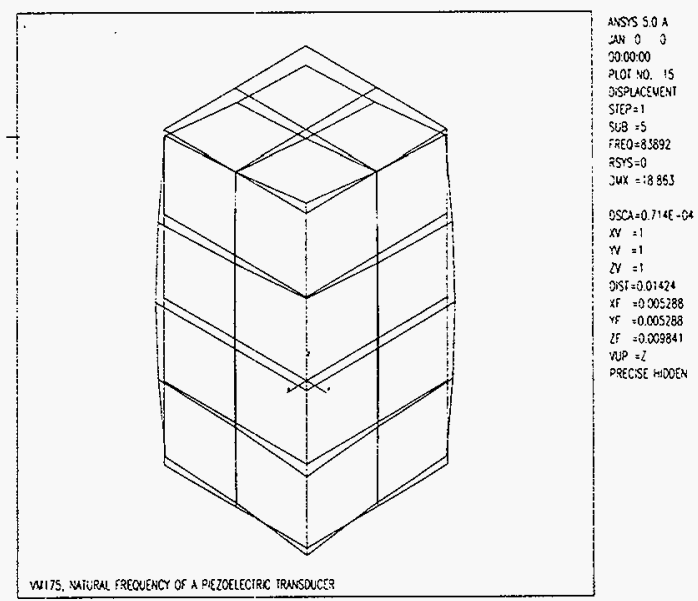

Figure 67

Problem VM175 - Plot 15

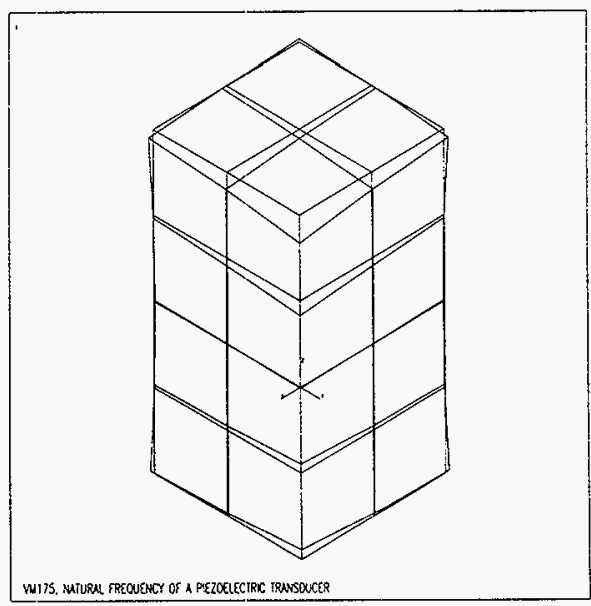

ALSTS $5.0 \mathrm{~A}$

यN 00

000000

PlOF No. 19

OSSPUCENENT

$S T E P=1$

SUP $=9$

EREO $=96453$

QSTS $=0$

6uX $=35.58$

$O S C A=0.357 E-x$

$x=1$

$w=1$

$N=1$

OSI $=0.013926$

$X 5=0.005289$

Yf $=0.005288$

If $=0.009077$

nPP $=2$

PRECSE HODEN

Figure 68

Problem VM175 - Plot 19 
Software Verification and Validation Report ANSYS Revision 5. OA, Version: PC 386/486
HNF-SD-SNF-CSWD-005, Rev. 0

Document Number: QA040.216 (P9O)

Revision 0

Page 90 of 95

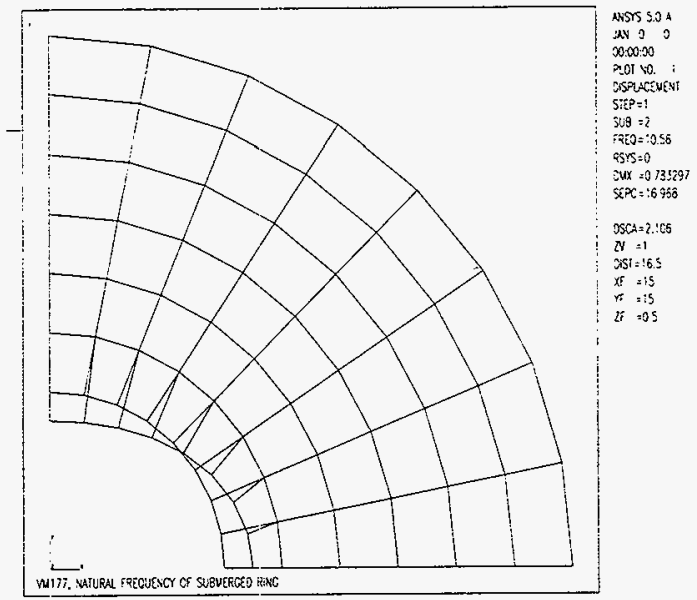

Figure 69

Problem VM177 - Plot 1

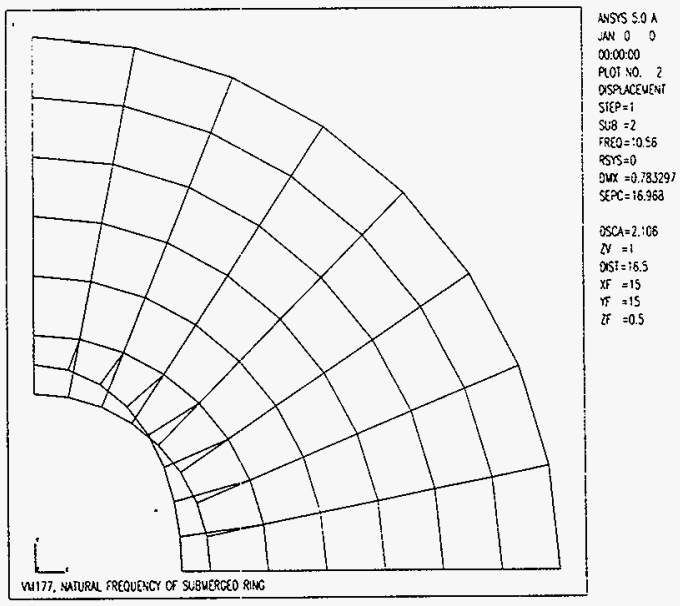

Figure 70

Problem VM177 - Plot 2 
Software Verification and Validation Report ANSYS Revision 5.0A, Version: PC 386/486

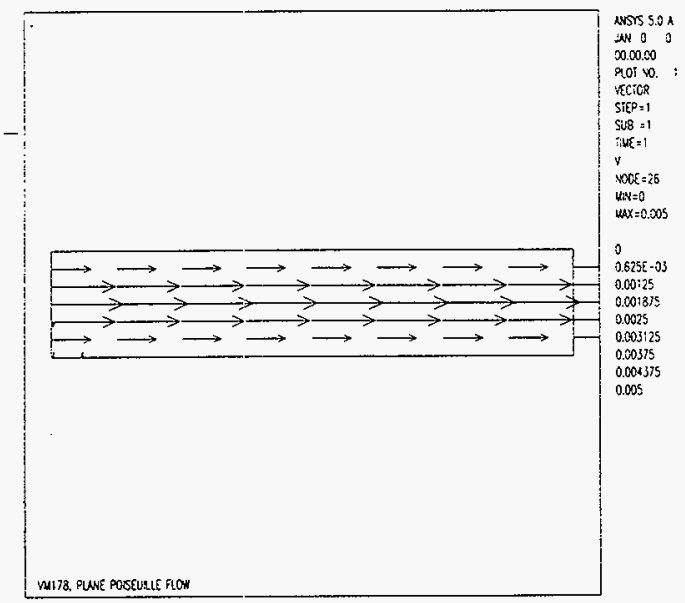

Figure 71

Problem VM178 - Plot 1

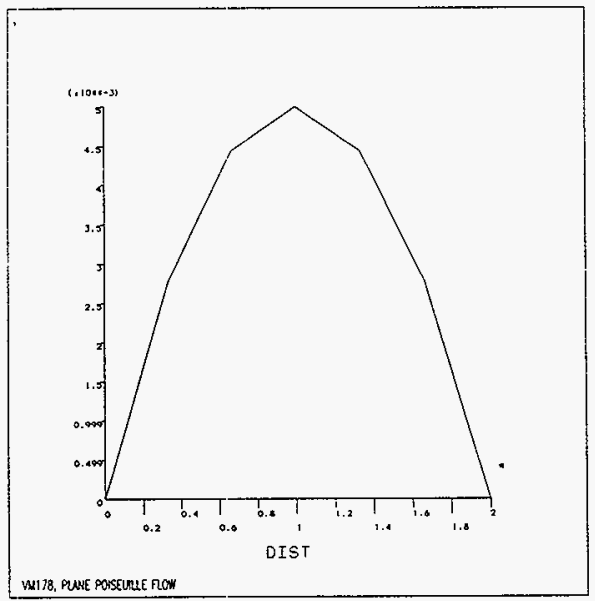

SIEP $=1$

$S 48=1$

대타 $=1$

PAIH PLOI

$4001=2$

$4002=10$

N $N=1$

OASI $=0.15$

$x=0.5$

$\pi=0.5$

if $=0.5$

Figure 72

Problem VM178 - Plot 2 
HNF-SD-SNF-CSWD-005, Rev. 0

Software Verification and Validation Report Document Number: QA040.216 (P9O) ANSYS Revision 5.0A, Version: PC 386/486

Revision 0

Page 92 of 95

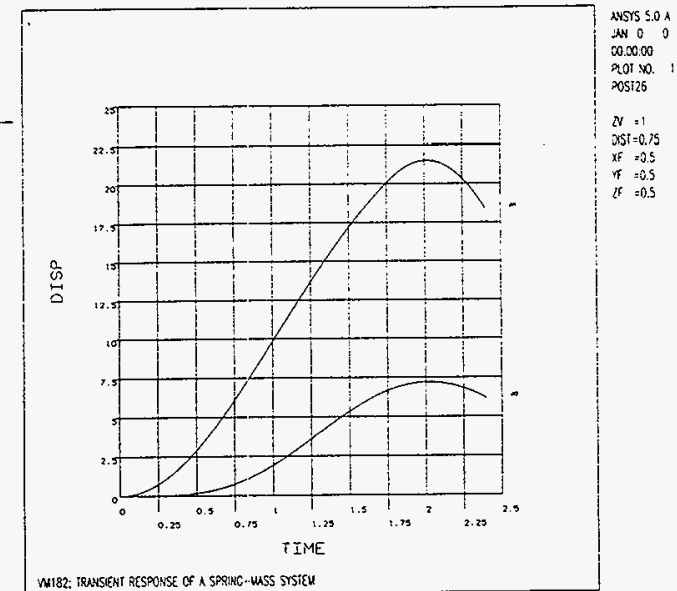

Figure 73

Problem VM182 - Plot 1

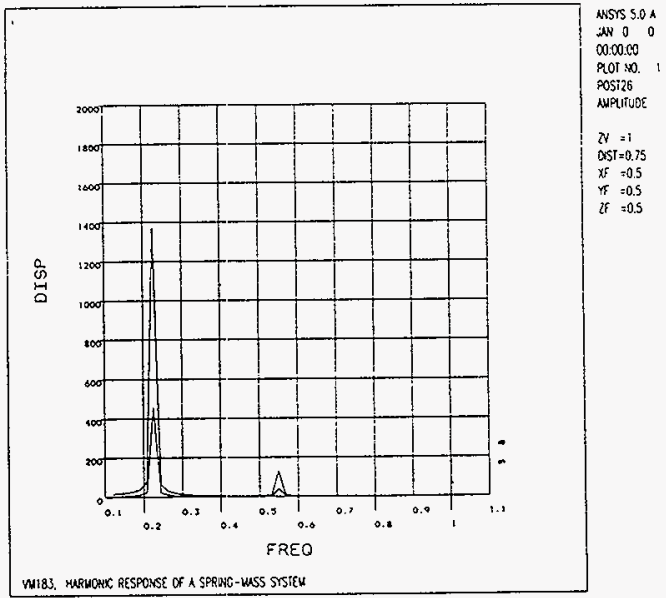

Figure 74

Problem VM183 - Plot 1 
Software Verification and Validation Report ANSYS Revision 5.OA, Version: PC 386/486
HNF-SD-SNF-CSWD-005, Rev. 0 Document Number: QA040.216 (P9O) Revision 0

Page 93 of 95
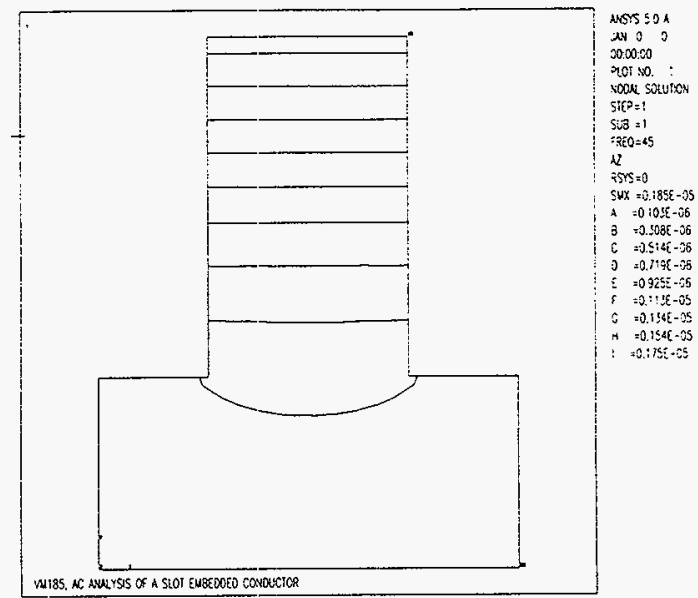

Figure 75

Problem VM185 - Plot 1
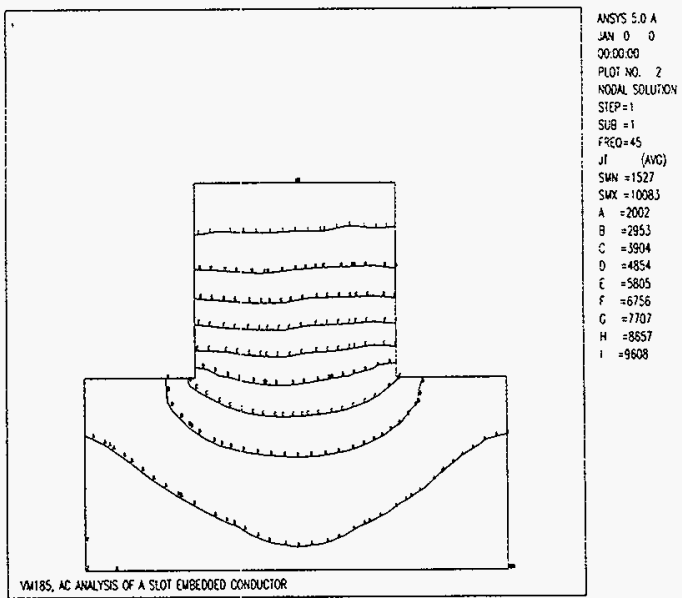

Figure 76

Problem VM185 - Plot 2 
Software Verification and Validation Report ANSYS Revision 5.0A, Version: PC 386/486
HNF-SD-SNF-CSWD-005, Rev. 0 Document Number: QA040.216 (P9O)

Revision 0

Page 94 of 95

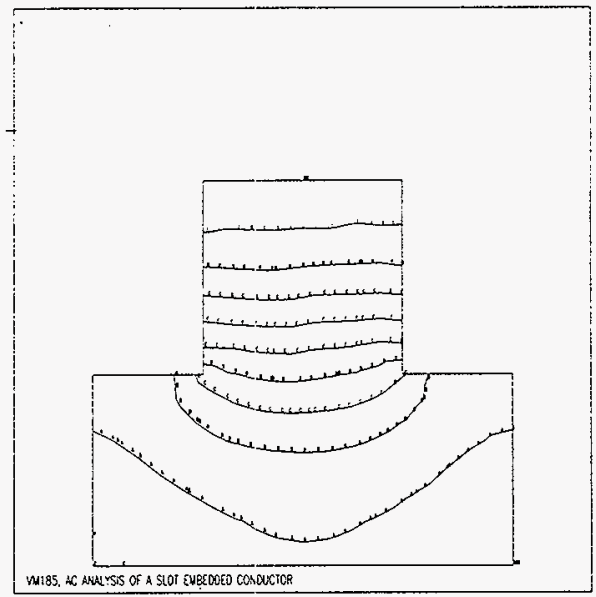

ass 550

dis 0

000000

pigi 10.

SCON SOLvine

$S: 5 P=1$

Si $=$ :

rREO $=45$

SE $\quad(A N C)$
SNA $=-8508$

SwX $=-5 ! 638$

$A=-0133$

$\hat{y}=-3 i 8 \hat{2}$

$c=-6231$

$0=-5281$

$=-4350$

$F=-3379$

$\hat{v}=-2+28$

H. $=-14 / 8$

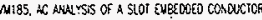

Figure 77

Problem VM185 - Plot 3

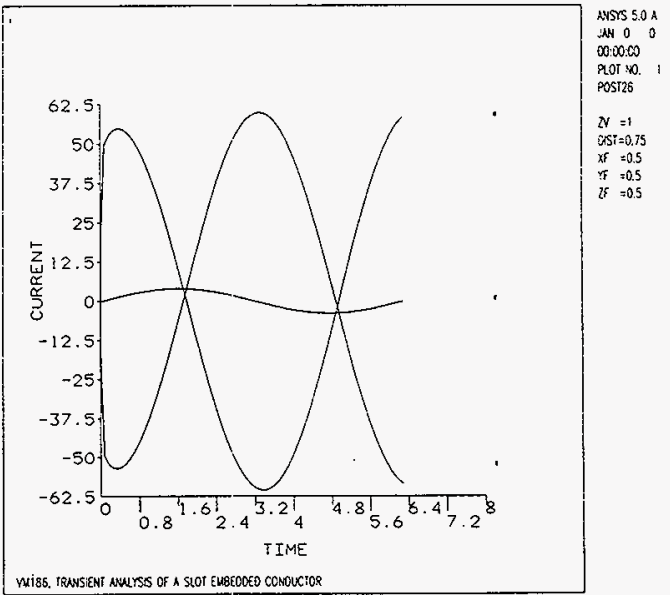

Figure 78

Problem VM186 - Plot 1 


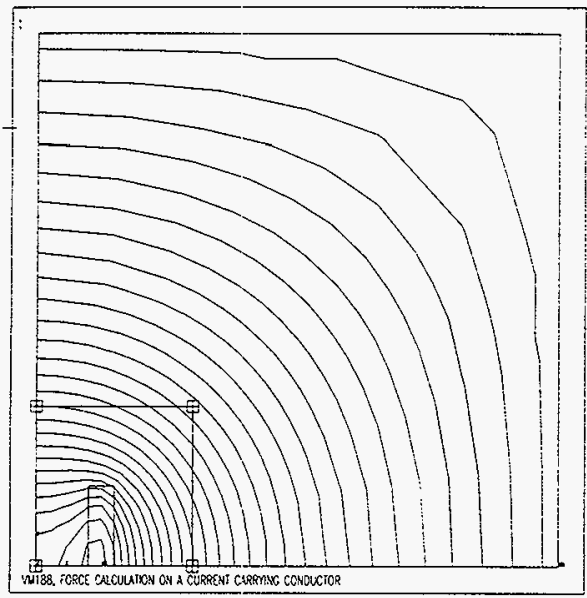

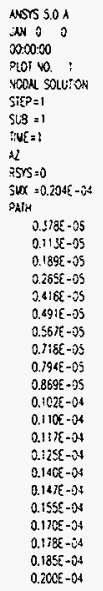

Figure 79

Problem VM188 - Plot 1 